\title{
27th International Congress of the European Association for Endoscopic Surgery (EAES) Sevilla, Spain, 12-15 June 2019
}

\section{KARL STORZ: EAES AWARD SESSION}

\section{O001-COLORECTAL-Malignant}

RANDOMISED CLINICAL TRIAL OF SELECTIVE DECONTAMINATION OF THE DIGESTIVE TRACT IN ELECTIVE COLORECTAL CANCER SURGERY (THE SELECT TRIAL)

G.S. Abis ${ }^{1}$, H.B.A.C. Stockmann ${ }^{2}$, H.J. Bonjer ${ }^{1}$, N. van Veenendaal ${ }^{1}$, M.L.M. van Doorn-Schepens ${ }^{3}$, A.E. Budding ${ }^{3}$, J.A. Wilschut ${ }^{4}$, M. van Egmond ${ }^{1}$, S.J. Oosterling ${ }^{2}$

${ }^{1}$ Surgery, VUMC, AMSTERDAM, The Netherlands; ${ }^{2}$ Surgery, Spaarne Gasthuis, HAARLEM, The Netherlands; ${ }^{3}$ Microbiology, VUMC, AMSTERDAM, The Netherlands; ${ }^{4}$ Statistics, VUMC, AMSTERDAM, The Netherlands

Aims: Infectious complications and anastomotic leakage affect approximately $30 \%$ of patients after colorectal cancer surgery. The aim of this multicenter randomized trial was to investigate whether selective decontamination of the digestive tract (SDD) reduces these complications of elective colorectal cancer surgery.

Methods: The effectiveness of SDD was evaluated in a multicenter, openlabel, randomised clinical trial in 6 centres in The Netherlands. Patients with colorectal cancer scheduled for elective curative surgery with a primary anastomosis were eligible.

Oral colistin, tobramycin, and amphotericin B were administered to the SDD group to decontaminate the digestive tract. Both groups received intravenous cefazoline and metronidazole for peri-operative prophylaxis. Mechanical bowel preparation was given for left sided colectomies, sigmoid and anterior resections. Anastomotic leakage was the primary outcome while infectious complications and mortality were secondary outcomes. This trial was registered with ClinicalTrials.gov number NCT01740947.

Results: In total, 228 patients were randomized to the SDD group and 227 to the control group until the trial was stopped after interim-analysis demonstrated that superiority was no longer attainable. Effective SDD was confirmed by interspace DNA profiling analysis of rectal swabs. Anastomotic leakage was observed in 14 patients $(6.1 \%)$ in the SDD group and in 22 patients $(9.6 \%)$ in the control group (odds ratio) [OR $0.61(0.30-1.22)]$. In the SDD group, fewer patients had one or more infectious complications than in the control group $(14.9 \%(\mathrm{n}=34)$ versus $26.9 \%(\mathrm{n}=61)$, [OR $0.48(0.30-0.76)]$. On multivariable analysis, SDD reduced infectious complications OR 0.472 (0.294-0.755).

Conclusion: SDD reduces infectious complications after colorectal cancer resection but did not significantly reduce anastomotic leakage in this trial.

\section{O002-COLORECTAL-Malignant \\ INTRACORPOREAL VERSUS EXTRACORPOREAL ANASTOMOSIS DURING LAPAROSCOPIC RIGHT HEMICOLECTOMY. Results FROM RANDOMIZED CONTROLLED TRIAL}

A. Rabal Fueyo ${ }^{1}$, J. Bollo Rodriguez ${ }^{1}$, C. Martinez Sánchez ${ }^{2}$, M. Solans Solerdelcoll ${ }^{1}$, N. de la Fuente ${ }^{1}$, D. Sacoto ${ }^{1}$, E.M. Targarona Soler ${ }^{1}$

${ }^{1}$ General Surgery, Hospital de la Santa Creu i Sant Pau, BARCELONA, Spain; ${ }^{2}$ Colorectal Surgery, Hospital de la Santa Creu i Sant Pau, BARCELONA, Spain

Aims: There are several studies that demonstrate the superiority of the intracorporeal (IA) vs extracorporeal (EA) anastomosis. But most reports are nonrandomized, retrospective, and carried out in heterogeneous groups of patients, which might induce patient selection bias.

Methods: We present the first randomized controlled trial, designed to evaluate the two interventions with thorough measurements of the postoperative variables and complications to improve the evaluation of the surgical technique. The primary endpoint is to compare the length of hospital stay. The secondary endpoints were the comparison of intraoperative technical and postoperative clinical events. We included patients aged $=18$ years old referred only for right colon cancer and requiring an elective laparoscopic right hemicolectomy.

Results: 140 patients were randomized. The characteristics of the patients were equivalent between groups. Surgical time was longer in IA vs EA (149 \pm 27 vs $123 \pm 36 \mathrm{~min})$. The length of resected colon was longer in IA vs EA $(25.2 \pm 5.7$ vs $22.6 \pm 7.8 \mathrm{~cm})$ with similar number of lymph nodes $(19.6 \pm 6$ vs $19.1 \pm 7)$. The length of wound was shorter in IA $(6.7 \pm 1.2 \mathrm{vs} 8.7 \pm 1.4 \mathrm{~cm})$. The postoperative analgesia was lower in IA ( $39 \pm 24.3 \mathrm{vs.} 53 \mathrm{vs.} 26)$, and the pain score was lower according to the EVA scale in group IA ( $1.8 \pm 1.8$ vs $2.9 \pm 2.2)$. The recovery of digestive functionality was earlier in IA ( 2.3 vs 3.3 days) with lower incidence of paralytic ileus (13\% vs $30 \%)$. Postoperative complications according to Clavien Dindo classification were lower in IA: grade I (10\% vs $27 \%$ ); grade II ( $18 \%$ vs $35 \%$ ); grade III (1.4\% vs. $7.2 \%)$.

Incidence of anastomotic leak was lower in IA (4.3\% vs. $7.14 \%$ ) with similar wound infection rates $(4.3 \%$ vs. $4.2 \%)$. Hospital stay was similar $(5.65 \pm 3.7$ vs $6.58 \pm 4.6$ days $)$.

Conclusions: IA in the laparoscopic right hemicolectomy is a surgical option that require a longer surgical time, but which provides a surgical specimen comparable to the extracorporeal anastomosis. IA is associated with lower perception of pain and analgesic requirements. IA is superior in terms of the earliest digestive functional recovery, with a lower morbidity. All these clinical advantages would lead to an earlier recovery. 


\section{O003-HEPATO-BILIAIRY \& PANCREAS-Gallbladder}

THE USE OF INDOCYANINE GREEN(ICG) FOR IMAGEGUIDED LAPAROSCOPIC CHOLECYSTECTOMY. COMPARISON OF TWO Methods OF ICG CHOLANGIOGRAPHY

S. Symeonidis, S. Mpitsianis, L.L. Loutzidou, K. Galanos-Demiris, M.G. Pramateutakis, E.. Kotidis, N. Antoniou, O.I. Ioannidis,

I. Mantzoros, S. Aggelopoulos, K. Tsalis

4th Department of General Surgery, General Hospital

"G. Papanikolaou", THESSALONIKI, Greece

Laparoscopic cholecystectomy is one of the most commonly performed operations worldwide. Bile duct injury (BDI) is a rare but very serious complication of the procedure, with a significant impact on quality of life and overall survival. The high frequency of BDI with laparoscopic cholecystectomy was first considered to be a consequence of the initial learning curve of the surgeon, but it later became clear that the primary cause of BDI is misinterpretation of biliary anatomy. Intraoperative cholangiography (IOC) has been advised by many authors as the technique reduces the risk of BDI. However, the procedure has inherent limitations and is therefore reserved for select cases. Fluorescent cholangiography using indocyanine green(ICG) is a novel approach, which offers real-time intraoperative imaging of the biliary anatomy. A comparative study was contacted by administering ICG intravenously or intrabiliary during the operation.

Forty patients scheduled to undergo an elective lap. cholecystectomy were randomly divided in two groups:

In Group A ICG was administered in a dose $2.5 \mathrm{mg}$ in $2 \mathrm{~mL}$ solution intravenously 1 hour before surgery.

In Group B ICG was injected intrabiliary in a $0.025 \mathrm{mg} / \mathrm{mL}$ solution mixed with the patient's bile.

Also, we observed and analysed the following parameters, liver function, B.M.I, ASA score and possible complications, before and after operation. Results: Group A. Intravenous ICG was administered in 20 patients. There was no any reaction and the extrahepatic biliary anatomy was identified well. There was no BDI or any complication related to the procedure.

Group B. ICG was injected intrabiliary in 20 patients during the laparoscopic procedure. In all but one patient the extrahepatic biliary tree was delineated very well. In one patient part of ICG solution was injected into the gallbladder wall and this resulted in a partially confusing image. There was no BDI and no postoperative complication

Conclusions: Fluorescence cholangiography can be used during laparoscopic cholecystectomy to obtain fluorescence images of the bile ducts following intrabiliary injection during the operation orintravenous injection $1 \mathrm{~h}$ before the procedure. The later technique is more easy to perform and does not require catheterization of the biliary tree.

\section{O004-HEPATO-BILIAIRY \& PANCREAS—Gallbladder}

FORMALIZING VIDEO EVALUATION OF THE CRITICAL VIEW OF SAFETY IN LAPAROSCOPIC CHOLECYSTECTOMY: A STEP TOWARDS ARTIFICIAL INTELLIGENCE ASSISTANCE

P. Mascagni ${ }^{1}$, C. Fiorillo ${ }^{1}$, T. Urade ${ }^{2}$, T. Emre ${ }^{3}$, T. $\mathrm{Yu}^{3}$, T. Wakabayashi ${ }^{4}$, E. Felli ${ }^{5}$, S. Perretta ${ }^{6}$, L. Swanstrom ${ }^{2}$, D. Mutter ${ }^{5}$, J. Marescaux ${ }^{4}$, P. Pessaux ${ }^{5}$, G. Costamagna ${ }^{1}$, N. Padoy ${ }^{3}$,

B. Dallemagne ${ }^{4}$

${ }^{1}$ Endoscopia Digestiva Chirurgica, Policlinico Universitario "A. Gemelli", ROME, Italy; ${ }^{2}$ IHU, STRASBOURG, France; ${ }^{3}$ Camma Group, ICube, University of Strasbourg, CNRS, IHU Strasbourg, STRASBOURG, France; ${ }^{4}$ IRCAD, STRASBOURG, France; ${ }^{5}$ Digestive and Endocrine Surgery, Nouvel Hopital Civil, University of Strasbourg, STRASBOURG, France;

${ }^{6}$ Digestive and Endocrine Surgery, IHU-Strasbourg, STRASBOURG, France

Aim: Surgical societies are united in promoting the Critical View of Safety(CVS) during laparoscopic cholecystectomy(LC). Nonetheless, reports have shown a discrepancy between the operative reports and the correct application of CVS, which may explain the stability of bile duct injury rates. Therefore, surgeons and computer scientists at our institution are developing a machine-learning algorithm to automatize CVS assessment. However, the lack of a consistent CVS video assessment framework limits the ability to generate data to train the artificial intelligence. Here we describe and test a method for CVS evaluation in videos.

Method: Between March and July 2016, 100 consecutive videos of LC performed at Nouvel Hospital Civil(Strasbourg, France) were recorded. Two independent reviewers assessed the achievement of CVS in the $60 \mathrm{~s}$ video sequences preceding clipping of cystic duct and artery. In addition to the 'Doublet View' method, a 'Binary' video evaluation method was tested: each of the 3 criteria composing the CVS 2 structures entering the gallbladder, clearance of the hepatocystic triangle and lower part of the cystic plate) was classified as achieved or not. If the 3 criteria were met, then the CVS was considered achieved. Inter-rater agreement for CVS and for each of the 3 criteria was evaluated.

Results: Twenty-two videos( 12 fundus first and 5 partial LC, and 5 broken videos) were excluded from the CVS analysis. CVS elements were assessable in all but one $60 \mathrm{~s}$ videos sequences $(98.72 \%)$. After mediation, CVS was achieved in 32/78(41.03\%) of LC. The cystic plate was identified in only $52.56 \%$ of videos. Inter-rater agreement using the Doublet View vs. the Binary method was as follows: $83.33 \%(?=0.54)$ vs. $88.46 \%(?=0.75) \quad$ for $\quad$ CVS achievement, $66.66 \%(?=0.48) \quad$ vs. $93.59 \%(?=0.79) \quad$ for the 2 structures, $65.38 \%(?=0.45) \quad$ vs. $82.05 \%(?=0.62)$ for the hepatocystic triangle and $61.53 \%(?=0.36)$ vs. $88.46 \%(?=0.77)$ for the cystic plate (Fig. 1$)$.

Conclusions: Reliable CVS assessment is crucial to generate consistent data for machine-learning algorithms aiming at decreasing bile duct injury after cholecystectomy. Our binary CVS video assessment method showed higher inter-rater reliability than the Doublet View, originally described for assessment of photos. Further studies are on going to validate the CVS assessment in videos and support our initial results. 


\section{O005-HEPATO-BILIAIRY \& PANCREAS-Liver}

\section{SURGICAL PROCESS MODELING STRATEGIES: MODELING OF MINIMALLY INVASIVE LIVER TREATMENT}

\section{Gholinejad, A.J. Loeve, J. Dankelman}

Department of Biomechanical Engineering, Delft University of Technology, DELFT, The Netherlands

The vital role of surgeries in healthcare requires a constant attention for improvement. Surgical process modeling is an innovative and rather recently introduced approach for tackling the issues in nowadays complex surgeries, involving complex logistics, much technology, and large teams. Surgical process modeling allows for evaluating the introduction of new technologies and tools prior to the actual development and is beneficial in optimization of the treatment planning and treatment performance in operating room. In this study, we first discuss the concepts associated with surgical process modeling, aiming to clarify them and to promote their use in future studies. Next, we apply these concepts to analyze the procedure of challenging interventions, minimally invasive liver treatment (MILT) methods, with the ultimate goal of improving and optimizing the treatment procedure. The procedure model of current treatment activities and planning of various MILT methods and the associated techniques, are analyzed and combined into a generic procedure model of MILT, which provides a firm foundation for qualitative and quantitative analysis of different MILT procedures. The generic procedure model is validated by data from Erasmus Medical Center (Rotterdam, The Netherlands) and Oslo University Hospital (Oslo, Norway). The proposed procedure model is designed to be a basis for improvement of the procedure and to determine how and where the new technologies can be best, effectively and efficiently, employed in the clinical practices prior to and/or during actual development of the new technologies for MILT. As a conclusion, the current work illuminates the importance of surgical process modeling for improving different aspects of treatment procedures and provides an overview of various modeling strategies that can be used to establish surgical process models. The generic procedure model of various MILT methods, including laparoscopic liver resection, laparoscopic liver ablation and percutaneous ablation, is introduced and validated which is a basis for introduction of the optimized procedure model of MILT methods in the clinical practice.\&\#13Funding: This work is part of the HiPerNav project that received funding from the European Union's Horizon 2020 Research and Innovation program under grant agreement No 722068.

\section{O006-HEPATO-BILIAIRY \& PANCREAS-Liver}

OPTIMAL TIMING FOR Introduction OF TOTAL LAPAROSCOPIC LIVING DONOR RIGHT HEPATECTOMY BASED ON THE EXPERIENCE WITH LAPAROSCOPIC HEPATECTOMY

\section{B.R. Lee, Y.R. Choi, General surgery}

Department of Surgery, Seoul National University Bundang Hospital, GYEONGGI-DO, Korea

Objective: To determine the most appropriate time to start total laparoscopic living donor right hepatectomy (TLDRH) based on the experience with laparoscopic liver resection (LLR).

Summary Background Data Accumulation of experience in LLR is essential before starting TLDRH to ensure donor safety.

Methods: We retrospectively reviewed data of 567 and 78 consecutive patients who underwent LLR and donor hepatectomy, respectively, between 2003 and 2017. Operative outcomes of laparoscopic major hepatectomy (LMH) were compared between two periods based on TLDRH introduction (Phase I 2003-2009 vs Phase II 2010-2017). Learning curve of LLR was evaluated using the cumulative sum (CUSUM) method to determine the optimal time of TLDRH introduction.

Results: A total of 132 LMHs (Phase I: 38 cases, Phase II: 94 cases) and 38 TLDRHs were performed. In LMH cases, hospital stay $(12.63 \pm 6.75: 9.61 \pm 8.20$ days, $\mathrm{P}=0.009)$ was significantly shortened, and EBL $(1122.89 \pm 1460.20: 931.88 \pm 1855.85 \mathrm{ml}, \mathrm{P}=0.024)$ was significantly decreased in Phase II. Although TLDRH was introduced after performing $38 \mathrm{LMHs}$, LMH learning curve was achieved after 73 cases in CUSUM analysis. However, when 73 LMH cases were performed, 15 TLDRH cases were already performed in our center. When comparing the operative outcomes before and after 15 TLDRH cases, operative time (min, $578.1 \pm 110.65$ vs. $422.3 \pm 230.6, \mathrm{P}=0.024$ ), hospital stay (days, $10.46 \pm 3.45$ vs. $9.09 \pm 4.63, \mathrm{P}=0.23)$, and $\mathrm{EBL}(\mathrm{ml}, 769.23 \pm 523.02$ vs $423.23 \pm 323.38, \mathrm{P}=0.026$ ) were significantly different.

Conclusion: Accumulating an experience of at least $73 \mathrm{LMH}$ cases is needed in low-volume LT centers before starting TLDRH to ensure donor safety. 


\section{O007-HEPATO-BILIAIRY \& PANCREAS-Pancreas}

SPLENIC PRESERVATION VERSUS SPLENECTOMY IN LAPAROSCOPIC DISTAL PANCREATECTOMY: A PROPENSITY SCORE MATCHED STUDY

A.L. Moekotte ${ }^{1}$, S. Lof ${ }^{1}$, S.A. White ${ }^{2}$, R. Marudanayagam ${ }^{3}$, B. al-Sarireh ${ }^{4}$, Z. Rahman ${ }^{5}$, Z. Soonawalla ${ }^{6}$, M. Deakin ${ }^{7}$, S. Aroori ${ }^{8}$, B. Ammori ${ }^{9}$, D. Gomez ${ }^{10}$, G. Marangoni ${ }^{11}$, M. Abu Hilal ${ }^{1}$

${ }^{1}$ HPB Surgery, University Hospital Southampton NHS Foundation Trust, SOUTHAMPTON, United Kingdom; ${ }^{2}$ HPB Surgery, Newcastle Upon Tyne Hospitals NHS Foundation Trust, NEWCASTLE, United Kingdom; ${ }^{3}$ HPB Surgery, University Hospitals Birmingham NHS Foundation Trust, BIRMINGHAM, United Kingdom; ${ }^{4}$ HPB Surgery, Morriston Hospital, SWANSEA, United Kingdom; ${ }^{5}$ HPB Surgery, Royal Free London NHS Foundation Trust, LONDON, United Kingdom; ${ }^{6}$ HPB Surgery, Oxford University Hospitals NHS Foundation Trust, OXFORD, United Kingdom; ${ }^{7} \mathrm{HPB}$ Surgery, Royal Stoke University Hospital, STOKE, United Kingdom; ${ }^{8}$ HPB Surgery, Plymouth Hospitals NHS Trust, PLYMOUTH, United Kingdom; ${ }^{9}$ HPB Surgery, Manchester University NHS Foundation Trust, MANCHESTER, United Kingdom; ${ }^{10}$ HPB Surgery, Nottingham University Hospitals NHS Trust, NOTTINGHAM, United Kingdom;

${ }^{11}$ HPB Surgery, University Hospitals Coventry and Warwickshire NHS Trust, COVENTRY, United Kingdom

Aims: The laparoscopic approach in distal pancreatectomy is associated with higher rates of splenic preservation compared to open surgery. Although favorable postoperative short-term outcomes have been reported in open spleen-preserving distal pancreatectomy when compared to distal pancreatectomy with splenectomy, it is unclear whether this observation applies to the laparoscopic approach. The aim of this study is to compare laparoscopic spleen preserving distal pancreatectomy (LSPDP) with laparoscopic distal pancreatectomy with splenectomy (LDPS), using propensity score matching.

Methods: This is a UK wide, propensity score matched study, including patients who underwent LSPDP or LDPS between 2006 and 2016. Short-term outcomes were compared between LSPDP and LDPS according to intention to treat. Additionally, risk factors for unplanned splenectomy were explored. Results: A total of 456 patients were included from eleven centers (229 LSPDP and 227 LDPS). The mean age of the cohort was $56 \pm 16$ years old and $293(64 \%)$ were female. The most common histopathologic diagnoses were neuroendocrine tumor (NET), Mucinous Cystic Neoplasm (MCN) and Intraductal Papillary Mucinous Neoplasm (IPMN). Splenic preservation was achieved in $184(80 \%)$ of the attempted LSPDP. We were able to match 173 LSPDP cases to 173 LDPS cases. After matching, the groups were well balanced in terms of tumor size, age and sex. No differences were seen in postoperative morbidity between the groups. The only identified risk factor for unplanned splenectomy was tumor size $=30 \mathrm{~mm}$.

Conclusions: A high splenic preservation rate was achieved with tumor size as a risk factor for unplanned splenectomy. Preserving the spleen during laparoscopic distal pancreatectomy is not associated with a lower postoperative morbidity compared to sacrifising the spleen. However, taking in consideration the long-term risks of post-splenectomy patients, the authors believe splenic preservation should be attempted in laparoscopic distal pancreatectomy for benign or low-grade malignant lesions as this study shows the approach is safe and feasible.

\section{GERHARD BUESS EAES TECHNOLOGY AWARD SESSION}

\author{
O008-ROBOTICS \& NEW TECHNIQUES-Basic \\ and Technical Research
}

AUGMENTED HYPERSPECTRAL IMAGING-THE

VISUALIZATION OF SMALL INTESTINAL ISCHEMIA AND ITS EVALUATION COMPARED TO INDOCYANIN GREEN-BASED EVALUATION

A. Studier-Fischer ${ }^{1}$, K.F. Kowalewski ${ }^{1}$, F.M. Schwab ${ }^{1}$, C. Haney $^{1}$, I. Gockel ${ }^{2}$, B.P. Müller-Stich ${ }^{1}$, F. Nickel ${ }^{1}$

${ }^{1}$ Department of General, Visceral and Transplantation Surgery, University Hospital of Heidelberg, HEIDELBERG, Germany; ${ }^{2}$ Department of Visceral, Transplantation, Thoracic and Vascular Surgery, University Hospital of Leipzig, LEIPZIG, Germany

Aims: The intraoperative real-time evaluation of small intestinal perfusion is essential for proper resection, but also for sparing of healthy tissue and for anastomotic integrity. The aim of this study was the visualisation of small intestinal ischemia with Hyperspectral Imaging (HSI) and the comparison to conventional indocyanin green-based (ICG) evaluation in terms of sensitivity.

Methods: The HSI camera records a 3 dimensional data cube from a 2 dimensional surgical situs obtaining wavelengths between 500 and $1000 \mathrm{~nm}$. The absorption at different wavelengths is tissue-specific and influenced by the amount of oxygenated haemoglobin and other pigments. A software calculates 4 different indices in real-time including an oxygenbased window. Artificial small intestinal ischemia was induced by a mesotomy of $15 \mathrm{~cm}$ in a porcine model. The intestinal site was recorded prior to and $2 \mathrm{~min}$ after ICG application $(5 \mathrm{mg})$ with the HSI camera and the conventional ICG camera for comparison. ICG emits wavelengths of $810 \mathrm{~nm}$ that enhance the signal in the oxygen-based window of HSI.

Results: In preliminary results $(n=10)$ with 3 levels of criticality of which visual evaluation formed the middle perfusion margin (visually evaluated perfusion margin: VEPM) the conventional ICG was the least critical modality showing ICG signal $1.9 \mathrm{~cm} 95 \%$ CI $[1.6 \mathrm{~cm}, 2.2 \mathrm{~cm}]$ away from VEPM towards mesotomic parts. HSI prior to ICG application was similar to VEPM. During augmented HSI (HSI after ICG application), sufficiently perfused parts became enhanced, demasking margin areas next to the mesotomy that would potentially lead to later necrosis and anastomotic insufficiency (HSI without ICG: $55.4 \%$ 95\% CI [51.1\%, 59.7\%], HSI with ICG: $79.2 \%$ 95\% CI [75.1\%, 83.3\%] $(\mathrm{p}<0.001)$. Augmented HSI depicted a perfusion margin with a distance of $-2.2 \mathrm{~cm} 95 \% \mathrm{CI}$ $[-1.8 \mathrm{~cm},-2.6 \mathrm{~cm}]$ away from VEPM.

Conclusion: HSI is a promising method for intraoperative identification of small intestinal ischemia. Conventional ICG is inclined to an overestimation of perfusion, but new approaches such as time-to-peak analyses are promising. When HSI is combined with ICG, sufficiently perfused intestinal areas are further enhanced, demasking critical margins that could otherwise lead to necrosis or anastomotic insufficiency. These preliminary findings need further validation. 
O009-ROBOTICS \& NEW TECHNIQUES-Basic and Technical research

\section{REAL-TIME, NEAR INFRARED FLUORESCENCE GUIDED IDENTIFICATION OF URETERS WITH NEW MATERIAL URETERAL CATHETER}

Y. Ushimaru $^{1}$, S. Katsuyama ${ }^{1}$, A. Oigawa ${ }^{2}$, K. Tanaka ${ }^{3}$, Y. Miyazaki $^{3}$, T. Makino ${ }^{3}$, T. Takahashi ${ }^{3}$, Y. Kurokawa ${ }^{3}$, M. Yamasaki ${ }^{3}$, M. Mori ${ }^{3}$, Y. Doki ${ }^{3}$, K. Nakajima ${ }^{3}$

${ }^{1}$ Department of Next Generation Endoscopic Intervention, Osaka University, Suita, OSAKA, Japan; ${ }^{2}$ R\&D center, Cardinal Health, FUKUROI CITY, Japan; ${ }^{3}$ Department of Gastroenterological Surgery, Osaka University, Suita, OSAKA, Japan

Background: Although ureteral catheters and ureteral fluorescence methods have been examined for the purpose of avoiding ureteral injury, they have not yet been standardized from the viewpoint of the complexity of the procedure and safety to living bodies. We jointly developed a nearinfrared (NIR) fluorescent ureteral catheter made of fluorescent resin for non-invasive detection of the ureters. The aims of this study were (1) to evaluate its bench-top performance, and (2) to assert its safety and potential usefulness in a series of animal models.

Methods: [Bench-top study] We verified whether the NIR fluorescent catheter was actually stimulated by NIR with the use of a commercially available laparoscopic fluorescence imaging system. In addition, the influence of the imaging distance and the shielding object, such as $1.5 \mathrm{~mm}$ sliced pig loin with multiple sheets, was evaluated. [Performance study] The fluorescent ureter catheter with fixed specification was then evaluated on 5 pigs, to validate its safety and potential usefulness. Non-fluorescent, fluorescent ureteral catheters were placed alternatively in the left and right ureters. Image $\mathbf{J}$ software was used to quantify fluorescence signals and signal-to-background ratio (SBR) for the intraoperative images.

Results: [Bench-top study] A fluorescent ureteral catheter was successfully identified at all distances. The fluorescent catheter decreased in fluorescence in inverse proportion to the distance, and the fluorescence decreased in inverse proportion to the thickness of the intervening shield (two-way ANOVA, $\mathrm{p}<0.01$ ). In situations where shields were present, catheter positions could not be recognized with non-fluorescent catheters, but the fluorescent catheters could be still recognized.[Performance study] It was confirmed that the fluorescent catheter fluoresces at all distances $(p<0.01)$. There was no individual difference $(p=0.21)$, there was no left / right difference in the ureter $(\mathrm{p}=0.79)$. The fluorescence of the fluorescent catheter decreased in inverse proportion to the distance $(\mathrm{p}<0.01)$.

Conclusion: This new fluorescent ureter catheter showed promising performance in providing ureteral identification with high specificity during laparoscopic surgery. Real time, sensitive visualization, and absence of invasive ureteral instrumentation inherent to this technique may reduce complications related to variety of pelvic surgery.

\section{O010—Robotics \& New TECHNIQUES—Basic and Technical} research

\section{THE USE OF A BLACK BOX IN THE OPERATING ROOM: LESSONS LEARNED OBSERVING SAFETY THREATS AND RESILIENCY}

A.S.H.M. van Dalen ${ }^{1}$, J. Jung ${ }^{2}$, E.J.M. Nieveen van Dijkum ${ }^{3}$, C.J. Buskens $^{1}$, W.A. Bemelman ${ }^{1}$, T.P. Grantcharov ${ }^{2}$, M.P. Schijven ${ }^{1}$

${ }^{1}$ Department of Surgery, Amsterdam Gastroenterology and Metabolism, Amsterdam UMC, University of Amsterdam, AMSTERDAM, The Netherlands; ${ }^{2}$ Department of Surgery, International Centre for Surgical Safety, St Michael's Hospital, University of T, TORONTO, Canada; ${ }^{3}$ Department of Surgery, Amsterdam UMC, University of Amsterdam, AMSTERDAM, The Netherlands

Introduction: The number of surgical adverse events is still too high. An important number of these adverse events occur within the operating room $(\mathrm{OR})$ and are in fact preventable. In order to reduce adverse events in the OR, we simply need to know what went well and what can be done better. The aim of this study was to analyze and debrief a predefined selection of surgical procedures, with the use of an operating room 'Black Box', to identify commonly observed safety threats and resilience support events. Methods: In the period 2017-2018, 35 predefined gastro-intestinal laparoscopic cases were recorded by the OR Black Box'. The postoperative Surgical Team Assessment Record (STAR) questionnaire was used. The recordings were analyzed by specifically trained raters, using the Systems Engineering Initiative for Patient Safety (SEIPS) model of work system and patient safety to identify relevant safety threat and resilience support events. Qualitative data analysis was used to identify the most commonly discussed events during the team debriefings.

Results: In only $26.5 \%(n=65)$ of times OR team members, when asked direct following surgery, indicated that they had noticed aberrations $(\mathrm{N}=234)$ during the case. A mean number of 52.5 (SD 15.0) relevant positive and negative events (e.i. aberrations) per surgical procedure were identified using the Black Box performance report. On average, 11.5 (SD 4.2) of events identified by the Black Box were rated as safety threats. Most events discussed during the team debriefings were related to communication.

Conclusion: These results once again highlighting the importance of clear and closed-loop communication in the operating room. Theatre staff underestimated the number of aberrations occurring in the OR, when asked to retrieve from memory. Postoperative structured team debriefing may be important for resolving incorrect assumptions between operating team members to avoid future unnecessary miscommunication. 


\section{O011-ROBOTICS \& NEW TECHNIQUES-Basic} and Technical research

\section{COGNITIVE CAMERA CONTROL FOR LAPAROSCOPIC SURGERY}

M. Wagner ${ }^{1}$, A. Bihlmaier ${ }^{2}$, H.G. Kenngott ${ }^{1}$, P. Mietkowski ${ }^{1}$ S. Bodenstedt $^{3}$, N. Nickel ${ }^{1}$, S. Speidel ${ }^{3}$, H. Woern ${ }^{2}$, T. Kroeger ${ }^{2}$, B.P. Müller-Stich ${ }^{1}$

${ }^{1}$ General, Visceral and Transplant Surgery, Heidelberg University Hospital, HEIDELBERG, Germany; ${ }^{2}$ Institute for Anthropomatics and Robotics, Karlsruhe Institute of Technology, KARLSRUHE, Germany; ${ }^{3}$ Translational Surgical Oncology, National Center for Tumour Diseases, DRESDEN, Germany

Aims: stablished surgical robots for laparoscopy, such as the DaVinci ${ }^{\circledR}$ or the Senhance ${ }^{\mathrm{TM}}$, are telemanipulators without any autonomous activities. Autonomous robots have been developed for laparoscopic camera guidance, but they follow simple rules such as keeping the instruments in the middle of the view. They do not adapt their behavior to different tasks, procedures or surgeons. We developed a robot for cognitive camera control that learns from experience, improves over time and adapts to the surgeons needs.

Methods: To enable robotic learning, a cognitive model was realized: the robot perceives its environment, interprets it according to a knowledge base and performs a context-aware action. By adding experience from robotic surgeries to the knowledge base the robot learns. For validation we used a phantom model of laparoscopic rectal resection. Here, $n=20$ operations were performed with human camera guidance. To gain experience from these operations, laparoscopic video as well as trajectories of laparoscopic instruments were recorded. Each video frame was rated for good, neutral and poor camera guidance quality. Afterwards, machine learning (random forest algorithm) was used to train the robot in three consecutive steps. First, we performed surgery with two different robots, Viky ${ }^{\circledR}$ by Trumpf (Viky) and Light Weight Robot 4 by KUKA (LWR), based on the experience from human camera guidance (trials: Viky $\mathrm{n}=5$, LWR1 $n=5$ ). Then, data from Viky was used to train LWR for another trial (LWR2, $\mathrm{n}=5$ ) and finally data from the trials LWR1 and LWR2 was used to train for LWR3 $(n=1)$.

Results: With increasing experience the duration of surgery decreased from $1704 \mathrm{~s} \pm 244 \mathrm{~s}$ for LWR1 to $1406 \mathrm{~s} \pm 112 \mathrm{~s}$ for LWR2 and $1197 \mathrm{~s}$ for LWR3. Rating of camera guidance (good/neutral/poor) improved with 38.6/53.4/7.9\% for LWR1, 49.4/46.3/4.1\% for LWR2 and 56.2/41.0/2.8\% for LWR3.

Conclusions: We developed a robot for cognitive camera control that learned from experience and improved its performance over time. The underlying cognitive model lays the foundation for a new generation of cognitive surgical robots that provide the right assistance at the right time and adapt to a surgeons needs just as a good human assistant.

\section{O012-ROBOTICS \& NEW TECHNIQUES-Education}

VALIDITY AND RELIABILITY EVALUATION OF THE EAES INTRAOPERATIVE ADVERSE EVENTS CLASSIFICATION FOR MINIMAL ACCESS SURGERY

N.J. Curtis $^{1}$, G. Dennison ${ }^{2}$, J.A. Conti ${ }^{3}$, G.B. Hanna ${ }^{1}$, N.K. Francis ${ }^{2}$

${ }^{1}$ Surgery and Cancer, Imperial College London, LONDON, United Kingdom; ${ }^{2}$ General Surgery, Yeovil District Hospital, YEOVIL,

United Kingdom; ${ }^{3}$ Colorectal Surgery, Portsmouth Hospitals NHS Trust, PORTSMOUTH, United Arab Emirates

Background: The EAES has recently published an intraoperative adverse event classification to assist the direct measurement and routine reporting of minimal access surgery interventions. We aimed to explore the clinically validity and reliability of the classification.

Methods: A prospective evaluation utilising case videos and clinical data from a completed multi-centre laparoscopic total mesorectal excision surgery randomised controlled trial was performed (ISRCTN59485808). Enacted adverse events identified with the observational clinical human reliability analysis technique were graded with the EAES classification by two blinded, independent assessors. Test-retest reliability was explored using grades previously applied during the development of the classification with intraclass correlation co-efficients calculated. Clinical validity was assessed using 30-day morbidity events, the Clavien-Dindo classification and the highest EAES grade per case.

Results: 77 laparoscopic cases (419 h of surgery) contained 1393 error events which were all successfully categorised. Excellent inter-rater and test-retest reliability was seen (ICC $0.957,95 \%$ CI $0.952-0.961, \mathrm{p}<0.001$ and ICC $0.893,95 \%$ CI $0.88-0.904, p<0.001$ respectively. $61 \%$ of patients experienced post-operative morbidity (median 1 event, range $0-5)$. Labelling analysed cases by their highest EAES classification grade gave $53 \%$ grade $2,43 \%$ grade 3 and $4 \%$ grade 4 procedures. $51 \%$ of grade 2 cases developed a morbidity event, but this significantly increased in grade 3 and 4 operations ( $70 \%$ and $100 \%, p=0.043)$. The number of complications and highest recorded Clavien-Dindo grade increased with each additional grade $(1.05 \pm 1.3$ vs. $1.48 \pm 1.3$ vs. $2.33 \pm 0.6$, $\mathrm{p}=0.145$ and median 1 vs. 2 vs. $3, p=0.023$ respectively). Anastomotic leak and re-operation were correctly captured by the allocated EAES grade ( $2.5 \%$ vs. $3.3 \%$ vs. $100 \%, \mathrm{p}<0.001$ and $5 \%$ vs. $0 \%$ vs. $66 \%, \mathrm{p}<0.001$ respectively). There was a significant rise in length of stay observed with increasing EAES grade (median 6 vs. 7 vs. 61 days, $\mathrm{p}<0.001$ ).

Conclusion: In the context of major laparoscopic surgery, the EAES intraoperative adverse classification is seen to be a clinically valid and reliable assessment method. 


\section{O013-ROBOTICS \& NEW TECHNIQUES-Education}

USE OF PRE-FRONTAL CORTEX ACTIVITY ESTIMATED BY FUNCTIONAL NEAR INFRARED SPECTROSCOPY, AS A MEASURE OF LEARNING CURVE: Results OF A RCT

S.A. Lui ${ }^{1}$, S. Wijerathne ${ }^{1}$, H. Khoe ${ }^{1}$, J.W. Low ${ }^{1}$, J. Ho $^{2}$, D. Lomanto ${ }^{1}, \mathrm{R} . \mathrm{Ho}^{3}$

${ }^{1}$ Surgery, NUHS, SINGAPORE, Singapore;

${ }^{2}$ Vascular Surgery, NUHS, SINGAPORE, Singapore;

${ }^{3}$ Psychological Medicine, NUHS, SINGAPORE, Singapore

Aims: Neurobiological feedback in surgical training could translate to better educational outcomes such as measures of learning curve. The variation in brain activation of medical students when performing laparoscopic tasks before and after a training workshop is not properly studied before and we planned to do this using functional near infrared spectroscopy (fNIRS) which is a non-invasive optical brain imaging tool that measures cortical oxygenation change which is used as a marker of pre-frontal cortex activity (PFCA).

Methods: This randomised controlled trial examined the PFC activity differences in two groups of novice medical students during the acquisition of 4 basic laparoscopic tasks. 'Trained-group' had standerdised oneto-one training on the tasks, while the 'Untrained-group' had no prior trainining and was just shown a video of the tasks. The PFCA was measured pre and post intervention using a portable fNIRS device. Primary outcome was the difference in the PFCA pre and post intervention. Secondary outcomes were the differences in PFCA between the 4 tasks and between the sexes.

Results: 16 trained and 16 untrained medical students with an equal sex distribution and a comparable age distribution were invovlved in the study. All students were right handed. Trained group had a significantly attenuated PFCA in the 'Precision-cutting' $(p=0.011)$ and 'Suture-insertion' $(p=0.025)$ tasks compared to the untrained group. Subgroup analysis based on sex revealed significant attenuation in PFCA in trained females compared to untrained females across 3 of the 4 laparoscopic tasks: 'Pegstransfer' $(p=0.013)$, 'Precision-cutting' $(p=0.034)$, 'Suture-insertion' $(p=0.03)$. No significant PFCA attenuation was found in male students who underwent training compared to untrained males.

Conclusion: A standardised laparoscopic training workshop promoted greater PFCA attenuation in female medical students compared to males. This suggests that female and male students respond differently to the same instructional approach. These results may have implications for surgical training and education such as a greater focus on one to one surgical training for female students and use of PFCA attenuation as a form of neurobiological feedback as a measure of learning curve in surgical training.
O014-ROBOTICS \& NEW TECHNIQUES-Education

ROBOT ASSISTED VERSUS LAPAROSCOPIC ADVANCED SUTURING LEARNING CURVE

$\underline{\text { E. Leijte }^{1}}$, I. de Blaauw ${ }^{2}$, C. Rosman ${ }^{1}$, S.M.B.I. Botden ${ }^{2}$

${ }^{1}$ Surgery, RadboudUMC, NIJMEGEN, The Netherlands;

${ }^{2}$ Pediatric surgery, RadboudUMC, NIJMEGEN, The Netherlands

Aims: Compared to conventional laparoscopy, robot assisted surgery is expected to have most potential in difficult areas and demanding technical skills as minimally invasive suturing. This study was performed to identify the differences in the learning curves of laparoscopic versus robot assisted advanced suturing

Method: Novice participants, with the knowledge of basic surgical procedures, were recruited and performed three suturing tasks on the EoSim laparoscopic augmented reality simulator or the RobotiX robot assisted virtual reality simulator. Each participant performed an intracorporeal suturing, tilted plane needle transfer and anastomosis needle transfer task. To complete the learning curve, all tasks were repeated for maximal twenty repetitions or until a plateau was reached three consecutive times. Clinical relevant and comparable parameters regarding time (seconds), movements and safety were recorded. Intracorporeal suturing was used to visualize and compare the learning curves between the groups.

Results: Forty-six participants completed the learning curve, of which 16 laparoscopically and 30 robot assisted. When comparing the suture time, the plateau was reached much faster in the robot assisted group (7-9 repetitions) than the laparoscopic group (10-12 repetitions) as shown in Figure 1 . There was a significant difference in 'time per suture', during the whole learning curve with median values of 637 versus 251 (first knot), 450 versus 147 (fifth) and 186 and 115 (eighteenth), all with a $\mathrm{p}<0.05$. However, the parameter 'adequate surgical knot' was reached earlier in the laparoscopic group than in the robot assisted group. First: $69 \%$ versus $60 \%$, fifth: $100 \%$ versus $70 \%$, and eighteenth: $100 \%$ versus $83 \%$. When assessing the 'needle out of view' parameter, the robot assisted group scored a median of 0.3 and $0.0 \mathrm{~s}$ during the first, respectively eighteenth knot, and the laparoscopic participants had their instruments out of view for 41 and $17 \mathrm{~s}$ during the first respectively eighteenth knot.

Conclusion: The learning curve of minimally invasive suturing can be reduced with the use of robot assisted surgery, with a specific reduction in operation time. The rate of adequate knots seemed to remain lower in robot assisted surgery, although this could be due to the virtual reality aspect of the simulator. 


\section{ORAL PRESENTATIONS}

\section{O015-BARIATRICS—Endoluminal}

\section{OBJECTIVE ASSESSMENT OF ENDOSCOPIC SLEEVE GASTROPLASTY APPEARANCE AND ITS RELATION TO WEIGHT LOSS IN A LARGE GROUP OF PATIENTS}

M. Pizzicannella ${ }^{1}$, C. Fiorillo ${ }^{1}$, P. Mascagni ${ }^{1}$, M. Vix ${ }^{2}$, D. Kadoche ${ }^{2}$, D. Mutter ${ }^{2}$, J. Marescaux ${ }^{3}$, S. Perretta ${ }^{1}$

${ }^{1}$ IHU, STRASBOURG, France; ${ }^{2}$ Digestive and Endocrine Surgery, Nouvel Hopital Civil, University of Strasbourg, STRASBOURG, France; ${ }^{3}$ IRCAD, IRCAD Research Institute against Digestive Cancer, STRASBOURG, France

Introduction: Endoscopic sleeve gastroplasty (ESG) is a novel promising bariatric endoscopy treatment. Gastric volume reduction and delayed gastric emptying are the mechanisms driving weight loss. However, little is known about the factors influencing the effectiveness of weight loss overtime. The present study aims at evaluating the correlation between endoscopic suture appearance and excess weight loss (EWL\%) at 6 and 12 months follow up.

Patients and methods: All patients who underwent follow-up endoscopy at 6 and 12 months after ESG were included. ESGs were classified in 3 groups according to endoscopic appearance of the gastric sutures: optimal (group 1) when all stitches were in place and tights; suboptimal (group 2) when one or more stiches were displaced; loose (group 3) when all the sutures were completely disrupted. BMI at enrollment and EWL\% at 6 and 12 months were recorded and compared to the endoscopic appearance.

Results: A total of 53 patients were included in the analysis. At 6 months, $25(47.2 \%)$ patients had an optimal ESG, $24(45.3 \%)$ had a suboptimal sleeve and $4(7.5 \%)$ had complete sutures failure.

BMI at enrollment and EWL\% were respectively $37.7 \pm 4.2$ and $36.6 \pm 21.3 \%$ for group $1,43.6 \pm 6.7$ and $22.77 \pm 18.7 \%$ for group 2 and $50.7 \pm 14.4$ and $7.8 \% \pm 16.5 \%$ for group 3 . Twenty five patients had 12 months EGDS: $5(20 \%)$ presented an intact ESG and were classified in group $1,15(60 \%)$ in group 2 and $5(20 \%)$ in group 3. Twelve months EWL\% was respectively $47.6 \pm 9.1 \%, 31.3 \pm 29.3$ and $12 \pm 14.4 \%$. Initial BMI significantly correlated with suture status at both 6 (rho $-0.528 ; p<0.001$ ) and 12 months (rho $-0.423 ; p=0.035$ ) follow-up. Furthermore, the sutures' appearance itself correlated with EWL\% at both time points (rho +0.416; $\mathrm{p}=0.002$ and rho $0.439 ; \mathrm{p}=0.028$ respectively).

Conclusion: Our preliminary results show that the aspect of the endoscopic suture has a significantly impact on EWL\% at 6 and 12 months after ESG. Furthermore, BMI at enrollment seems to predict endoscopic suture duration overtime. Larger studies and longer follow-up are needed to further validate our preliminary findings.

\section{O016-BARIATRICS—Endoluminal}

THE ROLE OF MULTIDISCIPLINARY APPROACH IN ENDOSCOPIC SLEEVE GASTROPLASTY

I. Boskoski, V. Bove, R. Landi, P. Familiari, A. Tringali, V. Perri, G. Costamagna

Digestive Endoscopy Unit, Fondazione Policlinico A. Gemelli IRCCS, ROME, Italy

Background and aim: Endoscopic sleeve gastroplasty(ESG) is a relatively novel endoscopic procedure that reduces the gastric lumen with proven less complications and less 6 months weight loss compared to laparoscopic sleeve gastroplasty (LSG). At present there are no studies investigating the role of multidisciplinary approach in ESG. The aims of the present study were to evaluate the role of multidisciplinary assessment(MA) prior ESG, weight loss outcomes, quality of live improvements and adverse events.

Material and methods: From May 2016 to May 2018 all patients that underwent ESG were retrospectively evaluated from a prospective database. Until September 2017 before ESG only psychiatric evaluation was requested, while after this date we adopted the guidelines of the Italian Society for Obesity Surgery and all patients were evaluated on a multidisciplinary fashion prior ESG. The multidisciplinary team was composed by:gastroenterologist, surgeon, psychiatrist, endocrinologist and dietitian. Patients were divided in two groups:group 1 were patients with ESG before MA and group 2 were patients with ESG after MA. We compared this two groups in terms of weight loss outcomes, quality of live improvements and adverse events. Quality of live was measured with the Bariatric Analysis and Reporting Outcome System(BAROS).All procedures were done with the Apollo Overstitch suturing system(Apollo Endosurgery) and a double channel gastroscope Olympus 2TGIF160(Olympus Japan).All procedures were done in general anesthesia and with insufflation of $\mathrm{CO} 2$. All patients had ambulatory visit $\mathrm{t} 1,3$ and 6 months after ESG and weight loss outcomes were measured in terms of Excess Weight Loss (\%EWL), the Total Body Weight Loss (\%TBWL) and BAROS scale were assessed. Statistical analysis was done with chi-square test and $<0.05$ value was considered significant.

Results: 31 patients were identified ( 20 female; mean age 45.4 , range 23-73). Mean BMI at inclusion was 41.6(range 31.6-62.4). Mean \%EWL and \%TBWL at 6 months was 37.1 and 16.7 respectively(Table 1).Non procedure related complications were observed.

Comparing the two groups there was significant $(\mathrm{P}<0.05)$ difference in terms of \%EWL and \% TBWL (Table 2), with better results in group 2. There was also a significant improvement in the BAROS scale in the patients in group 2.

Conclusions: MA before ESG has a fundamental role in terms of better procedure outcomes for both weight loss and quality of live in obese patients. 


\section{O017-BARIATRICS—Endoluminal}

STAPLE LINE LEAKS FOLLOWING LAPAROSCOPIC SLEEVE GASTRECTOMY: EFFICACY OF THE OVER THE SCOPE CLIP

I. Mizrahi ${ }^{1}$, R. Grinbaum ${ }^{1}$, R. Elazary ${ }^{1}$, T. Mordechay-Heyn ${ }^{1}$, H. Jacob ${ }^{2}$, J. Epstein ${ }^{2}$, N. Beglaibter ${ }^{1}$

${ }^{1}$ Surgery, Hadassah Medical Center, JERUSALEM, ISRAEL, Israel; ${ }^{2}$ Gastroenterology, Hadassah Medical Center, JERUSALEM, ISRAEL, Israel

Aims: The over-the-scope clip (OVESCO) is a novel endoscopic tool that enables non-surgical management of gastrointestinal defects. The aim of this study was to report our experience with OVESCO for patients with staple line leaks following laparoscopic sleeve gastrectomy (LSG).

Methods: A prospectively maintained IRB-approved institutional database was queried for all patients treated with OVESCO for staple line leaks following LSG from 2010 to 2018. Primary outcome was complete resolution of leak following OVESCO as defined by return to complete oral nutrition and no evidence of leak on imaging. Secondary outcome was the number of additional endoscopic or surgical procedures needed following OVESCO.

Results: Twenty-five patients ( 12 males, 13 females) were treated with OVESCO for staple line leaks following LSG. The median age was 35 years (range 18-62), and mean body mass index was $44 \mathrm{~kg} / \mathrm{m}^{2}$. Nine patients $(35 \%)$ were referred from an outside hospital. The median time from index operation to leak diagnosis and from leak diagnosis to OVESCO was 18 days (range 2-118), and 6 days (range 1-120), respectively. All patients had upper staple-line leaks near the gastroesophageal junction. Initial treatment included antibiotics -6 patients; computed tomography guided drainage and antibiotics -7 patients; and laparoscopic drainage -12 patients. OVESCO led to final resolution of leak in 8 patients (33\%) within 70 days of clip deployment (range 41-136). Leaks which persisted following OVESCO were eventually resolved with a combination of OVESCO and stent -5 patients $(21 \%)$, total gastrectomy and esophago-jejunostomy-10 patients (42\%), and endoscopic suturing - 1 patient $(4 \%)$. One mortality was noted in a patient who suffered multiorgan failure. The number of additional endoscopic sessions ranged from 1 to 10 (median 2). No procedure related complications were noted. All patients were treated with total parenteral nutrition and the total length of stay was 49 days (range 13-127).

Conclusions: Despite its low success rate, OVESCO should be part of the bariatric surgeon's non-surgical armamentarium in treating staple line leaks following LSG.

\section{O019-BARIATRICS—Endoluminal}

EARLY CLINICAL EXPERIENCE WITH PURELY PERCUTANEOUS IMAGE GUIDED CLEARANCE OF CBD STONES IN BARIATRIC PATIENTS

\author{
S. Mafeld ${ }^{1}$, R. Zener ${ }^{2}$, A. Okrainec ${ }^{3}$, E. Shlomovitz ${ }^{1}$
}

${ }^{1}$ Interventional Radiology / General Surgery, University Health Network, TORONTO, Canada; ${ }^{2}$ Interventional Radiology, University Health Network, TORONTO, Canada; ${ }^{3}$ General Surgery, University Health Network, TORONTO, Canada

Introduction: The population of post bariatric surgery patients is rapidly increasing worldwide. Due to the altered anatomy post Roux-en-Y gastric bypass (RYGB), conventional endoscopic management for choledocholithiasis is challenging. These patients are now commonly managed by means of a laparoscopic assisted ERCP. Although effective, this requires significant resource utilization and potential morbidity related to the need for surgical intervention. We present our preliminary experience with a purely percutaneous management of choledocholithiasis in bariatric patients post-RYGB.

Methods: A retrospective single center review identified five patients with choledocholithiasis after bariatric RYGB who underwent percutaneous CBD access and treatment by interventional radiology. Four patients underwent percutaneous transhepatic CBD access while one patient underwent percutaneous trans-cholecystic CBD access. In three of the five patients conscious sedation alone was sufficient to perform the procedure.

Results: All patients had radiologically confirmed choledocholithiasis and were clinically symptomatic prior to intervention. The biliary tree was successfully accessed percutaneously and cleared in all five patients. In the four patients where a percutaneous transhepatic access was utilized, three patients required only fluoroscopic balloon sphincterplasty and sweep of the CBD to clear the ductal stones, while the fourth required percutaneous cholangioscopy assisted lithotripsy for clearance. In the fifth patient with non-dilated intrahepatic bile ducts a trans-cholecystistic approach into the CBD was utilized with percutaneous cholangioscopic assistance to clear the ductal stones. All procedures were completed successfully with no post procedure complications.

Conclusion: Percutaneous clearance of CBD stones in bariatric patients presents a minimally invasive alternative to current surgical practice. The use of conscious sedation and the purely percutaneous approach may potentially reduce morbidity and resource utilization for this increasingly common clinical scenario.

\section{O018-BARIATRICS—Endoluminal}

\section{IS THE POSE AS EFFECTIVE AND RELIABLE AS THE} SURGICAL PROCEDURE?

R. Bademci ${ }^{1}$, R. Vilallonga ${ }^{2}$, P. Alberti $^{2}$, R. Renato ${ }^{2}$, C. Yuhamy ${ }^{2}$, S.S. Cordero $^{2}$, L. Posadas ${ }^{2}$

${ }^{1}$ General surgery, Istanbul medipol üniversitesi, ISTANBUL, Turkey; ${ }^{2}$ Bariatric surgery, Vall d'hebron, BARCELONA, Spain

Background: In cases of morbid obesity, treatment is generally applied as either a surgical or endoscopic approach. The number of Primary Obesity Surgery Endolumenal (POSE) procedures is increasing but the reliability and effectiveness is unclear as yet. The aim of this study was to present a series of cases that required revision surgery due to POSE failure and to reveal possible alternative surgeries.

Materials and methods: A retrospective comparison was made of the data of obese patients with POSE failure and conversion to surgical procedures between 2016 and 2018 in respect of operation, medical illness and BMI results.

Results: The patients comprised $60 \%$ females, $40 \%$ males with a mean age of $44.8 \pm 12.4$ years and mean follow-up period of $12.6 \pm 8.3$ months. On average, patients lost $24.1 \pm 8.9 \mathrm{~kg}$, with an average excess weight loss of $47.6 \%$.

Conclusion: No firm conclusions can be drawn from such a small group. Although SG seems to be a safe procedure and should be considered as the first technique to be applied following POSE failure, it is possible to perform gastric bypass on patients with this endoscopic precursor. 


\section{O020-BARIATRICS—Laparoscopic}

EFFICACY OF LOW OPIOID ANAESTHESIA PROTOCOL IN PATIENTS UNDERGOING LAPAROSCOPIC METABOLIC SURGERY IN A HIGH-VOLUME BARIATRIC CENTRE

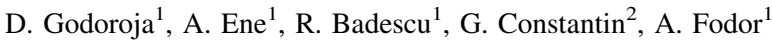

${ }^{1}$ Anaesthesia and Intensive Care, Ponderas Academic Hospital, BUCHAREST, Romania; ${ }^{2}$ Anaesthetist Ponderas Academic Hospital, BUCHAREST, Romania

Background and Aim: In morbidly obese patients, anaesthesiologists are recommended to use an obstructive sleep apnoea safe anaesthetic technique, minimising opioids. The primary goal of the study was to assess the efficacy of using a low opioid protocol for general anaesthesia in bariatric surgery.

Method: A protocol of low opioid general anaesthesia for obese patients undergoing laparoscopic bariatric operations has been introduced in our Center in 2016. The protocol was used by all anaesthetists and was based on using low dose of Fentanyl, adjuvants, and opioid free analgesics (Table 1). No intravenous morphine was administered at the end of the procedure. All patients received a protocolised opioid free multimodal postoperative analgesia and intravenous morphine only if the visual analogue score (VAS) was higher than 5. Cumulative opioid (iv $\mathrm{mg}$ morphine) consumption for $24 \mathrm{~h}$ and pain severity scores at rest and movement were recorded at $1,4,12$, and $24 \mathrm{~h}$ postoperatively.

Results: Between February 2016 and August 2017 in Ponderas Academic Hospital, 1227 patients were operated on for bariatric procedures. The descriptive statistical data showed that $70.18 \%$ were women and $29.82 \%$ men with a mean age of $40.63( \pm 12.03 \mathrm{SD})$ and the median BMI $\left(\mathrm{kg} \mathrm{m}^{-2}\right)$ of 39.7 (IQR-9.30). 247(20.13\%) patients presented severe obstructive sleep apnoea and received CPAP treatment. Out of 1227 patients only $361(29.43 \%)$ received intravenous morphine in the first $24 \mathrm{~h}$ after operation at a VAS higher than 5 . The median of cumulative consumption of morphine at $24 \mathrm{~h}$ in the morphine group was 6.00 (IQR 2.00). The pain scores at rest and with movement during all measured intervals were significantly low.

Women were more susceptible to the pain with increased consumption of morphine. In the CPAP group of patients the morphine used was statistic significantly lower $(\mathrm{p}<0.00001)$. Only $14 \%$ of the patients with severe OSA needed morphine comparing with $33 \%$ of the patients without OSA. Conclusion: The low opioid anesthesia protocol in bariatric patients proved to be efficient and its use should be extended in obese patients undergoing minimal invasive surgery.

\section{O021-BARIATRICS-Laparoscopic}

DOES LAPAROSCOPIC TRANSVERSUS ABDOMINIS PLANE \& RECTUS SHEATH BLOCKS REDUCE POSTOPERATIVE OPIOID USE? A RANDOMIZED CONTROLLED TRIAL

\author{
A. Jarrar ${ }^{1}$, N. Eipe ${ }^{2}$, A. Budiansky ${ }^{2}$, C. Walsh ${ }^{1}$, J. Mamazza $^{1}$ \\ ${ }^{1}$ General Surgery, The Ottawa Hospital, OTTAWA, Canada; \\ ${ }^{2}$ Anesthesia, The Ottawa Hospital, OTTAWA, Canada
}

Aim: The aim of this study is to evaluate the efficacy of a laparoscopically-guided, surgeon- performed transversus abdominis plane (TAP) and rectus sheath (RS) block in reducing pain while improving functional outcomes in patients undergoing bariatric surgery. there are limited evidence-based recommendations and no ideal analgesic regimen exists for patients with Morbid Obesity.

Methods: 150 patients elective laparoscopic Roux-En-Y gastric bypass (LRYGB) have been recruited to this double-blinded, placebo-controlled Randomized Control Trial from a provincial Bariatric Center of Excellence over a period of six months. Patients underwent objective prehabilitation with 6-min walk test (6MWT) and peak expiratory flow (PEF). Patients have been electronically randomized on a 1:1 basis to either an intervention or placebo group. At the end of the surgery, patients in the intervention arm receive a total of $60 \mathrm{~mL} 0.25 \%$ Ropivacaine, divided into four injections: two $20 \mathrm{~mL}$ each for TAP and two $10 \mathrm{~mL}$ each for RS block under laparoscopic visualization. The placebo arm receives normal saline in the same manner. All patients, providers and research personnel were blinded and standardized surgical and anesthetic protocols were followed, with careful adherence to established Enhanced Recovery after Bariatric Surgery (ERABS) protocols.

Results: baseline information for 150 patients is being collected and analyzed, Cumulative narcotic use was collected and all patients underwent PEF and 6MWT testing in addition to assessment of the quality of recovery using validated questionnaire (QOR-40), the data is being analyzed and we will present results through publication.

Conclusion: $30 \%$ reduction in narcotic use is a significant improvement in patient outcomes, this study adds to the body of evidence of best practices in enhancing recovery for bariatric patients. 


\section{O022-BARIATRICS-Laparoscopic}

LONG TERM Results AFTER LAPAROSCOPIC SLEEVE GASTRECTOMY WITH CONCOMITANT POSTERIOR CRUROPLASTY: FIVE-YEAR FOLLOW-UP UPDATE

\author{
C.E. Boru, M.G. Coluzzi, F. de Angelis, G. Silecchia
}

Division of General Surgery \& Bariatric Center of Excellence-IFSO EC, Sapienza University of Rome, Dept. of Medico-Surgical Sciences \& Biotechnologies, LATINA, Italy

Hiatal hernia $(\mathrm{HH})$ repair during laparoscopic sleeve gastrectomy (LSG) has been advocated to reduce the incidence of postoperative gastroesophageal reflux disease (GERD) and/or intrathoracic migration (ITM). The necessity of intraoperative repair in asymptomatic patients is still controversial. Previous, mid-term results of a prospective, comparative study evaluating posterior cruroplasty concomitant with LSG (group A 48 patients with simple vs. group B 48 patients with reinforced with bioabsorbable mesh) confirmed the safety and effectiveness of simultaneous procedures. Present aim was to report the 60 months follow-up update, evaluating GERD and esophageal lesions' incidence and HH's recurrence. Results: Follow-up of was completed in $79.5 \%$ of the patients.Recurrent GERD in $6 / 32(18.8 \%$, group A) and in $9 / 44(20.5 \%$, group B) was registered ( $\mathrm{p}>0.05)$. Grade A esophagitis and GERD was shown in 2 patients $(6.25 \%)$, respectively $2(4.5 \%)$ of each groups $(\mathrm{p}>0.05)$, and recurrent $\mathrm{HH}$ was confirmed later by contrast study and CT scan. Neither Barrett's lesions nor de novo GERD were found. A total of 12 patients $(12.5 \%, 8$ respective 4) were converted within five years for persistent/recurrent GERD, with only 1 case of de novo (group B, shown in the initial 21 months follow-up). Failure of the initial cruroplasty with ITM was recorded in 4 patients (13\% for group A and $7.4 \%$ for group B); hence, a repeat posterior, reinforced cruroplasty with bioabsorbable mesh was performed.

Conclusions: Accurate patient selection (absence of large $\mathrm{HH}$, absence of severe esophagitis, absence of severe long-lasting symptoms), proper sleeve technique (absence of tight sleeve), combined with posterior cruroplasty (simple or reinforced, based on hiatal defect's dimensions and quality of the crura) ensures effectiveness, with a rate of failure (recurrence) at five years of $9.1 \%$. Outcomes of LSG combined with posterior cruroplasty were satisfactory in all patients.

\section{O024-BARIATRICS—Laparoscopic}

\section{LAPAROSCOPIC NARBONA-ARNAU PROCEDURE TO CONTROL THE GERD AFTER LSG-3 YEARS Results OF A PROSPECTIVE STUDY}

\author{
I.C. Hutopila, C. Copaescu
}

General and Bariatric surgery, Ponderas Academic Hospital, BUCHAREST, Romania

Background: After the Laparoscopic Sleeve Gastrectomy (LSG) alone or associated with calibration of the esophageal hiatus, for some patients the reflux symptoms worsen postoperatively due to development of a hiatal hernia $(\mathrm{HH})$ or due to the recurrence of the $\mathrm{HH}$ previously repaired. For these situations, when the conservative treatment fails, are proposed some surgical solutions, one of them Cardiopexy with Teres ligament-Narbona Arnau.

Objective: Is to establish a standardized laparoscopic technique for cardiopexy using the Teres ligament (Narbona Arnau technique) and to analyze the procedure's outcomes.

Methods: The study was performed in a Bariatric and Metabolic Center of Excellence-Ponderas Academic Hospital. All the patients undergoing Narbona Arnau procedure to control GERD after LSG since 2014 were included and prospectively analyzed. The selection criteria included LSG patients, presenting $\mathrm{HH}$ and symptomatic GERD. Preoperative investigations were upper gastrointestinal endoscopy, radiological contrast study, $\mathrm{pH}-$ metry, computed tomography with oral contrast.

Results: 28 patients were included into the study. GERD and HH were preoperatively documented in all the cases. One patient was excluded after 2 years of follow up after being converted to a laparoscopic Roux-en-Y gastric bypass, for intense relux symptoms. No incidents during surgery. For 8 cases laparoscopic Narbona Arnau technique was performed concurrent with re-sleeve gastrectomy and gastric curvature plication. Without postoperative complications. Postoperative follow- up at 6 months, 1, 2 and 3 years, the percentage of patients without GERD symptoms and free of treatment with PPIs was $64,28 \%, 82,14 \%, 71,42 \%$, respectively $66.66 \%$. At 3 years postoperatively the upper GI endoscopy showed remission/ improvement of the degree of esophagitis for 17 patients. For the same period of follow-up, the Ph-metry highlighted a normal value of DeMeester score for $62.96 \%$ o patients (all the patients had preoperatively high De Meester scores). No objective signs of hiatal hernia recurrence at imagistic investigations and upper gastrointestinal endoscopy were encountered.

Conclusions: Complete preoperative evaluation is mandatory for choosing the optimal intervention. Laparoscopic Narbona Arnau technique after LSG is proved to be a good option for the treatment of symptomic GERD, but further studies with high-volume patients are necessary. 


\section{O025-BARIATRICS—Laparoscopic}

RISK FACTORS FOR RECURRENT AND CHRONIC MARGINAL ULCERS REQUIRING SURGICAL TREATMENT FOLLOWING ROUX-EN-Y GASTRIC BYPASS

\section{A. di Palma, A. Maeda, T. Jackson, A. Okrainec}

\section{Surgery, University Health Network, TORONTO, Canada}

Introduction: Marginal ulcer (MU) formation is a known complication following Roux-en-Y gastric bypass (RYGB) for weight loss. Although most respond to medical treatment, many patients have recurrent or chronic MU, with some requiring surgical revision. Although non-steroidal antiinflammatory drug (NSAID) use, smoking and $\mathrm{H}$. pyloriinfection have been proposed as risk factors for MU, little is known about what increases the likelihood of developing recalcitrant ulcers. The goal of this study was to identify potential risk factors for recurrent or chronic MU, including those requiring surgical revision.

Methods: All patients undergoing RYGB between 2011 and 2017 at an academic centre in Toronto, Ontario were included. Patients with a post-operative diagnosis of MU were identified from the institution's bariatric database and had their medical records reviewed. Patient characteristics, operative data and surgical outcomes were collected for statistical analysis.

Results: A total of 2,830 RYGB were performed during the study period. The incidence of MU was $6.9 \%$ with $4.5 \%$ having a single episode, $1.4 \%$ developing recurrent but medically-responsive MU and $1 \%$ undergoing revisional surgery. Patients requiring revision were significantly younger than patients with a single episode of MU or recurrent medically-responsive MU (39.2 vs. 44.5 and 41.8 years, $\mathrm{p}=0.027$ ). This effect disappeared however when a multivariate logistic regression model was applied, identifying previous smoking history (OR 7.98, 95\% CI 2.35-27.09) and therapeutic immunosuppression (OR 11.6, 95\% CI 1.18-114.07) as the primary risk factors for MU requiring surgical revision. NSAID use, H. pyloriinfection and a history of peptic ulcer disease did not significantly predict chronic or recurrent $\mathrm{MU}$ requiring surgical treatment.

Conclusions: Patients with a history of smoking prior to RYGB and patients on immunosuppressive medications were at significantly higher risk of developing MU requiring surgical revision in comparison to patients that responded to medical treatment. Further studies are needed to validate these findings and determine how they can inform peri-operative management of the bariatric patient.

\section{O026-BARIATRICS-Laparoscopic}

\section{BARBED VS CONVENTIONAL SUTURES IN BARIATRIC SURGERY: A PROPENSITY SCORE ANALYSIS FROM A HIGH VOLUME CENTER}

F. Pennestri ${ }^{1}$, F. Prioli ${ }^{1}$, P. Gallucci ${ }^{1}$, P. Giustacchini ${ }^{1}$, L. Ciccoritti ${ }^{1}$, L. Sessa' ${ }^{1}$, P. Aceto $^{2}$, R. Bellantone ${ }^{1}$, M. Raffaelli ${ }^{1}$

${ }^{1}$ U.O.C. Chirurgia Endocrina e Metabolica, Fondazione Policlinico Universitario A. Gemelli IRCCS, ROME, Italy; ${ }^{2}$ U.O.C. Anestesia delle Chirurgie Generali e dei Trapianti, Fondazione Policlinico Universitario A. Gemelli IRCCS, ROME, Italy

Aims: Laparoscopic intracorporeal suturing and knot tying for anastomosis are considered the most difficult laparoscopic skill to master. The introduction of barbed sutures seems to facilitate the practice, with bariatric surgery being one of the specialties that can most benefit from them. The aim of this study was to establish the safety and efficacy of barbed suture to close the gastrojejunal anastomosis (GJA) and the jejuno-jejunal anastomosis (JJA) in bariatric laparoscopic procedure procedures (Roux-en-Y gastric bypass-RYLGB) using propensity score matching (PSM) analysis. Methods: A retrospective analysis of patients who underwent primary bariatric procedures (RYLGB) between January 2012 and October 2018 was performed. PSM analysis was performed to overcome patient selection bias (age, gender, BMI, previously abdominal operations, comorbidities) between the two types of sutures (barbed sutures-BS, and conventional stutures-CS). The primary outcome measure were the operative time and the incidence of leak, bleeding and stenosis. The secondary outcomes were the post-operative hospital stay and the incidence of late complications (internal hernias and small bowel obstructions).

Results: A total of 969 patients were reviewed. After PSM, 322 (161 BS vs 161 CS) patients were apiece compared (chi-square $0.287, \mathrm{p}=0.862$ ). Median operative time was significantly shorter [65(25) vs 95(45) min $\mathrm{p}<0.001]$ for BS. Median Post-operative hospital stay was significantly shorter for BS [2(1) vs 5(1) days $\mathrm{p}<0.001)$. Post-operative 30th-days complications were comparable: one case of GJA's leakage for CS $(p=0.5)$ and one case of JJA's stenosis for BS $(p=0.5)$. Median follow-up was significantly shorter for BS $(p<0.001)$. At mean follow up of 26.06 months the rates of small bowel obstructions were comparable (BS 2(1.2\%) vs CS $1(0.6 \%)$, $p=0.562)$; instead the rates of internal hernias were significantly lower for BS $(0(0 \%)$ vs $7(4.4 \%)$, $\mathrm{p}=0.007)$.

Conclusions: Despite some of the results of the present study may be influenced by increasing surgical skill during the study period, barbed sutures appear to be effective in reducing the operative time, and as safe as conventional suture to close gastro-jejunal anastomosis and jejuno-jejunal anastomosis in bariatric surgery (RYLGB). Further studies are necessary to draw definitive conclusions

\section{O027-BARIATRICS-Laparoscopic}

PRELIMINARY WEIGHT LOSS DATA OF LAPAROSCOPIC ONE ANASTOMOSIS GASTRIC BYPASS VS. LAPAROSCOPIC ROUX-EN-Y GASTRIC BYPASS

M.J. García-Oria ${ }^{1}$, J.A. Rivera ${ }^{1}$, M. Artés ${ }^{1}$, J. Alvarez ${ }^{1}$, B. Olivan ${ }^{2}$, A. Sánchez ${ }^{1}$, P. Pla ${ }^{1}$, C. León-Gámez ${ }^{1}$, A. Equisoain ${ }^{1}$, L. Román ${ }^{1}$, D. Gonzalez ${ }^{1}$, X. Rial ${ }^{1}$, X. Remirez ${ }^{1}$

${ }^{1}$ Cirugía General y del Aparato Digestivo, Hospital Universitario Puerta de Hierro Majadahonda, MADRID, Spain; ${ }^{2}$ Endocrinología y Nutrición, Hospital Universitario Puerta de Hierro Majadahonda, MADRID, Spain

Aims: Laparoscopic One Anastomosis Gastric Bypass (OAGB) is an accepted bariatric technique, but few randomized prospective trials have compared it with Laparoscopic Roux-en-Y Gastric Bypass (LRYGB). We have conducted a study comparing these two techniques and herein we present the weight loss data in the first two years of follow-up.

Methods: Prospective Randomized Clinical Trial, (ClinicalTrials. gov Identifier: NCT02939664). One group of patients of the study was done a LRYGB, and the other group of patients was performed the OAGB. The investigators randomly assigned 10 patients to each group, $n=20$. We used the non-parametric Mann-Whitney test for statistical analysis of quantitative parameters considering a significant $\mathrm{p}<0.05$. Patients with BMI between $40-50 \mathrm{~kg} / \mathrm{m}^{2}$ were included in the study, and randomized in a 1:1 ratio to the LRYGB group or the OAGB group. Patients with gastroesophageal reflux disease were excluded from the study.

Results: The two groups are comparable in the preoperative parameters, and in terms of weight evolution we found a significantly different percentage of excess weight loss and percentage of excess body mass index loss $(\mathrm{p}<0.05)$ at $3,6,9,12$ and 18 months between the two groups, with greater weight loss in the OAGB group.

Conclusion: In the follow-up during the first year and a half the OAGB has better weight loss results than the BPGYL.

\section{O028-BARIATRICS—Laparoscopic}

DIABETES IMPROVEMENT AND RESOLUTION FOLLOWING LAPAROSCOPIC SLEEVE GASTRECTOMY VERSUS SLEEVE GASTRECTOMY WITH SINGLE ANASTOMOSIS SLEEVE ILEAL BYPASS

V.V. Grubnik ${ }^{1}$, O.V. Medvedev², V.V. Ilyashenko², S.A. Usenok ${ }^{2}$, V.V. Grubnyk ${ }^{2}$

${ }^{1}$ Department of surgery, Odessa medical university, ODESSA, Ukraine; ${ }^{2}$ Department of surgery \#1, Odessa medical university, ODESSA, Ukraine

The aim of this study was to compare the effectiveness of laparoscopic sleeve gastrectomy (LSG) with new surgical procedure: sleeve gastrectomy with single anastomosis sleeve ileal bypass (LSG + SASI) for the treatment of patients with diabetes mellitus type 2 (DM).

Methods: 48 obese patients with DM type 2 were divided in two groups: I group ( 25 patients) consisted from 15 women and 10 men age 36 to 64 years, mean body mass index (BMI) was $49.7 \pm 8.6 \mathrm{~kg} / \mathrm{m}^{2}$. LSG was performed in all patients of the I group. II group ( 23 patients) consisted from 14 women and 9 men, age 39-69 years, mean BMY was $51.8 \pm 7.4 \mathrm{~kg} / \mathrm{m}^{2}$. LSG + SASI was performed in all patients of the II group. There were no statistically significant difference between two groups in demographic, BMI, and comorbidities. Excess weight loss percentage (\%EWL), the total weight loss percentage (\%TWL) and diabetes improvement were analyzed.

Results: There were no serious complications and mortality in the both groups. There were no symptoms of malabsorption in the patients of II group after LSG + SASI. After 24 months the patients of the II group lost more \% TWL and \%EWL and the difference was statistically significan $(\mathrm{p}<0.05) \% \mathrm{EWL}$ in the I group was $69.7 \pm 9.2 \%$, in the patients of the II group $-80.7 \pm 13.4 \%$ $(\mathrm{p}<0.05)$. Resolution of DM type 2 was in $14(56 \%)$ patients from the I group and in $20(87 \%)$ from the II group $(\mathrm{p}<0.01)$.

Conclusion: Sleeve gastrectomy with with single anastomosis sleeve ileal bypass is more effective than LSG in the treatment of DM type 2 associated with obesity. 


\section{O029-BARIATRICS-Laparoscopic}

UTILITY OF INFLAMMATORY MARKERS IN DETECTION OF PERIOPERATIVE MORBIDITY AFTER BARIATRIC PROCEDURES-MULTICENTER STUDY

M. Wysocki ${ }^{1}$, P. Malczak ${ }^{1}$, M. Wierdak ${ }^{1}$, M. Janik ${ }^{2}$, M. Waledziak ${ }^{2}$, P. Lech ${ }^{3}$, N. Dowigiallo-Wnukiewicz ${ }^{3}$, M. Orlowski ${ }^{4}$, H. Razak Hady $^{5}$, M. Proczko-Stepaniak ${ }^{6}$, K. Major ${ }^{7}$, M. Pisarska ${ }^{1}$, J. Szeliga ${ }^{8}$, M. Pedziwiatr ${ }^{1}$, A. Budzynski ${ }^{1}$, P. Major ${ }^{1}$

${ }^{1}$ 2nd Department of General Surgery, Jagiellonian University Medical College, KRAKOW, Poland; ${ }^{2}$ Department of General, Oncological, Metabolic and Thoracic Surgery, Military Institute of Medicine, WARSAW, Poland; ${ }^{3}$ Department of General and Minimally Invasive Surgery, University of Warmia and Mazury, OLSZTYN, Poland; ${ }^{4}$ General and Vascular Surgery Department,, Ceynowa Hospital, CEYNOWA, Poland; ${ }^{5}$ Department of General and Endocrinological Surgery, Medical University of Bialystok, BIALYSTOK, Poland; ${ }^{6}$ Department of General, Endocrine and Transplant Surgery,, Medical University of Gdansk, GDANSK, Poland; ${ }^{7}$ Faculty of Health Sciences, Jagiellonian University Medical College, KRAKOW, Poland; ${ }^{8}$ Department of General, Gastroenterological, and Oncological Surgery, Nicolaus Copernicus University, TORUN, Poland

Aim: To determine if there are correlations between of C-reactive protein, procalcitonin and white blood count measured in first, second and third postoperative day after bariatric surgeries and postoperative morbidity (1-month and 12-months), length of hospital stay (LOS) and hospital readmissions.

Methods and Procedures: Retrospective cohort study of patients who underwent laparoscopic sleeve gastrectomy (SG), Roux-en-Y gastric bypass (GB) or mini-gastric bypass (MGB) for morbid obesity in seven referral bariatric centers. Patients were divided into two groups: complicated group - patients with postoperative complications (in 1 month or 12 months postoperative period) and non-complicated group-patients without postoperative morbidity. Primary endpoint: to determine utility of routinely tested inflammatory markers in prognosis of perioperative (30-days) and postoperative (12-months) morbidity rates. Secondary endpoints: utility of inflammatory markers to determine risk of LOS after bariatric surgeries and readmissions.

Results: 2125 patients, $68 \%$ females and 32\% males in median age of 43 years were included. Postoperative morbidity was $7.29 \%$ patients, of who 112 cases occurred in first postoperative month and 43 cases after first postoperative month during 12-months follow-up. Sex, age, BMI and co-morbidities did not differ complicated and non-complicated cases. In complicated group LOS was significantly longer as well as readmission rate. CRP (OR 1.02, 95\% CI: 1.01-1.03), WBC (1.23, 1.15-1.32), PCT (17.18, 3.09-95.46) on POD1 used as continuous variables were significantly increasing OR for perioperative morbidity. ROC analysis indicated suggested cut-off point of CRP on POD1 indicating for risk of perioperative morbidity for $23.8 \mathrm{mg} / \mathrm{L}, \mathrm{WBC}$ for $10 \times 10^{3} / \mathrm{mm}^{3}$, and PCT for $0.22 \mathrm{ng} / \mathrm{mL}$. In case of postoperative morbidity inflammatory parameters on POD1 were not significantly related to ORs. CRP (OR 1.02, 95\% CI 1.01-1.03), WBC (1.10, 1.02-1.20), PCT $(4.7,1.12-19.64)$ were increasing OR for prolonged LOS. CRP (OR 1.01, 95\% CI $1.00-1.03)$, WBC $(1.21,1.09-1.33)$ on POD1 were significantly increasing OR for readmissions. ROC analysis indicated suggested cut-off point of CRP on POD1 indicating for risk of readmissions for $94 \mathrm{mg} / \mathrm{L}$, WBC for $14.5 \times 10^{3} / \mathrm{mm}^{3}$. Conclusion: Even moderate increase in inflammatory parameters on POD1 should alert surgeon for possibility of increased chance for perioperative morbidity, prolonged LOS and hospital readmissions.

\section{O030-BARIATRICS—Physiology}

IMMUNOHISTOCHEMICAL ANALYSIS OF GLP1 AND GIP IN THE SMALL BOWEL, AND ITS RELATIONSHIP WITH THE PANCREATIC RECEPTOR OF GLP-1 IN THE WISTAR RAT

J. Falckenheiner ${ }^{1}$, A. Camacho ${ }^{2}$, J.A. Prada ${ }^{3}$, G. Perez Arana ${ }^{3}$, J.M. Pacheco ${ }^{1}$, M. Mayo ${ }^{1}$

${ }^{1}$ General Surgery, Puerta Del Mar Hospital, Cadiz, Spain;

${ }^{2}$ General Surgery, Puerto Real Hospital, Cadiz, Spain; ${ }^{3}$ Anatomy And Embriology, Cadiz University, Cadiz, Spain

Aims: Study the physiological mechanisms dependent on the enteropancreatic axis that accompanies bariatric surgery in a healthy animal model.

Analyze the relationship between Glucagon like Peptide 1 (GLP-1) and Glucose dependent insulinotropic polypeptide (GIP) in the small bowel.

Compare the impact of intestinal GLP-1 on its receptors in beta cells of the pancreas.

Methods: Healthy Wistar male rats between 12 and 14 weeks of age were used. Five groups were randomly assigned. The control groups were divided into Fasting Control (F) and SHAM (Surgical Control). The surgical groups were separated into Vertical Gastrectomy (GS), 50\% resection of the middle small bowel (RI50) and gastric bypass (GB). Three months later all the animals were sacrificed. The total number of positive GLP-1 and GIP cells in the duodenum, jejunum and ileum were studied. The total number of GLP-1 receptors (rGLP-1) expressed in pancreatic beta cells was also studied. The hormonal test was performed using immunohistochemical techniques, and were compared using the analysis of variance (ANOVA).

Results: An increase in the expression of GLP-1 was observed in the GB and RI50 in the duodenum and ileum. In the same way, a greater number of GLP-1 was identified in the GB group of the jejunum.

In the GIP study, there was a significant increase of this hormone in the duodenum of the GB and RI50 groups.

Regarding rGLP-1 in the pancreas, there is a statistically significant increase in the GB and RI50. Conclusions: We can confirm the existence of GLP-1 cells throughout the intestinal frame outside of their confinement in the ileum. The significant increase of GLP-1 and GIP cells in the malabsorptive groups demonstrate the importance of the premature passage of nutrients to the distal bowel as support for the 'Hindgut theory'. These results also offer us an indirect evidence of an intermediate and regulatory mechanism of hormonal homeostasis in the enteropancreatic axis.

Regarding the increase of pancreatic receptors in parallel with the intestinal expression of GLP-1 of a healthy rat, reinforces the idea of an active hormonal counter-regulator between the expression of GLP-1 and its physiological effects on the enteropancreatic axis .

\section{O031-BARIATRICS—Physiology}

\section{OUTCOME OF BARIATRIC SURGERY IN ELDERLY} PATIENTS $=65$ YEARS OLD

A. Almunifi ${ }^{1}$, S.D. Aldeghaither ${ }^{2}$, T. Debs ${ }^{3}$, I. Ben Amor ${ }^{3}$, J.G. Gugenhiem ${ }^{3}$, M. Ibrahim ${ }^{4}$

${ }^{1}$ Department of General Surgery, College of Medicine at Majmaah University, AL MAJMA'AH, Saudi Arabia; ${ }^{2}$ Department of General Surgery, Prince Mohammed bin Abdulaziz Hospital, Riyadh, Saudi Arabia., RIYADH, Saudi Arabia; ${ }^{3}$ Department of Digestive Surgery, Bariatric Surgery Center and Liver Transplant, CHU Nice, L'archet 2, NICE, France; ${ }^{4}$ Department of general surgery, Assuit university hospital, ASSUIT, Egypt

Introduction: Both obesity and life expectancy is increasing worldwide.

Objectives: The aim of the present study was to report the outcomes of bariatric surgery in patient's $=65$ years of age.

Methods: A retrospective review of prospectively collected data from patients aged $=65$ years who underwent LRYGP and LSG in our institute from 2006 to 2016. The data analyzed included age, preoperative and postoperative weight and body mass index, postoperative complications, and co-morbidities.

Results: A total of 47 patients $=65$ years $(66.5 \pm 0.2$ years $)$ underwent bariatric surgery in our institute. Of these 47 patients, 21 patients $(44.68 \%$ ) had undergone LRYGP, 20 patients $(42.55 \%)$ LSG, and 6 patients $(12.76 \%$ ) conversion of gastric band (5 patients) and Maison (one patient) to LRYGP. The mean preoperative weight and body mass index was $109.06 \pm 2.33 \mathrm{~kg}$ and $40.93 \pm 0.74 \mathrm{~kg} / \mathrm{m}^{2}$, respectively. The median length of follow-up was 12 months (range 1-48). The overall complications rate was $23.4 \%$. No mortality occurred. The mean percentage of excess weight loss and body mass index was $77.5 \pm 6 \%$ and $29.7 \pm 1$ at 12 months. The rate of resolution of diabetes mellitus, hypertension and obstructive sleep apnea syndrome was $70 \%, 57 \%, 75 \%$ and $100 \%$ respectively.

Conclusion: Bariatric surgery in carefully screened patients \pm 65 years can be performed safely and can achieve improvement in co-morbidities. 


\section{O032-BARIATRICS—Physiology}

CONTROVERSIES IN THE PREOPERATIVE WORKUP FOR BARIATRIC SURGERY: A REVIEW OF THE LITERATURE AND RECOMMENDATIONS FROM A BARIATRIC CENTER OF EXCELLENCE

\section{Garraud ${ }^{1}$, D. Lim², S. Liu ${ }^{2}$, K. Bain ${ }^{2}$, G. Ferzli ${ }^{2}$}

${ }^{1}$ General Surgery, Tuoro College of Osteopathic Medicine, BROOKLYN, United States of America; ${ }^{2}$ General Surgery, NYU Langone Hospital-Brooklyn (Lutheran), BROOKLYN, United States of America

Introduction: The preoperative workup for the bariatric patient varies from institution to institution and uncertainty exists in the literature as to the utility of certain aspects of the workup; such as routine esophagogastroduodenoscopy (EGD), screening adults and adolescence for obstructive sleep apnea (OSA), and management of asymptomatic cholelithiasis. In this submission we review the literature on controversial aspects of the bariatric preoperative workup, discuss the issues, and offer our institutional recommendations.

Methods: PUBMED search terms: 'EGD bariatric surgery,' 'cholecystectomy bariatric surgery,' 'obstructive sleep apnea bariatric surgery'. 850 total articles were returned and 20 from each category were selected by the authors for their direct relevance to the preoperative workup before bariatric surgery.

Results: After a review of the literature, we recommend OSA screening of adult bariatric patients with an approved questionnaire and treatment with continuous positive airway pressure therapy (CPAP) for 30 days preoperatively and continuation postoperatively. We recommend against routine screening and treatment of adolescence for OSA. We recommend against synchronous cholecystectomy with bariatric procedures for asymptomatic cholelithiasis. We recommend for the use of ursodeoxycholic acid pre and postoperatively for cholelithiasis prophylaxis. We recommend for the use of preoperative EGD in mucosal altering procedures, such as the roux-en-y gastric bypass, gastric sleeve, or duodenal switch. We recommend against a routine EGD before the gastric banding. We recommend routine proton pump inhibitor (PPI) use preoperatively when symptomatic.

Conclusion: When current literature is equivocal, clinical decision making and institutional guidelines target the best preoperative workup for each bariatric patient. The recommendations for our preoperative workup and algorithm for testing is driven by our clinical experience and best interpretation of available data.

\section{O033-BARIATRICS—Physiology}

INFLUENCE OF BASELINE GLYCATED HEMOGLOBIN LEVEL ON POSTOPERATIVE OUTCOMES OF BARIATRIC SURGERY

M. Wysocki ${ }^{1}$, M. Wierdak ${ }^{1}$, M. Janik ${ }^{2}$, M. Waledziak ${ }^{2}$, P. Lech ${ }^{3}$, N. Dowigiallo-Wnukiewicz ${ }^{3}$, M. Orlowski ${ }^{4}$, H. Razak Hady ${ }^{5}$, M. Proczko-Stepaniak ${ }^{6}$, K. Major ${ }^{7}$, P. Malczak ${ }^{1}$, M. Pisarska ${ }^{1}$, J. Szeliga $^{8}$, M. Pedziwiatr ${ }^{1}$, A. Budzynski ${ }^{1}$, P. Major ${ }^{1}$

${ }^{1}$ 2nd Department of General Surgery, Jagiellonian University Medical College, KRAKOW, Poland; ${ }^{2}$ Department of General, Oncological, Metabolic and Thoracic Surgery, Military Institute of Medicine, WARSAW, Poland; ${ }^{3}$ Department of General and Minimally Invasive Surgery, University of Warmia and Mazury, OLSZTYN, Poland; ${ }^{4}$ General and Vascular Surgery Department, Ceynowa Hospital, CEYNOWA, Poland; ${ }^{5}$ Department of General and Endocrinological Surgery, Medical University of Bialystok, BIALYSTOK, Poland;

${ }^{6}$ Department of General, Endocrine and Transplant Surgery, Medical University of Gdansk, GDANSK, Poland; ${ }^{7}$ Faculty of Health Sciences, Jagiellonian University Medical College, KRAKOW, Poland; ${ }^{8}$ Department of General, Gastroenterological, and Oncological Surgery, Nicolaus Copernicus University, TORUN, Poland

Introduction: The aim of this study was to investigate the influence of baseline glycated hemoglobin level (HbA1c) level in bariatric patients on postoperative outcomes. We found scarce of clinical data regarding influence of baseline $\mathrm{HbAlc}$ on bariatric surgeries postoperative morbidity and readmission what was inspiration to conduct this multicenter retrospective study.

Methods and Procedures: Retrospective cohort study analyzed patients who underwent laparoscopic: sleeve gastrectomy (SG), Roux-en-Y gastric bypass (RYGB) or mini-gastric bypass (MGB) for morbid obesity in seven referral bariatric centers. Patients were divided into groups depending on preoperative $\mathrm{HbA}_{1 \mathrm{c}}: \mathrm{HbA}_{1 \mathrm{c}}<5.7 \% ; 5.7-6.4 \%$ and $\geq 6.5 \%$. Primary endpoints: influence of $\mathrm{HbA}_{1 \mathrm{c}}$ level on perioperative (30-days) and postoperative (12-months) morbidity rates, operation time, length of hospital stay (LOS) and readmission rate.

Results: Study group included 2125, 68\% females and 32\% males. Median age was 43 (35-52) years. Median HbAlc was 5.7 (5.3-6.1). HbAlc $<5.7 \%$ was present in $49 \%$ patients, $\mathrm{HbAlc5.7-6.4 \%}$ in $35 \%$, and $\mathrm{HbAlc} \geq 6.5 \%$ in $16 \%$. Percentage of male patients increased in groups from $26 \%$ in $\mathrm{HbA}_{1 \mathrm{C}}<5.7 \%$ to $47 \%$ in $\mathrm{HbA}_{1 \mathrm{C}} \geq 6.5 \%$ significantly. Same tendency through groups we observed in case of BMI and age. Uncontrolled diabetes $\left(\mathrm{HbA}_{1 \mathrm{C}} \geq 6.5 \%\right)$ was present in $8.7 \%$ patients, while $7.62 \%$ patients were not on antidiabetic medications despite having $\mathrm{HbA}_{1 \mathrm{C}} \geq 6.5 \%$. Median operative time in patients was significantly longer than in $\mathrm{HbAlC}<5.7 \%$ and $\mathrm{HbAlC}$ $5.7-6.4 \%$. 30-days morbidity rate was $5.27 \%$ and did not differ groups significantly, as 12 -months morbidity rate (excl. 30-days) of $2.02 \%$. LOS did not differ groups significantly. Patients having $\mathrm{HbA1C}$ in range of $5.7-6.4 \%$ and with $\mathrm{HbAlC} \geq 6.5 \%$ did not have significantly increased odds for perioperative morbidity, 12-months postoperative morbidity as compared with those with $\mathrm{HbAlC}<5.7 \%$. Patients with $\mathrm{HbAlc} \geq 6.5 \%$ had increased OR for prolonged LOS as compared to those with $\mathrm{HbA1C}<5.7 \%$ (OR 1.45; 95\% CI 1.07-1.97). HbAlc did not influence OR for readmissions. Patients with baseline $\mathrm{HbA}_{1 \mathrm{c}} \geq 8 \%$ had significantly increased chances for hospital readmission (OR 3.53, 95\% CI 1.35-9.21).

Conclusion: Baseline level of HbAlc did not influence chance for perioperative morbidity, 12-months postoperative morbidity and prolonged LOS. Patients with $\mathrm{HbAlc} \geq 8 \%$ have increased chance for hospital readmissions. 


\section{O034-COLORECTAL_Benign}

IS THERE ANY ADVANTAGE PERITONEAL LAVAGE OVER ASPIRATION IN PERFORATED APPENDICITIS?

\section{N. Ozlem}

General surgery department, Ahievran University, KIRSEHIR, Turkey

Introduction: To date, prospective comparative studies are limited that, has compared peritoneal lavage with irrigation and aspirations with the only aspiration without irrigation in perforated appendicitis.

The purpose of this study was to evaluate the effect of peritoneal lavage on postoperative complications, perioperative stay and duration of perforation in perforated appendicitis patients.

Methods: Prospectively collected data from 279 consecutive patients with acute appendicitis were studied between march 2011 to august 2012 . Perforated appendicitis was revealed in 41 patients that were randomized into two groups. Only suction was performed in group 1, and peritoneal lavage with normal saline was performed in group 2. These two groups were compared with respect to postoperative complications and length of hospital stay and operation time. Chi-squared and t-tests were used to analyze the study data.

Results: There were no differences in patient characteristics at presentation. There were no perioperative or postoperative mortalities. postoperative complications developed in 10 patients, four in group 1 and six in group 2 . The overall complication rate for group 1 was $10 \%$, compared with $15 \%$ for group 2 $(p=0.46)$. The overall postoperative complication rate was statistically similar for both groups. There was no statistically significant difference in the length of hospital stay, but peritoneal irrigation resulted in a longer operation time in group 2.

Conclusion: These findings revealed that there is no advantage to lavage of the peritoneal cavity over suction alone during appendectomy for perforated appendicitis.

\section{O035-COLORECTAL_Benign}

LAPAROSCOPIC LAVAGE, PRIMARY ANASTOMOSIS OR HARTMANN PROCEDURE FOR DIVERTICULITIS: NETWORK META-ANALYSIS OF THE LADIES, DILALA AND SCANDINAVIAN TRIALS

S.A. Antoniou ${ }^{1}$, A. Andreou ${ }^{2}$, S. Hajibandeh ${ }^{3}$, S. Hajibandeh ${ }^{4}$, S. Antoniou ${ }^{5}$, M. Weitzendorfer ${ }^{6}$, I. Daniels ${ }^{1}$, N.J. Smart ${ }^{1}$

${ }^{1}$ Surgical Service, Royal Devon \& Exeter NHS Foundation Trust, EXETER, United Kingdom; ${ }^{2}$ Department of Colorectal Surgery, York Teaching Hospital NHS Foundation Trust, YORK, United Kingdom; ${ }^{3}$ General Surgery Department, Salford Royal Foundation Trust, SALFORD, United Kingdom; ${ }^{4}$ General Surgery Department, North Manchester General Hospital, MANCHESTER, United Kingdom; ${ }^{5}$ Department of Vascular \& Endovascular Surgery, The Royal Oldham Hospital, MANCHESTER, United Kingdom; ${ }^{6}$ Department of Surgery, Paracelsus Medical University, SALZBURG, Austria

Aims: The best treatment option for acute complicated diverticulitis is a matter of ongoing research. We aimed to synthesize available evidence on interventions in terms of network meta-analysis.

Methods: The databases of Medline, AMED, CENTRAL, OpenGrey were interrogated. Pairwise meta-analysis for each pair of interventions was performed using a random-effects model and network meta-analysis in Stata was performed using the mvmetacommand and self-programmed Stata routines. The restricted maximum likelihood method was used to estimate heterogeneity assuming a common estimate for the heterogeneity variance across the different comparisons. Differences between direct and indirect evidence were explored by comparing direct and indirect estimates by computing the inconsistency factor within each closed loop of evidence. The contribution of each direct comparison to each of the summary estimates was computed using the contribution plot. The ranking probabilities for all treatments of being at each possible rank for each intervention were computed using the mvmeta command in Stata. A hierarchy of the competing interventions was obtained using rankograms.

Results: There were no significant differences in terms of mortality and morbidity among Hartmann's procedure, laparoscopic lavage, sigmoidectomy with defunctioning loop ileostomy, and primary anastomosis, although a trend in favor of the latter in terms of mortality and morbidity was seen. Primary anastomosis had a 54\% probability of being the best treatment in terms of mortality and 58\% probability of being the best treatment in terms of mortality, the second best treatment being Hartmann's procedure in terms of morbidity and laparoscopic lavage in terms of mortality.

Conclusion: Evidence on the effects of treatment strategies for acute complicated diverticulitis is still inconclusive. Primary anastomosis seems an effective option with higher probability of being the best treatment compared to Hartmann's procedure, laparoscopic lavage, and sigmoidectomy with defunctioning loop ileostomy. 


\section{O036-COLORECTAL-Benign}

\section{NEOSTIGMINE FOR TREATMENT OF ACUTE COLONIC PSEUDO-OBSTRUCTION AS AN ALTERNATIVE TO COLONOSCOPIC DECOMPRESSION}

\author{
A.R.B. Kushairi, D. Igboin, N. Laskar, P. Daliya, E. Theophilidou, \\ A. Adiamah, A. Chowdhury \\ Department of Gastrointestinal Surgery, Queen's Medical Centre, \\ NOTTINGHAM, United Kingdom
}

Aims: Colonoscopic decompression is an effective option to definitively treat acute colonic pseudo-obstruction (ACPO). However, the availability of colonoscopy and frail condition of patients may limit the suitability of colonoscopy as a first-line option in all patients. Neostigmine, a reversible acetylcholinesterase inhibitor may effectively treat ACPO, but no up-to-date evidence supports this use. This systematic review and meta-analysis aims to investigate the use of neostigmine in treating acute colonic pseudo-obstruction as an alternative to colonoscopic decompression.

Methods: A comprehensive search of the major online databases: Medline, Embase and Cochrane databases were performed for randomised controlled trials, prospective and retrospective studies from 1946 to 2018 studying the use of neostigmine in acute colonic pseudo-obstruction. Our primary outcomes were dosage of neostigmine used, response to neostigmine, time to flatus, time to defecation, time to bowel sounds, abdominal circumference, colonic diameter, gastrointestinal motility, adverse effects of neostigmine and mortality. Quantitative pooling of data was performed using relative risk for dichotomous data with a random effects model.

Results: Eighteen studies were included in total, with 411 patients. Meta-analysis of response to neostigmine showed a significantly higher number of patients who responded to neostigmine compared to placebo [RR (95\% CI) 25.98 (6.63, 101.71), P $<0.00001]$. Patient response to colonoscopic decompression was greater than response to neostigmine [RR $(95 \% \mathrm{CI}) 1.53(1.23,1.91), \mathrm{P}=0.0001]$. Qualitatively, neostigmine had a success rate of $72.7-100 \%$ in ACPO patients and was associated with shorter time to defecation. Neostigmine was associated with significant reduction in abdominal circumference and colonic diameter. Adverse effects of neostigmine were rare and no mortality related to ACPO or neostigmine was seen.

Conclusion: ACPO is a rare condition and no conclusive large trial data exists on the therapeutic effect of neostigmine on ACPO. We conclude that colonoscopic decompression is superior to neostigmine in treating ACPO, however neostigmine is an effective and safe treatment modality for ACPO in patients unsuitable for colonoscopy.

\section{O037-COLORECTAL-IBD}

\section{A MULTIMODAL PREHABILITATION PROTOCOL AIMED AT PREVENTING EMERGENCYSURGERY AND IMPROVINGNUTRITIONAL ANDPHYSICALSTATUSINPATIENTSWITH BOWELOBSTRUCTION}

\author{
M. Fahim ${ }^{1}$, C.S. van Kessel ${ }^{1}$, D. Smeeing ${ }^{1}$, A. Braaksma ${ }^{2}$, A. Smits ${ }^{1}$
}

${ }^{1}$ Department of Surgery, St. Antonis Hospital, NIEUWEGEIN, The Netherlands; ${ }^{2}$ Department of Nutrition and Health, St. Antonis Hospital, NIEUWEGEIN, The Netherlands

Introduction: Patients with bowel obstruction have chronic insufficient intake due to the stenosis as well as pain and nausea, leading to malnutrition and weight loss. Furthermore, distension of the bowel wall may lead to worse healing and an increased risk of anastomotic leakage when emergency surgery takes place. These factors may explain the high morbidity and mortality rates. The goal of this study was to examine the effect of a multimodal prehabilitation protocol aimed at preventing acute surgery and improving nutritional and physical status before elective surgery.

Methods: All patient presenting with bowel obstruction in the period 2013-2017 were included. A combination of clinical symptoms and imaging or colonoscopy was used for diagnosis. Benign and malign causes of colon obstruction were included. The protocol consisted of a combination of 1. physical training, 2. the use of oral laxatives and 3. specific dietary adjustments and residue-low nutrition.

Results: A total of 61 patients were included. 44 patients $(72.1 \%)$ were treated for colorectal cancer. ASA score was moderate to severe (ASA III) in almost a fourth of the patients. Elective surgery was performed in 57 patients $(93.4 \%)$. Primary anastomosis was constructed in 49 patients $(86.0 \%)$. No bowel perforation, anastomotic leakages or 30-day mortality was observed. Rate of severe complications (Clavien-Dindo grade III or higher) was $6.6 \%$. Conclusion: Our study shows that patients with obstructive bowel disease can be safely treated with this study protocol and moreover, it results in good surgical outcome with majority of patients receiving elective laparoscopic resection with primary anastomosis and little postoperative complications. Therefore, we suggest to implement the use of this protocol for all patients with signs and symptoms of bowel obstruction.

\section{O038-COLORECTAL-IBD}

\section{LONG-TERM COMPARISON OF CONVENTIONAL VERSUS LAPAROSCOPIC SURGERY FOR ULCERATIVE COLITIS}

Gy. Lázár ${ }^{1}$, J. Tajti Jr. ${ }^{1}$, Zs. Simonka ${ }^{1}$, A. Paszt ${ }^{1}$, Sz. Ábrahám ${ }^{1}$, K. Farkas $^{2}$, T. Molnár ${ }^{2}$

${ }^{1}$ Department of Surgery, University of Szeged, SZEGED, Hungary; ${ }^{2}$ First Department of Internal Medicine, University of Szeged, SZEGED, Hungary

Aim: For the surgical treatment of ulcerative colitis (UC) laparoscopy is used more widely, but limited data are available on long-term results, such as postoperative quality of life and late complications. The objective of our study is to compare the mean 64 (1-158) month follow-up results of patients treated with open and minimally invasive surgical methods. Methods: Between 1 January 2005 and 1 December 2018 a total of 90 patients (49 women, $41 \mathrm{men}$ ) had undergone surgery for UC, out of which 28 (31.1\%) were emergency (total colectomy with mucous fistula) and $62(68.9 \%)$ were elective cases (proctocolectomy and ileal pouch-anal anastomosis). Laparoscopy was used in $65(72.2 \%)$ and conventional method in 25 (27.8\%) cases. Quality of life was examined with questionnaires: Functional Scoring System, Gastrointestinal Quality of Life Index (GIQLI) and Short Inflammatory Bowel Disease Questionnaire were used for testing gastroenterological conditions; Spielberger's State-Trait Anxiety Questionnaire, Beck Depression Inventory and Brief Illness Perception Questionnaire were performed to consider psychological status.

Results: At the early postoperative period in the laparoscopic group there were significantly shorter recovery of the bowel function (1.2 vs. 1.6) and shorter length of intensive care (2.1 vs. 2.5). During the long-term follow-up, significantly fewer complications were in the laparoscopy group such as septic condition $(7.6 \%$ vs. $64 \%)$, intestinal obstruction $(16.9 \%$ vs. $56 \%)$ and 'other' complications $(6.1 \%$ vs. $52 \%)$ such as hernia formation, anastomotic stenosis, per anum bleeding, and pouch-vaginal fistula. Trait anxiety was significantly lower in patients having undergone laparoscopic surgery compared with patients who had had open surgery $(\mathrm{P}=0.018)$ (average value of trait anxiety in patients with open surgery was $48.71, \mathrm{SD}=10.91$; this value was $40.22, \mathrm{SD}=9.82$ in the laparoscopic group). The incidence of abdominal pain was significantly less common (1.895 vs. $2.769 ; P=0.024)$ in the laparoscopic group based on the GIQLI. A significant correlation was found between the results of the psychological and gastrointestinal questionnaires.

Conclusion: Minimally invasive technique provides a better long-term outcome for patients with $\mathrm{UC}$, fewer late complications and a more balanced emotional condition.

\section{O040-COLORECTAL_Malignant}

\section{STEPWISE IMPROVEMENT OF SURGICAL QUALITY IN ROBOTIC LATERAL PELVIC NODE DISSECTION: LESSONS FROM 100 PATIENTS WITH LOCALLY ADVANCED RECTAL} CANCER

H.J. Kim, G.S. Choi, J.S. Park, S.Y. Park, Colorectal Cancer Center, Kyungpook National University Chilgok Hospital, DAEGU, Korea

Background: Lateral pelvic lymph node dissection (LPND) is a challenging procedure due to its technical difficulties and higher incidence of surgical morbidity. Owing to its technical difficulties, lateral pelvic lymph nodes (LPNs) may not be dissected completely and thus be remained in the narrow pelvis. Therefore, a steep learning curve is anticipated in doing LPND. However, no study has been conducted about the surgical acquisition of this complex procedure. Purpose: This study was aimed to evaluate the learning process in performing robotic total mesorectal excision (TME) with LPND for patients with locally advanced rectal cancer. Material and Methods: This study included 100 patients with rectal cancer who underwent robotic TME with LPND between 2011 and 2017. A cumulative sum analysis and moving average were performed on the basis of the number of unilateral retrieved LPNs for evaluating the learning curve.

Results: The number of retrieved LPNs steadily increased. The cumulative sum model suggested that the learning curve was divided into 4 phases based on 3 cut-off points as follows: learning I (33 patients), learning II (19 patients), consolidation (30 patients), and master (18 patients). At the beginning of the learning phase II, we started standardization of the technique and at the beginning of the consolidation phase, we adopted fluorescence-imaging. The operation time and blood loss were similar during the 4 phases. The unilateral number of retrieved LPNs was significantly greater in the master phase than in the other phases (12.8 vs. 4.9, 8.2, and 10.4, $\mathrm{P}<0.001)$. Urinary problems, including urinary retention and postoperative ?-blocker use, were more frequently observed in the learning phase I than in the master phase $(39.4 \%$ vs. $16.7 \%$, $\mathrm{P}=0.034$ ). During the median follow-up of 44.2 months, local recurrence in the pelvic sidewall occurred in 4 patients in learning phase I and in 1 patient in learning phase II.

Conclusion: The completeness of LPND has increased after adopting the standardized surgical technique and new imaging system, and accumulation of surgeon's experience. Further study is warranted to determine the oncologic result following each phase. 


\section{O041-COLORECTAL-Malignant}

THE USE OF ENDOLUMINAL VACUUM THERAPY IN RECTAL ANASTOMOTIC LEAKS: A SYSTEMATIC REVIEW

B. Mahendran, B. Rossi, S. Smolarek, N. Rajaretnam, Colorectal Surgery, University Hospitals Plymouth NHS Trust, PLYMOUTH, United Kingdom

Aims: The aims of this review was to assess the effectiveness of the endoluminal vacuum system (EndoSponge) in the treatment of rectal anastomotic leaks

Methods: A comprehensive systematic search of the literature was performed in keeping with PRISMA guidelines. Records on PubMed, Medline,?Embase?and Google Scholar were searched for all relevant articles. Primary outcome was defined as the rate of anastomotic salvage, with secondary outcomes including creation of new ileostomies, ileostomy closure, additional transrectal closures, length of hospital stay, and functional outcomes. Further subgroup analysis was performed for the primary outcome, comparing early and late initiation of therapy, and neo-adjuvant radiotherapy to the field.

Results: Sixteen studies met the inclusion criteria. There was a significant publication bias $(\mathrm{z}=3.53, \mathrm{p}=0.0004)$. All studies showed low heterogeneity $\left(\mathrm{I}^{2}=32.09\right)$ but were of low quality with a mean MINOR score of 11.5 (SD 1.5). A total of 266 patients were included, with 202 cases operated on for a malignancy. The median treatment failure rate was $11.8 \%$ (range $0-44 \%$ ), with random effects model of 0.17 (95\% CI $0.11-0.22)$. There was significant improvement with early therapy start (OR 3.48) and negative correlation with neo-adjuvant radiotherapy OR 0.56

$51 \%(107 / 210)$ of all diverting stomas were closed at the end of treatment period $34 / 266(12.8 \%)$ patients required an additional transrectal closure of the abscess cavity. Five studies reported functional outcomes in these patients, with different end points being used in each study. Outcomes were reported to be generally favourable amongst patients.

Conclusion: The EndoSponge works better with early initiation of therapy and in patients without pre operative radiotherapy. There is also an unexpectedly high rate of permanent ileostomies. The EndoSponge seems to be an useful method of anastomotic leak treatment in select group of patients, however the quality of available data is poor and it is impossible to draw a final conclusion. There is a need for further assessment of this therapy with well design randomised or cohort study.

\section{O042-COLORECTAL-Malignant}

FUNCTIONAL OUTCOMES AFTER LAPAROSCOPIC VERSUS ROBOTIC-ASSISTED SURGERY FOR RECTAL CANCER: A SYSTEMATIC REVIEW AND META-ANALYSIS

K.F. Kowalewski ${ }^{1}$, L. Seifert ${ }^{1}$, S. Ali $^{1}$, M.W. Schmidt ${ }^{1}$, S. Seide ${ }^{2}$, C. Tapking ${ }^{1}$, A. Shamiyeh ${ }^{3}$, T. Hackert ${ }^{1}$, Y. Kulu ${ }^{1}$, B.P. Müller-Stich ${ }^{1}$, F. Nickel ${ }^{1}$

${ }^{1}$ General, Visceral and Transplantation Surgery, University Hospital of Heidelberg, HEIDELBERG, Germany; ${ }^{2}$ Institute for Medical Biometry and Informatics, University Hospital of Heidelberg, HEIDELBERG, Germany; ${ }^{3}$ General and Visceral Surgery, Kepler Universitätsklinikum GmbH, LINZ, Austria

Introduction: Surgical resection is crucial for curative treatment of rectal cancer. Through improvements in treatment and minimally invasive techniques, 5-year survival improved to over $60 \%$ of patients. The most recently introduced surgical technique is robotic-assisted surgery (RAS). RAS and conventional laparoscopy (CL) seem equally effective in terms oncological control. However, RAS possibly provides further advantages e.g. 3D vision or the Endowrist function, which have the potential to maximize the precision of surgery and thus has benefits for functional outcomes such as sexual function as well continence. Therefore, the aim of this systematic review and meta-analysis was to compare functional outcomes of CL and RAS for rectal cancer.

Materials and Methods: This review was done according to the PRISMA and AMSTAR guidelines andregistered with PROSPER$\mathrm{O}(\mathrm{CRD} 42018104519)$. The search was planned with the PICO criteria and conducted on Medline (via PubMed), Web of Science and CENTRAL. Two independent reviewers first screened titles and abstracts and then eligible full-texts. Inclusion criteria were original studies, comparative studies for CL vs. RAS for rectal cancer as well as reporting of functional outcomes. Quality assessment was done with the Newcastle-Ottawa-Scale for non-randomized studies and the Cochrane tool to assess risk of bias for randomized trials.

Results: The search retrieved 9603 hits, of which 51 studies with 26225 patients met inclusion criteria. Preliminary results yielded a lower rate of urinary retention for RAS (Odds ratio (OR) [95\%-Confidence Interval (CI)] $0.64[0.45,0.91])$ while there were no differences for ileus (OR[CI]: $0.90[0.77,1.04])$. Erectile function (IIEF) showed no differences after 3 (Mean Difference (MD)[CI] 0.80[-1.63, 3.21], 6 (MD[CI] 1.60[-0.69, $3.89])$ and 12 months (MD[CI] $1.11[-1.70,3.93])$. In terms of urinary problems (IPSS) there were no differences 3 postoperative $(\mathrm{MD}[\mathrm{CI}]$ $-0.96[-2.16,0.23])$ and 6 month postoperative $\mathrm{MD}[\mathrm{CI}]$ $-0.92[-1.96,0.11])$, but advantages for the CL group after 12 months $\mathrm{MD}[\mathrm{CI}]-1.05[-1.89,-0.21])$.

Discussion: RAS and CL seem to provide similar functional outcomes after rectal cancer surgery. However, the results need to be interpreted carefully as none of the studies had any functional outcome defined as primary endpoint. Future studies should evaluate both surgical approaches in terms of functional outcomes and should be appropriately powered. 


\section{O043-COLORECTAL-Malignant}

LOOKING AT FUNCTIONAL Results: VALIDATION OF PORTUGUESE VERSION OF THE LOW ANTERIOR RESECTION SYNDROME SCORE

$\underline{\text { N.J. Rama }}{ }^{1}$, J.M. Pimentel ${ }^{2}$, P. Lopes Ferreira ${ }^{3}$, T. Jull ${ }^{4}$

${ }^{1}$ Colorectal Unit, Centro Hospitalar de Leiria, EPE, LEIRIA, Portugal; ${ }^{2}$ Colorectal Unit, CH Universitário de Coimbra, COIMBRA, Portugal; ${ }^{3}$ Centre for Health Studies and Research of the University of Coimbra (CEISUC), Faculty of Economics, University of Coimbra, COIMBRA, Portugal; ${ }^{4}$ Department of Surgery, Aarhus University Hospital, AARHUS, Denmark

Aim: We aim to perform a translation with cultural adaptation of the patient reported outcome tool, low anterior resection syndrome (LARS) Score, to the Portuguese language (LARS-PT) in the Portuguese population with rectal cancer, after proctectomy with anastomosis.

Methods: According the current international recommendations, we designed this study encompassing three main phases to validate LARS score. The questionnaire was completed by 154 patients from six Portuguese Colorectal Cancer Units, and 58 completed it twice.

Results: Portuguese version of LARS score showed a high construct validity and an internal consistency of 0.63 , considered a moderate score. Regarding the test-retest, the global ICC showed a very strong test-retest reliability. Looking at all five items, only items 3 and 5 present a moderate correlation. LARS score was able to discriminate symptoms showing worse quality of life, in patients submitted to preoperative radio and chemotherapy.

Conclusions: LARS questionnaire has been properly translated into European Portuguese, demonstrating high construct validity and reliability. This tool is a precise, reproducible, simple, clear and user-friendly tool for evaluating bowel function in rectal cancer patients after sphincter saving operation.

Keywords: Rectal neoplasms; bowel disfunction; low anterior resection syndrome score; quality of life; validation.

\section{O044-COLORECTAL-Malignant}

GUIDELINE ASSESSMENT PROJECT: FILLING THE GAP IN SURGICAL GUIDELINES. QUALITY IMPROVEMENT INITIATIVE BY AN INTERNATIONAL WORKING GROUP

S. Antoniou ${ }^{1}$, S. Tsokani ${ }^{2}$, D. Mavridis ${ }^{3}$, M. López-Cano ${ }^{4}$, D. Stefanidis $^{5}$, N.K. Francis ${ }^{6}$, N.J. Smart ${ }^{7}$, F.E. Muysoms ${ }^{8}$, S. Morales-Conde ${ }^{9}$, H.J. Bonjer ${ }^{10}$, M.C. Brouwers ${ }^{11}$

${ }^{1}$ Department of Vascular \& Endovascular Surgery, The Royal Oldham Hospital, MANCHESTER, United Kingdom; ${ }^{2}$ Department of Mathematics, University of Ioannina, IOANNINA, Greece; ${ }^{3}$ School of Education, University of Ioannina, IOANNINA, Greece; ${ }^{4}$ Abdominal Wall Surgery Unit, Department of General Surger, Hospital Universitari Vall d'Hebron, Universitat Autònoma de Barcelona, BARCELONA, Spain; ${ }^{5}$ Department of Surgery, Indiana University School of Medicine, INDIANAPOLIS, United States of America; ${ }^{6}$ Department of General Surgery, Yeovil District Hospital NHS Foundation Trust, YEOVIL, United Kingdom; ${ }^{7}$ Surgical Service, Royal Devon \& Exeter NHS Foundation Trust, EXETER, United Kingdom; ${ }^{8}$ Department of Surgery, Maria Middelares Hospital, GHENT, Belgium; ${ }^{9}$ Unit of Innovation in Minimally Invasive Surgery, University Hospital Virgen del Rocio, SEVILLA, Spain; ${ }^{10}$ Department of General Surgery, VU University Medical Center, AMSTERDAM, The Netherlands; ${ }^{11}$ McMaster University, McMaster University, HAMILTON, Canada

Aim: To identify clinical practice guidelines published by surgical scientific organizations, assess their quality and investigate the association between defined factors and quality. The ultimate objective was to develop a framework to improve the quality of surgical guidelines.

Methods: We searched MEDLINE for clinical practice guidelines published by surgical scientific organizations with an international scope between 2008 and 2017. We investigated the association between the following factors and guideline quality, as assessed using the AGREE II instrument: number of guidelines published within the study period by a scientific organization, the presence of a guidelines committee, applying the GRADE methodology, consensus project design, and the presence of inter-society collaboration.

Results: Ten surgical scientific organizations developed 67 guidelines over the study period. The median overall score using AGREE II tool was 4 out of a maximum of 7 , whereas $27(40 \%)$ guidelines were not considered suitable for use. Guidelines produced by a scientific organization with an output of at least 9 guidelines over the study period (odds ratio (OR) 3.79, 95\% confidence interval (CI) 1.01-12.66, $p=0.048)$, the presence of a guidelines committee (OR 4.15, 95\% CI 1.47-11.77, $p=0.007)$ and applying the GRADE methodology (OR 8.17, 95\% CI 2.54-26.29, $p<0.0001$ ) were associated with higher odds of being recommended for use.

Conclusion: Development by a guideline committee, routine guideline output and adhering to the GRADE methodology were found to be associated with higher guideline quality in the field of surgery. 


\section{O045-COLORECTAL-Malignant}

MEDIAL-TO-LATERAL VERSUS LATERAL-TO-MEDIAL COLORECTAL MOBILISATION DURING LAPAROSCOPIC COLORECTAL SURGERY: A SYSTEMATIC REVIEW AND META-ANALYSIS

\section{Eltair ${ }^{1}$, S. Hajibandeh ${ }^{2}$, S. Hajibandeh ${ }^{3}$}

${ }^{1}$ Colorectal Surgery, Sandwell Hospital, BIRMINGHAM, United Kingdom; ${ }^{2}$ General Surgery, Sandwell Hospital, BIRMINGHAM, United Kingdom; ${ }^{3}$ General Surgery, North Manchester General Hospital, MANCHESTER, United Kingdom

Objectives: To evaluate comparative outcomes of medial-to-lateral and lateral-to-medial colorectal mobilisation in patients undergoing laparoscopic colorectal surgery.

Methods: We conducted a systematic search of electronic databases and bibliographic reference lists. We applied a combination of free text and controlled vocabulary search adapted to thesaurus headings, search operators and limits in each of the above databases. Perioperative mortality and morbidity, procedure time, length of hospital stay, rate of conversion to open procedure, and number of harvested lymph nodes were the outcome parameters. Combined overall effect sizes were calculated using fixedeffect or random-effects models.

Results: We identified 8 comparative studies reporting a total of 1477 patients evaluating outcomes of medial-to-lateral $(n=626)$ and lateral-to-medial $(n=851)$ approaches in laparoscopic colorectal resection. The medial-to-lateral approach was associated with significantly lower rate of conversion to open ( $\mathrm{OR} 0.43, \mathrm{P}=0.001)$, shorter procedure time ( $\mathrm{MD}-32.25, \mathrm{P}=0.003$ ) and the length of hospital stay ( $\mathrm{MD}-1.54, \mathrm{P}=0.02)$ compared to the lateral-to-medial approach. However, there was no significant difference in mortality ( $R D 0.00, P=0.96$ ), overall complications (OR 0.78 , $\mathrm{P}=0.11$ ), wound infection ( $\mathrm{OR} 0.84, \mathrm{P}=0.60$ ), anastomotic leak ( $\mathrm{OR} 0.70, \mathrm{P}=0.26$ ), bleeding (OR $0.60, \mathrm{P}=0.50$ ), and number of harvested lymph nodes ( $\mathrm{MD}-1.54, \mathrm{P}=0.02$ ) between two groups. Sub-group analysis demonstrated that the lateral-to-medial approach may harvest more lymph nodes in left colectomy $(\mathrm{MD}-1.29, \mathrm{P}=0.0009)$. Removal of source of heterogeneity in sensitivity analysis showed that overall complications were lower in the medial-to-lateral group (OR $0.72, \mathrm{P}=0.49$ )

Conclusions: The medial-to-lateral approach during laparoscopic colorectal resection may reduce procedure time, length of hospital stay and conversion to open procedure rate compared to the lateral-to-medial approach. Moreover, it may probably reduce overall perioperative morbidity. However, both approaches may carry similar risk of mortality, and have comparable ability to harvest lymph nodes. Future high quality randomised studies may provide stronger evidence in favour of either approach.

\section{O046-COLORECTAL-Malignant}

BARBED SINGLE LAYER VERSUS HYBRID DOUBLE LAYER CLOSURE OF THE ENTEROTOMY IN LAPAROSCOPIC RIGHT COLECTOMY WITH INTRACORPOREAL MECHANICAL ANASTOMOSIS

\section{A. Cossu, U. Elmore, A. Vignali, R. Rosati,}

Gastrointestinal Surgery, San Raffaele Hospital, MILAN, Italy

Aim: To assess the safety and efficacy of single layer of barbed vs double layer 'hybrid' (interrupted and running) suturefor the closure of anastomotic stapler access enterotomyafter laparoscopic right colectomy with intracorporeal anastomosis.

Methods: from April 2014 to November 2018, 252 laparoscopic right colectomy with intracorporeal anastomosis were performed in our Surgical Department. All patients in both groups were perioperatively managed using an ERAS pathway. Seventy-two patients had the enterotomy closed with a single layer running suture of Filbloc ${ }^{\mathrm{TM}}$ (Assut Europe). These patients were matched with 72 patients who underwent intracorporeal right colectomy with enterotomy closed with a 'Hybrid' double layer technique (first layer interrupted stitches in Maxon $^{\mathrm{TM}}$ 3-0 (Covidien), second layer using a running suture in $\mathrm{PDS}^{\mathrm{TM}}$ 3-0 (Ethicon).Intraoperative variables, anastomotic leak rate, morbidity and mortality rates were analyzed.

Results: The two groups were homogeneous with respect to demographics, Body Mass Index (BMI), American surgical Association score (ASA) as well as for tumor stage. In the barbed group, median operating time was $121.5 \mathrm{~min}$ vs $140.7 \mathrm{~min}$ in the Hybrid group $(\mathrm{p}=0.02)$. Anastomotic leak occurred in $5(6.7 \%)$ patients in the hybrid vs $2(2.7 \%)$ patients in the barbed group $(\mathrm{p}=0.24)$ All patients required a reoperation. Intraoperative findings at shows in $2(0.4 \%)$ cases in the Hybrid group a leak at the enterotomy closure, while an intact staler access was observed in both patients in the barbed group. No difference was observed with respect to non-infectious complications between the two groups $(\mathrm{p}=0.55)$. Patients in the hybrid group experienced a longer hospital stay when compared to the barbed group $(\mathrm{p}=0.03)$. A re-admission occurred in the hybrid due an intraabdominal collection, while no re-admission was observed in the barbed group. No patient died in the postoperative period.

Conclusion: Our results shows that the use of knotless barbed suture for enterotomy closure after laparoscopic intracorporeal right colectomy is safe, reproducible and associated with shorter operative time.

\section{O047-COLORECTAL-Malignant}

VARIABILITY IN HEIGHT OF RECTAL TUMOURS; RADIOLOGY VERSUS ENDOSCOPY-WHO IS MORE ACCURATE?

\section{P.A. Blake, J.L. Waterman, P.N. Haray}

Colorectal Surgery, Cwm Taf University Health Board, MERTHYR TYDFIL, United Kingdom

Aims: The accurate measurement and staging of rectal cancer, in particular the distal margin of low rectal tumours, is of paramount importance to optimise oncological surgical resection whils preserving function. It is well recognised that the lower the tumour, the greater the technical challenges, operative time and the possibility of a temporary or permanent stoma. Accurate localisation of the tumour is also essential to assist the multi-disciplinary team when considering neo-adjuvant chemoradiotherapy (CRTx). The objective was to compare tumour height as reported on Magnetic Resonance Imaging (MRI) with endoscopic measurement.

Methods: a retrospective analysis of rectal tumour heights on pre-operative endoscopy and MR in patients undergoing radical colorectal surgery with curative intent over 3 years from January 2015. Rectal tumours were identified as within $15 \mathrm{~cm}$ of the anal verge (AV). All MRI measurements were reported by one of two specialist gastrointestinal radiologists. Measurements were taken from the lowermost point of the tumour to the AV. Endoscopic measurements were as recorded by 11 endoscopists including 2 rectal surgeons, 4 general surgeons, 4 gastroenterologists and a clinical nurse specialist endoscopist.

Results: Records of eighty one patients with histologically confirmed rectal adenocarcinoma were reviewed. Median age was 64 years (35 to 93). Twenty three patients had 2 or more endoscopies. On MRI the median tumour height from the AV was $10.75 \mathrm{~cm}(3.5-18 \mathrm{~cm})$. On endoscopy the median tumour height was $23 \mathrm{~cm}(1-45 \mathrm{~cm})$. On comparing endoscopy with MRI, the median difference was $12 \mathrm{~cm}(0-24 \mathrm{~cm})$. For over a third of patients $(36 \%)$ tumours were lower on MRI than endoscopy, median difference $12.25 \mathrm{~cm}(0.5-24 \mathrm{~cm})$. Only rectal surgeons documented tumour height in relation to the rectal folds. The majority of the repeat endoscopies were performed by surgeons to locate tumours more accurately pre-surgery. On no occasion was it documented whether the tumour had been measured during insertion or withdrawal of the endoscope

Conclusions: Precise localisation of rectal tumours is imperative to plan complex surgery and give informed counsel to patients. This study demonstrates the urgent need for a standardised protocol for all endoscopists to use while recording the distal extent of rectal tumours.

\section{O048-COLORECTAL_Malignant}

\section{LOW LIGATION OF IMA IN LAPAROSCOPIC ANTERIOR RESECTION FOR RECTAL CANCER REDUCES GENITOURINARY DYSFUNCTION}

\section{A. $\operatorname{Costanzi}^{1}$, G. Mari $^{1}$, J. Crippa ${ }^{2}$, D. Maggioni ${ }^{1}$}

${ }^{1}$ General and Emergency Surgery, ASST Monza-Desio Hospital, DESIO, Italy; ${ }^{2}$ Colorectal Surgery, Mayo Clinic, ROCHESTER, United States of America

Objectives: The aim of the present RCT was to compare the incidence of genitourinary (GU) dysfunction after elective laparoscopic low anterior rectal resection and total mesorectal excision (LAR + TME) with high or low ligation (LL) of the inferior mesenteric artery (IMA). Secondary aims included the incidence of anastomotic leakage and oncological outcomes.

Background: The criterion standard surgical approach for rectal cancer is LAR + TME. The level of artery ligation remains an issue related to functional outcome, anastomotic leak rate, and oncological adequacy. Retrospective studies failed to provide strong evidence in favor of one particular vascular approach and the specific impact on GU function is poorly understood.

Methods: Between June 2014 and December 2016, patients who underwent elective laparoscopic LAR + TME in 6 Italian nonacademic hospitals were randomized to high ligation (HL) or LL of IMA after meeting the inclusion criteria. GU function was evaluated using a standardized survey and uroflowmetric examination. The trial was registered under the ClinicalTrials.gov identifier NCT02153801.

Results: A total of 214 patients were randomized to HL (n 1/4 111) or LL (n 1/4 103). GU function was impaired in both groups after surgery. LL group reported better continence and less obstructive urinary symptoms and improved quality of life at 9 months postoperative. Sexual function was better in the LL group compared to $\mathrm{HL}$ group at 9 months. Urinated volume, maximum urinary flow, and flow time were significantly $(\mathrm{P}<0.05)$ in favour of the LL group a 1 and 9 months from surgery. Ultrasound measured post void residual volume and average urinary flow were significantly $(\mathrm{P}<0.05)$ better in the LL group at 9 months postoperatively. Time of flow worsened in both groups at 9 months compared to baseline. There was no difference in anastomotic leak rate $(8.1 \% \mathrm{HL}$ vs $6.7 \% \mathrm{LL})$. There were no differences in terms of blood loss, surgica 1 times, postoperative complications, and initial oncological outcomes between groups. Conclusions: LL of the IMA in LAR + TME results in better GU function preservation withou affecting initial oncological outcomes. HL does not seem to increase the anastomotic leak rate. 


\section{O049-COLORECTAL_Malignant}

TRANS-ANAL LATERAL PELVIC LYMPH NODE DISSECTION FOR LOCALLY ADVANCED LOW RECTAL CANCER

\section{A. Tanaka ${ }^{1}$, K. Uehara ${ }^{1}$, T. Aiba ${ }^{1}$, T. Mukai ${ }^{1}$, G. Nakayama ${ }^{2}$, N. Hattori ${ }^{2}$, Y. Kodera ${ }^{2}$, M. Nagino ${ }^{1}$}

${ }^{1}$ Surgical Oncology, Nagoya University Graduate School of Medicine, NAGOYA, Japan; ${ }^{2}$ Gastroenterological Surgery, Nagoya University Graduate School of Medicine, NAGOYA, Japan

Backgrounds: Chemoradiation therapy (CRT) is a standard treatment for locally advanced rectal cancer in the world. On the other hand, lateral pelvic lymph nodes (LPLN) dissection is a Japanese unique treatment for low rectal cancer. Although the technique is complicated and takes a longer operative time, the efficacies have been revised for patients with enlarged LPLNs even after CRT. Preoperative enlarged LPLN is a risk factor of lateral pelvic recurrence and enlarged LPLNs should be removed even after CRT. Colorectal surgeons should learn the technique. However, the most frequent site of LPLN metastasis occurs at the deep lateral pelvis, where is the farthest from the abdominal wall. In addition, vertical access of the forceps is unavoidable in laparoscopic surgery. Therefore, laparoscopic LPLD dissection is technically demanding. TaTME using the access from the anus is a promising method and rapidly spread in the world. The bottom of the lateral pelvis is the farthest from the abdominal wall but nearby the anus. Using the same platform of TaTME, trans-anal LPLD dissection (TaLPLND) enabled us parallel and shortest access to the deep lateral pelvis.

Surgical technique: After TaTME, we cut the inferior pubic ligament and enter the obturator space. The dissection along the internal obturator muscle is proceeded up. The obturator nerve is securely preserved and the peripheral obturator vessels are divided. Beyond the obturator nerve, the psoas muscle and external iliac vein are confirmed. The lymphatic chain connecting to the inguinal $\mathrm{LN}$ is divided. At the inside of the obturator space, the dissection along the vesicohypogastric fascia between the inferior vesical vessels and obturator fat is proceeded up. At the bottom of the obturator space, the obturator vein and artery are divided. Finally, the proximal obturator fat was divided at the bifurcation of the external and internal vein

Conclusions: This novel technique enabled us to access to the deep lateral pelvis easily. This approach has some potential advantages not only in total LPLN dissection in the difficult cases with obesity and narrow pelvis but also in picking up the enlarged LNs at the deep lateral pelvis. We show the actual technique.

\section{O050-COLORECTAL-Malignant}

\section{COMPLETE MESOCOLIC EXCISION DOES NOT INCREASE SHORT-TERM COMPLICATIONS IN LAPAROSCOPIC LEFT- SIDED COLECTOMIES}

\author{
M.J. Dewulf ${ }^{1}$, F.E. Muysoms ${ }^{1}$, B. Vandenberk ${ }^{3}$, B. Defoort ${ }^{1}$, \\ D. Claeys ${ }^{1}$, P. Pletinckx
}

${ }^{1}$ General Surgery, Maria Middelares, GENT, Belgium; ${ }^{2}$ Cardiovascular sciences, UZ Leuven, LEUVEN, Belgium

Background: After the implementation of Total Mesorectal Excision (TME) in rectal cancer surgery, oncological outcomes improved dramatically. With the technique of Complete Mesocolic Excision (CME) with Central Vascular Ligation (CVL), the same surgical principles were introduced to the field of colon cancer surgery. Until now, current literature fails to invariably demonstrate its oncological superiority when compared to conventional surgery, and there are some concerns on increased morbidity. The aim of this study is to compare short-term outcomes after left-sided laparoscopic CME versus conventional surgery.

Methods: In this retrospective analysis, data on all laparoscopic sigmoidal resections performed during a 3-year period (October 2015 to October 2018) at our insititution were collected. A comparative analysis between the CME group - for left-sided colon cancer-and the non-CME group—-for benign disease-was performed.

Results: One-hundred sixty-three patients met the inclusion criteria and were included for analysis. Data on $66 \mathrm{CME}$ resections were compared with 97 controls. Median age and operative risk were higher in the CME group. One leak was observed in the CME group (1/66) and 3 in the non-CME group (3/97), representing no significant difference. Operative times were significantly longer in the CME group $(210$ versus $184 \mathrm{~min}-\mathrm{p}<0.001)$, and a trend towards longer pathological specimens in the CME group was noted ( 21 vs $19 \mathrm{~cm}-\mathrm{p}=0.059)$. Regarding hospital stay, postoperative complications, surgical site infections and intra-abdominal collections, no differences were observed. There was a slightly higher reoperation $(6.2 \%$ versus $1.5 \%$ $\mathrm{p}=0.243)$ and readmission rate $(6.2 \%$ versus $4.5 \%-\mathrm{p}=0.740)$ in the non-CME group during the first 30 postoperative days.

Conclusions: CME does not increase short-term complications in laparoscopic left-sided colectomies. Significantly longer operative times were observed in the CME group.

\section{O051-COLORECTAL_Malignant}

\section{DO ELDERLY PATIENTS WITH COPD BENEFIT FROM LAPAROSCOPIC COLECTOMY?}

\section{Z. Zhang ${ }^{1}$,H.R. Zhuo ${ }^{2}$, Z. Liu $^{3}$, X.S. Wang ${ }^{3}$}

${ }^{1}$ Department of Surgery, Department of Environmental Health Sciences, Yale School of Medicine, Yale School of Public Health, NEW HAVEN, United States of America; ${ }^{2}$ Surgery, Yale School of Medicine, NEW HAVEN, United States of America; ${ }^{3}$ Colorectal Surgery, National Cancer Center/Cancer Hospital, Chinese Academy of Medical Sciences, BEIJING, China

Aims: Chronic obstructive pulmonary disease (COPD) is a common disorder in elderly patients. The physiologic derangement of the older patients with COPD is highly challenging and may result in postoperative complications. The aim of this study is to determine the impact of open colectomy and laparoscopic colectomy on postoperative outcomes in elderly patients with COPD. Methods: We identified 2509 elderly patients with COPD, who were 75 years or older and underwent elective colectomy from the American College of Surgeons National Surgical Quality Improvement Program (ACS-NSQIP) Colectomy Targeted database (2012-2017). Patients were excluded if they were emergent cases, or with American Society of Anesthesiologists (ASA) physical status classification of 5 , ventilation requirement, sepsis, renal failure or wound infection. Multivariate logistic regression models were employed to assess the impact of different surgery types on postoperative complications. Infectious complications included four complication types of wound infection, pneumonia, urinary tract infection, and sepsis; pulmonary complications included pneumonia, pulmonary embolism, and reintubation, and cardiac complications included myocardial infarction and cardiac arrest.

Results: In the identified 1976 elderly population with COPD, 856 (43.3\%) patients underwen open colectomy and $1120(56.7 \%)$ laparoscopic colectomy. Compared to open colectomy, laparoscopic surgery was associated with decreased risk of various complications including infectious complications, pulmonary complications, cardiac complications, anastomotic leak, surgical site infection, wound dehiscence, ileus, bleeding requiring transfusion, LOS $=4$ days, 30 days mortality and reoperation. After adjusting for potential confounders, significantly decreased risk associated with laparoscopic colectomy was seen for infectious complications (OR $0.41,95 \%$ CI $0.22-0.75$ ) and pulmonary complications (OR $0.57,95 \%$ CI $0.40-0.83$ ). Conclusion: Our study suggested a decreased risk of infectious and pulmonary complications associated with laparoscopic colectomy compared to open colectomy for older patients aged 75 years or older with COPD.

\section{O052-COLORECTAL-Stoma}

\section{CONVENTIONAL VERSUS MINIMALLY INVASIVE HARTMANN TAKEDOWN. A META-ANALYSIS OF THE LITERATURE}

\section{F. Guerra ${ }^{1}$, C. del Basso ${ }^{2}$, C. Coletta ${ }^{2}$}

${ }^{1}$ General Surgery, AOMN-Ospedale San Salvatore Pesaro, PESARO, Italy; ${ }^{2}$ General Surgery, Policlinico Umberto I, ROME, Italy

Background: Although end colostomy closure following Hartmann's procedure is a major surgery which is traditionally performed by conventional celiotomy, over the last decade there has been a growing interest towards the application of different minimally invasive techniques. Objective: We aimed at evaluating the relative outcomes of conventional surgery versus minimally invasive surgery by meta-analyzing the available data from the medical literature. Data Sources: The PubMed/MEDLINE, Cochrane Library and EMBASE electronic databases were searched through August 2018

Study Selection: Inclusion criteria considered eligible all comparative studies evaluating open versus minimally invasive procedures. Conventional laparoscopy, robotic and single-port laparoscopy were considered as minimally invasive techniques.

Main Outcomes: Measures Overall morbidity, rate of anastomotic failure, rate of wound complications, and mortality were evaluated as primary outcomes. Perioperative details and surgical outcomes were also assessed.

Results: The data of a total of 13,740 patients from 26 studies were eventually included in the analysis. There were no significant differences on baseline characteristics such as age, BMI and proportion of high-risk patients in the two groups of patients. As compared to the conventional technique, minimally invasive surgery proved significantly superior in terms of postoperative morbidity, length of hospital stay and rate of incisional hernia.

Limitations: The lack of randomization and the presence of different reporting biases within the primary studies may have affected the general results.

Conclusions: The current evidence from the literature suggests that minimally invasive surgery should be the preferred method of performing Hartmann's reversal.

Key-Words: Hartmann's reversal; minimally invasive surgery; diverticular disease; end colostomy closure; meta-analysis. 


\section{O053-COLORECTAL-Stoma}

SYSTEMATIC REVIEW, META-ANALYSIS AND METAREGRESSION OF INCISIONAL HERNIA POST REVERSAL OF ILEOSTOMY

\section{S.A. Antoniou ${ }^{1}$, N.L. Bullen ${ }^{1}$, S. Antoniou ${ }^{2}$, N.J. Smart ${ }^{1}$ \\ ${ }^{1}$ Surgical Service, Royal Devon \& Exeter NHS Foundation Trust, EXETER, United Kingdom; ${ }^{2}$ Department of Vascular \& \\ Endovascular Surgery, The Royal Oldham Hospital, MANCHESTER, United Kingdom}

Aim: Incisional hernia following closure of loop ileostomy is a pragmatic problem. Assessment of the incidence of this complication is limited by small sample sizes and inconsistent reporting among studies. The aim of this study was to provide an estimate of the incidence of incisional hernia following closure of loop ileostomy according to clinical and radiological diagnostic criteria and to investigate the association of bibliometric and study quality parameters with reported incidence. Methods: A systematic review of PubMed, Embase, CENTRAL, ISRCTN Registry and Open Grey from 2000 onwards was performed according to PRISMA standards. Reporting on the type of stoma and mesh reinforcement after closure was mandatory for inclusion, whereas studies on paediatric populations were excluded. Fixed effect or random effects models were used to calculate pooled incidence estimates. Meta-regression models were formed to explore potential heterogeneity. Results: Forty-two studies with7166 patients were included. Thepooled estimate of the incidence of incisional hernia after ileostomy closure site was $6.1 \%$ (95\% confidence interval, CI $4.4 \%$ to $8.3 \%$. Significant between study heterogeneity was identified $\left(\mathrm{P}<0.001, \mathrm{I}^{2}=87 \%\right)$ and the likelihood of publication bias was high $(\mathrm{P}=0.028)$. Mixed effects regression showed that both year of publication $(\mathrm{P}=0.034, \mathrm{Q}=4.484, \mathrm{df}=1.000)$ and defining hernia as a primary outcome $(\mathrm{Q}=20.298, \mathrm{P}<0.001)$ were related to effect size. Method of follow-up and quality of the studies also affected the reported incidence of hernia.

Conclusion: The incidence of incisional hernia at ileostomy closure site is estimated at $6.1 \%$. Reporting incisional hernia as primary or secondary outcome, the method of diagnosis, the year of publication and methodological quality are associated with reported incidence.Registration CRD42018092400.

\section{O054-HEPATO-BILIAIRY \& PANCREAS—Gallbladder}

\section{UTILITY OF FLUORESCENT CHOLANGIOGRAPHY IN LAPAROSCOPIC CHOLECYSTECTOMY FOR MODERATE CHOLECYSTITIS}

H. Kitamura, M. Kurokawa, T. Tsuji, D. Yamamoto, T. Takahashi, H. Bando

Department of gastroenterological surgery, Ishikawa prefectural central hospital, ISHIKAWA, Japan

Aim: Fluorescent cholangiography (FC) after intravenous injection of indocyanine green (ICG) is currently a promising new technique for improved intraoperative recognition of biliary anatomy. There have been few reports on the availability of FC in laparoscopic cholecystectomy (LC) for moderate cholecystitis. We aimed to evaluate the potential of FC during LC for moderate cholecystitis. Methods: Surgical outcomes of 100 cases (FC; $n=38$, control; $n=62$ ) that required LC for managing moderate cholecystitis during January 2009-October 2018 were analyzed retrospectively. We also reviewed video recordings of the laparoscopic procedure and analyzed the identification rate of the common bile duct, common hepatic duct and cystic duct. Surgical procedure: Intravenous injection of $2.5 \mathrm{mg}$ ICG was administered prior to the beginning of surgery. FC was used to identify extrahepatic biliary structures before and after partial and complete dissection of Calot's triangle. LC was performed using standard procedures.

Results: The visualization rate of the common bile duct, common hepatic duct, and cystic duct using FC were $61.2 \%, 35.5 \%$, and $12.9 \%$ before dissection, respectively and $93.3 \%, 80 \%$, and $63.3 \%$ after complete dissection, respectively. There were no significant differences in age, sex, body mass index, acute cholecystitis and CRP between FC and control groups. Median operative time was similar in FC $(117 \mathrm{~min})$ and control $(112 \mathrm{~min})$ groups $(\mathrm{p}=0.25)$. Estimated blood loss was significantly higher in FC group ( $50 \mathrm{ml}$ versus $3 \mathrm{ml} ; \mathrm{p}<0.01$ ). There was no post-operative bile leakage in the FC group, however there was one case with post-operative bile leakage that required reoperation in the control group. Abdominal abscess was observed in one patient in the $\mathrm{FC}$ group and four patients in the contro group. Conversion to open surgery was needed for one (2.6\%) patient in the FC group and six $(9.7 \%)$ patients in the control group $(\mathrm{p}=0.25)$. Partial cholecystectomy was performed for two $(5.2 \%)$ patients in the FC group and $10(16.1 \%)$ patients in the control group $(\mathrm{p}=0.13)$.

Conclusions: FC during LC may be useful for preventing bile leakage. However, persistence to dissect Calot's triangle using FC may increase bleeding.
O055-HEPATO-BILIAIRY \& PANCREAS—Gallbladder

TRANSDUCTAL VERSUS TRANSCYSTIC CBD STONE EXTRACTION: LESSONS LEARNT AFTER FOUR-HUNDRED LAPAROSCOPIC COMMON BILE DUCT EXPLORATIONS

L. Navaratne, J. al-Musawi, A. Isla

Upper GI Surgery, Northwick Park Hospital, LONDON, United Kingdom

Aims: Laparoscopic cholecystectomy and laparoscopic CBD exploration (LCBDE) is recommended for the treatment of CBD stones with gallbladder in situ. LCBDE can be achieved by transductal (via choledochotomy) or transcystic stone extraction. The aim of this paper was to report our experience of transductal and transcystic LCBDE from over four-hundred patients. Methods: A retrospective analysis was performed from our prospectively maintained database of 415 consecutive LCBDEs between February 1998 and December 2018. Patients were divided into two groups: transductal (TD) and transcystic (TC) LCBDE. Demographic, pre- and intraoperative data were collected. Outcome measures included successful stone clearance, mortality, morbidity, conversion to open surgery and post-operative length of hospital stay.

Results: The TD group consisted of 242 patients whereas the TC group had 173 patients. There were no significant differences in age, pre-operative bilirubin, number of CBD stones and operative times between the groups $(0.09,0.09,0.20$ and 0.38 respectively). TD group had larger CBD diameter when compared to the TC group $(12.8 \mathrm{~mm}$ vs $10.6 \mathrm{~mm}, \mathrm{P}<0.0001)$. Holmium laser lithotripsy was used significantly more in TC stone extraction $(16.8 \%$ vs $2.9 \%$ of cases, $\mathrm{p}<0.0001)$. TC LCBDE was associated with higher successful stone clearance rates $(99.4 \%$ vs $94.2 \%, \mathrm{p}=0.0057)$, less morbidity (6.4\% Clavien-Dindo I-II and 1.2\% Clavien-Dindo III-IV vs $21.9 \%$ Clavien-Dindo I-II and $5.0 \%$ Clavien-Dindo III-IV, $\mathrm{p}<0.0001)$, less conversion to open surgery ( $0 \%$ vs $2.1 \%$, $\mathrm{p}=0.0010$ ) and reduced post-operative length of hospital stay (median 1 vs 5 days, $\mathrm{p}<0.0001$ ). The incidence of post-operative pancreatitis was significantly less in the TC group when compared to the TD group $(0.6 \%$ vs $7.0 \%, p=0.0010)$. There was a trend towards a lower incidence of bile leak following TC when compared to TD stone extraction, however, this did not quite reach significance $(1.2 \%$ vs $5.0 \%, \mathrm{p}=0.0504)$. There was no difference between the groups in mortality Conclusion: TC stone extraction should be considered the gold standard approach to LCBDE. TC LCBDE is associated with higher success rates and reduced incidence of post-operative pancreatitis and bile leak. The use of holmium laser lithotripsy enables higher rates of TC exploration, where the alternative would have resulted in choledochotomy.

\section{O056-HEPATO-BILIAIRY \& PANCREAS-Gallbladder}

\section{COGNITIVE CHANGES WITH TRAINING AND EXPERTISE IN MINIMALLY INVASIVE SURGERY}

K.F. Koeppinger, K.F. Kowalewski, L. Seifert, M.W. Schmidt, F. Lang, F. Nickel, B.P. Müller-Stich

Department of General, Visceral, and Transplantation Surgery, University of Heidelberg, HEIDELBERG, Germany

Introduction: Laparoscopic surgery has benefits for patients, for surgeons however it prolongs the learning curve. Throughout the learning curve changes of cognition occur, which lead to an improved adaption to circumstances in the operative setting. In expert surgeons these changes have become implicit knowledge. Goal of this study was to analyse the cognitive changes with regard to growing expertise. Material and Methods: Surgeons with different levels of experience were enrolled (novices, intermediates, experts). Participants were asked to perform a standardised laparoscopic knot. The Thinking Aloud technique was used to record path of thoughts. For semantic annotation Atlas.ti software was used. Eye tracking (Pupil Labs, Germany, Berlin) was used to record number of eye movements and further parameters.

Results: With growing experience time needed for task completion decreased (novices $450+242.9 \mathrm{~s}$; intermediates $264+29.0 \mathrm{~s}$; experts $96.2+13.3 \mathrm{~s} ; \mathrm{p}=0.006)$. In eye tracking the total points of fixation correlated negatively with experience (novices: $4023 \pm 2345$; intermediates $249.3 \pm 33$; experts $104.3 \pm 22.1 ; \mathrm{p}=0.009$ ). Furthermore, differences not only occurred in quantitative analysis but in local distribution of view on the laparoscopic screen (img. 1). Semantic analysis of participant's recordings showed that experts referred to standardised procedures. In the novice group however, the starting position of the needle (PN) was not controlled properly (60\%) and the conclusion was not drawn until further mistrials $(40 \%)$. The intermediate group already possessed the knowledge that the correct PN was necessary $(80 \%)$. The ability to correctly manipulate the needle was not in the novice and the intermediate group $(0 \%, 20 \%)$. The expert group was capable to grab the needle correctly or to reposition it properly after evaluation.

Discussion: Results show cognitive differences between experience groups. Awareness and explicit targeting of the cognitive learning procsses, which are usually implicit, seems to make training more efficien and specific. Semantic annotation and eye-tracking help to decode the steps needed to reach expertise. The used methods open opportunies to design training sessions in a way which is more cut to the individual trainee with aimed feedback and exercises dependent on the current level of experience and expertise. 


\section{O057-HEPATO-BILIAIRY \& PANCREAS-Gallbladder}

EFFICACY AND SAFETY OF THE HOLMIUM LASER IN COMMON BILE DUCT EXPLORATION

\section{$\underline{\text { J. al-Musawi }}{ }^{1}$, L. Navaratne, A. Navarro-Sanchez, A. Martinez-Isla,} Upper GI Surgery, Northwest London Hospitals, LONDON, United Kingdom

Aims: The use of laser has become one of the adjuncts in laparoscopic common bile duct exploration (LCBDE) for the management of large and impacted stones, as well as to increase the transcystic rate.

The aim of this work is to review the efficacy and safety of the holmium laser in our practice of LCBDE.

Methods: Between 2014 and 2018, thirty six Laser Assisted Bile duct Exploration by Laparoendoscopy (LABEL) were performed, utilising holmium laser lithotripsy, in the same period 178 LCBDE were conducted. The primary outcome measures were the success rate of transcystic stone clearance using LABEL, which may have otherwise required a choledochotomy, and the feasibility of transductal stone clearance that otherwise would have been a failure requiring postoperative ERCP. Secondary outcome measures were general and laser related complications.

Results: During the study period 36 LABEL procedures were conducted. In the same period 178 patients underwent $\mathrm{LCBDE}$ (in $81 \%$ the transcystic route was used). Stone clearance was achieved in all but one case. The transcystic use of LABEL avoided choledochotomy in 28 patients, and in the 8 patients who required choledochotomy its use most likely avoided failure of the LCBDE.

Within our series, $6 \%$ (2/36) of patients developed medical complications (Clavien-Dindo I and II). There were no specific complications of laser application, despite having recorded mucosal damage after lengthy lithotripsies secondary to lateral deflection of the energy.

Conclusions: The Holmium laser is a safe and valuable adjunct in LCBDE, increasing its success rate and hopefully decreasing the complications by enhancing the transcystic route.

\section{O058-HEPATO-BILIAIRY \& PANCREAS—Gallbladder \\ EVALUATION OF LAPAROSCOPY IN SYMPTOMATIC GALLBLADDER REMNANT}

\section{M.K. Choudhury}

Laparoscopic Surgery, G I Surgery and Endo Laparoscopy Center, GUWAHATI, India

In difficult calot's dissection during cholecystectomy, part of the gallbladder is left behind to prevent bile duct or hepatic vascular injury. The gallbladder remnant subsequently remains embedded in fibrous adhesions with the surrounding structures. So laparoscopic adhesiolysis and removal of symptomatic gall bladder remnant poses a challenge.

We are presenting our experience of laparoscopic removal of fourteen symptomatic gallbladder remnants to evaluate its feasibility and safety.

Method: Fourteen cases were operated during the period from December 2012 to novembe 2017. Ultrasound was the routine diagnostic investigation. MRCP was done in all the cases to know the detailed biliary anatomy.

Ports were made as in conventional lap cholecystectomy. Left sub costal port was made in selected cases where difficulty was encountered in first entry. Post operative adhesions were carefully released. Fibrous adhesions around the gall bladder remnant and in the sub hepatic area were carefully released. Energy sources like bipolar and ultrasonic scissors were useful in these situations. Gall bladder remnant was dissected out up to the Cystic duct opening. Cystic duct was either clipped or ligated before excision. Operated area was re-examined for bleeding or bile leakage and irrigated with normal saline. Drainage was applied in selected cases.

Results: Out of 14 cases ( 11 cases of post lap cholecystectomy, 3 cases of post open cholecystectomy) nine cases were remnant gallbladder ( 3 cases with acute inflammation, 1 case with acalculous cholecystitis, one with type1 mirrizzi) and five cases were cystic duct remnant( 2 anomalous cystic ducts, one type1 mirrizzi) We had one minor bile duct injury at cystic duct-CBD angle which was repaired with a t-tube drainage. Another post operative sub capsular hematoma of right lobe of liver was encountered, treated conservatively and was resolved. Pre operative ERCP was done in two cases with CBD calculi. LCBDE was done in one case simultaneously. Average hospital stay was 2-4 days.

Conclusion: Laparoscopic removal of gallbladder remnant is difficult due to fibrous adhesions with the surrounding vital structures. Expertise and the assistance of technology are required for safe removal.
O059-HEPATO-BILIAIRY \& PANCREAS-Gallbladder

EFFICACY OF FLUORESCENT CHOLANGIOGRAPHY IN THE IDENTIFICATION OF CYSTIC DUCT ANATOMY IN COMPARISON TO MAGNETIC RESONANCE COLANGIOPANCREATOGRAPHY

A. Pesce $^{1}$, G. la Greca ${ }^{1}$, L. Esposto ${ }^{2}$, B. Basile ${ }^{2}$, S. Puleo $^{2}$, S. Palmucci ${ }^{2}$

${ }^{1}$ Department of Medical, Surgical Sciences and Advanced Technologies „G.F. Ingrass, University of Catania, CATANIA, Italy; ${ }^{2}$ Department of Medical, Surgical Sciences and Advanced Technologies, University Hospital Policlinico, CATANIA, Italy

Aims: Bile duct injury (BDI) represents the most serious complication of LC, with an incidence of $0.3-0.7 \%$ resulting in a significant impact on quality of life, overall survival and frequent medico-legal litigations. Near-infrared fluorescent cholangiography (NIRF-C) represents a nove intra-operative imaging technique that allows a real-time enhanced visualization of the extrahepatic biliary tree by fluorescence. The primary aim of this preliminary study was to evaluate the effectiveness of NIRF-C in the detection of cystic duct-common hepatic duct anatomy intraoperatively in comparison with pre-operative magnetic resonance cholangio-pancreatography (MRCP).

Methods: Data from 26 consecutive patients with symptomatic cholelithiasis or chronic cholecistitis, who underwent elective laparoscopic cholecystectomy with intra-operative fluorescent cholangiography and pre-operative MRCP examination between January 2018 and May 2018, were analyzed. Three selected features of the cystic duct-common hepatic duct anatomy were identified and analyzed by the two different imaging methods: insertion of cystic duct cystic duct-common hepatic duct junction and cystic duct course

Results: Fluorescent cholangiography was performed successfully in all twenty-six patients undergoing elective laparoscopic cholecystectomy. The visualization of cystic duct was reported in 23 out of 26 cases, showing an overall diagnostic accuracy of $86.9 \%$. The level of insertion, course and wall implantation of cystic duct were achieved by NIRF-C with diagnostic accuracy values of $65.2 \%, 78.3 \%$ and $91.3 \%$, respectively in comparison with MRCP data. No bile duct injuries were reported.

Conclusion: Fluorescent cholangiography can be considered a useful, safe and low-cost imaging modality comparable to MRCP for intra-operative visualization of the cystic duct-common hepatic duct anatomy during elective laparoscopic cholecystectomies.

\section{O060-HEPATO-BILIAIRY \& PANCREAS—Gallbladder}

\section{SELECTIVE HISTOLOGICAL EXAMINATION AFTER CHOLECYSTECTOMY; AN ANALYSIS OF CURRENT DAILY PRACTICE IN THE NETHERLANDS}

\section{B.J.G.A. Corten, W.K.G. Leclercq, R.M.H. Roumen, G.D. Slooter} Surgery, MMC Veldhoven, EINDHOVEN, The Netherlands

Background: The 2016 Dutch national guidelines on handling of a removed gallbladder for cholelithiasis favors a selective histopathologic policy (Sel-HP) rather than routine policy (RoutHP). The aim of this study was to determine the current implementation of the present guideline and the daily practice of Sel-HP.

Methods: Surgeons who were engaged in gallbladder surgery and were involved in local hospitals' gallbladder protocols completed a questionnaire study between December 2017 and May 2018. Data were analyzed using standard statistics.

Results: A $100 \%$ response rate was obtained $(\mathrm{n}=74)$. Approximately $64 \%$ of all gallbladder $(\mathrm{n}=22500)$ are currently examined microscopically. Sixty-nine $(93.2 \%)$ hospitals confirmed they were aware of the new guidelines, and $56(75.7 \%)$ knew the guideline was adjusted in favor of Sel-HP. Half of the hospitals $(\mathrm{n}=35,47.3 \%)$ has adopted a Sel-HP, and $39(52.7 \%)$ a RoutHP. Of the 39 hospitals who currently practiced a Rout-HP, 36 are open to a transition to a Sel-HP although some expressed the need for more evidence on safety or novel guidelines.

Conclusions: The current implementation of the 2016 Dutch guideline advising a selective microscopic analysis of removed gallbladders for gallstone disease is suboptimal. Evidence demonstrating safety and cost-effectiveness of an on demand histopathological examination will aid in the implementation process. 


\section{O061-HEPATO-BILIAIRY \& PANCREAS-Gallbladder}

NEW APPLICATION OF FLUORESCENCE CHOLANGIOGRAPHY FOR REVISION OF COMMON BILE DUCT IN CHOLEDOCHOLITHIASIS

\section{A.V. Malynovskyi, M. Maiorenko, I.I. Horbachuk}

Department of Robotic and Endoscopic Surgery, Odessa national medical university, ODESSA, Ukraine

Aims: Intraoperative cholangiography remains the 'gold standard' of revision of common bile duct (CBD) for choledocholithiasis. There might be some cases of transient choledocholithiasis combined with calculous cholecystitis with a narrow or borderline diameter of CBD, including cases of hepatitis. In these cases, the probability of finding the stones in CBD is very low, and the revision of the narrow CBD may be difficult. We have invented a new application of fluorescence cholangiography (FC) for assessment of the passage of bile through CBD. According to the new method, the fluorescence of indocyanine green was detected in the initial parts of small intestine. The aim of study was to assess the specificity and sensitivity of FC for the revision of CBD. Methods: Six patients underwent laparoscopic cholecystectomy for acute calculous cholecystitis combined with obstructive jaundice or biliary hypertension. One patient had progressive jaundice, 3 patients had transient jaundice, and 2 patients had fatty pancreatic necrosis and biliary hypertension.

Results: In 5 of 6 cases the fluorescence was detected in initial parts of small intestine, that proved normal passage of the bile. In all of these patients, the diameter of CBD was normal or borderline. In one of these cases, the jaundice subsided by the time of surgery and no revision was needed. In another 2 cases, where choledocholitiasis was combined with viral hepatitis, CBD drainage was performed via the cystic duct. The same was done for 2 patients with pancreatic necrosis. In one patient with progressive jaundice and dilated CBD, the fluorescence was not detected nether in CBD, nor in small intestine. Choledochoscopy showed stones, which were removed, and T-tube was placed. In all cases of CBD drainage, postoperative cholangiography showed no stones.

Conclusions: 1. In this limited series of cases, the new application of FC showed $100 \%$ of specificity and sensitivity in the revision of CBD. 2. FC may be sufficient to prove the absence of stones in cases of transient jaundice and narrow or borderline CBD. 3. More cases collection is needed to delignate the role of $\mathrm{FC}$ in revision of $\mathrm{CBD}$, including comparing with intraoperative cholangiography.

\section{O062-HEPATO-BILIAIRY \& PANCREAS—Gallbladder}

\section{LEARNING CURVE FOR SINGLE-PORT SURGERY-CUSUM ANALYSIS OF 1010 CASES}

M. Asakuma, R. Iida, Y. Imai, Y. Inoue, K. Koji, T. Shimizu, F. Hirokawa, K. Uchiyama

General and Gastroenterological Surgery, Osaka Medical College, TAKATSUKI OSAKA, Japan

Aims: To evaluate the learning curve of Single-Port Cholecystectomy (SPC) by cumulative summation (CUSUM) analysis. As reported in Br J Surg. (2011 Jul: 98), we started performing SPC in 2009 and developed 1010 cases by December, 2018. The study addresses the need for additional data about the CUSUM learning curve of SPC, which is a relatively new procedure. Methods: A total of 1010 SPC cases were included in this study. Cases of open conversion an port addition were excluded from the analysis. As a result, 920 pure SPC cases were evaluated by CUSUM analysis. In order to evaluate whether operative times were within the control limit, an Exponentially Weighted Moving Average (EWMA) analysis was used for each surgeon.

Results: Forty-eight surgeons took part in a total of 920 cases with a mean operative time of $89.4 \pm 31 \mathrm{~min}$. CUSUM analysis of each surgeon showed three phases: 20 cases in the developing phase, and 60 cases in the challenging phase were needed to reach the matured third phase. EWMA analysis showed only two of 47 surgeons were out of the upper limit of the developing phase. These two surgeons are the main developers of SPC at our institution. The operative times of the remaining 45 surgeons were within the control limit.

Conclusions: In the next decade, minimally invasive surgery will continue to change through the use of robotics, single-port, and/or reduced-port concepts. This study addresses the lack of basic data on single-port surgery. The learning curve of SPC is divided into three phases by CUSUM analysis. On average, it needs 20 cases to reach the developing phase and 60 cases to reach the matured phase, despite individual variations. A trainee will be a proctor through experience of 60 cases. According to EWMA analysis, when an institution introduces SPC, care needs to be taken as some cases may be out of the upper limit in the early phase. Properly supervised, however, SPC is a safe procedure that can be standardized.
O063-HEPATO-BILIAIRY \& PANCREAS-Gallbladder

COMPARISON OF ROBOTIC SINGLE-SITE CHOLECYSTECTOMY AND SINGLE-INCISION LAPAROSCOPIC CHOLECYSTECTOMY USING A PROPENSITY SCORE MATCHING

\section{S.H. Choi, J.W. Lee}

Surgery, Bundang CHA Medical Center, SEONGNAM-SI, Korea

Introduction: Robotic single-site cholecystectomy (RSSC) has been known to have some advantages such as reducing stress of the surgeon compared to single incision laparoscopic cholecystectomy (SILC). However, there are few studies comparing the perioperative outcomes of these two operative methods.

Patient and methods: Between March 2014 and February 2018, 145 RSSC and 268 SILC were performed for benign gallbladder disease in our center. Propensity score matching was performed to control variables including sex, age, body mass indes (BMI), diagnosis, American society of anesthesiologist (ASA) score and 145 cohorts were selected among the SILC group through 1:1 matching. The perioperative data of these 290 patients were analyzed retrospectively. The diagnosis was classified into acute cholecystitis, chronic cholecystitis, and gallbladder polyp.

Results: Patient demographics between the two groups were evenly matched. Total operation time including docking time was slightly longer in RSSC group ( $48.1 \mathrm{~min}$ vs. $42.6 \mathrm{~min}$, $\mathrm{P}<0.001$ ), but real working time except the docking or set-up was shorter in RSSC group (19.2 min vs. $23.5 \mathrm{~min}, \mathrm{P}<0.001$ ). Conversion to additional robotic arm or additional port was frequent in SILC group ( 0 vs. 5 cases, $P=0.03)$. Intraoperative bile spillage rate $(13.8 \%$ vs. $11.7 \%, \mathrm{P}=0.725)$ and postoperative hospital stay (1.8 days vs. 1.7 days, $\mathrm{P}=0.091)$ were comparable in both group.

Conclusion: Both surgical procedures performed safely. But the RSSC demonstrated the better performance of the operation with shorter working time and the advantage of overcoming unexpected difficulties during the surgery with low conversion rate compared to SILC.

\section{O064-HEPATO-BILIAIRY \& PANCREAS—Gallbladder}

\section{FLUORESCENCE CHOLANGIOGRAPHY VERSUS CONVENTIONAL LAPAROSCOPY IN DIFFICULT CHOLECYSTITIS}

\section{A.V. Malynovskyi, M. Maiorenko, S.Yu. Badion}

Department of Robotic and Endoscopic Surgery, Odessa national medical university, ODESSA, Ukraine

Aims: The most important technical step of laparoscopic cholecystectomy (LC) is achieving 'critical view of safety'. It may be difficult in severe acute cholecystitis with perivesicular mass, blocked gallbladder and long course of chronic cholecystitis. The aim of study was to compare fluorescence cholangiography (FC) and conventional laparoscopy for identification of anatomy in difficult LC.

Methods: FC was performed in 10 patients with severe acute and chronic cholecystitis (group 1). Conventional laparoscopy was used in 12 patients with severe acute and chronic cholecystitis (group 2). The ultrasound criteria to include the patients to group 1 were: thickening of gallbladder wall (more than $5 \mathrm{~mm}$ ), blocked gallbladder, intrahepatic gallbladder, and perivesicular mass. The clinical criteria to include the patients to group 1 were: progressive course of acute cheolesystitis, and history of multiple attacks in chonic cholecystitis. FC was performed using Karl Storz equipment. Indocyanine green intravenous injection was done 40 min before start of dissection.

Results: The mean duration of operation was $2 \mathrm{~h}(1.5-3 \mathrm{~h})$ in group 1, and 2,5 h $(1.5-5 \mathrm{~h})$ in group 2 . The mean time to achieve 'critical view of safety' was $25 \mathrm{~min}$ (15-45 min) in group 1 and $45 \mathrm{~min}(20-60 \mathrm{~min})$ in group 2 . Visual analogue scales of the comfort of dissection showed better scores in group 1 . No convertions and complications were observed in the both groups. Conclusions: 1. FC allows to decrease the time to achieve 'critical view of safety' and duration of the entire procedure of difficult LC. 2. FC makes dissection more comfortable for surgeon in difficult LC. 2. More cases collection is necessary to determine the indications for selective use of FC. 


\section{O065-HEPATO-BILIAIRY \& PANCREAS-Gallbladder}

PRIMARY DUCT CLOSURE WITH AND WITHOUT ENDOBILIARY STENT AFTER LAPAROSCOPIC COMMON BILE DUCT EXPLORATION FOR CHOLEDOCHOLITHIASIS

V.V. Grubnik ${ }^{1}$, V.V. Ilyashenko ${ }^{2}$, V.V. Grubnyk ${ }^{2}$, A.L. Kovalchuk ${ }^{3}$

${ }^{1}$ Department of surgery, Odessa medical university, ODESSA, Ukraine; ${ }^{2}$ Department of Surgery \#1, Odessa medical university, ODESSA, Ukraine; ${ }^{3}$ Surgical department, Ternopil medical university, TERNOPIL, Ukraine

Aim: Of the study was to compare outcomes of primary duct closure (PDC) with and without endobiliary stent.

Methods and Procedures: For period from 2013 to 2018 laparoscopic common bile duct exploration with choledochotomy was performed in 87 patients. In 15 patients, closure of choledochus was done with T-tube drainage. PDC was performed in 72 patients. In 37 patients (I group), PDC was done with anterograde insertion of endobiliary stent and in the 35 patients (II group) without stent. There were 54 women and 18 men, age from 29 to 82 years. Mean age was $63.7 \pm 12.5$ years. These patients had no signs of acute biliary pancreatitis, severe acute cholangitis, and suspected biliary neoplasia. The both of group were comparable regarding sex, age, comorbidities, diameter of common biliary duct, number of concrements. The groups were evaluated according to the following criteria: postoperative complications, postoperative biliary-specific complications, re-intervention (radiology, endoscopy, and surgery), and postoperative hospital stay.

Results: There were no serious intraoperative complications in the patients of both groups. Mean operative time was $92.4 \pm 22.5 \mathrm{~min}$ in the I group and $86.2 \pm 28.6 \mathrm{~min}$ in the II group $(\mathrm{p}>0.1)$. There were no significant postoperative complications such as postoperative bleeding, bile leakage or biliary stricture in the patients of I group. In the II group, 4 patients had bile leakage, lesions of leakage were retained stones in 3 patients and 1 patient had stricture of ampulla. Reinterventions were done in all four patients: endoscopic papillotomy —in three patients, relaparoscopy with insertion of T-tube in one patient. There were no mortalities in the patients of both groups. Mean hospital stay was $3.4 \pm 1.5$ days for I group and $6.5 \pm 1.2$ days for II group $(\mathrm{p}<0.05)$

Conclusions: PDC after laparoscopic common bile duct exploration is safe and feasible treatment modality. In the cases with multiple common bile duct stones and ampulla stenosis, anterograde insertion of endobiliary stent prevent biliary leakage.

\section{O066-HEPATO-BILIAIRY \& PANCREAS-Gallbladder}

\section{LAPAROSCOPIC CHODOCHODUODENOSTOMY-A} DELIBERATE AS WELL AS A RESCUE PROCEDURE

\section{A. Ali, A. Saha}

Dept of GI, HPB, Bariatric \& Minimal Access Surgery, Venkateshwar Hospital, NEW DELHI, India

Aims: To check the feasibility of Laparoscopic Choledochoduodenostomy (LCD) for failed $\mathrm{ERCP}$ as well as a rescue procedure for intraoperative complications.

Patients \& Methods: Fourteen patients underwent choledochoduodenostomy between April 2016 and November 2018. Eight underwent Open Choledochoduodenostomy (OCD) and six underwent LCD. Four had Gallstones alongwith Common Bile Duct (CBD) stones, while one had CBD stones presenting 5 years after Laparoscopic Cholecystectomy. One patient had a duodenal injury during a difficult dissection for Laparoscopic CBD exploration (LCDE). All patients had undergone a preoperative MRCP and an ERCP that failed to clear the CBD of stones, and a stent was placed. All patients had a CBD diameter of not less than $1.5 \mathrm{~cm}$. OCD were performed through a Kocker's incision. LCD were done using 4-5 ports. A $24 \mathrm{Fr}$ abdominal drain was placed in all cases, and removed at discharge.

Results: All patients were started on liquids next day of surgery. There were no anastomotic leaks, no bile collections. One OCD patient has a superficial surgical site infection. All LCD cases were discharged on day 3 post op and OCD were discharged between post-op day 3 and 5 . Conclusion: LCD is feasible and desirable in cases of large impacted CBD stones where ERCP has failed. At times, LCD can be used as a rescue procedure for some duodenal injuries during hepato-biliary surgeries.

\section{O067-HEPATO-BILIAIRY \& PANCREAS—Gallbladder} ENDOSCOPIC TREATMENT OF DIFFICULT BILE DUCT STONES

O.I. Lytvyn, P.V. Ogorodnik, A.G. Deinychenko, N.A. Yermak, O.I. Lytvyn

Laparoscopic surgery, National institute of surgery and transplantology, KYIV, Ukraine

The treatment of patients with bile duct stones is in a stage of evolution. In cases of difficult bile duct stones ordinary endoscopic methods (ERCP/balloon-basket extraction) fail. This study aimed at comparing the success rate and complications between mechanical lithotripsy (ML) and large balloon dilation (LBD) after endoscopic sphincterotomy.

Patient and Methods: Over a 8-year period, from 2010 to 2018, 1257 patients presented with difficult bile duct stones. The patients were divided into 2 groups : group A comprising 1120 patients treated by ML and group B comprising 137 patients treated by LBD. All patients underwent endoscopic sphincterotomy initially.

Results: Stones larger than $15 \mathrm{~mm}$, square-shaped stones, multiple bile duct stones and hard stones were observed in 718 cases. Anatomical variations which makes accessibility to the papilla challenging such as the presence of periampullary diverticulum, altered anatomy, narrow dista bile duct, duodenal or bile duct stricture occurred in 539 cases. The success rate for bile duct clearance was $92.5 \%$ and $94 \%$ for ML and LBD, respectively. The overall complication rate in this study was $10.8 \%$. The complication rate was $8.1 \%$ and $12.5 \%$ for LBD and ML, respectively. Patients treated by LBD, after EST, were prone to less adverse events than patients treated by lithotripsy after sphincterotomy and the difference was statistically significant $(P=0.04)$.

Conclusion: The LBD and ML are both acceptable in the treatment of patients with difficult bile duct stones. Endoscopic sphincterotomy followed by LBD is a safe and effective treatment for difficult bile duct stones in comparison with sphincterotomy followed by ML.

\section{O068-HEPATO-BILIAIRY \& PANCREAS-Gallbladder}

\section{INTRA-GALLBLADDER OR SYSTEMIC INDOCYANIDE GREEN INJECTION FACILITATE LAPAROSCOPIC CHOLECYSTECTOMY}

\section{Y.Y. Liu}

General surgery, Chang gung memorial hospital kaohsiung division, KAOHSIUNG, Taiwan

Purpose: Laparoscopic cholecystectomy(LC) is the one of most common procedure done by minimal invasive surgery worldwide but the common bile $\operatorname{duct}(\mathrm{CBD})$ injury still happened, especial in cholecystitis. Image guided surgery created new concept for fluorescent cholangiography to demonstrate the anatomy of CBD by using indocyanine green (ICG) intravenous injection before operation to decreased complication. The result is positive but the border of gallbladder can't be seen very well in systemic injection. In cholecystitis, the border between gallbladder and common bile duct is importan as well as CBD and cystic duct. We hypothesized injection of ICG into gallbladder directly will be helpful to identify cystic duct, CBD and the border of gallbladder as well as systemic injection. The purpose of this study was to evaluate feasibility of this image guide surgery

Material and method: This is a IRB proven prospective study. Total 120 patients were involved this study. Arm 1: ICG injected via Gallbladder; Arm 2: ICG injected via IV access; Arm 3: control. Three laparoscopic ports were introduced and the pneumoperitoneum $(12 \mathrm{mmHg})$ was established. A near-infrared optimized laparoscope was used to detect the ICG fluorescence signal arising from gallbladder, cystic duct and common bile duct before cholecystectomy According to the enhancement of ICG, the cholecystectomy was started from cystic duct in Calot's triangle. Time to gallbladder removed was recorded. Conversion rate, post-operative morbidity and mortality will be recorded as well .

Results: Overall, in all the patients divided into arm 1,2,3, the Hartman pouch was identified in $92.89,75 \%$ of cases, the cystic duct $: 84.7,55,3,45 \%$, the CBD in $78.2,90,60,3 \%$, and the CHD $63,83.3,33 \%$. No conversion, mortality and major complication including post-op bile leak in our study. Wound infection rate was $0.5 \%$ Median days for admission was 3.45 days.

Conclusions: Clinical translation of near-infrared fluorescence cholangiography has been successful with a visualization of biliary anatomy no matter injection from gallbladder or systemic circulation. It can be considered in difficult cases to increase the safety of laparoscopic cholecystectomy. 


\section{O069-HEPATO-BILIAIRY \& PANCREAS-Gallbladder}

C-REACTIVE PROTEIN IS THE BEST BIOMARKER TO PREDICT ADVANCED ACUTE CHOLECYSTITIS AND CONVERSION TO OPEN SURGERY

M. Bouassida, S. Zribi, G. Laamiri, I. Ben Smail, S. Sassi, M.M. Mighri, H. Touinsi,

Department of Surgery, Mohamed Tahar Maamouri Hospital, NABEUL, Tunisia

Aims: The 2018 Tokyo guidelines for acute cholecystitis (AC) use white cell count (WCC) as one of the severity criteria.

The aims of this study were to evaluate the discriminative powers of common inflammatory markers (neutrophil-to-lymphocyte ratio (NLR), and C-reactive protein (CRP)) compared with WCC for the severity of $\mathrm{AC}$, and the risk for conversion to open cholecystectomy and to determine their diagnostic cutoff levels. Methods: This was a retrospective cohort study. Over 5 years, 556 patients underwent laparoscopic cholecystectomy for AC. There were 139 advanced AC (gangrenous cholecystitis, pericholecystic abscess, hepatic abscess, biliary peritonitis, emphysematous cholecystitis), and 417 non advanced AC. The conversion rate to open cholecystectomy was $13.5 \%$.

Results: Predictive factors for advanced AC, in multivariate analysis were: body temperature (OR 2.8 [1.3-6]), CRP (OR 1.01 [1.004-1.015]), diabetes (OR 5.6 [1.44-22]).

Comparing areas under the receiver operating characteristic curves, it was the CRP that had the highest discriminative power in diagnosing advanced AC. Values of WCC for diagnosing advanced $\mathrm{AC}$ were equal to or above $12.1 \pm 10^{9} / \mathrm{L}$ ( $\mathrm{Se}=64 \%, \mathrm{Sp}=65 \%$ ), NLR 3.87 and above $(\mathrm{Se}=62 \%, \mathrm{Sp}=62.5 \%)$, and CRP concentration $60.5 \mathrm{mg} / \mathrm{L}$ or above $(\mathrm{Se}=71 \%, \mathrm{Sp}=71.4 \%)$. CRP was the only predictive factor for conversion in multivariate analysis $(\mathrm{OR}=1.008$ [1.003-1.013]. Comparing areas under the receiver operating characteristic curves, it was the CRP that had the highest discriminative power in term of conversion. Values of CRP for predicting conversion were equal to or above $76 \mathrm{mg} / \mathrm{L}$ or above ( $\mathrm{Se}=66 \% ; \mathrm{Sp}=66 \%$ ).

Conclusions: CRP is the only inflammatory marker predictive for advanced AC and for conversion to open cholecystectomy. CRP had the highest discriminative power in diagnosing advanced AC with a good sensitivity $(71 \%)$ and specificity $(71 \%)$.

We think that CRP should be considered as a severity criteria of acute cholecystitis instead of white cell count.

\section{O070-HEPATO-BILIAIRY \& PANCREAS-Gallbladder}

SURGICAL SITE INFECTION RATES POST LAPAROSCOPIC CHOLECYSTECTOMY AND ELECTIVE HERNIA REPAIR. IS THERE ROOM TO IMPROVE?

Mohamed S. Salama $^{1}$, Mahmoud S. Salama ${ }^{2}$, B. Eoghan ${ }^{1}$, S. Babur ${ }^{1}$, A. Ibrahim $^{1}$, A.R. Nasr ${ }^{1}$

${ }^{1}$ Surgery, Our lady of lourdes hospital, BETTYSTOWN, Ireland; ${ }^{2}$ Medical student, Trinity college, DUBLIN, Ireland

Introduction: Surgical Site Infection (SSI) impacts patient's morbidity, mortality and adversely impacts health care costs. It is used as a surrogate for quality and performance. Its incidence varies widely between hospitals and between surgeons (2.5-42\%) with NICE estimating an average SSI rate of $5 \%$. It is crucial for each healthcare system to conduct SSI surveillance. Aim: To quantify wound infection rate and risk factors for SSI in our hospital post elective laparoscopic cholecystectomy (LC) or hernia surgery (HS).

Methods: A prospective study was conducted from 01/04/2016 to 31/12/2016. All patients admitted for elective LC or HS were recruited. The surveillance methodology was based on National Healthcare Safety Network (NHSN) methods. A 30 and 90 day post discharge surveillance period was used for LC and HS respectively. Data collected preoperatively included: age, gender, BMI, comorbidities, ASA score, surgical technique, admission, surgery and discharge dates.

Results: The total patient cohort numbered 257 (LC 112 \& HS 145). In total 7 patients developed SSI (LC 3 \& HS 4 ) giving a rate of $2.7 \%$

Of 6 diabetic patients undergoing LC one developed a SSI and of the 9 diabetic patients undergoing HS one developed a SSI. In contrast, of the 101 non-diabetic patients undergoing LC two developed SSI, and of the 133 non-diabetic patients undergoing HS three developed SSI. 8 patients' diabetic status was not documented.

Of the 26 obese patients undergoing LC two developed a SSI and of the 20 obese patients undergoing HS one developed a SSI

Compliance with preoperative antibiotic timing (within 60 min prior to incision) was $69 \%$. SSI was observed in $3 \mathrm{LC} \& 2 \mathrm{HS}$ when antibiotics were administered $>60 \mathrm{~min}$. SSI was observed in 0 LC \& 2 HS for antibiotics administered $<60 \mathrm{~min}$.

Compliance with correct preoperative antibiotic choice was $71 \%$. SSI was observed in 1 LC \& 1 HS with correct antibiotic choice and 1 LC \& 2 HS in non-compliant group.

Conclusion: Every hospital should continuously monitor and audit SSI rates. We aim to improve our SSI rate by adopting zero tolerance tonon-compliance with timing and choice of prophylactic antibiotics.
O071-HEPATO-BILIAIRY \& PANCREAS-Gallbladder

COMPARISON OF POSTOPERATIVE PAIN IN VARIOUS PRESSURE PNEUMOPERITONEUM OF LAPAROSCOPIC CHOLECYSTECTOMY: A DOUBLE BLIND RANDOMIZED CONTROLLED STUDY

\section{T. Yoo, W.T. Cho,}

Surgery, Dongtan Sacred Heart Hospital Hallym University College of Medicine, HWASUNG-SI, Korea

Even though laparoscopic cholecystectomy(LC) is the gold standard procedure for cholelithiasis, patients are still suffering from various causes of pain. one of main causes is high pressure by pneumoperitoneum which makes peritoneal stretching and diaphragmatic irritation. However, there are few well-designed studies for evaluating pneumoperitoneum.

Therefore, we conducted a study to compare the postoperative pain after LC at serial differen pressure methods. A prospective randomised double blind study was done in 147 patients with benign gallbladder disease. They were divided into 3 groups. Each 49 patients underwent $\mathrm{LC}$ with different pneumoperitoneum method; Group A: far-low (6-8 mmHg), goup B: low (9-11 mmhg) and group $\mathrm{C}$ : standard pressure $(12-14 \mathrm{mmHg})$. Three groups were compared for pain intensity, duration, analgesic requirement and complications. Post-operative pain score was significantly leas in far-low pressure group as compared to low or standard pressure group during late periods (12, $24 \mathrm{~h}$ ). But, there were no pain score difference between far-low and low groups during early period $(1,2,4,8 \mathrm{~h})$ even though scores of standard group were significant higher than those of low group. Number of patients requiring rescue analgesic doses and intraoperative complications were not significantly different among 3 groups.

This study demonstrates reducing the pressure of pneumoperitoneum results in reduction in intensity of post-operative pain. This study also shows that low pressure technique is safe with comparable rate of intraoperative complications. However, in immediate postoperative period, there is limitaton of pain relief after low pressure surgery. Therefore, there may need new alternatives for pain.

\section{O072-HEPATO-BILIAIRY \& PANCREAS-Liver}

\section{STANDARDIZED GLISSONIAN APPROACH FOR} LAPAROSCOPIC ANATOMICAL HEPATECTOMY BASED ON NEW CONCEPT OF PROPER HEPATIC CAPSULE

\author{
T. Ikeda, K. Kimura, K. Kudo, S. Okano,
}

Center for Advanced Medical Innovation, Kyushu University, FUKUOKA, Japan

Background and aim: Anatomical hepatectomy with the Glissonian approach is widely accepted as an important technique to ensure surgical safety and curability of the carcinoma. However, the histomorphological structure of the hepatic connective tissue is not sufficiently understood by surgeons. This study aimed to clarify the hepatic connective tissue structure using modern tissue imaging and analytical techniques.

Materials and methods: In total 5000 stained thin slices were loaded onto the computer and were reconstructed as 3 Dimages and analyzed.

Results: When the liver capsule enters the liver at the hepatic hilum, it becomes a sheath which envelops the portal pedicle. The hepatocytes in a row that constitute the periportal limiting plate at the edge of the hepatic lobule are firmly supported by the framework of the reticular fiber. The hepatic lobule and the portal area are in contact via the periportal space of Mall. The framework of the limiting plate plays a role of a capsule of hepatic lobule (proper hepatic capsule) on the side in contact with the portal area. The binding site between the hepatic capsule and proper hepatic capsule (PPBS) is loose binding and is a layer that is easy to apply to surgical procedures.

In order to enter between the liver capsule which became the sheath of the portal pedicle and the proper hepatic capsule at the hepatic hilum, the liver capsule must be dissected to reach the surface of the proper hepatic capsule. Then, on the one hand, the portal pedicle is firmly gripped and pulled, on the other hand, the hepatic parenchyma covered by the proper hepatic capsule is pushed to expand between the portal pedicle and the liver parenchyma. At this time, the portal area (Glisson's sheath) branched from the sheath of the portal pedicle into the gap of the hepatic lobule breaks like a string. With this dissecting plane, dissecting layer can reach to the next branch of the portal pedicle without entering into the portal pedicle or liver parenchyma.

Conclusion: Understanding the connective tissue constituting the liver and conducting surgery turns the laparoscopic systematic hepatectomy into a standardized procedure. 


\section{O073-HEPATO-BILIAIRY \& PANCREAS-Liver}

LAPAROSCOPIC LIVER RESECTION VERSUS TRANSARTERIAL CHEMOEMBOLIZATION FOR HEPATOCELLULAR CARCINOMA IN BCLC B PATIENTS: A PROPENSITY SCORE ANALYSIS

U. Cillo $^{1}$, A. Bertacco ${ }^{1}$, E. Gringeri ${ }^{1}$, E. Fasolo ${ }^{1}$, L. Aldrighetti ${ }^{2}$, A. Guglielmi $^{3}$, V. Mazzaferro ${ }^{4}$, A. Ferrero ${ }^{5}$, S. Gruttadauria ${ }^{6}$, F. Calise ${ }^{7}$, U. Boggi ${ }^{8}$, F. Giuliante ${ }^{9}$, E. Jovine ${ }^{10}$, G. Belli ${ }^{11}$, G. Torzilli ${ }^{12}$, A. Frena ${ }^{13}$, F. Farinati ${ }^{14}$, F. Trevisani ${ }^{15}$

${ }^{1}$ Department of Surgery, Oncology and Gastroenterology, Hepatobiliary Surgery and Liver Transplantation, Padua University, PADUA, Italy; ${ }^{2}$ Ospedale San Raffaele-U.O. Chirurgia Epatobiliare, MILANO, Italy; ${ }^{3}$ A.O.U. Integrata Verona-Policlinico G.B. Rossi-Chirurgia Generale ed Epatobi, VERONA, Italy; ${ }^{4}$ Fondazione IRCCS Istituto Nazionale dei Tumori-S.C. Chirurgia generale indiriz, MILANO, Italy; ${ }^{5}$ Ospedale Mauriziano Umberto I-S.C. Chirurgia Generale ed Oncologica, TORINO, Italy; ${ }^{6}$ IsMeTT-U.O. Chirurgia Addominale e Trapianti Addominali, PALERMO, Italy; ${ }^{7}$ A. Cardarelli,,-Chirurgia Epatobiliare e Centro Trapianti di Fegato, NAPOLI, Italy; ${ }^{8}$ A.O.U. Pisana- U.O. Chirurgia generale e trapianti, PISA, Italy; ${ }^{9}$ A.Gemelli-U.O.C. Chirurgia Epatobiliare, ROMA, Italy; ${ }^{10}$ Ospedale Maggiore AUSL Bologna-Chirurgia generale A e d'urgenza, BOLOGNA, Italy; ${ }^{11}$ Ospedale S. M. Loreto Nuovo-ASL NA1 Centro- U.O. Chirurgia Generale ed Epatobili, NAPOLI, Italy; ${ }^{12}$ Istituto Clinico Humanitas-U.O.C. Chirurgia Epatobiliare, ROZZANO, Italy; ${ }^{13}$ Ospedale Centrale-S.S. Chirurgia Epatobiliare, BOLZANO, Italy; ${ }^{14}$ Department of Surgery, Oncology and Gastroenterology, Padua University, Padua, PADOVA, Italy; ${ }^{15}$ Dipartimento di Scienze Mediche e Chirurgiche, Department of Medical and Surgical Sciences Division of Semeiotics, Alma Mater S, BOLOGNA, Italy

Background: TACE represents the most widely used first line treatment across all disease stages. It represents, in general, the recommended treatment modality for BCLC B patients although this stage includes a very heterogeneous patient populationThe aim of this study is to compare outcome of laparoscopic liver resection (LLR)vs transarterial chemoembolization (TACE) in intermediate stage HCC patients.

Methods: Data of HCC patients treated with LLR at 13 Italian centers were analyzed and compared with those treated with TACE included in the ITA.LI.CA database in the period 2014-2017. Only BCLC B HCC patients were considered for the analysis: a propensity score analysis was used to match LLR and TACE patients.

Results: 52 HCC patients underwent LLR; patients treated with TACE in the same period were 124 . No difference in overall survival (OS) was found between the groups $(p=0.691)$ while proportion of recurrence was significantly lower in the LLR $(p=0.003)$. After propensy score analysis (40 patients each group) LLR provided 1,3 and 5 year OS of $97.1 \%$, $93.8 \%$ and $82.1 \%$ that resulted statistically significant $(\mathrm{p}=0.020)$ compared to TACE. Tumor recurrence, again, resulted significant between groups $(\mathrm{p}=0.087$ ). At the multivariate analysis LLR (HR 0.2) positively impacted survival while portal hypertension (HR 6.6) was found independently associated with a worse outcome.

Conclusions: The beneficial effect of LLR in term of survival and recurrence remains also in BCLC B patients when compared to TACE

\section{O074-HEPATO-BILIAIRY \& PANCREAS-Liver}

LAPAROSCOPIC LIVER RESECTION FOR NON-COLORECTAL NON-NEUROENDOCRINE METASTASES: PERIOPERATIVE AND ONCOLOGIC OUTCOMES

D. Aghayan ${ }^{1}$, P. Kalinowski ${ }^{2}$, A. Kazaryan ${ }^{3}, \AA$ A. Fretland ${ }^{4}$, M. Sahakyan $^{1}$, E. Pelanis ${ }^{1}$, B. Røsok ${ }^{4}$, B. Edwin ${ }^{1}$

${ }^{1}$ The Intervention Centre, Oslo University Hospital, OSLO, Norway; ${ }^{2}$ Department of General, Transplant and Liver Surgery, Medical University of Warsaw, WARSAW, Poland; ${ }^{3}$ Department of General Surgery, Fonna Hospital Trust, STORD, Norway; ${ }^{4}$ Department of Hepato-Pancreato-Biliary Surgery, Oslo University Hospital, OSLO, Norway

Background: Liver resection is a treatment of choice for colorectal and neuroendocrine liver metastases and laparoscopy is an accepted approach for surgical treatment of these patients. The role of liver resection for patients with non-colorectal non-neuroendocrine liver metastases (NCNNLM), however, is still disputable. Outcomes of laparoscopic liver resection for this group of patients have not been analyzed.

Material and methods: In this study, patients who underwent laparoscopic liver resection for NCNNLM at Oslo University Hospital between April 2000 and January 2018 were analyzed. Perioperative and oncologic data of these patients were examined. Postoperative morbidity was classified using the Accordion classification. Kaplan-Meier method was used for survival analysis. Median follow-up was 26 (4-109) months.

Results: Fifty-one patients were identified from a prospectively collected database. The histology of primary tumors was classified as adenocarcinoma $(n=16)$, sarcoma $(n=4)$, squamous cell carcinoma $(n=4)$, melanoma $(n=16)$, gastrointestinal stromal tumor $(n=9)$ and adrenocortical carcinoma $(n=2)$. The median operative time was $147(30-470)$ min, while the median blood loss was $200(20-4000)$ ml. Nine $(18 \%)$ patients experienced postoperative complications. There was no 90-day mortality in this study. Thirty-five $(68 \%)$ patients developed disease recurrence or progression. Seven (14\%) patients underwent repeat surgical procedure for recurrent liver metastases. One-, three- and five-year overall survival rates were $85 \%, 52 \%$ and $38 \%$, respectively. The median overall survival was 37 (95\% CI 25 to 49 ) months.

Conclusion: Laparoscopic liver resection for NCNNLM results in good outcomes and should be considered in patients selected for surgical treatment. 


\section{O075-HEPATO-BILIAIRY \& PANCREAS-Liver}

DEVELOPMENT AND IMPROVEMENT OF REAL-TIME NAVIGATION SYSTEM FOR LAPAROSCOPIC HEPATECTOMY

\section{T. Igami, Division of Surgical Oncology}

Department of Surgery, Nagoya University Graduate School of Medicine, NAGOYA, Japan

Background: Laparoscopic hepatectomy for an invisible small tumor even by intraoperative ultrasonography is technically demanding. In such situation, intrahepatic vessels are recognized as important indicators of determination of resection area and preoperative 3D liver images reconstructed by MDCT are utilized as surgical assist. We are going to try development of realtime navigation system for laparoscopic hepatectomy, which resembles a car navigation system. We report our real-time navigation system and surgical procedure.

Methods: Virtual 3D liver and body images are reconstructed using 'New-VES' system developed by Nagoya University Graduate School of Information Science-Mori's Office. These images correspond to maps of car navigation system. Some of patient's body parts are registered in virtual 3D liver and body images using a magnetic position sensor. Patient's body after registration corresponds to The Earth. A transmitter for magnetic position sensor, which corresponds to an artificial satellite, is placed about $30 \mathrm{~cm}$ above patient's body. A micro magnetic sensor, which corresponds to GPS antenna, fixes on the handling part of laparoscope. Laparoscopic hepatectomy is performed using both real operative and virtual monitors.

Fiducial registration error (FRE, which means an error between real operative and virtual lengths) is utilized to evaluate accuracy of real-time navigation system.

Results: e performed laparoscopic hepatectomy using this system in 21 patients. Mean FRE of initial 5 patients was $17.7 \mathrm{~mm}$. First improvement was that MDCT were taken using radiological markers for registration of body parts. Mean FRE of the 8 patients who utilized first improvement was $10.2 \mathrm{~mm}$ and decreased $(\mathrm{p}=0.014)$. Second improvement was that a micro magnetic sensor as an intraoperative body position sensor was fixed on the right-sided chest wall and meant that pre- and post- operative FRE was similar due to an intraoperatively automatic correction of gap of body position. Preoperative and postoperative mean FRE of the 8 patients who utilized second improvement were $11.1 \mathrm{~mm}$ and $10.1 \mathrm{~mm}$. Those mean FRE were statistically similar $(\mathrm{p}=0.250)$. Conclusions: Our real-time navigation system can assist laparoscopic hepatectomy. However, FRE is still large margins; therefore, further improvement of our system is necessary to represent an alternative to an intraoperative ultrasonography.

\section{O076-HEPATO-BILIAIRY \& PANCREAS-Liver}

\section{REGENERATING ACTIVITY OF REMNANT LIVER TISSUE FOLLOWING PARTIAL HEPATECTOMY IN THE RAT. LAPAROSCOPIC VERSUS OPEN TECHNIQUE}

P. Ypsilantis ${ }^{1}$, O. Ioannidis ${ }^{1}$, M. Lambropoulou ${ }^{2}$, C. Anagnostopoulos ${ }^{3}$, A. Totsi ${ }^{1}$, C. Zervas ${ }^{1}$, C. Simopoulos ${ }^{1}$

${ }^{1}$ Laboratory of Experimental Surgery and Surgical Research, School of Medicine, Democritus University of Thrace, Alexandroupolis, Greece, ALEXANDROUPOLIS, Greece; ${ }^{2}$ Laboratory of Histology and Embryology, School of Medicine, Democritus University of Thrace, Alexandroupolis, Greece, THESSALONIKI, Greece; ${ }^{3}$ Laboratory of Biochemistry, School of Medicine, Democritus University of Thrace, Alexandroupolis, Greece, THESSALONIKI, Greece

Aims: To compare laparoscopic versus open partial hepatectomy with regards to the regenerating activity, oxidative state, inflammatory response and histopathologic profile of the remnant liver tissue.

Methods: Ninety six Wistar rats were subjected to excision of the left lateral and the median hepatic lobes either laparoscopically (group LAP-HEP) or after midline laparotomy (group HEP), sham operation (group Sham) or no operation (group Control). At various timepoints postoperatively $(1 \mathrm{~h}-2 \mathrm{w})$ the relative liver weight, mitotic index (SER-10 antibody), oxidative state (TBARS levels), inflammatory response biomarkers (NF?B, ICAM-1 and VCAM-1) and the histopathologic index were assessed in the remnant liver parenchyma.

Results: Although relative liver weight did not differ between the hepatectomy groups, the mitotic index was higher in group LAP-HEP compared to group HEP. TBARS levels were increased in group LAP-HEP. VCAM-1 expression was more intense in group LAP-HEP compared to group HEP, whereas NF?B was equally overexpressed in the study groups and ICAM-1 in the hepatectomy groups. At $24 \mathrm{~h}$, histopathologic index was higher in group HEP than that in group LAP-HEP.

Conclusions: Laparoscopic partial hepatectomy conferred a more intense mitotic effect and oxidative stress, while a less pronounced inflammatory response and deterioration of the histopathologic profile of the remnant liver tissue than that of the open technique.

\section{O077-HEPATO-BILIAIRY \& PANCREAS-Liver}

\section{THE NEED FOR STRONGER COMMITMENT TO LAPAROSCOPY IN TECHNICALLY COMPLEX PROCEDURES: A DIFFERENTIAL BENEFIT-BASED APPRAISAL}

\author{
F. Ratti, F.C. Cipriani, G.F. Fiorentini, M. Catena, M. Paganelli, \\ A.L. Aldrighetti
}

Hepatobiliary Surgery Division, San Raffaele Hospital, MILANO, Italy

Background: Implementation of minimally invasive liver resection (MILS) programs starts, in a stepwise fashion, from procedures with a low degree of technical difficulty, given the higher feasibility at the beginning of the educational pathway. In this perspective, the strength of commitment to MILS is generally based on its feasibility, rather than on its benefit compared with open approach. Aim of the present study is to evaluate the differential benefit of laparoscopic over open technique according to the technical difficulty of the procedures and to define-according to this parameter-the strength of commitment to minimally invasive approach.

Material and methods: 936 MILS resections performed between 2005 and 2018 were stratified according to technical complexity (Low, Intermediate and High difficulty) and to approach (MILS or open) and then matched in a 1:1 ratio using propensity scores to obtain three pairs of groups (Pair 1:Low-MILS and Low-Open, including 274 cases respectively; Pair 2: Int-MILS and Int-Open, including 237 patients respectively; Pair 3: High-MILS and High-Open, including 226 patients respectively). Blood loss, postoperative morbidity and time for functional recovery were chosen as outcome indicators to calculate the differential benefit among pairs.

Results: MILS approach resulted in a statistically significant lower blood loss, reduced morbidity, reduced and shorter time for functional recover and length of stay within all pairs. Reflecting different degree of complexity, procedures showed significantly different blood loss, morbidity, rate of conversion and time for functional recovery among different Pairs. The evaluation of the differential benefit showed a greater advantage of laparoscopic approach in High degree procedures compared with Intermediate and Low degree, both in terms of blood loss $(-250 \mathrm{~mL}$ and $-200 \mathrm{~mL}$ respectively) and morbidity rate $(-5.7 \%$ and $-4.1 \%$ respectively). Conclusion: The favorable biological scenario associated with laparoscopic approach allows to obtain significant benefits in the setting of technically complex procedures, constituting the prerequisite for an adequate surgical outcome. The commitment towards MILS approach should be therefore stronger in this setting, where-overcome the limit of technical challenges-the advantage of laparoscopy seems to be enhanced.

\section{O078-HEPATO-BILIAIRY \& PANCREAS-Liver}

\section{A FEASIBILITY OF TOTALLY LAPAROSCOPIC LIVING DONOR RIGHT HEPATECTOMY}

\author{
Y.S. $\operatorname{Han}^{1}$, J.R. Han ${ }^{1}$, H.T. Ha ${ }^{1}$, J.M. Chun ${ }^{2}$
}

${ }^{1}$ Hepatobiliary Pancreas Surgery and Liver Transplantation, Kyungpook National University, School of Medicine, DAEGU, Korea; ${ }^{2}$ Liver Transplantation, Kyungpook National University, School of Medicine, DAEGU, Korea

Purpose: Although laparoscopic liver resection has progressively developed with increased surgical experience and the improvement of laparoscopes and specialized instruments, Only a limited number of centers have performed laparoscopic living donor hepatectomy to date because of concerns about donor safety,graft outcome and the need for expertise in both laparoscopic liver surgery and living donor liver transplantation(LDLT). For these reason, a totally laparoscopic living donor right hepatectomy (LDRH) technique has not been investigated for efficacy and feasibility. We describe the experiences and outcomes associated with LDRH in adult-to-adult LDLT in order to assess the safety of the totally laparoscopic technique in donors.

Methods: Between December 2014 and October 2018, we performed 97 cases of living donor right hepatectomy. Among them, 50 donors underwent totally laparoscopic living donor right hepatectomy and 47 donors underwent conventional open living donor right hepatectomy. We retrospectively reviewed the medical records to ascertain donor safety and the reproducibility of LDRH; intra-operative and post-operative results including complications were demonstrated after performing LDRH.

Results: The total operation time was longer $(367.0 \pm 74.3$ vs $323.5 \pm 62.5 ; \mathrm{P}=.002)$ and the warm ischemic time was also longer $(9.2+4.6$ vs $1.8 \pm 1.6 ; \mathrm{P}<.002)$ in LDRH group. However, the length of postoperative hospital stay was similar in both groups and no donors in LDRH group required blood transfusion, conversion to open surgery, or reoperation. There was no postoperative mortality. Postoperative complication of Clavien-Dindo classification III or more in LDRH group was identified in only one donor who had a minor bile leakage from the cutting edge of the right hepatic duct stump requiring endoscopic biliary stent insertion. All the liver function tests returned to normal ranges within 2 weeks.

Conclusion: In conclusion, our study reveals LDRH seems to be a safe and feasible procedure with acceptable outcomes. However, LDRH can be initially attempted after attaining sufficient experience in laparoscopic hepatectomy and LDLT techniques. 


\section{O079-HEPATO-BILIAIRY \& PANCREAS-Liver}

THE MODIFIED LIVER HANGING MANEUVER IN LAPAROSCOPIC MAJOR HEPATECTOMY: LEARNING CURVE AND EVOLUTION OF INDICATIONS

\section{J.H. Kim}

\section{Department of Surgery, Eulji University Hospital, DAEJEON, Korea}

Aims: Laparoscopic major hepatectomy is a technically challenging procedure and needs a steep learning curve. The liver hanging maneuver is a useful technique in liver surgery, especially in the case of large tumors or invasive tumors which is relatively contraindications for laparoscopic liver resection. The present study aimed to evaluate the learning curve and the evolution of indications for laparoscopic major hepatectomy using the modified liver hanging maneuver.

Methods: From January 2013 and September 2018, we retrospectively reviewed patients who underwent laparoscopic major hepatectomy using the modified liver hanging maneuver by a single surgeon. Our hanging technique involves the hanging tape was placed along the lateral side of inferior vena cava for right-sided hepatectomy or the ligamentum venosum for left-sided hepatectomy. The learning curve for operating time and blood loss was evaluated using the cumulative sum (CUSUM) method.

Results: Of the 53 patients, 18 patients underwent right hepatectomy, 26 underwent left hepatectomy and 9 underwent right posterior sectionectomy. CUSUM analysis showed that operative time and blood loss improved after the 30th laparoscopic major hepatectomy. These 53 consecutive patients were divided into two groups (early group: 1-30 cases, late group: 31-53 cases). The median operation time was decreased in the late group, but the difference was not statistically significant ( 270 vs. $245 \mathrm{~min}, \mathrm{p}=0.261$ ). The median blood loss was significantly lower in the late group ( 350 vs. $150 \mathrm{ml}, \mathrm{p}<0.001$ ). The large tumors (greater than $10 \mathrm{~cm}$ ) and tumors in proximity to major vessels were significantly higher in late group $(0 \mathrm{vs.} 17.4 \% \mathrm{p}=0.018,3.3 \mathrm{vs}$. $21.7 \% \mathrm{p}=0.036$, respectively). The tumors invading adjacent organ was higher in the late group, but the difference was not statistically significant. ( 0 vs. $8.7 \%, p=0.100)$.

Conclusion: This study shows that laparoscopic major hepatectomy has a learning curve of 30 cases. The step-wise training and standardized procedure of the modified liver hanging maneuver may shorten the learning curve. After standardization of our procedure, the indications have gradually been extended to large tumors or invasive tumors.

\section{O080-HEPATO-BILIAIRY \& PANCREAS-Liver}

\section{CHANGING TRENDS AND OUTCOME ASSOCIATED WITH} THE ADOPTION OF MINIMALLY-INVASIVE HEPATECTOMIES: EXPERIENCE WITH 500 CONSECUTIVE CASES

\section{B.K. Goh}

Hepatopancreatobiliary and Transplant Surgery, Singapore General Hospital, SINGAPORE, Singapore

Background: Several studies published mainly from pioneers and early adopters have documented the evolution of minimally-invasive hepatectomy $(\mathrm{MIH})$. However, questions remain if these reported experiences are applicable and reproducible today. This study examines the changing trends, safety and outcomes associated with the adoption of MIH.

Methods: Retrospective review of 500 consecutive patients who underwent MIH between 2006-2018 of which 460 cases (92\%) were performed since 2012. To determine the evolution of $\mathrm{MIH}$, the study population was stratified into 5 equal groups of 100 patients. Analyses was also performed of predictive factors and outcomes of open conversion.

Results: Five hundred patients underwent MIH of which 479 (97.8\%) were totally laparoscopic/ robotic. $118(23.6 \%)$ patients underwent major hepatectomy and $199(39.9 \%)$ had resection of tumors located in the posterosuperior segments. 32 patients $(6.4 \%)$ had previous liver resections. There were $45(9.0 \%)$ open conversions. Comparison across the 5 groups demonstrated that patients were older, had higher ASA score, had increased frequency of previous abdominal surgery and repeat liver resections. There was also an increase in the proportion of patients who underwent totally laparoscopic/robotic surgery, major liver resection, resection of $=3$ segments and multiple resections. Comparison of outcomes demonstrated that there was a significant decrease in open conversion rate, longer operation time and increased use of Pringles maneuver. Presence of cirrhosis and institution experience (1st 100 cases) were independent predictors of open conversion. Patients who required open conversion had significantly increased operation time, blood loss, blood transfusion rate, morbidity and mortality.

Conclusion: The case volume of MIH performed increased rapidly at our institution over time. Although the indications of MIH expanded to include higher risk patients and more complex hepatectomies, there was a decrease in open conversion rate and no change in other perioperative outcomes.

\section{O081-HEPATO-BILIAIRY \& PANCREAS-Pancreas \\ ROBOTIC VERSUS LAPAROSCOPIC DISTAL PANCREATECTOMY: A COMPARATIVE COSTS- EFFECTIVENESS STUDY}

V. Ferri, E. Vicente, Y. Quijano, D. Hipolito, B. Ielpo, E. Diaz, I. Fabra, L. Malave, R. Isernia, E. Pinna, R. Caruso

General Surgery, Sanchinarro University Hospital, MADRID, Spain

Introduction: The robotic surgery cost presents a critical issue which has been investigated only in few studies. In the literature there is not any study which evaluate the cost-effectiveness of the robotic distal pancreatectomy (RDP) over de laparoscopic distal pancreatectomy (LDP).

We have therefore performed a prospective comparative study of RDP and LDP performed at our centre with the aim to evaluate clinical and the cost-effective outcomes.

Matherial and methods: This is an observational, comparative prospective non-randomized study which includes patients that underwent RDP and LDP reaching a minimum of 6 months of follow up from February 2014 to March 2018, at the Sanchinarro University Hospital, Madrid. An independent company performed the financial analysis. Outcome parameters included surgical and post-operative costs, quality adjusted life years (QALY), and incremental cost per QALY gained or the incremental cost effectiveness ratio (ICER). The primary end-point was to compare the cost effectiveness differences between both groups.

Results: A total of 35 RDP and 31 LDP have been included. Conversion rate resulted to be significative higher in the LDP ( $3.6 \%$ vs $19.2 \%$; $\mathrm{p}=0.04)$. The overall rate of pancreatic leak was $10.7 \%$ in the RDP group and $15.4 \%$ in the LDP group ( $p>0.5$ ). The mean number of hospital stay days was significative higher in the LDP ( 8.9 days vs 16.9 days, $p=0.03)$. The mean operative time was higher in the RDP

( 294 vs $241 \mathrm{~min} ; \mathrm{p}=0.02$ ). The overall mean total cost was similar in both groups (RDP 9198.64€ versus LDP: 9399.74€; $\mathrm{P}>0.5)$. Mean QALYs at 1 year for RDP $(0.622)$ was higher than that associated with LDP $(0.60025)(\mathrm{p}>0.5)$. At a willingness-to-pay threshold of $20,000 €$ and $30,000 €$, there was a $63.58 \%$ and $76.69 \%$ probability that RDP was cost-effective relative to LDP.

Conclusion: This study provides data of cost-effectiveness between RDP and LDP approach showing benefit for the RDP.

\section{O082-HEPATO-BILIAIRY \& PANCREAS-Pancreas}

\section{MANAGEMENT OF ABERRANT RIGHT HEPATIC ARTERY IN ROBOTIC WHIPPLE PROCEDURE}

\section{C.M. Peng, Y.K. Yang}

General Surgery, Da Vinci MIS Center/Chung Shan Medical University Hospital, TAICHUNG, Taiwan

Purpose: The right hepatic artery (RHA) is the most common hepatic artery (CHA) variation. This variation may be problematic in in open, laparoscopic or robotic pancreaticoduodenectomy (PD). The vascular configuration described as normal is found in only $55-75.5 \%$ of cases, which means that a large percentage of patients present an anatomic variation. The importance of the presence of a variant hepatic artery in pancreatic surgery has been commented in several publications. An RHA that irrigates in the SMA has a close relationship with the head of the pancreas since its course is adjacent and occasionally passes through its parenchyma. The absence of collateral vascularization and the inadvertent sectioning of an RHA branch of the SMA during a pancreaticoduodenectomy (PD) can lead to ischemia and necrosis of the right liver lobe. Finally, once the gastroduodenal artery (GDA) is dissected, the RHA branch of the SMA becomes the main source of vascularization of the distal common bile duct.

Materials and methods: A total of 103 patients underwent da Vinci robotic pancreaticoduodenectomy (RPD) between January 2012 and July 2018 were analyzed by three surgeons. 27 patients underwent conventional RPD, 15 patients with pure RSPPD and 61 patients with RSPPD + 1 were performed. Besides, the PubMed database from 1950 to 2017 was systematically searched for comparative studies reporting management of the RHA during PD.

Results: A total of 103 patients were analyzed, of whom 17 (16.5\%) had a RHA. In our study with infiltration by the tumor mass, or in those with an intrapancreatic pathway, the artery should be sacrificed with the PD surgical specimen and later reconstructed. An aberrant RHA (ARHA) that was satisfactorily resolved with end-to-end arterial reconstruction without the use of vascular stent. We used vascular micro-bulldog clamps between ARHA to dissect the gastroduodenal artery (GDA), while preserving as much of its length as possible.

Conclusion: In study, postoperative and oncological outcomes seemed unaffected by the RHA in PD. The robotic system combined with single port platforms are able to overcome the current limitations even vascular anastomosis in ARHA. The robotic PD is safe and feasible in appropriately selected patients. 


\section{O083-HEPATO-BILIAIRY \& PANCREAS-Pancreas}

REINFORCED STAPLER VS. ULTRASONIC DISSECTOR FOR PANCREATIC TRANSECTION AND STUMP CLOSURE FOR DISTAL PANCREATECTOMY: A PROPENSITY-MATCHED ANALYSIS

M. de Pastena, A. Pulvirenti, L. Landoni, M. Fontana, C. Bassi, R. Salvia

General and Pancreatic Surgery, AOUI Verona, VERONA, Italy

Background: Postoperative pancreatic fistula (POPF) is the primary contributor to morbidity after distal pancreatectomy (DP). To date, no techniques used for the transection and closure of the pancreatic stump showed a clear superiority over the others. This study aimed to compare the rate of POPF after pancreatic transection conducted with the reinforced stapler (RS) and ultrasonic dissector (UD) following DP.

Method: Consecutive patients underwent DP from 2014 to 2017 were retrospectively reviewed. We included DPs where pancreatic transection was performed by RS or UD and excluded DPs extended to the pancreas head. To overcome the absence of randomization, we conducted a propensity matching analysis according to risk factors for POPF.

Results: Overall, 200 patients met the inclusion criteria. The RS was employed in 108 patients and UD in 92 cases. After the one-to-one propensity matching, 92 patients were selected from each group. The matched RS and UD cohort have no differences in baselines characteristics except for the mini-invasive approach, that was more common in the UD group (34\% vs. $51 \%$, $\mathrm{p}=0.025)$. Overall, 48 patients (26\%) developed a POPF, 46 a grade B $(25 \%)$ and $2(1 \%)$ a grade C. In the RS group the rate of POPF was $12 \%(\mathrm{n}=11)$ and the UD group $38 \%(\mathrm{n}=35)$ with a $\mathrm{p}<0.001$

Conclusion: The results of this study suggest that the use of RS for pancreatic transection, reduces the risk of postoperative pancreatic fistula. A randomized trial is needed to confirm these preliminary data.

\section{O084-HEPATO-BILIAIRY \& PANCREAS-Pancreas}

ROBOTIC SINGLE INCISION LONGITUDINAL PANCREATICOJEJUNOSTOMY FOR CHRONIC PANCREATITIS: COMPARISON OF CLINICAL OUTCOMES AND COST TO THE OPEN APPROACH

\section{C.M. Peng, General Surgery}

Da Vinci MIS Center/Chung Shan Medical University Hospital, TAICHUNG, Taiwan

Aim: This study compares clinical and cost outcomes of robot-assisted single port and open longitudinal pancreaticojejunostomy (RLPJ and OLPJ) for chronic pancreatitis. Single incision MIS needs more manual skills than conventional multiport operation. The advantage of better operation course is $3 \mathrm{D}$ vision and dedicate instrument. This paper aims to evaluate the feasibility and safety of the robot-assisted single incision with single port platform for chronic pancreatitis. Materials and methods: Clinical and cost data were retrospectively compared between open and RALPJ. We collected 21 patients since July, 2015 to September, 2018. The patient was supinely placed in reverse Trendelenburg position. The assistant surgeon was located between patient's legs. Under general anesthesia a trans-umbilical $4.0 \mathrm{~cm}$ skin incision was made. A single incision advanced access platform with Lagis port, Glove Port ${ }^{\circledR}$ (Nelis, S. Korea) and Gelpoint combined with the da Vinci Si and Xi Surgical System (Intuitive Surgical, Sunnyvale, CA, USA) pure or plus one was performed. The three arms, No. 1, No. 2, and da Vinci scope, were in dwelled through the Glove Port ${ }^{\circledR}$. Pneumoperitoneum of $12 \mathrm{mmHg}$ was established through the port. A rigid 30-degree up scope was used during operation.

Results: Twenty-one patients underwent LPJ: 5 open and 16 RALPJ. No robot-assisted cases converted to open were noted. Patients undergoing RALPJ had less intraoperative blood loss, a shorter surgical length of stay, less postoperative pain and lower medication costs. Operation supply cost was higher in the RALPJ group. No obvious difference in hospitalization cost was found.

Conclusions: Versus the open approach, RALPJ performed for chronic pancreatitis shortens hospitalization, less postoperative pain and reduces medication costs; hospitalization costs are equivalent. A higher operative cost for RALPJ is mitigated by a shorter hospitalization and less pain control. Robotassisted Puestow procedure using single port platform is feasible and safe method. The total procedures by da Vinci Robotic system are safe and easily performed in highly selected patients.

\section{O085-HERNIA-ADHESIONS_Abdominal wall hernia}

TOTALLY ENDOSCOPIC RETRO RECTAL APPROACH: THE 'TERRA' TECHNIQUE FOR DIASTASIS RECTI AND MIDLINE ABDOMINAL WALL HERNIAS ALL-IN-ONE REPAIR

F. del Castillo Diez ${ }^{1}$, L. García-Sancho Téllez ${ }^{2}$, C. Durán Escribano ${ }^{3}$, E. Alvarez Peña ${ }^{1}, M$. Heras Garceau ${ }^{1}$, J.A. Gonzalez Sanchez ${ }^{1}$, J. Díaz Dominguez ${ }^{1}$

${ }^{1}$ General Surgery, Hospital Universitario La Paz, MADRID, Spain; ${ }^{2}$ General Surgery, Hospital Universitario Infanta Sofia, MADRID, Spain; ${ }^{3}$ General Surgery, Hospital Quirón La Luz, MADRID, Spain

Aims: The concomitant presence of abdominal wall midline hernias and diastasis recti is frequent. Diastasis recti might be a risk factor not only for having but for recurrence of midline hernias. Most open surgical procedures not consider the treatment of both pathologies, nor laparoscopic most spread out approaches. The Author presents a novel endoscopic, extraperitoneal and retromuscular hernioplasty technique and its preliminary results.

Methods: A serie of 15 patients is presented. A CT abdominal wall study is performed preoperatively. They all presented abdominal wall midline hernias in presence of a $>3.5 \mathrm{~cm}$ concomitant diastasis recti. There were 8 females and 8 males. A totally endoscopic, extraperitoneal and retromuscular repair was performed, that included a midline anatomic restoration, tension-free hernia gap closure, omphaloplasty and skin treatment, if needed in every case. The tension-free massive-meshed hernioplasty included a bilateral totally endoscopic posterior components separation when needed. No drainages were used. All procedures included a bladder catheterization.

Results: All patient were dispatched within a period under $48 \mathrm{~h}$. No reoperations were needed in postoperative period. Postoperative pain was measured by an EVA scale. $85 \%$ of the patients have no pain medication after $24-48 \mathrm{~h}$ dispatching from hospital. $25 \%$ of the patients have a skin suffusion or hematoma. A male patient presented a temporary abdominal asymmetry due to a unilateral posterior component added to his technique. The mean following-up is to 6 month (1-12 months). No recurrence was observed.

Conclusions: Preliminary results demonstrate this new approach to be a safe, feasible and a reproductible procedure. The 'TERRA' novel technique could provide of a new minimally invasive approach to abdominal wall midline hernias repair in the presence of a diastasis recti. Only time and new results can predict the spreading out of this 'third way'.

\section{O086-HERNIA-ADHESIONS-Abdominal wall hernia}

'SLIM-MESH': SHORT/MID-TERM OUTCOMES IN 27 CASES OF GIANT/MASSIVE VENTRAL HERNIA

\section{S.A. Canton, S.D. Pianalto, C. Pasquali}

DiSCOG Department of Surgery, Oncology and Gastroenterology, University of Padua, PADOVA, Italy

Aims: We devised a sutureless technique ('Slim-Mesh', SM) to treat giant (GH $10-20 \mathrm{~cm}) /$ massive $(\mathrm{MH}=20 \mathrm{~cm}$ without loss of domain) ventral hernia in order to reduce intraand postoperative complications and to lower operative time.

Methods: Between September 2009 and October 2018, 27 patients with GH/MH were operated at Padua University-Hospital with the SM technique. Data on all patients were collected prospectively (67\%) and retrospectively (33\%).

Results: This study comprised 15 males and 12 females. Mean age was 62 years (range $33-82$ years) and mean body mass index was 30 . GH and $\mathrm{MH}$ were found intraoperatively in 22 and 5 cases respectively. Mean operative time for all hernias (GH/MH) was $122 \mathrm{~min}$ (range 70-240 min); $113 \mathrm{~min}$ for $\mathrm{GH}$ (range 70-155 min); and $169 \mathrm{~min}$ for $\mathrm{MH}$ (range 105-240 $\mathrm{min}$ ). In $51.8 \%$ of cases, hernia operative measurement was larger than preoperative size, especially in cases of incisional hernias (64.2\%). In $29.6 \%$ of cases, laparoscopy found additional abdomina wall defects previously undetected by physical examination and by US- and/or CT-scan. A composite mesh and a non-composite mesh (up to $30 \mathrm{~cm}$ in size) were used in $96.3 \%$ and $3.7 \%$ of cases respectively. The Ethicon SecureStrap?? Absorbable Fixation Device straps for SM fixation were employed in $77.8 \%$ of cases. Mean length of hospital stay was 2.8 days. Mean follow-up time was 33 months (range $1-109$ months). In our study, there was one early $(<30$ days) postoperative seroma $(3.7 \%)$, plus one late, small $(2 \mathrm{~cm})$ symptomless recurrence, but neither needed reoperation.

Conclusion: The sutureless SM technique facilitates intra-abdominal introduction, as well as the handling and fixation of large/very large meshes. This new approach is safe and fast, even in cases of $\mathrm{GH} / \mathrm{MH}$ repair. 


\section{O087-HERNIA-ADHESIONS-Abdominal wall hernia}

THE RIGHT APPROACH FOR REPAIRING VENTRAL HERNIA COMBINED WITH RECTUS MUSCLES SEPARATION; EXTENDED ENDOSCOPIC HERNIA \& LINEA ALBA RECONSTRUCTION GLUE

\section{Dudai, K. Ittah Gilboa}

Surgery, MERAV Medical Center, Hernia Excellence, TEL AVIV, Israel

Aims: Any Ventral Hernia (VH) combined with Rectus Muscle Separation (RMS) must be repaired along with repairing the RMS, otherwise there is a high risk for Hernia Recurrence. Open RMS repair is vast and traumatic surgery and Laparoscopy is not effective. At 2015 a new era of repairing Abdominal Wall Hernia by Assisted Endoscopy started with Wolfgang Reinhold's MILOS procedure. These procedures are somewhat complexed and real reconstruction of the Linea Alba (LA) was limited, which done better by Ferdinand Koeckerling's ELAR technique. We perfected the ELAR technique to be fully endoscopic with wide mesh fusing to the muscles immediately by Fibrin Glue: Extended Endoscopic Hernia \& Linea Alba Reconstruction Glue (eEHLARglue), achieving a low traumatic MIS for VH and RMS with excellent surgical and cosmetic results.

Methods: Our eEHLARglue is a totally endoscopic based technique used since 2017. Penetrating with Optiview trocar and $\mathrm{CO} 2$ pressure to the Anterior Rectus Sheet (ARS) level is followed by an extensive endoscopic dissection of the sub-cutaneous fat tissue from the ARS. Three trocars are inserted at the supra-pubic line enabling the dissection up to the Xiphoid and costal margins laterally. Any Hernia sac is dissected, and the content reduced back to the abdominal cavity. Relaxing Incisions of the ARS are performed longitudinally in the lateral aspect. The LA is reconstructed by running two layers of non-absorbable sutures from Xiphoid to Pubis. A light Mesh $30 \mathrm{X} 15 \mathrm{~cm}$ is applied over the repair and the mesh is fused immediately to the muscles by Fibrin Glue.

Results: 25 patients underwent the eEHLARglue with follow up of 24 months. All had significant RMS of 5-10 × 14-26 cm combined with primary or recurrent VH. Recovery was smooth with 1-3 days of simple analgesics and return to regular activity within 4-10 days. No one had recurrent $\mathrm{VH}$, but two males had limited RMS and two early cases Seroma formation. Conclusions: Our eEHLARglue enables endoscopic VH repair and LA reconstruction with extrastrength received by immediate mesh fusion to muscles with Fibrin glue. Thus, achieving low traumatic MIS, easy recovering and very effective results-a perfect solution for patients with VH combined with RMS.

\section{O088-HERNIA-ADHESIONS-Abdominal wall hernia}

TRANS-ABDOMINAL PRE-PERITONEAL VS TOTAL EXTRAPERITONEAL LAPAROSCOPIC INGUINAL HERNIA REPAIR-A META-ANALYSIS OF THE LITERATURE

\author{
K. Iles ${ }^{1}$, M. Portelli ${ }^{1}$, T. Bezzina ${ }^{2}$
}

${ }^{1}$ Department of Surgery, Mater Dei Hospital, MSIDA, Malta; ${ }^{2}$ Department of Health Sciences, Mater Dei Hospital, MSIDA, Malta

Background: Minimally invasive surgery is gradually becoming the mainstay of surgical treatment. Two techniques have been developed in management of inguinal herniae-the Transabdominal pre-peritoneal (TAPP) and the Total extraperitoneal (TEP) repair.

Method: A literature search was carried out on PubMed, MEDLINE, EMBASE and Google Scholar, using MESH terms 'Inguinal Hernia', 'Randomised Controlled Trials' (RCT), 'TEP', 'TAPP', 'Laparoscopy'. All RCTs published until December 2017, comparing TAPP and TEPP inguinal hernia repair were identified. Data was collected on post-operative pain, hospital stay, operative time and seroma formation.

Results: Twelve blinded prospective RCTs were used. When compared to TEP repair, TAPP repair has comparable Seroma formation rates $\left(\mathrm{Chi}^{2}=7.94 ;(\mathrm{P}=0.02) ; \mathrm{CI}-4.31,0.55\right.$; $\left.\mathrm{I}^{2}=75 \%\right)$ and Post-op pain at $24 \mathrm{~h}\left(\mathrm{Chi}^{2}=30.28\right.$; $\left.(\mathrm{P}=0.00001) ; \mathrm{CI}-0.31,0.06 ; \mathrm{I}^{2}=87 \%\right)$ However, TEP repair is associated with a significantly shorter operative time $\left(\mathrm{Chi}^{2}=502.95\right.$; $\left.(\mathrm{P}=0.00001) ; \mathrm{CI} 0.24,0.48 ; \mathrm{I}^{2}=98 \%\right)$, Post-op pain at 1 hour $\left(\mathrm{Chi}^{2}=11.26 ;(\mathrm{P}=0.004) ; 0.05\right.$, $\left.0.30 ; \mathrm{I}^{2}=82 \%\right)$ and shorter hospital stay $\left(\mathrm{Chi}^{2}=455.14 ;(\mathrm{P}=0.00001) ; \mathrm{CI} \quad 0.72,1.07\right.$; $\left.\mathrm{I}^{2}=99 \%\right)$.

Conclusion: TEP is significantly better than TAPP repair with regards to operative time, post-op pain at $1 \mathrm{~h}$ and hospital stay. There is no significant difference with regards to post-op pain at $24 \mathrm{~h}$ and seroma formation.

\section{O089-HERNIA-ADHESIONS_Abdominal wall hernia}

SINGLE-INCISION SURGERY AND TROCAR-SITE HERNIA: AN UPDATED SYSTEMATIC REVIEW AND META-ANALYSIS WITH TRIAL SEQUENTIAL ANALYSIS

A. Andreou ${ }^{1}$, J. Garcia-Alamino ${ }^{2}$, S. Hajibandeh ${ }^{3}$, S. Hajibandeh ${ }^{4}$, M. Weitzendorfer ${ }^{5}$, F.E. Muysoms ${ }^{6}$, F.A. Granderath ${ }^{7}$, G.E. Chalkiadakis ${ }^{8}$, K. Emmanuel ${ }^{9}$, M. Gioumidou ${ }^{10}$, S. Iliopoulou-Kosmadaki ${ }^{10}$, M. Mathioudaki ${ }^{10}$, K. Souliotis ${ }^{10}$, S. Antoniou ${ }^{11}$

${ }^{1}$ Department of Colorectal Surgery, York Teaching Hospital NHS Foundation Trust, YORK, United Kingdom; ${ }^{2}$ Nuffield Department of Primary Care Health Sciences, University of Oxford, OXFORD, United Kingdom; ${ }^{3}$ General Surgery Department, Salford Royal Foundation Trust, SALFORD, United Kingdom; ${ }^{4}$ General Surgery Department, North Manchester General Hospital, MANCHESTER, United Kingdom; ${ }^{5}$ Department of Surgery, Paracelsus Medical University, SALZBURG, Austria; ${ }^{6}$ Department of Surgery, Maria Middelares Hospital, GHENT, Belgium; ${ }^{7}$ Center for Minimally Invasive Surgery, Neuwerk Hospital, MÖNCHENGLADBACH, Germany; ${ }^{8}$ Department of General Surgery, University Hospital of Heraklion, HERAKLION, Greece; ${ }^{9}$ Department of General Surgery, Paracelsus Medical University, SALZBURG, Austria; ${ }^{10}$ School of Medicine, University of Crete, HERAKLION, Greece

Aims: Single-incision laparoscopic surgery (SILS) is a new technique that aims to minimize abdominal wall trauma and improve cosmesis. Concerns have been raised about the risk of trocar site hernia following SILS. This study aims to assess the risk of trocar site hernia following SILS compared to conventional laparoscopic surgery, and investigate whether current evidence is conclusive.

Methods: We performed a systematic search of MEDLINE, AMED, CINAHL, CENTRAL and OpenGrey. We considered randomized clinical trials comparing the risk of trocar-site hernia with SILS and conventional laparoscopic surgery. Pooled odds ratios with $95 \%$ confidence intervals were calculated using the Mantel-Haenszel method. Trial sequential analysis usingthe Land and DeMets methodwas performed to assess the possibility of type I error and compute the information size.

Results: Twenty-three articles reporting a total of 2471 patients were included. SILS was associated with higher odds of trocar-site hernia compared to conventional laparoscopic surgery (odds ratio $2.37,95 \%$ confidence interval $1.25-4.50, p=0.008$ ). There was no evidence of between study heterogeneity or small study effects. The information size was calculated at 1687 patients and the Z-curve crossed the O'Brien-Fleming a-spending boundaries at 1137 patients, suggesting that the evidence of higher risk of trocar-site hernia with SILS compared to conventional laparoscopic surgery can be considered conclusive.

Conclusions: Single-incision laparoscopic procedures through the umbilicus are associated with a higher risk of trocar-site hernia compared to conventional laparoscopic surgery. 


\section{O090-HERNIA-ADHESIONS_Abdominal wall hernia}

ROBOTIC INGUINAL HERNIA REPAIR: IS IT A NEW ERA IN THE SURGICAL MANAGEMENT OF INGUINAL HERNIA?

\section{E. Kakiashvili ${ }^{1}$, E. Brauner ${ }^{2}$, H. Gilshtain ${ }^{2}$}

${ }^{1}$ General Surgery, Galilee Medical Center, KIRIAT MOZKIN, Israel; ${ }^{2}$ General Surgery, Rambam Medical Center, HAIFA, Israel

Aim: robotic techniques relevance in inguinal hernia surgery is being examined. The study presents comparison of perioperative outcome between different surgical approaches for inguinal hernia.

Methods: retrospective cohort of 137 patients that underwent inguinal hernia repair at Rambam Medical Center during 2014-2016. Patients data was collected based on demographic characteristics, BMI, operating room time (ORT), Visual Analog Scale for Pain (VAS), postoperative need of analgesic, length of hospitalization ( $\mathrm{LOH}$ ) and perioperative complications.

Results: study population included 97 patients that underwent open inguinal hernia repair [12 bilateral (12.4\%); 85 unilateral (87.6\%)], 16 laparoscopic [8 bilateral (50\%); 8 unilateral (50\%)] and 24 robotic repair [17 bilateral $(70.8 \%) ; 7$ unilateral $(29.2 \%)]$.

Postoperative VAS level was significantly higher in open technique than in laparoscopic or robotic technique [median: 5.0 vs 2.0 vs $0 ; \mathrm{p}<0.001]$. Need of analgesics (per day) after surgery was also higher in open technique than in laparoscopic or robotic technique [median: 3.0 vs 1.5 vs $1.0 ; \mathrm{p}<0.001)$.

Operative room time (ORT) was much longer in robotic technique than in laparoscopic and or open technique [median: $92.5 \mathrm{~min}$ vs $79.0 \mathrm{~min}$ vs $44 \mathrm{~min} ; \mathrm{p}<0.001$ ).

Length of hospitalization was similar in each group (median1.0 day)

There was no different in terms of postoperative complications between three groups.

Conclusions: robotic inguinal hernia repair is technically feasible and safe procedure. Operative room time for robotic cases is significantly longer than laparoscopic and open techniques and there is substantial additional supply cost. There is clear benefit of robotic and laparoscopic techniques, comparing to open approach, in terms of patients postoperative recovery. Technical advantages of robotic technique and short learning curve may cause the more wide popularization of minimal invasive approach of the surgical management inguinal hernia.

\section{O091-HERNIA-ADHESIONS-Abdominal wall hernia THORACOSCOPIC SYMPATHECTOMY FOR PALMAR HYPERHIDROSIS WITH ELECTRO-COAGULATION VERSUS CLIPPING, IS THERE A DIFFERENCE?}

\section{A. Yehya}

Pediatric Surgery Department, Al-Azhar University Hospitals, CAIRO, Egypt

Background: Primary hyperhidrosis $(\mathrm{PH})$ is a neurological condition characterized by excessive sweating most often of the face, palms or axillae. Palmar hyperhidrosis is treated through sympathetic chain clipping or transection .We aiming to compare the efficacy and results obtained with both techniques.

Patients and Methods: Sixty Four patients underwent of 128 sympathetic procedures from March 2013 to February 2017. The patients were categorized into two groups: Right sided transection sympathectomy and left sided clipping. Patients were evaluated to compare the rates of success, satisfaction, compensatory sweating and recurrence either with transection or clipping of the T3 andT4 ganglion. Mean follow up was $15+7$ months.

Results: Sixty Four patients 24 males and 40 females undergoing electro-coagulation sympathectomy on the right side and clipping on the left side. With mean age was 15 years (range 13 to 18 years). All patients had balanced demographic data . No statistical difference between the two groups according rate of success. Compensatory sweating was observed in 28 patients $(43.75 \%)$ overall with 4 cases of severe unsatisfied compensatory sweating. Recurrence was reported in one case with transection and 2 cases in clipping. Satisfaction was occurred in 63 cases in transection group and 61 cases in clipping group .Pnumothorax was occurred in 2 cases in transection group compared to one case in clipping. No gustatory sweating and over dryness were reported in both groups.

Conclusion: Both thoracoscopic sympathetic transection and clipping of T3T4 ganglion are safe and effective procedure in palmar hyperhidosis treatment. with no differences regarding recurrence rate,satisfaction and incidence compensatory sweating.

Keywords: thoracoscopic sympathectomy,palmar hyperhidrosis, clipping, compensatory hyperhidrosis.

\section{O092-HERNIA-ADHESIONS_Abdominal wall hernia}

HYBRID APPROACH TO COMPLEX VENTRAL WALL HERNIA REPAIR: SINGLE INSTITUTIONAL COHORT STUDY

S. Jamel ${ }^{1}$, S. Huf ${ }^{2}$, K. Tukanova ${ }^{1}$, S. Markar ${ }^{1}$, S.M. Hakky ${ }^{1}$, S. Purkayastha ${ }^{1}$

${ }^{1}$ Department of Cancer and Surgery, Imperial College London, LONDON, United Kingdom; ${ }^{2}$ Imperial College London, LONDON, United Kingdom

Introduction: Primary ventral hernias and ventral incisional hernias pose a challenge for surgeons throughout the ages. Even though minimally invasive surgery and hernia repair have evolved rapidly, there is no standardized method that adequately decreases postoperative complications. Hybrid hernia repair is a surgical repair, which has not been adopted widely. It combines both a laparoscopic and open component allowing sac excision, primary defect repair as well as laparoscopic mesh insertion.

Aims: To evaluate the short-term and long-term outcomes of hernia recurrence for patient undergoing hybrid ventral repair (HVR) for the treatment of primary and incisional ventral hernias.

Methods: Between October- 2012 and June- 2013, hybrid VHR was performed in 24-patients a St Mary's Hospital, Imperial College London. The medical records of these patients were reviewed retrospectively for demographics, comorbidities, prior surgeries, body mass index (BMI), hernial defects, hybrid technique used; mesh selection, operative time, complications and recurrences over a 5-year follow-up.

Results: Twenty-four patients who underwent hybrid VHR were included with surgery performed by two surgeons. The mean age is 48 -years with a mean BMI of $33.1 \mathrm{~kg} / \mathrm{m}^{2} .88 \%$ had incisional hernias and $12 \%$ had primary hernias. The number of hernia defects ranged from 1 to 4 , with the average mesh size used was $15 \times 17 \mathrm{~cm}$. Extensive adhesionolysis was performed in 58\% of patients.

30-day postoperative complications; 2 patients developed post-operative seroma, paralytic ileus in 1 , pain control in 1 and urinary retention in 1 patient. There were no conversions to open procedures.

The mean length of hospital stay was 2-days. None of the patients developed chronic pain and only one recurrence over the 5-year follow-up period.

Conclusions: The hybrid technique for VHR is safe and feasible, and has important benefits over an open or purely laparoscopic approach, including a low rate of seroma formation, chronic pain and fiveyear hernia recurrence. Future investigation may include randomized controlled trials, to fully evaluate the benefits of hybrid VHR, with careful assessment of patient-centred end-points including quality of life and postoperative pain. 


\section{O093-HERNIA-ADHESIONS-Abdominal wall hernia}

\section{LAPAROSCOPIC SURGERY AND EFFICACY OF PREOPERATIVE DEXAMETHASONE}

K. Haxhirexha ${ }^{1}$, F. Dika-Haxhirexha ${ }^{2}$, N. Baftiu ${ }^{3}$, T. Emini ${ }^{1}$, B. Fejzuli ${ }^{4}$, A. Ademi ${ }^{1}$

${ }^{1}$ Surgery, Medical Faculty-University of Tetove, TETOVE, Macedonia; ${ }^{2}$ General medicine, Medical Faculty-University of Tetove, TETOVE, Macedonia; ${ }^{3}$ Anestesiology, Medical FacultyUniversity of Tetove, TETOVE, Macedonia; ${ }^{4}$ Surgery, Clinical Hospital-Tetove, TETOVE, Macedonia

Laparoscopic cholecystectomy is widely used operative technique and it's characterized with less postoperative hospitalization and side effects.

Duration of the hospitalization after laparoscopic surgery depends on several factors of which pain and physical weakness are the most important. Dexamethasone is well known; not only for its anti inflammatory effects but at the same time for analgesic and antiemetic effects, although the mechanism of this effects are not clarified yet.

Objectives: The aim of our study is the evaluation of analgesic effect of dexamethasone on reducing postoperative pain after laparoscopic surgery. Patients and Methods: In this study, 200 patients aged $25-74$ years old undergoing laparoscopic surgery, were classified into two groups, 100 patients in each group. The first group were treated with a intravenous injection of $8 \mathrm{mg}$ dexamethasone preoperatively and another dose the next day after operation. The second group received a intravenous injection of normal saline. We evaluated the dose of consumed analgesics and antiemetic's drug during the first $24 \mathrm{~h}$ in both groups.

Results: According to our experience results the total dose of tramadol in a postoperative period in dexamethasone receiving group was smaller than in normal saline group. Measure of postoperative pain was assessed using the paper-based VAS scale. Our result shows that the intensity of post operative pain in a period during first $36 \mathrm{~h}$, after surgery in a group of patients treated with dexamethasone was lower compared with the group of patients treated with normal saline. Nausea and vomiting during the first $36 \mathrm{~h}$ was significantly lower in the dexamethasone group than in the normal saline group.

Conclusion: Dexamethasone decreases postoperative pain, nausea and vomiting in patients after laparoscopic surgery as well as the need for analgesics.

Key words: dexamethasone, laparoscopic surgery, pain
O094-HERNIA-ADHESIONS-Abdominal wall hernia

SAFETY AND EFFECTIVENESS CLOSING MEDIUM-SIZE DEFECTS IN LVHR. DEFECT CLOSURE VS LAPAROSCOPIC INTRACORPOREAL RECTUS APONEUROPLASTY (LIRA)

J. Gomez Menchero $^{1}$, A. Gila Bohorquez ${ }^{1}$, E. Licardie Bolaños ${ }^{2}$, J.A. Bellido Luque ${ }^{3}$, J.M. Suarez Grau ${ }^{1}$, M. Sanchez Ramirez ${ }^{3}$, J. Garcia Moreno ${ }^{1}$, J. Landra ${ }^{1}$, I. Alarcon del Agua ${ }^{4}$, S. Morales Conde $^{4}$

${ }^{1}$ Surgery, Hospital de Riotinto, MINAS DE RIOTINTO, Spain;

${ }^{2}$ Surgery, Hospital Quiron Sagrado Corazon, SEVILLA, Spain;

${ }^{3}$ Surgery, Hospital Virgen Macarena, SEVILLA, Spain; ${ }^{4}$ Surgery, Hospital Virgen del Rocio Sevilla, SEVILLA, Spain

Aims: Closing the Defect (CD) during Laparoscopic Ventral Hernia Repair (LVHR) could be related to a reduction of seroma formation or bulging (hernia mesh) compared to conventional LVHR. But tension of the midline may contribute for some authors to a higher incidence of pain, recurrence in medium size defects and suggest to perform a Component Separation (CS) for restoring the midline in medium-large defects.We have developed a new technique for restoring the midline in medium ventral hernias (LIRA Technique) and weanalyzed our results in terms of pain and recurrence compared to our Conventional CD series (CCD).

Methods: We conducted a prospective controlled study of LVHR with CCD from January 2014 to December 2016 and a prospective controlled study performing LIRA technique from January 2015 to January 2017 . We analyzed and compared both techniques in medium size defects $(4-8 \mathrm{cms})$ in terms of postoperative pain (1,7 days, 1, 3 months and 1 year) using a Visual Analogue Scale (VAS), Bulging (return to prior distance among rectus muscles with the mesh in the sac in CT that didn't need surgical treatment)and recurrence (by physical examination and Tomography).

Results: CCD was performed in 42 patients (mean age was $58.10 \pm 13.15$ years old and mean BMI was $33.11 \pm 6.61 \mathrm{~kg} / \mathrm{m}^{2}$ ) and LIRA technique in 12 patients (Mean age was $56.5 \pm 10.5$ years old and mean BMI was $30.12 \pm 5.30 \mathrm{~kg} / \mathrm{m}^{2}$ ). The mean average Follow-up in both series was 1 year. Mean average VAS in CCD was $5.35 \pm 2.49$ ( 1 day), $2.01 \pm 2.13 \quad$ (7 days) $0.62 \pm 1.45 \quad(1$ month $) \quad 0.10 \pm 0.43$ ( 3 months) and 0 at 1 year. In LIRA series VAS was $3.9 \pm(24 \mathrm{~h})$ $1.08 \pm 1.78$ (7 days), $0.08 \pm 0.28$ (1 month), 0 (3 months) and 0 ( 1 year). There are 6 cases of Bulging in CCD series and 1 recurrence. Bulging and recurrence were absent in LIRA series.

Conclusions: LIRA technique might be a safe procedure in medium size defects for restoring the midline in LVHR, and could be related to a lower pain rate compared to CCD with no recurrence or bulging. 


\section{O095-HERNIA-ADHESIONS-Abdominal wall hernia}

NOVEL APPLICATION OF SEWING MACHINE PRINCIPLE; A NEW SIMPLIFIED INTRACORPOREAL SUTURING OF PEDIATRIC INGUINAL HERNIA

R. Shalaby $^{1}$, M. Abd-Alrazek ${ }^{1}$, A. al-Saied ${ }^{2}$, S. Mohamad ${ }^{1}$, A. Seddek ${ }^{1}$

${ }^{1}$ Pediatric Surgery, Al-Azhar University Hospitals, CAIRO, Egypt; ${ }^{2}$ Pediatric Surgery, Mansour University, MANSOURA, Egypt

Background: The desire of pediatric surgeon to reduce incision related morbidity and pain while achieving good cosmetic results has recently led to the introduction of single incision pediatric endo-surgery [SIPES] and needlescopic surgery. Intracorporeal suturing and knot tying during SIPES remains challenging. The aim of this study is to introduce a novel and simple technique for intracorporeal suturing of the pediatric inguinal hernia after needlescopic disconnection of hernia sac using just needles rather than laparoscopic instruments. It is an imitation of the principles of sewing machine.

Methods: The first author discussed the idea of the technique with the co-authors and a demonstration was done on a Silicon Pad before application of the technique on children with congenital inguinal hernia $[\mathrm{CIH}]$ for peritoneum closure after needlescopic disconnection of the hernia sac. The main outcome measurements were; feasibility of the technique, knot quality, suture placement accuracy, performance and suturing time and recurrence rate.

Results: The sutures were snugly applied to the ridges of Silicon Pad with good approximation and the knot was firmly tightened in all experiments. After applying and mastering the technique on a Silicon Pad, we shifted to use it on 373 children with 491 hernia defect. All operations were completed by the needlescopic technique without the need for insertion of any laparoscopic instruments. The time required for suturing of the peritoneum around internal inguinal ring [IIR] and knot tying, decreased considerably from $5 \mathrm{~min} 27 \mathrm{~s}$ in the first operation to less than $3 \mathrm{~min}$ after the fifth operation and stabilized at approximately 2 minute $30 \mathrm{~s}$. No major intraoperative complication and no recurrence.

Conclusion: The closure of the peritoneum around the IIR using needles mimicking what is happening in sewing machine suturing is feasible, safe and effective line of treatment of children with CIH. The cosmetic results are outstanding without any recurrence.

\section{O096-HERNIA-ADHESIONS-Abdominal wall hernia}

\section{RANDOMIZED PROSPECTIVE STUDY OF LAPAROSCOPIC} TRANS-ABDOMINAL PREPERITONEAL REPAIR (TAPP) VS THE OPEN REPAIR FOR BILATERAL INGUINAL HERNIA

V. Ferri, B. Ielpo, E. Vicente, Y. Quijano, D. Hipolito, E. Diaz, I. Fabra, L. Malave, R. Isernia, E. Pinna, R. Caruso,

General Surgery, Sanchinarro University Hospital, MADRID, Spain

Background and Aims: In the last decade there has been an increased interest in the laparoscopic repair approach of inguinal hernia showing benefits. We expect that even more benefits may exist for bilitateral inguinal hernias

However, in the scientific literature, benefits of laparoscopic versus open approach for bilateral inguinal hernia repair are still unknown.

The aim of this study is to compare the open Lichtenstein repair (OLR) and laparoscopic transabdominal preperitoneal (TAPP) repair.

Study design: This study was conducted at Sanchinarro University Hospital between March 2013 and May 2017.

Patients who presented with a primary, reducible bilateral inguinal hernia were included as patients which reach a minimum of one year of follow up. Outcome parameters included hospital stay, operation time, postoperative complications, immediate postoperative and chronic pain, recurrence. Quality of life according to the standardized SF36 questionnaire was recorded. The outcome parameters included surgical and post-operative costs, quality adjusted life years (QALY), and incremental cost per QALY gained or the incremental cost effectiveness ratio (ICER).

The primary end-point was to compare clinical outcome as well as cost effectiveness study between both groups.

Results: A total of 148 patients were enrolled (70 of them underwent TAPP and 78 OLR). Drop out occurred in 5 cases ( 2 of TAPP and 3 of OLR group).

Patient characteristics were statistically similar between the 2 groups. TAPP procedure had less early post-operative pain $(\mathrm{p}=0.037)$, a shorter length of stay $(\mathrm{p}=0.031)$ and less postoperative complications $(\mathrm{p}=0.002)$ when compared with the OLR approach. A slightly higher recurrence rate in the TAPP group was found. Additionally, there is a trend towards a higher postoperative quality of life and less chronic pain in the TAPP group.

Conclusions: TAPP procedure for bilateral inguinal hernia effectively reduces early postoperative pain, hospital stay and postoperative complications.
O097-HERNIA-ADHESIONS-Abdominal wall hernia

INCISIONAL VENTRAL HERNIA REPAIR WITH SELFGRIPPING MESH IN OPEN SUBLAY TECHNIQUE: PRELIMINARY Results

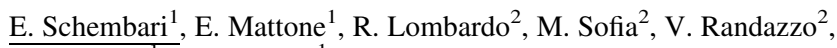
G. la Greca', D. Russello ${ }^{1}$

${ }^{1}$ Cannizzaro Hospital, University of Catania, CATANIA, Italy; ${ }^{2}$ Cannizzaro Hospital, CATANIA, Italy

Aim: The purpose of this study was to evaluate the long-term results in terms of safety and efficacy of a new technique to repair incisional ventral hernias with a self-gripping mesh, after a mean follow-up period of 15 months.

Methods: A retrospective, single-centre study was performed from June 2016 to June 2018 . Al patients undergoing elective incisional ventral hernia repair were included. Hernias were diagnosed based on clinical examination at the outpatient clinic. In case of doubtful diagnosis, CT-scan was used to confirm the diagnosis. The component separation technique and, when needed, TAR were performed. The self-gripping mesh was placed in sublay position (overlap $5 \mathrm{~cm}$ ) with the self-gripping surface face down. In all cases drainage tubes were placed in retromuscular and supraaponeurotic position. The following characteristics were collected: age, sex, Body Mass Index (BMI), smoking, comorbidities, number of previous surgical operations, defect size (EHS classification), mesh size, postoperative complications, duration of follow-up. All patients were interviewed by telephone every six months. When patients complained recurrence or other symptoms, visits were organized and when there was the doubt of recurrence a CT-scan was performed.

Results: A total of 40 patients were included in this study, 21 males, mean age was 59 years. $83 \%$ of patients had BMI $>25$, smokers and diabetics were respectively $28 \%$ and $9 \%$. The mean defect size was $115 \mathrm{~cm}^{2}$. Component separation technique was associated with TAR in 6 patients. In 11 cases the size of mesh was $20 \times 15 \mathrm{~cm}$, while in 7 patients the size of mesh was $30 \times 15 \mathrm{~cm}$ and in 11 cases this was $15 \times 15 \mathrm{~cm}$. In the other patients the mesh sizes were tailored to defect dimensions. Subcutaneous seromas occurred in 7 patients, they were treated conservatively in 5 cases and with percutaneous punction in 2 cases. Long-term follow-up demonstrated recurrences in one case, while in another one CT-scan revealed a bulging. No cases of mesh infection, pain or sensation of mesh

Conclusions: This study with a mean follow-up period of 15 months demonstrated that the use of self-gripping mesh in sublay position is safe and effective to treat incisional ventral hernias.

\section{O098-HERNIA-ADHESIONS-Adhesions}

\section{LAPAROSCOPIC TRANSABDOMINAL REAPAIR OF GIANT MORGAGNI HERNIA}

M. Ortenzi ${ }^{1}$, G.I. Lezoche ${ }^{1}$, G.I. Biondini ${ }^{1}$, A.N. Balla ${ }^{3}$, A.M. Paganini ${ }^{3}$, M.A. Guerrieri ${ }^{1}$

${ }^{1}$ Clinica Chirurgica, Università Politecnica delle Marche, ANCONA, Italy; ${ }^{3}$ Department of General Surgery and Surgical Specialties, Paride Stefanini, Sapienza University of Rome, ROMA, Italy

Aim: Morgagni hernias present technical challenges. The laparoscopic approach was described at first in 1992, however, as they are uncommon in adult life and, little data exist on the optimal method of surgical management. This study purpose was to analyse a method for laparoscopic repair of Morgagni giant hernias using laparoscopic primary closure.

Methods: This case series describes a method of laparoscopic Morgagni hernia repair using primary closure. In all patients a laparoscopic transabdominal approach was used. The content of the hernia was reduced into the abdomen and the diaphragmatic defect was closed with a running laparoscopic suture using a self-fixating suture. Clips were placed at the edges of the suture to secure the pledged sutures to both the anterior and posterior fascia. Demographic data as age, gender and BMI were collected. Operative data (operative time, rate of conversion, blood loss) and post-operative data (short and long term complications, length of hospital stay, need of readmission and reoperation) were recorded.

Results: Retrospectively collected data about 9 patients were analysed. There were $1(11.1 \%)$ male and $8(88.8 \%)$ females. The median BMI was $29.14 \pm 5.2 \mathrm{Kg} / \mathrm{m}^{2}$. Median operative time was $80 \pm 25 \mathrm{~min}$. There were no intraoperative complications nor conversion to open surgery. Patients began a fluid diet on the first post-operative day and were discharged after a median hospital stay of $3 \pm 1.87$ days. In a median follow up of 36 months we did not observe any recurrences.

Conclusions: Transabdominal laparoscopic approach with primary closure of the diaphragmatic defect is a viable approach for repair of Morgagni hernia. In our experience, the use of laparoscopic transabdominal suture fixed to the fascia allowed the closure of the defect laparoscopically with minimal tension on the repairs. 


\section{O099-HERNIA-ADHESIONS-Adhesions}

CAN WE PREDICT THE SUCCESS OF THE LAPAROSCOPIC APPROACH IN THE ADHESIVE SMALL BOWEL OBSTRUCTION?

C. Tellez Marques, E. Sebastian Valverde, E. Membrilla Fernandez, L. Grande Posa, I. Poves Prim

General surgery, Parc de Salut Mar-Hospital del Mar, BARCELONA, Spain

Aims: The laparoscopic approach in the acute adhesive small bowel obstruction and internal hernias (ASBO) has shown superior to laparotomy in terms of morbidity and hospital stay. Especially, in patients who present simple adhesions or internal hernias. According to this, the aim of the study is to determine those preoperative factors associated with simple adhesions and internal hernias, and consequently, improve the success of the laparoscopic approach in ASBO

Methods: A retrospective study of patients who underwent urgent surgery for ASBO was conducted from January 2007 to May 2016. We compare preoperative variables between single adhesions and internal hernias vs complex adhesions. A p value $<0.05$ was considered statistically significant.

Results: We analysed 262 patients who underwent surgery for ASBO, 78 (30\%) by laparoscopy and $184(70 \%)$ by laparotomy. Conversion rate in laparoscopy was $38.5 \% .49 .2 \%$ of patients presented a single adhesion or internal hernia; and $50.8 \%$ were considered complex adhesions. Sex and age did not correlate with the type of adhesions. Previous surgery $(\mathrm{p}<0.001)$, number of previous surgeries $(\mathrm{p}<0.001)$, ASA $(\mathrm{p}<0.001)$ and previous abdominal wall mesh $(\mathrm{p}=0.002)$ were significantly associated with complex adhesions. Laparoscopy as the only surgical history was significantly associated with simple adhesions $(p=0.033)$. Only appendectomy $(\mathrm{p}=0.139)$ or supramesocolic $(\mathrm{p}=0.076)$ previous surgeries tended to present single adhesions but it did not reach statistical significance. The need for intestinal resection was not related to the type of adhesions $(p=0.743)$. There was a significant correlation between the findings in the CT (computed tomography) and the type of adhesion found $(p=0.001)$. Signs of ischaemia on CT were related to the need for intestinal resection $(p<0.001)$. In the multivariate analysis, the number of previous surgeries, ASA and CT scan findings were identified as independent factors related to the type of adhesion.

Conclusions: According to our study, a lower number of previous surgeries, ASA I-II and internal hernia in the CT scan are associated with single adhesions and internal hernias. Patient selection is a key factor for the success of laparoscopic approach in ASBO

\section{O100-HERNIA-ADHESIONS-Emergency surgery}

AN INTERNATIONAL COMPARISON OF THE UTILISATION OF AND OUTCOMES FROM MINIMAL ACCESS SURGERY FOR THE TREATMENT OF COMMON ABDOMINAL SURGICAL EMERGENCIES

\section{S.R. Markar ${ }^{1}, \underline{\text { K. Tukanova }}{ }^{2}$, A. Vidal-Diez ${ }^{2}$, G.B. Hanna ${ }^{2}$}

${ }^{1}$ Surgery \& Cancer, Imperial College London, LONDON, United Kingdom; ${ }^{2}$ Surgery \& Cancer, St-Mary's Hospital, Imperial College London, LONDON, United Kingdom

Aims: There aims of this study were:

(i) To compare England with the United States in the utilisation of minimal access surgery (MAS) and in-hospital mortality from four common abdominal surgical emergencies (appendicitis, incarcerated or strangulated abdominal hernia, small or large bowel perforation and peptic ulcer perforation).

(ii) Within England to evaluate the influence of MAS upon in-hospital and long-term mortality.

Methods: Between 2006 and 2012, the rate of MAS and in-hospital mortality for four abdominal surgical emergencies were compared between the United States and England. Univariate and multivariate analyses were performed to adjust for underlying differences in baseline patient demographics.

Results: 132,364 admissions in England for four abdominal surgical emergencies were compared to an estimated 1,811,136 admissions in the United States.

After adjustment for patient demographics, MAS was used less commonly England for three conditions; appendicitis (odds ratio (OR) $0.30,95 \% \mathrm{CI}$ $0.30-0.31$ ), abdominal hernia (OR $0.18,95 \%$ CI $0.17-0.19$ ) and small or large bowel perforation (OR $0.48,95 \%$ CI $0.46-0.51$ ).

In-hospital mortality in multivariate analysis, was increased in England compared to the United States for three conditions; abdominal hernia (OR $1.91,95 \%$ CI 1.81-2.01), small or large bowel perforation (OR 2.33, $95 \%$ CI 2.25-2.42) and peptic ulcer perforation (OR 2.02, 95\% CI 1.91-2.14). In England, after adjustment for patient demographics, open surgery was associated with increased in-hospital mortality for three conditions; abdominal hernia (OR 1.80, 95\% CI 1.26-2.71), small or large bowel perforation (OR $1.59,95 \%$ CI 1.37-1.87) and peptic ulcer perforation (OR $2.31,95 \%$ CI 1.91-2.82). Similarly open surgery was associated with increased long-term mortality for three conditions; abdominal hernia (HR $1.32,95 \%$ CI 1.15-1.52), small or large bowel perforation (HR 1.30, 95\% CI 1.18-1.43) and peptic ulcer perforation (HR 1.69, 95\% CI 1.50-1.89). Conclusions: Minimal access surgery was used less commonly and inhospital mortality was increased in England compared to the United States for common abdominal surgical conditions. Given the benefits of MAS shown in this large study, strategies to enhance adoption of MAS in emergency conditions in England need to be optimised and include appropriate patient selection and improved surgeon MAS training and experience. 


\section{O102-HERNIA-ADHESIONS-Inguinal hernia}

SUTURE FIXATION VERSUS SELF-GRIPPING MESH FOR OPEN INGUINAL HERNIA REPAIR: SYSTEMATIC REVIEW, META-ANALYSIS AND TRIAL SEQUENTIAL ANALYSIS

S.A. Antoniou ${ }^{1}$, F. de Haes $^{1}$, S. Hajibandeh ${ }^{2}$, S. Hajibandeh ${ }^{3}$, F.E. Muysoms ${ }^{4}$, M. López-Cano ${ }^{5}$, J. Garcia-Alamino ${ }^{6}$, N.J. Smart ${ }^{1}$

${ }^{1}$ Surgical Service, Royal Devon \& Exeter NHS Foundation Trust, EXETER, United Kingdom; ${ }^{2}$ General Surgery Department, Salford Royal Foundation Trust, SALFORD, United Kingdom; ${ }^{3}$ General Surgery Department, North Manchester General Hospital,

MANCHESTER, United Kingdom; ${ }^{4}$ Department of Surgery, Maria Middelares Hospital, GHENT, Belgium; ${ }^{5}$ Abdominal Wall Surgery Unit, Department of General Surger, Hospital Universitari Vall d'Hebron, Universitat Autònoma de Barcelona, BARCELONA, Spain; ${ }^{6}$ Nuffield Department of Primary Care Health Sciences, University of Oxford, OXFORD, United Kingdom

Aims: Self-adhering meshes have been launched as material potentially reducing pain and surgery time. Meta-analyses of randomized trials (RCTs) have suggested no significant effect in terms of postoperative pain in open inguinal hernia repair. Aim of this study was to perform an update metaanalysis to consolidate the non superiority hypothesis of self-adhering mesh over traditional mesh and to investigate whether evidence is conclusive through trial sequential analysis.

Methods: The databases of Medline, Embase, CENTRAL and OpenGrey were interrogated to identify relevant RCTs. Incidence of pain and quantitative pain assessment through the Visual Analogue Scale (VAS) score were primary outcome measures. Duration of surgery was secondary outcome. The MantelHaenszel approach was used to synthesize data and sensitivity analyses included calculation of the Peto odds ratio, risk difference and random effects meta-analysis. The Lands \& DeMets method was used for trial sequential analysis with $\mathrm{a}=0.05, \beta=0.2$ and $\mathrm{O}$ 'Brien-Fleming a-spending boundaries. Relative risk reduction was calculated from low risk of bias studies. The analysis conformed to PRISMA standards.

Results: Thirteen RCTs with low to moderate risk of bias were identified. There was no difference in the incidence of pain at 3-12 months (risk ratio, RR 1.05, 95\% confidence interval, CI $0.75-1.49$ ), 2 years (RR 1.16, 95\% CI $0.43-3.12$ ), and 3-4 years (RR $0.93,95 \%$ CI $0.45-1.93$ ). VAS scores were also similar across follow up intervals. No difference in hematoma/seroma, wound infection, hospital stay, reoperation or recurrence was seen. Self-adhering mesh was associated with a mean operative time difference of $-7 \min (95 \%$ CI -10 to -4$)$. Trial sequential analyses consolidates non superiority in terms of long-term postoperative pain, the z-curve having passed the no effect boundary well beyond the information size.

Conclusion: Self-adhering mesh is not effective in reducing short-term and long-term postoperative pain after open hernia repair. This evidence is definitive and no further trials defining this endpoint are required. Reduction in operative time is negligible and the additional cost does not justify the routine use of self-adhering mesh in this context.

Registration: CRD42018069266

\section{O103-HERNIA-ADHESIONS-Inguinal hernia}

\section{OPEN AND LAPAROSCOPIC INGUINAL HERNIA SURGERY: A COST ANALYSIS}

F. Mongelli ${ }^{1}$, A. Ferrario di Tor Vajana ${ }^{1}$, M. Fitzgerald ${ }^{1}$, S. Cafarotti ${ }^{1}$, M. Lucchelli ${ }^{2}$, F. Proietti ${ }^{1}$, M. di Giuseppe ${ }^{1}$, D. la Regina ${ }^{1}$

${ }_{1}$ Surgery, Ospedale Regionale di Bellinzona e Valli, BELLINZONA, Switzerland; ${ }_{2}$ Medical Controlling, Ospedale Regionale di Bellinzona e Valli, BELLINZONA, Switzerland

Background: In the treatment of inguinal hernias, there is little hard evidence concerning the economic reimbursement in the diagnosis-related-group (DRG) era. Factors that affect whether a hospital may earn or lose financially depending on open or laparoscopic approach is still underexplored. The aim of this study is to provide a reliable analysis of in-hospital costs and reimbursements in inguinal hernia surgery. Methods: This retrospective study analysed the 1-year experience in inguinal hernia repair in patients undergoing open Lichtenstein (OL), laparoscopic totally extraperitoneal unilateral (UTEP) or bilateral (BTEP) hernia repair. Demographics, results, costs and DRG-based reimbursements were recorded and analysed. Results: During the study period, 39 patients underwent OL, 82 patients UTEP and 16 patients BTEP. The average total cost amounted to 4126 EUR in OL, 5134EUR in UTEP and 7082 EUR in BTEP groups $(\mathrm{p}<0.001 *)$. The hospital reimbursement amounted to 5486 EUR, 5252 EUR and 6555 EUR in the OL, UTEP and BTEP groups respectively $(\mathrm{p}<0.001 *)$. Finally, the mean hospital earnings were 1360 EUR, 118 EUR and -527 EUR for each patient in OL, UTEP and BTEP respectively $(\mathrm{p}<0.001 *)$. Conclusions: In-hospital costs were higher in UTEP and BTEP as compared to OL. The DRG-based reimbursement provided adequate compensation for patients with unilateral inguinal hernia, whereas hospital earnings were profitable in OL group only, and led an overall financial loss in the BTEP group. Surgeons should be conscious that clinical advantages of the laparoscopic approach are not adequately compensated for, from an economic point of view.

\section{O105-HERNIA-ADHESIONS—Inguinal hernia}

LAPAROSCOPIC IMPLANTATION OF MESENCHYMAL STEM CELL-COATED MESHES IN A SWINE MODEL OF ABDOMINAL HERNIA

F.M. Sanchez Margallo ${ }^{1}$, F. Marinaro ${ }^{2}$, R. Blazquez Duran ${ }^{2}$, M. Veloso Brun ${ }^{3}$, J.G. Casado ${ }^{2}$

${ }^{1}$ Scientific Direction, Minimally Invasive Surgery Centre, CÁCERES, Spain; ${ }^{2}$ Stem Cell Laboratory, Minimally Invasive Surgery Centre, CÁCERES, Spain; ${ }^{3}$ Surgery, Universidade Federal de Santa Maria, SANTA MARIA, Brazil

Aims: Umbilical hernias are common anatomical defects in swine which become a suitable model for surgical training and research in the field of surgical meshes. The aim of this study was to develop a surgical protocol for a successful laparoscopic implantation of stem cell-coated surgical meshes.

Methods: 9 Large White pigs, weighing 25-68 kg and with congenital abdominal hernia were anesthetized for the surgical procedures. Non absorbable polypropylene surgical meshes were coated with fibrin glue (FG) (control group) or with FG admixed with porcine bone marrowderived mesenchymal stem cells (FG/BM-MSCs). Approximation of hernia's borders was performed by intracorporeal suture. The meshes were carefully rolled inside the trocar for laparoscopic implantation. The surgical implantation was performed by laparoscopy using helicoidal staples. Laparoscopic inspections and biopsies of the tissue surrounding the mesh were performed at 7, and 30 days post-implantation. At day 30, the animals were euthanized and macroscopically evaluated. Ultrasonography was used at day 0,7 , and 30 to evaluate the size of the hernia. The biopsies were then processed for the histological analysis.

Results: Ultrasonography demonstrated that the mean size of umbilical hernias before mesh implantation was $2.49 \pm 0.99 \mathrm{~cm}$. A decrease in hernia mean size was observed at day 7 and 30 The laparoscopic procedures allowed a successful mesh implantation in all animals. In most of cases, the implantation site did not show excessive inflammation or tissue adhesions. But one animal showed hernia maintenance. One animal had peritoneal and implant-site infection. Foreign body reaction was noted in the histological analysis, although no significant difference was found between the control, and BM-MSC group.

Conclusions: The anatomical similarities between humans and pigs in umbilical hernias make this animal model useful to: I) improve minimally invasive surgical procedures for hernia treatment; II) evaluate new surgical meshes, and III) introducing stem cell therapy to hernia surgical repair. The laparoscopic approach is efficient and safe for the implantation of stem cellcoated meshes. Gene and protein expression analysis are required to evaluate the molecular changes between the conventional and the stem cell surgical approach. 


\section{O106-HERNIA-ADHESIONS-Inguinal hernia}

\section{RECURRENCES, COMPLICATIONS AND PATIENT SATISFACTION AFTER TRANSABDOMINAL} PREPERITONEAL HERNIA REPAIR (TAPP) IN 1120 CASES

\section{Tomala}

Klinik fuer Chirurgie, Schoen Klinik Neustadt, NEUSTADT IN HOLSTEIN, Germany

Background: The recurrence rates and the complication rates for groin hernia repair have been heterogeneous reported in the literature. Most of all the recurrence rates vary a wide range from 0.5 to $15 \%$. The aim of the study was to report recurrence rates, complication rates and patient satisfaction from 1120 single-center transabdominal pre-peritoneal inguinal hernia surgeries (TAPP) performed at the Schoen Klinik Neustadt between 2005 and 2014.

Methods: A follow-up questionnaire was sent out to all by TAPP operated patients in 2016 and the electronic medical files were investigated.

Results: The response rate to the follow-up questionnaire was $60.5 \%$. Patients reported a recurrence rate of $4.4 \%$ ( $95 \%$ confidence intervall (CI) $3.10 \%-6.30 \%$ ). If patients had at least 5 years of follow-up, the recurrence rate was $5.23 \%$, thus approximately 1.91 times higher when compared with patients with a follow-up between one and 5 years. In $9.86 \%$ of all surgeries, recurrence were operated. If patients had a recurrence at surgery, their odds for another recurrence was 3.30 times increased $(\mathrm{p}=0.01)$. Patients with a recurrence had a higher body mass index (median $1.61 \mathrm{~kg} / \mathrm{m}^{2}$ ). In $52.8 \%$ of patients, a medial inguinal hernia was recurrent, while lateral inguinal hernias were non-recurrent in $47.8 \%$ of operated patients. The risk of the complication (hematoma, seroma, wound infection) after TAPP was $4.80 \%$, and the odds for a recurrence was 3.36 times higher in case of complication $(p=0.001)$. The general satisfaction of the patients with the results of operation was $81.65 \%$ in the group of satisfied and very satisfied patients, and $13.76 \%$ in the group of dissatisfied and very dissatisfied patients. Regarding the patient satisfaction there was a big discrepancy between a patient group with recurrence, complication and current pain at the operated groin when compared with the group without recurrence, complication and current pain.

Conclusions: In accordance to the literature, there was a discrepancy between the proportion of by TAPP operated recurrences and the recurrence rate at follow-up. It may be explained by differences in follow-up times and the exclusion of hospitals with lower number of operated patients in the general reporting of recurrences.

\section{O107-HERNIA-ADHESIONS-Inguinal hernia}

\section{SPORTSMAN HERNIA THIGH NERVES ENTRAPMENT REPAIRED BY TEP INGUINAL LIGAMENT RELEASE \& REINFORCE TECHNIQUE}

\section{Dudai, K. Ittah Gilboa}

Surgery, MERAV Medical Center, Hernia Excellence, TEL AVIV, Israel

Aims: The pathologies of Sportsman Hernia (SH) are found in the Posterior Wall (PW), Conjoint Tendon and Inguinal Ligament (IL). As a consequence, high pressure is created in the Inguinal Canal (IC), on the Genital and Femoral branches of the Genitofemoral Nerve and the Lateral Cutaneous Nerves of thigh (GFLCN), causing entrapment during Sport Activities (SA). The aim of the SH surgical repair is to release those pressure who caused entrapments and then reinforce the PW. We are presenting a study that demonstrating the SH Thigh Nerves Entrapment (TNE) and its release by the TEP Inguinal Ligament Release \& Reinforce Technique (IL-RRT).

Methods: Since 1986 we are using our TEP IL-RRT for repairing SH; combining Pressure Release with PW Reinforcing. A vast release of inflammatory adhesions on the Pubic bone and $\mathrm{PW}$ is performed, followed by dividing the IL at the level of the internal ring releasing the pressure from the GFLCN below it, which causes the entrapment. To complete the procedure, we are reinforcing the PW with wide light PPP mesh. The IL-RRT is followed by the Athletic Muscles Rehabilitation Program. On 2017 we started a study in order to demonstrate the TNE and its release by the TEP IL-RRT. Any SH candidate for TEP IL-RRT was tested for the presence of hypersensitivity or hyposensitivity according to his skin Thighs Dermatomes. Postoperatively he was re-tested, and findings were compared to the preoperative records.

Results: $90 \%$ of the preoperative SH candidates clinically tested, demonstrated positive finding for TNE. While in the postoperative checkup, positive findings for TNE persisted in less than $2 \%$ of the patients.

Conclusions: The injured IL is a major factor of pressure created in the IC and entrapping the GFLCN below it. Dividing the IL in addition to PW reinforcement, add a great advantage for relieving pain during SA. Positive changes demonstrated in TNE clinical examination following the TEP IL-RRT, prove both the pathophysiology of TNE in SH and the efficiency of the TEP ILRRT to loosen it. That, in addition to excellent results of less than $0.5 \%$ persistence of the pain after returning to $\mathrm{SA}$.

\section{O108-ROBOTICS \& NEW TECHNIQUES-Bariatrics}

\section{INDOCYANINE GREEN FLUORESCENCE ANGIOGRAPHY IN ESOPHAGOGASTRIC AND BARIATRIC PROCEDURES}

E. Licardie $^{1}$, T. Yang ${ }^{2}$, I. Alarcón ${ }^{2}$, V. Camacho ${ }^{2}$, M. Sánchez ${ }^{2}$, F. López ${ }^{2}$, M. Socas ${ }^{2}$, A. Barranco ${ }^{2}$, J. Padillo ${ }^{2}$, S. Morales-Conde ${ }^{2}$

${ }^{1}$ Sevilla, Hospital Quirónsalud Sagrado Corazón, SEVILLA, Spain; ${ }^{2}$ Unit of innovation in Minimally Invasive Surgery and Unit of General Surgery, University Hospital Virgen del Rocío, SEVILLA, Spain

Aims: Fluorescence angiography with indocyanine green (ICG) is used as a marker in the assessment of tissue perfusion, being more frequently used in colorectal procedures. This technology has shown to be a good technique to reduce complications related to vascular supply to the anastomosis. In esophagogastric procedures blood supply to the gastric pouch, jejunum and esophagus could be evaluated by ICG fluorescence imaging. It could be also used in bariatric surgery to evaluated the anastomoses, during Gastric Bypass, and blood supply to the gastroesophageal junction and the angle of His during Sleeve gastrectomy.

Methods: We have collected data during 8 gastric resection due to adenocarcinoma and 53 bariatric procedures that were performed by the same surgeon, using ICG fluorescence to evaluate blood supply. The ICG was infused before performing the anastomosis in order to evaluate the need to change the transaction line (TL). We analyzed those cases in which the TL was changed and the number of leaks in those cases that we changed this line.

Results: All the 61 cases were performed by laparoscopic approach: 5 Subtotal Gastrectomy (SG), 3 Total Gastrectomy (TG), 26 Gastric Sleeve (GS) and 27 Gastric Bypass. There were no changes regarding the TL before performing the anastomosis in any of the four types of procedures (SG, TG GS, GB). In the analyzed data there is 1 anastomotic leak in one SG procedure (1.6\%).

Conclusions: ICG fluorescence angiography could be helpful in assessing blood supply during gastrointestinal anastomosis, although we have not find an influence in the results during bariatric and gastric procedures. However, we do not have the sufficient evidence to determine the value of this technology in this entities, being needed more volume and data to improve the significance of the results.

\section{O109-ROBOTICS \& NEW TECHNIQUES-Basic and Technical research}

FLUORESCENCE-BASED ENHANCED REALITY VS. HYPERSPECTRAL IMAGING TO ASSESS BOWEL PERFUSION

M. Barberio ${ }^{1}$, F. Longo ${ }^{1}$, F. Fiorillo ${ }^{1}$, B. Seeliger ${ }^{1}$, P. Mascagni ${ }^{1}$, E. Seyller $^{1}$, V. Agnus ${ }^{1}$, J. Marescaux ${ }^{2}$, M. Diana ${ }^{1}$

${ }^{1}$ Research Department, IHU, STRASBOURG, France; ${ }^{3}$ Research Department, IRCAD, STRASBOURG, France

Aims: Hyperspectral Imaging (HSI) combines a spectrometer with a camera to analyze the tissues' optical properties in a broad wavelength range, without the need for a contrast agent. It provides extensive real-time information about tissue physiology, including oxygen saturation (StO2). Fluorescence-based Enhanced Reality (FLER) is a software solution providing a dynamic, quantitative analysis of the signal evolution of a systemically administered fluorophore, during fluorescence angiography (FA). The aim of this study was to compare the performance of HSI and FLER to assess bowel perfusion, in a porcine, non-survival model of bowel ischemia. Methods: In 6 pigs, an ischemic small bowel segment was created and imaged after 1 hour of ischemia. The imaging modalities were applied sequentially to the same area.HSI was performed first, to acquire the StO2 spectra, by means of the TIVITA ${ }^{\mathrm{TM}}$ system (Diaspective Vision, Pepelow, Germany), which provides a spectral range of 500-1000 nm and a $5 \mathrm{~nm}$ resolution. Subsequently, FA was performed using a NIR-capable laparoscopic camera (D-Light P, Karl Storz, Germany), after intravenous injection of $0.2 \mathrm{mg} / \mathrm{kg}$ of Indocyanine Green (ICG; Infracyanine, Serb, Paris, France). The fluorescence flow was recorded during $40 \mathrm{~s}$, then the slope of the fluorescence flow was analyzed using a proprietary software to obtain a virtual perfusion cartography. The virtual cartography was overlaid onto real-time images to obtain the enhanced reality effect. Ten adjacent regions of interest (ROIs) were selected from HSI datasets and were superimposed to FLER-generated cartographies using a custom plug-in software function, allowing for a quantitative comparison of both imaging modalities. HSI was repeated after ICG injection.

Results: The $\mathrm{r}^{2}$ correlation coefficient between HSI-StO2 and the FLER slope was 0.79. A control HSI after ICG injection, the correlation coefficient dropped significantly $\left(\mathrm{r}^{2} 0.45\right)$. The interference of ICG on HSI imaging was clearly identified in the spectral curves.

Conclusion: StO2 given by HSI provided results comparable to those obtained with FLER in our bowel ischemia model, without the need to inject a contrast agent. ICG interferes with HSI datasets, disrupting $\mathrm{StO} 2$ values. 


\section{O110-ROBOTICS \& NEW TECHNIQUES-Basic} and Technical research

\section{ROBOTIC FUNDOPLICATIONS FOR THE TREATMENT OF GIANT HIATAL HERNIA}

\author{
M. Koshkin, O. Vasnev, A. Belousov, M. Baychorov, P. Agami
}

High-tech surgery, Moscow Clinical Scientific Centre, MOSCOW, Russia

Surgical treatment is one of the most effective options for treatment of giant hiatal hernia Laparoscopic approach became is a 'gold standard' over the time demonstrating all advantages of minimally invasive techniques over the open procedures. However the utility of robotic operations still remains controversial.

Aim of the study: Evaluate the initial experience of robotic fundoplication in compare to laparoscopic procedures.

Materials and methods: Since the January till the December of 2017 thirty operations were operated on. Mean age was 57.2 (44-76), among them $12(65 \%)$ were female and $6(35 \%)$ were males. Mean BMI was 29.4 (24.1-41.0). Laparoscopic procedures were performed in 8 patients (1st group), robotic procedures with DaVinci system were performed in 10 patients of the second group.

Nissen fundoplication modified was performed in 14 patients, Toupet fundoplication was used for 4 patients.

Results: The median operative time in laparoscopic group was $150 \mathrm{~min}$, in robotic group$131,2 \mathrm{~min}$. There were no statistical differences between two groups $(\mathrm{p}=0.93)$.

Blood loss was minimal in both groups.

Mean postoperative hospital stay was 4.08 days (2-7 days) in the 1st group and 3,6 days (2-6 days) in the second. There were no statistical differences between two groups $(\mathrm{p}=0.19)$. Postoperative course was uneventful in all patients of both groups.

Conclusion: Robotic fundoplication is safe and effective method of treatment of giant hiatal hernia

Robotic surgery shows a number of advantages. Future multi-center studies should standardize intraoperative techniques.

\section{O111-ROBOTICS \& NEW TECHNIQUES-Basic and Technical research}

\section{EFFECT OF ROBOT-ASSISTED SURGERY ON SURGICAL STRESS RESPONSE: WHAT DO WE REALLY KNOW? :SYSTEMATIC REVIEW}

\section{E. Yiannakopoulou}

Biomedical Sciences, University of West Attica, ATHENS, Greece

Surgical stress response is associated with systemic inflammatory syndrome, sepsis, multiorgan dysfunction syndrome. Robotic assisted surgery has been introduced to overcome the limitations of conventional laparoscopy. This technique has potential advantages over laparoscopy, such as increased dexterity, three-dimensional view, and a magnified view of the operative field. These advantages could result in limited intra-abdominal trauma and hence in attenuated surgical stress response over conventional laparoscopy.

Aims: This study aimed to synthesize data on the effect of robot assisted surgery on surgical stress response.

Methods: Electronic databases were searched with the search terms 'surgical stress', 'stress response', 'oxidative stress', 'robotic assisted surgery', 'c-reactive protein', 'interleukin 6', 'interleukin 10','cortisol',', oxidative stress markers', 'antioxidants', 'antioxidant status', 'MDA', 'glutathione', 'cortisol', 'acute phase response' up to and including March 2018.

Results: One hundred forty studies were identified and their title and abstract were reviewed. One randomized controlled trial, six non randomized comparative studies, one experimental study and one case report met inclusion criteria. Data were discordant. One prospective trial concluded that cortisol and IL-6 were lower in laparoscopic assisted distal gastrectomy compared with robot assisted distal gastrectomy In another study comparing robotic assisted laparoscopic radical prostatectomy with open radical prostatectomy based on plasma measurements of IL-6, IL-1a and C-reactive protein, it was demonstrated that robotic assisted laparoscopic radical prostatectomy induces lower tissue trauma than open radical prostatectomy. In another study, it was reported reduced expression of genes associated with surgical stress response in patients treated with robotically assisted radical prostatectomy compared with patients treated with open prostatectomy. The case report concerned a case of polymyalgia rheumatic after robotic assisted laparoscopic prostatectomy. The experimental trial demonstrated that cortisol and substance $\mathrm{P}$ were significantly higher with open thoracic approach versus robot assisted thoracoscopic oesophageal surgery.

Conclusion: Further research is needed to elucidate the effect of robotic surgery on surgical stress, based on a well standardized protocol for the measurement of surgical stress response.

\section{O112-ROBOTICS \& NEW TECHNIQUES-Basic} and Technical research

OPTIMIZATION OF CIRCULAR STAPLING LINE
COMPRESSION FOR SAFE ANASTOMOSIS DURING
COLORECTAL SURGERY IN VITRO STUDY

\section{G.M. Son, Surgery}

Yangsan Pusan national university hospital, YANGSAN SI, Korea

Purpose: Tissue compression is essential to prepare the tissue for proper staple formation. This study evaluates the risk factors of compression injury on the circular stapling line in vitro. Methods: To reproduce the artificial bowel wall, a collagen plate was prepared by mixing collagen extracted from porcine with glycerin. Artificial collagen plates with $4 \mathrm{~mm}$ and $6 \mathrm{~mm}$ in the thickness were made for dry and healthy condition and immersed plates in the tap water for 10 min to make wet and edematous condition. Circular stapler (CDH25A, Ethicon, USA) was applied in the collagen plates (dry and wet condition) and optimal compressions. Compression line was evaluated for compression injury score. Risk factors for excessive compressions and unacceptable injury were analyzed.

Results: In the dry condition, optimal compression didn't cause unacceptable injury. In the wet condition, excessive compressions were occurred in $47.1 \%$ with optimal approximation. unacceptable injury was significantly different in proper and excessive compression cases as $18.8 \%$ and $5.6 \%$, respectively. On the univariate analysis, thickness $(6 \mathrm{~mm})$, wet condition, proximal side, maximal compression, and excessive compression were associated with unacceptable injury. On the multivariate analysis using Logistic regression model, excessive compression was significant independent factor to cause tissue injury $(\mathrm{p}<0.001)$ and this significance was also proved in the optimal compression group $(\mathrm{p}=0.021)$

Conclusion: Excessive compression could result in unacceptable damage to the circular stapling line of thick tissue with edema and the careful guideline is required to reduce compression injury of stapling line for safe anastomosis for colorectal cancer patients.

Keywords: circular stapler, over-compression, compression injury, anastomosis, colorectal surgery

\section{O113-ROBOTICS \& NEW TECHNIQUES-Basic and Technical research}

NEEDLESCOPIC VERSUS SINGLE INCISION LAPAROSCOPIC APPENDECTOMY IN CHILDREN: A PROSPECTIVE RANDOMIZED TRIAL

M.A. Ismail Lotfallah ${ }^{1}$, A. Shams ${ }^{1}$, A.h. Abdelghaffar ${ }^{1}$, A.H. Fawzy ${ }^{2}$, M. Maged ${ }^{1}$

${ }^{1}$ Pediatric surgery, Al Azhar university, GIZA, Egypt; ${ }^{2}$ Pediatric surgery, Beni suef University, BENI SUEF, Egypt

Background: Minimal invasive appendectomy gained much popularity due to its better cosmoses, early recovery and less wound site infections. Single incision laparoscopic appendectomy (SILA) has many disadvantages such as, long operative time, bad ergonomics, surgical site infections, high conversion rate and port site hernia. Needlescopic appendectomy (NA) using Mediflex ${ }^{\circledR}$ facial closure needle expected to be more superior over SILA. Here in we compare our results of needlescopic appendectomy with single-incision one.

Material and methods: one hundred and twenty patients with acute non complicated appendicitis were randomly assigned to NA and SILA 60 children for each group during the period between January 2015 to October 2018. The main outcome measurements included, demographics, operative time, intraoperative complication, conversion rate, post-operative hospital stay, surgical site infection, port site hernia and cosmetic results.

Results: A total of 120 children underwent appendectomy. There were 60 children who underwent NA and 60 children who underwent SILA. There were no difference in age (11.5 vs 11.98 years, $\mathrm{P}=0.35$ ), weight ( $42.98 \mathrm{vs} 43.46 \mathrm{~kg}, \mathrm{p}=0.76)$ and hospital stay ( $1.51 \mathrm{vs} 1.55$ days, $\mathrm{P}=0.92)$ between the two groups. There were no intraoperative complication during the two surgical approaches. Operative time for NA group is significantly shorter than Single incision group (20.7 vs $38.2 \mathrm{~min}, \mathrm{P}=0.0001$ ). No single case of conversion in NA group and 18 cases needed conversion in SILA group. Seven cases of SILA showed surgical site infection. 2 cases of SILA group presented with port site hernia. The NA group was superior as regard ergonomics. The two groups showed equal excellent cosmetic results.

Conclusion: Needles scopic appendectomy and SILA are comparable as regard cosmetic results and hospital stay. NA proved to be safe, applicable, repetitive and superior over SILA as regard better ergonomics, less operative time, absence of surgical site infection and port site hernia. 


\section{O114-ROBOTICS \& NEW TECHNIQUES-Basic} and Technical research

\section{OBJECTIVE EVALUATION OF THE FLEXDEX® LAPAROSCOPIC INSTRUMENT: INITIAL EXPERIENCE IN A TRAINING SETUP}

$\underline{\text { F.M. Sanchez Margallo }}{ }^{1}$, J.A. Sánchez Margallo ${ }^{2}$

${ }^{1}$ Scientific Direction, Minimally Invasive Surgery Centre, CÁCERES, Spain; ${ }^{2}$ Bioengineering, Minimally Invasive Surgery Centre, CÁCERES, Spain

Aims: To objectively analyze the surgical performance and surgeon's ergonomics in the use of a novel flexible laparoscopic instrument during intracorporeal suture, and compare it with the use of a conventional laparoscopic needle holder.

Methods: Three experienced laparoscopic surgeons performed five laparoscopic sutures on an organic tissue using the novel flexible instrument (FlexDex $\left.{ }^{\circledR}\right)$ and five sutures using a conventional needle holder with axial handle. The new device is based on a mechanical design with no electrical components, which transfers the surgeon's hand, wrist, and arm movements to the instrument tip in an intuitive manner. The use of the instruments was organized in a random fashion. Prior to the study, participants conducted a 15-minute training session with the new flexible instrument. Execution time and quality of the suture were assessed for each repetition. Besides, flexion and radioulnar deviation of the wrist were recorded using an electrogoniometer (Biopac systems, Inc.) attached to the surgeon's hand and forearm. The intensity of the forearm's muscle activation was also analyzed by means of a MYO armband (Thalmic Labs). Results: Surgeons required more time to perform the intracorporeal suture using the novel laparoscopic instrument $(87.8 \pm 23.333 \mathrm{~s}$ vs. $56.467 \pm 8.733 \mathrm{~s} ; \mathrm{p}<0.001$ ), but the quality of the suture was similar with both instruments. The wrist flexion $\left(9.976 \pm 7.513^{\circ}\right.$ vs $\left.15.440 \pm 4.049^{\circ} ; \mathrm{p}<0.01\right)$ and wrist ulnar deviation $\left(21.565 \pm 5.19^{\circ} \mathrm{vs}\right.$ $27.401 \pm 3.19^{\circ} ; \mathrm{p}<0.01$ ) were significantly lower when using the flexible instrument. During the suturing tasks, the use of FlexDex ${ }^{\circledR}$ instrument led to a higher muscular activation of the flexor $(32.614 \pm 3.437$ vs $25.23 \pm 3.076$ RMS; $\mathrm{p}<0.001)$ and extensor $(23.341 \pm 1.869$ vs $20.017 \pm 1.307$ RMS; $p<0.001$ ) muscle groups of the forearm.

Conclusions: The presented novel instrument allows surgeons to perform robotic-like laparoscopic suturing. We believe that with a longer training period surgeons could potentially reduce surgical times with this device. The preliminary results of this study suggest that the use of this new instrument provides a quality of the suture similar to that obtained with a conventional laparoscopic needle holder and an ergonomically more adequate wrist posture.

\section{O115-ROBOTICS \& NEW TECHNIQUES-Basic and Technical research}

\section{HYPERSPECTRAL IMAGING IN VISCERAL SURGERY-A NOVEL METHOD FOR THE EVALUATION OF THE GASTRIC CONDUIT PERFUSION IN ESOPHAGECTOMY}

A. Studier-Fischer ${ }^{1}$, K.F. Kowalewski ${ }^{1}$, F.M. Schwab ${ }^{1}$, C. Haney ${ }^{1}$, I. Gockel ${ }^{2}$, F. Nickel ${ }^{1}$, B.P. Müller-Stich ${ }^{1}$

${ }^{1}$ Department of General, Visceral and Transplantation Surgery, University Hospital of Heidelberg, HEIDELBERG, Germany; ${ }^{2}$ Department of Visceral, Transplantation, Thoracic and Vascular Surgery, University Hospital of Leipzig, LEIPZIG, Germany

Aims: The intraoperative real-time evaluation of tissue perfusion is one key element for successful visceral surgery. Traditionally, tissue evaluation is performed visually by surgeons. Newer devices for objective quantification have in majority been based on the application of the fluorescent dye indocyanin green (ICG). A novel method derived from geographic research is Hyperspectral Imaging (HSI). The aim of this study was the evaluation of HSI as a promising method for the evaluation of tissue perfusion and its implementation in the evaluation of the gastric conduit during esophagectomy in a porcine model.

Methods: The HSI camera records a 3 dimensional data cube from a 2 dimensional surgical situs obtaining wavelengths between 500 and $1000 \mathrm{~nm}$. The absorption at different wavelengths is tissue-specific and influenced by the amount of oxygenated haemoglobin and other pigments. A software calculates 4 different indices in real-time including oxygen saturation. A porcine model $(\mathrm{n}=24)$ is used for esophagectomy with gastric conduit formation. Ischemia is induced artificially by magnets simulating staplers. Different shapes of the gastric conduit and anastomosis formation are evaluated for perfusion metrics in order to obtain recommendations for the optimal formation of esophagogastrostomy.

Results: In preliminary results the perfusion measurements showed impaired tissue oxyen saturation after longitudinal stapling in the distal gastric conduit (pre-stapling: mean $=62.5 \%, 95 \%$ CI $[58.9 \%, 66.1 \%]$, post-stapling after 2 min: mean $=52.2 \% 95 \%$ CI [49.9\%, $54.5 \%$, $\mathrm{p}<0.0001)$ whereas tissue oxygen saturation remained unchanged after transverse stapling in the gastric conduit (pre-stapling: mean $=71.2 \%$, $95 \%$ CI $[68.7 \%, 73.7 \%]$, post-stapling after 2 min: mean $=72.4 \%, 95 \%$ CI $[69.9 \%, 74.9 \%]$, n.s.).

Conclusion: HSI is a promising method for intraoperative evaluation of tissue perfusion that does not require application or injection of any agents. The preliminary results in this study showed that the gastric conduit receives its main blood supply from the gastroepiploic arteries and not via the mucosa. Further results from the current evaluations enable formation of an optimized gastric tube and esophagogastrostomy in esophagectomy. 


\section{O116-ROBOTICS \& NEW TECHNIQUES-Basic} and Technical research

\section{SINGLE PORT NEEDLESCOPIC LYMPHATIC SPARING VARICOCELE LIGATION IN CHILDREN USING MEDIFLEX INSTRUMENT: A NEW TECHNIQUE}

\section{M.A. Ismail Lotfallah ${ }^{1}$, A.H. Fawzy ${ }^{2}$, M. Maged ${ }^{1}$, A. Shams ${ }^{1}$}

${ }^{1}$ Pediatric surgery, Al Azhar university, GIZA, Egypt; ${ }^{2}$ Pediatric surgery, Beni suef University, BENI SUEF, Egypt

Background: varicocele is one of the most common causes of infertility. Many surgical interventions are used for varicocele ligation including open and conventional laparoscopic multiport or single incision techniques. The aim of the study is to present a new Needlescopic lymphatic sparing varicocele ligation using Mediflex ${ }^{\circledR}$ facial closure needle and 14 gauge vascular access cannula.

Material and Methods: twenty-two male children with bilateral varicocele of grade II-III. All children were counseled by Clinical examination, Doppler ultrasonography, abdominal ultrasonography, and routine laboratory investigations. Testicular lymphatics were delineated by subcutaneous injection of $1 / 2 \mathrm{~cm}^{3}$ methylene blue in anterior wall of the scrotum $20 \mathrm{~min}$ prior to surgery. The testicular vessels (both vein and artery) were ligated one $\mathrm{cm}$ above the deep inguinal ring using two Mediflex needles with preservation of lympatics. The main outcome measurements included; operative time, hospitalization, testicular atrophy, hydrocele formation, recurrence of varicocele and intra or postoperative complication.

Results: a total of twenty-two male children with grade II-III varicocele subjected to needlescopic lymphatic sparing technique. Twenty one were bilateral. 15 cases were grade III and 7 cases were grade II. Patient's age ranged between 8 and 16 years (mean 11.86 years \pm 2.96 ). The mean operative time was $32.59 \pm 9.42 \mathrm{~min}$. The mean hospital stay period was $1.77 \pm 0.75$ days. No single case of conversion to either open or multiport laparoscopic technique. No intra-operative complications. One case complicated by hydrocele which resolved by expectant treatment. Excellent cosmetic results were gained with good family satisfaction. Testicular volume measurements proved absence of testicular atrophy. Recurrence was detected in only one case, treated by selective embolization of abnormal communicating vein to the cremastric veins.

Conclusion: This study proved that needlescopic varicocele ligation technique is safe, effective, reproducible, less time consuming with fantastic minimally invasive cosmetic results.

\section{O117-ROBOTICS \& NEW TECHNIQUES-Colorectal}

\section{ROBOTIC VERSUS LAPAROSCOPIC SURGERY FOR RECTAL} CANCER: A COMPARATIVE COSTS-EFFECTIVENESS STUDY

R. Caruso, E. Vicente, Y. Quijano, H. Duran, B. Ielpo, I. Fabra, E. Diaz, L. Malave, R. Isernia, E. Pinna, V. Ferri

General Surgery, Sanchinarro University Hospital, MADRID, Spain

Background and Aims: Even if the clinical outcomes of robotic rectal resections are under investigation, the related robotic costs have not yet been well addressed, and the differences between the robotic rectal resection costs and the laparoscopic approach are still not well known We have therefore performed a prospective comparative study of robotic rectal resections (RRR) and laparoscopic rectal resections (LRR) performed at our centre with the aim to evaluate the cost-effective outcomes of robotic versus laparoscopic surgery.

Study design: This is an observational, comparative prospective non-randomized study which includes patients that underwent laparoscopic and robotic rectal resection reaching a minimum of 6 months of follow up from February 2014 to March 2018, at the Sanchinarro University Hospital, Madrid. An independent company performed the financial analysis and fixed costs were excluded. Outcome parameters included surgical and post-operative costs, quality adjusted life years (QALY), and incremental cost per QALY gained or the incremental cost effectiveness ratio (ICER). The primary end-point was to compare clinical outcome as well as cost effectiveness study between both groups.

Results: A total of 86 RRR and 112 LRR were included. The mean operative time was significantly lower in the LRR approach (336 versus $283 \mathrm{~min} ; \mathrm{p}=0.001$ ). The main pre-operative data, overall morbidity, hospital stay and oncological outcomes were similar in both groups, except for the readmission rate (RRR: $5.8 \%$, LRR: $11.6 \% ; \mathrm{p}=0.001$ ). The mean operative costs were higher for RRR ( 4285.16 versus $3506.11 € ; p=0.04$ ); however, the mean overall costs were similar (7279.31€ for RRR and $6879.8 €$ for the LLR; $p=0.44$ ). Mean QALYs at 1 year for RRR group $(0.5624)$ was higher than that associated with LRR $(0.5066)(\mathrm{p}=0.018)$. At a willingness-to-pay threshold of $20,000 €$ and $30,000 €$, there was a $61.18 \%$ and $64.09 \%$ probability that RRR group was cost-effective relative to LRR approach.

Conclusion: This study provides data of cost-effectiveness differences between RRR and LRR approach showing a benefit for the RRR

\section{O118-ROBOTICS \& NEW TECHNIQUES-Colorectal}

RANDOMIZED CLINICAL TRIAL ABOUT 3D VS ROBOTIC RECTAL RESECTION FOR CANCER: PRELIMINARY Results

M. Ortenzi ${ }^{1}$, R.O. Ghiselli ${ }^{1}$, A.N. Carrieri ${ }^{1}$, G.I. Lezoche ${ }^{1}$, A.N. Balla ${ }^{2}$, A.M. Paganini ${ }^{2}$, M.A. Guerrieri ${ }^{1}$

${ }^{1}$ Clinica Chirurgica, Università Politecnica delle Marche, ANCONA, Italy; ${ }^{2}$ Department of General Surgery and Surgical Specialties, Paride Stefanini, Sapienza University of Rome, ROMA, Italy

Aim: The efforts were aimed to the introduction of novel surgical technologies to overcome the intrinsic anatomical and technical constraints of rectal surgery. This was the case of the introduction into the clinical practice of laparoscopy and later on of robotic surgery for rectal surgery. However, whether robotic surgery is actually superior to laparoscopy is still debated. The aim of this study was to compare 3D laparoscopy and robotic surgery for rectal cancer on technical and oncological outcomes.

Methods: This was a single-center, prospective, randomized controlled trial. All patients more than 18 years of age undergoing elective surgery for rectal cancer situated from 5 to $10 \mathrm{~cm}$ from the anal verge were included. Patients undergoing abdominal perineal amputation and/or with T4 and/or M1 tumours were excluded. Patients were randomized before surgery into two arms: arms A (3D laparoscopy) and arm B (robotic), and gave their consensus to the study. Demographic data, data regarding the tumour, operative and post-operative data were collected. Patients with a follow up shorter than 24 months were excluded as well.

Results: Twenty patients were enrolled in arm A and 20 in arm B in the period time of one year. Patients' population of the 2 arms was homogeneous as concerns demographic characteristics and stage of the disease. Robot-assisted rectal resection results in comparable operative time (125.70 vs $170 \mathrm{~min} ; \mathrm{p}=0.068)$. The conversion rate was significantly lower for arm $\mathrm{B}(2$ vs $0 \mathrm{p}=0.0)$ Postoperative morbidity was comparable between groups. Hospital stay was comparable but time required to resolve post-operative ileus was shorter in arm B ( $2.5 \mathrm{vs} 1.2$ days, $\mathrm{p}=0.048)$. Overal survival and disease-free survival were comparable between arms ( $98.6 \%$ vs $98.3 \%, \mathrm{p}=0.989$ and $97.4 \%$ vs $97.6 \%, \mathrm{p}=0.856$, respectively)

Conclusions: 3D laparoscopy and robotic surgery are two viable options for rectal surgery. Robotic surgery can add some in terms of post-operative outcomes and ergonomics.

\section{O119-ROBOTICS \& NEW TECHNIQUES-Colorectal}

\section{A STUDY OF COMPLICATIONS AFTER ROBOTIC SURGERY FOR RECTAL CANCER}

Y. Tsuchiya ${ }^{1}$, Y. Okazawa ${ }^{1}$, K. Mizukoshi ${ }^{1}$, M. Kawai ${ }^{1}$, K. Sugimoto $^{1}$, H. Kamiyama ${ }^{1}$, M. Takahashi ${ }^{1}$, Y. Kojima ${ }^{1}$, Y. Tomiki ${ }^{1}$, T. Fukunaga ${ }^{2}$, Y. Kajiyama ${ }^{2}$, K. Sakamoto ${ }^{1}$

${ }^{1}$ Coloproctological Surgery, Juntendo University Faculty of Medicine, TOKYO, Japan; ${ }^{2}$ Gastroenterological Surgery, Juntendo University Faculty of Medicine, TOKYO, Japan

Aim: Currently, robotic surgery for rectal cancer is a surgical operation that is being performed worldwide. We also introduced robotic surgery in 2015. However, after robotic surgery, we observed a rise in creatinine kinase (CK), which is unlikely to happen in other surgeries. We studied the postoperative complications of rectal cancer patients who underwent either robotic surgery or laparoscopy during the same period of time.

Methods: From January 2016 to November 2018, 23 patients underwent surgery using robotassisted rectal resection (da Vinci Si 20 cases and Xi 3 cases) and 33 patients underwent laparoscopic rectal resection. In this study, abdominoperineal resection, intersphincteric resection, and lateral lymph node dissection were excluded.

Result: The operation time for the robotic surgery group was significantly longer than that for the laparoscopic group (424 min vs. $305 \mathrm{~min} ; \mathrm{p}<0.001$ ). The CK value of the robotic surgery group on 1POD was significantly higher than that of the laparoscopic group $(525 \mathrm{IU} / \mathrm{L}$ vs. $160 \mathrm{IU} / \mathrm{L}$; $\mathrm{p}<0.001$ ). In addition, one case of compartment syndrome was observed in the laparoscopic group. There were no significant differences in age, body mass index, intraoperative bleeding, tumor invasion depth, urination disorder, or postoperative hospital stay. In robotic surgery, it is considered that the increase in CK value is caused by the extended operation time, contact of the patient's cart with the left thigh of the patient, and the extra force applied to the abdominal wall caused by the displacement of the remote center.

Conclusion: In robotic surgery, it is suggested that the measurement of postoperative CK value is important. Therefore, an attempt to shorten the operation time and paying attention to the surgical field are necessary to improve the outcomes. 


\section{O120-ROBOTICS \& NEW TECHNIQUES-Colorectal}

\section{EFFICIENCY OF THE ROBOTIC PLATFORM IN SPHINCTER} PRESERVATION IN PATIENTS WITH LOW RECTAL CANCER

\section{T. Petropoulou, S.N. Amin}

Colorectal Surgery, Sheffield Teaching Hospitals, SHEFFIELD, UNITED KINGDOM, United Kingdom

Aim: The aim of this study is to investigate whether the robotic platform can have a positive impact in sphincter preservation in pts with low rectal tumours, undergoing robotic TME, in comparison with laparoscopic or open TME

We also analyzed and compared short term outcomes

Methods: A prospectively collected robotic database was reviewed and compared with the trust data. This includes all rectal resections which were performed with the robotic platform, over a period of 4 years, versus the trust data for the same period.

Results: 270 patients were analyzed.Groups were matched for distance from the anal verge.Demographics for the groups (sex, age, BMI) were similar.

The percentage of APER (abdominoperineal resection) rate was significant less in the robotic group $(11 \%$ vs $43 \%, \mathrm{p}<0.001)$ Median LoS, complication rate, CRM (+) rate for the robotic group was also statistically significant lower in all subgroup analyses. Specimen quality (TME grade, depth of mesocolon, LN harvested) was better for thre robotic group.

Conclusion: Robotic surgery for rectal cancer is safe and feasible, and could help surgeons perform ultra low rectal resections, rather than APER's and save patients sphincters. CRM $(+)$ rate is low, which could lead to improved oncological outcomes.

Key statement: This is one of the biggest databases for robotic rectal cancer resections in UK (awaits publication in peer reviewed journal) Our data for short term outcomes are favorable over previously published data.Specimen quality and long-term outcomes were better which could lead to improved oncological outcomes too.

\section{O121-ROBOTICS \& NEW TECHNIQUES-Colorectal}

VALUE OF ICG IN COLORECTAL ANASTOMOSIS: SPANISH MULTICENTER ANALYSIS

Y. Tao ${ }^{1}$, I. Alarcon ${ }^{1}$, V. Camacho ${ }^{1}$, G. Martin ${ }^{2}$, C. Moreno ${ }^{3}$, J. Noguera $^{4}$, X. Sierra ${ }^{5}$, B. Flor ${ }^{6}$, S. Morales-Conde ${ }^{1}$

${ }^{1}$ Unit of innovation in Minimally Invasive surgery. Unit of General Surgery, University Hospital Virgen del Rocio, SEVILLA, Spain;

${ }^{2}$ Unit of General and Digestive Surgery, University hospital Son Espases, PALMA DE MALLORCA, Spain; ${ }^{3}$ Unit of General and Digestive Surgery, Hospital La Mancha Centro, ALCÁZAR DE SAN JUAN, Spain; ${ }^{4}$ Unit of General and Digestive Surgery, University Hospital A Coruña, A CORUÑA, Spain; ${ }^{5}$ Unit of General and Digestive Surgery, C.C.S.Parc Tauli, BARCELONA, Spain;

${ }^{6}$ Unit of General and Digestive Surgery, University Hospital La Fe, VALENCIA, Spain

Aims: Anastomotic leak remains as one of the most important and life threatening post-operative complications in colorectal surgery. This complication has important consequences, both acute and long term, longer hospital stay, re-intervention, and increased morbidity and mortality. Among all different circumstances that have been related to this entity, blood supply is an important factor that might have influence. Fluorescence with indocyanine-green (ICG) is used as a marker in the assessment of tissue perfusion in colorectal surgery which might reduce the numbers of leaks.

Methods: A multicenter analysis of the experience of 5 centers in Spain is collected in order to assess the value of ICG in colorectal anastomosis. 379 colorectal procedures were performed using ICG to evaluate vascular supply in the anastomosis. ICG was infused before performing the anastomosis analyzing the number of cases in which the transection line (TL) was changed. We also analyzed the number of leaks in those cases that we changed this line.

Results: Out of the 379 cases performed, 15 cases were performed by open surgery, 319 by laparoscopy, 35 by single-port and 10 with Transanal total mesorectal excision(TATME). The following procedures were performed: 94 right colonic resection(RC), 9 splenic flexure partial resection(SF), 149 left colonic resection(LC), 3 Subtotal colectomy(SC), 2 total colectomy(TC), 6 Hartman reversal surgery(HR), 63 Low anterior resection with partial mesorectal-escision(LAR) and 47 Ultra low anterior resection with total mesorectal-escision(ULAR). Leak rate(LR) was $6.59 \%$ (3.19\%RC, 5.36\%LC, $33.33 \%$ SC, $11.11 \%$ LAR, $11.32 \%$ ULAR) Overall LR was $4.3 \%$ in colonic surgery and $11.2 \%$ in rectal surgery. The TL was changed due to ICG in $12.13 \%$ of the cases $(4.25 \% \mathrm{RC}, 11.1 \% \mathrm{SF}$, $16.77 \%$ LC, $50 \%$ TC, $7.93 \%$ LAR, $18.86 \%$ ULAR), being $11.9 \%$ in colonic resection and $12.9 \%$ in rectal resection. The relation between leaks and the cases in which the TL was changed, were $20 \%$ (33.3\% RC, 25\%LC, 33.3\%ULAR).

Conclusion: ICG fluorescence may play a role in anastomotic tissue perfusion assessment. The LR after colorectal surgery might decrease using ICG to detect the proper TL before to perform the anastomosis. However, we do not have the sufficient evidence to determine that the changing transaction line can lead to avoid leaks. 


\section{O122-ROBOTICS \& NEW TECHNIQUES-Colorectal}

EARLY CRP PEAK (DAY 2) PREDICTS SAFE DISCHARGE IN ROBOTIC TME

\section{P. Tejedor, J.S. Khan, F. Sagias}

Colorectal Surgery, Queen Alexandra Hospital, PORTSMOUTH, United Kingdom

Aims: To analyse the value of postoperative day $2 \mathrm{CRP}$ as an early predictor of safe discharge in robotic rectal cancer surgery.

Methods: A retrospective analysis was performed, including patients who had undergone robotic total mesorectal excision (TME) in a single centre over a 4-year period (May 2013-September 2017). Patients who had a permanent stoma (abdominperineal resections or Hartmann's procedure) were exluded from the study, leaving 144 patients for further analysis.

As the LOS is currently used as a performance tool in assessing outcomes in colorectal surgery (with a cut-off established at 5 days), we compared the CRP values in these 2 groups.

Results: Fourty one percent of patients were discharged home within 5 days. They had an earlier peak of CRP on postoperative day (POD) 2 (median 94.5, 80).

The group of patients that were discharge home after 5 days (59\%) had a CRP peak on POD 3 (median 151, 168).

On POD 3, the group of patients that went home within 5 days had a lower CRP (83-70- vs. 151-168-) compared to the group of patients that were discharge after 5 days, $p=0.001$ ). Conclusions: A CRP peak on POD 2 in robotic TME can predict an early and safe discharge (LOS within 5 days).

\section{O124-ROBOTICS \& NEW TECHNIQUES-Colorectal}

\section{THE IMPACT OF ROBOTIC TME ON SURVIVAL IN PATIENTS WITH RECTAL CANCER}

\section{P. Tejedor, F. Sagias, K. Flashman, J.S. Khan}

Colorectal Surgery, Queen Alexandra Hospital, PORTSMOUTH, United Kingdom

Aims: To compare the medium-term oncological outcomes of Laparoscopic Total Mesorectal Excision (L-TME) vs. Robotic Total Mesorectal Excision (R-TME) for rectal cancer.

Methods: A retrospective analysis was performed including patients who underwent L-TME or R-TME resection between 2011-2017. Patients with disease stage IV at diagnosis or R1 resection were excluded. 680 patients were initially included, and 136 cases of R-TME were matched based on age, gender, stage and time of follow-up with an equal number of patientswho underwent L-TME. We compared 3-year disease free survival (DFS) and overall survival (OS).

In adittion, a multivariate analysis was performed in order to idenfity independent prognostic factors for 3-year DFS and OS.

Results: Pathological outcomes were similar between groups. However, major complications were lower in the robotic group $(13.2 \%$ vs. $22.8 \%, \mathrm{p}=0.04)$, highlighting the anastomotic leakage rate, which was $7.4 \%$ in the R-TME vs. $16.9 \%$ in the L-TME group $(\mathrm{p}=0.01)$.

Overall, the 3-year DFS rate was 69\% in the laparoscopic group and $84 \%$ in the robotic group $(\mathrm{p}=0.02)$. The 3-year OS rate was 70\% in the L-TME groups and 97\% in the R-TME group ( $\mathrm{p}=0.000)$. For disease stage III, 3-year DFS was significantly higher in the R-TME group. OS was also significantly superior in the robotic group for every stage, reaching $86 \%$ in the stage III. In the multivariate analysis, R-TME was a significant positive prognostic factor for distant metastasis (OR 0.2 95\% CI 0.1, 0.6, p = 0.001) and OS (OR $0.295 \% \mathrm{CI} 0.07,0.4, \mathrm{p}=0.000$ ). Conclusions: R-TME for rectal cancer can achieve better oncological outcomes compared to L-TME, especially in stage III rectal cancers. The robotic approach has demonstrated to be a significant positive prognostic factor for local recurrence and overall survival, due to the better postoperative outcomes. However, a longer follow-up period is needed to confirm the oncologic findings.

\section{O125-ROBOTICS \& NEW TECHNIQUES-Education}

\section{O123-ROBOTICS \& NEW TECHNIQUES-Colorectal}

\section{INCREASED LYMPH NODE YIELD USING FLUORESCENCE- IMAGING TECHNIQUE DURING ROBOTIC LATERAL PELVIC LYMPH NODE DISSECTION}

\section{H.J. Kim, G.S. Choi, J.S. Park, S.Y. Park}

Colorectal Cancer Center, Kyungpook National University Chilgok Hospital, DAEGU, Korea

Background: PurposeLateral pelvic lymph node dissection (LPND) is suggested to treat suspected lymph node metastasis in pelvic side-wall in patients with rectal cancer who underwent preoperative chemoradiotherapy (CRT). However, technical difficulties make it possible that lateral pelvic lymph nodes (LPNs) are not dissected completely and, thus, remained in the narrow pelvis. Near-infrared fluorescence imaging (FI)-guided surgery is expected to help visualization and complete excision of nonvisible lymph nodes during cancer surgery. This study aimed to evaluate the efficacy of FI using indocyamine green (ICG) to identify LPNs during robotic LPND.

Methods: 31 rectal cancer patients who were suspected LPN metastasis and had received preoperative CRT were prospectively enrolled. ICG in a dose of $2.5 \mathrm{mg}$ was injected around tumor preoperatively. All procedures were performed with a totally robotic approach. After completing LPND, FI was checked again for identifying remained LPNs and resecting them completely.

Results: The LPNs were successfully detected in $25(80.6 \%)$ of the 31 patients. However, after accounting for eight cases, having finished adjusting ICG injection, the LPNs were successfully detected in $22(95.7 \%)$ of 23 patients. The FI-guided LPND group $(\mathrm{N}=25)$ showed similar mean operative time for unilateral pelvic dissection and complication rate, compared to patients who underwent conventional robotic LPND $(\mathrm{N}=62)$. However, the mean number of unilateral harvested LPNs was 10.2 in the FI-guided LPND group, which was greater than the mean of 6.6 in the conventional group. LPN metastasis was identified in $40 \%$ of the FI-guided LPND group, which was higher than that of the conventional group, $31.7 \%$.

Conclusion: FI-guided LPND identifies lymph nodes in pelvic side-wall with great reliability. This contributes to increased number of LPNs yield compared to conventional robotic LPND. This technique should be considered to dissect them completely by preventing subsequent missing of nonvisible LPNs.

\section{COMPARISON OF SELF- AND EXTERNAL ASSESSMENT OF LAPAROSCOPIC SKILLS OF RESIDENTS USING THE GLOBAL ASSESSMENT OF LAPAROSCOPIC SKILLS QUESTIONNAIRE}

\section{V.N. Uslar ${ }^{1}$, F. Piastowski ${ }^{1}$, T. Vajsbaher ${ }^{2}$, D. Weyhe ${ }^{1}$}

${ }^{1}$ University Hospital for Visceral Surgery, University of Oldenburg, OLDENBURG, Germany; ${ }^{2}$ Bremen Spatial Cognition Center, University of Bremen, BREMEN, Germany

Aims: In clinical settings, realistic assessment of one's own abilities can enhance performance and promote patient safety, especially in surgical residents, who inevitably have to acquire skills during real surgery. This study thus implemented the Global Assessment of Laparoscopic Skills (GOALS) questionnaire with the aim to explore divergences between resident self-evaluation and specialist's evaluation on laparoscopic performance, as a first step to implement the GOALS questionnaire as a tool for constructive and objective feedback.

Methods: Between July and October 2018, seven residents from the University Hospital for Visceral Surgery at the Pius-Hospital Oldenburg participated in this study. At the end of every laparoscopic operation where the resident acted as the primary surgeon, the resident and the supervising surgeon independently evaluated the resident's operative performance using the GOALS questionnaire. The five dimensions evaluated were depth perception, bimanual dexterity, efficiency, tissue handling and autonomy. A cumulative GOALS-Score (with 25 being the highes possible score) was calculated for $n=46$ laparoscopic operations. Resident's year of training, the level of case difficulty and the type of laparoscopic procedure performed was also analysed. Results: Residents overestimated their laparoscopic abilities in $64.4 \%$ of the operations (GOALS-Scores: residents: median $=16$, mean $=16.51$; specialists: median $=15$, mean $=$ $14.60 ; \mathrm{p}<0.001)$. Residents in the first three years of surgical training were more likely to overestimate their performance (residents: median $=16.5$, mean $=16.82$; specialists: median $=$ 13 , mean $=13.14 ; \mathrm{p}<0.001)$ than those with more than three years of surgical experience (residents: median $=16$, mean $=16.22$; specialists: median $=15$, mean $=16.00 ; \mathrm{p}=0.613$ ). GOALS score differences did not depend on case difficulty and laparoscopic procedure. Conclusions: Surgical residents tend to overestimate their intraoperative laparoscopic performance when compared to specialist evaluation. Overestimation was found to depend on one's own laparoscopic experience and seem to disappear with gained expertise. These results signify the importance of individually adapted training and the greater need for objective feedback for surgical residents. This approach could in return increase the skill acquisition rate of the resident and in return contribute towards enhancing patient safety. 


\section{O126-ROBOTICS \& NEW TECHNIQUES-Education}

\section{ASSESSING HUMAN FACTORS IN THE OPERATING THEATRE: DIFFERENCES IN PERCEPTIONS WITHIN THE SURGICAL TEAM}

A.S.H.M. van Dalen ${ }^{1}$, M. Goldenberg ${ }^{2}$, T.P. Grantcharov ${ }^{3}$, M.P. Schijven ${ }^{1}$

${ }^{1}$ Department of Surgery, Amsterdam Gastroenterology and Metabolism, Amsterdam UMC, University of Amsterdam, AMSTERDAM, The Netherlands; ${ }^{2}$ Department of Urology, International Centre for Surgical Safety, St Michael's Hospital, University of T, TORONTO, Canada; ${ }^{3}$ Department of Surgery, International Centre for Surgical Safety, St Michael's Hospital, University of T, TORONTO, Canada

Introduction: The delivery of safe surgical care is dependent of various, complex and interrelated factors. Substantial data exist regarding the impact of training in human factor skills on surgical outcomes. However, except for the standardized time-out process, the best way to go about improving these skills remains unclear. The aim of this study was to gain more insights in the theatre staff's perception of human factors and their importance on surgical outcome in the operating theatre.

Methods: The Surgical Team Assessment Record (STAR) questionnaire was used to study the role of human factors, such as communication, situational awareness and organization, contributing to surgical team performance. The self-assessment questionnaire was filled out by the theatre staff, directly after the surgical procedure. Conditional logistic regression was used to identify the impact of the role in the operating theatre on the yes versus no answers.

Results: Some 507 questionnaires were completed. The theatre staff rated their team's performance with a median of 4 (IQR 0.0, 5-point Likert scale). The surgical fellows $(\mathrm{N}=76)$ rated their personal factors significantly lower compared to the rest of the operating team (median 3 versus $4, P$-value $<0.0001)$. The staff surgeon $(\mathrm{N}=119)$ indicated significantly more often that there were many distractions $(51.3 \%$, yes $n=61)$ and noticed aberrations $(60.5 \%$, yes $\mathrm{n}=72)$ during the surgical procedure $(P$ value $<0.0001$ ) when compared to the rest of the operating team. Most aberrations reported by the surgeons were related to technical performance.

Conclusions: Human factors play an important role in the surgical environment. Situational awareness may be less developed in members of operating teams, compared to the surgeon of that team. Further work is needed to elucidate the impact of human factor skills on team performance. A team-based approach to safety interventions is recommended. Future studies should determine what type of aberrations and distractions are most relevant and valuable to embark on with team training.

\section{O127-ROBOTICS \& NEW TECHNIQUES-Education}

INVESTIGATION OF QUALITATIVE EVALUATION USING RUBRIC AND THE VR + AR TRAINING OF REGIONAL ANATOMY IN THE LAPAROSCOPIC TRAINING OF STUDENTS

T. Iwata $^{1}$, K. Yoshikawa ${ }^{2}$, J. Higashijima ${ }^{2}$, T. Miyatani ${ }^{2}$,

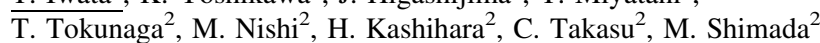

${ }^{1}$ Dept. of Digestive Surgery, School of Medicine, Tokushima University, TOKUSHIMA, Japan; ${ }^{2}$ Dept. of Digestive Surgery, Tokushima University, TOKUSHIMA, Japan

Background: The qualitative evaluation for laparoscopic training of medical students was performed using Rubric evaluation, and weak points in conjunction with the lack of anatomical knowledge were derived. To conquer these weak points, virtual reality (VR) + augmented reality (AR) training for understanding of regional anatomy was investigated.

Materials and methods: One hundred and six students in $5^{\text {th }}$ grade of Tokushima Univ. participated basic laparoscopic task training (gummy band ligation, beads transfer, delivery of beads, gauze excision) with training box and sham laparoscopic cholecystectomy with virtual simulator. Rubric evaluation, as qualitative evaluation, which includes the evaluation standards for each maneuver were performed before and after basic task training and sham operation. The group which self-evaluation was higher in a Rubric evaluation was investigated.

The 3D image of vessels and bile duct obtained from MDCT of real patient was projected in reality space with Microsoft HoloLens. Training of AR image using HoloLens was performed for understanding of regional anatomy. After training of regional anatomy with HoloLens, sham laparoscopic cholecystectomy was performed again, and quality of procedure was evaluated by Rubric. Anatomical questions were.

Results: Rubric evaluation in basic task training showed no difference between self-evaluation and evaluation by tutor before and after practice. In sham laparoscopic cholecystectomy, several students showed higher score than tutor, especially in part of extension of operation field by elevation of the Gall bladder, exposure of triangle of Calot, and exposure of cystic duct.

After AR training, all students showed high score in questions related regional anatomy during operation. Especially, rubric evaluation of students who showed high self-evaluation in sham operation showed same score with tutor.

Conclusions: As Rubric evaluation showed weak points of detailed parts of maneuver, and VR + AR was useful for understanding details of regional anatomy for laparoscopic training. 


\section{O128-ROBOTICS \& NEW TECHNIQUES-Education}

NEAR MISSES IN MINIMAL ACCESS SURGERY: COMMON AND UNDER REPORTED

N.J. Curtis ${ }^{1}$, G. Dennison ${ }^{2}$, J.A. Conti ${ }^{3}$, G.B. Hanna ${ }^{1}$, N.K. Francis ${ }^{2}$

${ }^{1}$ Surgery and Cancer, Imperial College London, LONDON, United Kingdom; ${ }^{2}$ General Surgery, Yeovil District Hospital, YEOVIL, United Kingdom; ${ }^{3}$ Colorectal Surgery, Portsmouth Hospitals NHS Trust, PORTSMOUTH, United Arab Emirates

Background: The EAES has recently published an intraoperative adverse event classification to aid reporting of minimally access surgery events. This includes capture of non-consequential errors. We aimed to investigate the clinical impact of these apparent 'near miss' events.

Methods: Case videos and clinical data from a completed multi-centre laparoscopic total mesorectal excision randomised controlled trial was utilised (ISRCTN59485808). The EAES classification was applied by two blinded assessors to all enacted adverse events identified on video analysis using the observational clinical human reliability analysis technique. The total number of grade 1 (non-consequential) errors were compared with the number and nature of 30 day morbidity events (graded with the Clavien-Dindo system) and length of stay. Results: 77 cases ( 419 h of surgery) contained 1377 error events of which $809(58.8 \%)$ were classified as EAES grade 1 (median 10 per case, interquartile range $7-13$, range 1-28). There were significantly more inconsequential errors recorded in patients that developed any early morbidity event than those who had an uneventful post-operative recovery (median 8.5 (IQR $6-12)$ vs. $11(9-14), p=0.005$ ). A stepwise increase in the sum of EAES grade 1 errors is seen for each additional 30 day morbidity event reported ( 8.5 vs. 11 vs. 11 vs. $12, p=0.047)$ and the highest Clavien-Dindo grade experienced ( 9 vs. 10 vs. 11 vs. 12 . $\mathrm{P}=0.067$ ). Positive correlation is observed between the sum of EAES grade A errors and length of post-operative stay $\left(r^{s}=0.36\right.$, $\mathrm{p}=0.001$ ).

Conclusion: In the context of major laparoscopic surgery, near misses are commonplace and correlate with surgical outcomes. This may represent a novel surrogate assessment method for intraoperative performance.

\section{O129-ROBOTICS \& NEW TECHNIQUES-Education}

\section{THE LAPLAT CURRICULUM: A PILOT STUDY OF} A SIMULATION-BASED TRAINING PROGRAMME IN DIAGNOSTIC LAPAROSCOPY FOR ABDOMINAL TRAUMA

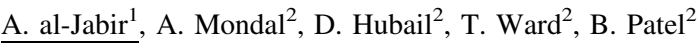

${ }^{1}$ Barts and the London School of Medicine and Dentistry, QMUL, Barts Cancer Institute, LONDON, United Kingdom; ${ }^{2}$ Barts Cancer Institute, Barts Cancer Institute, LONDON, United Kingdom

Aims: Diagnostic laparoscopy (DL) is an under-utilised procedure that can replace non-therapeutic exploratory laparotomies in many contexts. To date, no validated education programme for DL exists. This study seeks to evaluate the feasibility, acceptability and face, content, construct validity of the LAPLAT curriculum (Laparoscopic learning for abdominal trauma; a simulationbased curriculum for Trauma DL). This is in addition to the development of a novel 3D-printed bench-top model for abdominal inspection.

Methods: This prospective and observational pilot study involved 39 novice medical students and junior doctors. Surgeons from the UK and international $(n=8)$ were involved in a two stage Delphi-process to determine the components of the training course which were used to formulate a final curriculum. In the absence of an adequate model for abdominal inspection, a novel 3Dprinted abdominal inspection model was designed and produced. After an introductory familiarisation session as well as pre-course cognitive lectures, the novices performed 6 tasks on a virtual reality and bench-top simulator with 5 repetitions of each in a half-day session. Outcome measures for construct validity were total time to complete task, accuracy, percentage of horizon maintained and economy of movement. Face and content validity as well as acceptability was evaluated by a qualitative and quantitative survey.

Results: Face, content and construct validity as well as acceptability was established. Face validity was demonstrated in all components of the course (including pre- course cognitive content and technical tasks) in addition to content validity. All also met an acceptability threshold of 3/5 on a 5-point Likert scale. One-way ANOVA tests demonstrated construct validity in all tasks $(\mathrm{p}<0.0002)$ with learning curves in reducing time observed. Using a performance improvement metric, one-way ANOVA tests showed similar rates of improvement per participant between most tasks $(\mathrm{p}>0.05)$. The course was rated overall mean 8.86/10 ( \pm 1.05$)$. Conclusion: This pilot study has demonstrated the feasibility, acceptability and face, content and construct validity of the LAPLAT curriculum as well of the novel 3D-printed abdominal inspection model. Randomised controlled trials are needed to establish higher-quality evidence, as part of a wider curriculum with transfer needed to the clinical environment.

\section{O130-ROBOTICS \& NEW TECHNIQUES-Education}

THE ROLE OF RESIDENT'S 'WARMING UP' BEFORE LAPAROSCOPIC SWINE LIVER RESECTION USING A STANDARDIZED PROTOCOL

C. Popa ${ }^{1}$, C. Pestean ${ }^{2}$, C. Ober ${ }^{2}$, F. Graur ${ }^{1}$, M. Socaciu ${ }^{3}$, R. Couti ${ }^{4}$, T. al Momani ${ }^{5}$, R. Elisei ${ }^{5}$, F. Zaharie ${ }^{1}$, N. al Hajjar ${ }^{1}$, L. Oana ${ }^{2}$

${ }^{1}$ Surgery, Regional Institute of Gastroenterology and Hepatology, CLUJ-NAPOCA, Romania; ${ }^{2}$ Anesthesiology-Surgical Propedeutics, University of Agricultural Sciences and Veterinary Medicine, CLUJNAPOCA, Romania; ${ }^{3}$ Radiology, Regional Institute

of Gastroenterology and Hepatology, CLUJ-NAPOCA, Romania; ${ }^{4}$ Urology, Training and Research Center, Prof. Dr. Sergiu Duca, CLUJ-NAPOCA, Romania; ${ }^{5}$ General Surgery, Training and Research Center, Prof. Dr. Sergiu Duca, CLUJ-NAPOCA, Romania

Aims: To evaluate the benefits of systematical use of ex vivoliver model and CT imaging in the planning process for swine laparoscopic liver resections done by residents during training programs.

Methods: Twenty four general surgery residents were equally divided into two groups: first one which performed laparoscopic liver resections without planning stage and the second one which systematically used anatomical data from a swine liver model and interactive CT scans 3D reconstructions. The planning stage included an interactive tutorial for establishing the strategy for the next resection followed by performing open liver dissection and the same resection on an ex vivoswine model. A total of twelve models were used during this step. Afterwards, laparoscopic procedures were performed on sixteen anesthetized domestic pigs, two swine for every team, composed of three residents. Both groups were part of a dedicated and continuous training program and used the same 'step by step' protocol for resections.

Results: The average time for imagistic planning was $36.7 \mathrm{~min}$ and for open dissection and resection was $57.9 \mathrm{~min}$. All teams successfully completed the interventions and followed the standardized protocol without trainers' interventions and with no conversions. The second group obtained better results regarding the time needed for completion and blood loss. Also, when the planning stage was applied the resection was more accurate and less functional parenchyma was removed.

Conclusion(s): The 'warming up' by adding the imagistic and anatomical data to the core protocol offer more clarity before laparoscopic liver resections. This also makes an upgrade for our 'step by step' protocol and provides sufficient data to admit this planning stage as mandatory for laparoscopic liver resection on swine during a training program. 


\section{O131-ROBOTICS \& NEW TECHNIQUES-Education}

SITUATION AWARENESS IN MULTIMODAL LAPAROSCOPY TRAINING ENHANCES PERFORMANCE OF LAPAROSCOPIC PROCEDURES -A RANDOMIZED CONTROLLED TRIAL

L. Seifert $^{1}$, K.F. Kowalewski ${ }^{1}$, M.W. Schmidt ${ }^{1}$, B. Müller ${ }^{1}$, K.F. Köppinger ${ }^{1}$, B.P. Müller-Stich ${ }^{2}$, F. Nickel ${ }^{1}$

${ }^{1}$ Department of Surgery, Heidelberg University, HEIDELBERG, Germany; ${ }^{2}$ Department of General, Visceral and Transplant Surgery, University of Heidelberg, HEIDELBERG, Germany

Background: Conventional surgery which used to be gold standard for many years was replaced by Minimal Invasive Surgery (MIS) because it comes with benefits for the patients. For the surgeon it comes with obstacles, therefore many training curricula were established, aimed to train motoric skills. Situation awareness (SA) is a skill of effective information processing. It depends on common cognitive abilities and can be divided in three steps (1) perception of clues (2) connecting clues with knowledge for understanding their relevance (3) predicting possible outcomes. Good SA is required for adequate decision-making, consequently good SA is necessary in surgery to predict and avoid complications. This study aims to prove the potential benefit of SA training to the MIS trainings curriculum. Methods: This was a prospective, single-centre, two-arm, parallel-group randomized trial which was conducted during MIS training for medical students in year 3-5. Participants were blinded until training was finished and computer based randomized. Before and after the intervention the performance on a simulated cholecystectomy (SC) was measured with OSATS and GOALS. Video sessions showing difficulties during the $\mathrm{SC}$ were shown to both groups. Active participation in the intervention group was achieved by including questions into the videoclips, aiming to train the tree steps of SA. The control group got to see the same videoclips without interceptive questions.

Preliminary results: There was no significant result for either the GOALS score $(18.5 \pm 4.6$ / $18.7 \pm 5.4 ; \mathrm{p}=0.981)$ or the OSATS score $(62.23+12.79 / 57.25+9.36 ; \mathrm{p}=0.348)$ or the needed time $(64.5 \pm 22.0 / 62.8 \pm 15.8 ; \mathrm{p}=0.62)$.There were correlations between the self-assessed attention and GOALS score $\left(\mathrm{R}=0.1 ; \mathrm{R}^{2}=0.021\right)$ OSATS score $\left(\mathrm{R}=0.2 ; \mathrm{R}^{2}=0.0325\right)$ and the time $\left(R=-0.0836 ; R^{2}=0.007\right)$.The correlations between the right answered questions during the intervention and the different scores varied. The GOALS score had a slightly positive correlation ( $\mathrm{R}=0.1$; $\left.R^{2}=0.0126\right)$ whereas the OSATS score had a slightly negative correlation $\left(R=-0.3 ; R^{2}=0.06699\right)$. Time did not show a relation to the right answered questions yet $\left(R=-0.1 ; R^{2}=0.006\right)$.

Conclusion: The SA training doesn't show significant results in different scores and time yet, bu the individual assessment of the cognitive training requested through a questionnaire was higher in the group which trained situation awareness.

\section{O132-ROBOTICS \& NEW TECHNIQUES-Flexible surgery}

\section{ENDOSCOPIC FULL THICKNESS RESECTION FOR} TREATMENT OF GASTRIC SUBEPITHELIAL TUMORS

\section{P.W.Y. Chiu, H.C. Yip, A.Y. Teoh, S.M. Chan, S.K.H. Wong, E.K.W. Ng}

Department of Surgery, The Chinese University of Hong Kong, HONG KONG

Introduction: Submucosal tunnel endoscopic resections (STER) had been increasingly performed for treatment of gastric subepithelial tumors. One of the limitations for STER is the risk of incomplete tumor resection due to close dissection and bridging of tumor capsule. Endoscopic full thickness resection (EFTR) allowed complete resection of the tumor with margins to prevent recurrence. This study aimed to review the techniques and outcomes of EFTR for treatment of gastric subepithelial tumors.

Method: Patients who received endoscopic resection for gastric subepithelial tumors were recruited. The gastric subepithelial tumors were considered eligible for endoscopic resection with size $<40 \mathrm{~mm}$. All patients received preoperative assessments including EUS and CT scan to define the extend of tumors and the proportion of extra and intralumenal components. All the procedures were performed under general anesthesia with $\mathrm{CO} 2$ insufflation. EFTR started after injection with mucosal incision up to $50 \%$ of tumor circumference, followed by submucosal dissection to identify tumor margin. Further dissection was performed using ESD devices. After adequate exposure of lateral margins, incision into muscularis propria was performed to achieve full thickness resection. Luminal defects were closed by either clips, clip-loop crown method or Overstitch suturing.

Results: From 2012 to 2018, 10 patients received EFTR for gastric subepithelial tumors. The mean age was 60.6 years, and 4 were male. The GIST were located at greater curvature (4), cardia (3), lesser curve (2) and antrum (1). The mean size was $20.5 \mathrm{~mm}(10-50 \mathrm{~mm})$. Most of the EFTR were performed in operation theatre while two were done at endoscopy. The mean hospital stay was 4.5 days, and mean operative time was $98 \mathrm{~min}$ (34-180 mins). There was no conversion to laparoscopy. Closure of luminal defect were performed mostly with clips (5), followed by Overstitch (4) and clip and loop crown closure (1). Most patients resumed full diet on day 3 , and all the pathologies confirmed GIST tumors with clear resection margins.

Conclusion: Endoscopic Full Thickness Resection is technically feasible and safe procedure for treatment of gastric GIST. Future research should focus on refining the techniques of EFTR and closure of the defect.

\section{O133-ROBOTICS \& NEW TECHNIQUES-Flexible surgery}

THE USEFULNESS OF NOVEL, INFECTION-FREE, ADVANCE HEMOSTATIC MATERIAL FOR UPPER GASTROINTESTINAL ENDOSCOPIC SURGERY

S. Katsuyama ${ }^{1}$, Y. Miyazaki ${ }^{2}$, S. Kobayashi ${ }^{3}$, K. Yamamoto ${ }^{4}$, Y. Nakagawa $^{4}$, T. Takahashi ${ }^{2}$, K. Tanaka ${ }^{2}$, T. Makino ${ }^{2}$, Y. Kurokawa ${ }^{2}$, M. Yamasaki ${ }^{2}$, M. Mori ${ }^{2}$, Y. Doki ${ }^{2}$, K. Nakajima ${ }^{1}$

${ }^{1}$ Next Generation Endoscopic Intervention (Project ENGINE), Osaka University, SUITA, Japan; ${ }^{2}$ Gastroenterological Surgery, Osaka University, SUITA, Japan; ${ }^{3}$ Research \& Development, 3-D Matrix, Ltd., CHIYODA-KU, TOKYO, Japan; ${ }^{4}$ Research \& Development, FUSO Pharmaceutical Industries, Ltd., CYUOU-KU, OSAKA, Japan

Background: Hemostatic peptides have received increased attention. Self-assembling peptides (TDMs) comprise synthetic amphipathic peptides that immediately react to changes in $\mathrm{pH}$ and/or inorganic salts to transform into a gelatinous state. Since TDMs do not carry a risk of infection, their clinical application as new hemostatic agent is expected to increase. The first generation of these peptides (TDM-621) is currently used as a hemostatic agent in Europe. However, TDM-621 exhibits slow gel-formation and low retention capabilities on tissue surfaces. The second generation (TDM-623) was therefore developed to encourage faster gel-formation and better tissuesealing capabilities, and we subsequently verified its usefulness and increased performance relative to TDM-621 in preclinical open surgery.

Aim: The aim of this study was to verify the efficacy of TDM-623 in terms of its hemostatic effect in endoscopic surgery.

Materials and methods: Evaluation of the hemostatic effect in endoscopic surgery (anima study) was performed using eight female $(35 \mathrm{~kg}$ ) pigs in spine position. Following systemic heparinization, we established a bleeding model by utilizing flexible endoscopic grasping forceps on the anterior wall of the stomach and duodenum. In the hemostasis method, an endoscope with a distal hood was brought into contact with the bleeding point, and $1 \mathrm{~mL}$ TDM-623 was applied to the wound. After TDM-623 gelation, the endoscope was removed, and the acute hemostatic effect (after $2 \mathrm{~min}$ ) was confirmed. Histologic evaluation was subsequently performed on resected specimens.

Results: In the endoscopic bleeding model, 17 of the 23 cases $(73.9 \%)$ showed complete hemostatic effects on the anterior wall of the stomach, whereas on the anterior wall of the duodenum, 18 of 20 cases $(80 \%)$ showed complete hemostatic effects. Moreover, none of the gels were displaced from the anterior walls of the stomach and duodenum, and histologic evaluation confirmed no infiltration of inflammatory cells.

Conclusion: The new self-assembling peptide (TDM-623) displayed improved hemostatic effects relative to the previous generation (TDM-621) in endoscopic surgery. TDM-623 had potentia usefulness for upper gastrointestinal bleeding. Our future work will assess its usefulness for laparoscopic surgery. 


\section{O134-ROBOTICS \& NEW TECHNIQUES-Liver}

COULD INDOCYANINE GREEN FLUORESCENCE-GUIDED SURGERY BE THE NEXT FRONTIER OF HEPATIC SURGERY? A PROSPECTIVE SERIES

S. Molfino ${ }^{1}$, M.S. Alfano ${ }^{1}$, S. Benedicenti ${ }^{1}$, B. Molteni ${ }^{1}$, M. Botticini ${ }^{2}$, N. Portolani ${ }^{1}$, G.L. Baiocchi ${ }^{1}$

${ }^{1}$ Clinical and Experimental Sciences, Surgical Clinic, University of Brescia, BRESCIA, Italy; ${ }^{2}$ IGIER, Bocconi University, MILANO, Italy

Objective: Indocyanine green (ICG) is a dye used in medicine since the mid-1950 s for different applications in ophthalmology, cardiology and hepatobiliary surgery; thanks to its selective hepatic uptake and biliary excretion, it can be used to evaluate hepatic function in patients scheduled for hepatic resection surgery. The aim of this study is to evaluate the efficacy and the feasibility of ICG guided surgery in the intra-operative localization of liver tumors, comparing the pre-operative radiological aspect, the intra-operative visualization and the post-operative histopathological features of the tumors.

Materials and methods: ICG was intravenously injected for a routine liver function test (LiMON $®)$ in 58 patients who underwent hepatic resection surgery for primitive and secondary liver tumors in the period between November 2016 and September 2018. For each patient was performed an intraoperative visualization of the stain both in vivo and ex vivo, using a nearinfrared imaging system. All the images were recorded.

Results: A correct differentiation between liver parenchyma and tumor area was obtained in $89.1 \%$ of cases. Five patients were not evaluable due to widespread uptake or complete absence of uptake; it was probably the first cases enrolled in the study for which we were not able to set doses and timing of administration of ICG. In patients in which the method had been feasible, we observed a prevalence of nodular pattern in patients with hepatocellular carcinoma $(63 \%)$ and a predominance of rim pattern in both cholangiocarcinoma (80\%) and metastasis $(83 \%)$. Furthermore, in patients with HCCs well-intermediate differentiated (G1-G2) was found predominantly a nodular pattern $(82.9 \%)$, whereas in poorly differentiated ones was prevalent a rim appearance $(60 \%)$. Regarding radiological correlations, the only one patient who presented an atypical radiological feature in pre-operative evaluation, showed a lesion with no ICG captation in intra-operative visualization.

Conclusions: ICG fluorescence imaging is a safe, minimally invasive and quite inexpensive method, that can be easily administered for routine evaluation of pre-operative liver function. It can be a useful support tool in the intra-operative detection of liver tumors, especially in laparoscopic surgery where it is not possible to directly touch the tissue.

\section{O135-ROBOTICS \& NEW TECHNIQUES-Liver}

\section{PROSPECTIVE MULTICENTER STUDY FOR ROBOTIC ANATOMIC MAJOR LIVER RESECTION}

\section{S.H. Choi ${ }^{1}$, D.H. Han ${ }^{2}$, J.H. Lee ${ }^{3}$, G.H. Choi ${ }^{2}$, Y.R. Choi ${ }^{4}$, J.H. Lee \\ ${ }^{1}$ Surgery, Bundang CHA Medical Center, SEONGNAM-SI, Korea; ${ }^{2}$ Surgery, Severance Hospital, SEOUL, Korea; ${ }^{3}$ Surgery, NHIMC Ilsan Hospital, ILSAN, Korea; ${ }^{4}$ Surgery, Seoul National University Bundang Hospital, SEONGNAM, Korea; ${ }^{5}$ Surgery, Asan Medical Center, SEOUL, Korea}

Backgrounds \& aims: Robotic surgical system had been widely accepted in various surgical field with the expectations of overcoming the limitation of laparoscopic surgery. However, robotic liver resection had not generalized, so far. Thus, this study aimed to evaluate the feasibility and safety of robotic major liver resection by prospective multicenter study.

Methods: From July2017 to December 2018, five surgeons who were novice in robotic liver resection but experienced a lot in open and laparoscopic liver resection in five tertiary hospitals performed 46 cases of robotic major anatomical liver resection. Perioperative patient's clinical data and surgical data were prospectively collected.

Results: 22 cases of left hemihepatectomy, 1 case of extended left hemihepatectomy, 14 cases of right hemihepatectomy, 2 cases of right anterior sectionectomy, 6 cases of right posterior sectionectomy, and one cases of central bisectionectomy were performed. The most common indications were hepatocellular carcinoma for 21 cases following intrahepatic cholangiocellular carcinomas for 7 cases, liver metastases for 3 cases, sarcoma for 1 case, intraductal papillary neoplasms for 2 cases, mucinous cystic neoplasm for 1 case, hemangioma for 1 case, and intrahepatic duct stones for 10 cases. Surgical resection margins for all tumor cases were negative. Total average operation time was $378.58 \pm 124.31(190-696) \mathrm{min}$ and estimated intraoperative blood loss was $276.67 \pm 397.41 \mathrm{~mL}$ (minimal to $2600 \mathrm{~mL}$ ). In terms of severe surgical complication, there were 3 cases of postoperative fluid collection treated with drainage and one case of bile leakage treated with percutaneous trans-hepatic biliary drainage. Only one case out of 46 cases was converted to the conventional open left hemihepatectomy because of bleeding.

Conclusions: In this study, robotic anatomic major liver resection might be safely performed even by robotic beginners but advanced open and laparoscopic liver surgeons.

\section{O136-ROBOTICS \& NEW TECHNIQUES-Pancreas}

MINIMALLY INVASIVE VIDEO-ASSISTED APPROACH OF PANCREATIC NECROSIS DUE TO ACUTE PANCREATITIS

C. Gonzalez De Pedro, J. Tinoco Gonzalez, L. Tallon Aguilar, E. Perea Del Pozo, V. Duran Muñoz-Cruzado, A. Sanchez Arteaga, I. Ramallo Solis, F. Pareja Ciuro, J. Padillo Ruiz

Cirugia General, Hospital Virgen Del Rocio, SEVILLA, Spain

Objective: To present the systematization of the developed technique for video-assisted retroperitoneal access for pancreatic necrosectomy once the previous approaches according to the step-up approach have been exhausted.

Methods: Descriptive study of a cohort of 6 patients subjected to videoretroperitoneoscopy (VR) of 2016-2018. We realized a comparative study with the historical series of 20 open necrosectomies (ON) from 2011 to 2017. We analyzed hospital stay, number of interventions required and complications according to Clavien-Dindo.

Surgical technique: With the patient at $30^{\circ}$ on right lateral decubitus, access is gained through the path of the percutaneous drainage catheter after opening of the aponeuroses of the oblique and transverse muscles of the abdomen. A $15 \mathrm{~mm}$ laparoscopic trocar is inserted and a cavity is created with pneumoretroperitoneum at $15 \mathrm{mmHg}$. It is accessed with an optic of $0^{\circ}$ and $5 \mathrm{~mm}$, and the work space is extended with aspiration and hydrodissection. With $5 \mathrm{~mm}$ grippers, the necrotic material is removed, washed and drained. A two light silicone probe is left, one light for drainage and another one for washing.

Results: The mean age was $52.2 \pm 8.1$ for VR and $60.2 \pm 15.4$ years for ON $(\mathrm{p}=0.23)$, the median stay $34(28-60)$ vs. 38 (23-58) days, $p=0.497$, the median number of reoperations was 1 (1-2) vs. $2.5(1-3) \mathrm{p}=0.02$, and median complications according to the Clavien-Dindo grade of $2(1.75-4)$ vs. $4(2-5) \mathrm{p}=0.01$. They required ICU $2(33 \%)$ VR vs $17(85 \%) \mathrm{ON}(\mathrm{p}=0.01)$. The death rate was $0 \%$ in VR vs $20 \%$ in ON

Conclusions: The described technique provides a minimum invasion to the patient, allowing a necrosectomy under endoscopic vision, with an improvement of the results in terms of complications, at the expense of a median hospital stay of one month.

\section{O137-ROBOTICS \& NEW TECHNIQUES-Pancreas}

THE ROLE OF ROBOTIC-ASSISTED PANCREATIC SURGERY: LESSONS LEARNED FROM OUR INITIAL EXPERIENCE

R. Caruso, E. Vicente, Y. Quijano, H. Duran, B. Ielpo, I. Fabra, E. Diaz, L. Malave, R. Isernia, E. Pinna, V. Ferri

General Surgery, Sanchinarro University Hospital, MADRID, Spain

Background: Minimally invasive surgery has achieved worldwide acceptance in various fields, however, pancreatic surgery remains one of the most challenging abdominal procedures. In fact, the indication for robotic surgery in pancreatic disease has been controversial. The present study aimed to assess the safety and feasibility of robotic pancreatic resection.

Methods: We retrospectively reviewed our experience of robotic pancreatic resection done in Sanchinarro University Hospital. Clinicopathologic characteristics, and perioperative and postoperative outcomes were recorded and analyzed.

Results: From October 2010 to September 2018, 71 patients underwent robotic-assisted surgery for different pancreatic pathologies. All procedures were performed using the da Vinci robotic system. Of the 62 patients, 40 were male and 31 female. The average age of all patients was 63 years. Operative time was $340 \mathrm{~min}$. Among the procedures performed were 21 pancreaticoduodenectomies (PD), 33 distal pancreatectomies (DP), 17 tumor enucleations (TE). The mean hospital stay was 16.6 days in PD group, 8 days in DP group and 8.2 days in TE group. Pancreatic fistula occurred in 13 cases (20\%), 3 after PD, 4 after DP, and 6 after TE. Four patients had postoperative transfusion in PD group and one in DP group. Conversion to open laparotomy occurred in four patients $(8 \%)$. No serious intraoperative complications were observed. Conclusions: From our early experience, robotic pancreatic surgery is a safe and feasible procedure. Further experience and follow-up are required to confirm the role of robotic approach in pancreatic surgery. 


\section{O138-ROBOTICS \& NEW TECHNIQUES-Technology}

MODELLING OF CAPSULE-INTESTINE PRESSURE FOR A SELF-PROPELLED CAPSULE ROBOT FOR SMALL-BOWEL ENDOSCOPY VIA EXPERIMENTAL INVESTIGATION

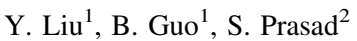

${ }^{1}$ College of Engineering, Mathematics and Physical Sciences, University of Exeter, EXETER, United Kingdom; ${ }^{2}$ Endoscopy Department, The Royal Devon and Exeter NHS Foundation Trust, EXETER, United Kingdom

Aim: This work aims to study the contact pressure between the moving capsule and a synthetic small intestine in order to provide design guidance for prototyping the self-propelled capsule robot for small-bowel endoscopy.

Method: Since small-bowel peristalsis consists of peristaltic contraction and wave distension, the contacts between the capsule and the small intestine are multimodal. We consider three contact cases for the capsule robot. Case 1: the capsule moves on a flat small intestinal surface; Case 2: the capsule moves in a collapsed intestine with a flat surface support; and Case 3: the capsule moves in a surrounded small intestine. By considering these three contact cases, experimental testing and finite element analysis (FEA) were conducted by measuring the contact pressure between the small intestine and the capsule.

Results: (1) The average contact pressure for Case 1 is $\mathrm{P} 1=0.8140[\mathrm{kPa}]$; (2) the contact pressure for Case 2, $\mathrm{P} 2=2.1759[\mathrm{kPa}]$, is larger than that of Case 1 ; (3) the pressure due to hoop stress, $\mathrm{Ph}=11.5886[\mathrm{kPa}]$, dominates the contact pressure of Case $3, \mathrm{P} 3=14.1400[\mathrm{kPa}]$ Therefore, the FEA result, $\mathrm{P} 2+\mathrm{Ph}$, fits the experimental result in Case 3 with a high accordance. Conclusion: Our experimental and numerical results show a high accordance, demonstrating the validity of the approach which can be used for shape optimisation and actuator design of the robot.

\section{O139-ROBOTICS \& NEW TECHNIQUES-Technology}

TRUE SINGLE PORT CHOLECYSTECTOMY WITH ICG CHOLANGIOGRAM AND ONLY ONE 15MM TROCAR USING THE NEW SURGICAL PLATFORM 'SYMPHONX'-FIRST HUMAN CASE SERIES

\section{H.F. Fuchs, R.R. Datta, R. Wahba, G. Dieplinger, F. Gebauer,} C.J. Bruns, D. Stippel

\section{Surgery, University of Cologne, COLOGNE, Germany}

Introduction: Minimally invasive single port surgery was associated with large incisions up to 2-3 cm, complicated handling due to the lack of triangulation, and instrument crossing.

Aim of this prospective study was to perform true singleport surgery (cholecystectomy) without the use of assisting trocars using a new surgical platform that allows for triangulation incorporating robotic features and to measure the perioperative outcome and cosmetic results.

Methods and procedures: The new technology has been introduced to our academic center as first European site after FDA and CE clearance. In patients with cholecystitis and cholecystolithiasis, the operation is performed through only one $15 \mathrm{~mm}$ trocar. For patients safety, intraoperative cholangiography using intravenous ICG and a standard Stryker $15885 \mathrm{~mm}$ camera was performed.

Results: Symphonx was used in $\mathrm{n}=11$ patients for abdominal surgery $(4$ females, median age 45.2 [32-77], median BMI 32.7 [29-35]. A total of 5 patients underwent surgery using no additional than the $15 \mathrm{~mm}$ trocar; in the remaining patients, one assisting instrument (3-5 mm) was used. Mean OR time was 103.6 [63-122] min. The postoperative course was uneventful in 10 patients, one patient required postoperative interventional drainage of a bilioma 1 month postoperatively. The cosmetic result was excellent. No intraoperative complications occurred. Conclusion: This is the first human case series using the SymphonX platform for abdominal surgery without assisting instruments.

Laparoscopic cholecystectomy in patients with cholecystitis and cholecystolithiasis using the symphonx platform through only one $15 \mathrm{~mm}$ trocar is feasible and safe. The cosmetic result is promising. Further recruiting of patients for validation of the new technology is necessary and ongoing.

\section{O140—ROBOTICS \& NEW TECHNIQUES-Technology}

HANDX, A HAND-HELD ELECTROMECHANICAL SMART LAPAROSCOPIC INSTRUMENT WITH ROBOT-LIKE FEATURE: FIRST CLINICAL TRIAL IN 30 HUMAN PROCEDURES

\section{A. Szold, A. Ben Yehuda}

\section{TEL AVIV, Israel}

Introduction: Traditional laparoscopic instruments have limited degrees of freedom and are not ergonomic. This results in severe limitations in performing complex, and even simple tasks in surgery, limiting many surgeons from performing a variety of minimally invasive procedures. Hand $\mathrm{X}^{\mathrm{TM}}$ is a hand-held, electromechanical smart instrument with robot-like features. The instrument is composed of a sophisticated user interface that enables unrestricted hand movement, and a novel, motor driven articulating tool that is controlled by the interface. The instrument is $5.5 \mathrm{~mm}$ in diameter, lightweight, and can be easily moved between laparoscopic trocars and perform complex motions in the surgical field. After the regulatory process was completed we have tested the device clinically through a structured, approved, clinical trial. Materials and methods: After IRB approval 30 patients were recruited to the trial. We have included a variety of procedures that require suturing and complex tissue manipulation. Two experiences surgeons performed all procedures. After completing each procedure the surgeons completed a detailed standard usability (SUS) questionnaire.

Results: 30 procedures were completed successfully without complications or device malfunction. There were 15 female and 15 male patients with an average BMI of 27. Procedures performed were 6 right hemicolectomis with intra-corporeal anastomosis, 3 paraesophageal hernia repairs and fundoplication, 3 diagnostic laparoscopies, 2 TAPP procedures, 10 ventra hernias with fascial suturing, and 6 laparoscopic cholecystectomies. The average performance score was $84.70 / 100$

Conclusions: The results suggest that the HandX device is safe and easy to use and may offer a simple solution for enhancing minimal invasive surgery capabilities and possibly reduce conversion rates while maintaining current standard surgery flow.The HandX could potentially extend the surgeon's abilities to access hard to reach anatomy and perform complex maneuvers and present a cost-effective alternative to large console-based robotic systems.

\section{O141-ROBOTICS \& NEW TECHNIQUES-Technology} APPLICATION OF DENTAL FLOSS-ASSISTED SUSPENSION TRACTION IN ENDOSCOPIC SUBMUCOSAL DISSECTION FOR RECTAL NEUROENDOCRINE NEOPLASM

\section{Q. Shi, Y.S. Zhong, P.H. Zhou}

\section{Endoscopy Center, Zhongshan hospital, SHANGHAI, China}

Objective: Endoscopic submucosal dissection (ESD) has become widely accepted treatment for rectum neuroendocrine neoplasm. The aim of this study is to evaluate the safety and efficacy of ESD with dental floss-assisted suspension traction for rectal neuroendocrine neoplasm.

Methods: We retrospectively reviewed the medical records of the patients, who underwent ESD for rectum neuroendocrine neoplasm at Endoscopy Center of Zhongshan Hospital, Fudan University. The data of operation time, R0 resection and adverse events were collected analyzed.In DFS-ESD group: after the mucosa was partly incised along the marker dots, the next step was to construct traction device, similar to others in ESD, with dental floss and hemoclip. The dental floss was tied to any arm of the metallic clip. The hemoclip was attached onto the incised mucosa, another hemoclip was attached onto normal mucosa opposite to the lesion in the same way. The submucosa was clearly exposed with the traction of dental floss and the resection could proceed.

Results: 37 patients were enrolled in the study. There were 17 patients treated by ESD with dental floss-assisted suspension traction and 20 patients treated by conventional ESD. The average tumor size was $(0.74 \pm 0.2) \mathrm{cm}$ in both group. The operation time was $17.9 \pm 6.6 \mathrm{~min}$ in conventional ESD group and $(14.7 \pm 3.3) \mathrm{min}$ in DFS-ESD group $(\mathrm{t}=1.776, \mathrm{P}=0.084)$ According to pathological grading about rectal neuroendocrine neoplasm, there were 17 grade 1 (G1) and 3 grade 2 (G2) in conventional ESD group while 13 grade 1 (G1) and 4 grade 2 (G2) in DFS-ESD group $(? 2=0.436, \mathrm{P}=0.509)$. Among 37 cases in this study, all the basal resection margins were negative, the en blot resection rate was $100 \%$ and the curative resection rate was $100 \%$. However, pathological results showed tumor tissue close to the burning margin in 5 cases of conventional ESD group and in 2 cases of DFS-ESD group $(? 2=0.364, \mathrm{P}=0.546)$.

Conclusions: ESD with dental floss-assisted suspension traction for rectum neuroendocrine neoplasm can assist exposing tumor borders, provide good vision during the procedure and offer clearer anatomic structure, so as to simplify operation, reduce operation time and ensure the negative basal margin. It is especially suitable to be promoted in primary hospitals. 


\section{O142-ROBOTICS \& NEW TECHNIQUES-Technology}

SEMI-AUTOMATED VS. EXPERT MANUAL 3D RECONSTRUCTION OF CENTRAL MESENTERIC VASCULAR MODELS. THE SURGEON'S VERDICT

\author{
J.A. Luzon ${ }^{1}$, B.V. Stimec ${ }^{2}$, R. Kumar ${ }^{3}$, O.J. Elle ${ }^{3}$, B. Edwin ${ }^{3}$, D. \\ Ignjatovic $^{1}$
}

${ }^{1}$ Department of Digestive Surgery, Akershus University Hospital, AKERSHUS, Norway; ${ }^{2}$ Anatomy Sector, Department of Cell Physiology and Metabolism, Faculty of Medicine, University of Geneva, GENEVA, Switzerland; ${ }^{3}$ The Intervention Centre, Oslo University Hospital, Rikshospitalet, OSLO, Norway

Aims: 3D reconstruction of patient-specific anatomy influences preoperative planning and surgeon performance. Though semi-automated applications facilitating fast reconstruction are described in the literature, quality assessment of CT-derived models from a clinician/surgeon's perspective is missing. The aim of this article is to compare semi-automated and manual 3D reconstructed central mesenteric vascular models quantitatively and qualitatively.

Methods: Pre-operative CT-datasets of 24 patients included in the clinical trial 'Safe Radical D3 Right Hemicolectomy for Cancer through Preoperative Biphasic MDCT Angiography' were reanalyzed. Manual reconstruction was through Mimics medical image processing software while semi-automatic was through a multistep algorithm with vessel-enhancement followed by activecontour segmentation. CT-datasets were delivered for semi-automatic segmentation in 4 batches (6 patients each). Manual segmented models were the reference group. Batches 1-3 contained datasets with usual anatomy while Batch 4 consisted of datasets with unusual anatomy. Feedback was provided after each batch reconstruction. Completeness of semi-automated reconstructed models was assessed quantitatively (root mean square [RMS]) with 3D image processing software CloudCompare and qualitatively (visual evaluation missing vessels) to the corresponding reference models. Vessels assessed were those of interest to the surgeon performing right colectomy.

Results: 48 models from 24 patients (13 females, age 44-77) were compared. Quantitatively, batch one: mean RMS $0.74+$ SD0.22, two: $0.54 \pm$ SD0.14, three: $0.51 \pm$ SD0.12, four: $1.20 \pm \mathrm{SD} 0.82$, overall: $0.73 \pm \mathrm{SD} 0.49 \mathrm{~mm}$. Qualitatively, only $2(8.3 \%)$ semi-automated models presented all vessels. Missing: right colic: $7 / 8(87.5 \%$, caliber $0.21 \pm$ SD0.05 cm), whole vessel missing $4 / 8(50 \%, 0.21 \pm$ SD0.64), partial $3 / 8(37.5 \%, 0.19 \pm$ SD0.29); middle colic: $18 / 24$ models (75\%, $0.28 \pm \mathrm{SD} 0.05)$, whole $7 / 24(29.2 \%, 0.25+\mathrm{SD} 0.03)$, partial 11/24 (45.8\%, $0.27+$ SD0.07); ileocolic: $10 / 24$ (41.7\%, caliber $0.335 \pm$ SD0.06), whole $1 / 24(4.2 \%, 0.37)$, partial 9/24 (37.5\%, $0.32 \pm \mathrm{SD} 0.06 \mathrm{~cm})$. Unusual anatomy: missing in 5/6 (83.4\%) models, $1 / 9$ $(11.1 \%)$ anomalies detected (accessory middle colic arteries, Tandler-Buhler anastomoses, multiple middle colic veins and pancreaticoduodenal vein). Detected vessel calibers were significantly higher than in the missing vessels (middle colic $\mathrm{p}=0.000$, right colic $\mathrm{p}=0.016$, ileocolic $\mathrm{p}=0.000)$. Vessel fusion was detected in 16/24 (66.7\%) models. Conclusion: Despite acceptable results of the quantitative analysis, results of the qualitative comparison indicate that semi-automatic generated central mesenteric vascular 3D-models, if used as roadmaps, could cause considerable confusion at surgery due to missing and/or fused vessels.

\section{3-ROBOTICS \& NEW TECHNIQUES-Technology}

REVEALING THE LEARNING CURVE FOR TISSUE MANIPULATION SKILLS IN LAPAROSCOPIC TRAINING

S.F. Hardon $^{1}$, L. van Gastel $^{1}$, T. Horeman ${ }^{2}$, H.J. Bonjer ${ }^{1}$, F. Daams ${ }^{1}$, D.L. van der Peet ${ }^{1}$

${ }^{1}$ Surgery, VU Medisch Centrum, AMSTERDAM, The Netherlands; ${ }^{2}$ BioMechanical Engineering, Delft University of Technology, DELFT, The Netherlands

Aims: Force feedback and assessment provides detailed insight into tissue manipulation skills. The aim of this study is to evaluate learning curves for basic laparoscopic skills based of force and motion learning curve patterns. Morevover, we aimed to detect the favourable time span for this curriculum for each individual trainee.

Methods: In this prospective cohort study, first year surgical residents participated in a three week at home training course. A mobile box training was equipped with Forcesense system for objective force, motion and time based assessment. The system provides seventeen unique metrics. The training goal was set by the mean score of proficient laparoscopic surgeons. Each repetition was captured and made available for analyses. Continuous force feedback was provided during training. Curve fitting was used to estimate the learning curve plateau and the number of repetitions needed to approach the plateau phase and to reach proficiency level. Finally, a comparisson between novices and experts was executed.

Results: A total of 2007 attempts, executed by 20 residents were captured and analyzed. Significant improvement of motion analysis parameters (e.g. path length and time) was observed for all training tasks, except for the fifth tasks. Tissue manipulation skills (i.e. maximum and mean applied force) significantly improved by training tasks 2,3 and 6 . Learning curve analysis revealed various shapes and lengths of the individual learning curves. A large range in learning curve plateaus was found between trainees and between tasks. Each trainee managed to accomplish the preset goals within three weeks.

Conclusion: Force- and motion based assessment provides insight into both tissue manipulation and instrument handling skills. When combined in learning curve analysis, these parameters effectively show progression towards proficiency for each individual trainee over time. We emphasize the variation in learning curves between trainees. Therefore, we recommend individually tailored courses provided with objective force- and motion-based learning curve tracking.

\section{O144-ROBOTICS \& NEW TECHNIQUES-Technology} MINIMALLY INVASIVE APPROACHES TO ABDOMINAL TRAUMA

A. Phaily ${ }^{1}$, A. $\mathrm{Ali}^{2}$, J. Thomas ${ }^{3}$

${ }^{1}$ Department of Surgery, Imperial College London, LONDON, United Kingdom; ${ }^{2}$ Department of Surgery, Royal National Orthopaedic Hospital, LONDON, United Kingdom; ${ }^{3}$ Department of Surgery, University Hospital of Wales, CARDIFF, United Kingdom

Aims: Novel technologies and scientific research have changed the nature of modern surgery and have resulted in the increasing use of minimally invasive surgery (MIS). Continued innovation in the fields of robotics, optics, miniaturisation, computing and developments in artificial intelligence hold promise that MIS will result in a paradigm shift in the future of surgery. This article assesses the role of MIS and its application in trauma, with a specific focus on laparoscopic surgery.

Methods: A literature search of the PubMed/MEDLINE, Cochrane Library, Web of Science and National Institute for Health and Care Excellence Evidence databases using entry sets of Medical Subject Headings (MeSH) for Abdomen (or Abdominal), Trauma and Laparoscopy (or Laparoscopic) as well as a comparative search of the grey literature was also carried out, as well as specific searches of Google Scholar for articles not available via the main databases

Results: 2623 unique articles were screened. Following abstract review, 64 articles were selected for further analysis. Full text analysis of the 65 eligible articles resulted in selection of 4 systematic reviews for inclusion in the analysis. Results show laparoscopy in abdominal trauma is associated with fewer complications, reduced hospital length of stay, less morbidity and a reduction in negative laparotomy rates in haemodynamically stable patients. Current systematic reviews are based on low quality studies with a great degree of heterogeneity. Alternative MIS methods such as endoscopy and angioembolisation have been successfully used as adjunct during non-operative management of abdominal trauma patients.

Conclusion: To increase preparedness for the inevitable rise in the worldwide trauma burden, a concerted effort must be made to focus research efforts on multi-centre, prospective studies on MIS in order to produce meaningful guidelines. Hybrid non-operative and minimally invasive techniques will have a greater role to play in future trauma management. Application of current and future technological innovations dedicated training in MIS and increased research efforts are still needed if we are to effectively combat the individual, societal and global burden of trauma. 


\section{O145-ROBOTICS \& NEW TECHNIQUES-Technology}

ARE CURRENTLY USED MEASUREMENTS OF FLUORESCENCE INTENSITY IN NEAR INFRARED FLUORESCENCE IMAGING DURING LAPAROSCOPIC CHOLECYSTECTOMY COMPARABLE?

J. van den Bos ${ }^{1}$, R.M. Schols ${ }^{2}$, S. van Kuijk ${ }^{3}$, F. Wieringa ${ }^{1}$, L.P.S. Stassen ${ }^{1}$

${ }^{1}$ Surgery, Maastricht University Medical Center, MAASTRICHT, The Netherlands; ${ }^{2}$ Plastic and reconstructive surgery, Maastricht University Medical Center, MAASTRICHT, The Netherlands; ${ }^{3}$ Clinical epidemiology and Health technology assessment, Maastricht University Medical Center, MAASTRICHT, The Netherlands

Aims: Investigate whether different calculation methods to express fluorescence intensity as target-to background ratio, described in current literature, are comparable and which method(s) match human perception.

Methods: Comparison of 3 calculation methods from current literature (Osirix, ImageJ and Photoshop) to measure fluorescence intensity during laparoscopic cholecystectomy measured at the exact same locations within recorded images of two categories: ex-vivo fluorescence imaging and in-vivo fluorescence imaging during laparoscopic cholecystectomy. Currently applied formulas to present fluorescence intensity (FI) in relation to the background (BG) signal are compared with the subjective assessment by the human observers. These three formulas are 'Signal contrast $=(\mathrm{FI}$ in fluorescence regions-FI in BG) $/ 255$ ', 'Target-to-background ratio $=(\mathrm{FI}$ of target $-\mathrm{FI}$ of BG) $/ \mathrm{FI}$ of BG', 'Signal-to-background ratio $=\mathrm{FI}$ of Cystic duct $/ \mathrm{FI}$ of liver' and 'Target-to-background ratio = (FI of Target-Noise $) /($ FI of BG-Noise $)$ '. Results: In our evaluation Osirix and ImageJ gave similar results, while Osirix values were always lower compared to ImageJ. Values obtained via Photoshop however are less evidently related to those obtained with Osirix and ImageJ. The formula Target-to-background ratio $=(\mathrm{FI}$ of Target—Noise) / (FI of BG-Noise)was less corresponding with human perception compared to other used formulas.

Conclusions: Fluorescent intensity measurement results from the programs Osirix and ImageJ are similar, allowing for comparison of results between these programs. Results using Photoshop differ from these, making direct comparison not possible. This is important when interpreting study results. We propose to report both target and background fluorescence intensity in manuscripts, so that calculations between articles can be made.

\section{O146-SOLID ORGANS-Adrenal}

POSTERIOR RETROPERITONEOSCOPIC VERSUS LAPAROSCOPIC TRANSABDOMINAL ADRENALECTOMY: PROSPECTIVE RANDOMIZED TRIAL

V.V. Grubnik ${ }^{1}$, V.V. Ilyashenko ${ }^{2}$, R.S. Parfentiev ${ }^{2}$, O.S. Burlak ${ }^{2}$, $\overline{\text { V.V. Grubnyk }}^{2}$

${ }^{1}$ Department of surgery, Odessa medical university, ODESSA, Ukraine; ${ }^{2}$ Department of Surgery \#1, Odessa medical university, ODESSA, Ukraine

Aim of the study was to compare results of laparoscopic transabdominal (LA) and posterior retroperitoneoscopic (RA) adrenalectomy.

Methods: From 2013 to 2017 years 86 patients with adrenal tumors were randomized in two groups: 44 patients with LA (group I), and 42 patients with RA (group II). Both groups were comparable with regard to demographic and clinical parameters, comorbidities, and size of tumors. Laparoscopic transabdominal adrenalectomy was performed employing the Gagner's technique. Retroperitoneoscopic adrenalectomy was performed using the Walz's technique. Results: There were no serious complications and mortality in both groups. Conversions were done in 4 cases $(9.0 \%)$ in group I, and in 1 case $(2.3 \%)$ in group II due to size of tumors and technical difficulties. Operative time, time to first intake, analgesic requirements, length of hospital stay, postoperative complications were all significantly lower in the RA group.

Conlusions: RA provides better perioperative outcomes compare to LA. RA is the preferred operation for not large adrenal tumors.

\section{O147-SOLID ORGANS-Adrenal}

RISK FACTORS FOR OPEN CONVERSION OF LATERAL TRANSPERITONEAL LAPAROSCOPIC ADRENALECTOMY: STUDY OF THE SPANISH ADRENAL SURGERY GROUP

D. Saavedra-Perez ${ }^{1}$, O. Vidal ${ }^{1}$, J.M. Martos ${ }^{2}$, A. de la Quintana ${ }^{3}$, J.I.

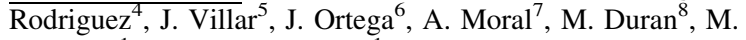
Valentini ${ }^{1}$, L. Fernandez-Cruz ${ }^{1}$

${ }^{1}$ Endocrine Surgery, Hospital Clinic of Barcelona, BARCELONA, Spain; ${ }^{2}$ Endocrine Surgery, Hospital Universitario Virgen del Rocio, SEVILLA, Spain; ${ }^{3}$ General and Digestive Surgery, Hospital Universitario Cruces, BIZKAIA, Spain; ${ }^{4}$ Endocrine Surgery, Hospital Universitari Josep Trueta, GIRONA, Spain; ${ }^{5}$ Endocrine Surgery, Hospital Universitario Virgen de las Nieves, GRANADA, Spain; ${ }^{6}$ Endocrine Surgery, Hospital Clínico Universitario, VALENCIA Spain; ${ }^{7}$ General and Digestive Surgery, Hospital Universitario de la Santa Creu i Sant Pau, BARCELONA, Spain; ${ }^{8}$ General and Digestive Surgery, Hospital Universitario Rey Juan Carlos, MADRID, Spain

Aims: Laparoscopic adrenalectomy has become the standard of care for treating adrenal tumors Conversion from laparoscopic adrenalectomy to an open approach during surgery may be necessary in some cases. This study aimed to identify risk factors for open conversion of laparoscopic adrenalectomy.

Methods: Retrospective analysis of all consecutive patients undergoing lateral transperitoneal laparoscopic adrenalectomy in six endocrine surgery units of the Spanish Adrenal Surgery Group (SASG) between January 2005 and December 2017. Demographic, clinical, surgical and histopathologic characteristics were recorded. Risk factors for conversion were assessed by logistic regression analysis.

Results: Of a total of 865 patients included in the study, $58(6.7 \%)$ required conversion to open surgery. In the univariate analysis, factors associated with open conversion from laparoscopic to open adrenalectomy were body mass index $(\mathrm{BMI})>30 \mathrm{~kg} / \mathrm{m}^{2}(\mathrm{p}=0.002)$, previous abdominal surgery $(\mathrm{p}=0.015)$, tumor size $>5 \mathrm{~cm} \quad(\mathrm{p}=0.001)$ and surgery for pheochromocytome $(\mathrm{p}=0.034)$. In the multivariate analysis, independent risk factors were BMI $>30 \mathrm{~kg} / \mathrm{m}^{2}$ (odds ratio $[\mathrm{OR}] 4.26,95 \%$ confidence interval $[\mathrm{CI}] 2.81-8.75 ; \mathrm{p}=0.001)$, tumor size $>5 \mathrm{~cm}(\mathrm{OR}$ $1.015,95 \%$ CI $4.24-28.31 ; \mathrm{p}<0.001=$ and surgery for pheochromocytoma $(\mathrm{OR} 2.96,95 \% \mathrm{CI}$ $1.89-11.55 ; \mathrm{p}=0.015$ ).

Conclusions: Obesity, tumor size, and pheochromocytoma as the type of adrenal tumor were predictive factors for introperative conversion from laparoscopic to open adrenalectomy. Preoperative assessement of these characteristics should be valuable to clinicians in discussing conversion risk with patients and for surgical planning. 


\section{O148-SOLID ORGANS-Adrenal}

FLUORESCENCE-ENABLED ASSESSMENT OF ADRENAL GLAND LOCALIZATION AND PERFUSION IN POSTERIOR RETROPERITONEOSCOPIC ADRENAL SURGERY IN A PRECLINICAL MODEL

B. Seeliger ${ }^{1}$, M.K. Walz ${ }^{2}$, P.F. Alesina ${ }^{2}$, V. Agnus ${ }^{1}$, M. Barberio ${ }^{1}$,

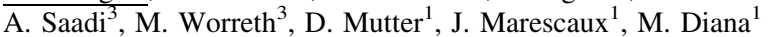

${ }^{1}$ Institute of Image-Guided Surgery, IHU-Strasbourg, STRASBOURG, France; ${ }^{2}$ Department of Surgery and Center of Minimally Invasive Surgery, Kliniken Essen-Mitte, ESSEN, Germany; ${ }^{3}$ Department of Surgery, Neuchâtel Hospital,

\section{NEUCHÂTEL, Switzerland}

Aims: The posterior retroperitoneoscopic adrenal access represents a challenge in orientation and working space creation.The aim of this experimental acute study was to evaluate the impact of computer-assisted quantitative fluorescence imaging on adrenal gland identification and perfusion assessment in the posterior retroperitoneoscopic approach.

Methods: Six pigs underwent synchronous $(n=5)$ or sequential $(n=1)$ bilateral posterior retroperitoneoscopic adrenalectomy (PRA, $n=12$ ). Fluorescence imaging was obtained via intravenous administration of $3 \mathrm{~mL}$ of Indocyanine Green (ICG) using two near-infrared camera systems. Fluorescence-based visualization of adrenal glands before vascular division $(n=4)$, after main vascular pedicle ligation (negative control, $\mathrm{n}=1)$ or after adrenal division $(\mathrm{n}=7)$ was followed by completion adrenalectomy. One of the animals had undergone ICG injection $3 \mathrm{~h}$ previously, during another study. The dynamic evolution of fluorescence signal intensity over time was recorded and analyzed using a proprietary software. The computed color-coded perfusion cartography was superimposed onto real-time images obtained by corresponding left $(\mathrm{L})$ and right $(\mathrm{R})$ camera systems. The slope of fluorescence signal intensity evolution over time in the regions of interest (ROI) served to assess adrenal perfusion by means of quantitative fluorescence signal analysis.

Results: In the retroperitoneum, the adrenal glands were promptly highlighted after primary intravenous ICG administration or showed an increase in fluorescence signal intensity upon reinjection (both glands in a recovery pig and one gland in the sequential approach). After left adrenal main vascular pedicle ligation, the gland displayed low perfusion (blue; ROIs A1-A2 in Figure 1), while a weak fluorescence signal after completion adrenalectomy suggests perfusion via collateral vessels. With intact vascular supply, the caudal segment of the right adrenal (A3) gland showed a significantly higher perfusion rate (red) than the ischemic cranial segment (A4). Quantitative analysis of logarithmic fluorescence intensity showed a statistically significant difference between perfused and ischemic zones $(p=0.005)$ allowing to assess gland vascularity. Kidneys $(\mathrm{K})$ and adrenal glands showed distinct perfusion curves (Figure 1). Conclusions: Prior to dissection, fluorescence imaging allows to easily discriminate the adrenal gland from surrounding retroperitoneal structures. During adrenal gland surgery, ICG injection complemented by a computer-assisted quantitative analysis helps to distinguish between wellperfused and low-perfused segments.

\section{O149-SOLID ORGANS-Adrenal}

\section{GIANT ADRENAL TUMORS:TECHNICAL CONSIDERATIONS AND SURGICAL OUTCOME}

A. Giordano, G. Alemanno, C. Bergamini, P. Prosperi, V. Iacopini, A. Dibella, A. Valeri

\section{Sod Chirurgia d'urgenza, AOU CAREGGI, FIRENZE, Italy}

Objectives: Giant adrenal tumors are tumors with size more than $6 \mathrm{~cm}$. These are rare cancer associated with malignancy in $25 \%$ of cases. The size of these tumors is an important topic in literature because of their higher probability of malignancy and possible technical limitations of laparoscopic approach. We report our center's experience on laparoscopic adrenalectomy. Materials and methods: In the last ten years we performed about 242 adrenalectomies for benign and malignant adrenal tumors. 45 of these were giant tumors. The medium size was $9.9 \mathrm{~cm}$ $(7-22 \mathrm{~cm}) .23$ tumors were on the left adrenal gland and 22 on the right. There were 20 women and 25 men, the average age was 55 ( $21-81$ years). 29 of these cancers were laparoscopically removed and 16 with open approach. 2 cases of open conversion.

Results: Betweenn the 29 tumors laparoscopically removed we recorded 6 cases of carcinoma, 2 endothelial cysts, 6 adenomas ( 3 with aldosterone and 2 with cortisol hypersecretion), 2 myelolipomas, 10 pheochromocytomas and 3 metastases from lung carcinoma. The surgical outcomes in these patients were optimal in terms of good pain control and hospital stay (median 3 days). The average time of the intervention was $110 \mathrm{~min}$ with very low blood less $(90 \mathrm{ml})$. No postoperative complications were recorded. The removal of the adrenal gland necessitated 3 or 4 trocars. in the dissection and resection phases we always used radiofrequency scalpel. The follow up after 12 and 24 months didn't show local recurrences.

Conclusions: Laparoscopic adrenalectomy offers significant advantages over the open approach. The size of these tumors is still at the center of debate for the choice of the technique. The tumor size is only a predictive parameter of possible malignancy. The laparoscopic approach is a safe and feasible method in terms of surgical and oncological outcomes also for the giant adrenal tumors, only if performed by expert surgeons and in high-volume centers. Vascular or adjacent organs infiltration is a contraindication to the laparoscopic approach.

\section{O150—SOLID ORGANS-Adrenal}

\section{ARE ADRENAL LESIONS $=6 \mathrm{CM}$ IN DIAMETER A CONTRAINDICATION TO LAPAROSCOPIC ADRENALECTOMY? A CASE CONTROL STUDY}

A. Balla ${ }^{1}$, L. Palmieri ${ }^{1}$, F. Meoli ${ }^{1}$, D. Corallino ${ }^{1}$, M. Ortenzi ${ }^{2}$, P.

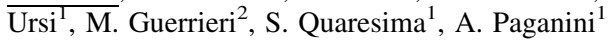

${ }^{1}$ Department of General Surgery and Surgical Specialties, Paride Stefanini, Sapienza University of Rome, ROMA, Italy; ${ }^{2}$ Department of General Surgery, Università Politecnica delle Marche, ANCONA, Italy

Aims: Adrenal gland size greater than $6 \mathrm{~cm}$ is considered a contraindication to laparoscopic adrenalectomy (LA). Aim of the present case-control study is to compare the surgical outcomes in patients undergoing LA for adrenal gland measuring $=6 \mathrm{~cm}$ versus $=5.9 \mathrm{~cm}$ in diameter. Methods: From January 1994 to August 2018, 552 LAs were performed in the two authors' centers which follow an identical treatment protocol. Eighty-one patients with an adrenal gland size $=6 \mathrm{~cm}$ (intervention group) were included in the study. Based on Body Mass Index (BMI) class (18-24.9, $25-29.9,30-34.9,35-39.9,>40 \mathrm{Kg} / \mathrm{m}^{2}$ ), lesion side (right or left), surgical technique (anterio transperitoneal for right and left-sided lesions, anterior transperitoneal submesocolic for left-sided lesions) and lesion type (Conn-Cushing, pheocromocytoma, primary adrenal cancer or metastases, other type of lesion), 81 patients with an adrenal gland lesion measuring $=5.9 \mathrm{~cm}$ in diameter were included (control group) and paired to the intervention group.

Results: Comparing the intervention and control groups, statistically significant differences were observed in mean lesion size $(3.305 \pm 1.157$ and $6.969 \pm 1.447, \mathrm{p}=<0.0001)$ and in mean operative time $(85.06 \pm 31.67$ and $101.42 \pm 52.43, p=0.0174)$. Four conversions to open surgery were observed in the control group $(4.94 \%)$ and 8 in the intervention group $(9.88 \%)(\mathrm{p}=0.3690)$. In the control group, 3 postoperative complications were observed (3.7\%) and in the intervention group 5 postoperative complications were observed $(6.17 \%)(\mathrm{p}=0.7196)$. Postoperative blood transfusions were required in 4 intervention group patients $(4.94 \%)$ and in none of the control group $(\mathrm{p}=0.1204)$. Mean postoperative hospital stay was $4.12 \pm 2.38$ in the control group and $4.62 \pm 2.46$ in the intervention group $(p=0.1957)$ Conclusions: The only significant difference between the two groups was the operative time which was longer in the intervention group. Conversion and complication rates were also higher in the intervention group but the difference was not statistically significant. Based on the present data, adrenal gland size measuring more than $6 \mathrm{~cm}$ in diameter is not a contraindication to a laparoscopic approach

\section{O151-SOLID ORGANS-Gynaecology}

\section{MEDICAL LIABILITY CLAim: s CONCERNING MINIMAL INVASIVE SURGERY}

\section{D.M.T.V. Klemann}

Gynaecologie, Zuyderland, MAASTRICHT, The Netherlands

Aims: Damage burden incidents have become a serious problem since the introduction of laparoscopic surgery. From 2007 to 2016, the increase of all medical claims in the Netherlands was +4.5\%; the damage burden off all incidents increased even $500 \%$. The primary aim of this supplementary study was to analyse absolute and relative numbers and sizes of medical claims after laparoscopic surgery $(\mathrm{N}=317$ in the period 2008-2017). In order to identify risk factors associated with medical claims related to laparoscopic surgery, we reviewed all claims related to laparoscopic gynaecologic surgery filed from 1993 to 2015. Methods: Data of medical claims of Centramed and MediRisk were used from 2007 till 2016 $(\mathrm{N}=15.335)$. Both are liability insurers for claims, and assume together $95 \%$ of all Dutch hospitals. To identify the claims related to laparoscopic surgery of all medical specialties, we searched the databases. Lapraroscopic related claims could be separately identified in the database of MediRisk. We reviewed the medical and legal charts of all claims related to laparoscopic gynaecologic surgery of both Centramed and MediRisk $(\mathrm{N}=328)$.

Results: Claims related to laparoscopic surgery decreased over a period of 10 years; in 2008 versus $2017,42(4 \%)$ versus 20 (2\%) of all claims were related to laparoscopic surgery. In all medical liability claims concerning laparoscopic surgery, general surgeons were responsible for 217 (68.5\%); gynaecologists for 79 (24.9\%); and orthopaedics and urologists for the remaining $6.6 \%$. The costs from these claims, differed from 2 to $13 \%$ of the total damage burden per year. The review of medical charts of claims related to laparoscopic gynaecologic surgery showed that $82 \%$ of claims were filed for visceral and/or vascular injuries (40\% bowel injuries, $20 \%$ ureter). $38 \%$ of the injuries were entry-related. A delay in diagnosing injuries was the primary reason for financial compensation.

Conclusion: Evaluating and learning from complications and claims will improve medical health care. In contrast to overall trends and developments considering medical claims, claims concerning laparoscopic surgery decreased, possible due to a rising learning curve. Considering laparoscopic surgery, extra caution is required at moment of entry and the early recognising complications and at pre-operative counselling from patients. 


\section{O152-SOLID ORGANS-Kidney}

LAPAROSCOPIC RADICAL NEPHRECTOMY FOR RENAL CELL CARCINOMA

\section{S. El-Shakhs}

General Surgery \& Surgical Oncology, Menoufia University, AL MINUFYA, Egypt

Aim: This prospective study was conducted to evaluate feasibility \& oncological safety in performing laproscopic radical nephrectomy for renal cell carcinoma.

Patients \& principle: This prospective study was conducted at Menoufia University Hospital from the period of January 2015 to January 2018 on 22 patient with RCC.

Methods: The patient was positioned either right lateral or left lateral according to the tumour site. We used to do it by 4 ports, $2 \times 10 \mathrm{ml}$ for camera \& clip applier, other $2 \times 5 \mathrm{ml}$ for surgeon left hand \& assistant. After exploration of the abdomen by camera, first step is a colon mobilization medially, followed by release of the upper border from liver on right side or splenopancreatic block on left side. Then the ureter \& gonadal vessels were identified \& transected. The attention was directed to the hilum where renal vein is identified followed by renal artery which is posterior. Clipping of the artery first then clipping of renal vein after being collapsed. Finally complete mobilization from the lateral wall, small incision for tumour excision. Drain was left for $24 \mathrm{~h}$. Patient started oral on the same day and discharged 2nd day morning.

Results: 7 patients had left renal tumours \& 15 had right. Twenty patients underwent total laparoscopic radical nephrectomy \& 2 were converted one for difficulty of mobilizing the fatty colon due to obesity, $2^{\text {nd }}$ for extensive adhesion that obscured the vena cava. No intraoperative blood was given except one \& this pt received another 2 units postoperative. Operative time was $175+23$ min. patient started oral on same day night except those who were converted started on 2 nd day post operative. The average number of harvested lymph nodes were $7+3$ nodes. All were renal cell carcinoma, 17 clear cell, 2 sarcomatoid \& last one with chromophobe. The mean tumour size was $9+3 \mathrm{~cm}$ ) \& the average was $5-17 \mathrm{~cm}$.

Conclusion: Laparoscopic radical nephrectomy proved to be feasible, safe with a smooth postoperative course.

\section{O154-SOLID ORGANS—Parathyroid}

\section{MINIMALLY INVASIVE VIDEOI-ASSISTED PARATHYROIDECTOMY WITH INTRAOPERATIVE NERVE STIMULATION}

V. Drakopoulos, N. Roukounakis, S. Voulgaris, V. Lygizos, I. Iliadis, P. Trakosari, A. Bakalis, V. Vougas, 1st Department of Surgery and Transplantation Unit, District General Hospital of Athens « Evangelismos », ATHENS, Greece

Introduction: Intraoperative nerve stimulation and neuromonitoring is a commonly accepted practice during endocrine surgical procedures in the neck. Minimally invasive thyroidectomy and parathyroidectomy and especially video-assisted parathyroidectomy are safely and successfully performed in selected patients with appropriate indications.

Material/Method: We present a video demonstrating our technique in a case of a Minimally Invasive Video-Assisted Parathyroidectomy (MIVAP), with intraoperative recurrent laryngeal nerve stimulation. We demonstrate the necessity of modification of the ordinary technique in order to get a positive identification of the nerve.

Conclusion: A modification of the standard technique of intraoperative nerve stimulation is mandatory during MIVAP

\section{O153-SOLID ORGANS-Parathyroid}

\section{CERVICAL PURE ENDOSCOPIC APPROACH FOR POSTERIOR MEDIASTINUM PARATHYROID ADENOMAS}

M. Rubio-Manzanares Dorado ${ }^{1}$, V. Pino Diaz ${ }^{1}$, J. Padillo Ruiz ${ }^{2}$, J.M. Martos Martínez

${ }^{1}$ Endocrine Unit, Hospital Virgen Del Rocio, SEVILLA, Spain; ${ }^{2}$ General Surgery Department, Hospital Virgen Del Rocio, SEVILLA, Spain

Aims: About one and half year ago, we proposed a novel pure endoscopic cervical approach to posterior mediastinum parathyroid adenomas that we called 'prevertebral cervical approach'.

Methods: Step by step technique is described for both right and left sided adenomas, by means of photographs and short video clips.

Results: From June 2015 to December 2018, 7 patients were operated using this technique. Five adenomas were right sided and 2 were left sided. Mean surgical time was $31 \pm 5$ min. There were neither intraoperative nor postoperative complications. Four patients presented a slight subcutaneous emphysema which did not cause complaints. All patients were discharged next day after surgery except for one patient with a previous neck open 4 glands removal for secondary hyperparathyroidism, which required calcium replacement. Calcium and parathyroid hormone levels were normalized in the other 6 patients after surgery.

Conclusions: This cervical pure endoscopic approach has shown, in our experience, a high feasibility and short surgical time, with excellent postoperative results regarding to patient comfort, length of stay and disease cure, offering besides a very reasonable procedure cost, and may result in a less aggressive surgical option when compared with thoracic approaches.

\section{O155-SOLID ORGANS-Spleen}

\section{LAPAROSCOPIC SPLENECTOMY IN PATIENTS WITH ABDOMINAL TRAUMA}

\section{P.A. Yartsev, M.M. Rogal, O.A. Chernysh}

General surgery, N.V. Sklifosovsky Emergency Medicine Research Institute, MOSCOW, Russia

The aim of the study was to determine indications and contraindications for laparoscopic splenectomy in abdominal trauma patients and to analyze results of the operations.

Patients and methods: The study involved 112 patients with spleen injury grade III who were admitted in our institute in the years of 2013-2018. The patients were divided on two groups. Laparoscopic splenectomy was performed in 62 patients (group I) and 'traditional' splenectomy was carried out in 50 patients (group II). There was no difference in the demographic data and trauma severity between the two groups.Non-invasive investigations, such as laboratory investigations, serial abdominal ultrasound examinations (US), X-ray in multiple views and computed tomography (CT) had been performed before the decision about necessity of an operation was made.

Results: Patients after laparoscopic operations had better recovering conditions compare to patients with the same injury after 'traditional' splenectomy. Neither surgery related complications no mortalities were registered in both groups. Laparoscopic splenectomy was more timeconsuming operation than 'traditional' splenectomy. We suggest that as experience of laparoscopic splenectomy is gained the operation time will be reduced.

Conclusion: Laparoscopic splenectomy is a safe feasible operation in patients with spleen injury. The operation is indicated in patients with spleen laceration more than $3 \mathrm{~cm}$ of parenchymal depth with moderate continuing bleeding or expanding hematoma and contraindicated in patients with hemodynamic instability and high bleeding rate (more than $500 \mathrm{~mL} / \mathrm{h}$ on serial US examinations). 


\section{O156-SOLID ORGANS-Spleen}

\section{LAPAROSCOPIC TREATMENT OF A LARGE SPLENIC HYDATID CYST}

\section{Calu, M. Oun, C. Paraianu, A. Miron}

Department of Surgery, Elias Emergency Hospital, BUCHAREST, Romania

The isolated hydatid disease of the spleen is a quite rare condition, liver and lungs being the most common locations. The treatment requires usually splenectomy, open or laparoscopic. There are few reports in the literature describing a spleen-preserving type of surgery.

We present a case of a female patient, 51 y.o., with a large cystic lesion of the spleen, $11 \mathrm{~cm}$ in diameter. Lab tests and CT scan confirmed that is a hydatid cyst. After Albendazole treatment and vaccination the patient was referred to us for surgical treatment. The procedure was performed under general anesthesia and laparoscopic approach was performed with the intention to preserve the spleen. After the cyst was identified and adhesiolysis was done, the area was isolated from the rest of the abdominal cavity with sponges with a betadine solution in order to prevent contamination. A needle aspiration of the cyst allowed the evacuation of $550 \mathrm{ml}$ of purulent content, an indicator of a dead cyst. Betadine solution was injected into the lesion. Laparoscopic excision of the cyst was performed using advanced electrocoagulation devices and the spleen removal was not deemed necessary. Two drainage tubes were placed in the remnant cavity. An abdominal ultrasound was performed in the third postoperative day and no collections were identified. The postoperative outcome was uneventful; the patient was discharged in the $6^{\text {th }}$ postoperative day. The conclusion is that in selected cases, with the cyst located in the anterior part of the spleen, with proper equipment and experienced laparoscopic teams, the cyst can be successfully treated without splenectomy.

\section{O157-SOLID ORGANS-Thyroid}

HOW DO WE MAKE NERVE MONITORING SAFE IN THYROID SURGERY ? EARLY PERIOD Results

T. Donmez, M.E. Gunes, C.A. Dural, C. Akarsu, N.A. Sahbaz, Y. Kesgin

General Surgery, Bakirkoy Dr.Sadi Konuk Training and Research Hospital, ISTANBUL, Turkey

Aim: Intraoperative neuromonitoring(IONM) can aid recurren laryngeal nerve(RLN) identification in thyroid surgery. However, its role in reducing the frequency of RLN injury controversial. The use of neuromuscular blocking agents(NMBD's) may affect IONM during thyroid surgery. A selective neuromuscular recovery protocol was evaluated in a retrospective clinical trial during thyroid neural monitoring surgery.

Methods: Seventy-five patients with American Society of Anesthesiologists physical class I-IIIII status who underwent propofol-remifentanil anesthesia for the surgery of the thyroid gland. Deep neuromuscular block was induced with rocuronium $1.2 \mathrm{mg} / \mathrm{kg}$. In Group1, Forty patients were enrolled for reversal of profound neuromuscular block during thyroid surgery (sugammadex $2 \mathrm{mg} / \mathrm{kg}$, after identification of vagus nerve). In group 2, thirty-five patients were enrolled profound neuromuscular block during thyroid surgery(without reversal of NMBD). TOF-Watch acceleromyograph was recorded in response to adductor pollicis muscle for ulnar nerve stimulation in patients with both groups; recovery was defined as a train-of-four (TOF) ratio $=0.9$.To prevent laryngeal nerve injury during the surgical procedures, all patients were neurophysiologically detected using IONM.

Results: The total duration of surgery was higher in group 2 than group $1(63.7 \pm 5.6$, $82.5 \pm 6.1 ; \mathrm{p}<0.001)$. The mean time to recovery of the TOF ratio to 0.9 was higher in group 2 than group $1(22.3 \pm 2.6,74.3 \pm 5.0 ; \mathrm{p}<0.001)$. The mean duration of vagus reverse (v1:3,5milisecond) was higher in group 2 than in group1(21.3 $\pm 1.7,42.9 \pm 5.1 ; \mathrm{p}<0.001)$. No significant difference was found between left and right $\mathrm{v} 1-\mathrm{v} 2$ and $\mathrm{r} 1-\mathrm{r} 2$ values in group 1 following nerve monitoring, whereas in group 2 , a significant difference was found between left $\mathrm{v} 1-\mathrm{v} 2$, left $\mathrm{r} 1-\mathrm{r} 2$ and right $\mathrm{v} 1-\mathrm{v} 2$ values $(0.19 \pm 0.07,0.32 \pm 0.1, \mathrm{p}<0.001 ; 0.20 \pm 0.06$ $0.28 \pm 0.06 \mathrm{p}<0.001 ; 0.29 \pm 0.06,0.40 \pm 0.08, \mathrm{p}<0.001$,respectively).

Conclusion: In the follow-up of nerve monitoring, a safe thyroid surgery can not be mentioned unless the effect of the neuromuscular blockade agent is overtaken. Agents that remove the NMBD's influence should be used to prevent recurrent nerve injury.

\section{O158-SOLID ORGANS-Thyroid}

\section{MINIMAL BLUNT DISSECTION FOR PRODUCING OPERATIVE FIELD IN BILATERAL AXILLO-BREAST APPROACH THYROIDECTOMY}

T.J. Liang, C.Y. Tsai, S.I. Liu, I.S. Chen, Surgery, Kaohsiung Veterans General Hospital, KAOHSIUNG, Taiwan

Aims: Bilateral Axillo-Breast Approach (BABA) thyroidectomy has superior cosmetic outcome but requires a larger working space for manipulation. There has been concerns about the extensive dissection and its damage to the breast. We present an easy and less traumatic technique to produce operative filed in BABA thyroidectomy

Methods: We developed a 'single bi-axillary tunneling' technique for working space creation in BABA thyroidectomy. All operations were performed by a single surgeon in the Kaohsiung Veterans General Hospital. The patient's data were retrospectively reviewed. Operative time, skin flap complications, and other surgical outcome parameters were recorded.

Results: With our technique, the entire working space creation was typically completed within 20-30 min. There was no infection, skin necrosis, flap perforation, and seroma formation. Only minor and small bruises were detected.

Conclusions: The single bi-axillary tunneling method is an easy technique for operative field production in BABA thyroidectomy. It minimizes skin bruises, improves patient satisfaction and remains time efficient.

\section{O159-UPPER GI-Benign Esophageal disorders}

AUTOLOGOUS AUGMENTATION OF HIATAL HERNIA REPAIR WITH PLATELET CONCENTRATE IMPROVES Results: IN PATIENTS WITH LARGE HIATAL HERNIAS

V.V. Grubnik ${ }^{1}$, V.V. Ilyashenko ${ }^{2}$, V.V. Grubnyk ${ }^{2}$, M.R. Paranyak. ${ }^{2}$

${ }^{1}$ Department of surgery, Odessa medical university, ODESSA, Ukraine; ${ }^{2}$ Department of surgery \#1, Odessa medical university, ODESSA, Ukraine

The aim of the study was to compare outcomes of paraesophageal hernias repair (PEHR) with mesh and autologous augmentation with platelet rich plasma (PRP) and without mesh in elderly patients.

Methods: 162 adult patients with large PEH undergoing laparoscopic repair from 2014 to 2018 years of our clinic were included. Patients were divided by age: group A (72patients) $=65$ years, group B (90patients) - age 66-87 years. In the I group (84 patients)—we used non-absorbable self-fixating ProGrip ${ }^{\mathrm{TM}}$ mesh for reinforcement of crura repair, II group (72 patients) - crura repair was done without mesh. In the patients of I group pieces of mesh were infiltrated by $2-4 \mathrm{ml}$ of autologous PRP. We determined type-I, and type-III collagens in patients of A and B groups. PRP was prepared from $20 \mathrm{ml}$ blood of the patient. The groups were evaluated according to the following criteria: dysphagia, patient's symptomatic outcome judgment according to the GERD-HRQL questionnaire and patient's satisfaction, hiatal hernia recurrence according to upper endoscopy and barium contrast swallow study.

Results: There were no mortality in the patients of both groups. Mean operative time was not significantly different in both groups. Major morbidities were significantly lower in the younger group when compared to the elderly patients (group B). There were similar operative time and blood loss between the groups. The mean length of hospital stay was shorter in-group A. Median length of follow-up was the same in both groups. Recurrence rate was significantly higher in the patients of II group [recurrence rate in-group I was 3.6\%, in II group-16.7\% $(\mathrm{p}<0.01)$ ] Recurrence rate was the same in younger and elderly groups of patients with mesh repair [group I A $-2 \%$, group I B $-4 \%(\mathrm{p}>0.05)$ ]. All groups demonstrated significant improvement in GERDHRQL score. The quantity of total, type-I, and type-III collagens was significantly lower in the elderly group of patients.

Conclusion: Laparoscopic PEH repair can be done safely and effectively in elderly patients. Mesh reinforcement of crura repair with autologous PRP significantly decrease recurrence rate in elderly patients. 


\section{O160-UPPER GI-Esophageal cancer}

\section{EXPERT STAKEHOLDER OPINION ON BARRIERS TO} QUALITY ASSURANCE OF OESOPHAGOGASTRIC SURGERY IN ONCOLOGY TRIALS AND POTENTIAL MITIGATING STRATEGIES $\frac{\text { J.W. Butterworth }^{1}}{\text { G.B. Hanna }^{1}}$ P.R. Boshier ${ }^{1}$, S. Mavroveli ${ }^{1}$, J.V. Reynolds ${ }^{2}$,

${ }^{1}$ Cancer and Surgery, Imperial College London, LONDON, United Kingdom; ${ }^{2}$ Surgery, St. James's Hospital and Trinity College, DUBLIN, Ireland

Introduction: Oeosphagogastric oncology trials have often lacked robust methods of monitoring and surgical quality assurance (SQA), leading to difficulty in interpretation of trial results. This study aims to assess expert opinion regarding challenges to SQA in oncology trials and potential mitigating strategies.

Method: A purposive international cohort of 71 expert stakeholders with experience in oncology trials were recruited including: 35 surgeons; 17 oncologists; 10 trial methodologists, and; 9 trial managers. Semi-structured interviews were thematically analysed using grounded theory. SPSS was utilised to assess differences between trial stakeholders' opinions.

Results: 389 emergent themes were identified and 74 consensus themes emerged on qualitative analysis of stakeholder responses. Key consensus challenges to implementation of SQA in oncology trials included: Insufficient resources; limitations of surgical volume in centre selection; differing oncological beliefs and resistance to change adoption; overly prescriptive protocols and standardisation contributing to difficulty in surgeon recruitment; and cultural factors leading to difficulties in providing and receiving feedback. Seminal consensus mitigating strategies to overcome challenges to SQA in oncology trials included: trial centre selection according to case volume $(\mathrm{n}=31,44 \%)$; requirement for specific centre attributes for inclusion in trials including specialist centre designation and participation in national audit $(\mathrm{n}=29,41 \%)$; consideration for surgeons learning curve in surgeon selection $(\mathrm{n}=33,46 \%)$; flexible standardisation of trial operating $(\mathrm{n}=22,31 \%)$; operation manual utilisation to aid standardisation of surgical interventions $(\mathrm{n}=34,48 \%)$; case monitoring using video $(\mathrm{n}=22,31 \%)$ or photographs $(\mathrm{n}=11,16 \%)$; direct intraoperative observation by an expert $(n=15,21 \%)$, and; histopathological assessment of resected specimens $(n=10,14 \%)$. Other methods of monitoring surgical quality advocated included: recording post-operative outcomes; lymph node yield; case report forms; and real time data monitoring $(\mathrm{n}=32,45 \%)$. Oncologists were significantly more likely to state the importance of standardisation of surgery in oncology trials $(\mathrm{p}<0.05)$, and trial methodologists significantly more likely to advocate consideration of surgeons' learning curve in surgeon selection $(\mathrm{p}<0.05)$.

Conclusion: Surveying international expert stakeholder opinion revealed a wide variety of perceived challenges across all domains of surgical quality assurance. Proposed mitigating solutions require consensus opinion to formulate a framework to aid design of SQA measures within future oncology trials.

\section{O162-UPPER GI-Esophageal cancer}

\section{NEW METHOD OF ESOPHAGO-GASTRO ANASTOMOSIS WITHIN MINIMALLY INVASIVE HYBRID IVOR LEWIS PROCEDURE}

O. Usenko ${ }^{1}$, A. Sydiuk ${ }^{1}$, A. Klimas ${ }^{1}$, O. Sydiuk ${ }^{2}$, G. Savenko $^{1}$

${ }^{1}$ Gastrointestinal surgery, O.O. Shalimov National Institute of Surgery and Transplantation, National Academ, KYIV, Ukraine; ${ }^{2}$ ICU, O.O. Shalimov National Institute of Surgery and Transplantation, National Academ, KYIV, Ukraine

Introduction: Reconstruction after esophagectomy together with the reliability of the esophagus-gastric anastomosis (EGA) constitute some of the most important problems in the surgery of the esophagus, because anastomosis leakage is one of the main reasons of lethal surgery.

Material and methods: The research included 60 patients who anderwent the minimally invasive hybrid Ivor Lewis procedure for malignant tumors of esophagus in Shalimov National Institute of Surgery and Transplantation (1 group comprised 30 patients with mechanical EGA end-to-side; 2 group comprised 30 patients with invaginated mechanical EGA).

The objects for estimation included post-operative mortality as well as the number of post-operative anastomosis complications: the number of EGA leakages in the early post-operative period and the number of post operative strictures in the EGA area three, six and twelve months after the surgery.

All patients underwent a course of neoadjuvant chemo/RT in accordance with NCCN guidelines.

Data processing was done by means of Statistic-licensed application programs for Windows.

Results and discussion: Patients belonging to both groups were comparable according to their age, sex, weight, height and surgery duration. Research group did not register a single case of EGA leakage while 2 patients in control group $(?<0,05)$. had the leakage which was stopped by means of 'Endovac' system. There were 2 cases of esophagus postoperative strictures which developed 3 months after the surgery in the research group which was less than in the control group which saw 6 cases of strictures of EGA $(?<0,05) .6$ months after surgery, the number of post-operative strictures increased in both groups, but was lower in the research group and amounted to 4 cases in the research group and 11 cases in the control group $(?<0,05)$. There were 5 cases of esophagus postoperative strictures which developed 12 months after the surgery in the research group which was less than in the control group which saw 13 cases of strictures of EGA $(?<0,05)$. Neither of the groups had any cases of post-operative mortality.

Conclusion: The study found that invaginated mechanical EGA within minimally invasive hybrid Ivor Lewis procedure reduces EGA leakages as well as EGA benign strictures. 


\section{O163-UPPER GI-Gastric cancer}

\section{IS THREE-DIMENSIONAL LAPAROSCOPIC SPLEEN PRESERVING SPLENIC HILAR LYMPHADENECTOMY BETTER THAN THAT OF TWO-DIMENSIONAL?}

C. Huang ${ }^{1}$, Q. Chen ${ }^{1}$, Z. Liu ${ }^{1}$, Q. Zhong ${ }^{1}$, C. Zheng ${ }^{1}$, P. Li ${ }^{1}$, J. Xie ${ }^{1}$, J. Wang ${ }^{1}$, J. Lin ${ }^{1}$, J. Lu ${ }^{1}$, L. Cao ${ }^{1}$, M. Lin ${ }^{1}$, R. Tu' ${ }^{2}$ Z. Huang ${ }^{1}$, J. Lin ${ }^{1}$

${ }^{1}$ Department of Gastric Surgery, Fujian Medical University Union Hospital, FUZHOU, China; ${ }^{2}$ Fujian Medical University Union Hospital, Fujian Medical University Union Hospital, FUZHOU, China

Background: Three-dimensional (3D) video systems for laparoscopy provide surgeons with additional information on spatial depth not found in two-dimensional (2D) video systems. Study Design: This study enrolled 156 spleen-preserving splenic hilar lymphadenectomy (LSPSHL) patients in a randomized controlled trial (ClinicalTrials.gov Identifier NCT02327481) at the department of gastric surgery at Fujian Medical University Union Hospital between January 2015 and April 2016. The short-term efficacies were compared between the treatment groups. The unedited videos of 80 LSPSHL (40 procedures each for $3 \mathrm{D}$ and $2 \mathrm{D}$ ) were rated for technical performance using the Generic Error Rating Tool.

Results: All patients in the cohort were successfully treated without conversion to open surgery. The data for 156 LSPSHL patients indicate the estimated blood loss (EBL) (3D vs $2 \mathrm{D}=66.3 \pm 79.3$ vs $99.0 \pm 119.7, \mathrm{P}=0.046)$ was significantly less in the $3 \mathrm{D}$ group. The postoperative recovery and complication rates were similar $(\mathrm{P}>0.05)$. There were no patient deaths within 30 days of surgery. Two observers analyzed 80 videos of LSPSHL. The results showed there were fewer grasping-errors made in the $3 \mathrm{D}$ group than in the $2 \mathrm{D}$ group when dissecting the inferior pole region of spleen (IPRS) $(\mathrm{P}=0.016)$ and the superior pole region of spleen (SPRS) ( $\mathrm{P}=0.022$ ). Additionally, the inter-rater reliability was high regarding graspingerrors in the IPRS (intraclass correlation coefficient (ICC) 0.92) and the SPRS (ICC 0.83). The ICC for the total number of errors was 0.82 . The mean of errors in the 3D group (3D vs $2 \mathrm{D}=20.4 \pm 4.2$ vs $23.3 \pm 6.3, \mathrm{P}=0.023$ ) was less than the $2 \mathrm{D}$ group.

Conclusions: Compared with 2D LSPSHL, 3D technology reduces EBL and technical errors during splenic hilar dissection.

\section{O164-UPPER GI-Gastric cancer}

\section{PROSPECTIVE RANDOMIZED CONTROLLED TRIAL FOR COMPARISON OF 2-DIMENSIONAL VERSUS 3-DIMENSIONAL LAPAROSCOPIC GASTRECTOMY FOR GASTRIC ADENOCARCINOMA}

\section{K.H. Lee, Y.S. Jo, S.H. Min, S.H. Ahn, D.J. Park, H.H. Kim, Y.S. Park}

Department of surgery, Seoul National Bundang Hospital, SEONGNAM-SI, Korea

Background: Due to the technological advance in resolution and stereoscopic depth, the 3-dimensional (3D) laparoscopic system has been widely used in real surgery. However, there have been few studies to confirm the clinical usefulness of the 3D laparoscopic gastrectomy. This study aimed to compare perioperative outcomes between the 2-dimensional (2D) and 3D laparoscopic gastrectomy for gastric cancer patients.

Method: This was a prospective, randomized controlled, single-center, and superiority trial. Patients with histologically confirmed gastric adenocarcinoma and $=$ cT4a tumor were randomly assigned (1:1) to the $2 \mathrm{D}$ or $3 \mathrm{D}$ group. The primary endpoint was the operation time. Secondary endpoints included estimated blood loss (EBL), amount of blood transfusion, number of retrieved lymph nodes, postoperative mortality, and open conversion rate.

Results: Between October 20, 2016 and August 8, 2018, 84 patients were randomly assigned to the $2 \mathrm{D}$ (44 patients) or 3D (40 patients) group. The operation time was significantly shorter in the $3 \mathrm{D}$ group $(128.2 \pm 4.5 \mathrm{~min}$ in $2 \mathrm{D}$ vs. $106.8 \pm 6.0 \mathrm{~min}$ in $3 \mathrm{D}, \mathrm{P}=0.005)$, so the study was closed on the basis of futility. Especially, the lymph node dissection time of station 6 was significantly shorter in the $3 \mathrm{D}$ group $(37.3 \pm 2.5 \mathrm{~min}$ vs. $26.9 \pm 1.8 \mathrm{~min}, \mathrm{P}=0.001)$. EBL $(26.4 \pm 3.5 \mathrm{~mL}$ vs. $19.8 \pm 2.9 \mathrm{~mL}, \mathrm{P}=0.16)$, and the number of retrieved lymph nodes $(61.1 \pm 3.6 \mathrm{vs}$. $62.7 \pm 3.0, \mathrm{P}=0.74$ ) were not significantly different between two groups. There was no blood transfusion and mortality in both groups, but one open conversion (due to tumor adhesion to pancreatic body) occurred in the 2D group.

Conclusion: The results of this prospective trial supported that the clinical usefulness and safety of 3D laparoscopic gastrectomy for gastric adenocarcinoma.

\section{O165-UPPER GI-Gastric cancer}

DEFINING AND PREVENTING EARLY RECURRENCE IN PATIENTS WITH GASTRIC CANCER AFTER RADICAL GASTRECTOMY:USING RANDOMIZED PHASE III TRIAL DATA

C. Huang ${ }^{1}$, J. $\mathrm{Lu}^{1}$, B. Xu ${ }^{1}$, C. Zheng ${ }^{1}$, P. $\mathrm{Li}^{1}, \mathrm{~J} . \mathrm{Xie}^{1}, \mathrm{~J} . \mathrm{Wang}{ }^{1}$, ${ }^{1}$ Department of Gastric Surgery, Fujian Medical University Union Hospital, FUZHOU, China; ${ }^{2}$ Fujian Medical University Union Hospital, FUZHOU, China

Background: Previous studies have shown that early recurrence (ER) after radical surgery significantly reduces long-term survival in patients with cancer. Currently, the definition and predictors of ER for patients with gastric cancer (GC) after radical gastrectomy are unclear. Objective: To establish an evidence-based cutoff to differentiate between ER and late recurrence (LR) and to explore the predictors for ER.

Methods: A total of 419 gastric cancer patients in a clinical trial (NCT02327481) from January 2015 to April 2016 were analyzed. A minimum P-value approach was used to evaluate the optimal cut-off value of recurrence-free survival to divide the patients into ER and LR cohorts based on subsequent prognosis. Potential risk factors for ER were assessed with Cox proportional hazards regression model. We performed decision curve analysis to evaluate the clinical utility of prediction model by calculating its net benefit in varied risk thresholds for screening.

Results: Ultimately, 401 patients were included this study, and the median follow-up time was 29 (3-41) months. The optimal length of recurrence-free survival to distinguish between ER $(n=44)$ and LR $(n=52)$ was 12 months. The median post-recurrence survival time of ER group and LR group was 3.5 months and 9.5 months, respectively, $\mathrm{p}<0.001$. Factors associated with ER included a C-reactive protein-albumin ratio $(\mathrm{CAR})=0.131$, stage III and postoperative adjuvant chemotherapy $(\mathrm{PAC})>3$ cycles. The decision curve analysis yielded a range of risk thresholds (1-70\%) at which the clinical net benefit of the risk model consisting of CAR and TNM stage was larger than that of only TNM stage. Further stratification analysis of phase III patients found for patients with CAR $<0.131$, PAC with 1-3 cycles $(50.0 \%$ vs $17.3 \%, \mathrm{p}=0.029)$ or $>3$ cycles $(50.0 \%$ vs $4.2 \%, \mathrm{p}<0.001)$ could significantly reduce the risk of ER. However, for patients with BMI $=0.131$, only PAC $>3$ cycles $(54.2 \%$ vs $16.0 \%, \mathrm{p}=0.004)$ rather than $1-3$ cycle $(58.3 \%$ vs $54.2 \%, \mathrm{p}=0.824)$ can benefit them.

Conclusions: A recurrence-free interval of 12 months is the optimal threshold for differentiating between ER and LR. Stage III GC patients with CAR $=0.131$ may not benefit from PAC with only $1-3$ cycles.

\section{O166-UPPER GI-Gastric cancer}

\section{ROBOTIC SPLEEN-PRESERVING SPLENIC HILAR LYMPHADENECTOMY FOR ADVANCED PROXIMAL GASTRIC CANCER:A FEASIBLE AND SIMPLIFIED PROCEDURE}

C. Huang, Q. Chen, Q. Zhong, C. Zheng, P. Li, J. Xie, J. Wang, J. Lin, J. Lu, L. Cao

Department of Gastric Surgery, Fujian Medical University Union Hospital, FUZHOU, China

Background: Robotic systems recently have been introduced to overcome technical limitations of conventional laparoscopic gastrectomy, especially for complex procedures.[\#_ENREF_1] We developed a set of procedural operation steps for robotic spleen-preserving splenic hilar lymphadenectomy, which is difficult and recommended in D2 lymph node (LN) dissection during total gastrectomy [\#_ENREF_2].

Methods: The robotic operative procedures of splenic hilar lymphadenectomy using the da Vinci (B) Si system were demonstrated in a step-by-step manner, with technical tips for each step, in the video clip. The above procedures were performed on 40 consecutive patients with stage cT2-3 proximal gastric cancer between July 2016 and September 2017. The learning curve was analyzed based on the cumulative sum method (CUSUM).

Results: The mean age and body mass index of patients were $55.3 \pm 10.4$ years (range 29-78) and $23.0 \pm 2.7 \mathrm{~kg} / \mathrm{m}^{2}$ (range 15.4-28.4), respectively. All spleen-preserving surgeries were successfully performed without open or laparoscopy conversion. Mean operation time of splenic hilar lymphadenectomy was $20.3 \pm 6.4 \mathrm{~min}$ (range 13.3-46.3); mean blood loss was $13.7 \pm 5.3 \mathrm{ml}$ (range 8.0-40.0). The overall average of $38.8 \pm 13.1 \mathrm{LNs}$ (range 19-81) was retrieved, including a mean $3.3 \pm 1.4$ (range $0-8)$ splenic hilar area LNs, with a $10 \%(4 / 40)$ metastatic rate. No immediate postoperative mortality was observed. 6 patients $(15.0 \%)$ experienced a complication after surgery; the operation-related complications consisted of one wound complications, one abdominal infection, and one anastomosis leakage. At a median follow-up of 12 months, one patient had experienced lung metastasis. According to the CUSUM, the cut-of point of splenic hilar LN dissection time and blood loss were 15th and 20th cases, respectively. Conclusion: Robotic surgery can improve the quality of surgery and promote the D2 LN dissection. This procedure is feasible and simplifies complicated splenic hilar lymphadenectomy. 


\section{O167-UPPER GI-Gastric cancer}

\section{LAPAROSCOPIC GASTRECTOMY AND METASTASECTOMY FOR STAGE 4 GASTRIC CANCER}

\section{S.H. Min}

Department of Surgery, Seoul National University Bundang Hospital, SEUNGNAM-SI, Korea

Background: This study was performed to analyze the safety and feasibility of laparoscopic gastrectomy in stage 4 gastric cancer patients by comparing with open surgery, matched with propensity scoring method and to investigate the role of maximum resection of primary gastric cancer and metastasectomy.

Methods: A total of 547 patients were pathologically proven stage 4 gastric cancer. 152 patients with proper surgery were eligible for analysis. After propensity score matching, a total of 78 patients were assigned to laparoscopy and open group.

Results: There were no significant differences between the laparoscopy and open group except for operation time and estimated blood loss. Complication rate did not show statistical significance. The 5-year survival rate was $18.6 \%$ and the median survival was 17.8 months. When compared between laparoscopy and open group, there was no difference between the two groups (23.1\% 19.7 months, vs. $21.7 \% 17.1$ months, $\mathrm{P}=.808)$. In the multivariate analysis of prognostic factor for overall survival, adjuvant chemotherapy and postoperative complication were independent prognostic factors. With REGATTA inclusion criteria applied to our data, the 5-year survival, 2-year survival and median survival was $27.8 \%, 55.6 \%$, and 26.8 months $(95 \% \mathrm{CI}$ $36.7-84.0$ ), respectively. The median ratio(MR) of the median survival between surgery plus chemotherapy group in the REGATTA study and our study was 1.874 , in favor of our study. Conclusions: Laparoscopic gastrectomy and metastasectomy in stage 4 gastric cancer patients is safe and feasible in terms of long-term oncologic outcome and complications. Maximum resection of primary gastric cancer and metastasectomy should be tried for better long-term oncologic outcome.

\section{O168-UPPER GI-Gastric cancer}

\section{NOMOGRAMS FOR PRE- AND POSTOPERATIVE PREDICTION OF LONG-TERM SURVIVAL FOR PROXIMAL GASTRIC CANCER PATIENTS}

C. Huang, Q. Chen, Q. Zhong, Z. Liu, M. Lin, C. Zheng, P. Li, J. Xie, J. Wang, J. Lin, J. Lu, L. Cao

Department of Gastric Surgery, Fujian Medical University Union Hospital, FUZHOU, China

Purpose: To develop nomograms for predicting long-term survival for proximal gastric cancer (GC) patients.

Background: The incidence of proximal GC is increasing, and methods or predicting the longterm survival of proximal GC patients have not been well established.

Methods: Between January 2007 and June 2013, we prospectively collected and retrospectively analyzed the medical records of 746 patients with proximal GC. The data were split $75 / 25$, with one group used for model development and the other group used for validation testing. A Cox regression analysis was used to identify the preoperative and postoperative risk factors for overall survival (OS).

Result: Among the 746 patients examined, the 3- and 5-year OS rates were $66.1 \%$ and $58.4 \%$, respectively. For the training set, the preoperative T stage (cT), N stage (cN), CA19-9, tumor size, ASA core, and 3-6-month weight loss were incorporated into the preoperative nomogram for predicting OS. In addition to these variables, LVI and the postoperative tumor size, T stage, $\mathrm{N}$ stage, blood transfusions and complications were incorporated into the postoperative nomogram. All the calibration curves for OS probability fitted well. In the training cohort, the preoperative nomogram achieved a C-index of 0.751 [95\% confidence interval (CI): $0.732-0.770]$ in predicting OS and accurately stratified patients into 4 prognostic subgroups (5-year OS rates: $86.8 \%$, $73.0 \%, 43.72 \%$ and $20.9 \%, \mathrm{P}<0.001)$. The postoperative nomogram had a $\mathrm{C}$-index of 0.758 in predicting OS and accurately stratified patients into 4 prognostic subgroups (5-year OS rates: $82.6 \%, 74.3 \%, 45.9 \%$ and $18.9 \%, \mathrm{P}<0.001$.

Conclusions: The nomograms accurately predicted the pre- and postoperative long-term survival of proximal GC patients.

\section{O169-UPPER GI-Gastric cancer}

FACTORS DETERMINING LENGHT OF HOSPITAL STAY FOLLOWING LAPAROSCOPIC TOTAL D2 GASTRECTOMY COMBINED WITH ERAS PROTOCOL

M. Pisarska ${ }^{1}$, M. Wierdak ${ }^{1}$, M. Dec ${ }^{1}$, M. Chrusciel $^{2}$, J. Kulawik ${ }^{1}$, P. Major ${ }^{1}$, A. Budzynski $^{1}$, M. Pedziwiatr ${ }^{1}$

${ }^{1}$ 2nd Department of General Surgery, Jagiellonian University Medical College, KRAKOW, Poland; ${ }^{2}$ Department of Endoscopic, Metabolic and Soft Tissue Tumor Surgery, University Hospital Krakow, KRAKOW, Poland

Aim: To determine the factors affecting length of hospital stay (LOS) in patient after laparoscopic total D2 gastrectomy (LTG) combined with ERAS protocol

Methods: AND PROCEDURES: The study enrolled 90 patients ( 60 men and 30 women, mean age $62.2 \pm 11.8$ years) who underwent elective LTG due to gastric adenocarcinoma between 2015 and 2017. Demographic and surgical parameters, compliance with ERAS protocol, recovery parameters, postoperative complications and readmissions were analyzed. Discharge from hospital after more than median hospital stay was considered as prolonged LOS (primary length of stay after surgery, excluding readmissions). We evaluated factors that potentially may influence LOS . Logistic regression models were used in univariate and corrected multivariate analyses, in order to identify the factors related to prolonged LOS.

Results: The median LOS after LTG in the studied group was 5 (4-8) days. 41 patients $(45.5 \%)$ required prolonged hospitalization. Univariate logistic regression showed that the following factors were related to prolonged LOS: Peritoneal drainage on the first postoperative day (POD1), Prolonged ( $>24 \mathrm{~h}$ ) catheterisation, No tolerating oral diet on POD-1 and postoperative complications, Multivariate logistic regression showed that only complications $(\mathrm{OR}=3.72 ; 95 \%$ CI: $1.15-12.03)$, No tolerating oral diet on POD-1 (OR $=6.44$; 95\% CI: 2.22-18.66), and Presence for peritoneal drainage on $1 \mathrm{POD}(\mathrm{OR}=4.06$; $95 \%$ CI: $1.26-13.14)$, prolonged LOS. Conclusions: Prolonged LOS following LTG with ERAS Protocol is strongly associated with the presence of postoperative complications, need for prolonged peritoneal drainage, and no tolerance of oral diet on POD-1. In our analysis, neither demographic nor operational factors had an impact on prolonged hospitalization.

\section{O170-UPPER GI-Gastric cancer}

\section{LAPAROSCOPIC SURGERY IN ADVANCED GASTRIC CANCER COMBINED WITH HIGH PRESSURE AEROSOL CHEMOTHERAPY FOR THE TREATMENT OF PERITONEAL CARCINOMATOSIS}

\section{N. Belev}

Surgical Department, Eurohospital, PLOVDIV, Bulgaria

Aim: Our aim is to established the effectiveness of combined radical laparoscopic gastric surgery with PIPAC in advanced gastric cancer for the prevention and treatment of PC.Object.Laparoscopic gastrectomy show better postoperative results in comparison to open surgery while having the same post surgical oncologic results. Peritoneal spread in gastric cancer is generally observed in 40 to $50 \%$ of the patients. Pressurized intraperitoneal aeorosol chemotherapy (PIPAC) is based on breaking up chemotherapy agents into particles of 10-15 microns and delivering them in a concentration of $10 \%$ of the systemic dose under pressure of $15 \mathrm{mmHg}$ for $30 \mathrm{~min}$. Prevention and therapy of $\mathrm{PC}$ is the main indication for PIPAC which have promising initial reults.

Material and methods: Patients with resectable advanced gastric cancer with high risk of PC are candidates for this study. In prospective 12 patients had laparoscopic gastrectomy ( 7 total and 5 subtotal) with D2 lymph node dissection. After finishing the reconstructive stage we applied PIPAC for $30 \mathrm{~min}$. This procedure was repeated after 30 days for a total of 3 times. We analyzed retrospectively 14 laparoscopic gastrectomies for a past period of 1 year (poorly differentiated, diffuse type) actively searching for PC

Results: Two postoperative complications $(16.6 \%)$ were established in the laparoscopic surgery group with PIPAC (internal pancreatic fistula, oesophago-jejuno anastomotic leak) and $3 \mathrm{com}$ plications $(21,4 \%)$ in the retrospective group (internal pancreatic fistula, duodenal stump leak, oesophago-jejuno anastomotic leak, all Clavien-Dindo-II). In 8(57\%) of the retrospective patients PC, was established at the end of the first year after surgery. Three of the patients in the aerosol group have reached the end of the first year with no data supporting PC, the others remain to be analyzed.

Conclusion: PIPAC has better pharmacological capabilities compared to HIPEC and systemic chemotherapy for the treatment of PC as in vitro and animal studies and clinical data support this statement. RCT are needed for complete and exact validation of this method. 


\section{O171-UPPER GI-Gastric cancer}

DOES UNTIMELY CHEMOTHERAPY AFTER RADICAL GASTRECTOMY AFFECT THE PROGNOSIS OF PATIENTS WITH II/III GASTRIC CANCER: CAN WE DO BETTER?

C. Huang, Q. Chen, Z. Liu, Q. Zhong, C. Zheng, P. Li, J. Xie, J. Wang, J. Lin, J. Lu, L. Cao

Department of Gastric Surgery, Fujian Medical University Union Hospital, FUZHOU, China

Purpose: To investigate the prognostic effects and risk factors of the omission and delay of postoperative chemotherapy of II/III gastric cancer (GC), with the goal of providing a reference for interventions of related departments.

Methods: The clinicopathological data of 1520 patients undergoing radical gastrectomy for II/III GC were collected and retrospectively analyzed. We defined the chemotherapy delayed until more than 60 days after radical gastrectomy and the complete omission of chemotherapy as unacceptable chemotherapy initiation (UAC group), while the chemotherapy conducted within 60 days of radical gastrectomy was defined as acceptable chemotherapy initiation (AC group). The survival between the two groups was compared, and the trends and risk factors of UAC were analyzed.

Results: The OS and DFS of the UAC group patients were significantly inferior to those in the AC group $(\mathrm{p}<0.001)$. The OS and DFS of the patients in UAC group were close to those of the patients without chemotherapy $(\mathrm{p}>0.05)$. Cox multivariate analysis demonstrated that UAC is an independent predictor of OS $(\mathrm{p}<0.05)$. Logistic analysis showed female sex, old age, a selfpaid status, a very low social status, a high ASA score, an intra-abdominal surgery history, and serious postoperative complications were independent risk factors of UAC $(\mathrm{p}<0.05)$. The radar chart shows the risk factors of UAC changed with time: the number of self-funded patients with an extremely low social status gradually decreased from 2011 to 2014 (0-3.2\%); however, it increased again in $2015(4.5 \%)$

Conclusion: UAC after radical gastrectomy is an independent risk factor for the prognosis of II/ III stage GC patients. However, no significant decline of UAC has been achieved recently and should call for the attention of both government and clinicians.

\section{O172-UPPER GI-Gastric cancer}

\section{UNCUT ROUX-EN-Y GASTROJEJUNOSTOMY IN TOTALLY LAPAROSCOPICDISTALGASTRECTOMYFOR GASTRICCANCER:TEN YEARS OF SINGLECENTEREXPERIENCE}

\section{C.H. Kim,}

Upper GI surgery, The catholic University of Korea, Incheon St. Mary's hospital, INCHEON CITY, Korea

Background: The aim of this study is to present our ten years of experience in terms of the evolution of surgical procedures and short and long term clinical outcomes of totally laparoscopic distal gastrectomy with uncut Roux-en-Y in comparison with Billroth-I technique.

Study Design: We retrospectively reviewed the medical records of 458 patients who underwent totally laparoscopic distal gastrectomy with uncut Roux-en-Y and Delta-shaped Billroth-I anastomosis from December 2004 to December 2014 at the Department of Surgery, Incheon St. Mary's Hospital, College of Medicine, The Catholic University of Korea. The evaluated parameters included patients and tumor characteristics, operative details, postoperative complications and endoscopic findings of the gastric remnant at postoperative 1 and 5 years.

Results: The total number of patients who underwent totally laparoscopic distal gastrectomy with uncut Roux-en-Y and Delta shaped Billroth-I anastomosis was 244 and 214, respectively. The mean reconstruction time was longer in uncut Roux-en-Y than in Delta shaped Billroth-I, $(30.5 \pm 14.5$ vs. $13.6 \pm 10.3 \mathrm{~min}, p<0.001)$. The uncut Roux-en-Y was used more cartridge than Delta shaped Billroth-I anastomosis $(6.9 \pm 1.2$ vs. $6.2 \pm 1.0, p<0.001)$. However there was no significant differences in operation time, estimate blood loss, number of retrieved lymph node and postoperative course between reconstruction methods. Postoperative complications more than Clavien-Dindo grade IIIa occurred in 22 cases (4.8\%) of postoperative early complications and 14 cases $(3.1 \%)$ of late complications. The endoscopic findings showed excellent short and long-term outcomes in terms of very low incidence of bile reflux and reflux-induced remnant gastritis in uncut Roux-en-Y compared with Delta shaped Billroth-I anastomosis. Conclusions: Uncut Roux-en-Y gastrojejunostomy was a useful reconstruction method with totally laparoscopic distal gastrectomy for cancer, especially for diverting enteral contents from the remnant stomach and preventing remnant gastritis. Therefore, it is recommended for young patients with early stage disease who have a long time to live after distal gastrectomy for cancer. Key words: uncut Roux-en-Y, Billroth-I, Totally laparoscopic surgery, Stomach

\section{O173-UPPER GI-Gastric cancer}

INDICATIONS FOR ADJUVANT CHEMOTHERAPY IN PATIENTS WITH AJCC (7TH ED.) STAGE IIA T3NOM0 AND T1N2M0 GASTRIC CANCER-AN EAST AND WEST, MULTICENTER STUDY

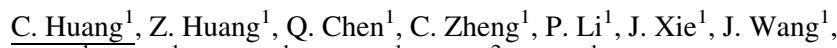

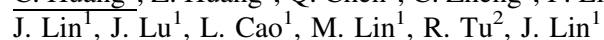

${ }^{1}$ Department of Gastric Surgery, Fujian Medical University Union Hospital, FUZHOU, China; ${ }^{2}$ Fujian Medical University Union Hospital, FUZHOU, China

Purpose: To determine the ideal indications of adjuvant chemotherapy(AC) in patients with stage IIa gastric cancer (T3NOM0 and T1N2M0) according to the $7^{\text {th }}$ American Joint Committee on Cancer (AJCC).

Method: 1593 gastric cancer patients with T3N0M0 or T1N2M0 stage were identified from Surveillance, Epidemiology,and End results (SEER) database in 1988.1-2012.12. Cox multiple regression, nomogram and decision curve was used.External validation was performed using database from Fujian Medical University Union Hospital $($ FJUUH $)(n=241)$ and Italy IMIGASTRIC center $(n=45)$.

Results: Cox multiple regression analysis showed that the risk factors that affected OS in Patients with $\mathrm{AC}$ were age $>65$ years old, T1N2M0, LN dissection number $=15$, tumor size $>20 \mathrm{~cm}$, and non-adenocarcinoma. Then a nomogram was constructed to predict 5-year OS and divided patients into a high-benefit from AC (points $=188$ ) or a low-benefit (points $>188$ ) according to a recursive partitioning analysis.OS was significantly higher for the high-benefit patients in the SEER database and the FJUUH dataset than in the non-AC patients (Log-rank $<0.05)$, and there was no significant difference in OS between low-benefit patients and non-AC patients among the three centers $(\log -\mathrm{rank}=0.154,0.470$, and 0.434 , respectively). The decision curve indicated that the best clinical effect can be obtained when the threshold probability is $0-92 \%$.

Conclusion: Regarding the controversy over whether T3N0M0 and T1N2M0 gastric cancer patients should be treated with AC, this study presents a predictive model that provides concise and accurate indications. These data show that high-benefit patients should receive AC.

\section{O174-UPPER GI-Gastroduodenal diseases}

\section{CLEAN-NET -A MODIFIED LAPAROSCOPIC WEDGE RESECTION TO AVOID EXCESSIVE SACRIFICE OF HEALTHY STOMACH}

E. Kanehira, Takashi Tanida, Kodai Takahashi, Yuichi Obana, Aya Kamei Kanehira, Kazunori Sasaki

Department of Surgery, Medical Topia Soka, SOKA, Japan

Background: To avoid excessive sacrifice of the tissue surrounding the gastric SMT (submucosal tumor) in stapling wedge resection, we carry out CLEAN-NET (combined laparoscopic and endoscopic approach for neoplasia with a nonexposure technique). We describe the operative technique of CLEAN-NET and demonstrate its clinical results.

Operative technique: The seromuscular layer above the tumor is dissected, while the mucosa is kept unbroken. When seromuscular layer is dissected all around the tumor, the full layer is lifted, and the mucosa is stretched. The mucosa is then transected with a stapling device to execute fullthickness resection of the specimen. Finally, the seromuscular defect is repaired by hand-sewn suture.

Results: Since December 2015, CLEAN-NET has been performed in 57 patients with gastric SMTs. All tumors were resected en-blocwithout rupture. The average operation time ranged from 50 to $220 \mathrm{~min}$ with an average of $101.7 \mathrm{~min}$. The postoperative course was uneventful. Microscopically the surgical margin was tumor-negative (R0 resection) in all cases. The margin width was small with an average of $5.4 \mathrm{~mm} \pm 2.5$.

Conclusions: CLEAN-NET is a useful option in the laparoscopic surgical treatment of gastric SMT, when excessive sacrifice of the healthy gastric wall surrounding the endophytic tumor should be avoided. 


\section{O175-UPPER GI-Reflux-Achalasia}

\section{REFLUXSTOP $^{\mathrm{TM}}$, A DEVICE FOR A POTENTIAL PARADIGM SHIFT IN ANTI-REFLUX SURGERY, KEEPING LES IN THE ABDOMEN WITHOUT COMPRESSING THE FOOD PASSAGEWAY}

\section{Forsell, M. Bjelovic}

Department of Minimally invasive Upper Digestive Surgery, University Clinic for Digestive Surgery, BELGRAD, Serbia

Aim: For the past 40 years prevailing surgical acid-reflux treatment has focused on supporting the LES, mainly performed by gold standard Nissen Fundoplication, where a wrap is formed around and compresses the LES. This procedure has disadvantages such as dysphagia, inability to burp and vomit, and long-term dystrophy of the wrap which becomes thin, inelastic, and fibrotic. Our aim was to; develop a new treatment for acid reflux which does not affect the food passageway, as well as avoid increasing reuse of PPI's long-term caused by the fibrotic degeneration of the fundus wrap.

Methods: A new device, RefluxStop ${ }^{\mathrm{TM}}$, is placed invaginated in the fundus to keep LES down in the abdomen and treats acid-reflux without affecting the food passageway.

In a multicenter clinical trial with 50 patients, the one-year results are presented using GERD$\mathrm{HRQL}$ as a screening tool and 24-hour $\mathrm{pH}$ monitoring at failure, defined as $<50 \%$ improvement since baseline in the GERD-HRQL score.

Results: The clinical results support a potential paradigm shift in the treatment of acid-reflux The GERD-HQRL score improved from 29 to 4 at 1 year, and the side effects relating to the compression of the food passageway disappear. The half-year surgical control 24-hour $\mathrm{pH}$ monitoring (total time $\mathrm{pH}<4$ ) reduced from $16.3 \%$ to $0.85 \%$. No ADE (adverse device events) occurred and no devices were explanted.

Conclusion(s): These results support that existing reflux treatments are based on the wrong assumption: the LES does not need to be supported and/or compressed to treat acid-reflux. Compression of the food passageway causes well-known side effects, and the result also indicates an improved efficacy compared to a literature review and meta-analysis on Nissen fundoplication.

\section{O176-UPPER GI-Reflux-Achalasia}

\section{AGE-RELATED OUTCOMES IN LAPAROSCOPIC HIATAL HERNIA REPAIR: IS THERE AN AGE LIMIT FOR ANTIREFLUX SURGERY?}

A. Addo, A. Park, A.S. Weltz, Z. Sanford, H.R. Zahiri,

R. Lu, A. Borda

General Surgery, Anne Arundel Medical Center, ANNAPOLIS, United States of America

Aim /Background: Minimally invasive antireflux surgery has been shown to be safe and effective for the treatment of gastroesophageal reflux (GERD) in elderly patients. However, there is a paucity of data on the influence of advanced age on long-term quality of life (QoL) and perioperative outcomes after laparoscopic antireflux surgery (LARS).

Method: A retrospective study of patients undergoing laparoscopic fundoplication for GERD or as a component of hiatal hernia surgery between February 2012 and June 2018 at a single institution was conducted. Patients were divided into four age categories, $<50</ \mathrm{i}>, 50-65<$ $\mathrm{i}>, 65-75</ \mathrm{i}>,=75<\mathrm{i}>$. Perioperative data and quality of life (QOL) outcomes were collected and analyzed. QoL information was obtained using the following four validated survey instruments: the Reflux Symptom Index, Gastroesophageal Reflux Disease-Health Related Quality of Life, Laryngopharyngeal Reflux QOL, and Swallowing QOL questionnaires. Results: A total of 492 patients $[<50(\mathrm{n}=</ \mathrm{i}>75), 50-65(\mathrm{n}=</ \mathrm{i}>179), 65-75(\mathrm{n}=<$ $\mathrm{i}>144),=75(\mathrm{n}=\langle/ \mathrm{i}>94)]$ with mean follow-up of 21 months were included in the final analysis. Advancing age was associated with increased likelihood of comorbid disease, including hypertension (OR 1.93, $\mathrm{p}<</ \mathrm{i}>.001$ ), dyslipidemia (OR 1.36, $\mathrm{p}=<\mathrm{i}>.001$ ), coronary artery disease (OR 1.70, $\mathrm{p}<</ \mathrm{i}>.005)$, arrhythmia $(\mathrm{OR}=3.23, \mathrm{p}=</ \mathrm{i}>.010)$, COPD (OR 3.00,p $<</ \mathrm{i}>.001)$, and renal disease (OR $1.97, \mathrm{p}=<\mathrm{i}>.010)$. Older patients were significantly more likely to present with advanced disease requiring Collis gastroplasty (OR 2.09) or concurrent gastrostomy tube placement (OR 3.20). Accordingly, older surgical patients required increased operative time (B 6.29, p < < $\mathrm{i}>.001)$, length of hospital stay $(B 0.56, \mathrm{p}<</ \mathrm{i}>.001)$ and increased likelihood of intraoperative complications (OR 2.94, $\mathrm{p}=<\mathrm{i}>.003$ ) and reoperations (OR 2.36, $\mathrm{p}<.05$ ). However, postoperative complication rates were parallel among all age groups. Additionally, patient-reported QoL outcomes and postoperative satisfaction trended favorably across all age groups.

Conclusions: Among older patients, there is a greater risk of intraoperative complications, reoperation rates as well as longer operative time and LOS after LARS, supporting more advanced disease. However, a long-term QoL benefit is demonstrated among elderly patients who have undergone this procedure parallel to younger groups. Rather than serving as an exclusion criterion for surgical intervention, advanced age among patients with pathologic reflux should not deter rendering therapy to this group. Enhanced outcomes are achieved through efforts towards preoperative optimization and utilization of advanced technical approaches.

\section{O177-UPPER GI-Reflux-Achalasia}

COMPLETE VS. PARTIAL FUNDOPLICATIONA COMPARATIVE MEASUREMENT OF FUNCTIONALITY AND EFFECTIVENESS UNDER LABORATORY CONDITIONS

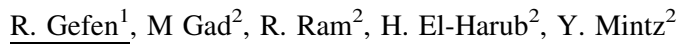

${ }^{1}$ General surgery, Hadassah Hebrew University Medical Center, JERUSALEM, Israel; ${ }^{2}$ General surgery, Hadassah medical center, JERUSALEM, Israel

Background: The type of fundoplication—complete or partial is still controversial for the surgical treatment of GERD. Laparoscopic Toupet $\left(270^{\circ}\right.$ wrap) fundoplication has less post op dysphagia and gas bloating compared to Nissen fundoplication $\left(360^{\circ}\right.$ wrap) and is advised to be the procedure of choice when esophageal manometry findings are abnormal, however it is considered by some less effective and more difficult to perform. The aim of this research was to determine in the functionality and efficacy of the different types of fundoplication

Methods: Explanted pigs stomachs weighing $45-60 \mathrm{Kg}$ were studied. Two different studies of the LES were performed: distensibility and failure point (occurrence of reflux according to volume added to the stomach). For both studies we first disrupted the lower esophageal sphincter using a Rigiflex ${ }^{\mathrm{TM}}$ dilating balloon. We then performed three different fundoplications- Nissen, Toupet, Dor and measured the distensibility of the EGJ after each fundoplication. The Failure point was determined following each fundoplication type.

Results: we used 12 pig stomachs for the distensibility study and 11 pig stomachs for the failure point study. There was no statistically significant difference between the Nissen and Toupe fundoplications when distensibility was measured, however the EGJ was more distensible following Dor fundoplication ( $\mathrm{p}=0.008$ for Nissen, 0.016 for Toupet). When the failure point was measured, Nissen fundoplication was significantly more effective than Toupet, and Toupet was significantly more effective than Dor $(\mathrm{p}=0.016, \mathrm{p}=0.017$ respectively)

Conclusions: We studied the differences between the mechanical effects on the EGJ following three different fundoplications, encompassing $360^{\circ}, 270^{\circ}$, and $180^{\circ}$ of the esophagus. We demonstrated that there is a significant difference between Dor fundoplication and Nissen/Toupe when distensibility was measured. There was no difference in the distensibility of the EGJ following a $360^{\circ}$ or $270^{\circ}$ wrap. There was, however a significant difference of effectiveness between all three fundoplications. These findings suggest that the $360^{\circ}$ and $270^{\circ}$ fundoplications have similar functionality while the $360^{\circ}$ wrap mechanically prevents possible reflux and suppor proponents of Toupet fundoplication rather than Nissen due to the similar functional results while decreasing the post op dysphagia and gas bloating complications. 


\section{O178-UPPER GI-Reflux-Achalasia}

THE USE OF ANTI-REFLUX MEDICATION AFTER ANTI-REFLUX SURGERY-A REGISTER-BASED COHORT STUDY

\section{J.S. Ljungdalh ${ }^{1}$, K.H. Rubin ${ }^{2}$, J. Durup ${ }^{3}$, K.C. Houlind ${ }^{4}$}

${ }^{1}$ Department of Surgery, Kolding Hospital, a part of Hospital Lillebaelt, KOLDING, Denmark; ${ }^{2}$ OPEN-Odense Patient Data Explorative Network, Department of Clinical Research, University of Southern Denmark and Odense University Hospital, Odense, Denmark, ODENSE, Denmark; ${ }^{3}$ Department of Surgery, Odense University Hospital, ODENSE, Denmark; ${ }^{4}$ Department of Vascular Surgery, Kolding Hospital, a part of Hospital Lillebaelt, KOLDING, Denmark

Aim: To describe patients undergoing surgical treatment of incident gastro-oesophageal reflux disease and the use of anti-reflux treatment in a Danish population-based cohort.

Methods: All adult Danes 2000-2015 undergoing upper endoscopy and receiving a diagnosis of GERD within 90 days were identified. Patients with previously diagnosed GERD, peptic ulcer-disease, Barrett's oesophagus or cancer of the gastrointestinal tract were excluded. In this study, only patients undergoing anti-reflux surgery within two years of GERD-diagnosis were subsequently included.

Age, sex, Charlson Comorbidity Index (CCI), anti-reflux surgery (primary and re-operative) and endoscopic dilatation were identified using the Danish National Patient Registry. Mortality was identified using the National Civil Registry. Pharmacological treatment of GERD (Proton pump inhibitors, $\mathrm{H}<$ su2 $<$ /su-blockers and other prescription anti-reflux drugs) as well as use of nonsteroid anti-inflammatory drugs (NSAID) and anti-thrombotic treatment were identified using theThe Danish National Prescription Registry. All data was linked on an individual level using the unique identification number that all Danish citizen are assigned to at birth or first immigration.

Results: A total of 674 first-time fundoplications were performed, hereof $98.1 \%$ performed laparoscopically $(\mathrm{n}=661)$ and $1.9 \%$ performed using open technique $(\mathrm{n}=13)$. At one-year followup, $4.9 \%(\mathrm{n}=33)$ had undergone endoscopic dilatation and $2.1 \%(\mathrm{n}=14)$ had undergone reoperation. The 90 -day mortality was $<0.5 \%$.

Patients had a median age of 46 years (18-80 years) and were predominately male $(57.9 \%-n=390)$. A total of $93.9 \%$ had CCI $0(n=633)$. Diagnoses were GERD with esophagitis $(66.9 \%-n=451)$, GERD without esophagitis $(31.5 \%, \mathrm{n}=212)$ and GERD without specification $(1.6 \%, \mathrm{n}=11)$.

Before initial endoscopy, $91,7 \%(n=618)$ used at least one type of anti-reflux drug, dropping to $32.2 \%$ $(\mathrm{n}=217)$ in the year after anti-reflux surgery. However, even when censoring patients with Barrett's esophagus or peptic ulcer disease after initial endoscopy and patients undergoing concomitant treatment with NSAIDs or antithrombotic drugs, $27.7 \%$ still used at least one type of anti-reflux drug after surgery. Conclusion: In this population-based study, anti-reflux surgery was safe and lowered the use of pharmacological treatment. However, even when adjusting for competing reasons for use of antireflux drugs, $27.7 \%$ used at least one type of anti-reflux drug one year after surgery.

\section{O179-UPPER GI-Reflux-Achalasia}

\section{THE NEW APPROACH TO PERFORM NISSEN} FUNDIPLICATION

\section{Paranyak, V. Grubnyk}

Surgery, Odessa national medical university, ODESSA, Ukraine

Nearly $10 \%$ of patients who undergo laparoscopic anti-reflux surgery at long-term follow-up need for surgical reintervention mostly because of hiatal hernia $(\mathrm{HH})$ recurrence, wrap migration or disruption. Purpose: The aim of our prospective study was to evaluate and compare several technics of wrap fixation and determine whether modified Nissen fundoplication (MNF) reduce failure rate in the long term follow up. Materials and methods: This was a prospective, randomized, controlled trial. From November 2012 to October 2014 one hundred and thirty-eight GERD patients who underwent anti-reflux surgery were divided into two groups. Excluded criteria for our study ware diagnosed hiatal hernia (HH) type III. In the I group which include 87 patients we performed the following manipulations: $\mathrm{NF}$ was supplemented with suturing wrap to the diaphragmatic crura (52 patients) on each side using two non-absorbable stitches. Such technique permit us to create more symmetrical wrap. In case of weak conditions of crura or short esophagus (35 patients) fundoplication wrap was sutured to the body of stomach using two non-absorbable stitches on each side. Control group (51 patients) underwent classic Nissen fundoplication (NF) without wrap fixation. All patients were assessed before and after surgery using validated symptoms and quality of life (GERD-HRQL) questionnaires, 24-h impedance-pH monitoring and barium-swallow.

Results: Baseline characteristics were similar between groups. There were no conversion to open procedure or mortality. Mean hospitalization was 2.7 days \pm 1.4 days. At 41,6 months (range 18--57) of followup, the overall rate of complications after MNF was $1,14 \%$ (1 HH reccurence) and NF 7,84\% (3 HH reccurence, 1 slipped wrap). Patient in MNF group show significant improvement in GERD-HRQL score, from $19.3 \pm 13.2$ (preoperatively) to $4.3 \pm 3.9$ (postoperatively) (p? < ?0.001). Complete PPI independence was achieved in $91 \%$. In the II group of patients mean GERD-HRQL score decline from $18.7 \pm 11.9$ (preoperatively) to $9.3 \pm 7.7$ (postoperatively), postoperative PPI treatment was necessary in $29 \%$. Conclusions: According to our study MNF minimized risk of slipped wrap and intrathoracic migration of the wrap and can make positive impact on reducing the failure rate of laparoscopic anti-reflux surgery.

\section{O180-UPPER GI-Reflux-Achalasia}

\section{NETWORK META-ANALYSIS OF LAPAROSCOPIC ANTIREFLUX PROCEDURES: A PROJECT OF THE EAES RESEARCH TALENT ACADEMY}

S.A. Antoniou ${ }^{1}$, A. Andreou ${ }^{2}$, D.I. Watson ${ }^{3}$, D. Mavridis ${ }^{4}$, N.K. Francis ${ }^{5}$, P. Chitsabesan $^{6}$

${ }^{1}$ Surgical Service, Royal Devon \& Exeter NHS Foundation Trust, EXETER, United Kingdom; ${ }^{2}$ Department of Colorectal Surgery, York Teaching Hospital NHS Foundation Trust, YORK, United Kingdom; ${ }^{3}$ Discipline of Surgery, College of Medicine and Public Health, Flinders University, ADELAIDE, Australia; ${ }^{4}$ School of Education, University of Ioannina, IOANNINA, Greece; ${ }^{5}$ Department of General Surgery, Yeovil District Hospital NHS Foundation Trust, YEOVIL, United Kingdom; ${ }^{6}$ Department of Surgery, York Teaching Hospital NHS Trust, YORK, United Kingdom

Aims: Comparative evidence across laparoscopic antireflux procedures does not exist. Aim of this project was to identify direct comparative evidence between laparoscopic antireflux procedures and synthesize evidence using network meta-analytical methods.

Methods: The databases of Medline, AMED, CENTRAL, OpenGrey were interrogated. Pairwise meta-analyses for each pair of interventions using a random-effects model and network metaanalysis in Stata was performed using the mvmetacommand and self-programmed Stata routines. Differences between direct and indirect evidence were explored by comparing direct and indirect estimates though computing the inconsistency factor within each closed loop of evidence. The ranking probabilities for all treatments of being at each possible rank for each intervention were computed using the mvmetacommand in Stata. A hierarchy of the competing interventions was obtained using rankograms. Quality of evidence was assessed using GRADE-NMA and the CINeMA application.

Results: Forty-three publications reporting on 32 randomized trials and some 1892 patients were identified. The network of treatments formed a closed loop between $270^{\circ}, 360^{\circ}$ and anterior $180^{\circ}$, and star network between $360^{\circ}$ and other treatments; and between anterior $180^{\circ}$ and other treatments. Laparoscopic $360^{\circ}, 270^{\circ}$, anterior $180^{\circ}$ and anterior $90^{\circ}$ were equally effective in the control of heartburn and this was supported by low quality of evidence according to GRADENMA. The odds for dysphagia were lower for anterior $90^{\circ}$ (high quality evidence), anterior $120^{\circ}$ (moderate quality evidence), $270^{\circ}$ (moderate quality evidence) and proton-pump inhibitors (moderate quality evidence) compared to $360^{\circ}$. The odds for gas-bloat were lower for $270^{\circ}$ and anterior $90^{\circ}$ compared to $360^{\circ}$ (low quality evidence). The odds for regurgitation, morbidity and reoperation were similar across treatments, albeit these were associated with very low quality evidence. Anterior $120^{\circ}$ had a $49 \%$ probability of being the best treatment in terms of dysphagia. Conclusion: Under consideration of treatment effect estimates, evidence quality as assessed with GRADE-NMA and other parameters, anterior $90^{\circ}$, anterior $120^{\circ}$ and $270^{\circ}$ should be preferred over $360^{\circ}$. Further research needs to focus on the comparison between $90^{\circ}$ and $120^{\circ} / 270^{\circ}$. 


\section{O181-UPPER GI-Reflux-Achalasia}

\section{TENSION CHARACTERISTICS AT THE DIAPHRAGMATIC HIATUS: A PROSPECTIVE OBSERVATION STUDY}

\section{Navaratne, A. Isla}

Upper GI Surgery, Northwick Park Hospital, LONDON, United Kingdom

Aims: We have recently demonstrated that the tension of crural closure can be reliably measured intraoperatively (ALSGBI Conference December 2018). The aims of this study were to further characterise tension at the diaphragmatic hiatus from our prospective pilot study of 72 patients. Methods: A prospective analysis was performed of patients undergoing laparoscopic hiatal hernia repair between April 2017 and December 2018. 72 patients underwent crural tension measurement intra-operatively. 24 patients had a pre-operative CT scan of the abdomen within one-year of surgery. Hiatal surface area (HSA) was measured intraoperatively and a Sauter-FH50 Universal Digital Force Gauge was used to measure the tension of crural closure during cruroplasty. Outcome measures included the mean tension of the crural closure and the presence of muscle splitting during the cruroplasty.

Results: For all patients, the mean crural tension measurement was $2.93 \mathrm{~N}$ and the mean HSA was $543 \mathrm{~mm}^{2}$. Pre-operative CT was positively correlated with post-dissection intra-operative HSA $(r=0.5402, p=0.0064)$, however, strength of association was weak $\left(r^{2}=0.2918\right)$ and CT consistently overestimated the size of hiatal defect intra-operatively (mean of differences $\left.404 \mathrm{~mm}^{2}, \mathrm{p}=0.0016\right)$. Crural tension was positively correlated with age $(\mathrm{r}=0.3321$, $\mathrm{p}=0.0044)$, hiatal height $(\mathrm{r}=0.6023, \mathrm{p}<0.0001)$, hiatal width $(\mathrm{r}=0.766, \mathrm{p}<0.0001)$ and HSA $(r=0.7753, p<0.0001)$. Crural tension was correlated to the hiatal width to height ratio to assess the shape of defect and there was positive correlation $(r=0.4072, p=0.0004)$. Tension was calculated for the posterior and anterior halves of the suture cruroplasty. Anterior tension was significantly higher when compared to posterior tension $(3.26 \mathrm{~N}$ vs $2.59 \mathrm{~N}, \mathrm{p}<0.0001) .16$ patients had evidence of muscle splitting during the cruroplasty. The group with muscle splitting were significantly older (66 vs $53, \mathrm{p}=0.0029)$, had larger HSA $\left(910 \mathrm{~mm}^{2}\right.$ vs $347 \mathrm{~mm}^{2}$, $\mathrm{p}<0.0001)$ and higher crural tension $(5.69 \mathrm{~N}$ vs $2.14 \mathrm{~N}, \mathrm{p}<0.0001)$. The lowest observed mean crural closure tension causing muscle splitting was $3.52 \mathrm{~N}$.

Conclusion: There is now a possibility to optimise this operation with objective measure 100 years after it was first described. Initial findings suggest that crural closure up to $\sim 4 \mathrm{~N}$ could be the permissible tension threshold for suture cruroplasty and higher tension may benefit from the use of mesh reinforcement.

\section{O182-AMAZING TECHNOLOGIES}

\section{NEW PLATFORM FOR FLEXIBLE ENDOSCOPIC SURGERY: LAPAROSCOPIC TRIANGULATION WITH TRADITIONAL ENDOSCOPES}

R. Broderick, K.H. Fuchs, A. Lee, R. Dominguez, B.J. Sandler, S. Horgan

Minimally Invasive Surgery, University of California San Diego, SAN DIEGO, United States of America

Background: Endoscopic Submucosal Dissection (ESD) and Endoscopic Full Thickness Resection (EFTR) are advanced endoscopic techniques which can be time consuming using traditional endoscopic instruments. A new endosurgery platform, designed by Fortimedix Surgical, was developed featuring flexible articulating instruments to use in combination with a standard flexible endoscope. The platform is intended to perform endoscopic cutting, dissecting, and hemostasis.

Aim: Evaluate feasibility of the platform in the upper GI-Tract.

Project Description: The platform was tested in a dry esophageal model as well as a second series with a porcine esophagus and stomach. The system has an external docking station affixed to the operative table to stabilize both flexible instruments for the right and left hand of the surgeon. At the tip of the endoscope, a cap containing instrument lumens is attached to allow advancing and removing the flexible instruments. The endoscope with the cap and instrument lumens attached is advanced via an overtube with outer diameter $18.5 \mathrm{~mm}$. In the first series, flexibility and range of motion of the endeffectors was assessed. Additionally, the ability to advance the instruments to the intraluminal target area from the docking station and along the scope was evaluated. In the second series, the functional capabilities of the system and instruments were evaluated in a porcine model.

Preliminary Results: : In the dry model, the platform was adequately deployed to the target then range of motion was tested as well as cutting and grasping gastric wall with instrumetn triangulation achieved. The grasping forceps provided enough force to pull the mucosal wall and expose the dissection plane. In the pig model, the distal esophagus and stomach could successfully be accessed and platform deployed. ESD was performed using newly designed flexible articulating scissors, dissection-hook, and graspers with good triangulation and sufficient grasping force with traction/counter-traction. The new Fortimedix Surgical endo-surgery platform applied to a standard flexible endoscope is feasible to perform ESD. Future studies are planned to determine learning curve and compare it to traditional endoscopic instruments.

\section{O183-AMAZING TECHNOLOGIES}

\section{THE NOVEL LAPAROSCOPIC POINTING DEVICE WITH TIP ILLUMINATION}

A. Takai, K. Sakamoto, M. Takashi, T. Ustunomiya, H. Masahiko, Y. Ueno, H. Inoue, K. Ogawa, Y. Takada

HBP surgery, Ehime University, TOON, Japan

Background: In laparoscopic surgery, we usually observe the organs in the same direction to avoid a mirror-image situation. Therefore, we are unable to recognize how far the dissection has proceeded on the other side of the target organs or lesions, especially when the plane of dissection is under the mesentery or organs. This becomes a problem not to understand how far the dissection has progressed and how much more dissection is needed.

Aim: To solve this problem, we developed a laparoscopic device with tip illumination.

Project description: The device is configured by the long and narrow part made of polycarbonate resin and a battery-powered light-emitting diode to illuminate the tip by shining light through the polycarbonate resin. During the surgery, the tip of the device is inserted into the deepest part of the dissection area, and the transmitted light indicates how far the dissection has progressed. The tip of the device has a prism structure and light is emitted in a direction perpendicular to its axis. Tip position can thus be more clearly identified even with insertion in the same direction as the laparoscopic view. To verify the utility of this instrument, laparoscopic surgeries were performed in a porcine model and cadavers.

Preliminary results: We performed some laparoscopic surgery such as the medial-to-latera approach to the white line of the left side of the descending colon for sigmoidectomy, dissection of the posterior surface of the pancreas to the upper edge of the pancreatic body or splenic artery for distal pancreatectomy, and the separation of the anterior surface of the inferior vena cava from the liver to the area between the right and middle hepatic vein for right hepatectomy. We quickly and easily identified the deepest part of the dissection area even if identification had been difficult using other techniques such as placing gauze in the deepest position, inserting forceps into the dissection area or simply depending on the experience of the operator.

\section{O184-AMAZING TECHNOLOGIES}

\section{A NOVEL EYE-TRACKING BASED ROBOTIC SCRUB NURSE: A STEP CLOSER TO THE FUTURE?}

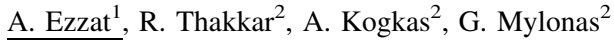

${ }^{1}$ Human-centred Automation, Robotics and Monitoring in Surgery,Faculty of Surgery, Imperial College London, LONDON, United Kingdom; ${ }^{2}$ Human-centred Automation, Robotics and Monitoring in Surgery (HARMS) Laboratory, Imperial College London, LONDON, United Kingdom

Background: Recent advancements within surgery have seen artificial intelligence transform traditional approaches. Robotic assistive devices have demonstrated particular success, as safe and cost effective, and are widely supported via industry and local government as a step closer to the future standard of practice. An example of seamless and touchless robotic assistive technology is based on touchless and interactive eye tracker glasses worn by the surgical team thereby enabling the team to perform wider surgical tasks, more efficiently and reduced human error. We introduce a perceptually-enabled, Smart Operating Room (Smart-OR) based on a novel real-time framework for theatre-wide 3D gaze localisation in a mobile fashion. This framework enables dynamic gaze based user interaction with a robotic scrub nurse to facilitate meaningful practical integration of human and technology intra-opertively.

Aims: We tested participant acceptability of a novel robotic scrub nurse during simulated surgery. Project description: Surgeons performed segmental resection of pig colon and handsewn end-to-end anastomosis while wearing eye-tracking glasses to select surgical instruments on a screen. The robotic scrub nurse(RN) picked up and transferred the instrument to the surgeon. The study compared human nurse(HN) vs RN. Gaze-screen interaction was based on a 3D gaze framework we developed with synergy of conventional wearable eye-tracking, motion capture system and fixed in space RGBD cameras for real-time 3D reconstruction of the environment. NASA-TLX and Van der Laan's technology acceptance questionnaires were collected and analysed using ANOVA.

Preliminary results: Overall, 7 teams of surgeons(ST) and scrub nurses(SN) participated. NASA TLX feedback for ST and SN revealed no significant difference between in mental, physical or temporal demand. Importantly, ST and SN reported no significant difference in task overal performance. ST reported more significant frustration with RN vs HN. Van der Laan's scores showed positive usefulness and satisfaction scores in using the RN platform. Overall, all outcomes were more positive by SN vs RN.

Conclusions: This is the first platform of its kind. Overall, quantitative and qualitative feedback was positive. The source of frustration has been understood and we believe it can be improved by appropriately modifying robot behaviour. Importantly, there was no difference on perception of performance. 


\section{O185-AMAZING TECHNOLOGIES}

\section{FLEXIBLE ENDOSCOPIC SUTURING IN THE GI TRACT}

K.H. Fuchs, K. Neki, A. Lee, R. Dominguez Profeta, B. Sandler, S. Horgan

\section{Surgery, UC San Diego, LA JOLLA, United States of America}

Background: Endoscopic tumor resections in the GI tract may be facilitated by more advanced instruments for dissecting and suturing. We have focused on developing an endoscopic suturing technique using a standard flexible pediatric endoscope with new, flexible instruments allowing for complex end-effector movements.

Aim: Perform flexible endoscopic suturing using a standard flexible scope in the GI tract

Project description: A standard flexible pediatric endoscope and a standard gastroscope were used for testing the new technique. Via an overtube, the endoscope and newly designed Fortimedix Surgical flexible instruments (needle holder; grasper) with a diameter of $5 \mathrm{~mm}$ were inserted into the esophagus. Suture training was performed in an experimental setting in a box in the dry lab and porcine model. The flexible needle holder was advanced into the esophagus next to the scope, and a suture of the esophageal wall was performed, followed by extracorporeal knot-tying with 3 knots. The test series consisted of training with both resident trainees and surgeons to evaluate the learning curve. Each participant performed sutures on the box model and in the pig-esophagus. Feasibility, duration of the different steps, and handling problems were documented.

Preliminary Results: Test series 01 (box training on esophago-gastric explant) with prototype 01 showed good feasibility. Suturing was possible in 9 out of 10 attempts. Median duration for single bite: 6 min (5-30); knot-tying: 5 min (2-8). Test series 02 (training in pig-model) with prototype 02 showed improved feasibility with better flexibility of instrument shaft: Median duration of double bite: 8 min (7-15); knottying: 2 min (1-5), overall duration intraluminal esophageal double bite suture and closing with 3 knots: median duration: $13 \mathrm{~min}(12-20)$.

Conclusions: The new flexible Endosuture instruments seem feasible to use and perform dependable intraluminal sutures. The training period and learning curve is short and the objective is to apply this system clinically for closure of perforations and fistulas.

\section{O187-AMAZING TECHNOLOGIES}

MODELLING A COLLABORATIVE ROBOT WITH THE IEEE 11073 SDC STANDARD FOR COMBINED FOCUSED ULTRASOUND AND RADIATION THERAPY

\section{J. Berger, M. Unger, L. Landgraf, A. Melzer}

Medical Faculty, University Hospital Leipzig, Innovation Center Computer Assisted Surgery, LEIPZIG, Germany

Background: Surgical robotics require a smooth integration into the operating room (OR). For this propose the IEEE 11073 SDC(Service-oriented Device Connectivity) standard has been developed in the OR.NET project. In preparation for a combined focused ultrasound and radiation therapy (FUS-RT) we have shown concepts and evaluations to position ultrasound and interventional devices with collaborative KUKA arms. However, the safe and intraoperative cooperation with multiple different OR-devices (e.g. an irradiation unit) requires a more sophisticated exchange of the robot's information and functionality.

Aim: To realize a safe clinical integration, the aim of this work is to implement and evaluate a dynamic connection between the KUKA robots and other devices using the vendor-independent SDC communication standard.

Project description: A KUKA LBR iiwa 7 R800 robot (KUKA AG, Germany) was modeled inside the SDC standard for medical device communication. The interconnection with other devices was implemented and evaluated on a mobile platform to position a Clarius L7 wireless ultrasound transducer (Clarius Mobile Health Corp, Canada). All necessary information of the robot was represented in the medical device description of the SDC standard to be shared via network. For each joint of the robot arm the position, torque, stiffness, damping, velocity and functional-states were represented, resulting in a total of 42 parameters. The software was implemented in $\mathrm{C}++$ on a standard PC accessing the KUKA controlle cabinet with ROS (Robot Operating System) via Ethernet. The accessibility of each parameter, as well as activation commands for planning and movement were tested with an SDC-consumer application. Preliminary results: The SDC-provider functionality of the robot was successfully implemented, allowing for dynamic changes of the robot state during interventions. All appliances (SDC standard compatible) in the robots network can react to state changes and send movement and planning commands to the robot via activations. After testing, $100 \%$ of the 42 defined parameters are safely accessible. Implementing the medical device communication for the KUKA robot enables its integration into any networked operation room that supports the SDC standard. It is, therefore, ready to be set up and evaluated for the application of FUS-RT in a clinical environment.

\section{O188-AMAZING TECHNOLOGIES}

\section{O186-AMAZING TECHNOLOGIES}

ENDOSCOPIC SUTURING USING A COMPUTERCONTROLLED MASTER AND SLAVE ROBOT: ANIMAL STUDY

\section{Cao ${ }^{1}$, H.L. Kaan ${ }^{2}$, X. Li ${ }^{1}$, P.T. Phan ${ }^{1}$, A.M.H. Tiong ${ }^{1}$,} K.Y. Ho ${ }^{3}$, P.W.Y. Chiu ${ }^{4}$, S.J. Phee ${ }^{1}$

${ }^{1}$ School of Mechanical and Aerospace Engineering, Nanyang Technological University, SINGAPORE, Singapore; ${ }^{2}$ General Surgery, National University Hospital, SINGAPORE, Singapore; ${ }^{3}$ Gastroenterology, National University Hospital, SINGAPORE, Singapore; ${ }^{4}$ Surgery, Chinese University of Hong Kong, HONG KONG, Hong Kong

Background: Ideally, endoscopic suturing should mimic surgical closure as the latter is stronger than most endoscopic closure devices. However, endoscopic suturing is challenging due to the confined endoluminal space and lack of dexterity of current endoscopic instruments. We have developed a novel robotic suturing device to overcome these problems.

Aim: This animal study aims to demonstrate the feasibility of this device in closing perforations.

Method: The trial was conducted on an anaesthetized live pig. A double-channel colonoscope was first inserted into the rectum. Following saline lift, a $10 \mathrm{~mm}$ submucosal incision was created in the rectum to simulate a perforation. The robotic suturing device and grasper were inserted into the two colonoscope channels, allowing the endoscope to remain in position for tool exchanges or needle reloading. Both the effectors were intuitively tele-operated by the user via a robotic master console.

This robotic suturing device manipulated a curved, double-point needle (with a $10 \mathrm{~cm}$ 3-0 Vicryl suture) to penetrate tissues at desired orientations. The needle could be switched between both jaws of the device through a locking mechanism. This facilitated passing the needle through tissues to form stitches or through suture loops to form surgical knots. The articulated joints and five degrees of freedom allowed dexterous steering to reach targets and triangulation with other tools in a confined space. The robotic grasper facilitated handling of tissue and suture

Result: A total of four running stitches were performed and secured with a surgical knot by passing the needle through suture loops. The suture was cut and the needle was removed by the robotic grasper through the channel. $11 \mathrm{~min}$ and $4 \mathrm{~min}$ were required to stitch and tie the knot respectively. There was no complication.

Conclusion: Our novel endoscopic robotic device can suture perforations resulting from complex endoscopic procedures. As our suturing method is similar to laparoscopic and robotic suturing, closure using our device is expected to be as strong as a surgical through-and-through closure. When developed further, this device can be used to close full-thickness resection sites and orifices in transluminal endoscopic surgery.

\section{STANDARDISATION OF FLUORESCENCE-GUIDED PERFUSION ASSESSMENT THROUGH AN UNSUPERVISED LEARNING ALGORITHM}

\section{A. Soares, S. Bano, D. Stoyanov, L.B. Lovat, M. Chand}

Wellcome / EPSRC Centre Interventional and Surgical Sciences, University College London, LONDON, United Kingdom

Background: Assessment of perfusion of the left colon with fluorescence during anterior resections for cancer changes surgical decisions in up to $19 \%$ of cases. Use of fluorescence has been shown to be associated with lower leak rates, and improved short- and long-term outcomes with reduced costs. Given the high incidence of colorectal cancer, fluorescence-guided perfusion assessment could be of great importance in contemporary surgical practice. However, there is currently no standardisation of this technique which represents a significant limitation to widespread adoption.

Aim: to standardise fluorescence-guided perfusion assessment in rectal anterior resection through a computer vision algorithm.

Project description: Videos were collected by a single surgeon in a referral centre for colorectal cancer treatment. Perfusion assessment was used before proximal colon division to identify the best location for transection. A bolus of indocyanine green was injected intravenously and a near-infrared camera used to assess perfusion through fluorescence.

Photographs of fluorescent imaging of the colon were analysed using a non-supervised learning al gorithm called 'K-means clustering' The first step was to digitally subtract all background pixels, leaving only the area of interest of the colon. This area was then subsegmented into 2 'clusters' corresponding to perfused and nonperfused areas. A mathematical model was applied based on the 2 sub-clusters centres to select the area for transection with optimal perfusion of the proximal colon.

Preliminary results: Representative images of proximal colon under perfusion assessment were presented to 8 expert surgeons. The optimal point for transection was selected based on their clinical judgement on previously delimited areas indicated by random letters. This was compared with the results from the automated segmentation using the algorithm (fig. 1). The area identified for section by the algorithm included the area selected by the expert surgeons in $87.5-100 \%$ of test cases.

These results need to be further validated due to high risk of overfitting. Next steps include the collection of multicentre data with a standardised fluorescence perfusion assessment. After robust training, the algorithm will be validated on real-time clinical data to ensure improved outcomes for patients, which is our ultimate goa 


\section{O189-AMAZING TECHNOLOGIES}

\section{A ROBOTIC PLATFORM FOR ENDOSCOPIC DISSECTION}

M. Brancadoro ${ }^{1}$, C. Quaglia $^{1}$, H. Abidi ${ }^{1}$, M.A. Bonino ${ }^{2}$, A. Menciassi ${ }^{1}$, A. Arezzo ${ }^{2}$

${ }^{1}$ BioRobotic Institute, Scuola Superiore Sant'Anna, PONTEDERA, Italy; ${ }^{2}$ Department of Surgical Sciences, Università di Torino, TORINO, Italy

Background: Endoscopic Submucosal Dissection (ESD) is a flexible endoscopic technique that allows for an en bloc removal of lesions of the gastrointestinal (GI) tract. These procedures are typically time consuming due to the difficult control of the tools, and they often require around $95 \mathrm{~min}$ for removing lesions, that can reach $3-4 \mathrm{~cm}$ in diameter. The probability of intestinal perforation exceeds $18 \%$ and the hemorrhage risk ranges from $3.5 \%$ to $15.5 \%$. A flexible robotic endoscope may offer a solution to overcome these limitations, by improving the degrees of freedom (DoF) and operational efficiency. Aim: Within this clinical panorama, the aim of this project is presenting the development of a novel miniaturized robotic device to be coupled to the tip of a traditional endoscope for the surgical dissection of GI neoplasms.

Project description: The robotic platform consists of the miniaturized robot, the actuator housing (hereafter called external platform), the control unit and the master console (i.e.two Geomagic Touch phantom) to allow the user driving and control (Figure 1a). During the operation, one surgeon stands close to the patient to maneuver the endoscope for exploring the GI tract and reaching the target area. Another surgeon operates the miniaturized robot through the master console, carrying out the surgical procedure. The robot has been designed to be coupled to the tip of traditional flexible endoscopes of $14.5 \mathrm{~mm}$ in diameter. It exploits the flexibility of the endoscope for navigation through the intestine and integrates two-active robotic arms (i.e.,cautery and gripper) extending the DoFs, and thus enhancing the efficiency during complex tasks such as manipulation and surgical tissue dissection. Furthermore, the endoscope provides the optical system for visual feedback and one working channels for conventional instruments.

Preliminary results: Firstly, a mock-up that faithfully reproduces the miniaturized robot has been realized using a 3D printer machine (ProJet MJP 3600, 3D System, Inc.) to verify the feasibility of the design solution. After verifying the potentiality of the 3D printed prototype, a final device, with the same features (i.e.,DoF and geometry) of the 3D printed prototype, has been designed, fabricated and assembled (Figure 1b).

\section{O190-AMAZING TECHNOLOGIES}

\section{AUGMENTED REALITY IN VASCULAR SURGERY-THREE DIMENTIONAL HOLOGRAPHIC IMAGING DURING ENDOVASCULAR AORTIC REPAIR}

\section{N. Wrzesinska, M. Szostek, J. Wawrzyniec, S. Willenberg}

Department of General, Endocrine and Vascular Surgery, Medical University of Warsaw, WARSAW, Poland

Background: Virtual and augmented reality has been widely used in many fields mainly for entertainment purposes. We think that it could be beneficial to use augmented reality in medical practice.

Aim: The aim of this study was to evaluate usefulness of 3D holographic images of patients anatomy displayed using augmented reality goggles during endovascular aortic repair (EVAR). Project description: One of the major challenges during endovascular procedures is working on two dimentional $\mathrm{x}$-ray images of three dimentional vascular anatomy. Using 3D holograms of patients anatomy could be beneficial during the EVAR procedure and could make the orientation in vascular anatomy easier for surgeon.

We performed two endovascular aortic repairs with the assists of Microsoft HoloLens -smart glasses using augmented reality. We used Carna Life application created by polish company MedApp. It was one of the first use of holograms during vascular procedures in the world (second and third stent-graft implantation using holographic imaging in the world).

Results: Two patients with abdominal aortic aneurysms, 79-years old male and 74-years old female, were operated on. Holograms of patient's anatomy made from preoperative angio CT scans by polish company MedApp were displayed during the procedures using Microsoft HoloLens. Holograms could be displayed in any place and configuration using augmented reality, which means that the images did not interfere with the surgeon's field of vision. Microsoft HoloLens use voice commends which permits the surgeon staying sterile.

Stent- graft implantations were successful. Both patients were discharged three days after the procedure and the hospitalization was uneventful.

Seeing precise patient's vascular anatomy reconstructions in three dimention certainly helped us to navigate in a vascular tree. We believe that in the future this technology would enable to reduce the operation time and need for radiation.

\section{O191-AMAZING TECHNOLOGIES}

USING THE BEST WEARABLE SENSOR IN FACILITATING HANDS-FREE, STERILE CONTROL IN THE OPERATING ROOM: THE TEDTRIAL

\author{
M. Jansen, H.A.W. Meijer, M.P. Schijven
}

Department of Surgery, Amsterdam UMC, location AMC, AMSTERDAM, The Netherlands

Background: Interaction with electronically controlled operating room (OR) systems embedded in modern surgical environments is everyday practice for surgeons performing Minimally Invasive Surgery (MIS). While there is a non-sterile operating nurse available in the OR, capable of interacting with these systems upon request by the surgeon, this indirect control is mostly slow, prone for error and disrupting surgical workflow. Facing an unanticipated and unwanted outcome may cause distress emotions. Distress emotions are undesirable when performing surgery, since they may impact available cognitive workload. Furthermore, they may result in negative communication, hampering OR-team empowerment and effective leadership. Both factors are known to negatively influence quality and safety in the OR.

Aim: The aim of the TedTrial is to investigate what setup best enables surgeons to interact with the endoscopic operating room setup during surgical procedures. As a result, disruptions of workflow, delays and errors may be reduced. Outcome parameters will be objectified using medical data recorder (MDR) derived output and biometric analysis using Hexoskin $\odot$. Subjective evaluation of outcome parameters is done using questionnaires.

Project description: The TedCube $\odot$ system is a plug-and-play device enabling wearable sensors to act as a wireless alternative for a regular computer mouse, therefore enabling direct hands-free and sterile control of the OR. The study is an observational trial with three different arms: Intervention group 1) direct interaction by surgeon with OR environment using TedCube $(\odot)$ and $\mathrm{Myo}^{\mathrm{TM}}$ armband, Intervention group 2) direct interaction of surgeon with OR environment using Tedcube $\odot$ and Plantronics $\odot$ wireless microphone headset. The third arm is the control group using indirect interaction of surgeon with OR environment using third-person computer interaction.

Main endpoint of study is the number of workflow disruptions due to the operation of laparoscopic OR equipment. Secondary endpoints are error rate, delay, team communication, subjectively reported frustration and satisfaction with the system and objectively measured stress as symptom of frustration and anger as distress emotions.

Preliminary results: Primary and secondary endpoints of study are compared among groups. It is anticipated that reduction of miscommunication, error and delay may result in a reduction of distress emotions. Trial start is expected Q1 2019. 


\section{O192-AMAZING TECHNOLOGIES}

\section{ANTICIPATING THE AUTOMATED INTRAOPERATIVE TISSUE RECOGNITION: INTRAOPERATIVE TISSUE CLASSIFICATION USING HYPERSPECTRAL IMAGING AND} MACHINE LEARNING

M. Barberio $^{1}$, V. Agnus ${ }^{1}$, F. Longo ${ }^{1}$, C. Fiorillo ${ }^{1}$, B. Seeliger ${ }^{1}$,

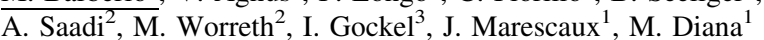

${ }^{1}$ Research, IHU, STRASBOURG, France; ${ }^{2}$ Surgery, Department of Surgery, Hospital of Neuchâtel-Pourtalès, NEUCHÂTEL, Switzerland; ${ }^{3}$ Department of General, Digestive, and Endocrine Surgery, University Hospital of Leipzig, LEIPZIG, Germany

Background: Iatrogenic injuries may occur despite a sound expertise in surgical anatomy. Hyperspectral imaging (HSI) is an emerging optical method, combining the use of a camera system with a spectrometer. HSI analyzes optical properties of tissues and acquires 3D data sets with two spatial dimensions ( $x, y)$ and one spectral dimension (?). The data sets contain information about tissue physiology, composition, and perfusion. Those spectral features coupled with machine learning algorithms might allow for automatic tissue recognition.

Aim: Assessing the ability of an HSI-based machine learning to discriminate the hyperspectral features of different tissues during neck and abdominal surgical procedures.

Methods and procedures: Fourteen pigs underwent laparotomy $(n=6)$ or neck dissection $(\mathrm{n}=8)$. Twenty data sets were acquired in vivo from abdominal organs and 20 from neck structures by means of a customized hyperspectral camera (Diaspective Vision, Germany). Different anatomical structures were manually outlined by a surgeon using an image manipulation software (GIMP). Each pixel contained a hyperspectral curve and each curve was composed of 100 bands (from 500 to $1000 \mathrm{~nm}$ with a $5 \mathrm{~nm}$ resolution). The curves were normalized using the standard normal variate method. A logistic regression machine learning (ML) algorithm was used to train the model to discriminate tissues, based on the HSI spectral features. The efficacy of the prediction model was tested using the $\mathrm{k}$-fold $(\mathrm{k}=10)$ cross-validation. Results: A large number of tissue-related hyperspectral curves could be extracted (4675 thyroid, 9417 vagal nerve, 48546 fatty tissue, 30486 cartilage, 16001 carotid artery, 81567 muscle, 5149 carotid vein, 7148 portal vein, 22973 biliary tract, 73940 gallbladder, 1874 hepatic artery, 16712 pancreas, 2412 duodenum, 34313 abdominal adipose tissue). The algorithm used 4 min to 'learn' all data sets, and prediction was provided as an immediate output.

Overall, prediction accuracy was 92 and $89 \%$ for neck and abdominal structures respectively. In particular, biliary ducts could be identified with a $93 \%$ accuracy and the vagal nerve with an $89 \%$ accuracy (See Figure 1 for details).

\section{O193-AMAZING TECHNOLOGIES}

\section{GAZE-CONTROLLED ROBOTIC FLEXIBLE ENDOSCOPY: A FEASIBILITY STUDY}

\section{N. Patel, A.A. Kogkas, B. Glover, A.W. Darzi, G. Mylonas}

Department of Surgery and Cancer, Imperial College London, LONDON, United Kingdom

Background: A gaze-controlled robotic endoscope is innovative technology with myriad potential applications in the rapidly advancing field of flexible endoscopy. Improvements to the current flexible device to allow examination of the gastrointestinal tract whilst minimising procedural discomfort and complications are desirable.

Aim: To use a gaze contingent framework to manipulate a flexible endoscope through a simulated upper gastrointestinal tract (UGIT) model.

Description: A flexible gastroscope (Karl Storz 13801 PKS) was attached to a UR5 6 axis robotic arm (Universal Robots), mounted onto a rail and placed on top of a surgical table. Two cogwheel shaped dials were 3D printed and placed onto the up/down and left/right wheels on the head of the gastroscope (Figure 1). Robotization of these controls was achieved by using two motors (DYNAMIXEL RX-24F) to steer the distal tip.

This system allows users to operate a robotised flexible endoscope using gaze control. Gaze interaction with the screen was based on a 3D gaze framework we developed with the synergy of conventional wearable eye-tracking, motion capture system and fixed in space RGB-D cameras for $3 \mathrm{D}$ reconstruction of the environment.

Users are able to control endoscope movements without handling the device. The distal tip of the gastroscope was controlled using eye gaze technology. The UR5 robot was used to enable shaft rotation (initiated by fixed head movements) and linear movements were triggered using a joystick handle (up for forward movement, down for endoscope withdrawal). Pause and retroflexion of the endoscope are achieved by moving the joystick left and right respectively.

Users were asked to navigate an endoscope through an UGIT model (Chamberlain group) simulating a diagnostic gastroscopy using gaze control and targeting ten points scattered through the stomach. Results: Four expert endoscopists and one novice used gaze control to successfully navigate a gastroscope through a simulated UGIT. All were able to intubate the oesophagus and accurately locate ten targets placed in the fundus, body, antrum and pylorus of the stomach.

Conclusion: Gaze control endoscopy is a feasible concept. It allows ergonomic, user-friendly and intuitive control whilst maintaining the benefits of a flexible endoscope

\section{O194-AMAZING TECHNOLOGIES}

\section{COMPUTING BIOPSY: MACHINE-LEARNING-TRIGGERED RADIOMICS EVALUATION OF HEPATIC TUMORS}

C. Gonzalez ${ }^{1}$, F. Ouhmich ${ }^{2}$, T. Wakabayashi ${ }^{3}$, V. Agnus ${ }^{2}$, B. Gallix ${ }^{4}$, D. Mutter ${ }^{5}$, P. Pessaux ${ }^{5}$, J. Marescaux ${ }^{3}$

${ }^{1}$ Imague-Guided Surgery, IHU Strasbourg, STRASBOURG, France; ${ }^{2}$ Research \& Development, IHU strasbourg, STRASBOURG, France; ${ }^{3}$ Surgery, IRCAD, STRASBOURG, France; ${ }^{4}$ Radiology, IHU strasbourg, STRASBOURG, France; ${ }^{5}$ Surgery, IHU strasbourg, STRASBOURG, France

Background: Image-guided needle biopsies and histopathological evaluation are the gold standard for the diagnosis of liver neoplasms. Most often, however, these are reserved for suspicious, but not diagnostic, situations. Radiomic may help to characterize tumor biology by correlating imaging features with relevant tumor-biology information. Features derived from radiomic analysis may provide complementary information to support clinical decisions, especially in situations where tissue analysis cannot be performed or is inconclusive.

Aim: The goal of our technology is to exploit computational capabilities for image analysis in order to identify radiomic features useful for characterizing liver lesions and to identify relevant information related to patient prognosis.

Project description: 17 patients derived from an internal database and 12 patients randomly extracted from the Cancer Archive Liver Dataset were included in this study. 56 lesions were extracted from those volumes using expert annotations (31 secondary vs 25 primary; 34 well differentiated vs 22 non-well differentiated). Lesions were then split into training and testing sets. First order statistical features were computed and a Lasso regression step was performed to reduce the number of features. Both logistic regression and random forest models were built using cross-validation to predict the target classes on the test set.

Preliminary results: Only 2 features namely the Energy and the Volume of the lesion were sufficient, when combined in either model, to predict the differentiation grade on the test set with an F1-Score of $0.74( \pm 0.07)$. We are currently working on the addition of higher order statistical features to the analysis in order to differentiate primary from metastatic tumors and identify complementary features that may assist clinical decisions in patients with inconclusive hepatic lesions.

\section{O195-AMAZING TECHNOLOGIES}

\section{DEVELOPMENT OF FUNCTIONAL STOMACH MODEL FOR TRAINING AND EVALUATION OF ENDOSCOPIC SKILLS (STOMED)}

\section{E.J. Barzola Navarro ${ }^{1}$, A. Glagolieva ${ }^{2}$, V. Galvez ${ }^{3}$, J. Salas ${ }^{4}$}

${ }^{1}$ Digestive Surgery, Universidad de Extremadura, BADAJOZ, Spain; ${ }^{2}$ Surgery, PL Shupyk National Medical Academy, KIEV, Ukraine; ${ }^{3}$ Chemistry, Universidad de Extremadura, BADAJOZ, Spain; ${ }^{4}$ Technology, Universidad de Extremadura, BADAJOZ, Spain

Objective of the technology or deviceIdeally, the use of medical simulators could provide trainees with initial background information about indications for procedures, endoscopic technique, and early hands-on training experience that could shorten the initial critical learning curve. Rationale for using ex vivo models is that in the beginning of the learning curve, the mos important issue is having an initial exposure to the basic movements and maneuvers. Our objective of is to create a stomach model from renewable polymer, which would closely simulate normal human stomach with gastric pathology for endoscopic diagnostic or interventional skill acquisition/evaluation.

Description of the technology and method of its use or application Stomach model is based in several steps; the first one is in the in-silicodesign of the overall shape, after that we 3D print the positive two halves of it. The interior detail is obtained shaping the $3 \mathrm{~d}$ printer parts with ceramic putty. Once concluded, this elaborated part will serve as a template in order to build injection bleeding moulds. In the injection bleeding moulding a mesh is placed between layers in order to provide structural attachment points as stiches or several pathological models that will be incorporated after the casting process. We have developed for these instance polyp moulds, fistulae structures in order to attach endoscopic clamps. The two halves are closed once the pathological models are placed inside via a thermic-fusing and stitching creating a leak proof stomach model.

Preliminary results if available: Our models were evaluated by 8 international experts in IRCAR/IHU France in Interventional Endoscopy Course and were favorable accepting for next trails in these prestigious institutions.

Conclusions: Future directionsA new endoscopic training model of stomach was made and will be evaluated and validated for feasibility in mastering diagnostic and interventional endoscopic skills.

Clinical trials will be necessary to compare the ability of the simulator to perform training compared with traditional methods of training in endoscopic procedures. 


\section{O196-AMAZING TECHNOLOGIES}

ROBOTIC FLEXIBLE ENDOSCOPE FOR MINIMALLY INVASIVE SURGERY WITH ENHANCED SAFETY

\section{Z. $\mathrm{Li}^{1}$, C.Z. Song ${ }^{1}$, X. $\mathrm{Ma}^{2}$, P.W.Y. Chiu ${ }^{1}$}

${ }^{1}$ Surgery, The Chinese University of Hong Kong, HONG KONG; ${ }^{2}$ Chow Yuk Ho Technology Centre for Innovative Medicine, The Chinese University of Hong Kong, HONG KONG

Background: Endoscopes are the eye of surgeons in minimally invasive surgery (MIS). Conventional endoscopes are mostly chopstick-like and are steered by the assistant. This limits the field of view and results in issues such as endoscope-instrument fencing, surgeon-assistant coordination. Existing robotic endoscope holder enables solo-surgery, however endoscope remains blocking the instrument movement and impairs the operational safety. Flexible endoscope such as the Endoeye provides angulation at the tip and could enlarge the field of view. However, its steering the view is much more complex compared to the rigid endoscope.

Aim: To provide an intuitive robotic flexible endoscope with enhanced safety.

Project description: In this work, we present a robotic flexible endoscope for MIS with enhanced safety. In the proof-of-concept system, it contains a flexible endoscope module and a robo manipulator. The endoscope contains a proximal rigid shaft and a distal flexible bending section. It is installed onto the patient side manipulator (PSM) of the da Vinci Research Kit (DVRK). Visual servoing is adopted to achieve autonomous instruments tracking. During the tracking process, movements of the manipulator as well as the endoscope are minimized to save space for the operation and avoid instrument-endoscope fencing. The endoscope could also be controlled by the surgeon. A foot pedal is used to switch between the Tracking-Mode and Control-Mode. Preliminary results: A prototype was developed and tested experimentally. In tracking a volume of $200 * 200 * 100 \mathrm{~mm}^{3}$, the spaces required by the flexible endoscope are $15.55 \%$ (inside the trocar) and $9.83 \%$ (outside the trocar) of that occupied by the rigid endoscope. Evaluation with the FLS tasks involved 10 subjects. All of the participants completed the tasks under the Tracking-Mode without failure. In the ex-vivo test with porcine stomach, the endoscope successfully guided the detection, dissection and knotting autonomously.

\section{O197-AMAZING TECHNOLOGIES}

\section{NEAR-INFRARED COATING OF EQUIPMENT (NICE) TO AID INTRAOPERATIVE CATHETER IDENTIFICATION-A PRECLINICAL TESTING OF NOVEL FLUORESCENT} COATINGS

B. Seeliger ${ }^{1}$, A.H. Ashoka ${ }^{2}$, V. Agnus ${ }^{1}$, M. Barberio ${ }^{1}$, A. Picchetto ${ }^{3}$, B. Andreiuk $^{2}$, S.H. Kong ${ }^{4}$, A.S. Klymchenko ${ }^{2}$, M. Diana ${ }^{1}$

${ }^{1}$ Institute of Image-Guided Surgery, IHU-Strasbourg,

STRASBOURG, France; ${ }^{2}$ Laboratory of Bioimaging and Pathologies, UMR 7021 CNRS, Faculty of Pharmacy, University of Strasbourg, STRASBOURG, France; ${ }^{3}$ Department of General Surgery, Surgical Specialties and Organ Transplantation, Sapienza University of Rome, ROME, Italy; ${ }^{4}$ Department of Surgery, Seoul National University Hospital, SEOUL, Korea

Background: Fluorescence imaging allows to visualize deep-seated anatomical structures, using a deeper tissue penetration of near-infrared (NIR) compared to visible light. The most commonly used fluorescent substance, Indocyanine Green (ICG), is not naturally excreted by the urinary system and requires retrograde stent placement and injection. Lighted catheters have been proposed to help visualise the ureter. Fluorescent dye-coated ureteral catheters could well represent a more effective and less expensive solution. ICG is unsuitable for coating materials. Aim: To develop a stable fluorescent coating for catheters to be used intraoperatively, working in the same NIR window as ICG, to facilitate its use with clinically available systems. Project description: The coating was developed based on Poly(methyl methacrylate) (PMMA), a biocompatible polymer, and on specifically designed fluorescent dyes exhibiting ICG-like optical properties.

Three NIR dyes (substances A, B, and C) were tested in order to find the optimal one, in terms of fluorescence signal intensity, and were compared to ICG in a polymer form and to an ICG-based reference card (Green Balance ${ }^{\mathrm{TM}}$ ). The fluorescent coating was applied onto 3 common ureteral stent materials: hydrophilic-coated Ultrathane ${ }^{\circledR}$, silicone-coated latex, and PVC. The coating process involved 3 cycles of immersion into the respective dyes blended in PMMA polymer (ICG, substances A, $B$, and $C$ ), followed by a drying phase. The various tubes were partly inserted into a porcine ureter, next to the ICG-based reference card. Images were taken in White Light and NIR modes using the D-Light P camera system (KARL STORZ), at a fixed camera-to-target distance. The fluorescence signal intensity was measured for the different regions of interest (each material/coating combination inside and outside of the ureter, reference card) using proprietary software and normalised against the reference card. Preliminary results: The signal intensity was significantly higher for all new substances as compared to ICG. Substance A showed the strongest fluorescence signal intensity among the tested coatings in all tested conditions and materials and was identified as the ideal candidate to undergo further evaluation and in vivo testing.

\section{O198-AMAZING TECHNOLOGIES}

EVALUATION OF GASTRIC SUBMUCOSAL ARCHITECTURE WITH EUS FINE NEEDLE BIOPSY, CONFOCAL MICROSCOPY AND MINIBIOPSY. AN EXPERIMENTAL PIG MODEL

\section{S.G. Lim, L. Sosa-Valencia, L. Swanstrom}

Institute for Image Guided Surgery, IHU-Strasbourg, STRASBOURG, France

Background: Endoscopic resection(ER) of early gastric cancers provides tremendous patient advantages. However, post-resection findings of deeper sub-mucosal(SM) and/or lympho-vascular invasion can necessitate a second, surgical intervention. We propose that pre-resection evaluation of the submucosal architecture under the tumour can provide critical information for staging and operative planning. We evaluate three techniques to assess the submucosal architecture underlying the gastric mucosa in a pig model.

Aim: To evaluate three needle-based methods of evaluating the SM before ER.

Project Description: 6 acute pigs were used. A simulation of sub-mucosal tumours (endoscopically and EUS visible bleb) by injecting the SM with $20 \mathrm{cc}$ of undyed $\mathrm{NaC}$. A linear EUS was use for all procedures. The tumours were marked and labelled according to geography.

Methodology: After creating the tumours, anterior lesions were evaluated using the following $19 \mathrm{G}$ needle-based modalities: Confocal microscopy $(\mathrm{CM})$ using the through-the-needle Cellvizio (Mauna-Kea) system; Mini-biopsy(MB) using the micro-biopsy forceps Moray (US Endoscopy) and Fine-needle biopsy(FNB).

Results: $18 \mathrm{CM}$ examinations were video recorded in all A positions. Submucosal vascular visualisation was possible in all cases, excellent in 17/18. MB was performed in 18 lesions with a total of 2 biopsies obtained from each lesion (total $=36$ ). FNB was performed once in the anterior lesions and twice in the posterior lesions with different needle brands. Therefore, there was a total of 54 biopsies collected. 2 passes were performed in each biopsy (total $=108$ ). Each pass constituted 20-25 insertion/withdrawal movements combined with fanning, slow pull technique, no suction and suction (10-20 cc air negative pressure) to collect the material. All material were sent to an animal anatomo-pathologist blinded to the acquisition method. Mean time of confocal examination was 15 min $8 \sec \left(6^{\prime} 02^{\prime \prime}-30^{\prime} 59^{\prime \prime}\right)$. MBtook a mean time of 5 min and FNB was a mean of $10 \mathrm{~min}$ for each biopsy. CM identified different patterns of vessels in relation to the probe position (superficial/reticular, middle cross-roads or deep/longitudinal). Conclusion: EUS-FNB, CM and MB are three potential methods to assess the sub-mucosal space underlying the gastric mucosa. CM offered the most architectural information but required more time to perform. These method's may have a role in better staging patients for appropriate ER. 


\section{O199-AMAZING TECHNOLOGIES}

3D MODELLING OF PELVIC ANATOMY FOR COLORECTAL CANCER STAGING AND DYNAMIC FUNCTION SIMULATION

A. Przedlacka ${ }^{1}$, S. Cox $^{1}$, N. Khan ${ }^{2}$, P. Paris ${ }^{1}$, F. Bello ${ }^{3}$, C. Kontovounisios ${ }^{1}$

${ }^{1}$ Department of Surgery and Cancer, Imperial College London, LONDON, United Kingdom; ${ }^{2}$ Radiology, Chelsea and Westminster Hospital, LONDON, United Kingdom; ${ }^{3}$ Centre for Engagement and Simulation Science, Imperial College London, LONDON, United Kingdom

Background: The overall and disease-free survival of patients with rectal cancer is dependant on its staging, and adequate selection of the treatment strategy. MRI has a proven efficacy in rectal cancer local staging and recognition of the adverse prognostic features. However, it can be difficult to utilise it as a navigation tool for surgeons, as it represents a complex three-dimensional pelvic space with a series of individual two-dimensional images. 3D image reconstruction has been successfully adopted in other surgical fields to overcome these limitations.

Aim: Our primary aim is to develop a bespoke automated generation of patient-specific 3D pelvic models, which will improve surgical planning and navigation, patient interaction and surgical education. True-size, rotatable 3D models will offer a more realistic three-dimensional representation of the surgical space and its complex relationships, allowing for a more confident surgical rehearsal and potentially better utilisation of minimally invasive techniques in rectal cancer management. Our secondary aim is to develop a large multipurpose database of the 3D models of male and female pelvis in health and in the disease.

Project description: Our multidisciplinary team consists of colorectal surgeons, radiologists specialising in pelvic MRI imaging and computer scientists. Virtual 3D pelvic models are generated based on standard 2D DICOM MRI images routinely used for rectal cancer staging, which guarantees the high fidelity of cancer delineation. Segmentation of the pelvic anatomy is performed with the use of ITK-SNAP, an open-access, multi-platform software. Machine learning technology is then employed to automate the 3D model generation, making it time-efficient, allowing for its clinical application.

Preliminary results: In the initial stage, using the manual segmentation, we have created ten models of normal male and female pelvic anatomy. A good inter-rater agreement level was found, which proves reproducibility of the approach applied. Various machine learning algorithms are being explored to fully automate the process of 3D model generation, which will allow for their use in clinical practice and in development of the 3D colorectal database. The technology will be further implemented in creation of dynamic models of functional pelvic floor disorders.

\section{O200-AMAZING TECHNOLOGIES}

HYBRID MAGNETIC GASTROJEJUNOSTOMY USING A FLUORESCENT MAGNETIC ANASTOMOTIC DEVICE: A FEASIBILITY STUDY ON THE PORCINE MODEL AND HUMAN CADAVER

R. Watanabe $^{1}$, M. Barberio ${ }^{2}$, A. Lapergola ${ }^{2}$, A. Klymchenko ${ }^{2}$, L.

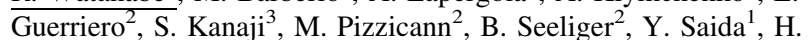
$\mathrm{Kaneko}^{4}$, M. Worreth ${ }^{5}$, A. Saadi ${ }^{5}$, J. Marescaux ${ }^{3}$, M. Diana ${ }^{2}$

${ }^{1}$ Surgery, Toho University Ohashi Medical Center, TOKYO, Japan; ${ }^{2}$ Institute of Image-Guided Surgery, IHU-Strasbourg, STRASBOURG, France; ${ }^{3}$ Research Institute against Digestive Cancer, IRCAD, STRASBOURG, France; ${ }^{4}$ Surgery, Toho University Omori Medical Center, TOKYO, Japan; ${ }^{5}$ Surgery, Neuchâtel Hospital, NEUCHÂTEL, Switzerland

Background: Laparoscopic gastrojejunostomies are time-consuming and require a specific training. Alternatively, sutureless anastomosis can be achieved by means of endoscopically delivered magnetic rings.

Objective of the study: Assessing the feasibility and reproducibility of an endo-laparoscopic gastrojejunostomy technique, using magnets coated with a fluorescent biocompatible polymer.

Methods and Procedures: Four pigs ( 2 acute, 2 survival models) and one cadaver were included in this study. The anastomotic device was composed of two magnetic rings $(25 \times 8 \times 6 \mathrm{~mm}$; attraction force 30 Newton), each one attached to a $75 \mathrm{~cm}$ long thread. The distal ring was inserted endoscopically into the first duodenum, and the extremity of the thread was clipped to the gastric mucosa. Twenty-four hours later, a two-port laparoscopy (12 mm, $5 \mathrm{~mm}$ ) was performed, using a Near-Infrared (NIR) laparoscope (D-Light-P; Karl Storz). The magnet's position in the jejunum was detected thanks to the transluminal fluorescence of the dye. Magnetic interaction with the metallic tip of the laparoscopic grasper allowed to catch the ring and bring the bowel loop to the future anastomotic site on the gastric wall. Simultaneously, the proximal magnet was delivered to the gastroesophageal junction using a flexible endoscope. The magnet was carefully advanced into the stomach allowing precise connection with the distal ring. In one cadaver the procedure was repeated. The sole variation was that, in order to reach the second jejunal loop, the distal magnet was placed using a gastroscope inserted through a transgastric port.

In two acute animals, the distal magnetic ring was introduced into the jejunum via an enterotomy. The anastomotic procedure (from the distal magnet detection via fluorescence to the magnetic connection using a hybrid approach) was reiterated 40 times. Survival animals were followedup for 10 days and underwent control endoscopies and CT-scans.

Results: The procedure was easy to standardize and reproducible, with a mean anastomotic procedure time of $2.62 \pm 1.42 \mathrm{~min}$. There were no technical problems and magnetic connection could be precisely directed in all cases, at both the anterior and posterior gastric wall. No complications occurred during the survival period and the anastomoses were patent by day 5. Transluminal fluorescence allowed for a rapid detection of the magnet. 


\section{O201-AMAZING TECHNOLOGIES}

\section{AN INNOVATIVE MEDICAL PLATFORM FOR} MAGNETICALLY-DRIVEN PAINLESS COLONOSCOPY: A ROBOTIC SOLUTION FOR COLORECTAL CANCER EARLYDIAGNOSIS AND TREATMENT

G. Ciuti ${ }^{1}$, A. Firrincieli ${ }^{1}$, F. Bianchi ${ }^{1}$, N. Gabrieli ${ }^{2}$, F.P. Falotico ${ }^{2}$, $\bar{J}_{\text {J. Ortega }}{ }^{1}$, M. Verra ${ }^{3}$, M. Bonino ${ }^{3}$, S. Arolfo ${ }^{3}$, E. Mazomenos ${ }^{4}$, P. Brandao $^{4}$, D. Stoyanov ${ }^{4}$, A. Koulaouzidis ${ }^{5}$, S. Schostek ${ }^{6}$, M.O. Schurr $^{6}$, A. Menciassi ${ }^{1}$, C.M. Oddo ${ }^{1}$, A. Arezzo ${ }^{7}$, P. Dario ${ }^{1}$

${ }^{1}$ The BioRobotics Institute, Scuola Superiore Sant'Anna, PONTEDERA, Italy; ${ }^{2}$ Mediate Medical, Mediate Srl, PISA, Italy; ${ }^{3}$ Molinette Hospital, University of Turin, TURIN, Italy; ${ }^{4}$ Computer Science, University College of London, LONDON, United Kingdom; ${ }^{5}$ UEDIN, University of Edinburgh, EDINBURGH, United Kingdom; ${ }^{6}$ OVE, Ovesco Endoscopy AG, TUBINGEN, Germany; ${ }^{7}$ Molinette hospital, University of Turin, TURIN, Italy

Colorectal cancer is the fourth most common cancer in high-income countries counting $>700.000$ deaths worldwide. Survival rate reaches $94 \%$ in case of early diagnosis, falling down to $11 \%$ in case of advanced stage. Conventional colonoscopy screening is limited by invasiveness, pain and often need of sedation. Wireless capsule endoscopy enables inspection without discomfort, but passive locomotion often leads to incomplete and/or false negative results.

The European Endoo Project (Grant Agreement 688592) aims to develop a novel system that overcomes most of the drawbacks of conventional colonoscopy, maintaining accurate and reliable diagnosis and therapy. The system is composed of an active robotic platform that magnetically drives a soft-tethered capsule; magnetic guidance is achieved through the magnetic localization of the capsule in combination with a closed-loop control that maintains an optimal and safe link between the capsule and the magnetic end-effector. A stereoscopic camera is integrated in the capsule for enhanced diagnosis though 3D reconstruction and automated detection of lesions/pathologies.

The different modules of the Endoo medical platform are illustrated in the figures. The robotic guidance systemconsists of an anthropomorphic manipulator that controls the capsule through an external permanent magnet. The robot, positioned on a dedicated trolley, is equipped with sensors for performing safe human-robot collaboration. The medical workstationincorporates: screens, buttons and pedals for visualization and command initiation, a joystick for system teleoperation and a back-end for fluidic control and data communication.

The soft-tethered capsuleembeds an internal permanent magnet, magnetic sensors, an accelerometer, white and infrared illumination and an HD stereoscopic vision system with two wide-angle customized optics. A controller serves as the main control unitfor performing real-time communication and closed-loop control of the robot, localization system, capsule and physician commands.

The synergistic cooperation of academic, industrial and clinical partners within the project allowed to develop and validate the system in in-vitro $\langle/ \mathrm{i}\rangle$, exvivoand preliminary cadaver sessions, performing comparisons with state-ofthe-art commercial colonoscopes. In conclusion, the Endoo medical platform provides: reduced procedural pressures, user-friendly procedures, similar functionalities and performances of commercial devices, comparable procedural times and considerably lower costs with a new painless approach.

\section{O202-AMAZING TECHNOLOGIES}

ROBOT-ASSISTED RADIO FREQUENCY ABLATION OF THE LIVER. FROM THE IDEA TO WORKING PROTOTYPE

D.N. Panchenkov ${ }^{1}$, D.D. Klimov ${ }^{2}$, A.A. Nechunaev ${ }^{3}$, A.S. Levin ${ }^{4}$, O.V. Zairatyans ${ }^{5}$, A.A. Vorotnikov ${ }^{4}$, Yu.V. Poduraev ${ }^{4}$, L.S. Prokhorenko $^{4}$, O.V. Levchenko ${ }^{6}$, V.V. Krylov ${ }^{6}$, O.O. Yanushevich ${ }^{7}$

${ }^{1}$ Minimally Invasive Surgery, A.I. Evdokimov Moscow State University of Medicine and Dentistry, MOSCOW, Russia; ${ }^{2}$ Medical Robotics Digital Technologies, A.I. Evdokimov Moscow State University of Medicine and Dentistry, MOSCOW, Russia; ${ }^{3}$ Endoscopic Surgery, A.I. Evdokimov Moscow State University of Medicine and Dentistry, MOSCOW, Russia; ${ }^{4}$ Robotics and Mechanotronics, Moscow State Technjlogy University STANKIN, MOSCOW, Russia; ${ }^{5}$ Pathology, A.I. Evdokimov Moscow State University of Medicine and Dentistry, MOSCOW, Russia; ${ }^{6}$ Clinical Medical Center, A.I. Evdokimov Moscow State University of Medicine and Dentistry, MOSCOW, Russia; ${ }^{7}$ Paradontology, A.I. Evdokimov Moscow State University of Medicine and Dentistry, MOSCOW, Russia

Background: This study is aimed at the comparison of the process of manual and robotic-assisted positioning of the electrode performing radiofrequency ablation with the usage of multifunctional robot-assisted surgical platform. under the control of the surgical navigation system. The main hypothesis of this experiment was that the use of a collaborative manipulator will allow to position the active part of the electrode relative to the center of the tumor more accurately and from the first attempt. We also check the stability of the electrode's velocity during insertion and consider some advantages in ergonomics using the robotic manipulator. Methods: Sphere-shaped tumor phantoms measuring $8 \mathrm{~mm}$ in diameter were filled with contrast and inserted in cow livers. 10 livers were used for the robotic experiment and an equal quantity for manual. The livers were encased in silicone phantoms. Analysis of CT data gave the opportunity to find the entry and the target point for each tumor phantom. This data was loaded into the surgical navigation system that was used to track and record the position of the RF-electrode during the operation for further analysis.

Results: Standard deviation of points from the programmed linear trajectory totaled in the average $0.3 \mathrm{~mm}$ for the robotic experiment and $2.33 \mathrm{~mm}$ for the manual operation with a maximum deviation of $0.55 \mathrm{~mm}$ and $7.99 \mathrm{~mm}$ respectively. Standard deviation from the target point was $2.69 \mathrm{~mm}$ for the collaborative method and $2.49 \mathrm{~mm}$ for manual method. The average velocity was $2.97 \mathrm{~mm} / \mathrm{s}$ for the manipulator and $3.12 \mathrm{~mm} / \mathrm{s}$ for the manual method, but the standard deviation of the velocity relative to the value of the average velocity was $0.66 \mathrm{~mm} / \mathrm{s}$ and $3.05 \mathrm{~mm} / \mathrm{s}$ respectively.Thus, in two criteria out of three, the manipulator is superior to the surgeon, and equality is established in one. Surgeons also noticed advantages in ergonomics performing the procedure using the manipulator.

Conclusions: This experiment was produced as part of the work on the developing of the robotic multifunctional surgical complex. We can confirm the potential advantages of using robotic manipulators for minimally invasive surgery in case of collaborative practice for cancer treatment. 


\section{O203-AMAZING TECHNOLOGIES}

\section{A NEW TEACHING TECHNOLOGY IMPROVES PERFORMANCE OF A LAPAROSCOPIC TRAINING TASK}

R.R. Postema ${ }^{1}$, L. van Gastel ${ }^{1}$, S.F. Hardon ${ }^{2}$, T. Horeman ${ }^{3}$, H.J. Bonjer $^{1}$

${ }^{1}$ Surgery VUMC, Amsterdam University Medical Centers, AMSTERDAM, The Netherlands; ${ }^{2}$ Surgery VUMC, Delft University of Technology, DELFT, The Netherlands; ${ }^{3}$ Biomechanical Engineering, Delft University of Technology, DELFT, The Netherlands

Background and aims: Laparoscopy has reduced tactile feedback compared to open surgery. In neuropsychological literature there is increasing evidence that visual and haptic information converge to form a mental representation of an object. Through the combination of these inputs, this representation is believed to be more refined and robust. We investigated whether tactile exploration of a lifelike anatomical object before executing a laparoscopic action on this object in a laparoscopic box trainer improves performance of this action.

Description: A randomized prospective cohort study with two groups $(\mathrm{A}+\mathrm{B})$ of ten laparoscopically naïve medical students was conducted. We compared the groups for baseline characteristics and performance, using a basic laparoscopic task (post and sleeve). To investigate the effect of haptic exploration, students performed ten repetitions of a laparoscopic needle action on a lifelike silicone caecum model (Applied Medical, Rancho Santa Margerita, USA). Group A did a pre-test visual exploration of the model. In group B manual exploration of the anatomical model was added to the visual exploration before executing the task. The box trainer was equipped with the Forcesense ${ }^{\mathrm{Tm}}$ (Medishield, Delft, The Netherlands) system for skill assessment using objective force, motion and time parameters.

Results: Baseline characteristics and-laparoscopic performance were comparable $(\mathrm{p}>0,05)$. Performances of 200 trials on the anatomical model were captured and parameter outcomes were compared between groups. Significantly less force (maximal force, maximal impulse, mean force and force volume) was exerted by the 'touch' group $(\mathrm{p}<0.000)$ (Fig. 1). This group also completed the task with less distance travelled by the instruments $(p<0,003)$. There was no significant difference in time needed to complete the task $(p=0,695)$.

Conclusion: This study showed that, when performing a laparoscopic task on an anatomical model, pre-task haptic exploration of the model results in the use of significantly less force and less movement. Adding haptic exploration to a laparoscopic training curriculum could therefore result in more efficient and more refined learning of laparoscopic actions. This, in turn, could lead to better, quicker and safer performance of laparoscopic operations.

\section{O204-COLORECTAL-Malignant}

\section{IS SMOKING COMPATIBLE WITH COLORECTAL ENHANCED RECOVERY AFTER SURGERY: 1 YEAR REVIEW}

\section{Okocha, N.J. Browning, T. El Jichi, S. Shafiq, H. Sumrien}

General surgery, NHS, BRISTOL, United Kingdom

Aim: To demonstrate the relation between smoking and length of hospital stay in Enhanced Recovery after Surgery (ERAS) patients, who underwent elective major colorectal surgery in a tertiary referral centre-North Bristol NHS Trust-(NBT).

Background: The NBT ERAS programme is well established and all patients who undergo colorectal resection are included. Smoking increases risk of poor postoperative outcomes, general morbidity and wound complications. NBT is a smoke-free trust, and it became noticeable that smokers mobilise earlier after surgery to get to smoking zones. Previous studies showed that less than $7.5 \%$ of smokers stop smoking in the perioperative period.

Methods: Prospective review of data collected from ERAS patients who had elective colorectal resection between January 2017 and January 2018. Outcomes collected included, type of surgery, open or laparoscopic \pm conversion, and length of hospital stay. Smoker and non-smoker groups were compared in terms of post-operative length of stay.

Results: Between January 2017 and January 2018, we performed 200 major colorectal resections, of these $40 \%$ were on Female patients (82) (males 118). Procedures included 51 right hemicolectomies (including extended), 70 anterior resections/TME, 10 APER, 14 Hartmann's reversals and 55 Left Hemicolectomies, Transverse colectomies, subtotal colectomies and segmental colectomies. 140 (69\%) cases were laparoscopic, $41(20 \%)$ open and $21(14 \%)$ were laparoscopic converted to open. Mean hospital stay 8 days, median 5 days, re-admission rate was $15.3 \%$ and SSI was $8.1 \%$. Mean age of smokers was 62, range 30-86. Mean age of non-smokers was 66, range (19-93). All smokers were advised to stop smoking prior to surgery during enrolment on our ERAS programme. Of the 200 patients 49 were smokers, 148 non-smokers and 3 patients' smoking status was unknown. Of the smokers $4 \%$ (2) ceased smoking with advice. Mean length of hospital stay for smokers was 6.81 and non-smokers was 7.13 days

Conclusion: Smokers remain a challenging group in terms of compliance and the majority of patients who are advised to stop smoking prior to surgery did not. Although smokers seemed to mobilise early postoperatively, that did not translate into a reduction of hospital stay.

\section{O205-AMAZING TECHNOLOGIES}

\section{EFFICACY ANS SAFETY OF GASTRIC ARTERY EMBOLIZATION FOR WEIGHT MANAGEMENT IN OBESE USING ENDOBAR LAMINA SYSTEM}

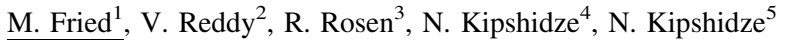

${ }^{1}$ Center for Treatment of Obesity and Metabolic Disorders, OB klinika, PRAGUE, Czech Republic; ${ }^{2}$ Mount Sinai Hospital, New York, USA; ${ }^{3}$ Lenox Hill Hospital, New York, USA; ${ }^{4}$ New York University, New York, USA; ${ }^{5}$ New York Cardiovascular Research, New York, USA

Background: Obesity is growing epidemic, associated with adverse cardiovascular and metabolic conditions. Number of weight loss strategies are unsuccessful and/or accompanied with risks. Ghrelin is a orexigenic hormone. Catheter-directed left Gastric Artery Bariatric Embolization (GABE) causes a reduction in plasma ghrelin levels and weight loss as demonstrated in animal models and several pilot clinical studies.

Aim: To evaluate the efficacy and safety of the Endobar Lamina embolization system (ELES) for the treatment of obesity.

Project description: 44 obese patients, BMI $35.0-55.0 \mathrm{~kg} / \mathrm{m}^{2}$ were randomized to blinded treatment with $\operatorname{GABE}(n=22)$ or sham procedure $(n=22)$. GABE was performed using ELES (Endobar Solutions, LLC, Orangeburg, NY, USA) and BeadBlock Embolic Bead 300-500 mm microspheres (Biocompatibles Limited, UK). Esophagogastroscopy was performed before GABE and 1-week post-procedure assessing gastric abnormalities. Weight and fasting plasma ghrelin were obtained at baseline, 1-, 3-, 6- and 12-months post-index procedure. After 6 months, the Sham group was unblinded and received GABE. Both GABE and Sham crossover to GABE groups were followed for 12 months and received Lifestyle Therapy (behavioral-diet education). Preliminary results: GABE was successful in all patients with no serious complications. Significant, progressive weight loss was observed at 6 and maintained at 12 months. Ghrelin in GABE group decreased by $22 \%(67.91 \mathrm{pg} / \mathrm{ml})$ compared to baseline and 12 months levels. Weight-loss was approximately $6.5 \%$ greater in the GABE group versus sham at 6 months (Table 1).

ITT $=$ Intent-to-treat, PP = Per-protocol

${ }^{\dagger}$ Analysis preformed using independent-sample t-test and ${ }^{*}$ Paired-sample t-test

Conclusions: GABE using ELES is safe, accompanied by significant and so far maintainable weight loss. GABE using the ELES demonstrated a reduction in ghrelin levels. 


\section{O206-UPPER GI-Gastric cancer}

\section{LAPAROSCOPIC VERSUS OPEN GASTRECTOMY FOR GASTRIC CANCER, Results: OF A MULTICENTER PROSPECTIVELY RANDOMIZED CONTROLLED TRIAL (LOGICA-TRIAL)}

R. Van Hillegersberg ${ }^{1}$, A. Van der Veen ${ }^{2}$, H.J.F. Brenkman ${ }^{2}$, M.F.J. Seesing ${ }^{2}$, L. Haverkamp ${ }^{2}$, M.D.P. Luyer ${ }^{3}$,

G.A.P. Nieuwenhuijzen ${ }^{3}$, J.H.M.B. Stoot ${ }^{4}$, K.W.E. Hulsewé ${ }^{4}$, B.P.L. Wijnhoven ${ }^{5}$, W.O. De Steur ${ }^{6}$, E.A. Kouwenhoven ${ }^{7}$, E.B. Wassenaar ${ }^{8}$, W. Draaisma ${ }^{9}$, S.S. Gisbertz ${ }^{10}$, D.L. Van der Peet ${ }^{11}$, J.P. Ruurda ${ }^{2}$, . LOGICA study group ${ }^{12}$

${ }^{1}$ Department of Gastrointestinal Surgical Oncology, University Medical Center Utrecht, UTRECHT, The Netherlands; ${ }^{2}$ Surgery, University Medical Center Utrecht, UTRECHT, The Netherlands; ${ }^{3}$ Surgery, Catharina Hospital, EINDHOVEN, The Netherlands; ${ }^{4}$ Surgery, Zuyderland Medical Center, HEERLEN AND SITTARD-GELEEN, The Netherlands; ${ }^{5}$ Surgery, Erasmus University Medical Center, ROTTERDAM, The Netherlands; ${ }^{6}$ Surgery, Leiden University Medical Center, LEIDEN, The Netherlands; ${ }^{7}$ Surgery, ZGT Hospitals, ALMELO, The Netherlands; ${ }^{8}$ Surgery, Gelre Ziekenhuizen, APELDOORN, The Netherlands; ${ }^{9}$ Surgery, Meander Medical Center, AMERSFOORT, The Netherlands; ${ }^{10}$ Surgery, Academic Medical Center and Cancer Center, AMSTERDAM, The Netherlands; ${ }^{11}$ Surgery, VU University Medical Center, AMSTERDAM, The Netherlands; ${ }^{12}$ The Netherlands

Introduction: Open gastrectomy is the preferred surgical approach for gastric cancer worldwide. This procedure is associated with considerable morbidity. Meta-analyses have shown an advantage in short-term outcomes of laparoscopic gastrectomy compared to open procedures, with similar oncologic outcomes. However, the included series are mostly from Asia with early gastric cancer. It is unclear whether these results can be extrapolated to the Western population with mostly advanced gastric cancer. In this randomized controlled multicenter trial from the Netherlands, we assessed the outcomes of laparoscopic versus open gastrectomy. Methods: Between 2015 and 2018, patients with resectable (cT1-4a, N0$3 \mathrm{~b}$, M0) gastric adenocarcinoma were randomly assigned to either laparoscopic (105 patients) or open (105 patients) gastrectomy, in 10 participating centers in the Netherlands. Inclusion criteria were age $=18$ years, European Clinical Oncology Group performance status 0, 1 or 2 and informed consent. The primary outcome was postoperative hospital stay (days). Secondary outcome were postoperative morbidity and mortality, oncologic outcome, readmissions, quality of life and cost-effectiveness. Results and conclusion: This is a late breaking abstract. The data are not yet mature at the moment of writing this abstract.

\section{O207-ROBOTICS \& NEW TECHNIQUES-Colorectal}

OBSERVATIONAL CLINICAL HUMAN RELIABILITY ANALYSIS OF TRANSANAL TOTAL MESORECTAL EXCISION: Results: FROM 100 CLINICAL CASES

$\underline{\text { M. Penna }}{ }^{1}$, R. Hompes ${ }^{2}$, N. Mortensen ${ }^{3}$, P.P. Tekkis ${ }^{4}$, G.B. Hanna ${ }^{5}$

${ }^{1}$ Academic Surgery, Imperial College London, LONDON, United Kingdom; ${ }^{2}$ Colorectal surgery, Amsterdam UMC, AMSTERDAM, The Netherlands; ${ }^{3}$ Colorectal surgery, University of Oxford, OXFORD, Uganda; ${ }^{4}$ Colorectal surgery, Imperial College London, LONDON, United Kingdom; ${ }^{5}$ Cancer and Surgery, Imperial College London, LONDON, United Kingdom

Aims: Transanal total mesorectal excision (TaTME) is the latest colorectal approach that continues to be in the spotlight. This study aims to describe the technique in depth by identifying and understanding technical advantages, errors and adverse events.

Methods: Detailed video analysis using Observational Clinical Human Reliability Analysis (OCHRA) was completed on 100 clinical TaTME cases performed by 27 international surgeons. Error frequency and error pathways leading to adverse events were described. TaTME expert surgeons were interviewed and engaged in a workshop to elicit error-reducing mechanisms.

Results: Overall technical errors and adverse events per procedure on average occurred $49 \pm 32.9$ (range 6-194) and $9 \pm 6.1$ (range 1-45) times respectively. Inadequate insufflation and poor camera optics were the most frequent set-up problems. Instrument handling errors consisted most commonly of excessive grasper movement during the pursestring phase (321 times total), inappropriate force applied (79 times) with the energy device during the rectotomy, inappropriate force with the grasper (74 times) and excessive movement with the energy device (117 times) during TME dissection. Incorrect dissection planes were created during TME dissection mostly due to insufficient retraction (127 times) which didn't allow adequate exposure of the tissue planes. The most frequently occurring consequence was bleeding (Mean: 6 times per procedure). Rectal perforation (7 cases), vaginal wall injury (4 cases), and prostatic injury ( 7 cases) were also recorded. Adverse events regularly occurred as a result of poor set-up/exposure, inappropriate retraction and/or instrument movement and incorrect plane surgery. Error-reducing mechanisms and 'technical tips' describe specific steps and actions, both set-up/equipmentrelated and technique-related, that aim to prevent errors from occurring and avoid adverse consequences. OCHRA and individual feedback with error-reducing mechanisms developed by this study have been implemented into the national training programme for TaTME.

Conclusion: TaTME is an advanced complex procedure during which technical errors and their consequences are not infrequent. TaTME requires knowledge of anatomy 'bottom-up', familiarity with its specialised equipment and technical skill working in a narrow space. Appropriate structured training and mentorship are therefore recommended. 


\section{O207-COLORECTAL-IBD}

INTRAOPERATIVE ANGIOGRAPHY WITH INDOCYANINEGREEN TO ASSESS ANASTOMOSIS PERFUSION IN PATIENTS UNDERGOING LAPAROSCOPIC COLORECTAL RESECTION: Results: OF A RANDOMIZED CONTROLLED TRIAL

G. Maggi ${ }^{1}$, P. De Nardi ${ }^{1}$, R. Maggiore ${ }^{1}$, L. Boni ${ }^{1}$, E. Cassinotti ${ }^{1}$, U. Fumagalli ${ }^{1}$, M. Gardani ${ }^{1}$, U. Elmore ${ }^{2}$, A. Vignali ${ }^{2}$, R. Rosati ${ }^{2}$

${ }^{1}$ San Raffaele Hospital, MILAN, Italy; ${ }^{2}$ Gastrointestinal Surgery Unit, San Raffaele Hospital, MILAN, Italy

Objective: Insufficient vascular supply is one of the main causes of anastomotic leak in colorectal surgery. ICG has been shown to provide information on tissue perfusion, identifying a well-perfused location for colonic and rectal transections and thus possibly reducing the leak rate. Objective of this study is to evaluate the usefulness of intraoperative assessment of anastomotic perfusion using intraoperative indocyanine-green dye (ICG) angiography in patients undergoing left-sided colon or rectal resection with colorectal anastomosis.

Methods: This randomized trial involved 252 patients undergoing laparoscopic left-sided colon and rectal resection randomized 1:1 to intraoperative ICG or to subjective visual evaluation of the bowel perfusion without ICG (ClinicalTrials.gov NCT02662946). The primary aim was to assess whether ICG angiography could lead to a reduction in anastomotic leak rate. Secondary outcomes were possible changes in the surgical strategy and postoperative morbidity.

Results: After randomization, 12 patients were excluded. Accordingly, 240 patients were included in the analysis; 118 in the study group, and 122 in the control group. ICG angiography showed insufficient perfusion of the colic stump, which led to extended bowel resection, in 13 cases $(11 \%)$. An anastomotic leak developed in 11 patients $(9 \%)$ in the control group and in 6 patients $(5 \%)$ in the study group $(\mathrm{p}=\mathrm{n} . \mathrm{s}$.).

Conclusion: Intraoperative ICG fluorescent angiography can effectively assess vascularization of the colic stump and anastomosis in patients undergoing colorectal resection. This method led to further proximal bowel resection in 13 cases, however its role in reducing anastomotic leak rate should be studied in further research.

\section{O208-BARIATRICS-Laparoscopic}

\section{BARIATRIC SURGERY PATIENT'S WEIGHT REDUCING EXPERIENCE PRIOR SURGERY}

G. Bilande ${ }^{1}$, M. Mukans ${ }^{2}$, E. Liepins ${ }^{3}$, O. Kozlovskis ${ }^{4}$, J. Zarinovs ${ }^{5}$, I. Troickis $^{3}$

${ }^{1}$ Department of Surgery, Jurmala hospital, JURMALA, Latvia; ${ }^{2}$ Surgery, Aiwa clinic, RIGA, Latvia; ${ }^{3}$ Surgery, Jurmala hospital, JURMALA, Latvia; ${ }^{4}$ Surgery, Sigulda hospital, SIGULDA, Latvia; ${ }^{5}$ Surgery, Riga 1 st hospital, RIGA, Latvia

Aims: The objective of the current study was to evaluate lifestyle habits and weight loss experience of obese patients prior to bariatric surgery.

Methods: This is 1 year quantitative cross-sectional study that was carried out in four clinics in Latvia. The subjects of this study were patients who applied for bariatric surgery and their participation in questionnaire was voluntary and random.

Results: There were 72 individuals ( 63 woman and 9 man; age 30-49 years). Maximum lifetime weight range was $132-96 \mathrm{~kg}$ with average patient BMI $37.6 \mathrm{~kg} / \mathrm{m}^{2}$. At least 1 co-morbity was admitted by $27 \%$ of patients. $54.8 \%$ of patients are eating regular breakfast. Most of subjects became obese in adolescence (26\%) or in adulthood (37\%). Only 14 patients succeeded to $(19.2 \%)$ reduce weight prior surgery; 20 subjects $(27.4 \%)$ weight was relatively stable, but rest patient's $(53.3 \%)$ weight increased. $98 \%$ of all patients had tried different weight loss methods before. There is linkage between patient's age and food consumption—subjects aged under 37 years regularly overeat $(61.3 \%)$, but elder ones are more likely to have regular meals $(72.2 \%)$. There is lack of physical activity-only $30.1 \%$ of patients are active more than $40 \mathrm{~min}$ a day. Over $70 \%$ of patients spend at least $4 \mathrm{~h}$ per day in sitting position.

Conclusions: The results highlight that all patients unsuccessfully tried to reduce weight by othe methods before bariatric surgery. The reason for poor preparation was irregular meals, breakfas avoidance, low physical activity and there also was impact of patient's age. Additional research and data are needed to explore how regular meals, adequate physical activity, combined with other weight reducing methods could give better results to prepare for bariatric surgery.

\section{EAES VIDEO AWARD SESSION}

\section{V001-BARIATRICS—Endoluminal}

ENDOSCOPIC SLEEVE GASTROPLASTY: HOW SURGEONS SEE BEYOND THE VISIBLE

M. Pizzicannella ${ }^{1}$, C. Fiorillo ${ }^{1}$, P. Mascagni ${ }^{1}$, M. Vix ${ }^{2}$, D. Kadoche ${ }^{2}$, D. Mutter ${ }^{2}$, J. Marescaux $^{3}$, S. Perretta

${ }^{1}$ IHU, STRASBOURG, France; ${ }^{2}$ Digestive and Endocrine Surgery, Nouvel Hopital Civil, University of Strasbourg, STRASBOURG, France; ${ }^{3}$ IRCAD Research Institute against Digestive Cancer, STRASBOURG, France

Endoscopic sleeve gastroplasty (ESG) is a promising endoscopic bariatric procedure carried out with the application of transmural sutures resulting in a gastric reduction and gastric shortening. Sutures are placed in $\mathrm{U}$ shape fashion, from the incisura to the fundus, which is preserved, using an over the endoscope suturing platform (OverStitch, Apollo Endosurgery, Austin, Texas, USA) The choice of right lankmarks for suturing the gastric wall is extremely important for the efficacy and safety of the procedure. Flexible endoscopy suffers from little anatomical reference points. Correct spatial relation to precisely target the insertion of the helix device used for retraction and correct orientation of the full thickhness tissue bite require a good undrestanding of the anatomy of the stomach and sourrounding organs including vascular structures that could be inadvertently injured (left lobe of the liver, gallbladder, spleen, short gastric vessels, pancreas, transverse colon). Surgeons by training can 'see' the anatomy beyond the gastric wall and undrestand whether they work in a safe layer or whether an underlying structure should be spared. This video illustrates all the potential risks realted with a wrong chioce of endoscopic landmarks when performing ESG with respect to gastric and abdominal anatomy.

\section{V002-HEPATO-BILIAIRY \& PANCREAS-Liver}

\section{ROBOTIC ANATOMICAL HEPATECTOMIES FOR DIFFICULT SEGMENTS}

\author{
S.H. ChoiJ.W. Lee, J.Y. Jang
}

\section{Surgery, Bundang CHA Medical Center, SEONGNAM-SI, Korea}

Introduction: Central bisectionectomy, anterior sectionectomy, and posterior sectionectomy are technically demanding procedures in minimally invasive approach because of difficult expoure and extensive parenchymal transection planes. With limited robotic instruments including absence of CUSA, these procedures have been rarely perfomed by robotic approach.

Method: Consecutive robotic central bisectionectomy, anterior sectionectomy, and posterior sectionectomy were performed. Patients were all males and were 67,71, and 41-years-old, respectively. Pathologic diagnoses were all hepatocellular carcinomas of each 4.4, 4.2, and $3.2 \mathrm{~cm}$ diameter. Operative settings were identical for the three kinds of procedure. The patients were placed in supine with a reverse Trendelenburg and right side elevation. Umbilical 12-mm camera port, three $8-\mathrm{mm}$ ports and additional $12-\mathrm{mm}$ assistant port were used. Glissonian approach and ICG fluorescence image clearly demarcated the resection planes. Parenchymal transection was performed using the Maryland bipolar dissector and harmonic scalpel. The rubber band self-retraction method and third arm of robot system helped for stable and excellent exposure of surgical planes

Result: There were no conversions to laparoscopic or open surgery. The operative time was 320 , 330 , and $290 \mathrm{~min}$ and estimated intraoperative blood loss was 200,330, and $250 \mathrm{ml}$. The pathologic surgical margin was $2.5,0.5$, and $3.6 \mathrm{~cm}$. The length of stay after surgery was 7,8 , and 6 days and there were no postoperative complications.

Conclusion: Robotic central bisectionectomy, anterior sectionectomy, and posterior sectionectomy are still demanding procedures with long operative time. However, these procedures could be performed safely in regard to short-term perioperative outcomes. Robot surgical system provided several benefits for anatomical hepatectomies including a stable and excellent operative field and clear surgical planes. 


\section{V003-HERNIA-ADHESIONS—Abdominal wall hernia \\ MINIMALLY INVASIVE REPAIR OF SUPRAPUBIC HERNIA, A CHALLENGE FOR THE SURGEON}

N. García Fernández, P. Garcia Muñoz, R.E. Licardie Bolaños², I. Alarcon del Agua, J. Tinoco Gonzalez, L. Tallon Aguilar, J. Padillo Ruiz, S. Morales Conde

Cirugía General Y Del Aparato Digestivo, Hospital Universitario Virgen Del Rocio, SEVILLA, Spain

Suprapubic hernias (less than $5 \mathrm{~cm}$ above the pubic arch in the midline) require important anatomical knowledge because of complexity of their repair and low incidence, by approximately $2 \%$ of all hernias. The problem to repair this type of hernias is that inferior margin of the defect is very close to pubic symphysis, consequently, mesh overlap is often inadequate. Treatment of suprapubic hernias is controversial because of limited evidence in the literatura.

This video shows the case of a 40-year-old female patient with suprapubic hernia with a defect of $3 \times 3 \mathrm{~cm}$. We performed a laparoscopic repair with a bilateral peritoneal flap of the groin region (as it is perfromed during TAPP) for proper view of the pubic symphysis, Cooper's ligaments, epigastric and major vessels, nerves and meticulous dissection the space of Retzius. The defect was repaired by reconstructing the middle line with a running sutures. Subsequently, titanium helical tacks were used to fix the mesh to the pubis and Cooper and following the double-crown technique having special attention when fixing the mesh near to inguinal chanal, due to the possibility of causing chronic pain. The peritoneal flap was fixed over the mesh with abdsorbable fixation devices and seal with fibrin glue.

Laparoscopic repair of suprapubic hernias can be considered as the first option in treatment, because it endeavors to join the advantages of a minimally invasive approach and it is associated to low recurrence. The main advantages are that allows a proper visualization the anatomy and a proper fixation of the mesh.

\section{V005-ROBOTICS \& NEW TECHNIQUES-Colorectal}

\section{IMPROVING ACCESS IN THE FEMALE PELVIS-A TECHNICAL TIP}

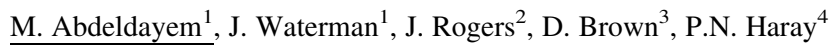

${ }^{1}$ Colorectal Surgery, Prince Charles Hospital, MERTHYR TYDFIL, United Kingdom; ${ }^{2}$ Obstetrics and Gynaecology, Prince Charles Hospital, MERTHYR TYDFIL, United Kingdom; ${ }^{3}$ Digimed ${ }^{\circledR}$, ESSEX, United Kingdom; ${ }^{4}$ Colorectal Surgery, University of South Wales, WALES, United Kingdom

Background: Laparoscopic Total Mesorectal Excision (TME), in a wide female pelvis is usually technically easier than in a narrow male pelvis. However, this is not always the case, as the uterus and adnexae may obscure the views and hinder safe dissection, especially in obese patients. Techniques such as graspers through additional ports or suspension with sutures through the broad ligament may potentially cause injury or need additional ports/assistants.

Aim: We present a novel technique using a self-retaining gynaecological uterine manipulator to improve access during deep pelvic laparoscopic surgery in female patients.

Technical tip: The operation is commenced in the standard manner for a laparoscopic rectal excision. Once pelvic dissection is commenced, whenever it is felt that uterine retraction would be advantageous (depending on the level of the rectal tumour, size of the uterus and ovaries, obesity etc.) a self-retaining uterine manipulator (as shown in the video) is used. The tip of this disposable device is introduced into the uterus after dilatation of the uterine cervix. Once the balloon at the tip has been inflated, the instrument is secure and hence there is no need for active manipulation by an assistant. The shaft can be rotated to allow anteversion/retroversion of the uterus to varying degrees as required to aid dissection. As the video depicts clearly, it acts as a self-retaining retractor for the uterus and is removed at the end of the operation. Though the procedure is being demonstrated by a gynaecologist in the video, the instrument is quite easy to insert and some of our colorectal team have been trained as well.

Conclusion: The self-retaining uterine manipulator is an efficient tool for uterine retraction in laparoscopic rectal surgery and we have been using it routinely in TME in females for the past 8 years, with no complications. This was previously published as a technical tip in the Journal of minimal access surgerybut has never been submitted for peer review as a video.

\section{V006-UPPER GI-Benign Esophageal disorders}

\section{TRANS-AXILLARY ENDOSCOPIC APPROACH OF ZENKER'S DIVERTICULUM-INNOVATIVE SURGICAL TECHNIQUE}

\author{
H. Cardoso-Louro, S. Graça, L. Lencastre, A. Fonte-Boa, J. Vilaça
}

\section{General Surgery, Hospital da Luz Arrábida, PORTO, Portugal}

Aims: Since the early $2000 \mathrm{~s}$ new cervical endoscopic approaches have been described. Zenker's diverticulum (ZD) is a false diverticulum at the level of the upper oesophageal sphincter resulting from a motor dysfunction between the lower pharyngeal constrictor muscle and the cricopharyngeal muscle (Killian triangle). The surgical treatment consists of cricopharyngeal myotomy with or without diverticulectomy.

The authors present a video of two clinical cases treated by trans-axillary endoscopic approach.

Methods: A 74 years-old male and a 73-year-old male presented with intermittent dysphagia and frequent reflux (class II of Lahey). One had a history of recurrent respiratory infections. The disease was characterized by oesophagogastroscopy (EGD) and oesophagogram.

Trans-axillary approach with areolar port. Step-by-step as follows: (i) dissection anteriorly to the pectoralis major muscle (ii) isolation of the anterior border of sternocleidomastoid muscle (iii) omohyoid muscle's isolation (iv) identification of the thyroid's upper pole (v) ZD isolation (vi) myotomy of the cricopharyngeal muscle (vii) ZD's resection with stapler and its withdrawn with sac.

Results: Both cases progressed without complications. Complete local recovery was verified in both cases one month after the procedure.

Conclusion: This technique seems feasible and reproducible, allowing ZD diverticulectomy with a better cosmetic result and perhaps lower surgical site infections (SSI). In the authors' knowledge, this approach to DZ has never been published. 


\section{V007-UPPER GI-Esophageal cancer}

MINIMALLY INVASIVE ESOPHAGECTOMY AND RADICAL LYMPH NODE DISSECTION WITHOUT RECURRENT LARYNGEAL NERVE PARALYSIS

K. Otsuka, M. Murakami, S. Goto, S. Akira, K. Fujimasa, M. Kohmoto, R. Kato, T. Ariyoshi, T. Yamashita, T. Aoki

Gastroenterological and General Surgery, Showa University, TOKYO, Japan

Introduction: Recurrent laryngeal nerve paralysis(RLNP) is considered a major postoperative complication of esophageal surgery. Although it depends on the extent of lymph node dissection performed, the incidence of RLNP after esophagectomy is reported from $8.3 \%$ to $40.9 \%$ By avoiding direct nerve injury, unreasonable traction force, thermal damage and other factors associated with RLNP, the incidence of RLNP should be minimized. We reviewed dissection techniques to limit the occurrence of RLNP, with specific attention to the anatomical layer around the recurrent laryngeal nerve. This technique is no touch, no traction and results in no injury to the recurrent laryngeal nerve, maintained in its anatomical position.

Methods: From September 2016 to December 2018, minimally invasive esophagectomy was performed in the left lateral decubitus position in 83 patients with esophageal cancer. The NoTouch Dissection technique for lymphadenectomy around the recurrent laryngeal nerve was performed and all patients evaluated for recurrent laryngeal nerve paralysis.

Results: Minimally invasive esophagectomy was completed in all patients without conversion to thoracotomy. Although an extended lymphadenectomy was performed in all patients, there were no grade II or higher complications (Clavien-Dindo classification) and no incidence of recurrent laryngeal nerve paralysis.

Conclusion: This No-Touch Dissection technique may reduce the incidence of recurrent laryngeal nerve paralysisafter minimally invasive esophagectomy with radical lymph node dissection.

\section{VIDEO PRESENTATIONS}

\section{V008-BARIATRICS-Laparoscopic}

\section{REVISIONAL SURGERY FOR EARLY GASTRIC LEAK AFTER} ROUX-EN-Y GASTRIC BYPASS (RYGB)

S. Sanchez-Cordero ${ }^{1}$, R. Vilallonga ${ }^{2}$, R. Roriz ${ }^{2}$, M. Kraft ${ }^{2}$, E. Caubet $^{2}$, O. Gonzalez ${ }^{2}$, Y. Curbelo ${ }^{2}$, J.M. Fort ${ }^{2}$, M. Armengol ${ }^{2}$

${ }^{1}$ General Surgery Department, Consorci Sanitari de l'Anoia, IGUALADA, Spain; ${ }^{2}$ Endocrine-Metabolic and Bariatric Surgery, Hospital Universitari Vall d'Hebron, BARCELONA, Spain

Background: Gastric leak occurs in 1-6\% of patients who undergo Roux-en Y gastric bypass (RYGB) for morbid obesity. The pathophysiology may be related to gastric ischemia, fistula, or ulcer.Gastric leak is a severe complication of gastric bypass (GBP) that is associated with significant morbidity and mortality. Fistula may have several clinical impacts, depending on patientrelated factors, fistula characteristics, onset time, and therapy proposal. Abdominal drainage, gastrostomy, and revisional surgery constitute the traditional approaches to dehiscence and fistula closure, with variable results.

Methods: We present a video of a clinical case of 44-year-old lady with body mass index of 45 $\mathrm{Kg} / \mathrm{m}^{2}$ who underwent Roux-en-Y gastric bypass and $48 \mathrm{~h}$ later presentedtaquicardia and right cuadrantum pain. The CTscan inform a apical leak at the gastric pouch level. The video shows the relevant aspects of a revisional surgery and the key points to drain the fistula and close de defect laparoscopically

Results: After 6 monts, the patient achieved succesful results, defined as a stabel clinical situation with image evidence of gastric fistula remision.

Conclusions: Gastric bypass (GBP) is one of the most efficient bariatric interventions in morbidly obese patients. The most severe risk of this procedure seems to be the staple line leak, and the management of this complication can be very arduous. Without any guidelines it is very difficult to determine the right procedure addressing the staple line leak after GBP.

\section{V009-BARIATRICS-Laparoscopic}

LAPAROSCOPIC COVERSION OF SLEEVE GASTRECTOMY TO ROUX EN Y GASTRIC BYPASS OR ONE ANASTOMOSIS GASTRIC BYPASS

L. Castagneto Gissey, G. Casella

Department of Surgical Sciences, Sapienza University of Rome, ROME, Italy

Laparoscopic sleeve gastrectomy (LSG) has become the most commonly performed operation worldwide as a primary bariatric/metabolic procedure. However, conversion to other surgical procedures such as Roux-en-Y gastric bypass (RYGB) or one anastomosis gastric bypass (OAGB) have been described as treatment options for inadequate weight loss after LSG and unresolved co-morbidities or complications such as leak, stricture, and severe gastroesophageal reflux disease (GERD). We present two clinical cases of weight regain and severe GERD and dysphagia, which account for the main indications to reversal of LSG to either OAGB or RYGB.

\section{V010-BARIATRICS-Laparoscopic}

\section{REPRODUCTIBLE TECHNIQUE TO PERFORM A LAPAROSCOPIC POLIPROPILENE MESH BANDED GASTRIC BYPASS}

M.J. García-Oria, A. Sánchez, E. Jimenez, P. Pla, C. León-Gámez, L. Román, X. Remirez, X. Rial, A. Equisoain, D. Gonzalez, V. Polaino, M. Suarez, E. Iglesias, J.A. Rivera

Cirugía General y del Aparato Digestivo, Hospital Universitario Puerta de Hierro Majadahonda, MADRID, Spain

Aims: We show in the video the surgical technique that we perform by laparoscopic aproach, in order to construct a Roux-en-Y polipropilene banded gastric bypass LRYGB-B.

Methods: We are performing this procedures within a prospective randomized trial that is design to compare the long term results of LRYGB-B versus the standard laparoscopic Roux-en-Y gastric bypass. The video shows our technique in a case of a 46 years old female with a BMI of 46 $\mathrm{Kg} / \mathrm{m} 2$. First we create a vertical gastric pouch of about $25-30 \mathrm{ml}$, and a polypropylene mesh $(10 \times 65 \mathrm{~mm}$ ) is placed $20-30 \mathrm{~mm}$ proximal to the anastomosis around the gastric pouch, with the help of a laparoscopic band retractor. After that a $150 \mathrm{~cm}$ Roux-en-Y limb is constructed in an antegastric antecolic fashion, been the lenght of the biliary limb $100 \mathrm{~cm}$. A $25 \mathrm{~mm}$ gastroyeyunal anastomosis is performed with a linear stapler, and the enterotomy and gastrostomy are closed with a $3 / 0$ barbed running sutures. Jejunojejunostomy anastomosis is constructed in similar fashion, but with a lenght of 30-45 mm. The Petersen space and the mesenteric defect are closed with polipropilene $0 / 0$ sutures.

Results: 31 patients has been operated following this technique, and there has been no complications related to the polipropilene band. (The ramdomized prospective trial is still ongoing). Conclusions:The video shows a reproductible easy way to perform a LRYGB-B using a polipropilene mesh. 


\section{V011-BARIATRICS-Laparoscopic}

GASTRIC BANDING FOR SEVERE DUMPING AFTER ROUXEN-Y GASTRIC BYPASS

\section{Ooms, J. Colpaert, G. Uijtterhaegen, S. Sagaama, B. Dillemans}

Department of Surgery, AZ Sint-Jan, BRUGGE, Belgium

Introduction: A 23-year old female patient presented at our clinic two years after initial Rouxen-Y gastric bypass. She had had a preoperative BMI of 31,5 and had a significant weight loss which resulted in a BMI of 21,4 at two years postoperatively. She currently suffered from severe dumping with glycaemia levels dropping to $30 \mathrm{mg} / \mathrm{dL}$. Pharmacological treatment with metformine, sandostatine and acarbose did not yield any results. On top of these problems she felt less restriction, could eat large portions and had gained $9 \mathrm{~kg}$ in the last three months.

Objective: The usual approach for severe dumping-related hypoglycemia would be to undo the gastric bypass. This patient however was extremely anxious to regain weight, so we sought other options. We assumed that by adding more restriction and slowing down the emptying of the gastric pouch we could alleviate some —if not all—of the dumping related symptoms and prevent further weight regain.

Methods: In this video we present the banding of a gastric pouch for severe dumping after Rouxen-Y gastric bypass.

Results: Although unconventional, the banding of the pouch yielded excellent results. The slowe pouch emptying and reduced portions resulted in a near complete remission of all symptoms. As an additional benefit we found a slight weight loss of four kilograms six weeks postoperatively. Conclusion: The usual treatment of severe dumping-related hypoglycemia would be an undo of the gastric bypass. In this case however the patient was extremely anxious to regain weight, being very pleased with the results her gastric bypass had yielded. In agreement with both the patient and treating endocrinologist we attempted a different approach. The slower pouch emptying and increased restriction offered another way to alleviate the dumping and deep hypoglycemia while concomitantly resulting in weight maintenance.

\section{V013-BARIATRICS-Laparoscopic}

HOW TO AVOID ENDOSCOPIC STENT MIGRATION: A NEW TECHNIQUE TO FACILITATE THE HEALING PROCESS AFTER LAPAROSCOPIC SLEEVE GASTRECTOMY

G. David, G. Cesana, F. Ciccarese, M. Uccelli, R. Giorgi, R. Villa, S. de Carli, G. Poli, A. Zanoni, A. Oldani, S. Olmi

General Surgery, Policlinico San Marco, ZINGONIA, BG, Italy

Aim: The aim of this video is to present a novel surgical technique to avoid stent migration after endoscopic placement in patients with leakage subsequent to laparoscopic sleeve gastrectomy (LSG).

Methods: This video shows the case of a patient (BMI $46,6 \mathrm{~kg} / \mathrm{m} 2$ ) who developed an upper gastric leakage 2 days after LSG. A CT scan showed a small leakage at the EG junction complicated by intra-abdominal abscess. A CT guided percutaneous drainage of the abscess was performed. A stent placement was attempted endoscopically three times and failed for migration. We decided to place laparoscopically a non adjustable gastric ring (NAGR) around the stomach, in order to avoid stent migration.First of all the stent is replaced endoscopically in order to cover the fistula tract. The patient is placed in a half sitting position and the pneumoperitoneum was obtained using a Veress needle in left subcostal space. A 4 port technique is used as in standard laparoscopic sleeve gastrectomy.The procedure starts with the mobilization of adhesions, the fistula is identified in the upper part of the tubule.The gastric tubule is isolated and the lesser omentum is opened. The blunt needle at the tip of the ring is passed retrogastrically, a tourniquet can be useful is the positioning turn out to be difficult. The NAGR is then closed over the gastric tubule containing the stent. A drain is finally placed.

Results: The stent was removed after 4 weeks. A gastrointestinal CT scan with oral contrast showed a complete resolution of leakage. After 6 months the patient was in a good condition with BMI $29,4 \mathrm{~kg} / \mathrm{m}^{2}$. The stent was endoscopically removed after 4 weeks. A gastrointestinal CT scan with oral contrast showed a complete resolution of the leakage. After 6 months the patient was in a good condition with BMI $29,4 \mathrm{~kg} / \mathrm{m}^{2}$.

Conclusions: This new technique is feasible and effective, as shown in this video; however the NAGR can lead to complications, so a strict follow up is needed and if any complication appears, should be considered to remove laparoscopically the ring.

\section{V014-BARIATRICS—Laparoscopic}

\section{CONVERSION TO A ROUX-EN-Y GASTRIC BYPASS FOR DYSPHAGIA AFTER GASTRIC PLICATION}

\section{PORTS SLEEVE GASTRECTOMY. VIDEO VIGNETTE AND 5 YEARS EXPERIENCE IN A SINGLE INSTITUTION}

D.J. Garcilazo Arismendi, E. Cuello Guzman, L. Lammers, S.T. Makkai-Poppa, M. Goergen, J.S. Azagra Soria

Department of General Surgery \& Urology, Mini-Invasive and Oncologic Surgery, Centre Hospitalier de Luxembourg, LUXEMBOURG

Background: Sleeve Gastrectomy (SG) has earned acceptance as one stage Surgical treatment of Obesity and nowadays is the most performed Bariatric procedure worldwide. Currently reduced port surgery is regaining momentum as the evolution of minimally invasive surgery.

Aims: We present a High Definition video to describe our technique of '3-Ports Sleeve Gastrectomy' which mimics all the fundamentals aspects of the conventional laparoscopic Sleeve Gastrectomy approach (5 Trocars) while incorporating some innovative technical features to reduce the quantity of ports ( 3 trocars); nonetheless maintaining triangulation and without the use of special equipment, a long learning curve or detrimental in exposure or ergonomics.

Methods: We present a high definition video describing our technique of 'Reduced Ports Sleeve Gastrectomy' inthe case of a 26-years old female with an initial Body Mass Index (BMI) of 79,1 $\mathrm{Kg} / \mathrm{m} 2$ withhistory of diabetes, arterial hypertension and obstructive sleep apnea syndrome; within a two-stage Gastric Bypass project for Obesity treatment

Results: Postoperative outcomes were uneventful. The patient shows a significant weight loss at 1 year follow-up with a change of BMI of $39,5 \mathrm{~kg} / \mathrm{m} 2$ (1-year BMI $39,6 \mathrm{Kg} / \mathrm{m} 2)$, a percent of total weight loss (\%TWL) of $50.5 \%$. (Initial weight: $200 \mathrm{~kg}-1$ year weight: $99 \mathrm{~kg}$ ) and a percent of excess weight loss (\%EWL) of $73,83 \%$; with complete resolution of the comorbidities. Conclusions:Our technique can be a useful, safe and feasible tool in selected patients in order to minimize parietal trauma and its possible complications, to improve cosmetic results and team dynamics and represent an alternative to conventional laparoscopic and single port surgery.
J. Colpaert, M. Ooms, K. Jacobs, I. van Campenhout, L. Maes, B. Dillemans

\section{Department of Surgery, AZ Sint-Jan, BRUGGE, Belgium}

Introduction: In this case, we will discuss the case of a 72 year old male patient who underwent a laparoscopic cruraplasty and gastric plication resulting in a weight loss of $12 \mathrm{~kg}$. Other medical history reported insulin-dependent diabetes, reflux esophagitis and sleeping apnea with CPAP. Two years after gastric plication the patient presented with passage problems, gastro-esophageal reflux and epigastric pain. To this end a swallow test was performed revealing a large fundus with a restricted passage of contrast. Due to the persistent complaints and the abnormal findings on barium swallow a surgical re-intervention was needed.

Objectives: Despite the current BMI of 27 and the age of the patient, conversion from a gastric plication to a Roux-en-Y gastric bypass was performed. Several other surgical options were considered, including an undo of the gastric plication or a dilatation with a resizing of the fundus. Methods: In the video we describe the laparoscopic approach for a conversion of a gastric plication to a Roux-en-Y gastric bypass.

Results: At 6 months follow-up the patient showed a weight loss of $8 \mathrm{~kg}$ and the resolution of his epigastric pain.

Conclusion: After a gastric plication, partial loosening of the sutures and stenosis are both wellknown complications. As presented in the video, it is apparent that a laparoscopic undoing of gastric plication is not as straightforward as it seems. Firm adhesions between folds can compromise the procedure and inhibit a complete separation of the tissues. We believe that in these cases the best surgical approach is to convert to a Roux-en-Y gastric bypass. earlier symptoms. The patient had a normal oral intake without any gastro-esophageal reflux or 


\section{V015-BARIATRICS-Laparoscopic}

IS IT POSSIBLE AND SAFE TO PERFORM A RESLEEVE GASTRECTOMY (RESG) FOR INCOMPLETE GASTRIC FUNDUS RESECTION POST-SG? OUR EXPERIENCE

\section{Palumbo ${ }^{1}$, E. Schembari ${ }^{1}$, M. Teodoro ${ }^{1}$, O. $\operatorname{Coco}^{1}$, G. la Greca ${ }^{1}$ S. Latteri ${ }^{1}$, A. Pesce ${ }^{2}$ \\ ${ }^{1}$ Chirurgia Generale, Cannizzaro, CATANIA, Italy; ${ }^{2}$ Department of General Surgery, Policlinico Hospital, University of Catania, CATANIA, Italy}

Laparoscopic sleeve gastrectomy (LSG) is a relatively new surgical approach in the weight loss surgeon's armamentarium. In literature there is a consensus about the importance of mobilizing completely the gastric fundus before transection. The ReSG (revised sleeve gastrectomyresleeve) may be a valid option for failure of primary LSG. We focused the attention on the consequences that can have an incomplete resection of gastric fundus during an operation of sleeve gastrectomy and how they can be solved by the repetition of this procedure.

A sleeve gastrectomy was performed in an obese 34 -year-old woman $(B M I=40)$. Three days after the operation, an upper GI x-ray with gastrografin did not show any abnormalities. Three months after the surgical procedure, the woman referred frequent episodes of vomiting and a significant weight loss ( 42 kilos). An upper GI x-ray with gastrografin demonstrated the presence of multiple communicating cavities of the gastric fundus. The esophagogastroduodenoscopy (EGD) showed that the gastric tube close to the esophagogastric junction was separated from a recess $(2-3 \mathrm{~cm}$ in diameter) by an incomplete septum. A severe hypokalemia and consequent ECG abnormalities were treated with intravenous infusion of potassium. Then, we performed a laparoscopic operation. The gastric tube was completely released along the suture line of the previous operation and, especially, the posterior surface of the upper part until the left crus of diaphragm became evident. Under the guide of the bougie, the recess was removed.

Results: The clinical course was regular, and the patient was discharged on third post-operative day after an upper GI x-ray with gastrografin which demonstrated the absence of leakage and a normal gastric tube. After 1 year, the patient was very satisfied with the operation.

Conclusions: The complete mobilization of the gastric fundus allows to see clearly which part should be resected to obtain an adequate gastric tube and facilitate a correct placement of the stapler. In our experience, in patients with a residual fundus, an upper GI x-ray with gastrografin and an EGD are needed to exclude the presence of stenosis. Then, a resleeve gastrectomy is an efficient and safe procedure to treat this post-LSG complication.

\section{V016-BARIATRICS-Laparoscopic}

\section{CONVERSION FROM SUPER-MAGENSTRASSE TO FUNCTIONAL OAGB WITH MINIMIZER RING AND INCISIONAL HERNIA REPAIR}

\author{
A. Peri ${ }^{1}$, M.S. Milani ${ }^{2}$, F.C. Bruno ${ }^{1}$, N. Mineo ${ }^{1}$, S. Malabarba ${ }^{1}$, \\ S. Carando ${ }^{1}$, A. Pietrabissa ${ }^{1}$
}

${ }^{1}$ General Surgery 2, IRCCS Policlinico San Matteo, PAVIA, Italy; ${ }^{2}$ General Surgery, IRCCS Policlinico San Matteo, PAVIA, Italy

Weight regain is one of the main problems in bariatric surgery. We have many surgical option but when we evaluate patients with long follow up and BMI of superobese patient before the first surgery, the weight recidivism can arrive up to $50-70 \%$ at 5 years.In most cases the first surgery is a restrictive procedure, and in many cases sleeve gastrectomy.Here we present a case of weight regain after laparotomic Super-Magenstrasse (that we consider like a sleeve gastrectomy except for remnant removal) with a big incisional hernia. After a complete multidisciplinary re-evaluation we decided to perform an OAGB (one anastomosis gastric bypass) but in this case we decided to create a functional exclusion to the duodenal transit by positioning a minimizer ring. This solution is effective in food diversion and guarantee gastric and duodenal endoscopic exploration in case of need. We think that this technique can represent an option to take in account for selected cases. At the end of bariatric procedure we perform a laparoscopic repair of incisional hernia with mesh in the hope to avoid future surgery and post operative small intestine herniation.

\section{V017-BARIATRICS-Laparoscopic}

LAPAROSCOPIC ADJUSTABLE GASTRIC BANDING EROSION ON LESSER CURVATURE: LAPAROSCOPIC MANAGEMENT

J. Trébol ${ }^{1}$, J.A. Gazo-Martínez ${ }^{2}$, P. Maté-Maté2 J.E. Quiñones-Sampedro ${ }^{1}$

${ }^{1}$ General and Digestive Tract Department, Complejo Asistencial Universitario de Salamanca, SALAMANCA, Spain; ${ }^{2}$ General and Digestive Tract Department, Hospital Universitario La Paz, MADRID, Spain

Aims: Erosion of Laparoscopic Adjustable Gastric Banding (LAGB) is uncommon and its clinical course is usually benign. We present and oligo-asymptomatic lesser curvature erosion diagnosed under scheduled LAGB removal repaired with laparoscopic suturing.

Methods: A 65 year-old female (BMI 35.38) with arterial hypertension and postoperative hypothyroidism was scheduled for a LAGB removal due to refractory vomiting. No information about LAGB type or surgical technique was available. A four port laparoscopic approach was performed sectioning the perigastric band with scissors over anterior gastric serosa. A longitudinal defect on the lesser curvature, in the place previously occupied by LAGB, was identified. Results: A laparoscopic suturing using single stitches with $3 / 0$ poliglactin 910 and $3 / 0$ polidioxanone barbed suture was performed (showed on video). An intraoperative gastroscopy was performed to confirm suture tightness. Oral intake was resumed once normal upper GI series were obtained three days later. The patient was discharged on the sixth postoperative day. LAGB was infected with Enterobacter cloacaeand Candida glabrata $</ \mathrm{i}>$. Normal endoscopy was obtained two months later.

Patient rejected additional bariatric procedures and in fact she has gained $10 \mathrm{~kg}$ two years later (BMI 39.14).

Conclusions: LAGB gastric erosion is uncommon (1.46-3\%). Intraoperative (such as perigastric approach) and patient related factors (smoking, alcohol...) have been described as risk factors The most frequent clinical presentation is weight loss failure; band and port issues (such as infection) are also frequent. Erosion is infrequent to present as an acute event $(<5 \%$ : peritonitis, abscess...) or asymptomatically $(<1 \%)$. Diagnosis is mostly performed under upper endoscopy The most common therapeutic technique is removal of the band (by endoscopy or surgery), repair of the stomach, if needed, and band replacement (at least three months later). Some authors have performed immediate replacement but the incidence of recurrent erosion seems to be higher. Other options are LAGB removal alone or conversion to different bariatric procedure. Fo endoscopic removal, it has been advised to wait until the band buckle is in the stomach and is sometimes very difficult. Replacement of the band is not associated with weight regain.

\section{V018-BARIATRICS-Laparoscopic}

\section{ENDOSCOPIC DETECTION AND LAPAROSCOPIC MANAGEMENT OF SUBMUCOSAL GASTRIC TUMORATION IN THE GASTROESOPHAGEAL JUNCTION DURING A ROUX-} EN-Y BYPASS

H. Segura-Marin ${ }^{1}$, B. Leal-Gonzalez ${ }^{2}$, G. Arredondo-Saldaña ${ }^{1}$, J.A. Diaz-Elizondo ${ }^{1}$

${ }^{1}$ General Surgery, Escuela de medicina del Tecnologico de Monterrey, MONTERREY, Mexico; ${ }^{2}$ Baritatric Surgery, Escuela de medicina del Tecnologico de Monterrey, MONTERREY, Mexico

Female patient of 45 years, Diabetic of 12 years of evolution, surgical history of $3 \mathrm{C}$-sections, last performed in 2001 and a conventional appendectomy in 2005. She reports 5 years of evolution presenting moderate intensity heartburn that was exacerbated during the night as well as submit occasional rejurgutation. The intensity of the symptoms is attenuated by maintaining a diet without irritants and improving feeding times. Denies hematochezia, unintentional reduction of weight, dysphagia or early satiety. The patient has suffered from obesity since childhood, after pregnancy she had progressive weight gain and difficulty in controlling blood sugar, so she is scheduled a gastric bypass Roux-en-Y . Preoperative endoscopy was performed, evidencing submucosal tumor in the gastroesophageal junction at 37 of the dentary arch, approximately $3 \mathrm{~cm}$ in diameter. An endoscopic ultrasound was performed, demonstrating subepithelial lesion of the gastroesophageal junction, hypoechoic, with well-defined borders, pseudobilobulated, $2.4 \mathrm{~cm}$ $\mathrm{x} 1.3 \mathrm{~cm}$, and dependent on the external muscular layer. A fine needle aspiration is performed in which spindle cells are identified, Leiomyoma is likely diagnosed. It is programmed for laparoscopic resection of submucosal gastric tumor, gastric bypass and laparoscopic cholecystectomy. A Tumor at the level of the gastro esophageal junction of approximately $2.5 \mathrm{~cm}$ is identified in the surgery, which can be resected by laparoscopy without complications. The patient is discharged after 2 days of postoperative stay. The final histopathological result: leiomyoma of $3.3 \mathrm{~cm}$ with free edges. CD4 (-) GOG1(-) Caldesmon (+)s100 (+). 


\section{V020-BARIATRICS-Laparoscopic}

LAPAROSCOPIC REPAIR OF A GIANT MORGAGNI HERNIA IN A MORBIDLY OBESE PATIENT: A DEMONSTRATION OF COMPLETE EXCISION OF THE SAC AND MESH

\section{AUGMENTATION}

\section{M.Y. Fanous}

Surgery, Aspirus Iron River Hospital, IRON RIVER, United States of America

Introduction: Minimally invasive repair of adult Morgagni hernia is gaining popularity. Given its rarity, there is no consensus regarding the dissection/excision of the sac or the use of mesh. Morbid obesity adds to the operative complexity and to the postoperative challenges.

Aim: To evaluate the safety and efficacy of laparoscopic repair of giant Morgagni hernia- with sac dissection and mesh augmentation- in morbidly obese patient.

Methods: This patient is a 60 year old female with BMI of 45 who has past medical history of diabetes, asthma, anxiety and gastroesophageal reflux disease. Patient had intermittent chest pain for 3 months. Extensive workup, including $\mathrm{CT}$ of the chest, revealed giant right sided Morgagni hernia.

Result: Patient underwent elective laparoscopic repair. Four ports were placed in the upper abdomen. She was placed in Reverse Trendelenberg position. The transverse colon and its omentum were reduced. The liver attachments to the diaphragm were divided. The diaphragmatic defect measured $6 \times 4 \mathrm{~cm}$. The sac was dissected and excised. This was achieved by scoring the inferior edge of the defect. The proper plane was easily identified laterally. A raytec gauze was placed to create space and to allow for diffusion of carbon dioxide. The sac was dissected of the mediastinal structures and excised. The defect was closed using interrupted permanent sutures tied extracorporeally with modified Duncan Loop. The repair was reinforced with coated mesh. Patient tolerated the procedure well. Her length of stay was two days. There were no postoperative complications. Patient was seen in clinic 5 months postoperatively as she reported abdominal pain with heavy lifting. CT showed no evidence of recurrence of Morgagni Hernia. Conclusion: Laparoscopic repair of adult giant Morgagni hernia- with complete dissection of the sac and mesh augmentation- in morbidly obese patient is safe and effective. Longer term follow is needed to evaluate the durability of this repair.

\section{V021-BARIATRICS-Laparoscopic}

\section{LIGAMENTUM TERES CARDIOPEXY AS A LATE ALTERNATIVE FOR GASTROESOPHAGEAL REFLUX DISEASE IN A PATIENT WITH HIATAL HERNIA AND CONVERTED SLEEVE GASTRECTOMY}

R. Vilallonga ${ }^{1}$, S. Sanchez-Cordero ${ }^{2}$, P. Alberti ${ }^{3}$, R. Roriz ${ }^{1}$ M. Kraft ${ }^{1}$, E. Caubet ${ }^{1}$, O. Gonzalez ${ }^{1}$, Y. Curbelo ${ }^{1}$, J.M. Fort ${ }^{1}$, M. Armengol ${ }^{1}$

${ }^{1}$ Endocrine-Metabolic and Bariatric Surgery, Hospital Universitari Vall d'Hebron, BARCELONA, Spain; ${ }^{2}$ General Surgery Department, Consorci Sanitari de l'Anoia, IGUALADA, Spain; ${ }^{3}$ General surgery department, Hospital Universitari Vall d'Hebron, BARCELONA, Spain

Background: Fifty percent of patients who have undergone gastric bypass, posterior reversal and sleeve gastrectomy and finally complete hiatoplasty presents symptomatic gastroesophageal reflux disease. Surgical reinforcement of the lower esophageal sphincter is necessary to prevent acid reflux. Here, we describe ligamentum teres cardiopexy, a surgical technique that reinforces the lower esophageal sphincter and restores its competence with a new valve, in patients with previous conversion of sleeve gastrectomy to gastric bypass and hiatal hernia repair.

Methods: We present the surgical techhnique performed to a patient with initial gastric bypas who underwent sleeve gasterctomy for hipoglycemias and hiatoplastia for severe GERD. Persistent GERD requested to undergo ligamentum teres cardiopexy. In this procedure, the ligamentum teres is released from its umbilical connection and the hernia reduced by manual traction, freeing the last $3-5 \mathrm{~cm}$ of esophagus in the abdomen. The distal ligamentum teres is fixed with one stitch to the apex of the angle of His, one at the gastroesophageal junction, and one joining the gastric fundus to the esophagus. The remainder of the ligamentum teres is fixed over itself with four to six stitches, forming a necktie cardiopexy. The procedure concludes with diaphragmatic crus closure.

Results: After 3 months, the patient achieved successful results, defined as resolution of GERD, no protonpump inhibitor (PPI) use, and manometry measurement over $12 \mathrm{mmHg}$ after surgery. Conclusions: Ligamentum teres cardiopexy combined with closure of the gastric crus is a late alternative treatment for gastroesophageal reflux disease in patients with previous sleeve gastrectomy and hiatal hernia.

\section{V023-BARIATRICS-Laparoscopic}

REDO VERTICAL BANDED GASTROPLASTY TO ROUX EN Y GASTRIC BYPASS-OUR TECHNIQUE

\section{B.A. Smeu}

General surgery, Ponderas Academic Hospital, BUCHAREST, Romania

Introduction: As metabolic surgery techniques evolve during the years, we have to face more and more patients with complications ands uboptimal results after the older/initial procedures. Vertical banded gastroplasty(VBG) is one of those procedures that gain momentum during the initial experience in bariatric surgery, but has proven to have dissapointing results and a lot of complications, nowadays surgeons having to deal with difficult revisional operations.

Aim in this video: we want to present from our experience the difficulties encountered during the revisional surgery, Rouxen Y Gastric Bypass (RYGBP)afterVBG, and the tips and tricks that will make this a safer and easier procedure.

Material and Methods: Between 2011-2018 we had 39 revisional surgeries after open VBG, all of them by laparoscopic approach, most of them redos to LRYGBP (34/39-87.1\%)and the rest revisions to sleeve gastrectomy $(5 / 39-12.9 \%)$. Most of the indications for revisional surgery after VBG were for complications (stenosis, dysphagia, band migration, gastro-gastric fistula) and suboptimal result with weight regain. We present our technique for LRYGBP after VBG emphasizing the possible pitfalls and tips and tricks needed for a safer and easier procedure. Results and Discussions: All patients were operated in ourCenter of Excelencefor Bariatric and Metabolic Surgery (COE) and went well postoperatively, without any major complications (no leaks, no mortality) and only minor and temporary complications: temporary dysphagia in 2 patients (4 and 7 PO days, remitted afterconservatory treatment), and 2gastro-jejunalanastomosis bleeding (endoscopic treatment). The mean operative time was $165 \mathrm{~min}$ (115 to $365 \mathrm{~min}$ ) and mean hospital stay 5,4 days ( 3 to 12 days).

Conclusions: Although the revision surgery after VBG is technique demanding, we consider it to be a safe procedure in the hands of experienced metabolic surgeons, with good postoperative results.

\section{V024-BARIATRICS—Laparoscopic}

\section{BARIATRIC SURGERY IN A CIRRHOTIC PATIENT WITH PORTAL HYPERTENSION}

\section{J. Colpaert, M. Ooms, G. Uijtterhaegen, B. Dillemans}

Department of Surgery, AZ Sint-Jan, BRUGGE, Belgium

Introduction: We present a 53 year old male patient with documented ethylic liver cirrhosis an portal hypertension. He weighed $124,2 \mathrm{~kg}$ for $170 \mathrm{~cm}$, resulting in a BMI of 43 . In the further preoperative workup we find fundal varicosis without esophageal varices on gastroscopy. This suggests mild to moderate portal hypertension and thus an increased surgical risk.

Objective: After thorough preoperative assessment and a review of the literature multiple treatment options were considered. The procedure of choice ended up being a laparoscopic adjustable gastric banding, with the objective to achieve optimal weight loss with the lowest risk for complications.

Methods: In this video we present the placement of an adjustable gastric banding in a patient with a cirrhotic liver and portal hypertension and the possible pitfalls.

Results: Postoperatively there were no complications and patient had a satisfying weight loss both 6 months and 1 year postoperatively. In a short review of the literature we've found that bariatric surgery is feasible in patients with portal hypertension as long as the patient is not decompensated or has bleeding varices.

Conclusion: Cirrhosis and portal hypertension are no absolute contraindication for banding, sleeve or RNY gastric bypass as long as the patient is not decompensated or has bleeding varices. The type of surgery is dependent on patient and surgeon-related factors. The aim should be to achieve optimal weight loss with the lowest possible surgical risk in this type of patients. 


\section{V025-BARIATRICS-Laparoscopic}

\section{CONVERSION FROM A N-SLEEVE TO A ROUX-EN-Y} GASTRIC BYPASS FOR INSUFFICIENT WEIGHT LOSS

M. Ooms, J. Colpaert, E. Melsens, L. Ruyssinck, L. Maes, B. Dillemans

\section{Department of Surgery, AZ Sint-Jan, BRUGGE, Belgium}

Introduction: In this case, we will discuss on a 54 year old female patient who had undergone a laparoscopic Nissen fundoplication 5 years ago due to GERD grade B. Because of morbid obesity a $\mathrm{N}$-sleeve gastrectomy was performed 1 year ago resulting in a weight loss of $12 \mathrm{~kg}$. At presentation she had regained all the lost weight, resulting in a BMI of 42,8 . The patient history also reported insulin-dependent diabetes and obstructive sleep apnea with CPAP. Gastroscopy was performed showing a large residual fundus but no esophagitis. On the subsequent upper GI series a relatively wide sleeve with an intact Nissen-collar was detected.

Objectives: A laparoscopic conversion to a Roux-en-Y gastric bypass was performed. Other potential surgical treatment options are a SADI procedure or a sleeve gastrectomy with transit bipartition (Santoro procedure)

Methods: In the video we describe the laparoscopic approach for a conversion of a N-sleeve to a Roux-en-Y gastric bypass.

Results: At 4 month follow-up the patient presented with a weight loss of $12 \mathrm{~kg}$. The patient had good restriction on oral intake and did not have any reflux-related symptoms or complaints. Conclusion: Conversion from a N-sleeve to a Roux-en-Y gastric bypass is a challenging procedure. The largest pitfall during the creation of the gastric pouch is to staple a double fold of the Nissen fundoplication. We believe that in these rare cases of weight regain after $\mathrm{N}$-sleeve, the best surgical approach is to convert to a Roux-en-Y gastric bypass.

\section{V026-BARIATRICS-Laparoscopic}

\section{NEED FOR ENDOSCOPIC SURVEILLANCE: CONVERSION FROM A ROUX-EN-Y GASTRIC BYPASS TO A SLEEVE GASTRECTOMY}

\section{Ooms, J. Colpaert, G. Uijtterhaegen, K. Jacobs, B. Dillemans}

Department of Surgery, AZ Sint-Jan, BRUGGE, Belgium

Introduction: A 44-year old female patient with extensive bariatric history presented at our hospital. She had had a gastric balloon in 2005 and a laparoscopic vertical banded gastroplasty (VBG) in 2006. Four years later, in 2010, a laparoscopic conversion to Roux-en-Y gastric bypass was performed because of weight regain. She now presents with satisfactory and stable weight loss over the last few years. She was recently diagnosed with a BRCA-1 mutation for which she underwent a bilateral ovarectomy and mastectomy. The patient's brother was also diagnosed with this mutation and died of pancreatic cancer at the age of 39. Genetic counseling advised a twoyearly follow-up because of an increased risk up to $10 \%$ of developing pancreatic cancer. Control gastroscopy showed a normal esophagus and gastric pouch. Control CT scan revealed hypertrophic stomach creases in the excluded stomach. These results prompted a laparoscopy-assisted gastroscopy of the excluded stomach which uncovered hypertrophic stomach glands and intestinal metaplasia on biopsy.

Methods: In this video we demonstrate the laparoscopic approach for complex revisional bariatric surgery. Conversion from RNY gastric bypass to a sleeve gastrectomy in a patient who already underwent a VBG. The focus of the video is on a manual gastro-gastrostomy with partial gastrectomy of the fundus and part of the stomach where the old VBG-band was placed. Results: After 1,5 months follow-up the patient had no complaints and a stable weight. Upper GI series shows a normal passage of contrast through the sleeve gastrectomy.

Conclusion: Endoscopic surveillance of the remnant stomach and echo-endoscopy of the pancreas is no longer possible after RNY gastric bypass. In cases where the need for such surveillance arises after a RNY bypass a patient-tailored approach is necessary. In our patient laparoscopic conversion from a RNY gastric bypass to a sleeve gastrectomy was performed. This approach keeps the patient's wish for weight loss intact while enabling further surveillance through natural-orifice endoscopy.

\section{V027-BARIATRICS—Laparoscopic}

ROUX LIMB STRICTURE AFTER LAPAROSCOPIC ROUX EN Y GASTRIC BYPASS: HOW TO CONVERT A RETRO-COLIC TO AN ANTE-COLIC ROUX LIMB ROUTE

\author{
S. Inamine $^{1}$, H. Nakazato ${ }^{2}$, T. Takaesu ${ }^{1}$, A. Gabe ${ }^{1}$, J. Oshiro $^{1}$ \\ ${ }^{1}$ Surgery, Ohama Daiichi Hospital, NAHA CTY OKINAWA \\ PREFECTURE, Japan; ${ }^{2}$ Surgery, Okinawa Red Cross Hospital, \\ NAHA, Japan
}

A 47 -year-old morbidly obese Japanese woman with a body mass index of $41 \mathrm{~kg} / \mathrm{m}^{2}$ suddenly complained of swallowing difficulty 4 months after laparoscopic Roux en Y gastric bypass surgery with retro-colic Roux limb route. An internal hernia of the defect of the transverse mesocolon was suspected by computed tomography, and emergency intervention was performed. The surgery revealed no internal hernia. However, strong inflammation and adhesion were observed between the transverse mesocolon and the retrocolicRoux limb. In addition, the Roux limb on the oral side of the adhesion site was dilated and bent.The adhesion between the transverse mesocolon and the flexed Roux limb was dissected, linearized and re-fixedby suturing to the transverse mesocolon. However, since the difficulty of oral intake persisted re-do surgery was performed again. After resecting the Roux limb involved in the severe inflammation, a 'new' Roux limb was lifted to the cephalad via the ante-colic route. Finally, the gastric pouch and Roux limb were re-anastomosed with 3-0 absorbable sutures in an interrupted full thickness single laye manner. In the present case, we experienced difficulty with both adhesiolysis and determining the accurate target line to resect at the 'old' gastrojejunostomy. However, blocking the blood flow of the 'old' Roux limb facilitated the accurate recognition of the target line.

\section{V028-BARIATRICS—Laparoscopic}

\section{MARGINAL ULCER AFTER GASTRIC BYPASS}

M. Pérez Reyes ${ }^{1}$, A. Rodríguez Cañete ${ }^{1}$, F.J. Moreno Ruiz ${ }^{1}$, C. Montiel Casado ${ }^{1}$, J.L. Fernández Aguilar ${ }^{1}$, P. Fernández Galeano ${ }^{1}$, I. Mirón Fernández ${ }^{1}$, J. Santoyo Santoyo ${ }^{2}$

${ }^{1}$ Esofagogástrica, Cirugía General y Ap Digestivo, Hospital Regional Universitario de Málaga, MALAGA, Spain; ${ }^{2}$ Hepatobiliopancreática, Hospital Regional Universitario de Málaga, MALAGA, Spain

Introduction: Marginal ulcer is one a serious complications after a bariatric gastric bypass Tobacco, non-steroidal anti-inflammatory drugs (NSAIDs) and Helicobacter pylori (HP) infection are known risk factors.

Methods: We present a 29-year-old women operated 3 years before of bariatric surgery with a gastrojejunal (GY) bypass technique due to intraoperative dehiscence of the staple line after attempting a vertical gastrectomy (sleeve). She has persistent vomiting and epigastralgia from 3 months after the intervention, affecting his quality of life.

Upper gastrointestinal endoscopy (UGE) was performed, describing an ulcer in the GY anastomosis. She started HP eradication treatment, treatment with proton pump inhibitors (PPIs), tobacco and NSAIDs were discontinued, but she had slight improvement. After 6 months the UGE was made again, which show peptic esophagitis and 2 marginal ulcers. The plasma gastrin level was normal.

Due to the persistence of symptoms despite conservative treatment, we decided reoperation by laparoscopy. We found herniated bowel in Petersen space, which were reduced and the space was closed. We proceeded to truncal vagotomy. The GY anastomosis was resected (fig. 1) and performed again. Finally, we perform antrectomy. The pathological anatomy showed ulceration. She was diacharged home on the 5th postoperative day without any complications.

Results: A marginal ulcer after bariatric surgery appears in the jejunal mucosa of the g-y anastomosis. The symptoms are epigastric pain, nausea and vomiting. Acid, tobacco, NSAIDs and HP infection has an important role in their development $<\sup >.</$ sup $>$ The first treatment is medical, discarding out the risk factors, but if it is not effective, it will be surgical, resecting the previous anastomosis. The usefulness of vagotomy is debatable, but the percentage of success increases. In our case, we perform antrectomy to avoid retained antrum syndrome. The hernia through Petersen space is a cause of intestinal obstruction and abdominal pain as the case presents. Although we believe that the symptoms were mainly caused by the marginal ulcer, the internal hernia was probably a symptomatic cause.

Conclusion: The treatment of a marginal ulcer is medical, eliminating the risk factors, but if it is not effective, the surgery is indicated. 


\section{V029-BARIATRICS-Laparoscopic}

HIATUS HERNIA REPAIR DURING LAPAROSCOPIC GASTRIC BYPASS

M. López Saiz, J. Riquelme Gaona, G. Rojas de la Serna, A. Morandeira Rivas, B. Muñoz de la Espada, C. Moreno Sanz

Digestive Surgery, General Hospital, La Mancha-Centro, ALCAZAR DE SAN JUAN, Spain

Aim: Hiatal hernia is often a pre-existing condition in morbidly obese patients, sometimes misdiagnosed, which can cause an intractable reflux, nausea and abdominal pain. In this video, we present the cases of two patients scheduled for bariatric surgery with a accompanying hiatal hernia. Material and methods: Patient 1: 49 years old men with a BMI of $38.2 \mathrm{~kg} / \mathrm{m} 2$, medical history of arterial hypertension, diabetes mellitus type 2, dyslipidemia and obtructive sleep apnea (OSA) and without clinical signs of gastroesophageal reflux. In the preoperative endoscopic study a hiatal hernia is observed.Patient 2: 61-year-old woman with a BMI of $42 \mathrm{~kg} / \mathrm{m}^{2}$, history of hypertension, type 2 diabetes mellitus, dyslipidemia, OSA in treatment with nocturnal continuous positive airway pressure, primary hypothyroidism and clinical gastroesophageal reflux. In the preoperative endoscopic examimation a hiatal hernia is detected.

Results: In both patients, a laparoscopic approach with five ports was carried out. The presence of a obvious hiatal hernia was confirmed in both cases. Hernia sac and distal esophagus were disected and the pillars of the hiatus were sutured, before performing the gastric bypass. Patients recovered uneventfully and were discharged on the third postoperative day.

Conclusions: The Roux-en- $Y$ gastric bypass is considered an adequate treatment in obese patients with gastroesophageal reflux disease. Although the bypass solves the reflux problems by itself, it is recommended to repair detected hiatal hernias.

\section{V031-BARIATRICS—Laparoscopic}

ROUX-Y TRANSMESOCOLIC GASTRIC BYPASS WITH REMNANT GASTRECTOMY FOR INTRACTABLE GERD AFTER SLEEVE

D.J. Garcilazo Arismendi ${ }^{1}$, E. Cuello Guzman ${ }^{2}$, L. Lammers ${ }^{1}$, B. Pascotto ${ }^{1}$, M. Goergen ${ }^{3}$, J.S. Azagra Soria ${ }^{4}$

${ }^{1}$ Department of General Surgery \& Urology, Mini-Invasive and Oncologic Surgery, Centre Hospitalier de Luxembourg, LUXEMBOURG; ${ }^{2}$ Centre Hospitalier de Luxembourg, LUXEMBOURG

Background: Sleeve Gastrectomy (SG) has gained momentum as one stage Surgical treatment of Obesity and nowadays is the most performed Bariatric procedure worldwide, nonetheless it's not exempt of acute or chronic complications difficult to solve. Although SG has shown some degree of efficacy in the treatment of Obese patient with GERD symptoms and some groups do not consider this as a contraindication for this technique, currently the evidence continues to grow towards SG as a promoting factor in the development of De Novo GERD symptoms. The laparoscopic Roux-en-Y gastric bypass (LRYGB) has shown to be the angular stone in the treatment of morbid obesity and also it's one of the most effective surgical procedures for GERD.This is the case of a 49-years old male with history of a Sleeve Gastrectomy within a two-stage Gastric Bypass project for Obesity treatment complicated with a invalidant De Novo GERD, 2 months after surgery.

Aims: The purpose is to present our team approach in the treatment of GERD after SG, associating LRYGB with Remnant Gastrectomy in order to increase the efficacy, adding up to the antireflux effects of the LRYGB plus the decrease of Gastrin levels induced by the removal of the gastric remnant.

Methods: We present a high definition video describing our technique of 'Roux-Y TransmesocolicGastric Bypass with Remnant Gastrectomy for GERD's treatment'.

Conclusions: Postoperative outcomes were uneventful. The patient shows a complete remission of GERD symptoms immediately after surgery.

\section{V032-BARIATRICS—Laparoscopic}

TRANSHIATAL SLEEVE GASTRECTOMY MIGRATION AND GERD: LAPAROSCOPIC HIATAL HERNIA REPAIR WITH REINFORCEMENT AND CONVERSION TO R-EN-Y GASTRIC BYPASS

C.E. Boru, P. Termine, G. Silecchia

Division of General Surgery \& Bariatric Center of Excellence-IFSO EC, Sapienza University of Rome, Dept. of Medico-Surgical Sciences \& Biotechnologies, LATINA, Italy

Background: Transhiatal sleeve migration, with consecutive gastroesophageal reflux disease (GERD) is a complication that leads to revisional surgery.

Methods: A fifty-five years old, morbid obese female patient with BMI $45 \mathrm{~kg} / \mathrm{m}^{2}$, hypertensive with OSAS and hypercholesterolemia, was operated by laparoscopic sleeve gastrectomy in 2010. She reached nadir in 2012 with BMI 28.7, with resolution of her comorbidities. From 2016 she complained from symptomatic GERD not responding to medical treatment,with evidence of transhiatal sleeve migration on radiological contrast study (Gastrografin ${ }^{\circledR}$ ), and on the CT scan of the hiatal area.

Results: we present the video of conversion to laparoscopic R-en-Y gastric bypass LRYGB, associated with reinforced cruroplasty with bioabsorbable mesh, with marked improvement of GERD symptoms after reoperation.

Conclusion: laparoscopic conversion from LSG to RYGB is feasible and useful for LSG complications. 


\section{V034-BARIATRICS-Laparoscopic}

\section{LAPAROSCOPIC CONVERSION OF A VERTICAL BANDED GASTROPLASTY INTO A ROUX-EN-Y GASTRIC BYPASS}

J.I. Ortiz de Elguea Lizárraga, R. Alatorre, J. Rojas, E. Flores Villalba, M. Guraieb Trueba, S. Lopez

Department of Surgery, Tecnologico De Monterrey, SAN PEDRO GARZA GARCÍA, Mexico

We present a clinical case of a 47 year old female. She had a vertical banded gastroplasty procedure (in another clinic) 9 years ago with an initial weight loss of $30 \mathrm{~kg}$ in a period of 2 months.

She was seen at our clinic because she was suffering from dysphagia to solids and general diffuse abdominal pain for the last month.

At physical exam we found a BMI of 39 and nothing else called our attention.

We did an upper GI endoscopy and EGD transit; we concluded that a gastric bypass would offer her the best results.

Therefore, we converted her vertical banded gastroplasty into a gastric bypass laparoscopically. She had an uneventful postoperative period and was discharged home without complications.

\section{V036-BARIATRICS-Laparoscopic}

LAPAROSCOPIC DUODENAL SWITCH AS A MALABSORTION SAFE AND EFFECTIVE TECHNIQUE AFTER LAPAROSCOPIC SLEEVE GASTRECTOMY

M.P. Carbonell Aliaga ${ }^{1}$, S. Pascual Camarena ${ }^{1}$, S. Delgado Rivilla ${ }^{2}$, X. Julian Argudo ${ }^{1}$, A. Crespí Mir ${ }^{1}$, A. de la Llave Serralvo ${ }^{1}$, M. Escales Oliver ${ }^{1}$, A. Sánchez López ${ }^{1}$, J.A. Cifuentes Ródenas ${ }^{1}$

${ }^{1}$ General Surgery, Hospital Universitari Son Llàtzer, PALMA, Spain; ${ }^{2}$ General Surgery, Hospital Universitario Mutua de Terrassa, TERRASSA, Spain

Aims: The LPS sleeve gastrectomy is the most common bariatric surgery technique because it has a low surgical complexity and acceptable weight loss results. However, $5-11 \%$ of patients present with an insufficient weight loss, weight regnances, reflux or dysphagia. In these cases, it is recommended to perform a second bariatric surgery to combine a component of malabsorption such as gastric bypass or duodenal switch. The video describes the technique of a laparoscopic biliopancreatic diversion with duodenal switch with a previous laparoscopic sleeve. The objective is to describe the safety of the technique and the subsequent success of it.

Methods: A 41-year-old female patient presented morbid obesity with a BMI of 49 after performing a laparoscopic sleeve gastrectomy in 2010. Initially, she presented a percentage of excess weight loss of $62 \%$, reaching a BMI of 33 after two years of follow-up. After this, she suffered reganancia of all the weight lost despite diet and exercise, presenting a BMI 49. A study was made with TEGD where no complications of the previous surgery or symptoms of gastroesophagical reflux or dysphagia were observed. The LPS duodenal switch is proposed in the obesity unit committee in 2016 , without immediate postsurgical complications. The patient presented a favorable postoperative period and was discharged three days postoperatively.

Results: At the present time, the patient has achieved a 98\% excess weight loss and has a BMI of 26.5. Presents good oral tolerance with 3 stools a day without urgency. It doesn't present protein deficioncies. Vitamin deficiencies are orally supplemented.

Conclusion: The LPS duodenal switch is a technique that can be performed after a sleeve gastrectomy safely in cases of insufficient weight loss or weight reganancia. The patients presented greater weight loss after the duodenal switch than after the gastric bypass, observing a lost of excess weight of $74 \%$ compared to $64 \%$. The differences being statistically significant.

\section{V037-BARIATRICS-Robotics}

\section{ROBOTIC CONVERSION OF ROUX-EN-Y GASTRIC BYPASS TO LOOP DUODENAL SWITCH}

\author{
D.S. Ranev, Y.J. Yatco, M. Roslin
}

Surgery, Lenox Hill Hospital-Northwell Health, NEW YORK, United States of America

Weight regain after gastric bypass is a challenging problem. A number of revisional surgical options have been reported. This is a case of a 48 year-old woman 10 years after LRYGB. Her initial BMI was 67 , lowest after surgery-28, at presentation -48 . The video shows a robotassisted laparoscopic conversion of RYGB to loop duodenal switch. The Roux limb is transected and dissected to the gastrojejunostomy. The gastrojejunostomy is resected and the gastric pouch is recreated over a bougie. The gastric blood suply is confirmed with ICG. A gastro-gastrostomy is created to restore gastric continuity and a sleeve gastrectomy is performed. The duodenum is devided and a duodeno-ileostomy is created $300 \mathrm{~cm}$ from the ileocecal valve. The remaining Roux limb is resected. The patient recovered uneventfully. Conversion of RYGB to loop duodenal switch requires creation of as little as two anastomoses, in comparison to standard DS, which requires four. It is a safe option for patients with weight regain after LRYGB. 


\section{V038-COLORECTAL-Benign}

\section{LAPAROSCOPIC APPENDECTOMY IN DIFFUSE PURULENT PERITONITIS DUE TO GANGRENOUS APPENDICITIS}

\section{Zdrojewski}

General Surgery Departament, Warmia Masuria University, BARTAG, Poland

18 years old male was admited to the ER due to lower abdominal pain and high fever for 6 days. Physical examination revaled rebound tenderness in every part of the abdominal wall and lab tests showed CRP $450 \mathrm{mg} / \mathrm{l}$, PCT $45 \mathrm{ng} / \mathrm{ml}$ and bilirubin level of $6 \mathrm{mg} / \mathrm{dl}$. He was admited to General Surgery department and qualified for the surgery. Laparoscopy showed diffused, purulent peritonitis with lots of adhesions. Further scouting of abdominal cavity revaled infalmated, perforated appendix. Appendectomy and lavage were performed. Patient was discharged on postoperative day 5, fully recovered, PCT level was normal and CRP showed downward trend.

During control visit, 2 weeks after surgery, patient didn't have any complaints, wounds were fully healed without any sings of infection and abddominal ultrasound showed no pathology.Laparoscopic approach is propper even for perforated appendicits with diffused, purulent peritonitis is associated with faster recovery, lesser postoperative pain and lower surgical site inffection and should be procedure of first choice.

\section{V039-COLORECTAL-Benign}

\section{SEGMENTAL LEFT COLECTOMY. A MODIFIED CAUDAL TO CRANIAL APPROACH}

\author{
M. Milone, M. Manigrasso, G. de Palma
}

Gastroenterology and Surgical Endoscopy, Federico II University, NAPLES, Italy

Background: We have designed a modified caudal-to-cranial approach to perform laparoscopic left colectomy preserving the inferior mesenteric artery for benign colorectal diseases.

Methods: IRB approval and informed consent have been obtained. A dissection is conducted to separate the descending mesocolon of the Gerota's plan from the medial aspect to the peritoneal lining to the left parietal gutter. The peritoneal layer is incised parallel to the vessel and close to the colonic wall. The dissection is continued anteriorly up to reach the resected parietal gutter. A passage into the mesentery of the upper rectum is created for the allocation of the stapler and the dissection of the rectum. These maneuvers permit to straighten the mesentery simplifying the identification and cutting of the sigmoid arteries. A caudal-to-cranial dissection of the mesentery is performed from the sectioned rectum to the proximal descending colon by a sealed envelope device. It can be very useful to mobilize the colon in any direction: laterally, medially, or upward. The dissection is performed along the course of the vessel up to the proximal colon, with progressive sectioning of the sigmoid arterial branches. The specimen is extracted by a pfannenstiel incision. The anastomosis is performed transanally with a circular stapler according to Knight-Griffin technique.

Results: We performed a laparoscopic segmental colectomy using this approach for 21 patients with benign sigmoid lesions: 13 diverticulitis, 3 flat polypoid lesions (no lift-up sign), and 5 bowel endometriosis. The mean operative time and blood loss were $161.4 \pm 15.7 \mathrm{~min}$ and $50 \pm 40 \mathrm{ml}$, respectively. There were not a single conversion to open surgery and no any leakage or stricture. Only 2 cases of intraluminal bleeding and 1 case of wound infection (treated conservatively) were observed.

Conclusion: We consider this approach to be safe and useful for segmental colectomy to be performed sectioning the sigmoid artery close to the colonic wall.
SUPRAPUBIC SINGLE-INCISION LAPAROSCOPIC RIGHT HEMICOLECTOMY FOR COLON CANCER

E.J. Barzola Navarro ${ }^{1}$, K. Ann Bob ${ }^{2}$, A. Glagolieva ${ }^{3}$, J.A. Flores Garcia ${ }^{1}$, G. Dapri ${ }^{4}$

${ }^{1}$ Digestive Surgery, Universidad de Extremadura, BADAJOZ, Spain; ${ }^{2}$ Department of Clinical Surgical Sciences, University of the West Indies, PORT OF SPAIN, Trinidad and Tobago; ${ }^{3}$ Surgery, PL Shupyk National Medical Academy, KIEV, Ukraine; ${ }^{4}$ Digestive Surgery, Hospital Saint Pierre, BRUSELLS, Belgium

Aims: To show a clinical case with a video of a patient was operated for colon cancer in hepatic angle by a single suprapubic incision (SSILRH).

Methods: A 44-year-old male assessed for abdominal pain and weight loss. On physical examination: a painful mass was detected in the upper right quadrant. The colonoscopy revealed an ulcerated lesion in the hepatic angle and the biopsy revealed a moderately differentiated adenocarcinoma. In the abdominal CT a mass of $3 \times 4 \mathrm{~cm}$ was observed (figure). The patient was operated with SSILRH technique, as shown in the attached video.

Results: The patient was placed in the supine position and with the legs separated. The surgeon is placed between the patient's legs. A transverse incision of the skin was made in the middle line of $3.5 \mathrm{~cm}$ to $1 \mathrm{~cm}$ above the pubis. The underlying fascia was divided transversely, the rectus abdominal muscle was exposed, a purse-string suture placed in the fascia. An $11 \mathrm{~mm}$ reusable trocar was inserted for the chamber, a $6 \mathrm{~mm}$ reusable flexible trocar was placed at the 9 o'clock position and another trocar was placed at the 3 o'clock position. The ileocecal valve was released from the peritoneal parietal foil, as well as the mesocolon right by a lateral to medial approach to the second portion of the duodenum. The hepatic angle was also dissected from lateral to medial. For the anastomosis, the $11 \mathrm{~mm}$ trocar was replaced with a $13 \mathrm{~mm}$ trocar and a stapler was placed. A $5 \mathrm{~mm} 30^{\circ}$ chamber was inserted through the $6 \mathrm{~mm}$ flexible trocar. The small intestine was divided as well as the proximal transverse colon with EndoGIA. An intracorporeal ileocolic anastomosis was performed. The piece was removed through the suprapubic incision. He was discharged after 5 days without complications. The histological studies confirmed a differentiated adenocarcinoma of $8 \times 7 \times 6 \mathrm{~cm}$. The surgical margins were free, without infiltrated lymph nodes $(0 / 26)$ with stage pT3N0.

Conclusion: The SSILRH technique allows a complete resection of the mesocolon and complies with the oncological principles.

\section{V041-COLORECTAL-Benign}

\section{GIANT DIVERTICULO DE COLON RIGHT: ¡WHAT SURPRISE!}

A.F. Aranzana Gómez ${ }^{1}$, J. Hernandez Gutierrez ${ }^{1}$, J. Malo Corral, B. Muñoz Jimenez, A. Trinidad Borras, S. Abad de Castro

${ }^{1}$ General Surgery, Complejo Hospitalario Toledo, TOLEDO, Spain; ${ }^{2}$ General Surgery, Hospital tres culturas, TOLEDO, Spain

Introduction and objectives: Giant colonic diverticula are infrequent lesions (10-15\%) They can present with abdominal pain, nausea, acute abdomen, symptoms of intestinal obstruction or asymptomatic with incidental diagnosis. Their diagnosis can be difficult. The objective is to demonstrate the safety and efficacy of the laparoscopic approach in this infrequent pathology.

Material and methods: We present a video of the surgical intervention of a 32-year-old patient, with functional dyspepsia, with a casual diagnosis of a pseudocystic mass of the right colon after performing a CT scan: giant diverticulum of the hepatic colon angle with fecaloid content inside it under tension The patient goes to the emergency room for acute abdominal pain, pending colonoscopy, antibiotic treatment is established, and a laparoscopic approach is decided upon after the patient's evolution.

Results: Intervention: complete laparoscopic approach, 4 trocars. Large size tumor in the right colon, diverticular in appearance, with stony content inside, with locoregional adenopathies, oncological radical right hemicolectomy, manual intracorporeal anastomosis, correct postoperative, hospital discharge. on the 4th day. Definitive pathological anatomy: giant diverticula on areas of intense mucosal ulceration, free edges.

Conclusion: The laparoscopic approach of the symptomatic diverticula of the right colon is safe and effective.

V040-COLORECTAL-Benign 


\section{V042-COLORECTAL-Benign}

TAMIS WITH TECHNIQUE OF STABILIZATION OF THE PNEUMORRECT WITH GLOVE INTERPOSED AS A RESERVOIR

M. López Saiz, J. Riquelme Gaona, G. Rojas de la Serna, A. López Sánchez, M. Manzanera Díaz, F.J. Cortina Oliva, J. Gonzales Aguila ${ }^{1}$, C. Moreno Sanz

Digestive Surgery, General Hospital, La Mancha-Centro, ALCAZAR DE SAN JUAN, Spain

Introduction: Minimally invasive transanal surgery (TAMIS) is a surgical technique whose established indications are the complete exeresis of rectal polyps that are not resectable endoscopically or early rectal neoplasms with good prognosis criteria. Transanal devices with gel platform facilitate dissection in this field. However, one of the drawbacks of this approach is the oscillation of the right nerve, which hinders dissection and prolongs the surgical time.

Material and methods: We present the case of a patient with a central depression neoformation, located $8 \mathrm{~cm}$ from the anal margin in the posterior aspect of the rectum in a male patient The lesion occupies $25 \%$ of the circumference and was considered unresectable endoscopically. The endoscopic biopsies showed a tubulovillous adenoma with moderate dysplasia. Results: An exeresis of full thickness of the rectal wall is performed, with subsequent suture of the defect. We show in the video the use of a glove interposed in the pneumoperitoneum gum to maintain the stability of the neumorectum and the technique of dissection and suture, as well as the stability of the neumorectum with this technique throughout the procedure.

Conclusion: The use of a glove as a reservoir to stabilize the nemorectum is an economical and easy-to-use method that can safely replace extra devices.

\section{V043-COLORECTAL-Benign}

\section{TOTALLY LAPAROSCOPIC RECTAL RESECTION AND HISTERECTOMY FOR DEEP INFILTRATING ENDOMETRIOSIS WITH NATURAL ORIFICE SPECIMEN EXTRACTION}

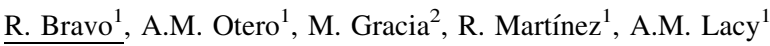

${ }^{1}$ Gastrointestinal Surgery, Hospital Clinic Barcelona, BARCELONA, Spain; ${ }^{2}$ Ginecology, Hospital Clinic Barcelona, BARCELONA, Spain

Aims: Endometriosis is a gynecologic disorder defined by the presence of endometrial glands and stroma outside the uterine cavity. Deep infiltrating endometriosis (DIE) invades $5 \mathrm{~mm}$ to the retroperitoneum of the pelvic sidewalls, the rectovaginal septum, or the muscularis of the bowel, bladder or ureters. The rectum is being the most common bowel site of involvement. For symptomatic DIE, medical therapy should always be the first-line treatment. Therefore, a minimally invasive approach using laparoscopy is considered the gold standard option and challenging aiming at complete disease excision. Also, there are several advantages of natural orifice specimen extraction when compared with abdominal incision that may directly impact the postoperative results of these young patients.

Methods: We report a case of a 36-year-old female with a 12-month history of chronic pelvic pain, dyschezia and rectal bleeding. These symptoms were refractory to hormonal, antispasmodic and opioid therapy. Magnetic resonance imaging reported a nodule $2 \times 2 \mathrm{~cm}$ invading the rectal wall $10 \mathrm{~cm}$ to the dentate lane. We performed a laparoscopy and we found the nodule at the uterine posterior wall invading the rectal anterior wall. The nodule was invading into the rectum in a large area so we proceeded with segmental resection and added hysterectomy and salpinguectomy because it was the preference of the patient. The anastomosis was created intracorporeally and the specimen was removed through the vagina performing in this way a totally laparoscopic procedure with natural orifice specimen extraction.

Results: The total operative time was $3 \mathrm{~h}$, the postoperative stay was uneventful and the patient was discharged on day four. The pathological report showed an endometrioma $4 \times$ $4 \mathrm{~cm}$ length predominantly involving colonic muscularis propria.

Conclusion: Laparoscopic surgery is a safe and feasible approach for the surgical management of deep infiltrating endometriosis of the rectum and the gold standard for female young patients that often need multiple surgeries. In addition natural orifice specimen extraction avoids potential complications of abdominal incisions.

\section{V044-COLORECTAL—Benign}

STATE-OF-THE-ART LAPAROSCOPIC RECTOPEXIES FOR COMPLETE RECTAL PROLAPSE: OUR MODIFIED RIPSTEIN AND RE-MODIFIED WELLS Methods:

T. Nishida, H. Ikuta, K. Nishimura, T. Kudo

Department of Surgery, Kasai City Hospital, KASAI CITY, Japan

Aims: The original Ripstein method of 1965, in which rectum was fixed posteriorly into the hollow of sacrum with T-shaped Teflon mesh tightly, showed good recurrence rate However, the complications of rectal stenosis, constipation, mesh penetration and mesh infection were reported. Then in 1987, McMahan and Ripstein modified the original Wells method of 1962 to fix $1 / 2$ back of rectum with rectangular Gore-Tex ${ }^{\circledR}$ sheet into sacrum, so as to reduce the complication of rectal stenosis. We that would know what shall be, must consider what has been. Therefore, we modified Ripstein method to keep good recurrence rate and get over the complications of original one. On the other hand, after the experience of 2 recurrent cases (8\%) after 25 modified Wells method, we clarified the cause of and remodified Wells method to overcome the recurrence.

Methods: We modified Ripstein method with T-shaped Bard ${ }^{\circledR} M e s h$. Horizontal side was made 1.2 times longer than the circumference of rectum including mesorectum to preven rectal stenosis after the mesh shrinkage of 7.7-18.9\%. Vertical side was made 1.2-2 times longer than the original method to sustain rectum physiologically along sacrum. And more, we re-modified Wells method with rectangular Bard®Mesh. Horizontal side was prolonged $9-11 \mathrm{~cm}$ so as to cover $4 / 5$ back of rectum and to be fixed accurately at sero-muscular tonic of ventral rectum. In both methods, mesh was fixed to sero-muscular tonic of ventral rectum with Endo Universal ${ }^{\mathrm{TM}}$ Stapler, and was fixed to sacrum or pre-hypogastric nerve fascia with Endo Universal ${ }^{\mathrm{TM}}$ Stapler (former) and/or AbsorbaTack ${ }^{\mathrm{TM}}$ (later).

Results: From February 2007 to October 2018, we underwent 100 rectopexies for complete rectal prolapse by 55 modified Ripstein, 25 modified Wells and 20 re-modified Wells methods. Pre/post-operative constipation was significantly reduced in every method. Pre/post-operative fecal incontinence was significantly reduced in re-modified Wells method. Recurrence rate were $0 \%, 8 \%$ and $0 \%$, respectively.

Conclusions: Modified Ripstein method is adapted to super-aged (85) female and applied to whom combined with other POP. Re-modified Wells method is adapted to under-aged ( $85>$ ) female and male. We would like to continue $0 \%$ of recurrence rate in both methods forever.

\section{V045-COLORECTAL-Benign}

KIDNEY MASS AND SIGMOID DIVERTICULAR STENOSIS: TWO FINE PROCEDURES AT THE SAME TIME

\section{A. Lo Conte ${ }^{1}$, G.V. Cunsolo ${ }^{1}$, M. Pezzatini ${ }^{1}$, G. di Natale $^{2}$, M. Gasparrini ${ }^{1}$}

${ }^{1}$ Week-Day Surgery, University, Sapienza, Ospedale Sant'Andrea, ROME, Italy; ${ }^{2}$ Urology, Clinica Mater Dei, ROME, Italy

Aims: We describe a case of a patient affected by a mass in the left kidney and a diverticular stenosis of the sigma.

Methods: A 65 years old woman complained abdominal pain in the left flank of the abdomen and in the left iliac fossa radiated to the hypogastrium, with fever and no passing flatus. Contrast enhanced computer tomography scan (CT-scan) showed a $7 \mathrm{~cm}$ mass of the superior pole in the left kidney and a colonic diverticulitis with thickness of the wall and a microperforation of the sigma. She underwent to medical therapy with resolution of the diverticulitis. After 4 weeks a laparoscopic nefrectomy and sigmoidectomy was planned. Patient was positioned on the right flank. This position was kept for both the procedures. We performed four trocar accesses along the left subcostal region and a periombelical incision for the specimen extraction.

Results: Post-operative course was uneventful. Patient was discharged in 7 post-operative day. Istopathological exam showed a renal cell carcinoma confined to kidney with no positive lymph nodes and a diverticular stenosis of the sigma.

Conclusion(s): Laparoscopy allowed to perform two fine procedures in a critical situation using few trocar incisions and obtaining good results. 


\section{V046-COLORECTAL-Benign}

COMBINED TRANSANAL AND LAPAROSCOPIC HARTMANN REVERSAL: A MINIMALLY INVASIVE OPTION FOR CHALLENGING CASES

\section{R. Bravo, A.M. Otero, R. Martínez, C. Gonzalez, R. Almenara, A.M. Lacy}

Gastrointestinal Surgery, Hospital Clinic Barcelona, BARCELONA, Spain

Background: Hartmann procedure consists in a sigmoidectomy followed by a terminal colostomy. Stoma is associated with complications and suboptimal quality of life, so the restoration of colonic continuity should be at least considered in any case. Open restoration has been associated with significant morbidity and mortality. Many authors have described the advantages of laparoscopic Hartmann reversal. We want to go a step further showing our experience using a combined laparoscopic and transanal approach in an attempt to improve the surgical technique in a patient with 5 previous abdominal surgeries and a rectovaginal fistulae.

Methods: The transanal and laparoscopic team work simultaneously. By the abdominal approach a pericolostomic incision is made, the distal affected colon is resected and a purse string suture is performed around the anvil of the EEA $31 \mathrm{~mm}$ Single-Use stapler with $4.8 \mathrm{~mm}$ Staples (Autosuture, Covidien). A $12 \mathrm{~mm}$ umbilical trocar is located for a $30^{\circ}$ camera and a GelPort Laparoscopic System (Applied Medical) with two $12 \mathrm{~mm}$ trocars is introduced through the colostomy wound. Hard pelvic adhesiolysis was performed and splenic flexure was also mobilized.The GelPoint path Transanal Access Platform (Applied Medical) is introduced through the anal canal with three trocars in a triangle position. The proximal rectum and mesorectum are dissected until the peritoneal reflexion. The previous stapler line with the resected tissue is then exteriorized throught the anus. The distal rectum is prepared with a circumferential purse string suture. The vaginal defect was sutured transanally. The proximal colon and the anvil are extracted through the rectal stump and connected to the circular stapler, performing an end-to-end anastomosis.

Results: The total operative time was $5 \mathrm{~h}$. The postoperative stay was uneventful and the patient was discharged on day 5.

Conclusions: As in patients with rectal cancer, dissection of the stump in Hartmann reversal procedure may be better and associated with shorter operative time. As with any new surgical procedure, it is probably too early to draw conclusions but nowadays transanal combined with laparoscopic approach seems to be a safe and feasible technique to perform a Hartmann reversal, especially in challenging cases.

\section{V047-COLORECTAL-Benign}

EMERGENCY LAPAROSCOPIC TREATMENT OF SIGMOID DIVERTICULITIS IN THE OBESE PATIENT: A CHALLENGE FOR THE COLORECTAL-SURGEON

\section{A. Costanzi, E. di Fratta, M. Gerosa, A. Miranda, A. Rosato, G. Mari, V. Berardi, D. Maggioni}

General and Emergency Surgery, ASST Monza-Desio Hospital, DESIO, Italy

Introduction: Diverticular disease is widespread in Western countries due to diet and lifestyle. Diverticular perforation with abscess formation is a common complicaton of diverticulitis and, if untreated or misdiagnosed may lead to several degrees of morbidity.

Case Report: A 48 year-old male patient, BMI 43, presented in the ED with left lower abdominal pain, vomiting and slight fever for 3 days. On P.E. he showed rebound tenderness on left iliac fossa. Neuthrofil leucocytosis $(21000 \mathrm{~mm} / \mathrm{c})$ and raised PCR $(201 \mathrm{mg} / \mathrm{dl})$ were present. Intravenous and endoluminal contrast enhanced $\mathrm{CT}$ revealed the presence of a large retroperitoneal fluid and gas collection, due to diverticular perforation, extended from pelvis to iliac bifurcation, involving the left urether. No hydrosoluble contrast media leakage or massive pnuemoperitoneum were present. After an initial conservative treatment without significan improvement an emergency laparoscopic left colectomy with primary anastomosis and laparoscopic retroperitoneal collection drainage was performed. The laparoscopic approach was very challenging due to the obesity of the patient and the presence of the abscess.

The patient was discharged on POD 12 after requiring re-intervention for dehiscence of the left iliac mini-laparotomy on POD 7.

Conclusion: Diverticular perforation in obese patients adds a further challenge to its laparoscopic treatment and deserves an aggressive surgical approach since its outbreak.

\section{V048-COLORECTAL-Malignant}

\section{LAPAROSCOPIC LEFT HEMICOLECTOMY WITH INTRACORPOREAL ANASTOMISIS FOR SPLENIC FLEXURE CANCER}

\section{P. Fabiano, E. Aycart}

General Surgery, Quiron salud Campo de Gibraltar, PALMONES, Spain

Although intracorporeal anastomosis has been demonstrated to be safe and effective after right colectomy, limited data are available about its efficacy after left colectomy for colon cancer located in splenic flexure. There are few studies comparing patients who underwent laparoscopic left colectomy with intracorporeal anastomosis or with extracorporeal anastomosis. Anyway literature shows that there is no significant difference between intracorporeal anastomosis and extracorporeal anastomosis about oncological result. As for right hemicolectomy, intracorporeal anastomosis seems to show a trend towards a faster recovery after surgery due to the shorter time to flatus and lower post-operative pain expressed in the mean VAS Scale. Laparoscopic left colectomy with intracorporeal anastomosis is associated with a lower rate of post-operative complications as for right colectomy. Literature results could suggest that a complete laparoscopic approach could be considered a safe method to perform laparoscopic left colectomy with the advantage of a guaranteed faster recovery after surgery. As usual further randomized clinical trials are needed to obtain a more definitive conclusion. We show a video of a 58 years old patient with a pure splenic flexure colon cancer who underwent to a laparoscopic left hemicolectomy with intracorporeal anastomosis.

\section{V049-COLORECTAL-Benign \\ SHOULD OCTOGENARIANS WITH HINCHEY IV DIVERTICULITIS BE TREATED BY LAPAROSCOPIC RESECTION?}

\section{A. Predrag, A. Csengeri, C. Cremona}

General Surgery, Mater Dei Hospital, MSIDA, Malta

Background: Despite the low volume of surgical cases at Mater Dei Hospital—Malta, a significantly large proportion of patients are admitted with diverticulitis, equating to approximately 18 surgical admissions a week. The majority of these cases are simple non-complicated diverticulitis-which are managed conservatively with intravenous antibiotics and analgesia. However in the emergency setting, cases with higher Hinchey scores are managed surgically—either via radiologically guided drainage or in the eventuality of diffuse peritonitis with pus or intraabdominal faeces-managed surgically via a Hartmann's procedure. With the initiation of the emergency laparoscopic service locally in 2012 -an average of 85 laparoscopic emergency surgeries are being done per year by one surgical firm. A recent audit of the same firm, titled-'A study of post-operative complications in a General Surgery Firm in 2018' showed that $14 \%$ of patients who underwent an emergency laparoscopic surgery experienced a Grade II or higher complications. (Clavien-Dindo Classification for Post-Operative Complications).

Case Presentation: Here we describe a case of a 81 year old asthmatic and hypertensive lady with an ASA score of III who presented to emergency after a right knee replacement with a four day history of lower abdominal pain. She was septic upon arrival to the resuscitation roomimmediately prompting the hospital's local septic management protocol. A CT scan of her abdomen showed a rectosigmoid perforation with free intra-abdominal air and fluid. The patien underwent laparoscopic Hartmann's procedure within $4 \mathrm{~h}$ of admission. After an uneventful postoperative recovery the patient was discharged home after a total of 4 days of hospitalisation. She was followed up at surgical outpatients with no adverse events over the course of the subsequent months.

Conclusion: This case exhibits the feasibility of laparoscopic Hartmann's procedure as a surgica modality for Hinchey Stage IV diverticulitis. The positive outcome supports the claim that for experienced surgeons Laparoscopic Hartmann's procedure remains a safe and viable option for elderly comorbid patients in the emergency setting. 


\section{V050-COLORECTAL-Benign}

\section{AN INSUAL CASE OF MESENTERIC CYST: LAPAROSCOPIC APPROACH WITH MINI INSTRUMENTS}

A.F. Aranzana Gómez ${ }^{1}$, J. Malo Corral ${ }^{1}$, J. Hernandez Gutierrez ${ }^{1}$, A. Muñoz Tébar ${ }^{1}$, B. Muñoz Jimenez ${ }^{1}$, A. Trinidad Borras ${ }^{1}$, S. Abad de Castro ${ }^{2}$

${ }^{1}$ General Surgery, Complejo Hospitalario Toledo, TOLEDO, Spain; ${ }^{2}$ General Surgery, Hospital tres culturas, TOLEDO, Spain

Introduction: Mesenteric cysts are a very infrequent pathology, they usually present an anodyne clinic, and their diagnosis is reached casually. Objectives: To demonstrate the safety and efficacy of the laparoscopic approach, in cases with intra-abdominal cysts of benign etiology, using material with mini-instruments, reducing surgical aggression, maintaining its safety and efficacy.Material and method: Clinical case: A 23-year-old man with no personal history of interest. In the last two months he presented episodes of pain in the right hypochondrium, exploration without findings, US-CT scan: a cystic tumor of $6 \mathrm{~cm}$. in hepatic colon angle compatible with uncomplicated benign mesenteric cyst, tumor markers and normal colonoscopy. Evidence of interest is exposed. Given the evolution it is decided tto. elective surgical.

Result: Intervention: laparoscopic approach, 4 trocars, two of $3.5 \mathrm{MM}$, OPTICS OF $5 \mathrm{MM} 30^{\circ}$, benign cystic tumor, with colloid content of more than $7 \mathrm{~cm}$. of diameter in antimesenteric border of colon, which is not possible to separate, mobilization and resection is carried out by ENDOGIA, including a portion of the colonic wall, appendectomy, extraction in a pocket. Good postoperative course, alt to 2 nd day. DEFINITIVE AP: Mesenteric cyst, absence of malignancy.

Conclusion: The laparoscopic approach is a valid and effective alternative in cases of benign intra-abdominal cystic pathology, the use of mini instruments reduces surgical aggression, favoring the recovery of the patient.

\section{V051-COLORECTAL-Malignant}

\section{ENDOSCOPIC PROSTHESIS POSITIONING IN PATIENTS WITH ULTRASLOW RECTAL STENOSIS WITH USE OF ULTRAFLEX PRECISION}

\section{S. Giungato, A.S. Pepe}

Emergency Surgery and Endoscopy, Castellaneta Hospital, TARANTO, Italy

Aims: Bowel endoscopic prosthesis is a procedure applied worldwide for treatment of intestinal occlusion by neoplasm or inflammatory stenosis. In our emergency department we have tried to performe this technique for treatment of ultralow stenosis.

Methods: In our center we have performed endoscopic treatment of ultralow rectal stenosis with positioning of Ultraflex Precision Prosthesis, $25 \mathrm{~mm} \times 8.7 \mathrm{~cm}$. We have observed: complications; pain; fecal inctinence; well-being of patients; time of hospitalization.

Result: We have treated 2 patients with Ultraflex Precision prosthesis. 1 Male, $70 \mathrm{yr}$, wuth doblue post-operative coloanal stenosi and 1 Women, with ultralow rectal neoplatis stenosis $(4 \mathrm{~cm}$ from anla verge). Both patients were discharge ater 3 days from prosthesis positionins without pain and complications. The first patient, with protection ileostomy, showed fecal incontinence before the operation and was performed prosthesis positioning because rectal losses of infected material and fever. Fecal incontinence was showed also after procedure but he had not fever. Second patient, $93 \mathrm{yr}$, with ultralow rectal tumor, after prosthesis positioning was submitted to radiotherapy and she decided for not to be operated and she survives after 6 months in ful well-being.

Conclusion: Endoscopic prosthesis positioning is a consolidated procedure for treatment of bowel obstruction. This study demonstrated that this procedure is safe and this kind of prosthesis is suitable for correct positioning.

\section{V052-COLORECTAL-Malignant}

TRANSANAL MINIMALLY INVASIVE SURGERY AS TREATMENT OF A RECTAL ADENOMA OCCUPYING 3/4 OF THE LUMEN

N. Mestres Petit, J.E. Sierra Grañon, C. Cerdán Santacruz, M. Santamaría Gómez, J. Escoll Rufino, M. Rufas Acin, J. Ortega Alcaide, J.J. Olsina Kissler

Department of Surgery, Hospital Arnau de Vilanova, LLEIDA, Spain

Aims: To remark the feasibility of TAMIS for treatment of big rectal lesions without invasive signs.

Methods: Single case video of TAMIS for rectal adenoma with high grade dysplasia

Results: We present a case of a 67 years old man with faecal occult blood test positivity that was diagnosed by colonoscopy of a villous lesion at $9 \mathrm{~cm}$ of anal verge. Biopsies were taken showing a tubulovillous adenoma with high grade dysplasia. A rectal MRI was done showing the lesion fixed to the postero-lateral left side of the lumen at $9 \mathrm{~cm}$ of anal verge. No pathological lymph nodes were reported. Extension study was negative. The case was presented in multidisciplinary committee agreeing in local excision. In October 2018 the procedure was done without incidents. The patient was placed in lithotomic position finding a lesion occupying of the lumen. Resection was done without incidents and posterior suture with 2 continuous barbed sutures. He presented an uneventful recovery being the patient discharged in $3^{\text {rd }}$ postoperative day. Definitive pathological findings showed a pTis with negative margins. After three months of followup the patient remains with good functional results and waiting for the first endoscopic revision.

Conclusions: TAMIS is a safe and feasible technique with low morbidity that gives us an alternative for early rectal cancer or big rectal lesions much less invasive than techniques used until now.

\section{V053-COLORECTAL-Malignant}

\section{TECHNICAL ASPECTS OF LAPAROSCOPIC D3 LYMPHADENECTOMY IN RIGHT COLON NEOPLASIA}

A. Rabal Fueyo ${ }^{1}$, J. Bollo Rodriguez ${ }^{1}$, D. Sacoto ${ }^{1}$, M. Solans Solerdelcoll ${ }^{1}$, C. Martinez Sánchez ${ }^{2}$, N. de la Fuente ${ }^{1}$, E.M. Targarona Sole $1^{2}$

${ }^{1}$ General Surgery, Hospital de la Santa Creu i Sant Pau, BARCELONA, Spain; ${ }^{2}$ Colorectal Surgery, Hospital de la Santa Creu i Sant Pau, BARCELONA, Spain

Complete mesocolon excision and D3 lymphadenectomy are two fundamental points in the oncological surgery of right colon cancer. Most of the adenopathic recurrences of colon neoplasia in tumors located in the hepatic angle and the ascending colon are located near the head of the pancreas and the vascular axis of the superior mesenteric vein due to an alleged incomplete dissection.

We present a case of right colon neoplasia where we performed a laparoscopic right hemicolectomy associated with a D3 lymphadenectomy. We use medial to lateral dissection of the mesocolon focused on the dissection of the superior mesenteric vein with the identification of ileocolic vascularization, right colic vessels and Henle's trunk. This approach is safe and facilitates a correct resection of the mesocolon, which is approached following the embryological plans and a vascular ligature near the bifurcation.

The performance of an extended lymphadenectomy allows a wider resection of the mesocolon and the excision of a greater number of lymph nodes, all of which can contribute to a greater survival. 


\section{V054-COLORECTAL-Malignant}

\section{USE OF INDOCYANINE GREEN IN LAPAROSCOPY CYTOREDUCTIVE SURGERY FOR PERITONEAL CARCINOMATOSIS FROM COLORECTAL CANCER: TWO CASES}

M.P. Gutierrez Delgado ${ }^{1}$, J. Carrasco Campos ${ }^{1}$, A. Rodríguez Cañete ${ }^{2}$, S. Mera Velasco ${ }^{1}$, J. Gonzalez Cano ${ }^{1}$, M. Pitarch Martinez ${ }^{1}$, A. Titos García ${ }^{1}$, J. Moreno Ruiz ${ }^{1}$, I. González Poveda ${ }^{1}$, M. Ruiz López ${ }^{1}$, J.A. Toval Mata ${ }^{1}$, J. Santoyo Santoyo ${ }^{1}$

${ }^{1}$ Cirugía General y Digestiva, Hospital Regional de Málaga, MALAGA, Spain; ${ }^{2}$ Esofagogástrica, Hospital Regional de Málaga, MALAGA, Spain

Aim: The aim of this study was to evaluate the role of fluorescence imaging using an injection of indocyanine green (ICG) $24-36 \mathrm{~h}$ before laparsocopic surgery, in the detection of peritoneal carcinomatosis (PC) due to colorectal cancer (CCR).

Background: Peritoneal metastasis (PM) occur in $30-40 \%$ of patients with CCR. Cytoreductive surgery (CS) followed by hiperthermic intraperitoneal chemotherapy (HIPEC) is the only potentially curative option in patients with limited PC. This treatment results are comparable to results obtained in patients surgically treated for liver metastases.

Fluorescence imaging guided surgery can improve tumor detection. ICG has been shown to be useful for identifying small subclinical tumors that were not identified at surgical exploration. Cases: Two patients who was diagnosed of CCR 2 and 5 years ago and who went under sigmoidectomy surgery, a CT scan of follow-up showed peritoneal nodule suggestive of PM. ICG, at $0 ' 25 \mathrm{mg} / \mathrm{kg}$ of patient weight, was intravenous injected $24-36 \mathrm{~h}$ before surgery. Exploratory laparoscopy was the selected approach in these cases, in one of them we could find under standard white light, a small liver tumor and a peritoneal nodule in left lower quadrant, both of them hyperfluorescent by infrared camera system. In the other case, ICG showed us a peritoneal metastasis on the small bowell. No more lesions were found in the abdominal cavity, and HIPEC was applied.

Results: Nodules were hyperfluorescent, and all of them were malignant. Both patientes, are currently undergoing chemotherapy and follow-up by Oncology.

Conclusion(s): The efficacy of PC treatment is related with a properly preoperative imaging diagnosis of the disease, but the poor sensitivity for identifying small peritoneal metastasis are the major obstacle to achieve a complete resection and that leads to peritoneal recurrence. Imageguided surgery using ICG, could represent an advance in the detection of small peritoneal nodules. There are only a few clinical studies that have analyzed the role of ICG for the staging of $\mathrm{PC}$, specially from CCR, and nearly in all of them the selected approach were exploratory laparotomy. This study presents a laparoscopy case, as a non-invasive way of CS in selected patients with limited PC.

\section{V055-COLORECTAL-Malignant}

TIPS AND TRICKS FOR LAPAROSCOPIC MANAGEMENT FOR THE APPENDICEAL MUCOID TUMOR: CASE REPORT AND REVIEW OF THE LITERATURE

M. Delgado Morales ${ }^{1}$, R. Pérez Quintero ${ }^{2}$, P. Rodríguez González ${ }^{2}$, N. Cisneros Cabello ${ }^{2}$, J. Guadalajara Jurado ${ }^{2}$

${ }^{1}$ Cirugía General, Hospital Infanta Elena, HUELVA, Spain; ${ }^{2}$ Cirugía General, Hospital Juan Ramón Jiménez, HUELVA, Spain

Aims: Mucinous appendiceal neoplasms (MAN) are rare, and they are detected in $0.2-0.3 \%$ of appendectomy specimens.

A new category, Tis, was created for low-grade appendiceal mucinous neoplasms (LAMNs) that invade or push into the muscularis propia by AJCC Cancer Staging 8th Ed. Management of these tumors depends on stage and histology.

Traditionally, laparotomy was the most recommended approach, however, if laparoscopy is safe, it could be used. The laparoscopic appendectomy should be done with 'not touch' technique and a radical approach has been recently proposed for its treatment.

The laparoscopic radical appendectomy should start by exploring complete abdominal cavity. Grasping of appendix should not be done. Complete resection of mesoappendix is obligated. Cequectomy with stapled endoGIA is necessary. The specimen must be extracted in an endobag. Methods: We report a case of a 64 year-old female patient with a personal history of three caesarean sections.

This patient was studied due to chronic abdominal pain. A computerized axial tomography was performed, showing an appendix increased in size and a thick wall. The colonoscopy evidence a lesion that protrudes from appendiceal base which is biopsied.

Results: A laparoscopic way was used and large and width appendiceal was viewed $(10 \times 2 \mathrm{~cm})$ Furthermore, a rounded right anexial tumor was also found. A radical not touch laparoscopic appendectomy with stapled cequectomy was done. The intraoperative study was mucinous appendiceal tumor without serose affection. The final result was pTisNx (LAMN) without resection margins affected.

After $48 \mathrm{~h}$ of admission, the patient is discharged without incidents.

Conclusion(s): Minimally invasive surgery in LAMNs is possible if it is performed with enough experience, following specific rules and tips to manage this tumors. A correct follow-up should be carried out using tumor markers and computer tomography (CT).

\section{V056-COLORECTAL-Malignant}

\section{A STREAMLINED APPROACH TO THE LAPAROSCOPIC MANAGEMENT OF BENIGN AND MALIGNANT COLOVESICAL FISTULAE}

M. Abdeldayem, C. Matthews, P. Blake, B. Gwilym, L. Maw, A.G. Masoud

Colorectal Surgery, Prince Charles Hospital, MERTHYR TYDFIL, United Kingdom

Introduction: Resection of both benign and malignant colovesical fistulae can be particularly challenging and carry with it specific surgical considerations. Often there is a large inflammatory mass sat within a narrow pelvis, limiting specimen mobility and consequently access to dissection plains. Additionally, with the underlying inflammatory process, the ureters may be displaced anatomically and be at risk of injury.

Aim: To demonstrate a streamlined and reproducible approach to the laparoscopic management of both benign and malignant colovesical fistula, with specific emphasis on the different modalities for bladder repair.

Method: The following method portrays an overall technique which is adapted dependant on the clinical scenario and specific intra-operative findings:

Approach to abdominal cavity in standard fashion.Identification of right ureter.Poster-medial mobilisation of the mass to facilitate delivery out of the pelvis followed by Visualisation of the left ureter on the medial AND lateral sides before division of the fistula.Division of the fistula in benign disease or resection of the bladder dome in malignant disease.Transverse laparoscopically sympathetic suprapubic skin incision.Vertical incision through Linea Alba to deliver bulky specimen.Intra/extracorporeal repair of bladder dome.

Results: All of the considered cases were successfully completed with a laparoscopic approach, irrespective of the malignant status of the disease in question.

Conclusion: Both benign and malignant colovesical fistula disease can make the laparoscopic approach to resection challenging, especially when encountering a bulky mass in a narrow male pelvis. The stepwise and streamlined approach considered here can help facilitate successful and safe laparoscopic completion without the necessity to convert to open. 


\section{V057-COLORECTAL-Malignant}

\section{COMBINED LAPAROSCOPIC ABDOMINOPERINEAL RESECTION OF RETRORECTAL TUMOR}

J. Ye Zhou, M. Solans, N. de la Fuente, M.C. Martinez, M.P. Hernandez, J. Bollo, E. Targarona

Cirugia General y Digestiva, Hospital de la Santa Creu i Sant Pau, BARCELONA, Spain

Background: Primary neoplasms of the retrorectal space are very rare. They are located in anatomically difficult area to be addressed, hence a complete evaluation of the lesion is required to determine the extent of resection and the appropriate surgical approach, which include posterior, abdominal and combined abdominoperineal, depending on the characteristics of the lesion. Objective: to show a combined laparoscopic abdominoperineal approach of retrorectal tumor. Method: we present a video of a combined laparoscopic abdominoperineal resection of a lowlying retrorectal tumor in a 73-year-old female without prior abdominal surgery.

Conclusion: Retrorectal tumors are infrequent. Their anatomical location can make difficult the surgical approach. Preoperative imaging can provide useful information for surgical planning. In the recent years, minimally invasive surgical approach has been proposed. Laparoscopic approach is feasible and safe, but it is important to select adequately the patients.

\section{V059-COLORECTAL-Malignant}

SINGLE-INCISION ROBOTIC RIGHT HEMICOLECTOMYVIDEO PRESENTATION

\section{T.H. Wang, T.C. Chang, Y.T. Chen, T.Y. Hsu}

Department of Colorectal Surgery, Taipei Medical University-Shuang Ho Hospital, NEW TAIPEI CITY, Taiwan

Aims: Single-incision laparoscopic colectomy (SILC) aims to achieve better cosmetic outcomes, less pain, and faster recovery compared to multi-port laparoscopic colectomy, but it also has several limitations, especially the technical difficulties. We report our experience with singleincision robotic right hemicolectomy via video presentation.

Methods: We arranged robotic-assisted single-incision right hemicolectomy for a 78-year-old female patient with ascending colon tumor. The operation was performed with Gloveport singleport device and a three-arm da Vinci robotic surgical system through a small midline umbilical incision. Colectomy was proceeded by a medial-to-lateral approach along with one or two accessory instruments for maintaining sufficient bowel traction or surgical field exposure. After vessel ligation, complete colon mobilization and right side omentum division, the robotic arms were undocked to perform anastomosis extracorporeally.

Results: The operation was performed successfully without drainage tube placement. The total operative time was $193 \mathrm{~min}$. The bowel movement returned on post-operative day 5, and the patient tolerated normal soft diet on post-operative day 7. She was hospitalized for 8 days after operation. The pathology report revealed colon adenocarcinoma (T1N0M0, tumor size $1.8 \mathrm{~cm}$ ), and 19 lymph nodes were harvested.

Conclusions: Single-incision robotic colectomy (SIRC) approach seems feasible and safe in treatment of ascending colon cancer. This surgical option provides less pain and wound scar for the patient. Moreover, it also achieves further benefits for the surgical procedures compared to SILC. Reasons being, first, it has better instruments flexibility and precision with endo-wrist, as well as less instruments clashing. Second, the improved camera stability achieved through the use of the robotic arm is unattainable through manual hand-controlled methods. Third, roboticassisted approach gives us an ergonomic environment, which enables the operator to control the arms while sitting by the console, and also to reassign them whenever they cross each other or block the surgical view. In spite of the advantages above, we still need to sincerely consider each patient's situation for proper management.

\section{V060-COLORECTAL-Malignant}

\section{APPLICATIONS OF INDOCYANINE GREEN ENHANCED FLUORESCENCE IN LAPAROSCOPIC COLORECTAL RESECTIONS}

\section{Santi ${ }^{1}$, L. Casali ${ }^{2}$, C. Franzini ${ }^{2}$, A. Rollo ${ }^{2}$, V. Violi ${ }^{1}$}

${ }^{1}$ Surgery, Hospital of Fidenza, University of Parma, FIDENZA (PR), Italy; ${ }^{2}$ Surgery, Hospital of Fidenza, FIDENZA (PR), Italy

Recently, Indocyanine Green (ICG) fluorescence has been introduced in laparoscopic colorectal surgery to provide detailed anatomical information.The aim of our study is the application of ICG imaging during laparoscopic colorectal resections: to identify sentinel lymph node, for studying its prognostic value on nodal status, to facilitate vascular dissection when vascular anatomy of the tumor site is unclear and to assess anastomotic perfusion to reduce the risk of anastomotic leak. After tumor identification $5 \mathrm{ml}$ of ICG solution $(0.3 \mathrm{mg} / \mathrm{Kg})$ is subserosal peritumoral injected. A Full HD IMAGE1 S camera, switching to NIR mode, in about 10 min displays fluorescence: the SLN is identified and the SLN biopsy (SLNB) is performed. When tumor is in difficult site, as hepatic or splenic flexure, $5 \mathrm{ml}$ of ICG solution $(0,3 \mathrm{mg} / \mathrm{Kg})$ is intravenous injected. In about $30-50 \mathrm{~s}$ a real-time angiography of tumor area is obtained; on this guide, vascular dissection and pedicle ligation is performed.After anastomosis, another $5 \mathrm{ml}$ of ICG solution is injected to confirm anastomotic perfusion. If there is an ischemic area, a new anastomosis is performed. From November 2016, 70 patients were enrolled: 22 left colectomy, 38 right colectomy, 2 transverse resections, and 8 resections of splenic flexure. In ten cases, intraoperative angiography led to the identification of vascular anatomy. In two cases the anastomotic perfusion wasn't good and the surgical strategy was changed. Four postoperative complications occurred, of which one anastomotic leak, due to a mechanical problem. From November 2017, 40 patients were enrolled to perform the SLNB: 23 right colectomy, 11 left colectomy, 1 transverse resection and 5 splenic flexure resections. The SLN was identified in 37 cases. 17 cases were found to be NO to the conventional examination and were subjected to ultrastaging. ICG-enhanced fluorescence imaging is a safe, cheap and effective tool to increase visualization during surgery. It's recommended to reduce the incidence of anastomotic leak, to facilitate the assessment of vascularization in order to perform oncological resections, and to perform the SLNB to study its clinical role on nodal status and for the SLN ultrastaging in order to identify the micrometastases. 


\section{V061-COLORECTAL-Malignant}

\section{LAPAROSCOPIC LATERAL LYMPH NODE DISSECTION FOR RECTAL CANCER-A SAFE, EFFECTIVE AND REPRODUCIBLE APPROACH}

\author{
J. Azevedo, C. Ordonez, B. Vailati, G. São Julião, R. Oliva Perez, \\ Colorectal
}

\section{Angelita and Joaquim Gama Institute, SÃO PAULO, Brazil}

Background: Surgical emptying of lateral pelvic lymph nodes (LLND) is a strategy used differently when compared the approaches to rectal cancer in the west and eastern countries. There is evidence that $=5 \mathrm{~mm}$ lymph nodes in lateral compartment should be removed, even in the setting of neoadjuvant chemoradiation. Minimally invasive surgery with nerve-sparing technique and sharp dissection with minimal bleeding may help overcome the significant complexity of the procedure that may have been a technical obstacle to implementation in the past. The standardization of the technique may help implementation with shorter learning curves and excellent surgical outcomes.

Methods: A 56-year-old male with distal rectal cancer underwent neoadjuvant CRT for a mrT3cN2M0 mrEMVI + mrCRM + disease. There was one left obturator node of $7 \mathrm{~mm}$ prior to CRT. Following 12 weeks of CRT completion, the patient underwent taTME for the primary disease followed by left lateral node dissection by laparoscopy.

Results: The present video illustrates the most relevant surgical steps to perform lateral node dissection. The procedure has been didactically divided into 7 steps. The left ureter is identified and retracted using a vessel loop (Step 1). Identification of the common iliac vein and dissection with subsequent identification of psoas and internal obturator muscles (Step 2). Identification and dissection of accessory vessels. (Step 3) Identification of obturator nerve and obturator vessels (Step 4). Blunt dissection of obturator nerve (Step 5). Identification and ligation of obturatory vessels. (Step 6) Umbilical artery is skeletonized to allow identification and clearance of fatty tissue along superior vesical arteries, internal iiliac artery/vein, inferior vesical artery and internal pudendal artery (Step 7). Postoperative course was uneventful.

Conclusion: Standardization of lateral-node dissection for rectal cancer has paramount importance. Laparoscopic lateral-node dissection for rectal cancer provides optimal anatomical view and allows safe dissection of the nodes of interest.

\section{V062-COLORECTAL-Malignant}

\section{TOTAL MESOCOLIC EXCISION FLUORESCENCE-GUIDED: IN SEARCH OF LYMPHATIC FLOW}

G.P. Martin-Martin, J.M. Olea, L. Fernandez, A. Ochogavia, M. Fernandez, J.J. Segura, N. Alonso, M. Gamundi, X. Gonzalez

Colorectal, Hospital Universitario Son Espases, PALMA, Spain

Aims: The aim of this video is to describe our technique using fluorescence to assess the lymph flow to ensure a complete mesocolic excision and central vascular ligation in order to provide expertise to contribute to the standardization of this new tool.

Methods: Laparoscopic right colectomy with total excision of the mesocolon was proposed in all cases. For the detection of lymph flow, we injected indocyanine green dye (1 milliliter of 25 milligrams dye dilution in 10 milliliter of distilled water) into the subserosal to submucosal layer around the tumor at 1 point with a 21-gauge injection laparoscopically after trocar insertion, and observed the lymph flow using a near-infrared system (Visera Elite II, Olympus) after injection. We also performed a total mesocolic excision with central vascular ligation in the region where the lymph flow was fluorescently observed.

Results: $7(100 \%)$ patients were included. No intraoperative or postoperative complications presented. No adverse effects were reported due to the infusion of indocyanine green. The lymph flow was visualized intraoperatively in a satisfactory way helping the surgeon in decision making to determine an appropriate separation line of the mesentery. The section line of the mesocolon was modified in $1(14 \%)$ case based on the findings obtained by fluorescence. The mean operative time was 160 (42) $\mathrm{min}$. The morphometric laboratory data of the specimens to audit the correct complete mesocolic excision were satisfactory according to the oncological standards. Conclusion: Fluorescence lymphography during colorectal surgery was feasible and reproducible with a minimum of added complexity. Fluorescence-guided surgery may be a helpful technique for determining an appropriate total mesocolic excision in colon neoplasms.

\section{V063-COLORECTAL-Malignant \\ COMPLETE MESOCOLIC EXCISION (CME) FOR RIGHT COLON CANCER: OUR TECHNIQUE}

M. Ballabio, S. Macina, M. de Francesco, L. Baldari, A. Spota, S. Petrucci, M. Della Porta, E. Cassinotti, L. Boni

General Surgery, Ospedale Policlinico di Milano-Università degli Studi di Milano, MILANO, Italy

Aims: This video shows our technique for complete mesocolic excision (CME) during right colectomy for cancer

Methods: In this video, a 62 years old patient underwent a laparoscopic right colectomy with CME for a cancer of the ascending colon diagnosed with a colonoscopy performed after positivity to fecal occult blood test (FOBT). After CT scan staging we obtained 3D printed models to clarify patient's vascular anatomy. Patient was placed in supine position, 4 trocars were inserted in left quadrants as for standard right colectomy. CME is performed by sharp dissection between the visceral fascia that covers the posterior lay of the mesocolon and the parietal fascia that covers the retroperitoneum (Toldt's fascia). The ileo-colic vessels are used as landmark to identify the right anterior surface of the superior mesenteric vessels. With a caudo-cranial approach, the mesocolon is sharply dissected and the root of tributaries venous is ligated, up to the inferior margin of the pancreas. The gastro-colic trunk is dissected out with ligation of the right colic vein, while the gastroepiploic vein is preserved (harvesting the sixth group lymph node). The pancreas-duodenum fascial plane is entered and all the lymphoid tissue around the vessel surface is harvested. Procedure is completed with ileo-transverse intracorporeal stapled anastomosis.

Results: In our experience, between April 2017 and December 2018, 46 laparoscopic right hemicolectomies with CME were performed. We had no major intraoperative vascular lesions. No patients needed intraoperative blood transfusion. Compared to our series of standard right colectomies we did not notice any significant difference in post-operative complications. The follow-up is too short to demonstrate if the CME approach has a better oncological outcome compared to standard right colectomy.

Conclusions: Laparoscopic CME is feasible, although it requires a higher expertise level of surgical know-how. The quality of evidence is limited and does not consistently support the superiority of CME as compared to standard right colectomy. Better data are needed before CME can be recommended as the standard of care for colon cancer resections.

\section{V064-COLORECTAL-Malignant \\ OUR TECHNIQUE OF LAPAROSCOPIC RIGHT HEMICOLECTOMY}

\section{H. Bando}

Gastroenterological Surgery, Ishikawa Prefectural Central Hospital, KANAZAWA, Japan

Aim: In case of right-sided transverse colon cancers, it is necessary to dissect the lymph nodes around the root of the middle colic vessels. But in this area there are dangerous organs, for example : pancreatic head, duodenum, and gastrocolic trunk. It is the point of our technique that we resect the accessory right colic vein and middle colic vein, and then dissect pancreas head and duodenum at early step of the operation.

Method: We perform the operation by five trocars. The first step is to transect the great omentum, and confirm the lower edge of pancreas. There are much adhesion between mesocolon of transverse colon and stomach, great omentum. It is very important to dissect the adhesion accurately. Secondly, the mesocolon is incised at lower edge of pancreas. It is possible to detect the lower edge of pancreas in obese people. The anterior surface of superior mesenteric vein is exposed. The accessory right colic and middle colic vein are resected. And then front face of surgical trunk, pancreas, and duodenum is dissected caudally as possible. The superior mesentery artery is resected below the mesocolon after flip up of transverse colon. This approach is safe and feasible, because the dangerous organs are handled by direct vision. By that, extraction of intestine is easy from small incision. Afer flip up of transverse colon, the mesenteric of ileum is incised. The roo of ileocecal vessels is exposed and these are resected. The peritoneum of the front of superior mesenteric artery is incised, and the lymph nodes around the surgical trunk are dissected. This dissected area is easily connected with the one done beforehand. Uniquely we resect the mesocolon and major omentum from the root of dissected vessels to resected side of transverse colon. And then right-side colon is dissected medial approach.

Conclusion: We dissect the dangerous organs in advance. That prevent major injury of them. 


\section{V065-COLORECTAL-Malignant}

\section{IMPROVED VISUALISATION AIDS SAFETY IN THE STEPWISE APPROACH TO LAPAROSCOPIC TOTAL MESORECTAL EXCISION}

\author{
J. Waterman ${ }^{1}$, M. Abdeldayem ${ }^{1}$, D. Brown ${ }^{2}$, P.N. Haray ${ }^{3}$
}

${ }^{1}$ Colorectal Surgery, Prince Charles Hospital, MERTHYR TYDFIL, United Kingdom; ${ }^{2}$ Digimed ${ }^{\circledR}$, ESSEX, United Kingdom; ${ }^{3}$ Colorectal Surgery, University of South Wales, WALES, United Kingdom

Background: Good visualisation of the operative field is a fundamental requirement for safe laparoscopic colorectal surgery. Over the past 25 years of the senior author's experience, camera systems have evolved from single to three chip, High Definition (HD) and most recently, the $4 \mathrm{~K}$ system. In parallel, the rest of the infrastructure such as cables, processors, monitors etc. have also undergone improvements, resulting in improved image quality.

Aim/Methods: We present a video of a case of Laparoscopic Total Mesorectal Excision (TME), performed with strict adherence to our previously published 'Stepwise Approach to Laparoscopic Colorectal Surgery' which places particular emphasis on safety aspects. TME was performed in a 54 year old male patient with history of previous abdominal as well as robotic prostatic surgery. The procedure was filmed with all components including the camera head, cables, processing unit, screens as well as the recording/mixing decks being $4 \mathrm{~K}$. Multiple external $4 \mathrm{~K}$ cameras were also used. Live transmission to a remote audience as part of our masterclass was achieved using appropriate bandwidth and projection on to $4 \mathrm{~K}$ screens.

Results: Feedback from the operating team as well as from the live audience was that the image quality was far superior to $\mathrm{HD}$ systems. The $4 \mathrm{~K}$ system accorded a degree of clarity well beyond usual expectations. The depth of field also appeared to be different initially, but within a few minutes of starting the procedure and acclimatisation, the effects were appreciable. The clarity of the image which showed the fine details of the dissection planes and anatomical landmarks as well as the vibrancy of the vasculature gave a distinct three-dimensional effect to the picture. This excellent visualisation added one more layer of safety and complemented our stepwise approach for a successful procedure. Conclusion: The laparoscopic $4 \mathrm{~K}$ system, in our practice, proved to be a beneficial visualisation tool to enhance the accuracy of dissection. Vital structures appeared to be more vivid and clearer with dissection planes being more easily apparent. In our opinion the Laparoscopic $4 \mathrm{~K}$ system when combined with a systematic approach enhances safety, especially in complex laparoscopic colorectal surgery.

\section{V067-COLORECTAL-Malignant}

\section{APPROACH TO LAPAROSCOPIC SURGERY UNDER ICG FLUORESCENCE NAVIGATION AGAINST REDO- COLECTOMY -FOR CONVINCING LAPAROSCOPIC RE-CME}

H. Takahashi ${ }^{1}$, M. Miyoshi ${ }^{1}$, H. Haraguchi ${ }^{1}$, T. Hata ${ }^{1}$, M. Chu ${ }^{1}$,

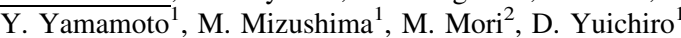

${ }^{1}$ Gastroenterological Surgery, Japan / Osaka University Graduate School of Medicine, SUITA, OSAKA, Japan; ${ }^{2}$ Department of Surgery and Science, Kyushu University, FUKUOKA, Japan

Accumulating evidence suggests that laparoscopic surgery for colon cancer has feasibility and efficacy equal to or over conventional laparotomy. For cases with pasthistory of laparotomy, especially history of colon resection, however, there is almost no evidence for laparoscopic recolectomy for metachronous colon cancer.

Since 2016, we have been used submucosal local injection of indocyanine green (ICG) around primary colorectal cancer by using intraoperative endoscopy, and complete mescolic excision (CME) have been convincingly carried out, which was clarified by completely resected ICG positive area. Although evidence on the oncological efficacy of ICG guided surgery has not yet been clarified, since it can be easily judged whether CME is performed clearly, it is considered that ICG guided surgery for primary colon cancer is useful for education.

Recently, we are applying this to ensure convincing CME for patients with colorectal cancer who had a history of colic resection. The representative case is as follows. A 60-year-old female was diagnosed as advanced sigmoid colon cancer, and laparoscopic sigmoidectomy with high tie of the inferior mesenteric artery was performed 10 years ago. Then she was diagnosed as the metachronous descending colon cancer. The feeding artery of the new tumor should be the left colic artery, however, the left colic artery was already resected and genuine feeding artery was not identified by preoperative examination. By injecting ICG into submucosa endoscopically during operation, it was clearly observed that the lymphatic flow from the tumor was directed to the inlet portion of the inferio mesenteric vein (IMV). Re-CME was performed by ligating the inlet of IMV. Intraoperative ICG was also useful for clarifying the borderline for adhesion detachment of pastoperation between the mesentery and retroperitoneum (Figure). Interestingly, ICG flow in the mesentery direct to of the anus side was disrupted clearly at the past anastomotic site.

We believe that laparoscopic surgery under ICG guidance is potential useful tool that can confirm evidence to date more intuitively in real time. Further studies, ideally randomized controlled trials, are required for define the oncological usefulness of ICG guided surgery for re-do colectomy. The operation movie will be presented at the meeting.

\section{V068-COLORECTAL-Malignan}

A NOVEL APPROACH FOR LAPAROSCOPIC LATERAL PELVIC NODE DISSECTION AFTER NEOADJUVANT CHEMORADIATION FOR LOCALLY ADVANCED RECTAL CANCER

\section{Z. Liu, X.S. Wang}

Department of Colorectal Surgery, National Cancer Center/Cancer Hospital, Chinese Academy of Medical Sciences, BEIJING, China

Background: Laparoscopic lateral pelvic node dissection (LLPND) is a minimally invasive alternative to open surgical therapy for advanced low rectal cancer patients. In this video, we demonstrate the technique of LLPND for rectal cancer patients with suspicion of LLN metastases after neoadjuvant chemo-radiation.

Methods: The principle of this approach is en bloc resection with bilateral peritoneum. The peritoneum is incised lateral to the ureter following the line between external and internal iliac vessels. In the next step, LLPND dissection of the regional lymph node and high ligation of inferior mesenteric vessels were performed. A contralateral LLPND was performed in the same manner as a mirrored technique. After extracting the specimen, an end-to-end double-stapled circular anastomosis was performed.

Results: The procedure was done safely without any complications. The surgical duration was 245 mins, and the blood loss was $50 \mathrm{~mL}$. The number of harvested lateral pelvic lymph nodes was 15 . The TNM stage was ypT4aN2M0.

Conclusion: This approach enables extended resection during lymph node dissection, allowing autonomic nerve preservation. It is maybe a helpful approach in the treatment of locally advanced rectal cancer with a lateral lymph node metastasis.

\section{V069-COLORECTAL-Malignant}

SIMULTANEOUS INTRAPERITONEAL AND INTRALUMINAL ICG FLUORESCENCE TESTING FOR LOW COLORECTAL ANASTOMOSIS

\section{Kryzauskas ${ }^{1}$, T. Poskus ${ }^{1}$, M. Jakubauskas ${ }^{1}$, A. Dulskas ${ }^{2}$,} E. Poskus ${ }^{1}$

${ }^{1}$ Clinic of Gastroenterology, Nephrourology and Surgery, Institute of Clinical Medicine, Faculty of Medicine, Vilnius University, Vilnius, VILNIUS, Lithuania; ${ }^{2}$ Surgery department, National Cancer Institute, VILNIUS, Lithuania

Aims: The aim is to present an inspection method where the anastomosis vascularity is testing simultaneously using the indocyanine green fluorescent angiography intraluminal and intraperitoneal.

Methods: Sixty-five year old female patient underwent standard laparoscopic-assisted low anterior rectal resection for rectal carcinoma. The proximal end of the bowel and the stump of the distal rectum were checked using near-infrared fluorescence imaging with D-Light camera. After making sure of adequate perfusion of the bowel, the end-to-end stapled anastomosis was performed under the laparoscopic visualisation. The D-port proctoscope was inserted into the anus. The second ICG injection was administered. The perfusion of the anastomosis in transabdominal way and viability of the mucosa in transanal way was evaluated with two D-Light cameras simultaneously. The anastomosis was determined $4 \mathrm{~cm}$ from the anal verge. An air-water leak and tension of the bowel tests were performed. After evaluation of anastomosis viability with fluorescence imaging, after negative air-water leak and tensions testing, the decision was made by surgeon not to perform preventive ileostomy.

Results: The patient had no complains for the first three days postoperatively. Nevertheless, CRP level was growing and was $69.6 \mathrm{mg} / \mathrm{l}$ on the second postoperative day, and $103.5 \mathrm{mg} / \mathrm{l}$ on the 4 th postoperative day. The patient complained of the pain in the right iliac area and below symphysis on the 4th postoperative day. The abdominal and pelvis computed tomography scan with ora contrast was performed which denied our thoughts about the anastomotic leakage. Intravenous cefuroxime and metronidazole antibiotics were prescribed. The CRP level was $16.3 \mathrm{mg} / \mathrm{l}$ on the 10 th postoperative day. The patient was discharged on the 11 th postoperative day without preventive ileostomy.

Conclusion: Using the original, standardized colorectal anastomosis inspection method we can determine which patient doesn't need the preventive ileostomy after low colorectal anastomosis. 


\section{V070-COLORECTAL_Malignant}

MODIFIED ANTERIOR RESECTION WITH PARTIAL MESORECTAL EXCISION AIMED TO IMPROVE THE SAFETY OF COLORECTAL ANASTOMOSIS

\section{Z.Z. Mamedli, S.S. Gordeyev, K.E. Dzhumabayev, I.S. Tatayev}

Colorectal cancer, N.N.Blokhin Russian Cancer Research Center, MOSCOW, Russia

The 2 important causes of anastomotic leak are local ischemia and staple line defect. The purpose of this study was to investigate the combination of methods aimed to reduce the risk of anastomotic leak after anterior resections for rectal cancer.

Methods: We retrospectively analyzed perioperative outcomes of the first 30 patients, who underwent modified laparoscopic anterior resection with partial mesorectal excision for rectal cancer without preventive stomy. Operative technique was modified and included routine preservation of the left colic artery (fig1)(aimed to improve anastomotic blood supply), manual suture invagination of the 'dog ears' (fig2) (aimed to reduce the risk of staple line defects), transperineal pelvic drainage and pelvic peritoneum reconstruction (aimed to reduce the risk of reoperation in case of leakage). Anastomotic leak rate, reoperation rate, left colic artery preservation rate, additional operative time (time required for left colic artery preservation, 'dog ears' invagination and pelvic peritoneum reconstruction), blood loss, morbidity and mortality were analyzed.

Results: $1(3.3 \%)$ patient developed an asymptomatic leakage, which was managed conservatively. There was no postoperative mortality and no reoperations. Median additional operative time was $56 \mathrm{~min}$ for the first 15 procedures and $41 \mathrm{~min}$ for the last 15 procedures. Left colic artery preservation was successful in $26(86.7 \%)$ patients. Median blood loss was $35 \mathrm{ml}$. Conclusions: Additional techniques used in our modification of laparoscopic anterior resection are safe and may lead to improved perioperative outcomes. However, they are associated with increased operative time, which may be reduced with a better learning curve.

\section{V071-COLORECTAL—Stoma}

\section{A STREAMLINED APPROACH TO LAPAROSCOPIC PARASTOMAL HERNIA REPAIR WITH INTRAPERITONEAL FUNNEL MESH. SHOULD WE ADOPT IT PROPHYLACTICALLY FOR END STOMAS?}

M. Abdeldayem, C. Matthews, P. Blake, B. Gwilym, L. Maw, A.G. Masoud

Colorectal Surgery, Prince Charles Hospital, MERTHYR TYDFIL, United Kingdom

Introduction: Parastomal hernias are a significant cause of post abdominal ostomy morbidity with an overall life-time incidence exceeding $80 \%$.

The complications can range from a bulge resulting in stoma bag leakage, to life threatening bowel obstruction. The PREVENT-trial sought to determine if prophylactic utilisation of polypropylene mesh would decrease the incidence of parastomal hernias, with initial results demonstrating that it was safe to use in permanent end stomas.

Aim: To demonstrate a reproducible and streamlined technique for laparoscopic parastomal hernia repair with intraperitoneal funnel mesh, and assess the outcomes with the Clavien-Dindo (CD) classification tool.

Method: 10 parastomal hernia repairs (7 colostomy, 3 ileostomy) were considered, with the following approach adopted for each:

Swab sutured in stoma orifice to prevent wound contamination.Sharp dissection of the stoma using parachute technique.Stoma end refreshed followed by change of gloves and instruments.Lateral stay sutures placed to tighten sheath later on.Pneumoperitoneum temporarily created to assess/divide adhesions.Funnel mesh placed in-situ, orientated in the optimal intraabdominal position, and sutured to the peri-colic fat to prevent slip.Medial suture placed to narrow the sheath further.Pneumoperitoneum re-created and mesh fixed in place with double crown laparoscopic tacks.Redundant portion of end stoma excised and stoma formed. Results: At median follow up of 12 months:

No Recurrence.No reported symptoms of pain or decreased stoma functionality.One superficial wound infection treated with drainage at bedside $(\mathrm{CD}=$ Grade 1$)$

Conclusion: Laparoscopic parastomal hernia repair with intraperitoneal funnel mesh for permanent end stomas yielded good outcomes in our patient cohort. A streamlined and reproducible approach ensures that the technique can be adopted for both prophylactic, primary and recurrent repair. Parastomal hernias are common and can be associated with significant morbidity. When taking this into account, in conjunction with the recommendations of the initial results of the PREVENT-trial, one may consider prophylactic utilisation of a mesh in patients receiving a permanent end stoma.
V072-HEPATO-BILIAIRY \& PANCREAS-Gallbladder

ROBOTIC-ASSISTED EXCISION OF COMMON BILE DUCT STRICTURE AND ROUX-AN-Y HEPATICOJEJUNOSTOMY

\section{E. Kakiashvili ${ }^{1}$, E. Brauner ${ }^{2}$}

${ }^{1}$ General Surgery, Galilee Medical Center, KIRIAT MOZKIN, Israel; ${ }^{2}$ General Surgery, Rambam Medical Center, HAIFA, Israel

29 year old, female patient referred to our institution with common bile duct stricture, caused by iatrogenic injury during laparoscopic cholecystectomy.

During last year, patient suffered from recurrent episodes of ascending cholangitis. Recently, she underwent ERCP and severe stricture of middle CBD was diagnosed. Plastic stent was inserted through the CBD. MRCP also showed severe stricture of CBD with dilatation of biliary tree, proximal to the stricture.

Due to severe and resistant (did not resolved by recurrent dilatation) structure of middle CBD, she was referred to operation.

Patient underwent da Vinci robot-assisted excision of the CBD stricture, hepaticojejunostomy and extracorporeal jejunojejunostomy of Roux-an-Y limb.

Total operating time was $320 \mathrm{~min}$. Day three after operation patient started regular diet and was discharged home on day four.

Final pathology has shoved part of CBD with severe inflammation.

\section{V073-HEPATO-BILIAIRY \& PANCREAS—Gallbladder}

\section{LAPAROSCOPIC MINIMALLY INVASIVE BILIARY RESECTION AND CHOLECYSTECTOMY FOR A KLATSKIN CHOLANGIOCARCINOMA INVOLVING THE CYSTIC DUCT}

E. Falsetti, T. Cipolat Mis, M.C. Cartillone, A. d'Alessandro, E. Chouillard

General And Minimally Invasive Surgery, Poissy-Saint-Germain-enLaye Medical Center, POISSY, France

Aims: Extrahepatic biliary duct resection for the treatment of Bismuth I and II stage Klatskin tumor is the standard surgical technique [1]

Methods: A 85 years old patient present at Emergency Room (ER) with right upper abdominal pain with an elevation of the inflammatory markers at the blood exams and fever. The patient was submitted to a computer tomography (CT) that shows a tumor involving the lower tract of the principal bile duct.

An endoscopic retrograde cholangio pancreatography (ERCP) with biopsy (Intraductal papillary neoplasm of the bile duct,IPNB with high-grade dysplasia) and stent placement was performed. Considering the good general conditions of the patient and an absence of vascular and nodal invasion at the preoperative imaging, a minimally invasive surgical resection of the biliary tract with cholecystectomy was performed.

Results: A four port laparoscopic biliary tract resection with cholecystectomy was performed with lymphadenectomy of the hepatic hilum. No vascular or liver infiltration was found. The hepatic hilum was completely skeletonized. The resection of the biliary duct was performed with adequate free margin. A biliary reconstruction with Roux-en-Y technique was performed and a fully laparoscopic hepatico-jejunal anastomosis was done. And abdominal retro anastomotic drain was placed. The operative time was $350 \mathrm{~min}$. The postoperative course was complicated by a low rate biliary leakage that was treated conservatively. The patient was discharged at 25 post operative day in good general conditions.

The histological examination revealed a moderately differentiated in situ cholangiocarcinoma of the principal bile duct with the involving of the cystic duct with free resection margin (pT1bNOR0).

Conclusions: Laparoscopic resection of the biliary tract is a challenging procedure that allows, in expert hands, to achieve in selected cases negative pathological margin, complete linfonode retrieval and entero-biliary bypass.

[1] Hilar cholangiocarcinoma: diagnosis, treatment options, and management Kevin C. Soares1, Ihab Kamel2, David P. Cosgrove3, Joseph M. Herman4, Timothy M. Pawlik1. 


\section{V074-HEPATO-BILIAIRY \& PANCREAS—Gallbladder}

ICG FLUORESCENCE IMAGING-GUIDED HUGE HEPATIC CYST EXCISION IN PATIENT AT INCREASED RISK OF BILE DUCT INJURY

\section{Y.S. Han, J.R. Han, H.T. Ha}

Hepatobiliary Pancreas Surgery and Liver Transplantation, Kyungpook National University, School of Medicine, DAEGU, Korea

Injury to the extrahepatic bile duct during bile duct or hepatic surgery can be reduced by better real-time visualization. Recently, indocyanine green (ICG) fluorescence imaging has been used in laparoscopic hepatobiliary surgery. We applied ICG fluorescence imaging in patient with huge hepatic cyst which severely deviated extrahepatic bile duct. The patient had received laparoscopic cholecystectomy and huge hepatic cyst stuck firmly with peri-hepatic structures including bile duct. ICG fluorescence imaging correctly identified the common hepatic duct and remnant cystic duct and allowed for more meticulous and easier dissection. Therefore, ICG fluorescence imaging may guide a safe and accurate dissection and excision in hepatobiliary surgery.

\section{V075-HEPATO-BILIAIRY \& PANCREAS-Gallbladder}

\section{ROBOTIC-ASSISTED EXTRACTION OF LARGE CBD STONES AND CHOLEDOCHODUODENOSTOMY}

E. Kakiashvili ${ }^{1}$, E. Brauner ${ }^{2}$

${ }^{1}$ General Surgery, Galilee Medical Center, KIRIAT MOZKIN, Israel; ${ }^{2}$ General Surgery, Rambam Medical Center, HAIFA, Israel

63 years old, female patient presented with recurrent right upper quadrant (RUQ) pain, without fever, nausea or vomiting.

30 years ago, patient underwent open cholecystectomy due to cholelithiasis. During last four years, she suffered from recurrent attacks of biliary colic or ascending cholangitis.

Patient several times underwent ERCP and extraction of stones from common bole duct (CBD).

At her last admission, ultrasound (US) revealed recurrent large stones in CBD with significant dilatation of extra and intra hepatic biliary duct (CBD up to $2 \mathrm{~cm}$ ).

Her blood laboratory examinations showed mild elevation of bilirubin and liver functional tests (LFT'S).

Patient underwent da Vinci robot-assisted choledochotomy, extraction of CBD stones and choledochoduodenostomy.

Total operating time (ORT) was $240 \mathrm{~min}$. Two days after operation patient started regular diet and was discharged home on day four.

\section{V076-HEPATO-BILIAIRY \& PANCREAS-Gallbladder}

\section{LAPAROSCOPIC COMMON BILE DUCT EXPLORATION} AFTER FAILED ERCP OUR SERIE OF 75 PATIENTS

A.L. Vargas Ávila, A.F. Palacio Vélez, G. Diazteran Aguilera, J. Garcia Cansino, V.G. Reyes Garcia, K.B. Molina Tavarez, J. Vargas Flores, J.F. Nagore Ancona, J.M. Hernandez Garrido, C.A. Cortazar Sanchez, N.N. Espinosa Queb, L.A. Guerrero Galindo, J.A. Dominguez Rodriguez, J.A. Gonzalez Luna, A. Jimenez Leyva, J. Sanchez Lora, S.J. Salgado Arzate, A. Castañeda Rodriguez Cabo

\section{General Surgery, ISSSTE, CIUDAD DE MEXICO, Mexico}

Aims: To report a serie of cases of LCBDE (laparoscopic common bile duct exploration) after failed ERCP (endoscopic retrograde cholangio pancreatography) in our hospital from January 2009 to June 2018

Methods: A retrospective cohort analysis was perform. Inclusion criteria were: a) Diagnosis of choledocolthiasis by clinics, imagenology and biochemestry measures. The variables were: Age, gender and tecnique employed: Cholecystectomy plus LCBDE, primary or recidivant choledocolithiasis, primary closure of choledocotomy, use of T-tube or transcistic catether; Intrahospital days and Morbimortality.

Results: Total patients who underwent ERCP were 2,321 and 3.2 percent (75 cases) had a firs failed ERCP and 13 of then were unsuccesfull in the second intent of ERCP. Intrahospitalary stay was more than 7 days in the 11 percent, in the 89.2 percent was 4 to 7 days, with and average of 6 days.

Conclusions: Before, during or after LCBDE, ERCP remains the gold standard for manegemen of Choledocolitiasis confirmed by clinics, laboratory and imagenology.

LCBDE is a very good option that requires experience and specific skills, and especialized equipment.

In 9 years the rate of sucess in our hospital was $95.3 \%$ and there were no posoperatory complications such as: Biliar peritonitis, pancreatitis or liver abscess.

\section{V077-HEPATO-BILIAIRY \& PANCREAS—Gallbladder}

FLUORESCENCE-GUIDED CHOLECYSTECTOMY: INFUSION ROUTES, TIMES AND DOSES IN DIFFERENT CLINICAL SITUATIONS

G.P. Martin-Martin ${ }^{1}$, X. Molina ${ }^{2}$, A. Pozo ${ }^{3}$, E. Palma ${ }^{2}$, J.M. Moron ${ }^{2}$, X. Gonzalez ${ }^{1}$

${ }^{1}$ Colorectal, Hospital Universitario Son Espases, PALMA, Spain; ${ }^{2}$ Hepatobiliopancreatic, Hospital Universitario Son Espases, PALMA, Spain; ${ }^{3}$ Imaging, Medical Systems Division, Olympus Iberia, BARCELONA, Spain

Aims: Easier intraoperative recognition of the biliary anatomy may be accomplished by using near-infrared (NIR) fluorescence imaging after an injection of indocyanine green (ICG). Neither radiological support nor additional intervention such as opening the cystic or common bile duct is required, making it an easy and real-time technique to use during surgery. The aim of this video is to describe our experience in fluorescence-guided cholangiography in different clinical situations. Methods: Intravenous injection of ICG is used to illuminate extrahepatic biliary anatomy. However, the simultaneous enhacement of liver parenchyma can disturb the visualization of clinical details. The key is in the used dose of ICG, the route of administration and the time since its infusion. In the first case, a scheduled cholecystectomy is shown in which a dose $(1 \mathrm{~mL}$ of $25 \mathrm{mg}$ dye dilution in $10 \mathrm{~mL}$ of distilled water) administered intravenously $3 \mathrm{~h}$ before the intervention was used. The second case shows an urgent cholecystectomy in which the dose ( $30 \mathrm{~mL}$ of $25 \mathrm{mg}$ dye dilution in $1000 \mathrm{~mL}$ of distilled water) was administered intragallbladder during surgery. All patients underwent laparoscopic cholecystectomy with traditional four-port technique. All procedures were performed using a 30-degree $10 \mathrm{~mm}$ laparoscope with NIR imaging capability (Visera Elite II, Olympus).

Results: There were no intraoperative or postoperative complications. There was no increase in operative time due to the use of ICG. In the first case, a clear identification of the cystic duct and the main bile duct was obtained thanks to the biliary excretion of the ICG and the intravenous clearance. In the second case, the identification of the cystic duct, the main bile duct and the cystic artery occurred due to the intravesicular absorption of ICG.

Conclusion: Fluorescence-guided cholecystectomy clarifies the dissection plane. It can be considered to increase the safety of laparoscopic cholecystectomy. Being aware of the doses, times and possible routes of administration is basic to universalize the technique and give it utility in different scenarios. 


\section{V078-HEPATO-BILIAIRY \& PANCREAS—Gallbladder}

\section{LAPAROSCOPIC APPROACH FOR A CASE OF MIRIZZI TYPE 2} AND CHOLEDOCHOLITHIASIS

A.L. Vargas Ávila, A.F. Palacio Vélez, J. Garcia Cansino, G. Diazteran Aguilera, V.G. Reyes Garcia, K.B. Molina Tavarez, J. Vargas Flores, J.F. Nagore Ancona, J.M. Hernandez Garrido, C.A. Cortazar Sanchez, N.N. Espinosa Queb, L.A. Guerrero Galindo, J.A. Dominguez Rodriguez, J.A. Gonzalez Luna, A. Jimenez Leyva, J. Sanchez Lora

\section{General Surgery, ISSSTE, CIUDAD DE MEXICO, Mexico}

Introduction: Mirizzi syndrome type 2 is an uncommon cause of obstructive jaundice caused by an inflammatory response to an impacted gallstone in Hartmann's pouch or the cystic duct with a resultant cholecystocholedochal fistula. The obstructive biochemical changes can be caused by direct extrinsic compression from the impacted gall stone or from the fibrosis caused by advanced chronic cholecystitis, or for the established fistula.

Objective: We present a case of a Mirizzi type 2 syndrome with choledocholithiasis which was solved by laparoscopy approach.

Material and Methods: A 28-year-old female patient with no past medical history. The history of present illness begans with the presence of icteric dye since the last 3 days; she received symptomatic treatment with poor improvement. A liver and biliary tract ultrasound was performed with report of a $12 \mathrm{~mm}$ coledochus, $5 \mathrm{~mm}$ wall gallbladder. Then an endoscopic retrograde clolangiopancreatography was performed with successful endoscopic sphincterotomy and removal of gallstones. But the patient jaundice persisted after the procedure. The patient underwent cholecystectomy and laparoscopic common bile duct exploration, where the findings were a Mirizzi type 2 according to the Csendez classification, chronic cholecistitis and choledocholithiasis.

Results: In this laparoscopic approach we performed a partial cholecystectomy, bile duct exploration with removal of residual gallstones. The closure of the choledocotomy was performed with simple knots using vycril 3.0. A subhepatic drainage was left. The patient showed adequate clinical evolution. After 4 days the patient was discharged.

Conclusions: It is important to properly identify the anatomy at the time of surgery to avoid injury of the common bile duct. Operative treatment of Mirizzi syndrome type 2 includes either laparoscopic or open subtotal cholecystectomy or placement of a T-tube or choledocoplasty.

\section{V079-HEPATO-BILIAIRY \& PANCREAS-Gallbladder}

FLUORESCENT CHOLANGIOGRAPHY AS A TEACHING TOOL FOR ELECTIVE LAPAROSCOPIC CHOLECYSTECTOMY

\section{A. Pesce $^{1}$, T.R. Portale ${ }^{2}$, B. di Stefano ${ }^{2}$, G. la Greca ${ }^{3}$, S. Puleo ${ }^{4}$}

${ }^{1}$ Department of Medical, Surgical Sciences and Advanced Technologies 'G.F. Ingrassia', University of Catania, CATANIA, Italy; ${ }^{2}$ Dpt of Medical, Surgical Sciences and Advanced Technologies „G.F. Ingrassia,,, University Hospital „Policlinico-Vittorio Emanuele,,, CATANIA, Italy; ${ }^{3}$ Department of Medical, Surgical Sciences and Advanced Technologies, CATANIA, Italy; ${ }^{4}$ Department of Medical, Surgical Sciences and Advanced Technologies, University Hospital Policlinico, CATANIA, Italy

Near-infrared fluorescent cholangiography (NIRF-C) is an innovative intra-operative imaging technique that allows a real-time enhanced visualization of the extrahepatic biliary tree by fluorescence. Thanks to the development of laparoscopes/endoscopes with light sources emitting infrared frequencies, it is possible to visualize anatomical structures (vessels, ureters, bile ducts, etc.) through the luminous intensity of substances (fluorescein, blue of methylene, indocyanine green) which are injected into the patient. This technology may be considered as an important teaching tool for laparoscopic surgery, especially for young surgeons in their surgical learning curve and it could lead to reduce the risk of iatrogenic bile duct injuries during laparoscopic cholecystectomy. The following video is characterized by a series of intra-operative images of biliary anatomy by fluorescence, having an important educational interest, while also detecting anatomical variations of the cystic duct.

\section{V080-HEPATO-BILIAIRY \& PANCREAS-Gallbladder}

SAFE STEPS IN LAPAROSCOPIC CHOLECYSTECTOMY FOR DIFFICULT GALLBLADDER ACCORDING TO TOKYO GUIDELINES 2018

A. Umezawa, Minimally Invasive Surgery Center, Yotsuya Medical Cube, TOKYO, Japan

Aims: Laparoscopic cholecystectomy(Lap-C) for cholecystolithiasis has become standard However, serious bile duct injury has been reported as a complication. Repeated colic and chronic inflammation in cholecystolithiasis lead to the so-called Difficult Gallbladder conditions, such as dense fibrosis and scarring of the tissue. Dissection of Calot's triangle includes the risk of bile duct injury. Critical View of Safety (CVS) is the most well-known land mark for safe cholecystectomy. In the revised Tokyo Guidelines 2018 (TG 18), important land marks and bailout procedures had been proposed. Those are for the Difficult Gallbladder which are not able to achieve CVS.

Methods land marks: Baseline of segment 4 of the liver and sulcus Rouvier should be confirmed. The gallbladder wall itself is also useful landmark. Bailout procedure: When the dissection of Calot's triangle is considered impossible, bailout procedures should be considered. Subtotal cholecystectomy which leave the neck is one of option. The fundus first technique is another approach. However, because fundus first technique has a possibility of leading to serious bile duct injury, it should stop by the neck. In this video, first case shows the importance of landmarks from near miss cases of misidentified injuries. Second Case shows bailout procedure, subtotal cholecystectomy with fundus first technique.

Result: In the atrophic gallbladder (case 1, near miss), it is liable to misidentify the junction of common bile duct as the gallbladder neck. The neck and common hepatic duct were lifted together easily. With confirming the landmark, misidentification was corrected and bile duct injury was avoided.In the case2, since the Calot's triangle was obscured due to repeated cholecystitis, dissection of gallbladder was performed from the bottom to the neck, and was excised with the cervical portion remained. The remaining neck was reconstituted.In each case, intraoperative cholangiography was performed, and it was confirmed that there was no bile duct injury. Without postoperative complications, those patients were discharged POD 2 as usual LapC.

Conclusion: During Lap-C for difficult gallbladder, the most annoying part is bile duct injury. Confirming landmarks and switching bailout procedures can be contributory to avoid bile duct injury and to achieve safe Lap-C.

\section{V081-HEPATO-BILIAIRY \& PANCREAS—Gallbladder}

\section{TRANSCYSTIC LASER LITHOTRIPSY OF COMMON} BILE DUCT

\author{
R.J. Costa, H.R. Fonseca, L.A. Graça, E.J. Maia
}

Cirurgia Geral, Centro Hospitalar Universitário de São João, PORTO, Portugal

Aims: Choledocholithiasis is an important cause of morbidity and is present in about $18 \%$ of patients submitted a cholecystectomy. His treatment should be done in the same operative time, avoiding the morbidity and hospitalization time and costs of multiple procedures.The transcystic approach is preferable to prevent morbidity associated to choledochotomy.Large stones can preclude this procedure. The use of laser lithotripsy to stone fragmentation is an option to provide transcystic extraction.

Methods: We present a video of Laparoscopic Transcystic Common Bile Duct (CBD) Exploration for Choledocholithiasis.

Results: Female patient, 65 years old with a previous hospitalization for acute cholangitis with choledocholitiasis.Submitted to laparoscopic cholecystectomy with intraoperative cholangiography that showed the presence of stone in distal CBD with $1 \mathrm{~cm}$ size. The use of Holmium laser lithotripsy made the stone fragmentation and provided his extraction by transcystic route using a basket.The patient was discharged at $4^{\text {th }}$ postoperative day, with no complications.

Conclusion: The use of laser lithotripsy for large CBD stones is safe and effective, making possible the transcystic approach and preventing the choledochotomy morbidity. 


\section{V082-HEPATO-BILIAIRY \& PANCREAS-Gallbladder MINIMALLY INVASIVE APPROACH FOR THE TREATMENT OF GALLBLADDER CANCER}

G.F. Faria, J.P. Gonçalves, A.F. Cocco, A. Cabral-Correia, A.M. Gouveia

Surgery, ULS Matosinhos / Hospital Pedro Hispano, MATOSINHOS, Portugal

Gallbladder adenocarcinoma is rare and extremely aggressive. Its' incidence is higher in elder females and its progression is rapid and silent with a dismal prognosis if diagnosed at advanced stages.

We present the case of a 77 years-old female with dyspeptic complaints. The abdominal ultrasound revealed a $2 \mathrm{~cm}$ solid lesion of the gallbladder suspect for malignancy. The CT confirmed the presence of a vegetant mass on the free border of the gallbladder fundus with $28 \times 13 \mathrm{~mm}$.

We performed a radical cholecystectomy with lymphadenectomy and liver bed excision. The post-operative period was complicated with a urinary tract infection, with full recovery after antimicrobial treatment. The histological sample revealed an adenocarcinoma of the gallbladder (T1bNOM0) and the patient remains asymptomatic and tumour free 9 months after the surgery.

Gallbladder cancer treatment depends of the stage and clinical presentation of the disease. Complete surgical excision is the only curative treatment and should include a limited hepatectomy and portal pedicle lymphadenectomy. Laparoscopic surgery might be an option in early stages, although it is challenging and requires both expertise in hepato-biliary and laparoscopic surgery.

\section{V084-HEPATO-BILIAIRY \& PANCREAS—Gallbladder}

CRITICAL VIEW OF SAFETY IN GRADE II ACUTE CHOLECYSTITIS-A YOUNG SURGEON POINT OF VIEW

C. Blajut, C. Cirlan, I. Budrugeac, M. Iordache-Petrescu, M. Iordache, F. Savulescu

General Surgery II, Dr. Carol Davila Central Military Emergency University Hospital, BUCHAREST, Romania

Aims: When training in the residency you watch your teacher perform laparoscopic cholecystectomy with ease, and even yourself perform several steps. But as a young surgeon, when confronted with a patient with acute cholecystitis, you're filled with emotions, and you do not know where to start the gallbladder dissection. The aim of this presentation is to show to young surgeons that you can, and must achieve, critical view of safety when performing laparoscopic cholecystectomy for acute cholecystitis. Methods: We present the case of a 42 years old female patient, BMI of 36.3 , who presented with a grade II (moderate) acute cholecystitis. Following Tokyo Guidelines, we initiated antibiotics and general supportive care, but without clinical improvement. The patient was proposed for laparoscopic cholecystectomy.

Results: At initial exploration we identified a $20 \mathrm{~cm}$ long gallbladder, with a thick wall, difficult to manipulate. We opted for an anterograde cholecystectomy, in our opinion the best option in acute cholecystitis. The dissection was started with hook electrocautery and then continued with a combination of blunt dissection with the aspirator and with the hook. When reaching the pedicle, blunt dissection was used in order to appreciate the anatomy of the cystic duct and cystic artery. After correct identification of these structures they ware clipped and cut. A drainage tub was then placed, and the abdomen deflated.

Conclusion(s): As a young surgeon, when dealing with acute you must maintain your calm, and try to achieve critical view of safety before transecting the cystic duct and cystic artery. This can be achieved with a combination of blunt and sharp dissection, keeping your camera clean and with a good collaboration with the assisting surgeon.

\section{V085-HEPATO-BILIAIRY \& PANCREAS-Gallbladder RETROGRADE CHOLECYSTECTOMY FOR COMPLICATED CASES IS NOT ALWAYS A SAFE APPROACH}

\section{H. Konstantinidis, C. Charisis}

Robotic General and Oncologic Surgical Department, Interbalkan Medical Centre, THESSALONIKI, Greece

Aims: Several tactics of safe dissection and identification of the Callot's triangle structures during a laparoscopic cholecystectomy have been proposed, with the 'critical view of safety' technique being the most accepted. Thus, in complicated cases, this approach is not always feasible and many surgeons turn to the retrograde cholecystectomy technique, in order to overcome the difficulties of the dissection. Our aim is to highlight the risks that may rise from that approach in complex cases of laparoscopic cholecystectomy.

Methods: We present video fragments of a laparoscopic cholecystectomy procedure in a case of acute cholecystitis, with gallbladder abscess and plastron, in which a retrograde approach was decided. We emphasize on the possible risks that this technique encapsulates and the mandatory surgeon's awareness, in order to avoid major complications.

Results: The retrograde cholecystectomy technique in cases of complex cholecystitis with severe inflammation can falsely misguide the surgeon to porta hepatis dissection. Considerable experience and proper operative strategy is required to avoid major incidents in these cases.

Conclusions: Difficult and sometimes impossible proper dissection of the Callot triangle during a laparoscopic cholecystectomy, oblige many surgeons to retrograde cholecystectomy technique. Severe inflammation may mislead a surgeon to the porta hepatis during this approach, occurring a very high risk of major vascular and extrahepatic biliary injuries. All surgeons who decide to utilize this particular technique should be aware of these risks and the strategy to overcome them. 


\section{V086-HEPATO-BILIAIRY \& PANCREAS—Gallbladder}

\section{PROPOSED METHOD FOR ADEQUATE SURGICAL} GALLBLADDER EXAMINATION

B.J.G.A. Corten ${ }^{1}$, W.K.G. Leclercq ${ }^{2}$, P.H. Zwam ${ }^{3}$, R.M.H. Roumen ${ }^{2}$, G.D. Slooter ${ }^{2}$

${ }^{1}$ Surgery, MMC Veldhoven, EINDHOVEN, The Netherlands; ${ }^{2}$ Surgery, MMC, EINDHOVEN, The Netherlands; ${ }^{3}$ Department of Pathology, PAMM, EINDHOVEN, The Netherlands

Background: The Dutch Surgical Society changed its national 'gallbladder' guideline 2016, regarding routine histopathologic examination after cholecystectomy in absence of macroscopic abnormalities. Thus, shifting the macroscopic examination of the gallbladder from the department of Pathology to the operating room. The surgeon is now asked to perform a macroscopic examination of the gallbladder, and decide whether additional histopathologic assessment is warranted. Up to this date, there is no clear guideline or protocol to perform a proper surgical examination. Leaving surgeons extemporaneous in regard to selective histopathologic gallbladder examination.

Methods: The present study describes a surgical approach for adequate macroscopic inspection of the gallbladder. This procedure was introduced in 2011 and implemented in 2012 following an evaluation of the existing literature in collaboration with the department of Pathology.

Results: Since incorporation of the selective policy we have performed over 2000 surgical macroscopic examinations of the gallbladder. As a result, we observed a significant decrease in histopathologic examination of the gallbladder following cholecystectomy. Whereas we observed a stable trend of gallbladder carcinoma in the same period.

Conclusions: Here, an easy and reproducible method is described for future macroscopic analysis by the surgeon following a cholecystectomy. In addition, we depict several frequent macroscopic abnormalities in order to provide surgical colleagues with some cases of abnormal macroscopic gallbladders.

\section{V087-HEPATO-BILIAIRY \& PANCREAS-Liver}

\section{CAUDAL APPROACH TO THE MIDDLE HEPATIC VEIN IN LAPAROSCOPIC LEFT HEPATECTOMY}

\author{
H. Cristino, V. Gomes, M. Almeida, L. Graça, J. Costa-Maia
}

Hepato-bilio-pancreatic, Centro Hospitalar são joão, PORTO, Portugal

The left hepatectomy is a demanding and difficult procedure, still limited to reference centers. The caudal approach and exposure of the middle hepatic vein is a reliable way to achieve a safely and reproductible left hepatectomy.

With this technique, exposing the middle hepatic vein, we believe that we can perform a safe and feasible laparoscopic left hepatectomy increasing the quality of this hepatectomy.

We present a 47-year-old woman with an intrahepatic and common bile ductlithiasiswhich was previously submitted to an ERCP. With an unsolved intrahepatic lithiasis the patient was proposed to alaparoscopic left hepatectomy.

\section{V088-HEPATO-BILIAIRY \& PANCREAS-Liver}

\section{TOTALLY MINI-INVASIVE ALPPS}

F. Ratti, F.C. Cipriani, G.F. Fiorentini, A.L. Aldrighetti, Hepatobiliary Surgery Division, San Raffaele Hospital, MILANO, Italy

The minimally invasive approach for ALPPS in a patient with a large hepatocellular carcinoma in a liver with severe steatosis is shown. During the first stage a partial ALPPS is performed. PVE is performed in postoperative day one. After 15 days from the first stage both liver volume and function (by HIDA scan) are re-assessed. Right hepatectomy (second stage of ALPPS) is then conducted by laparoscopic aproach.

\section{V089-HEPATO-BILIAIRY \& PANCREAS-Liver}

\section{SPLENIC PRESERVING TOTAL LAPAROSCOPIC} PANCREATODUODENECTOMY

H. Cristino, V. Gomes, M. Almeida, L. Graça, J. Costa-Maia

Hepato-bilio-pancreatic, Centro Hospitalar são joão, PORTO, Portugal

A 68 year old woman with a previous history of anxiety and catheter ablation to treat heart arrhythmias, was studied for for multiple pancreatic cysts incidentally discovered on a routine ultrasound.

An MRI was performed showing multiple cystic tumors throughout the pancreas, the largest of which was $15 \mathrm{~mm}$. This led to a suspicion of multi-focal, side-branch intraductal papillary mucinous neoplasm (IPMN), with minimal dilatation of the main pancreatic duct. An Echo endoscopy was subsequently performed indicating probable multifocal IPMN. A FNA was carried out during this procedure, with aspiration of cystic content which was sent for CEA analysis and cytology. Cytology was compatible with mucinous neoplasm with mild atypia and CEA $98 \mathrm{U} / \mathrm{ml}$.

A splenic preserving total laparoscopic pancreatoduodenectomy was proposed. The procedure was uneventful and the patient was discharged on the $5^{\text {th }}$ post-operative day. Pathology revealeded a $19 \mathrm{~mm}$ IPMN, with severe dysplasia and 3 foci of microinvasive ductal adenocarcinoma of $1 \mathrm{~mm}-\mathrm{pT} 1 \mathrm{~N} 0 \mathrm{R} 0$ 


\section{V090-HEPATO-BILIAIRY \& PANCREAS-Liver}

INDOCYANINE GREEN IMMUNOFLUORESCENCE GUIDED LAPAROSCOPIC PARTIAL HEPATECTOMY

\section{Y. Tai}

Department of Surgery, E-Da Hospital, KAOHSIUNG CITY, Taiwan

Obtaining negative tumor margin during laparoscopic hepatectomy has always been a very challenging topic for surgeons in that the surgeons are not able to palpate the tumor during laparoscopic surgery. Although intraabdominal echo is available, but it demands great experiences and skills. With the guidance of ICG immunofluorescence, surgeons can avoid failure of not obtaining enough negative margins nor resect too much healthy liver. ICG is often used to estimate the liver function prior to hepatectomy traditionally. It binds to plasma protein and has a peak absorbance at $780 \mathrm{~nm}$ and emits fluorescence with a wavelength of approximately $800 \mathrm{~nm}$. ICG is preferentially retained in or around biliary malignancies due to impaired biliary excretion of hepatocytes in the affected area. We performed ICG immunofluorescence guided laparoscopic partial hepatectomy on a 57 years old male who suffers from HCC located at segment 5 and 6. ICG was injected 3 days prior to the operation day. While evaluation of liver is performed, it also allowed us to use a high-end laparoscopic camera system equipped with integrated filters for detection of near-infrared fluorescence. During the surgery, we were able to clearly locate the borders of malignancies through the use of integrated filters combine with ICG injection. The pathology study also confirmed that the adequate tumor free margin $(>0.5 \mathrm{~cm})$ were obtained in both tumors and the patient's condition was stable as well.

ICG immunofluorescence guidance enables surgeons to obtain optimum result in tumor resection through laparoscopic surgery. It also has the ability to detect bile leakage. With the use of ICG immuofluorescence, surgeons will have higher chances to achieve adequate negative margins.

\section{V091-HEPATO-BILIAIRY \& PANCREAS-Liver}

\section{LAPAROSCOPIC PARENCHYMAL-SPARING LIVER} RESECTION INCLUDING CAUDATE LOBE FOR MULTIPLE COLORECTAL LIVER METASTASES

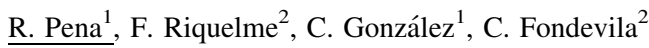

${ }^{1}$ General Surgery, Hospital Clinic, BARCELONA, Spain;

${ }^{2}$ Hepatobiliopancreatic Surgery, Hospital Clinic, BARCELONA, Spain

Background: Parenchymal sparing hepatic resection has the advantage of preserving valuable tissue in chemotherapy-treated livers, assuring an adequate future remnant volume without compromising long-term survival. Moreover, the laparoscopic approach offers the decreased postoperative morbidity of minimally invasive surgery. Whenever technically feasible, this kind of procedure should be considered a suitable alternative to the classic major hepatectomy for the treatment of multiple colorectal liver metastases. Methods: 69-year old male with a previous history of laparoscopic sigmoidectomy in November 2014 for a pT2N0M0 sigmoid adenocarcinoma. A control scanner three years later showed liver metastases in segments $\mathrm{V}$, VIII, II and caudate lobe. After chemotherapy (XELOX), control MRI and PET scans showed a good response. He was proposed for a laparoscopic parenchymal-sparing liver resection.

Results: Total operative time was $3 \mathrm{~h}$ and $45 \mathrm{~min}$ with no intraoperative complications. Patient presented a right atelectasis as the only postoperative complication and was resolved with respiratory therapy. He was discharged in 4 days. Pathology report showed that lesions on segment $\mathrm{V}$ and VIII had no viable tumor (100\% fibrosis) and lesions on segment II and caudate lobe had moderately differentiated adenocarcinoma. Margins were free in all the lesions. After a 6 month follow up, the patient has no recurrence and normal liver function tests.

Conclusion: Minimally invasive liver resection is possible in patients with multiple bilobar liver metastases and allows to perform parenchymalsparing surgery safely. Difficult localization of lesions such as the caudate lobe are not a contraindication for this type of surgery.
V092-HEPATO-BILIAIRY \& PANCREAS-Liver

ROBOTIC-ASSISTED COMPLETE EXCISION OF TYPE I CHOLEDOCHAL CYST AND ROUX-AN-Y HEPATICOJEJUNOSTOMY

\author{
E. Kakiashvili ${ }^{1}$, E. Brauner ${ }^{2}$
}

${ }^{1}$ General Surgery, Galilee Medical Center, KIRIAT MOZKIN, Israel; ${ }^{2}$ General Surgery, Rambam Medical Center, HAIFA, Israel

A 30 years old, female patient presented with recurrent right upper quadrant (RUQ) abdominal pain (during last two years), without nausea, vomiting or jaundice. Her blood laboratory examinations were within normal limits, including serum CA 19-9.

Ultrasonography (US) demonstrated a large cystic dilatation of common bile duct (CBD). An abdominal computed tomography scan (CT) and MRCP revealed a type I choledochal cyst, measuring $3.5 \mathrm{~cm}$ in diameter. Patient underwent da Vinci robot-assisted excision of the type I choledochal cyst, hepaticojejunostomy and extracorporeal jejuno-jejunostomy of Roux-an-Y limb.

Total operating time (ORT) was $325 \mathrm{~min}$. Three day after operation patient started regular diet and was discharged on day fife.

Pathology result confirmed choledochal cyst without evidence of malignancy.

V093-HEPATO-BILIAIRY \& PANCREAS-Liver

\section{LAPAROSCOPIC RESECTION FOR PERIHILAR CHOLANGIOCARCINOMA}

F. Ratti, F.C. Cipriani, G.F. Fiorentini, A.L. Aldrighetti

Hepatobiliary Surgery Division, San Raffaele Hospital, MILANO, Italy

Laparoscopic approach for perihilar cholangiocarcinoma is still poorly reported in the literature due to technical challenges secondary to the combination of major hepatectomy, lymphadenectomy and biliary confluence resection. Despite this, in selected cases it can be a good option to provide a short term benefit to patients. The video reports the case of a perihilar cholangiocarcinoma with involvement of left bile duct and therefore requiring left hepatectomy. 


\section{V094-HEPATO-BILIAIRY \& PANCREAS-Liver}

LAPAROSCOPIC ANATOMIC LIVER RESECTION OF SEGMENT 7 AND 8 USING INTRAHEPATIC GLISSONEAN APPROACH

Y. Ome, Hepato-Biliary-Pancreatic Surgery

Komagome Hospital, BUNKYO-KU,TOKYO, Japan

Aims: Segmentectomy is an anatomic liver resection, in which the tertiary branches of the Glissonean pedicles are selectively transected. However, the branching pattern of the tertiary branches varies depending on the case, particularly in segment 7 (S7) and segment 8 (S8). The extrahepatic approach to the Glissonean pedicle from the hepatic hilum is very difficult depending on the branching pattern. Furthermore, the distance of exposing the secondary branches that are to be preserved becomes longer, and there is an increased risk of biliary leakage and delayed biliary stricture due to excessive traction in laparoscopic surgery. Therefore, laparoscopic S7 and S8 segmentectomy are considered technically difficult. We standardized the intrahepatic Glissonean Pedicle Approach for laparoscopic S7 and S8 segmentectomy.

Methods: We standardized the intrahepatic Glissonean Pedicle Approach for laparoscopic S7 and S8 segmentectomy. We identify the targeted Glissonean pedicle intrahepatically after the parenchymal transection along the major hepatic vein or its branch running on the intersegmental plane, referring to the preoperative simulation by 3D imaging. (a)S7 segmentectomy; After the mobilization of the right lobe, the Glissonean pedicles of S7 (G7) can be approached from the dorsal side by transecting the parenchyma between the IVC and the right hepatic vein. After the division of the G7, the parenchyma is transected along the demarcation line and the RHV from the root side to the peripheral side. (b)S8 segmentectomy; First, the parenchyma is transected along the middle hepatic vein (MHV) from the root side to the peripheral. G8 is typically detected on the right dorsal side of the MHV. After the division of the G8, the liver parenchyma is transected along the demarcation line and the RHV from the root side to the peripheral side.

Results: We have experienced 11 cases of laparoscopic S7 segmentectomy and 26 cases of laparoscopic S8 segmentectomy.

Conclusion: Our approach to the G7 and the G8 is safe and very useful.

\section{V095-HEPATO-BILIAIRY \& PANCREAS-Liver}

\section{LAPAROSCOPIC ANATOMICAL SEGMENTECTOMY OF RIGHT ANTERIOR SECTION USING THE EXTRAFASCIAL AND TRANSFISSURAL APPROACH}

\section{J.H. Kim}

Department of Surgery, Eulji University Hospital, DAEJEON, Korea

Laparoscopic anatomical segmentectomy of right anterior section is technically demanding because it is difficult to dissect the deep tertiary branches of right anterior portal pedicle (RAPP). We present three cases of laparoscopic anatomical segmentectomy using the extrafascial and transfissural approach: 1) anatomical resection of segment 5,2) anatomical resection of the ventral area 3) anatomical resection of segment 8 dorsal area.

The extrafascial and transfissural approach means that the liver parenchyma along the fissure lines is opened, then the surgeon can confirm the Glissonean pedicles and territory directly. The extrafascial and transfissural approach in laparoscopic anatomical segmentectomy of right anterior section is feasible and effective because this technique can easily be approached to the deep tertiary branches of RAPP.
V096-HEPATO-BILIAIRY \& PANCREAS-Liver

\section{LAPAROSCOPIC LIVER RE-RESECTION FOR RECURRENT HEPATOCELLULAR CARCINOMA}

Y. Iimuro, A. Takano, Y. Tsukahara, K. Ikegame, H. Watanabe, K. Mastuoka, K. Furuya, M. Yasutome, M. Hada, Y. Miyasaka

Surgery, Yamanashi Central Hospital, KOFU, Japan

Repeated liver resection has significant role in patients with recurrent hepatocellular carcinoma (HCC) in several situations. Laparoscopic redo surgery is becoming safer along with advance in surgical technique. We have performed laparoscopic re-resection for limited intrahepatic HCC recurrence. The Aim of the present study was to investigate its significance comparing with first laparoscopic liver resections.

Subjects: Patients with limited intrahepatic HCC recurrence after open hepatectomy underwent laparoscopic liver re-resection $(n=12)$.

Methods: Adhesion between abdominal wall and visceral organs was carefully divided, after the first laparoscopic port was safely inserted. Adhesion between diaphragm and liver surface or between previous liver cut surface and colon or duodenum was also minimally dissected. Approach to the Glisson's pedicles at the hepatic hilum was often difficult due to previous surgical procedure, thus Pringle's maneuver was generally applied. Dissection of hepatic parenchyma approaching to the target Glisson's branch was often preceded under the ultrasound-guidance. Liver resection was performed using LCS, BiClamp, and CUSA using intermittent block of the hepatic inflow. Operation time, intraoperative bleeding, morbidity, mortality, and postoperative hospital stay were compared with those in patients who underwent first laparoscopic liver resection during the same period $(n=20)$. Results: Operation time was significantly longer in the re-resection group, possibly due to the adhesiolysis. Meanwhile, no significant difference was detected in intraoperative bleeding, morbidity, mortality and postoperative hospital stay between the first and the redo surgeries.

Conclusion: Laparoscopic liver re-resection seemed comparable to the first laparoscopic resection except for longer operation time. Considering the invasiveness of open hepatic re-resections, laparoscopic re-resection of $\mathrm{HCC}$ in selected patients is possibly feasible method.

\section{V097-HEPATO-BILIAIRY \& PANCREAS-Liver}

\section{PURE LAPAROSCOPIC LIVING DONOR RIGHT HEPATECTOMY USING REAL-TIME INDOCYANINE GREEN FLUORESCENCE IMAGE}

\section{S.H. Choi, J.W. Lee}

Surgery, Bundang CHA Medical Center, SEONGNAM-SI, Korea

Introduction: Indocyanine green near-infrared fluorescence image help determine the correct anatomical resection of the liver parenchyme as well as the exact point of the bile duct division. This video demonstrates the technique of pure laparoscopic living donor right hepatectomy and usefulness of the indocyanine green fluourescence image.

Methods: The donor was a 32-year-old gentleman who decided to donate part of his liver to his wife suffering from viral liver cirrhosis and hepatocellular carcinoma. His BMI was $20.3 \mathrm{~kg} / \mathrm{m}^{2}$ and the preoperatively estimated donor's right liver volume was $836 \mathrm{ml}$, representing $63.6 \%$ of his entire liver. With the recipient's weight of $57 \mathrm{~kg}$, the graft to recipient weight ratio (GRWR) was $1.6 \%$. The liver had classic hilar anatomy except that the right posterior intrahepatic duct seperately joined to the left main hepatic duct. After isolation and clamping of right hepatic artery and portal vein, indocyanine green of $2.5 \mathrm{mg}$ was injected intravenously.

Results: The total operation time was $370 \mathrm{~min}$ and the estimated blood loss was $150 \mathrm{~mL}$ without transfusion. Indocyanine green fluorescence image clearly demonstrated the anatomical demarcation between the lobes and visualized the running of the biliary tree. His postoperative course was uneventful and discharged postoperative day 7.

Conclusion: Real-time indocyanine green fluorescence image may be particularly helpful to delineate anatomical surgical plane and to determine the appropriate division point of hepatic duct during laparoscopic living donor hepatectomy. 


\section{V098-HEPATO-BILIAIRY \& PANCREAS-Liver}

LAPAROSCOPIC RIGHT HEPATECTOMY WITH MINIMALLY INVASIVE HAEMODYNAMIC MONITORING

F. Ratti ${ }^{1}$, R. Reineke ${ }^{2}$, F.C. Cipriani ${ }^{1}$, G.F. Fiorentini ${ }^{1}$, A.L. Aldrighetti ${ }^{1}$

${ }^{1}$ Hepatobiliary Surgery Division, San Raffaele Hospital, MILANO, Italy; ${ }^{2}$ Anaesthesiology and Intensive care, San Raffaele Hospital, MILANO, Italy

The correct management of intraoperative volemic status is essential in laparoscopic liver resection in order to control bleeding and to perform even complex procedures with a good profile of safety. Central venous pressure is not really reliable in laparoscopy, due to presence of the pneumoperitoneum and patient position. Monitoring of haemodynamic parameters via Vigileo system is a minimally invasive method to control stroke volume variation, cardiac output, cardiac index and oxygen delivery in order to optimize the anaesthesiological management by controlling venous bleeding and avoiding tissutal ischemia.

\section{V100-HEPATO-BILIAIRY \& PANCREAS-Liver}

\section{LAPAROSCOPIC UNROOFING OF SIMPLE GIANT LIVER CYSTS}

A.L. Vargas Ávila, A.F. Palacio Vélez, G. Diazteran Aguilera, S.J. Salgado Arzate, J. Garcia Cansino, V.G. Reyes Garcia, K.B. Molina Tavarez, J. Vargas Flores, J.F. Nagore Ancona, J.M. Hernandez Garrido, C.A. Cortazar Sanchez, N.N. Espinosa Queb, L.A. Guerrero Galindo, A. Jimenez Leyva, J. Sanchez Lora, J.A. Dominguez Rodriguez, J.A. Gonzalez Luna

\section{General Surgery, ISSSTE, CIUDAD DE MEXICO, Mexico}

Introduction: Polycystic liver disease (PLD) is the result of embryonic ductal plate malformation of the intrahepatic biliary tree. And usually remain silent during life. The symptoms associated with Polycystic liver disease include abdominal pain, abdominal distension and atypical symptoms because of voluminous cysts resulting in compression of adjacent tissue.

Objective: The objective of the following case is to present a patient with symptomatic polycystic liver disease, which was solved by laparoscopy approach and the management of its complications.

Material and Methods: The case reported is about a 62 years old female patient with abdominal pain in upper right quadrant associated to asthenia, adynamia and hyporexia. CT scan reported heterogeneous liver with multiple ovoid images with regular edges defined which the biggest one measure $102 \times 99 \times 137 \mathrm{~mm}$ with volume of $723 \mathrm{cc}$ on segment 2 and 3 , which comprises stomach, and the other one in segment 8 with a volume of $1453 \mathrm{cc}$ and others small sized located in segment 6,7 and $4 \mathrm{~B}$

Results: In this laparoscopic approach, we performed a cyst unroofing of the two biggest cysts as well as cholecystectomy because of firm and lax adhesions. The patient evolved with fever in the $5^{\text {th }}$ day postsurgical day and biliary leaking in a volume of $270 \mathrm{cc}$ in $24 \mathrm{hrs}$. An ERCP (Endoscopic retrograde cholangiopancreatography) was asked for that was carried out by finding leak at the intrahepatic biliary duct therefore; esphinterotomy with placement of plastic endoprotesis was performed. The patient evolved without complication and was discharged at the $10^{\text {th }}$ day. Conclusions: Only symptomatic polycystic liver disease needs to be treated. The choice of treatment is not yet standardized, for voluminous cysts the unroofing ideally by laparoscopy is the gold standard and the ERCP is the elected treatment when the biliary leak appears as a complication.

\section{V101-HEPATO-BILIAIRY \& PANCREAS-Liver}

\section{LAPAROSCOPIC LIVER RESECTION IN SEGMENT 7: HEPATIC VEIN FIRST APPROACH WITH SPECIAL REFERENCE TO SUFFICIENT RESECTION MARGIN}

\section{J.Y. Cho, B. Lee, H.S. Han, Y.S. Yoon, Y.R. Choi}

Surgery, Seoul National University Bundang Hospital, SEONGNAM, Korea

Introduction: Laparoscopic liver resection (LLR) for tumors located in the posterosuperio segments of the liver (Segments (S) 7 or 8) is a challenging procedure. Especially, LLR for S7 is difficult because the access of instruments is limited, bleeding control is not feasible, major LLR is sometimes required, and obtaining sufficient resection margin is not easy. To overcome this obstacles, we performed LLR in S7 with a lateral approach using intercostal trocars. To obtain competent resection margin, LLR through right hepatic vein (RHV) first approach was performed for $1.8 \mathrm{~cm}$ mass located near the RHV in a 58 year old female.

Case: After full mobilization of right liver including all short hepatic veins and caudate lobe, rotate the whole liver completely to the left side to approach to the root of RHV. One intercostal trocar was inserted to access the lesion. Parenchymal transection started from the confluence of hepatic vein and then, followed along RHV with ligating several small branches from RHV. Resection margin was demarcated after localization using laparoscopic ultrasonography. After completion of parenchymal dissection using CUSA and ultrasonic shears, hemostatic agents were applied and drain was inserted.

Operation time and estimated blood loss were $120 \mathrm{mins}$ and $400 \mathrm{ml}$. The patient was discharged without any complication on postoperative day 7. Final pathological assessment confirmed clear resection margin (Safety margin : $1.5 \mathrm{~cm}$ ).

Conclusion: Laparoscopic S7 segmentectomy with hepatic vein first approach technique is safe and recommended to obtain better resection margin. 


\section{V102-HEPATO-BILIAIRY \& PANCREAS-Liver}

\section{SIMPLE LIVER CYSTS, ¿WHEN IS SURGERY JUSTIFIED?}

G. Rojas de la Serna, M. López Saiz, J. Riquelme Gaona, V. Crespo Garcia del Castillo, A. Morandeira Rivas, C. Moreno Sanz

Digestive surgery, General Hospital, La Mancha-Centro, ALCÁZAR DE SAN JUAN, Spain

Aims: Simple liver cysts are the most common cystic lesions of the liver. Most are diagnosed casually in image tests such as ultrasound or computerized tomography, most of which are asymptomatic and do not require treatment. In symptomatic patients (abdominal distension with palpable mass, abdominal pain, dyspnea, jaundice, etc.) the clinical manifestations are usually due to the growth of the cysts or the compression of neighboring structures. Liver function tests are usually not altered. Intracystic complications occur in less than $5 \%$ of cases and malignancy is exceptional. In this video, we present the case of a symptomatic patient with polycystic liver disease including a large size hepatic cyst.

Material and Methods: 65-year-old woman with a personal history of arterial hypertension, SAOS, partial hysterectomy due to endometrial cancer, who was referred to our department complaining of supraumbilical pain and abdominal distension with palpable mases. Abdominal ultrasound showed cholelithiasis and multiple simple hepatic cysts. In CT scan, multiple hepatic cysts were found, the largest one of about $20 \mathrm{~cm}$ of larger diameter. Echinococcus granulosus serology test was negative. There was also no evidence of cancer disease in PET scan. Results: A laparoscopic approach was performed with four trocars, three of $5 \mathrm{~mm}$ and a Hasson trocar inserted thought a umbilical small incisional hernia. Aspiration and wide unroofing of the large size cyst and smaller accessible ones was done. The patient also underwent cholecystectomy with intraoperative cholangiography and umbilical eventroplasty. The patient recovered uneventfully and is asymptomatic one year after surgery.

Conclusion: Simple liver cysts rarely require treatment. In some cases, especially in large, complicated and symptomatic simple liver cysts, surgery is indicated. Laparoscopic fenestration treatment is the best choice.

\section{V103-HEPATO-BILIAIRY \& PANCREAS-Liver}

\section{LAPAROSCOPIC HEPATECTOMY CONTINUES GROWING}

M. Alba Valmorisco ${ }^{1}$, R. Perez Quintero ${ }^{2}$, R. Martinez Mojarro², D. Bejarano González-Serna ${ }^{1}$, J. Candón Vázquez ${ }^{1}$, M. González Benjumea $^{1}$, P. Beltrán Miranda ${ }^{1}$, R. Balongo García ${ }^{4}$

${ }^{1}$ General Surgery (Hepatobiliary surgery), Juan Ramon Jimenez Hospital, HUELVA, Spain; ${ }^{2}$ General Surgery, Juan Ramon Jimenez Hospital, HUELVA, Spain; ${ }^{4}$ Genial surgery (esophagogastric surgery), Juan Ramon Jimenez Hospital, HUELVA, Spain

Aims: Liver resection is the preferable initial treatment option for solitary or limited multifocal hepatocellular carcinomas.

Surgical indications for laparoscopic liver resection (LLR) are the most important consideration, like liver function, tumor size (diameter less than $5 \mathrm{~cm}$ ) and location (easy technical access like in the left lateral section or on the surface of the inferior region).

Partial liver resection or left lateral sectionectomy are the typical procedures for such tumors and are considered the best way to begin LLR. With accumulating experience and technical advancement, LLR has been performed for tumors larger than $5 \mathrm{~cm}$ and for others locations. Some requirements to perform LLR are to have experience in liver surgery and laparoscopic also, adequate technology and Intraoperative ultrasound.

Methods: A 69-year-old male smoker, ex-parenteral drug users with chronic HCV liver disease Child-A stage. He is diagnosed with a single lesion of $7 \mathrm{~cm}$ in segment III of the liver, biopsied twice without conclusive diagnosis and with a three-phase CT suggestive of hepatocarcinoma LIRADS 4 with data of portal hypertension (PHT) and mild ascites.

After the study is commented on tumor committee deciding surgical intervention.

Results: A laparoscopic resection of segment III was performed with 5 trocars. Liver is explored by intraoperative laparoscopic ultrasound. Vascular control was performed using the Pringle technique. Liver transection was done with Sonostar until identification of intraparenchymal segment III vascularization, which is sectioned with endoGIA $(45 \mathrm{~mm})$ with Seamguard. After the resection, we perform hemostasis control with electrocoagulation and hemostatic material. Intraoperative bleeding of $300 \mathrm{ml}$. Favorable postoperative evolution, high on the 5 th postoperative day.

AP: $7 \mathrm{~cm}$ trabecular hepatocarcinoma moderately differentiated $\mathrm{pT} 1 \mathrm{~b}, \mathrm{R} 0$ resection.

Conclusions: LLR allows major liver resections with low morbidity and mortality and the advantages of laparoscopic surgery. An efficient learning curve can be achieved by a parallel evolution of procedures and indications (according to modified BCLC staging system and treatment strategy). Studies suggest that LLR results in less blood loss, shorter postoperative hospital stays, lower abdominal wall trauma and lower incidences of ascites accumulation and postoperative liver failure. With respect to oncological considerations, tumor margins are adequately maintained during LLR.

\section{V104-HEPATO-BILIAIRY \& PANCREAS-Liver}

\section{LAPAROSCOPIC LEFT LATERAL HEPATECTOMY FOR LIVER METASTASIS}

V. Drakopoulos, S. Voulgaris, I. Iliadis, K. Botsakis, P. Trakosari, V. Vougas

1st Department of Surgery and Transplantation Unit, District General Hospital of Athens « Evangelismos », ATHENS, Greece

Introduction: Laparoscopic surgery is gaining acceptance in the treatment of liver metastasis Laparoscopic treatment of liver metastasis often presents technical difficulties and requires an extensive learning curve.

Material-Method: We present the case of a 62 year old woman presented with a liver metastasis in section 3 of the liver. The patient had been submitted to a laparoscopic low posterior resection in February 2018. Patient underwent laparoscopic left lateral hepatectomy, with the use of three trocars (umbilical $10 \mathrm{~mm}$, and two in the midclavicular line bilaterally.) Left lateral hepatectomy was conducted with the use of a linear stapler. The postoperative period was uncomplicated and the patient remains in good condition three months after surgery.

Conclusion: Laparoscopic approach seems to be safe for treatment of liver metastasis, offering better surgical field view and less postoperative complications. 5 year survival rate after laparoscopic hepatectomy is compared to the open approach.

\section{V105-HEPATO-BILIAIRY \& PANCREAS-Liver \\ LAPAROSCOPIC RIGHT HEPATECTOMY FOR HCC WITH IHD THROMBOSIS}

Y.Y. Liu

General surgery, Chang gung memorial hospital kaohsiung division, KAOHSIUNG, Taiwan

Purpose: Laparoscopic hepatectomy is a quickly growing method for liver tumor because of modern technology. But for the IHD thrombosis, it is still technique dependent. The video was tried to share our experience for special case.

Material and method: One 68 y/o female patient suffered from fever episode and image show S56 $3 \mathrm{~cm}$ HCC with right anterior IHD obstruction R/O tumor thrombosis, Hilum LN enlargement, double right portal vein, hilum adhesion with duodenum, no ascites . Lab data : NO-B, NOC Child A, AFP 1199, ICG clearance rate 4.5\%, PLT 174000 . Heart, lung function exam normal. The laparoscopic right total hepatectomy and hilum LN dissection was conducted. Results: Laparoscopic approached was performed. The hilum LN dissection was done with vessel and bile duct isolation. Hilum LN frozen show negative malignancy. Hemi-vessel contro was done with resecting the vessel. Right hepatectomy was done with preserving middle hepatic vein. The right anterior and posterior IHD was opened and tumor thrombosis was removed from right anterior IHD carefully. The stump of IHD was closed by suture separately. The total op time was $630 \mathrm{~min}$ with $345 \mathrm{cc}$ blood loss. Post op minimsl bile leakage was found in the drain at day 6. The patient discharged at day 14 with drain

Conclusions: Laparoscopic hepatectomy may be a feasible method for HCC even with IHD tumor thrombosis. 


\section{V106-HEPATO-BILIAIRY \& PANCREAS-Liver}

\section{LAPAROSCOPIC SUBTOTAL GASTRECTOMY WITH ASSOCIATED BILIARY ROUTE EXPLORATION}

A. Pinillos, A. Escartin, M. Pablo, M.G. Marta, J.T. Jaume, J. Ortega, J. Jorge

Cirugía General, Hospital Universitario Arnau de Vilanova, LLEIDA, Spain

Introduction: The progressive laparoscopic learning in gastric surgery and the great development of instruments and laparoscopic material that facilitates the realization of advanced procedures, has led to an increase in the use of laparoscopy in the treatment of gastric cancer. Material and methods: We present the case of a 75-year-old man without AMC with a history of ischemic heart disease who enters our surgery department for cholangitis secondary to choledocholithiasis. ERCP is requested during his admission that describes a gastric lesion from which a biopsy is taken, making it impossible to access Vater papilla to perform sphincterotomy and lithiasis extraction due to the existence of duodenal diverticula. The result of pathological anatomy of the gastric lesion was compatible with adenocarcinoma. Negative extension study. The clinical case is presented in a committee of multidisciplinary tumors and it is decided to perform surgical intervention of both pathologies.

A subtotal gastrectomy was performed with a Roux-en-Y reconstruction. Surgical time of 300 min. Choledochotomy was performed with lithiasis extraction, as well as intraoperative exploration of the bile duct and main conduits by means of a choledochoscope.

Results: Income of 9 days, with a Clavien II. The definitive pathological anatomy was an AI stage with a total of 22 isolated nodes without evidence of neoplasia in any of them, therefore it does not require adjuvant treatment. The patient is asymptomatic, with nutritional supplementation with follow-up in CCEE of Surgery.

Conclusions: In our case, there were no serious postoperative complications when performing gastric resection and bile duct exploration with drainage of the same. From the oncological poin of view, the number of lymph nodes extracted and the surgical margins are similar to those obtained in patients in whom we perform open surgery; therefore, although it is a single clinical case, laparoscopy in expert surgeons is a safe and effective technique.

\section{V107-HEPATO-BILIAIRY \& PANCREAS-Pancreas}

\section{TOTALLY LAPAROSCOPIC PUESTOW PROCEDURE IN A PATIENT WITH CYSTIC DUODENAL DYSTROPHY}

\section{Balescu ${ }^{1}$, A. Constantin ${ }^{2}$, C. Copaescu ${ }^{1}$}

${ }^{1}$ Visceral surgery, Ponderas Academic Hospital, BUCHAREST, Romania; ${ }^{2}$ Gastroenterology, Ponderas Academic Hospital, BUCHAREST, Romania

The Puestow procedure was initially proposed to alleviate the pain in patients with chronic pancreatitis and dilated Wirsung duct. Its objective is to provide an efficient drainage of the pancreatic fluids and, in the meantime, to preserve the pancreatic tissue and minimize the risk of endocrine and exocrine pancreatic insufficiency.

Aims: to describe the particular technical aspects and the efficacy of totally laparoscopic Puestow procedure in patients with cystic duodenal dystrophy.

Methods: A 37 years old patient presenting diffuse epigastric pain, vomiting and weight loss was diagnosed at endoscopic ultrasound and biopsy with cystic duodenal dystrophy. A conservative treatment was decided with octreotide and opioids. However, due to the persistence of symptoms surgery was performed.

Results: due to the association of a dilated Wirsung duct, the patient was submitted to a Puestow procedure. The surgical procedure was completed in a minimally invasive manner; after dissecting the anterior surface of the pancreas an intraoperative ultrasound was performed in order to identify the Wirsung duct. Therefore, the pancreatic parenchyma was transected along the Wirsung duct, a totally laparoscopic pancreato-jejunostomy on Roux en Y limb being performed. The early postoperative outcome was uneventful, the patient being discharged in the sixth postoperative day. At one month and six months follow up the need for opioid treatment significantly diminished. A kinking of the enteral anastomosis required a laparoscopic intervention one year after with a very good evolution after.

Conclusions: totally laparoscopic Puestow procedure seems to be a safe and efficient method in order to treat symptomatic patients with cystic duodenal dystrophy in whom a dilated Wirsung duct is present.

\section{V108-HEPATO-BILIAIRY \& PANCREAS-Pancreas}

\section{LAPAROSCOPIC SINGLE PORT (SILS) DISTAL PANCREATECTOMY WITH SPLENIC PRESERVATION FOR A IPMN OF PRINCIPAL PANCREATIC DUCT}

T. Cipolat Mis, E. Falsetti, M.C. Cartillone, A. d'Alessandro, E. Chouillard

General And Minimally Invasive Surgery, Poissy-Saint-Germain-enLaye Medical Center, POISSY, France

Aims: The approach to the intraductal papillary mucinous neoplasm (IPMN) is various, from a radiological follow-up with magnetic resonance (RM) to the surgical treatment with a pancreatic resection [1]. The surgical approach is various and depends on the localization of the lesion and on the surgical skills[2]

Methods: A 67 years old patient was admitted at the CHI Possy-Saint Germain-en-Laye with an acute pancreatitis. At the ecoendoscopy was found a pancreatic cystic at the junction of the pancreatic body and tail with a Wirusng diameter of $5 \mathrm{~mm}$. A second episode of acute pancreatitis occurred a few months later. After that episode the patient was submitted to a compute tomography (CT) that found a cystic lesion of $2 \mathrm{~cm}$ with an increasing dilatation of the Wirsung duct. The serum CA19-9 was $452 \mathrm{UI} / \mathrm{mL}$. A laparoscopic SILS distal pancreatectomy with spleen conservation was performed.

Results: A trans-umbilical incision was performed with the positioning of the GelPoint SILS platform with the placement of 3 trocars. A distal pancreatectomy with a spleen preservation and without a standard linfadenectomy was performed. The pancreatic stump was closed with an Endo-GIA $60 \mathrm{~mm}$ with Seamguard device. Any drain was placed. The post-operative course was uneventfull. A CT scan was performed in .... post-operative day which didn't show collections. The patient was discharged in -...... post'operative day. The histological examination shows an IPMN with low grade dysplasia. No invasive carcinomatoses cells were found.

Conclusions: The distal pancreatic SILS resection with spleen conservation is a feasible and safe technique that combine all the advantages of the minimally invasive laparoscopic approach with the esthetic advantages of the SILS approach.

[1] European evidence based guidelines on pancreatic cystic neoplasms. European Study Group on Cystic Tumours of the Pancreas. Gut. 2018 May;67(5):789-804. Epub 2018 Mar 24.

\section{V109-HEPATO-BILIAIRY \& PANCREAS-Pancreas}

\section{LAPAROSCOPIC RADICAL PANCREATO-DUODENECTOMY FOR PANCREATIC ADENOCARCINOMA}

\section{G.F. Faria, L. Freire, A.F. Cocco, A. Cabral-Correia, A.M. Gouveia}

Surgery, ULS Matosinhos / Hospital Pedro Hispano, MATOSINHOS, Portugal

Pancreato-duodenectomy is a complex surgery, requiring several anastomoses to reconstruct the digestive tract. Due to its technical complexity, the laparoscopic approach is not yet the goldstandard and there remains some controversy about its oncological safety. Worldwide experience is limited, and its safety and effectiveness are yet under evaluation.We present the clinical case of a 70 years-old woman with a prior history of epilepsy. She was studied due to painles obstructive jaundice and a $2 \mathrm{~cm}$ pancreatic head tumour was diagnosed on imaging, causing CBD and Wirsung channels' dilatation. The tumour was considered locally resectable and she was proposed for a radical pancreato-duodenectomy.We present the main steps of the surgery including the oncological resection with lymphatic basin clearance and totally laparoscopic reconstruction. The post-operative was uneventful, and the histologic sample revealed a ductal adenocarcinoma (T2) with an R0 resection and 0/30 lymph nodes invaded. Although technically demanding, laparoscopic pancreato-duodenectomy is safe and effective requiring teams with experience both in pancreato-biliary and laparoscopic surgery. 


\section{V110-HEPATO-BILIAIRY \& PANCREAS-Pancreas}

\section{LONGITUDINAL LAPAROSCOPIC PANCREATO-} JEJUNOSTOMY: A NEW APPROACH FOR AN OLD SURGERY

G.F. Faria, L. Freire, A.F. Cocco, A. Cabral-Correia, A.M. Gouveia, Surgery, ULS Matosinhos / Hospital Pedro Hispano, MATOSINHOS, Portugal

Chronic pancreatitis is characterized by a progressive pancreatic fibrosis with loss of endocrine and exocrine function. One of its main symptoms is debilitating pain. Surgical drainage of a dilated pancreatic duct is an option to consider in cases of refractory pain. Longitudinal pancreato-jejunostomy allows an effective decompression of the pancreatic channel and a significant improvement in the quality of life.

We present the clinical case of a 56 years-old lady with a prior history of gallstones. She was treated for an acute pancreatitis in May 2018, followed by recurrent relapses of pain and enzymatic elevation. She required opioid use for partial pain control and a significant $20 \mathrm{~kg}$ decrease on body weight due to 'fear of eating'. The endo-ultrasonography and the MRI revealed a chronic pancreatitis with an $8 \mathrm{~mm}$ Wirsung duct with ductal stones and an atrophic body and tail. We proposed a laparoscopic longitudinal pancreato-jejunostomy. The surgery was performed with 4 trocars, with the surgeon on the right side of the patient. We performed a trans-mesocolic $6 \mathrm{~cm}$ pancreato-jejunostomy. The post-operative was uneventful, and the patient was discharged on the 8 th post-operative day, asymptomatic.

Laparoscopic longitudinal pancreato-jejunostomy, although effective is a technically demanding surgery but brings the benefits of a minimally invasive approach.

\section{V111-HEPATO-BILIAIRY \& PANCREAS-Pancreas}

\section{LAPAROSCOPIC SPLEEN PRESERVATION DISTAL} PANCREATECTOMY: VIDEO COMPARISON OF SPLENIC VESSEL PRESERVATION AND WARSHAW TECHNIQUE AND CLINICAL OUTCOMES

\section{K. Mizunuma, Y. Ebihara}

Gastroenterological Surgery II, Hokkaido University Graduate School of Medicine, SAPPORO, Japan

Background: Preservation of spleen in distal pancreatectomy is also useful from the maintenance of platelets and the prevention of overwhelming post splenectomy infection. We have performed Laparoscopic Spleen Preservation Distal Pancreatectomy: LSPDP to benign and low-grade tumors of the pancreatic body tail. The aim of this study was to report our surgical experience with the method of SVP: splenic vessel preservation and WT: Warshaw technique of LSPDP, describe our techniques with videos.

Method: There are three points of our surgical technique. 1, Precede pancreatic dissection, improve the mobility of the pancreas. 2, Confirming the courses of splenic artery and classified them into two major types. 3, Preserving the left gastro-epiploic vessels and short gastric vessels.The postoperative cases of LSPDP which performed from April 2012 to September 2018 was retrospectively studied.

Result: Of 19 consecutive patients were performed LSPDP at our institute, 12 were SVP and 7 were WT. Ages, gender and BMI were similar for two groups. There were no significant differences in operative time, blood loss and length of stay after surgery. Comparing pathological finings, WT was associated with a slightly large tumor lesion (median $31 \mathrm{~mm}$ vs. $12.5, \mathrm{p}=0.08$ ). Among the median observation period of 27 months, splenic infarction was observed in 1 case in SVP and 2 cases in WT. However, they were focal splenic infarctions, they did not need surgery or drainage. There were no cases in which late onset of splenic artery occlusion or esophageal / gastric varices.

Conculusion: After performing LSPD, the function of the spleen was good in all cases. Both SVP and WT were safe and feasible procedures.

\section{V112-HEPATO-BILIAIRY \& PANCREAS-Pancreas}

\section{LAPAROSCOPIC FREY'S PROCEDURE WITH MANAGEMENT OF INTRAOPERATIVE COMPLICATION}

\section{P. Agami, R. Izrailov, A. Andrianov}

High Technology Surgery and Endoscopic Surgery, Moscow Clinical Scientific Center, MOSCOW, Russia

This is the case of a 61-years-old lady presenting with recurrent abdominal intractable pain she has been suffering from for the last 7 years. MSCT revealed pancreatic calcifications from $1 \mathrm{~mm}$ to 5-8 $\mathrm{mm}$ and dilatation of the main pancreatic duct in the body of the pancreas up to $4 \mathrm{~mm}$. The patient underwent laparoscopic local resection of the head of the pancreas combined with longitudinal Roux-en-Y pancreaticojejunostomy-a technique known as Frey's procedure. It is recognized as an effective therapeutic option for the surgical treatment of patients with persisten pain caused by chronic pancreatitis.After performing the posterior wall of the pancreaticojejunal anastomosis we've faced an intraoperative complication such as volvulus of the Roux limb causing serious ischemia of the limb. We were forced to remove all previous sutures in order to untwist the Roux limb, thereafter the pancreaticojejunostomy was started anew.The purpose of this video is to demonstrate that Frey's procedure can be performed in a minimally invasive fashion, which provides all the well-known advantages of this approach. We demonstrate that even such serious intraoperative complication as volvulus of the Roux limb can be managed without conversion. Our center has an experience of over 30 laparoscopic Frey's procedures, however this is the first case where we encountered with such complication and we believe this is an experience worth sharing.Yet we would like to underline that this approach should be used by highly skilled minimally invasive surgeons experienced in intracorporeal suturing which is the most challenging stage in Frey's procedure.

\section{V113-HEPATO-BILIAIRY \& PANCREAS-Pancreas}

\section{LAPAROSCOPIC SPLEEN PRESERVING DISTAL PANCREATECTOMY WITH SPLENIC VESSELS PRESERVATION}

\section{Tomulescu, I. Hutopila, C. Copaescu}

General Surgery, Ponderas Academic Hospital, BUCHAREST, Romania

Spleen preserving Distal Pancreatectomy (SPDP) is commonly applied in patients with benign or low-grade malignant tumors in the body and tail of the pancreas.

Two surgical techniques for SPDP have been described. The first technique was described by Kimura (spleen preserving distal pancreatectomy with splenic vessel preservation-SPDP-SVP) and preserves the main splenic artery and vein and excises the tail of the pancreas and those small, short vascular connections to the body;the second technique was described by Warshaw and involves resection of the splenic vein and artery before distal pancreatectomy, and conservation of theshort spleno-colic and gastric vessels to keep normal blood flow for the spleen (spleen-preserving distal pancreatectomy with splenic vessel resection-SPDP-SVR).

We present the case of a 50 years old female with $40 / 50 \mathrm{~mm}$ tumor of the pancreatic tail on ultrasonography. CT scan confirmed the tumor and endoscopic ultrasonography with FNA have shown a solid pseudopapillary tumor. Due to the low grade malignancy we have decided to perform a laparoscopic spleen preserving distal pancreatectomy with splenic vessels preservation (LSPDP-SVP). For LSPDP-SVP the difficulty is related with the splenic vessels dissection and manipulation. Primary dissection and control of main trunk of splenic artery and vein will help to quickly control bleeding during vascular rupture in small vessels dissection. Optimal stapling of any tissue requires an adequate tissue compression time to allow elongation of the tissue being compressed, smooth firing of the instrument, consistent staple line formation balanced against the risk of increased tissue tearing and excessive tensile strength. This is why, for pancreatic division, we prefer choosing a cartridge loaded with higher staplers. The pancreatic stump transection line is evaluated for bleeding and when it is needed, hemostatic clips are applied.

Histology report confirmed a Solid pseudopapillary tumor T3NoMxL0V0R0 at this moment with 12 month good follow up.

In conclusion LSPDP-SVP is safe, reproductible and demonstrated very good outcomes when certain indications are respected. 


\section{V115-HEPATO-BILIAIRY \& PANCREAS-Pancreas}

HEAD PANCREAS ADENOCARCINOMA TREATED BY COMPLETE LAPAROSCOPIC DUODENOPANCREATECTOMY

J. Tur-Martínez ${ }^{1}$, A. Escartín ${ }^{1}$, M. González ${ }^{1}$, P. Muriel ${ }^{1}$, A. Pinillos $^{2}$, J. Ortega ${ }^{2}$, J.J. Olsina ${ }^{1}$

${ }^{1}$ Hepatobiliary and Pancreatic Surgery Unit, Hospital Universitari Arnau de Vilanova, LLEIDA, Spain; ${ }^{2}$ General and Digestive Surgery Service, Hospital Universitari Arnau de Vilanova, LLEIDA, Spain

Aim: Advances in minimally invasive surgery has permitted to perform complex techniques by this approach, being the laparoscopic duodenopancreatectomy (LPD) one of these.

The aim of this communication is to present a surgical technique video for a complete laparoscopic PD, showing the most important steps of the resective and reconstructive phase, with the anastomosis realized completely by laparoscopy.

Methods: A surgical technique video is presented showing the main steps for the LPD and a complete laparoscopic reconstruction with an hepaticojejunostomy, duct-to-mucosa pancreatic-jejunostomy and a gastrojejunostomy.

Results: An 82 years old woman with past medial history of arteria hypertension, dyslipidemia, Type II Diabetes Mellitus and a breast cancer treated in 2009 with lumpectomy and axillary lymphadenectomy plus radiotherapy, recently diagnosed of and adenocarcinoma of the head of the pancreas. The CT scan showed a neoplasia localized in the head of the pancreas without extension to other organs. A laparoscopic PD was indicated after a multidisciplinary committee evaluation.

A supraumibical Hasson trocar was used for the pneumoperitoneum, three $12 \mathrm{~mm}$ trocars and two $5 \mathrm{~mm}$ trocars were used.

LPD was performed. The resective phase was done following the conventional steps of the open Whipple procedure and for the reconstructive phase, a Child limb was used for a termino-lateral hepatico-jejunostomy with an absorbable 4/0 monofilament; a duct-to-mucosa pancreatic-jejunostomy with an absorbable 5/0 monofilament and finally a latero-lateral mechanical gastro-jejunostomy was performed. Surgical time was 480 min. Postoperative course without complications and the patient was discharged on the 7th postoperative day.

Definitive anatomopathological exam: intraductal tubulopapilar neoplasia, $16 \times 16 \times 13 \mathrm{~mm}$, with wide high grade epithelial dysplasia. Free margins. pTisN0 (0/12)

Conclusion: Laparoscopic PD is a feasible procedure with a high technical requirement which should be performed in specialized centres with high experience in hepatobiliary surgery and in advanced laparoscopic procedures, because of its high morbidity and mortality.

\section{V116-HEPATO-BILIAIRY \& PANCREAS-Pancreas}

TOTALLY ROBOTIC WHIPPLE PANCREATODUODENECTOMY-OVERCOMING INTRAOPERATIVE DIFFICULTIES EXPERIENCE FROM 23 CASES

F. Antonakopoulos ${ }^{1}$, P. Athanasopoulos ${ }^{1}$, A. Ioannidis ${ }^{1}$, M. Konstantinidis ${ }^{2}$, K. Konstantinidis ${ }^{1}$

${ }^{1}$ General Surgery, Athens Medical Center, MAROUSI, ATHENS, Greece; ${ }^{2}$ Medical School of Athens, National Kapodistrian University of Athens, ATHENS, Greece

Introduction: Pancreaticoduodenectomy-Whipple procedure remains one of the most challenging operations in general surgery. Except from the cumbersome and meticulous dissection, it requires complex reconstructive procedures with numerous anastomosis. Furthermore the presence in patients history, of previous operative interventions in the area of interest, adds a lot to the degree of difficulty in completing such cases laparoscopically.

Aim: We present our experience of 23 totally robotic Whipple pancreatoduodenectomies during the last 4 years.

Methods: All patients underwent a totally robotic Whipple procedure using the da Vinci Si robotic system. Some of the patients had history of upper GI Surgery like Billroth-II Gastrectomies, or placement of metallic CBD stents.The phases of the operation were: entrance to the omental bursa, kocherization, evaluation of the pancreatic neck and portal vein, division of pancreatic head, lymph node harvesting, ligation of the gastroduodenal artery, dissection of the stomach at the pylorus, division of proximal jejunum, division of CBD, removal of CBD stent (if present) and cholecystectomy. Reconstruction included a pancreaticogastrostomy, a choledochojejunostomy and a gastrojejunostomy. In 5 cases a pylorus preserving technique was used.

Results: There where no conversions to open. Mean operative time was 8 h. Minimal need for transfusions.In all patients that underwent Whipple, the robotic platform proved useful in dissecting adhesions from previous surgeries and in handling the CBD when stents where placed. Also proved useful in intracorporeal construction of the anastomoses. Mean ICU stay was $14 \mathrm{~h}$.

Conclusions: Robotic assistance in Whipple may overcome limitations of laparoscopy and offer a minimaly invasive approach to this procedure potentially resulting in lower blood loss and less morbidity. We need further prospective randomized trials in order to determine the exact role of robotics in pancreatic surgery. 


\section{V117-HEPATO-BILIAIRY \& PANCREAS-Pancreas}

LAPAROSCOPIC SPLEEN-PRESERVING DISTAL PANCREATECTOMY WITH PRESERVATION OF SPLENIC VESSELS FOR BENIGN AND LOW-GRADE MALIGNANT PANCREATIC NEOPLASM

\section{A. Lytvynenko, I. Lukecha, Yu. Khilko}

Laparoscopic surgery, State institution « O.Shalimov National Institute of Surgery and Transplantology », KIEV, Ukraine

Aims: Distal pancreatectomy is the standard curative treatment for symptomatic benign, premalignant, and malignant disease of the pancreatic body and tail. The most obvious benefits of a laparoscopic approach to distal pancreatectomy include earlier recovery and shorter hospital stay. Spleen-preserving distal pancreatectomy should be attempted in case of benign disease. Laparoscopic spleen-preserving distal pancreatectomy (LSPDP) is expected to be less invasive than laparoscopic distal pancreatectomy with splenectomy. However, there are few reports regarding the details of the procedure for LSPDP, and its safety remains unclear. This study aimed to evaluate the feasibility and safety of LSPDP.

Methods: Retrospective analysis of surgery treatment of 48 patients was made. LSPDP was conducted in the period from 2014 to 2017 in the department of laparoscopy surgery of State institution O.Shalimov National Institute of Surgery and Transplantology. The average age was $45 \uparrow 13.4$ years, the body mass index (BMI) was $28.7 \pm 1$.

Results: Laparoscopic distal pancreatectomys was performed in $100 \%$ of cases, were attempted in 36 female and 12 male patients. Postoperative pathological examinations revealed 17 cases of serous cystadenoma in the body and tail of the pancreas, 2 case of serous oligocystic adenoma, 20 case of mucinous cystadenoma, 3 case of neuroendocrine tumor (insulinoma), and 6 case of solidpseudopapillary neoplasm. Complications related to the surgery were like acute pancreatitis with 3 -fold increase normal plasma amylase confirmed by CT -7 cases, fluid collection -4 cases, pancreatic fistula (grade A) - 3 cases. The operation time was $195.6 \mathrm{~min}$, (range 157-250 min) blood loss of $50.1 \mathrm{~g}$ (range $0-110 \mathrm{~g}$ ), mean hospital stay was 6.8 days (range 5-11 days). Conversion to laparotomy was in 1 case. Mortality was 0 .

Conclusion: Laparoscopic spleen-preserving distal pancreatectomy is minimally invasive, safe, and feasible for the management of benign pancreatic tail tumors, with the advantages of earlier recovery and less morbidity from complications.

\section{V118-HEPATO-BILIAIRY \& PANCREAS-Pancreas}

\section{LAPAROSCOPIC SPLENOPANCREATECTOMY FOR MUCINOUS CYST TUMOR}

A.F. Aranzana Gómez ${ }^{1}$, J. Hernandez Gutierrez ${ }^{1}$, J. Malo Corral ${ }^{1}$, B. Muñoz Jimenez ${ }^{1}$, A. Trinidad Borras ${ }^{1}$, G. Krasniki ${ }^{1}$,

P. Toral Guinea ${ }^{1}$, R. Lopez Pardo ${ }^{1}$, S. Abad de Castro ${ }^{2}$, M.A. Morlan Lopez ${ }^{1}$

${ }^{1}$ General Surgery, Complejo Hospitalario Toledo, TOLEDO, Spain; ${ }^{2}$ General Surgery, Hospital tres culturas, TOLEDO, Spain

Introduction and objectives: Cystic tumors of the pancreas are infrequent lesions (10-15\%). They can present with abdominal pain, nausea or jaundice or asymptomatic with incidental diagnosis. Their diagnosis and classification can be difficult. The objective is to demonstrate the safety and efficacy of the laparoscopic approach in this pancreatic pathology.Material and methods: We present a video of the surgical intervention of a 32-year-old patient, with functional dyspepsia, with a casual diagnosis of $18-\mathrm{mm}$ pancreatic LOE in the pancreas body after echoendoscopic study and CT, suggestive of cystadenoma / mucinous cystadenocarcinoma. Prophylactic anti-pneumococcal vaccine, against Haemophilus influenzae and against Neisseria meningitidis. Spleno-caudal pancreatectomy is proposed

Results: Intervention: complete laparoscopic approach, 4 trocars. Aperutra of the splenocolic and gastrocolic ligament for access in the transcavity of the omentum. Identification of the tumor, impresses serous cystadenoma. Liberation of the superior border of the pancreas of the splenic artery. Dissection of the inferior border of the pancreas and access to the retroperitoneum, dissection of the posterior face and elevation of the neck of the pancreas identifying superior mesenteric artery and vein and 3rd duodenal portion. Identification of splenic vein and dissection of it. Individualized section of vein and splenic artery with vascular Endogia. Body and tail section with endogia 60 purple charge. Identification of Gerota fascia and left adrenal gland are respected. Liberation of the spleen of the retorperitoneum. Removing the piece in a bag. Left pleural effusion secondary to left subphrenic collection resolved with conservative management. High to 8 th day. The anatomopathological result of the surgical specimen was serous cystoma, unilocular, large, free surgical border.

Conclusion: The laparoscopic approach of the cystic neoplasms of the pancreas is a valid option (if located in the body-tail of the pancreas). Applying the principles of oncological resection, the results are excellent provided that we avoid tumors of large size or with tumor invasion.

\section{V119-HEPATO-BILIAIRY \& PANCREAS-Pancreas}

\section{PANCREATIC PSEUDOCYST, MANAGEMENT AND LAPAROSCOPIC APPROACH}

M. Alba Valmorisco ${ }^{1}$, R. Martinez Mojarro $^{2}$, R. Perez Quintero ${ }^{2}$,

B. García del Pino ${ }^{3}$, J. Candón Vázquez ${ }^{1}$,

D. Bejarano González-Serna ${ }^{1}$, P. Beltrán Miranda ${ }^{1}$,

R. Balongo García ${ }^{4}$

${ }^{1}$ General Surgery (Hepatobiliary surgery), Juan Ramon Jimenez Hospital, HUELVA, Spain; ${ }^{2}$ General Surgery, Juan Ramon Jimenez Hospital, HUELVA, Spain; ${ }^{3}$ General Surgery (coloproctology surgery), Juan Ramon Jimenez Hospital, HUELVA, Spain; ${ }^{4}$ Genial surgery (esophagogastric surgery), Juan Ramon Jimenez Hospital, HUELVA, Spain

Aims: A pancreatic pseudocyst is an encapsulated, mature fluid collection occurring withing the pancreas that have a well-defined Wall minimal or no necrosis secondary to pancreatic injury and mediated by the enzimatic and inflammatory disruption of pancreatic tissue. It is a common complication of acute and chronic pancreatitis.

We present the case of a pancreatic pseudocyst located within the body of the pancreas due to recurrent necrotic pancreatitis.

The objective of this video is to show the minimally invasive surgical approach of this entity. Methods: A 47-year-old man without medical history was admitted to hospital in the Digestive Service on 3 times for acute necrotizing pancreatitis. After study in which is evidenced cholelithiasis and pseudocyst in pancreatic body of $6 \mathrm{~cm}$ maximum diameter and formation of two peripancreatic collections without signs of superinfection, cholecystectomy is indicated. Magnetic control cholangiography was performed after surgery and it showed an increase in the size of the pancreatic pseudocyst, suspecting Wirsung's duct disruption. Therefore, endoscopic retrograde cholangiopancreatography (ERCP) was performed by placing a plastic pancreatic prosthesis and performing a sphincterotomy.

After hospital discharge, the patient is re-admitted due to recurrent abdominal pain withou analytical alteration. TC abdominal observed an increase in the pseudocyst from 6 to $8 \mathrm{~cm}$.

This case was discussed in a multidisciplinary committee and surgical intervention was decided. Results: Laparoscopic approach is decided and four trocars were placed. Initially, a gastrostomy was performed with liquid outlet. An aspiration of the liquid and quistogastrostomy with $45 \mathrm{~mm}$ endoGIA was made.

The patient progresses favorably, being high on the tenth postoperative day, without complications. Conclusions: Almost every pancreatic pseudocyst improves spontaneously and needs no specific treatment. Draining is indicated when secondary symptoms to compression, complications or rapidly enlarging are found.

Depending on the complexity of the pseudocyst, its communication with Wirsung's duct and the existence of ductal injury, It may perform a percutaneous, endoscopic or surgical drainage. The goal of pancreatic debridement is to excise all dead and devitalized pancreatic and peripancreatic tissue while preserving viable functioning pancreas, controlling resultant pancreatic fistulas, and limiting extraneous organ damage. Only the surgical procedure is definitive. 
V121-HERNIA-ADHESIONS-Abdominal wall hernia

LAPAROSCOPIC REPAIR OF A SYMPTOMATIC LEFT SIDED BOCHDALEKS' HERNIA IN A YOUNG MALE WITH INTRATHORACIC MIGRATION OF THE LEFT KIDNEY, TRANSVERSE COLON AND SMALL INTESTINE

\section{Ruyssers, T. Gys, T. Lafullarde}

General Surgery, AZ Saint Dimpna Hospital, GEEL, Belgium

Case: A 29y old male presents with intermittent low retrosternal pain and progressive dyspnea with exercise since a couple of months. Cardiac investigation was negative and gastroscopy showed a grade B esophagitis. He was treated medically but with only partial response. On a thoraco-abdominal CAT-scan the diagnosis of a left sided Bochdaleks' hernia was made. The hernia includes the left kidney (with blood vessels and ureter), transverse colon and small intestine which are positioned in the left lower thoracic cavity with the left lung considerably compressed.

Method: Given the clear correlation between the patients' complaints and these anatomical findings, he was referred to our service of abdominal surgery.

We performed a laparoscopy with the patient in lithotomy position and the surgeon between the legs. The patient was tilted to his right side. Mobilization of the spleen was necessary to gain maximal access to the hernia. We were able to reduce all the herniated content, freed the margins of the defect, reduced the hernia sac and repositioned the kidney intra-abdominally. The defect was manually closed with non-resolvable stitches and covered with a mesh which was secured with tackers.

Result: Postoperatively the patient recovered well with adequate pain relief and pulmonary support. He could leave the hospital after 6 days. Control CAT-scan on day 5 postoperatively shows an intact lining of the diaphragm with normal positioning of the intra-abdominal organs. On follow-up 6 weeks after surgery the patient had regained normal activities and was symptom free.

Conclusion: A symptomatic left sided Bochdaleks' hernia in adults with an ectopic intrathoracic kidney is extremely rare. We hereby state that, during a laparoscopic repair, the kidney can also be safely reduced, which has almost never been described in literature yet, enhancing pulmonary recovery, improving access for mesh placement and thus diminishing recurrence rate.

\section{V122-HERNIA-ADHESIONS-Abdominal wall hernia}

COMPLETE ABDOMINAL WALL REPAIR WITH ENDOSCOPIC ANTERIOR COMPONENT SEPARATION AND LAPAROSCOPIC EVENTROPLASTY IN COMPLEX INCISIONAL MIDLINE HERNIAS

B. Bascuas Rodrigo ${ }^{1}$, J. Bellido Luque ${ }^{1}$, C. Dominguez Sanchez ${ }^{1}$, A. Bellido ${ }^{2}$, J. Gomez ${ }^{2}$, J.M. Suarez ${ }^{2}$, I. Sanchez-Matamoros Martin ${ }^{1}$, A. Nogales Muñoz ${ }^{1}$, F. Oliva Mompean ${ }^{1}$

${ }^{1}$ General Surgery, SAS, SEVILLA, Spain; ${ }^{2}$ General Surgery, Quiron Salud, SEVILLA, Spain

Aims: Large incisional hernias repair involves an actual problem for surgeons to face. Anterio component separation has been an important method allowing to close the fascia defects without tension while also having underlay mesh reinforcement.Therefore, we present a case of incisional hernia reparation performing endoscopic anterior component separation with advantages compared with open approach

Method: We present the case of a 31-year-old woman, BMI $40 \mathrm{~kg} / \mathrm{m}^{2}$, with previous laparoscopic gastric sleeve and posterior reintervention using open approach. The patient presented a $10 \mathrm{~cm}$ size incisional hernia M3W3. A CT scan was performed, confirming a midline incisional hernia containing colon, with an herniary defect of $11 \mathrm{~cm}$. Full minimal invasive abdominal wall repair was proposed. A $2 \mathrm{~cm}$ size incision was made in left iliac region to reach the aponeurosis of external oblique muscle. We placed a balloon trocar and subcutaneous pneumo-dissection with $8 \mathrm{mmHg}$ pressure was performed; then, we placed a $5 \mathrm{~mm}$ trocar in left lumbar space. The aponeurosis of external oblique muscle was incised and anterior component separation from inguinal to subcostal area was achieved. An extensive intermuscular dissection was performed to achieve complete midline closure. We performed the same procedure on the right side. Then, with laparoscopic approach using V-Loc $\mathrm{n}^{\circ} 0$ suture, we completely closed the midline. Eventually, we placed a 30x15 cm PTFE-c mesh fixed with a double crown of tackers and fibrin glue. Results: Postoperatory course was uneventful and the patient was discharged $24 \mathrm{~h}$ after surgery without any remarkable event during his postoperative stay. The patient has been followed up for 12 months without any complication or recurrence in CT scan, confirming the correct minimally invasive reconstruction of the abdominal wall.

Conclusions: Trends in abdominal wall reconstruction and complex-hernia repairs have advanced rapidly in recent years. The goal is to perform a complete abdominal wall repair with no tension in midline incisional hernias. Endoscopic anterior component separation and laparoscopic eventroplasty with closure of the defect, leads to a complete wall reconstruction without tension and avoids drawbacks due to primary close defect in those patients with herniary defects wider than $10 \mathrm{~cm}$.

\section{V123-HERNIA-ADHESIONS-Abdominal wall hernia}

TOTALLY ENDOSCOPIC SUBLAY ANTERIOR REPAIR (TESAR) FOR VENTRAL AND INCISIONAL HERNIAS

\section{F. Ferrara, F. Fiori, D. Gentile, D. Gobatti, M. Stella}

Department of Surgery, ASST Santi Paolo e Carlo, MILAN, Italy

Aims: Endoscopic technique is a valid and safe approach for the treatment of abdominal wal defects. To combine the advantages of complete endoscopic extraperitoneal surgery with those of sublay mesh repair we propose Totally Endoscopic Sublay Anterior Repair (TESAR), a safe and feasible approach for the treatment of ventral and incisional midline hernias.

Methods: From May to September 201812 patients were referred to our Unit for clinical and radiological diagnosis of midline ventral or incisional hernia and selected for TESAR. Exclusion criteria were: complicated ventral or incisional hernia (i.e. incarcerated hernia), maximum defect width $>5 \mathrm{~cm}$, contraindications to general anesthesia. The procedure consisted of suprapubic access with 3 trocars, complete endoscopic pre-aponeurotic dissection, isolation and reduction of the hernial sac, bilateral incision of the medial rims of recti aponeurosis and dissection of retromuscular plane to create the retromuscular space, sublay non-absorbable mesh positioning and anterior aponeurosis reconstruction. One drain was always placed in the retromuscular space and one drain in the subcutaneous space.

Results: All procedures were completed with endoscopic approach, with no conversion to laparoscopy or open surgery. No intraoperative complications were registered. Total mean operative time was $148 \pm 18.5 \mathrm{~min}$. No post-operative major complications were registered. Only one subcutaneous seroma was registered (8.3\%), and treated conservatively. The mean postoperative stay was $3.6 \pm 0.6$ days. At post-discharge clinical checkups drains were checked and removed when indicated. No wound complications nor recurrence were registered to date Cosmetic and functional results were successful in all patients.

Conclusions: TESAR is a safe and feasible technique for the extra-peritoneal sublay repair of ventral hernias with totally endoscopic approach. It provides accurate hernia repair with good outcomes in terms of resolution of symptoms and post-operative complications.

\section{V124-HERNIA-ADHESIONS-Abdominal wall hernia} COMPARISON OF STANDARD IPOM AND IPOM PLUS IN OUR HOSPITAL

\section{R. Mizuno, M. Kondo}

Surgery, Kobe City Medical Center General Hospital, KOBE CITY, Japan

Backgrounds: Abdominal incisional hernia is found in more than 10\% after abdominal surgery, and risk factors such as wound infection, obesity, elderly, high abdominal pressure are pointed out. Laparoscopic hernia repair using intraperitoneal onlay mesh (standard IPOM) is becoming widespread in Japan since the insurance release in 2012, and our hospital is actively working on it. Recently, IPOM plus procedure which also carries out fascia suture in addition to laparoscopic mesh placement has been introduced.

Aims: We report the clinical results of laparoscopic abdominal incisional hernia repair in our hospital.

Methods: We performed hernia repairs using a mesh for 36 cases from January 2014 to September 2018. Of these, 21 cases were standard IPOM and 15 cases were IPOM plus. There was no significant difference in the patient background such as gender, age, BMI, etc, and in the intraoperative findings such as hernia orifice diameters and adhesions. Surgical time, postoperative hospital stay, and the rate of complications such as seroma, mesh bulging, postoperative pain, hernia recurrence were compared and examined between the two groups.

Results: As a result, in IPOM plus group, the operation time was longer and the incidence rate of postoperative pain was higher, but the incidence of mesh bulging was significantly lower. Also, in some cases since 2018 , the ' $\mathrm{U}$ reverse stitch method ' is used as an ingenuity of fascia suture in IPOM plus.

Conclusions: Laparoscopic abdominal incisional hernia repair has the advantage of being able to reliably confirm the hernia orifice from the intraperitoneal side?it is excellent in the identification of the fragile part of the abdominal wall and in the visibility of the restoration range. With regard to the IPOM plus procedure which has been introduced in the last few years, although the operation time is extended, it has usefulness such as reduction of mesh bulging. From the viewpoint of cosmetic surgery, usage of IPOM plus will increase in the future. 


\section{V125-HERNIA-ADHESIONS-Abdominal wall hernia SINGLE-PORT RETRORECTAL INCISIONAL HERNIA REPAIR-A NEW APPROACH}

\section{S.H. Kang, S.H. Ahn, D.J. Park, H.H. Kim}

Department of Surgery, Seoul National University Bundang Hospital, 13620, Korea

Introduction: Incisional hernia is one of the most common complications after abdominal surgery. Several methods have been introduced, and yet, there is no consensus on the best method of repair. We present a novel method for hernia repair which uses the retromuscular sublay mesh repair through a single incision at the pubic area to improve cosmesis.

Methods: Medical records of patients who underwent single-port retrorectal incisional hernia repair from May 2018 to December 2018 were reviewed. Patients were placed in supine position and $3 \mathrm{~cm}$ incision was made in the pubic area below the panty line. A flap is made upwards until the defect is found and bilateral rectus sheathes are dissected. A mesh is then placed between the posterior rectus sheath and the muscle.

Results: A total of 30 patients with midline incisional hernia underwent single-port retro-rectal incisional hernia repair. Mean age was $59.0 \pm 12.5$ years with an average BMI of $23.4 \pm 2.7$. All the patients had midline hernia defect with an average of $3.4 \pm 2.2 \mathrm{~cm}$. Mean operation time was $59.6 \pm 30.1 \mathrm{~min}$ and estimate blood loss was $32.6 \pm 36.5 \mathrm{~mL}$. There was no postoperative complication, and $27(90 \%)$ patients were discharged on the day of surgery.

Conclusion: The single-port retrorectal incisional hernia repair is safe and effective while providing good cosmesis to selected patients with incisional hernia.

\section{V126-HERNIA-ADHESIONS-Abdominal wall hernia}

\section{TRANSABDOMINAL APPROACH FOR SUBLAY} RECONSTRUCTION OF VENTRAL HERNIA, LAPAROSCOPIC RIVES TECHNIQUE

M.V. Sosa ${ }^{1}$, M.P. Fernández ${ }^{2}$, A. Senent ${ }^{3}$, I. Alarcón ${ }^{4}$, L. Tallón ${ }^{3}$, J. Tinoco ${ }^{3}$, F.J. Padillo ${ }^{3}$, S. Morales-Conde ${ }^{4}$

${ }^{1}$ Servicio de Cirugía General y Digestiva, Hospital Universitario de Cabueñes, GIJÓN, Spain; ${ }^{2}$ Servicio de Cirugía General y Digestiva, Hospital Álvaro Junqueiro, VIGO, Spain; ${ }^{3}$ Servicio de Cirugía General y Digestiva, Hospital Universitario Virgen del Rocío, SEVILLA, Spain; ${ }^{4}$ Unidad de Innovación en Cirugia Mínimamente Invasiva. Servicio de Cirugía Genera, Hospital Universitario Virgen del Rocío, SEVILLA, Spain

Aims: Closing hernia defect during laparoscopic hernia repair is a vast extended technique nowadays. However, this technique is associated with mesh placemnt intraabdominally in contact by the abdominal content. Nowadays there is a trend to recontruct the midline and to avoid a mesh intraabdominally in those cases suitable for it, as a new step forward of minimally invasive abdominal wall reconstruction. Laparoscopic sublay approach with retromuscular placement of a mesh without mechanical fixation after reconstruction the linea alba migth be considered an option in primary hernias of the midline.

Methods: We present a case of a 47 year old male with an umbilcal hernia of 4 centimeter in diameter associated with rectus diastasis. A laparoscopic approach was performed, using one 12 and two 5 millimeter trocars placed on the left flank. The first step was to open the lateral side of the posterior fascia of the left rectus muscles, dissecting the retromuscular plane until we reach the linea alba getting into the preperitoneal space where the sac was diseected preserving the integrity of the peritoneum. The contralateral posterior fascia was also dissected all the way to the semilunaris line. The midline was closed, including th hernia defect, using a running double loop suture (Maxon-loop ${ }^{\circledR}$ ). A self gripping mesh (Progrip ${ }^{\circledR}$ ) is placed in the retromuscular space in a sublay position ( $21 \mathrm{~cm}$ long, $9 \mathrm{~cm}$ wide). Last, we close the fascia of the left rectus muscle using a barbed suture (V-Loc $\left.{ }^{\circledR}\right)$

Results: Surgical time was $80 \mathrm{~min}$, being discharged of the hospital on postoperative day 1. Pain was controlled with conventional analgesia and no postoperative complications, nor seroma was detected.

Conclusions: Sublay approach for ventral hernia can provide a midline reconstruction, reestablishing abdominal function and avoiding the use of intraabdominal meshes and traumatic fixation, decreasing postoperative complications and pain.

\section{V127-HERNIA-ADHESIONS-Abdominal wall hernia}

LAPAROSCOPIC APPROACH IN A LUMBAR INCISIONAL HERNIA AFTER A POSTERIOR MYELOMENINGOCELE SURGERY. A RARE CASE

J. Gómez Menchero ${ }^{1}$ A. Gila Bohórquez ${ }^{1}$, J.M. Suarez Grau ${ }^{1}$, J. Garcia Moreno $^{1}$, E. Licardie Bolaños ${ }^{2}$, J. Ferrufino Escobar ${ }^{1}$, J. Bellido Luque ${ }^{3}$, I. Alarcon del Agua ${ }^{4}$, S. Morales Conde ${ }^{4}$

${ }^{1}$ General Surgery, Hospital de Riotinto, MINAS DE RIOTINTO, Spain; ${ }^{2}$ Surgery, Hospital Quiron Sagrado Corazon, SEVILLA, Spain; ${ }^{3}$ Surgery, Hospital Virgen Macarena, SEVILLA, Spain; ${ }^{4}$ Surgery, Hospital Virgen del Rocio, SEVILLA, Spain

Aims: Lumbar hernia is one of the rare cases that most surgeons are not exposed to. Hence the diagnosis can be easily missed. This is often related to previous surgery as lumbotomies or primary in the superior lumbar triangle. This leads to delay in the treatment causing increased morbidity. We report a case of adquired lumbar hernia in a middle-aged woman repaired by laparoscopic approach.

Methods: A 60 years old woman with surgical history of a myelomeningocele surgery by posterior approach over 40 years ago, a laparoscopic left nephrectomy 2 years ago with a lef colostomy due to a left colon injury during this procedure. A Hartmann reversal by laparoscopic approach 6 months later. Patient showed a large lumbar mass over $6 \mathrm{cms}$ in the left lumbar region and a large scar near to spinal cord. It was soft in consistency, reducible and expansible on coughing and straining with defined borders. Computerized Tomography showed a large defect in the superior lumbar fascia over $6 \mathrm{cms}$ in the Grynfeltt-Lesshaft triangle with the left colon inside. Results: Patient was placed in a full lateral decubitus position. In order to optimize exposure, a lumbar roll was placed under the lumbar region. A capnoperitoneum $(12-15 \mathrm{mmHg}$ ) was built up. One $11 \mathrm{~mm}$ and two $5 \mathrm{~mm}$ trocars were used and positioned in the left mid axillary line. A 30 optic was used. Adhesions were removed and Toldt fascia was opened in order to expose the hernia defect bounded by quadrates lumborum, erector spinae muscles, 12 rib and serratus. Hernia content was carefully extracted from the sac using a Ligasure Maryland (Covidien Medtronic-USA). Hernia defect was measured and an intraperitoneal mesh (Dinamesh-IPOM FEG Textiltechnik mbH, Aachen, Germany) was positioned and sutured by tackers to the margins included the bone.

Patient was discharged in $48 \mathrm{~h}$ with a low pain rate and without complications.

There is not recurrence in 10 months follow-up.

Conclusion: Laparoscopy might be a safe and feasible approach for repairing lumbar hernias, either primary or adquired, with a low rate of pain and complications 


\section{V128-HERNIA-ADHESIONS-Abdominal wall hernia}

\section{DOWN TO UP LAPAROSCOPIC TRANSVERSUS ABDOMINIS} RELEASE

A. Senent-Boza ${ }^{1}$, I. Alarcón ${ }^{1}$, P. García-Muñoz ${ }^{1}$, N. GarcíaFernández $^{1}$, J. Tinoco ${ }^{2}$, L. Tallón ${ }^{2}$, F.J. Padillo ${ }^{2}$, S. Morales-Conde

${ }^{1}$ Innovation in Minimally Invasive Surgery Unit. General and Digestive Surgery Ser, University Hospital Virgen del Rocio, SEVILLE, Spain; ${ }^{2}$ General and Digestive Surgery Service, University Hospital Virgen del Rocio, SEVILLE, Spain

Aim: Laparoscopic posterior component separation with transversus abdominis release (TAR) allows an anatomo-functional reconstruction of complex abdominal wall defects with lower complication rates than open approach. However, it is a high technical level procedure. We present a modification of the technique to simplify it.

Methods: A 62 -year-old woman with a BMI of $36.73 \mathrm{~kg} / \mathrm{m}^{2}$, with a previous umbilical hernioplasty and three eventroplasties for recurrence of the same, presents with a new symptomatic recurrence with a M2-3W2 defect showed by CT

After pneumoperitoneum is done, three $5 \mathrm{~mm}$ trocars are placed on the left flank. The defect is delimited by drawing it over the skin of the patient with aid of an intramuscular needle and intraabdominal vision. Posterior fascia is opened longitudinally at its medial edge and the retromuscular space is dissected. The Poscrine arcuate line of Douglas and the epigastric vessels are identified. From this point, transversus abdominis fascia is sectioned cranially $1 \mathrm{~cm}$ medial to the semilunar line, preserving the neuro-vascular pedicles that reach the rectus abdominis laterally.

At supraumbilical level, transversus abdominis fibers advance behind rectus abdominis, so they need to be sectioned to access to the space below the ribs. Lateral dissection of this space enables a tensionfree closure at midline.

Once the procedure is repeated on the contralateral side using two $5 \mathrm{~mm}$ and one $12 \mathrm{~mm}$ trocars on the right flank, a continuous suture of the posterior fascia is performed with a barbed suture. The anterior fascia is closed with a slowly-absorbable monofilament loop-type suture.

Finally, a double-layer polypropylene mesh is placed at retromuscular level without any suture and fibrin glue is applied.

Results: The patient was discharged 24 hous after surgery. No recurrence has been presented to the moment.

Conclusions: The section of the aponeurotic plane from the arcuate line of Douglas enables a more accurate dissection of the retrotransversus plane without sectioning its fibers except for its cranial end, preserving the innervation and vascularization of the abdominal wall.

This technical modification aims to simplify a complex laparoscopic procedure allowing its estandarization

\section{V129-HERNIA-ADHESIONS-Abdominal wall hernia}

\section{TRANSABDOMINAL LAPAROSCOPIC TRANSVERSE} ABDOMINI RELEASE AS A TENSION-RELEASE MANEUVER FOR GAP CLOSURE DURING A LAPAROSCOPIC IPOM VENTRAL HERNIA REPAIR

F. del Castillo Diez ${ }^{1}$, C. Durán Escribano ${ }^{2}$, L. García-Sancho Téllez ${ }^{3}$, J.A. Gonzalez Sanchez ${ }^{1}$, J. Díaz Dominguez ${ }^{1}$

${ }^{1}$ General Surgery, Hospital Universitario La Paz, MADRID, Spain; ${ }^{2}$ General Surgery, Hospital Quirón La Luz, MADRID, Spain; ${ }^{3}$ General Surgery, Hospital Universitario Infanta Sofia, MADRID, Spain

Aims: The authors present a video with their standardized laparoscopic ventral hernia intraperitoneal mesh (IPOM) hernioplasty procedure but introducing a novel laparoscopic technique for tension releasing while hernia gap closure and midline anatomical restoration.

Methods: A 64 years old male patient with a BMI 31 presents a symptomatic ventral hernia recur-

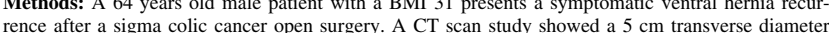
midline ventral hernia. A Laparoscopic IPOM hernia repair procedure is performed using $5 \mathrm{~mm}$ instruments and a $10 \mathrm{~mm}$ camera. When checking tension while midline restoration suturing, we instruments and a $10 \mathrm{~mm}$ camera. When checking tension while midline restoration suturing, we
decide to add a tension-releasing maneuver: A totally laparoscopic transverse abdomini muscle release decide to add a tension-releasing maneuver: A totally laparoscopic transverse abdomini muscle release
(TALTAR). This maneuver allow right rectus posterior sheath to advance some distance to the (TALTAR). This maneuver allow right rectus posterior sheath to advance some distance to the mesh is now placed and fixed. Case and technical details are shown in the video.

Results: The patient was discharged from hospital within a period of $5 \mathrm{~h}$ with a 4 rate in a EVA acute pain visual scale. In a 2 year follow-up, there has no been an anatomical or clinical recurrence. No chronic pain, anatomical recurrence, lateral asymmetry, umbilical or abdominal wall complications have been reported with this technique.

Conclusions: Depending on the patient characteristics, anatomical hernia factors and surgeon mini invasive experience, a TALTAR maneuver could be a safe and feasible option for releasing tension when midline anatomical laparoscopic closure. More studies are needed in order to standardized this approach.
V130-HERNIA-ADHESIONS-Abdominal wall hernia

SIMULTANEOUS LAPAROSCOPIC REPAIR OF A PARASTOMAL, MIDLINE INCISIONAL AND PERINEAL HERNIA

G.P. Protti, R. Villalobos, Y. Maestre, M.C. Mias, A. Escartin, J.J. Olsina

General Surgery, Arnau de Vilanova Hospital, LLEIDA, Spain

Aims: To present the case of a multioperated patient with 3 simultaneous hernias, that we managed to repair laparoscopically

Methods: A 45 year old male with a 29.4 BMI, hypertension and several past surgeries (Miles procedure, distal pancreatectomy with splenectomy and 2 hepatic tumorectomies), arrived at our outpatient clinic with 3 simultaneous hernias. The patient had a M2W1 incisional hernia, a type IV parastomal hernia and a perineal hernia. After discussing the different alternatives with the patient, a laparoscopic approach was selected, even though he had a complex surgical history.Under general anesthesia and with the help of three trocars, we first repaired the parastomal hernia, liberating it adhesions and reducing its contents. We placed an intraperitoneal $15 \mathrm{~cm} \times 20 \mathrm{~cm}$ composite mesh (Dynamesh) with the key-hole technique, fixed with absorbable tackers. We then proceeded to repair the midline incisional hernia with another intraperitoneal $20 \mathrm{~cm} \mathrm{x} 30 \mathrm{~cm}$ Dynamesh, fixed with absorbable tackers. This second mesh was simultaneously placed on the colostomy, on top of the previous mesh, in a Sugar the doble mesh parastomal repair technique. (5) with both absorbable tackers to the abdominal wall and Cooper ligaments and absorbable sutures to the peritoneum surrounding the iliac vessels. No intraoperative complications occurred.The patient had an uneventful postoperative recuperation, except for a postoperative ileus which resolved with medica treatment. He left the hospital after 12 days.

Results: 1 year after the surgery, he's had no clinical or radiological evidence of recurrence. He remains asymptomatic.

Conclusions: Patients with complex surgical histories can sometimes be operated laparoscopicaly.The simultaneous repair of multiple abdominal wall hernias or even perineal hernias is feasable and should be performed whenever possible.

\section{V131-HERNIA-ADHESIONS-Abdominal wall hernia}

TOTALLY ENDOSCOPIC EXTRAPERITONEAL TECHNIQUE FOR MIDLINE HERNIAS ASSOCIATED WITH DIASTASIS RECTI

J. Bellido Luque ${ }^{1}$, B. Bascuas Rodrigo ${ }^{1}$, A. Bellido ${ }^{2}$, J. Gomez ${ }^{2}$, J.M. Suarez ${ }^{2}$, I. Sanchez-Matamoros Martin ${ }^{1}$, A. Nogales Muñoz ${ }^{1}$, F. Oliva Mompean ${ }^{1}$

${ }^{1}$ General Surgery, SAS, SEVILLA, Spain; ${ }^{2}$ General Surgery, Quiron Salud, SEVILLA, Spain

Aims: When primary ventral hernia and simultaneous diastasis recti are diagnosed, there is no consensu among the international surgical community on the surgical treatment regarding indications or surgica technique. However, if diastasis recti is symptomatic of or is associated with midline hernias, the corrective surgery of both pathologies at the same time could be the most recommended option. When we only correct the herniary defect, we risk performing a reparation on an anatomically weak tissue, so the rate of hernia recurrence may increase. We propose a minimally invasive access using totally endoscopic retromuscula hernioplasty. By developing this technique, several advantages are provided, such as no peritoneal opening without intraabdominal access, no mesh fixation needed and simultaneous solving of both pathologies. Method: We present the case of a 50-year-old man, with BMI $35 \mathrm{~kg} / \mathrm{m}^{2}$ and no previous medical history cocical history with a $5 \mathrm{~cm}$ size supraumbilical diastasis recti associated. Full endoscopy retromuscular hernioplasty was with a $5 \mathrm{~cm}$ size supraumbilical diastasis recti associated. Full endoscopy retromuscular hernioplasty was
proposed. A $2 \mathrm{~cm}$ size incision was made in left hypocondrium, openned the anterior rectus sheath and proposed. A $2 \mathrm{~cm}$ size incision was made in left hypocondrium, openned the anterior rectus sheath and
retracted the rectus muscle. We placed a balloon trocar and open the homolateral retromuscular space after retracted the rectus muscle. We placed a balloon trocar and open the homolateral retromuscular space after
placing two $5 \mathrm{~mm}$ trocars in left lumbar space and epigastric position. We crossed-over the linea alba and placing two $5 \mathrm{~mm}$ trocars in left lumbar space and epigastric position. We crossed-over the linea alba and achieve contralateral retromuscular space. After this step, the hernia sac was reduced and we extended the dissection $5 \mathrm{~cm}$ caudal to the hernia ring. Both medial posterior rectus sheaths were sutured with running barbed suture $\mathrm{n}^{\circ} 0$ and a $20 \times 20 \mathrm{~cm}$ size light-weight, big pore, polipropilene mesh was placed in retromuscular space and unrolled properly with enough overlap. A drain was placed and the anterior rectus sheath incision was closed.

Results: The patient was discharged $24 \mathrm{~h}$ after surgery without remarkable events during his postoperative stay. He has been followed up for 8 months remaining asymptomatic.

Conclusions: Totally endoscopic retromuscular ventral hernia repair in men with umbilical hernia and diastasis recti associated, is feasible and reproducible procedure with several advantages compared to traditional laparoscopic IPOM in terms of pain and mesh position. 


\section{V132-HERNIA-ADHESIONS-Abdominal wall hernia}

PARASTOMAL AND MIDLINE ASSOCIATED HERNIAS: ADVANTAGES OF MINIMALLY INVASIVE SURGERY

B. Bascuas Rodrigo ${ }^{1}$, J. Bellido Luque ${ }^{1}$, C. Dominguez Sanchez ${ }^{1}$, A. Bellido ${ }^{2}$, J. Gomez ${ }^{2}$, J.M. Suarez ${ }^{2}$, I. Sanchez-Matamoros Martin ${ }^{1}$, A. Nogales Muñoz ${ }^{1}$, F. Oliva Mompean ${ }^{1}$

${ }^{1}$ General Surgery, SAS, SEVILLA, Spain; ${ }^{2}$ General Surgery, Quiron Salud, SEVILLA, Spain

Aims: Parastomal hernia (PH) is one of the most frequent long-term complications of stoma formation, occurring in 35\%-50\% of patients. Surgical treatment for parastomal hernia is the only cure but a fairly difficult field with a recurrence rate ranging from $24 \%$ to $54 \%$ of cases. Due to its advantages, the number of laparoscopic mesh repairs for parastomal hernia has gradually increased over the past decade. According to this common complication, we report a case of laparoscopic reparation of $\mathrm{PH}$ using the Sugarbaker technique.

Method: We present the case of a 65-year-old patient with surgical antecedent of laparoscopic low anterior resection due to rectal cancer, presenting in postoperative period an anastomosis leakage with severe peritonitis was identified and a laparotomy with end colostomy was performed. The postoperative course was uneventful. During the follow-up the patient showed a 6 centimetres size paraestomal hernia, being a M3W2 incisional hernia confirmed with CT scan.The patient underwent full laparoscopic hernia repair, performing a Sugarbaker technique, exposing parastomal hernia completely to measure the hernia ring size ( 6 centimetres) and the midline associated defect (5 centimetres). A $26 \times 36 \mathrm{~cm}$ size PTFE-c was selected to allow a 5-cm overlap over two defects.

Results: Using this approach, the bowel loop was pushed into the abdominal wall and appropriate place between the mesh edge and the abdominal wall is left to allow the bowel loop to pass through. Postoperatory course was uneventful and the patient was discharged $48 \mathrm{~h}$ after surgery without any remarkable event during his postoperative stay. He has been followed up for 18 months without realizing any clinical signs or alterations in CT scan.

Conclusions: Compared with traditional open surgical repairs, laparoscopic repair has certain advantages including its safe operation, postoperative rapid recovery, fewer complications, and lower recurrence rate. However, it still faces challenges regarding parastomal hernia treatment, and there is a need to improve existing surgical techniques.

V133-HERNIA-ADHESIONS-Abdominal wall hernia

\section{LAPAROSCOPIC VENTRAL HERNIA REPAIR WITH TRANSABDOMINAL RETROMUSCULAR MESH PLACEMENT WITHOUT TRAUMATIC FIXATION}

J. Bellido Luque, B. Bascuas Rodrigo, C. Dominguez Sanchez, I. Sanchez-Matamoros Martin, A. Nogales Muñoz, F. Oliva Mompean

\section{General Surgery, SAS, SEVILLA, Spain}

Aims: Nowadays, the principal disadvantages of laparoscopic approach in hernia repair are the use of intraabdominal meshes and traumatic fixation. First, intraabdominal meshes involve the contact of the prosthesis with the intestinal loops with the consequent risk of adhesion and fistula. Also, using helicoidal sutures in prosthetic fixation produces adhesions to the tackers and a non-negligible incidence of chronic pain. When it comes to lead to better results, placing the mesh in retromuscular space avoids the drawback of contact with the loops, and using self-fixation meshes may decrease the rate of acute and chronic pain. Accordind to this facts, we present a case of laparoscopic ventral hernia repair with transabdominal retromuscular mesh placement without traumatic fixation.

Methods: We present a 50-year-old patient with a $7 \mathrm{~cm}$ diameter hernia showed in preoperative CT scan, M3W2, with diastasis recti associated. The patient underwent laparoscopic surgery using transabdominal retromuscular route. One $11 \mathrm{~mm}$ and two $5 \mathrm{~mm}$ trocar were placed in left flank. The posterior rectus sheath on the left side is opened starting $5 \mathrm{cms}$ far from the left egde of the defect. Once the retromuscular space is dissected, the hernia ring is dissected and the hernia sac reduced, we continue with the dissection in retromuscular space on the side. Craniocaudal dissection is achieved $5 \mathrm{~cm}$ distal to the defect margins. The hernia defect with the anterior rectus sheath and the diastasis recti were closed using v-loc running suture. Self-adhesive mesh was subsequently placed. The mesh should be overlap $5 \mathrm{~cm}$ from the margins of the defect, covering the defect widely, with grips facing upwards. Finally, we closed the posterior rectus sheath with peritoneum on the left side with $\mathrm{v}$-loc running suture.

Results: The postoperative course was uneventful and the patient was discharged $24 \mathrm{~h}$ after the surgery. After 18 months of follow-up no clinical or radiological recurrence was showed. Conclusions: The combination of laparoscopic approach, retromuscular mesh placement and the use of self-fixation meshes, seems to be an actual useful solution, combining the advantages of each item and avoiding the use of intraabdominal meshes and helicoidal sutures.

\section{V134-HERNIA-ADHESIONS-Abdominal wall hernia}

ROBOTIC VENTRAL HERNIA REPAIR. TECHNICAL ASPECTS OF THE RIVES-STOPPA TECHNIQUE

F. Pulighe ${ }^{1}$, M. Podda ${ }^{1}$, D. Delogu ${ }^{2}$, F. Balestra ${ }^{1}$, M. Anania $^{1}$, $\overline{\text { M. Pazzona }}^{1}$, A. Cruccu ${ }^{1}$, G.P. Gusai ${ }^{1}$, M.L. Murru ${ }^{1}$, C. Massaiu ${ }^{1}$, C. de Nisco ${ }^{1}$

${ }^{1}$ General and Robotic Surgery, ATS Sardegna ASSL Nuoro, NUORO, Italy; ${ }^{2}$ General Surgery, ATS Sardegna ASSL Lanusei, LANUSEI, Italy

Aims: Laparoscopic ventral hernia repair has clear advantages over open repair, including less post-operative pain and earlier return to normal activity. However, a prolonged surgeon learning curve is necessary to perform this technique effectively. Robot assistance may improve outcomes of minimally invasive ventral hernia repair with improved three-dimensional visualization and enhanced dexterity with articulating instrumentation. We report a case of robotic Rives-Stoppa epigastric hernia repair in order to demonstrate the feasibility of the robotic approach.

Methods \& Results: A 58-year-old man came to our attention for the presence of a palpable mass in the epigastric region. The abdominal CT scan showed the presence of an epigastric hernia with herniation of omental content, and the presence of diastasis recti. The patient was then submitted to a Rives-Stoppa Robotic hernia repair under general anesthesia. The da Vinci-Si Surgical System (Intuitive Surgical Inc., Sunnyvale, CA, USA) was brought into position over the head of the patient and docked after placement of the ports. Three trocars were placed in the hypogastric region along the transtubercular line. A fourth trocar was placed in the left iliac fossa and used by the assistant. The operation started with an extended adhesiolysis and hernia reduction. Then, the retromuscolar dissection began by incising the posterior sheath starting from $4 \mathrm{~cm}$ above the pubic symphysis. An extended dissection of the Rives space was performed to create a correct housing for the mesh. The hernia defect and the diastasis recti were closed using a 1-0 absorbable barbed suture. A Phasix $\mathrm{ST}^{\mathrm{TM}}$ mesh (Bard Inc./Davol Inc., Warwick, RI) was positioned in the retromuscular plane, and was anchored with absorbable sutures and glue. The midline incision was closed using a 2-0 absorbable barbed suture. The operative time was 250 minute. The postoperative period was uneventful, and the patient was discharged home on the second post-operative day.

Conclusions: Robotic Rives-Stoppa ventral hernia repair is feasible, safe, and effective when a standardized approach is performed. Whether robotics may improve the outcomes of minimally invasive ventral hernia repairs, including lower recurrence rates, decreased post-operative pain, or shorter surgeons' learning curve, will require careful prospective investigation.

\section{V135-HERNIA-ADHESIONS—Abdominal wall hernia}

\section{LEFT BOCHDALECK HERNIA MINI INSTRUMENTS THORACOSCOPIC VATS REPAIR}

F. del Castillo Diez ${ }^{1}$, L. García-Sancho Téllez ${ }^{2}$, J.D. Sanchez Lopez ${ }^{3}$, E. Ferrero Celemin ${ }^{3}$, J.A. Gonzalez Sanchez ${ }^{1}$, J. Díaz Dominguez ${ }^{1}$

${ }^{1}$ General Surgery, Hospital Universitario La Paz, MADRID, Spain; ${ }^{2}$ General Surgery, Hospital Universitario Infanta Sofia, MADRID, Spain; ${ }^{3}$ Surgery, Hospital Universitario Infanta Sofia, MADRID, Spain

Aims: The authors present a video with a left chronic Bochdaleck hernia classical hernioplasty repair but performing a mini invasive thoracoscopic approach and $3 \mathrm{~mm}$ instruments.

Methods: A 73 years old female patient come to hospital due to chronic left dorsolumbar pain A CT scan study showed a chronic left diaphragmatic Bochdaleck hernia. A Lateral righ decubitus thoracoscopic repair is performed using $3 \mathrm{~mm}$ instruments and a $5 \mathrm{~mm}$ camera. Case and technical details are shown in the video.

Results: The patient was discharged from hospital within a period of $48 \mathrm{~h}$ with no pain and a clean chest X-ray. In a 2 year time follow-up, not an anatomical or clinical recurrence has been reported. Neither chronic pain or respiratory complications happened, with in this period of time. Conclusions: Depending on the patient characteristics, anatomical factors and surgeon mini invasive experience, left Bochdaleck hernia mini invasive thoracoscopic hernioplasty repair using $3 \mathrm{~mm}$ instruments could be a safe and feasible option. More studies are needed in order to standardized this approach. 


\section{V136-HERNIA-ADHESIONS-Abdominal wall hernia}

ONLY GLUE MESH FIXATION FOR LVIHR: PRELIMINARY EXPERIENCE

\section{R. Villalobos, Y. Maestre, G.P. Protti, M.C. Mias, J.J. Olsina}

General Surgery, Arnau de Vilanova University Hospital, LLEIDA, Spain

Abdominal wall surgery has expanded exponentially in the last decade. Many techniques have been developed, mainly in minimally invasive surgery. Laparoscopic ventral and incisional hernia repair (LVIHR) has become a common procedure because of its feasibility and safety but unfortunately, it is not free of complications. Chronic postoperative pain and bleeding are frequent complications, prolonging hospital stay and altering quality of life of the patients. Absorbable or non-absorbable tacks are the usual method of mesh fixation and sometimes combined with transfascial sutures to secure the mesh. These 2 mechanical fixations pierce the abdominal wall causing nerve or vessel injuries. Some studies showed no differences between absorbable tacks, non-absorbable tacks or transfascial sutures concerning postoperative remarkably high pain. Some authors consider that a non-penetrating fixation of the mesh getting an effective mesh-abdominal wall interface will reduce significantly the postoperative pain after a laparoscopic ventral hernia repair. Tissue glues are used in different medical treatments and also have been used successfully for extra peritoneal mesh fixation in laparoscopic inguinal hernia repair, open ventral hernia repair but not so in laparoscopic ventral hernia repair in spite of good results published in the literature. Cyanoacrylate and its derivatives are 'synthetic glues' and classified as medical devices with stronger adherent properties than fibrin glues. Experimental studies have reported good results compared with suture fixationand also tissue toxicity doesn't lead to an increased foreign body reaction. Some authors have studied the use of cyanoacrylate in laparoscopic inguinal hernia repair but unfortunately, clinical trial reports in ventral and incisional hernia repair were not found in the literature because the lacking of experimental studies that guarantee the safety of intra-abdominal mesh fixation and the interaction of the glue with the intra-abdominal tissue. Our group developed an experimental study demonstrating the feasibility, safety and effectiveness of the cyanoacrylate using for intraperitoneal mesh fixation and after this conclusion, started a clinical study. This video shows the methodology for laparoscopic mesh fixation with only glue in our first cases.

\section{V137-HERNIA-ADHESIONS-Abdominal wall hernia}

\section{EPIGASTRIC ENDOSCOPIC PRE-PERITONEAL HERNIA REPAIR}

\section{H. Cardoso-Louro, S. Graça, L. Lencastre, A. Fonte-Boa, J. Vilaça}

General Surgery, Hospital da Luz Arrábida, PORTO, Portugal

Aims: Small epigastric hernias, associated or not with the rectus abdominis diastasis, and small umbilical hernias are common in middle-aged women, particularly with past history of pregnancy. The aim of this video is to illustrate a new extraperitoneal approach to these clinical situations.

Methods: Patients between the ages of 40 and 60 years old, with epigastric hernia orifice up to $2 \mathrm{~cm}$, with or without associated umbilical hernia (up to $2 \mathrm{~cm}$ ), were chosen for this procedure. The surgery begins with a vertical umbilical incision for the umbilical hernia's correction, and dissection of the pre-aponeurotic plane. Two $3 \mathrm{~mm}$ trocars (mini-laparoscopy instruments) are introduced at both flanks to enlarge the pre-aponeurotic plane towards the xiphoid appendix. In this way epigastric hernial defects are isolated. The surgery proceeds with defect suturing with braded suture, midline invagination and mesh placement if necessary.

Results: All patients had an eventful post-operative period and were discharged home at postoperative day 1.

The aesthetic and functional results are optimal

Conclusion: For selected cases with high aesthetic motivation this technique seems to be feasible and with optimal cosmetic results. This technique allows the mesh placement both in-lay and onlay, protecting it from surgical site infections often present at the classical approac

\section{V138-HERNIA-ADHESIONS-Abdominal wall hernia}

\section{LAPAROSCOPIC REPAIR OF BOCHDALEK HERNIA}

D. Froylich ${ }^{1}$, F. Darawshi' ${ }^{2}$, D. Levi-Faber ${ }^{2}$, R. Galili ${ }^{2}$, D. Hazzan ${ }^{3}$, R. Hadad $^{4}$, E. Sharoni ${ }^{2}$

${ }^{1}$ Surgery B, Carmel Medical Center, HAIFA, Israel; ${ }^{2}$ Cardiothoracic Surgery, Carmel Medical Center, HAIFA, Israel; ${ }^{3}$ General Surgery, Carmel Medical Center, HAIFA, Israel

Aims: Bochdalek hernia is a rare entity in adults. Fewer than 200 have been reported in medica literature, the majority of which were incidentally diagnosed. As such, the optimal repair of a symptomatic hernia is unknown. We present a case of adult Bochdalek hernia repair.

Methods: A 30-year-old obese male patient with a 2 years of chronic dry cough and left lung opacity in chest $\mathrm{x}$-ray. A large posterior and lateral Bochdalek hernia with herniation of intestinal loops and fat to the left hemithorax was seen in chest and upper abdominal CT scan. The hernia extended to mid-thorax, caused significant atelectasis of left lung. Eighteen months later, due to appearance of chest and abdominal pain following a recent motor vehicle accident, a repeat chest CT was done and a slight enlargement of the hernia was shown.

Results: The patient was operated laparoscopically, positioned in a semi-right lateral decubitus with double lung intubation. A large left posterior and lateral diaphragmatic hernia which contained transverse and descending colon with omental fat was seen. They were pulled in to the intraperitoneal space carefully. The defect was measured to be $10 * 8 \mathrm{~cm}$. It was reduced to $7 * 7 \mathrm{~cm}$ by suturing with a non-absorbable $0 \mathrm{~V}$-Loc suture . Advancing the camera to the thoracic cavity showed the left lung to be severely atelectatic. After selective recruitment lung was well expanded. A Symbotex Composite $20 \times 25 \mathrm{~cm}$ mesh was fixed to the defect area by suturs and laparoscopic tacker. The operation and post-operative course were uneventful. Chest $\mathrm{x}$-ray demonstrated the bowel below the diaphragm. The patient was discharged on POD 3. At 8-month follow-up, chest X-ray was normal.

Conclusion: Adult Bochdalek hernia can be safely treated laparoscopically with mesh fixation.

\section{V139-HERNIA-ADHESIONS-Abdominal wall hernia}

PARASTOMAL HERNIA REPAIR: STANDARDIZED SUGARBAKER TECHNIQUE WITH MESH OF POLYPROPYLENE/PVDF

A.F. Aranzana Gómez, J. Malo Corral, J. Hernandez Gutierrez, B. Muñoz Jimenez, A. Muñoz Tébar, A. Trinidad Borras, J. de Pedro Conal

\section{General Surgery, Complejo Hospitalario Toledo, TOLEDO, Spain}

Objective: To demonstrate the safety and efficacy of the standardized laparoscopic approach in the treatment of large parastomal hernia. Currently, this approach is recognized as the one of choice in parastomal hernia pathology, being controversial which is the best technique of choice: Keyhole vs SugarBaker.

Material and method: Clinical case: A 76-year-old woman with a history of laparoscopic abdominoperineal amputation due to rectal neoplasia (pT2N0), a year ago, with symptomatic parastomal hernia with incarceration episodes and inflamation changes in the stomal orifice.Tac: large hernia parastomal with intestinal content inside. Surgical treatment is decided.

Result Intervention: Complete laparoscopic approach, right lateral partial decubitus, 4 trocars, dissection of the hernia defect and reduction of the content, partial mobilization of the pre-stomal colon, with bleeding at the level of the vascular origin, requiring careful hemostasis to avoid ischemia of the colostomy, herniorrhaphy with stitches with extracorporeal knotting, placemen of POLYPROPYLENE/PVDF mesh,fixed with irreabsorbable tackers with administration of biological glue at the edges of the mesh. Correct postoperative, discharge at the 3rd day. Asymptomatic and without hernia recurrence at one year of follow-up.

Conclusions: The technique of SugarBaker using a laparoscopic approach is a safe and effective alternative in the treatment of parstomal hernias. 


\section{V141-HERNIA-ADHESIONS-Adhesions}

LAPAROSCOPIC POSTERIOR COMPONENT SEPARATION IN COMPLEX INCISIONAL HERNIAS. ADVANTAGES OF MINIMALLY INVASIVE MIDLINE ABDOMINAL WALL RECONSTRUCTION

J. Bellido Luque ${ }^{1}$, I. Sanchez-Matamoros Martin ${ }^{1}$, B. Bascuas Rodrigo $^{1}$, A. Tejada ${ }^{2}$, C. Dominguez Sanchez ${ }^{1}$, A. Bellido ${ }^{2}$, I. Cornejo $^{3}$, F. Oliva Mompean ${ }^{1}$, A. Nogales Muñoz

${ }^{1}$ General Surgery, SAS, SEVILLA, Spain; ${ }^{2}$ Surgery Department, Quiron Salud, SEVILLA, Spain; ${ }^{3}$ Surgery Department, Hospital Universitario Virgen Macarena, SEVILLA, Spain

Objetives: laparoscopic ventral hernia repair provides advantages in term of low infection rates and postoperatory stay when is compared with open repair. Trends in laparoscopic abdominal wall surgery is to complete defect closure without tension in midline. Closing the defect in ventral hernias wider than $8-9 \mathrm{cms}$ creates high tension in midline and postoperatory pain. It's proposed different techniques to solve this drawback. Laparoscopic posterior component separation makes the defects closure easier with no tension and placing the mesh extraperitoneally.

Methods: 65 years old woman with previous total hysterectomy, a M3M4W3 midline incisional hernia was clinically diagnosed and confirmed with CT scan. Full laparoscopic abdominal wall repair with defect closure wa 列 side in the defect margin is freed. Once the lateral edge of the rectus sheath is reached, the posterior rectus sheath is incised, dividing the posterior aponeurotic sheath of the internal oblique muscle. This allows access to the plane between the internal oblique and the transversus abdominis muscles. It's is made the same steps in the left side with 3 trocar on the right flank.

The posterior rectus sheath both side is reapproximated in the midline and $20 \times 20 \mathrm{cms}$ Polipropilene mesh is placed and unfolded properly. It's fixed using cyanocrilate glue.

One drain is left in retromuscular position and $10 \mathrm{~mm}$ trocar wounds are sutured.

Results: Postoperatory course was uneventful. Hospital stay $24 \mathrm{~h}$. the drain was removed in day 3 after surgery. After 9 months follow-up no complication or recurrence were identified.

enent separation is an Conclusions: laparoscopic abent separation is an alternative to open procedure, providing no tension in midh pain when is compared with traditional IPOM.

\section{V142-HERNIA-ADHESIONS-Emergency surgery}

THE ROLE OF LAPAROSCOPIC SURGERY FOR DIAGNOSIS AND TREATMENT OF ACUTE ABDOMEN

\section{Hussein}

Surgery, American University of Beirut Medical Center, BEIRUT, Lebanon

Aims: The role of Laparoscopic surgery for diagnosis and treatment of Acute Abdomen Methods: This video will show the evidence of gangrenous jejunal segment due to superior mesenteric vein thrombosis in a patient with history of breast $\mathrm{CA}$ on hormonal treatment.In this video, the gangrenous segment was resected and primary anastomosis was done using EndoGIA $60 \mathrm{~mm}$. Results: A second look after $48 \mathrm{~h}$ revealed to be negative for any further ischemic bowel.

\section{V143-HERNIA-ADHESIONS-Emergency surgery}

\section{LAPAROSCOPIC REMOVAL OF BEZOAR POST LAPAROSCOPIC ROUX EN Y BYPASS}

\section{Hussein}

Surgery, American University of Beirut Medical Center, BEIRUT, Lebanon

Aims: Laparoscopic Removal of Bezoar post Laparoscopic Roux En Y Bypass.

Methods: The incidence of Bezoar post Laparoscopic Roux En Y Bypass is increased due to wide jejunal jejunal anastomosis that results in complete intestinal obstruction.The video will show the steps used to explore the abdomen and identification of the jejunal jejunal defect and Peterson defect and the removal of the Bezoar by Enterotomy then suturing of the small intestine.

Conclusion: Laparoscopic surgery is diagnostic and theraputic in Acute Abdomen.

\section{V144-HERNIA-ADHESIONS-Emergency surgery}

URGENT LAPAROSCOPIC APPROACH OF GASTRIC PSEUDOVOLVULATION IN THE MEDIASTINUM IN A PATIENT WITH GIANT HIATUS HERNIA

A. Gila Bohórquez, J. Gómez Menchero, J.M.

Suárez Grau, Surgery, Hospital General De Riotinto, HUELVA, Spain

Introduction: Gastric pseudo-volvulation is a rare entity of paraesophageal hernia that is characterized by migration of the stomach into the posterior mediastinum. This clinical-radiological picture has severe complications so in certain cases should be operated urgently. Another small group of patients are asymptomatic, although the current literature recommends their regulated surgical intervention We present a gastric pseudo-volvulation in the mediastinum, with a laparoscopic approach, showing that by systematizing the surgery, it is possible to perform this type of intervention with relative ease and safety

Material and methods: We present a video of an urgent laparoscopic approach in a female patient of 80 years with a personal history of hypertension, smoking and dyslipidemia. With a hiatus hernia diagnosed more than ten years ago. He went to the emergency department due to significant symptoms of heartburn and reflux, as well as incoerctable vomiting and difficulty feeding one week of evolution. A simple abdomen and postero-anterior chest radiograph was performed, showing a paraesophageal A simple abdomen and postero-anterior chest radiograph was performed, showing a paraesophageal
hiatus hernia with almost the entire stomach included in the mediastinum. A thoraco-abdominal axial hiatus hernia with almost the entire stomach included in the mediastinum. A thoraco-abdominal axial
tomography corroborated giant hiatus hernia with pseudovolvulation and incarceration data. Urgent tomography corroborated giant hiatus hernia with pseudovolvulation and incarceration data. Urgent
intervention was decided by laparoscopic approach in which hiatus hernia reduction and esophageal intervention was decided by laparoscopic approach in which hiatus hernia reduction and esophageal abdominalization were performed. Closure of pillars and reinforcement with bioabsorbable mesh. Gastric and gastropexy Toupet of anterior face to anterior peritoneum of abdominal wall. Results: The patient had a post-operative $48 \mathrm{~h}$ without incident, discharged with a crushed diet. The follow-up and evolution has been acceptable without notable complications.

Conclusion: The laparoscopic approach, in extreme cases of paraesophageal hiatus hernia with incarceration of the stomach and pseudovolvulation of it, is a correct, safe and effective alternative in experienced groups. 


\section{V145-HERNIA-ADHESIONS-Emergency surgery INCARCERATED HIATAL HERNIA-CASE REPORT}

\section{P. Rymkiewicz, M. Zdrojewski}

General, Minimally Invasive and Elderly Surgery, Municipal Hospital, OLSZTYN, Poland

Case report of incarcerated hiatal hernia. 30 years old female was admited to the hospital due to severe chest pain and vomiting for about six $\mathrm{h}$. Physical examination and lab test showed no abberations. Chest Xray revaled incarcerated stomach above the diaphragm. She was rushed to the OR. Laporoscopic approach was used, the stomach was removed from the chest and Nissen fundoplication was performed. Day after surgery patient was asymptomatic, got full oral diet. She was discharged on postoperative day two, without a need of any analgetics. Gastroduodenoscopy was performed 6 weeks after surgery and showed proper image of oesophagus, stomach and duodenum, neither signs of hiatal hernia nor inflamation were present. Laparoscopic approach is good way to treat incarcerated hiatal hernias and is related with shorter lenght of stay, lesser postoperative pain and better patient comfort. And it should be procedure of choice in this kind of cases.

\section{V147-HERNIA-ADHESIONS-Emergency surgery}

LAPAROSCOPIC FOREIGN BODY EXTRACTION: UNUSUAL MIGRATION OF EXTRA-ABDOMINAL METALLIC GUIDEWIRE

S. Alonso, A.M. Rodríguez, T. Ramos, M. Rodríguez, T. Rubio, J.E. Sánchez, M. Domínguez, E. Diego, J. López, CR. Díaz,

L. Muñoz-Bellvís

General Surgery, Complejo Asistencial Universitario de Salamanca, SALAMANCA, Spain

Introduction: Foreign bodies can enter inside the human body by different mechanisms such as ingestion, aspiration, trauma or in some cases due to medical procedures. They are potentially life-threatening events, the diagnosis could be challenging and its management depends on their location.

Case report: A 64-year-old male was referred to our hospital due to chronic abdominal pain. $\mathrm{He}$ had cholelithiasis, medical history of acute pericarditis and past surgical history of left adrenalectomy, left nephrectomy, distal pancreatectomy and colon resection due to an adrenal adenocarcinoma (stage T4NOM0).Abdominal radiograph showed a foreign body in the left lower quadrant of the abdomen, as an incidental finding. This was not detected in CT scans during ten years of oncology follow-up. CT scan revealed an extraintestinal metallic curved object in the right lower quadrant. This finding was not related to any surgical intervention or trauma. Diagnostic laparoscopy was performed: the foreign body seemed to be a guidewire, it was included into the omentum and almost stuck to the abdominal wall. The guidewire was reached and carefully extracted through a $10 \mathrm{~mm}$ trocar without any evidence of intra-abdominal organ injury. Then an elective cholecystectomy was also performed due to his medical history of symptomatic cholelithiasis.The procedure lasted $60 \mathrm{~min}$. The hospital discharge was on the third postoperative day and no complication was registered.

Conclusion: Is extremely rare to discover a guidewire that had migrated into the peritoneal space without abdominal injuries.This case report demonstrates the technical feasibility, safety and minimal postoperative morbidity associated with minimal invasive laparoscopic removal.

\section{V148-HERNIA-ADHESIONS-Inguinal hernia}

SELF-GRIPPING MESH LAPAROSCOPIC BILATERAL GROIN HERNIA TAP REPAIR PROCEDURE USING 3 MM MINI INSTRUMENTS IN A DAY-HOSPITAL PROGRAM: A NEW STANDARD?

F. del Castillo Diez, C. Valiño Fernández, F. Atahualpa Arenas, J.T. Castell Gomez, C. Duran Escribano

Surgery, Hospital Quiron La Luz, MADRID, Spain

Aims: The authors present a video with their standardized laparoscopic groin hernia transabdominal preperitoneal hernioplasty (TAP) procedure but using $3 \mathrm{~mm}$ instruments and $5 \mathrm{~mm}$ camera approach.

Methods: A 45 years old male patient with a BMI 30 presents a symptomatic bilateral groin hernia for 5 months. US study showed an indirect bilateral inguinal hernia. A Laparoscopic TAP hernia repair procedure is performed using $3 \mathrm{~mm}$ instruments and a $5 \mathrm{~mm}$ camera. A selfgripping mesh preperitoneal hernioplasty and peritoneal flap barbed-sutured hermetic closure was performed. Case and technical details are shown in the video.

Results: The patient was discharged from hospital within a period of $4 \mathrm{~h}$ with a 2 rate in a EVA acute pain visual scale. In a 2 year follow-up, there has no been an anatomical or clinical recurrence. No chronic pain, anatomical recurrence, umbilical or abdominal wall complications have been reported with in this period of time.

Conclusions: Depending on the patient characteristics, anatomical factors and surgeon mini invasive experience, a laparoscopic bilateral hernia repair using $3 \mathrm{~mm}$ instruments, could be a safe and feasible option. More studies are needed in order to standardized this approach. 


\section{V149-HERNIA-ADHESIONS-Inguinal hernia}

REFRACTORY INGUINODINIA AND GROIN HERNIA RELAPSE SOLVED BY LAPAROSCOPIC TRANS-ABDOMINOPREPERITONEAL REPAIR AND PREVIOUS MESH AND STITCHES REMOVAL

J. Trébol ${ }^{1}$, A.M. Rodríguez ${ }^{1}$, A.B. Sánchez-Casado ${ }^{1}$,

A. García-Plaza ${ }^{1}$, J.I. González-Muñoz ${ }^{1}$, A. Carabias-Orgaz ${ }^{2}$, L. Muñoz-Bellvis ${ }^{1}$

${ }^{1}$ General and Digestive Tract Department, Complejo Asistencial Universitario de Salamanca, SALAMANCA, Spain; ${ }^{2}$ Anesthesiology, Complejo Asistencial de Zamora, ZAMORA, Spain

Aims: Post-hermiorrhaphy chronic inguinal pain is a multifactorial complex complication that needs multimodal management. For some patients, pain can be persistent and disabling. Meshes can contribute to pain by causing inflammation or entrapping nerves

Management includes conventional analgesia, nerve blocks, nerve ablation and recently nerve stimulation. Surgery includes neurectomies (selective or triple), neurolysis or simple nerve divisions usually associated with previous mesh removal and replacement with a new one. We present a patient with refractory inguinodinia and posterior relapse managed successfully with laparoscopic previous mesh subtotal removal and TAPP, without neurectomy.

Methods: A 69 year-old male with hemochromatosis, cerebrovascular disease, hypothyroidism and eosinophilic esophagitis had bilateral Licthenstein repair one year before. This patient developed severe and impending groin pain (he needed a walking stick), diagnosed with neuralgia.

Chronic Pain Unit management ensued and 9 months later a hernia relapse occurred. Patient was offered laparoscopic TAPP repair and open groin exploration with mesh removal and eventual nerve transection

Results: During TAPP approach a direct hernia relapse was identified, the previous mesh was included on preperitoneal space and some non-absorbable sutures to inguinal ligament were identified. Stitches and nearly total mesh removal (only the part surrounding cord elements was left in place) were performed. $15 \times 15$ heavyweight polypropylene mesh was employed fixed with Gubran $2 ®$ and the flap was closed with running sutures. Patient was discharged uneventfully the same day. Seven months later he did not need analgesics and had no physical impairment. Conclusions: Post inguinal hernia repair chronic pain can be severe and disabling, and is becoming more prevalent. The origin is complex and meshes and sutures could play a role. The management is multimodal and demanding.

For refractory patients, surgery may be an option. Laparoscopic, open and mixed approaches have been employed. They usually combine mesh removal and substitution (often in different planes) and groin nerve therapies. Nowadays, triple neurectomy seems to be the most effective treatment (more than $90 \%$ pain relief). Generally, removal of mesh alone does not lead to lasting pain relief or has worse outcomes compared with associated neurectomy.

\section{V150-HERNIA-ADHESIONS-Inguinal hernia}

EXPLANT OR NOT TO EXPLANT? THAT IS THE QUESTION. INTERVENTION OF RECURRENT INGUINAL HERNIA WITH PREVIOUS MESH IN PREPERITONEAL AREA BY LAPAROSCOPY

J.M. Suárez Grau, J. Gómez Menchero, A. Gila Bohorquez, J.L. García Moreno, I. Duran Ferreras, P. Landra Dulato, J. Ferrufino General Surgery, Hospital Riotinto, MINAS DE RIOTINTO, Spain

Introduction: Mesh repair of inguinal hernia is sometimes followed by adverse effects such as mesh migration, chronic groin pain or recurrence. Removal of the mesh is necessary in selected cases. We affront this cases by TAPP intervention.

Methods: We present a video with two intreventions of inguinal recurrent hernia by laparoscopy (TAPP). We remar the points to decide explant the mesh or not to explant. The conditions to decide the explant were the proximity to the main vessels in inguinal area (espigastric and femoral vessels) and the plication of the mesh.

Results: and conclusion As we show in the video, the explant of the mesh is only conditioned by the plicature of the mesh for its migration and recurrence, accompanied usually with pain. We don't remove any time the mesh or the plug if it is in the triangle of doom with firm adhesions to the main vessels. We cover the previous mesh with a new ligthweigth 3D mesh and closing at the end the preitoneum over the new reparation.

\section{V151-HERNIA-ADHESIONS-Inguinal hernia}

LAPAROSCOPIC INGUINAL HERNIA REPAIR VIA TOTALLY EXTRAPERITONEAL APPROACH (TEP), UNDER GENERAL OR EPIDURAL ANESTHESIA

V. Drakopoulos, S. Voulgaris, K. Botsakis, V. Lygizos, I. Iliadis, K. Rekouna, P. Trakosari, A. Bakalis, V. Vougas

1st Department of Surgery and Transplantation Unit, District General Hospital of Athens « Evangelismos », ATHENS, Greece

Introduction: TEP technique isn't a controversial area in surgical practice for inguinal hernias anymore, but a fully accepted method. The use of general anesthesia has been the mainstay of laparoscopic hernia repair, but epidural anesthesia is not a contradiction to properly selected patients.

Material-Method: The approach of the extraperitoneal area achieved without use of a dilation balloon, but via the indroduction of the camera and the dissection of the regional structures.3 trocars ports were used: A $10 \mathrm{~mm}$ trocar through the umbilicus for the camera, exactly as in sils (single incision laparoscopic surgery), another one $5 \mathrm{~mm}$ is placed in the midline between the umbilicus and pubis, the last $5 \mathrm{~mm}$ trocar is placed in the midclavicular line ipsilateral with the hernia. The key for every operation was the tension free technique with placement and fixation of a mesh $10 \times 5 \mathrm{~cm}$. In 20/25 cases the mesh was placed with tacks on the inside of the inferior epigastric artery-vein complex. All patients were dismissed from the hospital in $24 \mathrm{~h}$, no drain was placed and no major postoperative complications took place.

Conclusion: TEP is a demanding technique with serious learning curve. The use of a dilation balloon for insertion in the extraperitoneal area is not prerequisite. TEP is an appropriate method both for first appearing and recurrent inguinal hernias. Epiduralanesthesia instead of general anesthesia is no a contradiction for properly selected patients.

\section{V152-HERNIA-ADHESIONS-Inguinal hernia}

\section{EFFECTS OF PREPERITONEAL CO2 INSUFFLATION DURING} TAPP INGUINAL HERNIOPLASTY

\author{
M. Zashev, A.M. Mihaylov, A. Trifunova, M. Vania, R. Gornev
}

\section{General Surgery, UH, Lozenetz, SOFIA, Bulgaria}

Aims: The aim of this study was to investigate the effects of preperitoneal carbon-dioxide $\left(\mathrm{CO}^{2}\right.$ insufflation during TAPP (transabdominal preperitoneal) repair.

Materials and methods: 20 male patients with inguinal hernia were include in our study. We obtain laparoscopic access at the umbilicus and introduce $10 \mathrm{~mm}$ port. Two $5 \mathrm{~mm}$ working ports are placed lateral. Diagnostic laparoscopy of the entire abdomen is necessary to rule out other pathology or contraindications for surgery. Using Aspiration Needle we insuflate carbon-dioxide $(14 \mathrm{mmHg})$ preperitoneal at the level of anterior superior iliac spine while decrease abdominal gas pressure to $8 \mathrm{mmHg}$. Same procedure is made lateral to the umbilical artery.

Results: We found that preperitoneal carbon-dioxide $\left(\mathrm{CO}^{2}\right)$ insufflation during TAPP facilitate the future parietalisation and even can reduce operating time in future improvements of the technique. There were no intraoperative complications related to this procedure. We did not found any potential risk of the technique when is use by trained surgeons.

Conclusion: Preperiotoneal insuflation of carbon-dioxide during TAPP is safe when special attention is paid to the key technical points and anatomical landmarks. It can facilitate the parietalisation, improve the operating time and reduce risk of peritoneal tears.

Bittner R, Arregui ME, Bisgaard T, et al. Guidelines for laparoscopic (TAPP) and endoscopic (TEP) treatment of inguinal hernia [International Endohernia Society (IEHS)]. Surg Endosc. 2011;25(9):2773-843

Lomanto D, Katara AN. Managing intra-operative complications during totally extraperitoneal repair of inguinal hernia. J Minim Access Surg. 2006;2(3):165-70. 


\section{V153-HERNIA-ADHESIONS-Inguinal hernia}

\section{PREPERITONEAL BOWEL INCARCERATION POST} LAPAROSCOPIC TRANSABDOMINO PREPERITONEAL INGUINAL HERNIA REPAIR SOLVED BY LAPAROSCOPY

J. Trébol ${ }^{1}$, I. Pascual-Migueláñez ${ }^{2}$, F. del Castillo ${ }^{2}$, S. Gortázar ${ }^{2}$, A. Carabias-Orgaz ${ }^{3}$

${ }^{1}$ General and Digestive Tract Department, Complejo Asistencial Universitario de Salamanca, SALAMANCA, Spain; ${ }^{2}$ General and Digestive Tract Surgery, Hospital Universitario La Paz, MADRID, Spain; ${ }^{3}$ Anesthesiology, Complejo Asistencial de Zamora, ZAMORA, Spain

Aims: Laparoscopic inguinal hernia repairs (LIHR) are performed more and more frequently because they offer some advantages; however, we cannot forget their specific complications. LIHR are associated sometimes with peritoneal tears that can lead to bowel obstruction. We present two cases of bowel obstruction related to peritoneal defects post TAPP procedure and review peritoneal closure, bowel obstruction and options to repair defects.

Methods: A 79 year-old male was scheduled for TAPP due to bilateral relapse. Two 10x15 $\mathrm{TiO}_{2} \mathrm{Mesh}^{\mathrm{TM}}$ fixed with Securestrap ${ }^{\circledR}$, employed also for peritoneal flap closure, were employed. Three days later he was readmitted with bowel obstruction with CT suggesting 'adhesions'.

A 56 year-old male had bilateral TAPP in another centre. Seven days later he presented with bowel obstruction. CT showed metallic tackers and suggested 'adhesions'

Results: First case: after four days of conservative treatment failure, a revisional laparoscopy showed ileum herniation through a peritoneal defect and firm adhesions to the mesh. Bowel was labouriously separated and the peritoneal defect closed with two running sutures. He was discharged on the $7<\sup >$ th $</$ sup $>$ postoperative day and three years later he is asymptomatic. Second: after two days of conservative treatment failure, on laparoscopy, ileum was filmy adhered to polipropilene mesh through a big defect on flap closure. Defect was closed with interrupted sutures. As tears persisted, an omental flap was created to cover the area. Patient was discharged on the 5th day and continues asymptomatic three years later.

Conclusions: LIHR bowel obstructions can be divided in adhesive disease and herniation. Herniation can be early (through peritoneal defects) or late (trocar site).

International guidelines recommends a thorough closure of peritoneal incision or bigger tears (Grade B). The closure can be achieved with staples, tacks, running suture, or glue. These last two methods are more time-consuming but less painful. Running suture seems to be the best, due to its low costs, tightness and low pain but sometimes can be technically difficult. Low intra-abdominal pressures $(=8 \mathrm{mmHg})$ facilitate suturing.

When a herniation appears, careful bowel management is needed and running sutures are recommended. If tears persist, an omental flap can be useful.

\section{V154-ROBOTICS \& NEW TECHNIQUES-Colorectal}

\section{TOTALLY TRANSANAL PROCTOSIGMOIDECTOMY: SINGLE PORT ROBOTIC TATME/TATA (SPRTATME)}

\section{J. Marks, J. Josse, B. Anderson, H. Schoonyoung}

Lankenau Medical Center, WYNNEWOOD, United States of America

Aims: Application of a Single Port robotic platform to perform an entirely transanal taTME/ TATA.

Methods: The following video demonstrates how a totally transanal proctosigmoidectomy is performed using a novel, Single Port (SP) robotic platform was used to carry out a totally transanal proctosigmoidectomy, Single Port robotic taTME/TATA. A 38-year-old female patient with a clinical T3N1b rectal cancer at the $3 \mathrm{~cm}$ level, status post neoadjuvant chemoradiotherapy ( $5580 \mathrm{cGy}$, Xeloda) is presented. Shown here is the open transanal dissection followed by docking of the SP robot, implementation of the single port instruments (fenestrated bipolar forceps, cadier, scissors, camera, clip applier) through a GelPoint Path to complete a totally transanal proctosigmoidectomy including transanal taTME, IMA/IMV transection, splenic flexure release, and left colonic mobilization, loop ileostomy, and handsewn coloanal anastomosis. Results: Blood loss was $100 \mathrm{cc}$. Pathology demonstrated a moderately differentiated, rectal adenocarcinoma. The total mesorectal excision was complete (Grade 3), margins were negative, and all $17 \mathrm{lymph}$ nodes were negative for metastatic carcinoma. The patient was discharged on postoperative day 4 after an uncomplicated hospital course. There was no postoperative morbidity or mortality. Conclusions: Application of the Single Port robot to transanal taTME/TATA (SPRtaTME) is presented here. While much work remains to be done to validate the SP robot's safety, this first demonstration of a totally transanal taTME/TATA establishes its feasibility and utility. This Single Port platform stands to greatly expand the application of natural orifice transluminal endoscopic surgery (NOTES). As shown, the SP robot offers more than sufficient visualization, technical control, and adequate reach to perform such an operation. We present an exciting new avenue by which to complete operations in an entirely transanal fashion, which are classically performed via a combined transanal and transabdominal approach.

\section{V155-ROBOTICS \& NEW TECHNIQUES-Colorectal}

SINGLE PORT ROBOTIC TRANSANAL ENDOSCOPIC MICROSURGICAL (SPRTEM)

\section{J. Marks, J. Salem, J. Josse, B. Anderson, H. Schoonyoung}

Lankenau Medical Center, WYNNEWOOD, United States of America

Aims: To share the initial experience using Single Port (SP) robotic TEM (RTEM). Methods: This video shows the utilization of a new robotic platform to perform Transanal Endoluminal Microsurgery, RTEM. Presented here is a 53 year old woman with a recurrent recta adenoma at the $6 \mathrm{~cm}$ level, status post a previous TEM resection in October 2017. Demonstrated is the utilization of the SP robot through a GelPoint Path in order to perform a partial fullthickness and full-thickness resection. The robot is introduced through a $25 \mathrm{~mm}$ in diameter cannula via a four-channel face-plate. The instruments' two-jointed mobility at the elbows and wrists as well as the novel navigation system are well demonstrated. The docking of the SP robot, utilization of the dissecting devices, and closure of the defect is shown.

Results: spRTEM was performed with a blood loss of $5 \mathrm{cc}$, and the patient was discharged on postoperative day 1 . There was no postoperative morbidity, mortality, or moderate/severe pain Pathology showed tubular adenoma with low-grade dysplasia in a non-fragmented specimen with negative margins circumferentially.

Conclusion: Initial Experience using the SP Robot for rTEM is demonstrated here. The robot provides wonderful visualization and operative control to the surgeon. Articulation of the robot's wrists and arms have the potential to facilitate technical aspects of the procedure. RTEM stands as an exciting development in the field of transanal endoluminal surgery.

\section{V156-ROBOTICS \& NEW TECHNIQUES-Bariatrics}

\section{ROBOTIC-ASSISTED ROBOTIC ESOPHAGECTOMY: INITIAL EXPERIENCE (VIDEO)}

\author{
M.V. Marino ${ }^{1}$, G. Gulotta ${ }^{2}$, A.L. Komorowski ${ }^{3}$
}

${ }^{1}$ Emergency and General Surgery, Azienda Ospedaliera, Ospedali Riuniti Villa Sofia-Cervello, PALERMO, Italy; ${ }^{2}$ Emergency and General Surgery, Policlinico Universitario, Paolo Giaccone, PALERMO, Italy; ${ }^{3}$ Department of Surgical Oncology, Maria Sklodowska-Curie Memorial Institute of Oncology Cancer Centre, KRAKOW, Poland

Introduction: The application of robotic approach in the esophageal surgical field is in its firs phase. The microsuturing and microdissection capabilites of the robotic system can potentially overcome the traditional limitation of the laparoscopic surgery thus enhancing the indications of minimally invasive surgery.

Methods: We have performed a retrospective analysis of our prospectively maintained database that included 16 patients who underwent robotic-assisted esophagectomy for malignant disease between 2014 and 2017

Results: Ten out of sixteen patients had squamous cell carcinoma meanwhile six had adenocarcinoma. Ten McKeown's and six Ivor Lewis were performed. The mean operative time was

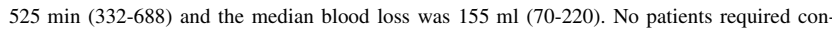
version nor intraoperative transfusion. The morbidity rate was $3 / 16(18.7 \%):$ a transitory laryngeal nerve paresis, a pneumotorax and pneumonia. The mean hospital stay was 8 (range 7-23) days. An R0 resection rate of $93.7 \%$ was achieved with a mean lymph node yield of 16 (1321 ). The 1 -year disease free survival was $82.8 \%$, wheres the the 1 -year overall survival was $88.5 \%$

Conclusions: Robotic assisted minimally invasive esophagectomy (RAMIE) is safe and feasible, it offers promising results while preserving a good oncology adequacy. 


\section{V157-ROBOTICS \& NEW TECHNIQUES-Basic} and Technical research

\section{ROBOTIC ASSISTED ESOPHAGEAL DIVERTICULECTOMY WITH HELLER MYOTOMY AND DOR FUNDOPLICATION}

\author{
F. Proietti ${ }^{1}$, M. Fitzgerald ${ }^{1}$, F. Mongelli ${ }^{2}$, M. di Giuseppe ${ }^{1}$
}

${ }^{1}$ Surgery, San Giovanni Hospital-EOC-Bellinzona $(\mathrm{CH})$, BELLINZONA, Switzerland; ${ }^{2}$ Surgery, Luzerner Kantonsspital, LUZERN, Switzerland

This video shows our technique for the treatment of an esophageal diverticolum using a robotic left sided transthoracic approach, followed by a heller myotomy and Dor fundoplication using a transabdominal approach

Our case is a 75 year old male, who suffered from severe dysphagia, halitosis and gastric reflux who on endoscopic and radiological investigations was found to have low grade and a $3 \mathrm{~cm}$ wide esophageal diverticulum, $7 \mathrm{~cm}$ from the Lower Esophageal Sphincter.

Initially conservative management was attempted, however following poor compliance and the perInitially conservative management was attempted, however following poor comp

sistance of symptoms after 1 year of therapy, surgical intervention was indicated.
The operation was performed using the minimally invasive robotic system of the DaVinci $\mathrm{Si} \circledast$, starting The operation was performed using the minimally invasive robotic syst
with the thorax time. The patient is positioned in left side decubitus.

pasitioned in left side decubitus. The camera-trocar is insert in the thorax via the fifth intercostal space the, two $8 \mathrm{~mm}$ and one $12 \mathrm{~mm}$ robotic trocars are added.

The lung is liberated from pleural adhesions and the esophagus is then prepared exposing the diverticulum which is successfully removed with an Endo-GIA ${ }$.

The esophageal muscle fibers, near the suture line is reinforced with separated Vicryl stitches and the resected piece is extracted via Endo-bag. A 16fr thoracic drainage tube is then placed and the trocar accesses repaired.

The patient is the put in supine position with a $15^{\circ}$ anti-trendelemburg angle. Three robotic trocars The patient is the put in supine position with a $15^{\circ}$ anti-trendelemburg angle. Three robotic trocars
(two $8 \mathrm{~mm}$ and one $12 \mathrm{~mm}$ ) are placed and the robot docking is made from the patient left shoulder. The lesser omentum is divided to visualize and prepare the Gastric-Esophageal Junction (GEJ) sparing the vagus nerve.

The Heller myotomy is then performed for $4 \mathrm{~cm}$ over the GEJ and $3 \mathrm{~cm}$ under it. The mucosal integrity is assured via laparoscopic and contemporary gastroscopic view.

The gastric fundus is attached to the distal esophagus completing the Dor fundoplication. Post-operative care comprehends the removal of the thoracic drainage during the first post-operative day, the pain management and the progressive realimentation.

The hospitalized period lasts 6 day and the patient was dismissed without complications occurred.

\section{V158-ROBOTICS \& NEW TECHNIQUES-Basic and Technical research}

\section{UNIPORTAL THORACOSCOPIC LOBECTOMY FOR A CONGENITAL CYSTIC ADENOMATOID MALFORMATION}

\section{F.A. Dobritoiu ${ }^{1}$, D. Dobritoiu ${ }^{2}$, D. Godoroja ${ }^{3}$, S. Slaniceanu ${ }^{4}$}

${ }^{1}$ Thoracic surgery, Ponderas Academic Hospital, BUCHAREST, Romania; ${ }^{2}$ Pediatric surgery, Ponderas Academic Hospital, BUCHAREST, Romania; ${ }^{3}$ Intensive care, Ponderas Academic Hospital, BUCHAREST, Romania; ${ }^{4}$ Pathology, Santomar OncoDiagnostic, CLUJ NAPOCA, Romania

The uniportal video assisted lung lobectomies gained popularity all over the world during the last 10 years. The technique is safely applied for peripheral pulmonary lesions, under $6 \mathrm{~cm}$, but more and more complex cases are being approached while the indications continue to evolve.

Our aim is to present the particular aspects of this technique in an 11-year-old female patient with a giant bullous lesion located in the lower lobe of the right lung.

The preoperative work-up for this case is presented and commented. A multidisciplinary surgical team consisting of thoracic and pediatric surgeons was involved. A single $3.5 \mathrm{~cm}$ length incision in the fourth intercostal space was used for the access. Due to the fact that the lesion involved almost the entire lobe and the margins were very close to the hilum, we have decided and performed a right lower lobectomy. Dissection and dimensions, forcing us to perform an 'artery first approach' in a very narrow space. No complications during or after surgery were encountered. The patient was discharged after four days and she went to school on the sixth day. Histopathological examination showed that the lesion was a type 1 CCAM (congenital cystic adenomatoid malformation).

Conclussion: The uniportal video assisted lung lobectomy was safety applied for a giant bullous lesion of the right lung.
V159-ROBOTICS \& NEW TECHNIQUES-Basic and Technical research

\section{LAPAROSCOPY IN TREATMENT OF DUNBAR SYNDROME}

N. Dowgiallo-Wnukiewicz, P. Lech, P. Rymkiewicz, M. Michalik

Department of General, Minimally Invasive and Elderly Surgery, University of Warmia and Mazury in Olsztyn, OLSZTYN, Poland

Aim: Dunbar syndrome, celiac trunk (CT) compression syndrome, caused by median arcuateligament is a rarely diagnosed disease because of its nonspecific symptoms, which cause adelay in the correct diagnosis. The aim of the study was to demonstrate the usefulness andadvantages of laparoscopic approach in the treatment of Dunbar syndrome.

Methods: We performed 3 laparoscopic release of CT in the Department of General, MinimallyInvasive and Elderly Surgery in Olsztyn in 2018. All of three patients suffered from severepain of abdominal cavity before the surgery.

Results: In two cases, there were a complete remission of the symptoms. In one case, there was animprovement. All patients reported relief of symptoms in the first days after the operation. There were no postoperative complications.

Conclusions: The laparoscopic treatment of Dunbar seems to be safe and feasible procedure. Thelaparoscopic surgery alone can often eliminate discomfort, while angioplasty and stentimplantation are no longer necessary.

\section{V160-ROBOTICS \& NEW TECHNIQUES-Basic} and Technical research

A CASE EXAMPLE OF THE TECHNICAL PROGRESSION IN THE MINIMALLY INVASIVE ROBOTIC RETROMUSCULAR VENTRAL HERNIA REPAIR

M. Fitzgerald $<$, F. Mongelli ${ }^{2}$, M. di Giuseppe ${ }^{1}$

${ }^{1}$ Surgery, San Giovanni Hospital-EOC-Bellinzona $(\mathrm{CH})$, BELLINZONA, Switzerland; ${ }^{2}$ Surgery, Luzerner Kantonsspital, LUZERN, Switzerland

Introduction: The advances in robotic surgery have permitted the application of such technology to various surgical fields, one of the last of these being hernia surgery. We present a case video of the treatment of a dual-hernia using a Robotic Retromuscular Ventral Hernia Repair(rRVHR) using the DaVinci $\mathrm{Si} \circledast$ robotic system.

The case report demonstrates the evolution of the Trans-abdominal Robotic Umbilical Prosthetic (TARUP) in that it utilises a 'double docking' technique to allow the positioning of a large retromuscular mesh.

Methodology: Our patient is a 50-year-old male who presented with chronic epigastric pain. The abdominal CT confirmed two abdominal wall hernias; an epigastric and supra-umbilical hernia with visceral contents and wall defect diameter of $6 \mathrm{~cm}$ and $2.5 \mathrm{~cm}$, respectively.

Using the minimally invasive robotic system of the DaVinci $\mathrm{Si}{ }^{\circledR}$ we adapted the well known retromuscular mesh technique. The operation was initially intraperitoneal with access to the retromuscular preperitoneale space using a right sided longitudinal incision.(as per standard TARUP technique). We proceed with the dissection of the retro-muscular space until the left lateral edges of sheath, creating a preperitoneal space for the placement of a specifically modified UltraPro polyprois positioned and the peritno subsequentr with ports placed in the retromuscular space. The mesh is positioned and the peritheum subsequently closed with a V-lock suture ${ }^{\circledR}$. Finally we opted for a negative pressure Jackson-pratt drain, inserte preperitoneally.

Results: The patient was discharged on the 2nd post-operative day without complication Follow up continued until 12 months post operatively during which the patient remained asymptomatic, without signs for hernia recurrance.

Conclusion: The technique highlighted in our video demonstrates the utility of the robotic system in hernia repair. Specifically the approach proved a success as it facilites the placement of the mesh totally extra-peritoneally with closure of the posteriore sheath without tension. The added adve are that the port-sites are distant from the mesh thus reducing infective risk. Additionally this technique allows the treatment of large peritoneal defects. 


\section{V161-ROBOTICS \& NEW TECHNIQUES-Colorectal}

ADVANCED ROBOTIC FELLOWSHIP LEADS IN SIMILAR PERFORMANCE TO THE MENTOR-A BLIND ASSESSMENT OF VIDEO FOOTAGE

\section{P. Tejedor, J.S. Khan, F. Sagias}

Colorectal Surgery, Queen Alexandra Hospital, PORTSMOUTH, United Kingdom

Aim: To analyse the performance of a robotic fellow during a robotic total mesorectal excision (TME) at the end of the fellowship, and subsequently compare it with their mentor.

Methods: The fellow is exposed to 2 robotic colorectal lists per week. During the fellowship, assessment of performance is recorded in a structured proforma covering aspects of autonomy, tissue handling and dissection.

At the end of the fellowship, areview of cases performed by the fellow and the mentor was carried out in a blindly manner (video footage).

Results: Robotic TME training was divided into modules in order of complexity and the trainee had to achieve sequential proficiency in each module, before progression.

Docking of daVinci robotic system.

Inferior mesenteric artery exposure and ligation, development of medial to lateral plane and inferior mesenteric vein division.

Left colonic and splenic flexure mobilization. Pancreas identification.

Rectal dissection (TME).

Qualitative assessments were recorded by the mentor; The fellow was 'able to perform with verbal help' most of the steps from early on. By the end of the fellowship, all steps were performed in a similar manner in terms of quality and oncological integrity when compared with the mentor.

Conclusions: At completion ofan advanced robotic colorectal fellowship, high quality trainees can perform every step of the TME dissection in a similar manner with the trainer, when assessed blindly, without compromising oncological integrity.
V163-ROBOTICS \& NEW TECHNIQUES-Colorectal

NERVE SPARING TME AND PELVIC NEUROANATOMY FOR COLORECTAL SURGEONS

\section{P. Tejedor, F. Sagias, J.S. Khan}

Colorectal Surgery, Queen Alexandra Hospital, PORTSMOUTH, United Kingdom

Aim: To describe the critical points in which the pelvic nerves can be damaged during a Total Mesorectal Excision (TME) for rectal cancer and the benefits of robotic surgery for identifying these points.

Methods: There are 4 critical points regarding pelvic neuroanatomy:

Superior hypogastric plexus (SHP): located in front of L5-S1. The ganglionic sympathetic fibres form the right and left sympathetic trunk, travel along the anterior surface of the aorta and coalesce in the SHP at the level of the inferior mesenteric artery (IMA).

Superior hypogastric nerves: they take an anterolateral course into the pelvis. There is an avascular 'holy plane' around the rectum between these two nerves.

Inferior hypogastric plexus (IHP): lies over the posterolateral pelvis, almost parallel to the internal iliac arteries. This can be identified at the lower end of the rectum.

Neurovascular bundles(of Walsh): in front of the Denonvillier's fascia, at 2 and 10 o'clock position. They are responsible for erectile function.

Results: Lack of knowledge or identification of key structures at these 4 points can lead to increased risk of nerve damage and translate into poor functional outcomes.

The IMA is dissected up to the origin from aorta and here the SHP can be seen. Care is taken to avoid any damage to these structures.

The TME plane is found at the back of IMA as the inner most dissectible layer between mesorectum pelvic fascia. Right and left superior hypogastric nerves are identified. Dissection is carried out posteriorly, laterally and anteriorly.

IHP is identified at the lower third of the rectum, when the dissection is about to reach the pelvic floor. Care should be taken in not to go too far lateral and damage this plexus.

In the anterior dissection, plane is carried in front of the Denonvilliers' fascia. The neurovascula bundles can be seen at 2 and 10 o'clock position and the surgeon has to be careful to stay inside that plane in order to avoid damage.

Conclusions: The precise dissection in robotic surgery results in minimal tissue damage and better visualization and preservation of the pelvic nerves.

\section{V162-ROBOTICS \& NEW TECHNIQUES-Colorectal}

\section{ROBOTIC RECTAL LOW ANTERIOR RESECTION WITH LOW TIE LIGATION USING TILEPRO SIMULTANEOUS DISPLAY} OF REAL TIME ULTRA SOUND NAVIGATION

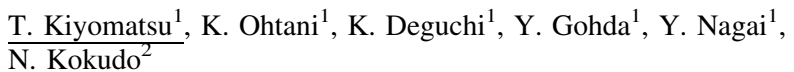

${ }^{1}$ Department of Colorectal Surgery, National Center for Global Health and Medicine, TOKYO, Japan; ${ }^{2}$ Department of Surgery, National Center for Global Health and Medicine, TOKYO, Japan

Aims: To find safe and simple method in robotic rectal low anterior resection with low tie arteria ligation and lymph node dissection around the root of inferior mesenteric artery.

Methods: We performed robotic rectal low anterior resection (RLAR) by daVinci Si system in eight patients with rectal cancer. We applied low tie arterial ligation, just caudally to the origin of the left colic artery in all cases. During the procedure, we used TilePro function of daVinci $\mathrm{Si}$ system which enabled to display two other visual informations through external inputs under the normal 3-dimensional surgeon console view. Preoperative 3D-CT vessel branching simulation video and intra-operative real time ultra sound navigation view were displayed simultaneously under normal operative camera view in the surgeon console.

Results: Left colic artery preservation was completely done in all 8 cases. The mean time to find and expose the left colic artery from the first incision in sigmoid mesentery was 5 min, which was drastically shorter than conventional method. This method needed lesser mobilization of inferior mesenteric artery (IMA), and may be less invasive to autonomic nerve around the root of IMA which is very important for ejaculation function.

Conclusion: Robotic rectal low anterior resection with low tie arterial ligation was performed safely and in short time, using TilePro intra-operative navigation method. Preoperative 3D-CT vessel branching simulation video and intra-operative real time ultra sound navigation view were very useful in the procedure. We present the method in video. 


\section{V164-ROBOTICS \& NEW TECHNIQUES-Colorectal}

NEW CONTRIBUTIONS OF ICG TO RECTAL SURGERY: LATERAL LYMPHADENECTOMY AND GHOST ILEOSTOMY

S. Alonso ${ }^{1}$, T. García ${ }^{2}$, I. Alarcón ${ }^{2}$, Y. Yang ${ }^{2}$, E. Licardie ${ }^{2}$, A. Senent $^{2}$, F. López ${ }^{2}$, A. Barranco ${ }^{2}$, M. Socas ${ }^{2}$, J. Padillo ${ }^{2}$ S. Morales-Conde ${ }^{2}$

${ }^{1}$ General Surgery, Complejo Asistencial Universitario de Salamanca, SALAMANCA, Spain; ${ }^{2}$ General Surgery, Unidad de Innovación en Cirugía Mínimamente Invasiva. H. Virgen del Rocío, SEVILLA, Spain

Aims: To describe and evaluate new contributions and eventual advantages of ICG fluorescence to perform an ICG guided bilateral pelvic lymph node dissection in a patient who underwent low-anterior-resection for rectal carcinoma. We also present the basic steps to avoid ileostomy during rectal surgery in which ICG and ghost ileostomy play an important role.

Methods: A 68-year-old male patient was referred to our hospital due to abdominal pain and significant changes in usual bowel habits.Colonoscopy showed a no obstructing $5 \mathrm{~cm}$ middle rectal mass, which was reported as an adenocarcinoma.CT scan and MRI revealed a $63 \times 52 \mathrm{~mm}$ polyp in the anterior rectal wall which was located $7 \mathrm{~cm}$ from the anal verge. It was involving mucosa and sub-mucosa with muscularis propia invasion. No pathological lymphadenopathies or hepatic metastatic disease were found (stage T2N0).A laparoscopic ultra-low-anterior resection plus ICG lateral lymphadenectomy with total mesorectal excision was performed. A complete splenic flexure mobilization was performed to achieve a safe tension-free anastomosis. Transection line of the proximal rectum was checked after ICG intravenous injection. ICG was injected around the tumor by inserting an anoscope, just before the surgery. After the dissection of the rectum, lateral lymphadenectomy was performed assisted by ICG. An end-to-side anastomosis was made. And a vascular loop was passed around the terminal ileum to create a ghost ileostomy. The procedure lasted $120 \mathrm{~min}$. Reactive protein $\mathrm{C}$ was monitored to identify an initial leak. The patient was discharge in postoperative day 7 and no complication was detected.

Results: Pathological exam reported a rectal adenocarcinoma. Pelvic lymphadenectomy results were: 2 negative nodes, 2 negative nodes and 10 negative nodes from right lymph node dissection, left lymph node dissection and rectosigmoid resection specimen respectively. No metastatic disease was found (stage T1NOM0).

Conclusions: In our experience, ICG fluorescence imaging system offers important contributions to rectal surgery furthermore than evaluating vascular supply to the anastomosis. Lymphatic mapping of the lateral lymph nodes and avoiding ileostomy could be a potential important use in the future. Larger studies and more specific evaluations are needed to confirm its role in colorectal surgery and to find its limitations.
V165-ROBOTICS \& NEW TECHNIQUES-Colorectal

TOTAL ROBOTIC SIGMOIDECTOMY FOR COLORECTAL CANCER

A.M. Otero, R. Bravo, F.B. de Lacy, A.M. Lacy

Gastrointestinal Surgery, Hospital Clinic, BARCELONA, Spain

Background: Robotic surgery for colorectal cancer is an emerging technique. Potential benefits as compared to conventional laparoscopic surgery have been demonstrated. Innovative robotic technologies have helped surgeons overcome many technical difficulties of conventional laparoscopic surgery such as hand-eye coordination, a two-dimensional view, and a restricted range of motion. Robotic-assisted surgery was established as a new approach to minimally invasive surgery, overcoming these limitations.

The following video shows a total robotic sigmoidectomy step by step on the basis of ourexperience.

Intervention: A 52-year-old male patient with no previous medical historyand a colon adenocarcinoma, $22 \mathrm{~cm}$ from the anal verge, no distant metastases. It was decided to perform a robotic sigmoidectomy.

Target anatomywas located andwe proceededto the exposure of the mesenteric vessels from medial to lateral. A cautery wasused to open the peritoneum,up to the origin of the inferior mesenteric artery, and caudally past the sacral promontory.The vessels weretransected by LigaSureTM. We performedthe complete release of the colon taking care to avoid injury to retroperitoneal structures.

We usedLigaSureTM to section the mesocolon in order to prepare the transection of the proximal colon. Indocyanine green was used to check the correct vascularization. An EndoGIA TriStapleTM was used to divide the colon. Subsequently, we sectioned the rectumand extracted the specimen through itwith no need to make any auxiliary incisions. We introduced the anvil of the suture device to perform the anastomosis. We sectionedand close the rectum with an EndoGIA TriStapleTM.

Finally we opened the proximal colon to introduce the anvil,making a pursestring to fix it and create a side to end anastomosis.

Outcome: The surgery took $110 \mathrm{~min}$. The patient started oral intake $6 \mathrm{~h}$ after surgery and left the hospital on the 3rd postoperative day. Pathological examination ruled out a colon adenocarcinoma pT1N0.

Conclusion: Total robotic sigmoidectomy is safe and feasible and can be a procedure of choice to achieve a good surgical qualityand avoid assistance incisions in patients with colon cancer. 


\section{V166-ROBOTICS \& NEW TECHNIQUES-Colorectal} ROBOTIC RESECTION FOR RECTAL REGROWTH IN AN OBESE PATIENT

M.F. Shah ${ }^{1}$, S. Penteleimonitis ${ }^{1}$, N. Irfan Ul Islam ${ }^{2}$, N. Figueiredo ${ }^{3}$, A. Parvaiz

${ }^{1}$ Department of surgery, Poole District Hospital Foundation Trust UK, POOLE, DORSET, United Kingdom; ${ }^{2}$ Minimally Invasive Colorectal Unit, Champalimaud Foundation, LISBON, Portugal; ${ }^{3}$ Colorectal Unit, Champalimaud Foundation, LISBOA, Portugal; ${ }^{4}$ Minimal Access and Robotic Colorectal Surgery, Poole Hospital NHS Trust, POOLE, United Kingdom

Surgery for rectal cancer has always been challenging. With the advent of neoadjuvan chemoradiotherapy for locally advance rectal cancer there have been patients having complete clinical response. With more and more data now advocating wait and watch policy for these patients which require close radiological and endoscopic follow-up but unfortunately around 30\% of them have regrowth of tumour which will require surgical intervention. The use of robot for cancer resections is becoming more frequent especially in narrow spaces like in an obese male pelvis. The reason being better 3-dimensional views, more angulation of the instruments and exclusion of tremors, which in turn leads to better dissection and preservation of hypogastric nerves.

In this video, we present a robotic low anterior resection for rectal re-growth in an obese 55-years old male patient. He was offered Neoadjuvant chemoradiotherapy after discussion in MDT. He had an complete response with chemoradiotherapy and was decided to offer him watch and wait regime. Unfortunately, he developed rectal re-growth in the first year of his follow up. Imaging showed T2 lesion with no distant metastasis and was later confirmed on histology as well. After MDT discussion he was offered robotic low anterior resection.

The video starts by showing the clinicopathological features of patient including his radiological and endoscopic images. Robotic port sites are shown. The edited video starts with rectal dissection after ligation of inferior mesenteric artery and vein with emphasis on narrow pelvis and preservation of hypogastric nerves, seminal vesicles and intact presacral fascia. Postoperative histology was ypT2No and patient was discharged home after 3 days with no postoperative complications.

\section{V167nores-ROBOTICS \& NEW TECHNIQUES-Colorectal}

\section{APPLICATION OF PARELLEL OVERLAP STAPLING IN LAPAROSCOPIC LEFT COLECTOMY COMBINED WITH NATURAL ORIFICE SPECIMEN EXTRACTION SURGERY}

\section{Zhang, J. Li}

Department of Gastrointestinal Surgery, Henan People's provincial Hospital, ZHENGZHOU, China

Background: Minimally invasive surgery for colon resection has improved patient outcome, however a minilaparotomy still is necessary to extract the specimen. This report describes a new approach that combine laparoscopic parellel overlap stapling left colectomy with natural orifice specimen extraction surgery, with the aim to minimize abdominal wall trauma

Method: Laparoscopic left colectomy for malignant diesease was performed using a standard five-port technique. After releasing the left colon via laparoscopy, divide the proximal and distal of specimen with 60-Echelon, and put distal sigmoid colon and proximal transverse colon together. Open sigmoid colon $6 \mathrm{~cm}$ apart from distal margin, and incise transverse colon at proximal margin. Take transverse colon and sigmoid colon side-to-side anastomosis via 60-Echelon. Incise posterior vaginal fornix to get into the abdominal cavity and extract specimen through vaginal. Outcome parameters such as complications, conversions, operative time, postoperative recovery, and postoperative pain were prospectively recorded in a database. Results: Surgery was performed for 17 patients with left-colonic carcinoma. No perioperative complications or conversions occurred. The median operating time was $157 \mathrm{~min}$. The median visual analogue scale score of postoperative pain was 1 , and 2 of 17 patients needed analgesia on postoperative day 1 . The median postoperative hospital stay was 6 days. For malignancies, tissue margins were oncologically adequate, the averge number of harvested lymph nodes were 16.9 . The 4-week follow-up period was uneventful.

Conclusion: The described technique, a combination of laparoscopic parellel overlap stapling and natural orifice surgery, has the potential to avoid incision-related morbidity of the minilaparotomy in laparoscopic left colon resections.
V168-ROBOTICS \& NEW TECHNIQUES-Education

\section{LAPAROSCOPIC COLORECTAL SURGICAL TRAINING- CHALLENGES AND SOLUTIONS}

$\underline{\text { M. Abdeldayem }}{ }^{1}$, J. Waterman ${ }^{1}$, D. Brown ${ }^{3}$, P.N. Haray ${ }^{4}$

${ }^{1}$ Colorectal Surgery, Prince Charles Hospital, MERTHYR TYDFIL, United Kingdom; ${ }^{3}$ Digimed $\AA$, ESSEX, United Kingdom; ${ }^{4}$ Colorectal Surgery, University of South Wales, WALES, United Kingdom

Background: Open surgical skills training has been well established over centuries, however there are some significant differences in laparoscopic surgical skills training. It is an obvious advantage that the trainee and the trainer have the same view; however, some of the hurdles include the differences in tactile feedback, hand eye co-ordination, spatial awareness, depth perception and maximizing assistance.

Aim: We present a video highlighting some of the key challenges faced in laparoscopic colorecta surgical training, show-casing our systematic, structured approach.

Our approach: We have developed a structured approach starting with junior surgical trainees and progressing through to consultant level as per the levels below:

Level 1: Attend courses/ workshops

Level 2: Master camera work

Level 3: Contra-lateral assisting

Level 4: Intermediate level trainee-start operating with trainer scrubbed. The trainer is an additional member of the scrub team and stands on the same side as the trainee (does not replace any assistant)

Level 5: Advanced level trainee-gradual progression from level 4. Trainer un-scrubbed but standing next to the monitor throughout the procedure.

Level 6: Trainer in theatre but out of sight of the trainee, with little interference

Level 7: Progression to Trainer-Once proficiency is achieved at level 5/6, the trainee is trained to become a trainer, for the junior and intermediate level trainees.

Within each level the complexity of the procedure increases as the trainee progresses through the level. Junior trainees (years 1-3 of surgical training) are taken through Levels 1-3, Intermediate (middle years of training) Level 4 or 5 and Advanced (last 2-3 years) up to levels 7. This way of training allows multiple members of the team to be trained simultaneously in every case. Each operating list is preceded by team briefings where the role of every member of the team is clearly identified and followed by individual and collective feedback.

Conclusion: This training ladder proved very successful through the years. The feedback from trainees at all stages has been consistently positive. Several trainees who have progressed to independent consultant practice, in the UK and abroad, are adopting this approach in their practice.

\section{V170-ROBOTICS \& NEW TECHNIQUES-Education} ROBOTIC-ASSISTED TOTAL GASTRECTOMY WITH HANDSEWN ANASTOMOSIS: INITIAL RESULTS: AND CONCLUSIONS: (VIDEO)

\section{M.V. Marino ${ }^{1}$, G. Gulotta ${ }^{2}$, A.L. Komorowski ${ }^{3}$}

${ }^{1}$ Emergency and General Surgery, Azienda Ospedaliera, Ospedali Riuniti Villa Sofia-Cervello, PALERMO, Italy; ${ }^{2}$ Emergency and General Surgery, Policlinico Universitario, Paolo Giaccone, PALERMO, Italy; ${ }^{3}$ Department of Surgical Oncology, Maria Sklodowska-Curie Memorial Institute of Oncology Cancer Centre, KRAKOW, Poland

Introduction: Despite the potential microsuturing capabilities of the robotic surgery, most of the esofago-jejunostomy after robotic total gastrectomy are still performed extracorporeal or through mechanical staplers. This can increase the cost of the procedure, the risk related to a improper functioning of the stapler.

Methods: We reviewed our prospectively maintained database analyzing patients from April 2015 to September 2017, who underwent robotic total gastrectomy with hand-sewn esophagojejunostomy for gastric cancer.

Results: A total of 18 patients were included in the study. The mean estimated blood loss was $140 \mathrm{ml}(60-257)$. The overall operative time was $365 \mathrm{~min}$ (277-421). Length of hospital stay was 6 days (5-13). No conversion was necessary nor anastomotic leakage occurred. The morbidity rate was $2 / 18(11.1 \%)$ and included a subhepatic abscess and wound infection trough Pfannenstie incision. A R0 resection rate was achieved in all cases. The mean of lymph node yield was 32 (14-39). The 1-year disease free survival was $74 \%$, the 1 -year overall survival $82.3 \%$.

Conclusions: The robotic-assisted hand-sewn esophago-jejunostomy is a safe and no time-consuming technique. It avoids the complication related to the stapler firing and it offers cosmetic benefit to the patient in terms of extraction site. 


\section{V171-ROBOTICS \& NEW TECHNIQUES-Flexible surgery}

COLONIC ENDOSCOPIC SUBMUCOSAL DISSECTION USING DILUMEN ENDOLUMENAL INTERVENATIONAL PLATFORM (EIP)

\section{P.W.Y. Chiu}

Department of Surgery, The Chinese University of Hong Kong, HONG KONG, Hong Kong

Introduction: Colorectal endoscopic submucosal dissection (ESD) is increasingly practiced for treatment of early colorectal neoplasia. However, colorectal ESD is difficult to perform due to lack of retraction as well as instability especially over hepatic flexure. Dilumen EIP is an external flexible sheath introduced during colonoscopy to stabilize environment for ESD. This video demonstrated the use of Dilumen EIP for performance of colonic ESD at the ascending colon

Method and Results: This is a 60 years old lady who received screening colonoscopy and found a $20 \mathrm{~mm}$ lateral spreading tumor (LST) Type 0 IIa lesion at ascending colon distal to ileocecal valve. Under general anesthesia, patient received colonic ESD using Dilumen EIP. Due to significant looping, the Dilumen device was introduced with the techniques of double balloon enteroscopy. After identification of the LST, the balloon in the front would be deployed to the proximal to the lesion while both balloons would be insufflated and created a stable environment. The ESD procedure started after submucosal injection with normal saline in mix with indigocarmine, epinephrine and hyaluronate. Mucosal incision was performed over the anal side of the lesion, and after adequate submucosal dissection, clips were applied to attach the mucosal flap to the sleeve of proximal balloon and achieved retraction. The submucosa was adequately exposed for dissection using Dual knife jet. This enhanced submucosal dissection especially at one area with significant fibrosis. After the procedure, complete closure of the mucosal defect was performed by clips and assisted by the front balloon. The pathology confirmed intramucosal adenocarcinoma with clear resection margins.

Discussion: The DiLumen EIP device stabilized the environment within the colon with the double balloon and provide adequate retraction for performance of colorectal ESD.

\section{V172-ROBOTICS \& NEW TECHNIQUES-Flexible surgery}

\section{INTRODUCTION AND RESULTS AS A SAFE OPERATIVE PROCEDURE FOR ROBOTIC GASTRECTOMY}

\section{Kondo}

Surgery, Kobe City Medical Center General Hospital, KOBE, Japan

Background: Robotic surgery has been widely spread all over the world, but robotic gastrectomy is not common and difficult because of complex anatomy and wide-ranging operation fields. In addition, it had been performed only under a few high-volume centers for reasons of the limitation of national health insurance in Japan, which means medical expenses not covered by insurance. The situation was changed from this April, so we started robotic gastrectomy to reduce complications more rather than laparoscopic gastrectomy. We report results and aim to present the methods in detail using da Vinci Si Surgical System.

Methods: We place five trocars, one is umbilical endoscopy port, and other four ports are placed at the reverse trapezoid, almost fan-shaped. Using the arm number 3 , the organ can be lifted up so that sharp lymphadenectomy is able to be done by almost a scissor as the arm number 1 while applying the countertraction by the arm number 2 . In order to achieve a clear and bloodless lymphnode dissection while maintaining the oncological safety, we think not only the ultrasonic coagulating scissor but also the electrocautery of the scissor is very essential in robotic surgery. Less postoperative complication such as pancreatic fistula or pancreatitis might be derived from robotic surgery because we can avoid pressing the pancreas during the suprapancreatic dissection of lymph nodes. The Billroth I reconstruction can be performed using da Vinci EndoWrist stapler under stable and inflexible surgical fields without needing help of surgical assistant.

Results: From October 2017 to December 2018, 25 patients with gastric cancer were operated robotic gastrectomy, included 3 total gastrectomy. There was no conversion to open surgery and no conversion to other procedures derived from intraoperative complications, and the overall operation time is gradually decreasing from the $14^{\text {th }}$ case.

Conclusions: We are now on the way of learning curve shortening operation time, but robotic gastrectomy is no less safer and adequate than laparoscopic surgery. We will show our robotic procedures including lymphadenectomy around subpyloric and suprapancreatic area, and reconstruction with several important points in our video
V173-ROBOTICS \& NEW TECHNIQUES-Flexible surgery

SINGLE-PORT DISTAL GASTRECTOMY WITH D2 LYMPHADENECTOMY USING A NOVEL ARTICULATING GRASPER

S.H. Kang, Y.S. Cho, S.H. Min, Y.S. Park, S.H. Ahn, D.J. Park, H.H. Kim

Department of Surgery, Seoul National University Bundang Hospital, 13620, Korea

Purpose: This report describes the benefits and drawbacks in the use of a novel articulating device (Artisential), which has a multi-degree wrist freedom like the Davinci endowrist, in performing complete single-port D2 lymph node dissection (LND) in single-incision distal gastrectomy (SIDG).

Methods: The Artisential was used in performing SIDG with D2 LND for patients with advanced gastric cancer. All operations were performed by a single surgeon using a threedimensional camera and a passive scope holder in place of a scopist. The Artisential was used mainly in the 4sb and suprapancreatic LND, an area that is relatively far from the single port. In certain cases when the pancreas needed to be pushed down, such as obese male patients, the intraabdominal organ retractor was used to lift the tissue and the Artisential to push the pancreas. Operative results and short-term outcome were analyzed. Results: Twelve patients underwent the procedure without any intraoperative events, conversion to conventional laparoscopy, or surgery-related complications including postoperative pancreatic fistula. All patients underwent single port D2 LND by complete exposure of the portal and splenic vein. Mean operation time was $181.9 \pm 42.5$ mins. and mean number of retrieved lymph nodes was $61.8 \pm 11.4$. The Artisential was found to be useful in grasping the tissues behind the pancreas and the major arteries throughout most of the LND. The articulating motion also allowed the narrow single-port field of view to be clearly seen without the instrument body obstructing the camera.

Conclusion: The use of Artisential in SIDG appears feasible and reproducible, and is mandatory in performing a complete D2 LND in SIDG.

\section{V174-ROBOTICS \& NEW TECHNIQUES-Flexible surgery} MEDROBOTICS FLEX® ROBOTIC SYSTEM FOR TRANSANAL EXCISION OF A LARGE RECTAL NEOPLASM: THE FIRST EUROPEAN EXPERIENCE

\section{A. Arezzo, M. Morino}

Department of Surgical Sciences, University of Torino, TORINO, Italy

The video shows a case of Laterally Spreading Tumour of the rectum with preoperative benign histology, Paris Classification 0-Is G (granular type), uT0N0 EUS stage, Kudo type IV, NICE type 2. The neoplasm measured $6 \times 7 \mathrm{~cm}$, and extended from 6 to $12 \mathrm{~cm}$ from the anal verge, mainly located on the posterior wall. According to our local policy the indication was a transanal full-thickness excision. This was performed with the Medrobotics Flex ${ }^{\circledR}$ Robotic System, used here for the first time outside the United States.The system technology utilizes an articulated multi-linked scope that can be steered along non-linear, circuitous paths in a way that is not possible with traditional, straight scopes. The maneuverability of the scope is derived from its numerous mechanical linkages with concentric mechanisms. This enables surgeons to perform minimally-invasive procedures in places that were previously difficult, or impossible, to reach. With the Flex ${ }^{\circledR}$ Robotic System, Surgeons can operate through a single access site and direct the scope to the surgical target. Once positioned, the scope can become rigid, forming a stable surgical platform from which the surgeon can pass flexible surgical instruments. The system includes on-board 3D HD visualization. The Flex ${ }^{\circledR}$ Robotic System contains two working channels to accept a number of different surgical and interventional instruments including monopolar and bipolar electrodes, scissors and graspers for tissue manipulation.The video shows the introduction of the dedicated rectoscope, the connection of the flexible robot, and the way to operate the device performing a full-thickness excision, including suturing of the rectal defect by means of two running sutures by a V-Lock $3 / 0$ thread. While illustrating the technique the authors will comment pros and cons of the use of the device. 


\section{V175-ROBOTICS \& NEW TECHNIQUES-Liver}

\section{MAJOR HEPATIC SURGERY WITH THE DA VINCI XI: A ROBOT-ASSISTED LEFT HEPATECTOMY}

L. Morelli, M. Palmeri, N. Furbetta, G. di Franco, D. Gianardi, G. Stefanini, S. Guadagni, C. d'Isidoro, M. Bianchini,

G. di Candio, F. Mosca

Department of Surgery, University of Pisa, PISA, Italy

Background: Hepatobiliary procedures using a minimally invasive approachare demanding, especially in major hepatectomies. The use of da Vinci surgical System allows to overcome some of the kinematics limitations of the direct manual laparoscopy maintaining the potential advantages of a minimally invasive approach. We herein present a case of left hepatectomy and local lymphadenectomy for hepatocellular carcinoma, carried out with the use of the da Vinci Xi. Methodology: A 72-years old man with a long-lasting HBV chronic infection and CT scan and MRI finding of a 4-cm solid neoplasia of the left hepatic lobe and gallbladder stones, was operated with the da Vinci Xi platform. The patient was placed in a supine position, with $15^{\circ}$ antiTrendelenburg inclination. The trocars were positioned according with the Intuitive indication for the upper quadrants surgery.

Results: The procedure was successfully completed in $360 \mathrm{~min}$.At first, an intraoperative US scan with the use of Tile-Pro technology was done to determinate the tumor extension. The hepatic parenchyma transaction and the local lymphadenectomy were performed with monopolar scissors and bipolar grasps. The left hepatic vein section was performed with an endoscopic vascular stapler. There were no surgical complications or need for conversion to laparoscopy or laparotomy. The post-operative course was uneventful and the patient was discharged 5 days after surgery.

Conclusion: The da Vinci Xi can facilitate some technically demanding procedures and ultimately widen the range of application of minimally invasive surgery such as hepatic surgery. Besides the well- known advantages provided by robotic surgery on 3D imaging, increased range of motion and augmented surgical dexterity, one of the most interesting and innovative features of robotic technology is the digitalization of the operative view; furthermore the Tile-Pro multiinput display allows the surgeon a 3D view of the operative field along with the ultrasound exam for a precise understanding of anatomy and vascularity and of tumor location.

\section{V176-ROBOTICS \& NEW TECHNIQUES-Liver}

\section{ROBOTIC LEFT LATERAL HEPATECTOMY. PRESENTATION OF SURGICAL TECHNIQUE}

\section{H. Konstantinidis, C. Charisis}

Robotic General and Oncologic Surgical Department, Interbalkan Medical Centre, THESSALONIKI, Greece

Aims: Minimally Invasive Techniques have well established their role in the field of hepatic surgery. During the last few years, robotic surgery as well, as the latest innovation of minimally invasive procedures, takes its position in this particular field with the benefits of overcoming the limitations of conventional laparoscopy. Our aim is to demonstrate the advantages of robotic surgery in procedures of hepatectomies, on occasion of a robotic hepatectomy performed by our

Methods: We present video fragments of a robotic left lateral hepatectomy procedure in an elderly female patient with a symptomatic gigantic haemangioma of the left hepatic lobe. We emphasize on the technical aspects and the advantages that the surgeon gains applying the robotic techniques in such procedures.

Results: The procedure was completed with minimal blood loss and the patient presented an uncomplicated post-operative course, with discharge on the third postoperative day, minimal need of analgesics and full recovery.

Conclusions: The excellent three-dimensional and high quality visualization that the robotic system offers, combined with the flexibility and the accuracy of the robotic instruments (especially on suturing), provide to the surgeon an important aid, in order to avoid serious complications, such as intraoperative bleeding and post-operative bile leaks. The restriction of the limitations of conventional laparoscopy is far more beneficial and promising for the evolution and the future of minimal invasive liver surgery.

\section{V177-ROBOTICS \& NEW TECHNIQUES-Pancreas}

ROBOT-ASSISTED APPLEBY PANCREATECTOMY WITH THE DA VINCI XI FOR A LOCALLY ADVANCED CANCER

L. Morelli, S. Guadagni, D. Gianardi, N. Furbetta, G. di Franco, G. Stefanini, M. Palmeri, C. d'Isidoro, M. Bianchini, G. di Candio, F. Mosca

Department of Surgery, University of Pisa, PISA, Italy

Aims: The new da Vinci Xi surgical cart allows multi-quadrant and complex surgical interventions in a minimally invasive fashion. We present a case of robotic Appleby left pancreatectomy using this platform and its specific operating bed.

Methods: A 73-years old woman with CT scan finding of a 30-mm hypo-vascular neoplasm of the pancreas body underwent surgery with the use of the new da Vinci Xi with four arms upper quadrants trocar' disposition.

Results: The procedure was successfully completed in $285 \mathrm{~min}$. The pancreatic body was mobilized in order to expose the portal-mesenteric axis. The gland was transected using a robotic endo-stapler as well as the splenic vein. After evaluating the patency of collateral circles with intra-operative ultrasound, the common hepatic artery and the celiac artery were transected. Then we increased the right tilted position and the neoplasia was detached from the gastric body by a tangential gastric resection using the robotic endo-stapler. Finally, the operation was accomplished with the transection of the posterior attachment of the spleen and the pancreatic tail. No conversion or intra-operative complications were recorded. The post-operative course was uneventful and the patient was discharged 6 days after surgery.

Conclusions: The da Vinci Xi with its specific tools helps in performing challenging procedures such as Appleby operation for locally advanced pancreatic cancer. In our experience, the robotic endo-stapler permits the operating surgeon to directly control the transaction phase whereas the specific operating bed allows to perform minimally invasive multi-quadrant surgery and to obtain a better exposition of the operating field.

\section{V178-ROBOTICS \& NEW TECHNIQUES-Pancreas}

THE USE OF THE NEW ENDOWRIST VESSEL SEALER EXTEND AND THE DA VINCI TABLE MOTION FOR THE DA VINCI XI IN PANCREATO-DUODENECTOMY

L. Morelli, N. Furbetta, D. Gianardi, M. Palmeri, S. Guadagni, G. di Franco, G. Stefanini, M. Bianchini, G. di Candio, F. Mosca

\section{Department of Surgery, University of Pisa, PISA, Italy}

Background: The new technologies of da Vinci Xi system can facilitate minimally invasive surgery in challenging abdominal procedures such as pancreato-duodenectomy, making them easier and faster. We present a case of pancreatic neuroendocrine tumor (pNET) of the head of the pancreas treated with robotic Whipple procedure with the da Vinci Xi platform.

Methodology: A 76-years old man with a finding of a pNET of the head of the pancreas, underwent a robot-assisted pancreato-duodenectomy with the use of the new EndoWrist Vessel Sealer Extend and the da Vinci-Integrated Table Motion (dVTM) for the Xi system. Patient was placed in a lithotomy position. Robotic trocars were placed on the transverse umbilical line. Results: The Whipple procedure was successfully completed in $570 \mathrm{~min}$. Thanks to the dVTM the patient's position changed during the intervention to improve the exposure, with the instruments left inside the abdomen and without undocking the robot. The dissection of the pancreatic head from the portal vein and the section of the retroportal lamina were performed with the use of the EndoWrist Vessel Sealer device. A personal modified end-to-side pancreatojejunostomy was carried out, with 5/0 Prolene and Gore-Tex double layer suture. No intra-operative complications occurred and no conversions to laparoscopy or laparotomy were required. The postoperative course was uneventful.

Conclusions: The use of the new fully wristed Vessel Sealer Extend makes easier difficult maneuvers such as the fine dissection of the pancreatic head from the portal vein and the section of the retroportal lamina, enabling an optimized approach for vessels sealing and cutting and tissue bundles. Moreover, the dVTM allows patient's movements without undocking the system or removing instruments from the abdomen, enhancing the surgical workflow. 


\section{V179-ROBOTICS \& NEW TECHNIQUES-Pancreas}

LAPAROSCOPIC ASSISTED TRANSGASTRIC ENDOSCOPIC NECROSECTOMY - A NOVEL STEP-UP APPROACH IN THE TREATMENT OF PANCREATIC WALLED-OFF NECROSIS

\section{Jureller ${ }^{1}$, J. Yang ${ }^{2}$}

${ }^{1}$ General Surgery, Montefiore Medical Center, BRONX, NY, United States of America; ${ }^{2}$ Gastroenterology, Montefiore Medical Center, NEW YORK, United States of America

Background: Necrotizing pancreatitis is a devastating illness which can develop in up to $20 \%$ of patients who suffer from pancreatitis. It carries great morbidity with an associated mortality rate between 8 to $39 \%$. Many of these patients require drainage of fluid collections to treat sequela related to pain, per-os tolerance, and source control of sepsis if infected. The step-up approach to treatment of this disease has trended towards minimally invasive techniques, considering the morbidity of open debridement. As such, many centers have implemented the use of transgastric debridement via debridene As suchement via anticulate necrotic Current endoscopic accessories designed for polypectomy or foreign body extraction, for example, are not optimal for performing necrosectomy. To overcome this obstacle, additional access sites can be utilized to assist debridement. We describe the first laparoscopic assisted transgastric endoscopic necrosectomy through a percutaneous gastrostomy in a 59 year old male with infected pancreatic necrosis secondary to biliary pancreatitis.

Aim: To investigate the feasibility of utilizing gastrostomy access to assist in debridement during endoscopic necrosectomy.

Methods: The patient previously underwent an open necrosectomy and gastrostomy tube placement for acute emphysematous pancreatitis. Post-operatively, there was a persistent and enlarging $12 \mathrm{~cm}$ for acute emphysematous pancreatitis. Post-operatively, there was a persistent and enlarging $12 \mathrm{~cm}$
infected walled-off necrosis (WON). Therefore, endoscopic cystogastrostomy was performed using a infected walled-off necrosis
lumen-apposing metal stent.

lumen-apposing metal stent.
Results: Frank pus was evacuated. Initial endoscopic necrosectomy was technically challenging due to the large volume of solid necrotic tissue. Repeat endoscopic debridement utilized a surgical laparoscopic grasper via the gastrostomy site to aide solid debris extraction (video). This allowed for complete necrosectomy and resolution of the WON. The patient did well and was discharged subsequently.

Conclusion: This is another emerging minimally invasive technique in the step-up approach for debridement and drainage of WON. The use of the gastrostomy as a utility port for accessory instruments not only enhanced the technical aspects of the procedure but increased its efficacy as well. Further experience is needed to validate the utility and reproducibility of this technique.

\section{V180—ROBOTICS \& NEW TECHNIQUES-Pancreas}

DESCRIPTION OF THE MINIMALLY INVASIVE SURGICAL TECHNIQUE FOR PANCREATIC NECROSECTOMY OF ACUTE PANCREATITIS: VIDEORRETROPERITONEOSCOPY

C. Gonzalez De Pedro, E. Perea Del Pozo, J. Tinoco Gonzalez, L. Tallon Aguilar, V. Duran Muñoz-Cruzado,

A. Sanchez Arteaga, I. Ramallo Solis, F. Pareja Ciuro, J. Padillo Ruiz

\section{Cirugia General, Hospital Virgen Del Rocio, SEVILLA, Spain}

Objective: The presentation of the minimally invasive surgical approach for pancreatic necrosectomy guided by videoretroperitoneoscopy or VAR (Video Assisted Retroperitoneoscopic), established in our center, as one of the option of the step-up approach treatment for acute necrotizing pancreatitis (ANP) Methods: The placement of the patient on the operating table should be in decubitus, with right lateral inclination, at $20-30^{\circ}$ on the horizontal surface.

The pancreatic cell is approached using the drainage catheter previously placed by radiological control (ultrasound or CT) as a guide, which will allow access to the cavity with safety.

An incision of $3-5 \mathrm{~cm}$ is made around the previously placed catheter, crossing the subcutaneous cellular tissue and muscular fascias, dissolving the musculature. It continues in a blunt dissection, until a loss of tissue and muscular fascias, dissolving the musculature. It continues in a blunt dissection, until a loss
resistance is appreciated which generally coincides with the outflow of necrotic or purulent material.

Once the retroperitoneal cell is accessed, a $15 \mathrm{~mm}$ trocar is placed and a pneumoretroperitoneum is Once the retroperitoneal cell is accessed, a $15 \mathrm{~mm}$ trocar is placed and a pneumoretroperitoneum is
performed. The $15-\mathrm{mm}$ trocar allows the joint use of a $5 \mathrm{~mm}$ and $0^{\circ}$ optic and the surgical material that performed. The $15-\mathrm{mm}$ trocar allows

allows debridement and cleaning.

The aspiration and hydrodissection of the necrotic material, and the extraction of the solid component of the necrosis are proceeded.

Once the collection is drained and the necrotic material removed, a wash and drain system is placed, like a 3-way Foley type probe.

Conclusions: In conclusion, the VAR is an alternative surgical technique, valid and reproducible in the treatment of ANP, which offers comparable results and even superior, in some series, to those of open surgery, with satisfactory results in terms of morbidity and postoperative mortality.

\section{V181-ROBOTICS \& NEW TECHNIQUES-Solid organs}

LUNG SUBSEGMENTECTOMY BY COMPLETE VIDEOASSISTED THORACOSCOPIC SURGERY WITH PREOPERATIVE CT ANGIOGRAPHY AND INTRAOPERATIVE BRONCHOSCOPY

T. Kusu, S. Nagaoka, Y. Nakahara, M. Hirota, T. Matsumoto, H. Takemoto, K. Takachi, S. Oshima

Surgery, Kinki Central Hospital, ITAMI, HYOGO, Japan

Aim: Lung subsegmentectomy is suitable for small and deep, non-palpable lung nodules. Since it is difficul to intraoperatively detect the arteries, veins and bronchi of the subsegment, as well as the intersubsegmenta borders, complete video-assisted thoracic surgery (VATS) for lung subsegmentectomy is challenging. We use preoperative three dimensional CT to detect the arteries, veins and bronchi of the subsegment before conducting complete VATS subsegmentectomy, and perform intraoperative bronchoscopy to detect the bronchi and intersubsegmental borders. I would like to describe our experience of complete VATS combined subsegmentectomy for a non-palpable lung nodule.

Methods and results: The patient was a 67-year-old woman. During health screening, a small groundMethods and results: The patient was a 67-year-old woman. During health screening, a small ground-
glass opacity was observed in her right lung on chest CT. The nodule was $15 \mathrm{~mm}$ in diameter and was glass opacity was observed in her right lung on chest CT. The nodule was $15 \mathrm{~mm}$ in diameter and was
located in $\mathrm{S}^{2 \mathrm{~b}}$ (horizontal subsegment of the posterior segment) near $\mathrm{S}^{3}$ (the anterior segment). We preoperatively diagnosed the lesion as well-differentiated adenocarcinoma, and planned combined subsegmentectomy for $\mathrm{S}^{2 \mathrm{~b}}$ and $\mathrm{S}^{\mathrm{a}}$ (lateral subsegment of the anterior segment) of the right upper pulmonary lobe. Before the operation, the locations of vessels were confirmed by three-dimensional CT angiography. Video-assisted thoracoscopic surgery was performed using four ports: two $1 \mathrm{~cm}$ ports in the 8th intercostal space in the post-axillary line and in the angulus inferior scapulae line for the operator, a $4 \mathrm{~cm}$ port in the 4th intercostal space in the mid-axillary line for the assistant, and a $1 \mathrm{~cm}$ port for the camera in the 6 th intercostal space in the mid-axillary line. The $4 \mathrm{~cm}$ port was a o specimen. Intraoperative bronchoscopy was used for detecting the subsegmental bronchi. She was diag-
nosed with primary lung cancer (adenocarcinoma in situ, nonmucinous) postoperatively. The tumor was nosed with primary lung cancer (adenocarcinoma in situ, nonmucinous) postoperatively. The tumor was
pathologically graded as TisNOMO. No tumor recurrence has been noted in follow-up of twenty two pathological
months.

months.
Conclusions: The combination of preoperative three-dimensional CT angiography, intraoperative bronchoscopy and complete video-assisted thoracoscopic surgery can be used for performing lung combined subsegmentectomy.

\section{V182-ROBOTICS \& NEW TECHNIQUES-Solid organs}

PELVIC LAPAROSCOPY FOR BOTH DIFFERENTIAL DIAGNOSIS AND TREATMENT OF A PELVIC MASS, A USEFUL TOOL

M.V. Sosa, J. García, E. Pérez-Prudencio, A. Arango, M. Soto, A. Cabrera, S. Moral, C.J. Álvarez-Laso, P. Lora

Servicio de Cirugía General y Digestiva, Hospital Universitario de Cabueñes, GIJÓN, Spain

Aims: Minimally invasive surgery is increasingly widespread for the diagnosis and treatment of abdominal pathology. Laparoscopy is a diagnostic resource for those cases in which mass biopsy is not approachable through image-guided puncture, and is often therapeutic in the same act. It avoids the morbidity and mortality associated with laparotomy, favoring the early treatment of malignan processes.

Methods: We present a case of a 71 year old male who was incidentally diagnosed with an oval-shaped pelvic mass in the right lateral wall of the pelvis, adjacent to the vascular bundle of the right external iliac at its origin $(5 \times 3 \times 5$ centimeter), without sign of infiltration of surrounding structures. No other pathological findings on the Abdominal Computerized Tomography and Magnetic Resonance Imaging were found Due to is bcalization, it was found. Due to its localiziin possibilities. To provide a definitive diagnosis the patient was subjected to an elective laparoscopic resection of the tumor. Surgical procedure was performed using a 12 millimiter and two 5 millimeter, umbilicus for the optical system and operative on hypogastrium and left iliac fossa respectively. Acleavage plan between the tumorand rightiliac vesselswas found. The exeresis of the masswas achieved, and it was extracted using an Endo-bag $®$ through the umbilical port site. A drain was put in the surgical bed. Results: The patient had a short, uneventful post-operative course, being discharged on postoperative day 1. Pathological examination revealed a lymphatic node with metastasis of poorly differentiated carcinoma, with suspected urothelial lineage. Cystoscopy was performed with the finding of a centimeter lesion on the right ureteral orifice with calcifications on the surface. Biopsies were taken, confirming the bladder origin of the tumor.

Conclusions: Both diagnostic and therapeutic laparoscopy is useful on pelvic masses because of the Conclusions: Both diagnostic and therapeutic laparoscopy is useful on pelvic masses because of the
direct vision into this narrow anatomical space, especially in obese patients, providinga detailed view direct vision into this narrow anatomical space, especially in obese patients, providinga detailed view
that makes easier to isolate and spear the anatomical structures surrounding the tumor, minimizing the risk of tumor rupture and bleeding. 


\section{V183-ROBOTICS \& NEW TECHNIQUES-Technology}

VALUE OF ICG FOR URETER IDENTIFICATION DURING LAPAROSCOPIC LEFT COLECTOMY DUE TO DEEP ENDOMETRIOSIS AND COLONIC CANCER

T. García, S. Alonso, I. Alarcón, E. Licardie, T. Yang, A. Senent, F. López, A. Barranco, M. Socas, J. Padillo, S. Morales-Conde

Servicio De Cirugía General Y Digestiva, Unidad Innovación En Cirugía Mínimamente Invasiva (Cirugía General Y Digestiva), SEVILLA, Spain

Aim: Indocyanine Green (ICG)-enhanced fluorescence has been introduced initially in laparoscopic surgery to provide detailed anatomical information during laparoscopic cholecystectomy and to evaluate vascular supply to garantee correct anastomotic perfussion in order to reduce the risk of anastomotic leak. The uses of ICG are increasing, specially in hepatic and oncological surgery in order to identify centinel lymph node and lymphatic mapping.We propose the use of ICG imaging during complex laparoscopic colorectal resection in cases presenting ureter obstruction, to prevent iatrogenic ureteral injury.

Methods: We present a case of a 42 year old female previously diagnosed of pelvic endometriosis with severe pain and symptoms related with episodes of pseudo-occlusion .A colonoscopy was performed finding sigmoid cancer in an area of endometriosis in a narrow colon with difficulties to perform a complete colonoscopy that could be related to the process of pseudo-occlusion. The biopsy was informed as an adenocarcinoma.The CT-scan showed a dilatated left ureter in an area next to the sigmoid colon.We propose a preoperative strategy with a bilateral double $\mathrm{J}$ stent insertion, finding a ureter obstruction caused by the endometriosis.ICG was injected through the ureteral catheter, guiding us during the surgery to avoid a iatrogenic ureteral injury.

Results: A laparoscopic left colectomy was performed. The ICG allows us to follow the ureter during the surgery, disecting the colon properly from the area attached to the ureter. The prestenotic area of the ureter was marked dilatated up to two centimeters allowing the ICG to identify it from the anatomic structures of the areas and guarateeing that there was not spill of ICG out of the ureter avoiding a postoperative leak of urine.

Conclussions: When tumors, or another entities like endometriosis, produce a ureteral occlusion, ICG could be injected through a J stent, allowing us to identify and to avoid an injury.ICG fluorescence imaging is a safe, cheap, and effective tool to increase visualization during surgery, offering additional information of the anatomy in colorectal surgery.

\section{V184-ROBOTICS \& NEW TECHNIQUES-Technology}

\section{PREOPERATIVE PLANNING EVOLUTION FOR THE} TREATMENT OF SPLENIC ARTERY ANEURISM

\section{A. Peri ${ }^{1}$, L. Pugliese ${ }^{1}$, E.M. Marone ${ }^{2}$, N. Mineo ${ }^{1}$, F. Argenti ${ }^{1}$, M. Botti ${ }^{1}$, S. Marconi ${ }^{3}$, F. Auricchio ${ }^{3}$, A. Pietrabissa ${ }^{1}$}

${ }^{1}$ General Surgery 2, IRCCS Policlinico San Matteo, PAVIA, Italy; ${ }^{2}$ Vascular Surgery, IRCCS Policlinico San Matteo, PAVIA, Italy; ${ }^{3}$ Departement of Civil Engineering and Architecture, University of Pavia, PAVIA, Italy

In this video we are going to show three cases of the robotic treatment of splenic artery aneurism and the evolution of the technology that we relied on for the preoperative planning and intraoperative navigation. Our preoperative evaluation evolved from a tridimensional virtual reconstruction with augmented reality to patient specific anatomical 3D printed models, initially made of rigid materials and afterwards made of malleable materials, in order to reproduce hollow anatomical structures such as vessels, feasible to simulate the planned surgical plan. The choice of a robotic approach, in selected cases, allowed to restore the continuity of the splenic artery after the exclusion or excision of the aneurism, in order to preserve the spleen.

\section{V185-ROBOTICS \& NEW TECHNIQUES-Technology}

SURGICAL TREATMENT OF TUMOR-INDUCED OSTEOMALACIA: TUMOR REMOVAL GUIDED BY INDOCYANINE GREEN FLUORESCENT ANGIOGRAPHY

A. Spota ${ }^{1}$, L. Baldari ${ }^{1}$, M. Ballabio ${ }^{1}$, M. de Francesco ${ }^{2}$, S. Macina ${ }^{2}$, S. Petrucci ${ }^{1}$, E. Cassinotti ${ }^{1}$, L. Boni ${ }^{1}$

${ }^{1}$ General Surgery, University of Milan-Policlinico Hospital, MILAN, Italy; ${ }^{2}$ General Surgery, Policlinico Hospital, MILAN, Italy

Aims: Tumor-Induced Osteomalacia (TIO) is a rare paraneoplastic syndrome in which patients presents bone pain, fractures and muscle weakness caused by the fibroblast growth factor 23 (FGF-23), a phosphate and vitamin D-regulating hormone. In TIO, FGF-23 is secreted by mesenchymal tumors that are usually benign but small and difficult to locate. When medical treatment is unsuccessful, surgical treatment is indicated and conclusive.This video shows our technique for TIO surgical treatment guided by indocyanine green (ICG) fluorescent angiography.

Methods: The patient is an 81-years-old woman with TIO confirmed by blood sampling of FGF23, gallium-68 PET/CT scan and abdominal CT scan which identified a highly vascularized $8 \mathrm{~mm}$ nodule in the mesentery along the ileo-colic vascular axis. The patient was scheduled for a diagnostic laparoscopy with TIO removal.The patient was placed in supine position. Three trocars were introduced in the left quadrants. Identified the last ileal loop, following ileo-colic vessels, a small mesenteric bulge was found. The ICG fluorescent angiography confirmed the localization of the ipervascularised nodule and helped to define its edges. The nodule was removed through monopolar energy and the hemostasis was optimized through bipolar energy. The specimen was extracted through an endobag using one of the trocar access.

Results: The postoperative course was uneventful and the patient was discharged on postoperative day 4. Histopathological examination showed an extrasurrenalic paraganglioma.

Conclusion: TIO are often difficult to locate for surgical removal. ICG fluorescent angiography allows to facilitate TIO localization and removal. The minimally invasive technique decreases perioperative morbidity and mortality. Laparoscopic removal guided by ICG angiography should be considered when TIO needs to be removed and is difficult to locate.

\section{V186-ROBOTICS \& NEW TECHNIQUES-Technology LAPAROSCOPIC RESECTION OF VOLUMINOUS LEFT PARAORTIC PARAGANGLIOMA}

M. De Francesco, S. Petrucci, L. Baldari, A. Spota, S. Macina, M. Ballabio, E. Cassinotti, L. Boni

General Surgery, Policlinic Hospital of Milan, MILAN, Italy

Aim: This video shows our technique to perform laparoscopic resection of a voluminous lef paraaortic paraganglioma.

Methods: The patient is a 16-years-old man with a recent medical history offever, lumbar pain and haematuria.Abdomen CT scan, performed during admission at emergency department, revealed a $8 \times 7 \mathrm{~cm}$ left paraaortic retroperitoneal mass with pseudo-aneurysm. After procedure of angiographic embolization (with spermatic artery sparing), the patient was scheduled for a laparoscopic resection of paraaortic tumor.

The patient was placed in the right flank position. Three trocars $(10 \mathrm{~mm})$ in the abdomina midline and one trocar in left hypocondrium were placed. At initial examination of the abdominal cavity, voluminous left paraaortic mass arising in the contest of left mesocolon was found, dislocating posteriorly kidney vessels. The parietal peritoneum was divided and the paraaortic lesion was dissected on the aortic plane from medial to lateral and from down to up, preserving the inferior mesenteric vessels; the mobilization was carried on to splenic vein. The vessels, supplying the mass and arising directly from aorta, was isolated and taped with vascular clips. On the inferior margin of the lesion a large vessel, probably connected with previously embolized pseudoaneurysm, was dissected with vascular linear stapler. The mobilization was completed through difficult dissection from aortic plane and mesocolic posterior surface. The colonic perfusion was verified with fluorescence angiography. Specimen was extracted through an endobag.A drain was left in pelvis. postoperative day 4.Histopathological examination showed a morphological and immunoistochemical pattern for benign paraganglioma.

Conclusion: Laparoscopic resection of paraaortic paragangliomas is feasible by skilled surgeon. The minimally invasive technique decreases perioperative morbidity and mortality. Carefu preoperative planning and surgeon's experience with vascular dissection and visceral mobilization are mandatory for a good outcome. 


\section{V187-ROBOTICS \& NEW TECHNIQUES-Technology}

ROBOTIC ASSISTED MINIMALLY INVASIVE ESOPHAGECTOMY (RAMIE) USING DA VINCI XI SYSTEM FOR TREATMENT OF SQUAMOUS ESOPHAGEAL CARCINOMA

\section{P.W.Y. Chiu, H.C. Yip, S.M. Chan}

Department of Surgery, The Chinese University of Hong Kong, HONG KONG

Introduction: We previously reported the use of Da Vinci Robotic Surgical System for performance of Robotic assisted minimally invasive esophagectomy (RAMIE) for treatment of squamous esophageal carcinoma in 20 patients. In this case, we reported the use of new Da Vinci $\mathrm{Xi}$ system for performance of RAMIE with 3 field lymphadenectomy

Method and results: This is a 62 years old gentleman who had squamous esophageal carcinoma located at mid esophagus. 8 weeks after neoadjuvant chemoradioltherapy, he received RAMIE. The procedure was performed with one lung ventilation. Firstly the mediastinal pleural was opened and the arch of azygos was controlled with hem-o-lock. Further dissection of the superior mediastinal nodes was performed following the right vagus nerve to the right recurrent laryngeal nerve. After RLN nodal dissection, the upper thoracic esophagus was mobilized and slinged to expose the left recurrent laryngeal nerve for nodal dissection. The thoracic duct was identified and controlled. Lymphadenectomy was performed with en-bloc resection of paraesophageal, para-aortic, subcarinal, paratracheal and pericardial nodes. The gastric mobilization was performed by laparoscopic approach and the esophago-gastric anastomosis was performed over left

Results: The total operative time was $520 \mathrm{~min}$, while the thoracic robotic part was $300 \mathrm{~min}$. The patient had transient left recurrent nerve palsy with compensation and he could tolerate diet 7 days after the procedure. He was discharged from hospital on 12 days.

Conclusion: RAMIE with three field lymphadenectomy could be safely performed using Da Vinci Xi system.
V189-SOLID ORGANS-Adrenal

COMPARING ANTERIOR TRANSABDOMINAL LAPAROSCOPIC APPROACH VERSUS POSTERIOR RETROPERITONEAL ENDOSCOPIC APPROACH, FOR ADRENAL GLAND TUMORS

E. Delgado-Oliver ${ }^{1}$, R. Diaz del Gobbo ${ }^{2}$, M. Manyalic-Blasi ${ }^{3}$, M.A. Lopez-Boado ${ }^{3}$, M. Valentini ${ }^{4}$, R. Rull ${ }^{3}$, C. Fondevila ${ }^{3}$, O. Vidal ${ }^{3}$

${ }^{1}$ General Surgery, Hospital Universitario Miguel Servet, ZARAGOZA, Spain; ${ }^{2}$ General Surgery, Hospital Mutua De Terrassa, BARCELONA, Spain; ${ }^{3}$ General Surgery, Hospital Clinic De Barcelona, BARCELONA, Spain; ${ }^{4}$ General Surgery, Fundació hospital asil de granollers, BARCELONA, Spain

Aims: Recent advances in technology and surgical instruments have allowed the development of better and less invasive approaches, such as natural orifice transluminal endoscopic surgery (NOTES) or single incision laparoscopic surgery (SILS), with the consequent decrease of perioperative morbidity and better cosmetic results. Thanks to these advances, posterior retroperitoneal adrenalectomy, known for years, is nowadays feasible, safe and reproducible technique. The objective of this presentation is to describe and compare laparoscopic transabdominal approach versus posterior retroperitoneal endoscopic approach, for adrenal gland tumors in both locations (right and left).

Methods: Four patients undergoing adrenalectomy, two of them with right adrenal pathology and two left. Minimally invasive access, endoscopic approach, is exposed in all of them. Results: In the first two surgeries, right gland is shown. Initially, transabdominal approach, which requires mobilization and separation of the liver to access the retroperitoneal space and subsequent proceed to adrenal extirpation. Later, right retroperitoneal approach is observed, with a meticulous sealing of the adrenal vein prior to complete the dissection of the gland, despite the small cavity created by $\mathrm{CO} 2$. In the second part, both left adrenal approaches are exposed. Transabdominal pathway is necessary to mobilize left colon and spleen to access a narrow space above the upper edge of the pancreas to locate adrenal gland. This is very different in posterior adrenal approach.

Conclusions: Posterior or retroperitoneal approach is feasible and safe, allowing access to adrenal glands, located in retroperitoneal space, without across peritoneal cavity and its disadvantages. Colon and small intestine mobilization is not necessary, with a lower rate of intestinal lesions and postoperative ileus. In the same way, liver or spleen mobilization is avoided.

\section{V190-SOLID ORGANS-Adrenal}

\section{CONGENITAL ABSENCE OF RIGHT ADRENAL VEIN DURING LAPAROSCOPIC ADRENALECTOMY FOR PHEOCHROMOCYTOMA}

\section{S. Pearlstein, D. Ranev, J. Teixeira}

Surgery, Northwell Health Lenox Hill Hospital, NEW YORK, United States of America

Aims: When performing a laparoscopic adrenalectomy, especially in the setting of pheochromocytoma, one of the most important steps is to gain control of the adrenal vein early on in the procedure before great manipulation of the adrenal gland. We present the case of a 78 year old female with episodic headaches and tachycardia and severe uncontrolled hypertension, found to have elevated plasma and urine metanephrines with CT scan localizing a $1.2 \mathrm{~cm}$ right sided adrenal nodule. The patient was prepared preoperatively with phenoxybenzamine until mildly orthostatic with dry mucous membranes and was taken for laparoscopic right adrenalectomy. Methods: After positioning our patient in left lateral decubitus, ports were placed inferior to the costal margin. The right lobe of the liver was mobilized and retracted cephalad and the IVC was exposed. Careful and meticulous dissection was carried up the IVC, however no main adrenal vein was encountered. The adrenal gland was then dissected circumferentially and was removed in an endoscopic retrieval bag. There was no difficulty in hemostasis and the patient was deemed to be hemostatic prior to withdrawal of the ports and extubation.

Results: Our patient had no issues with hemodynamic stability and her blood pressure was within normal ranges during and following the case. Her hemoglobin was stable postoperatively with 11.1 immediately post op and 9.9 on discharge. Her pre-op hemoglobin was 11.7.

Conclusions: Our video demonstrates a right adrenal gland that was congenitally missing a main adrenal vein. It is very possible that small venous branches were taken with dissection however we believe this report to be important to note in the literature for surgeons performing adrenalectomy. 


\section{V191-SOLID ORGANS-Adrenal}

\section{A MINIMALLY INVASIVE APPROACH TO AN ADRENAL CYST}

J. Hernandez Gutierrez, A.F. Aranzana Gomez, J.S. Malo Corral, B. Muñoz Jimenez, R. Lopez Pardo, P. Toral Guinea, G. Krasniqi

\section{General Surgery, C.H.Toledo, TOLEDO, Spain}

Aims: Adrenal cysts are the most frequently identified adrenal cysts, although they are a rare entity. Typically they are presented by abdominal pain or palpable mass, but nowadays, cystic lesions of the adrenal gland are more often discovered incidentally by radiologic studies. Adrenal cysts have an extensive differential diagnoses, which makes a difficult definitive diagnosis and a difficulty in later management. The management of an adrenal cyst can be summarized in three fundamental pillars: discard the functional status of the cyst, evaluation of eventual malignancy by images, and avoid possible complications (hemorrhage, infection), especially in large cysts .

Methods: Clinical case: A 45-year-old male patient, with no history, studied for nonspecific pain in the right hypochondrium, without other accompanying symptoms. An abdominal ultrasound was performed, a cystic lesion in HCD without being able to identify the origin was seen. Complementary explorations of interest are shown (CT), the biochemical study discards functionality of the lesion, negative serology for hydatidosis. The minimally invasive approach is the gold standard in the surgical treatment of adrenal pathology, so a laparoscopic approach is proposed for this patient.

Results: Full minimally invasive approach in left lateral decubitus position: 4 trocarslateral laparoscopic transabdominal approach. Identification of a large cystic tumor $(11 \mathrm{~cm}$.) which depends of the adrenal gland, medial dissection of the inferior vena cava, identification, clipping and section of the right adrenal vein, dissection of the tumor, requiring a puncture for its management, with intraoperative cytology to rule out malignancy, complete resection of the lesion with the gland, with drainage placement. Correct postoperative recovery, discharge on the 2 nd postoperative day. Definitive result of the specimen: Epithelial cyst. Asymptomatic at 1 year of follow-up.

Conclusion(s): The laparoscopic approach is a valid alternative in the treatment of large adrenal cystic lesions, the technical difficulty of this procedure, increased by the management of tumors, does not contraindicate this type of approach.

\section{V192-SOLID ORGANS-Gynaecology}

\section{ENDOSCOPIC RESECTION OF A DEEP ABDOMINAL WALL ENDOMETRIOSIS NODULE}

H. Cardoso-Louro ${ }^{1}$, L. Lencastre ${ }^{1}$, S. Graça ${ }^{1}$, A. Fonte-Boa ${ }^{1}$, H. Ferreira ${ }^{2}$, J. Vilaça ${ }^{1}$

${ }^{1}$ General Surgery, Hospital da Luz Arrábida, PORTO, Portugal; ${ }^{2}$ Gynecology, Hospital da Luz Arrábida, PORTO, Portugal

Aims: Endometriosis is a high incidence disease (approximately $10 \%$ of women) with a large impact on women's quality of life and fertility. Endometriosis nodules surgical treatment is necessary every time there is evidence of active disease.

The aim of this video is to present a minimally invasive technique for the resection of an endometriosis nodule from the abdominal wall.

Methods: A 41-years-old woman, with past history of endometriosis and a c-section, presents at the office with a palpable nodule at the rectus abdominis left lateral border, close to the umbilical scar. She had complaints of exuberant catamenial pain and magnetic resonance imaging (MRI) showed a $33 \mathrm{~mm}$ nodule compatible with endometriosis depot. This technique uses 3 trocars $(1 \times 10+2 \times 5 \mathrm{~mm})$ placed at the Pfannenstiel scar. Stepby-step as follows: (i) dissection of the pre-aponeurotic plane and isolation of the lesion (ii) lesion excision and its removal with sac (iii) closure of the aponeurotic defect braded suture.

Results: The post-operative period was uneventful and the patient was discharged home at post-operative day one.

The aesthetic result was excellent and the patient was asymptomatic one month after the procedure.

Conclusion: Endometriosis of the abdominal wall is related to previous c-section, is a rare event (incidence $0.1-0.4 \%$ ) and usually located in the subcutaneous fat underlying the scar. The presence of nodules in the depth of the muscle is much uncommon and particularly in this clinical case, the nodule was located $8 \mathrm{~cm}$ cephalad from the previous Pfannenstiel scar. This technique seems easy and reproducible in the authors' opinio.

\section{V193-SOLID ORGANS-Gynaecology}

\section{LAPAROSCOPIC APPROACH FOR THE TREATMENT OF MULTIPLE UTERINE FIBROMATOSIS OF THE PELVIS}

\section{H. Konstantinidis, C. Charisis}

Robotic General and Oncologic Surgical Department, Interbalkan Medical Centre, THESSALONIKI, Greece

Aims: General surgeons often face gynecological pathological findings, either along with other abdominal pathology, or as primitive cases that need laparoscopic expertise. With this particular presentation, our goal is to demonstrate the essential laparoscopic skills and the basic operative strategy that a general surgeon should be familiar with, in order to manage such cases. The presentation is made on occasion of a woman with multiple uterine fibromatosis of the pelvis, who was treated by our team.

Methods: We present video fragments of the laparoscopic excisional procedure for multiple uterine fibromyomatosis of the pelvis, highlighting the proper strategy in order to conclude the operation effectively and uneventfully, in a minimally invasive fashion. Results: Patients with multiple, large or other complex forms of uterine or pelvic fibromas can effectively be treated with a minimally invasive approach, with minimal blood loss, very fast recovery and minimal postoperative pain and complications.

Conclusions: The laparoscopic expertise of many general surgeons can prove itself crucial to the direction of the establishment of minimally invasive techniques for the treatment of complex gynecological pathology, that even up to this day are quite often managed with the conventional open surgery.

\section{V194-SOLID ORGANS—Gynaecology}

\section{LAPAROSCOPIC SURGERY IN NON-OBSTETRIC EMERGENCIES DURING PREGNANCY}

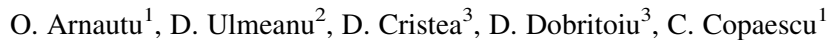

${ }^{1}$ Surgery, Ponderas Academic Hospital, BUCHAREST, Romania; ${ }^{2}$ Surgery, Regina Maria Private Healthcare-Baneasa Hospital, BUCHAREST, Romania; ${ }^{3}$ Pediatric Surgery, Ponderas Academic Hospital, BUCHAREST, Romania

$2 \%$ of pregnancies require emergency surgery for a non obstetric indication, including acute appendicitis, cholecystitis, adnexal torsion, choledoco-lytiasis, hernias, intestinal obstructions, oncologic pathology or other less frequent indications. Laparoscopic approach is the preffered surgical option for the patologies presented above.

Aims: to present the technical particularities and to analyze the outcomes of the emergency operations in pregnant women operated in hospital.

Method: A retrospective study including all the pregnant women operated in our hospital between 2015-2018 was performed. The preoperative workup and the surgical indication was discussed by a multidisciplinary medical team. The anesthesic and the obstetrical risk and their management was evaluated and specifically planned for each patient. The intraoperative and post-operative outcomes were recorded.

Results: 12 patients with gestational age between 16 weeks and 32 weeks who underwent emergency laparoscopic procedures were included in the study. Out of the 12 cases we have performed 5 appedectomies, 3 cholecystectomies, 4 adnexal torsions. With a 75 min mean operating time, we had no major intraoperative complications; the technical challenges are presented and discussed. The hospital stay was 1,5 days (1-3 days). No major complications were associated with the laparoscopic approach in these cohort. One pre-term labour in a 31 weeks gestational patient was post-operatively encountered.

Conclusion: Laparoscopic surgery can be the first option for pregnant woman with non obstetrical surgical emergencies; Challenges in diagnostic, management and surgical techniques of the multidisciplinary team are expected. 


\section{V195-SOLID ORGANS-Gynaecology}

\section{LAPAROSCOPIC TRANSPERITONEAL LUMBO-AORTIC LYMPHADENECTOMY FOR GYNAECOLOGICAL CANCER}

\section{K. Kothonidis}

General Surgery, APP SAMBRE ET MEUSE/ CHR VAL DE SAMBRE and CHU UCL NAMUR/ GODINNE, SAMBREVILLE, Belgium

The objective of this presentation is to demonstrate step by step the technique to the oncologic surgeon and gynaecologist in training, including some tips and pitfalls. This is a laparoscopic transperitoneal approach in a woman with advanced cervical cancer (FIGO IB2) that will be treated with exclusive radio-chemotherapy. The purpose of the laparoscopic lumbo-aortic ranges from 12 to $22 \%$ (depending of the existence of pelvic fixation or not).

The limits of this lymphadenectomy are: both ureters as the lateral limit of the dissection, iliac bifurcation as the caudal limit and renal veins as the cranial one. Since the tumour is cervical and not ovarian, both ovarian veins are not resected.

In the pathologic report, 25 lymph nodes were examined free of cancer spread. The patient have had a radio-chemotherapy with restriction of the irradiation field on the pelvis.

Lymphocele is a frequent complication that only sometimes needs treatment ranging from dietary changing to percutaneous drainage. If conversion to laparotomy for bleeding this technique loose its benefice but this is a rare complication. This technique is feasible and safe but requires advanced laparoscopic skills. The alternative approach is extra-peritoneal lymphadenectomy and may be helpful if history of previous abdominal surgery.

Other indication except cervical cancer FIGO > IB2 are: Endometrial cancer with type 2 histology (clear cell carcinoma, papillar/serous, carcinosarcoma of any FIGO stage, endometrioid adenocarcinoma in high risk FIGO I, in FIGO II directly or only if pelvic lymphadenectomy is positive and in stage FIGO III and IV. Ovarian cancer FIGO I and II (upgrading stage, possible survival benefit). lymph node staging is to define the irradiation field. In this indication false negative in PET CT

\section{V197-SOLID ORGANS-Spleen}

\section{LAPAROSCOPIC DIAGNOSTIC SPLENECTOMY: SPLENIC SARCOIDOSIS MIMICKING LYMPHOID DISEASE}

J.E. Sanchez Lara, J. Trebol Lopez, S. Alonso Batanero,

T. Rubio Sanchez, L. Muñoz Bellvis, G. Franch Arcas, M. Gonzalez Sanchez

General Surgery, Hospital Universitario de Salamanca, SALAMANCA, Spain

Sarcoidosis is a multisystem disease of unknown etiology characterized by the formation of noncaseating granulomas. Sarcoidosis should be considered in the differential diagnosis of lymphoid disease. Indications for diagnostic splenectomy includes a suspicion of a neoplasic process. The less invasive laparoscopic approach is the gold standard.

Case report: A 64-year-old female was referred to a General Surgery department to complete a study to rule out lymphoid neoplasia. Followed by Hematology for cytopenias. Biopsy of bone marrow and adenopathies were negative for lymphoid process. Patient presented CT with multiple solid $(8-10 \mathrm{~mm})$ lesions in spleen, in thorax showed no pathological changes.

Laparoscopic splenectomy was performed. Access with optical trocar, in mammary line. 3 triangle 5-mm trocars after pneumo under vision. Section with Ligasure of gastroesplenic ligament with short vessels and phrenic-splenic ligament. Identification and preservation of pancreatic tail. Section of splenic vessels at hilar level (branches) with Ligasure. Lower pole release. Release of posterior part with gerota and diaphragm. Incision by aid helps in bag without fragmenting. Review of hemostasis, extraction of trocars under direct vision. Intraoperative findings: Spleen with normal external appearance, not megalic.

Postoperative evolution: Satisfactory. First hours without incidents and with analytical control without anemization. Tolerance and mobilization starts without incidents.

The histopathology report shows granulomas formed by epithelioid histiocytes with the presence of multinucleated giant cells of the foreign body type, in some perisinusoidal granulomas the giant cells with the presence of asteroid bodies in their interior. The material has been revised with the extension of special studies.

Conclusion Epithelioid Granulomatosis, Non-Necrotizing, Which Suggests Sarcoidosis.

The procedure lasted $180 \mathrm{~min}$. The hospital discharge was on the next postoperative day and no complication was registered.

Conclusion: Splenectomy can be performed in a classic way, but at present the less invasive laparoscopic approach is the gold standard. Indications for splenectomy include splenic tumours of unknown origin, suspicion of a neoplastic process, and splenomegaly. Sarcoidosis should be considered in the differential diagnosis for lymphoid disease. Postoperative pathological examination confirms the diagnosis.

\section{V198-SOLID ORGANS-Spleen}

\section{V196-SOLID ORGANS-Spleen \\ LAPAROSCOPIC SPLENECTOMY FOR A SUSPICIOUS SPLENIC LESION}

\section{Ferrer Sales, D. Parente, M. Neves, P. Alves, N. Rama, V. Faria} General Surgery, Centro Hospitalar de Leiria, LEIRIA, Portugal

Objectives: Although extremely rare, isolated splenic metastases are being increasingly diagnosed due to the improvement of imaging, survival times, and surveillance of oncologic patients. This video alerts to the growing diagnostic dilemma with primary lesions of the spleen, particularly in patients with history of cancer, and reviews the laparoscopic splenectomy 'step-by-step'. Case-report: 58-year-old male patient diagnosed with rectal cancer (G2adenocarcinoma at $4 \mathrm{~cm}$ of the anal verge) after a colonoscopy for rectal bleeding. Thoracic and abdominal CTscan and pelvicMRI, showed a cT3N1 lesion, without distant metastases, except for a $15 \mathrm{~mm}$ suspicious splenic lesion. CEA-12.2 ng/mL. After neoadjuvant therapy, a complete response was verified at the 8 th week post-CRT with a stable splenic lesion, and a 'watch-and-wait' program was initiated with no evidence of disease at the 3rd month. PET-CTscan did not show active metabolic features, despite an increase in the splenic lesion. In MDT, elective laparoscopic splenectomy was proposed and afterwards performed uneventfully. With the patient in semi-right lateral tilt, we approached the spleen inferiorly by dividing the splenocolic ligament. Then we continued upwards, dividing the gastrosplenic ligament and exposing the splenic hilum, which was then carefully dissected, clipped and divided. Finally the splenorenal ligament was divided and the spleen was extracted within an endobag, through a small Pfannenstiel incision. Pathologic report revealed a splenic lymphangioma. The patient is currently under a 'watch and wait' protocol surveillance with no signs of regrowth or relapse disease after 1 year and 3 months of follow-up. Conclusion: One out of five colorectal carcinomas are metastatic at their presentation. Isolated metastases to sites other than liver, lung or axial skeleton, are extremely rare, but can be found in the spleen. Although the rare splenic secondary involvement is usually associated with breast, lung, melanoma, and gynecologic malignancies, if we consider solitary splenic metastases, colorectal and ovarian carcinomas are important sources. Also, imaging including percutaneous biopsy, is frequently insufficient to clarify the nature of splenic lesions. For all these reasons, the decision-making process about this issue can be a true challenge, and will probably end up with laparoscopic splenectomy. Therefore, surgeons must be familiarized with a standardized technique.

\section{LARGE SPLEEN CYST: LAPAROSCOPIC SPLENECTOMY}

A. Lo Conte, G.V. Cunsolo, M. Pezzatini, M. Gasparrini, M. Gasparrini

Week-Day Surgery, University, Sapienza, Ospedale Sant'Andrea, ROME, Italy

Aims: We describe an interesting case of a female patient affected by a suspected Echinococcus Granulosus large cyst of the spleen.

Methods: A 42 years old woman complained abdominal pain and a sense of gravity in the upper left abdominal quadrant. Computed tomography scan(CT-scan) showed a 13 centimetre $(\mathrm{cm})$ cys of the spleen with thickness of the wall and contrast enhancement uptake referred to an Echinococcus Granulosus cyst. The sierological blood test assessment, antigens and antybody markes, for Echinococcus Granulosus infection was negative. A laparoscopic procedure was planned. The patient was positioned on the right flank, four trocars were inserted along the left subcostal region of the abdomen: one 12 millimetre $(\mathrm{mm})$ trocar for camera, one $10 \mathrm{~mm}$ for the assistant, and two of $5 \mathrm{~mm}$ for instruments. A periombelical minilaparotomy was performed for the specimen extraction.

Results: Post-operative course was uneventful. Patient was discharged in third post-operative day. Istopathological exam showed a simple epithelial cyst of the spleen.

Conclusions: Laparoscopy is safe and feaseable in case of large cyst of spleen in condition of unclear nature of the cyst. Laparoscopy permits to explore the abdominal cavity and to assess the cyst characteristics in a lack overlap between the radiological exam and blood test examination. 


\section{V199-SOLID ORGANS-Spleen}

\section{LAPAROSCOPIC SPLENECTOMY FOR RECURRENT SPLENIC CYST AFTER LAPAROSCOPIC MARSUPIALIZATION}

\section{Leško, P. Pažinka, J. Radonak, M. Šoltés}

I.department of Surgery, University Hospital of L.Pasteur, KOSICE, Slovakia

We describe laparoscopic splenectomy for recurrent splenic cyst after laparoscopic marsupialization and partial resection of splenic cyst. The patient was a 31-year-old woman with abdominal discomfort and with a 24-cm palpable mass in the left upper and inferior quadrant. She undergone 9 years ago in another country a laparoscopic operation for splenic cyst. Abdominal computer tomography revealed a cystic lesion of the spleen with concomitant huge splenomegaly. Serology and oncological marker were negative. We performed laparoscopic splenectomy for the recurrent splenic cyst. The operation took $180 \mathrm{~min}$. Histologic examination of the resected spleen revealed a chronic hematoma. The patient had no abdominal symptoms during 12 months of follow-up. Postoperative long term follow-up and examination by ultrasound or computed tomography is required after surgical treatment for splenic cyst to exclude the possibility of recurrence after spleen-preserving surgery.

\section{V200-SOLID ORGANS-Spleen}

\section{MININVASIVE TREATMENT OF MEGASPLEENS: A CASE FOR HAND-ASSISTED SURGERY}

\author{
A. Peri, M. Botti, L. Pugliese, F.C. Bruno, N. Mineo, V. Gallo, \\ S. Malabarba, G. Costantini, A. Pietrabissa
}

\section{General Surgery II, IRCCS Policlinico San Matteo, PAVIA, Italy}

Hand-assisted surgery is a recognized technique that combines the advantages of laparoscopic approach with the tactile feedback of the laparotomic one. It proved beneficial especially for the treatment of megaspleens due to lymphoma localization, thanks to safer handling of splenic vessels, major bleeding control and more effective detachment of superior splenic pole from the diaphragmatic dome.

Here we show an hand-assisted splenectomy for megaspleen reaching the omolateral anterosuperior iliac spine due to lymphoproliferative disease, in which the hand, inserted through a right subcostal minilaparotomy, was very useful during the dissecting manoeuvres, the splenic artery recognition and ligation and the isolation of the superior pole of the spleen from the gastric fundus and diaphragm. In any case of huge spleens, the specimen bagging is very difficult to perform in a pure laparoscopic way, not to mention the inexistence of capable endobag; besides, a minilaparotomy would be necessary for the spleen extraction. Hand-assisted approach allow to overcome this not underestimable technical difficulty, reducing operative time with similar aesthetic and functional results to that of laparoscopic approach.

\section{V201-SOLID ORGANS-Thyroid}

TRANSAXILLARY ENDOSCOPIC LEFT LOBECTOMY WITH INTRAOPERATIVE ULTRASOUND-GUIDED ASPIRATION FOR A 4CM SOLID/CYSTIC THYROID NODULE_A CASE REPORT

M. Peyroteo ${ }^{1}$, S. Graça ${ }^{2}$, L. Lencastre ${ }^{2}$, A. Vasques ${ }^{3}$, A. Fonte Boa ${ }^{3}$, J. Vilaça ${ }^{2}$

${ }^{1}$ Surgical Oncology, IPO Porto, PORTO, Portugal; ${ }^{2}$ General Surgery, Hospital da Luz Arrábida, PORTO, Portugal; ${ }^{3}$ Anestesiology, Hospital da Luz Arrábida, PORTO, Portugal

Aims: Endoscopic thyroidectomy techniques have become established alternatives in surgical treatment of thyroid nodules. However the indications for these techniques are usually limited to nodules $=3 \mathrm{~cm}$. Our aim was to demonstrate the feasibility of this approach for bigger nodules, namely with a cystic component.

Methods: The authors present a case of transaxillary endoscopic left lobectomy with intraoperative aspiration for a $4 \mathrm{~cm}$ solid/cystic thyroid nodule.

Results: A 54 year-old female presented with a non-functioning $41 \mathrm{~mm}$ nodule with a cystic component in the left thyroid lobe. The biopsy result was compatible with a follicular lesion of undetermined significance. A transaxillary approach was made through the placement of three trocars: one of $5 \mathrm{~mm}$ periareolar in the left upper quadrant, one of $5 \mathrm{~mm}$ in the anterior axillary line and one of $11 \mathrm{~mm}$ in the axillary fold. Dissection with clear identification of the nodule was performed. The periareolar port site was then enlarged and a $11 \mathrm{~mm}$ trocar was placed to allow for the placement of the ultrasound. Ultrasound-guided aspiration of the cystic component was made and left lobectomy was completed. The recurrent laryngeal nerve identification was made with intraoperative neuromonitoring control. Postoperative period was uneventful and the patien was discharged in the first postoperative day.

Conclusion: This remote access approach is feasible and safe in patients with nodules $>3 \mathrm{~cm}$, especially when a cystic component is present. Intraoperative ultra-sound guided aspiration of cystic components is a valuable tool to reduce the size of the nodule and allow endoscopic thyroidectomy.

\section{V202-SOLID ORGANS-Thyroid}

\section{TRANSAXILLARY ENDOSCOPIC THYROIDECTOMY: WHAT} ADVANTAGES DOES IT OFFER?

M. Manyalich Blasi ${ }^{1}$, V. Gonzabay ${ }^{1}$, E. Delgado-Oliver ${ }^{2}$, B. Martin-Perez ${ }^{1}$, M.A. Lopez-Boado ${ }^{1}$, C. Fondevila ${ }^{1}$, O. Vidal $^{1}$

${ }^{1}$ General Surgery, Hospital Clinic de Barcelona, BARCELONA, Spain; ${ }^{2}$ General Surgery, Hospital Universitario Miguel Servet, ZARAGOZA, Spain

Aim: The evolution of technology and its application to the minimally invasive surgery of the thyroid gland offers new surgical techniques, like the transaxillary approach. This new procedure is still being implemented in our environment and has recently begun to be incorporated into our surgical practice. The objective of this case is to explain step by step how to carry out a right transaxillary endoscopic thyroidectomy and emphasize in the most relevant tips to take into account. Also, current indications and limitations of this technique will be addressed.

Methods: A 49-year-old woman is referred for evaluation of a right thyroid nodule without any associated symptomatology. The blood test shows normal thyroid profile. Cervical ultrasound is performed identifying a $3.5 \mathrm{~cm}$ single right nodule with well-defined edges and presence of peripheral vascularization. No other nodules are identified. Fine needle aspiracion (FNA) of the nodule describes a Bethesda III. After evaluation, a right transaxillary endoscopic thyroidectomy was performed.

Results: Dissection begins in the subcutaneous plane above the pectoralis major muscle until identification of the sternocleidomastoid muscle. Dissection continues towards the prethyroid muscles in order to perform a lateral approach of the thyroid gland. Section of the upper pole allows better exposure of the recurrent laryngeal nerve (RLN) which is being monitored intermittenly. Identification and preservation of the parathyroid glands is the next step. Surgery is completed with the section of the inferior pole of the thyroid along with the istmus. The postoperative period was uneventful and patient was discharged at $24 \mathrm{~h}$ after surgery. Final pathology revealed a $3 \mathrm{~cm}$ nodule without malignancy.

Conclusion: Surgical treatment of the thyroid gland by transaxillary approach may be indicated in previously selected patients with benign pathology, offering the advantages from minimally invasive techniques (shorter recovery time, shorter incision length, etc.). Further research is required to make a better assessment of the minimally invasive approaches in thyroid surgery. 


\section{V203-UPPER GI-Benign Esophageal disorders \\ THORACOSCOPIC ENUCLEATION OF AN ESOPHAGEAL LEIOMYOMA}

A. Herrero Fabregat, N. Perez Serrano, S. Aguas Blasco, A. Garcia Tejero, R. Gil Albarellos Marcos

General Surgery, Hospital San Pedro, LOGROÑO, Spain

We present the video of a thoracoscopic esophageal leiomyomaenucleation. It has been widely demonstratedthe advantages of theminimally invasive approach in surgery. Esophageal thoracoscopic surgery has been suggested as an alternative to open procedures, presenting less surgical trauma, lower risk of bleeding, less postoperative pain, lower wound infection and lower pulmonary morbidity, showing similar oncologic outcomes.

Although leiomyomas are the most commonof benign tumors of the esophagus, they are relatively rare, presenting an incidence of $10-40$ per 10.000 autopsy series.

In our case, the patient was diagnosed of leiomyoma located at the medium third of the esophagus. He referred a history of 6 months of dysphagia for solid and liquids and retrosternal pain. The complementary studies were esophagoscopy, esophagography, CT and endoscopic ultrasonography. The patient was operated by a thoracoscopy approach using 3 ports. It was completed the enucleation of the tumor following the closure of the muscular layer. Methylene blue test confirmed no leaks. The patient was discharged on third day postoperative developing no incidences. Pathology report: leiomyoma $6 \mathrm{~cm}$ size, actin and desmin positive; S-100, CD 34 and CD 117 negative.

We want to demonstrate the advantages of a minimally invasive approach in this kind of pathology.

V204-UPPER GI-Benign Esophageal disorders

\section{THORACOSCOPIC ENUCLEATION OF LARGE ESOPHAGEAL} LEIOMYOMA

L. Baldari ${ }^{1}$, S. Petrucci ${ }^{1}$, A. Spota ${ }^{1}$, M. de Francesco ${ }^{2}$,

M. Ballabio $^{1}$, S. Macina ${ }^{2}$, E. Cassinotti ${ }^{1}$, L. Boni $^{1}$

${ }^{1}$ General Surgery, University of Milan-Policlinico Hospital,

MILAN, Italy; ${ }^{2}$ General Surgery, Policlinico Hospital, MILAN, Italy

Aims: This video shows our technique to perform thoracoscopic enucleation of large esophageal leiomyoma.

Methods: The patient is a 53-years-old woman with a six months history of progressively worsening dysphagia. Chest CT scan revealed a $6 \mathrm{~cm}$ lesion of middle esophagus with extrinsic compression of mucosa and no increased FDG uptake on FDG-PET scan. Barium swallow study showed a lateral deviation of thoracic esophagus due to extrinsic compression. Endoscopic ultrasound confirmed the suspicion of esophageal leiomyoma. Patient was scheduled for a thoracoscopic enucleation of esophageal tumor. She was placed in prone position and one-lung ventilation was employed. Three trocars were placed in intercostals spaces on right hemithorax. Azygos vein was identified and transected between vascular clips. Esophagus was circumpherentially isolated from mediastinal structures. After myotomy, the lesion was dissected from submucosal-mucosal layer. Since air leak test excluded injury of internal layer, muscular layer was closed with a continuous suture. The specimen was extracted through an endobag. A drain was left in place.

Results: The postoperative course was uneventful and the patient was discharged on postoperative day 7. Final pathological examination confirmed esophageal leiomyoma.

Conclusion: Thoracoscopic surgery in prone position allows removal of large esophageal tumor with several advantages. The minimally invasive technique decreases perioperative morbidity and mortality.

\section{V205-UPPER GI-Benign Esophageal disorders}

\section{A CASE OF EMERGENT SURGERY FOR SPONTANEOUS ESOPHAGEAL PERFORATION WITH A MINIMALLY- INVASIVE ABDOMINAL AND LEFT THORACIC APPROACH (MALTA)}

S. Sekiya, Y. Ebihara, K. Tanaka, Y. Nakanishi, T. Asano, T. Noji, Y. Kurashima, S. Murakami, T. Nakamura, T. Tsuchikawa, K. Okamura, T. Shichinohe, S. Hirano

Gastroenterological Surgery 2, Hokkaido University Hospital, SAPPORO-CITY, Japan

Introduction: Spontaneous esophageal perforation is life threatening disease and requires emergent surgical treatment. Recently, the efficacy of minimally-invasive surgery such as laparoscopic and thoracoscopic surgery for esophageal perforation has been reported. We report a novel technique of minimallyinvasive abdominal and left thoracic approach (MALTA) for spontaneous esophageal perforation.

Case presentation: 64-year-old male, who had been under hemodialysis due to IgA nephropathy, complained of chest pain after vomiting several times. Since the CT scan showed left hydropneumothorax and pneumomediastinum, and the gastrografin study demonstrated extravasation from left side of esophagus, we diagnosed him with the spontaneous esophageal perforation and planed emergent surgery. The patient was placed in the reverse Trendelenburg position, and the legs were split, with the left side of the upper body lifted in order to perform thoracoscopy and laparoscopy simultaneously. First, we explored the thoracic cavity through a $12 \mathrm{~mm}$ port in the left 8 th intercostal space and added other 3 ports. We identified the rupture site $30 \mathrm{~mm}$ in size on the left wall of the lower esophagus and sutured the mucosa and the muscle layer with a running suture respectively. We covered the perforation section with pericardium fat and irrigated the cavity with physiological saline. Then transferred to the abdominal cavity, no contamination was found in the abdomina cavity. A feeding tube was inserted into stomach through the round ligament of the liver and the operation was completed. The total operative time was $173 \mathrm{~min}$ and the amount of intraoperative bleeding was $1300 \mathrm{ml}$ including pleural effusion. Postoperatively, the patient experienced left empyema pleurae but no other severe complications and was discharged on postoperative day 25 . Conclusion: We experienced a rare case of spontaneous esophageal perforation of a patient under hemodialysis. MALTA is an effective procedure for emergent esophageal operation because of great visual field of the chest and abdominal cavity without expanding contamination.

\section{V206-UPPER GI-Benign Esophageal disorders}

\section{TOTAL MINIMALLY INVASIVE ESOPHAGOGASTRECTOMY WITH COLONIC INTERPOSITION AND VASCULAR ASSESSMENT WITH ICG}

D. Momblán, V. Turrado-Rodríguez, A. Ibarzábal, R. Almenara, A. Torroella, X. Morales, A.M. Lacy

Gastrointestinal surgery, Hospital Clinic of Barcelona, BARCELONA, Spain

Introduction: Digestive caustic injury is associated with high morbidity and mortality with stenosis in the long term. Surgical treatment involves resection of the esophagus and reconstruction with the stomach, colon or jejunum. Coloplasty provides several advantages but its vascularization is complex and involves 3 anastomosis. Classically, vascular assessment was achieved by palpation through laparotomy and color evaluation. Indocyanine green (ICG) allows a minimally invasive intraoperative angiography in real time.

Methods: A 43-year-old female with medical history of caustic ingestion and subsequent esophagogastric stenosis, carrier of feeding jejunostomy.1. Thoracoscopy (prone position): dissection of the esophagus from the hiatus to the upper thoracic inlet.2. Laparoscopy: patient in the supine position, placement of five trocars. Total non-oncological gastrectomy, post-pyloric section of the duodenum and omentectomy were completed. Mobilization of the righ, transverse and descending colon. Measurement of the transverse colon with a tape (distance from the neck and the esophageal hiatus). Individualization of the righ, middle (with its branches) and left colic arteries and placement of clamps at the right colic, right branch of the middle colic and left colic arteries. $3 \mathrm{cc}$ of ICG were injected allowing for an assessment of the colon vascularization. Section of the right branch of the middle colic artery. Proximal section of the ascending and distal colon near the splenic angle, preserving the marginal arch. Silk point to join the staple line of the descending colon and the pylorys. Side-to-side mechanical antiperistaltic anastomosis between the distal endo of the coloplasty and the jejunum. Finally an anastomosis between the ascending and descending side-to-side mechanica anastomosis using an assistance incision in the left flank was performed.3. Cervical dissection extraction of the surgical especimen under laparoscopic control. Vascular assessment with ICG is performed before and after the side-to-side anastomosis is performed.

Results: There were no intraoperative complications. The patient was discharged on postoperative day 11 .

Discussion: We describe the first case of total minimally invasive colonic interposition with ICG assessment of the vascularization. This technique, although technically demanding, avoids the drawbacks of the open surgery and allows for a precise assessment of the vascularization of the graft. 


\section{V207-UPPER GI-Benign Esophageal disorders}

\section{VIDEO-ASSISTED TRANSORAL SURGICAL RESECTION OF GIANT ESOPHAGEAL FIBROVASCULAR POLYP}

E. Vicente, Y. Quijano, H. Duran, E. Diaz, I. Fabra, R. Caruso,

L. Malave, B. Ielpo, V. Ferri

\section{General Surgery, SANCHINARRO UNIVERSITY HOSPITAL,} MADRID, Spain

Introduction: Large pedunculated fibrovascular polyps are uncommon, mostly benign, intraluminal massess, usually located in the upper esophageal tract. Most frequent reported clinical manifestation is dysphagia, followed by regurgitation, chest pain and intestinal bleeding. CT scan, and MRI are the key in the diagnostic work-up revealing a sausage-shaped intraluminal mass. Endoscopy with ultrasonography and biopsy add important information for the diagnosis and pedicle location. Surgical excision is deemed due to potentially life-threating complication related to airway obstruction. The most frequent polyp resection is performed through cervical esophagotomy or by direct esophagectomy. However, this approach is related to a high morbidity and mortality rate. In the last years, few excisions have been reported by a endoscopical approach with a lower post operative complication.

Material and Methods: This video shows the surgical steps of a trans-oral endoscopic surgical resection of a giant $(23 \mathrm{~cm})$ pedunculated polyp in a 43 year old man. The procedure was performed under general anesthesia. A flexible endoscope probe was used and the distal end of the polyp was extracted through the oral cavity with a loop. The endo-GIA stapler was used to cut the base of polyp and finally removed. The anatomo-pathological study confirmed the diagnostic of a fibrovascular polyp with no evidence of malignancy.

Results and conclusions: The patient had an uneventful recovery with no recurrency at 3 years of follow up. This minimally invasive approach is a safe and feasible procedure to treat large esophageal fibrovascular polyps avoiding the complications related to more aggresive procedure.

\section{V208-UPPER GI-Benign Esophageal disorders}

IS MINIMALLY INVASIVE APPROACH SUITABLE FOR THE SURGICAL ENUCLEATION OF AN ESOPHAGEAL LEIOMYOMA?

S. Alonso, O. Abdel-Lah, J.E. Sánchez, T. Rubio, J. López,

J. Quiñones, F. Parreño, L. Hernández-Cosido, S. Carrero,

I. Jiménez, L. Muñoz-Bellvís

General Surgery, Complejo Asistencial Universitario de Salamanca, SALAMANCA, Spain

Introduction: Leiomyomas are the most common mesenchymal tumors affecting the esophagus and they usually grow in the mid to distal third of it. They tend to be asymptomatic, but sometimes they can grow to enormous size and produce dysphagia.

Case report: 61-year-old male asymptomatic patient was referred to our hospital due to an incidental finding. CT scan revealed a $41 \times 35 \mathrm{~mm}$ rounded submucosal tumor on the dorsal side of the lower third of the esophagus. Upper gastrointestinal endoscopy revealed a cystic lesion in the lower esophagus $37 \mathrm{~cm}$ from the incisor teeth, with normal overlying mucosa. An endoscopic-ultrasound-guided fine-needle-aspiration of the mass was performed, which was reported as a likely leiomyoma.Conservative treatment was performed, no growth was detected during eleven years of follow-up. But it became symptomatic, the patient complained of progressive dysphagia caused by compression so surgical resection was decided.Laparoscopic enucleation of esophageal leiomyoma was performed. The tumor was reached by transhiatal dissection. A careful dissection of the mass was performed, preserving the vagal branches. An intraoperative endoscopy was performed to verify the integrity of esophageal mucosa and that the tumor was completely resected. The muscular layer was sutured after enucleation using absorbable suture material and the hiatus was closed with non-absorbable suture material. A Dor fundoplication was also performed. A swallow test with a water-soluble contrast was obtained on postoperative day one. No pathological findings were found so the patient was asked to drink.Histopathological exam revealed a tumor measuring $70 \mathrm{~mm} \times 33 \mathrm{~mm} \times 17 \mathrm{~mm}$ consistent with leiomyoma.The procedure lasted $160 \mathrm{~min}$. The hospital discharge was on the third postoperative day and no complication was registered.

Conclusion: Surgical excision is the mainstay of treatment and is recommended for symptomatic leiomyomas and those greater than $5 \mathrm{~cm}$. This case report demonstrates the technical feasibility, safety and minimal postoperative morbidity associated with minimal invasive esophageal surgery.

\section{V209-UPPER GI-Benign Esophageal disorders}

\section{MINIMALLY INVASIVE SURGERY FOR MALLORY WEISS SYNDROME}

\section{López, A. Navarro-Sánchez, E. Esteban Perez Alonso, J.R. Juan Ramón}

General Surgery, Complejo Hospitalario Universitario Insular Materno Infantil, LAS PALMAS GC, Spain

Aim: Surgical treatment in Mallory Weiss Syndrome represents less than 5\% of cases. We present a video showing the laparoscopic management of Mallory Weiss Syndrome after endoscopic failure.

Methods: A 30 year-old male with past medical history of HIV, alcohol and cocaine consumption was referred to our unit with a history of upper gastrointestinal (GI) bleeding after vomiting. Physical examination demonstrated haemodynamic instability and anaemization on full blood count.

Results: After haemodynamic stabilisation, a gastroscopy was done showing hiatus hernia and active bleeding from a mucosal tear in the oesopgagogastric junction, adrenalin and polydocanol were admininistrated. A second look gastroscopy performed the same procedure as a prophylactic measure. CT scan demonstrated active bleeding from the oesophagogastric junction withou neither signs of perforation nor mediastinitis. Following failure of endoscopic application of clips, emergency laparoscopy was undertaken. A gastrotomy revealed active bleeding from a gastric mucosal tear between the clips applied endoscopically. Bleeding ceased with transfixion stitches. The gastrotomy was closed and the hiatus hernia repaired. Postoperative period was uneventful.

Conclusions: Endoscopic treatment is able to manage Mallory Weiss Syndrome in most cases Surgery is exceptional, if need it, minimally invasive procedures are feasible and safe with experience.

\section{V211-UPPER GI-Benign Esophageal disorders}

\section{UTILITY OF MINIMALLY INVASIVE SURGERY FOR THE APPROACH OF BENIGN ESOPHAGEAL STENOSIS}

A.F. Aranzana Gómez ${ }^{1}$, J. Hernandez Gutierrez ${ }^{1}$, J. Malo Corral ${ }^{1}$, B. Muñoz Jimenez ${ }^{1}$, R. Lopez Pardo ${ }^{1}$, A. Trinidad Borras ${ }^{1}$, S. Abad de Castro ${ }^{2}$, P. Toral Guinea ${ }^{1}$, G. Krasniki ${ }^{1}$, M.A. Morlan Lopez

${ }^{1}$ General Surgery, Complejo Hospitalario Toledo, TOLEDO, Spain; ${ }^{2}$ General Surgery, Hospital tres culturas, TOLEDO, Spain

Introduction: Total esophagectomy by means of minimally invasive surgery has proven to be a valid and effective alternative for performing this procedure. However, this procedure is not implemented in most centers.

Objective: Demonstrate the technique of a total esophagectomy by endoscopic surgery for a benign esophageal stenosis.

Material and methods: Clinical case: A 75-year-old female patient diagnosed with double esophageal peptic stenosis, treated on several occasions with endoscopic dilation by digestive, showing in the last endoscopy: severe esophagitis with stenosis impassable to $22 \mathrm{cms}$. Additiona tests of interest are exposed. Resolved: Intervention: Right thoracoscopy in prone position, dissection and complete mobilization of the thoracic esophagus, section of the azygos vein, pleura drainage. Laparoscopic time, 5 trocars, gastrolysis respecting the right gastroepiploic vessels, broad kocher until the cava is identified, vascular section of the vessels left gastric, full mobilization of the stomach, subxiphoid minilaparotomy, beginning of the cervical time with dissection of the cervical esophagus, section and fixation of this to a tube, externalization of the piece by abdominal route, creation of the gastric tubular with successive loads of GIA, ascending posterior mediastinal plasty with manual esophago-tubular anastomosis, with placement of drainages and feeding jejunostomy. Right operative with radiological control with gastrografin on the 6th day, discharge from hospital on the 11th day. Asymptomatic one year after surgery, with radiological control without alterations.

Conclusions: The approach of esophageal peptic stenosis with minimally invasive surgery is safe and effective, adding the advantages inherent to this type of technique. 


\section{V212-UPPER GI-Benign Esophageal disorders}

\section{ESOPHAGUS DUPLICATION CYSTS}

M. Pérez Reyes ${ }^{1}$, F.J. Moreno Ruiz, C. Montiel Casado,

J.L. Fernández Aguilar, A. Rodríguez Cañete, I. Mirón Fernández, P. Gutiérrez Delgado, J. Santoyo Santoyo ${ }^{2}$

${ }^{1}$ Esofagogástrica, Cirugía General y Ap Digestivo Hospital Regional Universitario de Málaga, MALAGA, Spain; ${ }^{2}$ Hepatobiliopancreática,, MALAGA, Spain

Introduction: Gastrointestinal duplication cysts are rare diseases that occur mainly in the ileum, jejunum, esophagus and colon. The prevalence at the esophageal is $0.01 \%$.

Methods: We present a 28 year old women studied by digestive by epigastralgia. Ultrasonography, upper gastrointestinal endoscopy(UGE), endoscopic ultrasound and computerized axial tomography(CT) were performed (Figs. 1,2), showing a cystic lesion in the gastric submucosa with a well defined, medial and superior to the lesser curvature of the stomach with exophytic growth. It causes extrinsic compression of the cardia in the gastric body. Within the differential diagnosis are gastric duplication cysts or gastrointestinal stromal tumor. A biopsy was taken, discarding the presence of neoplastic cells. Finally, a study of digestive transit showed extrinsic compression at the cardial level, which causes difficulty in passing contrast.

A laparoscopic approach was performed beginning with the dissection of the abdominal esophagus. The presence of a cystic lesion on the anterior face of the abdominal esophagus was identified (Figs. 3,4). We proceeded to the complete resection of cyst. The surgery was completed with $180^{\circ}$ fundoplication anterior Dor. The patient went home on the 3rd day without incident.

Results: Duplication cysts are congenital malformations of the gastrointestinal tract contiguous with the esophagus, which can communicate with the esophageal lumen. Most are diagnosed in childhood, but when it is diagnosed in adults, they used to be symptomatics. It is more frequent in men.

Although the pathogenic mechanism is unknown, it is caused by an anomaly during embryonic development. They are located in the thoracic esophagus, at the level of the lower and posterior mediastinum and, less frequently, in the abdominal esophagus, as in our case.

They can give digestive symptoms(epigastralgia, vomiting) or respiratory symptoms.

The diagnosis is made with EDA, CT, and Ecoendoscopy of choice, although it may be incidental The treatment of choice in symptomatic patients is complete resection or cystic enucleation. In asymptomatic surgical is not defined, because it can cause complications, and, malignization due to degeneration is very infrequent.

Conclusion: The most of duplication cysts are diagnosed in childhood, although it's more frequent in adults to be symptomatic. Surgical treatment can cure this disease.

\section{V213-UPPER GI-Benign Esophageal disorders}

\section{EXTENDED LAPAROSCOPIC HELLER MYOTOMY IN YOUNG PATIENT WITH HEPATIC ARTERY LEFT ABERRANT AND SEVERE ESOPHAGITIS}

\section{A.F. Aranzana Gómez, J. Hernandez Gutierrez, J. Malo Corral, B. Muñoz Jimenez, R. Lopez Pardo, A. Trinidad Borras, A. Muñoz Tébar, I. Fraile Alonso, M.A. Morlan Lopez}

General Surgery, Complejo Hospitalario Toledo, TOLEDO, Spain

Introduction: Achalasia is an infrequent pathology in young patients. ENDOSCOPIC PNEUMATIC DILATATION (DN) and MYOTOMY OFHELLER (MH) laparoscopic are the most defended procedures at present because they are effective andinsurance, currently showing very good results with the POEM. However, the choice between these becomes difficult in young patients, where the low incidence does not allow get series of long patients and decisions must be based on results achieved in adults.

Objetivos: To demonstrate the safety and efficacy of the laparoscopic approach in this infrequent pathology, pointing out the importance of having standardized the procedure to achieve better results.

Material and methods: Case report: A 19-year-old man with progressive dysphagia until almost complete afagia, with clinical, endoscopic, radiological and manometric diagnosis,compatible with typical primary Achalasia. Chagas negative serology,we show the complementary studies of interest.Dilatation is not performed, preoperative symptomatic treatment with calcium channel inhibitors. Intervention: laparoscopic approach, 5 trocars, aberrant left hepatic artery with signs of severe esophagitis,opening of the gastroesplenic-hepatic ligament, no retroesophageal window, dissection of the hiatus and inferior mediastinal, preservation and mobilization of the left hepatic artery and the anterior vagus,meticulous disection of the cardia,standardized myotomy: first proximal $8 \mathrm{cms}$. with adequate simultaneous traction of both edges of the myotomy, then distal myotomy including $2-3 \mathrm{~cm}$, including selectively the distal oblique fibers of LES, tutorization with Fouché and methylene blue to confirm good step and absence of leakage, Dor-type funduplication, 4 pts on each side, fixed to both pillars, hiatalmediastinal drainage. EGD on the 1st day of normal PO, dischargeat 3rd day, asymptomatic and with normal radiological control at 1 year of age.

Conclusions: Laparoscopic MH should be the first therapeutic option, in patients with primary Achalasia, even in young patients. The length of myotomy, especially distal to UEG is one of the most important aspects of surgery, most authors (Pellegrini) recommend that the myotomy extend $1-2 \mathrm{~cm}$ in the stomach, even up to $3 \mathrm{~cm}$ below the UEG to achieve an effective disruption of the EEI.The standardization of the procedure is fundamental to increase safety and effectiveness in these more complex cases

\section{V214-UPPER GI-Benign Esophageal disorders}

ROBOTIC TECHNIQUE FOR THE TREATMENT OF GIANT HIATAL HERNIAS WITH BRIDGING NON-ABSORBABLE MESHES IN CASES OF LARGE DIAPHRAGMATIC DEFECTS

\section{H. Konstantinidis, C. Charisis}

Robotic General and Oncologic Surgical Department, Interbalkan Medical Centre, THESSALONIKI, Greece

Aims: The surgical treatment of giant hiatal hernias is a complex and demanding procedure, not only in terms of performing the operation in a minimally invasive abdominal fashion by avoiding thoracic approaches, but also concerning the management of large hiatal defects which contribute to high recurrence rates. Our aim is to present our surgical technique for the reconstruction of such hiatal hernias, exploiting the benefits of the robotic approach and also to highlight the technical aspects of non-absorbable mesh placement in order to bridge effectively the hiatal defects.

Methods: We present video fragments from a procedures selected from a series of cases of robotic reconstruction of giant hiatal hernias performed by our team, in which a non-absorbable meshes were utilized to restore the hiatal gap. We emphasize on the clear benefits of robotic surgery in these cases and on the strategy of how to avoid high recurrence rates.

Results: All of our patients, who underwent reconstruction of giant hiatal hernias with this particular technique, experienced very good early post-operative results, very short hospital stay and no recurrence in a 12 -month follow-up.

Conclusions: The robotic approach for the treatment of large hiatal hernias offers great advantages to both surgeons and patients, by eliminating the restrictions of conventional laparoscopic surgery, minimizing intra-operative incidents and post-operative complications. Large hiatal defects are very effectively closed with the use of advanced suturing techniques and non-absorbable meshes in a tension-free bridging fashion.

\section{V215-UPPER GI-Esophageal cancer}

\section{STEP BY STEP INSTRUCTION VIDEO FOR SURGEONS IN TRAINING: LAPAROSCOPIC MCKEOWN ESOPHAGOGASTRECTOMY}

\section{Z.H.T. Teo ${ }^{1}$, M.O. Aung ${ }^{2}$}

${ }^{1}$ General Surgery, Tan Tock Seng Hospital, SINGAPORE, Singapore; ${ }^{2}$ Upper GI and Minimally Invasive Surgery Unit, General Surgery, Tan Tock Seng Hospital, SINGAPORE, Singapore

To provide a reference laparoscopic Mckeown esophagogastrectomy video for residents rotating through the upper gastrointestinal surgical unit in Tan Tock Seng Hospital, Singapore. This is with the aim of improving surgical outcomes for the patient as the whole surgical team is familiar with the steps of the surgery and also improve the learning of the residents with the provision of reference material.Residents are provided with the full length video of a Mckeown esophagogastrectomy and will have to edit the video to show the essential steps of the surgery. Steps of Surgery:

Azygos vein circumferentially dissected and divided between clips

Right pulmonary ligament divided

Pulmonary ligament lymph node excised

Mediastinal pleura divided from the azygos vein down to diaphragm

Left pleural divided to allow entry into the left pleural space

Penrose drain inserted around the esophagus to aid in retraction

Division of the contralateral inferior pulmonary ligament

Mobilisation of the esophagus from the crus of the diaphragm to the thoracic inlet

Para-esophagus lymph nodes excised

Chest tube $\times 2$ placed \& patient repositioned in supine

Mobilisation along the greater curve

Short gastrics divided

Further mobilisation of the posterior wall of stomach, in order to lift stomach anteriorly

Attachments to hepatoduodenal ligaments incised

Left gastric vessels divided \& divided between clips

Phrenoesophageal ligaments incised

Neck incision and NG sutured to transected cervical esophagus

Esophagus and stomach exteriorised through abdominal incisio

Conduit created using a series of staplers along stomach parallel to the greater curavature

Conduit passed from abdominal to neck incision

Anastomosis performed between conduit and cervical esophagus

Through the editing of the video, we have found that residents have become more familiar with the steps of the surgery and benefit more in their learning when they are assisting in the surgery. Through this project, we are aiming to lower the learning curve of residents and raise the standards of patient care. 


\section{V216-UPPER GI-Esophageal cancer}

MINIMALLY INVASIVE IVOR LEWIS ESOPHAGECTOMY WITH ROBOTIC ASSISTANCE AND ICG FLUORESCENCEMODULAR STEP UP APPROACH AND TWO ANASTOMOTIC TECHNIQUES

\section{H.F. Fuchs, C.J. Bruns}

\section{Surgery, University of Cologne, COLOGNE, Germany}

Introduction: Minimally invasive technologies have improved outcomes after esophagectomy and the use of robotic technology in Europe is rapidly increasing. Aim of this study is to evaluate the introduction of new technologies in a center of excellence for upper gastrointestinal surgery. Methods and procedures: A standardized teaching protocol of a complete OR team was performed in simulation and animal models at the center for the future of surgery (San Diego, CA) and IRCAD (Strasbourg, France) to receive certification as console surgeons. Starting 02/2017 the davinci xi and stryker ICG laparoscopy systems were introduced at our academic center (certified center of excellence for surgery of the upper gastrointestinal tract, $n>300$ esophageal cases/year). After simple training procedures based on our minimally invasive expertise were performed, difficulty was increased based on a modular step up approach to safely perform robotic thoracic assisted Ivor Lewis esophagectomy.

Results: From 02/2017 to $12 / 2018$, a total of 72 mainly upper gastrointestinal robotic cases including 40 esophagectomies for cancer were performed. All cases were performed safely without operation-associated complications. Level of difficulty was increased based on our modular step up approach without quality compromises. Video documentation using the new technology is provided and two anastomotic techniques are presented.

Conclusion: The standardized training protocol and our modular step up approach allowed safe introduction of the new technology used. All cases were performed safely without operationassociated complications.

\section{V217-UPPER GI-Esophageal cancer}

\section{MINIMALLY INVASIVE MCKEOWN ESOPHAGECTOMY}

S. Symeonidis, S. Mpitsianis, M.G. Pramateutakis, S.. Simeonidis,

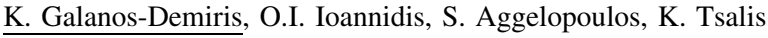

4th Surgical Department, General Hospital, G. Papanikolaou, THESSALONIKI, Greece

Aims: McKeown esophagectomy is commonly used for invasive esophageal carcinoma. As the morbidity and mortality rates for esophagectomy are persistently high, minimally invasive esophagectomy in prone position is expected to reduce respiratory postoperative complications. There is still limited experience for the use of minimally invasive approaches in patients undergoing surgery after neoadjuvant chemoradiation and many concerns about the feasibility, safety, and oncological outcomes of these procedures are still present.

Methods: We present the case of a 55-year-old female with a middle third esophageal squamous cell carcinoma, who received neoadjuvant chemoradiation. She underwent laparoscopic and thoracoscopic (prone position) McKeown esophagectomy with hand-sewn esophagogastric anastomosis through a left lateral cervical incision.

Results: The operation was completed successfully, with no conversion to open surgery. The operative time was $6 \mathrm{~h}$ with minimal blood loss and the patient was fed on day 5 and discharged on day 7 post-op. R0 resection was achieved and the number of total harvested lymph nodes was 44 (3 positive nodes, N2).

Conclusions: Minimally invasive McKeown esophagectomy in patients with esophageal cancer and prior chemoradiation is feasible and safe procedure with acceptable oncological outcomes.

\section{V218-UPPER GI-Esophageal cancer}

IVOR-LEWIS PRONE POSITION INTRATHORACIC NONROBOTIC THORACOSCOPIC ANASTOMOSIS: DIFFERENT OPTIONS, SAME OBJECTIVE

F. del Castillo Diez ${ }^{1}$, L. García-Sancho Téllez ${ }^{2}$, J.D. Sanchez Lopez ${ }^{3}$, E. Ferrero Celemin ${ }^{3}$, C. Durán Escribano ${ }^{4}$, J.A. Gonzalez Sanchez ${ }^{1}$, J. Díaz Dominguez ${ }^{1}$

${ }^{1}$ General Surgery, Hospital Universitario La Paz, MADRID, Spain; ${ }^{2}$ General Surgery, Hospital Universitario Infanta Sofia, MADRID, Spain; ${ }^{3}$ Surgery, Hospital Universitario Infanta Sofia, MADRID, Spain; ${ }^{4}$ General Surgery, Hospital Quirón La Luz, MADRID, Spain

Aims: The authors present a video with their experience involving intrathoracic anastomosis during minimally invasive Ivor-Lewis esophagectomy procedure: different options, in order to avoid major complications.

Methods: A serie of 25 patients is presented. They all presented indication for an esophagectomy, and no contraindication for a thoracoscopic approach: Three different types of thoracoscopic esophagogastric anastomosis and technical details are shown in the video.

Results: Preliminary results demonstrated that minimally invasive Ivor-Lewis esophagectomy procedure, provided of a better postoperative pain control and less respiratory complications. In order to standarise our procedure, the video shows how three different types of esophagogastric anastomosis are performed, depending on the patient characteristics, anatomical factors and safety and comfort for the surgeon: Manual termino-terminal, mechanical termino-terminal and mechanical latero-lateral.

Conclusions: In our way to standardization, we are still looking for the best type of anastomosis, even though, we find out that, manually performed anastomosis are easier to performed, when the section in esophagus is lower, involving medium and inferior third. In the other hand, mechanical termino-terminal anastomosis seemed to be an ideal option for upper sections. More studies are needed in order to standardized one anastomosis, for all cases.

\section{V219-UPPER GI-Esophageal cancer}

THORACOSCOPIC ESOPHAGECTOMY FOR ESOPHAGEAL SQUAMOUS CELL CARCINOMA WITH HYBRID POSITION (PRONE + LEFT LATERAL DECUBITUS POSITION)

\section{H. Kawakubo, S. Mayanagi, T. Ilino, Y. Kitagawa}

Department of Surgery, Keio University School of Medicine, TOKYO, Japan

Because esophagectomy with radical lymphadenectomy is highly invasive, thoracoscopic esophagectomy (TE) is attracting attention as a less invasive procedure. We first performed TE with the left decubitus position in 1996. In 2009 we developed a hybrid of the prone and left lateral decubitus positions for TE, and a total of 470 patients underwent TE with a hybrid position . We introduced TE with a hybrid position for the following three reasons: (1) Mobilization and lymphadenectomy around the middle and lower esophagus are easier in the prone position Thanks to artificial pneumothorax and the gravity, the middle and lower mediastinum are opened, and which give us good surgical field. (2) Lymphadenectomy along the left recurrent laryngeal nerve (RLN) is more reliable and precise when performed in the left lateral decubitus position. We can dissect lymph node around the RLN higher position in the upper mediastinum. (3) Unexpected events requiring conversion to thoracotomy (e.g., massive bleeding, injury of other organs, dense intrathoracic adhesion, resection of adjacent organs) are easier to deal with in the left lateral decubitus position.

The patient is fixed on the operating table with the semi-prone position and we can easily change patient positions from the left lateral decubitus position to the prone position and vice versa using rotation system of the operation table. The upper mediastinal procedure including lymphadenectomy along the right and left RLN is performed with the patient in the left lateral decubitus position, while the middle and lower mediastinal procedures are performed with the patient in the prone position with artificial pneumothorax $(7 \mathrm{mmHg})$. Theabdominal procedures wereperformed by hand-assisted laparoscopic surgery (HALS) and gastric tube reconstruction through aposterior mediastinal route was performed as a standard surgical procedure in our institution. The magnifying effect of thoracoscope enables us to perform more precise surgery and preserve nerve and vessels, and a hybrid position is thought to be feasible and effective methods. 


\section{V220-UPPER GI-Esophageal cancer}

\section{TOTALLY MININVASIVE IVOR LEWIS WITH ROBOTIC HAND-SEWN ANASTOMOSIS}

\section{A. Peri ${ }^{1}$, L. Pugliese ${ }^{1}$, F.C. Bruno ${ }^{1}$, N. Mineo ${ }^{1}$, M.S. Milani ${ }^{2}$, M. Filardo ${ }^{1}$, A. Pietrabissa ${ }^{1}$} ${ }^{2}$ General Surgery, IRCCS Policlinico San Matteo, PAVIA, Italy

Ivor Lewis esophago-gastrectomy is a standard procedure for the treatment of distal esophageal cancer. Among the years, the surgical community standardized the mininvasive abdominal phase. The thoracic phase is much more complex because usually all surgeons get in trouble in the phase of esophago-gastric anastomosis. In fact, is still very difficult and tricky to perform a mechanical circular anastomosis due to problems with the correct handling of the circular stapler through the minitoracotomy and is also difficult to place the anvyl in the proximal esophagus. The linear anastomosis (side-to-side) is a little bit easier but not so effective as the circular anastomosis in terms of leak rate. We think that robotic approach with its endowrist can allow us to overcome these limits and that a tailored double layer hand sewn esophago-gastric anastomosis could be the right choice. We treated 4 patients with this approach and they were all uneventful in the post operative period except for a case of chylothorax we treated successfully with lipiodol injection in inguinal lymphnodes. We need more cases to analyze the technique in terms of leak rate and major complications but we think this is a promising and cost-effective procedure for robotic approach.
${ }^{1}$ General Surgery II, IRCCS Policlinico San Matteo, PAVIA, Italy;

\section{V222-UPPER GI-Esophageal cancer}

\section{INDOCYANINE GREEN FLUORESCENCE IN ESOPHAGEAL CANCER SURGERY: USES AND APPLICATION}

A. Senent-Boza ${ }^{1}$, I. Alarcón ${ }^{1}$, T. Yang ${ }^{1}$, F. López-Bernal ${ }^{1}$, A. Barranco $^{1}$, M. Socas ${ }^{1}$, F.J. Padillo ${ }^{2}$, S. Morales-Conde ${ }^{1}$

${ }^{1}$ Innovation in Minimally Invasive Surgery Unit. General and Digestive Surgery Ser, University Hospital Virgen del Rocio, SEVILLE, Spain; ${ }^{2}$ General and Digestive Surgery Service, University Hospital Virgen del Rocio, SEVILLE, Spain

Aim: Uses and application of indocyanine green (ICG) fluorescence in the field of Surgery are growing exponentially. The safety and feasibility of its usage has been proven in several areas and various pathologies of surgery and surgeons are starting to incorporate it into their common practice. However, there are still several aspects to define regarding this technology. We present different uses of ICG in the specific area of esophageal cancer.

Methods: We used ICG fluorescence at different moments of a two-field minimally invasive esophagectomy.

First of all, peritumoral injection of ICG may offer a lymphatic mapping, both in the abdominal phase of the surgery and the thoracic one, improving lymph node dissection by allowing a more targeted and less morbid approach that includes all relevant nodal stations.

At the moment of the gastric section, intravenous injection provides assessment of gastric conduit perfusion, therefore optimizing the construction of the graft to avoid the inclusion of poorly perfused areas that may increase the risk of leak of the anastomosis.

Besides that, the esophagogastric anastomosis can be tested in the thoracic phase of the operation in order to check an adequate perfusion and prevent further complications.

Results: We consider that ICG fluorescence is a promising technology that could be easily introduced in the surgical routine of the esophageal surgeon as an instrument to assess the anastomosis perfusion. ICG is also feasible in detecting lymph node drainage from the esophagus, although its technique of application needs to be defined.

Conclusions: ICG fluorescence has opened a new world of possibilities in all the different surgical specialties. Its use in the esophagectomy is safe, simple and feasible. In a near future, its application to esophageal cancer surgery could improve survival by predicting and preventing anastomotic leak and guiding in a tailored lymphadenectomy. Further research is needed to demonstrate these promising applications.

\section{V223-UPPER GI-Esophageal cancer}

\section{INDOCYANINE-GREEN NEAR-INFRARED ANGIOGRAPHY FOR GASTRIC CONDUIT FASHIONING IN TOTAL MINIMALLY-INVASIVE IVOR-LEWIS ESOPHAGECTOMY}

\author{
P. Parise, A. Cossu, F. Puccetti, R. Cerchione, M. Palucci, R. Rosati
} Gastrointestinal Surgery Unit, San Raffaele Hospital, MILAN, Italy

Aim: Anastomotic leakage is one of the most dreaded complication after esophagectomy. Indocyanine-Green Near-Infrared Angiography (NIR-ICGA) intraoperative use has been recently introduced for visceral perfusion evaluation. In this video we present our technique for gastric conduit fashioning according to the NIR-ICGA blood supply evaluation in a total minimallyinvasive Ivor-Lewis esophagectomy.

Methods: A 48 years-old man affected by a Siewert 1 adenocarcinoma (cT3, N2, M0) underwent a preoperative neoadjuvant treatment according to CROSS protocol. At restaging CT-scan no more pathologic nodes were evident (cT3, N0, M0). The patient was submitted to a total minimally-Invasive Ivor-Lewis esophagectomy. Surgical procedure: After pneumoperitoneum induction and 4 trocars insertion, the lesser omentum was opened and a lymphadenectomy at stations $12 \mathrm{a}, 8,7,9$ and 11 performed. The esophagus was dissected at diaphragmatic hiatus with lymphadenectomy at station 1,2 and 111. The larger omentum was opened along the right gastroepiploic arcade, that was preserved, the short gastric vessels divided and gastric fundus mobilized. By evaluating the presence of an intense fluorescence at NIR-ICGA, a tailored partial tubulization of the stomach was performed with multiple linear stapler firing.

A right thoracoscopy was performed through 4 trocars. The azygos vein ligated and divided. The mediastinal pleura was opened and the esophagus was dissected entirely with an en-bloc excision of nodes at stations 110,112 and 108. Nodes at stations 105, 106, 107 and 109 were removed separately. The esophagus was sectioned above the azygos vein level and a purse-string fashioned. The cardia and the gastric tube were pulled up and a minithoracotomy performed. A new NIR-ICGA was repeated to verify the good blood supply and tailor the site of anastomosis on a well perfused area. The stomach was opened and a circular stapler inserted. After the end-to-side esophago-gastric anastomosis fashioning, the tubulization was completed by 2 linear stapler firing and the specimen removed.

Results: The post-operative course was uneventful and the pathologic examination revealed a cardial adenocarcinoma (ypT2,NO, R0)

Conclusions: NIR-ICGA is an interesting and easy-to use tool for surgeons. Nevertheless in literature is still not clear which is the best parameter to measure the blood supply. Large studied are needed.

\section{THORACOSCOPIC APPROACH OF ONCOLOGICAL} OESOPHAGECTOMY COMPLICATIONS AND ITS MANAGEMENT

M.Á. Sánchez-Gálvez, A. García-Vico, V. Ruiz-Luque, M.L.

Ruiz-Juliá, J. Aguilar-Luque, P. Martínez-García

\section{Surgery, Hospital Virgen de Valme, SEVILLA, Spain}

Introduction: The oesophagectomy is currently still mandatory in the curative treatment of the malignant oesphagic pathology. This procedure is defined by important morbidity and mortality. The minimally invasive approach aims to reduce the complications without repercussion on the oncological outcomes, however it's not exempt from them being a demanding surgical technique like it is.

Aim: We present the video of three complications after a three-stage oesophagectomy (McKeown-like) with the thoracic stage via thoracoscopy and the minimally invasive surgical solution for both of them.

Methods: All three cases represent a three-stage oesophagectomy for malignant esophageal pathology. The first one was a 50-year-old male who suffered an intraoperative left main bronchus injury. The second case was a 62-year-old male with no intraoperative complications whatsoever. Nonetheless, on the second postoperative day, milky drainage started to appear through the thoracic tube. The third and final case represents an intraoperative hemorrhage, which is the most common complication of this kind of surgery.

Results: The first case was diagnosed and treated intraoperatively with the use of an adhesive case was a chylothorax, diagnosed on the second postoperative day. It was treated initially with conservative measures. Due to bad evolution, he underwent surgery on the tenth postoperative day. We can see how we ligated the stump of the thoracic duct in the original surgery and then how we repaired the unexpected leak. After the second surgery, the patient was discharged on the sixth day. The last patient was also diagnosed and treated intraoperatively successfully, with no repercussion whatsoever in the postoperative time.

Conclusions: The minimally invasive surgery has many advantages in the upper gastrointestinal field. It is a demanding technique, so it is important to be able to treat the complications that may arise with this approach. matrix. During the postoperative period the patient showed no further complications. The second 


\section{V224-UPPER GI-Esophageal cancer}

\section{MINIMALLY INVASIVE MCKEOWN'S OESOPHAGECTOMY FOR ESOPHAGEAL SQUAMOUS CARCINOMA WITH COMPLETE PATHOLOGICAL RESPONSE}

\author{
$<\mathrm{u}>$ A. Tita $</ \mathrm{u}>^{1}$, M. Yapur $^{2}$, J.I. Moreno
}

${ }^{1}$ Digestive and Endocrine Surgery, Sanatorio NOSTI, RAFAELA, Argentina; ${ }^{2}$ Digestive and Endocrine Surgery Department, Sanatorio NOSTI, RAFAELA, Argentina

Introduction: Oesophageal cancer is the sixth leading cause of cancer death worldwide. Surgery offers the best chance of cure, however, esophagectomy leads to an extensive operation with high morbidity and mortality. In order to reduce complications, minimally invasive esophagectomy has been positioned as the procedure of choice. The objective of this video is to show the minivasive approach in a patient with esophageal middle $1 / 3$ carcinoma with complete pathological response.

Materials and methods: A 67-year-old woman with progressive dysphagia and weight loss. Upper Endoscopy shows a circumferencial lesion $25 \mathrm{cmform}$ the dental arch. Biopsy: Invasive Squamous cell carcinoma. Staging was completed with Thoraco-Abdominal CT, Endoscopic US and PET CT: identifying localized thickening of all esophageal layers in the middle $1 / 3$ esophagus, without mediastinal lymph nodes. Bronchoscopy: without tracheobronchial involvement. Neoadjuvant Therapy: Radiotherapy + Carboplatin / Paclitaxel. After 6 weeks re-staging is performed. Upper endoscopy: residual ulcer. CT shows tumor volume reduction. After nutritional and pre-rehabilitation support, minimally invasive surgery with 3-field approach (McKeown) is indicated. We present this video showing the feasibility of the thoraco-laparoscopic approach in malignant oesophageal pathology

Results: Patient in prone position, selective lung intubation, 3 trocars thoracoscopic approach in right hemithorax. Complete esophageal mobilization. The thoracic duct is clipped and divided. Azygous vein is section with linear stappler vascular load. Sub-carinal lymphadenectomy. Thoracic drain. 4 trocars laparoscopic approach. Abdominal Lindadenectomy. Section of Left Gastric Vessels and preparation of gastric tube. Cervical approach and exteriorization of the specimen. Latero-lateral esophagogastric anastomosis with linear stappler. Nasojejunal feeding tube placement. Jackson Pratt drains are left in cervical and transhiatal position. Uneventful recovery within the ERAS program. Barium swallow on 3rd day .Discharged on 6th day. Biopsy: residual ulcerated lesion, with granulation tissue, no atypia. Free margins. Subcarinal and abdominal nodes are negative.

Conclusions: Mininvasive esophagectomy is the ideal method to treat esophageal tumors with good clinical response. It is a well tolerated procedure, with fast recovery, low morbidity and good oncological results, thanks to the better exposure and ease to dissect the mediastinal structures.

\section{V226-UPPER GI-Gastric cancer}

TOTAL GASTRECTOMY WITH INDOCYANINE GREENGUIDED LYMPHADENECTOMY

I. Lledó ${ }^{1}$, I. Alarcón ${ }^{1}$, A. Senent ${ }^{1}$, T. Yang ${ }^{1}$, E. Licardie ${ }^{1}$,

F. Lopez-Bernal ${ }^{1}$, A. Barranco ${ }^{1}$, M. Socas ${ }^{1}$, F.J. Padillo ${ }^{2}$, S. Morales-Conde ${ }^{1}$

${ }^{1}$ Ud. de Innovación en Cirugía Mínimamente Invasiva. Servicio de Cirugía General, Hospital Universitario Virgen del Rocío, SEVILLA, Spain; ${ }^{2}$ Servicio de Cirugía General y Digestiva, Hospital Universitario Virgen del Rocío, SEVILLA, Spain

Aims: The use of ICG fluorescence is incrising in surgery, mainly as a test of vascular supply in colonic anastomoses. During the last years, other potential uses have been described, such as the identification of the sentinel lymph node and lymphatic mapping in oncological surgery. These advances could allow a better staging in order to decide the most appropriate treatment to each patient. Gastric cancer is one of the fields where this could play a key role in the near future. We present a case of a patient who underwent a laparoscopic total gastrectomy with ICG-guided D2 lymphadenectomy, where a personalized lymphatic mapping was performed.

Methods: A 37-year-old male patient underwent gastroscopy for gastric discomfort, and a gastric carcinoma was detected at the greater curvature of gastric body. Endoscopic biopsy was informed as diffuse type gastric adenocarcinoma. The preoperative staging was completed with echoendoscopy and CT-scan (T1bNOM0). We decided to perform a laparoscopic total gastrectomy with ICG-guided lymphadenectomy. The preoperative day, a gastroscopy was performed to inject $0.75 \mathrm{mg}$ of ICG in four submucosal areas around the tumor.

Results: Intraoperatively, the lymphatic mapping marked by ICG was checked, allowing the identification of the territory of drainage of the tumor to lymph nodes at the lesser curvature, the greater curvature and the splenic artery. A D2 lymphadenectomy and a total laparoscopic gastrectomy with Roux-en-Y reconstruction was performed. During the lymphadenectomy, we were able to observe marked lymph nodes in territory 11 , and also observed that the paraaortic lymph node behind the celiac trunk did not become green and the lymphadenectomy at this area was not continued. The patient presented no postoperative complications, and was discharged on the seventh postoperative day. The histological results showed a diffuse type gastric adenocarcinoma pT2N1 and 26 isolated lymph nodes, being one of them possitive (corresponding to the adenopathy marked at the greater curvature).

Conclusions: Lymphatic ICG-mapping in gastric cancer is a potential revolutionary advance that could ensure a correct lymphadenectomy, avoiding lymph node understaging. It is necessary to continue carrying out studies that will allow developing protocols to define appropriate lymphadenectomy based on ICG-mapping.

\section{V228-UPPER GI-Gastric cancer}

\section{TOTALLY LAPAROSCOPIC TOTAL GASTRECTOMY FOR GASTRIC CANCER}

B. Lluch ${ }^{1}$, P. Priego ${ }^{2}$, A. González Barranquero ${ }^{2}$, S. Sánchez Picot $^{2}$, L. Blazquez ${ }^{2}$, M. Cuadrado ${ }^{2}$, J. Galindo ${ }^{2}$

${ }^{1}$ General and Digestive Surgery, Hospital General Universitario Castellón, CASTELLÓN, Spain; ${ }^{2}$ Division of Esophagogastric and Bariatric Surgery, Ramón y Cajal University Hospital, MADRID, Spain

Background: Laparoscopic gastrectomy is gaining popularity in the last decades for treatment of gastric cancer. However, laparoscopic total gastrectomy (LTG) has not become as popular as laparoscopic distal gastrectomy because of the more difficult reconstruction technique. Anyway, this technically demanding procedure has largely demonstrated to be at least equal compare to conventional open gastrectomy (OG) in short and long-term studies.

Methods: A 51 years-old woman was admitted to our clinic with epigastric pain. The upper digestive endoscopy showed an ulcer of $1 \mathrm{~cm}$ of diameter with irregular edges in body of stomach. Ulcer biopsy indicated a moderate differentiated signet ring cell adenocarcinoma. The ecoendoscopy revealed a neoformation in the gastric body that extends out of the muscular layer, with pathological lymphadenopathies (uT3N1). CT scan did not revealed distant metastasis Scientific multidisciplinary committee decided neoadjuvant treatment with FLOT chemotherapy. Six weeks after chemotherapy was finished patient was operated.

Results: A total laparoscopic gastrectomy with D2 lymphadenectomy was performed. Operation time was $200 \mathrm{~min}$. The postoperative course was uneventful and patient was discharged home on 12 day post-op with normal digestive tolerance and an esophagogastric transit showed no anastomotic leak. The resected specimen showed a poor differentiated signet ring cell adenocarcinoma with 3 positive lymph nodes from 23 isolated (T2N2M0) and the margins had tumorfree.

Conclusion: Nowadays, LTG is believed to be a safe and feasible surgical technique for experienced surgeons in gastric cancer and minimally invasive surgery, comparable to OG. 


\section{V229-UPPER GI-Gastric cancer}

STRATEGY FOR LAPAROSCOPIC REPAIR OF PETERSEN'S HERNIA AFTER RADICAL GASTRECTOMY: A CASE REPORT

R. Matsui, N. Inaki, M. Ouchi, K. Nagakari, M. Fukunaga, Y. Ishizaki

Department of Gastroenterological Surgery, Juntendo University Urayasu Hospital, URAYASU, Japan

Introduction: Petersen's hernia is one of severe postoperative complication after gastrectomy, which may result in massive resection of small intestine. It is considered an essential proscedure to close the Petersen's defect for all cases after such reconstruction after gastrectomy as Roux-en Y method. We report a case of Petersen's hernia after radical gastrectomy, which was repaired laparoscopically.

Patient: The patient was 59-year-old male, who underwent laparoscopic distal gastrectomy for gastric cancer (D2 lymph node dissection followed by Roux-en-Y reconstruction) two years ago. The closure of Petersen's defect was not performed in the initial operation. He was aware of abdominal pain and visited emergency unit in our hospital. Abdominal CT scan showed internal hernia of Petersen's hernia.

Surgical procedure: In laparoscopic examination, dilation of small intestinal and mastoid ascites was observed. Massive small intestine including Y-limb entered into Petersen's defect from left to right side. We carefully pulled through the small intestine and confirmed absence of ischemic change in the whole small intestine. Then the Petersen's defect was closed by continuous suturing with 3-0 non-absorbable barbed suture.

Results: The operation time was $95 \mathrm{~min}$ and the estimated blood loss was $10 \mathrm{ml}$. Oral intake was started from the next day of the operation. There was no postoperative complication. The patient was discharged on the 10th postoperative day.

Conclusion: We could safely perform laparoscopic repair for Petersen's hernia. Regarding technical points in the procedure, it is important to judge the direction of the small intestine into the Petersen's defect, to manage the dilated small intestine gently, and to close the Petersen's defect by laparoscopic suturing.

\section{V230-UPPER GI-Gastric cancer}

\section{ADENOCARCINOMA OF THE GASTRIC POUCH AFTER RNY GASTRIC BYPASS}

J. Colpaert, M. Ooms, I. van Campenhout, J. Janssen, J. Lesaffer, B. Dillemans

Department of Surgery, AZ Sint-Jan, BRUGGE, Belgium

Introduction: In this case we present a 52-year old male with a history of morbid obesity, sleep apnea and psychiatric affliction including alcohol and nicotine abuse. In 2009 he underwent a laparoscopic Roux-en-Y gastric bypass. The results were satisfactory, with no complications post-surgery, and a steady weight loss over time (pre: $162 \mathrm{~kg}$, post: $125 \mathrm{~kg}$ ). In November 2017 , he presented with complaints of dysphagia and weight loss (12 kg in 3 months). Laryngoscopic examination by the otorhinolaryngologist was negative. He was referred to gastro-enterology for gastroscopy. Biopsies showed a mildly differentiated adenocarcinoma of the gastro-esophageal junction with submucosal invasion.

Objective: After negative staging assessment, multiple treatment options were considered. The route of choice ended up being a laparoscopic radical gastrectomy with esophago-jejunostomy, with the objective to achieve optimal oncological results.

Methods: The procedure is demonstrated in this video. The gastric pouch as well as the remnant stomach, greater and lesser omentum were resected laparoscopically. Due to the invasion of the carcinoma into the distal esophagus, a segment of the esophagus was resected as well. Following anatomopathological examination on frozen section, the resection margins were reported malignancy free.

Results: Postoperatively, there were no complications. CT scan with contrast showed no signs of leakage. Anatomopathological examination confirmed the tumor to be a mildly invasive and poorly differentiated adenocarcinoma with local signet-ring cell differentiation (pT1bN0). There was no need for adjuvant therapy. Oral intake was sound.

Conclusion: Adenocarcinomas of the gastric pouch are rarely seen following gastric bypass. This patient presented with complaints of dysphagia, and an adenocarcinoma was diagnosed. Consequently, the patient had a total gastrectomy at our hospital. The surgery was performed laparoscopically, and was executed with success. To conclude, it is feasible to treat adenocarcinomas after gastric bypass laparoscopically via total gastrectomy and omentectomy.

\section{V231-UPPER GI-Gastric cancer}

\section{LAPAROSCOPIC TOTAL GASTRECTOMY WITH MODIFIED D2 LYMPHADENECTOMY AND ROUX-AN-Y ESOPHAGOJEJUNOSTOMY}

\author{
E. Kakiashvili ${ }^{1}$, E. Brauner ${ }^{2}$, O.B. Ben Ishai $^{2}$
}

${ }^{1}$ General Surgery, Galilee Medical Center, KIRIAT MOZKIN, Israel; ${ }^{2}$ General Surgery, Rambam Medical Center, HAIFA, Israel

80 year old, female patient presented with upper abdominal discomfort and microcitic anemia An ulcerative lesion was found on gastroscopy examination in body of the stomach (near the grate curvature). Biopsy was done and pathology result showed poorly differentiated adenocarcinoma.

Chest computed tomography (CT) was without any significant findings. Abdominal CT showed the lesion in stomach without enlargement of regional lymph nodes.

Her blood laboratory examinations were within normal limits, including serum CEA.

Patient underwent laparoscopic Total Gastrectomy with modified D2 lymphadenectomy and Roux-en-Y esophagojejunostomy

Total operating time (ORT) was $218 \mathrm{~min}$.

Three days after operation, patient has developed none ST elevated MI and respiratory failure. She was intubated. On day 6 after operation she was extubated, on day 9 patient started regular diet and was discharged home on day 13 .

Final pathology result confirmed poorly differentiated adenocarcinoma of the stomach.

\section{V232-UPPER GI-Gastric cancer \\ LAPAROSCOPIC ROUX EN 'Y' D2 SUBTOTAL GASTRECTOMY}

A. Peri, L. Pugliese, F.P. Tinozzi, N. Mineo, V. Gallo, A. Pietrabissa General Surgery II, IRCCS Policlinico San Matteo, PAVIA, Italy

This video shows our favourite technique for laparoscopic D2 subtotal gastrectomy. We usually perform the procedure with 4 or 5 trocars; after the coloepiploic detachment we perform the gastric transection first! This manoeuvre provides a perfect view for the lymphadenectomy. At the end of our dissection we transect the duodenum with Seamguard reinforcement. Before going on with the reconstructive phase, we prepare the roux en ' $\mathrm{Y}$ ' with double loop technique, usually without dividing the mesentery. Then we remove the specimen through a periumbilical $3-4 \mathrm{~cm}$ minilaparotomy; we think it's important anyway to check margins and distance from the tumor before going on with the reconstructive phase. From the same minilaparotomy we retrieve the prepared limb for roux en $\mathrm{Y}$ and we perform a side to side mechanical linear anastomosis outside.Then we proceed with performing the anastomosis between the gastric pouch and the alimentary limb by laparoscopy. We like very much this technique for the increased exposition of tissues during lymphadenectomy. 


\section{V233-UPPER GI-Gastric cancer}

PROXIMAL GASTRECTOMY IN SUBCARDIAL GASTROINTESTINAL STROMAL TUMOR FEASIBLE BY LAPAROSCOPIC APROACH

M.A. Mayo-Ossorio ${ }^{1}$, A. Bengoechea-Trujillo ${ }^{2}$, M. Fornell-Ariza ${ }^{2}$, C. Peña- Barturen ${ }^{2}$, J. Varela-Recio ${ }^{2}$, S. Cerrato-Delgado ${ }^{2}$, A. FierroAguilar $^{2}$, J.M. Pacheco-Garcia ${ }^{2}$

${ }^{1}$ General. Surgery. Cirugía General Y Del Aparato Digestivo, Hospital Universitario Puerta Del Mar Cadiz, CADIZ, Spain; ${ }^{2}$ General Surgery, Hospital Universitario Puerta Del Mar Cadiz, CADIZ, Spain

Aims: Laparoscopic surgery for gastric gastrointestinal stromal tumors (GISTs) is now widely performed. Proximal gastrectomy is not very performed because the procedure is complicated, particularly by laparoscopic aproach. We present a 39 -year-old female patient diagnosed of a subcardial gastrointestinal stromal tumor who underwent laparoscopic proximal gastrectomy with good postoperative results.

Method: A 39-year-old patient studied for epigastric pain and fever. Upper gastrointestinal endoscopy was performed in which subcardial gastrointestinal stromal tumor is identified. A Body-TC was performed in which axillary, mediastinal adenopathies and images suggestive of hepatic metastases were identified. The biopsy confirms a gastrointestinal stromal tumor. The case discussed in a multidisciplinary committee and the PET-CT study was completed, Subcardial GIST T4N1M0 was diagnosed. Neoadyuvance was decided with imatinib for one month and surgery was performed using a laparoscopic approach. The approach was performed with 5 trocars $(11 \mathrm{~mm}$ supraumbilical, two $12 \mathrm{~mm}$ subcostal left and right, $5 \mathrm{~mm}$ subxifoid and $5 \mathrm{~mm}$ left flank). Gastrectomy was performed with D1 lymphadenectomy following the oncological principles of subcardial tumors. The piece was removed in a bag by extending the $11 \mathrm{~mm}$ port to mini-laparotomy. Esophagogastric anastomosis was performed by hand assisted circular mechanical suture. Methylene blue test was carried out. No nasogastric tube left, but drainage tutoring the esophagogastric anastomosis was left.

Results: The postoperative evolution was favorable. Oral tolerance without incidents at fourth postoperative day. The patient was discharged without incidences on the seventh postoperative day. The pathological study of the piece was reported as subcardial gastrointestinal stromal tumor $3 \mathrm{~cm}$ with respected surgical margins and 11 lymph nodes free of malignancy, postoperative diagnosis of T2NOM0. One month after surgery, the patient has adequate oral tolerance. She does not report gastroesophageal reflux and at 6 months remains asymptomatic and with good evolution.

Conclusions: Laparoscopic proximal gastrectomy is a technique that is not currently used but can be performed through a laparoscopic approach. It is a safe technique with good clinical and oncological results, especially in the early gastric cancer and gastrointestinal stromal tumors. However, long-term studies are necessary.

\section{V234-UPPER GI-Gastric cancer}

\section{ROBOTIC-ASSISTED TOTAL GASTRECTOMY WITH MODIFIED D2 LYMPHADENECTOMY AND ROUX-AN-Y ESOPHAGOJEJUNOSTOMY}

\section{E. Kakiashvili ${ }^{1}$, E. Brauner ${ }^{2}$}

${ }^{1}$ General Surgery, Galilee Medical Center, KIRIAT MOZKIN, Israel; ${ }^{2}$ General Surgery, Rambam Medical Center, HAIFA, Israel

67 year old, female patient presented with upper abdominal discomfort and pain, without nausea, vomiting or weight loss, started two month before admission.

An ulcerative lesion was found on gastroscopy examination (near the incisura angularis). Biopsy from the mass has showed signet ring cell adenocarcinoma, poorly differentiated, diffuse type. Endoscopic ultrasound (EUS) didn't show lymphadenopathy. Chest and abdominal computed tomography were without any significant findings.

Her blood laboratory examinations were within normal limits including serum CEA.

Patient underwent da Vinci robotic-assisted Total Gastrectomy with modified D2 lymphadenectomy and Roux-en-Y esophagojejunostomy.

Total operating time (ORT) was $200 \mathrm{~min}$. Three day after operation patient started regular diet and was discharged on day six after operation.

Final pathology result confirmed poorly differentiated adenocarcinoma with signet ring cells.

\section{V235-UPPER GI-Gastric cancer}

\section{SUBTOTAL LAPAROSCOPIC GASTRECTOMY WITH D2 LYMPHADENECTOMY}

\section{J. Maia Teixeira, T. Sanai, R. Pereira, B. Cruz}

Surgery, CHUA, FARO, Portugal

Laparoscopic gastrectomy is a perfectly safe option nowadays for the treatment of gastric cancer. Every year the percentage of the laparoscopic approach is rising not only in the east but also in the west.

We present a case of a 44 year old female patient with a Gastric Tumor of the antrum-G3 Adenocarcinoma with a cT2NOM0 staging.

We perform a Subtotal Laparoscopic Gastrectomy with a D2 Lymphadenectomy and Roux-en-Y anastomosis.

The patient begin clear liquids on the first post operative day and was discharged on the 5th The final anatomopathological result of the specimen was a Adenocarcinoma (G3) - pT1N0. There were 37 nodes resected all negative.

The case was discussed in multidisciplinary team and was decided for clinical follow up with no further treatments.

The patient was evaluated one month after surgery with no complaints and will continue the follow up.

\section{V236-UPPER GI-Gastric cancer}

\section{BREAKING NEWS}

\section{S. Giacopuzzi}

Upper GI Surgery, University of Verona, VERONA, Italy

Laparoscopic endoscopic cooperative surgery is an option in medium size submucosal cancers invading the muscular layer, mainly in border area were wedge resections are nor feasible.In this video we report a case of prepiloric GIST treated with NEWS Technique (nonexposed endoscopic wall-inversion surgery).We think that this technique is feasible and safe and should be considered a valid option with a view to preserving the organ. 


\section{V237-UPPER GI-Gastric cancer}

\section{ALMOST TOTAL LAPAROSCOPIC GASTRECTOMY (95\%) FOR GIANT GASTRIC GIST}

\author{
A. Ballestero, P. Priego, S. Sanchez, L. Blazquez, M. Cuadrado, \\ J. Galindo, E. Lobo
}

Cirugia general y del aparato digestivo, Ramon y cajal hospital, MADRID, Spain

Aims: Gastrointestinal Stromal Tumors (GIST) are the most frequent mesenchymal tumors of the digestive tract. The most common location is the stomach in the $70 \%$ of the cases. The treatment of choice is the surgical approach. It is under discussion the laparoscopic approach in large tumors.

Methods: A 72-year-old man was studied for elevated ferritin. It was identified abdominal heterogeneous mass in the epigastrium with a measure of $10 \times 9 \mathrm{~cm}$. Complementary tests were performed observing in the endoscopy a extrinsic compression image in the gastric antrum without alterations in the mucosa. The endoscopic ultrasound confirmed the presence of an heterogeneous mass of $8 \mathrm{~cm}$ with well-defined borders compatible with GIST. In the computerized axial tomography the lesion was observed in contact with the left hepatic lobe withou apparent dependence. Another $13 \mathrm{~mm}$ nodular lesion was identified in the anterior wall of the gastric antrum of the same nature. FNA of the tumor was performed with findings suggestive of low-grade mesenchymal injury. Exploratory laparoscopy was performed, identifying a large lesion which was depending of the gastric body but not attached to the left hepatic lobe neither pancreatic body. Almost total laparoscopic gastrectomy was performed according to the usual technique with 5 trocars with digestive reconstruction in Roux-en-Y.

Results: The procedure was successfull. Upper GI tract was performed on the seventh postoperative day without evidence of leakage. After oral tolerance the patient was discharged on the ninth postoperative day. The anatomopathological result was Gastrointestinal Stromal Tumor (GIST) of $10.5 \mathrm{~cm}$ in greatest diameter and another nodule of $1 \mathrm{~cm}$ in antral location, positive c-Kit, Ki 67 1-2\%, pT4N0 with a mitotic index of 2 mitosis / $50 \mathrm{cmga}$.

Conclusions: Gastric GIST is a rare tumor which surgical resection is the best treatment. A laparoscopic approach is feasible even in large tumors if it is performed in specialized centers and by experienced surgeons.

\section{V238-UPPER GI-Gastroduodenal diseases}

PERCUTANEOUS ENDOSCOPIC INTRAGASTRIC SURGERY FOR SUBMUCOSAL TUMORS AT GASTRIC CARDIA : TIPS FOR ENSURING THE BENEFIT AS AN ORGAN PRESERVING APPROACH

\section{K. Doden ${ }^{1}$, N. Inaki ${ }^{2}$, T. Tsuji ${ }^{1}$, D. Yamamoto ${ }^{1}$, H. Kitamura ${ }^{1}$}

${ }^{1}$ Department of Gastroenterological, Ishikawa Prefectural Central Hospital, KANAZAWA, Japan; ${ }^{2}$ Department of Surgery, Juntendo University Urayasu Hospital, URAYASU, Japan

Aim: Laparoscopic wedge resection or partial resection is a safe and feasible stomach preserving approach to gastric submucosal tumors (SMT) such as gastrointestinal tumors (GIST), and it has been widely performed recently. However, it should not be applied to the tumors at cardia in order to avoid stenosis or disruption of anti-reflux mechanism. We have introduced Percutaneous endoscopic intragastric surgery (PEIGS) for SMT at cardia since 2013 to preserve function of cardia. We will report the tips, techniques, and clinical result of our PEIGS.

Methods: From September 2013 to August 2018, seven patients with SMT at cardia underwent PEIGS in our hospital. We insert the $12 \mathrm{~mm}$ port at umbilicus and investigate the abdominal cavity at first. Then the incision is extended to $2.5 \mathrm{~cm}$ and Lap-Protector ${ }^{\mathrm{TM}}$ is equipped with the site to perform mini-laparotomy. Using peroralendoscopy, a stomach was insufflatedand incision is made at an anterior wall of gastric body under direct vision. Additional Lap-Protector ${ }^{\mathrm{TM}}$ is placed into the stomach so that the stomach is fixed on the abdominal wall. It enables us to keep direct access to gastric lumen and stable operative field. EZ access ${ }^{\mathrm{TM}}$ is attatched on LapProtector $^{\mathrm{TM}}$, and intragastric operation is started. Subsequently, two trocars are inserted using Funada's gastropexy instrument. Tumor is dissected by using energy devices, avoiding injury of capsule of the tumor. The defect of the gastric wall is closed by intragastric suturing. We should take particular care not to damage EGJ during the suturing, inserting peroral endoscopy in and out.

Results: The mean operation time was $145(117-149) \mathrm{min}$ and the amount of intraoperative bleeding was 5.0(3.0-7.5) $\mathrm{ml}$. The maximum diameter of tumors was $30(15-30) \mathrm{mm}$. One case was low risk GIST and otherwise were leiomyoma. The postoperative course was uneventful in all cases, without leakage or stenosis. Total hospital stay was 9(8.5-9) days. No patient had symptoms of esophagitis.

Conclusions: PEIGS for SMT at gastric cardia is a feasible and safe approach, preserving function of cardia. Our procedure achieves great stability and excellent visualization during the operation, which may have led to the fine surgical results.

\section{V239-UPPER GI-Gastroduodenal diseases}

FOCUS ON STATION NUMBER 6 IN LAPAROSCOPIC LYMPH NODE DISSECTION USING ICG

\section{S. Giacopuzzi}

Upper GI Surgery, University of Verona, VERONA, Italy

Laparoscopic lymphadenectomyin gastric cancer is considered a feasible and safe procedure Data on the compliance of lymphadenectomy in the various lymph node stations is not yet well understood; moreover it is not clear if there are particular conditions relate to the patients impairing the oncological results. This video reports the use of the ICG for the lymph node dissection of station number 6 in a case of obese patient and a case of a cirrhotic patient. Fist patient, M.A., was a 67 year old man with distal cancer cT2N0 and a 32 BMI. Second patient, T.D., was a 56 year old man with distal cancer cT2N0 and a alcoholic cirrhosis Child B7. In both cases, intraoperative endoscopy was performed 20 to $30 \mathrm{~min}$ before the onset of lymph node dissection. $0.1 \mathrm{mg}$ of ICG was injected into the submucosal layer in 4 quadrants of the primary tumor. A laparoscopic subtotal gastrectomy was conduced with D2 lymphadenectomy. Lymph note navigation were analyzed by novadaq ${ }^{\circledR}$ detector. Using navigation system we removed the $\mathrm{n} 6$ basin. In both cases dissection were effective and pancreatic surface were easily detectable.

Number of lymph nodes retrieved was 8 in the case of obese patient and 3 in the case of cirrhotic patient. Pathological TNM were pT3N0 $(0 / 50 \mathrm{~N}+)$ in the first case and pT3N1 $(2 / 40 \mathrm{~N}+)$ in the second. No $\mathrm{N}+$ metastases were detected in $\mathrm{n} 6$ station for both cases. No pancreatic fistula was recorded.

ICG lymph node navigation system should be considered a valid support for the surgeon for completion of a correct lymphadenectomy in surgical challenging cases.

\section{V240_UPPER GI_Gastroduodenal diseases}

\section{MORGANI'S HERNIA: TWO TIPES OF MESH FOR LAPAROSCOPIC APPROACH}

J. Hernandez Gutierrez, A.F. Aranzana Gomez, J.S. Malo Corral, B. Muñoz Jimenez, G. Krasniqi, R. Lopez Pardo, P. Toral Guinea

General Surgery, C.H.Toledo, TOLEDO, Spain

Aims: Morgagni hernia is the rarest of congenital diaphragmatic hernias (2-3\%). Its presentation is rare in adults and its finding is usually incidental. It was first described by Giovanni B. Morgagni in 1769. It is located in the anterior region of the diaphragm. It is caused by a congenital defect in the fusion of the transverse septum of the diaphragm and the costal arches. The need for surgery depends on the presentation, it is recommended early repair before the development of complications. Classically, the surgical approach was thoracotomy or laparotomy. Currently, the tendency is to use a minimally invasive approach, which has shown good results, lower morbidity and faster recovery. The need to repair the defect with a mesh is controversial, recommended when it is not possible to close the defect without tension. The objective of this VIDEO is to demonstrate the safety and efficacy of the laparoscopic approach for the repair of this type of hernia, as well as the different types of mesh that can be used.

Methods: 2 Clinical cases. The iconography of interest is exposed

78 year old female with history of Chronic TEP anticoagulated, clinical respiratory difficulty. CT and EGD shows Morgagni hernia that contains omentum and transverse colon.

76 year old male with history of COPD. Respiratory distress due to Morgagni hernia. Results: Complete endoscopic approach. Reduction of hernial content into the abdominal cavity, dissection of the hernial sac. Measurement of the hernial defect, being impossible to close the defect so a hernioplasty with mesh was performed, in the first clinical case an Omyra mesh was used and in the other case a Ventralight ST mesh. Correct postoperative period. Asymptomatic and no recurrence in follow up at 24 months after surgery.

Conclusion(s): The laparoscopic approach is safe and effective in the surgical treatment of patients with Morgagni hernia. The use of stapling techniques with tackers can be dangerous due to the proximity of the heart and the subsequent risk, so we recommend fixing the mesh with loose stitches in contact with the hernial pericardium. 


\section{V241-UPPER GI-Gastroduodenal diseases}

LAPAROSCOPIC SEGMENTAL DUODENO-JEJUNAL RESECTION FOR A GASTRO-INTESTINAL STROMAL TUMOUR

\author{
T. Cipolat Mis, A. d'Alessandro, E. Falsetti, M.C. Cartillone, \\ E. Chouillard
}

General and Minimally Invasive Surgery, Poissy-Saint-Germain-enLaye Medical Center, POISSY, Italy

Aims: The treatment of the non-metastatic gastro intestinal stromal tumour (GIST) is the surgical resection[1]. The duodenal gastro-intestinal stromal tumour (GIST) is a relatively rare clinical entity. From all the resected GIST, only 5\% are duodenal [2]. The clinical presentation could vary from the most common acute gastro intestinal (GI) bleeding, chronic anaemia, but also acute abdomen caused by tumour rupture and intestinal obstruction.

Methods: A 69 years old patient present at the Emergency department of the CHI Poissy-St Germain-en-Laye (Paris, France) with acute gastrointestinal bleeding. At the laboratory exams the haemoglobin was $9.1 \mathrm{~g} /$ $\mathrm{dL}$. The patient perform a computer tomography (CT) which shows two hyper vascularised lesion at the 4th duodenum; this lesions has an intra and extraluminare growing. The CT scan didn't show any other abdominal lesions. The patient were submitted to a minimally invasive surgical operation with the multiport laparoscopic technique.

Results: The resection of the 3th and 4th duodenum and of the first 5 centimetre of jejunum was performed with a four trocar laparoscopic technique. A latero-lateral duodeno-jejunal mechanical anastomosis was performed. The operative time was $90 \mathrm{~min}$. The patient start with oral alimentation on the third post-operative day after a CT scan with oral contrast that was negative for anastomotic dehiscence and collections. The post-operative course was globally uneventful and the patient was discharged at fifth post-operative day without complications.

The histological examination shows a low risk GIST, CD117 positive and with a Ki-67 inferior of $2 \%$ (classification TNM 7th edition pT1).

Conclusions: Laparoscopic distal duodenal resection for a non-metastatic GIST is a safe and feasible surgical procedure if conducted by expert surgeons. [1] Surgical management of gastrointestinal stromal tumours P. Gervaz,O. Huberand,P. Morel British Journal of Surgery 2009;96: 567-578

[2] Gastrointestinal stromal tumor X. Zhao, C. Yue J Gastrointest Oncol. 2012 Sep; 3(3): 189-208.

\section{V243-UPPER GI-Gastroduodenal diseases}

\section{ROBOTIC ENUCLEATION OF LEYOMIOMA FROM GASTROESOPHAGEAL JUNCTION}

\section{E. Kakiashvili ${ }^{1}$, E. Brauner ${ }^{2}$}

${ }^{1}$ General Surgery, Galilee Medical Center, KIRIAT MOZKIN, Israel; ${ }^{2}$ General Surgery, Rambam Medical Center, HAIFA, Israel

26 year old, female patient presented with upper abdominal pain and moderate difficulty during swallowing, started four month before admission.

Gastroscopy revealed sub mucosal mass in gastroesophageal junction. Endoscopic ultrasound (EUS) showed $3 \mathrm{~cm}$ sub mucosal mass. Cytology was taken from the lesion and result confirmed smooth muscle tumor (most probably leyomioma)

Her blood laboratory examinations were within normal limits.

Patient underwent da Vinci robotic enucleation of leyomioma from gastroesophageal junction.

Total operating time (ORT) was $135 \mathrm{~min}$. During operation, frozen section has confirmed diagnosis of leyomioma.

Day after operation patient has started regular diet and was discharged home.

Final pathology report has confirmed diagnosis of leyomioma.

\section{V244-UPPER GI-Gastroduodenal diseases}

MANAGEMENT AND MINIMALLY INVASIVE APPROACH OF A GASTRIC VOLVULUS CASE

M.Á. Sánchez-Gálvez, V. Ruiz-Luque, M.L. Ruiz-Juliá, J. Aguilar-Luque, P. Martínez-García

\section{Surgery, Hospital Virgen de Valme, SEVILLA, Spain}

Aims: Gastric volvulus are a rare condition that sometimes represent a diagnostic challenge for surgeons. Here we present the video of a recent case of a gastric volvulus in our area that was treated with a minimal invasive approach.

Methods: We report the case of a 58-year-old woman who was admitted in the emergency room (ER) with epigastric transfixing pain and impossibility to vomit that had started $8 \mathrm{~h}$ prior to the admission. The physical exam showed good vital signs, and her abdomen was soft, with a tendency to tenderness with palpation in the epigastrium without guarding or rigidity. Her lab tests were normal and the conventional radiology showed a double gastric bubble. We run an urgent computed tomography scan (CT scan) and a upper gastrointestinal (GI) endoscopy that showed a big type II hiatal hernia that was complicated with a gastric volvulus.

Results: First, a nasogastric (NG) tube was placed for decompression of the stomach at the time when the endoscopy was made. The patient experienced a great improvement of the pain with that initial measure and remained stable. After almost a day of appropriate resuscitation, she underwent urgent surgery: we performed a hernia reduction, resection of the hernia sac, hiatal closure and a gastropexy and Nissen fundoplication. The patient suffered no complications in the immediate postoperative time and was discharged after six days.

Conclusion: Gastric volvulus are an uncommon emergency that we need to keep in mind. A simple abdomen $\mathrm{x}$-ray can be very helpful, given that the double gastric bubble sign is a typical sign of this condition. It's always mandatory to perform an upper GI endoscopy in order to get to the diagnose and place a NG tube promptly. The surgery can be safely delayed in stable patients with no signs of ischemia, and a laparoscopic approach is associated with a shorter hospital stay and good long-term outcomes in this kind of patients.

\section{V245-UPPER GI-Gastroduodenal diseases}

\section{LAPAROSCOPIC SUBTOTAL GASTRECTOMY WITH GASTROINTESTINAL ANASTOMOSIS (GIA) FOR GASTROINTESTINAL STROMAL TUMOR (GIST)}

V. Drakopoulos, S. Voulgaris, V. Lygizos, I. Iliadis, K. Rekouna, P. Trakosari, V. Vougas

1st Department of Surgery and Transplantation Unit, District General Hospital of Athens « Evangelismos », ATHENS, Greece

Introduction: Laparoscopic surgery is gaining acceptance in the treatment for gastric cancer. Laparoscopic treatment of gastric cancers and restoration of gastrointestinal tract after excision often present technical difficulties and require a large learning curve.

Material-Method: We present the case of a 69 years old presented with melena diarrhea, anemia (Ht 24.5\%) and weight loss. An upper GI endoscopy revealed a mass in the pyloric antrum (gastrointestinal stromal tumor). Patient underwent laparoscopic subtotal gastrectomy with gastrointestinal anastomosis, with the use of three trocars (umbilical $10 \mathrm{~mm}$, and two in the midclavical line bilateral.) Gastrointestinal anastomosis was conducted with the use of a linear stapler and additional laparoscopic stitches. The postoperative period was uncomplicated and remains in good condition six months after surgery.

Conclusion: Laparoscopic approach seems to be safe for treatment of gastric cancer, offering better surgical field view and less postoperative complications. 5 year survival after laparoscopic gastrectomy for gastric adenocarcinoma is compared with the open approach. 


\section{V246-UPPER GI-Gastroduodenal diseases}

PROSTHETIC REINFORCEMENT AS A TECHNICAL RESOURCE DURING LAPAROSCOPIC TYPE IV HIATAL HERNIA REPAIR

J. Riquelme-Gaona, M.L. Herrero-Bogajo, G. Tadeo-Ruiz, M. Lopez-Saiz, G. Rojas-de la Serna, A. Morandeira-Rivas, C. Moreno-Sanz

General Surgery, Hospital General La Mancha Centro, ALCAZAR DE SAN JUAN, Spain

Aims: During laparoscopic treatment of hiatal hernias the dissection can be complicated, but even more so the closure of the pillars, especially in giant hiatus hernias with a large defect. The use of prosthesis is controversial due to the lack of long-term studies and the possibility of secondary complications. The aim of this video is to demonstrate the safety of mesh hiatoplasty in hiatus hernia surgery.

Methods: We present the case of a 78-year-old woman with hypertension, hypothyroidism and right hemicolectomy for neoplasia 18 years ago. The patient presented with malnutrition, with a weight loss of $15 \mathrm{~kg}$ in the last months. A gastroscopy was performed in which a large hiatus hernia, that caused gastric volvulation, was shown. The upper gastrointestinal oral contrast study revealed esophageal tertiary waves and good passage to the gastric chamber, with an organoaxial volvulation of the stomach that was completely included in the thoracic cavity.

Results: Through a five trocar laparoscopic approach, a large paraesophageal type IV hiatal hernia $(7 \times 6 \mathrm{~cm}$ hiatal orifice) with complete herniation of the stomach and greater omentum to the mediastinum was observed. After reduction of the hernia content, complete dissection with partial resection of the sac was performed. An extended mediastinal dissection of the esophagus, with descent of the esophagogastric junction until achieving an abdominal esophagus of 3-4 cm, was carried out. After posterior and anterior phrenorrhaphy with nonabsorbable sutures, dislacement of the right pillar without diagragmatic opening was evidenced. It was decided to reinforce the hiatorraphy using a $\mathrm{C}$ shape composite mesh fixed with nonabsorbable sutures. The procedure was completed with a Nissen-type fundoplication. Postoperative course was uneventful and the patient remains without hernia recurrence 9 months after the intervention. Conclusion: Prosthetic reinforcement in hiatal hernia repair can be a very useful resource in large hiatal defects in which a stress-free hiatus closure cannot be achieved. However, its use must be individualized according to the characteristics of the patient, the quality of the tissues involved and the result of simple hiatorraphy.

\section{V247-UPPER GI-Reflux-Achalasia}

\section{SURGICAL SIMULATION FOR HELLER MYOTOMY} TRAINING

M. López Saiz ${ }^{1}$, J. Riquelme Gaona ${ }^{1}$, G. Rojas de la Serna ${ }^{1}$, V. Crespo García del Castillo ${ }^{1}$, G. Tadeo Ruiz ${ }^{1}$,

I. Díaz-Güemez Martín-Portugéss ${ }^{2}$, M.C. Tejonero Alvarez ${ }^{3}$, A. Morandeira Rivas ${ }^{1}$, C. Moreno Sanz ${ }^{1}$

${ }^{1}$ Digestive Surgery, General Hospital „La Mancha-Centro,,, ALCAZAR DE SAN JUAN, Spain; ${ }^{2}$ Laparoscopy Unit, Jesús Usón Minimally Invasive Surgery Center, CÁCERES, Spain; ${ }^{3}$ Digestive Surgery, Hospital Santa María del Puerto, CÁDIZ, Spain

Aims: Heller myotomy is an advanced laparoscopic surgical technique for the treatment of Achalasia, a rare disease in which long time is needed to achieve the learning curve. Surgical simulation, using animal models with an anatomy similar to humans, could help improving surgeon performance in a shorter time. The aim of our video is to show an ex-vivo and in-vivo animal model for Heller myotomy training.

Methods: A cadaveric porcine model was developed using a tissue block including the esophagus and the stomach, without the diaphragm, mounted in a physical laparoscopic simulator. Training procedures were also performed in an in vivo porcine model. Experiments were carried out in the ‘Jesús Usón' Minimally Invasive Surgery Centre in Cáceres.

Results: The surgical technique is described step by step, first in the esophagus-stomach ex-vivo model and later in the live animal model.

Conclusion: Surgical simulation using cadaveric an live animals offers a realistic representation, allows training in a safe environment, and can be very useful for advanced laparoscopic training in low incidence pathologies.

\section{V249-UPPER GI-Reflux-Achalasia}

\section{LAPAROSCOPIC REPAIR OF ESOPHAGIC PERFORATION AFTER LAPAROSCOPIC NISSEN FUNDOPLICATION}

E. Laviano, M.T. Gonzalez-Nicolas, M. Sanchez, S.J. Genzor, E. Puerta, J.R. Oliver, A. Bueno

Cirugia General y Digestiva, Hospital Miguel Servet, ZARAGOZA, Spain

Introduction: Esophagic perforation is one of the least frequent complications after laparoscopic Nissen fundoplication, but it remains one of the most dreaded because of its morbidity and mortality rates and its technically difficult reparation.

Aims: To present how the authors dealt with an iatrogenic esophagus perforation post laparoscopic Nissen fundoplication.

Methods: The authors report the clinical case of a 65-year-old woman who underwent a laparoscopic Nissen fundoplication because of a symptomatic large hiatus hernia in a differen hospital. The second postoperative day and after resuming oral intake, she presented respiratory and hemodynamic instability. She needed a chest tube that drained purulent content. The patient was referred to our hospital for clinical management. An urgent CT scan with oral contrast was performed without showing any leakage.

Results: In spite of the results, as the patient was unstable, she underwent an emergent diagnostic laparoscopy. It was found a small anterior esophagus perforation with right mediastinic collection. A running suture of the perforation was carried out and the Nissen fundoplication was converted to a Dör fundoplication. The operative time was $120 \mathrm{~min}$. She went to the intensive care unit for ten days. Five days after the surgery, she was given methylene blue with no exteriorization through the drainages. As postoperative morbidity, she suffered from a righ pneumonia and a thoracic collection that was treated by thoracic surgeons. The patient was finally discharged on the 64th postoperative day. She did well at home. She attended follow-ups without clinical reflux or any other particular condition.

Conclusions: Esophagic perforation is a life-threatening complication that can be managed laparoscopically if it is detected soon after surgery and an expertise is available.

\section{V250-UPPER GI-Reflux-Achalasia}

STEPS AND TRICKS DURING REVISONAL SURGERY AFTER FAILED HELLER'S MYOTOMY FOR ACHALASIA. ANALYSIS OF THREE CASES

M.C. Balagué ${ }^{1}$, B. Gonzalo ${ }^{1}$, S. Fernandez-Ananín ${ }^{1}$, C. Guarner ${ }^{2}$, I. Gomez ${ }^{1}$, E. Pareja ${ }^{1}$, A. Basterra ${ }^{1}$, D. Sacoto ${ }^{1}$, A. Rabal Fueyo ${ }^{1}$, E.M. Targarona ${ }^{1}$

${ }^{1}$ Digestive Surgery, Hospital Sant Pau, BARCELONA, Spain; ${ }^{2}$ Endoscopy, Hospital Sant Pau, BARCELONA, Spain

Surgical treatment of achalasia fails in $10-20 \%$ of patients. The most frequent responsible cause is a previous incomplete myotomy, followed by fibrosis and aspects related with antireflux procedure. Revisional surgery can represent a greater difficulty and a challenge. In this video we show 3 revisional surgeries after Heller's myotomy with Dor fundoplication for Achalasia. All cases presented a recurrence of the symptomatology and a revisional surgery was proposed. Surgeries were characterized by the presence of a herniation of the previous fundoplication, fibrosis around the prior myotomy and/or the formation of a pseudodiverticulum. We show the steps followed and the aspects to consider during the dissection. These cases demonstrate that laparoscopic reoperation for achalasia is feasible, even after open surgery. 


\section{V251-UPPER GI-Reflux-Achalasia}

\section{A NEW TECHNIQUE FOR LYMPH NODE DISSECTION ALONG PROXIMAL SPLENIC ARTERY DURING LAPAROSCOPIC GASTRECTOMY FOR GASTRIC CANCER: LEFT-LATERAL APPROACH}

S. Kanaji, S. Suzuki, M. Yamamoto, G. Takiguchi, Y. Matsuda, K. Yamashita, T. Matsuda, T. Oshikiri, T. Nakamura, Y. Kakeji, Division of Gastrointestinal Surgery

Department of Surgery, Graduate School of Medicine, Kobe University, KOBE, Japan

Background: Recently, laparoscopic lymph node (LN) dissection for gastric cancer can be performed safety by technical improvement. However, there is still technical difficulty in LN dissection along proximal splenic artery (SA), because frequent rupture of central LNs due to vulnerable supporting tissue and ruptured $\mathrm{LNs}$ pulled further in deep point around suprapancreas. To overcome these problems, we develop the new method for LN dissection along proximal SA done from patient's left side.

Methods: After dissecting LNs around right side of celiac artery (CA) as previous reports, operator move to patient' left side. First, suprapancreatic membrane is cut around the point where SA push out to head side of pancreas. The dorsal landmark of LN dissection along SA, splenic vein or back side of pancreas, is more easily can be identified than around CA. The LNs along the proximal SA is dissected toward the center of CA exposing the dorsal landmark. The dissection for LNs around CA can be done without rupture by preserving the suprapancreatic membrane around CA to the final phase of $\mathrm{LN}$ dissection.

Results: From April 2016, we performed this method in 25 cases. The median operation time was $43 \mathrm{~min}$ and retrieved lymph node were 16 during suprapancreatic lumphadenectomy using this approach. There were no operative complications with $<\mathrm{u}>></ \mathrm{u}>$ Grade II including pancreatic fistula. In all cases, LNs around proximal SA and left sides of CA were removed without rupture and could exposed the dorsal landmark.

Conclusion: Present method is enable to early identification of the dorsal landmark and preventing the rapture of central LNs preserving the suprapancreatic membrane around CA. The leftlateral approach can be a valid option of $\mathrm{LN}$ dissection along proximal SA.

\section{V252-UPPER GI-Reflux-Achalasia}

LAPAROSCOPIC MESH REPAIR OF LARGE HIATAL HERNIA AND UPSIDE-DOWN STOMACH WITH INTRAOPERATIVE TREATMENT OF AN ESOPHAGEAL LESION

\section{J. Dohmen, J. Arensmeyer, J.C. Kalff, P. Lingohr}

\section{Department of Surgery, University of Bonn, BONN, Germany}

Aims: Upside-down stomach (UDS) is a rare type of large paraoesophageal hernia, characterized by migration of the entire or large parts of the stomach into the posterior mediastinum. UDS is associated with severe complications like strangulation or volvulus development, possibly leading to acute gastric outlet obstruction and incarceration. Surgical repair is the only curative treatment option and therefore recommended for these patients. Standard procedure includes a hiatoplasty followed by an anti-reflux procedure. In a variety of studies, the use of mesh proved to be superior with respect to reduction of anatomical recurrences.

Methods: A 78-year old woman presented to us with reflux symptoms, dysphagia, dyspnea and tachyarrhythmias. She reported a weight loss of $14 \mathrm{~kg}$ in the last 6 months. CT scan and esophagogastroscopy showed a large paraoesophageal hernia, measuring approximately $10 \mathrm{~cm}$, with intrathoracic UDS.

Results: We performed a laparoscopic hernia repair with dissection of the hernia sac from the posterior mediastinum, tension-free intrabdominal reposition of stomach and distal esophagus and hiatoplasty with biologic mesh (Tutomesh ${ }^{\mathrm{TM}}$ ) augmentation. Finally, a Toupet fundoplication was performed to recreate the antireflux valve. In consequence of pronounced adhesions, a lesion of the muscularis of the distal esophagus occurred during surgery. The esophageal mucosa was unaffected. We treated the lesion laparoscopically with a simple interrupted suture (Vicryl $^{\mathrm{TM}}$ 3-0). An intraoperative Patent Blue V leak test did not identify any leaks.

The recovery was uneventful. The patient was discharged on day 12 after surgery and was free of symptoms in her follow-ups.

Conclusions: Patients with large hiatal hernias and UDS can be treated successfully and effectively with laparoscopic mesh repair. Intraoperative complications can be handled laparoscopically in a safe manner.

\section{V253-UPPER GI-Reflux-Achalasia}

\section{TOUPET OF NEED IN OLDER PATIENT WITH PARAESOPHAGEAL GIANT HIATAL HERNIA WITH LEFT ABERRANT HEPATIC ARTERY}

A.F. Aranzana Gómez, J. Malo Corral, J. Hernandez Gutierrez, P. Toral Guinea, A. Trinidad Borras, R. Lopez Pardo, B. Muñoz Jimenez, A. Muñoz Tébar, G. Krasniki, I. Fraile Alonso

General Surgery, Complejo Hospitalario Toledo, TOLEDO, Spain

Introduction: The laparoscopic approach of giant paraesophageal hiatal hernias with associated gastric volvulus is always a challenge for the surgeon given the complexity of its effective and safe resolution.

Objective: To demonstrate the efficacy of laparoscopic approach in complex cases of paraesophageal hernias, such as the one presented in the video, with an aberrant left hepatic artery and where it is necessary to perform an intraoperative endoscopy, modifying the anti-reflux procedure with a laparoscopic TOUPET, as well as the STANDARDIZATION of our technique, with the technical steps that we make successively.

Material and method:Clinical case: 75-year-old woman, with symptomatic hiatal hernia with progressive intolerance and dysnea. EGD: the stomach rotated in a giant hiatal hernia. Gastroscopy not completed due to endoscope loop formation within giant hiatal hernia with gastric volvulation. CT: large hiatal hernia. It is not possible to perform preoperative manometry.

Results: Intervention: Laparoscopic approach.HH of large paraesophageal size, Gastric walls very thickened and adhered to the mediastinum, aberrant left hepatic artery that difficult the dissection.Reduction of all contents and the sac, EXTENDED MEDIASTINAL ESOPHAGEAL DISECTION, up to vein pulmonary and get enough abdominal esophagus and rule out the presence of an short esophagus, posterior and anterior tutorized MODIFIED HIATORRAPHY with stitches in ' $U$ ' with non-absorbable suture on Teflon reinforcement patches.It is not possible to tutor the esophagus with the Fouché tube, performing intraoperative endoscopy that is normal, performing Toupet fixed to both pillars, intramediastinal drainage.EGD at the 1st day, good postoperative course with minimal pleural effussion resolved with physiotherapy. Discharge at 3th day.Asymptomatic and without radiological recurrence after 14 months of follow-up. Conclusions: In giant and paraesophageal hiatal hernias, modified primary hiatorraphy together with mediastinal esophageal dissection extended can be an effective and safe alternative, STANDARDIZATION of the procedure facilitates the realization of the same increased safety and effectiveness,especially in cases of greater complexity. Intraoperative endoscopy is mandatory if proper esophagogastric tutoring is not achieved.

\section{V254-UPPER GI-Reflux-Achalasia}

\section{REPAIR OF A GIANT HIATAL HERNIA: IATOPLASTY WITH BIO A PATCH REINFORCEMET}

A. Peri, M. Botti, L. Pugliese, F.C. Bruno, N. Mineo, V. Gallo, M. Filardo, G. Costantini, A. Pietrabissa

\section{General Surgery II, IRCCS Policlinico San Matteo, PAVIA, Italy}

Iatoplasty followed by Nissen fundoplication represent the gold standard treatment of hiatal hernia; however it has been reported high hernia recurrence rate, especially in case of giant hiatal defects. In order to reduce recurrence rate, various techniques of hiatoplasty reinforcement have been implemented, such as prosthetic materials apposition. However, in Literature have been reported various mesh complications such as esophageal and proximal stomach erosion and late esophageal perforation after ischemia, especially in case of synthetic non absorbable materials.In this video we are going to show the repair of a huge hiatal hernia by hiatoplasty and positioning of an absorbable biosynthetic 'Bio A' mesh which is replaced by connective soft tissue over six months, therefore decresing complications and recurrence rate.As usual, we start with the mobilization of gastric fundus and isolation of diaphragmatic pillars by sectioning the aderences between them and herniated viscera. We proceed then with intrabdominal esophagus mobilization and higher mediastinal dissection in order to obtain an adequate esophageal lenght. After the exposition of the hiatus, we approximate the pillars with some non absorbable stitches and we reinforce the hiatus positioning a ' $U$ ' shaped Bio A mesh over the esophagus, simply fixed to crura with single stitches. Then we go on performing Nissen fundoplication.The use of this prosthetic material appears to be cost-effective; first series in Literature show very low complication rate and less recurrences of hiatal hernia than hiatoplasty without reinforcement. 


\section{V255-ROBOTICS \& NEW TECHNIQUES-Colorectal}

\section{BILE DUCT INJURY REPAIR AFTER CHOLECYSTECTOMY: LAPAROSCOPIC ROUX-EN-Y HEPATICOJEJUNOSTOMY}

\section{Artiles, L.L. Pereira, M.P. Tice, H. Asbun}

Baptist Health Hospital, MIAMI, United States of America

Background: Laparoscopic cholecystectomy (LC) is the standard procedure for the management of symptomatic cholelithiasis or acute cholecystitis in patients without specific contraindications. Bile duct injuries (BDI) occur in $0.4-1 \%$ of LC but are a serious complication associated with high postoperative morbidity and usually require major surgical reoperation.

Material \& Methods: We present a case of Strasberg type E3 inadvertent post-cholecystectomy laparoscopic reconverted lesion and its amazing laparoscopic repair.

Result(s): A 65-year-old female patient with acute cholecystitis and underwent a LC converted to open due to hepatic bleeding. The operative report informed that the Critical View of Safety was assured. On the third postoperative day presented with jaundice, a bilirubin of $10.9 \mathrm{mg} / \mathrm{dL}$ and bilious drainage on the Jackson-Pratt drain. An endobiliary stent was placed. ERCP and HIDA scan confirmed the BDI. The patient was then reefer under our institution. A percutaneous transhepatic biliary drainage was placed assuring adequate external drainage. She was stabilized and a delayed surgical repair was planned.

Two months after, she underwent a laparoscopic extensive adhesiolysis, exposure of the biliary tree with Roux-en-Y hepaticojejunostomy and closure of distal bile duct stump. An intraoperative injury of the right hepatic artery was repair. The patient had an uneventful postoperative period. A postoperative doppler demonstrated intact hepatic circulation. She has remained asymptomatic to the present.

Conclusion(s): Roux-en-Y hepaticojejunostomy can be successful performed laparoscopically in experienced hands. This video demonstrates the technical steps of the procedure, including resolution of intraoperative complications.

\section{V256-UPPER GI-Reflux-Achalasia}

\section{IDENTIFICATION AND DISSECTION OF THE COMPARTMENTS OUTSIDE THE SAC OF GIANT HIATAL HERNIA}

\section{M.Y. Fanous}

Surgery, Aspirus Iron River Hospital, IRON RIVER, United States of America

Meticulous dissection of the sac of giant hiatal hernia is imperative for successful outcome. This should be performed in the extra-saccular plane. Failure to identify the sac is a major factor in converting laparoscopic to open hiatal hernia repair.

This video demonstrates the technique of laparoscopic identification and complete dissection of the sac of a totally intrathoracic stomach. Identification of the sac is performed centrally by scoring the peritoneum overlying the arch of the diaphragmatic hiatus. The inferior edge is retracted and series of blunt dissection is undertaken. Once the areolar tissue of the avascular plan is visualized; a raytec sponge is placed and advanced cephalad to expose the extra-saccular space. This raytec is kept in place to allow carbon dioxide to infiltrate and further delineate the anatomy. We then proceed with dissection at the left crus and right crus.

Complete reduction of the stomach can be achieved without grasping it. This can be performed by applying caudal retraction on the sac. This maneuver exposes the plane outside the sac. This space is divided into two compartments (right and left) separated by a septum which indicates the proximal extent of the sac. This plane is avascular and blunt dissection can easily free the hernia sac from the mediastinal structures and the pleura.

This was followed by excision of the sac and hiatal repair which is reinforced with bioabsorbable mesh. The proximal short gastric vessels were then divided and a standard Toupet fundoplication was performed.

\section{V257-UPPER GI-Reflux-Achalasia}

TOTALLY LAPAROSCOPIC REPAIR OF A LARGE LEFT DIAPHRAGMATIC HERNIA

V. Turrado-Rodriguez, D. Momblan, J.J. Espert, A. Ibarzábal, J. Farguell, X. Morales, A.M. Lacy

Gastro intestinal surgery, Hospital Clinic of Barcelona, BARCELONA, Spain

Introduction: Diaphragmatic hernias, other than hiatal hernias, are rare and usually congenital or traumatic. They may include adjacent organs such as stomach, spleen, colon, pancreas. The classical treatment consisted on open surgery with hernia repair \pm mesh reinforcement. With the increasing expertise on laparoscopic surgery, laparoscopic repair may offer a less traumatic surgery with faster recovery and less short-term complications.

Methods: A 59 year-old woman with medical history of clear cell renal carcinoma to whom a left laparoscopic nefrectomy was performed on november 2008, with recurrence in the retropertineum in 2014 (surgical resection) and nowadays with liver recurrence. The patient went to the Emergency Department complaining of two days of epigastric pain with nausea and vomits. A plain chest X-ray was performed showing a possible left diaphragmatic hernia. CT scan was performed showing a big left posteromedial diaphragmatic hernia in which the stomach, tail of the pancreas, spleen and splenic flexure of the colon were included. A laparoscopic diaphragmatic hernia repair was indicated.

Results: The patient was placed in the antiTrendelemburg position with both legs open and arms closed. Using five trocars and a 3D flexible tip camera, and exploration of the hernia was performed, showing the intrathoracic presence of the full stomach, the spleen, and the transverse colon with the splenic flexure. Carefull dissection allowed for the reduction of the stomach and, further diseccion of adherences allowed the reduction into the abdominal cavity of the spleen, that was completely introduced into the hernia and in a horizontal position, and colon. Resection of the hernial sac was performed. Aproximation of the borders of the hernia with Maxon 2/0 suture was performed. A titanium mesh was placed over the closed defect and was fixed with glue. The postoperative evolution was uneventful, with oral intake resumed on postoperative day 2 and hospital discharge on postoperative day 5 .

Conclusions: Laparoscopic repair of gigant diaphragmatic hernias is feasible eventhough it should be performed by an expert laparoscopic team. Careful dissection that allows reduction of the organs into the abdominal cavity without lesions is necessary.

\section{V258-UPPER GI-Reflux-Achalasia}

\section{REVISONAL SURGERY AFTER FAILED HELLER'S MYOTOMY FOR ACHALASIA}

A. Rabal Fueyo ${ }^{1}$, S. Fernández-Ananín ${ }^{1}$, C. Balagué Ponz ${ }^{1}$, D. Sacoto ${ }^{1}$, I. Gómez Torres ${ }^{1}$, B. Gonzalo Prats ${ }^{1}$, A. Basterra ${ }^{1}$, E. Pareja Nieto ${ }^{1}$, C. Guarner Argente ${ }^{2}$, E.M. Targarona Soler ${ }^{1}$

${ }^{1}$ General surgery, Hospital de la Santa Creu i Sant Pau, BARCELONA, Spain; ${ }^{2}$ Gastroenterology, Hospital de la Santa Creu i Sant Pau, BARCELONA, Spain

Surgical treatment of achalasia fails in 10-20\% of patients. The most frequent responsible cause is the performance of an incomplete myotomy at primary surgery. Other reasons for failure are fibrosis and related with antireflux procedure. In this video we show the case of a 36-year-old woman who underwent at 11-year-old a Heller's myotomy with a Dor fundoplication for Achalasia by open surgical approach. Due to the recurrence of the symptomatology, a revisional surgery was proposed. Surgery was characterized by the presence of a herniation of the previous fundoplication, fibrosis around the prior myotomy and the formation of a pseudodiverticulum These case demonstrate that, although sometimes it is difficult and challenged, laparoscopic reoperation for achalasia is feasible. 


\section{V259-UPPER GI-Reflux-Achalasia}

\section{RECURRENCE OF HIATAL HERNIA POST BOEREMA PROCEDURE}

A.F. Aranzana Gómez, J. Malo Corral, J. Hernandez Gutierrez,

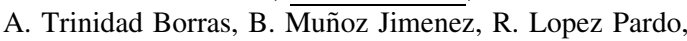
P. Toral Guinea, G. Krasniki, M.A. Morlan Lopez

General Surgery, Complejo Hospitalario Toledo, TOLEDO, Spain

Introduction-objectives: The mixed giant hiatus hernias with paraesophageal component are hernias of difficult surgical correction, the laparoscopic approach of these implies a greater experience of the surgical team, given the complexity involved in its management, being the recurrence a complication that neither the use of meshes in this surgery has been able to avoid. In very high-risk patients, the gastric anterior pexy (BOEREMA) may be an alternative to treat or alleviate the symptoms of these large hiatal hernias, although the frequency of recurrence with this technique is very high. Material and methods.

Clinical case: A 74-year-old woman with a psychiatric history (Sd. Diogenes), severe malnutrition, DM, HBP. Diagnosed of giant hiatus hernia, mixed by sliding, with paraesophageal component by gastric semivolvulation with episodes of incarceration, complementary tests of interest are provided. Enter by gastric volvulation table, place SNG and establish parenteral nutrition, deciding surgery, making approach laparoscopic, where complete reduction of the hernial content is made, with gastric anterior pexia to the wall. Initial correct postoperative period, with normal control EGD at 6 months and good oral tolerance. Subsequently, she presented complete recurrence of hernia and new gastric semivolvulation with vomiting and intolerance to intake. At this moment the patient is in better general conditions, deciding definitive surgery.

Results: Intervention. Complete laparoscopic approach, 5 trocars, complete recurrence of giant hiatus hernia that contains almost all of the stomach, removal of previous fixation of the pexy, reduction of the content, dissection of the phagia hiatus, exposing enough abdominal esophagus, both pillars to crura and vagos, complete resection of the hernia sac, tutorial hiatorraphy on Teflon patches, Nissen fixed to pillars, intramediastinic drainage. Postoperative correct, being asymptomatic and with control EGD without recurrence two years after the intervention.

Conclusions: The laparoscopic approach of large hiatal hernias with curative intent in high-risk patients may be a valid alternative in the surgical approach of this pathology.

\section{V260-UPPER GI-Reflux-Achalasia}

\section{LAPAROSCOPIC PARTIAL FUNDOPLICATION IN A PATIENT WITH SITUS INVERSUS TOTALIS}

A.L. Vargas Ávila, S.J. Salgado Arzate, M.V. Diaz Mier, A.F. Palacio Vélez, J. Garcia Cansino, V.G. Reyes Garcia, K.B. Molina Tavarez, J. Vargas Flores, J.F. Nagore Ancona, J.M. Hernandez Garrido, C.A. Cortazar Sanchez,

N.N. Espinosa Queb, L.A. Guerrero Galindo, J.A. Dominguez Rodriguez, J.A. Gonzalez Luna, A. Jimenez Leyva, J. Sanchez Lora

General Surgery, ISSSTE, CIUDAD DE MEXICO, Mexico

Aim: Situs inversus totalis is a rare genetic disorder characterized by the transposition of the thoracoabdominal organs in the sagittal direction, resulting in a 'mirror image' and a specular inversion of all the asymmetric structures of the body. Situs is estimated to occur in $1 / 8,000$ $1 / 25,000$ live births. Most patients with situs live a completely normal life.

Objective: The objective of the following case is to present a patient with type-1 hiatal hernia, Barrett's Esophagus without dysplasia and situs inversus.

Method: A laparoscopic partial fundoplication was performed on a 47-year-old male patient with a history of situs inversus totalis, who was seen in our general surgery service presenting a clinical history of retrosternal pain, heartburn and regurgitation.

An endoscopy was performed, which reported hiatal hernia type 1 and incompetence of lower esophageal sphincter, with squamocolumnar junction biopsies with report of Barrett's Esophagus without dysplasia.

Results: Surgical time was programmed, for Toupet type fundoplication; there were lax adhesions from omentum to wall, the lax hiatus and already known situs inversus.

Partial funcuplication was performed, with the usual technique adapted for structural anatomical abnormality, postoperative course without complications, oral initiation at the next day, drainage penrose type was set draining just a little serohematic liquid, diet was progressed and patient was discharged on the third postoperative day without complications.

Conclusion: Situs inversus is the mirror image of situs solitus, which presents subdivision in situs inversus totalis, which is the most usual form, characterized by the mirror location of the intraabdominal and thoracic organs including the heart; in the case presented, the patient was referred with Situs inversus and Barrett's Esophagus, performing laparoscopic fundoplication.

The gold standard of surgical management is laparoscopic in benign esophageal pathology.

\section{V262-UPPER GI-Reflux-Achalasia}

THE LAPAROSCOPIC APPROACH TO THE TREATMENT OF REFLUX ESOPHAGITIS RESULTING FROM GIANT HIATAL HERNIA, TYPE IV. A CASE REPORT

\section{Agapov, E.A. Gallyamov, V.V. Kakotkin}

General surgery, Medical Research Educational Center. M.V. Lomonosov Moscow State University., MOSCOW, Russia

Gastroesophageal reflux disease (GERD) is a condition that reduces the quality of life and can causedisorders associated with acid reflux, such as bronchial asthma, Barrett's esophagus and esophagealadenocarcinoma. GERD is often caused by existing of hiatal hernia. In rare instances GERD is associatedwith type IV hiatal hernias in which the part of stomach and other organs migrate into mediastinum.Nowadays this condition can cause problems for some surgeons.Patient was a 64-year-old man. He was diagnosed with hiatal hernia in 1992. The condition had beenhaving asymptomatic course until 2003. Patient takes omeprazole $20 \mathrm{mg}$ for 15 years. He startedexperiencing chest pains when inhaled and dyspnoea in June 2018. Co-morbidities were: arterialhypertension, chronic obstructive pulmonary disease (COPD) and obesity (body mass index was $43.9 \mathrm{~kg} / \mathrm{m}^{2}$ ). The posterior mediastinum contained the part of stomach, large bowe and small bowelaccording to chest roentgenography. Sizes of esophageal hiatus were $145 \times 98 \mathrm{~mm}$. In our clinical centerwas performed laparoscopic removal of hernia, cruroraphy, mesh repair of the esophageal hiatus andNissen fundoplication in 2018 July. During the surgery stomach, the part of small intestine, greatomentum and transverse colon were relocated into abdominal cavity. After cruroraphy, repair of theesophageal hiatus with prolene mesh was performed. The patient was in intensive care during $9 \mathrm{~h}$.Total enteral feeding was initiated on second day. Patients had been discharged within 5 days aftersurgery. Patient was re-examined by a surgeon in September 2018, there were no signs of a reccurence. This case report shows an efficiency and feasibility of the laparoscopic approach to the treatment GERDassotiated with a large defect in the phrenoesophageal membrane, allowing other organs, such as colon,great omentum and small intestine to enter the hernia sac.

\section{V263-UPPER GI-Reflux-Achalasia}

\section{HIATAL HERNIA AND FLOPPY CALIBRATED NISSEN PROCEDURE USING 3 MM MINI INSTRUMENTS IN A DAY- HOSPITAL PROGRAM}

F. del Castillo Diez ${ }^{1}$, L. García-Sancho Téllez ${ }^{2}$, J.D. Sanchez Lopez ${ }^{2}$, E. Ferrero Celemin' ${ }^{2}$, J.A. Gonzalez Sanchez ${ }^{1}$, J. Díaz Dominguez ${ }^{1}$

${ }^{1}$ General Surgery, Hospital Universitario La Paz, MADRID, Spain; ${ }^{2}$ General Surgery, Hospital Universitario Infanta Sofia, MADRID, Spain

Aims: The authors present a video with their standardized laparoscopic hiatal hernia repair and anti-reflux Nissen procedure but using $3 \mathrm{~mm}$ instruments and $5 \mathrm{~mm}$ camera approach.

Methods: A 42 years old female patient with a BMI 29 presents a symptomatic gastro esophageal reflux disease (GERD) for 20 years. Manometry and Ph-metry showed a lack of esophageal motor disorders and a severe acid pattern with a 96.35 DeMeester Score. Panendoscopy study showed a $3 \mathrm{~cm}$ hiatal hernia and a Los Angeles-grade 2 esophagitis. She decided not to go with PPI treatment anymore. A Laparoscopic hiatal hernia repair and standardized Nissen procedure is performed using $3 \mathrm{~mm}$ instruments and a $5 \mathrm{~mm}$ camera. Case and technical details are shown in the video.

Results: The patient was discharged from hospital within a period of $6 \mathrm{~h}$ with a 3 rate in a EVA acute pain visual scale. In a 2 year follow-up, there has no been an anatomical or clinical recurrence. No chronic dysphagia, anatomical recurrence or abdominal wall complications have been reported with in this period of time.

Conclusions: Depending on the patient characteristics, anatomical factors and surgeon min invasive experience, hiatal hernia and anti-reflux mini invasive standardized repair using $3 \mathrm{~mm}$ instruments, could be a safe and feasible option. More studies are needed in order to standardized this approach 


\section{V264-COLORECTAL-Stoma}

ROBOTIC ASSISTED LAPAROSCOPIC RETROMUSCULAR PARASTOMAL HERNIA REPAIR: THE PAULI TECHNIQUE

\section{G F K Onghena, F Muysoms, P Pletinckx}

General Surgery, AZ Maria Middelares Ghent, GHENT, Belgium

Background and Aims: Parastomal herniation a very frequent complication in stoma patients. In isolated parastomal hernias (type I or III)* a laparoscopic Sugarbaker repair with intraperitoneal mesh is our preferred technique. If a concomitant incisional hernia is present (type II or III)* we currently opt for a retromuscular mesh repair as described by Pauli. We adopted a minimal invasive approach using the robotic platform.

Methods: We performed a robot-assisted laparoscopic Pauli repair for a wide incisional henria needing component separation and a small parastomal hernia (Type II)*. A non-slit retromuscular mesh was placed after a bilateral TAR (transversus abdominus release) and lateralization of the colon.

Results: The operation was performed robot-assisted laparoscopically with $2 \times 3$ trocars without the need to convert to an open procedure. The procedure lasted $300 \mathrm{~min}$. The patient was discharged from the hospital on post-operative day three. One month after the repair our patient presented with a parastomal skin infection for which she received surgical cleaning and wound dressing. CT scan three months postoperative shows good positioning of the mesh with a reinforced abdominal wall.

Conclusions: The modified Sugarbaker repair in parastomal herniation is feasible following a Pauli approach (retromuscular mesh positioning) completely laparoscopic, albeit robotically assisted. Short-term follow up is promising. Long-term postoperative follow-up in these patients is needed.

* European Hernia Society classification

\section{V266-UPPER GI-Benign Esophageal disorders}

\section{ENDOSCOPIC RESECTION OF GIANT OESOPHAGEAL POLYP}

F. Jackson-Spence, M. Bonomaully, C. Mcardle, S. Randhawa, D. Mukherjee, H. Malik

Upper GI Surgery, Barking, Havering and Redbridge University Trust, LONDON, United Kingdom

Aims: The giant fibrovascular polyp is one of the rarest benign lesions of the oesophagus. The most common locations of origin are the upper oesophagus or crico-pharyngeal region. The lesion is mor common in elderly population, particularly men. Symptoms include dysphagia, odynophagia Management options include surgical resection or endoscopic removal with endoloop. We aim to demonstrate the optimal management of these rare lesions using an endoscopic approach.

Method: We demonstrated the management of 75-year old patient with a giant oesophageal polyp, excised by minimally invasive endoscopic resection. The patient was placed in supine position and tracheal intubation was performed under general anaesthetic before an endoscopic approach was taken. The oesophago-duodenoscopy visualised a cresenteric shaped lumen due to an intraluminal mass occupying two thirds of the oesophageal diameter. The procedure was a multidisciplinary approach with the upper GI surgical and gastroenterology consultants. The polyp stalk was located in the oesophagus at the level of T2 and an endoloop was manipulated to surround the polyp. The polyp was then separated from the stalk by cauterisation and resected from the patient. The stalk was then injected with adrenaline to prevent haemorrhage.

Results: The excised specimen was a $15 \mathrm{~cm}$ polyp with stalk originating from the T2-T3 level Histology confirmed diagnostic suspicions of a benign pedunculated fibrovascular polyp. The polyp was covered by non-keratinising squamous epithelium with a discoloured tip demonstrating ulceration. There was no evidence of dysplasia or neoplastic process.

Conclusion: Due to the rarity of these lesions, there is a paucity of studies in the literature regarding diagnosis and management of giant fibrovascular oesophageal polyps. An alternative approach would be a surgical resection, but we have demonstrated a successful minimally invasive excision of a giant oesophageal fibrovascular polyp, with shorter time under general anaesthetic, recovery time and reduced post-operative analgesic requirements.

\section{V265-HERNIA-ADHESIONS-Abdominal wall hernia}

\section{LAPAROSCOPIC REPAIR OF A LARGE MORGAGNI HERNIA WITH INTERMITTENT GASTRIC INCARCERATION}

\author{
D. Munteanu, C. Popa, I.C. Puia, A. Munteanu, N. al Hajjar
}

Surgery, Regional Institute of Gastroenterology and Hepatology, O. Fodor, CLUJ-NAPOCA, Romania

Aim: To present the diagnosis, technique and outcome of a rare abdominal wall hernia. Introduction out of the rare congenital diaphragmatic hernias, Morgagni hernia is the most uncommon. It consists of the intrathoracic herniation of the abdominal viscera, through a congenital defect of the anterior diaphragm.

Methods: Case presentation

Results: A 67-year-old Caucasian female patient was admitted on emergency due to a progressive alteration of her physical condition and weigh loss, caused by intermitent and intense epigastric pain and vomisments. Symptomes occurred several years prior to admittance, but used to be mild and rare. During the last months, the described episodes became more intense, lasted longer and occurred more frequently. Percutaneous ultrasound raised the suspicion, while thoraco-abdominal CT revealed an enormous intrathoracic Morgagni hernia and gastric volvulus inside the hernial sack. After a careful preoperative preparation (restoring of the nutritional and hydric inballances, amelioration of respiratory parameters), laproscopy confirmed the diagnosis; we gently reintegrated the herniated organs from the thoracic hernia into the abdominal cavity (small bowel, large omentum, transverse-doloco-colon, and thisted stomach); a laparoscopic exploration of the hernial cavity was followed by a thorough hemostasis. Do to patient's frailty, we decided to leave the hernial sack in situ. Surgical direct repair of the defect technique was done by using extracorporeally tied separate sutures through separate skin incisions. The postoperative outcome was completelly uneventful; patient was discharged on postoperative day 5 . Barium contrast at 3 mounths followup showed a slight esophageal diskynesia, but normal gastroduodenal passage; due to aerocolia, the normal position of the transverse colon could be confirmed aswell; no signs of reccurrence where detected.

Conclusions: although very rare, a Morgagni hernia should be suspected and included in the differential diagnosis of patinets with dispeptic syndrome and epigastric/thoracic symptomes. Thoraco-abdominal CTscanning is the imagistic technique of choice. Laparoscopic approach became the gold standard, being far mora addvantageous as compared to laparotomy or thoracoscopy. Direct suture with extracorporeally separate sutures through separate skin incisions was chosen for being less time consuming; for the same resons, the hernial sack was left in situ, with no consequences so far.

\section{POSTER PRESENTATIONS}

\section{P001-AMAZING TECHNOLOGIES}

MEDROBOTICS FLEX® ROBOTIC SYSTEM FOR TRANSANAL EXCISION OF A LARGE RECTAL NEOPLASM: THE FIRST EUROPEAN EXPERIENCE

\section{A. Arezzo}

Department of Surgical Sciences, University of Torino, TORINO, Italy

The video shows a case of Laterally Spreading Tumour of the rectum with preoperative benign histology, Paris Classification 0-Is G (granular type), uTONO EUS stage, Kudo type IV, NICE type 2. The neoplasm measured $6 \times 7 \mathrm{~cm}$, and extended from 6 to $12 \mathrm{~cm}$ from the anal verge, mainly located on the posterior wall. According to our local policy the indication was a transanal full-thickness excision. This was performed with the Medrobotics Flex ${ }^{\circledR}$ Robotic System, used here for the first time outside the United States.

The system technology utilizes an articulated multi-linked scope that can be steered along nonlinear, circuitous paths in a way that is not possible with traditional, straight scopes. The maneuverability of the scope is derived from its numerous mechanical linkages with concentric mechanisms. This enables surgeons to perform minimally-invasive procedures in places that were previously difficult, or impossible, to reach. With the Flex ${ }^{\circledR}$ Robotic System, Surgeons can operate through a single access site and direct the scope to the surgical target. Once positioned, the scope can become rigid, forming a stable surgical platform from which the surgeon can pass flexible surgical instruments. The system includes on-board 3D HD visualization. The Flex ${ }^{\circledR}$ Robotic System contains two working channels to accept a number of different surgical and interventional instruments including monopolar and bipolar electrodes, scissors and graspers for tissue manipulation.

The video shows the introduction of the dedicated rectoscope, the connection of the flexible robot, and the way to operate the device performing a full-thickness excision, including suturing of the rectal defect by means of two running sutures by a V-Lock $3 / 0$ thread. While illustrating the technique the authors will comment pros and cons of the use of the device. 


\section{PO02-AMAZING TECHNOLOGIES}

SINGLE BIDIRECTIONAL BARBED SUTURE WITH MIDLINE LOCKING MECHANISM FOR TRANSANAL TRANSVERSE WOUND CLOSURE

\section{A. Arezzo \\ Department of Surgical Sciences, University of Torino, TORINO, Italy}

The video shows the use of a barbed suture of novel concept. This first prototype thread developed together with Assut Europe (Roma, Italy) is characterised by a bidirectional 3/0 barbed suture, of $24 \mathrm{~cm}$ length overall, with two needles $22 \mathrm{~mm}$ in diameter, and $1 / 2$ circumference. In order to fix the thread to the tissue, on the exact midline of the thread a small $(2 \mathrm{~mm})$ ball of the same material of the thread is fused with heat. This button as the entire thread is made of Polydioxanone, a monofilament material for long-term absorption, self-retaining. This small advancement offera a consistent advancement in endorectal surgery helping in making transverse wound closures as easy as never before. The first stitch is placed on the transverse midline of the rectal wall defect, this way approximating proximal and distal edges. The button keeps the thread under tension allowing the completion of a running suture towards one of the two lateral ends of the would. When the first end is reached the needle is cut and the other needle is grabbed in order to perform the other half of the running suture keeping the right tension on the thread, with no risk of lumen stenosis. The two lateral ends of the suture are self-blocked passing them through the last stitch. No need for clips, knotting or buttonholes to pass through. While illustrating the technique the authors will comment pros and cons of the use of the device.

\section{P003-AMAZING TECHNOLOGIES}

\section{SAFE PROFILE AND ONCOLOGICAL OUTCOMES OF GASTRIC GASTROINTESTINAL STROMAL TUMORS (GISTS) RO-BOTIC RESECTION: SINGLE CENTER EXPERIENCE}

\section{G. Pernazza ${ }^{1}$, C. Maggioni ${ }^{2}$, R. Mancini ${ }^{1}$, L. Ioni ${ }^{1}$}

${ }^{1}$ UOSD Chirurgia Generale Robotica, AO San Giovanni Addolorata Hosp, ROMA, Italy; ${ }^{2}$ Surgery, School of Medicine and SurgeryUniversity of Florence, FLORENCE, Italy

Background: The usefulness of robotic surgery has been largely reported; however, there are not enough reports on GIST's treatment. The aim of this study is to report a single center experience on gastric GIST's robotic resection. Gastrointestinal stromal tumor (GISTs) are the most common mesenchymal tumors (overall incidence 1-3\% of all gastrointestinal malignant tumor). They are most frequently located into the stomach. Complete surgical resection still remains crucial for patients with GISTs. In cases of difficult localization of tumor (e.g. posterior wall, His angle) and bigger tumor (more than $5 \mathrm{~cm}$ in diameter), there still exist apparent difficulty and limitation to conduct laparoscopic resection. Robotic assistance provides wide movements of its arms and was recognized particularly useful in case of difficult tumor localization, especially for those positioned at the posterior side of the stomach wall or around the lesser curvature.

Methods: Six consecutive patients were analyzed focusing on safety (conversion/complications rate; hospital stay), oncological (margin resection, recurrence rate) and feasibility (operative time, technical tip and tricks) profile of robotic approach.

Results: The mean operative time was $173 \pm 39 \mathrm{~min}$, the mean hospital stay $3 \pm 1$ days. Conversion rate was nihil. No intra and post-operative (mean follow-up 12 months) complications were registered. In all cases, the resections were classified as R0.

Conclusions: Our experience supports the usefulness of robotic system in case of GIST located at anatomically difficult gastric portion, such as lesser curvature or fundus close to GEJ, confirming the excellent oncological outcomes (100\% R0) and the encouraging safety profile $(0 \%)$. Regarding the operative time our data are similar or better as compared to those reported by the previous literatures and didn't differ by the most recent published data for laparoscopic gastric resection. The anatomical hand-sewn reconstruction performed by precise hand-sewn suture instead of the usage of mechanical staplers represents a real great advantages of robotic resection. In our series, no patients suffered from stenosis or leakage after operation.

\section{P004-AMAZING TECHNOLOGIES}

\section{LAPAROSCOPIC NISSEN FUNDOPLICATION WITH BARBED} SUTURE: PRELIMINARY Results:

E. Ferrero-Celemín ${ }^{1}$, A. Cuadrado-García ${ }^{1}$, S. Núñez-O’Sullivan ${ }^{1}$, L. García-Sancho ${ }^{1}$, J.D. Sanchez-Lopez ${ }^{1}$, M. García-Virosta', F. del Castillo-Diez ${ }^{2}$, A.L. Picardo-Nieto ${ }^{1}$

${ }^{1}$ Servicio de Cirugía General y del Aparato Digestivo, Hospital Universitario Infanta Sofía, Univeridad Europea de Madrid, SAN SEBASTIAN DE LOS REYES, Spain; ${ }^{2}$ Servicio de Cirugía General y del Aparato Digestivo, Hospital Universitario La Paz, MADRID, Spain

Background: We describe the use of a different suture from those historically used in the elaboration of a widely spread surgical technique such as the Nissen fundoplication, for the treatment of pathological gastroesophageal reflux or symptomatic hiatal hernias. In our unit we have implemented the use of $2 / 0$ irreabsorbable barbed suture to close the diaphragmatic pillars and the $360^{\circ}$ fundoplication with the same continues suture.

Aim: The objetive of the use of the irreabsorbable barbed suture in the Nissen fundoplication is to shorten the surgical times, which would achieve benefits for the patient and the institution, increasing the number of ambulant patients and the number of patients to be operated the same surgical session. Project description: French position. 5 supraumbilical $5 \mathrm{~mm}$ trocars. Wide dissection of the hiatus and the distal esophagus until sufficient esophagus was obtained in the abdominal cavity. The hiatorraphy is then carried out with an irreabsorbable running barbed suture. The $360^{\circ}$ fundoplication is performed with the same technique. Both the hiatorraphy and the fundoplication are calibrated with a $12 \mathrm{~mm}$ Foucher tube.

Preliminary results: A pilot study of the first 46 cases of Nissen fundoplication with barbed suture, between January 2015 and December 2018. Average age was 52.84 years, 25 women (54.35\%).

In $2015,5 \%$ of the Nissen fundoplications were carried out with barbed suture, in $201636 \%$, in $201750 \%$, while in 2018 this material was used in $100 \%$ of the cases.

We did not have intraoperative or postoperative complications in relation to the use of barbed suture. We have shortened our surgical times: barbed suture: $40 \mathrm{~min}$, not barbed: $50 \mathrm{~min}$. The average hospital stay was: barbed suture 1.3 days ( $40 \%$ ambulatory surgery).

4 patients have again symptoms of gastroesophageal reflux without pathological pHmetry, one patient had to be reoperated for dysphagia ( 85 year old patient with esophageal motor disorder). Conclusions: Barbed suture is safe, easy to use and since it does not need intracorporeal knots, it makes the surgery faster. The increase in its use in our hospital is related to the absence of complications, good short-term results, easy reproducibility and shortening of surgical time.

\section{P005-AMAZING TECHNOLOGIES}

\section{SLIM-MESH TECHNIQUE}

\section{S.A. Canton}

DiSCOG Department of Surgery, Oncology and Gastroenterology, University of Padua, PADOVA, Italy

Background: In 2009, in our Lab at Padua University, I devised a new sutureless laparoscopic technique which I called 'Slim-Mesh' (SM), to treat ventral hernias (VH), including large/giant/massive (L/G/M) types (those larger than $10 / 15 / 20 \mathrm{~cm}$ in size).

Aim: The primary goal was to reduce intra- and postoperative complications in laparoscopic $\mathrm{VH}$ repair, as well as operative time.

Project description: SM also eliminated the transabdominal full-thickness stitches in order to prevent their many intra- and postoperative complications: hematomas, organs iatrogenic lesions, intraoperative bleeding, postoperative chronic pain (1.4-28\%), mesh breakdown, suture site infections, thin skin scars, etc. The SM technique is divided into 4 surgical phases: (1) first laparoscopic step: laparoscopic exploration of the whole abdominal cavity with adhesiolysis when necessary, measurement of SM overlap for various sizes of VH according to the SM technique, the marking with methylene blue of 4 peritoneal axial points (PAP), and measurement of peritoneal (internal) SM application area (SMAA); (2) marking corresponding skin axial points (SAP) and measurement of skin (external) SMAA; (3) handling the prosthesis on the operative bench as per the SM technique; (4) last laparoscopic step: intra-abdominal SM introduction and fixation according to the SM approach

Preliminary results: Since 2009-November 2018, 83 patients with VH were treated in our center, with this new SM technique. Fifty one percent males and $49 \%$ females, averaging 57 years, were included in our prospective (60\% of cases from September 2016) or retrospective study. Overall, mean operation time for the 83 patients was $100 \mathrm{~min}$ (range 55-240 min). In our series we had one intraoperative complication (1.2\%) with transient bradycardia, and one case of shortterm urinary retention during hospitalization. Two early (whitin 30 days after hospital discharge) postoperative complications were detected: one $(1.2 \%)$ case of abdominal pain, and one case of seroma. Mean length of hospital stay was 2.6 days. Mean follow-up time was 34 months. Late surgical complications included: 5 cases $(6 \%)$ of $\mathrm{VH}$ recurrence, 3 of which undervent surgery, in the remaining 2 cases a small $(<2 \mathrm{~cm})$ symptomless recurrence occured, and 2 cases $(2.4 \%)$ of $10 \mathrm{~mm}$ trocar site incisional hernia. 


\section{PO06-AMAZING TECHNOLOGIES}

INDOCYANINE GREEN AS A BEACON LIGHT IN LAPAROSCOPY: A NEW APPLICATION IN TRANSPLANT SURGERY. A CASE REPORT

G. Giuseppe ${ }^{1}$, D. Iovino ${ }^{1}$, G. Soldini ${ }^{1}$, E. Zani ${ }^{1}$, F. Masci ${ }^{1}$, V. Raveglia ${ }^{1}$, C. Parise ${ }^{1}$, F.M. Carrano ${ }^{1}$, E. Ferri ${ }^{1}$, L. Latham ${ }^{1}$, D. Confalonieri ${ }^{1}$, G. Saredi ${ }^{2}$, M. Tozzi ${ }^{3}$, G. Carcano ${ }^{1}$

${ }^{1}$ General, Emergency and Transplant Surgery, Insubria University, VARESE, Italy; ${ }^{2}$ Urology, Insubria University, VARESE, Italy; ${ }^{3}$ Vascular Surgery, Insubria University, VARESE, Italy

Introduction: Indocyanine green (ICG) fluorescence imaging system is a now a consolidated complementary technique for several surgical fields. The development of post transplant lymphocele following lymph spread could affect kidney function; between therapeutic options, the literature reports a $12 \%$ overall conversion rate from laparoscopic to open surgery with a major risk of damaging the urinary tract.

Objective: The goal of the present study was to demonstrate that intraoperative ICG fluorescent imaging is a safe technique that can be used in laparoscopy establishing the exact location of the lymphocele and reducing intraoperative risks.

Method: Fifty milligrams of ICG dissolved in $20 \mathrm{~mL}$ of saline solution was injected via percutaneous drainage placed into the lymphocele to decompress transplanted kidneys 2 weeks before a laparoscopic lymphocele marsupialization procedure.

Results: During the first exploratory laparoscopy, in the flank and right iliac fossa, near the 2 renal grafts, fluorescence was identified in 3 raised areas that were the internal side of the lymphocele lobes. The lymphocele wall was dissected and $300 \mathrm{~mL}$ of serous fluid was aspirated after puncturing. A $5 \mathrm{~cm}$ breach was then made in the cyst wall using the Ultracision harmonic scalpel (Ethicon US). Afterwards, a pedicle of the omentum in the lymphocele core was interfered with and fixed by 2 stitches.

Conclusions: Laparoscopic surgery seems to be the preferred surgical option for the treatment of primary symptomatic lymphocele after kidney transplantation. Intraoperative ICG fluorescent imaging is a safe technique to establish the exact location of the lymphocele and reduces the risk of damaging urinary structures during surgery.

\section{P007-AMAZING TECHNOLOGIES}

INDOCYANINE GREEN AND EARLY OUTCOME OF KIDNEY TRANSPLANTATION: GRAFT'S REPERFUSION ANALYSIS BASED ON INTRAOPERATIVE FLUORESCENT ANGIOGRAPHY

G. Giuseppe ${ }^{1}$, F. Benedetti ${ }^{1}$, E. Zani ${ }^{1}$, D. Iovino ${ }^{1}$, G. Soldini ${ }^{1}$,

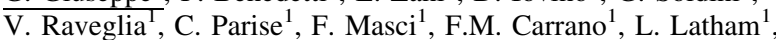
C. Baglieri ${ }^{1}$, G. Saredi ${ }^{2}$, M. Tozzi ${ }^{3}$, G. Carcano $^{1}$

${ }^{1}$ General, Emergency and Transplant Surgery, Insubria University, VARESE, Italy; ${ }^{2}$ Urology, Insubria University, VARESE, Italy; ${ }^{3}$ Vascular Surgery, Insubria University, VARESE, Italy

Introduction: Since the introduction of extended criteria donors, the number of marginal organs employed for transplantation has dramatically increased along with delayed graft function (DGF) rate and the number of 'fragile' transplants (1). It is clear how also the post-operative management of the transplanted patient plays a key role in the good outcome of the procedure (2). In our study we tried to find intraoperative factors able to guide the optimization of post-operative therapies toward better transplant outcomes.

Methods: We employed Indocyanine Green (ICG), an hydrosoluble fluorescent dye, in order to perform an intraoperative fluorescent angiography and evaluate graft blood supply after reperfusion from a quantitative point of view. Pictures of the transplanted kidney are taken respectively 5,15 and 45 min after reperfusion with a dedicated high definition fluorescent camera (Karl Storz Endoscopes $\left.{ }^{\circledR}\right)$ after the injection of 3 doses of ICG properly diluted in saline solution (Verdye $(\mathbb{B})$. Images were then analysed with a specific open source software (ICY bioimage analysis ${ }^{\circledR}$, Quantitative Image Analysis Unit, Institut Pasteur, Paris) and correlated with the post-operative conduct of the graft.

Results: 12 patients so far underwent the procedure ( 8 men and 4 women), with a mean age of 46.33 years (min. 27-max. 66). Of these, 2 experienced delayed graft function (intended as the need of dialysis during the first post transplantation week). The patients were divided in two subgroups based on the post-operative course. The mean value of perfusion score for DGF group was 80.33 , while the mean value for standard group was 206.66 .

Conclusions: This method proved safe and risk-free, easily reproducible and without the need for a different toolkit than the one of a modern operating theatre. The preliminary analysis shows a strong correlation between the perfusional data so far obtained and the early outcome of the graft. Thus opening the way to further analysis aimed to a future better management of post-operative immunosuppressant and support therapy. These results are quite encouraging, even if our study is at an initial stage.

\section{P008-AMAZING TECHNOLOGIES}

\section{HOSPITAL PREPAREDNESS DURING MASS CASUALTY EVENTS-A SYSTEMATIC REVIEW}

$\frac{\text { A. Phaily }}{\text { H. Wang }}{ }^{3}$ O. Adeboyejo ${ }^{2}$, A. Hakim ${ }^{2}$, A. d'Cunha Kamath ${ }^{2}$,

${ }^{1}$ Department of Surgery, Imperial College London, LONDON, United Kingdom; ${ }^{2}$ Department of Surgery, Royal Berkshire Hospital, READING, United Kingdom; ${ }^{3}$ Department of Surgery, Royal National Orthopaedic Hospital, LONDON, United Kingdom

Aims: The dawn of the 21st century ushered in an era of increasing terrorism and a wave of mass casualty events across cities in Europe and the United States. The scientific community must now consider how best to prepare for such events and analyse previous responses. Are lessons learned or the same mistakes repeated in reported mass casualty events?

Methods: A systematic literature review of the PubMed/MEDLINE, Scopus and Cochrane databases from December 2012 to December 2017 using Medical Subject Headings (MeSH) for 'Mass casualty/casualties' and 'analysis' or 'review'. In-depth analysis of responses to 3 separate events in Boston (2013), Paris (2015) and London (2017) was carried out with a focus on the preparedness, accomplishments and challenges of each.

Results: From 612 potentially relevant citations, 72 underwent further analysis and 8 met the inclusion criteria. Lessons learned include greater collaboration between emergency services, improved crisis command and control structures and rapid augmentation of surge capacity. Although preparedness has improved, recurring failures in internal hospital systems, communication and resource allocation were noted.

Conclusions: Lack of a standardised and validated method of analysis of emergency responses remains a barrier to dissemination of lessons learned. The need for greater transnational academic collaboration in mass casualty response and preparedness is now undeniable.

Lastly, this review recommends increasing collaboration and training between emergency services, the formation of hospital networks to deal with multiple critical victims and drawing upon the experiences from military combat situations.

\section{P009-AMAZING TECHNOLOGIES}

\section{THE IMPACT OF ARTIFICIAL INTELLIGENCE ON ENDOSCOPY - A DISRUPTIVE TECHNOLOGY OR UNIQUE OPPORTUNITY?}

$\frac{\text { A. Phaily }}{{ }^{1}}$, A. d'Cunha Kamath ${ }^{2}$, A. Hakim ${ }^{2}$, O. Adeboyejo ${ }^{2}$,

${ }^{1}$ Department of Surgery, Imperial College London, LONDON, United Kingdom; ${ }^{2}$ Department of Surgery, Royal Berkshire Hospital, READING, United Kingdom; ${ }^{3}$ Department of Surgery, Royal National Orthopaedic Hospital, LONDON, United Kingdom

Background: Developments in Artificial Intelligence (AI) has made it a disruptive technology in fields as varied as heavy industry, transportation and computing. This past decade has seen further strides in AI development with its application being inevitability linked to medical diagnosis once regulatory hurdles are passed.

Aim: This article analyses the current feeling amongst endoscopist radiologists and asks whether the coming paradigm shift could benefit to patients and clinician alike.

Project Description

A systematic literature review of MEDLINE, Scopus and Cochrane databases from 01/08/2008 to 01/08/2018 was conducted to identify studies related to AI and its application to radiology. Articles were assessed using PRISMA and on strength of evidence. A questionnaire survery of clinicians was also performed

Preliminary Results: There is a paucity of robust high level evidence assessing AI application in real world healthcare systems. Results from available studies show that AI algorithms and deep learning methods excel in performing tasks associated with pattern recognition, quantitative assessment and time critical analyses.

Conclusions: Results show a persistent dread across specialities regarding AI. Rather than be seen as threat, AI should be embraced by clinicians as it will ease the ever-increasing daily workload faced. This will enable clinicians to focus their skills on patient centred activities, interventional procedures and development. Despite current regulatory hurdles, AI implementation in medicine is unavoidable. This coming revolution presents a unique opportunity for endoscopist and radiologist to refocus their expertise in novel areas. 


\section{P010-AMAZING TECHNOLOGIES}

CAN ELECTRIC NOSE BREATH ANALYSIS IDENTIFY ABDOMINAL WALL HERNIA RECURRENCE AND AORTIC ANEURYSMS? A PROOF OF CONCEPT STUDY

C.S. van Steensel ${ }^{1}$, E.H.H. Mommers ${ }^{1}$, L. van Kooten ${ }^{1}$, S.W. Nienhuijs ${ }^{2}$, T.S. de Vries Reilingh ${ }^{3}$, S.O. Breukink ${ }^{1}$, B.M.E. Mees ${ }^{4}$, G.W.H. Schurink ${ }^{4}$, N. Bouvy ${ }^{5}$

${ }^{1}$ Surgery, Maastricht University Medical Centre, MAASTRICHT, The Netherlands; ${ }^{2}$ Surgery, Catharina Hospital, EINDHOVEN, The Netherlands; ${ }^{3}$ Surgery, Elkerliek Hospital, HELMOND, The Netherlands; ${ }^{4}$ Vascular Surgery, Maastricht University Medical Centre, MAASTRICHT, The Netherlands; ${ }^{5}$ Maastricht University Medical Centre, MAASTRICHT, The Netherlands

Background: Hernia recurrence is observed in up to $32 \%$ of all ventral hernia patients. Previous studies have shown that patients who develop hernia recurrences are likely to have an decreased collagen I:III ratio. The same observation is made in patients with an aortic aneurysm, predisposing both for incisional and recurrent ventral hernia formation. If patients with weak collagen could be identified in a reliable, low cost, non-invasive manner, it would greatly enhance preventive options such as prophylactic mesh placement after abdominal surgery.

Aim: The purpose of this study is to evaluate if an electric nose (Aeonose $₫$, the eNose company) can distinguish recurrent hernia patients and aortic aneurysm patients from healthy controls based on volatile organic compound analysis.

Project description: From February to July 2017 a three-armed proof of concept study was conducted at three hospitals, in three groups of patients; recurrent ventral hernia, aortic aneurysm, and healthy controls. Patients were measured once at the outpatient clinic using an electronic nose based on three metal oxide sensors. Measurement data were compressed to low-dimensional vectors using a TUCKER 3 like algorithm, and used to train an artificial neural network (ANN) to provide a classification between patients $(+1)$ and healthy controls $(-1)$

Preliminary resultsA total of 64 patients (Hernia $n=29$, Aneurysm $n=35$ ) and 37 controls were included in the study. Based on receiver operating curve (ROC) analysis, the ANN could differentiate between recurrent hernia patients and controls with the following details: area under the curve (AUC) 0.74 , sensitivity 0.79 , specificity 0.65 . Aortic aneurysm patients and healthy controls could be differentiated with an AUC of 0.84 , sensitivity of 0.83 , and specificity of 0.81 . Conclusions: The Aeonose ${ }^{\circledR}$ eNose can reliably distinguish patients with weak collagen (recurrent hernia and aortic aneurysm patients) from healthy controls. Validation of these results in a prospective cohort study is required before clinical application of the device.

\section{P011-AMAZING TECHNOLOGIES}

\section{COMBINED THORACOSCOPIC AND ENDOSCOPIC SURGERY FOR A LARGE ESOPHAGEAL SUBMUCOSAL TUMOR}

H. Okamoto, Y. Taniyama, T. Sakurai, M. Hikage, C. Sato, K. Takaya, T. Konno-Kumagai, N. Ujiie, T. Kamei

\section{Surgery, Tohoku University Hospital, SENDAI, Japan}

Background: Laparoscopic and endoscopic cooperative surgery (LECS) has been performed gastric submucosal tumor (SMT) or duodenal tumor. Although minimally invasive surgery using thoracoscopy has been the usual approach for esophageal SMT, the treatment method combined thoracoscopy and endoscopy has not been established. In addition, submucosal endoscopic tumor resection (SET) for esophageal SMT was reported using the technique of submucosal tunnel. Aim and Project description: We planned to resect large esophageal SMT located in the upper or middle thoracic esophagus by a combined endoscopic and thoracoscopic approach. Because SET is only recommended for tumors up to $40 \mathrm{~mm}$ in size owing to the limited submucosal space available and the left thoracic approach is restricted by the aortic arch and the trachea.

Preliminary results (Case presentation): A 47-year-old woman was diagnosed with a benign schwannoma of length $60 \mathrm{~mm}$ originated from either the submucosal or the muscular layer of the middle thoracic esophagus by endoscopic ultrasonography, enhanced computed tomography, and ultrasound-guided fine-needle aspiration biopsy. Since the tumor had increased in the 5 years and she had a symptom of dysphagia, we planned to resect it by a combined endoscopic and thoracoscopic approach. On endoscopy in the supine position, a submucosal tunnel was created $40 \mathrm{~mm}$ proximal to the cranial edge of the tumor, and its only oral end was dissected from the mucosal and muscular layers. This was followed by the resection of the entire tumor by left-sided thoracoscopic procedure in the prone position. Endoscopic mucosal closure was achieved by using clips. No postoperative complications were observed. Large benign esophageal tumors can be safely excised with minimally invasive surgery by using a combination of endoscopy and thoracoscopy.

\section{P012-AMAZING TECHNOLOGIES}

\section{SAFETY AND FEASIBILITY OF PURASTAT® IN LAPAROSCOPIC COLORECTAL SURGERY}

$\underline{\text { M. Ortenzi }}{ }^{1}$, A. Haji ${ }^{2}$

${ }^{1}$ Clinica Chirurgica, Università Politecnica delle Marche, ANCONA, Italy; ${ }^{2}$ Colorectal, King's College Hospital, LONDON, United Kingdom

Background: Haemorrhage remains a major cause of morbidity and death in all surgical specialties. In laparoscopic surgery. Relatively small amounts of blood can obscure the view of the operative field and increase the risk of injury to surrounding structures. Excessive bleeding often leads to longer hospital stays, increased healthcare service utilisation, and higher healthcare costs, among other negative consequences.

Aim: The aim of this study was to analyse the feasibility of PuraStat ${ }^{\circledR}$, a new synthetic haemostatic device, made of self-assembling peptides in laparoscopic colorectal surgery.

Project description: This was a prospective observational non-randomised study. Consecutive patients undergoing laparoscopic colorectal surgery were enrolled. Informed consent was obtained from all patients Inclusion criteria was the need employ a secondary method of haemostasis when traditional methods such as conventional pressure or utilization of energy devices to control the bleeding were either insufficient or not recommended/appropriate due to proximity to ureter, pelvic/sacral veins and other important structure.

Preliminary Results: Twenty patients were enrolled (12 males $(60 \%)$ and $8(40 \%)$ females) Mean age was 61 years $( \pm 2,4$ years). All patients were undergoing elective laparoscopic colorectal cancer surgery (4 right hemicolectomy, 5 sigmoid colectomy, 11 anterior resection). We utilised $3 \mathrm{mls}$ of PuraStat ${ }^{\circledR}$ in all patients. The mean area of application was $5,35 \mathrm{~cm}^{2}$ $\left( \pm 2.25 \mathrm{~cm}^{2}\right)$ with the amount of PuraStat ${ }^{\circledR}$ needed per centimetre being $0.56 \mathrm{mls}$. The mean time to apply the product was $40 \operatorname{secs}( \pm 17 \mathrm{~s})$, whereas the mean time to achieve haemostasis was 17,5 secs $( \pm 3.5 \mathrm{~s})$. There were no post operative complications in this cohort of 20 patients. Mean operative time overall was $185 \mathrm{~min}( \pm 45.2 \mathrm{~min})$. None of the patients experienced delayed post-operative bleeding and the mean hospital stay was 5 days $( \pm 3.4)$. In conclusion, according to the purpose of this preliminary study, we have demonstrated that PuraStat ${ }^{\circledR}$ can be easily used in laparoscopic surgery and it is a safe, effective haemostatic agent. This is a feasibility study and additional controlled studies would be useful in the future.

\section{P013-AMAZING TECHNOLOGIES}

\section{LAPAROENDOSCOPIC INTERVENTIONS ON COMMON BILE DUCT IN THE PATIENTS WITH CHOLECYSTITIS AND CONCOMITANT BILE DUCT STONES}

A.P. Ukhanov, D. Zakharov, S. Zhilin, S. Bolshakov, A. Leonov, V. Ambartzumjan

Surgical, Central municipal clinical hospital, VELIKIY NOVGOROD, Russia

Aim: To study the feasibility and effectivity of laparoendoscopic interventions in cholecystocholedocholithiasis

Materials and methods: 2175 patients with acute cholecystitis, aged from 18 years to 100 years, were operated on for the period from 2007 to 2018. Among them laparoscopic cholecystectomy was performed in $1875(86.2 \%)$. The combination of acute cholecystitis with suspected stones or presence of stones in the extrahepatic bile ducts were detected in $224(10,3 \%)$ of patients. During last three years the laparoscopic method of surgery in the presence of common bile duct stones was carried out. After performance of intraoperative cholangiography and visualization of stones in the common bile duct laparoscopic, choledochotomy and bile duct stones extraction was undertaken in 56 patients, using flexible choledochoscopy control. In all patients with gallbladder stones was then performed laparoscopic cholecystectomy.

Results: Laparoendoscopic intervention on common bile duct was successfully performed in 48 patients $(85.7 \%)$ and the operation was completed by common bile duct drainage by Kehr. In 8 patients due to technical difficulties conversion to open surgery was carried out. Postoperative morbidity in the form of bile leakage were diagnosed in 9 patients (16.1\%). In three cases they stopped spontaneously in 5-6 days after the operation. 6 patients were operated on repeatedly and additional suturing on choledocholithiasis was carried out.

Postoperative mortality was $2.4 \%$. The death of the patient of 92 years was caused by acute cardiovascular failure.

Conclusion: Our moderate experience of laparoendoscopic interventions on the common bile duct in patients with cholecystitis and choledocholithiasis shows good feasibility of this miniinvasive interventions. Further accumulation of experience with the development of indications and contraindications and the improvement of surgical technique is necessary for a comprehensive assessment 


\section{P014-AMAZING TECHNOLOGIES}

LAPAROSCOPIC APPENDECTOMY IS A GOLDEN STANDARD IN THE TREATMENT OF ALMOST ALL FORMS OF ACUTE APPENDICITIS

\author{
A. Ukhanov, D. Zakhrov, S. Zhilin, S. Bolshakov, A. Leonov, \\ V. Ambartzumjan
}

Surgical, Central municipal clinical hospital, VELIKIY NOVGOROD, Russia

Aim: Improvement of the results of the treatment of acute appendicitis on the basis of wide use of laparoscopic appendectomy.

Material and methods: From 2007 till 2018 in 1334 patients (63.6\%) with acute appendicitis laparoscopic appendectomy was carried out and 762 patients (36.4\%) patients were operated on by open method. Among patients operated endovideosurgically 363 had complications of acute appendicitis, in particular, $86(6.4 \%)$-periappendicular infiltration, $49(3.7 \%)$-periappendicular abscess, $162(12.1 \%)$-local and $66(4.9 \%)$ generalized peritonitis

Results: Conversion rate was $2,8 \%$ (38 patients). The postoperative morbidity $3.6 \%$ (48 patients $\}$ including bleeding into the abdominal cavity in $2(0.15 \%)$ patients, diffuse peritonitis in $3(0.2 \%)$, abscess of the abdominal cavity in $26(1.9 \%)$, suppuration of the trocar wound in 10 $(0.7 \%)$, early adhesive small bowel ileus in $7(0.5 \%)$ patients. There were no mortality among the patients operated laparoscopically. The number of wound suppuration after laparoscopic appendectomy was 7.5 times less than after open operations. The period of hospital stay after laparoscopic interventions was 1.6 times shorter than in patients undergoing open surgery. Conclusion: The broad use of endovideosurgical technology in treatment of acute appendicitis is preferable and shows that laparoscopic appendectomy is becoming the gold standard of the treatment of almost all forms of acute appendicitis

\section{P015-AMAZING TECHNOLOGIES}

\section{ENDOVIDEOSURGICAL TREATMENT OF ACUTE CHOLECYSTITIS AND ITS COMPLICATIONS IN MUNICIPAL HOSPITAL}

\author{
A. Ukhanov, D. Zakarov, S. Zhilin, S. Bolshakov, A. Leonov, V.
}

Ambartzumjan, Surgical, Central municipal clinical hospital, VELIKIY NOVGOROD, Russia

\footnotetext{
Aim: Improve the results of the treatment of patients with acute cholecystitis and its complications. Materials and methods: 2006 patients with acute cholecystitis aged from 18 to 100 years were operated on in the period from 2007 till and 2018 in municipal hospital. Among the operated patients $977(48.7 \%)$ were older 60 years. Destructive forms of gall-bladder inflammation (phlegmonous, gangrenous cholecystitis, empyema of the gall bladder, paravesical infiltration or abscess) were detected during surgery in 1340 patients (66.8\%). Laparoscopic cholecystectomy (LCE) was performed in $1672(83.3 \%)$ and in 27 patients LCE was combined with choledocholithotomy and choledochoscopy due to concomitant choledocholithiasis and cholangitis. 334 patients $(16.7 \%)$ patients were operated on by laparotomy.

Results: Among the patients who were operated on laparoscopically the conversion to open access was carried out in $52(3.1 \%)$, herewith in $6(22.2 \%)$ patients during laparoscopic interventions on the common bile duct. Postoperative intraabdominal complications were occured in 56 patients (3.3\%), including bleeding in 4 $(0.2 \%)$, peritonitis in $1(0.06 \%)$, intra-abdominal abscesses in $19(1.1 \%)$, acute intestinal obstruction in 1 $(0.06 \%)$, bile leakage in $18(1.1 \%)$, duodenal fistula in $1(0.06 \%)$, suppuration of trocar wounds in $12(0.7 \%)$ patients. Postoperative mortality was $0.7 \%$ (12 patients). All deceased patients were elderly and causes of death were extraabdominal complications of the cardiovascular and respiratory systems.

A comparative analysis of the results of the operation with open surgery (334 patients) showed that the use of laparoscopic method in the treatment of patients with acute cholecystitis allows five times to reduce postoperative mortality and three times diminish postoperative morbidity. Conclusions: Laparoscopic surgery in the treatment of acute cholecystitis and its complications is a priority method and this trend should be continued in the treatment of concomitant choledocholithiasis
}

\section{P016-AMAZING TECHNOLOGIES}

TAKING TRAINING TO THE NEXT LEVEL. HIGH FIDELITY LIVE ANIMAL MODELS FOR HANDS-ON THERAPEUTIC ENDOSCOPIC ULTRASOUND (TEUS)

L. Sosa-Valencia ${ }^{1}$, S.G. Lim ${ }^{1}$, L. Swanstrom ${ }^{1}$, F. Habersetzer ${ }^{2}$, J. Huppertz ${ }^{3}$, S. Koch ${ }^{4}$, T. Ponchon ${ }^{5}$, L. Palazzo ${ }^{6}$, M. Gimenez ${ }^{1}$, A. Garcia ${ }^{1}$, S.G. Lim ${ }^{1}$

${ }^{1}$ Institute for Image Guided Surgery, IHU-Strasbourg, STRASBOURG, France; ${ }^{2}$ Hepato-digestif, Nouvel Hôpital Civil, STRASBOURG, France; ${ }^{3}$ Image Guided Surgery, Nouvel Hôpital Civil, STRASBOURG, France; ${ }^{4}$ Gastroenterology, CHU-Besancon, BESANCON, France; ${ }^{5}$ Gastroenterology, CHU-Lyon, LYON, France; ${ }^{6}$ Gastroenterology, Clinic de Trocadero, PARIS, France

Background: EUS is difficult to learn and has a steep learning curve. Therapeutic EUS (TEUS) is even more so. Simulators, ex-vivo models and phantoms are the most common current teaching modalities but are felt by many to be unsatisfactory for high-level training.

Aim: We designed a training curriculum for TEUS that uses high-fidelity animal models and present a validation study performed by 4 TEUS experts.

Project Description: 3 different simulated pathologies were created in each of 9 acute pigs. 4 TEUS experts performed 15 therapeutic procedures in two or more animals over two days. Each intervention was evaluated simultaneously using a structured survey by an non-expert observer Data included demographics and procedure details as well as likert-scale evaluation of the quality, realism and education utility of the simulations. Global evaluation of the experience was captured from the experts as written comments. All data was consurrently registered and subsequently analysed by two blinded surgical educators.

Methodology: Three types of models were created using surgical access: 1-Tumors (injection of 4 types of Hydrogel), 2-Retro-gastric Collections (5-7 cm long intestinal loops filled with oatmeal, oil-water and gel), 3-Obstructions (bile duct and ureteral ligations 2 days prior to experience). Gastric, pancreatic and liver tumor models were used for FNA and FNB practice. Retrogastric fluid collections and choledochal/ureteral Obstructions were used for cyst gastrostomy, hepaticogastrostomy, gallbladder drainage and kidney drainage.

Results: Experts Age: 45-63, Median intervention time $22 \min$ (5-45), Total of 60 interventions evaluated, Overall quality of experience: 37 (68\%) ranked 8-10 (Excellent), 14 (27\%) from 7-4 (good), 3 (5\%) from 1-3 (poor), 54/60 procedures were successfully completed. Models were rated good to excellent quality (7-10) in $42(70 \%)$, poor quality in $8(13 \%)$. For $17 \%(10)$ of the interventions the model was considered not good enough to be repeated (solid retrogastric tumor and peripheral hepatic lesion).

Conclusion: High-fidelity live animal models with simulated pathologies are considered to be excellent training tools by experts and may provide a better learning experience for TEUS

\section{P017-AMAZING TECHNOLOGIES}

THE USE OF LAPAROSCOPIC SUTURING OF PERFORATED ULCERS OF STOMACH AND DUODENUM

A. Ukhanov, D. Zakharov, S. Zhilin, S. Bolshakov, A. Leonov, V. Ambartzumjan

Surgical, Central municipal hospital, VELEKIY NOVGOROD, Russia

Aim: Improvement of the results of the treatment of perforated ulcers of stomach and duodenum by use of laparoscopic suturing.

Material and methods: 272 patients with perforated ulcers of the stomach and duodenum were operated on from 2013 till 2018. Age of the patients varied from 18 till 78 years. Laparoscopic suturing of the perforation was carried out in 72 cases and 200 patients were operated on by laparotomy.

Results: Conversion rate was $4.2 \%$, postoperative morbidity-6.9\% (5 patients). There was no postoperative mortality in laparoscopic group, but, among 200 patients operated on by laparotomy, 15 died $(7.5 \%)$

Conclusion: The use of laparoscopic suturing of perforated ulcers of stomach and duodenum has definite advantages over the open method and, in the absence of contraindications, should be used more widely in emergency surgery hospitals. 


\section{P018-AMAZING TECHNOLOGIES}

NEAR INFRARED FLUOROSCENCE CHOLANGIOGRAPHY AS AN ESSENTIAL ADJUNCT TO EMERGENCY LAPAROSCOPIC CHOLECYSTECTOMIES

\section{F. di Maggio ${ }^{1}$, K. Shetty ${ }^{2}$, M. Bonomo ${ }^{2}$}

${ }^{1}$ Upper GI Surgery, St Thomas Hospital, LONDON, United Kingdom; ${ }^{2}$ General Surgery, St Thomas Hospital, LONDON, United Kingdom

Background: Emergency cholecystectomy is the gold standard treatment for acute cholecystitis according to NICE recommendations. The procedure is feasible but carries a higher risk of iatrogenic injury to the bile duct, which should be considered preventable.

Intraoperative fluorescence cholangiography following injection of indocyanine green (ICG) has been reported to aid identification of the extrahepatic bile duct. Data focused on its feasibility in the context of emergency cholecystectomies are currently missing.

Aim: To establish the feasibility and potential benefits of intraoperative ICG cholangiography in the settings of emergency cholecystecomies.

Project: Fluorescent ICG was used to enhance the biliary anatomy intraoperatively for 15 consecutive emergency laparoscopic cholecystectomies at our Institution.

Primary outcomes of surgery were considered the length of hospital stay, conversion to open and complications rate, including bile duct injury. Secondary outcome was operating time.

Preliminary Results: No iatrogenic bile duct injuries or conversion to open surgery were recorded. ICG demonstrated intra-op biliary anatomy allowing greater confidence to the surgeon performing emergency cholecystectomies, irrespective of duration of symptoms. All patients went home within $24 \mathrm{~h}$ in the absence of complications.

In our short videos, we present scenarios in which this technology helped: to distinguish a significantly dilated cystic duct from the CBD, to identify an anterior cystic artery in the contest of acute inflammation, to identify a Luksha duct, to exclude a leak after endoloops positioning on cystic duct.

Intra-operative augmented visualisation of biliary anatomy with indocyanine green cholangiography is an essential technology tool with the potential to extend the 72 hour window of safety for emergency cholecystectomies, with significant logistics benefits.

\section{P019-AMAZING TECHNOLOGIES}

\section{UNIPORTAL INTRAGASTRIC SURGERY FOR SUBCARDIAL PREMALIGNANT LESIONS}

\section{R. Sanchez-Santos ${ }^{1}$, E. Mariño Padin ${ }^{2}$, S. Gonzalez Fernandez ${ }^{1}$,} I. Dominguez Sanchez ${ }^{2}$

${ }^{1}$ General Surgery, Complejo Hospitalario Universitario de Vigo, VIGO, Spain; ${ }^{2}$ General Surgery, Complejo Hospitalario Universitario de Pontevedra, PONTEVEDRA, Spain

Introduction: Endoscopic resection of subcardial polyps has its limitations; especially when it is necessary to dry out the entire gastric wall. Uniportal intragastric surgery is a good alternative for the exeresis of subcardial premalignant lesions with gastric preservation.

Patient and method: We present a video with two cases.

Technique: we perform a laparoscopy to explore the entire abdominal cavity, then we open a $1.5 \mathrm{~cm}$ hole in the great curvature; a $2 \mathrm{~cm}$ incision is made in left hypochondrium and the uniportal device is placed inside de stomach. We inject serum in the submucosa with a endoscopic needle. When submucosa is completely separated from muscular lay; a submucosal exeresis can be made; but when there is not a complete separation after injection, then we perform a entire wall resection with a $1 \mathrm{~cm}$ circular margin. If a complete wall resection is made, then we close the defect with a barbed suture.

Results: Case 1: 45 years old male with a $1,5 \mathrm{~cm}$ subcardial polyp, preoperative biopsy was informed as severe dysplasia. In the laparoscopy we saw an unknown lesion in the great curvature that looked like a GIST. We placed the uniportal device intragastric and proceed to the submucosal serum injection. As the submucosal lay was completely separated from muscular, then we performed a submucosal exeresis. We close the gastrotomy and made the resection of the great curvature lesion with endostapler. The pathological analysis confirmed the severe dysplasia in subcardial lesion and GIST in the great curvature lesion. Patient was discharged with no complications after 2 days.

Case 2: 58 years old male with a $2 \mathrm{~cm}$ subcardial polyp. Preoperative ecoendoscopy suggested a muscular layer infiltration but only severe dysplasia was found in the previous biospsy. Laparoscopy did not found more lesions, and uniportal intragastric device was placed. A complete wall resection was made, and the defect was closed with manual barbed suture. Pathologyst confirmed severe dysplasia and unaffected margin. Patient was discharged with no complications after 4 days.

Conclusion: Uniportal intragastric surgery is feasible and safe and may be useful for subcardial premalignant lesions when endoscopic resection is very difficult or not feasible.

\section{P020-AMAZING TECHNOLOGIES}

ICG IN REVISIONAL BARIATRIC SURGERY: IS THERE ENOUGH EVIDENCE TO MAKE INTRAOPERATIVE DECISSIONS?

\section{R. Sanchez-Santos ${ }^{1}$, E. Mariño Padin ${ }^{2}$, S. Gonzalez Fernandez ${ }^{1}$,} I. Dominguez Sanchez ${ }^{2}$

${ }^{1}$ General Surgery, Complejo Hospitalario Universitario de Vigo, VIGO, Spain; ${ }^{2}$ General Surgery, Complejo Hospitalario Universitario de Pontevedra, PONTEVEDRA, Spain

Introduction: The role of ICG in bariatric surgery is still unclear. Knowing the lack of perfusion in the gastric pouch could be of great interest in revisional surgeries; but the question remains: is the current icg technology reliable enough to make intraoperative decisions in bariatric surgery? Methods: We have carried out a check of tissue perfusion with icg fluorescence in several cases of primary and revisional bariatric surgery. A solution of $0,1 \mathrm{mg} / \mathrm{kg}$ was injected intravenously and icg fluorescence was performed. We looked for the correct staining of the entire gastric pouch and the intestinal loop trying to rule out areas of tissue ischemia.

Results: The 15 cases in which the test was performed showed a minimal delay of 10-15 s between the intestinal loop stain and the pouch. A correct staining was observed in all but one case shown in the video. Is the case of a 46 years-old male patient who was operated 8 years earlier in another center; at that time he underwent a sleeve gastrectomy. We evaluated the patient for persistent gastroesofhageal reflux of 5 years of evolution with esophagitis. We offered revisional surgery to perform gastric bypass and hiatal closure. Fundus was dilated so a funduplasty was performed instead of using endostappler in the vertical side of the pouch. Manual anastomosis gastric bypass was performed. When the icg test was performed, a corner of the pouch does not stain green (an area of $1,5 \mathrm{~cm}$ ). So the decision was to resect that part and redo the anastomosis or wait and see. It was decided not to resect and the patient was discharged two days later with no complications and good outcome with a 12 months follow up. Conclusion: ICG fluorescence may be useful in bariatric surgery in the future but more evidence is needed of its usefulness in making intraoperative decisions.

\section{P021-AMAZING TECHNOLOGIES}

\section{A COMPARISON OF Results: OF APPLICATION OF LAPAROSCOPIC MANUALLY ESOPHAGOENTEROSTOMY AND ESOPHAGOENTEROSTOMY WITH MECHANICAL ANASTOMOTIC DEVICES}

\section{Agapov, E.A. Gallyamov, V.A. Kubyshkin, V.V. Kakotkin}

General surgery, Medical Research Educational Center. M.V. Lomonosov Moscow State University, MOSCOW, Russia

Background: Nowadays a total gastrectomy is the most common surgery to treat stomach cancer There are a number of techniques for reconstruction of the digestive tract after total gastrectomy. Special features of laparoscopic esophagoenterostomy are a technical difficulity, necessity of dissection of posterior mediastinum and several complications associated with anastomotic leakage. Since the introduction of the first mechanical stapling devices, a debate started about whether mechanical staplers or manual suture produce better results.

Aim: to compare results of application of laparoscopic manually esophagoenterostomy proposed by our surgical team and esophagoenterostomy created with mechanical stapling anastomotic devices.

Project description: 57 patients operated by a surgical team of Medical Research and Educational Center (Moscow State University) between February 2015 and October 2018 were included in this study: patients underwent laparoscopic total gastrectomy. Manual suture anastomoses were performed on 23 patients (the 1-st group) and stapled anastomoses were done on 34 patients (the 2-nd group).

Preliminary results: The method of reconstructive surgery do not results in terms of postoperative complications $(4.35 \%$ in 1 -st group versus $2.94 \%$ in second, $\mathrm{p}=0.08$, not significant). However duration of reconstructive surgery in the group with mechanical stapling anastomotic devices was shorter $(\mathrm{Tm}$ in 1-st group $=217 \mathrm{~min}(184-302)$ versus $201 \mathrm{~min}(162-311)$ in second group, duration of surgery in the first group was shorter in 1.08 times that second group (95\% C $1.03-1.13, \mathrm{p}=0.02))$. Patients were in intensive care during $20.2(17-42) \mathrm{h}$ in first group and $21.1(16.2-46) \mathrm{h}$ in second group [proportion $0.96(95 \% \mathrm{CI} 0.92-1.01, \mathrm{p}=0.05)$ ]. Total enteral feeding (sipping) was initiated on third day in both groups. Patients had been discharged within 7.21 (6-13) days after surgery in first and 7.23 (6-12 days) in second group [proportion 0.99 $(95 \%$ CI $0.95-1.02, \mathrm{p}=0.05)]$

Laparoscopic manually esophagoenterostomy proposed by our surgical team does not have disadvantages in comparison to stapling anastomotic devices and these methods can be alternative to each other. 


\section{P022-AMAZING TECHNOLOGIES}

THE IMPACT OF SSRIS ALONE OR WITH CONCURRENT ASPIRIN OR NSAIDS USE ON POST-ENDOSCOPIC SPHINCTEROTOMY BLEEDING

S. Anastasiadis ${ }^{1}$, G. Lazaraki ${ }^{1}$, G. Chatzimavroudis ${ }^{2}$, T. Katsinelos ${ }^{3}$, A. Xanthis ${ }^{1}$, S. Terzoudis ${ }^{1}$, J. Kountouras ${ }^{1}$, P. Katsinelos ${ }^{1}$

${ }^{1}$ Gastroenterology, Ippokration Hospital, THESSALONIKI, Greece; ${ }^{2}$ Gennimatas hospital, Ippokration Hospital, THESSALONIKI, Greece; ${ }^{3}$ Pharmacology, Aristotle University, THESSALONIKI, Greece

Background: Observational studies have shown an increased risk of upper gastrointestinal bleeding in users of selective serotonin receptor inhibitors (SSRIs), probably by inhibiting platelet aggregation, and patients, particularly in old age, undergoing pancreatobiliary procedures commonly use these drugs. We retrospectively investigated the impact of SSRIs alone or with combined aspirin (ASA) or non-steroidal anti-inflammatory drugs (NSAIDs) use on the incidence of post-endoscopic sphincterotomy (post-ES) bleeding.

Methods: A total of 3058 patients were included: in group A, 457 patients constantly received SSRIs or with combination ASA or NSAIDs use until the day of ES and 2659 patients (group B) who never had been on SSRIs (1925 patients) or had been on ASA (613 patients) or NSAIDS (121 patients). Patient assessment included indication of endoscopic retrograde cholangiopancreatography (ERCP), comorbid diseases, detailed drug history before and after ES, procedural details, and risk factors for post-ES bleeding. Primary outcome was defined as the incidence type and severity of post-ES bleeding.

Results: There was no statistical difference on age, sex, indication for ERCP, comorbid diseases, technical characteristics and results of therapeutic ERCP among the two groups. The incidence of post-ES bleeding was $3.9 \%$ in group A and $3 \%$ in group B, a difference not statistically significant $(\mathrm{p}=0.754)$. Likewise, there was no difference on type $(\mathrm{p}=0.145)$ and severity of bleeding $(\mathrm{p}=0.754)$ between the two groups. Multivariate analysis showed the precut technique as the only independent risk factor for post ES hemorrhage ( $p=0.001$, odds ratio $2.56,95 \%$ confidence interval 1.23-3.63)

Conclusion: The results of this study showed no influence on the incidence of post-ER bleeding with SSRIs alone or with combined ASA or NSAIDs use.

\section{P023-AMAZING TECHNOLOGIES}

\section{ANIMAL VALIDATION OF SENTINEL LYMPH NODE DETECTION BY ICG LYMPHOGRAPHY IN COLORECTAL SURGERY}

J.F. Noguera ${ }^{1}$, L. Castro ${ }^{1}$, L. García $^{1}$, A. Miguez ${ }^{1}$, A. Gómez ${ }^{1}$, A. Centeno ${ }^{2}$

${ }^{1}$ Surgery, Complejo Hospitalario Universitario A Coruña, A CORUÑA, Spain; ${ }^{2}$ Centro Tecnológico de Formación, Complejo Hospitalario Universitario A Coruña, A CORUÑA, Spain

Background: Lymph node status is one of the key prognostic factors in patients with colorectal cancer, and remains the most important selection criteria for adjuvant chemotherapy. It is believed that at least $30 \%$ of node negative patients will suffer disease recurrence within the first 5 years after surgery. This may be due to understaging of lymph node status. Sentinel lymph node mapping is widely used for staging of breast cancer and melanoma, with injection of colloid Tc 99 and isosulfan blue (IB). However, indocyanine green (ICG) fluorescence guidance is a new technical approach to this issue, with promising results for detection of aberrant lymphatic drainage outside of the planned resection. The ICG lymphography has the advantage of offering a good visualization of the lymphatic channels but there are problems in order to identify the lymphatic nodes.

Aim: The objective of the experimental study is to investigate the possibility to detect the sentinel lymph nodes after the injection of different solutions with indocyanine green in the subserosal colonic layer in the pig.

Project description: Twelve female Large White pigs were operated with laparosocpic approach and Spies optic filter (Karl Storz, Germany). Indocyanine green was injected in the subserosa of the colonic wall ( $1 \mathrm{ml}$ at 2 points). Lymphatic flow was observed at 1-3-5-10-15 and $20 \mathrm{~min}$, searching for the migration of the ICG by the lymphatic channels and its introduction in the sentinel nodes. Preliminary results. The identification of the sentinel nodes is very difficult with the solution of ICG-sterile water. With this technique we can see the lympjatic channels but not the lymphatic nodes. The adition of 5\% human albumin as a transporter of the ICG is very helpful for the correct identifiaction of the lymphatic channels at 5-10-15 $\mathrm{min}$ and the correct visualization of the lymphatic nodes at $20 \mathrm{~min}$ after the bowel injection. Addition of other transporters like dextran solutions may be helpful too but the time to the correct visualization is longer.

\section{P024-AMAZING TECHNOLOGIES}

\section{SIMULTANEOUS LAPAROSCOPIC CHOLECYSTECTOMY AND INTRA-OPERATIVE ERCP FOR CALCULAR OBSTRUCTIVE JAUNDICE. A SINGLE CENTER EXPERIENCE OF 120 CASES}

\section{El Sorogy, A. El Nakeeb}

Surgery, Gastro-Intestinal Surgery Center, Mansoura University, MANSOURA, Egypt

Background: Upto $20 \%$ of patients diagnosed with chronic calcular cholecystitis have concomitant common bile duct (CBD) stones. Managment classically involved cholecystectomy and CBD exploration.Recent innovations and refinements in surgical technologies and techniques lead to shift towards minimally invasive procedures including:laparoscopic CBD exploration, pre-operative ERCP and laparoscopic cholecystectomy and intra-operative ERCP.

Aim: Evaluate the feasibility and success rate of combined laparoscopic cholecystectomy (LC) and intra-operative ERCP for management of concomitant gall bladder and CBD stones. Project description: Patients treated for concomitant gall bladder and CBD stones were included. LC and intraoperative transcystic cholangiogram (TCC) were performed in most of the cases. Intraoperative ERCP was done for cases with proven CBDstones. ERCP was performed by the operating surgeon himself.

Preliminary results: In the period between 2012 and 2018, 120 patients with concomitant gall bladder and CBD stones were included. LC was successful in all cases. Intraoperative TCC revealed passed CBD stones in 9 cases $(7.5 \%)$ so intra-operative ERCP was performed only in 111 patients. ERCP was successful in 107 cases where complete CBD clearance was achieved in 102 cases of them $(95.3 \%)$. The failed 4 cases underwent either open or laparoscopic CBD exploration. Post-operative complications included bleeding papillotomy ( 2 cases), one of them requiring endoscopic control, 3 cases of acute pancreatitis ( 2 mild and one severe) and 1 case or retained $\mathrm{CBD}$ stone requiring an additional setting of ERCP.

Conclusion: Laparoscopic cholecystectomy with intra-operative ERCP is a safe and feasible option for management of concomitant gall bladder and CBD stones. Combining laparoscopy and endoscopy makes both procedures easier and enhances the outcomes. ERCP is a powerfull tool in the hands of a well trained hepatobiliary surgeon.

\section{P025-AMAZING TECHNOLOGIES}

\section{IMPACT OF THE MYOTOMY EXTENT ON GASTRIC SIDE ON SURGICAL OUTCOME AFTER LAPAROSCOPIC HELLER'S CARDIOMOTOMY FOR ACHALASIA}

\section{Abdelgawad, M.S. Elsorogy, A.N. El Nakeeb}

Gastroenterology surgical center, Faculty of medicine, Mansoura University, MANSOURA, Egypt

Background: Laparoscopic Heller cardiomyotomy (LHM) with Dor fundoplication represents the most commonly accepted surgical management for achalasia. The ideal extent of myotomy on the gastric side remains a matter of continuous debate.

Aim: The aim of this study was to compare the impact of the extent of myotomy on the gastric side on the outcome of LHM.

Project description: Patients suffering from achalasia who underwent LHM between June 2009 and December 2017 at the Gastro-intestinal surgical center (GISC), Mansoura University, Egypt, were identified through a retrospective review of a prospectively collected database. The patients were classified according to the length of the gastric myotomy into 3 groups (Group I: $<1.5 \mathrm{~cm}$, group II: $1.5-2.5 \mathrm{~cm}$ and group III: $>2.5 \mathrm{~cm}$ ).

Preliminary results: 212 patients (94 males and 118 females) with achalasia treated by LHM and Dor fundoplication included in the study. No statistically significant differences were found among the 3 groups as regards preoperative data, intraoperative mucosal perforation, operative time, blood loss and hospital stay. Preoperative oesophageal manometery were performed for all cases among different groups. Postoperative PH metery and oesophageal manometery were performed in 53/69 (76.8\%) in group 1, 80/113 (70.8\%) in group 2, and 23/30 (76.7\%) in group 3 There was significant difference among the three groups as regards postoperative LES and postoperative PH metery.The incidence of persistent dysphagia was significantly higher in the group I. Postoperative GERD symptoms were significantly higher in group III (23.3\% $\mathrm{p}<0.0001)$. Recurrent achalasia was significantly higher in Group I (11 patients $15.9 \%, 8$ patients in group II $(7.1 \%)$ and nil in group III $(\mathrm{p}<0.02)$

In conclusion: Longer myotomy on the gastric side $(>2.5 \mathrm{~cm})$ ensures complete division of the LES with better outcomes in term of resolution of dysphagia but may be associated with higher postoperative GERD. Therefore, a myotomy length of 1.5 to $2.5 \mathrm{~cm}$ on the gastric side provides a balance between relieve of dysphagia and development of postoperative GERD. 


\section{P026-AMAZING TECHNOLOGIES}

\section{STRANGULATED INGUINAL HERNIA MANAGED BY LAPAROSCOPIC APPROACH AS DAY CASE}

\section{J.A. Butt}

Colorectal Surgery, Letterkenny University Hospital, LETTERKENNY, Ireland

70 years male patient waiting for elective surgery for inguinal hernia repair presented with painful lump in right groin and moderate abdominal distension.

$\mathrm{C} / \mathrm{T}$ scan of his abdomen revealed a large groin hernia with signs of small bowel obstruction and collapsed distal bowel. Emergency Theatre was organised for this patient with anaesthetic assessment prior to his surgery.

Initial plan of local exploration with possibility of small resection was changed once he was under full anaesthetic with muscle relaxation. His abdominal girth provided an opportunity to utilise laparoscopic intervention as an initial approach.

Laparoscopy with 0 Degree lens revealed moderately distended loops of small bowel and a large omental mass along with a loop of small bowel incarcerated in right direct inguinal hernia site.Table tilting improved the exposure of the operative field and whole omental mass and small bowel loop was fully reduced back into abdominal cavity.

Learning aspect of this technique relates to looks of small bowel loop which looked almost gangrenous on initial inspection but as the time passed while removing the incarcerated omental mass,colour of small bowel changed to a more healthy appearnce.By instilling warm saline into the abdominal cavity for wash out purposes further enhanced the perfusion status of small bowel and carefull inspection of the incarcerated segment of small did not show any breach of luminal integrity of bowel.At this point,a standard TAPP repair of direct inguinal defect was performed using a UltrPro light weight mesh using Absorbatechs.Peritoneum was fully closed excluding mesh from any contact with peritoneal cavity.

This patient made an uneventful recovery within $24 \mathrm{~h}$ and discharged home. Histology of Omental mass showed only oedematous peritoneal fat.

\section{P027-AMAZING TECHNOLOGIES}

\section{INTERNATIONAL ROBOTIC COLORECTAL REGISTRY}

\section{A. Przedlacka, S.Y. Qiu, P. Paris, C. Kontovounisios}

Department of Surgery and Cancer, Imperial College London, LONDON, United Kingdom

Background: Robotically assisted surgery is a rapidly developing modality of minimally invasive surgery with proven advantages in the management of cancer. Despite its increasing prevalence, there is still an ongoing debate regarding its future role in colorectal surgery. While the prospective randomised multi-centre studies provide research evidence for its potential efficacy, an assessment of its effectiveness and realistic outcomes in everyday clinical practice can add an important perspective to this discussion. The International Robotic Colorectal Registry will allow to compile and pool the international robotic colorectal experience.

Aims: The aim of the International Robotic Colorectal Registry is to monitor the safety and outcomes, as well as the quality of specimen of robotically assisted colorectal surgery for malignant and benign diseases of the colon and rectum. The primary endpoint is a composite oncological failure. The secondary endpoints include anastomotic leak, resection margin involvement, conversion rate, operative time, post-operative 30-day morbidity and mortality, long term oncological outcomes, quality of life, functional outcomes and cost-effectiveness. Project description: The International Robotic Colorectal Registry is a multicentre web-based, online secure database. The Registry has been awarded an ethical approval by a relevant national committee. All surgeons performing robotic or robotically-assisted surgery are invited to participate. The data collected includes patient demographics, cancer characteristics, operative details, histology of the specimen, wound healing, post-operative therapy, readmission, quality of life and functional (bowel, urinary and sexual) outcomes. All the sensitive patient information is encrypted before its introduction into the database.

Preliminary results: So far, twenty robotic colorectal centres have joined the International Robotic Colorectal Registry. The preliminary results will be published once 600 patients have been enrolled. Univariate and multivariate analyses will be performed to identify possible risk factors for poor outcome.The possibility to record open, laparoscopic or other minimally invasive colorectal procedures will facilitate comparison of the outcomes of the robotically assisted surgery and other modalities. The Registry will also allow each surgeon enrolled to monitor their skill progression and outcomes over the time. Results will be published in surgical literature and presented internationally.

\section{P028-AMAZING TECHNOLOGIES}

RETROSPECTIVE STUDY: ARGON PLASMA VS POLYPECTOME SNARE TIP COAGULATION: EFFECT ON RECURRENCE RATE AFTER RESECTION OF LATERALLY SPREADING TYPE LESIONS

$\underline{\text { S. Anastasiadis }}{ }^{1}$, G. Lazaraki ${ }^{1}$, G. Chatzimavroudis ${ }^{2}$, S. Terzoudis ${ }^{1}$, A. Xanthis ${ }^{1}$, J. Kountouras ${ }^{1}$, P. Katsinelos ${ }^{1}$

${ }^{1}$ Gastroenterology, Ippokration Hospital, THESSALONIKI, Greece; ${ }^{2}$ Gennimatas hospital, Ippokration Hospital, THESSALONIKI, Greece

Background: Endoscopic mucosal resection (EMR) is an established technique for treating large laterally spreading type (LST) lesions $=20 \mathrm{~mm}$. The aim of our study was to compare the use of argon plasma (APC) versus snare-tip coagulation on the recurrence rate of large LST lesions. Methods: All patients with large LST lesions resected by EMR between January 2006 and December 2014 were enrolled. After piecemeal resection, patients underwent either APC or snare-tip coagulation of the rim of the resection area and any residual adenomatous tissue. Follow up included colonoscopy and biopsies. Medical records, including characteristics of patients and polyps, complications and recurrence were retrieved and collected.

Results: One hundred one patients were included in the final analysis. They were divided into the APC group $(n=50)$ and the snare-tip coagulation group $(n=51)$. The 2 groups were similar concerning patients' characteristics, size of polyps and histology. Post-polypectomy coagulation syndrome was observed in 8 patients (7.9\%) (APC group: $n=5$ and snare tip group: $n=3$ ). EMRrelated bleeding occurred in 9 patients $(8.9 \%)$ (APC group: $\mathrm{n}=4$ and snare tip group: $\mathrm{n}=5$ ). Total recurrence rate was $14.85 \%(16 \%$ and $13.7 \%$ in APC and snare-tip groups, respectively, $\mathrm{P}=0.34$ )

Conclusion: The effectiveness of snare-tip coagulation is comparable with that of APC with respect to recurrence rate after resection of large LST lesions. It thus represents a cost-effective alternative to APC.

\section{P029-UPPER GI-Esophageal cancer}

BENIGN GASTRIC OUTLET OBSTRUCTION: STILL A SURGICAL DISEASE. LAPAROSCOPIC MANAGEMENT IN A MAJOR CENTRE IN OMAN

\section{R. Almehdi}

\section{Surgery, The Muscat Hospital, MUSCAT, Oman}

Background: Gastric outlet obstruction (GOO) due to benign strictures is an uncommon surgical entity today. This paucity relates to the decrease in its aetiological factors in the modern era as well as to advances in both prevention and medical as well as endoscopic treatments of such condition. The most common of causes relating to peptic ulcer disease, has been subdued for decades with quality acid control medications. On the other hand advances in gastroscopic dilatations skimmed even more the frequency of these cases from arriving to surgical intervention.

Aim: This presentation gives an update on the standing of this pathology and its surgical management today. It will also shed a light on our early experience in this condition at the Royal Hospital of Muscat in Oman.

Project description: A case series of all patients with GOO, who were surgically managed between 2010 and 2015

Results: There were a total of 16 patients, 10 males and 6 females. The cause of obstruction was Peptic ulcer disease in 10, Corrosive injury in 2, Iatrogenic perforation in 1 and Idiopathic hypertrophic stenosis in 2 . Emergency presentation was seen in 4. Management included Jaboulay Pylorpolasty in 2, Resection in 10 (Distal gastrectomy in 9, Total Gastrectomy in 1) or a Bypass (Gastrojejunostomy) in 4. In 14 of the above, the procedure was done by Laparoscopy. Post operatively, temporary gastric paresis delayed recovery in 5 , however all symptoms resolved in 15 . There were no recurrences at minimum of 2 years of follow up.

In spite of advances in medications and gastroscopy interventions, we still seem to identify this condition within our population. Although infrequent, they demand awareness from surgeons since they could be managed successfully, especially laparoscopically, with minimal morbidities and early recovery. The introduction of advanced laparoscopy to the unit's setup in recent years, made such option feasible with satisfactory and durable outcomes. 


\section{P030_UPPER GI-Benign Esophageal disorders}

WHEN LESS IS ENOUGH: LAPAROSCOPIC MANAGEMENT OF DUODENAL GIST WITH LATERAL DUODENECTOMY AND ROUX EN Y DUODENO-JEJUNOSTOMY-A CASE REPORT

\author{
R. Almehdi ${ }^{1}$, K. al Harrasi ${ }^{2}$
}

${ }^{1}$ Surgery, The Muscat Hospital, MUSCAT, Oman, ${ }^{2}$ Surgery, The Royal Hospital, MUSCAT, Oman

Background: GISTs of the Upper GI are found mainly in the stomach (60-70\% of cases) and small intestine $(30 \%)$

Duodenal GISTs however, comprise a smaller subset with a frequency of 6 to $21 \%$.

The optimal surgical procedure for Duodenal GIST is still evolving. Since wide margins and extensive

lymphadenectomy are not required, restrain from more radical resections in this area would be a valid option.

Aim: This is a Video case report of a patient with a GIST involving the third part of the duodenum treated by laparoscopic lateral duodenectomy and end-side Roux-en-Y duodenojejunostomy

Case report: 55 years lady presented with recurrent mid abdominal postprandial pain with anorexia, nausea and occasional vomiting An Ultrasound showed well defined hypoechoic mass of $3 \times 2.5 \times 2.2 \mathrm{~cm}$ at the Right para-aortic region. CT scan defined the mass as retroperitoneal, intimately related to the Pancreas uncinate process and the third part of duodenum with no clear cleavage line between them. An MRI endorsed the diagnosis of GIST of the duodenum. She was operated upon through a Laparoscopic lateral duodenectomy including the GIST at the third part of the duodenum. A frozen section confirmed the clear margins . Reconstruction was done by a Roux-en-Y Duodenojejunostomy with the alimentary limb taken $30 \mathrm{~cm}$ from the DJ flexure. She had an uneventful post operative recovery and was discharged well. The histology confirmed a Low grade GIST tumour hence no further treatment was needed. At follow up six months later, she was doing well and gaining weight.

Conclusion: Complex anatomy of the pancreatico-duodenal area makes conserving the duodenum for tumours rather than a major resection a challenging option. In our case however, with the disease in the third part being of a moderate size, a lateral duodenal wall resection including the mass was possible rather than a segmental resection. This procedure could be an ideal choice for benign, moderate sized tumours in the third and fourth part of the duodenum.

\section{P031-AMAZING TECHNOLOGIES}

\section{OVERLAP ANASTOMOSIS IN LAPAROSCOPIC COLECTOMY}

\section{K. Tanabe, S. Mori, K. Baba, Y. Kita, S. Natsugoe}

Digestive Surgery, Kagoshima University Hospital, KAGOSHIMA, Japan

Background: During laparoscopic colectomy, laparoscopic lymph node dissection and extracorporeal intestinal anastomosis is commonly performed. An umbilical incision of 4-6 cm and wide-range mobilization of the intestinal tract is required for extracorporeal anastomosis. Previously, we introduced intracorporeal overlap anastomosis in June 2017 as a minimally invasive treatment. Here, we report its short-term outcomes.

Aim: We retrospectively compared the surgical outcomes of 21 cases of extracorporeal anastomosis and 8 cases of intracorporeal anastomosis, all of which were performed between June 2017 to May 2018.

Procedures: After lymph node dissection and sufficient mobilization of the intestinal tract, the proximal and distal intestines were resected perpendicularly to the intestinal tract with a 60-mm linear stapler. The anastomosis was performed after the specimen was extracted from an umbilical incision. The opposite sides of the mesenteric margin $3 \mathrm{~cm}$ from the staple line of the one intestinal tract, and $7 \mathrm{~cm}$ from the staple line of the other intestinal tract, were marked, and then the respective intestinal tract was positioned to join the opposite mesenteric sides together. An insertion hole was made in the intestinal tract at the marked site. Side-to-side anastomosis with a linear stapler was performed, and then the insertion hole was closed with a linear stapler after several temporary sutures.

Preliminary results: In the extracorporeal anastomosis group, the mean operation time, blood loss, and post-operative days were $270 \mathrm{~min}, 127 \mathrm{~mL}$, and 13.2 days, respectively. Furthermore, there were three intraoperative cases of bleeding (14.3\%), and two postoperative cases of lymphorrhea $(9.5 \%)$ that occurred. However, in the intracorporeal overlap anastomosis group, the mean operation time, blood loss, and post-operative days were $284 \mathrm{~min}, 75 \mathrm{~mL}$, and 12.5 days, respectively. Additionally, there were no cases of intraoperative complications, and only one postoperative case of lymphorrhea $(12.5 \%)$

Conclusion: Intracorporeal overlap anastomosis in laparoscopic colectomy is safe and feasible, and can be used as a minimally invasive treatment.

\section{P032-AMAZING TECHNOLOGIES}

EXPERIMENTAL MODEL FOR PHANTOM LIVER USING A 3D PRINTED MOLD

R.C. Elisei ${ }^{1}$, F. Graur ${ }^{2}$, N. al Hajjar ${ }^{2}$, C. Popa $^{2}$, E. Mois ${ }^{2}$, C. Vaida ${ }^{3}$, D. Pisla

${ }^{1}$ General Surgery, Bistrita Emergency County Hospital, BISTRITA, Romania; ${ }^{2}$ General Surgery, Regional Institute of Gastroenterology and Hepathology, Prof. O. Fodor, CLUJ-NAPOCA, Romania; ${ }^{3}$ CESTER, Technical University, CLUJ-NAPOCA, Romania

Nowadays 3D-printing it's not a new technology any more but with an exponential developing. There are beliefs that in $202710 \%$ of everything that will be produced will be 3d-printed. In medical field this technology knows the same exponential developing. First used in orthopedics and maxilo-facial surgery now 3d-printind is used in many other fields for different reasons, like preoperative training models, surgical special instruments, in medical education, etc.. Liver surgery is in continuous developing and this is the reason why we need experimental liver mode for training and testing. A best liver experimental model should heave liver consistency, to be flexible, to heave the same ultrasound feedback, to be cheap and easy to be reproduced. This is why we developed a liver experimental model made of gelatin by a simple recipe, using a $3 \mathrm{~d}$ printed mold, created after a human liver ct-scan. First was made the segmentation of the liver After segmentation we create the $3 \mathrm{~d}$ virtual liver model and the negative image of the liver, which was used for creation of the 2 pieces of the liver mold, with connections between them and a hole on the top to pour the gelatin solution. After the solidification of the gelatin liver we can open the mold and use it to create a new experimental liver. It can be used silicone solution and any other liquid or semi-liquid solution to create an experimental liver model. The 3d-printed mold can be used several times without any other costs.

\section{P033-AMAZING TECHNOLOGIES}

\section{LAPAROSCOPIC CHOLECYSTECTOMY IN THE ELDERLY PATIENTS-A COUNTY HOSPITAL EXPERIENCE}

\section{A. Cotirlet ${ }^{1}$, L. Gavril ${ }^{2}$, E. Popa ${ }^{3}$}

${ }^{1}$ Surgery, University „Vasile Alecsandri,„Bacau/ Moinesti Hospital, MOINESTI, Romania; ${ }^{2}$ Intensive care, University „Gr. T. Popa,Iasi/ IRO Iasi, IASI, Romania; ${ }^{3}$ Surgery, Moinesti Hospital, MOINESTI,

\section{Romania}

Background: Advancing age with its associated co-morbidities increases the likelihood of postoperative complications as well as conversion rate during laparoscopic cholecystectomy. The present study assesses the safety and applicability of laparoscopic cholecystectomy in the elderly patients of 65 years old and above.

Methods: This study is an analysis of 173 patients, over 65 years of age, who underwent laparoscopic cholecystectomy from January 2014 to December 2018 in our surgery department. The data included demographic details, co-morbidities, underlying biliary pathology, indications for surgery, operative and postoperative complications, morbidity and mortality, and hospital stay.

Results: Laparoscopic cholecystectomy for 173 elderly patients with a mean age of 69.56, out of whom $52(30.05 \%)$ were males and $121(69.94 \%)$ were females. Co-morbid conditions were identified in $53.17 \%(\mathrm{n}=92)$ patients $)$. There were $67(38.72 \%)$ emergency laparoscopic cholecystectomies and $106(61.28 \%)$ patients were operated electively,mean operative time being $65 \mathrm{~min}$. Fourteen $(8.09 \%)$ patients required conversion, 23 (13.29\%) patients developed postoperative complications, the mean hospital stay was 5.30 days.

Conclusion: There is no undue risk in laparoscopic cholecystectomy in the elderly population and the procedure can be regarded as safe as in patients below 65 years of age. 


\section{P034-AMAZING TECHNOLOGIES}

\section{PRELIMINARY RESULTS OF A NEW ENVIRONMENT FOR SURGICAL TRAINING VIRTUAL REALITY CONTENTS CREATION}

P. Sánchez-González ${ }^{1}$, I. Oropesa ${ }^{2}$, M. Rodríguez ${ }^{2}$, D. CambaLamas $^{2}$, L. Wauben ${ }^{3}$, J. Dankelman ${ }^{3}$, F.W. Jansen ${ }^{4}$, G. Wéber ${ }^{5}$, E.J. Gómez ${ }^{2}$

${ }^{1}$ ETSI Telecomunicación. Center for Biomedical Technology, Universidad Politécnica de Madrid, MADRID, Spain; ${ }^{2}$ ETSI Telecomunicación, Universidad Politécnica de Madrid, MADRID, Spain; ${ }^{3}$ Department of Biomechanical Engineering. Faculty of Mechanical, Maritime and Mat, Delft University of Technology, DELFT, The Netherlands; ${ }^{4}$ Leiden University Medical Center, Leiden University Medical Center, LEIDEN, The Netherlands; ${ }^{5}$ Department of Surgical Research and Techniques, Semmelweis University, BUDAPEST, Hungary

Current pedagogical needs in medical education are closely related to (1) ethical concerns on learning and training with real patients and substitutives such as animals, and (2) reconciling time devoted to learning with clinical practice, considering the European Work Time Directives. Simulation in medical education is and has been the preferred route to address both pedagogical needs. Virtual simulation has proven to be a valid tool for training; however, current systems restrict usage to tasks and modules offered, without possibility of personalization. We present the Minimally Invasive Surgery Simulator Scenario Editor (MIS-SIM) an environment where users can create, edit and run virtual reality tasks designed for medical training. The environment features an editor allowing users to develop learning tasks, defining its learning objectives and task goals in an easy way.

A first proof of concept has been implemented for surgical training and training activities (demostrators and short courses) have been carried out in three European sites: Spain, The Netherlands and Hungary.

During training activities, 10 different exercises have been created and uploaded to the contents' database. Trained technical skills include handeye and bimanual coordination, instrument handling and pulling. Preliminary results with 30 users have shown MIS-SIM training potential, although some functionalities should be made easier. Personalization has been highlighted as the key added value of MIS-SIM with respect to the current competitions in the market: the ability for target users to use virtual reality based learning tools while remaining in complete control of the learning process.

MIS-SIM aspires to break the barrier between VR and medical education by empowering users to create their own tasks. With MIS-SIM teachers/course creators and learners (healthcare professionals \& future healthcare professionals) will benefit from an innovative tool to (1) create personalised medical learning contents tailored to preferred learning styles, allowing the creation of individualize learning paths; (2) improve the efficiency of training by focusing on the training needs of the learners and (3) share and sell VR-based didactic contents.

\section{P035-AMAZING TECHNOLOGIES}

CURRENT STATUS AND DEVELOPMENTS OF THE EUROPEAN KNOWLEDGE ALLIANCE FOR EDUCATIVE EDUCATION OF SURGICAL AND INTERVENTIONAL SKILLS (EASIER)

C. Tiu ${ }^{1}$, P. Sánchez-González ${ }^{2}$, M. Chmarra ${ }^{3}$, D. Gutiérrez ${ }^{4}$, C. Guzmán-García ${ }^{5}$, L. Sánchez-Peralta ${ }^{6}$, G. Wéber ${ }^{7}$, F. Sánchez-Margallo ${ }^{8}$, B. Pagador ${ }^{9}$, J. Dankelman ${ }^{10}$, K. Juhos ${ }^{7}$, I. Oropesa ${ }^{11}$, A. Negoita Tiu ${ }^{1}$, C. Mettouris ${ }^{12}$, A. Yieratziotis ${ }^{12}$, G. Papadopoulos ${ }^{12}$, E. Gómez ${ }^{11}$

${ }^{1}$ Research, Medis Foundation, CAMPINA, Romania; ${ }^{2}$ Research, 2Biomedical Engineering and Telemedicine Centre, ETSI Telecomunicación, Center f, MADRID, Spain; ${ }^{3}$ Department of Biomechanical Engineering, Faculty of Mechanical, Maritime and Materials Engineering ( $3 \mathrm{mE}$ ),Delft University, DELFT, The Netherlands; ${ }^{4}$ Reserach, EverisConsultancy Ltd., Valencia, España;, VALENCIA, Spain; ${ }^{5}$ 2Biomedical Engineering and Telemedicine Centre, Universidad Politécnica de Madrid, MADRID, Spain; ${ }^{6}$ Research, 6Centro de Cirugía de Mínima Invasión Jesús Usón, CACERES, Spain; ${ }^{7}$ Department of Surgical Research and Techniques, Semmelweis University, BUDAPEST, Hungary; ${ }^{8}$ Research, Centro de Cirugía de Mínima Invasión Jesús Usón, CACERES, Spain; ${ }^{9}$ Centro de Cirugía de Mínima Invasión Jesús Usón, Centro de Cirugía de Mínima Invasión Jesús Usón, CACERES, Spain; ${ }^{10}$ Department of Biomechanical Engineering, Faculty of Mechanical, Maritime and Materials Engineering, DELFT, Spain;

${ }^{11}$ Biomedical Engineering and Telemedicine Centre, Universidad Politécnica de Madrid, MADRID, Spain; ${ }^{12}$ Computer Science Department, University of Cyprus, NICOSIA, Cyprus

Aims: Currently surgical training is largely based on the improvement of Technology Enhanced Learning solutions. The progress of engineering and the diversification of training facilities outside the operating theater results in an even greater contribution of technology in the future. The main reasons to encourage these changes are increased efficiency of simulators and directly increased patient's safety. The goal assumed by the EASIER Project is to develop multi-skill, online platforms for minimally invasive surgical (MIS) procedures-based on common pedagogical principles with reference value in a multinational space. The platform will allow the connection of external assets (such as simulators) to centralize all training data from residents. This work presents the milestones of the project during its first year of life.

Methods: The Consortium's activity started with a Knowledge Elicitation process organizing brainstorms and workshops including experts in MIS and interventional techniques, from Spain, Romania and Hungary. This experience led to the formulation of a questionnaire that was implemented online and sent via email to surgeons and residents from the participating countries.

Results: Accumulated experience was used to define the pedagogical needs of the platform. The pedagogical needs form the starting point for defining the technical requirements and specifications. Based on them, the design of the platform has been achieved, including its architecture and communication protocol between external assets design, facilitated by the use of state of art educational standards.

Discussions and conclusions: Next steps include the implementation of the EASIER platform, as well as the definition of cases studies selected by the clinical partners in Spain, Romania and Hungary to solve applications of the platform dedicated to cholecystectomy, lumbar puncture and arthroscopy. A pedagogical model, built by the experience of the Consortium, is being used to guide instructional design of the course. Finally, results will be validated in a multi-center validation study.The EASIER project will create a training platform with reference value for European surgery. The structure of the Consortium, based on the confluence between collectives with clinical, technological and pedagogical experience, will generate a complex learning tool in surgery embodying technology-based training systems with clinical experience. 


\section{P036-AMAZING TECHNOLOGIES}

\section{LAPAROSCOPIC TAPP GROIN HERNIA REPAIR: A TUNISIAN SINGLE CENTER EXPERIENCE IN 5 YEARS}

\section{M.A. Bourguiba ${ }^{1}$, G. Elkebir ${ }^{2}$, M.A. Moussi ${ }^{1}$, M Ben Moussa ${ }^{1}$}

${ }^{1}$ General surgery, Faculty of Medecine Of Tunis, TUNIS, Tunisia; ${ }^{2}$ General surgery, Charle nicolle Hospital, TUNIS, Tunisia

Background: The treatment of groin hernia is an important part of our daily surgical activity.

Aim: We proposed to evaluate outcomes of the Laparoscopic Trans abdominal pre peritoneal treatment (TAPP) of the groin hernia.

Project description : one hundred and fifty patients who underwent a TAPP for a groin hernia were included in a retrospective study between January 2014 and November 2018.

Results: The gender ratio was 5 . The average age was 57,4 years. Twenty percent of patients had a history of abdominal surgery. The operative indication was a unilateral hernia in $25 \%$ of cases, associated with an umbilical hernia in $40 \%$ of cases, a recurrent groin hernia in $30 \%$ of cases and a bilateral inguinal hernia in $45 \%$ of cases. The conversion rate was $1.33 \%$. The hernias were classified according to the EHS classification in L3 type in $31 \%$ of cases, L2 in $24 \%$ of cases, L1 in 19\% of cases, M2 19\%, L2 R in $20 \%$ of cases and F1R in $15 \%$ of cases. A contralateral inguinal hernia was discovered in $15 \%$ of patients. A polypropylene mesh $15 \times 15 \mathrm{~cm}$ was fixed by a stapling in $59 \%$ of cases and by a suture in $41 \%$ of the cases. The average operation time was $55 \mathrm{~min}$. The hospital stay average was 0,7 day. An antalgic treatment was prescribed in $15 \%$ of patients. The average time to return to normal physical activity was 5 days. A postoperative seroma was noted in $14 \%$ of patients. No cases of mesh suppuration were noted. Chronic pain was noted in two patients. No recurrence was noted with an average follow-up of 25 months.

Conclusion: laparoscopic treatment of the groin hernia by TAPP had good results concerning the postoperative pain, early recovery of physical activity and aesthetic damage. A larger setback is needed in our study to evaluate the recidivism rate.

\section{P037-AMAZING TECHNOLOGIES}

\section{LAPAROSCOPIC REVERSAL OF HARTMANN PROCEDURE: A SINGLE SURGEON EXPERIENCE}

M.A. Bourguiba ${ }^{1}$, F. Souai ${ }^{1}$, M. Ghalleb ${ }^{2}$, A. Khemir ${ }^{1}$, M.A. Moussi ${ }^{1}$, M. Ben Moussa ${ }^{3}$

${ }^{1}$ General surgery, Faculty of Medecine Of Tunis, TUNIS, Tunisia;

${ }^{2}$ Oncologic surgery, Faculty of Medecine Of Tunis, TUNIS, Tunisia;

${ }^{3}$ General surgery, Charle Nicolle Hospital, TUNIS, Tunisia

Background: The laparoscopic reversal Hartmann procedure is a challenging technique showing promising results in comparison to the open procedure.

Aim: Report a single surgeon experience showing promising results.

Project description: we report retrospectively twenty patients who had laparoscopic reversal hartman procedure between January 2015 and September 2018

Preliminary Results: It's a twenty patient's case series. The median age was 57 years old. The majority of patients had a previous open surgery $(18 / 20)$. The most commun etiology was pelvic colon volvulus (10/20) and sigmoid neoplasic occlusion (6/20). The mean operative time was $158.2 \mathrm{~min}$ (75-200 $\mathrm{min})$. No per-operative complication was reported. Only one procedure was converted to laparotomy due to severe pelvic adhesions. All patients ambulated on postoperative day 1 . The Mean time to the recovery of the bowel movement was Two days ( 1 to 4 days). The mean time to Oral analgesia intake was 1.56 days ( 1 to 3 ). The mean time of hospital stay was 5.66 Days (4 to 8). Two patients had complication in a mean time of 18 days after the surgery.

Conclusion: The laparoscopic reversal Hartmann procedure is a safe technique showing promising results but in the hand of trained laparoscopic surgeon.

\section{P038-AMAZING TECHNOLOGIES}

AN ULTRASOUND COURSE FOR SURGEONS—PREMISES, IMPLEMENTATION, STRATEGIES

C. Tiu $^{1}$, A. Keidar ${ }^{2}$, T. Lango ${ }^{3}$, S. Antoniou ${ }^{4}$, G. Filip ${ }^{5}$, V. Surlin ${ }^{6}$, C. Duta $^{7}$, V. Bintintan ${ }^{8}$, A. Carap ${ }^{9}$, B. Socea ${ }^{9}$, A. Melzer ${ }^{10}$

${ }^{1}$ Research, Medis Foundation, CAMPINA, Romania; ${ }^{2}$ Surgery, Beilinson Hospital, TEL AVIV, Israel; ${ }^{3}$ SINTEF, SINTEF, TRONDHEIM, Norway; ${ }^{4}$ Surgery, 401 General Military Hospital, ATHENS, Greece; ${ }^{5}$ Sugery, Ponderas Hospital, BUCHAREST, Romania; ${ }^{6}$ Surgery, UMF Craiova, CRAIOVA, Romania; ${ }^{7}$ Surgery, UMF Timisoara, TIMISOARA, Romania; ${ }^{8}$ Surgery, UMF Cluj, CLUJ, Romania; ${ }^{9}$ Surgery, UMF Carol Davila, BUCHAREST, Romania; ${ }^{10}$ IMSAT, University Dundee, DUNDEE, United Kingdom

Background: Surgeon's training in ultrasound is viewed and understood differently in different parts of the world. If in United States the need for surgeons' training was accepted and taken over by the American College of Surgeons, in Europe the practice is completely different from one country to another, from one city to another, from one department to another within the same premises hospital.

In some European countries, surgeons currently use ultrasound for diagnosis-especially in urgency, for follow-up, intraoperative, or as guidance for many surgical gestures.

During this time, access to ultrasound of other surgical specialties-gynecology, urology, ophthalmology-is considered natural.

Material and Method: Once the decision to initiate an ultrasound course for surgeons was taken, a team of experts with technical or clinical expertise in ultrasound was organized. At the initiative of the Technology Commission, the courses were to be organized at the EAES congresses or others communication events endorsed by EAES.

Starting from the importance of each ultrasound application in surgery, it was decided to develop different modules to solve different training needs. At this time, the course offered at Seville covers the capitols like Abdominal Ultrasound, Guided Punctures and Trauma. A module dedicated to intraoperative ultrasound is under construction and will be available in November 2019.

The course has a skill abilities dominant character, two thirds of it being thought of as a hands-on application on stationary, classical ultrasounds with large screens and also on small size wireless actual devices.

Results: After his debut in Frankfurt in 2017, the course was resumed in London and in Bucharest, twice. In this process, new modules and better teamwork skills have been developed. The participants' satisfaction quizzes, coming from all continents, were really encouraging. For the intraoperative ultrasound module the team approach is unique. Students will have the opportunity to practice on live animals both laparoscopic and open abdominal procedures

Conclusions: The ultrasound for surgeons course initiated by EAEAS was received with interest. The team will seek to inspect the real needs of training surgeons in this field and will complement and diversify the current platform 


\section{P039-AMAZING TECHNOLOGIES}

\section{ENDOSCOPIC VACUUM-ASSISTED CLOSURE IN UPPER GASTROINTESTINAL TRACT SURGERY-A SINGLE CENTRE EXPERIENCE}

\author{
M. Stašek, R. Aujeský, R. Vrba, J. Chudácek, L. Bébarová, \\ C. Neoral
}

Department of Surgery I, University Hospital Olomouc, OLOMOUC, Czech Republic

Introduction: Endoscopic vacuum-assisted closure (EVAC) is a relatively new method for endoscopic therapy of ruptures, fistulas, and leaks of the gastrointestinal tract. Despite the common use in rectal leaks, the use in the upper gastrointestinal tract (UGI) is less frequent. Success rate is very convincing, but the indications are still emerging and the technical complexity requires adequate evaluation.

Aim: Evaluation of the effectiveness of endoscopic vacuum-assisted closure in postoperative conditions in UGI surgery

Project description: Prospectively observed case series of postoperative conditions in the UGI tract with endoscopic treatment using EVAC in a 2-year period.

Preliminary results: A total of 26 procedures $(3-12, \pm 5.2)$ were carried out in 5 patients. The indications included acute ( 2 leaks following esophageal resection, 1 rupture of the strictured anastomosis following pneumatic dilatation) and 2 chronic conditions (esophagopleurobronchial and gastropleurobronchial fistulas following the resection of esophageal diverticulum and sleeve gastrectomy). The initiation of the therapy was in 13,18 and 1 day in case of acute conditions, and after 2 years of the duration of the unsuccessful therapy in 2 chronic cases. The successful closure was observed in 2 patients, 1 patient passed from MODS and ARDS. In 1 case, the initiation of EVAC was provided as a combined surgical and endoscopic intervention (CT proven distant intraabdominal abscesses). In 2 chronic cases, 1 was discontinued due to the haemophagocytic syndrome of unknown etiology, in the second one, success in reduction of the lesion and symptomatology with long term duration was observed following just 3 applications of EVAC, despite minimal remanent leakage. The success is to our experience linked to early initiation of the therapy and presumes complex intensive care. The future investigation should specify the timing including preemptive use of EVAC and the combination of EVAC with other endoscopic, interventional and surgical therapeutic modalities.

\section{P040-AMAZING TECHNOLOGIES}

\section{LEFT THORACOSCOPIC 3D MANAGEMENT OF SECONDARY} EPIPHRENIC DIVERTICULUM IN COEXISTING ACHALASIA AND GIANT PARAESOPHAGEAL HERNIA- VIDEO PRESENTATION

E. Baili, A. Meropouli, I. Vagios, E. Kyros, A. Papalampros, T. Liakakos, A. Charalabopoulos

1st Department of Surgery, Laiko General Hospital, ATHENS, Greece

Background: Esophageal pulsion epiphrenic diverticula generally arise from elevated intraluminal pressure due to esophageal motility disorders such as achalasia or rarely due to a hiatal hernia. The latter can exacerbate an underlying achalasia-related dysphagia and patients' overall psychological and physical status, by affecting their nutritional state.

Aim: Herein, we report a case of a 63- year-old Caucasian paraplegic male with recurrent dysphagia, following an open transabdominal hernia reduction with posterior cruropexy for a type IV paraesophageal hernia performed 35 days earlier. Subsequent work-up with CT, upper endoscopy and barium esophagram confirmed the presence of a large 6-7 cm left epiphrenic diverticulum in the left hemithorax. A comprehensive review of patient's medical records, including an esophageal manometry test, confirmed the co-existence of achalasia in combination with a left epiphrenic diverticulum and a giant paraesophageal hernia. After MDT discussion and keeping in mind the wheel-chair bound patient's history with a recent strenuous laparotomy and a worrisome pulmonary status, a 3D left-thoracoscopic esophageal diverticulectomy with a long esophagomyotomy was scheduled.

Project description: The patient was placed in a right semi-prone position, four thoracoscopic ports were introduced, and an exploration of the left chest cavity was performed. After division of the left inferior pulmonary ligament, the lung was retracted anteriorly, and the mediastinal pleura was divided. The esophagus was dissected proximally and distally, exposing the diverticulum's neck. A gastroscope was intra-operatively used to calibrate the esophagus and to guide the length of the myotomy. The esophagomyotomy was then initiated by dividing both the longitudinal and circular muscle layers. Diverticulectomy was finally accomplished.

Preliminary results: Operative time was $100 \mathrm{~min}, \mathrm{EBL}$ was $<50 \mathrm{ml}$, with no intraoperative complications. A water-soluble leak test demonstrated no contrast extravasation. The patient was allowed a semi-solid diet and discharged on the 8th postoperative day. At 3 months' follow-up, no dysphagia or reflux is reported. Several important technical principles are highlighted through this video presentation, one of which is that the management of benign esophageal disorders in a referral specialised centre with sufficient experience, comes with patients' most favourable outcomes.

\section{P041-AMAZING TECHNOLOGIES}

ROBOTICALLY ENHANCED ANATOMY ENABLES TO PERFORM COMPLETE ROBOTIC ESOPHAGECTOMY WITH PRECISE MEDIASTINAL LYMPH NODE DISSECTION

$\underline{\text { H. Daiko }}{ }^{1}$, J. Kanamori ${ }^{1}$, J. Oguma ${ }^{2}$

${ }^{1}$ Esophageal Surgery Division, National Cancer Center Hospital, TOKYO, Japan; ${ }^{2}$ National Cancer Center Hospital, National Cancer Center Hospital, TOKYO, Japan

The aim of this feasible study is to investigate complete robotic esophagectomy with tota mediastinal lymph node dissection (RETM) only by the robotic arms.

Methods: The patient is placed hemi-prone position with one lung ventilation by blocking balloon tube under general anesthesia. The robotic trocar for 1st arm of da Vinci Xi Surgical System is placed in the 10th intercostal space (ICS) on the scapular line, the trocar of 2nd arm is placed in the 7th ICS on the posterior axillary line, 3rd arm trocar is placed in the 5th ICS on the middle axillary line, 4th robotic arm trocar is placed 3rd ICS on the middle axillary line, and assistant trocar only for taking in and out of gauze is placed in the 8th ICS on the middle axillary line. On the upper mediastinal lymph node dissection, robotic camera exchanges from 2 nd to $3 \mathrm{r}$ robotic arm to close and identify anatomical structures. Esophagectomy with lymph node dissection starts from middle and lower mediastinum to upper mediastinum including along bilatera recurrent laryngeal nerves. All procedure perform under close-up view along the robotic enhanced anatomy to preserve organ functions.

Results: 20 patients with esophageal squamous cell carcinoma in 2018 underwent RETM Median age and BMI were 68 and 24, respectively. Perioperative complication rate was $47 \%$, including recurrent laryngeal nerve palsy was $20 \%$, pneumonia was $11 \%$, and leakage was $5 \%$. And median hospital stay after RETM was 14 days.

Conclusion: RETM according to robotically enhanced anatomy was technically feasible and safe.

\section{P042-AMAZING TECHNOLOGIES}

\section{RESOLUTION BY ENDOSCOPY OF IATROGENIC OCCLUSION OF PERFORATED DUODENAL ULCER}

P. Olivares ${ }^{1}$, L. Aguilera ${ }^{2}$, R. Dominguez ${ }^{2}$, V. Mendoza ${ }^{3}$,

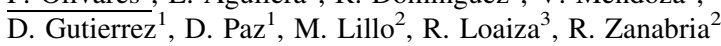

${ }^{1}$ Cirugía, Hospital Urgencia y Asistencia Pública, SANTIAGO, Chile; ${ }^{2}$ Cirugía y Endoscopia, Hospital Urgencia y Asistencia Pública, SANTIAGO, Chile; ${ }^{3}$ Endoscopia, Hospital Urgencia y Asistencia Pública, SANTIAGO, Chile

Background: Complete stenosis of the duodenal lumen secondary to a surgical suture in the treatment of a duodenal ulcer is a rare complication. The usual surgical resolution corresponds to a gastrojejunostomy associated or not, to an antrectomy with vagotomy, as a treatment for the peptic disease. The endoscopic resolution of this complication requires the use of complex maneuvers and specific therapeutic instruments.

Aim: To describe the endoscopic resolution of iatrogenic occlusion after raffia of perforated duodenal ulcer.

Description: A 43-year-old man was admitted to the emergency service for four days of pain and abdominal distension associated with abundant retention vomiting. Performed ten days ago of a perforated duodenal ulcer, in which manual raffia was performed in two planes and drainage. Abdomen and pelvis $\mathrm{CT}$ showed great distension and diffuse thickening of the gastric wall. The endoscopy showed abundant gastric retention content, pylorus, and bulb edema, and complete closure of the duodenal lumen, secondary to suture material; it was possible to count three suture threads. With a tipped papillotome and electrocautery, all the suture threads were sectioned, identifying a filiform opening through which a hydrophilic guide is inserted under fluoroscopy until it is sure to overcome the stenosis; We dilated the trajectory with a dilator of $10 \mathrm{~mm}$ in diameter and $4 \mathrm{~cm}$ in length. With a contrast medium, we observed an adequate trajectory and installed a partially covered duodenal metal stent (Hanaro Stent) of $14 \mathrm{~cm}$ in length by $20 \mathrm{~mm}$ in diameter in order to sustain the dilation. He was sent to home with inhibitors of the proton pump. After two weeks, the Stent was removed, without complications. He was controlled two weeks after withdrawal, and, with pharmacotherapy, the patient was asymptomatic, making a norma life.

Conclusion: In this case, the result was positive. Satisfactory results can prevent major surgery, which reduces the risk and possible complications. 


\section{P043-AMAZING TECHNOLOGIES}

\section{CYSTIC DUCT CONTROL USING GLUE IN SUBTOTAL CHOLECYSTECTOMY - NEW TECHNIQUE RESULTS}

\section{Klimovskij, D. Jenner}

General Surgery, Conquest Hospital, East Sussex NHS Healthcare Trust, ST LEONARDS ON SEA, United Kingdom

Background: Laparoscopic cholecystectomy can be challenging and make sweat even an experienced surgeon choosing subtotal way to complete the operation. There are debatable ways how to deal with the gallbladder stump actively draining bile through the cystic duct. Closing the stump may create a small neo-gallbladder, leaving it open - a controlled bile fistula, which may require further ERCP and stenting. We would like to share a reasonably easy way to close the cystic duct by applying tissue glue in its orifice.

Methods: 45 laparoscopic subtotal cholecystectomies with closure of cystic duct orifice using Dermaflex glue were performed.

Results: 17 (37.8\%) male patients; 22 (47.5\%) had preoperative diagnosis of acute cholecystitis, $10(22.5 \%)$ - history of common bile duct stones, $2(5 \%)$-pancreatitis, $11(25 \%)$-biliary colic. Median age was $64.4(10 \%)$ —emergency operations, $36(90 \%)$ —elective. Median BMI-31. Operating time median-72 min. Subhepatic drain in the gallbladder stump was kept for 3 days. 3 patients had persistent bile drainage on day 3 and underwent ERCP postoperatively with only 1 showing a true leak through cystic duct. Average hospital stay -1.2 days, with the vast majority discharged as day cases.

Conclusions: Laparoscopic subtotal cholecystectomy and cystic duct orifice closure using the tissue glue is a safe and reasonably easy way to deal with difficult gallbladder and reduce the time of resolution of bile fistula.

\section{P044-AMAZING TECHNOLOGIES}

\section{ROBOT EMULATOR GRASPER}

\section{Perez Berdasco}

Commercial, Servocad Microtronics SL, SANT FRUITOS DE BAGES, BARCELONA, Spain

A new range of non-invasive medical tools with a remarkable improvement on the existing market. A manual laparoscopy, with the important novelty of having a bending head with a high degree of movement. This head can get multiple spatial positions to work in surgery and is very easy to use, with the same scissors thimble who controls it, so its performance and learning is very simple and intuitive. The tools can be easily reusable and they can be cleaned and sterilized by ordinary methods, very ergonomic and lightweight. The generic type models we initially have developed are focused in general surgery, but gradually we will develop new applications and different heads for specific medical conditions such as arthroscopy, laryngoscopy, otolaryngology, ophthalmology, orthopedics. Its operation is simple and functional, simply moving the scissors thimbles, where they have a dual role, combining the head tilt and the action of opening and closing of this is achieved.The design of this tool allows us to work with some degrees of unparalleled freedom from the existing tools.

Our instruments replicate the movements of the robot with a simple handheld mechanical instrument, our philosophy is to position our instruments in between a long empty field between the surgeon and the robot. The tip of our instruments are providing the surgeons with angulations impossible to reach with the traditional instruments unless applying huge movements from the hands of the surgeon

We consider that this devices will have a very fast acceptance from the market as this robotic type movements can be managed by the surgeon through the traditional $5 \mathrm{~mm}$ trocars, without the need to change to a new surgical technique, just with the traditional method and a very brief training.

\section{P046-AMAZING TECHNOLOGIES}

\section{MINIMALLY INVASIVE THORACOSCOPY APPROACH OF THE MIDESOPHAGEAL DIVERTICULUM}

M. Dominguez Bastante, M.J. Alvarez Martin, M. Mogollon Gonzalez, J. Triguero Cabrera, B. Espadas Padial, J. Villar del Moral

General Surgery, Hospital universitario Virgen de las Nieves, GRANADA, Spain

Background: Diverticula of the middle thoracic esophagus are infrequent, its etiology may be secondary to traction or pulsion mechanisms. When the etiology is a mechanism of pulsion, they are associated with esophageal motor disorders and its prevalence is estimated between $0.02 \%$ and $0.77 \%$ of the population. They are rarely symptomatic and the diagnostic is usually incidental. The most common symptoms are episodes of food impaction, chest pain or bronchoaspiration.Diverticulectomy and esophageal myomectomy by minimally invasive approach is the treatment of choice in those with large size or associated symptoms. Aim: to describe a clinical case of esophageal diverticulum solved by minimally invasive surgery approach

Clinical case description: A 74-year-old patient with a history of epilepsy and hypothyroidism consulted for atypical chest pain and dysphagia to liquids and solids.

A study with esophagogastroduodenoscopy was performed: $27 \mathrm{~cm}$ from the dental arch, a large wide-mouth diverticulum was identified. We complete the study with an esophagram with barium: voluminous diverticulum in the right lateral face of the middle esophageal and a thoracic CT scan showing esophageal diverticulum located in the carina, from $5.6 \times 4.5 \mathrm{~cm}$ to $13 \mathrm{~cm}$ from the esophagogastric junction.

Due to the suspicion of associated motor disorder, high resolution manometry was performed showing a significant motor disorder with alteration of peristalsis and exit obstruction with incomplete relaxation of the inferior sphincter and superior hypertonic sphincter.

Preliminary results: The patient underwent surgery: diverticulectomy and complete esophageal myotomy by thoracoscopy minimall invasive approach. The patient evolved favorably and was discharged after 5 days with a previus control esophagram without pathological findings. Currently, it remains asymptomatic 6 months after surgery.

\section{P047-AMAZING TECHNOLOGIES}

\section{RECTUS DIASTASIS LAPAROSCOPIC RECONSTRUCTION (RDLR) THROUGH LAP-T MINI-INVASIVE TECHNIQUE WITH SOPRA-PUBIC GHOST ACCESS}

\section{G. Pozzi}

Minimally Invasive Advanced Surgery, Quisisana Clinic, ROMA, Italy

Background: Diastasis of Rectus Abdominis Muscles (DRAM) is still commonly treated through open surgery, with $30-40 \mathrm{~cm}$ wide incisions and extended retro-muscular o pre-peritoneal dissection, whit high incidence of seromas, significant pain, long recovery and higher risk of infection compared to laparoscopic approach. Laparoscopic treatment of DRAM is still underused.

Aim: Mini-Invasive restoration of anatomy and physiologic functionality of the abdominal wall affected by Diastasis Recti, by re-approximating the rectus muscles through laparoscopic running suture \& mesh repair. The repair is executed with a sopra-pubic approach, using miniaturized instruments and camera, seeking for the maximum achievable result with the minimal trauma to the patient.

Project Description: 453 cases from November 2007 to January 2017, minimum follow-up of 12 months. Elected patients under 65 years old, BMI $<30$, ASA1-ASA2, with maximum interrectal distance of $10 \mathrm{~cm}$. According to the innovative LAP-T (Laparoscopic AbdominoPlasty Technique), rectus muscles are re-approximated from xiphoid to pubis using laparoscopic running self-locking, PDS sutures to restore anatomy and physiologic function of the abdominal wall. Unlike the standard access to the abdominal cavity executed with 3 lateral access, the LAP$\mathrm{T}$ technique is performed through 3 sopra-pubic aesthetic approaches, using one $3 \mathrm{~mm}$ and one $5 \mathrm{~mm}$ bariatric $(45 \mathrm{~cm})$ instruments laterally, and one $8 \mathrm{~mm}$ camera in the middle.The entire procedure is performed in gas-less laparoscopy, with laryngeal mask and intra-peritoneal liquid anesthesia. The repair is consolidated placing an intra-peritoneal semi-absorbable mesh. Preliminary results: In all cases abdominal functioning was successfully restored; no higher pain related to the continuous laparoscopic suturing has been reported compared to bridge IPOM laparoscopic repair, while allowing for a more physiologic outcome and a stronger repair. The use of miniaturized instruments allowed for minimal tissue trauma and accurate surgical gestures; the tiny trocar sites did not require skin suturing and might reduce the risk of trocar hernias. No intra operative bleeding, no seroma formation, chronic pain, nor mesh infection have been recorded. $98 \%$ follow up at 24 months, $89 \%$ at 36 months with no recurrences observed. The LAP-T technique allowed for a sound and anatomic reconstruction, reduced trauma, faster recovery and more satisfactory aesthetic results. 


\section{P048-AMAZING TECHNOLOGIES}

\section{NEAR-INFRARED INDOCYANINE-GREEN FLUORESCENCE IMAGING FOR TATME IN RECTAL CANCER- A CASE REPORT WITH REVIEW OF THE LITERATURE}

\section{J. Grosek, J.A. Košir, A. Tomažic, G. Norcic}

Dept. of Abdominal Surgery, University Medical Centre Ljubljana, LJUBLJANA, Slovenia

Background: Anastomotic leakage is a serious complication, associated with significant morbidity and mortality. One possible cause of anastomotic leakage is insufficient vascular supply. Markers of sufficient perfusion include pink color of the bowel wall, visible peristalsis, palpable pulsations and bleeding from the marginal arteries. These signs are subjective and may be misinterpreted even by experienced surgeons.

Aim: The assessment of bowel perfusion with the use of indocyanine-green fluorescence angiography might be helpful in decreasing the number of anastomotic leaks.

Project description: We report a case report of a middle-aged patient without significant medical history who was treated by transanal total mesorectal excision (TaTME) for rectal carcinoma. The patient underwent neoadjuvant treatment with radiochemotherapy. During the surgical procedure, indocyanine-green fluorescence angiography showed adequate perfusion of the bowel. The postoperative phase was uneventful and the patient was discharged home on the 9th postoperative day.

Preliminary results: Indocyanine-green fluorescence angiography is a safe, cost-effective and feasible tool for assessment of tissue perfusion during colorectal resections.

\section{P049-AMAZING TECHNOLOGIES}

\section{TRAINING IN LAPAROSCOPIC SUTURE WITH VISUAL FORCE FEEDBACK USING SENSOR FORCEFILM TECHNOLOGY}

\section{F.M. Sanchez Margallo ${ }^{1}$, J.A. Sánchez Margallo ${ }^{2}$}

${ }^{1}$ Scientific Direction, Minimally Invasive Surgery Centre, CÁCERES, Spain; ${ }^{2}$ Bioengineering, Minimally Invasive Surgery Centre, CÁCERES, Spain

Background: To properly learn how to perform a laparoscopic suture, along with safe tissue handling, applying an appropriate magnitude of the force on the tissue is essential. For this reason, it is fundamental to investigate and validate if training with real-time visual force feedback improves the suturing performance of laparoscopic novice surgeons. Capturing all of the forces applied in laparoscopic in surgery in an unobtrusive way has been difficult in the past. SensOR has supplied a novel force-sensing film (ForceFilm) that can detect all of the forces applied with laparoscopic instruments without changing the surgical workflow or operation of the instruments.

Aim: To evaluate the effect of visual force feedback on surgical performance, applied force and surgeon's ergonomics during training of laparoscopic suturing using the SensOR technology (SensOR Medical Laboratories Ltd.)

Methods: Twenty novice laparoscopic surgeons participate in this study. They perform a laparoscopic suture on an ex vivo stomach tissue from a pig. Participants are assigned, in a random fashion, to either group that receives visual force feedback (A) or the control group (B) without visual force feedback. Five training trials (T1-T5) are carried out in order to assess the learning curve. In addition, an evaluation pretest (T0) and posttest (T6), without visual force feedback but recording the force applied, will be performed after the training trials. The applied force on the tissue and visual force feedback of each instrument are provided by means of the SensOR technology. It accurately measures the forces exerted on the tissue from the instrument tip and wirelessly communicates the force information to the surgeon via visual force-feedback. During each trial, several parameters are evaluated such as execution time, applied force, surgical performance, and mental and physical workload.

Preliminary results: Laparoscopic training using visual force feedback leads to an improvement of suturing skills with a reduction of the applied force and therefore providing a potentially positive effect on patient outcomes and surgeon's ergonomics.

\section{P050-AMAZING TECHNOLOGIES}

\section{LAPAROSCOPIC 3D MANAGEMENT OF DISTAL GASTRIC ADENOCARCINOMA AND GIANT PARAESOPHAGEAL HERNIA WITH COMPLETE INTRATHORACIC STOMACH- VIDEO PRESENTATION}

E. Baili, A. Alexandrou, A. Syllaios, S. Davakis, D. Schizas, A. Charalabopoulos, E. Felekouras

1st Department of Surgery, Laiko General Hospital, ATHENS, Greece

Background: Hiatal hernia is a frequent disorder, characterized by a protrusion of any abdominal structure other than the esophagus into the thoracic cavity through a widening of the diaphragmatic hiatus. Current anatomic classification is mainly based on the location of the gastroesophageal junction and the presence of a true hernial sac, differentiating sliding from paraesophageal hernias. There is no solid evidence to support an association between gastric carcinogenesis and PEH. However, chronic reflux is considered as one of the strongest risk factors of developing adenocarcinoma of the esophagus and proximal stomach.

Aim: Herein, we report a case of an 83- year-old Caucasian female with dysphagia, regurgitation and heartburn accompanied by an iron deficiency anemia, a remarkable total body weight loss and recurrent lower respiratory tract infections due to microaspirations. Subsequent work-up with CT, upper endoscopy and barium esophagram confirmed the presence of synchronous distal gastric adenocarcinoma and a giant paraesophageal hernia with complete intrathoracic stomach. After MDT discussion and keeping in mind the patient's age and comorbidity, a 3D laparoscopic distal gastrectomy with a synchronous hernia reduction with posterior cruropexy was scheduled. Project Description: The patient was placed in a supine position, five thoracoscopic ports were introduced, and a diagnostic laparoscopy of the abdominal cavity was performed. The stomach was identified through the dilated hiatus into the left thorax. The hernia sac was dissected away from mediastinal structures, then excised to untwist the stomach. After reduction of the stomach to abdominal cavity, a total D1 + gastrectomy with a Roux-en-Y reconstruction was performed. Preliminary results: Operative time was approximately $280 \mathrm{~min}$, EBL was $<80 \mathrm{ml}$, with no intraoperative complications. A water-soluble leak test demonstrated no contrast extravasation. The patient was allowed a semi-solid diet and discharged on the 11th postoperative day. Final histopathological confirmed a low grade gastric pyloric adenocarcinoma pT4aN3bMx, with adequate resection margins. At 3 months' follow-up, no dysphagia, reflux or recurence is reported. Several important technical principles are highlighted through this video presentation, one of which is that management of combined upper gastrointestinal disorders in a referral specialised centre with sufficient experience, comes with patients' most favourable outcomes.

\section{P051-AMAZING TECHNOLOGIES}

THERMO-ADJUSTABLE ENDOSCOPE HEATER SYSTEM (EHS) TO SOLVE PROBLEMS OF LENS CONDENSATION AND FOGGING DURING SURGICAL INTERVENTIONS

I. Alarcón ${ }^{1}$, J.L. Ruiz ${ }^{2}$, R. Mañero ${ }^{2}$, E. Eugenio ${ }^{1}$, M. Sanchez ${ }^{1}$, S. Morales-Conde ${ }^{1}$

${ }^{1}$ Innovation and Minimally Invasive Surgery, Hospital Quirón Sagrado Corazón, SEVILLA, Spain; ${ }^{2} \mathrm{R}+$ D Department, Noruco SL, SEVILLA, Spain

Maintenance of optimal vision during minimally invasive surgery is crucial to maintaining operative awareness, efficiency and safety. Hampered vision is commonly caused by laparoscopic lens fogging (LLF) and lens condensation which has prompted the development of various antifogging fluids and warming devices. Numerous tricks have been proposed to overcome this issue, such as heating the scope into a sterile thermos flask filled with hot water, or using one of the commercially available antifogging solutions. However, whether one method is superior to another remains elusive. As most surgeons know, none of these tips are totally efficient, as they don't treat the cause: the temperature difference.

Taking into account this need, we have developed EHS (Endoscopy Heater System), a thermoadjustable system by microcontroller, which is implemented in the manufacturing process of the rigid endoscope focused on laparoscopy. The technology enables the self-modulation of the temperature of the endoscope within the different conditions during the surgery, avoiding the $100 \%$ of laparoscopic fogging. With the adoption of EHS surgeons get a clear field of vision avoiding continues repetitions of extraction and insertion of the endoscope in the body during the intervention. In this way the risks of the patients are reduced with a more efficient and shorter duration procedure. EHS also represents an alternative that meets sustainability criteria by reducing energy costs and eliminating much of the waste currently generated by this procedure. Therefore, this innovation will disrupt the laparoscopic device market by enhancing safety and effectiveness without introducing new components that could complicate surgical procedures. 


\section{P052-AMAZING TECHNOLOGIES}

\section{TREATMENT OF LOWER-GI POST-SURGICAL FISTULAS WITH OVESCO}

\section{Lara Palmero, S. Gomez Modet, A. del Castillo Cuesta}

General surgery, SAS, ALGECIRAS, Spain

Introduction: Anorectal fistulas remain an ongoing challenge to colorectal surgeons still leading to the development of new techniques and devices. The Ovesco is a new device for transanal anorectal fistula closure, which consists of a clip and a clip applicator.

Case report: We presented the case of a 63 year old women with chronic coloenteric fistula. Conservative treatment was unsuccessful. The orifice was then closed with two subsequent clips, and the patient recovered well. To our knowledge, this is the first successful case of coloenteric fistula treatment with Ovesco

Discussion: Ovesco system is a technique that enables the closure of gastrointestinal defects (perforation sites, leaks, fistulas). After the system application, the patient can be treated at home as was the case with our patient. A successful closure of the leak or fistula is possible when no extraluminal abscess is present. In our case, we had a cavity (previous sinus or abscess) that drained into the small bowel, thereby forming the coloenteric fistula. This allowed us to succeed with a fistula closure, as the cavity could drain into the small bowel

Conclusions: Looking through the reports, one notes that the success rate of the OTSC system procedure for insufficiency of anastomosis or colorectal fistula was $57-100 \%$, but only nine successful reports of chronic colorectal fistula were found). A $100 \%$ success rate is reported if the clip is placed within a week of occurrence of the leak . On considering the financial side, clips could reduce costs and time of hospitalization and avoid patients having to undergo a surgical repair. The major advantage of Ovesco clips seems to be their ability to grasp more tissue compared to the standard clips and their strong grip on the wound margins because of their sharpened teeth. The drawback of the clips in fistula sealing is their incomplete grasp when the tissue is fibrotic. Most authors agree that Ovesco is not very appropriate for fistulas larger than $12-15 \mathrm{~mm}$.

\section{P054-AMAZING TECHNOLOGIES}

A MOTORIZED AND COMPUTERIZED LAPAROSCOPIC TOOL THAT CAN BE CUSTOMIZED TO THE SPECIFIC SURGEON AND PROCEDURE

\section{A. Szold}

Assia Medical Group and Assuta Medical Center, TEL AVIV, Israel

Aim: Surgeons have different levels of skill and use instruments for different tasks, but laparoscopic instrument are commonly simple mechanical instruments that allow limited degrees of motion, and the same instruments are used regardless of the surgeon or the task. Robotized articulating instruments so far have added degrees of freedom, but perform in a standard way for all users and procedures.

Technology: Human Xtensions has developed a $<\mathrm{u}>$ hand-held $</ \mathrm{u}>$ motorized smart laparoscopic instrument, that was recently introduced in human procedures. The device has several features that enable to customize it to the user and procedure. The degrees of freedom can be reduced from 7 to 5 , the scale of rotation motion has 3 options that can control both speed and range of rotation, a feature especially useful for the variable types of suturing tasks.

Results: The variable features were tested in different procedures requiring suturing and grasping. The combination of all optional settings made the instrument customizable to the different skill levels of the surgeons. As such, it enabled to control the complexity of the device and take the surgeon through the learning curve until full control of all features was achieved. In addition, the combination of different controls was used for performing specific tasks requiring different levels of maneuverability.

\section{P053-AMAZING TECHNOLOGIES}

NEW TECHNIQUE FOR HERNIA INGUINAL SURGERY WITH ROBOTIC GRASPER AND MANGETIC DEVICES

\author{
C. Cadaval Gallardo ${ }^{1}$, G. Dominguez ${ }^{2}$, J. Petrone ${ }^{2}$
}

${ }^{1}$ Pediatric surgery, Hospital Universitario Virgen del Rocío, SEVILLA, Spain; ${ }^{2}$ General surgery, Fundación Hospitalaria Buenos Aires, BUENOS AIRES, Argentina

Inguinal hernia repair is one of the most performed interventions in minimally invasive surgery. In this opportunity we report a new technique through the use of innovative devices such as the robotic clamp and magnetic devicesWith this technique and thanks to the magnetized devices and the robotic clamp we have demonstrated to reduce the surgical time between 10-20 min as well as to optimize the ergonomics of the surgeon.We explain the technique with a demostrative video and exposition of the devices that are necessary for make it.With this new technique we get a greater capacity of mabiobra for dissection of the peritoneum and later a greater facility for the suture of the same in the repair of the inguinal hernia.

\section{P055-BARIATRICS—Endoluminal}

\section{SHOULD WE ROUTINELY OGD ALL BARIATRIC PATIENTS AS PART OF THEIR PRE-OPERATIVE WORK UP?}

C.A.W. Macano, R. Edmonds, N. Coleman, M. Robba, A. Antequera

\section{General Surgery, GHA, GIBRALTAR, Gibraltar}

In September 2016, the results of a BOMSS survey regarding the routine use of pre-operative bariatric surgery were published. They found that $10 \%$ of units surveyed considered routine preoperative OGD completely unnecessary. As part of newly launching bariatric services in a single isolated centre we protocoled that all bariatric patients had to undergo pre-operative OGD, including a CLO test, and reviewed if the OGD findings had influenced our surgical choice of operation and any necessary treatment before surgery.

All patients embarking on the bariatric programme since its launch in Jan 2017 to Sept 2018 were included and had an OGD. The results of these OGDs and all the CLO tests were reviewed. These OGDs were all performed by a single consultant to minimise any potential subjective differences. Of the 45 patients, 7 (16\%) tested CLO positive of which 3 had normal findings on OGD. 9 patients had a hiatus hernia, 5 gastritis, 8 oesophagitis, 12 gastritis and oesophagitis, 9 had other findings e.g. ulcers, polyps' or nodules.

The 7 positive CLO patients underwent eradication of $\mathrm{H}$ pylori. Studies have shown that this is a treatable and preventable cause of gastritis/gastric cancers and potential surgical complications causing prolonged hospital stay in $22 \%$ of patients. Knowing about the presence of a hiatus hernia prior to surgery also contributed to the surgical planning, including allowing time for the concurrent correction of the hiatus hernia in the operation. All patients with demonstrable oesophagitis (44\%) had their operative choice changed to Roux En y gastric bypass thus aiming to prevent post-operative reflux which would have been exacerbated had they undergone a sleeve gastrectomy instead. Carrying out a pre-operative OGD had a significant impact in operative choice and additional treatment before surgery and therefore should be advised in all patients. 


\section{P056-BARIATRICS-Laparoscopic}

MORE DISTAL SMALL BOWEL PERFORATION THAN USUALLY KNOWN FROM BLUNT ABDOMINAL TRAUMA (TRAFFIC ACCIDENT) IN A PATIENT WHO HAD SLEEVE GASTRECTOMY

\section{N. Ozlem}

General surgery department, Ahievran University, KIRSEHIR, Turkey

The majority of fatalities worldwide in people under the age of 35 years are caused by trauma ${ }^{1}$. Blunt mechanisms account for 78.9 to $95.6 \%$ of injuries ${ }^{2-5}$,with the abdomen being affected in 6.0 to $14.9 \%$ of all traumatic injuries.

This case contribute to the literature: a patient with sleeve gastrectomy has distorted anatomy at duodenogastric junction, if has BAT, her/his small bowel perforation(SBP) will occur on more distal segment. This a unique case before unpublished. 33 years old female who had sleeve gastrectomy 3 years ago presented to emergency department sustained blunt abdominal trauma (BAT). When she arrived pyschical exam (PE) revealed an abdominal guarding, tenderness, normal vital signs but those increased $8 \mathrm{~h}$ later. wbc values also increased $8 \mathrm{~h}$ later. $\mathrm{hb}$ was normal.FAST showed in $3 \mathrm{~cm}$ thickness fluid early in Douglas pauch (DP), $50 \mathrm{~mm}$ in supravesical, $33 \mathrm{~mm}$ diameter in DP $4 \mathrm{~h}$ later. Abdominal ct: $37 \mathrm{~mm}$ diameter fluid in interloop, DP free air at 5 th $\mathrm{h}$ from the accident. A diagnostic laparoscopy(DL) was done with diagnossed acute abdomen.There were a SBR-located $60 \mathrm{~cm}$ from treitz, intraperioneal fibrin deposits and fluid- repaired with a primary suture.the patient discharged on 5 days without any event. Repeat CT scans are recommended for patients with initial suspected bowel injury. We could not do this; cause ct exam could taken in rush hours only but we did repeatly PE that peritoneal iritation signs increased,resulted a DL,surgical therapy. According to the literature; DL may be a good treatment option in these patients, to reduce morbidity or mortality, time to surgery has been emphasized. Long interval between presentation and surgery was found to be associated with complications. Very few reports of isolated jejunal transection following blunt abdominal trauma have been published in literature. The literature mentioned; the patients with SBP are hemodinamically stable on arrival to the hospital like our case are, a rupture of the jejunum was seen just distal to the duodenal-jejunal flexure but there were a perforation $60 \mathrm{~cm}$ below treitz ligament and caused me to think the patient had sleeve gastrectomy and some brid around gastric and duodenal proximal jejunal part of intestines and also caused a new descended treitz ligament. Normally external forces across to spine produce a blast effect on small bowel between treitz and ileocolic ligament.

\section{P057-BARIATRICS—Laparoscopic}

\section{SINGLE ANASTOMOSIS SLEEVE ILEAL (SASI) BYPASS:AN OPTION FOR WEIGHT REGAIN AFTER SLEEVE GASTRECTOMY}

\section{I.A.M. Hassan, M. Alhaifi}

\section{Bariatric Surgery, Sidra Hospital, KUWAIT, Kuwait}

Introduction: SASI Bypass is a Novel Metabolic/Bariatric Surgery operation based on Minigastric bypass and Santoro's operation.It can be offered for patients with weight regain after Sleeve gastrectomy.

Sleeve gastrectomy (SG)is a commonly performed bariatric procedure.Weight regain following $\mathrm{SG}$ is a significant issue.Yet,the understanding of this phenomenon is still unclear.Rates of regain ranged from $5.7 \%$ at 2 years to $75.6 \%$ at 6 years.SASI bypass was an option for some candidates having SG done 2 years back and failed to achieve the required weight loss or having weight regain.In SASI bypass, Resleeve gastrectomy of the dilated gastric pouch is done followed by a side to side gastro-ileal anastomosis.

The aim of this study is to report the clinical results and the outcomes of SASI bypass as a therapeutic option for patients with weight regain after SG

Methods: We conducted a retrospective study for 25 morbidly obese patients having history of SG done more than 2 years back and failed to achieve and/or to maintain the required BMI. Exclusion criteria:Patients with recent history of laparotomy(less than 12 months). Procedure was done at Sidra Hospital in kuwait from November 2016 to November 2018. Using 5 ports, Resleeve Gastrectomy was performed over $36 \mathrm{fr}$ bougie tube starting $6 \mathrm{~cm}$ above the pylorus then gastro-ileal anastomosis (side to side)was performed $6 \mathrm{~cm}$ above the pyloric ring to an ileal loop counted $250 \mathrm{~cm}$ from the ileocaecal valve.

Data was collected from the patients including:Weight loss progress,laboratory full results. Discussion and Results: During the study period:25 morbidly obese patients with a mean BMI of $44 \pm 6 \mathrm{Kg} / \mathrm{m}^{2}$ were evaluated. -\%EWL(excess weight loss)reached $85 \%$ at one year. -Diabetes was cured in the 2 known diabetic patients (type2)within 6 months,and the one known type 1 diabetic patient had better control and less insulin daily doses(results were guided by glycated haemoglobin results every 3 months). Follow up laboratory results were normal in $88 \%$ of patients (all were kept on regular vitamins and proteins supplementation)-one patient had postoperative leak(day1)from the anastomotic line that was treated conservatively.

Conclusion: SASI Bypass is a promising operation that offers a good weight loss for morbidly obese patients having weight regain after SG

\section{P058-BARIATRICS-Laparoscopic \\ EFFECTS OF SLEEVE GASTRECTOMY ON ECTOPIC FAT DEPOSITION}

\section{Castagneto Gissey, J. Casella Mariolo, G. Casella}

Department of Surgical Sciences, Sapienza University of Rome, ROME, Italy

Aims: We aimed at studying the effect of sleeve gastrectomy (SG) on non-alcoholic fatty liver disease (NAFLD), insulin resistance and ectopic fat deposition in monocytes.

Methods: Sixteen morbidly-obese subjects underwent liver biopsy during SG and blood drawn at fast to isolate $\mathrm{CD} 14++\mathrm{CD} 16+$ monocytes by flow cytometry and to analyze plasma glucose and insulin before and 1-year after SG.

Results: Liver histology showed different degrees of NAFLD from NAFL to NASH. Monocytes Plin2, a protein coating lipid droplets (LD) which avoids triglycerides enzymatic-hydrolysis, correlated with liver Plin2 expression $(\mathrm{R}=0.7 \mathrm{P}=0.006)$. Monocyte Plin2 mean fluorescence intensity (MFI) decreased from $68.4 \pm 6.3$ to $46.4 \pm 3.3(\mathrm{P}=0.02)$ after surgery. Plin2-MFI significantly and positively correlated with the degree of hepatic steatosis $(R=0.60 ; P=0.03$ ). The Bland-Altman plot showed a good agreement between NAFLD severity and monocyte Plin2 levels. Moreover, monocytes Plin2MFI significantly and positively correlated with plasma glucose concentrations $(\mathrm{R}=0.60 ; \mathrm{P}=0.002)$, HOMA-IR $(\mathrm{R}=0.60 ; \mathrm{P}=0.0005)$ and $\mathrm{BM}$ $(\mathrm{R}=0.80 ; \mathrm{P}=0.02)$. Comparable amounts of $\mathrm{LD}$ were found in monocytes and liver, stained with Nile Red.

Then, we studied insulin signalling in monocytes before and after SG. Plin2 expression correlated with Akt-Ser473 phosphorylation $(\mathrm{R}=0.60 ; \mathrm{P}=0.01$ ), which strongly correlated with HOMAIR $(\mathrm{R}=0.80 ; \mathrm{P}<0.0001)$.

Liver damage leads to reactive oxygen and nitrogen species and other pro-inflammatory mediators release with stimulation of extracellular matrix formation leading to fibrosis. Matrix metalloproteinase (MMP) isoform-9 is linked to NASH. Liver MMP9 protein expression strongly correlated with MMP9 serum levels $(\mathrm{R}=0.70 \mathrm{P}=0.002)$. Furthermore, liver MMP9 expression positively correlated with heat shock protein (HSP)70 hepatic expression $(\mathrm{R}=0.80 ; \mathrm{P}=0.001)$ Conclusions: Our study demonstrates a good agreement between the degree of liver steatosis and monocytes fat accumulation as well as between Plin2 levels in liver and circulating monocytes. This suggests that ectopic fat deposition is a generalized feature of insulin resistance in obesity. SG reverses monocyte fat accumulation and restores insulin signalling, which correlates well with insulin sensitivity. Moreover, circulating MMP9 levels significantly dropped after SG suggesting that the state of generalized inflammation characterizing obesity normalizes.

\section{P059-BARIATRICS-Laparoscopic}

IF A PATIENT HIDE HIS/HER OPERATION WHO HAS IN THE PAST WHAT THE SURGEON WILL BE IN TROUBLE ?

\section{N. Ozlem}

General surgery department, Ahievran University, KIRSEHIR, Turkey

LAGB can improve the quality of life but it is not without complications. Chevallier JM et al' serie include no deaths, $19.2 \%$ of cumulative rate of complications. we present a patient,who has an LAGB hiden in past medical history, may cause a complication not seen before in the literature.In laparoscopic sleeve gastrectomy operation of $52 \mathrm{Y}$ female with BMI: $43.2 \mathrm{~kg} / \mathrm{m}^{2}$. her stomach stapled,a foreign tube like body was seen on cut surface of the stomach.the foreign body seen in dissected stomach wall is the tube is in placed a gastric banding insuflation tube.A laparatomy was made and the tube is extracted her stomach sutured primarily,nasogastric decompression,peritoneal drainage was made.Her peritoneum was drained.She has a septic condition, leave in ICU for a long period.Her general status being well and discharged from hospital in 50 days.we learn after the operation not before;she had a gastric adjustable banding and extraction the gastric band, but the tube of gastric band is not removed. Alkhaffaf et al present a case of fistulation of the LAGB tubing into the jejunum a review of the published data to identify the salient learning points with this and similar rare complications Fistulation from LAGB tubing is a rare complication that tends to follow removal of an infected port. The clinical presentation is nonspecific, rendering the preoperative diagnosis difficult. The tube and band can be removed laparoscopically, with closure of the small bowel fistula site. Securing the tubing to the abdominal wall fascia after intentional detachment from the port might reduce the incidence of this complication.Katherine $\mathrm{J}$ et al report a late and rare complication of a small bowel obstruction in a 52-year-old woman from an LAGB placed for 2 years. Although not a common complication, one that could easily see the safety record of LAGB patients tarnished if this smal subgroup of patients is not acted upon promptly by emergency departments' unfamiliar LAGB surgery. In our case we already made esophagogastroduodenoscopy before operation, ofcourse take past medical history from the patient.the patient hide past operation (gastric banding and removing band and port but leaving insuflation tube). 


\section{P060-BARIATRICS-Laparoscopic}

DO MALES FARE BETTER IN REVISIONAL SURGERY AFTER A FAILED BAND? A RETROSPECTIVE COHORT STUDY SURVEYING SLEEVE POST BAND

\section{Z. Perry ${ }^{1}$, T. Zionni ${ }^{2}$, S. Atias ${ }^{2}$, L.1. Lantsberg ${ }^{2}$, B. Kirshtein ${ }^{2}$ \\ ${ }^{1}$ Simulation Center, Montreal General Hospital, MONTREAL, Canada; ${ }^{2}$ Surgery A, Soroka University Medical Center, BEER- SHEVA, Israel}

Introduction: Sleeve gastrectomy is becoming the most popular bariatric procedure in the world Studies in the field of bariatrics, have frequently been gender imbalanced, with males representing only $20 \%$ of examinees. Long-term gender-oriented results of sleeve after previous band, have not been addressed sufficiently. The aim of the current study was to examine the long-term gender association with outcome of sleeve as a revisional procedure post band.

Methods: A retrospective analysis of a prospectively maintained database of revisional laparoscopic sleeve gastrectomies (LSG) after LAGB was performed between January 2006 and March 2015. All patients who underwent a sleeve gastrectomy after LAGB surgery from 2006 to 2015 . Data analyzed included bougie size, weight loss, postoperative complications and long-term outcomes. Outcomes included BMI reduction, evolution of comorbidities, complications, reoperations, and QOL were compared according to the Bariatric Analysis and Reporting Outcome System (BAROS).

Results: 114 patients who had LSG after a band were included, of which $26(22.8 \%)$ were diabetics. $74(64.9 \%)$ were females. Mean age and body mass index (BMI) were 37.8 years $( \pm 12.9)$ and $43.02 \mathrm{~kg} / \mathrm{m}^{2}( \pm 6.15)$, respectively. Mean Follow up time was 22.83 months ( \pm 8.44$)$. There was no difference in the two groups regarding follow-up rate. Basic demographics were the same, and other long-term results were similar between the groups. Regression models for both post-op complications and failure as defined by BAROS score did not show that gender is a risk factor.

Discussion and Conclusions: In our study, revisional sleeve surgery were similar. We did not see any significant difference in post-op complications, success of the operation as defined by BAROS, or subjective feeling of the patients. We do believe that Gender-specific outcomes should be taken into consideration in optimizing patient selection and preoperative patient counseling, and that in the case of a sleeve post a band gender is not a risk factor for complication or failure of the procedure.

\section{P062-BARIATRICS-Laparoscopic}

IMPACT OF MESENTERIC DEFECT CLOSURE DURING LAPROSCOPIC ROUX-EN-Y GASTRIC BYPASS

\author{
A. Almunifi ${ }^{1}$, S.A. Alqahtani ${ }^{1}$, T. Debs ${ }^{2}$, I. Ben Amor ${ }^{2}$, J.G. \\ Gugenhiem $^{2}$
}

${ }^{1}$ Department of General Surgery, College of Medicine at Majmaah University, AL MAJMA'AH, Saudi Arabia; ${ }^{2}$ Department of Digestive Surgery, Bariatric Surgery Center and Liver Transplant, CHU Nice, L'archet 2, NICE, France

Objective: The internal hernia is a rare but a potentially fatal complication of Laparoscopic Roux-En-Y Gastric Bypass (LRYGB). The aims of this study are:

(1) To determine the impact of mesenteric defects closure on the incidence of internal hernia after LRYGB;

(2) To determine the symptoms, characteristics and management of internal hernias after LRYGB.

Methods: A retrospective study for a total of 2093 LRYGB which was carried out from 1998 to December 2013 at Nice University Hospital 'L'Archet 2' in the Department of Gastrointestinal Surgery and Liver Transplantation.

Until December 2004, 421 patients were operated on without closure of the mesenteric defects (Group A). From January 2005 to December 2013, 1672 patients were operated on with closure of the mesenteric defects at Petersen's Defect (PD) and at the jejuno-jejunal anastomosis (JJA), using tight non-absorbable continuous sutures (Group B). The incidence of internal hernias was compared between the two periods.

Results: Out of the 2093 patients who underwent LRYGB, 20 patients $(0.95 \%)$ developed a symptomatic internal hernia that required primary surgical intervention, 7 patients $(1.66 \%)$ in Group A all at JJA versus 13 patients $(0.78 \%$ ) in (Group B) 6 at (JJA), 5 at PD and 2 at PD, JJA, This incidence was significantly lower in (Group B) $(p=0.0021)$. The median interval between LRYGB and reoperation was 53 months in group A and 26 months in group B. The median percentage of excess weight loss $(\% \mathrm{EWL})$ was $61 \%$ vs $67 \%$, respectively $(\mathrm{p}=0.79)$. The median percentage of total weight loss (\%TWL) was $39 \%$ vs $37 \%$, respectively ( $\mathrm{p}=\mathrm{NS}) .14$ patients, $70 \%$ (5 in group A), were admitted to the Emergency Room with acute abdomen pain. A CT scan was performed in 8 patients, $40 \%$, and showed signs of occlusion in all cases. The most common symptoms were abdominal pain and vomiting. The surgery was performed using laparoscopy in 8 patients, $40 \%$, and using laparotomy or conversion in 12 patients, $60 \%$.

Conclusions: The closure of mesenteric defects during LRYGB is recommended because it is associated with a significant reduction in the incidence of internal hernia.

\section{P061-BARIATRICS-Laparoscopic \\ SHORT TERM OUTCOME OF LAPAROSCOPIC GASTRIC PLICATION IN CLASS I OBESTIY}

\section{A. Elgeidie}

Digestive surgery, Gastrointestinal surgery center, MANSOURA, Egypt

Background: Class I obestiy patients are not eligible for bariatric surgery till now. Those patient may have tried unsucceful non-surgical means. Less invasive options are needed to overcome their over-weight probelm. Laparoscopic gastric plication (LGP) may be the most suitable option. This work tests the efficacy and safety of LGP in patients with class I obestiy.

Patients and Methods: A retrospective analysis of our database was carried out on class I obese patients (BMI between 30 and $35 \mathrm{~kg} / \mathrm{m}^{2}$ ) who underwent LGP. Short term outcome was recorded. Results: In the period between June 2013 and November 2017, 84 patients were included. The mean age was 27.3 years and 75 of them were females. The mean BMI was $31.2 \mathrm{~kg} / \mathrm{m}^{2}$ ranging between 30.6 and $34.8 \mathrm{~kg} / \mathrm{m} 2$. Associated comorbity was reported in $32.1 \%$ of them. The technique of LGP was standardized in all patients and all surgeries were performed by one surgical team. \%EWL was 30.6, 45.7 and 53.9 at 3,6 and 12 months respectively. No mortality or conversion to open surgery was detected. Major complications were detected in 2 patients in the form of leak that was corrected by undoing plication in one case and reversal to sleeve in the other one. One patient was reversed to single anastomosis gastric bypass after 3 years. Resolution/improvement of comorbidities was reported in $45 \%$ of cases.

Conclusion: LGP may be a safe and effective less invasive surgical option for patients with class I obesity. Further studies with longer follow up and larger population sample, and studies comparing LGP to other less invasive options are required.

\section{P063-BARIATRICS-Laparoscopic \\ LONG TERM RESULTS OF THE SLEEVE GASTRECTOMY}

F. Martinez-Ubieto, A. Bueno-Delgado, E. Monsalve, I. Barranco Dominguez, C. Gracia-Roche, J. Martinez-Ubieto

\section{Surgery, Viamed Montecanal Hospital, ZARAGOZA, Spain}

Our study intends to analyze the long term results of 112 sleeve gastrectomies performed by 3D laparoscopic approach.

Materials and methods: A prospective cohort study was conducted to perform gastric sleeve for morbid abesity. All surgeries were performed by the same surgeon over a period of two years. The operating surgeon is a senior most laparoscopic surgeon with vast experience in laparoscopic surgery. During two years period, 112 cases were operated using 3D laparoscopy system. Scientific calculation was done using SPSS release 18.0 windows software.

Results: 112 patients, 74 female (66\%) and 39 male (43\%), with median age of $42.3 \pm 12.6$ (15-70)

The excess weight lost (EWL) was 68\% in the first year, $72 \%$ in the second, $73 \%$ in the third, $71 \%$ in the fourth, $70 \%$ for the fith year, $68 \%$ for the sixth and $67 \%$ for the seventh.

Postoperative complications were 3 stenosis of the sleeve always located in the incisura and treated with endoscopic dilatation except one that required conversion to OAGB. Three leakages, all of them reoperated with drainage and introducing prosthesis by endoscopy in the same act. We have never had a postoperative bleeding of the sleeve.

Conclusions: 3D gastric sleeve laparoscopy is a safe and feasible technique for morbid obesity and related pathologies. The EWL is correct in long time.

Complications are rare but is necessary to have a good level of suspicious in order to a rapid solution.

The worst complication is the leak of the sleeve. The oversewn of the gastric section is a good technique to avoid this complication. 


\section{P064-BARIATRICS—Laparoscopic}

LAPAROSCOPIC REVISION OF GASTRIC PLICATION TO LAPAROSCOPIC MINI GASTRIC BYPASS

\section{Hussein}

Surgery, American University of Beirut Medical Center, BEIRUT, Lebanon

Aims: Laparoscopic Revision of Gastric Plication to Laparoscopic Mini Gastric Bypass Methods: The video will show the steps used for unfolding of the plication followed by partial gastrectomy and creation of mini bypass $200 \mathrm{~cm}$ from the ligament of treitz Results: All bariatric procedures are associated with failure rate. Laparoscopic Gastric plication as new Bariatric procedure is associated with failure rate up to $30 \%$.

\section{P065-BARIATRICS-Laparoscopic}

\section{NEW APPROACH FOR THE TREATMENT OF SLEEVE GASTRECTOMY LEAK WITH LAPAROSCOPIC ROUX EN Y BYPASS TO THE LEAK SITE AS ONE STEP PROCEDURE}

\section{Hussein}

Surgery, American University of Beirut Medical Center, BEIRUT, Lebanon

Aims: Leak is one of the common complications of laparoscopic sleeve gastrectomy that result prolongation of hospital stay, morbidity and even mortality.

Methods: I report new approach for the treatment of 17 leaks presented to me post laparoscopic sleeve gastrectomy with laparoscopic Roux En Y bypass to the leak site at the level of gastroesophageal area. This new approach is possible and feasible, and avoids stenting due to high failure rate, prolonged hospitalization and saves life of patients.

Results: All leaks healed 7 days from surgery due to well vascularized small intestinal patch, except for 2 leaks that healed after 2 weeks of conservative treatment.

\section{P066-BARIATRICS-Laparoscopic}

ONE ANASTOMOSIS MINI GASTRIC BYPASS FOR REVISION OF SLEEVE GASTRECTOMY IN PATIENTS WITH GERD: PRELIMINARY Results:

\section{R. Villa}

General and Bariatric Surgery, Policlinico San Marco, BERGAMO, Italy

Aims: analyse the effect of One Anastomosis (Mini) Gastric Bypass (MGB/OAGB) in the treatment of gastro-esophageal reflux in patients previously submitted to laparoscopic sleeve gastrectomy (SG).

Methods: a retrospective analysis was performed on the data of patients who underwent MGB/ OAGB after a previous SG at Policlinico San Marco, Italy, from January 2014 to June 2017. A total of 40 patients, 36 female and 6 males $(85 \% \mathrm{~F} / 15 \% \mathrm{M})$ underwent MGB/OAGB after SG due to the development of significant gastro-esophageal reflux disease (GERD), refractory to proton pump inhibitors (PPI), detected with the GERD questionnaire (GERD-Q) and esophagogastroduodenoscopy (EGDS). In three patients (5\%) a weight regain was also observed (mean BMI $41.3 \mathrm{~kg} / \mathrm{m}^{2}$, range $39.3 \mathrm{~kg} / \mathrm{m}^{2}-42.5 \mathrm{~kg} / \mathrm{m}^{2}$ ). Mean patients age was 40.6 (35-60 years old). Before SG none of the patients had declared symptoms of GERD or was subjected to a therapy with PPI, preoperative EGDS did not show signs of esophagitis. Mean BMI of the 37 patients who developed GERD without weight regain was $31.4 \mathrm{~kg} / \mathrm{m}^{2}\left(28 \mathrm{~kg} / \mathrm{m}^{2}-33.7 \mathrm{~kg} / \mathrm{m}^{2}\right)$ at the time of surgery, with a medium EWL\% of $51 \%(68.3-42.5 \%)$. Patients were treated unsuccessfully with PPI for at least six months before programming revisional surgery. Mean GERD-Q score was 12.

Results: after MGB/OAGB, with a mean follow up of 19 months (24-15 months), mean BMI was $28.5 \mathrm{~kg} / \mathrm{m}^{2}$ and GERD-Q score was 5 . However, five patients out of 40 (13\%) developed an anastomotic ulcer or a grade $\mathrm{C}$ esophagitis. We did not observe any post-operative immediate complication nor any death.

Conclusion: MGB/OAGB is a simple, effective and safe surgical procedure for patients who underwent a previous SG and who developed GERD, with satisfactory results in the short and medium post-operative time, even if there is still concern regarding the complications linked to biliary reflux.

\section{P067-BARIATRICS-Laparoscopic}

MIGRATION OF LAPAROSCOPIC ADJUSTABLE GASTRIC BAND

\section{V.S. Kyosev}

Surgery, Military Medical Academy, SOFIA, Bulgaria

Aims: Laparoscopic Adjustable Gastric Band (LAGB) was one of the common techniques in bariatric surgery worldwide. The advantages included the possibility of regulation, ease of placement, acceptable weight loss and low rate of perioperative complications. A late complication of LAGB is penetration of the gatric band through the gastric wall and migration into the lumen of the stomach. Hereby, we present three cases of gastric band migration following LAGB Methods: From 2013 to 2017 we observed 3 cases of gastric band migration in between 5 and 7 years after LAGB placement. The patients were hospitalized in surgical department complaining of sudden sharp epigastric pain, nausea and vomiting, with symptoms onset in the last few days. All patients underwent abdominal ultrasound examination, X-ray investigation of the abdomen with oral contrast administration, fibrogastroscopy. In 2 cases the imaging studies revealed gastric band migration into the stomach's lumen and in 1 case-obstruction of the jejunum by the gastric band. All patients underwent laparoscopic surgery.

Results: Two of the patients underwent gastrotomy, extraction of the gastric band and Roux- and Y-gatric bypass. The patient with jejunal obstruction underwent laparoscopic enterotomy, extraction of the gastric band and cholecystectomy due to concomitant cholecystitis. Two of the patients had no additional perioperative complications and were discharged at the 5th postoperative day. One patient developed fever, left pleural effusion and partial insufficiency of the gastrointestinal anastomosis in the early postoperative period without the need of surgical treatment. The patient was discharged on 20th postoperative day. All patients were prescribed a diet and monthly blood test of ion balance.

Conclusions: LAGB was one of the most common treatment methods due to the epidemic spread of morbid obesity in Western countries. Detailed knowledge on possible LAGB complications is essential for the treatment of these patients. The diagnosis of LAGB complications is often delayed due to its relative rarity and nonspecific clinical manifestations, but in most of the cases it requires emergency surgery for management of life-threatening conditions. 


\section{P068-BARIATRICS-Laparoscopic}

\section{LAPAROSCOPIC MINI GASTRIC BYPASS FOR METABOLIC SYNDROME IN MORBIDLY OBESE PATIENTS}

\section{O. Usenko, O. Tyvonchuk, V. Moskalenko, Y. Voitiv}

Gastrointestinal tract surgery, Shalimov's National Institute of Surgery and Transplantology, KYIV, Ukraine

Background: The International Diabetes Federation (IDF) estimates that ' $25 \%$ of the world's population has MetSyn, although this estimate varies widely due to the age, ethnicity, and gender of the population studied. Conservative treatment of metabolic syndrome is ineffective, especially in patients with morbid obesity. The results of surgical treatment of morbid obesity have shown that a consistent, adequate reduction in body weight is accompanied by improvement of the course of type 2 diabetes, lipid metabolism normalization and blood pressure.

Methods: retrospectively reviewed result of treatment 37 patients, whom performed laparoscopic mini gastric bypass for morbid obesity from October 2013 till November 2018. There were 18 $(48.6 \%)$ men and $19(51.4 \%)$ women.

Average age $43.7 \pm 9.5$ years; average BMI was $44.8 \pm 8.4 \mathrm{~kg}^{2}$ the length of small bowel is run to $200-250-300 \mathrm{~cm}$ distal to Treitz' ligament according to patients BMI $\left(200 \mathrm{~cm}-40-45 \mathrm{~kg}^{2}\right.$, $250 \mathrm{~cm}-45-50 \mathrm{~kg}^{2}, 300 \mathrm{~cm}->50 \mathrm{~kg}^{2}$ ) There was 18 patients with T2DM, 17 with dyslipidemia and 29 with arterial hypertension, what lead to metabolic syndrome in $20(54 \%)$ patients.

Results: There was conversion in 2 patients (short mesentery of the small intestine). Such postoperative complication like anastomotic leak in 1 patient $(2.7 \%)$ and staple line bleeding in 1 patient $(2.7 \%)$, which was managed laparoscopically.

Compensation for type 2 diabetes was achieved in $9(50 \%)$ patients, improvement was recorded in $7(38.9 \%)$, dyslipidemia in $4(23.5 \%)$ and $7(41.1 \%)$ patients, arterial hypertension in 16 $(55.1 \%)$ and $9(31 \%)$ patients respectively, what led to metabolic syndrome resolution in 11 $(55 \%)$ patients.

The liquid is allowed to take after 1 day. Average postoperative hospitalization- $4.1 \pm 1.9$ days. $\%$ EWL for 48 months $69 \%$.

Conclusions: Laparoscopic mini gastric bypass is an effective method of surgical correction of body weight and metabolic disorders in patients with morbid obesity and allows to receive an adequate and stable correction of arterial hypertension, lipid and carbohydrate metabolism, which are components of a metabolic syndrome.

\section{P069-BARIATRICS—Laparoscopic}

\section{STUDY OF THE ROLE OF GASTRISAIL DEVICE IN LAPAROSCOPIC SLEEVE GASTRECTOMY}

\section{M.R. Elkeleny ${ }^{1}$, M. Elriwini ${ }^{2}$, A.H. Ghazal ${ }^{3}$}

${ }^{1}$ GIT and bariatric surgery, Faculty of medicine, Alexandria University, ALEXANDRIA, Egypt; ${ }^{2}$ GIT surgery, Faculty of medicine, ALEXANDRIA, Egypt; ${ }^{3}$ GIT and bariatric surgery, Faculty of medicine, ALEXANDRIA, Egypt

Introduction: Over time laparoscopic sleeve gastrectomy LSG has become the most popular bariatric operation worldwide. A critical step during LSG is ensuring sleeve-size consistency. GASTRISAIL device (gastric positioning system) is a three in one surgical device replacing the standard bougie used in LSG for the application of suction, decompression and to serve as a sizing guide for gastric sleeve creation. The aim of this study is to evaluate the possible merits of Gastrisail device in LSG over the standard laparoscopic sleeve gastrectomy.

Methods: A prospective study of 40 patients randomly divided into two groups: Group A composed of twenty patients who undergo LSG with the use of Gastrisail and Group B composed of twenty patients who undergo LSG with the standard bougie without the use of Gastrisail comparing both according to operative time, consistent sleeve formation, delineation and visualization, intraoperative and post-operative complication rates, the lenght of hospital stay,gastric pouch design and percentage of excess weight loss (\%EWL).

Results: Regarding intraoperative time, the mean time was $72.0 \pm 13.58$ and $79.0 \pm 11.74$ for group A and B respectively, While no patients in group B had consistent sleeve formation,12 patients $(60 \%)$ had consistent sleeve formation. Delineation and visualization were accomplished in $100 \%$ of group A patients, was not accomplished at all in group B patients. The alignment of the stomach was reached in 12 patients in Group A but no patients at all in group B, the mean of hospital stay was $2.20 \pm 0.42$ and $2.40 \pm 0.84$ for group A and B respectively, the smaller tube design illustrated by gastrograffin $\mathrm{x}$-ray at 3rd post-operative day was accomplished in 8 patients $(80 \%)$ and 2 patients $(20 \%)$ in group A and B respectively. there was no significant difference in $\%$ EWL in both groups.

Conclusion: the use of Gastrisail device is superior to the standard LSG in consistent sleeve formation, visualization, delineation and good alignment and accomplishment of a small tube design while no significant difference in $\% \mathrm{EWL}$.

\section{P070-BARIATRICS—Laparoscopic \\ REVISION SURGERY AFTER SLEEVE GASTRECTOMY - EXPERIENCE AND PERSPECTIVES IN JAPAN}

T. Naitoh, N. Tanaka, H. Imoto, H. Musha, T. Aoki, A. Yamamura, S. Ohnuma, F. Motoi, T. Kamei, M. Unno

Surgery, Tohoku University Graduate School of Medicine, SENDAI, Japan

Bariatric surgery has spread all over the world. Since Japan has few patients with morbid obesity compared with Western countries, it has been implemented only in limited facilities. However, bariatric surgery in Japan is rapidly spreading recently, and many facilities are about to install bariatric surgery. Effects of bariatric surgery are known to last for a long time, but some cases require reoperation which is called revision surgery due to late complications or rebound Because of thick subcutaneous and visceral fat, open surgeries are not even always a good solution to make surgery easier in morbid obese patients and all procedures must be completed laparoscopically. Therefore, especially in revision surgery, the incidence of complications tends to be increased. As the number of bariatric cases to be increased in Japan, cases requiring revision surgery is likely to increase. In revision surgery, it is necessary to select the procedure according to patient condition, and it is necessary to familiar well with those procedures. We will present cases that underwent revision surgery in our department and show the clinical outcome.

We have done four revision surgeries after sleeve gastrectomy so far. Operative indications are 2 mid-gastric stenosis and 2 rebounding disease. For stenosis cases, we performed roux-en Y gastric bypass with distal stomach resection, and for rebounding cases, we performed re-sleeve gastrectomy with duodenal-jejunal bypass.

Average interval from initial operation to revision surgery is 69 months in rebounding cases, and 9 months in stenosis cases. Duration of operation was $269 \mathrm{~min}$ in average, and mean estimated blood loss was $18 \mathrm{ml}$. No postoperative mortality was observed. In rebounding cases, excess BMI loss at 1 year after surgery was $40.9 \%$ in average, and both cases achieve diabetes remission at 1 year. One cases of mid-gastric stenosis required a nutritional support with formula diet temporally.

In particular after sleeve gastrectomy, revising to Roux-en $\mathrm{Y}$ gastric bypass, re-sleeve gastrectomy, and adding the duodenal-jejunal bypass will be the main techniques. Along with an increase of bariatric surgery in Japan, it is necessary to acquire sufficient knowledge and skills to carry out revision surgery.

\section{P071-BARIATRICS-Laparoscopic}

\section{LAPAROSCOPIC SLEEVE GASTRECTOMY AFTER ADJUSTABLE GASTRIC BAND REMOVAL AND GASTRIC PLICATION}

V. Drakopoulos, V. Lygizos, S. Voulgaris, I. Iliadis, P. Trakosari, A. Bakalis, V. Vougas

1st Department of Surgery and Transplantation Unit, District General Hospital of Athens « Evangelismos », ATHENS, Greece

Background: Revision procedures after Laparoscopic Adjustable Gastric Band placement and Laparoscopic Greater Curvature Plicationare often necessary in cases of inadequate weight loss or weight regain. Inflammation and foreign body reaction make the revision procedure technically demanding.

Introduction: Laparoscopic Greater Curvature Plication (LGCP) andLaparoscopic Adjustable Gastric Band (LAGB) related complications often require revision procedures with band removal and/or conversion to Laparoscopic Sleeve Gastrectomy (LSG) or Roux-en-Y Gastric By-pass (RYGB). The optimal method of revision remains controversial.

Objectives: We present our 4-year experience concerning Laparoscopic Adjustable Gastric Banding (LAGB) removal, Laparoscopic Greater Curvature Plication (LGCP) and laparoscopic sleeve gastrectomy (LSG).

Methods: We present the case of a 45 year old woman who underwent LSG after LAGB removal and LGCP. The patient underwent preoperative endoscopy and barium swallow, with no sign of stomach perforation or erosion. We emphasize that the patient, had undergone three operations of gastric band placement, gastric band removal and gastric plication before sleeve gastrectomy. However, a successful LSG was achieved.

Results: No severe postoperative complications were mentioned.

Conclusion: Weight loss in the first year was $70 \%$ of the excess weight.Sleeve gastrectomy after gastric band removal and gastric plication, for morbid obesity seems to be safe and efficient, especially in casesof absence of gastric erosion. 


\section{P072-BARIATRICS-Laparoscopic}

\section{SURGICAL MANAGEMENT OF LATE AND SERIOUS COMPLICATIONS AFTER BARIATRIC SURGERY}

\author{
A. Paszt ${ }^{1}$, L. Andrasi ${ }^{1}$, L. Andrasi ${ }^{1}$, M. Vas ${ }^{1}$, Sz. Abraham ${ }^{1}$, \\ Zs. Simonka ${ }^{1}$, Z. Szepes ${ }^{2}$, Gy. Lazar ${ }^{1}$ \\ ${ }^{1}$ Department of Surgery, University of Szeged, SZEGED, Hungary; \\ ${ }^{2} 1$ st Department of Internal Medicine, University of Szeged, \\ SZEGED, Hungary
}

Aims: Minimal invasive gastric banding surgery is one of the most frequently performed and successful surgical treatment in patients with morbid obesity. There are controversial international data about patients' follow up and therapeutic strategy for possible late complications. Methods: In our retrospective analysis, between 2008 and 2018, at the University of Szeged

Department of Surgery, 19 patients were observed with serious septic complications many years after gastric banding operation. We detected a female dominance ( 16 female, 3 male) in patients with a mean age of 41.6 years. The leading symptoms were: dysphagia, upper abdominal tenderness and pain, spontaneous fistula formation, fever, masked septic signs, bowel and urinary obstruction. Patients underwent video-endoscopy, chest and abdominal CT (computed tomography), fistulography and cystoscopy.

Results: In still morbid obese patients, laparoscopic procedures were performed with a conversion rate of 50\%: atypic gastric and cardia resection in 4 cases, gastric suture in 9 cases, small bowel resection and suture in $4-4$ cases. In one case, fistulectomy, abscess evacuation and combined urinary bladder suture and drainage were carried out. The duration of the surgeries were over $2 \mathrm{~h}$ with minimal blood loss $(<200 \mathrm{ml})$. The foreign bodies were completely removed in every case. Intraoperative complication was not occurred. Early physiotherapy were promoted, oral feeding were gradually built up from the 2 th postoperativ day depending on the type of the operation. Early postoperative complications included recurrent fistula formation $(\mathrm{n}=2)$ and wound infection $(\mathrm{n}=11)$. All the fistulas were closed after conservative treatment. Average hospital stay was 8 days, regular check-ups were held on the $3 \mathrm{rd}$, 6th and 12 th months of follow up.

Conclusion: Gastric banding is the most common, routine and safe technique for the treatment of morbid obesity. The development of late, severe septic complications draws attention to the crucial importance of follow up. The surgical management of these patients is recommended in specialized centers in regard to difficult operative conditions and atypic treatment options.

\section{P073-BARIATRICS—Laparoscopic}

\section{SINGLE ANASTOMOSIS DUODENO-ILEAL BYPASS WITH/ AFTER SLEEVE GASTRECTOMY: INITIAL EXPERIENCE OF A HIGH-VOLUME SINGLE INSTITUTION}

L. Sessa ${ }^{1}$, F. Pennestri ${ }^{1}$, P. Gallucci ${ }^{1}$, P. Giustacchini ${ }^{1}$, L. Ciccoritti ${ }^{1}$, C. Modesti ${ }^{2}$, R. Bellantone ${ }^{1}$, M. Raffaelli ${ }^{1}$

${ }^{1}$ U.O.C. Chiruriga Endocrina e Metabolica, Fondazione Policlinico Universitario A. Gemelli IRCCS, ROME, Italy; ${ }^{2}$ U.O.C. Anestesia delle Chirurgie Generali e dei Trapianti, Fondazione Policlinico Universitario A. Gemelli IRCCS, ROME, Italy

Aims: Single anastomosis duodeno-ileal bypass with sleeve gastrectomy (SADI-S) has been proposed as an alternative to biliopancreatic diversion with duodenal switch (BPD-DS) in order to maintain the outcome of the original procedure simplifying the technical complexity and to avoid potential complications. Moreover, it potentially represents the more natural second step bariatric procedure after sleeve gastrectomy (SG). We aimed to report the initial experience with SADI-S of our high volume bariatric Center.

Methods: retrospective analysis of patients who underwent bariatric procedure between July 2016 and November 2018 was conducted. The primary aim was the evaluation of the safety of SADI-S, defined as the rate of postoperative complications. The secondary endpoint was the bariatric efficacy of the procedure, defined as percentage excess weight loss (\%EWL).

Results: Among 813 patients who underwent bariatric procedures at our institution 36 (4.4\%) patients were scheduled for SADI-S. All patients had multiple comorbidities. Initial indication for SADI-S was failed SG in 8 patients (median pre-SG BMI $52.1 \mathrm{Kg} / \mathrm{m}<$ sup $>2</$ sup $>$; median 39 months after initial operation respectively) and primary procedure in 28 patients (median pre-operative BMI 56.5 $\mathrm{Kg} / \mathrm{m}^{2}$ ). The surgical procedure was accomplished with robotic-assisted approach in 4 cases (median operative time $198 \mathrm{~min}$ ) and with laparoscopic 4 trocars standard approach in the remaining 32 cases (median operative time $130 \mathrm{~min}$ ). The duodeno-ileal anastomosis was fashioned using a double layer hand-sewn running sutures. No patients showed early post-operative complications, the median postoperative stay was 3 days. At a mean follow up of 12 months the median \%EWL was 66.1. To date no patients experienced surgical. One patient develop Wernicke encephalopathy 6 months after surgery, but he was non-compliant to multivitamin supplementation.

Conclusions: At least in a high volume bariatric Center SADI-S, both as second step after SG and as primary surgical option, seems to be a safe and effective bariatric metabolic procedure based on solid physiopathologic principles. On the other hand, longer follow-up is necessary to support the use of this procedure as a better alternative to BPD-DS.

\section{P074-BARIATRICS-Laparoscopic \\ RARE INTERESTING BARIATRIC PROCEDURES COMPLICATIONS AND ITS MANAGEMENT}

\section{$\underline{\text { M.R. Elkeleny }}{ }^{1}$, A. Abo Khozima ${ }^{2}$}

${ }^{1}$ GIT and bariatric surgery, Faculty of medicine, Alexandria University, ALEXANDRIA, Egypt; ${ }^{2}$ GIT Surgery department, Faculty of medicine,Alexandria university, ALEXANDRIA, Egypt

Four bariatric cases 1. Female patient with intragastric balloon, minor leak from the balloon leading to ballon migration to the jejunum;hance, small bowel obstruction occured. Emergency diagnostic laparoscopy was done, enterotomy and extraction of the balloon, direct repair of enterotomy and balloon extraction through $10 \mathrm{~mm}$ port site. 2. Male patient presented after 5 days of LSG with small bowel obstruction due to entrapment of small bowel loop through one of the port sites;therefor, emergency laparoscopy was done with reduction of the herniated segment and closure of the port site. 3. Female patient presented with stricture of the OGJ after re-sleeve gastrectomy managed by balloon dilatation which recur after 2 weeks .she was managed by expandable metallic stent for 6 weeks with good response and the stent was removed.4.38-year-old male patient presented with sever peripheral neuropathy following 5 months after sleeve gastrectomy, and the patient was getting worse; thus, he used wheel chair. he has been making good progress on vitamin B complex injections.

\section{P075-BARIATRICS-Laparoscopic}

\section{HISTOPATHOLOGICAL FINDINGS IN 44 CASES OF LAPAROSCOPIC SLEEVE GASTRECTOMY. IS THERE A NEED FOR PREOPERATIVE GASTROSCOPY?}

N. Kontoravdis ${ }^{1}$, S. Artsitas ${ }^{2}$, A. Matsas ${ }^{1}$, S. Theodoroleas ${ }^{1}$, S. Papadimatos $^{1}$, K. Nisotakis ${ }^{2}$, N. Salemis ${ }^{2}$

${ }^{1}$ 2nd Surgical Department, 417 Share Fund Army Hospital, ATHENS, Greece; ${ }^{2}$ 2nd Surgical Department, 401 General Army Hospital, ATHENS, Greece

Aims: Conditions of the GI tract, such as gastroesophageal reflux disease or gastritis are common among obese population. Although symptomatic obese patients have to be evaluated with UG endoscopy prior to surgery, there are no clear recommendations about the asymptomatic cohort of them.

The aim of our study was to compare histopathological findings of gastric specimens to preoperative clinical symptoms and to conclude about the need for UGI endoscopy as a routine prior to surgery.

Methods: The last two years, 44 morbid obese patients were selected to undergo laparoscopic sleeve gastrectomy (LSG) in our institution. For the needs of our study, all of them had UGI endoscopy and were reviewed for upper GI symptoms. Histopathological reports obtained according to our protocol, after surgery.

Results: Gastric histology from specimens revealed: no findings in 16/44 patients (36.4\%), gastritis in 19/44 patients (43.2\%) and focuses of incomplete intestinal metaplasia without dysplasia in $8 / 44$ patients(18.1\%). Finally, two minor leiomyomas with low cellular proliferation rate were fully excised in a patient's specimen.

There was no inconsistency between preoperative symptoms and gastric histology, while leiomyomas found were no reported to UGI endoscopy due to size.

Conclusions: Some of the patients with clinical features of food intolerance, gastroesophagea reflux disease, and peptic ulcer disease had finally findings in histopathology of their stomachs. History of Helicobacter pylori infection implements a raised incidence of mucosa pathology as well.

Because only one case revealed carrying significant pathology (leiomyomas), we consider that is safe to proceed with surgery in an otherwise asymptomatic patient based on his previous medical records and blood tests. 


\section{P076-BARIATRICS-Laparoscopic}

PROPHYLACTIC CHOLECYSTECTOMY DURING LAPAROSCOPIC SLEEVE GASTRECTOMY IN MORBIDLY OBESE PATIENTS. RESULTS FROM A SERIES OF 306 PROCEDURES

\section{Parianos, C. Aggeli, S. Roditis, A. Thanasa, I. Margaris}

3rd Department of Surgery, General hospital of Athens, ATHENS, Greece

Background: The prevalence of cholelithiasis in morbid obesity is $19-45 \%$ among different series. Moreover there is an increased risk of gallstone formation during rapid weight loss. Laparoscopic sleeve gastrectomy has become one of the most performed procedures worldwide in the last decade. The management of the gallstones at the time of laparoscopic sleeve gastrectomy is under debate. We report our experience in the treatment of patients with morbid obesity and cholelithiasis with or without symptoms.

Methods: 306 patients underwent laparoscopic sleeve gastrectomy between 2008 and 2018 . Preoperative ultrasonography was performed for all patients and gallstone presence was recorded. Patients with cholelithiasis underwent concomitant cholecystectomy. Age, gender, BMI and operative time were collected. Perioperative complications, length of hospital stay and the incidence of symptomatic disease in patients after sleeve gastrectomy with no previous history of cholelithiasis were noted.

Results: Laparoscopic sleeve gastrectomy was performed in 306 patients with morbid obesity. Cholelithiasis was detected preoperatively in 41 patients who underwent laparoscopic sleeve gastrectomy. Only 18 of these patients had symptomatic cholelithiasis. Laparoscopic cholecystectomy was combined with sleeve gastrectomy in 36 of the 41 patients. The sleeve procedure was performed using 5 ports. An additional $5 \mathrm{~mm}$ port was placed in the right lateral abdominal wall in patients receiving concomitant cholecystectomy. Concomitant cholecystectomy required an additional 15-29 min. Four patients with normal preoperative abdominal ultrasound, developed cholelithiasis after the weight loss, and underwent laparoscopic cholecystectomy. One female patient with gastric leak was reoperated and after a long hospital stay has developed cholecystitis. She was successfully treated with percutaneous cholecystostomy.

Conclusions: Laparoscopic cholecystectomy during laparoscopic sleeve gastrectomy is safe and prevents possible postoperative events of cholecystitis.

Keywords: Sleeve gastrectomy. Prophylactic cholecystectomy. Obesity

\section{P077-BARIATRICS—Laparoscopic}

\section{FEASIBILITY, SAFETY AND EFFICACY OF LAPAROSCOPIC SLEEVE GASTRECTOMY IN CLASS I OBESE PATIENTS WITH BMI 30-34.9}

\section{A. Elnabil-Mortada, H. Elmaleh}

Department of Surgery, Ain Shams University, CAIRO, Egypt

Background: Class I (Mild obese) patients (BMI 30-34.9) are routinely managed by diet with life style modifications or endoscopic solutions. In spite of that, failure of weight loss or weight regain is quite common among this group. Laparoscopic sleeve gastrectomy (LSG) has proved itself to be a safe effective weight loss procedure in patients with $\mathrm{BMI}=35$, but its currently not strongly recommended for lower BMI. In our study we aimed see if LSG in class I obesity patients have the same safety, efficacy and feasibility profile as it had been demonstrated in higher BMI.

Patients \& methods: Between January 2015 and October2017, 250 obese patients with $\mathrm{BMI}=30$ were enrolled in a study. Patients were classified into 2 groups: Group A patients with BMI $\left(30-34.9 \mathrm{~kg} / \mathrm{m}^{2}\right)$ and Group B with BMI $=35$. Both groups underwent LSG and followed up for at least 1 year with surgical outcomes compared between the 2 groups.

Results: Eighty patients (32\%) belonged to Group A with 61\% female patients, 170 (68\%) Group B with $73.5 \%$ female. Mean preoperative excess weight was $21.5 \pm 4.9 \& 62.3 \pm 23.6 \mathrm{~kg}$, mean preoperative BMI was $32.7 \pm 1.4 \& 47.8 \pm 8.2 \mathrm{~kg} / \mathrm{m}^{2}$ respectively. The incidence of severe complications requiring invasive intervention was significantly higher in higher BMI group. At one year follow up mean EWL\% was $106 \pm 21.5 \& 79.9 \pm 12.2$, mean TWL $\%$ was $24.8 \& 37$, postop BMI was $24.6 \pm 1.5 \& 30 \pm 4.1$ respectively considering ideal weight to BMI 25 , resolution of comorbidities was observed in more than $80 \%$ of the patients both groups with normal $\mathrm{Hb}$, albumin, Iron,ca, vitamin D3, B12 levels.

Conclusion: laparoscopic sleeve gastrectomy is a feasible, safe and effective option in class obese patients with or without comorbidities, further larger studies are needed aiming to modify current recommendations regarding patients with low BMI

Keywords: Laparoscopic Sleeve Gastrectomy, Class I obesity, BMI 30-35.

\section{P078-BARIATRICS-Laparoscopic}

\section{SPLENIC ABSCESS AS AN EARLY COMPLICATION FOLLOWING LAPAROSCOPIC SLEEVE GASTRECTOMY-A CASE REPORT}

N. Kontoravdis ${ }^{1}$, R. Mourelatou ${ }^{1}$, S. $\operatorname{Artsitas}^{2}$, A. Matsas ${ }^{1}$, K. Manesis ${ }^{1}$, D. Kalliouris ${ }^{1}$, S. Papadimatos ${ }^{1}$, K. Nisotakis ${ }^{2}$

${ }^{1}$ 2nd Surgical Department, 417 Share Fund Army Hospital, ATHENS, Greece; ${ }^{2}$ 2nd Surgical Department, 401 General Army Hospital, ATHENS, Greece

Aims: Splenic abscess following laparoscopic sleeve gastrectomy (LSG) is a rarely seen complication. The aim of our study was to present a case of splenic abscess in a morbid obese patient who underwent LSG. As the main concern in these cases is leakage from the staple line, we present our diagnostic and treatment approach.

Methods: A 42-year-old, female morbid obese patient (BMI $56.6 \mathrm{~kg} / \mathrm{m}^{2}$ ), without any predisposing risk factors, underwent elective LSG in our department. Following an uneventful course, she was discharged at the $2^{\text {nd }}$ postoperative day. However, at the $20^{\text {th }}$ postoperative day, she readmitted to our unit with high temperature of $38.4^{\circ} \mathrm{C}$, left upper quadrant tenderness and leukocytosis. Contrast computed tomography (CT) revealed an abscess at the upper pole of the spleen $4,5 \mathrm{~cm}$ in maximum diameter, without leakage from the staple line.

Results: The patient was treated with broad-spectrum antibiotics and radiological percutaneous drainage of the abscess. Although there was a partial clinical improvement, a week later, a new CT scan revealed the continuous presence of the abscess. Despite the stable general condition of the patient a laparoscopic splenectomy was performed and a gradual recovery was followed. Conclusion: The presence of splenic abscess without splenic trauma or leakage from the gastric staple line, is an extremely rare complication and only a few cases have been previously reported. The cause has not yet clarified, but the proposed mechanism involves infarction of the spleen, due to vascular compromise and subsequent infection. Most of the reported splenic abscesses were diagnosed during the late postoperative period. In our report we present a case of early onset, hence highlighting the need of clinical awareness for early diagnosis and treatment.

\section{P079-BARIATRICS-Laparoscopic}

\section{POSTOPERATIVE PULSE OXIMETRY WITHOUT OBSTRUCTIVE SLEEP APNEA SCREENING IN BARIATRIC SURGERY: OUTCOMES OF 5000 CASES}

S.L. van Veldhuisen, I. Arslan, I.M.C. Janssen, F.J. Berends, E.J. Hazebroek, E.O. Aarts

\section{Surgery, Rijnstate Hospital, ARNHEM, The Netherlands}

Introduction: Obese surgical patients with obstructive sleep apnea (OSA) have a higher risk of peri- and postoperative desaturations and subsequent morbidity and mortality. Currently, the bes perioperative management of patients without known OSA remains unclear. Although routine OSA screening has been advocated, sleep studies are costly and time consuming. We hypothesized that bariatric patients can be safely monitored on a surgical ward by continuous postoperative pulse oximetry without preoperative screening for OSA.

Objectives: To evaluate outcomes of continuous postoperative pulse oximetry without preoperative OSA-screening, and to compare the results to outcomes of patients with OSA and continuous positive airway pressure (CPAP) treatment.

Methods: All patients who underwent bariatric surgery between 2011 and 2017 were included in this single-center retrospective cohort study. All patients were postoperatively monitored with continuous pulse oximetry on the surgical ward. Patients with less than two documented saturation measurements were excluded. Patient files were reviewed for OSA diagnosis, CPAP usage and perioperative details. Primary outcomes were 30-day complication rates, Intensive Care Uni admissions due to cardiopulmonary causes and postoperative desaturations of $\mathrm{SpO}_{2}<90 \%$. Secondary outcomes were ICU admissions following all causes, length of stay and rates of reoperation and readmission.

Results: In total, 5203 patients were included. 675 Patients (13\%) were preoperatively diagnosed with OSA, $511(9.8 \%)$ were CPAP users. Complications occurred in $7.2 \%$ of patients without OSA and in $9.6 \%$ with OSA $(\mathrm{p}=0.028)$. Desaturations were documented in $1.4 \%$ and $4 \%(\mathrm{p}<0.001)$, respectively. In both groups, 1 patient was admitted postoperatively to the ICU for cardiopulmonary causes that could be related to OSA $(\mathrm{p}=0.119)$. Both recovered without further complications. ICU admissions, regardless of cause, occurred in $0.42 \%$ of patients without OSA and in $1.18 \%$ with OSA $(\mathrm{p}<0.001)$. No significant difference between groups was observed in complications based on Clavien Dindo classification, length of stay, reoperation- and readmissions-rates.

Conclusions: These findings suggest that continuous postoperative pulse oximetry without preoperative OSA-screening is a safe perioperative management strategy for bariatric surgical patients. Future studies are needed to assess cost-effectiveness of pulse oximetry vs. routine preoperative OSA-screening in a prospective clinical setting. 


\section{P080-BARIATRICS-Laparoscopic}

MINIMALLY INVASIVE Method: S OF SURGICAL TREATMENT OF COLOSTASIS

\section{K. Rustemova, B. Aitmoldin, A.R. Shakeeva, G.K. Nurusheva}

\section{Surgery, City Hospital 2, ASTANA, Kazakhstan}

Background: The pathology of colon is one of the most pressing and socially significan problems of modern health care, because it leads to reduction of the working population employed in manufacturing, in some cases to disability and reduced quality of life. Mini invasive surgery of the colon has a great advantage: speed recovery, shorter hospital stay and better cosmetic results, the quickest return of patients to work. As a result, mini invasive endovideosurgery is firmly established in clinical practice of coloproctology.

Objective: The choice of optimal surgical method for treatment of colostasis, achievement of favorable outcomes of treatment.

Materials and methods: CT, MRI, installation of 'ZORING', endoscopic stand 'Karl Stors', argon-plasma coagulator company 'Karl Stors'. Laparoscopic setup with manual assistance: GelPort (Applied Medical). Morphological, microbiological research methods, clinical and laboratory studies

Results: Since the beginning of 2013, on the basis of Department of General Surgery and Coloproctology of 'City Hospital ?2', 57 operations were performed in the department of purulen surgery: 42-laparoscopic left-sided hemicolectomy, 7-laparoscopic right-sided hemicolectomy, 8 -sigma resection. In 21 cases, the operation was performed on new formation of sigmoid colon, in 11 cases on dolichosigmoid. Operated ?n 42 female patients, 15 male patients from 15 to 63 years. The average patient stay in the clinic is 6-7 days. Patients were activated on the 2nd day after surgery. All patients are under the supervision of surgeons.

Control in a month: defecation is normal, up to 1 time per day (delays reached a month before the operation), improvement of overall health, improvement of memory, attention, no signs of chronic fatigue, improvement overall appearance (hair shine, improved color and skin condition) that did not plays a small role for women and, accordingly, improve the quality of life. Conclusion: During the past 15 years, laparoscopic surgeries in coloproctology have taken their rightful place in the surgical treatment of colon diseases

\section{P081-BARIATRICS-Laparoscopic}

\section{HIPERINSULINEMIC HYPOGLYCEMIA AFTER GASTRIC BYPASS: REVERSAL TO NORMAL ANATOMY WITH SLEEVE GASTRECTOMY}

\section{R. Roriz-Silva ${ }^{1}$, J.M. Fort ${ }^{1}$, R. Vilallonga ${ }^{1}$, J.M. Balibrea ${ }^{1}$,} $\overline{\text { E. Enriq Caubet }}^{1}$, O. Gonzales ${ }^{1}$, M.K. Carré ${ }^{1}$, M.D. Romero ${ }^{2}$

${ }^{1}$ Unit of Endocrine-Metabolic and Bariatric Surgery, Hospital Vall d'Hebron, BARCELONA, Spain; ${ }^{2}$ Hospital General Universitari de la Vall d'Hebrón, Hospital Vall d'Hebron, BARCELONA, Spain

Introduction: Laparoscopic Roux-en-Y gastric bypass (RYGB) is one of the most important bariatric surgical procedures performed worldwide and it can produce an important loss of weight with reversal of metabolic disorders like diabetes and dyslipidemia. Even though it has good results, some complications occur after gastric bypass. A rare but serious complication of RYGB is the so-called postprandial hyperinsulinemic hypoglycemia. Its prevalence has been estimated less than $1 \%$ of cases and its pathophysiology remains unclear.

Methods: The aim is to present a case series of reversal surgery in patients with severe hiperinsulinemic hypoglycemia after RYGBP in the Hospital General Universitari de la Vall d'Hebron. Unit of Endocrine-Metabolic and Bariatric Surgery (EAC-BS Center of Excellence for Bariatric and Metabolic Surgery by IFSO). It is a retrospective analysis of a prospective database same surgical team. We present in this study, the main features of those patients.

Results: Between 2011 and 2018, 13 patients underwent a laparoscopic reversal procedure to normal anatomy and age mean was 57 year ( 22 years to 70 years). Mean preoperative body mass index (BMI) was $31.2 \mathrm{Kg} / \mathrm{m}^{2}$ (range $28-39.4 \mathrm{Kg} / \mathrm{m}^{2}$ ) and 10 were women. All patients presented hypoglycemia symptoms 5 years after and the longest was 15 years after the procedure. The first step of the standard approach was a laparoscopic reversal to normal anatomy with resection of the alimentary RYGB limb in 7 cases. A concomitant sleeve-like gastrectomy (SG) was added. Four patients presented postoperative complications: gastrogastric anastomosis leak (1), colitis with intra-abdominal collection (conservatively managed), Intraperitoneal bleeding and one case of mesenteric venous thrombosis. Average hospital stay was 9.8 days. No mortality. At mid-term, all patients recovered from their initial condition. Two patients had weight regain (mean BMI at the last control was 29.8). Most patients developed gastroesophageal reflux.

Conclusions: The results of the laparoscopic reversal with SG after a RYGB are acceptable, showing a clinical improvement of the hypoglycemic syndrome in all cases. Due to the high risk of developing GERD, we recommend to consider adding a hiatoplasty to the procedure.

\section{P082-BARIATRICS-Laparoscopic}

IS GASTRIC BYPASS STILL THE REDEMPTION AFTER CONSECUTIVE, FAILED BARIATRIC PROCEDURES? A VIDEO REPORT OF A COMPLICATED LSG

\section{C.E. Boru, G. Silecchia}

Division of General Surgery \& Bariatric Center of Excellence-IFSO EC, Sapienza University of Rome, Dept. of Medico-Surgical Sciences \& Biotechnologies, LATINA, Italy

Introduction: Laparoscopic sleeve gastrectomy is the most performed bariatric procedure, bu complications might interfere with patient's long-term evolution based on its compliance and tolerance, surgical attitude and unpredictable evolution.

Materials: we present the case of a female obese patient, with type II diabetes mellitus and blood hypertension, with multiple, sequential bariatric minimally-invasive interventions: sleeve gastrectomy in 2012 complicated by postoperative acute gastric dilation and mediogastric stenosis, reoperated for viscerolysis and cholecystectomy, with endoscopic gastric dilations, initially converted to functional one anastomosis gastric bypass $(200 \mathrm{~cm}$ limb), with a non-adjustable gastric ring positioned instead of stapled division. The last operation was complicated 12 months after by persistent biliary gastro-esophageal reflux, chronic abdominal pain, and gas bloat syndrome. In 2018 the patient underwent conversion to laparoscopic R-en-Y gastric bypass, with gastro-enteral anastomosis resection, band removal and viscerolysis.

Results: Conversion to R-en-Y was complicated by biliary leakage post-viscerolysis, treated with laparoscopic approach in the $9<\sup >$ th $<$ sup $>$ po day. After multiple surgical and endoscopic interventions, the patient presents short-term favorable outcomes, with no reflux or abdominal pain, with further weight loss and diabetes improvement.

Conclusion: bariatric surgery has unpredictable evolution in same cases, and conversion to R-enY seems to be the best solution.

\section{P083-BARIATRICS—Laparoscopic}

\section{REVISION SURGERY AFTER FAILED LAPAROSCOPIC GASTRIC PLICATION FOR MORBID OBESITY}

C. Borz ${ }^{1}$, T. Bara ${ }^{1}$, M.B. Borz ${ }^{1}$, T. Bara ${ }^{1}$, E. Elthes ${ }^{1}$, S. Gunea ${ }^{1}$, M. Denes ${ }^{1}$, A. Torok ${ }^{1}$, D. Marian ${ }^{1}$, P.C. Borz ${ }^{2}$

${ }^{1}$ 2nd Department of Surgery, Emergency County Hospital Tg.Mures, TG.MURES, Romania; ${ }^{2}$ 2nd Department of surgery, University of Medicine and Pharmacy, TG.MURES, Romania

Introduction: Laparoscopic gastric plication(LGCP) along with mini gastric bypass are new bariatric procedures. The golden standard for bariatric surgery remain the sleeve gastrectomy(SG) and gastric bypass. LGCP is widely used in developing countries due to its lower cost and good results.

Material and methods: We performed in our department 120 LGCP for morbid obesity. Excess weight loss (\%EWL) was 55\% at 6 month after surgery and $65 \%$ at one year. In 12 cases revision surgery was needed for different complications and in 22 cases for inadequate weight loss or weight regain after 18 month follow up. In 8 cases we performed sleeve gastrectomy (in 3 cases after taking down the plication) and in 14 cases we performed a re-plication in one row.

Results: The rate of revision surgery was $28 \%$ overall and $18 \%$ for inadequate weight los (excess weight loss $<50 \%$ ) or weight regain. Major complications occurred only in one patient (leak with abscess) but it was solved by laparoscopy. Minor complications as vomiting and nausea appeared in 5 patients $(22 \%)$ and were solved with medication. After one year follow up $\%$ EWL in these cases was $85 \%$.

Conclusions: Revision surgery after LGCP is possible. A new plication or SG was the option in our series with good results. Further studies are needed to evaluate the use of LGCP in the armamentarium of bariatric surgery. 


\section{P084-BARIATRICS-Laparoscopic}

\section{LAPAROSCOPIC REVERSAL OF GASTRIC BYPASS IN A PATIENT WITH MALNUTRITION}

P. Priego ${ }^{1}$, A. Puerta ${ }^{2}$, A. González Barranquero ${ }^{1}$, S. Sánchez Picot ${ }^{1}$, $\overline{\text { L. Blazquez }}^{1}$, M. Cuadrado ${ }^{1}$, J. Galindo

Division of Esophagogastric and Bariatric Surgery, Ramón y Cajal University Hospital, MADRID, Spain

Background: Roux-en-Y gastric bypass (RYGB) is one of the most commonly performed bariatric procedures around the world. However, RYGB it sometimes carries the risk of rare but serious long-term complications such as malnutrition and liver failure. We report a case of laparoscopic reversal of RYGB. Methods: In March 2017, a laparoscopic RYGB was performed for a 53-year-old female without comorbidities and with a BMI of $54 \mathrm{~kg} / \mathrm{m}^{2}$. All laboratory test results at the preoperative evaluation were within the normal range. Abdominal ultrasound revealed moderate hepatic steatosis and oral endoscopy a hiatal hernia with grade B esophagitis.

One year later, patient experienced an important weight loss of $75 \mathrm{~kg}$ (from 155 to $80 \mathrm{~kg}$ ) with a BMI of $28 \mathrm{~kg} / \mathrm{m}^{2}$. However, patient presented general weakness, abdominal pain, ascitis lower extremitiy edema, anemia, progressive caloric and protein malnutrition, vitamin (A,D), mineral (copper) and folic acid deficiencies, nonalcoholic steatohepatitis (NASH) and liver function was progressive worsening. Results: A laparoscopic reversal of gastric bypass was performed. The operation was successfully performed via laparoscopy. Operating time was $70 \mathrm{~min}$. Postoperative was uneventful and patient discharge home at day 6. Hepatic biopsy revealed NASH with steatohepatitis of $80 \%$ (Fibrosis F2-3/4). Eight months after reversal of gastric bypass, patient has improved her clinical situation (no asthenia), maintains of weight $(80 \mathrm{~kg})$ and has improved her nutritional status and liver function parameters. Conclusion: Laparoscopic reversal of RYGB is technically feasible and might be performed safely after thorough preoperative evaluation in carefully selected patients with malnutrition and liver failure.

\section{P085-BARIATRICS-Laparoscopic}

\section{LAPAROSCOPIC SLEEVE GASTRECTOMY FOR THE TREATMENT OF PATIENTS WITH MASSIVE HERNIAS WITH LOSS OF DOMAIN}

R. Roriz-Silva ${ }^{1}$, R. Vilallonga ${ }^{1}$, S. Sánchez-Cordero ${ }^{2}$, Y. Curbero ${ }^{3}$, M.D. Romero ${ }^{4}$ J. $\mathrm{Meza}^{3}$

${ }^{1}$ Unit of Endocrine-Metabolic and Bariatric Surgery, Hospital Vall d'Hebron, BARCELONA, Spain; ${ }^{2}$ General Surgery, Consorci Sanitari de l'Anoia, Igualada, Barcelona, IGUALADA, Spain; ${ }^{3}$ Department of anesthesiology, Hospital de barcelona, BARCELONA, Spain; ${ }^{4}$ Hospital General Universitari de la Vall d'Hebrón, Hospital Vall d'Hebron, BARCELONA, Spain

Introduction: The repair of a complex abdominal hernia has an important morbimortality. We propose laparoscopic sleeve gastrectomy as the first step in the treatment of hernias with loss of domain in patients with morbid obesity.

Clinical case: A 56-year-old female, with an Incisional hernia due to infraumbilical laparotomy and comorbidities of DM2, HTA, HBP, SAOS. Weight113.6 kg, Size 1.62, BMI $43.6(09 / 2015)$ We proposed surgery in two times:

$1^{\circ}$ Sleeve gastrectomy

$2^{\circ}$ Repair of the incisional hernia \pm abdominoplasty

Weight 59.7, Size 1.62, BMI 22.9 PSP 93.2\%(12/2017)

CT Scan: Sleeve gastrectomy changes. Hypogastric eventration containing intestinal loops. Cystocele and colpocele. Bilateral ovarian cysts.

The postoperative course was uneventful.

Conclusion: Laparoscopic sleeve gastrectomy it's a safety obesity procedure before major abdominal hernia repair.

It's a minimally invasively technique with an absence of anastomoses.

These factors prevent fewer complications, without using the small bowel, and skin problems and allow resolution of obesity-associated co-morbidities.

Body weight loss after surgery may be an opportunity to repair the severe loss of domain incisional hernia. Bibliography Borbély, Y., Zerkowski, J., Altmeier, J., Eschenburg, A., Kröll, D. and Nett, P. (2018). Complex hernias with loss of domain in morbidly obese patients: role of laparoscopic sleeve gastrectomy in a multi-step approach

Raziel, A., Sakran, N., Szold, A. and Goitein, D. (2013). Concomitant bariatric and ventral/ incisional hernia surgery in morbidly obese patients. Surgical Endoscopy, 28(4), pp.1209-1212. Iljin,A.,Szymanski,D.,Kruk-Jeromin,J. andStrzelczyk,J.(2008). The Repairof Incisional HerniaFollowing Roux-enY Gastric Bypass—With or Without Concomitant Abdominoplasty?. Obesity Surgery, 18(11), pp.1387-1391.

4. Chan, D., Talbot, M., Chen, Z. and Kwon, S. (2013). Simultaneous ventral hernia repair in bariatric surgery. ANZ Journal of Surgery, 84(7-8), pp.581-583

\section{P086-BARIATRICS-Laparoscopic}

\section{GASTRO-GASTRIC GREATER CURVATURE HERNIATION AFTER GASTRIC PLICATION FOR MORBID OBESITY}

\section{H. Elbernawi ${ }^{1}$, A. Kadhem ${ }^{2}$}

${ }^{1}$ General surgery, Benhazi medical center, BENGHAZI, Libia, ${ }^{2}$ General surgery, Royal bahrain hospital, MANAMA, Bahrain

Obesity is a worldwide epidemic with an increasing incidence trends and as a consequence obesity related health problems become priority to healthcare authorities in all the countries laparoscopic gastric plication is an emergent restrictive procedure which claimed to be low cost because they do not need staplers and carries less complications as compared to laparoscopic sleeve gastrectomy. We present here a 37 years female who was operated for morbid obesity four months back where she underwent laparoscopic gastric plication with no immediate post operative complication and her wight loss was adequate.

Two days before presentation to our emergency department she started to complains of sever attacks or upper abdominal pain and vomiting clinical examination was unremarkable apart of abdominal tenderness in left upper abdomen. all blood routine were normal and all inflammatory markers were within normal range.CT abdomen showed large cystic lesion around the greater gastric curvature containing fluid and raised possibility of collection.

Patient was admitted to hospital, in despite of medical treatment her pain persists and necessitate immediate laparoscopic exploration. gastro-gastric hernia at the greater curvature through loosen Ethibond suture that was used to plicate the stomach in the previous surgery.

We released the suture to liberate the strangulated stomach which is not gangrenous. re-plication was not possible because of the extensive gastric wall edema and as preoperative discussion with the patient she refused conversion to sleeve gastrectomy no intervention was done.

Post surgery patient was free of symptoms and tolerating oral diet and discharged home on third post operative day with no complications.

Gastro-gastric herniation could progress to gastric wall gangrene which will result in high morbidity and even mortality. high index of suspicion is required to diagnose the condition

Preoperative patient counseling is important to explore the surgical options if deemed necessary to convert to another bariatric procedure.

\section{P088-BARIATRICS-Laparoscopic}

\section{REVISION IN 1000 CONSECUTIVE PATIENTS WHO HAD SLEEVE GASTRECTOMY FOR MORBID OBESITY IN A SINGLE CENTER}

\section{K. Chouillard, A. d'Alessandro, L. Chahine}

General And Digestive Surgery, Poissy Medical Center, POISSY, France

Background: Bariatric surgery is the best available, long-term treatment for morbid obesity Currently, laparoscopic sleeve gastrectomy (SG) is the most commonly performed bariatric procedure in France. Despite its safety and efficacy, long-term complications of SG are not rare including gastro-esophageal reflux disease (GERFD), twisting, stenosis, insufficient weight loss, and weight regain. The goal of this study was to analyze the pattern and short-term results of surgical revision in patients with SG.

Methods: Revisional bariatric surgery, regardless of its motivation, was always a multidisciplinary decision after clinical, biological, endoscopic, and radiological assessment. Patients who had revisional surgery after SG were retrospectively identified and subsequently divided in 4 subgroups according to preoperative body mass index (BMI) $(<$ or $>50 \mathrm{~kg} / \mathrm{m}<\sup >2<$ sup $>$ ) and the presence or not of GERD. The minimal follow up period was 12 months.

Results: Between December 2004 and September 2015, 1000 patients had SG. 114 patients were lost to follow-up (11.4\%). The rate of excess weight loss (EWL) $>50 \%$ was $68 \%$. Out of 886 patients, 94 had a revisional surgery $(10.6 \%)$ for inadequate weight loss, GERD, or stenosis, respectively. The performed procedures included Roux en Y Gastric Bypass (60\%), re-Sleeve (18\%), Duodenal Switch (12\%), Single Anastomosis Duodeno-Ileal Shunt (6\%), and seromyotomy (4\%). Median interval from the initial SG to conversion was 29 months (18-81). Median operating room time was $170 \mathrm{~min}$ (range 100-290). Median length of stay was $72 \mathrm{~h}$ (range 48-120). Mortality rate was nil. Overall complication rate was $8 \%(8 / 94)$. The follow-up rate at 12 months was $96 \%$. Satisfaction score at 12 month was $88 \%$.

Conclusions: Revisional options after SG are safe and lead rarely to complications. Nowadays, SG could be considered as the first step of a potentially multi-step bariatric pathway. Longe follow-up is needed to establish the algorithm of choice of the optimal surgical option for post SG revision. 


\section{P089-BARIATRICS-Laparoscopic}

\section{IMPLEMENTING ERAS FOR LAPAROSCOPIC SLEEVE} GASTRECTOMY

${ }^{1}$ Anesthesiology, Beth Israel Deaconess Medical Center, BOSTON, United States of America

${ }^{2}$ Surgery, Beth Israel Deaconess Medical Center, BOSTON, United States of America

Laparoscopic sleeve gastrectomy has become the most popular metabolic operation in the United States for weight loss. Our average length of stay in hospital was 2.6 days. Enhanced recovery pathways for coloretal and cardiac operations have show faster recuperation than non ERAS clinical pathways. The aim of our study was to implement an ERAS for sleeve gastrecomy at an academic medical center.After literature review, we created a multidisciplinary team (anesthesiologists, bariatric surgeons, dieticans, nurse educators, pharmacist) to establish protocol for diet, medications, CPAP, TAP block. Goal was narcotic sparring. Protocol was adopted at two MBSAQIP accredited hospitals.All patients undergoing sleeve gastrectomy were offered ERAS pathway. Outcome variables were tracked by MBSAQIP nurse data extractor. Need for narcotics and length of hospitalization were recorded.Preliminary results showed improved outcome with narcotic sparring ERAS protocol. $60 \%$ of patients were discharged post operative day 1 . Most patients were discharged without prescription for narcotics. ERAS with multimodal pain management shortens hospital stay.
B. Jones ${ }^{1}, \underline{\text { D. Jones }}{ }^{2}$, A. $\mathrm{Ku}^{1}$

\section{P091-BARIATRICS-Laparoscopic}

INDOCYANINE GREEN ENHANCED FLUORESCENCE (ICG)-A VALUABLE TOOL IN PREVENTING POSTOPERATIVE LEAKAGE IN METABOLIC SUGERY

\section{S.I. Filip, I. Hutopila, C. Copaescu}

General Surgery, Ponderas Academic Hospital, BUCHAREST, Romania

Introduction: Leakage remains one of the most dreadful complications in metabolic surgery. The main cause of leakage is poor tissue oxygenation due to inadequate vascular perfusion. The study of intraoperative tissue perfusion in real time due to ICG enhanced fluorescence could provide valuable information for the surgeon in order to prevent postoperative fistula. Aim: to present our experience in using ICG enhanced fluorescence in laparoscopic bariatric surgery

Material and method: In 30 cases of gastric sleeve, 12 cases of gastric bypass and in 10 cases of revisional surgery or redo cases we used intraoperative ICG mediated fluorescence to assure the optimal vascularization of the involved tissues.

In our video we present intraoperative aspects before and after using ICG in different cases.

Results: In all cases of primary gastric sleeve and gastric bypass with intraoperative use of ICG we did not encounter inadequate perfusion.

In one case of redo gastric bypass after failed vertical banded gastroplasty for morbid obesity despite intraoperative laparoscopic normal aspect of the gastro-jejunal anastomosis, ICG mediated fluorescence allowed to identify an unexpected ischemic anastomosis and we could prevent consecutive postoperative leakage.

Discussion: Presented cases are discussed and result with referral to literature is made. Conclusion: Intraoperative use of ICG is a valuable tool in assessing the perfusion of the tissues and provide essential information for the surgeon in order to avoid postoperative leakage.

\section{P092-BARIATRICS-Laparoscopic}

\section{P090-BARIATRICS—Laparoscopic}

SURGICAL TREATMENT OF THE GASTROBRONCHIAL FISTULA AFTER GASTRIC SLEEVE

\section{Andrei ${ }^{1}$, C.A. Copaescu ${ }^{2}$}

${ }^{1}$ Bariatric Surgery, Ponderas Academic Hospital, BUCHAREST, Romania, ${ }^{2}$ Surgery, Ponderas Academic Hospital, BUCHAREST, Romania

Background: Gastrobronchial fistula is a rare but complex and difficult to manage complication after bariatric surgery.The treatment can be endoscopic, surgical or combined.

We aim to present the management and the particular aspects of the surgical technique in a gastrobronchial fistula after gastric sleeve

The mean time between intervention and diagnosis is 6.7-7.2 months.

Methods: Between 2011 and 2018, 4253 laparoscopic gastric sleeve resections were performed in our Bariatric Center. We had one case of gastrobronchial fistula associated with an inferior lobe abscess of the left lung, diagnosed 3 months after the gastric sleeve. The patient was subject for medical treatment for $24 \mathrm{~h}$, than a laparoscopic intervention was performed in order to drain the lung abscess and the gastric fistula and to place a feeding jejunostomy. 2.5 months after this intervention (5.5 months after gastric sleeve) a laparoscopic Roux-en-Y fistulojejunostomy was performed. The evolution was monitorized with blood tests, upper GI contrast series and CT scans.

Results: The surgical drainage of the lung abscess, along with the antibiotherapy, controlled the infection and allowed the lung cavity to reduce in size, and thus the drainage tubes introduced in the thorax through the diaphragmatic orifice were retracted progressively. Also, the feeding jejunostomy allowed a proper nutrition for the patient with a good recovery. However, 2.5 months after the drainage intervention, the gastric fistula was not healed, and a decision to interrupt the communication with the lung cavity was made, by creating a laparoscopic fistulojejunostomy. After this, the evolution was favorable, with the healing of the lung cavity, oral feeding was permitted and the jejunostomy was suppressed.

Conclusions: The treatment of the gastrobronchial fistula is complex (medical, endoscopic o surgical), phased and long lasting until healing. Surgery was our initial choice for treatment due to the existence of the lung abscess, which needed to be drained.

Key words: gastrobronchial fistula, lung abscess, laparoscopy, fistulojejunostomy

\section{POSTOPERATIVE BLEEDING RISK IN BARIATRIC SURGERY. COMPARISON OF TWO TECHNIQUES OF STAPLED LINE REINFORCEMENT}

\section{B.I. Banescu, C. Copaescu}

Surgery, Ponderas Academic Hospital, BUCURESTI, Romania

Aim: to compare the effectiveness of two different techniques used to control the postoperative bleeding in bariatric surgery: over sewing the entire gastric stapled line and placing hemostatic clips on the bleeders along the stapled lines .

Methods: Our active protocol for controlling bleeding sources started in 2012 in Ponderas Academic Hospital. During the first period (between 2012 and 2014) hemostasis was provided by placing clips at the level of the gastric staple line. During the second period (from 2015 until present) hemostasis was provided by over sewing with a monofilament running suture the entire gastric stapled line.

Results: Between 2012 and 2018, 4237 bariatric patients were operated in our Center. During the first period (2012-2014), including 1270 patients, hemostasis with clips has been performed in all cases. However, among these cases nine patients required reoperation for early postoperative bleeding. In five cases a bleeding source from the stapled line was identified while in 4 cases no identifiable source was found. During the second period (2015 to present) 2967 patients were submitted to bariatric surgery and hemostasis was performed by over sewing with a running suture. Among these cases reoperation for postoperative bleeding was needed in 13 cases $(0.4 \%)$, but no bleeding from the staple line being encountered $(0 \%)$. The difference has statistical significance. No significant complications related to the use of this type of reinforcement were encountered.

Conclusions: Over sewing the gastric stapled line in bariatric surgery is superior to hemostatic clip application in preventing the postoperative bleeding from the stapled line postoperative bleeding. A protocol of active search of the bleeders during the bariatric procedure should be implemented and respected in all the cases. 


\section{P093-BARIATRICS-Laparoscopic}

\section{STUDY OF FIVE CASES WITH INTESTINAL ENDOMETRIOSIS PERFORMED LAPAROSCOPIC SURGERY}

\section{H. Kondo, S. Yamaguchi}

Gastroenterological Surgery, Saitama Medical University International Medical Center, HIDAKA-SHI, SAITAMA, Japan

Intestinal endometriosis is a rare disease which is associated with about 10 to $30 \%$ of patients with endometriosis, and it is favorable to the rectum and sigmoid colon. Here we report 5 cases (shown in the table) underwent laparoscopic resection for intestinal endometriosis. There were no postoperative complications in all cases, and all patient was discharged on $5-8<\sup >$ th $</$ sup $>$ postoperative day. Before the operation, 2 of 5 patients were diagnosing intestinal endometriosis, and it was difficult to preoperatively diagnose. Among them, the symptoms at the time of menstruation were clear was one case. In case of submucosal tumor, preoperative diagnosis seems difficult. Additional image examination at menstruation may be useful for diagnosis. D2 dissection was performed for case 1,2, 4 because malignant disease could not be denied as a preoperative diagnosis. 2 of them were strongly doubted endometriosis in surgical findings. In intestinal endometriosis surgery, pelvic adhesions and fibrosis are often advanced. In the sigmoidectomy, the average operation time was $152 \mathrm{~min}$ and the blood loss was $10 \mathrm{ml}$. In the rectal resection, the average operation time was $282 \mathrm{~min}$ and the blood loss was $17 \mathrm{ml}$. In case 1 and 5 , pelvic adhesion was severe, residual rectum could not be straightened, and side to side anastomosis was performed. In intestinal endometriosis surgery, intestinal anastomosis method should be considered flexibly. Conclusion: Laparoscopic surgery for intestinal endometriosis was safe, but technically difficult because of fibrosis and adhesion. It is important to accurately diagnose from clinical symptoms and image also intraoperative findings. Anastomotic method should be decided according to the case.

\section{P094-BARIATRICS—Laparoscopic}

\section{ENDOSCOPIC MANAGEMENT OF OBSTRUCTION AFTER LAPAROSCOPIC SLEEVE GASTRECTOMY}

\section{F. Turcu ${ }^{1}$, C. Balahura ${ }^{2}$, I. Doras ${ }^{2}$, A. Constantin ${ }^{2}$, C. Copaescu ${ }^{1}$}

${ }^{1}$ General Surgery, Ponderas AH, BUCHAREST, Romania; ${ }^{2}$ Gastroenterology, Ponderas AH, BUCHAREST, Romania

Aims: Owing to the increase in the number of laparoscopic sleeve gastrectomy (LSG), a rare complication, the symptomatic obstruction (SO) will be more often encounter. The purpose of this study is to evaluate the safety and effectiveness of endoscopic pneumatic dilation for the treatment of SO after LSG

Methods: We have prospectively analyzed the data of a series of 46 patients with LSG who were referred to endoscopy for SO in Ponderas Academic Hospital from May 2011 to September 2018. The average age was $47 \pm 10$ years and the average body mass index (BMI) before LSG was $38.6 \pm 7.8 \mathrm{Kg} / \mathrm{m}^{2}$.

Results: From the 46 patients referred to endoscopy 10(21.7\%) have had previous reinterventions: 6 for stapler line leakage with abscess and/or fistula, 2 for migration of the gastric tube and 2 for twisted gastric tube. There have been 79 procedures ( 34 with through the scope balloons, 33 with $30 \mathrm{~mm}, 10$ with $35 \mathrm{~mm}$ and 2 with $40 \mathrm{~mm}$ balloons), an average of $1.7 \pm 1.1$ per patient. We have encounter 1 perforation but any hemorrhage or death. We have loose from follow up 2 patients and one was done in the last month. The follow up for the remaining 43 patients is $654 \pm 317$ days. Over all we have $90 \%$ (39 from 43 patients) good results with endoscopic dilation.

Conclusion: Endoscopic pneumatic dilation is a safe and effective procedure and should be the front line choice in the management of SO after LSG.

\section{P095-BARIATRICS-Laparoscopic}

SURGICAL COMPLICATIONS IN SLEEVE GASTRECTOMYWHAT SHOULD ONE EXPECT

\section{Slavu, L. Alecu, D. Mihaila, A. Tulin, V. Braga}

General Surgery Department, Emergency Clinical Hospital, Prof. Dr. Agrippa Ionescu, BUCHAREST, Romania

Aim: The aim of the study was to identify and highlight some of the complications one can encounter in bariatric surgery-specific-sleeve gastrectomy and discuss the therapeutic options one has at his disposal. Methods: The study was retrospective. We identified a number of 260 patients which had a sleeve gastrectomy done in our clinic for a 2 year period. Of these 10 had important surgical complications encountered during the surgery or in postoperative care.

Results: The group included 260 patients, with an average BMI of $>40$ $\mathrm{kg} / \mathrm{m}^{2}$. Average hospital stay was 7 days, with an average of 4.5 days which increased to 30 days when fistulas were encountered. The most frequent surgical complications were bleeding from the gastric suture (6) and gastric fistula (4 cases). Other complications encountered were wound hematoma. Surgery was required in 4 of the 6 cases of bleeding and 3 of the fistula cases required reintervention. One case was resolved with endoscopic stenting.

Conclusions: Laparoscopic gastrectomy is considered a safe procedure with good results for the patient. Although complications are rare they pose a series of technical difficulties for the surgeon due to the weight of the patient and frequent comorbidities which come with obesity. A thorough understanding of the symptoms and good follow-up ensures the best results.

\section{P096-BARIATRICS-Laparoscopic}

\section{A ONE-STEP CONVERSION FROM GASTRIC BANDING TO LAPAROSCOPIC ROUX-EN-Y GASTRIC BYPASS IS AS SAFE AS A TWO-STEP CONVERSION: A COMPARATIVE ANALYSIS} OF 1383 PATIENTS

\section{J. Colpaert, B. Dillemans}

Department of Surgery, AZ Sint-Jan, BRUGGE, Belgium

Aims: To achieve additional weight loss or to resolve band-related problems, a laparoscopic adjustable gastric banding (lagb) can be converted to a laparoscopic roux-en-y gastric bypass (rygb). There is limited data on the feasibility and safety of routinely performing a single-step conversion. We assessed the efficacy of this revisional approach in a large cohort of patients operated in a high-volume bariatric institution. To the best of our knowledge this series represents the largest single-center study on conversion from lagb to rygb

Methods: Between october 2004 and december 2017, a total of 1383 patients who underwent lagb removal with rygb were identified from a prospectively collected database. In all cases, a single-stage conversion procedure was planned. The feasibility of this approach and peri-operative outcomes of these patients were evaluated and analyzed.

Results: A single-step approach was successfully achieved in 920 (86.5\%) of the 1383 patients. During the study period, there was a significant increase in performing the conversion from lagb to rygb single-staged. No mortality or anastomotic leakage was observed in both groups. Only 49 patients $(3.6 \%)$ had a $30-\mathrm{d}$ complication: most commonly hemorrhage $(\mathrm{n} ?=? 23 / 49)$, with no significant difference between the groups.

Conclusion: Converting a lagb to rygb can be performed with a very low morbidity and zero-mortality in a high-volume revisional bariatric center. With increasing experience and full standardization of the conversion, the vast majority of operations can be performed as a single-stage procedure. Only a migrated band remains a formal contraindication for a one-step approach. 


\section{P097-BARIATRICS—Physiology}

\section{GASTROESOPHAGEAL REFLUX DISEASE EVALUATION} BEFORE AND AFTER BARIATRIC SURGERY: PRELIMINARY Results:

A. Balla $^{1}$, L. Palmieri ${ }^{1}$, D. Corallino ${ }^{1}$, F. Meoli ${ }^{1}$, D. Badiali ${ }^{2}$, A. Pronio $^{1}$, P. Ursi ${ }^{1}$, S. Quaresima ${ }^{1}$, A. Paganini ${ }^{1}$

${ }^{1}$ Department of General Surgery and Surgical Specialties, Paride Stefanini, Sapienza University of Rome, ROMA, Italy; ${ }^{2}$ Department of Internal Medicine and Medical Specialties, Sapienza University of Rome, ROME, Italy

Aims: The aim of the present study is to evaluate the evolution of gastroesophageal reflux disease (GERD) in obese patients who are candidate to bariatric surgery.

Methods: Patients underwent laparoscopic sleeve gastrectomy (LSG) or gastric bypass (LGB) based on preoperative GERD assessment by esophagram, endoscopy, 24 h-pH-manometry and GERD Health Related Quality of Life (HRQL) questionnaire. Patients with esophagitis were excluded from the study. The questionnaire was administered again 6 and 12 months after surgery. Endoscopy, esophagram and $24 \mathrm{~h}$-pH-manometry were repeated one year after surgery.

Results: From July 2017 to November 2018, 13 patients were enrolled. Nine patients underwent LSG. The mean pre-operative HRQL score was 5 (range 0-34). At pH-manometry, mean percentage time of acid reflux in orthostatism was 2.2 (range 0.2-5) and in clinostatism 3.4 (range 0-20.1). The mean DeMeester score at the distal electrode was 15 (range 0.7-78.9). Four patients underwent LGB. The mean pre-operative HRQL score was 1.2 (range 0-4). At pH-manometry, mean percentage time of acid reflux in orthostatism was 30.3 (range 4.3-84.8) and in clinostatism 31.4 (range 0-95.2). The mean DeMeester score at the distal electrode was 153.5 (range 18.1-416). Six months after surgery the mean HRQL score, was 2 $(0-6)$ in 5 patients underwent to LSG and $0.6(0-2)$ in 3 patients underwent LGB. Twelve months after surgery the mean postoperative questionnaire score was $6(0-12)$ in 2 patients who underwent LSG. At pH-manometry the mean percentage time of acid reflux in orthostatism was 7.3 (range 6-8.6) and in clinostatism 5.3 (range 0.3-10.4). The mean DeMeester score at the distal electrode was 33.1 (13.7-52.5).

Conclusions: In asymptomatic patients, complete GERD evaluation before bariatric surgery allows better selection of surgical procedure, to reduce the postoperative occurrence of severe or de novo GERD. Postoperative GERD evaluation provides useful data regarding the impact of LSG on gastroesophageal reflux. A larger patient sample size is required.

\section{P098-BARIATRICS—Physiology}

GASTROESOPHAGEAL REFLUX DISEASE QUESTIONNAIRE IN BARIATRIC POPULATION: DIAGNOSTIC USEFULNESS

L. Navarro Morales ${ }^{1}$, I. Alarcon del Agua ${ }^{1}$, T. Yang ${ }^{1}$, N. García Fernandez $^{1}$, M. Socas Macias ${ }^{1}$, F. Lopez Bernal ${ }^{1}$, J. Padillo Ruiz ${ }^{1}$, S. Morales Conde ${ }^{2}$

${ }^{1}$ Cirugía General Y Del Aparato Digestivo, Hospital Universitario Virgen Del Rocio, SEVILLA, Spain; ${ }^{2}$ Surgery, Hospital Virgen del Rocio, SEVILLA, Spain

Introduction: Gastroesophageal reflux disease (GERD) is a chronic and frequent disorder with wide socio-sanitary and individual consequences. Classically, it is characterized by the presence of heartburn and acid regurgitation, being diagnosed by anamnesis. However, this is not always the case. Currently, clinical questionnaires have awaken interest because they are simple, reproducible and practical instruments that can help to quickly reach the diagnosis and guide the treatment.

One of them, Reflux Disease Questionnaire (RDQ). has been validated for the diagnosis of GERD in Spanish.

The aim of this study is to assess the the results of RDQ questionnaires compared to the results of pH-metry and gastroscopy taken as gold standards in GERD diagnosis, in a group of patients candidates to Bariatric Sugery, were preoperative GERD plays an important role in the decision of the surgical technique.

Methods: 63 obesity patients completed both Spanish validated versions of RDQ and $\mathrm{pH}$ test, and only 52 obesity patients with gastroscopy and RDQ. We compared the Sensitivity, Specificity, Positive predictive value(VPP), Negative predictive value(VPN), Positive likelihood ratio(LR +$)$, Negative likelihood ratio(LR -$)$ between $\mathrm{RDQ}$ and $\mathrm{pH}-$ metry or gastroscopy. RDQ was considered positive if questionnaire score was $>40.32$. $24 \mathrm{~h} \mathrm{pH}$-metry was considered positive if Demeester score was $>14.7$. Finally, if the patient was diagnosed esophagitis with gastroscopy, we considered that result as positive.

Results: Between the Ph-metry and the RDQ scores, the sensitivity of RDQ in detecting reflux was $25.0 \%$ and the specificity was $70.0 \%$. The Positive predictive value(VPP) was $81.25 \%$, the Negative predictive value(VPN) was $15.22 \%$, the positive likelihood ratio(LR +) was $88.33 \%$, the Negative likelihood ratio(LR -) was $107.14 \%$. Between gastroscopy and RDQ scores, the sensitivity of RDQ was $12.5 \%$ and the specificity was $71.11 \%$. The Positive predictive value(VPP) was $7.14 \%$, the Negative predictive value(VPN) was $82.05 \%$, the positive likelihood ratio(LR +) was $43.27 \%$, the Negative likelihood ratio(LR -) was $123.05 \%$.

Conclusion: The use of RDQ to diagnose GERD cannot validated in bariatric patients compared with objective diagnostic tests. New cut off point for diagnosis of GERD in bariatric population should be reassessed. 


\section{P099-BARIATRICS—Physiology}

SHORT-TERM RESULTS OF LAPAROSCOPIC VERTICAL GASTRECTOMY VERSUS LAPAROSCOPIC ROUX-Y BYPASS IN BARIATRIC SURGERY UNIT

\author{
M. Fornell Ariza, A. Mayo Ossorio, A. Bengoechea Trujillo, J.M. \\ Pacheco Garcia \\ Unidad de Cirugia Esofagogastrica y Bariatrica, Hospital \\ Universitario Puerta del Mar, CADIZ, Spain
}

Aims: vertical calibrated gastrectomy (usually know as gastric sleeve) as unique technique gives better results than the Roux $\mathrm{Y}$ bypass in terms of improvement of anthropometric measures, reduces comorbidities and has a lower rate of postsurgical complications, with an improvement of quality of life.

Material and methods: An observational, longitudinal, retrospective and comparative study with 95 patients, aged 18-65 years,during a period of 3 years. Everyone must comply with the Protocol of the unit. Demographics of the population and the anthropometric data will be measured in the Presurgical consultation, the month and the year after the surgery: weight, height, BMI, weight loss percentage,BMI percentage and percentage of excess weight lost. We took data on the cardiovascular risk by the Framingham SCORE. The quality of life is measured by BAROS scale. Mayor Comorbidities are hypertension, diabetes, Dyslipidemia. Complications will be measured in absolute frequencies. For de statistical study, we apply type T student or Chi square being statistically significant $\mathrm{p}$ equal to or less than 0.05 .

Results: There was not statistically significant difference between the 2 techniques of surgery month $(p=0,83)$, but they were evident to the year of the same ( $p 0.003)$. Not gender or age differences were apparent. Mayor Complications did not appear in gastrectomy (no leaks), highlighting the number of bleeds with this surgical technique. The bypass there were two leaks. There was no statistically significant difference in cardiovascular risk $(p=0,07)$ between the two techniques. There was a more significant decrease in number of comorbidities in gastrectomy against the bypass, with a total disappearance of patients with Dyslipidemia. There were no statistically significant differences in BAROS score, although it was higher in gastrectomy.

Conclusions:-The vertical gastrectomy as unique technique can be considered superior in the short term, as well as safe, according to the AEC quality parameter.

We think it will be necessary to continue their studies into the medium-long term.

\section{P100—BARIATRICS—Physiology}

\section{THE IMPACT OF THE BARIATRIC SURGERY IN PANCREATIC BETA CELL POPULATION AND CARBOHYDRATE METABOLISM IN A HEALTHY RAT MODEL}

J. Falckenheiner $^{1}$, A. Camacho ${ }^{2}$, G. Perez Arana ${ }^{3}$, J.A. Prada $^{3}$

${ }^{1}$ General Surgery, Puerta Del Mar Hospital, CADIZ, Spain; ${ }^{2}$ General Surgery, Puerto Real Hospital, CADIZ, Spain; ${ }^{3}$ Anatomy And Embriology, Cadiz University, CADIZ, Spain

Aims: Analyze the impact of different bariatric surgeries technics in carbohydrate metabolism and pancreatic beta cell population of none obese adult Wistar rats.

Methods: We used twenty healthy not obese adult Wistar rats divided in five groups randomly assigned. Each with $n=4$. The control groups were divided into Fasting Control $(\mathrm{F})$ and SHAM (Surgical Control). The surgical groups were separated into Vertical Gastrectomy (GS), 50\% resection of the middle small bowel (RI50) and gastric bypass (GB). In each group was assessment: beta cell mass modifications, pancreatic islets histomorphometry, proliferation, apoptosis and neogenesis in beta-cell pancreatic population; intraperitoneal glucose test tolerance, body weight and food intake. Statistical analysis as evaluated using mann whitney test. Results: the malabsorptive and restrictive group have a significantly smaller increase weight than the control groups. The intraperitoneal tolerance glucose test reports incremental glucose area under curve (AUC) was significantly higher in the malabsorptive group and lower in the restrictive group compare to the control groups during the second $(\mathrm{p}<0.01)$ and third $(\mathrm{p}<0.05)$ month of the study. The beta-cell mass was significantly higher in the RI50 group compared with control groups respectively. There was a significantly increased number of beta-cell per pancreatic insulin positive area in GS and GB. Proliferation was significantly increased in RI50 and GB group, and significantly decreased in SG compared. There was no significantly difference during apoptosis assessment among surgical and control groups. In neogenesis differences between groups were assessed qualitatively by the presence PDX - 1 expression, being higher in RYGB.

Conclusions: The endocrine pancreas in our model is altered by the anatomical and functional conditions arising from surgical techniques. Carbohydrate metabolism conditions are affected by temporary adaptive processes due to surgical alternatives. There is a hyperplasia and hypertrophy of the beta cells in surgeries with a malabsorptive component, as well as greater neogenesis These results could explain part of the existing relationship between the enteropancreatic axis and the existing incretins.

\section{P101-BARIATRICS—Physiology}

THE IMPORTANCE OF THE PSYCHOLOGICAL EVALUATION IN PRE- AND POSTOPERATIVE BARIATRIC EVALUATION PROGRAM

\author{
M. Buza, C. Copaescu
}

General Surgery, Ponderas Academic Hospital, BUCHAREST, Romania

Introduction: Nowadays, we have high volumes of obese patients for whom surgery is the answer, but unfortunately the psychological evaluation has no standard recomandation in preoperative evaluation of bariatric patients. It is argued that surgery success, in addition to the operation itself, relies on behavioral changes and that one of the goals of the preoperative assessment is to prepare the patient for the postoperative period, aiming to optimize surgical results.

Aim: Although no formal standard exists in the literature, there is growing recognition of the important elements to be addressed and the appropriate means for collecting the necessary data to determine psychological readiness for these procedures.

Methods: Information regarding the components of the clinical interview and the specific measures used for psychological testing are discussed. Given the limited data on predicting success after surgery, determining psychological contraindications for surgery is addressed. Additionally, the multiple functions served by the psychologist during this assessment procedure are highlighted along with the value of this procedure in the patients' preparation for surgery as well as the postoperative follow-up. In our Center of Excelence for Bariatric and Metabolic Surgery (COE) we introduced since 2013 a mandatory pre- and postoperative psychological evaluation for all patients addressing the metabolic program.

Results: Psychological evaluation of patients before bariatric surgery is a critical step, not only to identify contraindications for surgery, but also-and more so- to better understand their motivation, readiness, behavioral challenges, and emotional factors that may impact their coping and adjustment through surgery and the associated lifestyle changes. Postoperative follow-up is necessary.

Conclusion: The psychological evaluation of the patient undergoing bariatric surgery is an invaluable piece of the larger pre- and post-surgical assessment, aiming better results in the short and long term after bariatric surgery.

\section{P102-COLORECTAL-Benign}

\section{LAPAROSCOPIC EXCISION OF MESENTERIC CHYLOUS (LYMPHATIC) CYST}

\section{E. Imam, H. Fahmy}

General surgery, Royal commission medical center, YANBU INDUSTRIAL CITY, Saudi Arabia

Introduction: A mesenteric cyst is defined as a benign abdominal tumors that is located in the mesentery of the gastrointestinal tract, identified in $\sim 1$ of 100,000 hospital admissions. Mesenteric chylous cysts are rare pathologic entities that often present with unspecific symptoms. The preoperative diagnosis requires all the common abdominal imaging techniques. Usually the correct diagnosis may be made only at the operation stage or during the histological examination. All mesenteric cyst should be resected in order to avoid their complications, complete surgical resection is recommended and curative in the majority of cases with a low risk of local recurrence.

The laparoscopic approach is the gold standard in the treatment of intraabdominal Mesenteric chylous cyst. Laparoscopic resection provides less pain, shorter hospital stay, and early recovery for the patient.

Case report: We report a case of 28-year-old Saudi woman who presented to our clinic complaining of upper abdominal pain and mass in the epigastrium for one week, no history of nausea, vomiting, or recent changes in bowel habits. Her medical and family histories were clear and she had never had any abdominal interventions. Abdominal palpation revealed a smooth-surfaced mass palpable in the left upper quadrant, ultrasonography and with computed tomography of the abdomen revealed an approximately $93 \times 72 \times 66 \mathrm{~mm}$ unilocular cyst closely related to the mesentery in the left side of upper abdomen not related to the pancreas. The cyst was excised by laparoscopy complete surgical excision to avoid recurrence within healthy borders, it is contained milky white fluid. The histopathological findings were chronic inflamed mesenteric cyst. A review of the literature considering this rare entity was also performed to evaluate our treatmen strategy.

Conclusion: Mesenteric chylous cysts represent a diagnostic challenge and they should be considered when a physician encounters an intraabdominal mass. Usually the correct diagnosis may be made only at the operation stage or during the histological examination. The treatment of choice is the complete surgical excision that can be safely performed by laparoscopy 


\section{P103-COLORECTAL_Benign}

\section{A COMPERATIVE ANALYSIS OF THE PATIENTS WHO OPERATED WITH LAPAROSCOPIC OR OPEN Method: FOR ACUTE APPENDICITIS}

\section{N. Ozlem}

General surgery department, AHIEVRAN UNIVERSITY, KIRSEHIR, Turkey

Laparoscopic appendectomy is an operation that is done in increasing rate, between all other operations made for acute appendicitis. aim is to make a comperative analysis in laparoscopic and open appendectomy was done in our clinic

Material method: between april 2015 -november2017 in our clinic the files of the patients who have laparoscopic and open appendectomy retrospectively was review; the demographics and operative time and length of hospital stay was recorded and compared.statistical data analysessed with spss.

Results: the patients were divided to the patients had laparoscopic appendectomy and the patients had open appendectomy.there are $63(67.8 \%)$ male and 30(32.2\%) female and $93(\mathrm{M}+\mathrm{F})$ in total patients in laparoscopic appendectomy group. Open appendectomy group have $60(41.7 \%)$ female and $84(58.3 \%)$ male, $144(\mathrm{~F}+\mathrm{M})$ in total patients. Laparoscopic appendectomy group consist more female patients than male ones this difference is statistically significant $(\mathrm{p}<0.001)$. the mean age in laparoscopic appendectomy group patients is $35.8 \pm 14.8(17-71)$ the mean age of open appendectomy group of patients is $33.8 \pm 16.1$ (15-84). There is no significant difference between the two group of patients age statistically $(\mathrm{p}=0.327)$. the mean operative time in lap appendectomy group of patients is $78.01 \pm 37$ (29-240)min. In open appendectomy group of patients this time is $51.6 \pm 17.6(24-110) \mathrm{min}$. Operative time is statistically significant longer in laparoscopic appendectomy group of patients. $(\mathrm{p}<0.001)$. length of hospital stay of laparoscopic appendectomy group of patients $2.39 \pm 1.68$ (1-9) day. Lohs of open appendectomy group of patients is $2.02 \pm 1.73(1-11)$ day.but there is not statistically significant differencesses between the groups of patients lohs. $(\mathrm{p}=0.107)$

Conclusion: laparoscopic appendectomy is preffered to aid the diagnosiss in female patients group.but laparoscopic appendectomy cause a longer operative time significantly, but it does not have a shorter length of hospital stay.

\section{P104-COLORECTAL-Benign}

\section{CASES OF LAPAROSCOPIC SURGERY FOR APPENDICEAL DIVERTICULITIS}

H. Murase ${ }^{1}$, H. Iseki ${ }^{1}$, A. Ogawa ${ }^{1}$, K. Kamachi ${ }^{1}$, A. Mitsuoka ${ }^{1}$, M. Hayashi ${ }^{1}$, S. Park ${ }^{1}$, A. Fukuuchi ${ }^{1}$, R. Oono ${ }^{2}$

${ }^{1}$ Surgery, Tokyo Shinagawa Hospital, TOKYO, Japan; ${ }^{2}$ Surgery, Nitobe Memorial Nakano General Hospital, TOKYO, Japan

Background: Diverticulum of appendix is relatively rare, and appendiceal diverticulitis was reported to have a higher risk of perforation than appendicitis. In the US and Europe, because of the high risk of perforation, preventive appendectomy is recommended to appendiceal diverticulosis, even if the patient has no abdominal pain.

Methods: We retrospectively reviewed the records of 672 post-operative patients, who were diagnosed appendicitis or appendiceal diverticulitis on the pathological findings in our institution from January 2012 to October 2018. All patients were performed computed tomography (CT) before operation. 652 patients underwent laparoscopic surgery, including appendectomy, cecal resection, ileocecal resection and right hemicolectomy, while 20 patients underwent open surgery. Total of 12 cases of appendiceal diverticulitis were analyzed in our study.

Result: 11 patients had abdominal pain before surgery. 4 patients were diagnosed appendiceal diverticulitis by preoperative CT. All patients underwent laparoscopic surgery (10 appendectomy, 1 cecal resection, and 1 ileocecal resection). On the pathological findings, perforation of appendix was found in 5 patients and the pseudo type of diverticula with no muscle layer was found in all patients. 660 patients with appendicitis were treated surgically during the same period. Among them, a perforation of appendix was found in 78 cases. The perforation rate was $11.8 \%$. On the other hand, the perforation rate of appendiceal diverticulitis was $58.3 \%$ in our study.

Conclusion: The perforation rate of appendiceal diverticulitis was higher than of appendicitis in our study. For the examination of the treatment strategy, including preventive appendectomy, the accumulation of more cases will be expected.

Case presentation: A 59-year-old man was referred to our hospital with right lower quadrant abdominal pain for 2 days. His fever was $38.4^{\circ} \mathrm{C}$. His white blood cell count was $30,200 / \mu l$, and C-reactive protein level was $8.3 \mathrm{mg} / \mathrm{dl}$. CT revealed multiple diverticula of cecum and appendix. Micro-abscess and free air were found around appendix. We diagnosed this case as appendiceal diverticulitis and laparoscopic appendectomy was performed. A perforation was found in resected appendix. Microscopic study revealed a pseudo-diverticulum. The inflammation of appendix was stronger in serous membrane side than in mucosa side. This finding accorded with appendiceal diverticulitis.

\section{P105-COLORECTAL—Benign}

SINGLE ACCESS LAPAROSCOPIC LEFT COLECTOMY WITH OR WITHOUT INFERIOR MESENTERIC ARTERY PRESERVATION: AN UPDATE OF OUR PREVIOUS Results:

\section{G. Merola ${ }^{1}$, U. Bracale ${ }^{2}$, J. Andreuccetti ${ }^{3}$, G. Pignata ${ }^{4}$}

${ }^{1}$ Gastroenterology, Endocrinology and Surgical Endoscopy, AUOP Federico II, NAPOLI, Italy; ${ }^{2}$ Gastroenterology, Endocrinology and Surgical Endoscopy, University Federico II, NAPOLI, Italy; ${ }^{3}$ General and Mininvasive surgery, San Camillo Hospital, TRENTO, Italy; ${ }^{4}$ General Surgery II, Civil Hospital of Brescia, BRESCIA, Italy

Introduction: In order to reduce the abdominal trauma and the length of scar incisions (also during laparoscopic surgery) many approaches during the last decade has been proposed, such as Single Access Laparoscopic Surgery (SALS). The aim of our paper was to update the data of our previous paper with a greater cohort of patients and a longer follow-up, also showing the single access laparoscopic left colectomy (SALC) technique in particular with inferior mesenteric artery preservation IMAP (Valdoni's technique)

Materials and methods: We made a retrospective analysis from October 2009 and October 2016 of all patients who underwent a SALS approach for colorectal disease in the department of General and Mininvasive Surgery of San Camillo Hospital of Trento. Statistical analysis was performed using IBM SPSS Statistics 23. Continuous data were expressed as mean \pm standard deviation (SD). Categorical data were expressed as absolute number and percentage. The results are presented as 2-tailed values with statistical significance if $\mathrm{p}$ values $<0.05$

Results: From October 2009 until October 2016, 72 SALC for colorectal surgery were performed in our unit. Of this 72, 58 were for left colectomy. In 12 cases we performed an IMAP. The SALC with IMAP were performed only in case of benign disease. The mean operative time was $149.74 \pm 27.93$. Only one intraoperative complication were recorded, that was a splenic capsule tear, resolved with apposition of fibrillar haemostats. According to Clavien Dindo classification there were in particular 2 grade II complications, a bleeding solved with blood transfusion and one pancreatitis solved with medical therapy; 2 grade IIIa complications that was anastomotic bleeding solved endoscopically (the two complications raised in patients with IMAP) and 2IIIb complications due to anastomotic leakage which needed reoperation. The mean length of incision was $3.64 \pm 0.86 \mathrm{~cm}$. Logistic regression did not show any correlation between IMAP and any complications.

Conclusion: In conclusion, SALC is a safe but very challenging technique which need a longer learning curve than the conventional laparoscopic one. In laparoscopic colectomy, also, IMAP seems to be safe and effective without correlation with post-operative complications also if performed in single access laparoscopic approach. 


\section{P106-COLORECTAL-Benign}

ACUTE APPENDICITIS IN A PATIENT WITH INTESTINAL MALROTATION AND HYPERSPLENISM, ABOUT A CASE

\author{
L.A. Vega Rojas ${ }^{1}$, S. Sánchez Cordero ${ }^{1}$, K. Oh-Uiginn ${ }^{2}$,

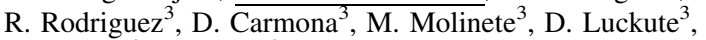 \\ C. Galmes ${ }^{3}$, J. Camps ${ }^{3}$
}

${ }^{1}$ General Surgery, Consorcio Sanitario de la Anoia, IGUALADA, Spain; ${ }^{2}$ General Surgery, Hospital de Igualada, IGUALADA, Spain; ${ }^{3}$ General Surgery, IGUALADA, Spain

Aims: To describe an infrequent anatomical variation that can give rise to diagnostic and therapeutic difficulties.

Methods: Patient with Ivermark syndrome (Situs ambiguus and polysplenia) with acute appendicitis and bibliographic review

Results: A 41-year-old male who consulted for flank and right hypochondrium pain of $18 \mathrm{~h}$ of evolution, associated with nausea without vomiting, no fever noir other symptoms. To the physical examination good general condition. Painful to palpation selectively on the flank and right hypochondrium, with involuntary defense and positive decompression at this level. The signs of Rovsing and Psoas were negatives.

In the analytical performed leukocytes of 17,000 with neutrophils in $82 \%$ and RPC (Reactive Protein C) in $22 \mathrm{mg} / \mathrm{L}$.

Abdominal CT (Computed Tomograph): Cecum and the ilio-cecal valve were visualized at the subhepatic level with tubular structure on the side and seemed to correspond to the cecal appendix which is increased in size $(12 \mathrm{~mm})$, with findings suggestive of acute appendicitis. Sigma and descending colon located in right hemiabdomen. Second per duodenal portion located anterior to the superio mesenteric artery. Superior mesenteric vein located to the left of the superior mesenteric artery, rotating around it, (radiological signs compatible with intestinal malrotation). No free fluid collections nor pneumoperitoneum.

Laparoscopic appendectomy on phlegmonous acute appendicitis without incidents. Correct post-operative course, being discharged at $48 \mathrm{~h}$; the pathological anatomy was reported as acute appendicitis in phlegmonous.

Conclusions: Ivermark syndrome is a genetic alteration with a multifactorial inheritance pattern, characterized by an alteration in the situation of the mesenteric vessels, which leads to abnormal rotation of the intestine during the embryonic period and alteration of the situation of different intra-abdominal organs, without a specific pattern that is pathognomonic, is associated with congenital heart anomalies between 50 and $90 \%$. Reaching adulthood only between 5 and $10 \%$ of them.

A case of acute appendicitis is presented in a patient with this anomaly, which can lead to diagnostic and therapeutic difficulties due to the anatomical variations involved. Abdominal tomography is the image method that provides the best performance for the diagnosis of acute pathologies in this type of patients.

\section{P107-COLORECTAL_Benign}

\section{SURGICAL MANAGEMENT FOR DIVING-INDUCED ACUTE ISCHEMIC COLITIS}

\section{J.K. Ju, H.D. Kwak}

Surgery, Chonnam National University Hospital, GWANGJU, Korea

Background: The clinical manifestations which occur in relation to decompression during scuba diving are variable. Mild symptoms have often been reported in gastrointestinal tract. This is one of the severe cases with gastrointestinal barotrauma. Ischemic colitis caused by air embolism very rare, therefore it is to be reported and discussed.

Case presentation: A 58-year-old man visited our emergency room with diffuse abdominal pain and bloody diarrhea 2 days ago. The patient was a skilled diver who took seafood through diving for 30 years. Two days before presenting, the patient had severe abdominal pain just after diving for $2 \mathrm{~h}$ at a depth of 30 meters. He was immediately transferred to a local hospital for hyperbaric oxygen therapy, but there was no improvement with the symptom. Abdomen CT angiography showed terminal ileal, ascending, sigmoid colonic and rectal decreased enhancement with wall thickening. Sigmoidoscopy showed diffuse huge ulcerative lesions and ischemic changes on mid rectum and sigmoid colon. Emergent subtotal colectomy and temporary loop ileostomy were done, and pathologic findings revealed diffuse mural infarct with serosal abscess formation in whole colon and transmural infarct in terminal ileum

Conclusion: Surgical approach could be one of the treatment options, though it depends on severity of the symptoms and the patients' conditions.

Figure legends

Figure 1. Resected specimen showed multifocal necrosis in terminal ileum, colon and rectum. Figure 2. (A) Thinning mucosa, fat necrosis, infiltration of inflammatory cells, and necrosis of serosa show typical transmural infarct findings in terminal ileum, (B) Mucosal crypt necrosis, inflammatory cell infiltration across all layers, and serosa suppurative inflammation and abscess show typical necrotizing inflammation in colon.

\section{P108-COLORECTAL—Benign}

\section{LAPAROSCOPIC TRANSANAL RESECTION OF RECTAL VILLOUS ADENOMA THROUGH GELPOINT PATH}

\section{Hussein}

Surgery, American University of Beirut Medical Center, BEIRUT, Lebanon

Aims: Laparoscopic Trans-anal Resection of Rectal Villous Adenoma through Gel point path Methods: I report my experience at the American University of Beirut Medical Center of 2 cases of villous adenoma $7 \mathrm{~cm}$ from the anal verge by trans-anal resection using Gelpoint path.The video showed the technical steps used for resection using Enseal followed by hook dissection alternating with hydro dissection and later suturing of the rectal mucosa.

Results: Patient was discharged $48 \mathrm{~h}$ post surgery.

\section{P109-COLORECTAL-Benign}

\section{LAPAROSCOPIC SEGMENTAL RESECTION OF SPLENIC FLEXURE FOR INTUSSUSCEPTION CAUSED BY COLONIC LIPOMA}

S. Martínez-Núñez, J. Pintor-Tortolero, N. García-Fernández, M.J. Cuevas-López, L. Navarro-Morales, F. Moreno-Suero,

L. Tallón-Aguilar, J. Padillo-Ruiz

General and Gastrointestinal Surgery Department, Virgen del Rocío Universitary Hospital, SEVILLA, Spain

Colonic lipomas are extremely uncommon benign tumours, with an incidence ranging between $0.035 \%$ and $4.4 \%$. Although they are most frequently asymptomatic, when colonic lipomas are $>$ $2 ? \mathrm{~cm}$, they may present symptoms such as constipation, abdominal pain or rectal bleeding. Most colonic lipomas typically occur in middle aged women and are located in the ascending colon and the caecum, while occurrence in other parts of the colon and rectum is rare. In this case report, we describe a lipoma that caused descendent bowel intussusception.

A 55-year-old male presented with longstanding history of constipation. Personal history of interest included active smoker, hypertension, hypercholesterolemia, psoriasis with joint affectation and Reiter syndrome. He had had no previous surgery. He attended the Emergency Services on 17th July 2018 with a two-day bowel obstruction, without fever or nausea, being attended by our Surgical Emergency Unit. He had been assessed during the previous months by Gastroenterology, with a colonoscopy that showed a $4 \mathrm{~cm}$ submucosal lesion that partially occluded descendent bowel, with inconclusive biopsy.

An abdominal contrast-enhanced computed tomography (CT) was performed, confirming a welldefined mass located in splenic flexure of descendent bowel, conditioning a large bowel intussusception, nevertheless with no obstructive acute signs.

The surgery was scheduled a few weeks later, performing a laparoscopic segmental resection with primary anastomosis including oncologic margins. The patient evolved satisfactorily in the postoperative period an was discharged six days after the surgery without any complications. Likewise, he was monitored on a regular basis at our outpatient department and was free of symptoms at the 1-month follow-up visit. The histological analysis revealed a $5 \mathrm{~cm}$ ulcerated lipoma affecting $60 \%$ of bowel circumference. The molecula study, using fluorescent in situ hybridation (FISH) showed no MDM2 gene amplification.

Laparoscopic segmental resection of the large bowel is a safe and feasible technique for the treatmen of large bowel intussusception caused by a colonic lipoma. The complete removal of the lipoma will condition the prognosis. Furthermore, in the future, endoscopic surgery using colonoscopy could be employed when having a certain preoperative diagnosis of lipoma. 


\section{P110-COLORECTAL-Benign}

ROLE OF CBC PARAMETERS IN PREDICTING THE DIAGNOSIS OF ACUTE APPENDICITIS

M.S. Umerani, K. Alkhawaja, F.E. al Anazi,

Z.J. Arulanantham, E. Zakarneh, A. Abouleid, M.Y. Taha

\section{GENERAL SURGERY, KING FAHD MILITARY MEDICAL COMPLEX, DHAHRAN, Saudi Arabia}

Introduction: Acute appendicitis is one of the most common abdominal surgical emergency, the diagnosis of which mostly relies on conventional methods such as physical examination and blood tests. The use of ultrasonography and CT abdomen aids in more precise diagnosis especially in patients with atypical presentation or in elderly.

Aim: This study aims to evaluate the ability of the neutrophil/lymphocyte ratio (NLR), platelet/lymphocyte ratio (PLR) and mean platelet volume (MPV) in predicting the diagnosis of acute appendicitis.

Methods: Retrospective analysis of prospectivly maintained data of all patients (98) admitted with acute appendicitis to the emergency department at a tertiary hospital in the Middle East between January 2016 till September 2016. Medical records and database of patients, who had appendicectomy for clinically and radiologically proven appendicitis, were reviewed. The retrieved data included patient's demographic and laboratory values of white blood cells (WBC), Neutrophil (N), Lymphocyte (L), and Platelet (P) along with their ratios for comparison.

Results: SPSS 23 version was used for tabulating the data. The recommended cutoff value of the NLR, PLR and MPV in predicting the diagnosis of acute appendicitis was decided by using receiver operating characteristic (ROC) curve analyses. At least for NLR, the confidence interval (CI) was 0.47 which is 47 percentage of the positive values, since the confidence limit was between 35 to $58 \%$. Our results showed that the laboratory parameters were fairly significant since the confidence interval was 0.47 in predicting the diagnosis in our population.

Conclusion: Although appendicitis is a clinical diagnosis but laboratory parameters specially NLR, PLR and MPV can be used as an adjunct in the diagnosis of acute appendicitis. Literature is scarce concerning the validity of such parameters in our part of the world and prospective randomized controlled trials are needed to prove the efficacy of such rationale.

\section{P111-COLORECTAL-Benign}

\section{LAPAROSCOPIC RIGHT HEMICOLECTOMY IN TUMORAL LESION OF THE APPENDICULAR OSTIUM. UNUSUAL ANATOMOPATHOLOGICAL FINDING}

J. Gomez Sanchez, M.S. Zurita Savedra, C. Gonzalez Puga, B. Mirón Pozo

Cirugia General y del Aparato Digestivo, Hospital Clinico San Cecilio, GRANADA, Spain

Objective: Tumors of the cecal appendix represent a subset of colonic neoplasms whose early diagnosis is a real clinical challenge. Correspond to $0.5 \%$ of all gastrointestinal tumors and their prognosis depends on the type of injury, being the most frequent variety the carcinoid type. Appendix involvement in endometriosis is rare, accounting for $3 \%$ of all endometriosis cases, and sometimes mimicking cecal tumors.

Methods: A 43-year-old woman with a history of Hypothyroidism due to autoimmune thyroiditis and atrophic gastritis with gastric neuroendocrine tumors resected by endoscopy that in the Digestive Unit reviews, TAC with double contrast was requested, showing a lobulated lesion in the cecum adjacent to the ileocecal valve, with contrast enhancement of approximately $27 \times 21 \times 20 \mathrm{~mm}$, suggestive of tumor. The colonoscopy evidenced a protruding appendicular osteum with inflammatory aspect that was biopsied. The pathological anatomy of the biopsy reports chronic congestive colitis with edema of the own blade and minimal acute activity, with moderate local eosinophilia.The case was presented in the multidisciplinary oncology committee and it is decided, due to the patient's background, to perform surgery on the lesion. Laparoscopic right hemicolectomy was performed, with extracorporeal latero-lateral mechanical anastomosis with EndoGia Signia ${ }^{\circledR} 60 \mathrm{~mm}$

Results: The patient evolves favorably, with good oral tolerance and depositional habit. She is sent home at the sixth postoperative day. The pathological anatomy reports tumor injury in the appendicular ostium compatible with endometriosis at the base of the cecal appendix implantation, ruling out malignant tumor pathology.

Conclusions: Gastrointestinal tract endometriosis represents 3-15\% of cases, being most frequently located in the rectal-sigmoid region. Appendix involvement in endometriosis is rare, accounting $2-3 \%$ of all endometriosis cases and presents a preoperative diagnostic challenge, because sometimes mimicking a carcinoid cecal tumor. In our case, due to the patient's history, we assumed that the cecal lesion was a carcinoid tumor, so we performed a laparoscopic right colectomy, but if we had known that it was an endometriosis, we could have performed an appendectomy, although in both cases the laparoscopic approach gives us some benefits compared to the open approach

\section{P112-COLORECTAL-Benign}

INTRACORPOREAL ANASTOMOSIS IN MIS RIGHT COLECTOMY: THE MULTICENTRIC PORTUGUESE EXPERIENCE

N.J. Rama ${ }^{1}$, I. Sales ${ }^{1}$, D. Parente ${ }^{1}$, M. Neves ${ }^{1}$, M. Coelho ${ }^{1}$, V. Faria ${ }^{1}$, I.C. Gil ${ }^{1}$, P. Alves ${ }^{1}$, P. Clara ${ }^{1}$, S. Amado ${ }^{1}$, J. Costa Pereira ${ }^{2}$, C. Costa Pereira ${ }^{3}$, J. Costa $^{4}$, N. Vilela ${ }^{5}$

${ }^{1}$ Colorectal Unit, Centro Hospitalar de Leiria, EPE, LEIRIA, Portugal; ${ }^{2}$ Colorectal Unit, Braga Hospital, BRAGA, Portugal; ${ }^{3}$ Colorectal Unit, Centro Hospitalar Tâmega Sousa, PENAFIEL, Portugal; ${ }^{4}$ Colorectal Unit, Centro Hospitalar Entre Douro Vouga, FEIRA, Portugal; ${ }^{5}$ Colorectal Unit, Hospital de Santarém, SANTARÉM, Portugal

Background: Laparoscopic colectomy (LC) is considered a safe and effective surgical technique regarding short- and long-term outcomes. Recently, there has been a growing interest for the implementation of intracorporeal anastomosis technique (IA), usually considered more challenging than extracorporeal one (EA). However, evidence regarding the outcomes of both techniques is still lacking.

Methods: The authors designed a multicentric retrospective comparative study, including all the 450 consecutive patients who underwent laparoscopic right colectomy with EA or IA, from September, 2014 to October, 2018. Four Portuguese colorectal units were enrolled and the anastomotic techniques was chosen accordingly surgeons' preference. The analysed outcomes included length of stay, postoperative complications (ileus, anastomotic failure and surgical site infection), reoperation rate, readmission rate and 30-day mortality.

Results: IA procedure was performed in $60 \%$ of patients, with global conversion rate of $5.6 \%$. The IA group had less ileus (6.7\% versus $17.9 \%, \mathrm{p}=0.025)$, surgical site infections (SIS's) $(6,1 \%$ versus $18.7 \%, p=0.0007)$ and incisional hernia $(1.7 \%$ versus $6.9 \%, p=0.0292)$, but without a statistically significant differences in anastomotic failure, readmisson rate or mortality. Conclusion: Our study reveals better outcomes for IA technique, regarding SSI's, ileus and late parietal complications. Therefore, this procedure seems to be feasible and apparently safest than EA technique. Key words: laparoscopy, colectomy, surgical anastomosis, retrospective study

\section{P113-COLORECTAL-Benign}

\section{COMPUTED TOMOGRAPHY AT 6TH MONTH AFTER SURGERY PREDICT CHRONIC ANASTOMOTIC COMPLICATIONS}

\section{S. Kim, S.I. Kang, J.H. Kim}

Department Of Surgery, College Of Medicine, Yeungnam University, DAEGU, Korea

Aims: The natural history and predictive factors associated with chronic anastomotic complications have not been clearly studied. The aim of this study was to evaluated the predictive factors related to chronic anastomotic complications

Methods: From January 2010 to December 2016, a total of 53 patients who underwent anastomotic leakage were enrolled in this study. All patients underwent anterior resection with or without defunctioning stoma due to colorectal cancer. The patients received follow-up by clinical examination and abdominopelvic computed tomography (CT). They underwent a follow-up CT every 6 months for the first 1 year and then every 12 months for the next 2 years after that. Complicated group (CG) underwent chronic anastomotic complications. Normal group (NG) didn't underwent chronic anastomotic complications like stricture, fistula, chronic sinus, etc. Results: There were no significant differences in gender, age, preoperative chemoradiotherapy and operation type between two groups. Low rectum lesion and defunctioning stoma at the time of primary surgery were more frequent in CG $(p=0.013,0.021)$. There were no significant differences in type of anastomotic leakage, international leakage grade and CT findings at the time of diagnosis of anastomotic leakage. However, abnormal CT findings at the time of 6 month were more frequent in CG group $(\mathrm{P}<0.0001)$. In multivariate analysis, abnormal CT finding at the 6th months was only significant factor related to chronic anastomotic complications. Conclusions: Abnormal CT findings at the 6th month associated with prediction of chronic anastomotic complications. 


\section{P114-COLORECTAL-Benign}

\section{LAPAROSCOPIC URGENT APPENDECTOMY: OUR EXPERIENCE IN EARLY DISCHARGE}

B. Bascuas Rodrigo ${ }^{1}$, J.A. Lopez Ruiz ${ }^{1}$, A. Aguilar Marquez ${ }^{1}$, Y. Lara Fernandez ${ }^{1}$, B. Marenco de la Cuadra ${ }^{1}$, A. Cano Matias ${ }^{1}$ M. Sanchez Ramirez ${ }^{1}$, M. Retamar Gentil ${ }^{1}$, Y. Benabdeljalil ${ }^{2}$, F. Oliva Mompean ${ }^{1}$

${ }^{1}$ General Surgery, SAS, SEVILLA, Spain; ${ }^{2}$ Traumatology, SAS, SEVILLA, Spain

Aims: Acute appendicitis is the most common cause of acute abdomen requiring surgical intervention in the world. Nowadays, standard treatment of acute appendicitis involves a surgical approach, eitherlaparoscopic or open.The purpose of the present study is to evaluate the safety of a discharge within less than $24 \mathrm{~h}$ after performing appendectomy as a result of an uncomplicated acute appendicitis.

Methods: We retrospectively reviewed our prospectively collected database of patients who underwent surgery for acute uncomplicated appendicitis at our institution between January 2015 and March $2017(\mathrm{n}=309)$. Patients have been divided into 2 groups according to the length of their postoperative stay (Group 1 post-operative stay of $24 \mathrm{~h}$ or less, Group 2 post-operative stay of more than $24 \mathrm{~h}$ ). The following data concerning patient and surgery were recorded:age, gender, operative time, surgical approach (open or laparoscopic), rate of post-operative complications, reoperation and readmissions within 30 days after surgery. $\langle\mathrm{b}\rangle$

Results: No significant differences were observed in age, gender, operative time, or surgical approach between Group 1 and $2(\mathrm{p}>0.05)$. Nor were there statistically significant differences in post-operative complications studied (intraabdominal abscess, surgical wound infection, bleeding, paralytic ileus and evisceration), reoperation or readmission rate. Nevertheless, the difference in post-operative stay between the two groups was statistically significant $(\mathrm{p}<0.05)$. In Group 1 an average post-operative stay of $21 \mathrm{~h}$ and $43 \mathrm{~min}$, with a median of $22 \mathrm{~h}$ and $18 \mathrm{~min}$, was observed; in Group two the average stay was $73 \mathrm{~h}$ and 21 min with a median of $47 \mathrm{~h}$ and $41 \mathrm{~min}$. Conclusions: Patients who undergo appendectomy (open or laparoscopic) for acute uncomplicated appendicitis, without surgical incidents and an adequate social/family network, can be discharged in less than $24 \mathrm{~h}$ without a higher risk of post-operative complications or readmissions than patients with longer postoperative stays. It will be necessary to conduct more prospective studies with higher level of evidence that could corroborate our results.

\section{P115-COLORECTAL-Benign}

\section{LAPAROSCOPIC APPROACH IN MEDIAN ARCUATE LIGAMENT SYNDROME}

J. Bellido Luque, B. Bascuas Rodrigo, C. Dominguez Sanchez, I. Sanchez-Matamoros Martin, A. Nogales Muñoz, F. Oliva Mompean

\section{General Surgery, SAS, SEVILLA, Spain}

Aims: Median Arcuate Ligament Syndrome (MALS), also known as the celiac axis compression syndrome, is a rare condition caused by to the compression of the celiac trunk and the nerves located in this area (celiac plexus) by the median arcuate ligament. It is believed that MALS is caused by the median arcuate ligament compression of the celiac plexus nerves over the celiac trunk, but another probably cause may be the lack of blood flow to the organs supplied by the celiac artery, however, this theory is controversial. The first clinical sign of MALS is the apparition of postprandial abdominal pain in the upper abdomen. This typical pain forces patients to avoid eating, which can lead to loss weight (often more than 20 pounds). Other associated symptoms may include nausea, diarrhea, vomiting and delayed gastric emptying (a delay in food moving from the stomach into the small intestine). In relation to this uncommon condition, we present a clinical case of laparoscopic management of MALS.

Methods: We present a 23-year-old patient with complaints of recurrent epigastric pain, postprandial vomiting and loss weight. Blood tests and gastroscopy were performed to help ruling out more common causes of his symptoms, such as gastroesophageal reflux disease (GERD), gastritis or gastroparesis. As a part of the differential diagnosis, MALS was suspected and a mesenteric doppler ultrasound was ordered to check blood flow through the celiac trunk and evaluate a possible compression of the celiac plexus. Also, an angio-CT scan was also performed to confirm the diagnosis. Once the MALS was diagnosed, we decided to perform a laparoscopic approach as definitive surgical procedure.

Results: The patient was discharged $48 \mathrm{~h}$ after surgery with no remarkable events during his postoperative stay. He has been followed up during 6 months, remaining asymptomatic. Conclusions: Laparoscopic approach in MALS offers a superior visualization during the surgery and involves lower morbidity in compare to open approach, which makes it an optimal treatment for this condition.

\section{P116-COLORECTAL-Benign}

INITIAL EXPERIENCE WITH THE EPSIT (ENDOSCOPIC PILONIDAL SINUS TREATMENT) TECHNIQUE FOR THE TREATMENT OF PILONIDAL SINUS IN NORTHERN GREECE

M.G. Pramateftakis ${ }^{1}$, E. Kanavos ${ }^{2}$, S. Simeonidis ${ }^{1}$, E. Kotidis ${ }^{1}$, S. Angelopoulos ${ }^{1}, \mathrm{~K}$. Tsalis ${ }^{1}$, L. Loutzidou ${ }^{1}$

${ }^{1} 4$ th Surgical Department, Aristotle University of Thessaloniki, THESSALONIKI, Greece; ${ }^{2}$ Department of Laparoendoscopic Surgery, Iaso General Hospital, LARISA, Greece

Aim: Pilonidal sinus is a common disease with annoying and often painful symptoms. Traditiona surgical techniques for its treatment are characterized by either intense postoperative pain and prolonged wound-healing periods (wide resection, marsupialization) or unsatisfying aesthetic results (advancement or rhomboid flaps). 'Endoscopic pilonidal sinus treatment' (EPSiT) is a new minimally invasive technique which utilises the Meinero scope, primarily designed for the endoscopic treatment of complex perianal fistulas in a technique known as VAAFT. We presen our experience and outcomes in three treatment centers in Northern Greece.

Methods: Between July 2015 and November 2018 we treated 61 patients with pilonidal sinus using the EPSiT technique. The mean age of patients was 30 , and $85 \%$ of them were male. 4 patients were treated in the acute phase with the presence of pilonidal abscess. All operations were performed by two laparoendoscopic surgeons specifically trained in the technique. Most patients were treated on a day-case basis. Postoperative wound care included daily tract irrigation with $10 \mathrm{ml}$ of saline for a total of 10 days.

Results: There were no immediate postoperative complications. Medium postoperative pain was 2.8 on a VAS scale. $91 \%$ of patients were discharged on the same day, 4 patients remained in hospital for one day mainly due to social reasons. Return to daily activities was immediate. In a maximum follow-up of 24 months we observed 5 recurrences.

Conclusions: EPSiT is a promising minimally invasive technique for the treatment of pilonida sinus. What makes it mostly attractive is the minimal amount of postoperative pain, the excellent cosmetic result and the fast recovery with return to daily activities.

\section{P117-COLORECTAL_Benign}

\section{ACUTE CHYLOUS PERITONITIS DUE TO MECKEL'S DIVERTICULITIS: A CASE REPORT}

\section{M.M. Akhter Rahman, R. Seton, N. Warnaar}

General Surgery, Colchester General Hospital, COLCHESTER, United Kingdom

Introduction: Isolated acute chylous peritonitis is a rare event. When presented as an acute abdomen warranting surgical intervention, it is often difficult to determine the cause pre-operatively. Here, we report a case of acute chylous peritonitis due to Meckel's diverticulitis presented with the clinical features suggestive of acute appendicitis.

Presentation of the case: A 32-year-old female presented with abdominal pain and clinical features consistent with acute appendicitis underwent diagnostic laparoscopy. She was found to have four-quadrant chylous peritonitis and ileus caused by an inflamed Meckel's diverticulum adhered underneath a loop of small bowel and mesentery leaking chyle. After uneventful postoperative recovery, she was discharged at post-operative day two with oral antibiotics and was advised to take a low-fat diet.

Discussion: Till date, only a handful number of cases have been reported with chylous peritonitis, and aetiology includes blunt abdominal trauma, abdominal malignancy, cirrhosis, pancreatitis, radiation, tuberculosis, carcinoid syndrome, abdominal aortic surgery, peritoneal dialysis, and lymph node dissection. Preoperatively investigations including USS and CT are often inconclusive. Identifying the underlying cause by either laparoscopy or laparotomy is definitive and remains the choice of treatment for patient's presenting with features of peritonitis. Cytological and biochemical examination of fluid is often helpful to establish the underlying cause. Majority of the patients presenting with acute chylous peritonitis treated surgically recovers entirely with no further recurrences. Non-surgical options include dietary modifications including TPN, use of somatostatin and paracentesis.

Conclusion: Here we have presented a case of acute chylous peritonitis with Meckel's diverticulitis in a young 32-year-old female. Diagnostic laparoscopy remains the diagnostic tool and treatment of choice which is specific and associated with less morbidity and length of hospital stay 


\section{P118-COLORECTAL-Benign}

\section{LAPAROSCOPIC TREATMENT OF PERFORATED DIVERTICULITIS WITH PURULENT PERITONITIS}

F.J. Buils Vilalta, J.J. Sánchez Cano, J. Domènech Calvet, R. Prieto, P. Martínez, D. del Castillo

General and Digestive Surgery, University Hospital of Sant Joan. Faculty of Medicine Rovira i Virgili, REUS, Spain

Aims: Perforated diverticulitis with purulent peritonitis (Hinchey III) has traditionally been treated with surgery including colon resection and stoma (Hartmann procedure) with considerable postoperative morbidity and mortality. Laparoscopic lavage has been suggested as a less invasive surgical treatment.

Methods: A 78-year-old woman with a 10-day history of abdominal discomfort exacerbed during the last $48 \mathrm{~h}$. CT scan showed neumoperitoneum accompanied by free fluid and a $6 \mathrm{~cm}$ collection adjacent to descending colon showing diverticula suggestive of covert perforation. After $48 \mathrm{~h}$ of non-response to medical treatment, associated with the impossibility of percutaneous drainage through interposition of intestinal loops, colon and lumbar vessels, urgent surgical intervention is decided.

Results: Laparoscopic lavage of all 4 quadrants was performed with saline, $3 \mathrm{~L}$ or more, of body temperature, until clear fluid was returned. Two non-suction J-Pratt drains were placed. Intravenous antibiotics were continued for a minimum of $72 \mathrm{~h}$, then oral antibiotics were continued for 1 week. Oral fluids were commenced on the first postoperative day and solids were subsequently introduced, depending on clinical progress.

Conclusion: Laparoscopic management is reasonable alternative to the traditional open resection for Hinchey grade II-III perforated diverticulitis with generalized peritonitis. This approach has a low mortality rate despite patient co-morbidity and disease severity. Benefits include stoma avoidance and minimal wound infection. Subsequent elective resection is probably unnecessary and readmission in the medium term is uncommon.

\section{P119-COLORECTAL_Benign}

\section{MANAGEMENT OF POST PULL THROUGH CONSTIPATION AND FECAL INCONTINENCE}

\author{
M.A. Ismail Lotfallah ${ }^{1}$, A.H. Fawzy ${ }^{2}$, M. Maged ${ }^{1}$, \\ A. Shams ${ }^{1}$, A.h. Abdelghaffar ${ }^{1}$ \\ ${ }^{1}$ Pediatric surgery, Al Azhar university, GIZA, Egypt; ${ }^{2}$ Pediatric \\ surgery, Beni suef University, BENI SUEF, Egypt
}

Background: Constipation and fecal incontinence are common annoying complications after puth through procedures for Hirschsprung disease (HSD). Many causes could be the etiology of these problems. Perineal descent syndrome could be the major hidden cause of these complications. The aim of this study is to evaluate the role of perineal descent syndrome in the development of post pull through constipation and fecal incontinence in addition to evaluate the role of laparoscopic rectopexy for treatment of these problems. $<\mathrm{b}>$ Patient and methods: $</ \mathrm{b}>380$ patients treated with pull through for HSD over the period of five years. 62 out of the 380 patients presented with constipation and fecal incontinence. 23 patients with constipation and 39 patients with fecal incontinence. Rectal exam, anorectal manomety, defecography, contrast enema, rectal biopsy, EMG, proctoscopy and endorectal ultrasound were performed to all patients. Patients with stricture, missed aganglionic segment, injured internal anal sphincter, and loss of the sensory mucosa above the dentate line were excluded from the study. Anterior wall rectopexy was performed for anterior wall rectocele. Posterior wall rectocele was treated by retro rectal mesh rectopexy. EMG is repeated 7 weeks and 7 months after surgery. Outcome measurements included constipation, fecal incontinence and pudendal nerve latency.

Results: 62 cases of post pull through constipation and fecal incontinence. 23 patients with constipation and 39 patients with fecal incontinence. 7 patients with stricture, 3 patients with missed aganglionic segment, 2 patients with loss of anal sensory sensation and 2 patients with injured anal sphincter were excluded from the study. Defecography showed 40 patients with anterior rectocele ( 22 males and 18 females) and 8 patients with posterior rectocele ( 2 males and 6 females). The patients mean age $8.93 \pm 2.4$ years. EMG showed prolonged pudendal nerve conduction in all cases. Anterior wall and retro rectal rectopexy were performed laparoscopically without complications. Constipation was resolved in all patients after surgery. All patients showed fully control in defecation. Pudendal nerve latency decreased in all patients.

Conclusion: perineal descent syndrome proved to be a major hidden cause of post pull-through constipation and fecal incontinence. Laparoscopic rectopexy showed a good solution of these complications.

\section{P120-COLORECTAL—Benign}

\section{LAPAROSCOPIC EXCISION OF A MESENTERIC CYSTIC LYMPHANGIOMA}

J. Varela Recio ${ }^{1}$, T. Gomez Sanchez ${ }^{1}$, S. Ayllon Gamez ${ }^{1}$, C. Peña Barturen ${ }^{1}$, S. Cerrato Delgado ${ }^{1}$, M.A. Mayo Ossorio ${ }^{2}$, A. Valverde Martinez ${ }^{1}$, M.J. Castro Santiago ${ }^{1}$, M.D.

Casado Maestre ${ }^{1}$, J.M. Pacheco Garcia ${ }^{1}$

${ }^{1}$ Cirugía General Y Digestivo, Hospital Puerta Del Mar Cadiz, CADIZ, Spain; ${ }^{2}$ Cirugía General Y Aparato Digestivo., Hospital Puerta Del Mar Cadiz, CADIZ, Spain

Introduction: Cystic lymphangioma is a rare entity of benign tumor originated by endothelial cells of the lymphatic vessels consider it a tumor of congenital origin, formed during embryonic development. Perhaps this explains that this pathology is more frequent in pediatric ages especially between 0 and 2 years. The most common locations are head, neck and axillary region.

It is diagnosed as incidental finding or compression caused to the surrounding structures. Clinical case: A 69-year-old man with no history of clinical or surgical interest, who began a digestive study after starting marked dyspepsia of 4 weeks of evolution.

Blood analysis: no alterations. Abdominal TC: retroperitoneal tumoration to study.Pancreas MRI cystic lesion $6.5 \times 3.3 \times 4.5 \mathrm{~cm}$ that imprints in the second duodenal portion. It extends to the mesentery root posterior to the superior mesentery vessels without interfering with them. Characteristics: thin wall without signs of stratification, multiple internal septa that delimit multiple cysts. Inside liquid content without signs of restriction.In dynamic postgadolinium series, the wall is enhanced. Diagnosis compatible with MESENTERIC CYSTIC LYMPHANGIOMA

After decision in a multidisciplinary committee, surgical treatment was performed: Laparoscopic retroperitoneal mass resection in the mesentery root, in an american position with 4 trocars. (image) Conclusion: The immediate postoperative course with good evolution and the patient was hospital discharged on the fourth day.

After subsequent revisions, the patient also did not present complications in the late postoperative period. Result anatomopathological study: CYSTIC LYMPHANGIOMA IN MESENTERIC ROOT of $4.3 \times 3 \times 2.5 \mathrm{~cm}$.

Cystic lymphangioma is a rare entity. The surgical indication is determined by the size and symptomatology, and consists of the complete exeresis of the tumor. The laparoscopic approach is feasible in these cases, allowing a broad visualization of the anatomy, accessibility to the retroperitoneum in the context of a minimally invasive approach and a better recovery of the patient, without providing an increase in morbidity compared to the conventional.

In this way we defend as a technique of choice laparoscopic surgery against these rare tumors for the general surgeon in the abdominal cavity, betting on a minimally invasive surgery.

\section{P121-COLORECTAL-Benign}

\section{REDUCED PORT LAPAROSCOPIC POSTERIOR SUTURE} RECTOPEXY FOR FULL-THICKNESS RECTAL PROLAPSE

C. Kusunoki ${ }^{1}$, M. Uemura ${ }^{1}$, M. Miyake ${ }^{1}$, M. Miyazaki ${ }^{1}$, T. Kato ${ }^{1}$, M. Ikeda ${ }^{2}$, S. Maeda ${ }^{1}$, T. Hamakawa ${ }^{1}$, N. Hama ${ }^{1}$, K. Nishikawa ${ }^{1}$, A. Miyamoto ${ }^{1}$, M. Hirao ${ }^{1}$, M. Sekimoto ${ }^{1}$

${ }^{1}$ Surgery, National Hospital Organization Osaka National Hospital, OSAKA, Japan; ${ }^{2}$ Division of Lower GI Surgery, Hyogo College of Medicine, HYOGO, Japan

Aims: Laparoscopicposterior sutured rectopexy is one of the accepted treatment options for fullthickness rectal prolapse. Recently, reduced port surgery(RPS) has beenan emerging concept that, compared with conventional multiple port surgery (MPS), yields reduced postoperative pain and improved cosmesis. The aim of the study is to evaluate the feasibility and safety of RPS for fullthickness rectal prolapse.

Methods: RPS was performed by single-incision plus one puncture, using internal organ retractor(IOR) to secure operative field. Straining one IOR by $3-4$ strings in $3-4$ directions makes it possible to retract the internal organs three-dimensionally. This multi-directional flexible retraction could secure good operative field. From 2012 to 2018, 32 patients (RPS: 22 cases, MRS: 10 cases) underwent laparoscopicposterior suture rectopexyfor total rectal prolapse. Shortterm outcomes were compared between the two procedures.

Results: There was no significant difference between RPS and MPS in median operative time ( $175 \mathrm{vs}$ 167.4 min, respectively, $p>0.05$ ). The median blood loss volume was not significantly different between RPS and MPS groups ( $7.7 \mathrm{vs} 8.0 \mathrm{ml}, p<0.05$ ). The duration of median hospital stay afte surgery was not significantly different between two groups ( $12.9 \mathrm{vs} 13$ days, respectively, $p>0.05$ ). The frequency of complications after surgery were not different between them.

Conclusions: Reduced port Lap-rectopexy can be a good therapeutic option for total rectal prolapse. A prospective, randomized, controlled trial should be conducted to confirm the superiority of this procedure over MPS. 


\section{P122-COLORECTAL-Benign}

PILOT STUDY FOR THE CHARACTERIZATION OF A MURINE MODEL OF HYPERPLASTIC GROWTH IN COLON

\section{J. Bote Chacón $^{1}$, B. Moreno-Lobato ${ }^{1}$, F.M. Sánchez-Margallo²}

${ }^{1}$ Animal Modelling, Minimally Invasive Surgery Center Jesus Uson, CÁCERES, Spain; ${ }^{2}$ Scientific direction, MInimally Invasive Surgery Center Jesus Uson, CÁCERES, Spain

Introduction: The PICCOLO project proposes a new compact, hybrid and multimodal photonics endoscope based on Optical Coherence Tomography (OCT) and Multi-Photon Tomography (MPT) combined with novel red-flag fluorescence technology for in vivo diagnosis and clinical decision support. For its development it includes different phases of validation. Within this framework, the present study has as main objective: To characterize a model of rat colonic hyperplasia, which will be used for the development and validation of the previously mentioned endoscopic technology. Secondary objectives: Procure the reproducibility of the model chosen and determine the optimal time, after induction of the model.

Material and Methods: 12 animals (Rattus norvegicus), Wistar, Males and Females $<1$-yearold, randomly distributed.

Group $1(\mathrm{n}=2)$ : By laparotomy, a non-resorbable suture (Silk 4/0), not stenosing, is placed through the wall of the colon. Group $2(\mathrm{n}=2)$ : By endoscopy, a $0.3 \mathrm{~mm}$ long segment of a polymeric catheter is inserted, which is fixed to the wall of the colon by means of a suture. Group $3(n=2)$ : By means of endoscopy, a self-expanding and uncoated metallic stent are placed in the colon. Group $4(\mathrm{n}=2)$ : A superficial laser resection of the colonic mucosa is performed by endoscopy. Group $5(n=4)$ : As an extension of the most optimal model.

Weekly, the animals were anesthetized again to perform a colonoscopy, which determined the degree of mucosal growth in descending colon and colonic biopsies were extracted weekly (4 weeks). Results: Group 1. Growth around the sutures after the second follow-up, diagnosed as hyperplastic polyps after a histopathological analysis. Group 2: The catheter disappeared. Group 3: Stent caused mechanical intestinal obstruction. Group 4: The injuries caused by the laser were resolved weekly. Group 5: The model chosen was Group 1, In all of them we found growths after the second follow-up (We are waiting for the histopathology results for these animals.

Conclusion: We have obtained a surgical model of colonic hyperplasia that solves the limitations of other traditional models that only produce hypertrophy. This proposed model allows its reproducibility for subsequent studies.

\section{P123-COLORECTAL-Benign}

\section{EARLY OUTCOMES OF THE LAPAROSCOPIC TREATMENT OF GENERALIZED APPENDICULAR PERITONITIS}

Y. Ben Safta ${ }^{1}$, A. Mabrouk ${ }^{2}$, M.D. Maatouk ${ }^{3}$, M.T. Bouzidi ${ }^{2}$,

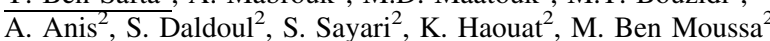

${ }^{1}$ Surgery A, Charles Nicolle Hospital, Tunis El Manar University, TUNIS, Tunisia; ${ }^{2}$ Surgery, Charles Nicolle Hospital, TUNIS, Tunisia; ${ }^{3}$ Surgery A, Charles Nicolle Hospital, TUNIS, Tunisia

Aim: The Role of laparoscopy in the management of generalized appendicular peritonitis is controversial. This is due mainly to the lack of scientific data. Through this study and a laborious bibliography research, we proposed to report our experience in terms of postoperative results, in the laparoscopic treatment of generalized appendicular peritonitis and to try to identify the risk factors associated with the occurrence of global morbidity and conclude on the feasibility of this technique in its treatment.

Methods: We conducted a retrospective study including all cases of generalized appendicular peritonitis managed laparoscopically, in the general surgery department of Charles Nicolle hospital between January 2006 and December 2016

Results: We identified 93 patients. The mean age was 31.7 years. One fifth of the cases required a midline conversion $(20.4 \%)$. The mean operative time was $146.6 \pm 36,7 \mathrm{~min}$. The overall morbidity rate was $15 \%$ including 7 surgical complications. There were no deaths. In uni-variate analysis, comorbidity, CRP $>200 \mathrm{mg} / \mathrm{l}$, operative time exceeding $170 \mathrm{~min}$ and midline conversion were significantly associated with postoperative morbidity. Co-morbidity, diabetes, ASA score $>2$, delay of consultation $>3$ days, intra-abdominal abscess and operative time exceeding $170 \mathrm{~min}$ were significantly associated with medical complications. The univariate analysis also revealed that $\mathrm{CRP}>200 \mathrm{mg} / \mathrm{l}$ and midline conversion were predictive of surgical complications. The multivariate analysis identified the midline conversion as the only independent factor significantly associated with post operative morbidity (Odds Ratio $=6.57,95 \%$ confidence interval [1.28-33.7]). Conclusion: Based on our results, it appears reasonable to continue the laparoscopic management of diffuse appendicular peritonitis. However, enhance this technique is basic in order to reduce midline conversion rate and to shorten operative time, which can lead to post operative complications.

\section{P124-COLORECTAL—Benign}

SAFETY OF ELECTROCOAGULATION IN THE TREATMENT OF MESOAPPENDIX IN LAPAROSCOPIC APPENDECTOMY

A.J. Díaz Martín, J. Reguera Rosal, A.I. Aguilar Márquez, A. Cano Matías, B. Marenco de la Cuadra, E. Pérez Margallo, M. Retamar Gentil, M. Sánchez Ramírez, F. Oliva Mompeán, J. López Ruiz

Emergency Surgery Unit, Hospital Universitario Virgen Macarena, SEVILLA, Spain

Aims: Currently, acute appendicitis is the most common surgical emergency. Laparoscopic appendectomy is the usual procedure to treat acute appendicitis. The aim of this study is to evaluate the safety of electrocoagulation in the treatment of mesoappendix in laparoscopic appendectomy. Methods: We have retrospectively studied a prospective database of operated patients of appendecectomy in Emergency Surgery Unit. We have reviewed laparoscopic appendectomies from June 1st, 2014 to December 31st, 2017. The mesoappendix was electrocoagulated in every laparoscopic appendectomy. The statistical analyses has been done with SPSS 24.0 version. Results: Our group consists of 294 patients of which $59.2 \%$ were male and $40.8 \%$ were female. The average age was 37.94 years with a standard deviation of $18.14 \%$ and p 75 was 51.5 years. The most common total stay was 1 day (100 patients). The usual post-operative stay was one day (144). We classified the diagnosis in complicated apendicitis ( 82 patients) and no complicated apendicitis (198 patients). The conversion rate was $3.1 \%$ (9). The main surgical complications were: surgical wound infection (1.4\%); intraabdominal abscess (6.8\%); and bleeding (1\%). Only one of the patients that suffered bleeding had complicated appendicitis. The medical complications were catheter sepsis $(0.7 \%)$; respiratory infection $(0.3 \%)$; cardiologicals $(0,3 \%)$; and paralytic ileus $(4.1 \%)$.

Conclusions: The treatment of mesoappendix with electrocoagulation is safe and effective since the complications rate is very low. Even so, it would be necessary to conduct more prospectives randomized studies in order to get enough evidence about the treatment of mesoappendix with monopolar electrocoagulation.

\section{P125-COLORECTAL-Benign}

TRANSANAL PROCTECTOMY USING TAMIS: A NEW TECHNIQUE FOR THE RECONSTRUCTION OF RESTORATIVE PROCTOCOLECTOMY WITH ILEAL POUCH-ANAL ANASTOMOSIS

M.Á. García Martínez ${ }^{1}$, M. Alcaide Lucena ${ }^{1}$, M. Zurita Saavedra ${ }^{1}$, C. González Callejass ${ }^{1}$, P. de Castro Monedero ${ }^{1}$, C. González Puga ${ }^{1}$, C. Garde Lecumberri ${ }^{1}$, J. Gutiérrez Sainz ${ }^{1}$, J. Gómez Sánchez ${ }^{2}$, B. Mirón Pozo ${ }^{1}$

${ }^{1}$ Cirugía General y del Aparato Digestivo, Hospital Universitario San Cecilio de Granada, GRANADA, Spain; ${ }^{2}$ General Surgery, H. U. San Cecilio, GRANADA, Spain

Introduction: The difficulty of resection of the rectum is determined by its anatomical relationships, intimately in contact with the bladder, seminal vesicles, prostate and urethra in the case of the male, vagina in the woman and nerve structures that will give defecatory, genital and urinary functionality This structure creates a big impediment due to problems of visualization and difficult dissection, in such a way that conventional surgical techniques instigates a series of complications derived from this difficulty. We propose a new approach in rectal surgery in patients with inflammatory bowel disease Material and methods: A 49-year-old man with a history of ulcerative colitis developed a severe acute outbreak refractory to treatment. A total laparoscopic colectomy with a terminal ileostomy was performed in 2016. In 2018 he was notified for reconstruction. We evidenced a rectal stump of about $10 \mathrm{~cm}$ with signs of inflammatory disease at the mucosal level. A transanal proctectomy was performed with confection of 'J-pouch' and ileoanal anastomosis about $4 \mathrm{~cm}$ from the anal margin by laparoscopy. The postoperative courses favorably, being discharged on the sixth day Currently in follow-up in Digestive and General Surgery, he is asymptomatic and he has an optimum level of quality of life valued by the SF-36 12 weeks after the intervention.

Conclusions: Our service introduces the transanal approach to the performance of proctectomy in cases of inflammatory disease, a technique that provides clear advantages by improving visualization and the identification of anatomical structures. In this way, a safe dissection of the pelvis is achieved, adjusted to the serosa of the rectum, with preservation of the mesorectum and the hypogastric plexus, and with the consequent improvement of the genital and urinary function. The result is an equally safe surgery, which implies little increase in operative time and with better and shorter postoperative recovery.The conservation of the pelvic innervation avoids disorders of ejaculation, vaginal lubrication and bladder and rectal motility. The transanal approach for the performance of proctectomy provides benefits in terms of the preservation of the hypogastric plexus, minimizing the anatomical difficulties involved in rectal surgery and maintaining urinary and sexual function. 


\section{P126-COLORECTAL-Benign}

'KEYHOLE ALL THE WAY': A 7-YEAR EXPERIENCE OF LAPAROSCOPIC APPENDICECTOMIES FOR BOTH SIMPLE AND COMPLICATED APPENDICITIS

\section{K.S. Tong ${ }^{1}$, J. Gan ${ }^{1}$, R. Doshi ${ }^{1}$, D. Larkin ${ }^{2}$, A. Warsi ${ }^{1}$}

${ }^{1}$ General Surgery, Furness General Hospital, BARROW-INFURNESS, United Kingdom; ${ }^{2}$ General Surgery, Stepping Hill Hospital, STOCKPORT, United Kingdom

Aims: To evaluate the feasibility and outcomes of laparoscopic appendicectomies in both simple and complicated appendicitis, given the increasing trend towards a laparoscopic approach in the last four decades for the treatment of acute appendicitis. We present data from a district general hospital over a 7-year period.

Methods: We retrospectively analysed a single consultant's continually updated database of laparoscopic appendicectomies between 01/03/2012 and 15/12/2018 (82 months). Patient demographics, investigations, intraoperative findings and postoperative outcomes were recorded and analysed. Complicated appendicitis was defined as the formation of appendiceal mass or abscess with or without perforation and peritonitis.

Results: 81 cases of laparoscopic appendicectomies were identified during the specified period. The median patient age was 30 (range $10-89$ years). True positive rates for USS and CT were $33 \%$ and $84 \%$, respectively. The rate of negative appendicectomies was $14 \%$. Among the patients with histologically-confirmed appendicitis, about half had complicated appendicitis. A registrar-led approach was taken in four-fifths of cases, with the supervising consultant physically present. There were no conversions to open surgery. $90 \%$ of patients were discharged within $48 \mathrm{~h}$ ( $33 \%$ within day 1) with no complication within 30 days. Conclusion: A laparoscopic approach to appendicectomy without conversion to open was safe and feasible in all simple and complicated appendicitis in our unit, despite the high rate of complicated cases, achieving excellent postoperative outcomes.

\section{P127-COLORECTAL-Benign}

\section{LAPAROSCOPIC APPROACH FOR COMPLICATED APPENDICITIS IS CONVERSION TO OPEN NECESSARILY SAFER?-A DISTRICT GENERAL HOSPITAL EXPERIENCE}

\section{Idama, J. Gan, K. Tong, D. Larkin, A. Warsi}

General Surgery, University Hospitals Morecombe Bay NHS Foundation Trust, BARROW-IN-FURNESS, United Kingdom

Background: Laparoscopic appendicectomy (LA) is increasingly performed in preference to open appendicectomy (OA) over the last 40 years with several advantages. However, complicated appendicitis may need conversion to open in $3-13 \%$. We present our data of 71 consecutive laparoscopic appendicectomies with no conversion.

Methods: We reviewed a single consultant's prospectively maintained database of LAs a our trust from 1/3/2012 to 13/2/2018 (72 months) making this Group 1, compared with other appendicectomies undertaken at the hospital from a period of 6/2016-12/2017 (18 months) becoming Group 2 . Group 1 comprised a Surgeon with bariatric training and advanced laparoscopic experience, having undertaken extensive training for 2 years post Certificate of Completion of Training. Group 2 consisted of 5 surgeons with competent laparoscopic experience and no bariatric training. Patient demographics, investigations, intraoperative findings and postoperative results were recorded and analysed. Complicated appendicitis was defined as appendix mass, abscess or mass + abscess, and perforation with peritonitis.

Results: Our results are reflected as per the table attached. The median age in Group 1 and 2 are 28 and 37 years old respectively. $47 \%$ of cases in Group 1 were complicated appendixes, while $51 \%$ of appendixes in Group 2 were complicated. The conversion rates for Group 1 and Group 2 were $0 \%$ and $12.9 \%$ respectively. In terms of complications, the complication rate in Group 1 was $2.81 \%$. In Group 2, $18.2 \%$ of cases that were converted to open surgery, and $22.2 \%$ of cases in which OA was performed, had complications. $91 \%$ of patients in Group 1 were discharged within $48 \mathrm{~h}$, compared to $60 \%$ in Group 2.

Conclusion: Despite the high rate of complicated appendicitis (49\%) in our trust, LA was possible in all cases of Group 1 without needing to convert to open surgery compared to $21.4 \%$ of total open cases in Group 2 .Group 1 showed good post operative results compared to data from Group 2, with reduced complication rates and reduced length of stay.

\section{P128-COLORECTAL-Benign}

TAMIS RESECTION OF A LARGE POLYP LOCATED $20 \mathrm{CM}$ FROM THE ANAL VERGE

J. Valdes-Hernandez, C. Dominguez, J. Gomez-Rosado, J. Cintas-Catena, F.J. del Rio, A. Perez-Sanchez, C. Torres, F. Oliva, L.C. Capitan-Morales

Colorectal Surgery Unit, Virgen Macarena University Hospital, SEVILLA, Spain

Transanal Minimally Invasive Surgery (TAMIS) has been used for the treatment of rectal neoplasms such us benign polyps and early rectal cancer. When the tumour is located in the upper rectum or close to the rectosigmoid junction, this approach may be technically dificcult.We present a video of a TAMIS resection of a large polyp located $20 \mathrm{~cm}$ from the anal verge. After properative examination and CT and MRI were performed, the patient was prepared for surgery, and a Trasnanal Minimally Invasive Surgery was proposed.Resection of the polyp was performed with the aim of an EndoGIA and conventional laparoscopic materials. Total resection of the polyp with free margin was possible. The postoperative pathology report confirmed a high grade displasia villo-tubular adenoma with a lesion free margin. TAMIS resection of tumours located above the rectosigmoid junction may be a safe and feasible technique in selected patients.

\section{COLORECTAL-Benign}

LAPAROSCOPIC SACRORECTOPEXY AND PREPERITONEAL LATERAL SUSPENSION FOR POSTERIOR AND APICAL PELVIC ORGAN PROLAPSE

M. Toledano-Trincado ${ }^{1}$, M. Bailon-Cuadrado ${ }^{1}$, J. Sanchez-Gonzalez ${ }^{1}$, M.L. Martin-Esteban ${ }^{2}$, P. Concejo-Cutoli ${ }^{2}$, J.R. Gomez-Lopez ${ }^{2}$, M.A. Montenegro-Martin², J.C. Martin-del Olmo ${ }^{2}$, D. Pacheco-Sanchez ${ }^{1}$

${ }^{1}$ General and Digestive Surgery, Rio Hortega University Hospital, VALLADOLID, Spain; ${ }^{2}$ General and Digestive Surgery, Hospital of Medina del Campo, MEDINA DEL CAMPO, Spain

Aims: Pelvic organ prolapse (POP) is a very relevant problem for women's quality of life and has a prevalence of about $5 \%$ defined by symptoms and up to $50 \%$ when established by physical examination. Nowadays, sacrorectopexy for posterior POP and sacrocolpopexy for apical POP are considered the gold standard techniques. Recently, we have seen that laparoscopic lateral suspension is a feasible procedure for apical POP, obtaining a success rate higher than $90 \%$ at one year. These results are similar to what we can achieve with sacrocolpopexy.

Methods: We herein present the case of a 70-year-old woman with apical and posterior POP, this was provoking an important impact on her quality of life, with obstructive defecation (needing digitations) and urinary incontinence. We proposed sacrorectopexy for her posterior POP and laparoscopic lateral suspension for her apical POP. In the video we can see how we perform a ventral mesh sacrorectopexy, following d'Hoore technique; and a laparoscopic lateral suspension with preperitoneal dissection, following the technique described by the team headed by Dubuisson and Veit-Rubin. We used 4 laparoscopic ports (12, 5, 2.9 and $2.5 \mathrm{~mm}$ ) Results: Patient was discharged home on the second postoperative day and has not had any sign of recurrence or extrusion after more than two years of follow-up. In addition, she has not suffered lower urinary tract symptoms, constipation or pain.

Conclusions: We present a case in which we have carried out a laparoscopic lateral suspension instead of a sacrocolpopexy for an apical POP, obtaining good short-term and long-term results. We consider it is very soon to assess this technique's efficacy and it has to be validated in studies with larger source of patients. Nevertheless, we think this procedure might become an excellent alternative to sacrocolpopexy for apical POP 


\section{P130-COLORECTAL-Benign}

\section{LAPAROSCOPIC MANAGEMENT OF COMPLICATED ACUTE DIVERTICULITIS. A CASE SERIES IN COUNTY HOSPITAL}

I. Plata Pérez, P. Nicolás Martinez, B. Cantarero Jimenez, I. Muñoz Nuñez, M. Maes-Carballo, A.B. Vico Arias

\section{General Surgery, Santa Ana Hospital, MOTRIL, Spain}

Aims: Laparoscopy is a minimally invasive approach with low morbidity.

The aim is to show the usefulness of the laparoscopic approach for massive intra-abdominal abscesses, which it is controversial. We report three patients who underwent emergency laparoscopy for peritonitis or massive intra-abdominal abscesses not amenable to percutaneous approach that were suspected to be caused by acute diverticulitis.

Methods: All patients had diagnosis of acute diverticulitis (Hinchey II-III grade) with pelvic abscesses situated between sigma and bladder or diffuse peritonitis. The patients with Hinchey II grade had failed conservative management with antibiotics.

They underwent emergency laparoscopy under general anaesthesia, with three abdominal ports. Intra-abdominal abscess cavities were exposed and the purulent exudate was sampled and aspirated. Copious irrigation was performed under direct vision and thorough examination without other findings. The procedure was completed laparoscopically in all cases.

Results: All patients had favourable evolution. One of them had a properly drained faecal fistula which changed to a purulent fistula on the twentieth postoperative day. This patient underwent laparoscopic left colectomy three months later because he had have a new episode of acute diverticulitis. Other two cases showed very good clinical evolution, without evidence of fistula in postoperative period and they were complete asymptomatic one month later.

Conclusion: In our experience laparoscopic drainage is a feasible, safe, and effective for the treatment of pelvic abscesses and diffuse peritonitis secondary to acute diverticulitis.

\section{P131-COLORECTAL-Benign}

\section{FOLLOW-UP AFTER SACROPROMONTO FIXATION FOR SOLITARY RECTAL ULCER}

\author{
N. Pinheiro, A. Ziegler
}

\section{Surgery, Hospital Sirio Libanes, SAO PAULO, Brazil}

Introduction: Solitary rectal ulcer syndrome (SUSR) is characterized as a rare disease whose pathophysiology remains uncertain. It was first described in 1829 by Cruveilhier and his clinicopathological feature was reported in 1969 by Mandigan and Morson, where he is associated with defective disorders, internal rectal prolapse, and psychological changes. According to works about $26 \%$ of the patients are asymptomatic. When symptomatic the diagnosis can be made through physical examination, clinical history and, often, confirmed by endoscopy with biopsies. Treatment depends on the severity of the symptoms and the existence of associated rectal prolapse. According to the literature, conventional surgical options include local excision, rectal mucosectomy, retopexy, and segmental colonic resection.

Rolato: A 28-year-old male complaining of anal bleeding at bowel movements 10 years ago. He performed, several times, conservative treatment, but without improvement. He sought proctological care and underwent colonoscopy, in which he showed an ulcerated lesion on the anterior wall of the distal rectum. New investigation with videodefecogram revealed colorectal intussusception with associated mucosal prolapse, being considered the factor causing the ulcer. Elected by the sacropromontofixação. Evoluiu with improvement of anal bleeding, mucorrhea and anal discomfort. After a proctological examination, which was normal, a control colonoscopy performed after 5 months of surgery revealed rectal mucosa, with residual scarring and disappearance of the submucosal nodule present in the initial examination. Reassessed after 12 months, the patient is asymptomatic.

Conclusion: Rectal solitary ulcer whose causal factor was a colorectal prolapse (intussusception) with mucosal exteriorization through the anal canal, which was individually treated with sacropromontofixation

\section{P132-COLORECTAL-Benign \\ DETERMINANTS OF TREATMENT AND OUTCOMES OF DIVERTICULAR ABSCESSES}

\section{J.P. Mali, P.J. Mentula, A.K. Leppäniemi, V.J. Sallinen}

Department of Abdominal Surgery, Helsinki University Central Hospital, HELSINKI, Finland

Approximately $15-20 \%$ of patients diagnosed with colonic diverticulitis have an intra-abdominal abscess as a complication. Abscess diameter of $3-6 \mathrm{~cm}$ is generally accepted as a cut-off determining the choice of treatment between antibiotics alone and percutaneous drainage.

The aim of this study was to analyze the treatment choices and outcomes of patients with diverticular abscesses. This was a retrospective cohort study which was conducted in Helsinki University Hospital, an academic teaching hospital functioning as secondary and tertiary referral center. Patients with computer tomography-verified acute left-side colonic diverticulitis with intra-abdominal abscess were collected from a database containing all patients treated for colonic diverticulitis in our institution during 2006-2013. Altogether, 241 suitable patients were included in analyses. Those treated primarily with percutaneous drainage or antibiotics alone (29 and 150 patients, respectively) were further compared in regards to treatment results. The main measured outcomes were need of emergency surgery and 30-day mortality.

Abscesses under $40 \mathrm{~mm}$ were mostly treated with antibiotics alone with high success rate (93 out of $107,87 \%$ ). In abscesses over $40 \mathrm{~mm}$, the use of emergency surgery increased and use of antibiotics alone decreased with increasing abscess size, but the proportion of successful drainage remained at $13-18 \%$ regardless of abscess size (Figure 1). There were no differences in failure rate, 30-day mortality, need of emergency surgery, permanent stoma, recurrence, or length of stay in patients treated with percutaneous drainage versus antibiotics alone, even when groups were adjusted for potential confounders. White blood cell count $=15.0 * 10^{9} / \mathrm{l}$, abscess diameter $=50 \mathrm{~mm}$, and corticosteroid medication were independent risk factors for failure of treatment with antibiotics alone. Patients without these risk factor had $95 \%$ and patients with one risk factor had $78 \%$ success with antibiotics alone.

Percutaneous drainage as treatment for large abscess does not seem to be superior to treatmen with only antibiotics. Majority of patients with abscesses over $60 \mathrm{~mm}$ in diameter undergo surgery as primary intervention.

\section{P133-COLORECTAL-Benign}

\section{CHRONIC APPENDICITIS; A CLINICAL ENTITY OR A DIAGNOSTIC MYTH}

\section{G. Ayiomamitis, P. Stamatakos, I. Sianis, V. Iakovaki,} O. Dardagani, N. Paschalidis

1st Surgical Department, Tzaneio General Hopsital, PIRAEUS, Greece

Introduction: Even today, 'chronic appendicitis' is a clinical term that is not widely accepted nor well documented amongst the Medical Community. Its etiology is the presence of a mass (e.g. fecal mass, hyperplasia of lymphatic tissue, etc.) that continuously and partially obstructs appendix lumen. It is presented as a low intensity, intermittent, with exacerbations and remissions, abdominal pain that is located at the right iliac region. The pain lasts up to several month and it is usually underestimated by the patient. Its diagnosis is based on imaging examination. Appendectomy is the treatment of choice for chronic appendicitis. The operation is challenging for the surgeon who has to cope with an intensively inflamed area around the appendix without the ease of access to that area.

Purpose: To present our laparoscopic approach to a chronic appendicitis case and to review the literature.

Case Report: A 56-year-old woman is hospitalized due to chronic appendicitis. The patient was treated conservatively with the use of intravenous antibiotics in two separate Hospital admissions dated 2 and 4 months back respectively. Eight weeks after the last exacerbation, she underwent a laparoscopic appendectomy.

Results: Even though the procedure was planned six months after the first episode, the laparoscopy revealed a severe inflammation of the appendix, which was extended to the caecum and the surrounding preperitoneal tissues. Although the difficultness of the operation it was completed successfully laparoscopically. The histological examination confirmed without any doubt the existence of 'chronic appendicitis'. The patient was discharged uneventfully the third postoperative day.

Conclusions: Chronic appendicitis is an existing clinical entity that the surgeon may come through during his career. In the hands of experienced laparoscopic Surgeon, the laparoscopic approach is feasible and safe. 


\section{P134-COLORECTAL-Benign}

\section{TRANS-ANAL PROTRUSION OF VENTRICULO-PERITONEAL SHUNT CATHETER WITH SILENT COLON PERFORATION}

L. Navarro Morales, F. Moreno-Suero, V. Duran-Muñoz Cruzado, V. Pino-Díaz, F. Pareja-Ciuro, J. Padillo-Ruiz, L. Tallón-Aguilar

Cirugía General Y Del Aparato Digestivo, Hospital Universitario Virgen Del Rocio, SEVILLA, Spain

Introduction: Ventriculo-peritoneal shunting (VPS) used in the treatment for hydrocephalus is associated with several complications. The exact cause of such extrusion is not known. Visceral perforation is an unusual but serious complication with consequeces such as peritonitis, meningitis or encephalitis. Management involves prompt removal of shunt, intravenous antibiotics, an adequate recovery gap so that cerebrospinal fluid culture is sterile and then followed by shunt replacement on opposite side. Aim: Multidisciplinary approach of extrusion of VPS through anus by laparoscopic and external ventricular drainage.

Case exposure: A 49-year-old woman had a VPS inserted 11 year ago after excision of gangliocytoma due to Lhermitte-Duclos disease.

She was admitted in the emergency department without symptos after trans-anal protrusion of VPS catheter. The neurological and abdominal evaluation was normal.

Laboratory tests did not reveal disorders and abdominal CT-scan suggested perforation, itshowed the insertion of the end of the catheter in sigma, without pneumoperitoneum or intraabdominal free fluid. Cranial CT-scan did no describe sings of hydrocephalus.

The patient underwent an emergencysurgical intervention. First of all, antibiotic therapy was initiated and neurosurgery's team was performed an external ventricular drain and they disconnected the proximal catheter side.

After that, an exploratory laparoscopy was performed. It revealed a microperforation and collection beside to an appendix' base due to the proximity with the catheter. Additionally, the catheter was freed from adhesions at the point of entry into the colon and after careful dissectionwe release the VPS from colon with a $1.5 \mathrm{~cm}$ transmural trajectory at the sigmoid level. No free fluid was seen and rest of the bowel appeared normal.

The distal end was removed through the anus and the proximal end through a laparoscopic port. We performed a laparoscopic segmental cecum resection and an extracorporeal colo-colonic anastomosis was performed for a mini-Pfannestiel laparotomy of assistance. There were no complications in the postoperative period, being discharge on the 5th day.

Conclusion: The multidisciplinary approach and the laparoscopic support in the diagnosis and treatment of patients with colon perforation caused bay VPS catheter is a feasible and safe option in third level centers.

\section{P135-COLORECTAL-Benign}

\section{TROCAR PLACEMENT IS IMPORTANT FOR LAPAROSCOPIC} SURGERY FOR DIFFUSE APPENDICULAR PERITONITIS

\section{A. Sazhin ${ }^{1}$, G. Ivakhov ${ }^{1}$, I. Ermakov ${ }^{1}$, S. Titkova ${ }^{2}$ \\ ${ }^{1}$ Faculty surgery, Russian State National Medical University,} MOSCOW, Russia; ${ }^{2}$ Experimental surgery department, Russian State National Medical University, MOSCOW, Russia

Background: acute appendicitis continues to be the most common source of complicated intraabdominal infection worldwide. The high incidence of postoperative complications and dissatisfaction with the results of treatment in cases of complicated appendicitis and peritonitis gave the reason for conducting this study.

Aim: to evaluate the effect of different laparoscopic trocars position in case of laparoscopic appendectomy for diffuse appendicular peritonitis for the incidence of postoperative complications Methods: the results of laparoscopic treatment of 116 patients with acute appendicitis complicated by diffuse peritonitis were analyzed. The first group consisted of 37 (32\%) patients operated by triangulation access (type 1 trocar placement according SAGES guidelines for laparoscopic appendectomy (SAGES QLA). The second group consisted of 79 (68\%) patients operated by sectorisation access (type 4 SAGES QLA). Postoperative complications were classified by Clavien-Dindo classification.

Results: the duration of the operation for the analyzed groups was $91.1 \pm 29.9$ vs $84.5 \pm 24.9$ min. There were no deaths among this group of patients. The incidence of postoperative complications for both group was $36.2 \%$. Postoperative complications in the triangulation and sectorisation group were $43 \%$ and $21.6 \%$ respectively (p 0.043). Clavien-Dindo IIIB complications were noted in $4.3 \%(\mathrm{n}-5)$ patients and presented with intra-abdominal abscesses (IAA). All patients with IAA were operated in sectorisation group.

Conclusion: sectorisation trocar placement increases the incidence of intra-abdominal complications for laparoscopic appendectomy for diffuse appendicular peritonitis.

\section{P136-COLORECTAL-Benign}

\section{LAPAROSCOPIC VERSUS OPEN APPENDECTOMY IN THE MANAGEMENT OF PERFORATED ACUTE APPENDICITIS}

\section{M.F. Avram ${ }^{1}$, I.O. Avram ${ }^{2}$, D. Koukoulas ${ }^{3}$, F. Cadariu ${ }^{1}$, S. Olariu ${ }^{1}$}

${ }^{1} 1$ st Surgery Department, University of Medicine and Pharmacy „Victor Babes, Timisoara, TIMISOARA, Romania; ${ }^{2}$ Klinik für Allgemein-, Viszeral-, Thorax- und Tumorchirurgie, Caritasklinikum Saarbrücken, SAARBRUCKEN, Germany; ${ }^{3}$ Gastroenterology, City Hospital, LUGOJ, Romania

Aims: Due to the severity of inflammation and complexity of anatomy laparoscopic appendectomy (LA) was contraindicated in perforated appendicitis in the early days. As surgeons gained more experience with the technique, LA became feasible for patients with perforated appendicitis. We analyse the outcomes of LA compared to open appendectomy $(\mathrm{OA})$ for perforated appendicitis. Methods: We compared two lots of patients operated in our department for perforated appendicitis in 2018, one lot underwent OA (21pts), the other LA ( $7 \mathrm{pts})$. We analysed for each group sex distribution, age, operation time, time until oral intake, surgical site infections rate, intraabdominal abscess rate, length of hospital stay and 30 days readmissions rate.

Results: LA - the appendiceal base was divided using a laparoscopic stapler in 1 patient and endoloops for the other patients. OA was performed through a Mc Burney incision, 2 patients needed a midline incision because of free perforation and generalized peritonitis. In the LA group patients were younger compared to OA group (mean age $33.5 \pm 14.2$ years vs $41.5 \pm 8.4$ years), there was a female predominance in both groups, operating time was longer for the LA group compared to $\mathrm{OA}$ $(105 \pm 33 \mathrm{~min}$ vs $74 \pm 23 \mathrm{~min})$. We have to mention that one LA was converted due to atypical anatomy that proved to be an intestinal malrotation. Time until oral intake was shorter for the LA group ( $23 \pm 2 \mathrm{~h}$ vs $35 \pm 9 \mathrm{~h}$ for the OA group). Surgical site infection occurred in 2 patients from the LA group and 5 patients in the OA group. No intrabdominal abscess was noted in the LA group, was present in the OA group. The mean length of hospital stay was 5 days for LA and 6 days for OA group. The rate of readmission at 30 days was the same, $14.28 \%$ for both LA and OP groups. Conclusions: Laparoscopic appendectomy was feasible and safe for complicated appendicitis, although it required a longer operating time, it had the benefits of a shorter hospital stay, fewe immediate postoperative complications, shorter time until oral intake and shorter hospital stay.

\section{P137-COLORECTAL-Benign}

\section{WHAT IS THE LIMIT FOR THE LAPAROSCOPIC APPROACH OF A COMPLICATED COLON DIVERTICULITIS?}

A.F. Aranzana Gómez, J. Malo Corral, J. Hernandez Gutierrez, P. Toral Guinea, B. Muñoz Jimenez, R. Lopez Pardo, G. Krasniki, M.A. Morlan Lopez

\section{General Surgery, Complejo Hospitalario Toledo, TOLEDO, Spain}

Introduction: The diverticular disease of the colon is a chronic entity with a variety of abdomina symptoms that can present with recurrent episodes of acute diverticulitis (AD). The prevalence of diverticulosis is not influenced by gender and increases with age, which, according to the increase in life expectancy, explains the accumulation of cases in Western countries. The classic diagnostic-therapeutic algorithm of the disease is It has been based on the Hinchey classification, the use of antibiotics and the intervention of Hartmann (IH) at the acute time and elective colectomy in the multirecurrent cases. The use of laparoscopy with washing and drainage is actualymore extended in cases with peritonitis.

Objectives: To demonstrate the safety and efficacy of the laparoscopic approach, in cases with diverticular disease complicated by severe inflammatory plastron with 'covered' perforation, with several recurrent episodes.

Material and method: Case report: A 46-year-old man with AP-Diverticulitis 10 years ago with complete resolution and normal control colonoscopy. He presents in the last two months three compatible episodes of acute DIverticulitis, exploration with plastron-mass in hypogastrium without defense, TAC-marked thickening of a segment of $10 \mathrm{cms}$. of medium sigma, collection not drainable in mesosigma, of $3 \mathrm{~cm}$, which loses the plane of cleavage with loops of thin neighbors with a linear tract that suggests fistulization. Evidence of interest is exposed. Given the evolution, it is decided surgical elective treatment.

Result Intervention: preoperative ureteral double catheterization, laparoscopic approach, is exposed by video, rectosigmoid resection by diverticular plastron, with negative IO biopsy, mechanical colorectal anastomosis. Good postoperative course, discaharge at 5th day. DEFINITIVE AP: PERFORATED DIVERTICULITIS, absence of malignancy.

Conclusion: The laparoscopic approach is a valid and effective alternative in cases of complex and severe diverticular disease. 


\section{P138-COLORECTAL-Benign}

TAMIS APPROACH FOR A RECTAL STENOSIS NON TREATABLE BY ENDOSCOPY

J. Valdes-Hernandez, J. Cintas-Catena, J. Gomez-Rosado, F.J. del Rio, C. Torres, A. Perez-Sanchez, F. Oliva, L.C. Capitan-Morales, Colorectal Surgery Unit, Virgen Macarena University Hospital, SEVILLA, Spain

Aim: TAMIS resection has been described for the treatment of rectal neoplasms, wether benign or early malignant tumours. Since TAMIS appearance, many different indications have been reported.We aim to show an special indication as seen in this video of a TAMIS resolution of a rectal stenosis non treatable by endoscopy.

Method: We present a video of a female patient, previously treated for a large rectal adenoma treated by trasnanal apporach, with a postoperative sepsis which required lateral colostomy and trasanal drainage. After surgery, the patient suffered from a rectal stenosis which couldn' $t$ be solved by endoscopy, so the patient was sent back for a surgical treatment.We decided to performed a trasnanal apporach by TAMIS and a long and circunferiential stenosis around $6 \mathrm{~cm}$ from the anal verge was seen.We performed a rectotomy by electrocautery in the posterior rectal wall until the perirectal fat was seen and the stenosis was passed. A dilatation with a foley catether was also performed.

Results: Postoperative course was uneventful and after 6 months she was prepared for colostomy closure with no complications and remains asymptomatic nowadays.

Conclusion: TAMIS approach of rectal stenosis may be a safe and feasible technique in selected cases if conservative treatments fail.

\section{P139-COLORECTAL-Benign}

\section{SURGICAL TREATMENT OF IATROGENIC ENDOSCOPIC COLON PERFORATION}

\section{A.M. Maghiar, G. Dejeu, O.A. Maghiar, T.A. Maghiar \\ Surgery, Universitatea Oradea,Spital Clinic Pelican, ORADEA, Romania}

Iatrogenic endoscopic colon perforation it is a severe, but rare complication of colonoscopy. The incidence of this complication is estimated to be $0.016-0.8 \%$ for diagnostic colonoscopies and $0.02-8 \%$ for therapeutic colonoscopies. The management of these complications depends on the size of the lesion,the time elapsed between the lesions were produced and diagnostic of the lesions and associated pathology. The treatment can be consevative,endoscopic or surgical(clasic/ laparoscopic) In our sevice in last 10 years we treated 5 cases with iatrogenic colon perforation after diagnostic colonoscopies. All lesions were at sigma level.

One case was admission in our service at 3 days after a diagnostic colonoscopy. The pacient was operated clasic,in emergency,we found a fecaloid peritonitis,a perforation at sigma level.We made a colostomy, lavage, drainage but the pacient died after 4 days. In 4 cases we made the operation at maximum $2 \mathrm{~h}$ after the lesion was diagnosticated by the endoscopist(directly visualisation).We didn't made radiologic investigation.The pacients were operated laparoscopic,we made suture,lavage,drainage. Evolutions of the pacients were good.

Conclusion: Iatrogenic colonic perfortion are rare,but severe complication. Laparoscopic surgery can be a choice in treatment of this complication

\section{P140-COLORECTAL-Benign \\ LAPAROSCOPIC APPROACH TO COMPLICATED DIVERTICULITIS WITH COLOVESICAL FISTULA}

\section{Ferrer Sales, M. Neves, D. Parente, P. Alves, N. Rama, V. Faria}

General Surgery, Centro Hospitalar de Leiria, LEIRIA, Portugal

Introduction: Complicated diverticulitis with fistula is responsible for about $20 \%$ of surgical procedures in diverticular disease and is commonly found in patients with diverticulitis of the sigmoid colon. Colovesical fistulas are the most frequent $(65 \%)$, with highest incidence in males. Only a third of these patients have a history of diverticulitis. In most cases, treatment is surgical, and colectomy is performed, whether or not in association with vesical recession.

Case report: 61 year old male with pneumaturia and fecaluria for the preceding 4 months. The colonoscopy identified a diverticulitis of the sigmoid colon and the subsequent pelvic MR suggested a colovesical fistula. The cystoscopy was not able to identify any fistulous opening, bu a double $\mathrm{j}$ catheter was placed in the left ureter, as surgical treatment had been proposed. A subsequently abdominal pain motivated a preoperative CTscan which revealed a pneumoretroperitoneum and a fluid collection near the left ureteral tract. The multidisciplinary team on the case decided to perform a percutaneous nephrostomy, followed by an exploratory laparoscopy. The fistula tract was identified and a laparoscopic sigmoidectomy with partial cystectomy was performed, as well as a ureterorenoscopy (with double J replacement). There were no intra or postoperative complications, and the patologic repport had no signs of malig nancy. Video of the surgical procedure is presented.

Conclusion: A laparoscopic approach to complicated diverticulitis with colovesical fistula is safe and effective when performed by experienced colorectal surgeons.

\section{P141-COLORECTAL-Benign}

\section{DIVERTICULAR DISEASE—SURGICAL TREATMENT}

\section{Ferrer Sales, D. Parente, M. Neves, P. Alves, N. Rama, V. Faria}

General Surgery, Centro Hospitalar de Leiria, LEIRIA, Portugal

Introduction: Diverticular disease is characterized by its high prevalence, being one of the most frequent causes for hospital admission when it comes to gastrointestinal pathology. Even though it is more frequent in older patients, there has been an increase in incidence amongst lower age groups. The approach to the disease has also suffered changes in the last few years, showcasing tendency for less invasive options, deferring elective surgery to later in the course of the disease. This study examines the therapeutic approach to diverticular disease in our Hospital.

Methods: Retrospective analysis of demographic data, therapeutic options and surgical outcomes in patients admitted for diverticular disease between January2015 and June2018.

Results: 154patients $(\mathrm{n}=154)$ were included in the study:75(49\%)were male and79 $(51 \%)$ were female, with an average age of 61 years. 135patients $(88 \%)$ underwent medical treatment, with surgery reserved for the remaining 19 patients, 11 of which were emergencies, with the other8 being elective(5\%).About $89 \%$ of patients were only admitted once, $9 \%$ were admitted twice and $2 \%$ had3or more episodes. For the single-admission group, the most common treatment was medical(93\% of cases), as was the case for the group with3or more episodes(in which 58\%o cases were subjected to medical treatment). In the patient group with2episodes,53\%were submitted for surgery, most of which elective.As far as the surgically operated group is concerned, no statistically significant differences were found with regards to patient sex. Age, however, was significantly greater for the group that underwent emergency surgery vs that submitted to elective (65.2 years vs 45.2 years,p $<0.001$ ). The most common procedure overall was colic recession, with Hartmann's operation standing out as the most frequent for the emergency surgery group. Length of hospital stay was again higher for the emergency group (vs elective; 14 vs $5, \mathrm{p}<0.001$ ), as well as the morbidity rate. No statistically significant differences were found with regards to mortality rate.

Conclusion: Knowledge of the natural history of diverticular disease led to changes in the approach to treatment, with a tendency to adopt a less aggressive therapeutic. Despite controversy around aspects such as selection of patients for elective surgery, among others, it is key to the approach to diverticular disease that existing recommendations are taken into account, treatment is individualized and outcomes are closely monitored. 


\section{P142-COLORECTAL-Benign}

\section{SECURITY OF THE USE OF ENDOLOOP IN NON- COMPLICATED AND COMPLICATED ACUTE APPENDICITIS}

G. Anguiano, V. Duran Muñoz-Cruzado, J. Tinoco-Gonzalez, A. Sánchez-Arteaga, L. Tallon-Aguilar, F. Pareja-Ciuro, J. Padillo

General Surgery, Hospital Universitario Virgen Del Rocío, SEVILLE, Spain

Aims: The objective of this analysis is to establish if there is differences after the procedure of laparoscopy appendicectomy comparing the use of endoloop (EL) vs. endostapler (ES) in complicated and non-complicated acute appendicitis.

Methods: We performed a retrospective analysis of a prospective database of 499 patients from February 2012 to June 2018. We divided the patients in two groups: depending on that the procedure of the appendicectomy was with endoloop or endostapler. The groups were created selecting 229 patients in order to be homogeneous as to perforation appendix rate, thus a propensity score was performed for sex, age and perforation rate. An univariant analysis was carried out in regard to the differences in the use of EL vs ES in the apparition of abdominal complication, as well as hemorrhage, ileo, surgical wound infection, collection, reintervention or hospital readmission.Qualitative variables were expressed in terms of absolute frequencies and percentages and mean values and standard deviation were used to express quantitative variables. The analysis of data was performed by means of contingency tables applying Fisher's exact test or Chi-squared test for qualitative variables and variance analysis or Student'-t test for quantitative variables.

Results: The present study includes 229 patients (136 males). 93 patients $(45.6 \%$ males) the appendicectomy was performed with EL and in 136 with ES (65.45\% males); $p=0.065$. The average age was 39.6 (17.8) years old (EL: 38.28 (16.4) vs. ES 40.7 (18.19); $\mathrm{p}=0.089$. The perforation of the appendix was $44.5 \%$ (EL $39.2 \%$ and EG 60.78\%); $\mathrm{p}=0.7$. The abdominal complications rate was $14.12 \%$ (EL $39.4 \%$ and ES $60.6 \%$ ); $p=0,878$. There were no differences as regards to the experience of the surgeon (fellow vs. resident $)(p=0.117)$, hemorraghe $(\mathrm{p}=0.516)$, ileo $(\mathrm{p}=0.805)$, Surgical wound infection $(\mathrm{p}=0.99)$, coleccion $(\mathrm{p}=0.737)$, reintervention $(\mathrm{p}=0.66)$, hospital stance $(\mathrm{p}=0.488)$ or postoperative hospital readmission $(\mathrm{p}=0.689)$.

Conclusion: The use of endoloop and endostapler in laparoscopic appendicectomy do not offer differences in regards to postoperative complications in non-complication and complicated acute appenditing.

\section{P143-COLORECTAL-Benign}

\section{SPONTANEOUS RECTAL PERFORATION... RARE PRESENTATION}

M. Robba ${ }^{1}$, N. Coleman ${ }^{1}$, M. Sapundijeski ${ }^{1}$, C. Macano ${ }^{1}$, E. Mansour $^{2}$

${ }^{1}$ Surgery, St Bernards Hospital, GIBRALTAR, Gibraltar; ${ }^{2}$ Colorectal Surgery, St Bernard Hospital, GIBRALTAR, Gibraltar

Introduction: Intramural haematomas can develop anywhere within any the gastrointestinal tract3. These are most frequently associated with blunt trauma above the level of the sigmoid colon and very rarely occur in the rectum 1 . Spontaneous, non-traumatic haematomas are a rare clinical condition usually secondary to haematological blood disorders or anticoagulant therapy2. Case Summary: A 56-year old gentleman presented to the emergency department with a 2 day history of worsening lower abdominal pain and bloody stool. He presented twice within the previous week with worsening, generalised abdominal pain. The patient had been taking regular aspirin and clopidogrel following insertion of coronary artery stents. On clinical examination, he was guarding with a distended, generally tender lower abdomen but all observations were stable, afebrile. An initial computer tomography of the abdomen reported pneumoperitoneum with haemorrhagic ascites; a differential diagnosis being perforated sigmoid colon with a large localised haematoma. The patient underwent an emergency laparotomy and Hartmann's procedure (appendicectomy, sigmoid colostomy and rectal stump). He recovered well with no significant post-operative complications. Histology reported the rectal perforation macroscopically associated with an opened haematoma and no evidence of malignancy. The appendix shows reactive appendicitis with serousal inflammation.

References: 1) McClenathan JH, Dabadghav N. Blunt rectal trauma causing intramural haematoma; report of a case. Dis Colon Rectum. 2004:47:380-382. [PubMed]2) Hughes CE, Conn J, Sherman JO. Intramural haematoma of the gastrointestinal tract. Am J Surg. 1977;133:276-279. [PubMed]3) Zhu-Lin Li, Zhen-Jun Wang, Jia-Gang Han. Spontaneous perforation of an intramural rectal haematoma: Report of a case. World J Gastroenterol. 2012 May 21; 18(19): $2438-2440$

\section{P144-COLORECTAL-IBD}

A FIVE YEARS COMPLETED FOLLOW UP OF COLORECTAL CANCER RELATED ULCERATIVE COLITIS UNDERGOING SURGERY AMONG SURVIVOR : A CASE REPORT

B. Ida Bagus ${ }^{1}$, M. Ida Bagus ${ }^{2}$, I.A. Setyawati ${ }^{3}$

${ }^{1}$ Surgery, Sebelas Maret University, KARANGANYAR, Indonesia; ${ }^{2}$ Medical Faculty, Sebelas Maret University, KARANGANYAR, Indonesia; ${ }^{3}$ Medical Faculty, Pendidikan Ganesha University, SINGARAJA, Indonesia

Background: Ulcerative colitis (UC) is one of the risk factor of developing sporadic colorectal cancer. Approximately $15 \%$ of UC patients develop an acute attack of severe colitis, and $30 \%$ of these patients require colectomy. One third of the patients will not respond to steroid therapy. Thus, a long-term follow-up has been recommended.

Case Report: We reported a single case of completed 5 years follow of colorectal cancer related ulcerative colitis on 54 years old female patient undergoing emergency operation ( 2 staged total colectomy and J-pouch Ileo-Rectal anastomosis) after 1 year no responsed of medical treatmen before, presenting with bloody diarrhea and anemia. There was no post operative complication reported. Pathologic finding was early adeno carcinoma, closed follow up was done each year and for another five years later, no progression of the disease was found in this period and the patients has good quality of life after this procedures.

Keywords: ulcerative colitis, surgery, 5 years follow up

\section{P145-COLORECTAL-IBD}

\section{YEARS EXPERIENCE WITH LAPAROSCOPIC SURGERY FOR INFLAMMATORY BOWEL DISEASE}

\section{Kasalicky ${ }^{1}$, E. Koblihova ${ }^{1}$, M. Bortlik ${ }^{2}$}

${ }^{1}$ Surgical department, Central Military Hospital Prague, PRAGUE, Czech Republic; ${ }^{2}$ IBD clinic, Iscare IBD, PRAGUE, Czech Republic

Aim: Laparoscopic colorectal surgery (LCS) in patients with inflammatory bowel disease (IBD) is becoming a standard and feasible surgical method worldwide. $20 \%$ of patients with Crohn's disease (CD) and $80 \%$ patients with ulcerative colitis (UC) will require an operation during their life. Over the last decade, there have been many studies documenting the safety and feasibility of the laparoscopic approach for IBD in well-selected patients.

Methods: Patients with a CD with the tight stenosis in the distal ileum and/or ileo-colon or various colon and rectum stenosis, patients with UC with ineffective medical therapy, steroid dependence or dysplasia underwent the LCS. From 2009 to 2018, 247 ileocolic resections, 63 hemicolectomies, 74 subtotal colectomies and 35 restorative proctocolectomies with ileopouchanal anastomosis were performed either totally laparoscopically or laparoscopically assisted $(\mathrm{n}=419)$. The average time of the procedure was $105 \mathrm{~min}(55-295 \mathrm{~min})$, average blood loss $125 \mathrm{ml}(0-350 \mathrm{ml})$ and the conversion to laparotomy was in $8.2 \%$. Average return time of the bowel function was 3.5 days (2-9 days) and the average hospital stay was 7.1 days (6-11 days). Complications occurred in 24 patients (5.7\%). 3 cases of the early ileus due to adhesions, 2 cases of the anastomotic bleeding threated conservatively, 1 case of the instrumental perforation of the small bowel, 7 cases of the incisional hernia in minilaparotomy and 11 wound infections occurred.

Conclusion: In well-selected patients with IBD, thanks to superior short- and long-term outcomes, the laparoscopic approach should be considered a safe and effective method when performed by experienced surgeons. Supported by MO1012. 


\section{P146-COLORECTAL-IBD}

\section{CHOICE OF Method: OF SURGICAL TREATMENT OF ACUTE APPENDICITIS BY PREGNANCY}

V.A. Bombizo, P.N. Buldakov, D.N. Ustinov, A. Averkina, Surgical department, Regional Clinical Hospital of Emergency Medical Care, BARNAUL, Russia

Introduction: Acute appendicitis occurs in $0.03-5.2 \%$ of cases and is considered the most common surgical disease in pregnant women. To date, there is no consensus on the choice of method of surgical treatment of acute appendicitis by pregnancy.

Research objective: To study the results of surgical treatment in pregnant women with acute appendicitis. To determine the possibility of using laparoscopic appendectomy at various periods of pregnancy.

Materials and methods: During the period of 3 years (2015-2017), 86 pregnant women with acute appendicitis at various periods of gestation were operated in the surgical departments of the Regional Clinical Hospital of Emergency Medical Care.

Laparoscopic access was performed in 78 women: in I trimester-in 39; in II-in 32; and in III-in 7.

Appendectomy with McBurney's access was performed in 7 women: in II trimester-in 2 patients and in III-in 5 patients.

Midline laparotomy was performed in 1 patient with diffuse peritonitis, caused by gangrenous-perforated appendicitis on the 36th week of pregnancy.

Results: All patients lacked complications in the postoperative period; The duration of hospital stay ranged from 4 to 10 days, depending on the method of surgical treatment and the need for correction of comorbidities; In 1 patient with diffuse peritonitis,delivery was performed by caesarean section during midline access appendectomy.

Conclusion: In $90.7 \%$ of cases in pregnant women with acute appendicitis, it is possible to perform appendectomy with laparoscopic access regardless of the gestational age; In cases of diffuse peritonitis in patients with acute appendicitis by pregnancy, the use of 'open' surgeries is preferable.

\section{P147-COLORECTAL-IBD}

\section{HEADACHE AS A MARKER OF QUALITY OF LIFE} IN ULCERATIVE COLITIS AFTER LAPAROSCOPIC SURGERY

\author{
J. Tajti Jr., Sz. Ábrahám, Zs. Simonka, A. Paszt, Gy. Lázár
}

\section{Department of Surgery, University of Szeged, SZEGED, Hungary}

Aims: Tension-type headache is a very common disease with a high socio-economic impact as its lifetime prevalence is $30-78 \%$ in the general population. Limited data are accessible on quality of life in patients with surgically treated ulcerative colitis. The aim of our study is to examine quality of life, concerning headache, among ulcerative colitis patients who had undergone laparoscopic and conventional surgery.

Methods: Between 1 January 2005 and 1 March 2016, surgery was performed due to ulcerative colitis in 75 patients. During this retrospective analysis the average duration of the follow-up was $46(1-124)$ months. The presence of headache was evaluated by the use of Brief Illness Perception and Headache Questionnaires.

Results: Among the primary headaches $(\mathrm{n}=27)$, tension-type headache occurred in 19 $(70.4 \%)$ cases, and $8(29.6 \%)$ patients had migraine. Among tension-type headache cases 17 $(89.5 \%)$ patients experienced episodic tension-type headache and $2(10.5 \%)$ suffered from chronic tension-type headache. Patients with headache had obtained a significantly higher score on Brief Illness Perception Questionnaire. Headache influenced quality of life and daily activities slightly to moderately in $79.1 \%(n=34)$ of the patients.

Conclusions: According to our study tension-type headache is common among patients with ulcerative colitis. This observation raises the question whether stress plays role in the pathogenesis of both diseases, which influences and worsens considerably quality of life. Tension-type headache negatively influences quality of life. Neurological examination, psychological and psychiatric guidance are worth considering in patients with ulcerative colitis.

\section{P148-COLORECTAL-IBD}

COMPARATIVE EVALUATION OF THE Results: OF OPEN APPENDECTOMY AGAINST LAPAROSCOPIC APPENDECTOMY IN THE PRACTICE OF THE DEPARTMENT OF GENERAL SURGERY

Z. Mekhtikhanov ${ }^{1}$, Z. Mekhtikhanov $^{2}$, M.K. Abdulzhalilov ${ }^{3}$,

${ }^{1}$ 2-nd Medical Faculty, KNMU, KHARKIV, Ukraine; ${ }^{2}$ Surgical Department, City Hospital, DERBENT, Russia; ${ }^{3}$ Surgical, City Hospital, MACHACHKALA, Russia

Aims: Comparison of laparoscopic and open appendectomy

Methods: A prospective analysis of the treatment results of 1006 patients with acute appendicitis. Time period-July 2012 to September 2018. Laparoscopic appendectomy was performed in $347(34.5 \%)$ patients and $659(65.5 \%)$ were operated on using the open method.

Open appendectomy was performed in the traditional way-shelter appendicular stump by dome of the cecum. Laparoscopic appendectomy was preceded by diagnostic laparoscopy, which allows you to fully examine the abdominal cavity. In laparoscopic appendectomy mobilization of the appendix depended on the anatomical situation, inflammatory changes in the ileocecal region. Antegrade mobilization was performed in 272 patients (78.4\%), retrograde removal in 48 cases (13.8\%), combined mobilization in 27 patients (7.8\%).

An important step in laparoscopic appendectomy is the treatment of the appendicular stump. We used ligation the base of the appendix with intracorporal suture in $59(17.0 \%)$ patients, endoloop ligation-in 153 (44.1\%), endoclipation in 135 (38.9\%) patients.

Results: Groups of patients who underwent laparoscopic appendectomy and open appendectomy were matched by sex, age, pathology of the appendix and the extent of peritonitis. Complications after open appendectomy were present in $28(4.2 \%)$ patients, including bleeding into the abdominal cavity in 1 , diffuse peritonitis in 3 , abscess of the abdominal cavity in 2 , adhesive intestinal obstruction in 3 , suppuration of wounds in 17 , subcutaneous eventration-in 2 patients.

After laparoscopic appendectomy, complications occurred in 12 patients (3.4\%): abdominal abscess in 1 , adhesive intestinal obstruction in 1 , suppuration of trocar wounds in 10 patients.

Discussion: These studies have shown that the total number of complications in performing laparoscopic and open appendectomy is approximately the same, but the number of intraabdominal complications in performing open appendectomy more than in laparoscopic appendectomy. Early adhesive intestinal obstruction was observed three times more often after open appendectomy than with laparoscopic appendectomy.

Conclusion: Laparoscopic appendectomy is accompanied by a smaller number of intra-abdominal complications, faster activation of patients and a reduction the period of their inpatient stay.

If there are contraindications to open appendectomy, laparoscopic appendectomy is the surgery of choice for acute appedicitis and its complications. 


\section{P149-COLORECTAL-IBD}

HAND-ASSISTED VERSUS LAPAROSCOPIC RESTORATIVE PROCTOCOLECTOMY FOR ULCERATIVE COLITIS AND FAMILIAL ADENOMATOUS POLYPOSIS

A. Kohyama, K. Watanabe, H. Suzuki, H. Karasawa, S. Ohnuma, T. Kamei, M. Unno, T. Naitoh

Department of Surgery, Tohoku University Graduate School of Medicine, SENDAI, MIYAGI, Japan

Background: Restorative proctocolectomy (RP) is an established surgical treatment for ulcerative colitis (UC) and familial adenomatous polyposis (FAP). Recent reports have showed that laparoscopic restorative proctocolectomy (Lap-RP) had more cosmetic advantages compared to open surgery or hand-assisted laparoscopic restorative proctocolectomy (HALS-RP).

Aim: The aim of this study was to evaluate the short-term results of Lap-RP compared with HALS-RP.

Methods: A retrospective study was conducted using a prospectively maintained database to compare a consecutive series of 38 patients who underwent Lap-RP with 45 patients who underwent HALS-RP from February 2000 to August 2018 in our institution. Patient characteristics, perioperative parameters, and the surgical outcomes were assessed. MannWhitney $\mathrm{U}$ and Fisher exact tests were used for statistical analysis.

Results: There were no statistically significant differences of clinical backgrounds between the two groups; disease ( 57.9 vs $66.7 \%$ UC; $\mathrm{p}=0.49)$, gender $(60.5$ vs $55.6 \%$ males; $\mathrm{p}=0.66)$, age (median age 29 vs 32 years; $\mathrm{p}=0.72)$ and cases with cancer $(18.4$ vs $15.6 \%$ $\mathrm{p}=0.78$ ). We had no conversions to open surgery. No mortality was found in this study. The amount of median blood loss and the incidence of surgical site infection (SSI) was significantly lower in the Lap-RP group (207 vs $260 \mathrm{ml} ; \mathrm{p}=0.019,2.6$ vs 24.6\%; $\mathrm{p}=0.005$, respectively). The median operative time was significantly longer (542 vs 471 $\min \mathrm{p}=0.0012)$ and the incidence of small bowel obstruction (SBO) (36.8 vs $13.3 \%$; $\mathrm{p}=0.02$ ) was higher in the Lap-RP group. The other parameters were comparable in the two groups as follows; median post-operative hospital stay ( $21 v s 20$ days; $\mathrm{p}=0.66$ ), and morbidity (Clavien-Dindo grade III) (36.8 vs $24.4 \%$, p = 0.24).

Conclusions: Lap-RP was favorable outcome in the blood loss and SSI, although the operative time and the incidence of SBO were problems to overcome in the future.

\section{P150-COLORECTAL-IBD}

\section{LAPAROSCOPY FOR CROHN'S DISEASE-10 YEARS} EXPERIENCE

\section{E. Koblihova, M. Kasalicky}

\section{Surgery, Central Military Hospital, PRAGUE, Czech Republic}

Aim: Over $80 \%$ of patients with Crohn's disease (CD) will require a surgical resection within 10 years of their diagnosis and one quarter will have another resection for disease recurrence. Laparoscopy should by preferred approach in surgery in $\mathrm{CD}$ due to reduced morbidity, faster recovery time, shorter hospital stay and reduction in adhesions and hernial formation.

Methods: Patients with CD with the tight stenosis in the distal ileum and/or ileo-colon or various colon stenosis were indicated for the laparoscopy. From January 2009 to November 2018 we performed 246 ileocolic resections, 63 hemicolectomies and 72 subtotal colectomies either totally laparoscopically or laparoscopically assisted. The average time of the procedure was $92 \mathrm{~min}(55-240 \mathrm{~min})$. The average return time of the bowel function was 3.5 days (2-9 days) and the hospital stay was from 6 to 10 days. Complications occurred in 14 patients $(3.7 \%)$. In 3 cases early ileus developed due to adhesions, in 1 case was anastomotic bleeding threated conservatively, the incisional hernia in minilaparotomy occurred in 6 cases and 4 wound infections occurred.

Conclusion: Minimally invasive surgery is becoming a gold standard in CD. It is safe and feasible in well-selected patients thanks to short- and long-term outcomes. Laparoscopic approach for recurrent disease is still in debate. Supported by MO1012.

\section{P151-COLORECTAL-IBD}

IS THERE A PLACE FOR LAPAROSCOPY IN THE DIAGNOSIS OF CROHN DISEASE?

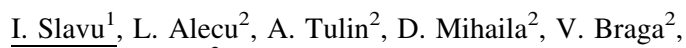
S. Constantinoiu ${ }^{3}$

${ }^{1}$ General Surgery Department, Emergency Clinical Hospital, Prof. Dr. Agrippa Ionescu, BUCHAREST, Romania; ${ }^{2}$ Department of Surgery, Emergency Clinical Hospital, Prof. Dr. Agrippa Ionescu, BUCHAREST, Romania; ${ }^{3}$ General Surgery Department, Saint-Mary Hospital, BUCHAREST, Romania

Aim: The aim of this pilot study was to observe as the title implies if one can rely on macroscopic visual elements to visible at laparoscopy to correctly diagnose and identify Crohn Disease so the patient can benefit from all advantages of laparoscopy, keeping in mind that a large part of these patients are young and require multiple surgical interventions during their lifetime. Our literature review failed to identify any large studies who addressed this issue.

Methods: our study was retrospective in nature and configured as a pilot study extended over a period of 1 year and included patients operated in our clinic with the help of laparoscopy for clinical suspicions of Crohn Disease in which other investigations failed to confirm the diagnosis or the symptoms showed no remission under medical treatment. Results: We identified a number of 6 patients in which Crohn Disease was confirmed via laparoscopy and the following macroscopic visual elements were constantly encountered and associated with the disease: enlarged reactive lymph nodes found only in the mesentery associated with the affected bowel, rigid intestinal loops which could not be compressed with laparoscopic instruments, fat-wrapping of the intestinal serosa.

Conclusions: Keeping in mind the beforementioned results, we consider this study to be a pilot study that raises this issue and tries to find a new role for minimally invasive approaches in Crohn Disease. There are macroscopic visual elements encountered constantly in Crohn Disease which can confirm or refute the diagnosis.

\section{P152-COLORECTAL-IBD}

WHEN IS LAPAROSCOPY AVOIDED IN THE TREATMENT OF SURGICAL COMPLICATIONS OF CROHN DISEASE?

I. Slavu' ${ }^{1}$ L. Alecu' ${ }^{2}$ A. Tulin ${ }^{2}$, D. Mihaila ${ }^{2}$, V. Braga ${ }^{2}$,

S. Constantinoiu ${ }^{3}$

${ }^{1}$ General Surgery Department, Emergency Clinical Hospital, Prof. Dr. Agrippa Ionescu, BUCHAREST, Romania; ${ }^{2}$ Department of Surgery, Emergency Clinical Hospital, Prof. Dr. Agrippa Ionescu, BUCHAREST, Romania; ${ }^{3}$ General Surgery Department, Saint-Mary Hospital, BUCHAREST, Romania

Aim: The aim of the study was to observe when laparoscopy is avoided when treating surgical complications of Crohn Disease.

Methods: We did a retrospective study which included all of the patients diagnosed and operated in our clinic for complications of Crohn Disease during a period of 3 years. Results: We identified a number of 62 patients operated for complications of Crohn Disease. Of these 15 were operated by minimally invasive procedures. We observed that laparoscopy was avoided in the case of intestinal fistulas $(p=0,02)$. Also when sepsis associated the surgical complication—laparoscopy was avoided $(\mathrm{p}=0,01)$. Age under 43 years represented another factor to avoid laparoscopy $(p=0,04)$.

Conclusions: Although laparoscopy offers numerous advantages careful selection of the patients is of utmost importance so the safety of the procedure can be ensured. 


\section{P153-COLORECTAL-Malignant}

\section{LAPAROSCOPIC RESECTION OF A HYPOGLYCEMIA- INDUCING RETROPERITONEAL FIBROSARCOMA}

\section{Abdelwahab, H. Fahmy}

\section{Surgery, Royal Commission Medical Center, YANBU} INDUSTRIAL, Saudi Arabia

Retroperitoneal sarcoma represents approximately $12-15 \%$ of all sarcomas and less than $0.5 \%$ of all neoplasia. Radiotherapy and chemotherapy still do not represent valid therapeutic alternatives; therefore radical surgery remains the only valid option. Complete surgical resection is the only potential curative treatment modality for retroperitoneal sarcomas. The ability of complete resection of a retroperitoneal sarcoma with tumor grading remains the most important predictor of local recurrence and disease-specific survival. Hypoglycemia is a rare but potentially lifethreatening presentation of soft tissue tumors the etiology of hypoglycemia may be difficult to diagnose, assays for insulin-like activity (ILA) were found to be high in the extract of tumor tissue, while insulin was not detected in significant concentration neither in the same extract nor in his serum. The most likely mechanism of hypoglycemia appears to be production of insulin like substance and increased utilization of glucose by the tumor. Laparoscopic surgery represents an alternative technique for radical resection of such tumors rather than traditional surgery. Only few cases of retroperitoneal tumors resected laparoscopically were reported in the literature.

We report a rare case of 53 years old male presented to ED unconscious due to hypoglycemia.he was resuscitated and admitted for further investigations. Hypoglycemic attack recurred again during the same evening of admission. Initial investigations were within normal except for serum glucose $35 \mathrm{mg} / \mathrm{dL}(2.0 \mathrm{mmol} / \mathrm{L})$. His TSH, glucagon \& cortisol levels were within normal, insulin and C-peptide levels were undetectable. Only hypokalemia $(2.3 \mathrm{mEq} / \mathrm{L})$. He tested negative for the anti-insulin antibodies. His abdominal ultrasound as well as his CT scans showed the presence of a large retroperitoneal tumor $(15 \mathrm{~cm} \times 12 \mathrm{~cm} \times 7 \mathrm{~cm})$ with a heterogeneous contrast effect. A glucose supplement was required to maintain the plasma glucose level within normal limits during which complete resection of the tumor which was performed laparoscopically.

Diagnosis of such hypoglycemia inducing Retroperitoneal Fibrosarcoma represents great challenge especially when patients presents only with hypoglycemia and no other abdominal symptoms, management using minimal invasive technique to resect and remove such tumors from the retroperitoneal region shows superiority in recovery and limitation of complications when done by experienced surgeons.

\section{P154-COLORECTAL_Malignant}

\section{EXTRAPLEURIC INTRABDOMINAL SOLITARY FIBROUS TUMOR: CASE REPORT AND LICTERATURE REVIEW}

\section{N. Mariani, A. Pisani Ceretti, C. Froiio, M. Longhi,} M. Giovenzana, E. Opocher

\section{General Surgery, ASST Santi Paolo e Carlo, MILAN, Italy}

Solitary Fibrous Tumor (SFT) is a rare fibroblastic mesenchymal neoplasm, tipically arising from the pleura, less frequently from other anatomic sites. SFT is an indolent neoplasm, but it have been described cases of greater aggressiveness in terms of local recurrences and more rarelly of distant metastases. Among the various extrapleural sites, intrabdominal, retroperitoneal localization is the most common site, followed by the pelvis soft tissues and parenchymatous organs. The most common clinical finding of intraabdominal localization is a palpable mass, and the pain is the most frequently associated symptom. The diagnosis is performed by imaging, but the histological as well as immunohistochemistry characterization of the lesion is the latest goal. Furthermore, histological features are used to attempt to identify the patient with a hight risk of malignant evolution of the tumor. The gold standard treatment is surgical approach, meanwhile there are no evidences about the efficacy of any adjuvant treatment. We present the case of a 62-year-old man affected by symptomatic TFS arising from mesosigma treated by surgical radical excision. Finally, we propose a review of the literature of last decade.

\section{P155-COLORECTAL-Malignant}

NATURAL ORIFICE SPECIMEN EXTRACTION SURGERY
(NOSES) FOR LAPAROSCOPIC RIGHT HEMICOLECTOMY

\section{Z. Liu, X.S. Wang}

Department of Colorectal Surgery, National Cancer Center/Cancer Hospital, Chinese Academy of Medical Sciences, BEIJING, China

Background: Laparoscopic right hemicolectomy involves making an additional incision to remove the specimen and perform the anastomosis. Recently, natural orifice specimen extraction surgery (NOSES) has been reported as an alternative approach without any additional incisions or extensions, may lead to better outcomes compared to conventional laparoscopic right hemicolectomy. In this video, we aimed to evaluate the safety and feasibility of NOSES for laparoscopic right hemicolectomy.

Methods: We describe the technique with transvaginal specimen extraction and D3 lymph node dissection in laparoscopic right hemicolectomy by this video. We performed intracorporeal anastomosis combined with a transvaginal route of specimen extraction after medial-to-lateral mobilization. Transverse transvaginal posterior colpotomy was performed under aid with visualization. The specimen was pulled into the sterilized plastic bag, passed transvaginally. The vaginal incision was then closed with a running suture.

Results: The operation time was $230 \mathrm{~min}$ and the hospital stay was 6 days. An excellent postoperative recovery was demonstrated and has shown future potential for less incision. The pathologic TNM stage is T3NOMO.

Conclusions: This video has shown that laparoscopic right hemicolectomy with the NOSES technique is feasible and safe for selected cases. The long-term benefits of this procedure need to be more evaluated.

\section{P156-COLORECTAL-Malignant}

AIR LEAK TEST CAN NOT REDUCES THE RATE OF ANASTOMOTIC LEAKAGE FOLLOWING COLORECTAL ANASTOMOSIS: A PROPENSITY MATCHED ANALYSIS

\section{S.H. Song, G.S. Choi, J.S. Park, S.Y. Park, H.J. Kim}

Colorectal cancer center, Kyungpook National University Chilgok Hospital, DAEGU, Korea

Aims: Intraoperative air leak test (ALT) is frequently performed to detect mechanically defect on anastomosis. But anastomotic leak is caused by multifactorial reasons. Therefore, there is debate about whether the ALT is effective. The aim of this study is show that ALT is not useful to prevent anastomotic leak following colorectal anastomosis.

Methods: We reviewed the prospectively collected records of colorectal cancer patients ( $n=2334$ ) from January 2012 to January 2018 at Kyungpook National University Hospital. We selected 1,191 patients who undergoing colorectal anastomosis. Exclusion criteria were patients with stoma, open surgery, synchronous tumor, metastatic tumor, and others. We performed propensity score analysis and compared postoperative outcomes for ALT and no ALT groups in a 1:1 matched cohort. Covariates included age, gender, BMI, tumor height, preoperative chemoradiation status, and number of linear stapler use.

Results: 201 patients were allocated to each group and there were no significant differences. No ALT group was associated longer operation times and more blood loss than ALT group ( $135.4 \mathrm{~min}$ vs. $126.8 \mathrm{~min}, \mathrm{p}=0.042,50.9 \mathrm{ml}$ vs. $35.1 \mathrm{ml}, \mathrm{p}=0.002)$. Intra-operative transfusion rate, anastomotic level, procedure type (laparoscopic, robotic), and postoperative hospital stay are similar in both groups. In ALT group, 1 patient was positive result in ALT (0.5\%) and treated by rectal tube drainage, in result, anastomotic leak did not occurred. 200 patients were negative result in ALT (99.5\%), but anastomotic leak occurred in 9 patients (4.5\%). Postoperative anastomotic leak rate is same in both groups (4.5\%). Depending on the need for reoperation, major leak rate is also same in both groups (2.0\%). In other morbidities, there were no significant difference in both groups. The rate of major complications (Dindo's complication grade above 3 ) is also similar in both groups ( $5.0 \%$ vs. $5.5 \%, \mathrm{p}=0.645)$. On multivariate analysis, ALT was not risk factor for anastomotic leak. Male sex (OR 9.57, 95\%CI 1.25-73.47) and low tumor height $(<7 \mathrm{~cm})(\mathrm{OR} 3.97,95 \% \mathrm{CI} 1.44-10.95)$ were the independent risk factors for anastomotic leak. Conclusions: Our study shows that air leak test may not be useful to prevent anastomotic leakage following colorectal anastomosis. Further randomized trials are warranted to validate our results. 


\section{P157-COLORECTAL_Malignant}

\section{APPLICATIONS OF INDOCYANINE GREEN ENHANCED FLUORESCENCE IN LAPAROSCOPIC COLORECTAL RESECTIONS FOR CANCER}

\section{Santi ${ }^{1}$, L. Casali ${ }^{2}$, C. Franzini ${ }^{2}$, P. Orsi ${ }^{2}$, V. Violi ${ }^{1}$}

${ }^{1}$ Surgery, Hospital of Fidenza, University of Parma, FIDENZA (PR), Italy; ${ }^{2}$ Surgery, Hospital of Fidenza, FIDENZA (PR), Italy

Recently, Indocyanine Green (ICG) fluorescence has been introduced in laparoscopic colorectal surgery to provide detailed anatomical information.The aim of our study is the application of ICG imaging during laparoscopic colorectal resections: to identify the sentinel lymph node (SLN) to search micrometastases that can be missed with the conventional pathological exam, and to assess anastomotic perfusion to reduce the risk of anastomotic leak.

After tumor identification $5 \mathrm{ml}$ of ICG solution $(0.3 \mathrm{mg} / \mathrm{Kg})$ is subserosal peritumoral injected. A Full HD IMAGE1 S camera, switching to NIR mode, in about 10 min displays fluorescence: the SLN is identified and the SLN biopsy (SLNB) is performed.After the transection $5 \mathrm{ml}$ of ICG solution is injected to confirm the stumps perfusion. If there is an ischemic area, a new resection is performed.After the anastomosis is performed, another bolus of ICG is intravenous injected to confirm the anastomotic perfusion. When the sentinel node is negative for cancer metastases by conventional histological examination, ultrastaging is performed by serial sections. When no micrometastases are identified on these sections, immunohistochemical techniques are applied.

From November 2016, 70 patients were enrolled: 22 left colectomy, 38 right colectomy, 2 transverse resections, and 8 splenic flexure resections. In two cases, one left colectomy and one right colectomy, the anastomotic perfusion wasn't good and the surgical strategy was changed. Four postoperative complications occurred, of which one anastomotic leak, due to a mechanical problem. From November 2017, 40 patients were enrolled to perform the SLNB: 23 right colectomy, 11 left colectomy, 1 transverse resection and 5 splenic flexure resections. The SLN was identified in 37 cases. 17 cases were found to be N0 to the conventional examination and were subjected to ultrastaging. The serial sections showed micrometastases in two cases. In the other cases the immunohistochemistry was performed but the exam is still in progress. ICG-enhanced fluorescence imaging is a safe, cheap and effective tool to increase visualization during surgery. It's recommended to assess the anastomotic perfusion in order to reduce the incidence of anastomotic leak, and to perform the SLNB for the SLN ultrastaging in order to identify micrometastases.

\section{P158-COLORECTAL_Malignant}

\section{TRANSANAL ENDOSCOPIC MICROSURGERY IN THE PATIENT WITH EARLY RECTAL CANCER: ROLE OF THE SENTINEL NODE CONCEPT}

V.V. Grubnik $^{1}$, S.P. Degtyrenko ${ }^{2}$, V.V. Ilyashenko ${ }^{2}$, V.V. Grubnyk ${ }^{2}$

${ }^{1}$ Department of surgery, Odessa medical university, ODESSA, Ukraine; ${ }^{2}$ Department of Surgery \#1, Odessa medical university, ODESSA, Ukraine

Aim: of the study was elaboration of indications for transanal endoscopic microsurgery (TEM) in the patients with early rectal cancer based on sentinel lymph node (SLN) concept. Methods: For the last 3 years, TEM was performed on 28 patients' with early rectal cancer. There were 19 women and nine men, age 68 to 87 . Localization of tumors was $4-12 \mathrm{~cm}$ from anus. Mean size of tumors was $3.8 \mathrm{~cm}$. Full thickness excision was performed in all patient with suturing of mucosa. During follow-up in three patients' metastasis in lymph nodes of mesorectum were detected. All of these patients were re-operated: laparoscopic colectomy with total mesorectal excision (TME) was done. For the last year in 9 patients with early stage rectal cancer we used indocyanine green (ICG) with fluorescent imaging for mapping sentinel lymph node. ICG was injected in four quadrants to submucosa around the tumor. During the laparoscopy, SLN was detected and removed with morphological examination.

Results: Among nine patients in 8 patients, SLN was negative. TEM was performed in these patients with good results. After 10-12 months no recurrence or metastasis were detected in these patients. In two patients with positive SN laparoscopic TME was performed with low colorectal anastomosis. Anastomotic complication was occurred in one patient.

Conclusion: TEM procedure is highly effective in selected group of patients with early rectal cancer. Mapping and examination of SLN can clarify indication for the TEM in the patients with early rectal cancer.

\section{P159-COLORECTAL-Malignant}

\section{LAPAROSCOPIC NATURAL ORIFICE SPECIMEN EXTRACTION USING NEEDLESCOPIC INSTRUMENTS FOR COLON CANCER}

K. Sakamoto $^{1}$, Y. Kojima ${ }^{1}$, Y. Tsuchiya ${ }^{1}$, Y. Okazawa $^{1}$,

M. Kawai ${ }^{1}$, S. Ishiyama ${ }^{1}$, K. Sugimoto ${ }^{1}$, H. Kamiyama ${ }^{1}$,

M. Takahashi ${ }^{1}$, T. Fukunaga ${ }^{2}$, Y. Kajiyama ${ }^{2}$

${ }^{1}$ Coloproctological Surgery, Juntendo University, TOKYO, Japan; ${ }^{2}$ Gastroenterological Surgery, Juntendo University, TOKYO, Japan

Purpose: Laparoscopic surgery for colorectal cancer provides better short-term benefits and similar long-term outcomes compared with conventional open surgery. Unlike minimally invasive surgery, natural orifice specimen extraction (NOSE) can provide additional advantages by reducing morbidity and postoperative pain related to the surgical extraction site.

This study aimed to evaluate the efficacy and safety of a NOSE procedure using needlescopic instruments for colon cancer surgery.

Methods: Between November 2013 and February 2018, 6 patients underwent laparoscopic NOSE using needlescopic instruments.

The first port for the camera was placed at the umbilicus. A $5-\mathrm{mm}$ or 12-mm port was inserted in the right lower quadrant. A 3-mm or 5-mm port was inserted in the right upper quadrant. Individual needlescopic forceps for the assistant were inserted into left upper and lower quadrant ports. Thus, a total of 5 ports were placed.

The superior rectal artery and inferior mesenteric vein were ligated with clips, and colonic mobilization was performed using a medial to lateral approach.

After rectal stump irrigation, the distal rectum was transected using an endoscopic linear stapler. The proximal colon and associated mesentery were transected. After the rectal stump was opened, a wound retractor was pulled through the anus and inserted in the rectal lumen. The resected specimen was transanally extracted through this route. An anvil was intracorporeally attached to the proximal colon, and the open rectal stump was reclosed using an endoscopic linear stapler; colorectal anastomosis was then performed using a double-stapling technique.

Results: Of the 6 patients, 4 were male and 2 were female, with a median age of 71 years (44-76 years). Median body mass index was 21.8 . The tumor site was in the sigmoid colon in 4 patients and rectosigmoid colon in 2 patients. Median operative time was $309 \mathrm{~min}$ and blood loss was $13 \mathrm{~mL}$. There was no conversion to open surgery. No postoperative complication was observed. Median postoperative hospital stay was 8 days (7-12 days).

Conclusions: NOSE surgery using needlescopic forceps is an easily performed type of reducedport surgery with a conventional port arrangement. This procedure is feasible for the selected patients. 


\section{P160-COLORECTAL_Malignant}

ADVANTAGE OF TEMPERARY LOOP TRANSVERSE COLOSTOMY AND LAPAROSCOPIC LEFT HEMICOLECTOMY IN SPLENIC FLEXURE COLON CANCER OBSTRUCTION: 3 CASE REPORTS

\section{$\underline{\text { K.H. Lee }}{ }^{1}$, Y.H. Sul ${ }^{2}$}

${ }^{1}$ Surgery, Chungnam National University Hospital, DAEJEON, Korea; ${ }^{2}$ Surgery, Choongbuk National University Hospital, CHEONGJU, Korea

Introduction: Splenic flexure colon cancer accompanying obstruction is usually managed stent insertion as a bridge to surgery and left hemicolectomy, or subtotal colectomy. However, stent insertion can fail more often than in sigmoid colon because it requires longer colonoscopic approach in the circumstance of impossible bowel preperation. Although subtotal colectomy has advantage in the aspect that it is 1-stage treatment, it needs open surgery in most cases, right colon has to be sacrificed without oncologic neccesity, and preoperative staging and evaluation can be insufficient Despite colostomy is reluctant procedure when considering quality of life, in splenic flexure colon cancer obstruction, we can obtain prompt stabilization of patient state, suffient time to preoperative staging and evaluation, and also we can achieve minimally invasive surgery by using colostomy site as mini-laparotomy and close colostomy before discharge. Colostomy site, tumor location, and minilaparotomy site for next radical surgery have to be considered comprehensively before making colostomy incision. Colostomy site has to be appropriate as mini-laparitomy site for feasibility of laparoscopic left hemicolectomy and the colostomy has to be included in the specimen with caution to prevent unneccessary lengthening of the specimen. We experienced 3 cases which were treated succefully in this strategy and report them.

Cases: 87 years old woman with cT4aN1M0 splenic flexure colon cancer obstruction underwent loop transverse colostomy and laparoscopic left hemicolectomy on the $12<\sup >$ th $<$ sup $>$ day postoperively. 73 years old with cT4aN1M0 proximal descending colon cancer obstruction underwent loop transverse colostomy and laparoscopic left hemicolectomy on the $12<\sup >$ th $</$ sup $>$ day postoperively. 82 years old woman with cT3N1M0 proximal descending colon cancer obstruction underwent loop transverse colostomy and laparoscopic left hemicolectomy on the $15<\sup >$ th $<$ /sup $>$ day postoperively. No patients underwent significant complication. Result: Temperary loop transverse colostomy and laparoscopic left hemicolectomy via colostomy site in splenic flexure colon cancer obstruction has advantage of quick stabilization of patient's status, suffient preoperative staging and evaluation, achieving minimally invasive surgery, and also rapid colostomy closure before discharge.

\section{P161-COLORECTAL_Malignant}

OUR TATME PROCEDURE FOR LOCALLY ADVANCED LOW RECTAL CANCER FOLLOWING CHEMORADIOTHERAPY

Y. Nakamoto $^{1}$, R. Okamoto ${ }^{1}$, F. Kimura ${ }^{1}$, H. Yanagi ${ }^{1}$, T. Nakajima ${ }^{1}$, H. Yoshie ${ }^{2}$, N. Yamanaka ${ }^{1}$

${ }^{1}$ Surgery, Meiwa Hospital, NISHINOMIYA, Japan, ${ }^{2}$ Surgery, Yoshie Clinic, ITAMI, Japan

Background: Short-course chemoradiotherapy using hyper-fractionation method (SCRT; 25 Gy/ 10 fraction/5 days $+\mathrm{S}-1$ or Xeloda) is performed to secure circumferential resection margin (CRM) due to tumor shrinkage, reduction of cancer cells with viability, reduction of radiation hazard for resectable locally advanced lower rectal cancer (more T3 or N1). The patient underwent radical surgery after one month of SCRT. For more locally advanced lower rectal cancer (T4 or N2), induction chemotherapy is performed before SCRT. For patients with poor efficacy of chemotherapy, we also do normal 25 fraction 45 Gy radiotherapy.

Methods: We introduced TaTME from last August, and 18 cases were performed so far. In all cases temporary stoma has been constructed, and intersphincter resection (ISR) is based on partial ISR avoiding total ISR considering postoperative anal function. If possible, colonic J-pouch is added, and pelvic floor repair may be added for ESR cases and older people. At first one team preceded with the anal operation and shifted to the abdominal procedure, now it is done with two teams with the advantage of getting good visual field from both sides when there is difficulty identifying the right dissecting layer. TaTME is very useful in cases such as large tumor, obesity, and narrow pelvis. Furthermore, when it is difficult to identify the dissecting layer by scarring after CRT, it is more possible to control the CRM/DRM of cancer.

Results: 14 cases of ISR, 3 case of APR, 1 case of TPE were performed, and in 10 of these cases lateral lymph node dissection was also performed (one side 5, both sides 5). Postoperative complications were 2 anastomotic leakage, 4 pelvic floor infection, 2 perineum infection, and 1 bowel obstruction.

Conclusions: TaTME for locally advanced lower rectal cancer is useful even after chemotherapy and SCRT.

\section{P162-COLORECTAL-Malignant}

COST ANALYSIS OF SINGLE-INCISION VERSUS CONVENTIONAL LAPAROSCOPIC SURGERY FOR COLON CANCER: A PROPENSITY SCORE-MATCHING ANALYSIS

\author{
C.W. Kim ${ }^{1}$, H. Hur ${ }^{2}$, B.S. $\mathrm{Min}^{2}$, K.Y. Lee ${ }^{2}$, N.K. Kim ${ }^{2}$
}

${ }^{1}$ Surgery, Kyung Hee University Hospital at Gangdong, SEOUL, Korea; ${ }^{2}$ Surgery, Severance Hospital, Yonsei University College of Medicine, SEOUL, Korea

Background: Although many studies have demonstrated similar perioperative outcomes for single-incision laparoscopic surgery (SILS) and conventional laparoscopic surgery (CLS) for colon cancer, few have directly compared the costs of them. We aimed to compare costs between SILS and CLS for colon cancer.

Methods: We analyzed the clinical outcomes and overall hospital costs of patients who underwent laparoscopic surgery for colon cancer from July 2009 to September 2014 at Severance Hospital; 288 were used for analysis after propensity score matching. The total hospital charge, including fees for the operation, anesthesia, preoperative diagnosis, and postoperative management was analyzed.

Results: The total hospital charges were similar in both groups ( $\$ 8770.40$ vs. $\$ 8352.80$, $P=0.099$ ). However, the patients' total hospital bill was higher in the SILS group than in the CLS group ( $\$ 4184.82$ vs. $\$ 3735.00, P<0.001)$ mainly due to the difference of the cost of access devices. There was no difference in the additional costs associated with readmission due to late complications between the two groups ( $\$ 2383.08$ vs. $\$ 2288.33, P=0.662)$.

Conclusions: SILS for colon cancer yielded similar costs as well as perioperative and long-term outcomes compared with CLS. Therefore, SILS can be considered a reasonable treatment option for colon cancer for selective patients.

\section{P163-COLORECTAL-Malignant}

TECHNICAL CONSIDERATIONS DURING TRANSANAL TOTAL MESORECTAL EXCISION_OBSERVATIONAL STUDY OF 25 CASES

D.K. Penchev, P. Ivanov, G. Kotashev, V. Kyosev, V. Hristova, V. Krasimir, G. Grigorov, V. Mutafchiyski

Endoscopic Surgery, Military Medical Academy Sofia, SOFIA, Bulgaria

Aims: Technology improvements in medicine allow the development of new minimally invasive approaches. Despite every single advantage of these new devices they also can cause technical problems and difficulties for the surgical team. Well known from last few years-laparoscopic assisted transanal total mesorectal excision for distal rectal cancer is perfect example for a quite new procedure, based on the combination of forgotten old surgical principles and technology advances. The aim of the study is to analyze the rate of technical problems during the procedure and to measure the impact of them on the operative time.

Methods: We conducted prospective observational study related to technical problems during the procedure. For the period between september 2017 and november 2018 in the department of Endoscopic endocrine surgery and coloproctolgy at Military Medical Academy—Sofia have been performed 25 laparoscopic assisted transanal total mesorectal excisions. We used standard local preoperative work up and postoperative care protocols. We defined technical problem as intraoperative event different from complication leading to delay in operative time. Every technical problem during the procedure was recorded and time for resolving the problem was measured in seconds. Results: Overall technical problems occurred in 11 of the cases. Most of them were related to the insufficient smoke evacuation during the 6 cases. The second most common technical problem were the excessive rectal stump spasms during the procedure-this complication occurred in 3 of the patients. Mean delay of the procedure related to technical problems is $21 \mathrm{~min}$. In our series we experienced only one intraoperative complication which was specimen perforation during the dissection. Three complications occurred in postoperative period-two urinary retentions and one perianastomotic abscess, without need of reoperation.

Conclusion: Technical problems during the procedure can be source of delay in operative time. Correct use of devices in operating room is the key to reduce technical issues. Technical problems can increase the rate of intraoperative near miss events and complications during the transanal total mesorectal excision. 


\section{P164-COLORECTAL_Malignant}

THE REAL (RECTAL ANASTOMOTIC LEAK) SCORE FOR PREDICTION OF ANASTOMOTIC LEAK AFTER RECTAL CANCER SURGERY

A. Arezzo ${ }^{1}$, M. Migliore ${ }^{1}$, P. Chiaro ${ }^{1}$, S. Arolfo ${ }^{1}$, C. Filippini ${ }^{1}$, D. di Cuonzo ${ }^{2}$, R. Cirocchi ${ }^{3}$, M. Morino ${ }^{1}$

${ }^{1}$ Department of Surgical Sciences, University of Turin, TURIN, Italy; ${ }^{2}$ Cancer Epidemiology Unit, San Giovanni Batista Hospital, TURIN, Italy; ${ }^{3}$ Department of General Surgery, University of Perugia, Terni Hospital, PERUGIA, Italy

Aims: Anastomotic leak after rectal cancer surgery constitutes a severe complication associated with poorer oncologic outcome and quality of life. Preoperative assessment of the risk for anastomotic leak is a key component of surgical planning, including the opportunity of creating a defunctioning stoma.

Methods: Studies on rectal cancer surgery published between 2000 and 2015 were systematically reviewed according to the Preferred Reporting Items for Systematic Reviews and Meta-Analyses of Individual Participant Data (PRISMA-IPD) guidelines. With the aim to generate a score for anastomotic leak, all available per-operative covariates were used as independent factors in a logistic regression model with anastomotic leak as dependent variable. A Receiver Operating Characteristic curve (ROC) analysis was generated. We selected as threshold the value that allowed a missing rate of anastomotic leak $<2 \%$. The predictive power of the previously selected cut-off was validated in an independent set of patients.

Results: Twenty-six centers provided individual data on 9735 patients. With a threshold value of the ROC corresponding to 0.0791 in the training set, the area under the ROC curve (AUC) was $0.585(\mathrm{p}<0.0001)$. Sensitivity and specificity of the model's probability $>0.0791$ to identify anastomotic leak were $79.1 \%$ and $32.9 \%$, respectively. Accuracy of the threshold value was confirmed in the validation set with $77.8 \%$ of sensitivity and $35.2 \%$ specificity.

Conclusions: We trust that, with further refinement using prospective data, this nomogram based on preoperative risk factors may assist surgeons in decision making. The score is now available online (http://www.real-score.org)

\section{P165-COLORECTAL_Malignant}

\section{THE USE OF MINIMALLY INVASIVE ACCESS IN COLORECTAL SURGERY}

\author{
O. Usenko, O. Tyvonchuk, Y. Voitiv, V. Moskalenko
}

Gastrointestinal tract surgery, Shalimov's National Institute of Surgery and Transplantology, KYIV, Ukraine

Background: The experience of the use of minimally invasive interventions in 83 patients with malignant tumors of the colon and rectum, diverticulosis of the sigmoid colon has been analyzed. The peculiarities of performing surgical interventions with miniinvasive access, depending on the type and localization of the process are shown.

Methods: Laparoscopic access during the colectomy was used in 83 patients treated in the department of surgery of the gastrointestinal tract during the 2015-2018. Most patients-76 (92\%) operated on malignant tumors of different parts of the colon and rectum (adenocarcinoma G2,3). In $7(12.5 \%)$ cases laparoscopic interventions were performed in patients with diverticular colon disease. In the group of patients with colorectal cancer localization of the tumor in the right parts was observed in $27(36 \%)$ patients, in the left-in $30(39 \%)$, in the rectum-19 $(25 \%)$

Results: In the adenocarcinoma of the sigmoid colon, performed a left laparoscopic hemicolectomy (19 cases) and resection of the sigmoid colon (11). Was executed high clipping and intersection of the lower mesenteric vessels, aorto-iliac lymphatic dissection. In the standard scope, lymph node dissection was performed with removal and testing of not less than 12 epi-, para- and mesocolical lymph nodes ( $\max 21)$. The average length of the laparoscopic stage is $125 \pm 22 \mathrm{~min}$.

Laparoscopic right hemicolectomy (27 cases) was performed in accordance with the principles of CVL (Central Vascular Ligation) and CME (Complete Mesocolic Excision). Intracorporal ileotransversoanastomosis was formed by a semimanual method with EndoGia Universal and V-Lock suture material. The average length of the laparoscopic stage was $125 \pm 25 \mathrm{~min}$, the open phase was $56 \pm 14$.

In the tumor of the lower and middle ampullary parts of the rectum (19 cases) after neoadjuvant chemoradiotherapy, was executed a laparoscopic total mesorectumectomy.

Conclusions: The use of minimally invasive technologies in colorectal surgery provides a complete revising of the abdominal organs, adequate scope of resection and lymph nodes dissection in surgical interventions.

\section{P166-COLORECTAL-Malignant}

USEFULNESS OF RETRO-MESENTERIC MEDIAL APPROACH IN SINGLE PORT COMPLETE MESOCOLIC EXCISION WITH RIGHT SIDE COLON CANCER

M. Fukunaga ${ }^{1}$, K. Nagakari ${ }^{2}$, S. Yoshikawa ${ }^{2}$, M. Ouchi ${ }^{2}$,

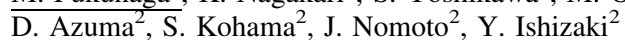

${ }^{1}$ Gastrointestinal Surgery, Juntendo Tokyo Koto Medical Center, TOKYO, Japan; ${ }^{2}$ Gastrointestinal Surgery, Juntendo University Urayasu Hospital, URAYASU, Japan

Background: It is thought that complete mesocolic excision (CME) improves the oncologic outcomes for colon cancer. But, precise mesenteric mobilization from retroperitoneum and safe ligations at the origins of central vessels are considered to be technically difficult in single por surgery(SPS). To resolve this problem, we utilize retro-mesenteric medial approach for right side colon cancer. Herein, we introduce this technique and assess its outcomes.

Operative procedure: The multi-trocar platform is placed in the umbilical site. 3D laparosopy is inserted from one of this channels. The surgeon manipulates instruments via the other 2 channels 1st step: Right colonic mesentery is mobilized medial to lateral from the head of the pancreas and retroperitoneum along the embryonic plane. 2nd step: The origins of ileocolic and right colic vessels are divided and central lymph node dissection is achieved. 3rd step: hepatic flexure is taken down from cranial. And right lateral attachment is dissected away and CME is achieved. 4th step: Specimen is extracted and anastomosis is performed using a functional end to end anastomosis extracorporealy.

Results: From April 2009 to December 2018, 125 consecutive patients underwent SPS-CME with right side colon cancer. There were 52 in Stage I, 32 in Stage II, 30 in Stage III and 11 in Stage IV. The mean operative time was $207 \mathrm{~min}$. The mean estimated blood loss was $42 \mathrm{ml}$ There was no conversion to open surgery. Additional port was placed in 4 patients $(3.2 \%)$. Intraoperative bleeding was occurred in 1 patient. Anastomotic leakage was observed in 1 patient $(0.8 \%)$, intestinal obstruction $1(0.8 \%)$ and wound infection in $3(2.4 \%)$.

Conclusion: These results suggest that retro-mesenteric medial approach in single port surgery with right side colon cancer is useful and safe technique. 


\section{P167-COLORECTAL_Malignant}

QUALITY OF LIFE AFTER SINGLE PORT LAPAROSCOPIC SURGERY FOR COLON CANCER: POSTOPERATIVE 1-YEAR OUTCOMES OF RANDOMIZED CONTROLLED TRIAL (SIMPLE TRIAL)

B.M. Kang ${ }^{1}$, H.J. Kim ${ }^{2}$, B.H. Kye ${ }^{3}$, S.C. Lee $^{4}$, K.Y. Lee ${ }^{5}$, S.J. Park ${ }^{5}$,

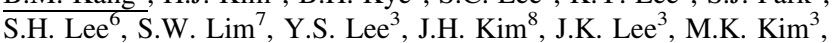
J.G. $\mathrm{Kim}^{3}$

${ }^{1}$ Surgery, Chuncheon Sacred Heart Hospital, Hallym University College of Medicine, CHUNCHEON, Korea; ${ }^{2}$ Surgery, St. Vincent's Hospital, College of Medicine, The Catholic University of Korea, SUWON, Korea; ${ }^{3}$ Surgery, Seoul St. Mary's Hospital, College of Medicine, The Catholic University of Korea, SEOUL, Korea; ${ }^{4}$ Surgery, Daejeon St. Mary's Hospital, College of Medicine, The Catholic University of Kor, DAEJEON, Korea; ${ }^{5}$ Surgery, Kyung Hee Medical Center, Kyung Hee University School of Medicine, SEOUL, Korea; ${ }^{6}$ Surgery, Kyung Hee University Hospital at Gangdong, Kyung Hee University School of Medici, SEOUL, Korea; ${ }^{7}$ Surgery, Hallym Sacred Heart Hospital, Hallym University College of Medicine, ANYANG, Korea; ${ }^{8}$ Surgery, Incheon St. Mary's Hospital, College of Medicine, The Catholic University of Kor, INCHEON, Korea

Aims: This multicenter, randomized controlled trial (SIMPLE trial) aimed to investigate the quality of life (QoL) and patient satisfaction of single port laparoscopic surgery (SPLS) for colon cancer, compared with multiport laparoscopic surgery (MPLS).

Methods: Patients with histologically diagnosed adenocarcinoma in cecum, ascending and sigmoid colon were eligible for this trial. Eligible patients were randomly assigned to the SPLS or MPLS group at a ratio of 1:1. QoL was measured with the EORTC QLQ-C30 third edition (Korean version) preoperatively and postoperatively at month 1, 3, 6 and 12. In addition, patient satisfaction was surveyed with a five-point questionnaire at postoperative 12 month. To exclude the impact of adjuvant chemotherapy on QoL, subgroup analysis for patients with or without adjuvant chemotherapy were carried out. (ClincalTrials.gov identifier: NCT01480128)

Results: Total 359 patients were randomly allocated into the SPLS group $(n=179)$ and MPLS group $(n=180)$. In total patients, global health status and five functional scale steadily increased and nine symptom scales also gradually improved over time. But, nausea/ vomiting and appetite loss temporally deteriorated at postoperative 3 month. Pain score was significantly worse in the MPLS group (11.6 in the SPLS group vs. 17.6 in the MPLS, $\mathrm{p}=0.002)$ at postoperative 1 month and appetite loss score was significantly worse in the SPLS group (19.9 vs 13.5, p = 0.017) at postoperative 3 month. Except for that domains, all the other items of QoL between groups were not different until postoperative 12 months. Patient satisfaction was significantly higher regarding the operation $(\mathrm{p}=0.025)$ and the abdominal wound $(\mathrm{p}=0.025)$ in the SPLS group. In patients without adjuvant chemotherapy, some items of QoL (Global health status, physical functioning, role functioning, emotional functioning, fatigue and pain) were significantly better in the SPLS group at postoperative 1 month. Since postoperative 3 month, all of QoL domains (except pain score) were similar between groups.

Conclusion: Although postoperative pain was temporarily better in the SPLS, most of QoL domain were similar between the SPLS and the MPLS group until postoperative 12 month. In patients without adjuvant chemotherapy, SPLS showed better outcomes in some of functional scales and symptom scores at postoperative 1 month.

\section{P168-COLORECTAL-Malignant}

MULTIMODAL ANALGESIA USING ACETAMINOPHEN FOR LAPAROSCOPIC COLORECTAL CANCER SURGERY

Y. Kojima ${ }^{1}$, K. Sakamoto ${ }^{1}$, Y. Tomiki ${ }^{1}$, A. Okuzawa ${ }^{1}$, T. Makoto ${ }^{1}$, K. Sugimoto $^{1}$, M. Kawai ${ }^{1}$, Y. Okazawa ${ }^{1}$, K. Mizukoshi ${ }^{1}$, Y. Makino ${ }^{1}$, K. Amemiya ${ }^{1}$, T. Hagiwara ${ }^{1}$, T. Fukunaga ${ }^{2}$

${ }^{1}$ Coloproctological surgery, Juntendo University, TOKYO, Japan;

${ }^{2}$ Gastroenterological Surgery, Juntendo University, TOKYO, Japan

Introduction: Laparoscopic surgery causes less postoperative pain compared with pain after laparotomic surgery, and its low invasiveness should be considered for pain control We have previously controlled postoperative pain by epidural anesthesia. In this study we compared postoperative multimodal analgesia centering on acetaminophen in patients who underwent laparoscopic colorectal cancer surgery with the conventional method.

Subjects: The subjects were 39 patients who underwent laparoscopic colorectal cancer surgery between January 2018 and June 2018. Surgery was performed under epidural anesthesia in 24 patients and multimodal analgesia in 15: periodic acetaminophen administration + transverse abdominis plane (TAP) block in 6, periodic acetaminophen administration + local anesthesia of the wound in 2, and periodic acetaminophen administration + intravenous patient-controlled analgesia (IVPCA) in 7. The operating roomoccupying time, postoperative pain (NRS), frequency of taking analgesics as needed, and postoperative nausea were investigated for 3 days after surgery and the duration of urethral catheter placement and postoperative intestinal movement were investigated in the epidural anesthesia and multimodal analgesia groups.

Results: While the time from entering the operating room to initiation of surgery was significantly shorter, the time from completion of surgery to leaving the room was significantly longer in the multimodal analgesia group. There was no difference in the operating room-occupying time. The frequency of postoperative pain was significantly lower in the multimodal analgesia group on postoperative day (POD) 2 . The frequency of taking analgesics as needed was significantly lower in the multimodal analgesia group on POD1, 2, and 3. No significant difference was noted in the duration (number of days) of urethral catheter placement or postoperative nausea between the 2 groups. Regarding postoperative intestinal movement, discharge of gas occurred significantly earlier in the epidural anesthesia group. The total number of incidents of complications in the epidural anesthesia group was 12

Discussion: In laparoscopic colorectal cancer surgery, the effect of multimodal analgesia centering on periodic administration of acetaminophen without epidural anesthesia for postoperative analgesia was sufficient compared with the effectiveness of epidural anesthesia. This approach to analgesia may be useful because none of the potential complications of epidural anesthesia occur. 


\section{P170-COLORECTAL_Malignant}

\section{DIGITAL 3-DIMENSIONAL VIRTUAL MODEL OF THE COLORECTAL CT SCAN IMAGEN OF PATIENTS WITH COLORECTAL CÁNCER AND ITS APPLICATION IN SURGICAL PRACTICE}

$<\mathrm{u}>$ N. Pérez-Serrano $</ \mathrm{u}>^{1}$, J.F. Trebolle ${ }^{2}$, F.M. Sanchez Margallo $^{3}$, J.R. Blanco Ramos ${ }^{4}$, B. Romero Fernández ${ }^{5}$, I. Fernandez $\mathrm{Marzo}^{5}$, S. Aguas Blasco

${ }^{1}$ General Surgery, Public Hospital San Pedro Logroño, ALFARO (LA RIOJA), Spain; ${ }^{2}$ General Surgery, Hospital Royo Villanova, ZARAGOZA, Spain; ${ }^{3}$ Cirugía Minima Invasiòn, Centro de Cirugía de Mínima Invasión Jesús Usón, CÁCERES, Spain; ${ }^{4}$ CIBIR, Hospital San Pedro, LOGROÑO, Spain; ${ }^{5}$ General Surgery, Hospital San Pedro, LOGROÑO, Spain

In the last years the application of new technologies like $3 \mathrm{D}$ vision or virtual reality have provided to surgeons the possibility of establish a preoperative surgical plan of each surgery and of each patient. These advances are specially useful in minimally invasive colorectal surgery due to the variability in location, anatomical relationship with other organs and vascular variants of these type of surgeries. The aim of our work is to built a digital 3-Dimensional virtual model of the colorectal CT scan imagen of patients with colorectal cancer. The virtual models are obtained from the preoperative CT scan. The CT scans that we use to this work are General Electric Healthcare Revolution GSI ${ }^{\circledR}$ and Siemens Somatom Perspective $64 \circledast$ and the size of each image is $1 \mathrm{~mm}$. A Medical Software let us build a reconstruction of colorectal digital images where a radiologist has marked the exact image of the tumor so we obtain a 3D reconstruction which can provide an enhanced understanding of crucial anatomical details like the exact location of the tumor and the relationship with other organs and structures of the patient which can be selectively displayed or hidden.

This information has an important applicability into clinical practice since it lets surgeons estimate the colorectal anatomy, tumor size and relationships, providing key landmarks to choose the most appropiate surgery, the best trocar location and a safer dissection specially in some cases whose location can change the kind of surgery radically.

We present some cases where virtual models were crucial for the preoperative and intraoperative surgical plan, showing the potential interest of these $3 \mathrm{D}$ reconstructions in colorectal surgery. In conclusion the $\mathrm{CT}$ scan colorrectal image reconstruction can provide an enhanced understanding of crucial anatomical details of the colon and tumor location and relations which could contribute to choose the best surgical option and to improve safety in colorectal surgery.

\section{P171-COLORECTAL_Malignant}

\section{ANASTOMOTIC LEAK IN COLORECTAL CANCER SURGERY: LOOKING BACK TO LEAPING FORWARD}

\section{N.J. Rama ${ }^{1}$, N. Figueiredo ${ }^{2}$, I. Sales ${ }^{1}$, D. Parente ${ }^{1}$, M. Neves ${ }^{1}$, M. Coelho ${ }^{1}$, V. Faria ${ }^{1}$ \\ ${ }^{1}$ Colorectal Unit, Centro Hospitalar de Leiria, EPE, LEIRIA, Portugal; ${ }^{2}$ Colorectal Unit, Champalimaud Foundation, LISBOA, Portugal}

Background: Anastomotic leakage (AL) after colorectal procedures are a common surgical experience and represents a significant burden both for patients and surgeons. The incidence of AL has been reported to vary between $0.5 \%$ to up to $21 \%$, with rates for the colon and rectum of 3-7\% and $13-18 \%$, respectively. They, not only add to potential postoperative patient morbidities and to overall costs of postoperative patient care, but also are considered a quality indicator in colorectal surgery. Aim: We aimed to evaluate the clinical burden associated with anastomotic leaks following colorectal surgery.

Methods: We conducted a retrospective analysis of 641 colorectal patients who underwent conventional or laparoscopic colorectal surgery for colorectal cancer (CRC), from January 1st, 2013 to December 31st, 2016 in a Single Colorectal Centre (Centro Hospitalar de Leiria). Patient demographics, intraoperative and postoperative aspects were collected and analysed. All statistical analysis will be conducted using Stata Software (StataCorp LP).

Results: In our cohort of 641 pts, 35 developed a clinical AL (5.46\%), mostly males (90\%), with an average age of $71 \pm 10.57$. Male gender and conversion were independent risk factors. The group with AL had a higher LOHS (25.2 days vs, $6.59-\mathrm{p}<0.0001)$. 6 out of $35 \mathrm{AL}$ have been detected after the discharge. The mean diagnostic day was the eighth, and mode estimated at day 5. When compared with a control group, WCC, Eosinophils and CRP were statistically significant different in AL group, at day 3 and 5.

Conclusion: In the present study, no statistically significant risk factors for AL in CRC surgery were detected, except for male gender and conversion. Clinical methods and biomarkers were useful for early diagnosis. Technology combined with experience and common sense may be the embodiment of the clinical method.

\section{P172-COLORECTAL-Malignant}

\section{ANASTOMOSIS LEAK AND INDOCYANINE GREEN FLUORESCENCE IMAGING IN COLON-RECTAL SURGERY. A SINGLE CENTER STUDY}

P. Losurdo, D. Cosola, T. Cipolat-Mis, L. Bonadio, A. Biloslavo, N. de Manzini

General Surgery, University of Trieste, TRIESTE, Italy

Background: Anastomotic leakage (AL) is one of the most important complication of colorecta cancer surgery. The aim of this study was to evaluate the clinical utility of intraoperative ICG fluorescence imaging during laparoscopic and open colorectal surgery and to investigate the risk factors associated with the AL.

Methods: We retrospectively analyzed 216 patients underwent to rectal and left colon surgery, consecutively enrolled in the Colon-Rectal Disease Registry of Trieste from 2016 to 2017. Results: Among 216 patients undergoing colorectal surgery and anastomosis, symptomatic and nonsymptomatic AL occurred in 30 patients (14\%). Anastomotic distance from the dentate line is considered to be an important factor related to AL. 78\% of patient without AL has an anastomotic height less than $5 \mathrm{~cm}$ from the dentate line (22\%vs78\%; p-value 0,030). The baseline characteristics of patients experiencing AL (30 patients), compared to patients without AL (184 cases), were mostly similar, although patients with AL presented a longer time of surgery $(252 \pm 76 \mathrm{~min}$ vs. $209 \pm 75 \mathrm{~min}$, p-value: 0,004$)$, a higher ASA score ( 3 vs. 2 , p-value: 0,05 ) and were more Radio-chemo treaded (respectively RT $56 \%$ vs. $26 \%$, p-value: 0,005 and CT $47 \%$ vs. $23 \%$; p-value: 0.011 ). In addition, ICG fluorescence intensity was insufficient in the $18 \%$ of patient with $\mathrm{AL}$ compared to patients without $\mathrm{AL}$ (p-value: 0.055). Fluorescence imaging not-significantly reduce the $\mathrm{AL}$ rate in patients undergoing surgery for colorectal cancer but can lead a change in the planned anastomotic level. Because of these, only 2 patients (p-value 0.04) developed AL after the changed of the anastomotic level.

Conclusions: Hypoperfusion is a well-recognized cause of anastomotic leakage. Patients with AL are classically older, with a lot of comorbidities and a high expected post-operatory complication, reinforcing the importance of solid strategies to reduce AL. ICG fluorescence can help to confirm adequate perfusion in those cases where there is a clinical impression of inadequate perfusion, and therefore indicate that the resection margins do not need to be extendedfurther.

\section{P173-COLORECTAL-Malignant}

\section{COLORECTAL CANCER SCREENING PROGRAM IMPROVES BOTH SHORT AND LONG TERM OUTCOMES: A SINGLE CENTER EXPERIENCE IN TRIESTE}

P. Losurdo, M. Giacca, S. Fracon, A. Pasquali, N. de Manzini General Surgery, University of Trieste, TRIESTE, Italy

Background: Colorectal cancer is the third most common type of non-skin cancer in both men and women. The aim of our study is to show the results we had in terms of improvement of survival and clinical outcomes in patients undergoing surgery for CRC since the screening program started in the city of Trieste.

Methods: We analyzed patients who underwent surgical treatment for CRC between January 2011 and march 2015 in the service of General Surgery, Cattinara hospital, Trieste. The current screening program in Friuli Venezia Giulia region collects all men and women aged 50-69. For this reason, out of a total of 441 patients we compared 92 patients who were found to have a CRC during the screening program (SC) to 119 patients who presented with symptoms and were diagnosed when aged 50-69 (NO-SC). Adhesion to the regional program was $60.3 \%$ in the last year.

Results: $41(45 \%)$ patients in the SC group were diagnosed as AJCC stage I vs $32(27 \%)$ patients in the NO-SC group; $13(14 \%)$ vs $24(20 \%)$ were stage II, $13(14 \%)$ vs $31(26 \%)$ were stage III and $3(3 \%)$ vs $17(14 \%)$ were stage IV $(\mathrm{p}<0.002)$. We found a significant difference in surgical approach: $82(89 \%)$ SC vs $67(56 \%)$ NO-SC patients had laparoscopic surgery $(\mathrm{p}<0.002)$. In the NO-SC group, $19(16 \%)$ patients underwent resection in an emergency setting. Only $5(5 \%)$ patients in the SC group had postoperative complications vs $17(14 \%)$ patients in the NO-SC group $(\mathrm{p}=0.03)$. Considering all patients, we had a 2 -year Overall Survival (OS) of $86 \%$, being $95 \%$ in the SC group and $80 \%$ in the NO-SC group $(\mathrm{p}=0.002)$. Likewise, the whole 2-year Disease Free Survival (DFS) was $77 \%$, whereas it was $90 \%$ in the SC group and $66 \%$ in the NOSC group $(\mathrm{p}<0.002)$.

Conclusions: Our regional screening program has significantly improved early diagnosis and quickened surgical treatment of CRC. Thanks to this, we obtained an earlier stage at diagnosis, a less invasive surgical approach, and a lower rate of complications and emergency surgery need were obtained also with an improvement in both OS and DFS. 


\section{P174-COLORECTAL_Malignant}

\section{EARLY ANASTOMOTIC LEAK DIAGNOSIS AFTER COLORECTAL SURGERY: PRELIMINARY RESULTS FROM PROSPECTIVE OBSERVATIONAL STUDY}

\section{N.J. Rama ${ }^{1}$, N. Figueiredo ${ }^{2}$, J.M. Pimentel ${ }^{3}$, M. Guarino ${ }^{4}$, R. Castro ${ }^{5}$,} A. Rocha $^{6}$, F. Castro-Poças ${ }^{7}$

${ }^{1}$ Colorectal Unit, Centro Hospitalar de Leiria, EPE, LEIRIA, Portugal; ${ }^{2}$ Colorectal Unit, Champalimaud Foundation, LISBOA, Portugal; ${ }^{3}$ Colorectal Unit, $\mathrm{CH}$ Universitário de Coimbra, COIMBRA, Portugal; ${ }^{4}$ ESEL, Escola Superior de Saúde -Leiria, LEIRIA, Portugal; ${ }^{5}$ Clinical Pathology Department, Centro Hospitalar de Leiria, EPE, LEIRIA, Portugal; ${ }^{6}$ Colorectal Unit, Centro Hospitalar do Porto, PORTO, Portugal; ${ }^{7}$ Gastroenterology Department, Centro Hospitalar do Porto, PORTO, Portugal

Background: Anastomotic leakage (AL) after colorectal resections is a common surgical experience and the most frequent major adverse outcome in this type of procedure. It represents a significant burden both for patients (pts) and surgeons. This dreadful complication is associated with high mortality, reoperation rate, health care costs and increased hospital length of stay. It's crucial to identify accurate biomarkers (BMK), and some markers of inflammation and gut damage might be useful.

Aim: We would like to timely identify a pt with AL after colorectal surgery. We intend to determine de accuracy and predictive value of clinical criteria and some BMK [eosinopenia (EO), C Reactive Protein (CRP), Procalcitonin (PCT) and Calprotectin (CLP)] for the early diagnosis of colorectal anastomotic failure.

Methods: A prospective observational study included, from Mars 15th, 2017 to October 31st, 2018, 198 consecutive patients (pts) with documented colorectal disease requiring an open or laparoscopic procedure, with anastomosis, at the Colorectal Unit of the Centro Hospitalar de Leiria, EPE. The primary endpoints of this study were AL, other postoperative complications, length of stay and 30-day mortality. All statistical analysis was conducted using Stata Software. Results: In the first eighteen months we collected 198 pts, 55\% were males, with an average Charlson of 4, and $20 \%$ underwent a proctectomy. Pts which developed AL (10 pts-5.05\%) had a higher LOHS and readmission rate (15.3 vs 7.14 days $-\mathrm{p}=0.0001$, and 6.2 vs $25 \%$, respectively). Clinical criteria showed an acceptable predictive accuracy ( 0.89 for clinical condition and 0.81 for abdominal pain). Receiver operating characteristic (ROC) curve shows that on the $3 \mathrm{rd}$ postoperative day (POD) EO count has higher accuracy for AL detection (0.78) than the others BMK. However, at POD5 PCT has the higher accuracy (0.81). Plasma calprotectin is under measurement process.

Conclusion: From our preliminary results, EO count and PCT seems to have a good accuracy for AL diagnosis, in 3rd and 5th POD, respectively. Levels of CRP and PCT below $61 \mathrm{mg} / \mathrm{dl}$ and $0.17 \mathrm{ng} / \mathrm{ml}$ seem to be useful for AL exclusion and might be useful as additional criteria for discharge protocols after colorectal surgery.

\section{P175-COLORECTAL-Malignant}

INTRACORPOREAL VERSUS EXTRACORPOREAL ANASTOMOSIS IN LAPAROSCOPIC LEFT COLECTOMY FOR LEFT-SIDE COLON CANCER

S. Masubuchi ${ }^{1}$, J. Okuda ${ }^{2}$, H. Hamamoto ${ }^{1}$, W. Osumi ${ }^{1}$, M. Ishii ${ }^{1}$,

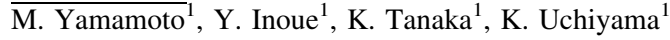

${ }^{1}$ General and Gastroenterological Surgery, Osaka Medical College, TAKATSUKI, Japan; ${ }^{2}$ Cancer center, Osaka Medical College, TAKATSUKI, Japan

Background: Laparoscopic colectomy for colon cancer with intracorporeal anastomosis (IA) is a procedure that is being increasingly performed.

Objective: This study aimed to evaluate short-term outcomes of IA compared with extracorporeal anastomosis (EA) in laparoscopic left colectomy for left-side colon cancer.

Design: In this retrospective study, propensity score matching was performed to reduce the effects of confounding factors between groups, including gender, age, BMI, ASA classification, medical history, TNM classification and maximum tumor diameter.

Settings: A database maintained at our institute was used to identify patients during the period from May 2013 to November 2017.

Patients: Overall, 98 patients were enrolled in this study, of whom 39 were treated by IA, and 42 were treated by EA; 17 patients were excluded due to anastomosis with double-stapling technique.

Results: After propensity score matching, 20 patients in the IA group and 20 patients in the EA group were compared. There were no significant differences in operative time, estimated blood loss, complications, and postoperative course. The mini-laparotomy incision was significantly shorter in the IA than in the EA group $(3.3$ vs. $5.0 \mathrm{~cm}, \mathrm{p}<0.01)$. Although takedown of the splenic flexure to fully mobilize the left hemicolon was mandatory in the EA group, it was performed in only 5 patients $(25.0 \%)$ in the IA group. Furthermore, the number of days to firs flatus was shorter in the IA group than in the EA group ( 1 vs. 2 days, $\mathrm{p}<0.05)$.

Conclusions: A totally laparoscopic colectomy with IA for the treatment of left-side colon cancer is technically feasible and can be performed with good cosmetic outcomes and decreased time to first flatus, suggesting faster recovery.

\section{P176-COLORECTAL-Malignant}

CLINICAL AND ONCOLOGICAL SAFETY OF SINGLEINCISION LAPAROSCOPIC SURGERY IN PATIENTS OF OVER 80 YEARS WITH COLON CANCER

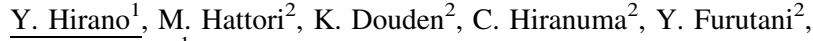
S. Yamaguchi ${ }^{1}$

${ }^{1}$ Gastroenterological Surgery, Saitama Medical University International Medical Center, HIDAKA, Japan; ${ }^{2}$ Surgery, Fukui Prefectural Hospital, FUKUI, Japan

Introduction: Surgeons are increasingly being faced with the problem of treating elderly colon cancer patients. We evaluated the outcome of SILC in patients of over 80 years with colon cancer with a propensity score matched comparison to assess its perioperative and long-term oncological outcomes.

Methods: This retrospective cohort study analyzed our experience with SILC for colon cancer over 5 years. Eighty-seven patients of over 80 years with colon cancer who electively underwent SILC were included in this study (elderly group). Eighty-seven patients were then chosen out of a collective of 257 patients less than 80 years old in a propensity score matched design (younge group). Short-term clinical outcomes in both groups were compared and verified its long-term oncological outcome.

Results: American Society of Anesthesiologists Score and post-operative complication rate were significantly higher in elderly group. However, the other short-term clinical outcomes including post-operative hospital stay were equivalent in two groups. The rates of 5-year cancer specific survival were $78.0 \%$ in elderly group and $70.9 \%$ in younger group, respectively, and the 5-year overall survival rates were $64.6 \%$ and $66.8 \%$, respectively. No significant differences were seen between two groups.

Conclusions: Our initial experiences suggested the oncological and clinical safety of SILC in patients of over 80 years with colon cancer. However, further studies are needed to demonstrate the advantages of this procedure compared to conventional laparoscopic colectomy. 


\section{P177-COLORECTAL_Malignant}

\section{THE ROLE OF SCOPIST ON SAFE AND SMOOTH LAPAROSCOPIC COLECTOMY}

T. Arita, M. Nakanishi, Y. Kuriu, Y. Murayama, M. Kudou, E. Otsuji, Division of Digestive Surgery

Department of Surgery, Kyoto Prefectural University of Medicine, KYOTO, Japan

Aims: Recently, laparoscopic colectomy has been popular worldwide. In Japan, JSES (Japan Society for Endoscopic Surgery) has established board-certified technical specialists to promote the development and spread of the laparoscopic surgery since 2004, however, the acceptance rate for certification is less than $30 \%$ within the past 3 years. Numerous discussion and education for operating surgeon have been noted, by contrast, less attention has been paid to the role of scopist for safe and smooth laparoscopic surgery. In this presentation, we demonstrate a standard routine of scopist in our institute.

Methods: Flexible scope has been adopted since 2007. Holding horizontal view with retroperitoneal structures such as gonadal vessels, urinary duct, Gerota's fascia, hypogastric nerve, abdominal aorta is important to keep oriented constantly during the operation. Calm movement is prefered as rapid movement leads to dizziness to operating surgeon. Close view by holding scope with both hands is recommended in delicate part such as vessel ligation, while distant view is more suitable in dynamic scene. Front-wided view can provide proper orientation and direction to the operating surgeon.

Conclusions: Recognition and providing the proper view to the surgeon may lead to safe and smooth operation. Thoughts of brushing up on the skill of scopist are necessary for maturation of laparoscopic team.

\section{P179-COLORECTAL-Malignant}

TIPS FOR LAPAROSCOPICAL SURGERY FOR TRANSVERSE COLON CANCER

M. Nakanishi, Y. Kuriu, Y. Murayama, T. Arita, M. Kudou, T. Kosuga, R. Morimura, H. Konishi, A. Shiozaki, H. Ikoma, T. Kubota, H. Fujiwara, K. Okamoto, E. Otsuji, Digestive Surgery, Kyoto Prefectural University of Medicine, KYOTO, Japan

Aim: Some clinical trials have reported the safety and efficacy of laparoscopic colectomy for colon cancer. On the other hand, transverse colon cancer was excluded in these trials because of the difficulty of laparoscopic colectomy for transverse colon cancer. In this presentation, we report the tips for laparoscopic colectomy for transverse colon cancer. Tips: In our department, 87 transverse colon cancers has been resected by laparoscopically so far. To complete CVL and CME, lymph nodes around middle colic artery should be resected, however many important structure, duodenum, pancreas, superior mesenteric vein (SMV) and so on, may be obstacles. This is most difficult point for this surgery. Our surgery is as follows.

Mobilization of ileum and ascending mesocolon from caudal sideConfirm duodenum and pancreasExpose SMV and ligation root of ileocecal artery and veinDissect lymph nodes around SMV and ligation of middle colic artery and accessary right colic veinConfirm pancreas from caudate side of transverse mesocolon and incise the peritoneum along the caudal side of the pancreasDissect lymph nodes sufficiently by dissection from both side of transverse mesocolonMost important point.

To dissect lymph nodes safely, confirmation from both side of transverse mesocolon is necessary and dissection should be performed along important structure, SMV, pancreas and so on.

Conclusion: In our results, laparoscopic surgery for transverse colon cancer was performed safely compared to open surgery.

\section{P180-COLORECTAL-Malignant}

\section{LONG-TERM ONCOLOGICAL SAFETY OF SINGLE-INCISION LAPAROSCOPIC SURGERY FOR COLON CANCER}

\section{$\underline{\text { M. Hattori }}{ }^{1}$, Y. Hirano ${ }^{2}$, Y. Furutani ${ }^{1}$, C. Hiranuma ${ }^{1}$, K. Douden ${ }^{1}$}

${ }^{1}$ Surgery, Fukui prefectural Hospital, FUKUI, Japan;

${ }^{2}$ Gastroenterological Surgery, Saitama Medical University International Medical Center, HIDAKA, Japan

Introduction: There have been no studies demonstrating the adequate long-term oncological safety of single-incision laparoscopic surgery (SILS) for colon cancer. A consecutive experience from its introduction of SILS for colon cancer is reviewed, and its 5 -year oncological outcomes are evaluated retrospectively.

Methods: Two hundred eighty-eight patients (148 women) with a mean age of 71.5 years were treated with the SILS procedure for stage I to III colon cancer. Exclusion criteria of SILS included T4b, unresolved bowel obstruction, tumor perforation and severe medical illness.

Results: Of these 288 patients, 20 patients $(6.9 \%)$ required an additional port insertion mainly for rectal transection and drainage route in sigmoid colon cancer patients. The median follow-up interval was 52 months. The 5 -year relapse free survival for stage I, stage II and stage III disease were $95.8 \%, 80.2 \%$ and $61.6 \%$, respectively. The 5-year overall survival for stage I, stage II and stage III disease were $97.4 \%, 85.3 \%, 72.9 \%$ and $40.9 \%$, respectively. The 5-year cancer-specific survival in patients diagnosed pathologically $\mathrm{T} 1$, T2, T3 and T4 were $100 \%, 100 \%, 92.1 \%$ and $73.9 \%$, respectively. There were significant differences between pT3 and pT4.

Conclusions: Our initial experiences showed that SILS colectomy can be applied to the treatment of colon cancer with good long-term oncological outcomes. However, we should pay more attention when we treat the pT4 tumors. 


\section{P181-COLORECTAL_Malignant}

LONG-TERM ONCOLOGICAL OUTCOMES AFTER SINGLEINCISION PLUS ONE PORT LAPAROSCOPIC ANTERIOR RESECTION FOR RECTAL CANCER

\author{
Y. Furutani ${ }^{1}$, Y. Hirano ${ }^{2}$, M. Hattori ${ }^{1}$, C. Hiranuma ${ }^{1}$, K. Douden ${ }^{1}$ \\ ${ }^{1}$ Surgery, Fukui Prefectural Hospital, FUKUI, Japan, \\ ${ }^{2}$ Gastroenterological Surgery, Saitama Medical University \\ International Medical Center, HIDAKA, Japan
}

Introduction: We have developed and previously reported single-incision plus one por laparoscopic anterior resection of the rectum (SILS + 1-AR) as a reduced port surgery in which we can utilize the incision for drainage as an additional access route for laparoscopic procedures including the transection the lower rectum. A consecutive experience from its introduction of SILS + 1-AR for rectal cancer is reviewed, and its 5-year oncological outcomes are evaluated retrospectively.

Methods: One hundred and forty-one patients (53 female) with a mean age of 67.6 years adopted the SILS + 1 procedure for rectal cancer. A Lap protector (LP) was inserted through a $2.5 \mathrm{~cm}$ transumbilical incision; an EZ-access was mounted to the LP and three 5-mm ports were placed. A 12 -mm port was inserted in the right lower quadrant.

Results: One hundred and thirty-six patients (96.5\%) completed with SILA + 1-AR. The tumor locations in the rectosigmoid, rectum above the peritoneal reflection (Ra), and rectum below $(\mathrm{Rb})$ were 44,63 and 29, respectively. The median follow-up interval was 42 months. The 5 -year cancer-specific survival for stage I, stage II, stage III and stage IV disease were $100 \%, 100 \%$, $96.9 \%$ and $57.7 \%$, respectively. The 5 -year overall survival for stage I, stage II, stage III and stage IV disease were $95.2 \%, 90 \%, 96.9 \%$ and $57.7 \%$, respectively.

Conclusions: Our initial experiences showed that SILS + 1-AR can be applied to the treatment of rectal cancer with good long-term oncological outcomes.

\section{P182-COLORECTAL_Malignant}

IS THE LAPAROSCOPIC APPROACH SAFE IN COLOVESICAL FISTULAE?

N. de la Fuente Hernández, J. Ye Zhou, M. Martínez Sánchez, M. Solans Solerdelcoll, A. Rabal Fueyo, P. Hernández Casanovas, J. Bollo Rodríguez, E. Targarona Soler

Colorrectal Surgery, Hospital De La Santa Creu I Sant Pau, BARCELONA, Spain

Aims: Colovesical fistulae came from inflammatory disease or cancer and do have a significant morbidity. The most common location is the sigmoid colon and the most common aetiology is diverticulitis. The treatment of choice is a surgical procedure. The aim was studying compare laparoscopic approach in patients diagnosed by benign (diverticulitis) and malignant (colon adenocarcinoma) colovesical fistulae.

Methods: From January 2001 to March 2005 all characteristics of surgical patients with diverticular and colon adenocarcinoma colovesical fistulae were reviewed. Patient details (sex, age, symptoms, diagnosis, medical history and anaesthetic risk), surgical approach, hospital stay and complications were recorded. Both groups were compared with significance level set at $\mathrm{p}<0.05$. Results: Nine laparoscopic $(71 \%)$ and 4 open approaches $(29 \%)$ in diverticular colovesical fistulae were performed, with a conversion rate of $33 \%$. The procedure done was sigmoidectomy. There were also performed 3 laparoscopic (14\%) and 16 open approaches $(72 \%)$ in colon adenocarcinoma colovesical fistulae. The procedures done were sigmoidectomy, pelvic exenteration, left colectomy, low anterior resection and loop colostomy. Comparison between the two groups didn't show significant differences in characteristics but did show significant differences regarding the approach, with more cases performed by open approach in colon adenocarcinoma colovesical fistulae $(\mathrm{p}=0.03)$. Conversion rate didn't show significant differences. Patients diagnosed for malignant colovesical fistulae had more complications, 15 cases (68\%), $10(45 \%)$ I-II and 5 (23\%) III-IV-V according to Clavien Dindo classification, manifesting significant differences $(\mathrm{p}=0.03)$. Laparoscopic approach didn't show significant differences regarding complications.

Conclusions: Generally, surgical approach with colonic resection and partial or total cystectomy is the treatment of choice in colovesical fistulae, although vesical resection can be avoided if it is suspected benign aetiology. Whenever laparoscopic approach is performed by experienced surgeons, is feasible in colovesical fistulae and the morbidity and mortality numbers are acceptable. Laparoscopic approach allows the advantages of a minimally invasive treatment but implies clinical trials to stablish stronger evidence.
P183-COLORECTAL-Malignant

\section{CLINICAL EFFECTIVENESS OF SINGLE-INCISION LAPAROSCOPIC RIGHT HEMICOLECTOMY WITH INTRACORPOREAL RESECTION FOR RIGHT-SIDED COLON CANCER}

M. Yamamoto ${ }^{1}$, M. Asakuma ${ }^{1}$, K. Tanaka ${ }^{1}$, S. Masubuchi ${ }^{1}$,

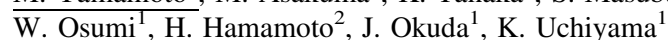

${ }^{1}$ Departments of General and Gastroenterological Surgery, Osaka Medical College, TAKATSUKI, OSAKA, Japan; ${ }^{2}$ Hiroki, Osaka Medical College, TAKATSUKI, OSAKA, Japan

Aims: Laparoscopic right hemicolectomy became the standard of care for treating cecum, ascending and proximal transverse colon cancer in many center. Most centers use multiport laparoscopic colectomy with extracorporeal resection and anastomosis (MCE). Single-incision laparoscopic colectomy with intracorporeal resection and extracorporeal (SCI) remains controversial. The aim of the present study is to compare these two techniques using propensity matching analysis.

Methods: This study analyzed 171 patients who underwent laparoscopic right hemicolectomy including 119 MCE surgeries and 52 SCI surgeries from December 2015 to December 2017 Short-term outcomes were recorded. Postoperative pain was evaluated using a visual analogue scale (VAS) and postoperative analgesic use as outcome measure.

Results: The length of skin incision in the SCI group was significantly shorter than in the MCE group: median (range) $3(2-10) \mathrm{cm}$ verses $4(3-8) \mathrm{cm}(\mathrm{P}<0.0001)$. The VAS score after surgery was significantly less in SRHI than in MRHE. Significantly fewer patients required analgesia after SRHI after surgery. There were no significant differences in operative time, intraoperative blood loss, the number of lymph nodes removed and postoperative courses between the groups. The cost effectiveness was significantly cheaper in SRHI than in MRHE.

Conclusions: SCI for right colon cancer is safe and technically feasible. SCI reduces the length of skin incision and postoperative pain compared with conventional MCE.

\section{P184-COLORECTAL-Malignant}

\section{CLINICAL OUTCOMES OF LAPAROSCOPIC SURGERY AFTER NCRT FOR ADVANCED LOWER RECTAL CANCER}

K. Kojo, M. Shimazu, H. Miura, T. Yamanashi, T. Sato, T. Nakamura, M. Watanabe

Surgery, Kitasato University Hospital, SAGAMIHARA, Japan

Background: The laparoscopic approach for lower advanced rectal cancer is recommended only in clinical trial in Japanese clinical guidelines for colorectal cancer due to concerns about technical difficulty and possibility of positive circumferential resection margin. On the other hand, a lot of study have shown that neoadjuvant chemoradiotherapy (NCRT) can reduce tumor size and recurrence, and increase the tumor resection rate.

Aim: This study was designed to clarify the utility of laparoscopic surgery for advanced lower rectal cancer after Neoadjuvant chemoradiotherapy (NCRT).

Patients and Methods: We investigated 3-year disease-free survival rate, operative outcomes and recurrence risk factor in 73 patients with lower rectal cancer (cT2-4, N0-2) who underwent laparoscopic surgery after NCRT from 2010 to December 2017 in Kitasato University hospital. Results: Of 73 patients, 43 patients underwent low anterior resection (LAR), 4 patients underwent intersphincteric resection (ISR) and 27 abdominoperineal resection (APR). There were 7 anastomotic leakage, and 1 urinary disorder and 2 sexual dysfunction. ypCR rate was $24.7 \%$, but 15 patients $(20.5 \%)$ had recurrence ( 7 liver, 7 lung and 2 lymph node and 1 local recurrence; There is some overlapping). ypT4 and lymph node metastasis were detected as a recurrent risk factor. The 3-year relapse-free survival rate (RFS) was $79.5 \%$ and the 3-year overall survival rate (OS) was $92 \%$.

Conclusion: In this examination, ypT4 and lymph node metastasis were risk factor for recurrence. The operative outcomes, 3-year RFS and the 3-year OS are relatively good results. We will conduct further follow-up, and it is necessary to investigate a long term prognosis. Laparoscopic surgery is warranted for rectal cancer after NCRT. 


\section{P185-COLORECTAL_Malignant}

ANASTOMOTIC LEAK IN COLORECTAL CANCER SURGERY: FROM DIAGNOSIS TO MANAGEMENT OR FAILURE

N.J. Rama ${ }^{1}$, N. Figueiredo ${ }^{2}$, I. Sales ${ }^{1}$, D. Parente ${ }^{1}$, M. Neves ${ }^{1}$, M. Coelho ${ }^{1}$, V. Faria ${ }^{1}$, J.M. Pimentel ${ }^{3}$, P. Alves ${ }^{1}$, P. Clara ${ }^{1}$, S. Amado

${ }^{1}$ Colorectal Unit, Centro Hospitalar de Leiria, EPE, LEIRIA, Portugal; ${ }^{2}$ Colorectal Unit, Champalimaud Foundation, LISBOA, Portugal; ${ }^{3}$ Colorectal Unit, $\mathrm{CH}$ Universitário de Coimbra, COIMBRA, Portugal

Background: Prevention and treatment of anastomotic leak (AL), has received interest, in last decades. Failure-to-Rescue (FTR) concept reflets the estimated mortality rate in the group of patients (pts) who developed a specific postoperative complication. FTR rate differs among distinct institutions and suggests that different management strategies can influence the survival. Thereby, as performance metric for colorectal surgery, we cannot only consider mortality or AL ratio, but also the rate of pts who died due to a specific surgical complication.

Aim: We aimed to characterize the management approach in the group of pts with AL complication. Methods: We conducted a retrospective analysis of 641 colorectal patients who underwent conventional or laparoscopic colorectal surgery for colorectal cancer (CRC), from January $1^{\text {st }}, 2013$ to December 31st, 2016 in one Portuguese Colorectal Unit (Centro Hospitalar de Leiria). We analysed the $35 \mathrm{pts}$ who developed AL. Patient demographics, intraoperative and postoperative aspects were collected and analysed. All statistical analysis will be conducted using Stata Software.

Results: In our cohort, 35 developed a clinical AL $(5,46 \%)$, mostly males (90\%), with an average age of $71+/-10,57$. The group with AL had a higher LOHS $(25,2$ days vs, $6,59-\mathrm{p}<0.0001) .6$ out of $35 \mathrm{AL}$ have been detected after the discharge. The mean diagnostic day was the eighth, and mode estimated at day 5. Mortality rate in AL group was $15 \%$ (5 pts). $20 \%$ of pts had intensive care (IC) management, with a mean length of stay of 15,85 days. $68 \%$ of pts received parental nutrition for a mean period of 8,5 days. $80 \%$ of pts had an ostomy as an AL management option, and two thirds were not closed. Mean time for ostomy closure was 440 days.

Conclusion: In the present study, mortality rate in $\mathrm{AL}$ group was high but still quite remarkable the rate of rescue, probably related with the carefully management protocol, including IC and nutritional support. Provider's ability to treat this group of pts seems to have a positive impact in survival and can be used as an important measure of service quality.

\section{P186-COLORECTAL-Malignant}

\section{LAPAROSCOPIC SURGERY FOR SYNCHRONOUS COLORECTAL CANCER: DOUBLE SEGMENTAL RESECTION OR EXTENDED COLECTOMY?}

A. Rabal Fueyo ${ }^{1}$, C. Martinez Sánchez ${ }^{2}$, J. Bollo Rodriguez ${ }^{1}$, M. Solans Solerdelcoll ${ }^{1}$, N. de la Fuente ${ }^{1}$, D. Sacoto ${ }^{1}$, E.M. Targarona Soler ${ }^{1}$

${ }^{1}$ General Surgery, Hospital de la Santa Creu i Sant Pau,

BARCELONA, Spain; ${ }^{2}$ Colorectal Surgery, Hospital de la Santa Creu i Sant Pau, BARCELONA, Spain

Introduction: Synchronous colorectal neoplasia presents an incidence ranges from $2 \%$ and $7 \%$. Classically its surgical treatment consisted in the realisation of a subtotal colectomy (STC), however, several authors have proposed that in certain occasions the realisation of two segmental resections with two anastomoses was not accompanied by an increased risk of anastomotic failure.

The objective of this study was to compare the feasibility and safety of the laparoscopic approach of synchronous colorectal neoplasia using two different techniques: STC versus two segmental resections with two anastomoses.

Methods: We retrospectively reviewed the clinical data of patients over 18 years of age who underwent colorectal surgery between 1998 and 2018 at a single center. We included patients with a synchronous colorectal neoplasia who underwent laparoscopic surgery, either STC or double resection (DR)

Results: A total of 24 patients met the inclusion criteria. Mainly males ( $86 \%)$ with an average age of 75 years, with a scale of the American Association of Anesthesiologists superior to II in 53\% and with an average body mass index of $29 \mathrm{~kg} / \mathrm{m} 2$. The mean operative time was $251 \mathrm{~min}$ in the DR and 281 $\mathrm{min}$ in the STC, the STC resulted in a higher conversion rate (23\% vs $11 \%)$ and intraoperative bleeding ( $39 \%$ vs $22 \%$ ), in addition to a postoperative period with more complications, only $15 \%$ of the patients undergoing STC didn't present any complication while $67 \%$ of the patients with a DR didn't present any complication. 38\% of the STC presented anastomotic failure and only $11 \%$ of the DR. The mean hospital stay was 8 days in the DR and 18.5 in the STC.

In the DR, an average of $47 \mathrm{~cm}$ of colon was resected with an average of 24.8 lymph nodes, while in the STC, $127 \mathrm{~cm}$ of colon was resected with an average of 24.2 resected nodes.

Conclusions: The double resection with two anastomosis is a less aggressive surgery, with fewer complications and a shorter hospital stay, providing similar oncological results.

\section{P187-COLORECTAL-Malignant}

\section{YEARS EXPERIENCE INT THE SURGICAL TREATMENT OF SPLENIC FLEXURE NEOPLASIA: LESS IS MORE?}

A. Rabal Fueyo ${ }^{1}$, J. Bollo Rodriguez ${ }^{1}$, C. Martinez Sánchez ${ }^{2}$, D. Sacoto $^{1}$, M. Solans Solerdelcoll ${ }^{1}$, N. de la Fuente ${ }^{1}$, E.M. Targarona Soler ${ }^{1}$

${ }^{1}$ General Surgery, Hospital de la Santa Creu i Sant Pau, BARCELONA, Spain, ${ }^{2}$ Colorectal Surgery, Hospital de la Santa Creu i Sant Pau, BARCELONA, Spain

Aims: Splenic flexure neoplasia represents a challenge because of its complex vascularisation and lymphatic drainage. Many surgeons prefer extended resections, while others prefer segmental resection. The aim of our study is to compare three surgical techniques for the treatment splenic flexure cancer

Methods: We retrospectively review patients with splenic flexure neoplasia who underwen elective surgery in a single tertiary hospital from 1998 to 2018 .

In this years we have used three different techniques: subtotal colectomy (STC), left colectomy (LC) and segmental resection (SR). We reviewed demographic data, operative time, surgical approach, morbidity, hospital stay, recurrence and survival.

Results: 124 patients were included. 55 (44\%) LC, 41 (33\%) STC and 28 (23\%) SR. The characteristics of patients were equivalent between groups. $61 \%$ of patients were operated by laparoscopy, the laparoscopic approach was $93 \%$ SR, $61 \%$ in the STC and $58 \%$ in the LC. There were no differences in conversion rate or in the operative time.

There were no differences in morbidity, re-operations or hospital stay.

Regarding tumor stage there were no differences between the three groups. As for the resected nodes, we found a mean of 21 in STC, 16 in LC and 14 SR with no statistical difference. There were no differences in the affected nodes among groups. In our patients we didn't find differences in the recurrences rate or in the distant metastases rate.The average follow-up was 76 months (range: 30-114), with no differences in overall survival.

Conclusion: Segmental resection of splenic flexure neoplasias is safe and feasible, with no differences in morbidity or in the oncological outcomes compared with more aggressive surgeries.

\section{P188-COLORECTAL_Malignant}

\section{EVALUATION OF PERFUSION IN COLORECTAL ANASTOMOSIS BY USING FLUORESCENT IMAGING WITH INDOCYANINE GREEN (ICG)}

A. Gila Bohórquez, J. Gómez Menchero, J.M. Suárez Grau, J.L. García Moreno

\section{Surgery, Hospital General De Riotinto, HUELVA, Spain}

Introduction: The evaluation of perfusion in colorectal anastomosis is still a field of study and progress for the development of new modalities that allow reducing the ratio of dehiscence or anastomotic leakage (AL) in said surgery. Our objective with this work is to highlight the utility of indocyanine green (ICG) in the said evaluation after colo-rectal surgery.

Methods: We present a series of 85 cases of colorectal surgery (benign and malignant disease) intervened in the period between 2014 and 2018. The population sample has been homogenized according to age criteria, risk factors and comorbidity. A retrospective database has been developed with the SPSS v.22 software for the evaluation of the results obtained. The primary outcome measure was AL rate with at least 1 month of follow-up.

Results: A significant reduction in the incidence of $\mathrm{AL}$ was observed in patients who underwent colo-rectal surgery $(p=0.005)$. Low AL rates were shown in rectal cancer surgery $(p=0.02)$. There was no significant decrease in the AL rate when colorectal procedures for benign and malignant disease were combined.

Conclusions: The use of the image by fluorescence with indocyanine green is a safe, reproducible and relatively simple method with which to evaluate the perfusion of the colorectal anastomosis as well as reduce the rate of anastomotic leak in the postoperative period. Large well-designed randomized control trials are needed to provide evidence for its routine use in colorectal surgery. 


\section{P189-COLORECTAL_Malignant}

\section{INCOMPLETE COLONOSCOPY. WHAT'S NEXT? A SINGLE CENTRE'S EXPERIENCE}

\section{M.S. Salama, M. Hamza, E. Burke, M. Mohamed, A.R. Nasr}

Surgery, Our lady of lourdes hospital, BETTYSTOWN, Ireland

Introduction: Currently colonoscopy is the gold standard investigation for colonic evaluation. Although caecal intubation is one of its quality indicators, it is not attained in up to $20 \%$ of cases. This remains a significant concern. Limited data are available on the follow-up of patients with incomplete colonoscopy.

Aims: To assess colonoscopy completion rate, the reasons for incomplete colonoscopy, and the methods used to complete colonic evaluations after incomplete colonoscopy

Methods: We performed a retrospective study of incomplete colonoscopies in our unit over a one year period (2017). Parameters recorded were demographics, colonoscopy indications, bowel preparation quality, sedation, incomplete colonoscopy reasons, further investigations (Repeat colonoscopy, CT colonography), and final findings. Exclusion criteria included previous colonic surgery.

Results: Total number of patients: 3,411, \{males 1634(47.9\%); (females 1,777(52.1\%)\}.212(6.2\%) had incomplete colonoscopy \{male $65(30.7 \%$ ), (female 147 (69.3\%)\}. Completion rate: $93.8 \%$. Reasons for failure: Looping (33\%), Patient discomfort (24\%), Poor bowel prep (20\%), Colonic pathology $(9.9 \%)$, Redundant bowel (2.8\%), Withdrawal on patient request $(2.4 \%)$, and others (18\%). $132(62.3 \%)$ cases of incomplete colonoscopy had CT Colonography and $42(20 \%)$ were rescoped. Picolax was used for bowel preparation in 3,089 (91\%), and Moviprep in 322 (9\%). The mean age for complete colonoscopy was 56 years and for incomplete colonoscopy was 75 years. Findings of CT Colonography in 132 cases of incomplete colonoscopy were: Normal: 83 (62.8\%), Diverticular disease: $22(16.6 \%)$, Tortuous colon: 6 (4.5\%), Stricture of Sigmoid colon: $3(2.3 \%)$, Polyps: $9(5.1 \%)$, Inflammatory bowel disease (IBD): 1 (0.8\%) and Tumour: $5(3.8 \%)$. Results: of 42 cases re-scoped: Normal: 16 (38\%), Polyps: 7 (16.6\%), Failed: 10 (23.8\%), Stricture: 1 (2.4\%), IBD: 2 (4.8\%), Tumour: 1 (2.4\%), Lipoma: 1 (2.4\%) and Diverticular disease: $4(9.5 \%)$. All patients who failed second colonoscopy had CT Colonography (Tortuous bowel: 7 , Sigmoid stricture secondary to Diverticulitis: 3 ).

38 patients were not further investigated as per clinical judgement.

Conclusions: (1) A thorough and complete colonoscopy is critically important in detecting colorectal cancer. (2) If colonoscopy fails, other alternatives have to be considered. While repeat colonoscopy can be successful, CT colonography may be considered in certain cases.

\section{P190_COLORECTAL_Malignant \\ PATIENT REPORTED OUTCOMES AFTER LAPAROSCOPIC SURGERY FOR RECTAL CANCER}

D.S. White, R.K. Singh, S. Allen, R. Elangovan, E. Osenda, M. Dunstan, I.C. Jourdan, T.A. Rockall, A. Scala

Colorectal Surgery, Royal Surrey County Hospital, GUILDFORD, United Kingdom

Aims Colorectal cancer survivors suffer many problems affecting quality of life [QOL] ranging from psychological distress to urological, bowel and sexual dysfunction. Follow-up programs focus mainly on detection of recurrence rather than quality of life assessment. There is a move to rebalance follow-up to assess QOL more formally. With this study, we wish to build up a body of evidence such that patients get the support they require, secondly that they can incorporate QOL in decisions regarding management strategies, and thirdly evidence is emerging that PROMS improves survival in cancer patients.

Method: Patients undergoing laparoscopic TME [LAR] or abdominoperineal resection [LAPER] between 2005-2015 in a single centre were identified from a pre-existing database and sent patient reported outcome questionnaires [PROMs]. Measures collected were EORTC-QLQC-30 and CR29. Comparisons were made between the following subgroups; LAPER vs. LAR, chemotherapy vs. no chemotherapy, radiotherapy vs. no radiotherapy, stoma vs. no stoma, and complications vs. no complications.

Results: 141 patients were identified. 118 [83.7\%] responded. 110 completed the questionnaires. Mean age 67 years (47-88 years). Median follow up 58 months (21-162 months). For global QOL scores, $28 \%$ of patients described their quality of life as perfect and $83.6 \%$ as good. Median QOL score was 6 of a maximum 7.Few statistically significant differences were found between subgroups. Significance was deemed $\mathrm{P}<0.05$. LAPER patients reported better symptoms relating to diarrhoea. LAR patients reported better scores for sexual interest, but worse scores for sexual dysfunction. Patients who had undergone chemotherapy reported a better overall quality of life score. Chemotherapy patients however, reported worse scores for pain on urination, problems with taste, and perianal pain. Radiotherapy patients reported worse scores for faecal incontinence. Patients without stomata reported better scores for sexual interest

Conclusion: These results compare favorably with published data. Few statistically significant differences between groups suggest varying modalities of treatment broadly result in similar QOL. This data highlights a need for well-delivered support programmes for specific issues, for example stoma care and sexual dysfunction. Future studies will need to include a baseline questionnaire to truly measure the impact of surgery and measure quality in an increasingly elderly and comorbid population.

\section{P191-COLORECTAL-Malignant}

\section{LAPAROSCOPIC RESECTION WITH COMPLETE MESOCOLIC EXCISION FOR SPLENIC FLEXURE CANCER: LONG-TERM FOLLOW-UP DATA FROM A MULTICENTRE RETROSPECTIVE STUDY}

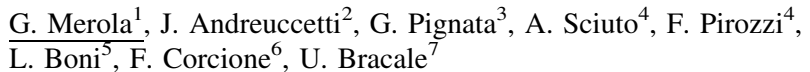

${ }^{1}$ Gastroenterology, Endocrinology and Surgical Endoscopy, AUOP Federico II, NAPOLI, Italy; ${ }^{2}$ General and Mininvasive surgery, San Camillo Hospital, TRENTO, Italy; ${ }^{3}$ General Surgery II, Civil Hospital of Brescia, BRESCIA, Italy; ${ }^{4}$ General Surgery, Santa Maria delle Grazie, POZZUOLI, Italy; ${ }^{5}$ General Surgery, Ca Granda Hospital, MILANO, Italy; ${ }^{6}$ General Surgery, University Hospital Federico II, NAPLES, Italy; ${ }^{7}$ Gastroenterology, Endocrinology and Surgical Endoscopy, University Federico II, NAPOLI, Italy

Splenic flexure cancer (SFC), comprising the tumours raised in the distal transverse colon and proximal descending colon, account for 2 to $5 \%$ of all surgically treated colorectal cancers. In CME for SFC, dissection of both the transverse and descending mesocolon must be considered. However, the use of laparoscopic surgery as a curative treatment for SFC, has never been investigated in adequate controlled trials, because of difficulty in deciding on the appropriate operative procedure, as well as technical difficulties with laparoscopic lymph node dissection. The aim of this multicenter study is to evaluate the oncologic effectiveness of laparoscopic segmental resection with $\mathrm{CME}$ with for cancer located at the splenic flexure. We performed a retrospective analysis of all cases of SFC treated with a laparoscopic segmental resection with CME in five different institution. Intra and post operative were evaluated. 112 patientes were evaluated, the mean operative time was $155.17 \pm 48.54 \mathrm{~min}$. A total of 6 (5.4\%) conversions occurred, 2 due to splenic artery lesion, one for difficult adesyolisis and three due to locally advanced tumour. Recurrence was observed in 13 $(11.6 \%)$ patients. There was a significant association between disease stage and recurrence $(\mathrm{p}<0.001)$ with a higher proportion of stage IV patients in the recurrence group ( $46.1 \%$ vs $7.1 \%$ ). At 30 days follow-up no mortality were recorded. During a median follow-up of 43 months (range 12-149), 13 deaths occurred (all of them for disease progression). Keplan Mayer curves showed a compareble suvival with other colo-rectal cancer. In conclusion, laparoscopic segmental resection with CME and CVL seems to be an oncologically safe and effective procedure for treatment of SFC. It may be regarded as the standard surgical method for elective management of this disease. In the future, more tailored patient- and tumor- specific segmental resection might be achieved with the use of routine lymph node road mapping.

\section{P192-COLORECTAL-Malignant}

\section{LAPAROSCOPIC VERSUS LAPAROTOMIC APPROACHES IN COLORECTAL CANCER SURGERY: LYMPHADENECTOMY ASSESSMENT}

M. Maes-Carballo, I.M. Múñoz-Núñez, P. Nicolás-Martínez, B. Cantarero-Jiménez, I. Plata-Pérez, P. Vázquez-Barros

General Surgery, Hospital Santa Ana de Motril, MOTRIL, Spain

Introduction: Nowadays, colorectal cancer is one of the neoplasms with higher prevalence and mortality. Lots of studies have demonstrated lymph node involvement and distant metastases are the main prognostic and therapeutic decision-making factors.

It is very important to establish a minimum number of lymph nodes to analyse for a correct staging. It has been established as 12 .

The treatment of colorectal cancer is essentially surgical. The review of the medical literature indicates that laparoscopic colorectal surgery is a safe procedure that has not found significan differences in the survival rate from open surgery.

Aim: The aim of our study is to compare the outcomes of laparoscopic and open resection for colorectal cancer surgery evaluating lymph node assessment.

Methods: The patients were collected in our hospital during the period from 1/11/2017 to 1/12 2018 and the number of lymph nodes obtained in lymphadenectomy has been studied comparing the laparoscopic and laparotomy approaches.

Results: 81 interventions were performed. 55 were laparotomic, 20 were laparoscopic and 5 converted laparoscopic (fig 1). The average number of nodes found in these interventions was 15, 36 . Nowadays, the recommendations to obtain a proper lymphadenectomy is to find more than 12 lymph nodes. Analysing our procedures, 61 surgeries had obtained a good lymphadenectomy. According to the approach, $62,3 \%$ of the interventions (38) are laparotomy, $31,2 \%$ (19) are laparoscopic procedures and 6,5\% (4) are by reconverted laparotomy (Figure 2).

The average number of lymph nodes isolated was similar. Laparotomy approach found 16,45 nodes while 13,5 nodes were found in laparoscopy. Converted laparoscopy found 12,2 (Figure 3). Conclusion: The treatment of colorectal cancer is essentially surgical. Today, there are a lot of studies that support that laparoscopic surgery has a survival rate similar to laparotomy surgery. According to our study, the data collected indicates that the number of isolated lymph nodes in both approaches is very similar. To sump up, laparoscopic colorectal surgery is safe and has demonstrated oncologica adequacy comparable to open approach and better short-term outcomes due to a less invasive approach. 


\section{P193-COLORECTAL_Malignant}

INTEGRATED COLORECTAL CANCER CARE PROCESS: ANALYSIS OF QUALITY INDICATORS FOR ASSISTANCE IMPROVEMENT IN A REGIONAL HOSPITAL

M. Maes-Carballo, I.M. Múñoz-Núñez, I. Plata-Pérez, P. Nicolás-Martínez, B. Cantarero-Jiménez, P. Vázquez-Barros

General Surgery, Hospital Santa Ana de Motril, MOTRIL, Spain

Introduction: Colorectal cancer is the most frequent neoplasm in Spain. More than 34,000 cases are diagnosed each year. Nowadays, early diagnosis and advances in treatment (multidisciplinary and increasingly personalized) are allowing a substantial improvement in survival and quality of

Aim: Examination of the utility of collecting clinical and care data for the analysis of the main quality indicators of the PAICCR (Integrated Colorectal Cancer Care Process) and identification of areas for improvement.

Methods: Series of 73 patients treated in our hospital from January 2018 to November 2018. Sociodemographic, clinical and healthcare variables were collected prospectively, as well as compliance with the quality standards proposed for the PAICCR.

Results: The most common approach was open $(67.12 \%)$, with almost $10 \%$ of converted laparoscopy. This percentage of laparotomies decreases by $10 \%$ in scheduled surgery.The average length of hospital stay was 10 days, with insignificant differences according to the approach. This increases in the case of complications (anastomotic leak, wound infection,...).An analysis of the quality indicators was carried out:Surgical site infection indicator:The percentage of infection of the abdominal wound is greater in emergency procedures (19\%) than in programmed surgery $(11 \%)$; the percentage of infection of the perineal wound of APR is $40 \%$.Anastomotic leakage indicator: $12.24 \%$. All these patients had drainage but only in one case was effective.Eviscertion indicator: $3 \%$.Postoperative hospital stay indicator: 8.7 days in patients without complications. It is increasing to more than 17 days in those with complications.Mortality indicator: $9.5 \%$.

Conclusions: - The appearance of postsurgical complications does not seem to depend on the approach. However, emergency or programmed surgeries have different percentage of complications.

- Our study does not show the use of drainage is effective for the early diagnosis of anastomotic leakage.

- The average hospital stay is almost 9 days, being recommended according to the PAICCR less than 7 days.

\section{P194_COLORECTAL_Malignant}

\section{EXPERIENCE OF THE FREE ACCESS ${ }^{\text {TM }}$ IN SINGLE-INCISION LAPAROSCOPIC RIGHT HEMICOLECTOMY}

\section{S. Kitashiro, S. Okushiba, Y. Kawarada, Y. Suzuki}

Surgery, Tonan Hospital, SAPPORO, Japan

Background: The single port right hemicolectomy was first used in 2008 by Bucher. Singleincision laparoscopy has gained significance recently. The umbilicus has been the preferred access site for Single-incision laparoscopy by Free access ${ }^{\mathrm{TM}}$.

Method: Between June 2013 and September 2018, 155 patients presenting with malignant disease underwent single-incision laparoscopy for right hemicolectomy. The median age was 72 years. Seven percent had undergone previous abdominal surgery, and the median body mass index was 22 (range, 18-34). Free access ${ }^{\mathrm{TM}}$ with three trocar was used. The access site was used for extracorporeal linear stapled anastomosis and specimen extraction.

Result: The average surgical time was about $168 \mathrm{~min}$. In almost all operations, we successfully managed to get an adequate operative field. There were no additional trocar. Only 1 patients were postoperative complication above Clevian Dindou Grade III. Median blood loss was $5 \mathrm{~mL}$. Median lymph node number was 18 (range, 11-42). The median hospital stay was 15 days. There was one mortality in this series. Morbidity, including wound infection, was $4 \%$.

Conclusions: This represents the largest experience with single-port laparoscopic right hemicolectomy to date. This technique was used with acceptable morbidity and mortality and without compromise of conventional oncologic parameters by colorectal surgeons experienced in minimally invasive technique. These findings support the use of Free access ${ }^{\mathrm{TM}}$ in a single-port approach for patients requiring right hemicolectomy.

\section{P195-COLORECTAL-Malignant}

LAPAROSCOPIC LOW ANTERIOR RESECTION FOR RECTAL CANCER WITHOUT DEFUNCTIONING ILEOSTOMY

V. Drakopoulos, M.C. Papadopoulou, K. Botsakis, S. Petsa-Poutouri, K. Sarafi, N. Morfis, K. Rekouna, E. Daskalaki, V. Vougas

1st Department of Surgery and Transplantation Unit, District General Hospital of Athens « Evangelismos », ATHENS, Greece

Background: Laparoscopic low anterior resection highlights the advantages of laparoscopic surgery (better surgical field, less bloodloss, less postoperativepain, better cosmeticresult) Defunctioning ileostomy prevents anastomotic leakage in low rectal cancers, butincreases morbidity, degrades thequality of life and requires a second surgery for its closure.

Method: In the last 24 months we performed 8 laparoscopic low anterior resections for rectal cancer, whithout performing any protectiveileostomy, afterchecking the anastomosis intraoperatively(5 men, 6 women. Average age: 65 years). The typical placement of trocars included one supraumbilical $5 \mathrm{~mm}$ trocar, two right sided $10 \mathrm{~mm}$ trocars in the midclavicular line, one $5 \mathrm{~mm}$ in the left midclavicular line and one $5 \mathrm{~mm}$ trocar in the suprapubic midline which is also used for specimen removal, after a $2 \mathrm{~cm}$ transverse extension of the incision. We present themain stages of the procedure (dissection andmesorectal excision, division of the rectum with linear stapler using the 'Chinese hat-Parnex' technique, creation of an end-to-end intracorporeal anastomosisusing circular stapler under direct laparoscopic vision).

Results: No major postoperative complication was observed. The mean operative time was 250 $\min (180-300)$ and free surgical margins were achieved. In one case a conversion to open surgery occured. The average length of hospital stay was 8 days (7-9).

Conclusions: The laparoscopic approach facilitates access to the middle and lower rectum, tota mesorectal excision and avoidance of ileostomy if possible. It is a demanding operation with extended learning curve, and requires adequate experience in laparoscopic surgery and colorectal surgical oncology.

\section{P196-COLORECTAL-Malignant}

CLINICAL OUTCOMES OF LAPAROSCOPIC SURGERY FOR SUBMUCOSALLY INVASIVE RECTAL ADENOCARCINOMA (PT1) : A SINGLE CENTER EXPERIENCE

W. Osumi, K. Tanaka, M. Yamamoto, S. Masubuchi, H. Hamamoto, M. Ishii, A. Hirata, J. Okuda, K. Uchiyama

General and Gastroenterological Surgery, Osaka Medical College Hospital, TAKATSUKI, Japan

Background: In colorectal cancer, local excision is an attractive treatment option, but additional resection is considered when lymph node metastasis(LNM) is expected at high rate. In lower rectal cancer, advanced surgery techniques are required, so it is often difficult to make judgments. The aim of the current study is to assess the reliability of laparoscopic surgery for submucosally invasive rectal adenocarcinoma (pT1) analyzing short-term outcomes and long-term survival. Method: This cohort study analyzed 217 patients who underwent laparoscopic rectal resection for submucosally invasive rectal adenocarcinoma (pT1). Conversion rate and functional and oncologic outcomes were analyzed. Data on long-term results and survival were evaluated. Result: Surgical procedure was low anterior resection / intersphincteric resection / abdominoperineal resection: 190/23/4, and Conversion to open surgery was needed for $6(2.8 \%)$ patients. Sphincter-preserving procedures were performed in $204(97.2 \%)$ patients. There were no perioperative mortalities and positive resection margin. The mean length of hospital stay was 10.5 days. Complications beyond Clavien-Dindo Grade III occurred in $14(6.4 \%)$ patients,the anastomotic leakage rate was $3.6 \%(8 / 217)$. The positive lymph node metastasis rate was $12.9 \%$ (28/217). High tumor budding $(\mathrm{p}=0.006)$, lymphatic invasion $(\mathrm{p}<0.0001)$, and mucinous /poor histological differentiation $(\mathrm{p}=0.01)$ were significantly associated with lymph node metastasis on univariate analysis. On multivariate analysis, only lymphatic invasion was associated with lymph node metastasis $(\mathrm{p}<0.001)$.The median follow-up time was 50 months (range, 6-151 months), recurrence free survival rates was $96.3 \%$ (209/217).

Conculusion: The outcomes of this study suggest that laparoscopic surgery can be used for safe and radical resection of submucosally invasive rectal adenocarcinoma (pT1)?and the absence of lymphatic invasion, budding, and mucinous /poor histological differentiation are each associated with low risk of LNM. Risk stratification models integrating these factors need to be investigated further. 


\section{P197-COLORECTAL_Malignant}

\section{SARCOPENIA IS NOT AMELIORATED WITH LAPAROSCOPIC SURGERY WITHIN AN ENHANCED RECOVERY SETTING}

\section{$\underline{\text { D.S. White }^{1}}$, A. Day ${ }^{2}$, T.A. Rockall ${ }^{1}$}

${ }^{1}$ Colorectal Surgery, Royal Surrey County Hospital, GUILDFORD, United Kingdom; ${ }^{2}$ Colorectal Surgery, East Surrey Hospital, REDHILL, United Kingdom

Aim: Surgery and neo-adjuvant chemotherapy induces loss of skeletal muscle mass and function, leading to the development of sarcopenia (defined as $>5 \%$ skeletal muscle (SM) loss in patients with a BMI of $>20 \mathrm{~kg} / \mathrm{m}^{2}$ ). This is a poor prognostic indicator of outcome. We wished to establish if minimally invasive surgery within an enhanced recovery setting, reduced the incidence of this negative prognostic indicator and what the incidence of sarcopenia was in a typically low risk, well-nourished cohort of patients.

Method: A prospectively kept database from a single centre was used to identify patients undergoing colorectal resection between 2008 and 2010. All patients included in the database underwent surgery within an enhanced recovery setting. Baseline CT scans were compared to the next CT scan performed at six months or one year depending on risk stratification, and availability. Analysis was performed using Tomovision SliceOmatic software, with a single slice at the third lumbar vertebra. Skeletal muscle was measured using a Hounsfield unit [HU] range of $-29 \mathrm{HU}$ to $+150 \mathrm{HU}$. A significant reduction in skeletal muscle mass was $5 \%$.

Results: 250 patients were reviewed. 76 open resections, 174 laparoscopic. Mean age 70 years [24-95 years], 58.7\% male. Dukes stage: A-20\%, B-37\%, C-32\%, D-11\%. There were no statistical differences between open and laparoscopic surgery groups for; ASA, BMI, age, sex, tumour stage, complication rate or use of adjuvant or neo-adjuvant chemotherapy. 106/174 (61\%) laparoscopic patients and 48/76 (63\%) open surgery patients had scans available for review. 9/48 developed sarcopenia in the open surgery group.-18.7\%. 33/106 developed sarcopenia in the laparoscopic group-31.1\%

Conclusions: This study highlights the complex nature of sarcopenia, as well as its common incidence. Minimally invasive surgery had a higher incidence of sarcopenia than that of open surgery when both were performed within an enhanced recovery setting. Despite colorectal patients being a typically well-nourished cohort at low risk of complications, there may well be benefit from interventional strategies such as perioperative immunonutrition or pre-habilitation to reduce the incidence of this poor prognostic indicator.

\section{P198-COLORECTAL_Malignant}

\section{THE ATTEMPT OF EARLY REMOVAL OF URINARY CATHETER AFTER ROBOTIC RECTAL SURGERY}

T. Aiba ${ }^{1}$, K. Uehara ${ }^{1}$, A. Tanaka ${ }^{1}$, K. Hiramatsu ${ }^{2}$, T. Aoba ${ }^{2}$, C. Maeda $^{2}$, T. Kato ${ }^{2}$, M. Nagino ${ }^{1}$

${ }^{1}$ Division of Surgical Oncology, Department of Surgery, Nagoya University Graduate School of Medicine, NAGOYA, Japan; ${ }^{2}$ General surgery, Toyohashi Municipal Hospital, TOYOHASHI, Japan

Backgrounds: Urinary dysfunction is frequently observed after rectal resection and justifies urinary drainage. The concept of enhanced recovery after surgery (ERAS) has been widely spread from the early $2000 \mathrm{~s}$. However, the optimal duration of postoperative urinary drainage is unknown. Aims: The aim of this study was to comprehend short-term outcome of early removal of urinary catheter after robotic rectal surgery (RRS).

Patients and Methods: (Patients) The data of 44 consecutive patients who underwent RRS at two hospitals between April 2015 and November 2017 were retrospectively reviewed. The main indication of RRS was the patients who need rectal mobilization with autonomic nerve preservation regardless of benign or malignant disease.

Perioperative management: None of the patients received epidural anesthesia for postoperative analgesia. Our basic principle was to remove urinary catheter on postoperative day (POD) 1 . After removal of urinary catheter, trans-urethral catheterization (TUC) was performed in the following situations:1) no autonomous urination over $6 \mathrm{~h}$ after removal 2) the decrease in urine volume $(<150 \mathrm{~mL} / 6 \mathrm{hr}) 3)$ the appearance of subjective symptoms like abdominal distension. When TUC was required even once, residual urine volume was measured with ultrasonic examination device since then.

Results: Twenty seven male and 17 female were included. The median age of patients and BMI were 67 years old and $22.7 \mathrm{~kg} / \mathrm{m} 2$, respectively. The surgical procedures included anterior resection $(n=33)$, intersphincteric resection $(n=4)$, abdominoperineal resection $(n=5)$, Hartmann's procedure $(n=1)$, and total coloproctectomy $(n=1)$. Only one patient received lateral pelvic lymph node dissection. Urinary catheter was removed on POD1 in 40 cases $(90.9 \%)$, on POD2 in 4 cases $(9.1 \%)$. Although TUC was needed in three cases $(6.8 \%)$ immediately after removal, TUC was no longer needed within three days in all three patients. Late dysuria was observed in two cases $(4.5 \%)$, and bladder overdistension was suspected in these two cases. Conclusions: Our study showed that urinary catheter could be safely removed on POD1 after RRS. However, careful follow-up observation to avoid bladder overdistension is essential after removal.

\section{P199-COLORECTAL-Malignant}

\section{LAPAROSCOPIC INTERSSPHINCTERIAN RECTAL RESECTION WITH LOW ENDOANAL ANASTOMOSIS FOR LOWER RECTAL CANCERS}

\author{
F. Zaharie ${ }^{1}$, G. Ciorogar ${ }^{2}$, A. Vaduva ${ }^{2}$, I. Balint ${ }^{2}$, C. Popa ${ }^{2}$,

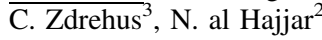

${ }^{1}$ Regional Institute of Gastroenterology nd Hepatology, Octavian Fodor, University of Medicine and Pharmacy, Iuliu Hatieganu, CLUJNAPOCA, Romania; ${ }^{2}$ Surgery Clinic no 3 , University of Medicine and Pharmacy, Iuliu Hatieganu, CLUJ-NAPOCA, Romania; ${ }^{3}$ ATI, University of Medicine and Pharmacy, Iuliu Hatieganu, CLUJNAPOCA, Romania

Introduction: Intersphincterian low rectal resection is a valid alternative to lower rectal cancers located at about $4-7 \mathrm{~cm}$ from the anus.

Methods: We present 19 cases from our personal experience for tumors localized 4-7 cm from the anus. 13 of them required preoperative radiochemotherapy. In 12 cases, abdominal surgery was performed laparoscopic, 7 having the surgical specimen extracted transanal. Lone Star device was used for the perineal procedure in all cases. 6 cases required a manually, separate wires anastomosis; the others 13 cases benefited from mechanical anastomosis performed endoanal with $29-31 \mathrm{~mm}$ circular stapler. We performed complete mesorectum excision in all cases, ligation at the origin of inferior mesenteric artery, complete mobilization of left splenic flexure and lateral protective ileostomy. All pacients underwent inspection rectoscopy before transit reintegration, and 16 cases were reintegrated over a period of 3-12 weeks, except for 3 cases which developed a colo-anal fistula, that closed under conservative treatment over a period of 3-9 months.

Results: There were no postoperative anal incontinence. In one case, a relative anal stenosis occured, which required endoscopic dilation. There was 1 case of tumor recurrence and required abdominoperineal resection.

Conclusion: Literature data sustain a 3-4/1 ratio for very low rectal resection versus rectum amputation. The limit resection under the tumor is accepted as $0.5 \mathrm{~cm}$. Very good functional results by considering oncological principles, is a sustainable argument for choosing this kind of procedure as an alternative of rectum amputation.

\section{P200-COLORECTAL-Malignant}

LONG-TERM SURGICAL AND ONCOLOGICAL OUTCOME IN T2NO VERSUS NEOADJUVANT T3NO LOW RECTAL CANCER RESECTION:NON INFERIORITY RETROSPECTIVE STUDY

\section{Bergamini, A. Giordano, C. Agostini, A. Minuzzo, G. Alemanno}

Chirurgia d'Urgenza, Azienda Ospedaliero-Universitaria Careggi, FIRENZE, Italy

Objective: Current management of sub-peritoneal rectal T2N0 adenocarcinoma is up-front surgery. No sufficient data demonstrate that neoadjuvant chemoradiotherapy (nCRT) in T2N0 could be beneficial. The absence of indications to a nCRT in that subgroup makes it impossible to perform an iso-stage comparison study T2N0 vs T2NO + nRCT and, consequently, reduces the probability of an approval by the local Ethical Committee for prospective study comparing those two groups.

To obviate this problem, we have performed a retrospective study to demonstrate the noninferiority of $\mathrm{T} 3 \mathrm{~N} 0+\mathrm{nCRT}$ with respect to $\mathrm{T} 2 \mathrm{~N} 0$ regarding the surgical outcome, quality of life (QoL) and mortality.

Method: SA retrospective, monocentric, non-inferiority study analyzing rectal resections (Miles or RAR) for low rectum adenocarcinoma was performed including pT2N0 and pT3N0 + nRCT (capecitabine $825 \mathrm{mg} / \mathrm{mq}$ BID 25 day $+1,8 \mathrm{GyX} 25$ days radiotherapy L5-S1 pelvic filed). Objectives were: operatory outcome, QoL (LARS, VATC scale) and oncological outcomes. Results: Between 2008 and 2017, 37 patients (T2N0) and 38 patients (T3N0 + nCRT) underwent a surgical procedure, comparable in terms of median age $(69.4$ vs $71.84 \mathrm{p}=0.32)$, mean time between diagnosis and procedure ( 2.68 vs 2.66 months $\mathrm{p}=0.963)$ and Charlson comorbidity index ( 3.32 vs $3.71 ; p=0.34)$. Differences were found only for a higher rate of robotic procedure in $\mathrm{T} 2 \mathrm{~N} 0$ ( $35.1 \%$ vs $7.9 \%$; $\mathrm{p}=0.004)$. TME were similarly performed in RAR $(56.7 \%$ vs $65.7 \% ; \mathrm{p}=0.422)$ and Miles ( $8.1 \%$ vs $18.4 \% ; \mathrm{p}=0.189)$. Similar follow-up (30.92 vs 40.68 moths; $\mathrm{p}=0.099)$, local recurrence $(2.7 \%$ vs $7.9 \% ; \mathrm{p}=0.32)$, distance recurrence $(10.8 \%$ vs $15.8 \% ; \mathrm{p}=0.53)$, mortality $(10.8 \%$ vs $23.7 \% ; \mathrm{p}=)$ and LARS score $(15.81$ vs $9.96 ; \mathrm{p}=0.054)$ were found, as well as the more detailed and specific VATS score.

Discussion: Despite a lower stage, T2N0 have no better outcomes in terms of local recurrence, distance recurrence, mortality and quality of life with respect to the T3N0 + nCRT. Such results may constitute the bssics for a future evaluation of the benefit of nCRT application T2 N 0stage. 


\section{P201-COLORECTAL—Malignant}

DETECTION OF MICROMETASTASES IN COLORECTAL CANCER WITH THE EX-VIVO FLUORESCENCE SENTINEL LYMPH NODE MAPPING

A. Picchetto ${ }^{1}$, C. Santi ${ }^{2}$, M. Diana ${ }^{3}$, L. Casali ${ }^{4}$, V. Violi $^{2}$, G. d'Ambrosio 1

${ }^{1}$ Department of General Surgery and Organ Transplantation, Sapienza University of Rome, ROMA, Italy; ${ }^{2}$ Department of General Surgery, Hospital of Fidenza, Ausl of Parma, University of Parma, FIDENZA, Italy; ${ }^{3}$ IHU-Strasbourg, Institute of Image-Guided Surgery, IRCAD, Research Institute against Cancer of the Digestive System, STRASBOURG, France; ${ }^{4}$ Department of General Surgery, Hospital of Fidenza, Ausl of Parma, FIDENZA, Italy

Background and Aim: Near-Infrared Fluorescence (NIRF) sentinel lymph node (SLN) mapping can improve the nodal staging in several type of tumors, including gastro-intestinal, head and neck, breast and melanoma. The ultrastaging of ColoRectal Cancer (CRC) SLN was proposed to identify the micrometastases in Node Negative Disease (NND or N0) patients, in order to upstage those patients as N1 (according to the last TNM edition).

In the few studies conducted on CRCs, the reported rate of SLN micro-metastases is up to $20-30 \%$. The aim of this ongoing prospective study is to assess the predictability of the ex-vivo NIRF SLN mapping and of the research of micrometastases in NND CRC patients to propose adjuvant chemotherapy.

Materials and methods: Fifty-eight patients undergoing standard oncological CRC laparoscopic resection have been prospectively enrolled in two centre. As previously described by the Authors, the intact surgical specimen was extracted and opened longitudinally and $1 \mathrm{ml}$ of Indocyanine Green (ICG; $5 \mathrm{mg} / \mathrm{ml}$ ) was injected submucosally at four corners around the tumor in order to identify the lymphatic pathway and the SLNs. Each SLN presenting as negative at conventional histological analysis, was further investigated with ultrastaging techniques including serial sectioning and additional immunohistochemistry, in order to detect the presence of micrometastases. Results: Thirty patients were N + , and 28 were NND. Overall, a total of 1085 lymph nodes were retrieved. A total of 117 SLN were identified (mean 2.01 per case) and 54 of those were NND. After ultrastaging investigations, 4 micrometastatic cases were found in NND patients. The patients were so upstaged to N1.

SLN located deeper in the mesenteric and mesorectal fat could easily be identified by NIRF (even after nChRT).

Conclusions: In our preliminary series, the ex-vivo NIRF SLN mapping rightly predicts the status of loco-regional nodes, as confirmed by the histological investigations. The micrometastases' identification let selected patients to undergo the adjuvant treatment with the aim to reduce the risk of recurrence.

\section{P202-COLORECTAL-Malignant}

SHORT AND LONG TERM Results: OF SELECTIVE LAPAROSCOPIC LATERAL PELVIC LYMPHADENECTOMY FOR LOWER RECTAL CANCER

S. Yamaguchi, Y. Hirano, T. Ishii, H. Kondo, K. Hara, A. Suzuki, N. Obara, L. Wang, S. Sakuramoto

Department of Gastroenterological Surgery, Saitama Medical University International Medical Center, HIDAKA, Japan

Introduction: Lateral pelvic lymphadenectomy (LPL) for rectal cancer is still controversial in Western countries. We performed selective LPL for only the patients with suspicious lateral lymph node metastasis. Purpose of this study is to assess the short and long term results of selective LPL.

Methods and patients: Consecutive 29 patients were performed R0 laparoscopic rectal cancer resection with LPL since January 2009 to February 2015. Mean follow-up period was 58.4 months.

Results: Mean age was 62.3 y.o. and gender was male: 22 and female: 7. Preoperative chemoradiation was performed for 5 patients. Preoperative T stage was cT2: 2, cT3: 23, cT4: 4 Laparoscopic procedures were LAR: 12, ISR: 12, APR: 5 and bilateral LPL was performed for 3 patient and the other 26 patients underwent unilateral LPL. All patients were performed lymphadenectomy around the origin of the IMA. Except 1 patient, all patients were created diverting or permanent stoma. Median operative time was $333 \mathrm{~min}$ (230-611) and median blood loss coun was 50 g. Postoperative complications were anastomotic leak: 1 , ileus: 3 , urinary tract infection: 1, neurogenic bladder: 1 . Median postoperative hospital stay was 10 (7-32) days. Pathological lateral lymph node metastasis was observed in 13 patients (37.7\%). Node positive number was: $1=11,2=1,3=1$. Cumulative 5 year OS and 3 year RFS were $91.7 \%, 61.5 \%$ in lateral node positive group and $79.5 \%, 75.0 \%$ in node negative group, respectively. Local recurrence was observed in 6 patients (20.6\%) and $23.1 \%$ (3/13) in lateral node positive group and $18.8 \%(3 / 16)$ in lateral node negative group. Four of 6 local recurrence were lateral lymph node recurrence. Two patients recurred the other lateral side of previous LPL, then they were laparoscopically resected and no recurrence $(52,58$ months). Two patients recurred the same side after LPL were not curable because of liver metastasis and extensive invasion to the common iliac vessels. Conclusion: Selective LPL for rectal cancer was safe and good local control for lateral lymph node positive patients. Also curable local recurrence resection was possible for non-treated lateral lymph node recurrence.

\section{P204-COLORECTAL_Malignant}

FEASIBILITY OF LAPAROSCOPIC SURGERY AS A BRIDGE TO SURGERY AFTER SELF-EXPANDABLE METALLIC STENT INSERTION FOR OBSTRUCTIVE LEFT SIDED COLORECTAL CANCER

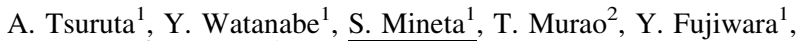
A. Shiotani ${ }^{2}$, T. Ueno ${ }^{1}$

${ }^{1}$ Digestive Surgery, Kawasaki Medical School, KURASHIKI, OKAYAMA, Japan, ${ }^{2}$ Internal Medicine, Kawasaki Medical School, KURASHIKI, OKAYAMA, Japan

Purpose: To evaluate the feasibility and safety of laparoscopic colorectal surgery as a bridge to surgery after self-expandable metallic stent insertion for obstructive left sided colorectal cancer. Methods and Materials: Between July 2012 and October 2018, 40 patients who underwent colorectal surgery after colonic stent insertion for left-sided colorectal cancer were retrospectively analyzed.

Results: Laparoscopic surgery group was 27 patients and open surgery group was 13 patients. Regarding gender, age, the colorectal obstruction scoring system classification score, and duration of stent insertion till operation, there were no significant differences between the laparoscopic group and the open group. Duration of operation was significantly more in the laparoscopic group and blood loss was significantly less in the laparoscopic group. Postoperative hospital stay was significantly shorter in the laparoscopic group. There was no significant difference about perioperative complications between the two groups. 3-year disease free survival was $67.0 \%$ in both the laparoscopic group and the open group.

Conclusion: Laparoscopic surgery as the bridge to surgery for obstructive left-sided colorectal cancer after self-expandable metallic stent insertion was feasible and safe. Considering postoperative course, surgical outcome in laparoscopic surgery would be better than that in open surgery. Laparoscopic surgery after colonic stent insertion for left-sided colorectal cancer was comparable to open surgery in terms of long-term outcome. 


\section{P205-COLORECTAL-Malignant}

\section{LAPAROSCOPIC SUBTOTAL COLECTOMY FOR SINCHRONOUS CAECAL AND SIGMOID HGD ADENOMAS IN INTESTINAL MALROTATION}

\author{
A. Costanzi, M. Gerosa, E. di Fratta, A. Miranda, A. Rosato, G. Mari, \\ D. Maggioni
}

General and Emergency Surgery, ASST Monza-Desio Hospital, DESIO, Italy

Intestinal malrotation is an embriologic anomaly generally discovered in the first months of life due to bowel obstruction. Adult presentation is rare and its association with colon cancer is far more rare. We report a case of a 70 years old man affected by asymptomatic intestinal malrotation incidentally found during an abdominal computed tomography (CT) performed for retroperitoneal colonic perforation in a patient with an endoscopically diagnosed Aenocarcinoma of the caecum and a large polyp of the descending colon. Preoperative vascular anatomic study allowed us to plan a laparoscopic approach safely also with adequate lymphoadenectomy. The abdominal cavity was entered throught a right flank $12 \mathrm{~mm}$ optical trocar on the transverse umbilical line. Three additional $5 \mathrm{~mm}$ trocars were placed in right iliac fossa, right and left hypocondrium respectively. Exploratory laparoscopy confirmed midgut malrotation and a fresh flogistic area at the descending colon perforation site. Caecum and ascending colon were on midline and attached due to adhesions to sacral promontory. Ileocolic artery (ICA), middle colic artery (MCA) and IMA were selectively ligated but not at their origins due to aberrant anatomy. Laparoscopic subtotal colectomy with intracorporeal stapled ileosigmoid anastomosis were carried out (EndoGIA $45 \mathrm{~mm}$, double layer 3/0 Polyglicolic Acid suturing of the breech). The anisoperistaltic nature of the anastomosis is due to the disposition of the mesenterium which did not allow an isoperistalting orientation of the two resected stumps. The specimen was extracted throught a Pfannestiel incision. The postoperative course was complicated by intestinal obstruction conservatively treated with slow bowel function's restoration. The patient was discharged from the hospital in 15th postoperative day. Unexpectedly specimen histology revealed two villous adenomas with High Grade Dysplasia. 17 lymphnodes were retrieved from the specimen (pTisN0). To date our case is the only fully laparoscopic colonic resection reported in literature in malrotation as well as the first intracorporeal stapled ileo-sigmoid anastomosis for such disease.

\section{P206-COLORECTAL-Malignant}

\section{USE OF INFLAMMATORY BIOMARKERS TO PREDICT INFECTIOUS COMPLICATIONS AFTER COLORECTAL SURGERY}

L. Fernandez ${ }^{1}$, P.V. Vieira ${ }^{1}$, I. Nasir ${ }^{1}$, H. Domingos ${ }^{1}$, R. Carvalho $^{2}$, A.M. Martinho ${ }^{2}$, F.. Moura ${ }^{2}$, J.F. Cunha ${ }^{1}$, A. Parvaiz ${ }^{1}$, N. Figueiredo ${ }^{1}$

${ }^{1}$ Digestive Surgery, Champalimaud Foundation, LISBON, Portugal; ${ }^{2}$ Intensive Care Unit, Champalimaud Foundation, LISBON, Portugal

Background: Inflammatory parameters have been used as indicators of postoperative complications in abdominal surgery. The aim of this study was to evaluate the utility of sequential postoperative serum inflammatory biomarkers for early identification of postoperative infectious complications following minimally invasive colorectal surgery(MICS).

Patients \& Methods: Retrospective review of consecutive patients undergoing MICS, between 07/2016 and 07/2018. WBC count, CRP (C Reactive Protein), PCT (Procalcitonine) and Albumin were measured at post-operative day one (POD1) and POD3. Patients who developed any infectious complication were compared to the remaining cohort.

Results: A total of 114 patients were included. 26(22.8\%) had an infectious complication. Patients with surgical infections showed significantly higher CRP levels on POD1 $(8.1 \pm 4.9$ vs $5.7 \pm 3.3 \mathrm{p}<0.01)$ and POD3 $(15.8 \pm 9.2$ vs $8.1 \pm 5.0 \mathrm{p} 0.01)$ and CAR (CRP/Albumin ratio) levels on POD1 $(2.4 \pm 1.7$ vs $1.7 \pm 1.1 \mathrm{p}<0.01)$ and POD3 $(5.4 \pm 3.5$ vs $2.4 \pm 1.6 \mathrm{p}<0.01)$ than patients without complications. A CAR cut-off value of 2.19 on POD3 presented the highest area under the curve $(\mathrm{AUC}=0.79)$ for predicting postoperative infectious complications with a sensitivity of $88 \%$ and specificity of $61 \%$.

Conclusions: CRP concentration and CAR on POD1 and POD3 could be used for early identification of patients at higher risk of infectious complications after MICS. Routine postoperative measurement of these inflammatory parameters may improve clinical decision management during early postoperative period.

\section{P207-COLORECTAL-Malignant}

\section{ROBOTIC SURGERY FOR RECTAL CANCER: A SINGLE CENTER EXPERIENCE OF 80 CONSECUTIVE CASES}

F. Savulescu, C. Blajut, C. Cirlan, I. Budrugeac, M. Iordache, M. Iordache Petrescu

General Surgery II, Dr. Carol Davila Central Military Emergency University Hospital, BUCHAREST, Romania

Aims: Minimally invasive techniques have revolutionized the field of general surgery over the few last decades. The available literature on minimally invasive colorectal cancer surgery demonstrates that this approach is feasible and associated with better short term outcomes than open surgery while maintaining equivalent oncologic safety. Despite its advantages, in complex procedures such as rectal surgery, laparoscopy has not achieved a high penetration rate because of its steep learning curve, its relatively high conversion rate and technical challenges.The aim of this study was to present a single center experience with robotic surgery for rectal cancer focusing mainly on early and mid-term postoperative outcome.

Methods: A series of 80 consecutive patients who underwent robotic rectal surgery between December 2014 and September 2018 in 'Dr. Carol Davila' Central Military Emergency University Hospital, in Bucharest, Romania was analyzed retrospectively in terms of demographics, pathological data, surgical and oncological outcomes.

Results: Seventy-five patients underwent robotic sphincter-saving resection, and five patients underwent robotic abdominoperineal resection. There ware two conversions. The median operative time for sphincter-saving procedures was $210 \mathrm{~min}$. The median time for robotic abdominoperineal resection was $180 \mathrm{~min}$. The median number of retrieved lymph nodes was 22 . The median hospital stay was 6 days. In-hospital mortality was nil. The overall morbidity was $20 \%$. The median length of follow-up was 23 months.

Conclusions: Our preliminary results suggest that robotic-assisted surgery for colorectal cancer can be carried out safely and according to oncological principles. Robotic surgery is advantageous for both surgeons (in that it facilitates dissection in a narrow pelvis) and patients (in that i affords a very good quality of life via the preservation of sexual and urinary function in the vast majority of patients and it has low morbidity and good midterm oncological outcomes). In recta cancer surgery, the robotic approach is a promising alternative and is expected to overcome the low penetration rate of laparoscopy in this field.

\section{P208-COLORECTAL-Malignant}

\section{ARTIFICIAL NEURAL NETWORK PREDICTION OF PROLONGED LAPAROSCOPIC COLORECTAL CANCER OPERATIVE TIME}

N.J. Curtis ${ }^{1}$, A. Dias ${ }^{2}$, E. Salib ${ }^{3}$, G. Dennison ${ }^{4}$, R. Dalton ${ }^{2}$, A.S. Allison ${ }^{2}$, J.B. Ockrim ${ }^{2}$, N.K. Francis ${ }^{4}$

${ }^{1}$ Surgery and Cancer, Imperial College London, LONDON, United Kingdom; ${ }^{2}$ Colorectal Surgery, Yeovil District Hospital, YEOVIL, United Kingdom; ${ }^{3}$ Medical Statistics, Aid Medical, LIVERPOOL, United Kingdom; ${ }^{4}$ General Surgery, Yeovil District Hospital, YEOVIL, United Kingdom

Aim: Prolonged operative timing is likely to negatively impact clinical outcomes following laparoscopic colorectal resection. Pre-operative prediction of those likely to undergo longer procedures can assist theatre planning and post-operative care. We aimed to apply artificial neural networks (ANN) as a predictive tool for prolonged operating time in laparoscopic colorectal surgery.

Methods: A dedicated, prospectively populated database of elective laparoscopic colorectal cancer surgery with curative intent was utilised. Primary endpoint was the prediction of operative times. We defined prolonged operative time as over $5 \mathrm{~h}$ based on our previous work. Variables included in the network were: age, gender, ASA, BMI, stage, location of cancer, neoadjuvant therapy, time from diagnosis and planned stoma. A multi-layered perceptron ANN model was trained and tested alongside uni and multivariate analyses.

Results: 590 consecutive patients with complete data were included (59\% male, mean age and BMI 70 years and 26 respectively). 161 (29\%) were ASA III. 261 (47\%) had rectal cancer and $8.5 \%$ underwent neoadjuvant treatment. Mean operative time was 213 min (95\% CI 206-221) with $465(78.8 \%)$ of less than $5 \mathrm{~h}$ and $16 \%$ conversion rate. ANN correctly identified those having surgery less $<5 \mathrm{~h}(91.2 \%$; AUC 0.843 and $93.3 \%$; AUC 0.893$)$ of the training and testing cohorts respectively with an overall accuracy of $84.5 \%$. The ANN findings were accurately cross-validated with a logistic regression model

Conclusion: Artificial neural networks using demographic and tumour data successfully predicts the likelihood of prolonged laparoscopic surgery. This finding could assist the personalisation of pre-operative care and theatre utilisation. 


\section{P209-COLORECTAL_Malignant}

CONCOMITANT PRIMARY LEIOMYOSARCOMA OF JEJUNUM AND RENAL ONCOCYTOMA: A CASE REPORT AND LITERATURE REVIEW

M.H. Mirza, A. Coyle, N.H. Soomro, R. Burke, R. Mitru, M. Aremu

General Surgery, Connolly Hospital Blanchardstown, DUBLIN, Ireland

Background: Leiomyosarcoma of small bowel is quite rare, comprising 2-3\% of all small bowel tumors. It usually presents with non-specific symptoms and early recognition is crucial. Here we report a case of jejunal leiomyosarcoma with concurrent renal oncocytoma and review of the literature.

Case Presentation: A 51 year old gentleman presented to emergency department with abdominal pain and right sided abdominal tenderness, with no associated systemic features. After initial workup, $\mathrm{CT}$ abdomen and pelvis that showed a $12 \mathrm{~cm}$ mass in the right abdomen and an incidental $5 \mathrm{~cm}$ left renal mass. After MDT discussion, he underwent laparotomy and en-bloc resection of Jejunal mass with primary anastomosis, and left nephrectomy. Histology of jejunal mass revealed an intermediate grade leiomyosarcoma with clear margins and confirmatory immunohistochemistry. Renal mass was an oncocytoma. No evidence of distant metastases was found on staging CT. Patient recovered fully. MDT recommended 6 monthly imaging follow-up and no adjuvant treatment.

Discussion: Leiomyosarcoma of small bowel is rare with only 26 cases reported between 2000 and 2012. Only after the advent of novel Immunohistochemical techniques in late $1990 \mathrm{~s}$, their differentiation form GISTs became possible. Also no case of a co-existing renal oncocytoma has been reported to date. Oncocytoma is benign and surgical resection is curative. NCCN guidelines for intra-abdominal sarcomas recommend complete oncological resection, intense follow-up and selective use of adjuvant chemo-radiation.

Conclusion: The management of small bowel leiomyosarcoma is centred on surgical resection with adjuvant treatment in selected cases and requires regular follow up. Tumour grade and extent of surgical resection are main predictors of prognosis.

Key words: Leiomyosarcoma, oncocytoma, small bowel, surgical resection

Abbreviations: MDT: Multi-disciplinary team, CT: computed tomography, NCCN: National Comprehensive Cancer Network

Disclosures: none

Conflict of interests: none

\section{P210-COLORECTAL_Malignant}

\section{THE IMPACT OF POSTOPERATIVE INFLAMMATION ON RECURRENCE IN PATIENTS WITH COLORECTAL CANCER}

D. Matsubara, T. Arita, M. Nakanishi, Y. Kuriu, M. Murayama, M. Kudou, T. Kobayashi, K. Shoda, T. Kosuga, H. Konishi, R. Morimura, A. Shiozaki, H. Ikoma, T. Kubota, H. Fujiwara, K. Okamoto, E. Otsuji, Division of Digestive Surgery Department of Surgery, Kyoto Prefectural University of Medicine, KYOTO, Japan

Aims: Postoperative inflammation have been reported as one of the independent prognostic factors in several types of malignancies.The aim of this study is to clarify the impact of laparoscopic approach on postoperative inflammatory status after surgery for colorectal cancer, and to analyze the association between postoperative inflammation and prognosis in patients with colorectal cancer. Methods: A total of 636 patients with stage 1-lll colorectal cancer (CRC) who underwent curative surgery were retrospectively analyzed. The maximum CRP value measured between the times of surgical resection and discharge was defined as 'max CRP'. The optimal cut-off value of max CRP that best predicts RFS was determined to be $10 \mathrm{mg} / \mathrm{dL}$ by the minimum p-value approach. Patients were divided into two groups according to max CRP level: low CRP group $(<10 \mathrm{mg} / \mathrm{dl}$ : $\mathrm{n}=482)$ and high CRP group. $(=10 \mathrm{mg} / \mathrm{dl}: \mathrm{n}=154)$

Results: max CRP level in patients with laparoscopic surgery (group L, $\mathrm{n}=559$ ) were significantly lower than that in patients with open surgery (group $\mathrm{O}, \mathrm{n}=77 ; 6.79 \pm 5.83$ vs $10.28 \pm 7.05$; $\mathrm{p}<0.001)$. Patients in high CRP group showed significantly worse RFS than patients in low CRP group. $(\mathrm{p}=0.004)$ Even among patients without postoperative complications, max CRP level in group $\mathrm{L}$ was significantly lower than that in group $\mathrm{O}(5.89 \pm 4.49 \mathrm{vs} 8.79 \pm 6.00 ; \mathrm{p}<0.001)$, and high CRP group revealed worse RFS when compared with low CRP group. $(p=0.0001)$ Besides, survival analysis stratified by surgical approach demonstrated that the 5-years RFS in high CRP group was significantly worse in patients who underwent laparoscopic surgery when compared to their counterparts $(\mathrm{p}=0.005)$. Multivariate logistic regression analysis revealed that undergoing open surgery, as well as older age, male sex, presence of complications, was independent risk factors for elevated max CRP (OR $2.10,95 \%$ CI $1.01-4.32, \mathrm{p}=0.046)$

Conclusions: Our study suggested that postoperative inflammation might be one of crucial mechanisms of poor prognosis in patients with colorectal cancer. Less invasive surgery such as laparoscopic surgery might improve prognosis by reducing postoperative complications or suppressing inflammatory responses.

\section{P211-COLORECTAL-Malignant}

\section{LAPAROSCOPIC INTERSPHINCTERIC RESECTION COMBINATION WITH PULL-THROUGH DELAYED COLOANAL ANASTOMOSIS ENABLES NO-STOMA SURGERY FOR ULTRALOW RECTAL CANCER}

K. Tanaka ${ }^{1}$, M. Yamamoto ${ }^{1}$, S. Masubuchi ${ }^{1}$, W. Osumi ${ }^{1}$,

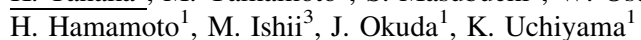

${ }^{1}$ Department of General and Gastroenterological Surgery, Osaka Medical College, OSAKA, Japan; ${ }^{3}$ Surgery, Osaka Medical College, OSAKA, Japan

Aims: The aim of this study is to compare laparoscopic intersphincteric resection (LapISR) reconstruction with delayed coloanal anastomosis (CAA) using pull-through technique and conventioal LapISR with diverting stoma in a series of consecutive rectal cancer patients. Methods: From July 2014 to November 2018, a total of 103 consecutive cases with low recta cancers underwent Lap ISR at our department. 23were reconstructed with delayed ( 7 days after Lap ISR) coloanal anastomosis (CAA) using pull-through technique (PT group) and 80 patients with immediate CAA with diverting stoma (DS group). The stomas of DS group were closed 3-12 month after Lap ISR. Patients' characteristics, operative and post operative outcomes, short-term technical and functional results were analyzed.

Results: Male (69.6\% vs $65.0 \%$; n.s.), Age $(58.6 \pm 1.58$ vs $62.4 \pm 0.85 ; \mathrm{p}<0.05)$ ), NAC NACRT $(52.2 \%$ vs $41.3 \%$; n.s.) length of skin incision $(3.5 \pm 0.27$ vs $4.4 \pm 0.14 ; \mathrm{p}<0.005)$ lateral lymphnode dissection ( $13.0 \%$ vs $4.3 \%$; n.s.), left flexure take down $(13.0 \%$ vs $11.3 \%$; n.s.), operation time ( $339 \pm 23$ vs $363 \pm 13$; n.s.), blood loss ( $34 \pm 42$ vs $90 \pm 23$; n.s.), curative resection ( $95.5 \%$ vs $94.4 \%$ ), conversion to open surgery ( 0 vs 0 ), postoperative complication (13.4\% vs $10 \%$; n.s.) (PT group vs DS group; respectively), Three patients of PT group were created diverting stoma 7-15 days after LapISR, because two bowel ischemia and one anastomotic leakage after delayed CAA. Lap ISR was performed without stoma for 20 patients $(87.0 \%)$ in PT group. The stoma closure ratio was $86.6 \%(58 / 67$ 2014-2017) in DS group. The Wexner score within 12 months postoperatively was almost same ( $10.1 \pm 2.6$ vs $9.7 \pm 0.9$;). Conclusions: Lap ISR combination with pull-through delayed CAA was feasible. This technique enables no-stoma surgery for the patients with ultralow rectal cancer.

\section{P212-COLORECTAL-Malignant}

\section{A RETROSPECTIVE REVIEW ON CONVERSION FROM LAPAROSCOPIC TO OPEN SURGERY FOR COLORECTAL CANCER}

T. Kobayashi, T. Arita, M. Nakanishi, Y. Kuriu, Y. Murayama, M. Kudou, D. Matsubara, E. Otsuji

Digestive Surgery, Kyoto Prefectural University of Medicine, KYOTO, Japan

Aims and methods: Recently, Laparoscopic surgery (LS) for colorectal cancer has been common worldwide. In our institute, LS has been adopted since 2007, and about $85 \%$ of patients with colorectal cancer underwent LS. However, approximately $5 \%$ of them had conversion to open surgery (COS) and 10\% had open surgery (OS). We investigated the risk factors for COS and short- and long-term result of patients who underwent COS comparing with LS and OS.

Results: 558 consecutive patients who underwent colorectal cancer resection between 2012 and 2016 in our hospital were involved in this study. The number of LS/COS/OS was 480/25/53 cases. The reasons for COS were other organ infiltration ( 9 cases), severe adhesion ( 9 cases), uncontrollable bleeding ( 3 cases), obesity ( 1 case) and others ( 3 cases). Patients with higher age $(\mathrm{p}=0.03)$, past surgical history (except for appendectomy and caesarean section) $(\mathrm{p}=0.04)$, preoperative ileus $(\mathrm{p}=0.04)$, cT4 and deeper $(\mathrm{p}<0.01)$, and larger tumor size $(\mathrm{p}<0.01)$ received COS. Regarding tumor localization, $\operatorname{COS}$ rate was $4.6 \%$ in cecum and ascending colon, $6.5 \%$ in transverse colon, $13.3 \%$ in descending colon, $5.0 \%$ in sigmoid colon, and $3.1 \%$ in rectum. In terms of short-term results, COS group was associated with larger intraoperative hemorrhage $(\mathrm{p}<0.01)$ and postoperative paralytic ileus $(\mathrm{p}=0.04)$ when compared with LS group. In contrast, when comparing $\mathrm{COS}$ with OS, short-term results such as operation time, blood loss, SSI, anastomotic leakage, and postoperative intestinal paralysis were statistically equal, and furthermore, 3-year overall survival rate was equivalent (COS: 0.677, OS: 0.683 , $\mathrm{p}=0.75$ ).

Conslusions: LS showed favorable results when compared with COS and OS, however, the results of COS were almost equal to OS. COS may be permissible in the point of safety and oncological validity. 


\section{P213-COLORECTAL-Malignant}

\section{THE Results: OF LAPAROSCOPIC RESECTION FOR COLORECTAL CANCER IN PATIENTS WITH PREVIOUS ABDOMINAL OPERATIONS}

\section{Y. Shingu, E. Sakamoto, S. Norimizu, H. Otsuji, K. Yamauchi}

Digestive surgery, Japanese red cross Nagoya Daini Hospital, NAGOYA, Japan

Background: Previous abdominal operations increase the difficulty of laparoscopic resection for colorectal cancer and may affect consequences. However, little study has been pursued to investigate the effect of previous abdominal operations on outcomes of laparoscopic colorectal surgery for cancer. The aim of this study was to evaluate the impact of previous abdominal operations on laparoscopic resection for colorectal cancer in terms of short and long-term outcomes.

Methods: Between January 2001 and December 2017, 2408 patients who underwent laparoscopic resection for colorectal cancer with curative intent were enrolled in this study. They were divided into two groups: 671 patients with previous abdominal operations (PAO group) and 1737 without previous abdominal operation (NPAO group). Their clinical records in our prospectively maintained database were collected, and were compared between two groups.

Results: The two groups were homogeneous for clinical feature. Conversion rates of PAO and NPAO group were $7.0 \%$ and $2.8 \%$, respectively, with statistical difference $(\mathrm{p}<0.001)$. There were no significant difference in mean operating time, mean estimated blood loss, and mean number of retrieved lymph nodes between the two groups. Morbidity rates were also comparable (PAO 25.4\% vs. NPAO 26.6\%, p = 0.577). Five-year overall survival rates in PAO and NPAO group were $84.8 \%$ and $83.3 \%$, respectively, indicating no statistical significance.

Conclusion: Although laparoscopic surgery in PAO group has a trend toward more conversions, it does not affect the short-time and long-time outcomes. The results of this study suggest that laparoscopic resection for colorectal cancer in patients with previous abdominal operations is not inferior to the same procedure in patients without previous operations in terms of safety and tumor clearance.

\section{P215-COLORECTAL-Malignant}

\section{COLONOSCOPIC SCREENING IN EARLY DETECTION OF COLORECTAL CANCER IN HIGH RISK GROUPS}

\section{$\underline{\text { M.R. Elkeleny }}{ }^{1}$, A.A. Elkhatib ${ }^{2}$}

${ }^{1}$ GIT and bariatric surgery, Faculty of medicine, Alexandria University, ALEXANDRIA, Egypt; ${ }^{2}$ GIT surgery, Faculty of medicine, ALEXANDRIA, Egypt

Background : Colorectal cancer (CRC) is the third most common malignant neoplasm worldwide and the second most common cause of cancer death in the United States. In Egypt there was low incidence of CRC and high proportion of young-onset disease. Colonoscopy is commonly used to screen for colorectal neoplasia. Screening colonoscopy reduces CRC incidence and mortality. The aim of this work is to evaluate colonoscopy as screening tool for early detection of colorectal cancer in high risk groups.

Methods: The study involved 200 patients, 92 males and 108 females, age ranged from 20 to 83 (mean $53.42 \pm 15.50$ ). They were presented to the Gastrointestinal Surgery Unit, Main Alexandria University Hospital complaining of lower gastrointestinal tract symptoms and/or at high risk of colorectal cancer. During a 9-month period, 2 experienced endoscopists performed 200 colonoscopies, were screening colonoscopy was done in subjects who had not previously undergone

Results: Complete colonoscopy was done in $94 \%$ of cases and histopathological examination of the biopsies obtained were performed. Colorectal polyps were seen in 54 patients $(27 \%)$ and more likely in males. Of these adenomatous polyps were the most common (81.5\%), constitute $22 \%$ (range of the percentage of subjects with adenomas, 18 to $22 \%$ ). of all cases. Colonic masses were seen in 22 cases $(11 \%)$ commonly seen in males. Of these adenocarcinoma was the most common $(81.8 \%)$, constitute $9 \%$ of all cases. Inflammatory and/ or ulcerative colonic lesions were seen in 14 patients $(7 \%)$. Of these $42.9 \%$ were diagnosed as non-specific colitis, constitute $6.7 \%$ of all cases. $35.7 \%$ as ulcerative colitis, constitute $5.6 \%$ of all cases and $21.4 \%$ as Crohn's disease, constitute $3.3 \%$ of all cases. Colonic diverticulosis was diagnosed in 8 cases and internal piles in 10 cases.

Conclusions: In this prospective study, we observed greater rates of detection of adenomas among endoscopists. Screening colonoscopy on symptomatic and/or high risk group for CRC is valuable in early detection and the prevention of CRC. Large sample size and long period of screening colonoscopy was needed. Limitation of our study was the small sample size and no use of high detention endoscopy.

\section{P216-COLORECTAL-Malignant}

\section{P214-COLORECTAL_Malignant}

\section{A STUDY OF POST-OPERATIVE COMPLICATIONS IN AN EMERGENCY GENERAL SURGERY FIRM IN 2018}

\section{Cremona, P. Andrejevic, A. Csengeri}

\section{General Surgery, Mater Dei Hospital, MSIDA, Malta}

Aim: To provide a basic statistical assessment of the post-operative complications within a 30-day period of a local laparoscopic general surgery firm using the Clavien-Dindo classification. The data collected pertains to all surgeries performed by this surgical firm over the course of 2018.

Methods: Trainees working in this firm were responsible for data collection. Patients who underwent emergency surgery during the calendar year of 2018 had the following details collected - the presence or absence of a complication in the 30-day post-operative period, the type of complication and description of complication along with the grade of the complication (see Fig. 1.). Patients who underwent intermediate to major surgery were followed up at outpatients and were specifically asked for the occurrence of complications from the point of discharge up until the outpatient appointment. With one centralised national hospital-the people who were discharged and subsequently experienced considerable or major complications invariably represented back to hospital via the A\&E department.

Results: A total of 148 emergency surgeries were performed by this surgical firm in 2018, $63 \%$ of these being done laparoscopically. Of these 148 cases-29 patients experienced post-operative complications within the first 30 days after their procedure. This equated to a complication rate of $19.59 \%$. The most common complications were abdominal pain, nausea \& vomiting, and wound infection. There were 8 complications for each of these 3 categories. Post-operative bleeding occurred in 5 cases with fistulas or leak of an anastomosis occurring in 3 cases. Death of a patient occurred in 3 instances once as a result of post-operative bleeding from the site of anastomosis after a Whipple's procedure, the 2nd occurred subsequent to post-operative bleeding from a peptic ulcer and in the 3rd case occurred in an instance of faecal peritonitis as a result of anastomotic failure after a Roux-en-Y bypass for a patient with pancreatic malignancy. Conclusion: The Davien-Clindo classification proved to be simple, efficient and useful in analysing post-operative outcomes. The results indicate that despite the emergency setting \& elderly cohort of patients-minimally invasive surgery proved to be a safe and viable option

\section{LAPAROSCOPIC TOTAL PELVIC EXENTERATION FOR LOCALLY ADVANCED COLORECTAL CANCER}

M. Uemura ${ }^{1}$, M. Miyake ${ }^{1}$, M. Miyazak ${ }^{1}$, T. Kato ${ }^{1}$, M. Ikeda $^{2}$, M. Hamakawa ${ }^{1}$, S. Maeda ${ }^{1}$, N. Hama ${ }^{1}$, K. Nishikawa ${ }^{1}$, A. Miyamoto ${ }^{1}$, M. Hirao ${ }^{1}$, T. Mizushima ${ }^{3}$, H. Yamamoto ${ }^{3}$, Y. Doki ${ }^{3}$, M. Sekimoto

${ }^{1}$ Department of Surgery, National Hospital Organization, Osaka National Hospital, OSAKA CITY, Japan; ${ }^{2}$ Division of Lower GI Surgery, Hyogo College of Medicine, HYOGO, Japan; ${ }^{3}$ Department of Gastroenterological Surgery, Osaka University, OSAKA, Japan

Background: Complete radical resection of locally advanced colorectal cancer is the mos important factor for achieving cure. Total pelvic exenteration (TPE) is often employed to secure negative resection margins. Although minimally invasive approaches have become more common in the field of pelvic malignancies, the safety and efficacy of laparoscopic TPE (Lap-TPE) have not been well elucidated. The aim of this study was to evaluate the feasibility of Lap-TPE for colorectal cancer. Surgical procedure of Lap-TPE is also presented here. recurrent colorectal cancer. To assess the feasibility and safety of Lap surgery, the records of 52 patients (35, open surgery; 17, laparoscopic surgery) with primary colorectal cancer were reviewed.

Results: The mean intraoperative blood loss volume was significantly less in the Lap group than in the Open group (735 vs. $4447 \mathrm{ml}$, respectively, $p<0.01$ ). The mean operative time was not significantly different between the Lap group and the Open group (738 vs 679 min, respectively, $p=0.276$ ). The incidence of severe postoperative complication (grade 3 or higher in the ClavienDindo classification) was lower in the Lap group (4/17 (24\%) vs 16/35 (46\%), respectively). The mean postoperative hospital stay was significantly shorter in the Lap group than that in the Open group (39 vs. 454 days, respectively, $p=0.022$ )

Conclusions: Lap-TPE can be a safe and feasible procedure.
Methods: From 2001 to 2017, totally 111 patients underwent TPE for locally advanced or 


\section{P217-COLORECTAL_Malignant}

\section{SYSTEMIC LIGHT CHAIN AMYLOIDOSIS PRESENTING WITH PSEUDO-OBSTRUCTION OF THE SMALL BOWEL: A CASE REPORT AND LITERATURE REVIEW}

\author{
A. Coyle, R. Burke, H. Mirza, N. Soomro, R. Mitru, M. Aremu \\ General Surgery, Connolly Hosiptal, BLANCHARDSTOWN, \\ DUBLIN, Ireland
}

Background: Amyloid light chain (AL) amyloidosis is a rare protein deposition disorder with an incidence ranging between 3-8 cases per million people. It can present insidiously with localized or multisystem symptoms and usually occurs later in life. Prognosis is poor as AL typically presents at an advanced stage. Intestinal pseudo-obstruction is a rarely reported complication of $\mathrm{AL}$ amyloidosis. Here we report a case of $\mathrm{AL}$ amyloidosis which was identified during surgery for intestinal pseudo-obstruction.

Case presentation: A 56 year old male presented to the Emergency Department with a 4 month history of abdominal pain and distension, as well as marked swelling of his lower limbs. This had worsened in the previous 2 weeks and he had developed intermittent diarrhoea. CT showed ileitis with marked dilation of the proximal small bowel. Laparatomy revealed small bowel that was grossly distended that rapidly developed multiple petechiae and subsequent haematomas upon handling. Two days later a repeat laparotomy was performed and $3.45 \mathrm{~m}$ of ishaemic small bowel was resected. Histology showed amyloid deposition with positive congo red staining. Subsequent cardiac events led to an echo being performed that showed concentric left ventricular hypertrophy attributed to amyloid deposition within the myocardium. Free serum light chain ratio was sent and confirmed the diagnosis of $\mathrm{AL}$ amyloidosis. He has recently been started on a treatment regimen consisting of cyclophosphamide and dexamethasone.

Discussion: Systemic AL amyloidosis frequently involves the gastrointestinal tract, typically presenting with chronic diarrhoea and associated malabsorption. Only 1 case presenting with pseudo-obstruction has been reported in the literature. AL amyloidosis presents insidiously with non-specific symptoms depending on which organs are affected. Treatment aims to prevent further deposition of protein within the organs. Prognosis is determined by the organs that are affected and the extent of protein deposition within them. Cardiac involvement holds the worst prognosis ultimately causing sudden cardiac death. The mainstays of management are early identification and treatment implementation to prevent protein build up and subsequent organ failure.

Conclusion: A diagnosis of amyloidosis should be considered in patients with intestinal pseudo-obstruction to expedite the diagnosis of $\mathrm{AL}$ amyloidosis and improve survival.

\section{P218-COLORECTAL-Malignant}

EVALUATION OF SURGICAL DIFFICULTY IN LOCALLY ADVANCED MID-LOWER RECTAL CANCER THROUGH THE EUMARCS MRI-BASED PREDICTIVE SCORE

E. Reitano ${ }^{1}$, F. Pigneur ${ }^{2}$, A. Martinez-Perez ${ }^{3}$, G.C. Vitali ${ }^{4}$, F. Landi ${ }^{5}$, S.A. Gomez-Abril ${ }^{3}$, M. Assalino ${ }^{4}$, F. Ris ${ }^{4}$, A. Luciani ${ }^{2}$, F. Brunetti ${ }^{1}$, N. de' Angelis ${ }^{1}$

${ }^{1}$ Digestive Surgery, Henri Mondor Hospital, AP-HP, University of Paris Est, UPEC, PARIS, France; ${ }^{2}$ Department of Radiology, Henri Mondor Hospital, AP-HP, University of Paris Est, UPEC, PARIS, France; ${ }^{3}$ Department of General and Digestive Surgery, Hospital Universitario Doctor Peset, VALENCIA, Spain; ${ }^{4}$ Department of Abdominal Surgery, Geneva University Hospitals and Medical School, GENEVA, Switzerland; ${ }^{5}$ Department of Colorectal Surgery, Hospital Universitario Vall d'Hebron, BARCELONA, Spain

Aim: In the management of locally advanced rectal cancer (LARC), the achievement of a complete total mesorectal excision (TME) with clear resection margins was demonstrated to be the main predictor of overall and disease-free survival. Predicting surgical difficulty in LARC patients may be of particular importance to choose the best surgical approach. This study proposes a MRI-based score to identify preoperatively LARC patients with a high risk of having a difficult surgery.

Methods: This is a retrospective study based on the European MRI and Rectal Cancer Surgery (EuMaRCS) database, including patients with mid-low LARC who were treated with neoadjuvant chemoradiation therapy and laparoscopic TME with primary anastomosis. Data on pre-treatment and restaging through magnetic resonance imaging were available for all patients. Surgical difficulty was defined as high or low grade taking in to account operative (e.g. duration of surgery), and postoperative factors (e.g. hospital stay). Score accuracy was evaluated by estimating sensitivity, specificity and area under the receiver operating characteristics curve (AROC).

Results: Seventeen (12.5\%) of 136 LARC patients were graded as high surgical difficulty The EuMaRCS score was developed using the following significant predictors of surgical difficulty: BMI $>30$, interspinous distance $<96.4 \mathrm{~mm}$, ymrTstage $=\mathrm{T} 3 \mathrm{~b}$, and male sex The score ranged from 0 to 10 . The cut-off score to best differentiate patients with a high probability of difficult surgery was $=3$ points. This cut-off value showed the best balance in sensitivity and specificity. The EuMaRCS score demonstrated high accuracy (AROC: 0.0802)

Conclusions: The EuMaRCS score was found to be sensitive and specific in predicting surgical difficulty in LARC patients who were candidate for laparoscopic TME. The score has the advantage of considering patient and cancer related characteristics that can be all assessed preoperatively and it can be useful in the decision making process. This score has not yet been externally validated. 


\section{P219-COLORECTAL-Malignant}

\section{LAPAROSCOPIC MANAGEMENT OF LOCALLY ADVANCED RECTAL CANCERS: PROPENSITY SCORE MATCHED STUDY}

N. Irfan Ul Islam ${ }^{1}$, S. Panteleimonitis ${ }^{2}$, M.F. Shah $^{2}$, N. Figueiredo ${ }^{1}$, A. Parviaz ${ }^{3}$

${ }^{1}$ Minimally Invasive Colorectal Unit, Champalimaud Foundation, LISBON, Portugal; ${ }^{2}$ Minimally Invasive Colorectal Unit, Poole Hospital, POOLE, United Kingdom; ${ }^{3}$ Director of European Academy of Robotic colorectal Surgery (EARCS), Champalimaud Foundation, LISBON, Portugal

Background: Recently published two non-inferiority randomised control trials has raised questions on laparoscopic surgery for rectal cancer, showing lower quality pathological specimens to those achieved using an open technique. Locally advanced rectal cancers add to the level of difficulty for laparoscopy approach. Our study was aimed to assess feasibility of laparoscopic rectal surgery, comparing short term outcomes, quality of surgical specimen, morbidity and mortality, between propensity score match groups of locally advanced and early rectal cancers. Methods: Prospectively acquired data from consecutive patients undergoing laparoscopic surgery for rectal cancer at the minimally invasive colorectal unit in United Kingdom between 2006 and 2014. Locally advanced rectal tumours were identified as T3B or T4 with pre-operative MRI scans. All the patients were operated by the same team and the procedures were performed laparoscopically. 1:1 propensity score matching was performed to create a perfect match in terms of tumour height. Results: Total of 369 laparoscopic rectal resections were performed during the study period, out of which 87 patients had locally advanced (LA) disease and were propensity-score matched for tumour height with non-locally advanced (NLA) patients. Median operative time was higher for the LA surgery group ( $270 \mathrm{~min}$ Vs $250 \mathrm{~min} \mathrm{p}=0.024)$. However, conversion to open surgery $(p=0.621)$, readmission $(p=0.295)$, re-operation $(p=0.747)$, clinical anastomotic leak $(\mathrm{p}=0.589)$ and 30 -day mortality rates $(\mathrm{p}=0.497)$ were all equivalent between the two groups. R0 resection was achieved in $89 \%$ of LA group as compare to 94\% of NLA group ( $p=0.177$ ). Conclusion: This study demonstrate that standardised approach to laparoscopy is safe and feasible in locally advanced rectal cancers. Comparable post-operative short-term clinical and pathological outcomes were seen between LA and NLA groups.

\section{P220-COLORECTAL_Malignant}

\section{INITIAL SHORT-TERM RESULTS IN COLORECTAL CANCER SURGERY AFTER THE IMPLEMENTATION OF A SCREENING PROGRAM IN A COMMUNITY HOSPITAL}

M. Roldon Golet ${ }^{1}$, I. Goded Broto ${ }^{1}$, V. Zambrana ${ }^{1}$, M. Garcia Dominguez ${ }^{1}$, A. Utrilla Fornals ${ }^{1}$, G. Inaraja Perez ${ }^{2}$, V. Borrego Estella ${ }^{3}$

${ }^{1}$ General and Digestive Surgery, Hospital General San Jorge, HUESCA, Spain; ${ }^{2}$ Vascular Surgery, Hospital Universitario Miguel Servet, ZARAGOZA, Spain; ${ }^{3}$ General and Digestive Surgery, Hospital Clínico Universitario, ZARAGOZA, Spain

Aims: The application of colorectal cancer screening programs, has showed a decrease in recurrence and mortality. For this reason, these programs are being implemented at a national level in the different Spanish regions, as has happened in our community.To present the initial short-term results on the morbidity of the immediate postoperative period to 90 days of colon cancer, mortality and hospital stay after the implementation of a screening program in our center. Methods: A retrospective study was performed. 73 patients aged between 60 and 69 years were included in the study, diagnosed with colon cancer. They underwent minimally invasive surgery, in most cases, with any type of colonic resection, from January 2010 to December 2017. All patients were diagnosed, conventionally or through a screening program, the latter according to the plan implemented in our community. The sample was divided into two groups of patients according to the way of being diagnosed (Group Si screening $=25$ patients, Group No screening $=48$ patients) and they were compared according different variables: Dependent factors of the patient, factor of type colon cancer, factors of colon cancer resection and follow-up. Results: Both groups were comparable in all study variables. Regarding the variables included in the follow-up, no statistically significant differences were found in terms of postoperative mortalityClavien-Dindo V. However we found differences statistically significant in postoperative morbidity $(\mathrm{p}=0.006)$ and in its classification according to Clavien Dindo I-IV $(\mathrm{p}=0.018)$. The complications analyzed independently, such as anastomotic dehiscence $(\mathrm{p}=0.023)$ or postoperative ileus $(\mathrm{p}=0.033)$, have also presented significant differences, unlike surgical wound infection $(\mathrm{p}=0.115)$. Conclusion: At our center, the application of the screening program has not influenced in the initial stage of colon cancer or its surgical approach. However, we have found a lower overall morbidity rate and minor complications, justified by a lower incidence of anastomotic dehiscence and postoperative ileus.

\section{P221-COLORECTAL-Malignant}

\section{LAPAROSCOPIC VERSUS OPEN COLECTOMY FOR CANCER-A SINGLE INSTITUTION EXPERIENCE}

N. Shussman, S. Sultani, O. Cohen Arazy, B. Helou, P. Arnos, A.J. Pikarsky

Surgery, Hadassah Hebrew University Medical Center, JERUSALEM, Israel

Background: Colorectal carcinoma is one of the most common malignancies. Surgery is the only definitive method to achieve cure for this illness and can be performed via an open or a laparoscopic approach. The pros and cons of each approach have been discussed extensively, with the oncologic efficiency of the laparoscopic approach being one of the leading topics.

Objective: The aim of this study was to establish oncological non-inferiority of the laparoscopic approach to colorectal cancer. Primary outcome measure was defined as number of harvested lymph nodes. Secondary outcome measures were medium-term disease free and overall survival as well as length of hospital stay, time to oral feeding and short- and long-term complication rate. Methods: This was a single center retrospective chart review. All consecutive patients who underwent colon or rectal resection due to colorectal carcinoma at Hadassah Medical Center between the years 2014-2017 were included. Patients who were operated on for recurrent disease or who had metastatic disease at the time of surgery were excluded. Patients were divided into three groups according to the surgical approach: laparoscopic, open or converted.

Results: 391 patients were included. 133 patients were included in the laparoscopic group, 242 in the open group and 16 in the converted group. The average number of harvested lymph nodes was 24.81 in the laparoscopic group, 29.13 in the open group and 30.66 in the converted group $(p=0.051)$. Medium-term oncological outcomes were the same for all groups. Time to oral feeding, length of hospital stay, short- and long-term complication rate were all significantly improved in the laparoscopic group.

Conclusions: We were unable to prove non-inferiority of the laparoscopic approach regarding the number of harvested lymph nodes. However, all surgical approaches yielded a high number of harvested lymph nodes which is most probably oncologically sufficient, as reflected by the non-existen difference in medium-term oncological follow up. This study supports previous studies showing the superiority of the laparoscopic approach regarding short term recovery and overall complications rates.

\section{P222-COLORECTAL-Malignant}

\section{SPOTLIGHT ON STANDARDISED LAPAROSCOPIC TOTAL MESORECTAL EXCISION APPROACH: A MULTI-CENTRE ANALYSIS OF DATA FROM 379 CONSECUTIVE PATIENTS}

N. Irfan Ul Islam ${ }^{1}$, M. Bullock ${ }^{2}$, A. Hemandas ${ }^{3}$, T. Qureshi ${ }^{4}$,

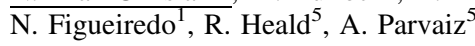

${ }^{1}$ Minimally Invasive Colorectal Unit, Champalimaud Foundation, LISBON, Portugal; ${ }^{2}$ Department of Academic Surgery, University Hospital Southampton, SOUTHAMPTON, United Kingdom; ${ }^{3}$ Department of Colorectal Surgery, Milton Keynes University Hospital NHS Foundation Trust, MILTON KEYNES, United Kingdom; ${ }^{4}$ General and Colorectal Unit, Poole Hospital, POOLE, United Kingdom; ${ }^{5}$ Laparoscopic and Robotic Colorectal Unit, Champalimaud Foundation, LISBON, Portugal

Aims: Two non-inferiority randomised control trials have questioned the utility of laparoscopic surgery for rectal cancer by failing to prove that pathological markers of high quality surgery are equivalent to those achieved by open technique. We intend to present short and long-term postoperative outcomes from the largest single surgeon series of consecutive patients undergoing laparoscopic TME for rectal cancer. We describe the standardised laparoscopic technique developed by the principal surgeon, and the short-term outcomes from three surgeons who were trained in and subsequently adopted the same approach.

Methods: Prospectively acquired data from consecutive patients undergoing surgery for rectal cancer by the principal surgeon (AP) at the minimally invasive colorectal unit in Portsmouth between 2006 and 2014 were analysed along with data acquired between 2010 and 2017 from surgeons (TQ,NF,AH) at three further international centres. End-points were overall and diseasefree survival at 5 years, and early post-operative clinical and pathological outcomes.

Results: 263 consecutive patients underwent laparoscopic TME surgery by the principal surgeon (AP). At 5 years Overall Survival was $82.9 \%$ (Dukes' $\mathrm{A}=94.4 \% ; \mathrm{B}=81.6 \% ; \mathrm{C}=73.7 \%$ ); Disease-free survival was $84.0 \%$ (Dukes' $A=93.3 \%$; $B=86.8 \%$; $C=72.6 \%$ ). Post-operative length of stay, lymph node harvest, mean operating time, rate of conversion, incomplete resection, major morbidity and 30 day mortality were not significantly different between the principal surgeon and those he had trained when subsequently in independent practices.

Conclusion: Laparoscopic TME produces excellent long-term survival outcomes for patients with rectal cancer. A standardised approach has the potential to improve outcomes by setting bench-marks for surgical quality, and providing a step-by-step method for surgical training. 


\section{P223-COLORECTAL-Malignant}

\section{LAPAROSCOPIC COLONIC RESECTIONS-WHO IS THE REASON OF COMPLICATIONS?}

\section{L.L. Panaiotti, A.Yu. Olkina, T.S. Lankov, A.S. Petrov, A.M. Karachun}

Surgical department of abdominal oncology, National Medical Research Centre of Oncology named after N.N. Petrov, SAINT-PETERSBURG, Russia

Aims: Although $21^{\text {st }}$ century is an era of standardized surgical procedures, all experienced surgeons have their own unique technique. The aim of this study was to identify factors predicting early and late postoperative complications.

Methods: We analyzed 66 consecutive cases of elective laparoscopic colonic resections for colon cancer performed in our center between February 2017 and September 2018. Median follow-up was 10.1 months. All operations were performed by consultant colorectal surgeons.

Results: Analysis of association of tumor location (sigmoid, right or left colon), operation time, blood loss, extraction site, type of surgical sutures used for wound closure with postoperative complications or specimen quality either did not show significant correlation or could not be conducted due to data nature. Unexpectedly, a significant difference was demonstrated between two surgical teams in terms of hernias. Majority of cases-44 (64.7\%) were performed by Surgeon 1 (S1), Surgeon 2 (S2) operated on $22(35.3 \%)$ patients, nevertheless minilaparotomy closure was usually performed by junior members of the team. Conversion rate was $4.5 \%$ for $\mathrm{S} 1$ and $18.2 \%$ for S2 $(\mathrm{p}=0.089)$. Operation time and blood loss were smaller in $\mathrm{S} 1$ group compared to S2 $\quad(153.6 \pm 62.5 \mathrm{~min} \quad$ vs $179.3 \pm 55.2 \mathrm{~min}, \quad \mathrm{p}=0.037$ and $59.7 \pm 45.7 \mathrm{ml} \quad$ vs $100.0 \pm 74.0 \mathrm{ml}, \mathrm{p}=0.027$ respectively). Specimen quality and early postoperative complications did not differ. Postoperative hernia rate was $2.3 \%$ for S1 and $22.7 \%$ for S2 ( $\mathrm{p}=0,013)$. Both surgeons used the same specimen extraction sites and materials for wound closure. Hernias were more frequent after vertical minilaparotomy-25\% ( 1 of 4 patients), and in converted patients $33,3 \%$ ( 2 of 6 patients), compared to 5, 5\% (3 of 56) in transverse minilaparotomy group. There was no association of hernias and wound infections.

Conclusions: Our study demonstrates, that besides consultant dependent surgical surrogates, steps which are often performed by other members of surgical team (such as wound closure) may contribute to complication rate as well. More thorough supervision of wound closure may be needed.

\section{P224-COLORECTAL_Malignant}

\section{OPEN VERSUS LAPAROSCOPIC RIGHT COLECTOMY FOR THE TREATMENT OF COLON CANCER: LONG TERM SURVIVAL IN A CONSECUTIVE SERIES OF 230 PATIENTS}

N. Irfan Ul Islam, M.I. Ashraf, M.F. Shah, O. Shakeel, A.A. Malik, A.W. Anwer, M.T. Pirzada, S. Khattak, A. Syed

\section{Surgical Oncology, SKMCH \& RC, LAHORE, Pakistan}

Background: With the introduction of complete mesocolic excision (CME) with central vascular ligation and methodical lymph node dissection, the overall survival of the afflicted patients has improved significantly and with the introduction of new technologies, now treatment strategies are evolving. Serious questions are being raised about the utility of laparoscopic surgery in colorectal cancer treatment.The aim of our study was to evaluate the early surgical outcomes and long-term survival of open versus laparoscopic right hemicolectomy.

Methods: We analyzed the medical record files of all the patients who presented to our hospital with the diagnosis of right sided colon carcinoma from Jan 2006 to Aug 2018 and underwent laparoscopic / open right hemicolectomy. Demographics, operative findings and histopathological reports were all recorded and analysis was performed on SPSS 21

Results: Total of 230 patients were operated during the study period, out of which 115 were operated laparoscopically and 115 open with a male to female ratio of $2: 1$. Median age was 50 for lap and 51 for open $(\mathrm{p}=0.694)$. BMI of the patients was higher on the laparoscopic side ( $23 \mathrm{Vs}$ 22 for open surgery group, $p=0.034$ ). Once the learning curve was achieved, around $45 \%$ of the laparoscopic procedures were carried out for transverse colon tumor and extended right hemicolectomy was performed $(\mathrm{p}=0.019)$. Blood loss was less in laparoscopic group $(65 \mathrm{mls})$ as compare to $112 \mathrm{mls}$ for open group $(\mathrm{p}=0.0001)$. Tumor size was comparable in both the groups $(\mathrm{p}=0.08)$, as well as proximal margin $(\mathrm{p}=0.07)$, and mesenteric margins $(\mathrm{p}=0.557)$. The number of retrieved lymph nodes (median 18.5 for lap vs 18 for open group, $\mathrm{p}=0.426$ ) were comparable between the two groups. Considering early surgical outcomes, length of stay was shorted in laparoscopic group ( $6 \mathrm{Vs} 7$ days in open surgery, $\mathrm{p}=0.0001$ ), whereas, Clavien-Dindo complications $(\mathrm{p}=0.98), 30$-day morbidity and mortality including 30-day re-intervention between the 2 arms were comparable. The overall 5 year survival is around $90 \%$ in Laparoscopic arm whereas, open arm has only $70 \%$.

Conclusion: There is no difference in early surgical outcomes of laparoscopic versus open right hemicolectomy, whereas, laparoscopic surgery has better long-term survival.

\section{P225-COLORECTAL-Malignant}

COMPLETE MESOCOLIC EXCISION VERSUS STANDARD LAPAROSCOPIC COLECTOMY IN RIGHT-SIDED COLON CANCER: SHORT-TERM RESULTS FROM A SINGLE ITALIAN CENTER

\section{Pedrazzani, C. Conti, G. Turri, E. Lazzarini, L. Gerard,} A. Guglielmi

Chirurgia generale ed epatobiliare, AOUI Verona, VERONA, Italy

Aims: Laparoscopic complete mesocolic excision (CME) right hemicolectomy is considered a demanding procedure and it is actually adopted in few centers from the West. The aim of the present study is to analyze the safety of laparoscopic CME right hemicolectomy and to compare its short-term results with standard right hemicolectomy in a single Western center.

Methods: Prospectively collected data from 56 patients who underwent laparoscopic CME right hemicolectomy between June 2014 and November 2017 were retrospectively analyzed (CME group) and compared with data from 49 patients submitted to standard laparoscopic right hemicolectomy between April 2013 and November 2017 (S group).

Results: No differences were observed between the CME and the standard right hemicolectomy groups in terms of clinical characteristics. In the CME group, $39.3 \%$ of patients were $=75$ years old, $28.6 \%$ of patients were ASA class $3,46.4 \%$ of patients had $=2$ comorbidities, $30.4 \%$ of patients had BMI $>28$ and $14.3 \%$ of patients had $=2$ previous abdominal surgeries.

No differences were observed in terms of duration of surgery ( $215 \pm 59 \mathrm{~min}$ vs. $208 \pm 58 \mathrm{~min}$ $\mathrm{P}=0.573)$ and intraoperative complications $(5.4 \%$ vs. $4.1 \% ; \mathrm{P}=0.759)$ between $\mathrm{CME}$ and $\mathrm{S}$ groups; mean blood loss was lower in the CME group $(50.5 \pm 45.9 \mathrm{~mL}$ Vs $75.7 \pm 62.6 \mathrm{~mL}$ $P=0.029)$. The percentage of overall $(42.9 \%$ vs. $46.9 \% ; P=0.412)$ and severe (ClavienDindo $=3)$ complications $(8.9 \%$ vs. $8.2 \% ; \mathrm{P}=0.875)$, redo surgery $(3.6 \%$ vs. $8.2 \% ; \mathrm{P}=0.414)$ and readmission $(3.6 \%$ vs. $6.1 \%$; $\mathrm{P}=0.662)$ was comparable between CME group and $\mathrm{S}$ group. A significant difference was observed in the length of specimen $(329 \pm 79 \mathrm{~mm}$ vs. $270 \pm 98 \mathrm{~mm}$ $\mathrm{P}<0.001)$ as well as in the length of proximal $(159 \pm 96 \mathrm{~mm}$ vs. $121 \pm 70 \mathrm{~mm} ; \mathrm{P}=0.028)$ and distal margins $(134 \pm 64 \mathrm{~mm}$ vs. $110 \pm 61 \mathrm{~mm} ; \mathrm{P}=0.05)$ in favor of the $\mathrm{CME}$ group. The number of lymph nodes harvested was slightly higher in the CME group $(21.9 \pm 9.6$ vs. $25.7 \pm 10.2$; $\mathrm{P}=0.055)$ as it was for the percentage of cases with less than 12 retrieved lymph nodes $(8.2 \%$ vs $1.8 \% ; \mathrm{P}=0.143$ ), although these differences did not reach statistical significance.

Conclusions: This study represents one of the few Western experiences demonstrating the safety of laparoscopic CME right hemicolectomy. CME technique showed good short-term results and better quality specimens when compared with the standard procedure.

\section{P226-COLORECTAL-Malignant}

\section{REAL-TIME HISTOLOGICAL DIAGNOSIS FOR PERITONEAL DISSEMINATION OF COLORECTAL CANCER USING} LAPAROSCOPIC ENDOCYTOSCOPY (EC)

F. Ishida ${ }^{1}$, S. Shimada ${ }^{1}$, Y. Mori ${ }^{1}$, M. Misawa ${ }^{1}$, K. Ichimasa ${ }^{1}$,

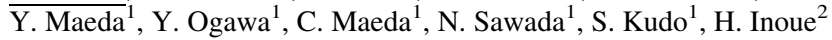

${ }^{1}$ Digestive Disease Center, Showa University Northern Yokohama Hospital, YOKOHAMA, Japan; ${ }^{2}$ Digestive Disease Center, Showa University Koto Toyosu Hospital, Japan

Aim and background: Peritoneal dissemination of colorectal cancer (PC) makes the complete resection of cancer lesions impossible. In such cases, multidisciplinary therapy is essential with mainly chemotherapy. Preoperative diagnosis of PC is usually uncertain by CT or MRI image. For diagnosis of PC needs surgical materials with laparotomy. But the laparotomy and resection of PC with general anesthesia tends to make impossible for immediate chemotherapy. Less invasive diagnosis of $\mathrm{PC}$ is necessary and expected.Endocytoscopy (EC) makes the histologica diagnosis with precise images gained by high magnification (x 520). As a preliminary examination, EC diagnosis for resected specimens of PC were evaluated.

Methods: Two cases of PC diagnosed in operation were evaluated. Under general anesthesia, laparotomy was conducted. Peritoneal dissemination lesions obviously diagnosed as PC were resected. Immediately the lesions were stained by methylene blue solution for 120 to180 s. EC observation was done according EC classification1) and ECV classification2).

Results: In two cases, EC observation was successfully done. Images of dilated surface microvessels of a nonhomogeneous caliber or arrangement were observed in NBI EC corresponding to EC-V3. Histopathological diagnosis of resected specimens was metastatic colorectal carcinoma in peritoneum in both cases.

Conclusions: Histological diagnosis for PC is gained by EC with resected specimen. As the result of this investigation, EC examination via camera port in laparoscopic operation might be possible for diagnosis for PC of colorectal cancer in vivo.

https://www.ncbi.nlm.nih.gov/pubmed/27876633Kudo T, Kudo SE, Mori Y et al https://www. ncbi.nlm.nih.gov/pubmed/27876633 2017 Mar;85(3):628-638. doi: 10.1016/j.gie.2016.10.039. Epub 2016 Nov 19

https://www.ncbi.nlm.nih.gov/pubmed/26071058Kudo SE, Misawa M et al https://www. ncbi.nlm.nih.gov/pubmed/26071058 2015 Nov;82(5):912-23. doi: 10.1016/j.gie.201 


\section{P227-COLORECTAL-Malignant}

\section{COMPLICATIONS IN LAPAROSCOPIC COLORECTAL RESECTIONS-BLACK VIDEO AND CLASSIFICATION OF MISTAKES}

\author{
L.L. Panaiotti, A.S. Petrov, A.Yu. Olkina, T.S. Lankov, \\ A.M. Karachun
}

Surgical department of abdominal oncology, National Medical Research Centre of Oncology named after N.N. Petrov, SAINT-PETERSBURG, Russia

Aims: The aim of this presentation is to demonstrate and analyze surgical complications, arising during laparoscopic colorectal resections for cancer and to analyze the reasons of adverse events. Methods: We demonstrate videos from our surgeries, where different types of complications occurred and share our classification of types of mistakes, that may lead to intraoperative complications and ways to prevent them.

Results: We divide mistakes in laparoscopic colorectal resections into two large groups-'false strategy' and 'dangerous techniques'. The first includes poor diagnosis, too extensive or insufficient extent of surgery and improper enthusiasm in using platforms. Prevention of first type mistakes is in thorough training and peer-review of each consultant practice. Second type of mistakes includes two subtypes : 'faulty habits'-use of unsafe techniques (blind port insertion, poor vascular exposure prior to clipping, not obtaining 'critical views', unsafe use of energy and stapling devices etc.) and 'failure in a certain case'-when despite correct general approach a complication occurred (misinterpretation of fascial layers or vessels). Prevention of 'faulty habits' lies in supervised training in high volume colorectal departments including dedicated surgical devices training. To avoid 'failure in a certain case' standardization of surgical procedure is essential, as the most efficient way to prevent this type of mistake is 'pattern recognition'ability of a surgeon to compare the picture he sees during a procedure with a 'standard' view, he used to have during previously performed standard surgeries-this is apparentely impossible when every procedure is done differently. Regular reviews of own surgeries recording and other surgeons' procedures may also fascilitate pattern formation.

In case a complication occurres we use the four step course of action: preservation of the view, temporary control, decision on conversion, permanent control.

Conclusion: As popularity of laparoscopic colorectal resections is growing rapidly the number of intraoperative compliactions is increasing as well. We demonstrate videos of complications and our approach to classification of possible mistakes. Systematic aproach to reasons, underlying certain mistakes helps to produce a strategy to reduce intraoperative complication rate.

\section{P228-COLORECTAL-Malignant}

\section{PROPHYLACTIC USE OF DRAINAGE IN COLORECTAL SURGERY}

B. Cantarero Jiménez, M. Maes Carballo, P. Nicolas Martinez, I. Plata Pérez, I. Muñoz Nuñez, P. Vázquez Barros

General Surgery, Hospital Santa Ana Motril, GRANADA, Spain

Introduction: The drains placement inside the abdominal cavity has traditionally been carried out to evacuate hematic remains or postoperative collections. There is no scientific evidence of the prophylactic use of drainage in elective colorectal cancer (CCR) surgery to avoid anastomotic complications or other complications. However, it is traditionally used. When the anastomotic leak is produced, it is generally agreed that drainage system should be used for therapeutic purposes.

Aims: The aim of this study is to evaluate the effectiveness of the use of prophylactic drainage in elective surgery of CCR. We would check if they avoided the appearance of complications, and if they are useful when the anastomotic leak appears.

Methods and results: We analyzed the data collected in our hospital from 1/11/17 to 12/12/18 We studied the number and type of interventions in which prophylactic drainages were placed, the appearance of anastomotic complications and if these drains were effective.

93 interventions were performed during this period of time. $72 \%$ of these procedures had used prophylactic drainage (67 interventions). This percentage was up to $100 \%$ in patients who have performed a left colon surgery as a sigmoidectomy or rectal procedure. During this period, there were 10 cases of anastomotic leakage. In all of them had been placed drainage but only 3 of them were effective.

Conclusions: We have seen that prophylactic drainage is a common practice independently of the location of the anastomosis. The last multimodal rehabilitation guidelines recommended the nonuse of drains systematically above the peritoneal reflection with a high level of scientific evidence. They cause discomfort to the patient and delay early mobilization. However, it may be useful to use drains in the first $24 \mathrm{~h}$ of a pelvic floor procedure.

There is not enough evidence to show sistematic drainage after colorectal anastomosis prevents complications of the anastomosis or other complications.

\section{P229-COLORECTAL_Malignant}

\section{LAPAROSCOPIC RESECTION FOR SPLENIC FLEXURE CANCER: HOW WE DO IT}

M. Ortenzi ${ }^{1}$, R.O. Ghiselli ${ }^{1}$, A.N. Reggiani ${ }^{1}$, A.N. Balla ${ }^{2}$, A.M. Paganini ${ }^{2}$, M.A. Guerrieri ${ }^{1}$

${ }^{1}$ Clinica Chirurgica, Università Politecnica delle Marche, ANCONA, Italy; ${ }^{2}$ Department of General Surgery and Surgical Specialties, Paride Stefanini, Sapienza University of Rome, ROMA, Italy

Aims: Colonic cancers of the splenic flexure is uncommon and associated with poor prognosis Several studies were published aimed to identify the optimal surgical option for the best oncological outcomes. However, whether an extended colectomy or a segmental resection is required is still controversial. The aim of this study is to analyse the outcome of the two different approaches through the experience of a single centre.

Materials and methods: Retrospective data of consecutive patients with diagnosis of colonic cancer situated at the splenic flexure of our department between 2004 and 2017 were analysed. Based on type of surgical procedure, patients were enrolled in arm A (segmental resection) and arm B (extended resection). Arm A patients were treated with segmental resections with a wide mobilisation of the transverse and descending colon and ligation of the left colic artery, sparing the middle colic artery and the inferior mesenteric artery. Functional lateral to lateral anastomosis was performed extracorporeally. Arm B patients were treated with more extended colectomies, both associated with central vascular ligation.

Results: Out of 200 patients included, 141 were allocated in arm A and 59 in arm B. Patients population of the 2 arms was homogeneous as concerns demographic characteristics and stage of the disease. Operative time was comparable (108,9 min Vs $119 \mathrm{~min}, \mathrm{p}=0,332)$. The length of the specimen was significantly shorter in arms A $(15,7 \mathrm{Vs} 32,1, \mathrm{p}=0,0351)$. The number of harvested lymphnodes did not differ between the two groups $(12,5 \mathrm{Vs} 17 \mathrm{p}=0,167)$ Postoperative short term complications was comparable in both arms $(17 \mathrm{Vs} 1, \mathrm{p}=0,692)$. No postoperative mortality was observed. Overall 5-year survival and disease free survival rates were similar in arm A and B (81.3\% Vs $83.05 \%, \mathrm{p}=0,321$ and $78,6 \%$ Vs $80,5 \%, \mathrm{p}=0,534)$. Hospital stay was similar in the two groups $(\mathrm{p}=0,99)$.

Conclusions: Despite a shorter length of surgical specimen after limited resections, postoperative complications, lymph node harvest, and survival were comparable in both.In our opinion the extracorporeal anastomosis is functional to both the achievement of a cleaner operative field and a better control of the resection margins.

\section{P230-COLORECTAL-Malignant}

\section{TAMIS RESECTION ON RECTAL NEUROENDOCRINE TUMOURS}

J. Valdes-Hernandez, M. Lopez-Cantarero, F.J. del Rio, J. Cintas-Catena, C. Torres, A. Perez-Sanchez, J. Gomez-Rosado, F. Oliva, L.C. Capitan-Morales

Colorectal Surgery Unit, Virgen Macarena University Hospital, SEVILLA, Spain

Incidence of Neuroendocrine tumours in the rectal area has increased in recent years.Before the onset of minimally invasive colorectal surgery, these lesion had to be treated by a more radical technique when not suitable for endoscopic resection.Selection of the cases is mandatory in order to achieve good results not only surgical, but also oncological. We present our series of 3 Neuroendocrine tumoirs treated by TAMIS approach, including technical aspects, deffect closure techniques and data regarding pathological findings.All cases were low grade carcinoid tumours. Resection with free margin was obtained in all cases. Defect closure was performed in all cases. The tumours were settled 9,10 and $15 \mathrm{~cm}$ form the anal verge. Postoperative course was uneventful, ann No adyuvant therapy was needed.TAMIS apporach for rectal neuroendocrine tumours is a safe and feasible technique. Proper selection of the cases is mandatory in order to achieve good results. 


\section{P231-COLORECTAL-Malignant}

BARBED SINGLE LAYER VERSUS HYBRID DOUBLE LAYER CLOSURE OF THE ENTEROTOMY IN TOTALLY LAPAROSCOPICALLY RIGHT COLECTOMY WITH INTRACORPOREAL ANASTOMOSIS

A. Cossu, R. Quattromani, U. Elmore, A. Vignali, R. Rosati, Gastrointestinal Surgery, San Raffaele Hospital, MILAN, Italy

Aim: To assess the safety and efficacy of single layer of barbed vs double layer 'hybrid' (interrupted and running) suture for the closure of anastomotic stapler access enterotomy after laparoscopic right colectomy with intracorporeal anastomosis.

Methods: from April 2014 to November 2018, 252 laparoscopic right colectomy with intracorporeal anastomosis were performed in our Surgical Department. All patients in both groups were perioperatively managed using an ERAS pathway. Seventy-two patients had the enterotomy closed with a single layer running suture of Filbloc ${ }^{\mathrm{TM}}$ (Assut Europe). These patients were matched with 72 patients who underwent intracorporeal right colectomy with enterotomy closed with a 'Hybrid' double layer technique (first layer interrupted stitches in Maxon ${ }^{\mathrm{TM}}$ 3-0 (Covidien), second layer using a running suture in $\operatorname{PDS}^{\mathrm{TM}}$ 3-0 (Ethicon). Intraoperative variables, anastomotic leak rate, morbidity and mortality rates were analyzed.

Results: the two groups were homogeneous with respect to demographics, Body Mass Index (BMI), American surgical Association score (ASA) as well as for tumor stage. In the barbed group, median operating time was $121.5 \mathrm{~min}$ vs $140.7 \mathrm{~min}$ in the Hybrid group $(\mathrm{p}=0.02)$. Anastomotic leak occurred in $5(6.7 \%)$ patients in the hybrid vs $2(2.7 \%)$ patients in the barbed group $(\mathrm{p}=0.24)$. All patients required a reoperation. Intraoperative findings show in $2(0.4 \%)$ cases in the Hybrid group a leak at the enterotomy closure, while an intact staler access was observed in both patients in the barbed group. No difference was observed with respect to noninfectious complications between the two groups $(\mathrm{p}=0.55)$. Patients in the hybrid group experienced a longer hospital stay when compared to the barbed group $(\mathrm{p}=0.03)$. A re-admission occurred in the hybrid due an intraabdominal collection, while no re-admission was observed in the barbed group. No patient died in the postoperative period.

Conclusion: our results shows that the use of knotless barbed suture for enterotomy closure after laparoscopic intracorporeal right colectomy is safe, reproducible and associated with shorter operative time

\section{P233-COLORECTAL-Malignant}

\section{LYMPHADENECTOMY GUIDED BY INDOCIANIN-GREEN (ICG) IN COLORECTAL CANCER}

M.L. García Jiménez, L. Castro Diez, D.P. Bravo Beltrán, C. Mosquera Fernández, J. Aguirrezabalaga González, J.F. Noguera Aguilar

General Surgery, Complejo Hospitalario Universitario A Coruña, A CORUÑA, Spain

Aims: Lymph node status is one of the key prognostic factors in patients with colorectal cancer, and remains the most important selection criteria for adjuvant chemotherapy. It is believed that at least $30 \%$ of node negative patients will suffer disease recurrence within the first 5 years after surgery. This may be due to understaging lymph node status. Sentinel lymph node mapping is widely used for staging of breast cancer and melanoma, with injection of colloid Tc99 and isosulfan blue (IB). However, indocyanine green (ICG) fluorescence guidance is a new technical approach to this issue, with promising results as it is not influenced by body mass index or lymphatic invasion. Intraoperative fluorescence ICG navigation also aims for detection of aberrant lymphatic drainage outside the planned resection. The ICG lymphography has the advantage of offering a good visualization of the lymphatic channels but there are problems to identify the lymphatic nodes. Our objective with this study is to rate the use of the intraoperative lymphogram in cases of elective colorectal surgery to evaluate if there were changes in the surgical attitude regarding the performance of lymphadenectomy.

Methods: Indocyanine green was injected into the submucosal layer around the tumor at 2 points with?a 23-gauge localized injection before lymph node dissection and the lymph flow was observed at 1, 3 and $5 \mathrm{~min}$ after injection, using a near-infrared camera system. In addition, a complete mesocolic excision with central vascular ligation guided the region where the lymph flow was observed to be fluorescent.

Results: The following table summarizes the 10 procedures carried out as well as the lymphadenectomy performed before and after the use of ICG. In brief, after the application of intraoperative ICG it was observed that in $20 \%$ of patients additional lymph nodes were obtained after the expansion of the surgical plan, moreover $10 \%$ affected lymph nodes were spotted after the expansion of the surgical plan.

Conclusions: Intraoperative real-time visualization of the lymph flow using indocyanine green fluorescence imaging during laparoscopic colon cancer surgery is feasible and a helpful technique for lymph node mapping which may lead to intraoperative changes in lymphadenectomy.

\section{P234-COLORECTAL-Malignant}

\section{TAMIS RESECTION OF TUMORS LOCATED AROUND THE RECTOSIGMOID JUNCTION}

J. Valdes-Hernandez, A. Garcia, J. Gomez-Rosado, M. Lopez-Cantarero, J. Cintas-Catena, F.J. del Rio, C. Torres, A. Perez-Sanchez, F. Oliva, L.C. Capitan-Morales

Colorectal Surgery Unit, Virgen Macarena University Hospital, SEVILLA, Spain

TAMIS resection of rectal tumours has proven to be a sefe and feasible technique, specially for lesion located in the mid and low rectum. When the tumour is located in the upper rectum, and specially near the colorectal junction, TAMIS resection may be more difficult, not only due to technical aspects, but also due to the risk of a free perforation, specially when a full thickness resection is performed.We present our results of 6 TAMIS resections of lesions located around the colorectal junction. Four resections where performed with the aim of an endostpaler in order to achieve full resection without the risk of a free colonic perforation.In 3 cases, an abdominal combined laparoscopic exploration was made, in order to help and assure proper resection of the lesion as well as avoiding intraoperative complications.Distance from the anal verge ranged from 12 to $20 \mathrm{~cm}$.Postoperative course was uneventful in all cases, and a complete specimen resection was obtained in all cases.TAMIS resection of tumours located in the rectosigmoid junction may be a safe and feasible technique in selected patients. 


\section{P235-COLORECTAL_Malignant}

\section{ICG FOR LAPAROSCOPIC RESECTIONS OF THE COLON}

\section{J. Rejholec $^{1}$, J. Moravík ${ }^{1}$, P. Timoshin ${ }^{2}$}

${ }^{1}$ General Surgery, Regional Healt-Hospital Decin, DECÍN, Czech Republic, ${ }^{2}$ General Surgery, DECÍN, Czech Republic

Aims: Uncomplicated healing of anastomoses in colorectal surgery is the basis for early adjuvan oncology therapy. The basis for proper healing is good blood flow. We use by robotic surgery foor control the Firefly by Intuitive. Since January 2018, ICG has used for blood flow in laparoscopic bowel surgery for the D-Light system of Storz.

Method: Use of ICG wants to accurately determine the resection line for free colon operation based on good blood circulation. We use ICG Pulse in two batches and color detection using D Light from Storz to verify blood flow. The first dose is given after the skeletalisation of the intestines intraabdominally and the second after the colic anastomosis to verify its vitality.

Results: In the period under review we performed 85 laparoscopic operations on the free colon, $82 \%$ of the operations were elective. We had $5.88 \%$ leakage across the set, however, in the subset of elective operations, we had only a leak of $0.142 \%$.

Conclusion: In an unselected set of colorectal operations, leakage was $5.88 \%$, but only 0.142 for elective operations. In our group there was a clear effect of using ICG in elective laparoscopic resections with an intracorporal anastomosis, the effect was not shown in others, probably due to leakage were factors other than blood flow.

\section{P236-COLORECTAL_Malignant}

\section{WHICH COLIC BRANCHES OF SUPERIOR MESENTERIC ARTERY ARE MOST COMMON-COMPARISON OF RUSSIAN AND TURKISH RIGHT COLON CANCER PATIENT COHORTS}

S.K. Efetov ${ }^{1}$, I.A. Tulina ${ }^{2}$, K.B. Puzakov ${ }^{1}$, V.D. Kim ${ }^{1}$, E. Sahin ${ }^{3}$, E. Kaplan ${ }^{4}$, V. Shchegelsky ${ }^{1}$, C. Kayaalp ${ }^{4}$, P.V. Tsarkov ${ }^{1}$

${ }^{1}$ Colorectal surgery, I. M. Sechenov First State Moscow Medical University, MOSCOW, Russia; ${ }^{2}$ Colorectal and minimally invasive surgery, I. M. Sechenov First State Moscow Medical University, MOSCOW, Russia; ${ }^{3}$ Colorectal surgery, Inonu University, Turgut Ozal Medical Center, MALATYA, Turkey; ${ }^{4}$ Surgery, Inonu University, Turgut Ozal Medical Center, MALATYA, Turkey

Objective: Right hemicoloctomy (RHCE) is the first choice in treating the right colon cancer. Complete mesocolic excision with extended lymph node dissection at the roots of superior mesenteric artery (SMA) branches enables removal of all lymphatic tissue and prevents local recurrence. Previously variability of SMA branches was demonstrated. The aim of presented study was to compare the distribution of SMA branches in two ethnically different cohorts

Methods: Preoperative CT scans with vascular 3D reconstruction were assessed in 100 patients (28-93 years) from Russia and 95 patients (24-88 years) from Turkey with right colon cancer operated in 2015-2018. The distribution of ileocolic artery (ICA), right colic artery (RCA) and middle colic artery (MCA) was investigated. Results: ICA and MCA could be found on CT scans in all patients, whereas RCA had significantly different distribution between patient cohorts: it was visible in 93 (98\%) of Turkish patients and only in $32(32 \%)$ of Russian patients $(\mathrm{p}=0.001)$.

Conclusion: These results suggest that there might be ethnical differences in SMA branches distribution. In Turkish patients all named SMA branches ate visible on CT scans in 98\%, whereas in Russian patients only in 32\%. The majority of patients from Russia don't have RCA. ICA and MCA could be found in all patients regardless ethnicity. Knowing the variant of SMA branching before the operation can help plan extended lymph node dissection.

\section{P237-COLORECTAL-Malignant}

NATIONAL TRAINING PROGRAM FOR MINIMALLY INVASIVE COLORECTAL AND ANAL SURGERY (S-MICRASLAPSERB) IN SERBIA-PRELIMINARY Results:

M.C. Ceranic ${ }^{1}$, B.D Djukanovic ${ }^{2}$, M.J. Janic ${ }^{3}$, A.. Gluhovic ${ }^{4}$, I. Krdzic ${ }^{5}$, D. Slavkovic ${ }^{6}$, J. Ahmed ${ }^{7}$, A. Parvaiz ${ }^{8}$

${ }^{1}$ Department for Colorectal and Pelvic Surgery,, First Surgical Clinic, Clinical Center of Serbia, BELGRADE, Serbia; ${ }^{2}$ Department for colorectal surgery, University Clinical Hospital Center 'Bežanijska Kosa', BELGRADE, Serbia; ${ }^{3}$ Department for colorectal surgery, General Hospital „Pancevo,, PANCEVO, Serbia; ${ }^{4}$ Clinic for Abdominal, Endocrine and Transplantation Surgery, Clinical Center of Vojvodina, NOVI SAD, Serbia; ${ }^{5}$ Department of Colorectal Surgery, University Clinical Hospital Center, Zvezdara, BELGRADE, Serbia; ${ }^{6}$ Clinic for General Surgery, Military Medical Academy, BELGRADE, Serbia; ${ }^{7}$ Department for colorectal surgery, Royal Bournemouth Hospital, BOURNEMOUTH, United Kingdom; ${ }^{8}$ Minimal Access and Robotic Colorectal Surgery, Poole Hospital NHS Trust, POOLE, United Kingdom

The National Training Programme in laparoscopic colorectal surgery (S-MICRAS-LAPSERB) in Serbia was set up to introduce standardized and structured training in laparoscopic colorectal surgery. Method: An assessment based structured training programme (LAPSERB) started in 2015. Series of hands on supervised workshops were conducted for four different hospitals using the structured training by single trainer. This study aims at retrospective analysis of prospectively collected data for patients undergoing colorectal resections. We look at short-term clinical and pathological outcome of patients within laparoscopic colorectal resections performed in National training program. Results: During the period November 2015 until November 2018, laparoscopic colorectal resection was performed in 746 ( 426 male and 320 female) patients. Mean age of patients was $65.6(21-88)$. The most common indication was colorectal cancer (645 patients, $86.4 \%$ ), 80 (10.7\%) patients were operated due to the colorectal polyps not suitable for endoscopic resection and $21(2.8 \%)$ was operated due to IBD There were 174 (23.3\%) right colonic, 557 (74.6\%) left colonic /TME and $13(1.7 \%)$ other resections. Average number of lymph node harvested in patients with colorectal carcinoma was 16.5 (2-65). There were $6 / 645(0.9 \%) \mathrm{R} 1$ resections Mean duration of hospital stay was 6.5 days (2-18). Postoperative complications were encountered in $103 / 746$ patients $(13.8 \%)$. Overall, mortality rate was $1.2 \%(9 / 746)$ Conclusions: This study demonstrates successful and safe adoption of laparoscopic technique for colorectal resections. Short term clinical and pathological outcomes are compared to published data and shows wider adoption at the National level. Standardization of operative technique and structured training remains the key in success.

\section{P238-COLORECTAL-Malignant}

\section{FATWO-FEMAL ADNEXAL TUMOR OF PROBABLE WOLFFIAN ORIGIN}

\section{J. Moravík, J. Rejholec}

Surgical, Krajská zdravotní, a.s.—Nemocnice Decín, o.z., DECIN, Czech Republic

Introduction: Femal Adnexal Tumor of Probable Wolffian Origin (FATWO) was first described in 1973. It is a tumor of mesonephiris Wolffian duct origin. FATWO is rare tumor which is usualle benign. In the literature has been reported 71 cases and only 8 cases of recurrent disease. Next rare tumor in pelvic localisation is Sex Cord-gonadal stromal tumor of Sertoli cells. Methods: We present case report of women who presented metastatic FATWO with duplicity of Sertoli tumor.

Results: 60-year old women underwent 15 years ago exstirpation of tumor in the left broad ligament. Histologically there was rare benign FATWO. This year was indicated adnexectomy at Gynecology Department. During the operation was done bilateral adnexectomy and discovered tumor of anterio wall on upper rectum. Microscopic examination showed Sertoli tumor on the left ovary. Afterward we completed next examinations. Colonoscopy without any abnormality. On CT scan was tumor $7 \mathrm{~cm}$ without contact to rectum wall, without distant metastasis. The same was described on rectal ultrasonography-normal wall of rectum, tumor probably from uterus. At diagnostic laparoscopy was tumor mass $7 \mathrm{~cm}$, with necrosis arising from anterior wall of the rectum. Next small metastasis on pelvic peritoneum. We performed debulking of this big tumor and metastasectomy, there was no infiltration to muscularis propria of the rectum. Patient did not have any postoperativ complications. Microscopic examination of the rectal tumor and small peritoneal metastasis showed metastatic FATWO. After 6 weeks she underwent laparoscopic second look operation. There were smal metastasis on pelvis peritoneum. We removed two biggest metastasis and the rest was destroyed wit J plazma. Microscopic examination showed in this metastasis Sertoli tumor.

Conclusion: Our patient has metastatic FATWO and Sertoli tumor. FATWO is so rare, that in the literaure is not enough information for observation or adjuvant therapy. In one case was described imatinib mesylae (Gleevec) therapy with good results. Surgeons must be ready to meet new diagnosis. 


\section{P239-COLORECTAL_Malignant}

\section{ADOPTION OF LAPAROSCOPIC RIGHT HEMICOLECTOMY TECHNIQUE FOR COLON CANCER: COMPARATIVE STUDY WITH OPEN SURGERY AND EARLY CLINICAL OUTCOMES}

M.C. Ceranic ${ }^{1}$, S. Panteleimonitis ${ }^{2}$, S. Latincic ${ }^{1}$, O.S. Skrobic ${ }^{3}$, D. Gunjic ${ }^{4}$, J.V. Vasiljevic ${ }^{1}$, M. Pavlov ${ }^{1}$, A. Parvaiz

${ }^{1}$ Department for Colorectal and Pelvic Surgery, First Surgical Clinic, Clinical Center of Serbia, BELGRADE, Serbia; ${ }^{2}$ Department for colorectal surgery, University of Portsmouth, PORTSMOUTH, United Kingdom; ${ }^{3}$ Department for Esophagogastric Surgery, First Surgical Clinic, Clinical Center of Serbia, BELGRADE, Serbia; ${ }^{4}$ 5Department for Minimally Invasive Upper Digestive Surgery, First Surgical Clinic, Clinical Center of Serbia, BELGRADE, Serbia;

${ }^{5}$ Minimal Access and Robotic Colorectal Surgery, Poole Hospital NHS Trust, POOLE, United Kingdom

Objective: Laparoscopic colorectal surgery has become a gold standard with studies reporting better short term outcomes with comparable oncological results when compared to open surgery. The goal of this study was to evaluate the adoption of laparoscopic approach for right hemicolectomy and compare the early postoperative outcome of patients receiving open right hemicolectomies.

Methods: Between January 2015 to April 2018, 83 patients with diagnosis of right colon adenocarcinomas underwent right hemicolectomies. The data was analysed for patients demographic, histology, type of surgical approach, intraoperative details (length of surgical procedure, blood loss, blood transfusion, conversion rate) and short-term post-operative outcomes including complications.

Results: Laparoscopic resection (LS) was performed in 41 patients, while 42 patients had open right hemicolectomy (OS). Demograhic data between the two groups is comparable. Average duration of laparoscopic right hemicolectomy was $190 \mathrm{~min}$. (150-230 $\mathrm{min})$, while the average time for the open procedure was $130 \mathrm{~min}(100-190 \mathrm{~min}) \mathrm{p}<0.01$. Postoperative complications in the form of prolonged postoperative ileus were diagnosed in 7 patients $(16.66 \%)$ from OS group and one patient from LS group $(2.43 \%) \mathrm{p}<0.05$. In comparison to LS patients, the average time spent in hospital was significantly longer in group OS (8.2: 4.5 days) $\mathrm{p}<0.01$. An average number of excised mesocolic lymph nodes in the dissected colon was 21 in the LS group (12-38), compared to $23(14-42)$ in the OS group. Conclusion: Our experience demonstrates that it is safe to adopt laparoscopic approach for right colon resections. Although the median operating time is longer in lap group, complications, length of stay, blood loss and use of analgesia id significantly lower in Laparoscopic group when compared to open technique.
P240-COLORECTAL_Malignant

TRANSANAL EXPULSION OF AN ISCHEMIC COLON SEGMENT AFTER ANTERIOR RECTAL RESECTION WHITOUT ANASTOMOSIS DISRUPTION: AN IMPOSIBLE COMPLICATION?

E. Muñoz Caracuel, R. Escalera Pérez, G. Salguero Segui, E. Gutierrez Cafranga, J.L. Estebam Ramos, S. Mac Mathuna, J.D. Franco Osorio, F.J. Garcia Molina

Cirugía General y del Aparato Digestivo, Hospital de Jerez de la Frontera, JEREZ DE LA FRONTERA, Spain

Introduction: Postoperative ischemic colitis is a life-threatening vascular gastrointestinal condition, that mainly occurs after cardiovascular surgery. We present a surprising case following a laparoscopic rectum resection.

Case Report: A 77-year-old diabetic patient with upper rectal adenocarcinoma undergoing laparoscopic anterior rectal resection (partial mesorectum excision) and mechanical anastomosis following chemotherapy / radiotherapy. After $48 \mathrm{~h}$ postsurgery he presented abdominal pain, distension and fever. On adominal computed tomography (CT) scan (contrast enema) no anastomotic leakage (AL) finfings were revealed. Neither digital palpation nor proctosigmoidoscopy (3th day) showed AL signs. The patient clinical situation improve with conservative treatment (antibiotics, digestive rest ...), C-reactive protein levels decreased and the blood cultures were negative. On the 11th day he was discharged presenting semiliquid stools.

Eight days later he needed hospital readmission: air and feculent/purulent discharge from the previos abdominal drainage orifice. CT scan: no evidence of dehiscense found although rectum and sigmoid colon distention and an image of a 'large fecaloma' were observable. On the $4^{\text {th }}$ day of hospitalization he expulsed a large malodorous segment of tissue with necrotic asppearance (image) through the anus with surprising histologic features: 'complete-thickness necrotic colonic wall'. Further rectosigmoidoscopy: complete anastomosis, signs of ischemic colitis proximate to the anastomosis and a fistulous orifice.

Surprisingly, the patient progressed favorably, being discharged the 12th day for ambulatory control with a low debit enterocutaneous fistula. Histopathological diagnosis: ypT3 N0 M0.

Follow up: The fistula discharge quantity increased maintaining diarrheal stools through anus along with persistent anemia and malnutrition. A exploratory laparotomy was schedule. Fistulous tract towards a small stenotic segment of colon inmediately proximal to the colorectal anastomosis was identify and resected. Finally a terminal colostomy was performed. Subsequent postoperative without incidents. Currently the patient is asymptomatic.

Comments: It seems indisputable that a colon segment, proximal to the anastomosis, was necrosed and expelled through a colorectal anastomosis. The mechanism seems inexplicable to us. It is even more disconcerting that there was no disruption of the anastomosis. 


\section{P241-COLORECTAL-Malignant}

ASSESSMENT OF ANOMALOUS LYMPHATIC DRAINAGE WITH LINFOGRAPHY GUIDED BY INDOCYANINE GREEN IN THE RIGHT COLON CARCINOMA

J. Bellido Luque ${ }^{1}$, I. Cornejo ${ }^{2}$, A. Bellido ${ }^{3}$, A. Tejada ${ }^{3}$, J.M. Suarez ${ }^{3}$, J. Gomez ${ }^{3}$, I. Sanchez-Matamoros Martin ${ }^{1}$, F. Oliva Mompean ${ }^{1}$, A. Nogales Muñoz ${ }^{1}$

${ }^{1}$ General Surgery, SAS, SEVILLA, Spain; ${ }^{2}$ Surgery Department, Hospital Universitario Virgen Macarena, SEVILLA, Spain; ${ }^{3}$ General Surgery, Quiron Salud, SEVILLA, Spain

Objectives: Fluorescence-guided surgery has emerged as a new imaging modality to improve the detection of liver and lymph node metastasis in colorectal cancer. In right-sided colon cancer, the standard lymphadenectomy should reach the ileocolic vessels and the right branch of the middle colic vessels. The purpose of this study is to perform an objective estimation of lymphatic drainage and metastatic Lymphonodes in right-sided colon carcinoma through indocyanine green (ICG) lymphography.

Methods: Patients with right-sided colon adenocarcinoma were included, excluding those in stage IV, T4 and those who underwent urgent surgery. $2 \mathrm{cc}$ of ICG peritumoral were injected using a peripheral intravenous catheter at the beginning of the intervention. The lymphatic drainage mapping of the tumor was identified. Lymphadenectomy of the ileocolic vessels and right branch of the middle colic vessels was performed extending it to the left branch and origin of middle colic vessels if it was shown in the mapping.

Results: 16 patients were included. The average age was 58 . In 10 patients the tumor was located in the ascending colon and in 6 patients in the hepatic angle. In 11 patients, the mapping showed lymphatic drainage to ileocolic vessels and right branch of the middle colic vessels. In 5 patients $(31 \%)$ it showed drainage to the left branch and origin of the middle colic artery, therefore extended lymphadenectomy was performed at that level. In 14 patients, the postoperative period was uneventful. 1 patient presented infection of the surgical wound and another patient developed a $6 \mathrm{~cm}$ perianastomotic collection treated with percutaneous drainage. The anatomopathological report showed nodal metastasis in 4 of the 5 patients $(80 \%)$ in whom lymphatic drainage was observed in the territory of the middle colic vessels with ICG. These patients presented the tumor in the hepatic angle. Therefore, 4 of the 16 patients with right-sided colon carcinoma (25\%) presented nodal metastasis in the territory of the middle colic vessels.

Conclusions: Fluorescent lymphography may improve the results of lymphadenectomy in colon cancer. In patients with tumors of the hepatic angle, lymphadenectomy extended to the left branch and origin of middle colic vessels, could be an adequate alternative.

\section{P242-COLORECTAL-Malignant}

\section{VASCULAR ANATOMY OF THE RIGHT COLON RELATED TO} RIGHT COLECTOMY

\section{N.A. Pepenin, K.O. Zadorozhna, K.S. Burmich}

General surgery, O. O. Bohomolets National Medical University, KYIV, Ukraine

Introduction: Over the last decade, the common principles of surgical treatment in colon surgery are central vascular ligation (CVL) and complete mesocolic excision (CME). However, the superior mesenteric vessels anatomy, while performing the right colectomy is characterized by wide variability, which can lead to complications, especially during minimally invasive surgical intervention. Objective. The purpose of this study is describing vascular variations around the superior mesenteric artery and vein-middle colic, right colic and ileocolic vessels, Henle trunk in the laparoscopic right colectomy.

Materials and methods: The study was held in the 'Dobrobut' clinic and $O$. O. Bohomolets National Medical University, department of general surgery (Kyiv, Ukraine) during the 2016-2018 period. 24 patients were included to the study, 13 females (45.8\%), 11 males $(54.2 \%)$ in the average age of 71,4 $\pm 9,8$ years. All the patients underwent the laparoscopic right colectomy (CME + CVL) with D3 lymph node dissection. Recorded video materials from each laparoscopic right colectomy were analyzed during the study.

Results: Ileocolic vessels were the most stable. There were typical anatomical position in al cases. $58.3 \%$ of cases, ileocolic vein was identified anteriorly to the ileocolic artery, while $41.7 \%$ being posteriorly. Right colic vein was absent in $29.1 \%$ of cases. Right colic vein drainage was to Henle trunk and inferior mesenteric vein in $62.5 \%$ and $37.5 \%$ respectively. The right colic artery was present in $75 \%$ of patients, it 's origin was superior mesenteric artery in $94.4 \%$ and $5.6 \%$ the middle colic artery. The middle colic vein was present and drained to superior mesenteric vein in $100 \%$ of cases. Same as the middle colic artery with the superior mesenteric artery origin. Henle trunk was present in $91.7 \%$, gastro-pancreato-colic trunk in $45.5 \%$ of cases gastro-pancreatic trunk in $40.9 \%$, gastro-colic in $13.6 \%$.

Conclusions: Knowing the options of surgical vessels anatomy, while performing the right colectomy, altogether with surgeons preparation, using the CT-scan data can reduce the risk of iatrogenic damage and complications risks.

\section{P243-COLORECTAL-Malignant}

\section{GENERAL SURGERY RESIDENTS PERFORMING} COLONOSCOPY FOR COLORECTAL CANCER SCREENING

M. Matyja ${ }^{1}$, A. Pasternak ${ }^{2}$, M. Szura ${ }^{3}$, M. Wysocki ${ }^{1}$, P. Major ${ }^{1}$, M. Pedziwiatr ${ }^{1}$

${ }^{1}$ 2nd Department of General Surgery, Jagiellonian University Medical College, KRAKOW, Poland; ${ }^{2}$ Department of Anatomy, Jagiellonian University Medical College, KRAKOW, Poland; ${ }^{3}$ Department of Experimental and Clinical Surgery, Jagiellonian University Medical College, KRAKOW, Poland

Aims: Colonoscopy is a gold standard for colorectal cancer (CRC) screening. Training in endoscopy one of the essentials of general surgery residency program. Patients need to receive care at the highest level possible, nevertheless surgical trainees should gain experience. The aim of our study was to analyze the effectiveness of colonoscopy performed by general surgery residents by evaluating endoscopic quality indicators.

Methods: The analysis included 2542 patients aged 40-65 who underwent screening colonoscopy between October 2014 and June 2018 in the 2nd Department of Surgery, Jagiellonian University Medical College, Cracow, Poland. The patients were examined by general surgery residents. All the residents were accredited to perform endoscopy examinations by the Association of Polish Surgeons. Quality indicators such as adenoma detection rate (ADR), cecal intubation rate (CIR) and patient tolerance scale (4-point scale) were evaluated

Results: The overall ADR was: $29.31 \%$. Cecal intubation rate was: $95.98 \%$. Patient tolerance for exam was very good in $75.16 \%$ cases, good in $14,48 \%$, medium in $6.55 \%$, poor in $3.81 \%$. No severe complications were noted.

Conclusion: General surgery residents are able to perform high quality and effective screening colonoscopy in a proper learning environment. Surgical trainees still need to enhance their technique to improve patient tolerance. Our results may become a foundation to improve the quality and training in endoscopy of general surgery residents.

\section{P244-COLORECTAL_Malignant}

\section{THE IMPACT OF PERIOPERATIVE FLUID THERAPY ON SHORT-TERM OUTCOMES AFTER LAPAROSCOPIC COLORECTAL CANCER SURGERY COMBINED WITH ERAS PROTOCOL}

M. Pisarska, M. Wierdak, N. Gajewska, G. Torbicz, P. Major, A. Budzynski, M. Pedziwiatr

2nd Department of General Surgery, Jagiellonian University Medical College, KRAKÓW, Poland

Introduction: The Enhanced Recovery After Surgery (ERAS) protocol was designed to accelerate convalescence, reduce morbidity and shorten the length of hospital stay (LOS). One of its major interventions is balanced perioperative fluid therapy. The impact of this single intervention on short-term outcomes is widely discuss.

Aim: The aim of this study was to assess the impact of perioperative fluid therapy on short-term outcomes.

Material and methods: The analysis included consecutive prospectively registered patients operated laparoscopically for colorectal cancer between November 2012 and January 2018. Patients were divided into two groups: balanced $(=2500 \mathrm{ml})$ or unbalanced $(>2500 \mathrm{ml})$ perioperative fluid therapy. All patients were treated according to ERAS protocol. Study outcomes were: recovery parameters, morbidity rate, LOS, 30-day readmission rate.

Results: Group 1 consisted of 361 and Group 2 of 80 patients. There were no statistically significant differences between the groups in terms of demographic and operative parameters. Morbidity was lower in Group $1(27.4 \%$ vs $38.8 \%, p=0.044)$. Patients in Group 1 were discharged home earlier than in Group 2 ( 4 vs 5 days, $\mathrm{p}<0.001$ ). Moreover, we observed differences in recovery parameters between the groups: tolerance of an oral diet on the $1 \mathrm{~s}$ postoperative day $(76 \%$ vs. $59 \%, \mathrm{p}=0.002)$ and patient mobilization on the day of surgery $(90 \%$ vs. $78 \%, \mathrm{p}=0.005) .30$-day readmission rate was lower in group $1(7.8 \%$ vs. $15 \%, \mathrm{p}=0.041)$ Conclusion: A balanced perioperative fluid therapy on the day of surgery may be associated with faster convalescence, lower morbidity rate, shorter LOS and lower 30-day readmission rate. 


\section{P245-COLORECTAL_Malignant}

\section{LAPAROSCOPIC VS. OPEN EMERGENCY COLONIC RESECTIONS. A PROPENSITY MATCHED STUDY}

\author{
P. Tejedor ${ }^{1}$, E. Kyle ${ }^{1}$, N. Carter ${ }^{2}$, V. Celentano ${ }^{1}$, J. Conti ${ }^{1}$, \\ S. Mercer ${ }^{2}$, J.S. Khan ${ }^{1}$, F. Sagias
}

${ }^{1}$ Colorectal Surgery, Queen Alexandra Hospital, PORTSMOUTH, United Kingdom; ${ }^{2}$ Upper GI Surgery, Queen Alexandra Hospital, PORTSMOUTH, United Kingdom

Aims: To compare short- and long-term outcomes of emergency laparoscopic colonic surgery (LCS) vs. open colonic surgery (OCS) for cancer.

Methods: A retrospective analysis was performed including patients who underwent LCS or OCS for cancer treated as emergency in a single centre between 2014 and 2018. Patients who underwent palliative surgery were excluded. LCS were 1:1 propensity score-matched based on PPOSUM and stage of disease with OCS. Short-term outcomes included oncological quality, length of hospital stay (LOS) and postoperative mortality. For long-term outcomes, 3-year overall and disease free survival (OS and DFS) rates were analyzed.

Results: During the study period, a total of 406 emergency colorectal resections were performed. Of them, $25 \%(n=101)$ were coloniccancers. 38 LCS were matched to an equal number of OCS.Median age was 71 (21) years and 62\% were females. Median follow-up was 18 (22) months. The majority of resections were right hemicolectomies $(47 \%)$, followed by sigmoid resections $(36 \%)$ and subtotal colectomies $(17 \%)$

Operative time (188 (90) LCS vs. $164(80)$ OCS, $\mathrm{p}=0.027)$ ) and median lymph node harvest $(22$ (14) LCS vs. 23 (10) OCS, $p=0.235$ ) were similar between groups. However, the rate of R1 resections ( $2.6 \%$ vs. $13.2 \%, \mathrm{p}=0.089)$, and the median $\operatorname{LOS}(9$ (9) vs. 18 (13) days, $<\mathrm{b}>\mathrm{p}=$ $0.007</ \mathrm{b}>$ ) favoured laparoscopy. The 90 -day mortality rate was significantly lower for the LCS $(2.6 \%$ vs. $23.7 \%,<\mathrm{b}>\mathrm{p}=0.007</ \mathrm{b}>)$.

The 3 -year OS rate was $39 \%$ for the LCS vs. $42 \%$ in the OCS group $(\mathrm{p}=0.303)$. The 3 -year DFS rate for the LCS was $48 \%$ vs. $65 \%$ for the OCS $(p=0.322)$.

Conclusions: Laparoscopic surgery for emergency colonic cancer resections can achieve better short-term outcomes compared to open surgery, without compromising long-term oncological outcomes, when performed by experienced colorectal surgeons. However, a longer follow-up period is needed to confirm these findings.

\section{P246-COLORECTAL-Malignant}

\section{LAPAROSCOPIC TOTAL MESORECTAL EXCISION (TME) FOR MID AND DISTAL RECTAL CANCER. OUTCOMES IN 266 PATIENTS. A SINGLE CENTER EXPERIENCE}

\section{E. Koutroumanos ${ }^{1}$, Y. van Molhem ${ }^{2}$}

${ }^{1} 1$ st Department of General Surgery, Tzaneio General Hospital of Piraeus, PIRAEUS, Greece; ${ }^{2}$ Department of General Surgery, OLV Hospital, AALST, Belgium

Background: Total mesorectal excision (TME) offers the best reported rates for local recurrence and survival in patients with rectal cancer. Our series from a single high-volume center, assessed the feasibility, safety and long-term oncologic adequacy of laparoscopic total mesorectal excision Methods: We reviewed the prospective database of 266 consecutive unselected patients undergoing laparoscopic TME for rectal cancer between 1995 and 2009 at the Department of General Surgery, Onze-Lieve-Vrouwziekenhuis Hospital (OLV), campus Aalst, Belgium. The objective of the present study was to evaluate the effectiveness of laparoscopic TME, with an emphasis on perioperative variables and long-term oncological outcomes.

Results: 266 pts with mid and distal rectal cancer up to $10 \mathrm{~cm}$ from the anal verge had laparoscopic TME resection. 161 patients $(60.5 \%)$ underwent a sphincter-preserving surgery and the remaining 105 patients $(39.5 \%)$ had an abdominoperineal resection. End-to-end anastomoses: 68 pts $(42 \%)$, J-colonic pouch: 92 pts $(58 \%)$. Female: $32 \%$ (85), Male: $68 \%$ (181). 30-day mortality occurred in only one patient $(0.3 \%)$. 30-day morbidity: $19.5 \%$ (52 pts). Mean follow-up time: 48 months. Lymph nodes harvested: Mean 9, Median 8 (1-25). pCRM positive $(<1 \mathrm{~mm})$ : $5.6 \%$ (15pts). Distal margin (cm): Median (cm) $2.5(0.1-8) .58 \%$ (154 pts) received neoadjuvant radiotherapy. Anastomotic leak: $4.1 \%$ (11pts). Conversion rate: $3.7 \%$ (10 pts). Local recurrence : $5.6 \%$ (15 pts), 5-year overall survival: $87.4 \%, 5$-year disease-specific survival: $77.5 \%$. Conclusion: Our results were comparable to the previous studies published concerning laparoscopic TME for mid and distal rectal cancer tumors. The study demonstrate that laparoscopic approach to mid and distal rectal cancer is a feasible and safe method, without serious oncologic disadvantages at specialized high-volume centers

\section{P247-COLORECTAL-Malignant}

INTENSIFIED REHABILITATION IN COLORECTAL SURGERY IN OUR CENTER

B. Cantarero Jiménez, P. Nicolas Martinez, M. Maes Carballo, I. Muñoz Nuñez, I. Plata Pérez, P. Vázquez Barros

\section{General Surgery, Hospital Santa Ana Motril, GRANADA, Spain}

Introduction: The RICA clinical pathway (intensified recovery in abdominal surgery), also called surgical multimodal rehabilitation, is the application of a series of perioperative measures and strategies in those patients who are going to undergo a surgical procedure with the objective of reducing secondary stress to the surgical intervention. In this way, we achieve a better recovery of the patient and significantly reduce complications and morbidity. Objective: S To analyze, through our database of patients undergoing CRC, the percentage of postoperative ileuses and the following quality indicators: the postsurgical hospital stay, the anastomotic leak, and the infection of the surgical site. To check if the implantation of the RICA pathway has meant an improvement in our postoperative hospital stay and with that, a lower sanitary cost.

Methods and results: We analyzed the data collected from those patients who underwent $\mathrm{CCR}$ in our hospital between 01/11/2017 and 12/12/18, during which time we implemented the RICA clinical pathway. The average hospital stay was 8 days. Of the 78 patients, $8.97 \%$ presented anastomotic leak, $12.82 \%$ infection of the surgical wound and $19.23 \%$ paralytic ileus. We have verified how the average hospital stay increases with the appearance of anastomotic leak (18.86 days), infection of the surgical wound (16.10 days) and paralytic ileus (16.80 days). When we divided this 12-month period into two halves to see the impact of the implantation of the clinical pathway, we obtained the following results: The post-surgical hospital stay in the period from $01 / 11 / 2017$ to $05 / 01 / 2018$ was 8.33 . The stay from $05 / 01 / 2018$ to $12 / 12 / 2018$ was 5.33 .

Conclusions: The implantation of the RICA clinical pathway is providing us with important advantages in our clinical practice, with greater postoperative comfort and an improvement in our quality indicators, such as the decrease in the average hospital stay of our patients. On the other hand, after starting its implementation we have encountered the resistance to change clinical habits and the one that requires a multidisciplinary participation, so adherence to this is being progressive, and requires periodic audits to reinforce and consolidate our achievements, and identify our points of improvement.

\section{P248-COLORECTAL-Malignant}

SEVERAL USES OF GEL POINT PATH OVER PATIENTS WITH RECTAL NEOPLASMS

E. Rubio, J. García Borda, J. Alcalde, M. Labalde, C. Nevado, O. Garcia Villar, N. Yagüe, K. Shirai, E. Ferrero

\section{Surgery, H.U 12 de Octubre, MADRID, Spain}

Introduction: Transanal endoscopic microsurgery (TEM) is a minimally invasive technique However, TEM has not yet achieved widespread use. Recently, transanal minimally invasive surgery (TAMIS) using single-port surgery devices has been reported. Initially facilitated by existing single-port surgery devices, two platforms for transanal access, the GelPOINT ${ }^{\circledast}$ Path (Applied Medical, Rancho Santa Margarita, CA, USA) and the SILS ${ }^{\mathrm{TM}}$ Port. The GelPOINT $^{\circledR}$ Path is the only platform to be specifically designed for TAMIS y TaTME.

Objetive: In the present study, usesa GelPOINT ${ }^{\circledR}$ Path was performed in 35 patients with lower rectal neplasms.

Results: Complete full-thickness excision was performed in all cases of TAMIS and free margins over rectal cancer. On two cases no neoplasm was visualizad. The patient characteristics, operative techniques and operative outcomes were evaluated. The mean age of the patients was 63.0 years (range 48-76). The mean operating time were $186 \mathrm{~min}$ (range 55-110). 23 patients was selsted for TaTME, 10 for TAMIS and two patients for evaluation and biopsy if was necesary. Additional transabdominal rectal resection was not performed, and adjuvant chemoradiotherapy was performed in all cases. TAMIS using a GelPOINT ${ }^{\circledR}$ Path was revealed to be easy and safe to perform. Although only a small number of cases were treated, and the operation was demonstrated to be sufficiently feasible.

Conclusion: Gelpoint path is a good tool for colorectal surgery in TaTME, TAMIS and evalluation of anastomoses or de novo lesions 


\section{P249-COLORECTAL-Malignant}

\section{LOW ANTERIOR RESECTION SYNDROME SCORE- PORTUGUESE VALIDATION}

\section{Ferrer Sales, D. Parente, M. Neves, P. Alves, N. Rama, V. Faria}

General Surgery, Centro Hospitalar de Leiria, LEIRIA, Portugal

Introduction: Several improvements in rectal cancer treatment, in the last decades, resulted in a markedly increased survival. Nevertheless, surgery remains the prevalent treatment and 60 to $90 \%$ of operated patients experience some kind of functional abnormalities. As nowadays we acknowledge the importance to focus not only on survival rates but also on quality of life, we craved for a precise, reproducible, simple, clear and user-friendly tool for evaluating bowel function in rectal cancer patients after sphincter saving operation. Therefore, we performed a thorough translation with cultural adaptation of the patient reported outcome tool, Low Anterior Resection Syndrome (LARS) Score, to the Portuguese language (LARS-PT) and population.

Methods: According to the current international recommendations, we designed this study encompassing three main phases: (i) cultural and linguistic validation to European Portuguese; (ii) feasibility and reliability tests of the version obtained in the previous phase; and (iii) validity tests to produce a final version. The questionnaire was completed by 154 patients from six Portuguese Colorectal Cancer Units, and 58 completed it twice.

Results: The Portuguese version of LARS score showed high construct validity. Regarding the test-retest, the global Intraclass Correlation showed very strong test-retest reliability. Looking at all five items, only items 3 and 5 presented a moderate correlation. LARS score was able to discriminate symptoms showing worse quality of life in patients submitted to preoperative radio and chemotherapy.

Conclusion: LARS questionnaire has been properly translated into European Portuguese, demonstrating high construct validity and reliability. This is a precise, reproducible, simple, clear and user-friendly tool for evaluating bowel function in rectal cancer patients after sphincter saving operation. Therefore, his sistematic use should be implemented.

\section{P250_COLORECTAL_Malignant}

\section{RISK FACTORS AND TREATMENT OF DIAPHRAGMATIC HERNIA AFTER IVOR-LEWIS OESOPHAGECTOMY FOR CANCER}

F. Puccetti, P. Parise, U. Elmore, A. Cossu, G. Marcocci,

R. Quattromani, R. Rosati, Gastrointestinal Surgery, Ospedale San Raffaele, MILAN, Italy

Oesophagectomy is the mainstay of curative treatment for oesophageal cancer and post-oesophagectomy diaphragmatic hernia $(\mathrm{PODH})$ represents a potentially life-threatening surgical complication characterized by an underestimated occurrence rate and unknown related risk factors. This study analyses the experience of two tertiary designated centers in order to evaluate key elements concerning development and treatment of PODH.

A cohort of consecutive patients affected by a clinically resectable oesophageal cancer (any T, any N and M0) underwent Ivor-Lewis oesophagectomy between March 1997 and April 2017 according to three different approaches: totally open incision procedure (OILO), hybrid (HILO) and totally mininvasive to esophagectomy (MILO). All population was retrospectively observed in the context of a postoperative calendarised follow-up in order to record the incidence and postrepair results of $\mathrm{PODH}$

414 patients underwent Ivor-Lewis oesophagectomy for cancer and 22 (5.3\%) developed PODH within a median follow-up period of 16 months (6-177). Surgical repair was generally applied by the mean of laparoscopic cruroplasty (77\%) with a conversion rate of $24 \%$. Postoperative morbidity did not include early recurrences but exclusively cardio-pulmonary complications (5 patients) with one case of respiratory failure leading to death. The discharge was reached after a median hospital stay of 6 days (2-95) while 3 recurrences (14\%) occurred over a median followup period of 10.1 months. A wide univariate analysis identified statistically significant associations between PODH occurrence and the administration of preoperative chemoradiotherapy, the complete pathological response (CPR) and a lymph node harvest (LNH) larger than 33 stations (p-value of $0.016,0.001$ and 0.024 respectively). The strong influence of an extended LNH was confirmed by the multivariable analyses (0.026) along with CPR which should however be considered as longer survival-related bias.

The minimally invasive surgery and the neoadjuvant chemoradiotherapy represent a considerable part of multimodal treatment for oesophageal cancer presenting a not statistically significant association with PODH development while a LNH including more than 33 nodes resulted to be an independent risk factor mirroring the extent of surgical demolition in oesophagectomy.

\section{P251-COLORECTAL-Malignant}

THE SAFETY AND EFFECTIVENESS OF ROBOTIC VERSUS LAPAROSCOPIC TME IN PATIENTS WITH RECTAL CANCER. INITIAL ANALYSIS

L. Barbulescu

General Surgery, Ponderas Academic Hospital, BUCHAREST, Romania

Aim: To asses the safety and effectiveness of robotic total meso-rectal excision vs laparoscopic total meso-rectal excision and to analyse the primary outcomes.

Methods: The operative, post-operative and oncological outcomes were evaluated to assess the effectiveness of both techniques of TME. In our center were performed 30 robotic rectal resections and 48 laparoscopic resections from January 2018 to present.

Results: The RTME was associated with longer operation time, early bowel movements, lower risk of conversion and shorter hospitalization. The statistical equivalence was seen between RTME and LTME for non-oncological variables like blood loss, morbidity and reintervention risk. The oncological variables such as number of harvested nodes and positive circumferential resection margin risk were also comparable in both groups. The length of distal resection margin was similar in both groups.

Conclusion: RTME in patients with rectal cancer was associated with a lower rate of conversion and less incidence of urinary retention. The operative time in RTME was significantly longer than in LTME. The initial oncological and function outcomes of RTME seem to be equivalent with LTME.

\section{P252-COLORECTAL-Malignant}

COMPARATIVE EFFECTIVENESS OF OPEN, LAPAROSCOPIC AND ROBOTIC APPROACH ON THE PATHOLOGIC SPECIMEN OF TOTAL MESORECTAL EXCISION FOR RECTAL CANCER. A SYSTEMATIC REVIEW AND NETWORK META-ANALYSIS

\section{Athanasiou}

General Surgery, Ipswich Hospital, IPSWICH, United Kingdom

Aims: Two randomized controlled trials failed to show non-inferiority of the laparoscopic total mesorectal excision (LTME) compared to open. LTME becomes particularly challenging in low rectal cancers and in narrow pelves. Many surgeons report that robotic TME (RTME) may be beneficial in that setting. Our aim was to systematically review the literature and compare the pathologic outcomes of open, laparoscopic and robotic TME for rectal cancer

Methods: Medline, Embase, Scopus, Cochrane library and Web of Knowledge databases were searched for randomized controlled trials (RCT) reporting patholologic outcomes of open, laparoscopic or robotic TME with no language restriction. Our primary outcome was quality of TME on macroscopic assessment of the specimen. Secondary outcomes included positive circumferential resection margin, distance to radial margin, number of lymph nodes and positive radial margin. The included studies were quality assessed and the Jadad score was reported. The Grade approach was used to rate the certainty of each network estimate.

Results: Fourteen RCTs were included in our study. Seven RCTs compared the OTME to the LTME, six compared the LTME and RTME and one study the OTME to the RTME. No statistical significant difference was found in quality of TME when the the LTME was compared to the OTME OR $=1.36(0.99,1,85)$ or the RTME OR $=1.33(0.82,2.16)$. No difference was found in pCRM for the laparoscopic OR $=1.23(0.90,1.69)$ or the robotic approach $\mathrm{OR}=0.87(0.44$ 1.75 ) when compared to open. Distance to radial margin and number or lymph nodes didn't differ between the groups.

Conclusions: No significant advantage on pathologic specimen quality has been found with the robotic approach. The LTME doesn't seem to compromise the quality of the specimen. 


\section{P253-COLORECTAL_Malignant}

\section{SHORT TIME RESULT OF LAPAROSCOPIC RESECTION OF ADJACENT ORGAN CT4B COLORECTAL CANCER}

H. Samura ${ }^{1}$, J. Arakaki ${ }^{2}$, K. Sugata ${ }^{2}$, Y. Hori ${ }^{2}$, Y. Nagamine ${ }^{2}$, $\overline{\text { F. Kohagura }}^{2}$, H. Motonari ${ }^{2}$, S. Kameyama ${ }^{2}$, T. Ishimine ${ }^{2}$

${ }^{1}$ Division of Digestive and General Surgery, Urasoe General Hospital, OKINAWA, Japan; ${ }^{2}$ Department of Surgery, Urasoe General Hospital, OKINAWA, Japan

Colorectal cancer often invade adjacent organs and It is known that prognosis improves with resection of the involved organ.

We report our experience of invaded adjacent organ resection, which include seminal vesicle, uterine and bilateral appendages, posterior wall of the vagina and bladder wall.

Method: Although the range of resection is predicted by image study preoperatively, at the time of operation, it was decided by palpation with a forceps. Each operation is evaluated by operation time, blood loss, blood transfusion volume, postoperative complication, postoperative hospital stay, and short term prognosis.

Result: Resection cases of seminal vesicle, posterior vaginal wall, uterine and bilateral appendages and bladder wall were $5,4,4$ and 1 , respectively.

The results are shown in the order of seminal vesicle / vaginal posterior wall / uterine / bladder. Median age was 70, 67, 54 and 74 years old. The median operation time was 541,630,623, 249 $\mathrm{min}$, the median blood loss was $580,340,310,10 \mathrm{ml}$, and only one case of uterine and bilateral appendages resection required the blood transfusion. The average postoperative hospital stay was $40,38,45,15$ days.

Nine cases have postoperative complication, that include delayed wound healing, anastomotic leakage and rectovesical fistula, postoperative ileus, chyle ascites and neurogenic blodder. All of those were improved with conservative treatment.

The mean hospital stay in complication cases was 48 days (38-88) and 19 (14-29) days without complications.

The median observation period was 773 days (166-2166), and there was no local recurrence. All of the case of stage IV were dead.

There was no local recurrence and all patient without stage IV are alive, it seems that the resection range was sufficient.

Conclusion: Even with adjacent organ invasion colorectal cancer, it was possible to determine the resection line by palpation with laparoscopic forceps manipulation, and possible to resect margin free of cancer.

\section{P254-COLORECTAL-Stoma}

\section{LAPAROSCOPIC LOW RECTAL RESECTION WITH/WITHOUT DIVERTING ILEOSTOMY}

\section{P. Ihnát, M. Tesar, P. Ostruszka, P. Gunková, P. Vávra}

Department of Surgery, University Hospital Ostrava, OSTRAVA, Czech Republic

Background: The construction of diverting ileostomy (DI) is recommended to avoid septic complications of anastomotic leakage. The aim of our study was to assess the benefits and risks of DI constructed during laparoscopic low anterior resection (LAR).

Methods: Retrospective clinical cohort study was conducted in University Hospital Ostrava, Czech Republic. All patients undergoing laparoscopic LAR with TME because of rectal cancer within a 6-year study period were assessed for study eligibility.

Results: A total of 151 patients ( 73 patients without DI, 78 patients with DI) after laparoscopic LAR were enrolled into the study and underwent analysis. Both study subgroups were comparable in terms of demographic and clinical features. Postoperative 30-day morbidity was significantly lower in patients without DI $(23.3 \%$ vs. $42.3 \%, \mathrm{P}=0.013)$. Anastomotic leakage frequency was higher in patients without DI $(9.6 \%$ vs. $2.5 \%, \mathrm{P}=0.090)$; surgical intervention was necessary in $6.8 \%$ of patients without DI.

Stoma-related complications were noted in $53.8 \%$ of patients with DI; some patients had more than one complication. Surgical intervention because of stoma-related complications was needed in 9 patients (11.5\%). Distinctive complications of DI laparoscopic construction (small bowel obstruction due to DI semi-rotation around its longitudinal axis) was noted in 3 patients (3.8\%). Mean stoma period (interval between LAR and DI reversal) was more than 10 months in our study; only $19.2 \%$ of patients were reversed without delay ( $=4$ months). Postoperative morbidity after DI reversal was $16.6 \%$; re-laparotomy was needed in $2.5 \%$ of patients.

Conclusions: Despite benefits of DI in protecting low rectal anastomosis, ileostomy construction remains fraught with many stoma-related complications and long stoma periods associated with significantly decreased quality of life

\section{P255-COLORECTAL-Stoma}

\section{LAPAROSCOPIC SINGLE PORT HARTMANN REVERSAL AFTER SINGLE PORT LAPAROSCOPIC HARTMANN PROCEDURE WITH UMBILICAL COLOSTOMY}

\author{
E. Falsetti, T. Cipolat Mis, M.C. Cartillone, A. d'Alessandro, \\ E. Chouillard
}

GENERAL AND MINIMALLY INVASIVE SURGERY, PoissySaint-Germain-en-Laye Medical Center, POISSY, France

Aims: Single port laparoscopic is a minimally invasive surgical technique that joint the cosmetic advantages with the well recognized benefits of the standard laparoscopic approach [1]. We describe a laparoscopic single port Hartmann reversal in a patient by the use of the umbilical colostomy site for surgical access [2].

Methods: A 42 years old patient was submitted to a laparoscopic single port Hartmann procedure with an trans-umbilical colostomy for a recurrent sigmoid volvulus that was treated at the beginning by endoscopic de-rotation. After three months the patient was reevaluated for a Hartmann reversal with a laparoscopic single port technique.

After routine skin preparation and laparoscopic setup, the colostomy is mobilized from its mucocutaneous border, and the anvil of a circular stapler is secured to the distal lumen. By the use of a GelPoint system with 3 trocars, the intra-abdominal adhesiolisis in performed. The splenic flexure is mobilized to achieve a sufficient mobilization of the left colon that allows the fashion of a tension free anastomosis. The rectal stump is mobilized to the mid rectum, starting from the posterior mesorectal fascia around to the anterior rectal wall. A tension-free colorectal anastomosis is secured with a standard circular $31 \mathrm{~mm}$ stapling device inserted transanally. The colostomy wound is closed. The operative time was $60 \mathrm{~min}$.

Results: The postoperative course was uneventful, the patient was discharged at forth postoperative day, oral intake started on postoperative day three.

Conclusions: Single port laparoscopic Hartmann reversal thought the umbilical stoma site is a minimally invasive surgical option that is safe in selected patients and offer the best cosmetic results.

[[1] Single-port access laparoscopic reversal of Hartmann operation. Borowski DW, Kanakala V, Agarwal AK, Tabaqchali MA, Garg DK, Gill TS Dis Colon Rectum. 2011 Aug;54(8):1053-6 [2] Preliminary experience with umbilical stoma in transumbilical single-port colorectal surgery d'Alessandro A, Kari N, Alameh A, Pasquier N, Tarhini A, Vinson Bonnet B, Noun R, Chouillard E Tech Coloproctol. 2018 Apr;22(4):301-304

\section{P256-COLORECTAL-Stoma}

\section{MINI INVASIVE LOOP ILEOSTOMY ON DEMAND IN LAR. OUR INITIAL EXPERIENCE}

\section{A. Dibra, E. Etmont}

Departement of General Surgery, University of Medicina Tirana, TIRANA, Albania

The progressive evolution of surgical techniques and oncologic protocols on rectal cancer disease facilitates surgeons to challenge the skills for anus preservation in low rectal cancer surgery. The laparoscopic surgery is already one of the best ways to reach the pelvic floor and to try procedures, which were previously difficult to apply through open surgery.

The anastomotic leakage has particularly high occurrence if the anastomosis is performed in the anal or distal rectum area.

It is evident that although the fecal diversion does not decrease post operatory mortality, it significantly reduce the risk of anastomotic leak and the risk of a second major surgery when the leak occur.

Diverting stomas are low-risk procedures from a technical point of view, but they potentially expose the patients to postoperative morbidity, impacting the patients' quality of life.

It is not easy to decide whether the fecal diversion is needed or not. This decision must be made on a case to case basis, trying to apply the stomas only when they are really needed. We report our initial experience by living a transmesenterial cotton loop around the pre terminal ileum which extremities are turned out usually through the lateral trocar wound in laparoscopy or by applying a dedicated mini incision in open surgery.

The purpose is to perform (in case of suspected fistula), a mini invasive diverting procedure, by widening the loop wound and by pulling up the ileum in a lateral loop ileostomy.

We applied this procedure to 12 consecutive patients with low colorectal anastomosis and in two of them we performed a lateral loop ileostomy with good results. We believe this can be an alternative that needs to be standardized. 
P257-HEPATO-BILIAIRY \& PANCREAS—Gallbladder

THE ODD GALLBLADDER-A RARE CASE OF

GALLBLADDER AND LYMPH NODE SARCOIDOSIS: A CASE REPORT AND REVIEW OF THE LITERATURE

\section{H.J. $\operatorname{Tan}^{1}$, J.T.L. Ho ${ }^{1}$, A.K.H. Chiow ${ }^{2}$}

${ }^{1}$ General Surgery, Mohh, SINGAPORE; ${ }^{2}$ General Surgery, Changi General Hospital, SINGAPORE

Purpose: Sarcoidosis is a chronic, multisystem inflammatory disorder with unknown aetiology characterised by noncaseating granulomas within involved organs. Gallbladder involvement in sarcoidosis is extremely rare and literature review revealed only 7 reported cases to date. In this paper, we present a case of gallbladder associated sarcoidosis.

Method: A 67-year-old lady was known to the clinic for regular surveillance of liver steatosis and incidental gallbladder polyps. The largest polyp was $4 \mathrm{~mm}$ at presentation in 2008 and has grown to $7 \mathrm{~mm}$ in 2017. In view of worsening symptoms of biliary colic and growing polyps, a laparoscopic cholecystectomy was performed.

Results: Laparoscopic cholecystectomy was unremarkable and specimens of the gallbladder and lymph nodes were sent for histology. Histological examination revealed chronic cholecystitis with polypoid cholesterolosis of the gallbladder and noncaseating granulomata within a lymph node, which strongly suggest sarcoidosis.

Conclusion: In conclusion, we report a case of incidental finding of gallbladder sarcoidosis over the course of treatment of biliary colic and symptomatic gallbladder polyps. Therefore, the definitive treatment for patients with symptomatic gallbladder sarcoidosis is a cholecystectomy.

\section{P258-HEPATO-BILIAIRY \& PANCREAS-Gallbladder}

FINDING THE CALOT \& BILE DUCT INJURY: EVALUATION OF GALLBLADDER LITHOTOMY AND DISCONNECTION (GLAD) AS A PRIMARY INTENT PROCEDURE

\section{Bekheit, M. Habib}

HPB Surgery, Aberdeen Royal Infirmary, ABERDEEN, United Kingdom

The surgical management of cholelithiasis can be associated with significant morbidities. Despite the relatively low incidence of bile duct injuries during laparoscopic cholecystectomy, the total number is large due to the high frequency of the operation. The subtotal cholecystectomy with its variants is a well known bailout strategy to the surgical community. However, there is no agreement on when and how to perform these procedures. Indeed, the majority of surgeons will adopt these solutions when there is a struggle to identify the critical view of safety. This struggle results increases the risk of injuries. We hypothesize that a primary intent gall bladder lithotomy and disconnection (GLAD) when the dissection of the GB pedicle is anticipated difficult dissection is a safe and feasible strategic option.

Methods: out 347 patients elevtively admitted to Aberdeen Univesity Hospital with gall stone disease between March 2017 and November 2017, 75 consecutive patients were operated with GLAD procedure based on intraoperative criteria. The primary outcome was the operative time. Secondary outcomes were length of hospital stay, the criteria to do this procedure will be explained, the outcomes will be listed.

\section{P259-HEPATO-BILIAIRY \& PANCREAS-Gallbladder}

LAPARASCOPIC COMPLETED CHOLECYSTECTOMIES;THE REASONS AND THE Results: OF NOT TO CONVERT TO OPEN

\section{N. Ozlem}

General surgery department, Ahievran University, KIRSEHIR, Turkey

Judment, training, experience of the surgeon(SE) influence the results of laparoscopic cholecystectomy (LC). A laparoscopic to open cholecystectomy conversion rate(LOCR) in consecutive 210 cases operated by same surgeon was searched in 2010-2018. Mean ages :53.6.F:156(\%74.285), M:54(\%25.714). Mean operative time(MOT):93.47(30-271),93.194 are for elective laparoscopic cholecystectomy(ELC).MOT: 97.272(40-210)mins for urgent laparoscopic cholecystectomy(ULC). Total hospital stay(pre + postop)(LOS):3.65(1-11)days. LOS of most of the patient (pre + postop):2(36\% rate). The mean postoperative hospital stay (POLOS): 1.5(1-6)days.out of 148(70.47\%) were discharged in POD1.OT for female (89.25 min) are shorter than for male patients $(94.89 \mathrm{~min})(\mathrm{p}<0.05)$.OT prolong $\operatorname{LOS}(\mathrm{p}<0.05)$. Urgently operative time(UOT) is statistically significantly longer than ELC ones $(\mathrm{p}<0.05)$. OTin male LC is not longer than in female $(\mathrm{p}<0.05)$. Lee NW et'l reported their LOCR is $41 / 346(11.9 \%)$.this rate is $17 \%$ in the patients who has upper gastrointestinal tract surgery(UGITS) before. our LOC is in no patients who has with or without UGITS.LOCR of Le VH et'l is $2 \%$ in 3371 LC. the cause of LOC in $60 \%$ of patients who could operated with open aproach is to have acute cholecystitis(AC). in 20 thousand patients literature review are of LOCR is $5.5 \%$.the reasons are infection, adhesion, anatomic difficulty.we could completed laparoscopic in our patients who have $\mathrm{AC}(14.35 \%$ of ourcases). Lee $\mathrm{VH}$, other authors suggest the results of $\mathrm{L}$ surgery will be better when SE increase but undesirable hemorrhage, iatrogenic injuries will continue, declare:LOC is not failure to finish the operation without any accident in difficulty situations. in our series there is not any injury hemorrhage. Lengley BL et'l their LOCR is 5.8\% in 70/ 1193, stressed the complete dissection was not made in $49 \%$ of the patients,suggest 4 port placement, to tilt the gallbladder should made before LOC in LC. Our OT was long,some cases completed with 4 trocars so our LOCR is zero. Robb WB et'l report no effect on LCO in LC in patient who has UGITS before; If a 10 stage check list could use LOCR will decrease from 8.7 to 2.2.our data is obtained retrospectively,lost about risk factors of our case. The minimum SE should be $>200$ patients in LC.when SE increase LOCR may also increase. Mahabaleshwar V et'l report MOT is mean $27 \mathrm{~min}$, longer than ours.this. SE, case difficulty may be reason of these differences. Lee WN et'l concluded the reason of LCO is the patient is male gender.this conclusion could not made by me. $92 \%$ of patients have robotic surgery of Spinoglio et'l discharged in PO1.our patients in rate of 79\% discharged in PO1. Lengley et'l,others LCO increase LOS,cost.on the other hand LC without early conversion may cause bigger complication medicolegal problems. Their OT is the same with me; the reason is late LCO. Lengley stressed much more dissection on calo triangle result a decrease in LOCR.A meticulous dissection could not made on calot triangle result a in rate of mean $4.9 \pm 2.5$ LOS.Lengley et'l belive that the surgeon feel better him/her self in a difficult case when continue laparoscopic, a LC with longer OT do not result more complication than that in OC.actually there is an advantage about health care cost and the patients. 


\section{P260-HEPATO-BILIAIRY \& PANCREAS-Gallbladder}

LAP(LC) AND OPEN CHOLECYSTECTOMY(OC) RESULTS OF OUR EDUCATION AND RESEARCH CLINIC (EARC) NEWLY ESTABLISHED IN RURAL AREA IN MIDDLE ANATHOLIA

\section{N. Ozlem}

General surgery department, Ahievran University, KIRSEHIR, Turkey

1492 OC between2009 to 2017, 985 LC between 2013-2017was made. Demographics, operation types(to begin OC/LC in last years,the effect of being female/ male sex on the results of OC/ LC(POLOS),converting from a state hospital(SH)to EARC were assesed.From 2009 to 2017 the LC/OC rate decreased dramatically(from $163 / 89=$ OC/LC in 2013 to $19 / 172$ OC/LC). 243 of $73.99 \%$ of the patients who operated with open method are female, of $26.1 \%$ is male. $77.36 \%$ of the patients operated with laparoscopy are female, $23.44 \%$ of them are male.in year 2013 the mean age of the patients who operated with open method is 51.89 this rate increases 51.97 in 2017.The patients who has LC in 2013 is in a mean age 47.13 year,mean age of the patients who LC in 2017 increases 48.8. mean POLOS of the patients who have LC in women are 2(0-18) days,median LOS is 1.5 days. mean POLOS in men who haveLC are 2(124)days.medianPOLOSare 2 days.mean operative time(OT) of LC is $73.56 \mathrm{~min}$ in men and $66.81 \mathrm{~min}$ in women.LC unfortunatelly begin in our hospital in rural area, middle anatolia in about 2013.LC/OC rates increased as soon as possible.The mean age of the patients who has LC were high in a short time with converting to be ERC. Young,female patients selected to make LC in the begining.But now elderly and the patients with acute cholecystitis, with upper abdominal operation history operated sequently.there is no differences between women and men in mean POLOS. Mean OTof men for LC is longer than womens'.No mortality was seen.Agabiti N et al the complications of LC/OC operations were studied and state the OR(operation related) of complications after LC versus OC was $0.60(\mathrm{p}<0.001)$ for SRC and $0.52(\mathrm{p}<0.001)$ for SC.In relation to SRC,the advantage of LC was consistent across age categories,severity of gallstones and previous upper abdominal surgery, whereas there was no advantage among people with emergency admission (OR 0.94, $\mathrm{p}=0.764)$.For SC,no significant advantage of LC was seen among very old people (OR $0.99, \mathrm{p}=0.975)$ and among those with previous upper abdominal surgery $(\mathrm{OR} 0.86, \mathrm{p}=0.905)$. two patients(clip slippage,gallbladder cavityabscess)with LC patients make longer our POLOS

\section{P261-HEPATO-BILIAIRY \& PANCREAS-Gallbladder}

THE COMBINATION OF ENDOSCOPIC AND LAPAROSCOPIC TECHNIQUES IN THE TREATMENT OF PATIENTS WITH ACUTE CHOLANGITIS

\section{V.A. Vovk}

Surgical, Regional Clinic Hospital, Kharkiv, Ukraine, KHARKIV, Ukraine

Background: Therapeutic measures for acute cholangitis must necessarily envisage two main directions: elimination of biliary hypertension and the fight against infection in the biliary tract. Without timely, adequate treatment, this disease is fatal. In acute cholangitis, any surgical intervention that provides sufficient drainage of the biliary tree is considered adequate, since it eliminates one of the principal links (biliary hypertension) in the chain of the pathogenesis of acute cholangitis. With the achievement of the elimination of the acute process, correction of the causes of acute cholangitis is given in order to prevent subsequent biliary complications. The use of laparoscopic surgical procedures in the event of acute cholangitis in most cases is limited only to laparoscopic cholecystectomy after the elimination of cholangitis.

Material and Methods: Laparoscopic and video-assisted surgeries were performed in 125 patients with acute cholangitis at different times in relation to endoscopic transduction interventions. In total, these patients underwent 151 video laparoscopic and 102 endoscopic surgical interventions. Among them, endoscopic: endoscopic papillosphincterotomy (EPST)-102, including: mechanical lithotripsy (MLT) - 87, lithoextraction (PE) - 25; and videolaparoscopic: laparoscopic cholecystectomy (LCE) - 113, laparoscopic cholecystostomy (LHS) - 12, laparoscopic dissection and drainage of cholangiogenic liver abscess-9, laparoscopic and videoassisted drainage of the choledoch -8 , laparoscopic and video-assisted choledocholithotomy -5 , laparoscopic and video-assisted cholecystojejunostomy -4

Results and conclusions: Postoperative complications were in 9 patients $(7,2 \%)$. There were no lethal outcomes. Laparoscopic surgeries and endoscopic transpapillar interventions were performed in a different sequence depending on the presence of destructive changes in the gallbladder and complemented each other. Operative interventions using video-laparoscopic technology in most cases make it possible to eliminate biliary hypertension as one of the pathogenetic factors of acute cholangitis and to eliminate the cause of its occurrence.

\section{P262-HEPATO-BILIAIRY \& PANCREAS-Gallbladder}

\section{APPLICATIONS OF INDOCYANINE GREEN ENHANCED} FLUORESCENCE IN LAPAROSCOPIC COLECISTECTOMY

\section{Santi ${ }^{1}$, L. Casali ${ }^{2}$, L. Tartamella ${ }^{2}$, V. Violi ${ }^{1}$}

${ }^{1}$ Surgery, Hospital of Fidenza, University of Parma, FIDENZA (PR), Italy; ${ }^{2}$ Surgery, Hospital of Fidenza, FIDENZA (PR), Italy

Indocyanine green is a molecule that becomes fluorescent when excited struck with light of a specific wavelength in the infrared spectrum (NIR-Infrared), allowing the visualization of anatomical structures in which it has accumulated.

The aim of the study is the application of ICG enhanced fluorescence in laparoscopic cholecystectomy in order to identify the anatomy of the biliary tract, to reduce the risk of iatrogenic lesions and the conversion rate.

The study involves laparoscopic cholecystectomy for cholecystitis and gallstones of main biliary tract. The evening before the surgery, a vial of ICG $(25 \mathrm{mg})$ diluted in $100 \mathrm{ml}$ of saline solution was intravenous injected. During the procedure, after opening the Calot triangle, switching to the NIR mode on the camera, the anatomy of the biliary tract and in particular of the main biliary tract is visualized. The cystic duct and cystic artery are isolated, their section between clips is cut and the cholecystectomy is performed.

From January 201811 patients were enrolled: 9 cases of acute cholecystitis, 2 cases of gallstones of main biliary tract, undergoing preoperative ERCP. In 8 cases of cholecystitis, the angiography allowed the visualization of the main biliary tract. In one case, an abnormal course of the cystic duct was identified. In two cases of gallstones of common bile duct, it favoured the visualization of the biliary tract anatomy. All cases were completed with laparoscopic technique. There were no intra- and post-operative complications.

ICG-enhanced fluorescence is a safe, effective, cheap and rapid tool that can also be applied in small hospitals with no need for training. Its use does not extend the time of surgery and allows the visualization of the anatomy of the biliary tract, especially in situations where it can be altered by reducing the conversion rate and potentially the risk of iatrogenic lesions of the main biliary tract.

\section{P263-HEPATO-BILIAIRY \& PANCREAS-Gallbladder} CHOLECYSTOMEGALY: MANAGEMENT AND TREATMENT

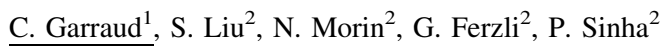

${ }^{1}$ General Surgery, Tuoro College of Osteopathic Medicine, BROOKLYN, United States of America; ${ }^{2}$ General Surgery, NYU Langone Hospital—Brooklyn (Lutheran), BROOKLYN, United States of America

Case Presentation: Patient is a 22 year old female with no significant past medical or surgical history presented to the emergency department with a 2 day history of worsening sharp right upper quadrant pain with associated nausea, vomiting, and PO intolerance. The pain started a few months prior, however it was self-limited with diet modifications. An ultrasound demonstrated a contracted gallbladder with a $15 \mathrm{~mm}$ gallbladder wall. White blood cell count was within normal limits and total bilirubin was slightly elevated to $1.8 \mathrm{mg} / \mathrm{dL}$. No palpable mass was noted on physical exam. An MR cholangiopancreatography was performed which demonstrated a dilated gallbladder measuring $11.5 \times 2.5 \mathrm{~cm}$, a severely thickened gallbladder with a small intramural collection and multiple gallstones. The patient proceeded with a laparoscopic cholecystectomy. Intraoperatively, the omentum was densely adhered to the gallbladder and needle decompression of the gallbladder was unsuccessful due to the wall thickness. The gallbladder was subsequently removed without any complications. Patient's remaining hospital course was uncomplicated. Surgical pathology returned demonstrating acute on chronic cholecystitis.

Discussion: Cholecystomegaly or 'Giant Gallbladder' disease is a rare pathology encountered in the surgical world. There have been few reported cases, most of which occurred in the elderly (>65 years). Kuznetsov et al. defined an enlarged gallbladder to have a volume of 200-300 cc and a giant gallbladder as exceeding $1500 \mathrm{cc}$ (the average weight of the liver). The etiology remains unknown, however certain factors exist to allow the gallbladder to reach this size withou life-threatening sequela. Preoperative imaging, such as MR cholangiopancreatography, is important to differentiate biliary pathology and delineate anatomy. Removal of the gallbladder is recommended to prevent the development of complications like cholangitis or bowel obstruction. The cause of cholecystomegaly still remains uncertain and warrants further research. The management and treatment remains similar to acute cholecystitis. 
P264-HEPATO-BILIAIRY \& PANCREAS—Gallbladder

LAPAROSCOPIC EXPLORATION AND STONE EXTRACTION OF COMMON BILE DUCT STONES

\section{Hussein}

Surgery, American University of Beirut Medical Center, BEIRUT, Lebanon

Aims: Laparoscopic Exploration and Stone Extraction of Common Bile Duct Methods: CBD Stone is a frequent presentation in-patient with cholelithesis and treated in $99 \%$ with ERCP, failure of ERCP necessitate surgical intervention. I report 5 cases done at the American University of Beirut Medical Center successfully that were treated by Laparoscopic exploration through 4 trocars and the use of choledochoscope for stone extraction using dormia basket and Fogarty Catheter 5 French and insertion of T-Tube with no complication. Results: The video will show the various steps used for completion of the procedure. Conclusion: Laparoscopic Exploration of CBD for stone extraction is feasible in Advanced Laparoscopic Centers

P266-HEPATO-BILIAIRY \& PANCREAS-Gallbladder

OUTCOME OF MINI-LAPAROSCOPIC CHOLECYSTECTOMY VS CONVENTIONAL LAPAROSCOPIC CHOLECYSTECTOMY AT SOUTHERN PART OF SINDH PAKISTAN

\section{A.H. Abro}

General Surgery, Liaquat University of Medical \& Health Sciences, Jamshoro, HYDERABAD, Pakistan

Objective: To determine the outcome of mini-laparoscopic cholecystectomy vs conventiona laparoscopic cholecystectomy at Southern part of Sindh Pakistan

Patients and Methods: The comparative case series study of one year (June 2017-May 2018) was conducted in department of surgery LUMHS Jamshoro. All the patients having age $=18$ year of age, either gender presented with history of abdominal pain, nausea and vomiting and were diagnosed as cholelithiasis included in the study and were planned either for mini-laparoscopic cholecystectomy and conventional laparoscopic cholecystectomy were explored for outcome while the patients with empyema gallbladder, gangrene, mucocele gallbladder and adhesions were excluded from the study.

Results: During one year study period, total five hundred patients were diagnosed as cholelithiasis with means age $53.21 \pm 6.83$ (SD). Of five hundred, $127(25.4 \%)$ were underwent for mini-laparoscopic cholecystectomy with $15(11.8 \%)$ were males and $112(88.1 \%)$ were females. The outcome were measured as postoperative pain (VAS) $1.61 \pm 0.92$, size of wound (umbilical $10 \mathrm{~mm}$, epigastrium $5 \mathrm{~mm}$ and subcostal $2 \mathrm{~mm}$ ), excellent cosmetic results, mean \pm SD for hospital stay (hrs) and operative time (minutes) was $12.86 \pm 5.73$ and $30.83 \pm 10.85$, early return to work 115 $(90.5 \%)$, minor oozing $07(5.5 \%)$, port size hernia $2(1.5 \%)$.

Remaining 373 (74.6\%) were underwent for conventional laparoscopic cholecystectomy with 38 $(10.1 \%)$ were males and $335(89.8 \%)$ were females. The outcome were measured as postoperative pain (VAS) $3.53 \pm 1.95$, size of wound (umbilical $10 \mathrm{~mm}$, epigastrium $10 \mathrm{~mm}$ and subcostal $5 \mathrm{~mm}$ ), mean \pm SD for hospital stay (hrs) and operative time (minutes) was $49.95 \pm 8.95$ and $30.83 \pm 7.72$, early return to work $310(83.1 \%)$, port size hernia $10(2.6 \%)$ along with zero $(0 \%)$ mortality. Conclusion: It has been concluded that mini-laparoscopic cholecystectomy is superior and feasible than conventional laparoscopic cholecystectomy and has decreased early postoperative incisional pain, avoided late incisional discomfort and safe procedure with nearly scarless wounds with superior cosmetic effect especially for young female patients.

\section{P267-HEPATO-BILIAIRY \& PANCREAS-Gallbladder}

\section{P265-HEPATO-BILIAIRY \& PANCREAS-Gallbladder}

\section{THE IMPROVED TECHNIQUE OF MINI-LAPAROSCOPIC CHOLECYSTECTOMY FOR CHRONIC AND ACUTE CHOLECYSTITIS}

\section{A.V. Malynovskyi, M.M. Galimon, S.Yu. Badion}

Department of Robotic and Endoscopic Surgery, Odessa national medical university, ODESSA, Ukraine

Aims: Mini-laparoscopic cholecysectomy (MLC) is considered to be the best variant of minimizing surgical trauma and improving cosmesis in laparoscopic cholecystectomy. The most challenging techniqual step of MLC is clipping the cystic duct. It may be impossible or unsafe when diameter of cystic duct exceeds $3 \mathrm{~mm}$, which is common in severe chronic colecystitis or acure cholecystits. There is very limited data in the literature about the use of MLC in acute cholecystits. The aim of study was to access the first results of new technique of MLC.

Methods: Five women with the mean age of 39 years (32-48) underwent MLC. The 1st 10-mm troacar was inserted in the umbilicus and used for the camera and removal of the gallbladder. The 2nd 5-mm troacar was inserted in subxyphoidal area and used for the main working instruments, including medium-large polymer clip-applier (Hem-o-lok type). The 3rd and 4th 3-mm troacars were placed in right subcostal area and used for mini-graspers (Karl Storz). In initial 4 procedures we used conventional 5-mm clip-applier with adopted medium-large titanium clips. To improve safety, we aplied 5-mm Hem-o-lok type clip-applier for the last patient with acute cholecystitis. In this case the diameter of cystic duct was $3,5 \mathrm{~mm}$. The clipping was performed successfully. The 3-mm drain was placed via subcostal troacar incision. Also, in this case we applied original technique of removal of the bladder using wound retraction instrument (Karl Storz).

Results: In all the cases there were no intra- or postoperative complications. The mean duration of procedures was $120 \mathrm{~min}(100-180 \mathrm{~min})$. The postoperative stay was 2 days in every patient. The patients estimated their pain on postop day 2 as 'almost absent' and cosmethic results 1 mo postop as 'exellent'.

Conclusions: 1. New technique of MLC alowed to perform the clipping of cystic duct safely, which is essential in acute calculous cholecystitis. 2. MLC produces almost complete postoperative pain and excellent cosmetic results. 3. More cases collection is needed to study the possibilities and limitations of MLC, including in acute cholecystitis.

\section{OUTCOME OF IMMEDIATE VERSUS LATE LAPAROSCOPIC CHOLECYSTECTOMY IN ACUTE CHOLECYSTITIS AT TERTIARY CARE HOSPITAL HYDERABAD / JAMSHORO SINDH PAKISTAN}

\section{A.H. Pathan}

General Surgery, Liaquat University of Medical \& Health Sciecnes, Jamshoro, HYDERABAD, Pakistan

Objective: To determine the outcome of immediate versus late laparoscopic cholecystectomy in acute cholecystitis at tertiary care hospital Hyderabad / Jamshoro sindh Pakistan

Patients and Methods: The descriptive case series study of one year (2016 -2017) was conducted in department of surgery LUMHS Jamshoro. All the patients having age $=18$ year of age, either gender presented with history of abdominal pain, nausea and vomiting and were diagnosed as acute cholecystitis (cholillthiasis) included in the study and were planned for laparoscopic cholecystectomy and were explored for outcome as immediate (within $48 \mathrm{~h}$ ) and late components ( $>6$ weeks). The frequency and percentage was calculated for categorical variables and mean \pm SD was calculated for numerical variables. As this was descriptive case series so there was no any statistical test of significance was applied.

Results: During one year study period, total one hundred patients were diagnosed as acute cholecystitis with means age $55.72 \pm 8.95$ (SD). Of one hundred, $80 \%$ were females and $20 \%$ were males. The immediate outcome reported as tissue fragile $10 \%$, pancreatitis $2 \%$, slipage of ligature of cystic duct $5 \%$, empyema gallbladder $5 \%$, mucocele $15 \%$ and gangrenous gallbladder $2 \%$ while the late outcome reported as adhesions $70 \%$, cholecystoduodenal fistula and Mirizzi syndrome $1 \%$ and $6 \%$, gallstone ileus $2 \%$, perforated gallbladder $8 \%$ and cholidochiolithiasis $20 \%$ while the mean \pm SD for hospital stay (days) in immediate as $1.51 \pm 0.32$ while in late outcome (days) during acute cholecystitis $4.50 \pm 0.55$ and after surgery (6 weeks later) as $2.85 \pm 1.21$ respectively.

Conclusion: It has been concluded that early LC for acute cholecystitis with cholelithiasis is safe, low cost and feasible intervention and offering the additional benefit of shorter hospital stay and reduce the economical burden. 


\section{P268-HEPATO-BILIAIRY \& PANCREAS-Gallbladder}

NEAR-INFRARED CHOLANGIOGRAPHY CAN FACILITATE A LAPAROSCOPIC CHOLEDOCHOLITHOTOMY EVEN AFTER UPPER ABDOMINAL SURGERY

\section{Y.Y. Liu}

General surgery, Chang gung memorial hospital kaohsiung division, KAOHSIUNG, Taiwan

Background: The treatment of common bile duct (CBD) stones is challenging while unclear hepatic hilum anatomy especial experience of previous laparotomy. A minimally-invasive approach choledocholithotomy is feasible, but can be difficult and converted for the unclear anatomy of the biliary tree. Near-infrared (NIR) cholangiography by systemic administration of Indocyanine Green (ICG) can enhance the visualization of the biliary tree anatomy but is limited by the high intensity of background fluorescence signal coming from the liver. NIR fluorescence cholecysto-cholangiography by direct biliary tree administration of the ICG can enhance the biliary tree without background noise signal. We created the NIR cholangiography via different route according to patient situation : systemic circulation or biliary tree injection to see the feasibility of those application.

Material and method: Ten patients who suffered from obstructive jaundice due to CBD stone and 5 patients received percutaneous biliary tree drainage as first treatment and 2 patients received endoscopic biliary tree drainage. Those patients received laparoscopic choledocholithotomy as definite treatment after acute infection phase. 5 patients received biliary tree ICG injection via drain tube and 5 patients by systemic injection. Visualization and fluorescence patterns around CBD was recorded. Results: In our series, one patient received previous gastrectomy and 4 patients had previous biliary tree surgery. 2 Patients received PTGBD and 3 patient received PTCD for biliary decompression. The near infra-red cholangiography of ten patients can show the clear margin of CBD. There were no intra or postoperative complications. Median time to CBD localization after ICG injection was $5.2 \mathrm{sec}$ for biliary tree injection and $35 \mathrm{~min}$ for systemic injection. One patient converted to open method due to big stone impaction in distal CBD. No mortality and mobidity were found.

Conclusions: In conclusion, fluorescence cholangiography through direct biliary tree or systemic ICG injection could provide visualization of CBD even inflammatory or post operative adhesion status, and direct injection via biliary tree can have real-time and noise-free image comparing systemic injection.

\section{P269-HEPATO-BILIAIRY \& PANCREAS-Gallbladder}

INDEX LAPAROSCOPIC CHOLECYSTECTOMY, SINGLE CENTRE EXPERIENCE AFTER IMPLEMENTING AN ACUTE CARE SURGERY PROGRAM

\section{M.Y. Kayyal, L. Simmons}

Surgery, Cork University Hospital, CORK, Ireland

Background: Laparoscopic cholecystectomy (LC) has become the gold standard for the treatment of gallstone disease. Multiple studies have confirmed its safety, LC at index admission is still not widely practiced in Ireland. We present our experience in performing index cholecystectomy at CUH after the start of acute care surgery program in May 2017.

Aim: The aim of this study is to determine the safety of laparoscopic cholecystectomy at index admission, complications,re-admissions, and LOS.

Methods: Electronic records, Theatre records and Imaging reports were searched to enroll all patients who underwent LC for gallstone disease at index admission from May17 to October18. Patient demographics, indication for surgery, postoperative complications, readmission an conversion rate were recorded.In addition timings of MRCP AND ERCP, imaging findings, and LOS were also noted.

Results: A total of 117 patients underwent LC during the study period. Median age was 47 years (18-79). Male to female ratio was 1: 1.78. 75(64\%) patients had acute cholecystitis, $12(10 \%)$ had acute biliary pancreatitis, $10(8.5 \%)$ biliary colic and $9(7.6 \%)$ had cholecystitis with signs of CBD obstruction.

$7(5.9 \%)$ patients had obstructive jaundice and one with adenomyomatosis. 50 patients $(42 \%)$ had preop MRCP while 23 (19\%) underwent pre-op ERCP. All except 3 patients undergoing ERCP had preprocedure MRCP. 2 patients had pre-op cholangiograms. In terms of complications, $2(1.7 \%)$ patients had bile leak and one $(0.85 \%)$ had re-operation. One patient had the post-op hematoma which was drained percutaneously, One patient had procedure abandoned because of bradycardia upon induction of anesthesia. There was no Common bile duct injury, no conversion to open and no 30 days mortality was reported. The average length of hospital stay has been 6 days. (2to18 days). Conclusions: Laparoscopic cholecystectomy at index admission for cholecystitis, choledocholithiasis, and biliary pancreatitis, has been a safe and feasible treatment option in our hospital. A safe practice can be ensured by adherence to a care pathway and a multidisciplinary, consultant-led service. Index cholecystectomy service can be provided safely across the country to prevent diseaserelated morbidity and multiple re-admissions in patients awaiting interval surgery.

\section{P271-HEPATO-BILIAIRY \& PANCREAS-Gallbladder}

WHEN TO USE THE TWO-STAGE SURGERY TO TREAT
CHOLEDOCHOLITHIASIS: THE SIZE DOES MATTER

C.C. Hung, C.Y. Hsu, I.S. Chen

Surgical Department, Kaohsiung Veterans General Hospital, KAOHSIUNG, Taiwan

Aims: The treatment of choledocholithiasis has been provided by various of studies worldwide The most common accepted minimal invasive treatment was two-stage treatment using endoscopic retrograde cholangiopancreatography before or after laparoscopic cholecystectomy $(\mathrm{ERCP}+\mathrm{LC})$, and one-stage treatment with laparoscopic exploration of the common bile duct(LCBDE). In fact, despite several large studies have been published in recent years, the debate for the ideal treatment of choledocholithiasis is way from being concluded. We aim to find the proper treatment option for the patients with variable sizes of choledocholithiasis. Methods: We retrospectively analyzed 136 patients who underwent treatments for cholidocholithiasis in our institute between January 1, 2011 and July 31, 2016. The patients who received either ERCP and LC in the same admission, and the patient who received LCBDE, irrespective of trans-cystic(LTCBDE), or choledochotomy(LCD), were included. The data was analyzed with Chi-square test and Mann-Whitney U test.

Results: The stone size of the ERCP + LC group is significantly smaller than the LCBDE group. We further analyzed the ERCP failure case, and the group of stone size $>=9.5 \mathrm{~mm}$ has a significantly higher rate of procedure failure. The failure rate is increasing with the stone size.

Conclusions: Both the treatment of LCBDE and ERCP + LC have similar safety and success rate, and the rate of residual stone was also similar in both group. However, the failure rate for ERCP is significantly increased when stone size is larger than $9.5 \mathrm{~mm}$ in this study.

Keywords: Laparoscopic, Endoscopic, Choledocholithiasis

\section{P272-HEPATO-BILIAIRY \& PANCREAS-Gallbladder}

DIFFICULTY OF SURGICAL TREATMENT IN
XANTHOGRANULOMATOUS CHOLECYSTITIS:
DIFFERENTIAL DIAGNOSIS WITH GALLBLADER
CARCINOMA

I.M. Muñoz Núñez, S.C. Alonso García, I. Plata Pérez, B. Cantarero Jiménez, M. Maes Carballo, F. Herrera Fernández

Cirugía General y Aparato digestivo, Hospital Santa Ana, MOTRIL (GRANADA), Spain

Aims: The xanthogranulomatous cholecystitis (XC) is a rare entity that can cause doubt in the choice of surgical treatment, because of differential diagnosis with gallbladder carcinoma (GC). Methods: A 70-year-old patient presented acute abdominal pain in the right upper quadrant, nausea and low-grade fever with signs of peritonitis. He had elevated PCR, leukocytosis with neutrophilia. Abdominal ultrasound showed an acute xanthogranulomatous cholecystitis. A laparoscopic cholecystectomy was decided but it was converted to open surgery due to the difficulty in the dissection, with fundus embedded in the hepatic bed and intraoperative finding of hilar adenopathic conglomerate .The postoperative period was torpid, with abdominal pain, jaundice, elevated bilirubin and enzymes of cholestasis. Postoperative abdominal tomography showed injury in the IV segment of the liver suggestive of neoplasia. Metastatic adenopathic conglomerate at the hepatic hilum caused extrinsic biliary obstruction with hepatic failure later so an internal-external drain was placed in the bile duct. The patient was died a week later. The pathological anatomy reported a stage four of GC Results: XC is a rare, non-neoplastic, inflammatory and destructive entity of the gallbladder wall, considered a variant of chronic lithiasic cholecystitis. It may be due to extravasation of bile or ulceration of the mucosa, causing an inflammatory reaction and fibrosis, with xanthomatous cells. The prevalence is 1 to $2 \%$ in the resected gallbladders. It is more frequent in 60-70 years oldfemales. Its clinical presentation does not have specific characteristics that differ from cholelithiasis, except for the weight loss. Radiologically it is characterized by nodular thickening and increased attenuation of the vesicular wall with signs of cholecystitis, indistinguishable from a VC. The xanthogranulomatous inflammatory foci infiltrate the hepatic parenchyma, having an invasive behavior; hence, it mimics a neoplastic disease. The confusion in diagnostic and the risk of GC (up to $10 \%$ ) makes treatment contentious.

Conclusions: The XC can simulate an advanced GC that sometimes makes us wonder if we should perform a radical surgical treatment; when presented in an emergency situation, our therapeutic decision can focus on solving the acute problem and be conditioned by the patient's general condition. 


\section{P273-HEPATO-BILIAIRY \& PANCREAS-Gallbladder}

TRASNSUMBILICAL SINGLE PORT LAPAROSCOPIC SURGERY; A RETROSPECTIVE CASE SERIES FROM A SINGLE GROUP IN MEXICO CITY

\author{
A. Martínez Oñate ${ }^{1}$, A. Martínez Salas ${ }^{2}$ \\ ${ }^{1}$ Surgery, Private Practice, MEXICO CITY, Mexico; ${ }^{2}$ Urology, \\ Hospital Manuel Gea González SS, MEXICO CITY, Mexico
}

Single Port Transumbilical Laparoscopic Surgery (SPTLS) is a techinque that has been around for about 18 years. Although the enthusiasm for this type of surgery seems to have diminished in recent years it is expected to rise considering the recent development of sophisticated devices for its execution. We report retrospectively our 8 year experience with 253 procedures performed by SPTLS technique. in a private practice setting in Mexico City.

Procedures include Cholecystectomy (107), Appendectomy (69), Inguinal hernia TAPP and TEP (26), Hiatus and esophageal (15), Sleeve gastrectomy (2), Colon (6), GYN (28) . 4 different access platforms were employed. We explain our selection criteria for the application of the technique and describe the evolution of the instruments employed during the past 8 years, from laparoscopic conventional to curved and bendable; regular scopes to extra long telescopes with different angles. OR time, TOP bleeding, conversion rate, the need to employ an extra trocar, complications, pathology reports, scheduled or urgent kind of surgery and length of hospital stay were recorded from the beggining; patients variables such as BMI, ASA status, TEP risk, satisfaction with the procedure and other were recorded We describe the evolution of our technique, and our learning curve with cholecystectomies. We compare our group of SPTLS Transvaginal Assisted Laparoscopic Hysterectomy (TVALH) patients vs TVALH Multiport patients. We explain the feasibilty, and efficiency of the procedure in our hands compared to other series.

\section{P274-HEPATO-BILIAIRY \& PANCREAS-Gallbladder}

\section{THE EFFECT OF C-REACTIVE PROTEIN LEVEL ON OPEN CONVERSION RATE IN LAPAROSCOPIC CHOLECYSTECTOMY FOR ACUTE CHOLECYSTITIS}

Y. Matsui, E. Tanaka, S. Ishida, K. Horita, M. Yamada, R. Mikami, T. Murakami, T. Nitta, T. Harada

\section{Surgery, Kobe City Medical Center West Hospital, KOBE, Japan}

Background: In Japan, the severity of acute cholecystitis(AC) is assessed by the severity classification of the Tokyo Guidelines 2018 (TG18). The value of C-reactive protein (CRP) is not included in the severity classification criteria. The first line treatment, according to TG18, for mild (grade I) to moderate (grade 2) AC is laparoscopic cholecystectomy, but laparoscopic surgery may not be feasible in some cases due to adhesion or local inflammation of the gall bladder.

Aim: The aim of this study is to assess the effect of CRP on the open conversion rate in laparoscopic cholecystectomy for acute cholecystitis.Method: We conducted a retrospective study. 41 patients who were diagnosed with AC and treated with emergent laparoscopic cholecystectomy between June 2017 and May 2018 in our institution are included. We set the cutof value for CRP at $20 \mathrm{mg} / \mathrm{dL}$ and compared the open conversion rate. Secondary endpoints are amount of bleeding, operation time, post-operative course (peak in body temperature and inflammatory markers) and the frequency of complications according to the Clavien-Dindo classification.

Results: 10 out of 41 patients had a CRP value greater than or equal to $20 \mathrm{mg} / \mathrm{dL}$. The median CRP values for the CRP $<20$ group and CRP $=20$ group were 4.4 and 23.1 , respectively. The open conversion rate of the $\mathrm{CRP}=20$ group was significantly higher than that of the $\mathrm{CRP}<20$ group $(3 / 10,1 / 31, p=0.01)$. The most common reason for these conversions was local adhesion (3/4). There were no differences in the amount of bleeding, operation time, post-operative course, and frequency of complications with Clavien-Dindo grade II or higher.

Conclusion: A CRP value of $20 \mathrm{mg} / \mathrm{dL}$ is a possible cutoff point for the feasibility of laparoscopic cholecystectomy in acute cholecystitis.

\section{P275-HEPATO-BILIAIRY \& PANCREAS-Gallbladder}

CLINICAL VALUE OF FLUORESCENT CHOLANGIOGRAPHY FOR THE PATIENTS WITH INFRAPOTAL TYPE OF THE RIGHT POSTERIOR BILE DUCT

T. Igami, Division of Surgical Oncology

Department of Surgery, Nagoya University Graduate School of Medicine, NAGOYA, Japan

Background: Reports about clinical value of fluorescent cholangiography using indocyanine green (ICG) during single-incision laparoscopic cholecystectomy (SILC) were increasing. We report clinical value and pitfalls of fluorescent cholangiography during SILC for the patients with the infraportal type of the right posterior bile duct.

Methods: Our SILC procedure utilized the SILS-Port with an additional 5-mm forceps through the umbilical incision. Before SILC, $1 \mathrm{~mL}$ of ICG $(2.5 \mathrm{mg})$ was administrated by intravenous injection. For fluorescent cholangiography, ICG fluorescent laparoscope system was used.

Results: We performed fluorescent cholangiography during SILC in 13 patients with the infraportal type of the right posterior bile duct. All procedures were completed successfully. The interval from the injection of ICG to the first obtained fluorescent cholangiography before the dissection of Calot's triangle ranged from 40 to $60 \mathrm{~min}$. Detectability of infraportal type of the right posterior bile duct before dissection in Claot's triangle was $23.1 \%(\mathrm{n}=3)$ and that during dissection in Calot's triangle was $53.8 \%(\mathrm{n}=7)$. The infraportal type of the right posterior bile duct could be identified under fluorescent cholangiography only when it joined into the common hepatic duct.

Conclusions: Utilization of fluorescent cholangiography can lead SILC to safe even for the patients with the infraportal type of the right posterior bile duct. Its benefit is emphasized when the infraportal type of the right posterior bile duct joins into the common hepatic duct.

\section{P276-HEPATO-BILIAIRY \& PANCREAS-Gallbladder}

\section{A RARE COMPLICATION OF EMERGENCY LAPAROSCOPIC} SURGERY: ACCIDENTAL SECTION OF VENTRICULOPERITONEAL SHUNT

B. Bascuas Rodrigo, A. Aguilar Marquez, Y. Lara Fernandez, B. Lopez Duran, E. Perea del Pozo, F. Pilo Uceda, F. Oliva Mompean General Surgery, SAS, SEVILLA, Spain

Aims: Due to the development of laparoscopic surgery and the progress made in surgical treatment ofhydrocephalus, surgeons may come across patients with ventriculoperitoneal (VP) shunt, as candidates for laparoscopic procedures. According to this fact, we report a case of an unusual complication of laparoscopy surgery that can appear in this kind of patients.

Methods: We present a case of a 66-year-old man with medical history of normotensive hydrocephalus with VP shunt, that came to the emergency room complaining of abdominal pain and fever since two days. Blood test showed an elevation of infection parameters and inflammatory markers, and the ultrasound study revealed an emphysematous cholecystitis. Therefore, we decide to carry out an emergency laparoscopic cholecistectomy. The patient did not present any adverse event during the surgery or the immediate postoperative period, being discharged the third postoperative day and evaluated ambulatory one month after the surgery with no complications. Two months after surgery, the patient returned to the emergency room presenting alteration in consciousness and fever.

Results: During the study of the pacient, an abdominal CT was performed, showing a complete section of the VP shunt in the subcutaneus space of the upper abdominal wall and intraperitoneal migration of the remaining catheter. The patient was transferred to Neurosurgery to carry out an emergent replacement of the ventriculoperitoneal shunt. After surgery and intravenous antibiotic treatment, the patient evolved favourably and was discharged a few days later.

Conclusions: The rate of serious complications associated with a laparoscopic approach is overall low and up to $50 \%$ of them occur during the abdominal access for camera or port placement and may not be recognized until postoperative period. VP shunts should not be a contraindication for laparoscopic surgery. However, laparoscopy approach must be carry out with good anesthetic and monitoring facilities and taking several previous considerations, such as verifying the proper functioning of the VP shunt, identifying the path of the catheter within the abdominal wall to avoid inadvertent damage to the catheter during trocar placement and ensuring that the intraperitoneal portion of the catheter is not twisted or obstructed prior to decompression of the abdomen. 


\section{P277-HEPATO-BILIAIRY \& PANCREAS-Gallbladder}

\section{LAPAROSCOPIC CHOLECYSTECTOMY. THE DANGER IS ALWAYS PRESENT}

\section{A.F. Aranzana Gómez, J. Malo Corral, J. Hernandez Gutierrez, A. Trinidad Borras, B. Muñoz Jimenez,} R. Lopez Pardo, P. Toral Guinea, G. Krasniki, M.A. Morlan Lopez General Surgery, Complejo Hospitalario Toledo, TOLEDO, Spain

Objective: Demonstrate the safety and efficacy of the standardization of the surgical procedure, to avoid iatrogenic and vascular injuries during laparoscopic cholecystectomy, required when unexpected findings such as the presence of a chronic cholecystitis along with unexpected undiagnosed cirrhosis are present, risk factors of intraoperative complications

Material and method: Clinical case. A 59-year-old man with no medical-surgical history, abdominal pain compatible with recurrent biliary colic. Clinical and analytical examination without alterations, sonographic study: Vesicle distended with multiple stones, thickened wall, normal bile duct, normal rest.

Result: Intervention in a case-day regimen, in another hospital, with no possibility of performing intraoperative cholangiography. Laparoscopic approach, 4 trocars, sclero-atrophic cholecystitis, severe macronodular liver cirrhosis, Calot's triangle dissection, individualization of the elements of the hilum, above the groove of Rouviere, critical vision of Strasberg, evidencing cystic duct with implantation in right hepatic duct with vascular anomaly that presents right hepatic artery engulfing the exit of the cystic duct. Clipaje duct and cystic artery, completing cholecystectomy and liver biopsy.Normal postoperative, discharged to the 2 nd day. Pathological anatomy. chronic cholecystitis, cirrhosis grade $b$.

Conclusions: The presence of unexpected findings as risk factors during a regulated cholecystectomy, requires extreme care for possible iatrogenic injuries during surgery, standardization of the procedure facilitates the realization of it

\section{P278-HEPATO-BILIAIRY \& PANCREAS—Gallbladder}

\section{NEW PORTS PLACEMENT FOR ROBOTIC CHOLECYSTECTOMY}

\section{T. Yoo, W.T. Cho}

Surgery, Dongtan Sacred Heart Hospital Hallym University College of Medicine, HWASUNG-SI, Korea

Introduction: Since advantages of robotic surgery is being more emphasized, robotic cholecystectomy (RC) cases are increasing. Ajou group had introduced a method called which technique places the trocars transversally on the bikini line and it makes cosmesis and pain beneficial. However, RC with low incision port has several limitations. Therefore, we changed port placement which may be a one of safe tehniques for RC.

Method: This study retrospectively reviewed data for patients who received RC with port changing method (RCPC, $n=33$ ) and RC with low incision port (RCLI, $n=81$ ) from February 2016-February 2017 and surgical variables were analyzed.

Results: Patients in both groups had similar demographic features and indications for surgery. The RCPC group required no conversions to conventional robotic surgery and no additional operation, whereas the RCLI group had one incisional hernia (1.2\%) and two bowel perforation $(2.4 \%)$ cases. Length of stay $(4.29 \pm 0.72$ vs. $5.13 \pm 0.93$ days, respectively; $\mathrm{p}=0.123) \mathrm{did}$ not significantly differ between the RCPC and SCLI groups. However, the RCPC group had shorter operative time $(71.30 \pm 48.88$ vs. $74.70 \pm 30.16 \mathrm{~min} ; \mathrm{p}=0.772)$ than the RCLI group, although the parameters mentioned above were not statistically significant.

Conclusion: Robotic cholecystectomy with bikini line incision has some limitations even though it has cosmetic benefits. Whereas robotic surgery with changing port method is one of safe and feasible procedures for performing robotic cholecystectomy. Also nothing more to say that it gains cosmesis effect and escapes complications.

\section{P279-HEPATO-BILIAIRY \& PANCREAS—Gallbladder}

\section{DIAGNOSTIC AND SURGICAL TACTICS IN YATROGENIC INJURIES OF THE EXTRAHEPATIC BILIARY DUCTS}

V.V. Mischenko

Mini surgery, Odessa medical university, ODESSA, Ukraine

The aim of the study was to optimize the diagnostic and therapeutic tactics for yatrogenic injuries of the extrahepatic bile ducts.

Methods: 15 patients were examined. Typical manifestations were jaundice, cholangitis, biliary peritonitis, external biliary fistula, subhepatic abscess.Cholecystectomy main cause of damage.A visual, manual and X-ray examination of the hepato-choledochus and cholangioscopy were performed. Ultrasound, endoscopic retrograde cholangiopancreatography, fistuloholangiography or percutaneous transhepatic cholangiography play a leading role in diagnosing.

The results: High damage to the bile duct was detected in $53.3 \%$ of patients, low-in 46.7\%.Percutaneous transhepatic drainage under ultrasound control was performed in $66.7 \%$ of patients.Emergency laparotomy, sanation of the abdominal cavity and external drainage of the bile ducts were performed with bile peritonitis. Recovery operations produced $60.0 \%$ of patients. Reconstructive interventions were performed in $40.0 \%$ of patients after $6-8$ weeks after the first stage. The covery operations were successful in $66.7 \%$ of patients. $33.3 \%$ of the sick had complications in the form of biloma. A scar stricture formed in $33.3 \%$ of patients after 4-6 months. 1 patient underwent recanalization of the stricture zone with a dilatation balloon through interchangeable transhepatic drainage. Balloon dilatation was performed retrogradely through the large duodenal papilla in 2 patients. Deaths in the postoperative period was not observed.

Conclusions: The surgical team should be strengthened by an experienced surgeon when intraoperative diagnosis of yatrogenic damage to the bile ducts. The operation should be completed by external drainage of the bile duct and the abdominal cavity in the absence of an experienced specialist.Recovery operations are shown only with lateral injury of the ducts.The patient must be sent to a specialized institution for radical surgical treatment after stabilization of his general condition.

\section{P280-HEPATO-BILIAIRY \& PANCREAS-Gallbladder}

\section{SUBHEPATIC COLLECTION AFTER LAPAROSCOPIC CHOLECYSTECTOMY}

K. Haxhirexha ${ }^{1}$, F. Dika-Haxhirexha ${ }^{2}$, L. Zylbehari ${ }^{2}$, T. Emini ${ }^{1}$, A. Ademi

${ }^{1}$ Surgery, Medical Faculty-University of Tetove, TETOVE, Macedonia; ${ }^{2}$ General Medicine, Medical Faculty-University of Tetove, TETOVE, Macedonia

Laparoscopic cholecystectomy is the most used surgical technique to treat cholecystolithiasis. The most common complications occurring in the postoperative period during LC are bleeding, bile duct leakage, damage to the bile duct, damage to the intestine, abdominal wall hematoma etc. Aim of the study Sub-hepatic bile collections, biloma and hematoma are rare complications and we present our experience in treatment this complications.

Material and methods: from 750 laparoscopic cholecystectomy performed in our clinic, three patients (two women and one men) to whom it was performed laparoscopic cholecystectomy, came back two weeks later after they were released from the hospital because of epigastric discomfort, fever and nausea.

Results: Clinical examination after rehospitalization showed tenderness in the epigastrium and right subcostal region. In all patients were measured high levels of leukocytosis and CRP . An ultrasound examination of the abdomen revealed a large hypoechoic collection in the sub hepatic space, after the abdominal CT scan was performed, the density of the collection did not indicate the presence of blood in two patients.

Percutaneous drainage of the collection in both patients was realized under US guidance and 8-10 Fr catheter was inserted in the sub hepatic region. In the first patient $800 \mathrm{cc}$ of bile-stained liquid, and in the second patient $650 \mathrm{cc}$ of biliary liquid was drained. In a third patient $16 \mathrm{~h}$ after surgery signs of significant hypotension and limited tenderness at the right subcostal region occurred. A complete blood count (CBC) showed a decrease in the level of haemoglobin to $10.4 \mathrm{~g} \%$. Ultrasound examination revealed a fluid collection in the sub hepatic space, which is also confirmed by computed tomography. Laparotomy was performed and the large sub hepatic hematoma was evacuated. After that the $18 \mathrm{Fr}$ abdominal drain was inserted into the sub hepatic space. The postoperative course of all three patients was not complicated.

Conclusion: Sub hepatic biloma and hematoma are rare complications of laparoscopic Cholecystectomy, while early diagnosis followed by percutaneous drainage or open laparotomy is the only way to resolve these complications.

Key words: Laparascopic cholecystectomy, biloma, hematoma 


\section{P281-HEPATO-BILIAIRY \& PANCREAS-Gallbladder}

YEAR EXPERIENCE WITH RENDEZVOUS TECHNIQUE IN AN EMERGENCY HOSPITAL IN SANTIAGO DE CHILE

\section{Aguilera, D. Gutierrez, F. Moraga, D. Paz, M. Lillo, A. Dominguez, P. Olivares}

Emergency, Hospital De Urgencia Asistencia Publica, SANTIAGO, Chile

Introduction: The optimal treatment for cholecystolithiasis associated with choledocholithiasis remains controversial. The rendezvous technique combines laparoscopic cholecystectomy and endoscopic sphincterotomy in one stage. Fewer complications have been reported associated with cannulation of the duodenal papilla compared to the 2-stage resolution. Its use is infrequent in public hospitals in the country due to the lack of availability of endoscopist. We present the results obtained with this technique in our emergency center.

Objective: To evaluate in a descriptive way the experience with rendezvous technique in our center, causes, success rate, operative time, complications, hospital stay and mortality.

Methodology: Cohort study retrospective. Patients operated on laparoscopic cholecystectomy associated with endoscopic sphincterotomy by rendezvous technique in the Public Assistance Emergency Hospital of Santiago de Chile between February and November of 2018. The statistical analysis was performed through SPSS V - 24. Sociodemographic characteristics, admission diagnoses, cannulation success, biliary clearence, surgical time, complications, hospital stay and mortality were evaluated.

Results: 29 patients underwent the procedure in the period. 51.7\% (15) female. Average age of 44 years. $17.2 \%$ (5) ASA I, $65 \%$ (19) ASA II and 17.2\% (5) ASA III. Most frequent diagnosis of admission: acute cholecystitis $72.4 \%$ (21), choledocholithiasis $24.1 \%$ (7) and acute pancreatitis $3.4 \%$ (1). The cannulation of the papilla was achieved in $100 \%$, biliary clearence of $89.7 \%$ (26). A biliary stent was installed in $10.3 \%$ (3). $3.4 \%$ (1) required conversion to open surgery. The average surgical time was $158 \mathrm{~min}$. Acute mild pancreatitis $10.2 \%$ (3), hemoperitoneum $3.4 \%$ (1). The average number of days of hospitalization was 7.6 days. There was no mortality at 30 days.

Conclusion: In the emergency setting the rendezvous technique has an adequate success rate of cannulation and clearence of the bile duct, an acceptable surgical time, few complications, these being more frequent in those patients with inflammation of the gallbladder and without associated mortality at 30 days. There is a need for controlled randomized studies with a greater number of patients recruited and follow-up to determine the usefulness of this technique.

\section{P282-HEPATO-BILIAIRY \& PANCREAS-Gallbladder}

EASY AND EFFECTIVE METHOD OF INTRAOPERATIVE CHOLECYSTOGRAPHY DURING LAPAROSCOPIC CHOLECYSTECTOMY

\section{Y. Mihara, T. Kanazawa, M. Takahashi, K. Kawabe}

Surgery, JR tokyo general hospital, TOKYO, Japan

Intraoperative cholangiography could serve as a fundamental solution to avoid the bile duct injury during laparoscopic cholecystectomy. However, it is difficult to identify the cystic duct to which the contrast catheter should be inserted in cases with high degrees of adhesion around the Calot's triangle. In these cases, it is not possible to conduct cholangiography from the cystic duct. For these types of cases, intraoperative cholecystography may serve as an option. However, since the bladder is a bag-like organ that expands when liquids are entered, directly inserting a contrast dye into the bladder would make the bladder itself expand, which makes it impossible for to maintain enough pressure in the contrast dye to flow into the cystic duct, extrahepatic bile duct, and intrahepatic bile duct. Also, since it is difficult to control leakage of the contrast dye from the catheter insertion site, it is not possible to obtain enough images to sufficiently understand the anatomical characteristics of the bile duct in many cases. Therefore, cholecystography is not generally recognized as a method to be used during surgery. In our facility, we insert the contrast catheter through the bladder after stretching the gallbladder neck as much as possible, hold the gallbladder neck with a removable intestinal clamp, and then apply the contrast dye to the bile duct. Through this method, it is possible to insert enough contrast dye into the cystic duct, extrahepatic bile duct, and intrahepatic bile duct to understand the anatomical characteristics of the bile duct, allowing us to obtain appropriate images of the biliary tract. Because this method uses equipment that is highly versatile, we believe that it is inexpensive and convenient. During this presentation, we will also conduct a case presentation of the methods of bladder contrasting that we utilize in our facility during laparoscopic cholecystectomy.
P283-HEPATO-BILIAIRY \& PANCREAS-Gallbladder

IMPROVING ERGONOMICS OF GALL BLADDER RETRIEVAL AT LAPAROSCOPIC CHOLECYSTECTOMY - THE DELHI MANEUVER

A. $\operatorname{Ali}^{1}$, A. Saha ${ }^{1}$, S. Kalhan ${ }^{2}$

${ }^{1}$ Dept of GI, HPB, Bariatric \& Minimal Access Surgery, Venkateshwar Hospital, NEW DELHI, India; ${ }^{2}$ Institute of Minimal Access Surgery, Sir Ganga Ram Hospital, NEW DELHI, India

Introduction: Retrieval of a thick walled gallbladder during a difficult laparoscopic cholecystectomy (LC) for an acute or chronic calculous cholecystitis can be exasperating. It increases operative time and often necessitates enlargement of $10 \mathrm{~mm}$ port to deliver the specimen. The 'in-situ cholecystotomy', which we wish to call the 'Delhi Maneuver' is very helpful in improving the ergonomics of specimen retrieval, saves time and conserves cosmesis.

Patients \& methods: One hundred and ten patients of Acute or Chronic Calculous cholecystitis were placed randomly in 2 groups. A disposable transparent plastic bag was used in all cases to retrieve the gallbladder specimen through the $10-12 \mathrm{~mm}$ port using a Rampley's sponge holding forceps. Retrieval was done using conventional technique in 60 patients (Group B). The Delhi Maneuver was used in the remaining 50 patients (Group A). It involved cutting the gall bladder inside the plastic bag in a certain fashion, delivering the gallstones in the bag, and removal of gallbladder preceding the stones. The retrieval time, number of insertions of sponge holder, any rupture of plastic bag as well as the number of cases needing port enlargement were noted. Results: The average time taken by Delhi Maneuver (group A) was 9 min as compared to was 14 min by conventional method (group B). The number of insertions of sponge holder ranged from 3-11 in group A (mean 5) and 5-18 in group B (mean 13). Four patients needed port enlargement in group A $(8 \%)$ while 17 patients needed enlargement in group B $(28.3 \%)$. There were 2 incidences of bag rupture in group A (4\%) and 3 in group B (5\%).

Conclusion: The Delhi Maneuver improved the ease and speed of specimen extraction at Laparoscopic Cholecystectomy for thick walled gallbladders. It also decreased the need for port enlargement for specimen retrieval.

\section{P284-HEPATO-BILIAIRY \& PANCREAS-Gallbladder}

MANAGEMENT OF BILE DUCT INJURIES, 10 YEARS' EXPERIENCE IN A PRIVATE HOSPITAL IN MEXICO

H. Segura-Marin, G. Arredondo-Saldaña, J.A. Diaz-Elizondo, E. Flores-Villaba

General Surgery, Escuela de medicina del Tecnologico de Monterrey, MONTERREY, Mexico

The bile duct injuries are a very complex desease to confront, the inciian managment is to clasificate the injury and to identifie the mechamism of the injury. It's important for the optimal heal of the patient to have a multidisciplinary approach including internal medicine, surgery, endoscopy and interventional radiology specialists. The laparoscopic cholecystectomy responsible for $80 \%-85 \%$ of them.This is a retrospective study on the incidence, classification and management of bile duct injuries in a private sector hospital in Monterrey NL. Mexico. In this study, 17 bile duct injuries were identified in 10 years of experience in a single center. were categorized using the Strasberg classification. Variables were evaluated such as type of injury, mechanism of injury, hospital stay, if the surgery was scheduled or of emergency, the moment in which the surgeon evidenced the injury, the way in which the surgeon became aware of the injury performed. The type of management that was given to this lesion was also studied and the days of intrahospital stay and the number of reinterventions or procedures performed were compared.The average age of the patients was 53 years, 10 patients belonged to the female sex, Although there were lesions of all kinds in this work, there was a greater incidence in Strasberg type A lesions, which represented $41 \%$ of the lesions. The most common diagnosis presented was cholecystolithiasis. In 7 surgeries the evidence and repair of the bile duct was in the same intervention. 13 surgeries were by laparoscopy and 4 open cholecystectomy. There were 3 hepatic jejunum anastomosis and $3 \mathrm{~T}$-tubes. 


\section{P285-HEPATO-BILIAIRY \& PANCREAS-Gallbladder}

\section{LAPAROSCOPIC MANAGEMENT OF POST- CHOLECYSTECTOMY BILE LEAK}

\section{A.M. Harris, Q. Azeem,}

General Surgery, Hinchingbrooke Hospital, HUNTINGDON, United Kingdom

Aims: Bile leak is a rare but recognised complication after laparoscopic cholecystectomy. This usually occurs after a difficult procedure complicated by adhesions, unusual anatomy or if the surgeon is inexperienced or unfamiliar with the anatomy. This video aims to demonstrate the laparoscopic diagnosis and treatment of this complication particularly for surgical trainees. Methods: We report a case of significant bile leak occurring soon after a straightforward laparoscopic cholecystectomy due to very short cystic duct (CD). The procedure was carried out uneventfully but the $\mathrm{CD}$ was clipped flush with the bile duct. The patient was discharged on the day of surgery feeling well but readmitted with abdominal pain $48 \mathrm{~h}$ later.

Results: After readmission the patient underwent a CT demonstrating only a small amount of fluid suggestive of a small collection. She was treated conservatively but suddenly deteriorated and a repeat CT confirmed significant intraperitoneal fluid. A diagnostic laparoscopy was carried out urgently confirming a CD stump bile leak where the clips had sloughed off causing the leak. Two litres of bile was aspirated with copious irrigation and a latex t-tube inserted into the CBD Patient made a full and rapid recovery.

Conclusions: This is a rare complication and learning opportunities for trainees are therefore infrequent. This video demonstrates a successful laparoscopic approach to management of postoperative bile leak showing t-tube insertion technique and highlighting the need for careful $\mathrm{CD}$ closure techniques during laparoscopic cholecystectomy when the duct is very short.

\section{P286-HEPATO-BILIAIRY \& PANCREAS-Gallbladder}

SPYGLASS CHOLEDOCHOSCOPE: A CASE REPORT AND THE EXPERIENCE OF DIFFICULT BILE DUCT STONES MANAGEMENT IN A PRIVATE HOSPITAL OF MEXICO

\section{G. Arredondo ${ }^{1}$, H. Segura ${ }^{1}$, M. Rodarte Shade ${ }^{1}$, J. Jaquez ${ }^{2}$}

${ }^{1}$ General Surgery, Escuela de Medicina y Ciencias de la Salud del Tecnológico de Monterrey, MONTERREY, Mexico; ${ }^{2}$ Endoscopy, Hospital Zambrano Hellion TecSalud, MONTERREY, Mexico

About $10-15 \%$ of bile duct stones could not be extracted using conventional endoscopic tech niques (baloon, sphincterotomy). There is lower success rate in elderly patients; among the biggest challenges are intrahepatic stones, size of stone is large, etc.

Aims: To present the case of a recurrent intrahepatic lithiasis and its management using Spyglass choledochoscopy.To expose, other cases and the main outcome and complications of other difficult cases of bile duct stones that solvedusing this Choledochoscope vs. the traditional one and the beneffits.

Methods \& results: We present a case of 85 years old male who presented with cholangitis caused by an intrahepatic stone that required multiple sessions of endoscopic retrograde cholangiopancreatography with Spyglass for clearance. One year later, he presented again with cholangitis, that required another session of Spyglass lithotripsy and cholecistectomy.

Conclusions: Besides ERCP, there are different approaches to treat difficult bile duct stones, as transhepatic percutaneous drainage, surgical techniques, or other endoscopic techniques (doubleballoon, enteroscopy). ERCP and sphincterotomy are the first step of endoscopic treatment with more than $90 \%$ of success rate, and a low mortality and morbility rate; other steps include some lithotripsy techniques, or the use of biliary stent as a bridge before definite treatment. Spyglass is a visualization \& intervention system used when common ERCP has been unsuccessful, and it is first line for better and direct image of biliary ducts, with $12^{\circ}$ range of motion, with multiple advantages like the concomitant use of lithotripsy devices.

\section{P287-HEPATO-BILIAIRY \& PANCREAS-Gallbladder}

CHOICE OF THERAPEUTIC APPROACH IN ACUTE CHOLECYSTITIS IN THE OCTOGENARIAN PATIENTS

G. Cakmak ${ }^{1}$, F. Altintoprak ${ }^{2}$, K. Ozdemir ${ }^{3}$, M.Y. Uzunoglu ${ }^{1}$, N. Firat ${ }^{1}, \mathrm{~K}$. Gundogdu ${ }^{1}$, F. Celebi ${ }^{3}$

${ }^{1}$ General Surgery, Sakarya University Research and Educational Hospital, SAKARYA, Turkey; ${ }^{2}$ General Surgery, Istinye University, School of Medicine, ISTANBUL, Turkey; ${ }^{3}$ General Surgery, Sakarya University, School of Medicine, SAKARYA, Turkey

Aims: The number of elderly people has increased, because of the strong association between age and gallstone disease, both prevalence and incidence of this disease are increasing. This presentation aims to review our current management options of octogenerian patients with acute cholecystitis.

Methods: We retrospectively analyzed 173 octogenerian patients who were admitted to the our hospital with the diagnosis of acute cholecystitis between January 2013 and October 2018. The patients were initially allocated to four different treatment groups as follows: immediate surgery, delayed surgery, medical treatment and cholecystostomy. Differences in the outcomes between the treatment groups were evaluated.

Results: There were 67 males $(38.8 \%)$ and 106 females $(61.2 \%)$ with a mean age of 85.7 years (range 80-90 years). The patients had different co-morbid diseases, especially hypertension (65, $37.5 \%)$ cardiovascular disease $(43,24.8 \%)$ and diabetes mellitus $(29,16.7 \%)$. Mostly patients had Grade 3 cholecystitis $(81,46.8 \%)$. Treatment options as follows: immediate surgical intervention in thirty-three $(19 \%)$ patients, cholecystostomy in $26(15 \%)$ patients, delayed surgery in $23(13.2 \%)$ patients and medical treatment in $88(52.8 \%)$ patients. Mortality rates according to treatment options were as follows: 4 out of 33 patients $(12.1 \%)$ in the immediate surgery group, 3 out of 88 patients $(3.4 \%)$ in the medical treatment group, 0 out of 23 patients $(0 \%)$ in the delayed surgery group and 3 out of 26 patients (11.5\%) in the cholecystostomy group. In the immediate surgery group; twenty four surgeries were performed as laparoscopic $(72.7 \%)$ with 7 cases (29.1\%) of conversion to open surgery and the remaining 9 patients $(27.3 \%)$ underwent open surgery. There were 27 postoperative complications in all patients (82/170, except medical treatment) as follows: intraabdominal abscess (10 patients, $12.1 \%)$, bilioma ( 8 patients,9.7\%), liver bed bleeding ( 4 patients, $4.8 \%$ ), haemobilia ( 3 patients, $3.6 \%$ ) and cystic stump leakage ( 3 patients,3.6\%). Subtotal cholecystectomy was performed in thirteen patients $(39.3 \%)$ in immediate surgery group. Fourty one patients $(24.1 \%)$ followed in intensive care unit on postoperative period or medical treatment course.

Conclusion: The evaluation for the initial treatment in acute cholecystitis in octogenarian patients should include a systematic determination of the degree of cholecystitis, patients comorbidities and a surgical risk assessment. 


\section{P288-HEPATO-BILIAIRY \& PANCREAS-Gallbladder}

\section{OUTCOMES OF LAPAROSCOPIC CHOLECYSTECTOMY IN} ELDERLY PATIENTS WITH ACUTE CHOLECYSTITIS

A. García Reyes ${ }^{1}$, C. Franco Peñuelas ${ }^{1}$, M. Retamar Gentil ${ }^{1}$, A. Cano Matías $^{1}$, B. Marenco de la Cuadra ${ }^{1}$, M. Sánchez Ramírez ${ }^{1}$, E. Pérez Margallo $^{1}$, J. Reguera Rosal ${ }^{1}$, F. Oliva Mompeán ${ }^{2}$, J.A. López-Ruiz ${ }^{1}$

${ }^{1}$ Emergency surgery, Hospital Universitario Virgen Macarena, SEVILLA, Spain; ${ }^{2}$ Colorectal surgery, Hospital Universitario Virgen Macarena, SEVILLA, Spain

Aim: To analyze the outcomes in patients with acute cholecystitis (AC) older than 75 years and younger.

Methods: A retrospective observational study where were analyzed patients older than 75 years who underwent urgent surgery for $\mathrm{AC}$ who fulfilled an indication for surgery according to Tokyo guidelines 2018 .

The type of cholecystitis, stay and postoperative complications, the type of intervention, the conversion rate, the need for reoperation and re-admissions in patients older than 75 years were analyzed and compared with those of patients operated on for cholecystitis younger than 75 years.

Outcomes: A total of 289 patients were registered, 55 older than 75 years $(19 \%)$ and 234 younger $(81 \%)$.

In 128 cases, cholecystitis were complicated (44.3\%), 34 cases older than 75 years $(26.56 \%)$ and in 94 cases younger than 75 years $(73.44 \%)$.

The approach was laparoscopic in $89 \%$ of the cases older than 75 years, with a conversion rate of $10.2 \%$, not finding statistically significant differences with younger than 75 years $(91 \%$ laparoscopies with $4.2 \%$ of conversions)

$18 \%$ of patients older than 75 years had some type of postoperative complication, not finding statistically significant differences in patient younger than 75 years $(17 \%)$; being the most frequent complication the intrabdomintal abscess $(3.66 \%$ of patients $>75$ years, and $4.27 \%$ of those $<75$ years $=$ " span $=">$ being not statistically significant with $95 \%$ CI.

Any patient older than 75 years required re-entry after discharge, compared to 8 patients younger than 75 years who were re-entered, not being statistically significant; and any patient older than 75 years required reintervention, while it was necessary to reoperate 3 patients younger than 75 years $(1 \%)$, being not statistically significant.

Mortality was very low, finding 1 case in older than 75 years $(1.8 \%)$ and 1 case in younger $(0.4 \%)$, not obtaining statistically significant differences. The postoperative stay in patients younger than 75 years of age has a median of 3 days and in older than 75 years a median of 4 days, not finding statistically significant differences with $95 \%$ CI

Conclusions: Laparoscopic cholecystectomy is safe and effective in the treatment of elderly patients with (AC), there being no differences with younger patients.

\section{P289-HEPATO-BILIAIRY \& PANCREAS-Gallbladder}

SURGICAL MANAGEMENT OF ACUTE CHOLECYSTITIS AT INDEX ADMISSION

G. Dedemadi, P.L. Chalkias, E. Bouka, I. Georgieva, K. Karkalemis, S. Adamopoulos, G. Dedemadi

Amalia Fleming Surgical Department, Sismanogleio-Amalia Fleming Hospital, ATHENS, Greece

Aim: Acute cholecystitis occurs in $20 \%$ of patients with cholelithiasis. Laparoscopic cholecystectomy should be offered to all patients, exception of high risk of morbidity or mortality patients. The aim is to present the management of acute cholecystitis at index admission and compare with elective management of cholelithiasis.

Methods: An 8-year retrospective study was conducted from January 2010 to November 2018. Patients presenting with cholelithiasis and acute cholecystitis, were identified. Data on patient demographic, length of hospital stay, peri-operative complications, and conversions to open procedure were recorded. Index cholecystectomy has been defined as that performed in patients with acute cholecystitis 10 days from onset of symptoms or 4 to 6 days of diagnosis.

Results: A total of 1747 patients underwent cholecystectomy; 1435 were performed electively and 312 at index admission. $312(28.3 \%)$ patients with acute cholecystitis underwent index cholecystectomy. In the elective cholecystectomy group 1389 patients underwent laparoscopic procedure, 29 open and 13 conversions to open, while in the index cholecystectomy group, 242 laparoscopic, 28 open and 40 conversions to open. Index cholecystectomy was associated with higher conversion rate $12.8 \%$ vs $0.9 \%$ in the elective cholecystectomy group. 11 patients with acute cholecystitis and 1 patient with chronic cholecystitis had a subtotal cholecystectomy, performed using laparoscopic (50\%), open $(33.3 \%)$ and laparoscopic converted to open $(16.7 \%)$ procedures. Men and elder patients were more likely to undergo index cholecystectomy for acute disease than patients in elective cholecystectomy group 53.2 vs $40.06 \%$, and 64.52 (median 24-91) vs 56.49 (median 19-90), respectively. Length of hospital stay was shorter in elective cholecystectomy group 5.56 (median 3-39) days vs 9.69 (median, (3-28) days. Complication rate was similar $4.48 \%$ vs $3.06 \%$ among patients. Bile duct injuries were minor, and repaired after conversion to an open procedure. One death was encountered after index cholecystectomy.

Conclusions: Index admission laparoscopic cholecystectomy can be performed safely in patients with acute cholecystitis and should be considered the gold standard for management of these patients as mortality, morbidity, complications and conversion rate remain relatively low. A higher conversion rate to an open procedure must be accepted when treating complicated disease. 


\section{P290-HEPATO-BILIAIRY \& PANCREAS-Gallbladder}

RE-LAPAROSCOPY IN DIAGNOSTIC AND MANAGEMENT OF POSTOPERATIVE BILE LEAK AFTER EXTRAHEPATIC BILIARY OPERATIONS

O.I. Lytvyn, O.Y.u. Usenko, M.Ye. Nichitaylo, P.V. Ogorodnik, O.I. Lytvyn, A.G. Deynichenko

Laparoscopic surgery, National institute of surgery and transplantology, KYIV, Ukraine

Introduction: Significant bile leak is an uncommon but serious complication of laparoscopic cholecystectomy. Our study aims to evaluate the efficacy of relaparoscopy in treating symptomatic bile leak and biloma formation.

Material and methods: 125 patients presenting with postoperative bile leak after different operations on extrahepatic biliary tree from January 1993 to December 2018 were reviewed retrospectively (In total, 23,590 laparoscopic surgical interventions were performed for the period under study). The sites of bile leaks were the cystic duct stump in thirty seven patients, the bile ducts of Luschka in fifty two, liver beds in 16 cases after hepatectomy, in 13 had small injury of CBD, and seven patients with tubular stenosis of the common bile duct.

Results: Three main approaches of mini-invasive treatment of bile leakage was used: (1) percutaneous puncture with or without drain under CT-scan or ultrasound guidance in 45 patients; (2) endoscopic management in 50 patients (in 35 patients $(70.0 \%)$ were managed with ERCP alone and fifteen $(30.0 \%)$ were treated with a percutaneous intervention followed by ERCP. Endobiliary stent placement was performed after ES in 23 patients and without ES in twenty seven patients (3) relaparoscopy has been performed in 30 patients, in cases of biliary peritonitis. Conclusions: Relaparoscopy was the ultimate method of treating postoperative complications of laparoscopic surgery in $94.3 \%$ of patients. In general, this method, as well as laparoscopic intervention, is highly effective in the diagnosis and correction of postoperative complications, with minimal surgical trauma for the patient, with great therapeutic effect and subsequent rapid social rehabilitation of patients.

\section{P291-HEPATO-BILIAIRY \& PANCREAS—Gallbladder}

LAPAROSCOPIC CHOLECYSTOGASTROANASTOMOSISNEW OR FORGOTTEN Method: FOR UNRESECTABLE PERIAMPULLARY CANCER

\author{
Iu. Mikheiev, V. Yareshko, K. Otarashvily
}

Surgery and minimally invasive technologies, State Institution, Zaporizhia Medical Academy of Post-Graduate Education Ministr, ZAPORIZHIA, Ukraine

Introduction: Laparoscopic operations have already become routine, even for pancreatoduodenectomy for periampular cancer. For unresectable cases, endoscopic bibliary stenting or hepaticojejunostomy are usually used. These methods are quite expensive and may be accompanied by complications.

Materials and methods: Laparoscopic cholecystogastroanastomosis was performed in 72 patients with unresectable periampullary cancer. There were 34 females and 38 men and average age was 72,6 . The indications for surgery in all patients was unresectable periampullary cancer and biliary hypertension with preserved patency of the cystic duct. The level of bilirubinemia ranged from 89 to $520 \mu \mathrm{mol} / \mathrm{l}$ (the average level was $179,8 \mu \mathrm{mol} / \mathrm{l}$ ). We used 3-port technique. Optical trocar was placed in the right iliac region, one $10 \mathrm{~mm}$ above the navel and one $5 \mathrm{~mm}$ in the right hypochondrium After punction gallblaber and aspiration of bile, we cut the apex of the gallbladder and gastric antrum up to $2.5 \mathrm{~cm}$ and performed cholecystogastroanastomosis with barbed-suture V-Loc.

Results: We had not conversion to open surgery. The average operation time was $37 \mathrm{~min}$. Postoperative stay was average 4 days and on median follow-up of 12 month. Post-operatively, there were no major morbidity and nil mortality. We had 2 cases of leakage of bile through drainage for up to 3-5 days, which spontaneously stopped. All patients showed a decrease in the level of bilirubinemia. 12 patients were later radical operated (pancreatoduodenectomy), while they did not have such phenomena as cholangitis, pancreatitis, inflammation of the hepatoduodenal ligament elements, which we often observe after endoscopic biliary stenting.

Conclusions: Laparoscopic cholecystogastroanastomosis is safe, effective and feasible for patients with periampular cancer and obstructive jaundice.

\section{P292-HEPATO-BILIAIRY \& PANCREAS-Gallbladder}

THE EFFECTIVENESS OF THE " ONE STAGE APPROACH" FOR THE MANAGEMENT OF COMMON BILE DUCT STONES-A CASE SERIES. RESULTS OF A SINGLE SURGICAL UNIT

P. Christopoulos, A. Bush, R.M. Jones, S. Sinha, K. Bowling, G. Srinivas, S. Andrews

South Devon Upper GI Unit, Torbay General Hospital, TORQUAY, United Kingdom

Aims: Surgeons with the expertise and resources to perform laparoscopic common bile duct exploration often prefer the 'one stage approach' over endoscopic retrograde cholangio-pancreatography (ERCP) for the management of common bile duct (CBD) stones. This case series aims to evaluate the effectiveness of LCBDE in a single benign upper gastrointestinal (GI) unit.

Methods: All patients with suspected and confirmed pre-operatively CBD stones who underwent a LCBDE between January 2015 and October 2018 were included. LCBDE was performed on the basis of pre-operative suspicion of CBD stone confirmed by intra-operative imaging.

Results: 187 patients with confirmed choledocolithiasis had LCBDE during this time period. The indications for LCBDE were deranged liver function tests, dilated CBD or confirmed stones on preoperative imaging. Median age was 63 (Range 19-91), 67\% of whom were female. $36 \%$ of patients had confirmed CBD stones pre-op. $70 \%$ of cases were performed as emergencies and conversion rate to open was $6.5 \%$. Choledocotomy was performed in $60 \%$ of cases. In $17 \%$ of these t-tube was left in situ. Transcystic approach was used in the remaining $40 \%$. Despite positive intraoperative imaging no stones were found on CBD exploration in 11 cases $(6 \%)$. In 3 patients stones were unable to be cleared with LCBDE. The overall morbidity was $20 \% .11 \%$ of patients had gallstone related complications. Overall mortality was $1 \%$ (due to bile leak). 19/187 patients required re-intervention with re-look laparoscopy $(n=6)$ or ERCP $(n=13) .3$ patients re-presented within 3 months with CBD stones. Overall median length of stay was 5 days Conclusions: Our case series demonstrates that LCBDE is an effective and safe treatment for choledocolithiasis in both the elective and emergency settings. Complication rates are comparable with therapeutic ERCP (10\% specific complications) followed by laparascopic cholecystectomy (10\% 30 day morbidity).

\section{P293-HEPATO-BILIAIRY \& PANCREAS-Gallbladder}

PERCUTANEOUS CHOLECYSTOSTOMY FOR THE TREATMENT OF ACUTE CHOLECYSTITIS IN THE CRITICALLY ILL AND ELDERLY

J. Routledge, M. Chaplen, S. Sinha, K. Bowling, S. Andrews, G. Srinivas

Upper GI, General Surgery, Torquay Hospital, TORQUAY, United Kingdom

Aims: Percutaneous cholecystostomy (PC) is a well-established procedure in acute cholecystitis (AC) especially in high-risk patients. The purpose of this study is to evaluate the clinical efficacy and outcomes of PC in elderly and critically ill patients who have acute cholecystitis. Methods: Retrospective review on all patients who underwent PC for AC between January 2015 and October 2017 at Torbay hospital.

Results: 34 patients were included with a median age of 77 years. Technical success was $100 \%$ and there were no procedure-related mortalities. Significant post-procedural improvement on inflammatory markers were observed in terms of white cell count and CRP. Subsequently, 16 (47\%) patients underwent elective cholecystectomy, 4 open and 12 laparoscopic. Two patients are currently waiting for cholecystectomy. 16 patients $(47 \%)$ were managed conservatively and not offered surgery due to high risk, 3 of these patients developed further episodes of biliary infections managed with antibiotics. The overall 30 -day mortality was $11 \%$ and were all in the non-surgical group.

Conclusion: Percutaneous cholecystostomy is a viable treatment option for elderly and critically ill patients presenting with acute cholecystitis. It has a high success rate with minimal procedurerelated complications. However, the majority of patients who subsequently had a cholecystectomy had a better outcome. 


\section{P294-HEPATO-BILIAIRY \& PANCREAS-Gallbladder}

PROGNOSTIC VALUE OF PREOPERATIVE NEUTROPHIL-TO LYMPHOCYTE RATIO FOR PREDICTION OF SEVERE CHOLECYSTITIS

D. Micic $^{1}$, S. Mijatovic ${ }^{1}$, B.. Oluic ${ }^{1}$, D. Krstina ${ }^{1}$, D. Rujevic ${ }^{1}$, S. Polovina ${ }^{2}$

${ }^{1}$ Surgery, Clinic for Emergency surgery, Emergency center, Clinical center Serbia, BELGRADE, Serbia; ${ }^{2}$ Endocrinology, Clinic for Emergency surgery, Emergency center, Clinical center Serbia, BELGRADE, Serbia

Background: The predictive value of preoperative neutrophil-to-lymphocyte ratio (NLR) in patients with cholecystitis has not been established. The aim of this study was to investigate preoperative NLR in patients with cholecystitis and to identify a relevant NLR value that discriminates between simple and severe cholecystitis.

Methods: This study consisted of 136 patients who underwent laparoscopic cholecystectomy due to cholecystitis. The Receiver Operating Characteristic (ROC) analysis was performed to identify the most useful NLR cut-off value in relation to the severity of cholecystitis. The patients were divided into two groups according to the cut-off NLR value: high NLR group $\left({ }^{3} 4.18, \mathrm{n}=23\right)$ and low NLR group $(<4.18, \mathrm{n}=113)$. Severe cholecystitis was defined as state which including inflammation, empyema, gangrene, perforation of gallbladder, adhesions or difficulty in dissecting Calots triangle.

Results: High NLR group had significantly more frequent severe cholecystitis $(p<0.0001)$ and higher C-reactive protein level (CRP) and white blood cells count (WBC) $(\mathrm{p}<0.0001)$. There was no difference in homeostatic model assessment-insulin resistance index (HOMA-IR) between both groups before the operation $(\mathrm{p}<0.634)$. The incidence of severe cholecystitis was $16.9 \%$. The NLR of 4.18 could predict severe cholecystitis with $78.3 \%$ sensitivity and $74.3 \%$ specificity. Spearman's correlation revealed significant association between the preoperative NLR and HOMA-IR on day $1,(\mathrm{r}=0.254, \mathrm{p}=0.030)$ and between preoperative NLR and CRP on day $1(\mathrm{r}=0.355 ; \mathrm{p}<0.0001)$

Conclusions: NLR $=4.18$ was significantly associated with severe cholecystitis. The preoperative NLR in patients undergoing cholecystectomy due to cholecystitis could be a useful surrogate marker of severe cholecystitis.

Keywords: Cholecystitis, Inflammatory biomarkers, neutrophil-to-lymphocyte ratio, prognosis

\section{P295-HEPATO-BILIAIRY \& PANCREAS-Gallbladder}

\section{IS LAPAROSCOPIC CHOLECYSTECTOMY AS SIMPLE AS IT} LOOKS?

\section{O. Andronic, D.N. Paduraru, A. Bolocan, D. Ion}

General Surgery and Emergency III, Emergency Universitary Hospital of Bucharest, BUCURESTI, Romania

Aims: Today, laparoscopic cholecystectomy is the gold standard in acute cholecystitis management, being the laparoscopic surgery most commonly performed worldwide. Medical practice has shown that any seemingly 'banal' intervention can be complicated by the most complex repercussions on patient evolution. In this context, we asked ourselves: what are the particularities of the learning curve in the case of laparoscopic cholecystectomy?

Methods: Our study was retrospective, unicentric, descriptive, analyzing in a comparative manner cases of acute cholecystitis from 2007-2008 in relation to 2017-2018. The cases of 3 distinct teams were analyzed. The analysis focused on a number of surgical intervention parameters: the ratio of open / laparoscopic cases, the duration of the surgery, the conversion rate and a number of postoperative parameters: the number of complications, the number of hospitalization days and other variables.

Results: The study included 214 cases, divided into two lots: the first group of patients in the 2007-2008 period, respectively the 2 nd group covering the period 2017-2018. The results indicate a significant increase in the percentage of laparoscopically resolved cases and improved postoperative evolution by decreasing both the number of days of hospitalization and the number of complications. As far as the duration of the interventions is concerned, it did not show any significant variation. Taking into account the fact that this year's cases were included, the results are partial and will be finalized soon.

Conclusion: Although the easiest laparoscopic surgery, laparoscopic cholecystectomy often presents difficulties that can be real pitfalls even for experienced surgeons.

\section{P296-HEPATO-BILIAIRY \& PANCREAS-Gallbladder}

RETROSPECTIVE ANALYSIS OF ENDOSCOPIC RETROGRADE CHOLANGIOPANCREATOGRAPHY (ERCP) SUCCESS RATE AND COMPLICATIONS

\author{
S. Krivan ${ }^{1}$, P. Kiritharamohan ${ }^{2}$, B. Ramesh ${ }^{2}$, D. Whitelaw ${ }^{1}$
}

${ }^{1}$ Upper Gastrointestinal and Bariatrics, General Surgery, Luton and Dunstable Hospital, LUTON, United Kingdom; ${ }^{2}$ General Surgery, Luton and Dunstable Hospital, LUTON, United Kingdom

Introduction: Endoscopic retrograde cholangiopancreatography (ERCP) has evolved from a diagnostic test to an advanced therapeutic procedure. ERCP can treat biliary, pancreatic and ampullary diseases. Post-ERCP complications include pancreatitis up to $15 \%$, cholangitis $1-5 \%$, haemorrhage and perforation up to $2 \%$.

Materials-Methods: This is a retrospective analysis of ERCP data collected at Luton and Dunstable University Hospital from 01.10.2017 until 30.09.2018. Overall, 236 procedures were revised after excluding diagnostic ERCPs. Data was collected regarding successful procedures, successful first cannulation, as well as second and third attempts. Indications, findings, successful interventions and complications were monitored.

Results: In total, 240 ERCP were collected from 01.10.2017 until 30.09.2018. Four diagnostic procedures were excluded. Of the remaining, $168(80 \%)$ were successful, $179(85.2 \%)$ were successful at first cannulation, 23 had a second attempt with success rate almost $80 \%$ and 3 had a third successful attempt. Dilated common bile duct (CBD) secondary to passed stones was diagnosed in $15.2 \%$ with a clear duct on cholangiogram. $41.5 \%$ had CBD stones with successful stone/debris extraction in $91.8 \%$ of the cases and $17.8 \%$ showed CBD stricture with successful stenting in $83.3 \%$. Three cases were performed for bile leak post laparoscopic cholecystectomy with successful stenting, 9 relooks for stent removal and $4.6 \%$ with stent occlusion/cholangitis for stent replacement $(63.6 \%)$. Complications rate were $3.8 \%$ for pancreatitis, $3.3 \%$ for cholangitis, $2.9 \%$ for haemmorhage and $1.69 \%$ for perforation. All were treated conservatively and improved apart from one elderly patient with multiple comorbidities, who deceased post ERCP perforation. Conclusion: ERCP is a therapeutic technique for both benign and malignant pathology of the biliary tree. The success rate is high and increases in specialised units with trained endoscopists. Complication rate remains low requirung conservative management in most cases.

\section{P297-HEPATO-BILIAIRY \& PANCREAS-Gallbladder}

\section{SURGICAL OUTPUT OF ACUTE CHOLECYSTITIS IN OUR HOSPITAL}

T. Yazawa, R. Sato, T. Abe, T. Okada, T. Kakita, M. Oikawa, T. Tsuchiya

Gastrointestinal surgery, Sendai City Medical Center, Sendai Open Hospital, SENDAI-SI, Japan

Introduction: In our hospital, we have tried to perform early surgery against acute cholecystitis. Tokyo guideline 2018, which is surgical management of acute cholecystitis by Japanese Society of Hepato-Biliary-Pancreatic Surgery, extends the adaptation of early operation for a part of severity Grade III cholecystitis. We analyzed our surgical results of cholecystitis retrospectively and examined its validity.

Materials and methods: There were 174 patients who underwent laparoscopic cholecystectomy (LC) after diagnosis of acute cholecystitis at the first visit, in the period from 1 January 2013 to 31 December 2015. We examined the time from diagnosis to operation and these postoperative complications for these cases.

Results: 174 cases were operated by laparoscopic procedure; male: female $=123: 51$, average age 64.5 years (22-94), severity Grade of cholecystitis ((I: II: III $=135: 25: 14)$. Time from diagnosis to surgery was as follows, 116 cases $(67 \%)$ within $72 \mathrm{~h}, 28$ cases (16\%) within $72 \mathrm{~h}$ from 1 week, and 30 cases (17\%) over 1 week. Even in cases diagnosed as Grade III, surgery was performed within $72 \mathrm{~h}$ in 7 of 14 cases. Regarding Clavien-Dindo classification, Post operative complications of Grade II or higher were confirmed in 15 cases (8.6\%). Details were as follows; Respiratory complication ( 8 cases, $4.6 \%$ ), cholangitis (including choledocolithiasis) (3 cases, $1.7 \%$ ), transverse colon perforation ( 1 case, $0.6 \%$ ), pseudomembranous colitis ( 1 case, $0.6 \%$ ). Conclusions: In our hospital, We operated about $70 \%$ of acute cholecystitis cases within $72 \mathrm{~h}$ after diagnosis by laparoscopic procedure, and those courses were almost in good condition. Laparoscopic surgery for acute cholecystitis including severity Grade ? should be performed as soon as practicable, if the condition would meet. 


\section{P298-HEPATO-BILIAIRY \& PANCREAS-Gallbladder}

\section{EMPHYSEMATOUS CHOLECYSTITIS: A RARE} COMPLICATION FOLLOWING ERCP

\section{K.A. Malik}

Presenting a case report following an ERCP developing emphysematous cholecystitis in multiple comorbid patient who was successfully treated conservatively. A rare entity for education and highlighting management aspects.
Surgery, Wrexham Maelor Hospital, WREXHAM, United Kingdom

\section{P300-HEPATO-BILIAIRY \& PANCREAS-Liver}

LI-RADS SCORE IN HEPATOCELLULAR CARCINOMA DIAGNOSIS AND MANAGEMENT: A RETROSPECTIVE STUDY

N. Mariani ${ }^{1}$, M. Barabino ${ }^{1}$, C. Fochesato ${ }^{1}$, R. Santambrogio ${ }^{1}$, G. Franceschelli ${ }^{2}$, A.S. Angileri ${ }^{2}$, E. Opocher ${ }^{1}$

${ }^{1}$ General Surgery, ASST Santi Paolo e Carlo, MILAN, Italy; ${ }^{2}$ General Radiology, ASST Santi Paolo e Carlo, MILAN, Italy

Nowadays, the diagnosis of liver cancer is primarily radiological, as recommended by the principal international societies. In doubtful cases or due to the clinician needs, diagnostic evaluations can eventually be completed with a liver biopsy. The goal is to perform the examination, or the examinations, that guarantee the most elevated sensibility and specificity levels being as little invasive as possible. Nevertheless, even using the best radiological tools, the diagnosis is not certain, due both to device limitations and radiology experience. Recently, various diagnostic algorithms have been proposed, relating with contrast enhancement characteristics, different radiological techniques, blood examinations and cross evaluations from different radiologists. One of the most recent algorithm purposed is Liver Imaging Reporting and Data System (LIRADS), that evaluates CT and MRI imaging to classify hepatic lesions in different diagnostic categories, in order to perform a better and more precise diagnosis of HCC or other liver benign or malignant lesion. Through a retrospective study, we evaluated and compared preoperative imaging and post-operative histological reports. Results reveal that LIRADS routine use increases HCC diagnosis up to $95 \%$.

\section{P301-HEPATO-BILIAIRY \& PANCREAS-Liver}

\section{DUCT OF LUSHCKA. SOURCE OF COMPLICATIONS}

A. Antequera, C. Macano, M.. Lopez, M. Robba, R. Edmons, N. Coleman

Surgery, St Bernards Hospital, GIBRALTAR, Gibraltar

Subvesical bile ducts usually called 'ducts of Luschka' have gained increased clinical relevance in the era of laparoscopic cholecystectomy. The variability in anatomic location of subvesical bile ducts puts them in danger during hepato-biliary operations. Its prevalence varies between $3 \%$ and $10 \%$. The origin and drainage of these ducts were limited mainly to the right lobe of the liver, but great variation could be seen. Some authors think of them as small bile ducts that drain directly into the body of the gallbladder; others consider them to be networks of miniscule bile ducts between the liver capsule and the gallbladder. Recent studies suggest that clinically relevant bile leaks complicate approximately $0.4-1.2 \%$ of cholecystectomies. Injury to a subvesical duct is one of the most common causes of cholecystectomy associated bile leak and occurs as often as major bile duct injuries and leaks from the cystic duct stump. Indeed, recent studies suggest that about $27 \%$ of clinically relevant bile leaks are caused by inadvertent injury to a subvesical bile duct. There are four types of subvesical bile ducts, including (1) superficial variations of segmental and sectorial bile ducts, (2) superficial or intercommunicating accessory bile ducts, (3) hepaticocholecystic ducts, and (4) aberrant bile ducts.We present a case of 73 year old patient who developed a coleperitoneum after a routine daycase colecystectomy due to the inadvertent injury of a hepatocholecystic duct. A superior comprehension of ductal anatomy is essential in preventing and managing operative injury to the subvesical ducts, although some times is unavoidable.

\section{VALIDATION OF DIFFICULTY SCORING SYSTEM OF LAPAROSCOPIC LIVER RESECTION FOR HEPATOLITHIASIS}

\section{J.Y. Cho, J. Kim, H.S. Han, Y.S. Yoon, Y.R. Choi, B. Lee}

Surgery, Seoul National University Bundang Hospital, SEONGNAM, Korea

Background: We previously developed a modified difficulty scoring system (DSS-IHD) of laparoscopic liver resection (LLR) for patients with underwent LLR for hepatolithiasis.

Methods: DSS-IHD was based on the extent of liver resection (2 to 4), stone location ( 1 to 5 ), atrophy of liver parenchyma ( 0 to 1 ), ductal stricture $<1 \mathrm{~cm}$ from the bifurcation (0 to 1$)$, and combined choledochoscopic examination for remnant IHD (0 to 1 ).

Results: The DSS-IHD ranged from 3 to 12 and divided to 3-level groups of low group (score $3 \sim 5 ; \mathrm{n}=26$ ), intermediate group $(6 \sim 8 ; \mathrm{n}=72$ ), and high group $(9 \sim 12 ; \mathrm{n}=23)$. The mean operation time $(187.4 \pm 80.2$ vs. $348.8 \pm 147.6$ vs. $367.8 \pm 141.1 ; P<0.001)$ and the mean blood loss $(354.2 \pm 301.3$ vs. $849.4 \pm 968.4$ vs. $1083.0 \pm 955.2 ; P=0.004)$ were significantly different among groups. The mean hospital stay was also marginally different among groups $(7.6 \pm 3.5$ vs. $13.4 \pm 11.6$ vs. $13.2 \pm 11.2 ; P=0.054)$. When we simply divided patients by right and left LLR, there were significant differences in DSS-IHD (8.9 $\pm 2.0 \mathrm{vs}$. $6.5 \pm 1.6 ; P<0.001)$, operation time $(472.4 \pm 185.3$ vs. $289.7 \pm 124.5$; $P<0.001)$ and blood loss $(1810.5 \pm 1269.7$ vs. $596.8 \pm 656.1$; $P=0.001$ ) between right and left LLR group.

Conclusions: The surgical difficulty varies among patients undergoing LLR for IHD stones. The DSS-IHD predicts well the surgical difficulties of LLR for patients with IHD stones. intrahepatic duct (IHD) stone. We validated DSS-IHD in patients who 


\section{P302-HEPATO-BILIAIRY \& PANCREAS-Liver}

PARENCHYMAL-SPARING LIVER RESECTIONS (OPEN AND LAPAROSCOPIC) IN METASTATIC COLORECTAL CANCER

\section{O.O. Kvasivka, K.V. Kopchak, HPB surgery}

National cancer institute, KYIV, Ukraine

Background: Modern chemotherapy regiments and biological agents with various surgical strategies increase number of patients with resectable colorectal liver metastasis (CRLM). Difficult group to cure is patients with bilobar metastasis. There are two different surgical strategies treat these patients: parenchymal-sparing liver resection (PSLR), hemihepatectomy in different modifications(PVE, ALPPS, two stage). Because of high probability of early intrahepatic recurrence and necessity to perform further resections such division of surgical strategies is practical.

Method: Between 2012 and 2018 from a total cohort of 239 patients resected for CRLM, 73 (30.5\%) were with bilobar liver metastasis. In 51 (69.9\%) of them the resection was carried out in one stage for all nodules, 7 of them from laparoscopic access, in the other $22(30.1 \%)$ cases hemihepatectomy in different modifications was performed. In 2018 we start laparoscopic liver parenchymal-sparing resection.

Results: R0 resection in PSLR group were 38 (74.5\%), R1-13 (25.5\%), in hemihepatectomy group: R0 $-15(68.1 \%), \mathrm{R} 1-7$ (31.9\%) respectively. Overall complication rate in PSLR group was $15(29.4 \%)$, in hemihepatectomy group $-7(35 \%)$, among them post resection liver failure (PRLF) in PSLR group-2 (2,8\%), in hemihepatectomy group-3 (14.2\%). There were $3(5.8 \%)$ cases of biliary fistula in PSLR group and $3(14.2 \%)$ in hemihepatectomy. In-hospital mortality was $1(1.9 \%)$ in PSLR, $2(10 \%)$ in hemihepatectomy group.

Protocol of surgical intervention included intraoperative ultrasound examination of the liver and determine relationship between metastases and main structures. We perform ligament lymph node dissection if on CT or MRI was found positive lymph nodes. For parenchymal transection we use crash-clamp technic.

Conclusions: Although of more technical complicities, larger total wound area in case of PSLR that can probably lead to increase of biliary fistula, the more segmental brunches preserved the more variety of decisions surgeon can make in recurrence settings. Parenchymal sparing liver resections in case of bilobar liver metastasis should be the main choice of radical surgical strategy and allow to increase the chance of repeat resection in case of liver recurrence.

\section{P303-HEPATO-BILIAIRY \& PANCREAS-Liver}

THE USE OF MINIMALLY INVASIVE INTERVENTIONS IN THE TREATMENT OF CHOLANGIOGENIC LIVER ABSCESSES

V.A. Vovk ${ }^{1}$, V.V. Boyko ${ }^{2}$

${ }^{1}$ Surgical, Regional Clinic Hospital, Kharkiv, Ukraine, KHARKIV, Ukraine; ${ }^{2}$ Surgical, Institute of General and Emergency Surgery, KHARKIV, Ukraine

Objective: Improving the surgical treatment of patients with cholangiogenic abscesses of the liver through the application of minimally invasive technologies.

Material and method: In the presented study presented results of treatment of 49 patients with biliary liver abscesses. Surgical interventions for hepatic abscesses were performed simultaneously with the elimination of the primary pathological process of the biliary system, which caused the occurrence of cholangitis, or in the near future (up to 3 days) after biliary drainage drainage. Among 49 patients with biliary liver abscesses, treated with minimally invasive methods, 29 revealed abscesses of the right hepatic lobe, 15-abscesses of the left hepatic lobe, 5-abscesses and right and left hepatic lobes. Single abscesses were detected in 39 patients, and in 10-two or more abscesses. In terms of liver abscesses, more than $3 \mathrm{~cm}$ were detected in 43 patients, more than $5 \mathrm{~cm}$ in 6 patients. Drainage of the biliary tract was carried out endoscopically transpapillary and (if the endoscopic approach was unsuccessful) with transcutaneous transhepatic approach.

Results: Drainage under ultrasound guidance was performed on 21 patients with solitary and 7 patients with two or more cholangiogenic abscesses of the liver.

Laparoscopic interventions were performed on 21 patients.

Among the patients operated on using minimally invasive technologies, 7 occurred complications (14.3\%). 1 patient died due to the development of biliary sepsis (2.0\%).

Conclusion: Percutaneous drainage of liver abscesses under ultrasound control is appropriate not only for single abscesses, but also for their larger number, which has many advantages over other interventions. It was proved possibility of simultaneous drainage of liver abscess and bile duct. Percutaneous drainage of the liver abscess, drainage of the biliary tract and laparoscopic surgical intervention are complementary aspects in the treatment of liver abscesses of biliary origin After laparoscopy residual calculus can be removed endoscopically in more favorable conditions after stabilization of the patient's condition is achieved and the infection-associated disorders are eliminated.

In case of localization of abscesses in the marginal segments of the liver, laparoscopic atypical resection of the liver with an abscess is most desirable.

\section{P304-HEPATO-BILIAIRY \& PANCREAS-Liver}

\section{ROLE OF ROBOTIC SURGERY IN THE MANAGEMENT OF BENIGN HEPATOBILIARY DISEASES}

\section{E. Kakiashvili ${ }^{1}$, E. Brauner ${ }^{2}$, O.B. Ben Ishai ${ }^{2}$}

${ }^{1}$ General Surgery, Galilee Medical Center, KIRIAT MOZKIN, Israel; ${ }^{2}$ General Surgery, Rambam Medical Center, HAIFA, Israel

Background: Recently robotic surgery has emerged as one of the most promising surgical advances. Despite its worldwide acceptance in many different surgical specialties, the use of robotic assistance in the field of hepatobiliary (HBP) surgery remains relatively unexplored.

Our study presents single institution's initial experience of robotic assisted surgery for treatment of benign hepatobiliary pathologies.

Methods: A retrospective analysis of a prospectively maintained database on clinical outcomes was performed for 26 consecutive patients that underwent robotic assisted surgery for benign HBP disease at Rambam Medical Center during 2013-2015.

Results: There were 26 robotic assisted surgical procedures performed for benign HBP pathologies during the study period. There were 3 anatomical robotic liver resections for symptomatic hemangiomas, 9 cases of giant liver cyst, 5 robotic assisted surgery for type choledochal cyst, 2 case of benign (iatrogenic) common bile duct (CBD) stricture, 3 cases of robotic (CBD) exploration due to large intra choledochal stones and 6 cases of cholecystectomy for cholelithiasis. The median postoperative hospital stays for all procedures were 3.5 days (range 1-6 days). General morbidity (minor) was $2 \%$. There was no mortality in our series.

Conclusion: Robotic surgery is feasible and can be safely performed in patients with differen benign HBP pathologies. Further evaluation with clinical trials is required to validate it's real benefits.

\section{P305-HEPATO-BILIAIRY \& PANCREAS-Liver \\ LAPAROSCOPIC TREATMENT OF NON-INFECTIOUS HEPATIC CYSTS}

M.V. Venianaki, E. Theodorakis, K. Lasiothiotakis, I.P. Petrakis, E. Chrysos

General Surgery, University Hospital Of Heraklion Crete Greece, HERAKLION, Greece

Introduction: Benign, non-infectious hepatic cystic lesions are mostly incidental findings on imaging. Simple cysts and polycystic liver disease account for $2.5-5 \%$ and approximately $10-15 \%$ of them are symptomatic. Only symptomatic cysts are indicated to be treated surgically.SCOPEOur scope is to present our experience regarding laparoscopic treatment of benign cystic hepatic lesions analysing the technical ability, the limits and the effectiveness of the method.

Patients \& Methods: Between 1994 and 2018, 27 patients underwent laparoscopic hepatic cys deroofing in our department. Indications for laparoscopic surgery included solitary, simple liver cysts in 23 cases and polycystic liver disease in 5 cases. Only cysts localized in the forward hepatic segments were considered suitable for laparoscopic management. Patients with cholangitis, khirrosis and severe cardiac and pulmonary disease were excluded.

Results: All 27 operations were completed laparoscopically. Mean duration of surgery was $68 \mathrm{~min}$. Mean cystic diameter was $15 \mathrm{~cm}$ and all cystic lesions were drained along with broad deroofing. No deaths or significant postoperative complications resulted. Plural infusion was noted in two cases, self-limited haemorrhage came up in one case and also self-limited bile-leak resulted in one case. Mean hospital stay was determined in 4,4 days. Postoperative follow up showed no signs of recurrence or operation-related complications.

Discussion: Most of non-parasitic cystic lesions are asymptomatic in approximately $90 \%-95 \%$ and need no treatment. Due to low incidence of symptomatic hepatic cysts patients are treated on an individual base worldwide. The choice of surgical approach depends on the lesion's extent, its location at the liver and surgeon's experience. Currently laparoscopy has become the procedure of choice for deroofing in terms of postoperative morbidity including all the benefits of laparoscopic approach itself, such as limited postoperative pain, decreased blood loss and length of hospital stay.

Conclusion: Laparoscopic treatment of non-paracitic, benign hepatic cystic lesions is feasible and safe with careful selection of cases and it is related with low morbidity and limited hospita stay.Macedo et al., World J Hepatol, 2013Vardakostas et al., Eur Rev Med Pharmacol Sci, 2018Vasquez et al., J Clin Rev Case Rep, 2018Gall et al., HPB 2009 


\section{P306-HEPATO-BILIAIRY \& PANCREAS-Liver}

\section{LAPAROSCOPIC UNROOFING FOR SYMPTOMATIC LIVER CYST}

H. Kashiwagi, J. Kawachi, N. Isogai, R. Shimoyama, K. Miyake, K. Murata, N. Kume, N. Shinozaki

Surgery, Shonan Kamakura General Hospital, KAMAKURA, Japan

Most liver cysts are asymptomatic and tend to have a benign clinical course. However, symptomatic or complicated liver cysts sometimes require surgical intervention. Needle aspiration is safe and can be the lease invasive procedure, this procedure is however associated with a high failure rate and rapid recurrence. Surgical approach is the crucial and provides definitive treatment for such cysts. Thirteen cases were nominated from Shonan Kamakura General Hospital between January 2015 and December 2018. Mean age and Body Mass Index (BMI) were 67.8 and 20.8 , respectively. All patients have had any complaint such as upper abdominal pain, dyspnea, and fever. Two cases were clinically diagnosed as the infectious cyst and serum CRP was elevated before surgery. Additional cholecystectomy was planned for one case of chronic cholecystitis with gallbladder stones. All cases were prompted the reduced port surgery (RPS) and 4 cases were performed RPS with trans-vaginal approach (Hybrid NOTES) and 4 case was chosen in Single Port Surgery. Cyst unroofing was performed for all cases. Mean operation time and blood loss of all cases were $122.3 \mathrm{~min}$. and $62.7 \mathrm{ml}$, respectively. No surgical complication has been occurred in all cases, an infectious cyst case was however required additional drainage for infectious control after surgery. Although statistic difference was not shown, fewer blood loss and shorter hospital stay was seen in non-infectious cases, compared to laparotomy cases. Mean hospital stay after surgery of whole cases, non-infectious cases, infectious cases was 5.5, 2.5, 22.5 days, respectively. No recurrence of any symptom was shown in any cases in observation period (10-1392 days). Laparoscopic unroofing is the definitive treatment for the complicated or symptomatic liver cyst. However, for the infectious cyst, infection control such as intensive drainage and/or administration of antibiotic before surgery may be needed to avoid additional treatment, leading to longer hospital stay. Laparoscopic unroofing of liver cyst can be the firs choice for symptomatic or complicated liver cyst. Also, reduced port surgery can be nominated to achieve less invasiveness.

\section{P307-HEPATO-BILIAIRY \& PANCREAS-Liver}

\section{SIMULTANEOUS MINIMALLY INVASIVE SURGERY OF SYNCHRONOUS COLORECTAL CANCER WITH LIVER METASTASES}

\section{T.J. Tsai, C.C. Chen, T.Y. Cheng, C.M. Chen}

Surgery, Koo Foundation Sun Yat-Sen Cancer Center, TAIPEI, Taiwan

Aims: To evaluate the perioperative and oncological outcomes of simultaneous minimally invasive surgery of colorectal cancer with synchronous liver metastases.

Methods: 30 patients with simultaneous laparoscopic and robotic-assisted liver and colorectal resection for synchronous liver metastases were enrolled from September 2005 to June 2018. Operative and peri-operative data were retrospectively evaluated.

Results: The mean operation time was 415 mins, with the mean blood loss $213 \mathrm{ml}$. Mean hospital stay was 9 days. There was no surgical mortality. Morbidity rate was $9.4 \%$. The 5 -year disease free survival rate was $19.9 \%$ and 5-year overall survival rate was $52.2 \%$.

Conclusions: Simultaneous minimally invasive surgery can be safely performed for synchronous colorectal cancer with liver metastases with acceptable morbidity and short-term oncological outcome.

\section{P308-HEPATO-BILIAIRY \& PANCREAS-Liver}

\section{HEPATOCELLULAR CARCINOMA. LAPAROSCOPIC SEGMENTECTOMY}

A. Valverde Martínez, C. Peña, S. Cerrato, M.D. Casado, M.J. Castro, J.M. Pacheco

General Surgery, Hospital Universitario Puerta del Mar, Cádiz, CÁDIZ, Spain

Introducction: Laparoscopic liver resection (LLR) has been increasing since it was first reported in 1991. Three international expert consensus conferences on LLR surgery were held in Louisville, KY, USA, in 2008, Morioka, Japan in 2014 and Southampton, UK, in 2017. While most initial minimally invasive liver resections were typically done for benign lesions in anterior o lef segments, LLR is currently being applied for major anatomic resections, malignancy, cirrhosis and liver donor hepatectomy.

Clinical case report: This is a 78-year-old male patient with a history of HTA and liver cirrhosis due to Hepatitis B Virus. Hepatocarcinoma is diagnosed in liver segment VI with a size of $3 \mathrm{~cm}$ In the digestive study the patient presents a CHILD A stage, MELD $<9$, without signs of portal hypertension. Complete analytical with normal AFP and CEA 19.9 markers. After presentation of the patient in a multidisciplinary committee and being a stadium according to the early BCLC classification, laparoscopic surgery with segment VI resection was decided.

Discussion: Laparoscopic liver resection is becoming widely accepted for the treatment of hepatocellular carcinoma. Liver resection is a first-line option in very early and early-stage disease. Many meta-analysis have shown that LLR is better than open liver resection in terms of short-term outcomes for patients with Child-Pugh A cirrhosis, solitary tumors, and minor resections. In the long-term setting, the results demonstrate that a minimally invasive approach is comparable to an open approach in terms of overall. In conclusion, the current evidence conclude than LLRs for HCC are safe and may be considered a standard practice in specific settings.

\section{P309-HEPATO-BILIAIRY \& PANCREAS-Liver}

\section{MINIINVASIVE TECHNOLOGIES: AN EFFECTIVE AND PROMISING TREATMENT APPROACH FOR PATIENTS WITH TUMOR LESION OF PROXIMAL BILE DUCTS}

A.L. Alyanov ${ }^{1}$, A.V. Mamoshin ${ }^{1}$, A.V. Borsukov ${ }^{2}$, V.Yu. Ivanov ${ }^{3}$, A.V. Abolmasov ${ }^{1}$, A. Abdulkarimova ${ }^{1}$, A.V. Dunaev ${ }^{1}$

${ }^{1}$ Orel Regional Clinical Hospital, Orel State University named after I.S. Turgenev, OREL, Russia; ${ }^{2}$ Research and Minimally Invasive Technologies, Smolensk Regional Clinical Hospital, SMOLENSK, Russia; ${ }^{3}$ A.I.Evdokimov Moscow State University of Medicine and Dentistry, Federal Scientific and Clinical Center for Specialized Medical Service and Medic, MOSCOW, Russia

Aims: to analyze and evaluate the possibilities and effectiveness of antegrade mini-invasive technologies in the diagnosis and treatment of patients with malignant lesions of the proximal bile ducts.

Methods: 14 patients with cholangiocarcinoma of proximal bile duct (named Klatskin tumor), complicated by obstructive jaundice were examined in Orel regional clinical hospital in 2010-2015 years.

Results: There were 6 women (43\%) and 6 men (57\%). The age of patients ranged from 43 to 82 years. The patients underwent complex examination including abdominal ultrasound, Esophagogastroduodenoscopy, and some of them underwent CT (computed tomography). Al patients in the first stage were performed antegrade external drainage of biliary tracts with X-rays of the biliary tracts, and specifying the level and extent of the block.Total 28 miniinvasive interventions were hold. Two patients in connection with the uncoupling of equity ducts were performed antegrade bilobar stenting with preliminary split external bile release.There were complications after carried out interventions in 10 cases, which were associated with dislocation of holangiostomic drainage in 5 patients (35.7\%); with acute cholecystitis in 1 patient (7.1\%); with hydrothorax in 2 patients (14.2\%); perihepatic biloma in 1 case $(7.1 \%) .1$ patient $(7.1 \%)$ had a recurrence of obstructive jaundice due to germination of endobiliary stent in the late period after stenting. Lethal outcome appeared in 1 patient.

Conclusions: ultrasound examination allows us to determine the level of obstruction of the biliary tract, to substantiate the tactical position in the application of mini-invasive technologies. Antegrade miniinvasive technologies in the treatment of tumor lesions of the proximal bile ducts allow timely and effectively stop biliary hypertension and to determine further treatment strategy. Acknowledgements This study was supported by the Russian Science Foundation under project ? 18-15-00201. 


\section{P310-HEPATO-BILIAIRY \& PANCREAS-Liver}

\section{SHORT-TERM SURGICAL OUTCOMES OF LAPAROSCOPIC REPEAT HEPATECTOMY FOR RECURRENT LIVER TUMOR}

F. Hirokawa, K. Uchiyama, M. Asakuma, K. Komeda, T. Shimizu, Y. Inoue, S. Kagota, A. Tomioka

General and Gastroenterological Surgery, Osaka Medical College, TAKATSUKI, Japan

Background: Repeat hepatectomy is an effective treatment, with long-term surgical outcomes for recurrent HCC and colorectal liver metastasis(CRLM). However, the efficacy of a minimally invasive surgical approach for recurrent liver tumor is not yet confirmed. The purpose of this study is to examine the efficacy of laparoscopic repeat hepatectomy(LRH) compared with open repeat hepatectomy $(\mathrm{ORH})$ for recurrent liver tumor. We retrospectively analyzed the clinicopathological features and short-term surgical outcomes between LRH and ORH.

Methods: From 2006 to 2018, 158 patients with liver cancer underwent repeat hepatectomy. O those patients, 113 patients underwent partial hepatectomy, 37 patients were undergone laparoscopically, and 76 patients underwent open hepatectomy. We compared the clinicopathological and surgical parameters in the LRH group with those in the ORH group.

Results: There were no significant differences in patients' gender, age, viral infection status, Child-Pugh classification, tumor size, tumor number, and tumor location in the two groups. The operative times were similar, but blood loss was significantly lower in LRH group (68 vs. $310 \mathrm{ml}$, $\mathrm{p}<0.001)$. The postoperative hospital stay was significantly shorter in the LRH group $(9.0 \mathrm{vs}$. 11.5 days, $\mathrm{p}=0.016)$. Postoperative complications $(\mathrm{CD}=3 \mathrm{a}$ ) were observed only in the $\mathrm{ORH}$ group, with a complication rate of $9.2 \%$.

Conclusions: We demonstrate that LRH reduces blood loss and postoperative complications compared with ORH. LRH might be a feasible and effective procedure for the selected patients.

\section{P312-HEPATO-BILIAIRY \& PANCREAS-Liver}

\section{LAPAROSCOPIC RIGHT POSTERIOR SECTIONECTOMY IN ONE CENTER}

Y.S. Koh, C.K. Cho, Y.H. Hur, E.K. Park, H.J. Kim, K.H. Kim, $\overline{\text { H.S. Cho }}$

HBP Surgery, Chonnam National University Hwasun Hospital, HWASUN-GUN, JEOLLANAM-DO, Korea

Laparoscopic approach to the liver has become an integral part of surgery. Two consecutive international consensus meeting recommends major hepatectomy has been on the expert hands. Tumors located in the right posterior section are considered to be difficult for laparoscopic resection.

Patients and Methods: Since 2005, until 2017, CNUHH has been performing 260 laparoscopic hepatectomies including 67 major hepatectomies. Among 67 major ones, there are $36 \mathrm{RH}, 13 \mathrm{LH}$ $16 \mathrm{RPS}, 2 \mathrm{CH}$, and 1 AS. We analyze data on patient demographics, tumor characteristics, operative date, and posterior outcome retrospectively.

Results: During 2014-2017, 16 laparoscopic RPS were performed. The diagnosis were HCC in 13 and CRLM in 3 patients. Median operative time was $405 \mathrm{~min}$, and median blood loss was $850 \mathrm{~mL}$. No blood transfusion was occurred. Median tumor size was $38 \mathrm{~mm}$, and median resection margin was $12.3 \mathrm{~mm}$. Six of the 16 patients (38\%) were cirrhotic on pathology. There was no conversion and was no postoperative mortality. Median hospital stay was 11.6 days.

Conclusion: Laparoscopic RPS is known challenging procecedure. Strict preoperative planning and operative procedure is mandatory. Even though it should be performed by the experienced hands both on hepatic surgery and laparoscopic skill, it can be an good option for treatment of the tumor locating over right posterior section.

\section{P311-HEPATO-BILIAIRY \& PANCREAS-Liver}

\section{INTRAOPERATIVE ULTRASOUND IN ASSESSMENT OF HEPATIC DEPOSITS IN INTRAABDOMINAL MALIGNANCIES}

\section{M.R. Elkeleny ${ }^{1}$, A. Faried ${ }^{2}$}

${ }^{1}$ GIT and bariatric surgery, Faculty of medicine, Alexandria University, ALEXANDRIA, Egypt; ${ }^{2}$ GIT surgery department, Faculty of medicine,Alexandria university, ALEXANDRIA, Egypt

Background: The liver is the most common site of metastatic disease with up 40-50\% of all cancers having the potentiality for sending liver metastasis during the disease. Consequently, increasing value for surgical resection of hepatic deposits of different types of cancers, the need for accurate evaluation of the extent of hepatic metastasis was established for choosing the most suitable patients for surgery and in planning the extent of hepatic resection.

The aim of this work is to evaluate the role of intra-operative ultrasound in the detection of hepatic deposits in intra-abdominal malignancies with special emphasis on its accuracy, sensitivity, specificity.

Patients and method: This study was carried out on thirty patients who were admitted to the Gastrointestinal Surgery Unit, Main Alexandria University Hospital with intra-abdominal malignancies for whom elective open surgical intervention was recommended in the period from 1st of September 2017 till the 31th of March 2018

Results: in the present study consisted of 17 males (56.7\%) and 13 females (43.3\%). Their mean age at admission was $52.77 \pm 9.12$ years. Six of the included patients $(20 \%)$ were found to have hepatic lesions by using IOUS including the four cases $(13.3 \%$ ) already detected by preoperative imaging. Two cases $(6.67 \%)$ were newly discovered in the operative room by using IOUS.

Conclusion: The current study has proved that IOUS demonstrates superior lesion detection ove the various non-invasive preoperative imaging modalities causing significant impact on change of the planned surgical strategy

\section{P313-HEPATO-BILIAIRY \& PANCREAS-Liver}

\section{USEFULNESS OF ENDORACTOR IN LAPAROSCOPIC RADIOFREQUENCY ABLATION}

S. Tsuchida ${ }^{1}$, S. Asari ${ }^{1}$, K. Kanemitsu ${ }^{1}$, T. Wakahara ${ }^{1}$, T. Maeda ${ }^{1}$, S. So ${ }^{1}$, N. Ueno ${ }^{1}$, A. Toyokawa ${ }^{1}$, M. Sasako ${ }^{1}$, S. Kim ${ }^{2}$, S. Kim ${ }^{2}$

${ }^{1}$ Surgery, Yodogawa Christian Hospital, OSAKA, Japan;

${ }^{2}$ Gastroenterology, Kobe Asahi Hospital, KOBE, Japan

Purpose: Previously we developed a new sponge (named Endoractor) as an organ retraction device in laparoscopic surgery in 2009 and have reported that it is useful in various surgical procedures including rectal surgery

We confirmed that it is also useful in laparoscopic radiofrequency ablation of the liver in terms of pulling and protecting organ, so we report it

Materials and methods: A case is an 82-year-old female with liver cirrhosis. She had primary hepatocellular carcinoma in S8 lesion with a diameter of $1.8 \mathrm{~cm}$ very close to the inferior vena cava and middle hepatic vein root and in S3 lesion with a diameter of $2.0 \mathrm{~cm}$

We thought she could not put up with hepatic resection because of her poor hepatic reserve capacity. And we could not expect treatment effect by embolization therapy since contrast effect was poor. So we decided to select ablation therapy

In the puncture and ablation of the S8 tumor, since there was concern about the thermal damage of the middle hepatic vein and the cooling effect by the inferior vena cava, we would dissect the right coronary mesentery sufficiently and pull the liver apart from the inferior vena cava and the middle hepatic vein as much as possible using our Endoractor

Also, in the puncture and ablation of the S3 tumor, it was feared that the stomach would be thermally damaged, so we would place Endoclactor between the liver and the stomach to protect the stomach

Results: When ablating the S8 tumor, we could pull the liver securely without slipping, so we did not cause thermal damage to the middle hepatic vein. And there was no cooling effect by the inferior vena cava, so we could obtain sufficient cautery margin.

In ablation of S3 tumor, we were able to puncture by stabilizing the lateral segment of the liver on our Endoractor, and avoid thermal damage of the stomach

Conclusion: It seems possible to perform safe and reliable puncture and ablation by using our Endoractor as well in laparoscopic radiofrequency ablation 


\section{P314-HEPATO-BILIAIRY \& PANCREAS-Liver}

\section{LAPAROSCOPIC TREATMENT FOR RECURRENT LIVER HYDATID DISEASE-A CASE REPORT}

V.M. Surlin ${ }^{1}$, I. Georgescu ${ }^{1}$, D. Margaritescu ${ }^{1}$, A. Nicolaescu ${ }^{1}$, S. Bordu ${ }^{1}$, D. Radulescu ${ }^{1}$, A. Ciocalteu ${ }^{2}$, S. Patrascu ${ }^{1}$

${ }^{1}$ Surgery, Emergency County Hospital Craiova, CRAIOVA, Romania; ${ }^{2}$ Gastroenterology, Emergency County Hospital Craiova, CRAIOVA, Romania

Surgical reinterventions in patients with complicated hepatic hydatid cysts usually occur as a result of diagnostic or technical failures during the initial procedure. According to recent studies, the most common complication after liver hydatid cyst surgery is local sepsis at the residual cavity and long-term biliary leak. We report the case of a 21-year-old male with a history of liver hydatid disease four years before the current episode, admitted in our Surgical Department for intense upper right quadrant pain. Abdominal ultrasonography, CT and MRI scans revealed three cysts in the gastrosplenic ligament, in liver segments VII-VIII, and II-III respectively, sized between 4 and $8 \mathrm{~cm}$. The intraoperative aspect during laparoscopy was strongly suggestive for liver hydatid disease. Laparoscopic fenestration with tunneling for the hepatic cyst in segment VIII, partial cystectomy in the left liver lobe and ideal cystectomy in the gastrosplenic ligament were performed. Postoperatively, the patient displayed a constant biliary drainage output of $500-600 \mathrm{ml}$ from the cavity remnant in the segment VIII. Conservative therapy for external biliary fistula and concomitant treatment with Albendazole for 3 months were initiated. Evolution was slowly favorable with decreased biliary drainage to $200 \mathrm{ml}$ two months after surgery and complete symptom resolution five months after hospital discharge.

\section{P316-HEPATO-BILIAIRY \& PANCREAS-Liver \\ LAPAROSCOPIC TREATMENT OF THE COMPLICATED HEPATIC CYST. REPORT OF A CLINICAL CASE}

P. Dabán-López, J.A. Ubiña-Martínez, C. Moreno-Cortés, J. Gómez Sánchez, M.D. Hernández-García, E. Dabán-Collado, B. Mirón-Pozo

\section{General Surgery, H. U. San Cecilio, GRANADA, Spain}

Aims: The simple cyst's treatment is necessary only when symptoms appear; as its size is not an indication of the need for surgery. Complicated hepatic cyst can be approached through conservative or surgical management.

Methods: 76-year-old-woman with personal history of ulcerative colitis is admitted to the department of Internal Medicine with the diagnosis of a year progressive dyspnea of duration of one year. She refers upper quadrant pain of several days of duration that was relieved with omeprazol.

The exploration showed a liver swelling of a size of 7 fingers. Blood test of admission results GGT: 78, LDH: 290; rest of parameters were normal.

Abdominal CT showed: large hepatic cyst (18x17,8x22 cm size), with no malignity signs, that occupies practically the whole right liver, causing subsegmentary atelectasis of the middle lobe, superior and inferior cava vein compression, and displacement of right kidney, pancreas and right atrial. Due to breath involvement, a percutaneous drainage is performed achieving clinical improvement and reduction of the size of the injury. The patient was released but a cyst superinfection occurred; once this problem was solved, the drainage was removed.

Results: In light of the complication, surgical treatment was decided, which confirmed the large cyst located in right posterior hepatic segments with tight diaphragmatic adhesions. We carried out the cyst evacuation and a wide laparoscopic resection of the cyst walls, until the posterior area of the cava vein, combining supra and infrahepatic access. The patient was released on the sixth postoperative day and continues asymptomatic.

Conclusions: Simple cysts can be approached in a no surgical way (punction-aspiration with/ without sclerosing products injections) or in a surgical way (cyst wall fenestrations, cystectomy or liver resections). A conservative treatment will obtain symptomatic relief but with a high risk of recurring.

Recurrence is the main drawback of unroofing. Cystectomy is the better option but may be too complicated depending on the cyst's location. To our patient, we carried out a wide laparoscopic unroofing (even though its posterior localization) to minimize recurrence possibilities. In conclusion, laparoscopic resection of the cyst wall is a simple and effective approach in symptomatic or complicated cases.

\section{P317-HEPATO-BILIAIRY \& PANCREAS-Liver}

\section{SINGLE-INCISION LAPAROSCOPIC ANATOMICAL RIGHT POSTERIOR SECTIONECTOMY: A CASE REPORT}

\section{S.H. Chuang, Division of General Surgery}

Department of Surgery, MacKay Memorial Hospital, Hsin-Chu Branch, HSIN-CHU CITY, Taiwan

Background: Single-incision laparoscopic surgery or laparoendoscopic single-site surgery is emerging as an alternative to conventional multiple-incision laparoscopic surgery. It has a potential benefit of less postoperative pain and faster recovery compared with conventional multiple-incision laparoscopic surgery. Single-incision laparoscopic hepatectomy (SILH) has been reported in only a few small series and the majority were minor resections.

Case Report: A 54 y/o male patient is a case of chronic viral hepatitis B and early cirrhosis of liver. Two atypical hepatocellular carcinomas (up to $2.4 \mathrm{~cm}$ in diameter) located at the junctions of segments $6 \& 7$ and segments $5 \& 6$ were impressed by liver magnetic resonance imaging (MRI) We performed single-incision laparoscopic anatomical hepatic resection of the right posterior section via a 5 -cm transverse incision on the right middle abdominal wall. Inflow control was carried out with an extra-Glissonian approach before parenchymal transection. The Glissonean pedicles of segments 6 and 7 were divided by linear staplers respectively as well as a major branch of the right hepatic vein in segment 7 . The operative time was $580 \mathrm{~min}$ and the estimated blood loss was $150 \mathrm{ml}$. The pathologic examination revealed two foci of hepatocyte dysplasia with a safe margin of $4 \mathrm{~cm}$. The patient was discharged eight days after the surgery uneventfully.

Conclusion: Single-incision laparoscopic anatomical right posterior sectionectomy is feasible and safe by experienced laparoscopic surgeons. It provides a fast recovery but needs a long operative time. 


\section{P318-HEPATO-BILIAIRY \& PANCREAS-Liver}

\section{LAPAROSCOPIC AND ENDOSCOPIC OPERATION IN THE PATIENTS WITH CIRROSIS COMPLICATED BY VARICEAL BLEEDING}

\section{Yu.V. Grubnik, Yu.V. Yuzvak, V.A. Fomenko}

Surgery department 3, Odessa National Medical University, ODESSA, Ukraine

The mortality in the patient with liver cirrhosis is very high. The aim of this work was to decrease mortality and morbidity by using endoscopic local heamostasis and laparoscopic operations, in the patients with bleeding from cirrhosis by variceal bleeding.

Methods and material: We observed 692 patients with cirrhosis complicated by variceal bleeding during 12 years. There were 260 patients with Child Phue A, 279 ones with Child Phue B, 153 ones with Child Phue C. All the patients were performed prolonged endoscopic heamostasis with conservative therapy. The main methods that we used were the ligation in 345 cases, sealing in 50 cases, sclerotherapy in 158 cases. In 18 cases we couldn't stop the bleeding with band ligation method and introduce the Danis stents into esophagus and stopped the bleeding successfully. To prevent the re-bleeding we performed the laparoscopic dissection the abdominal part of esophagus with suturing the venous vessels, coagulations and dissection of short gastric vessels between stomach and spleen, clipping the left gastric artery and vein in the 67 patients. In 29 patients we performed laparoscopical suturing the variceal veins by introducing the laparoscopic trocars into the stomach. In 35 cases with varices vien of stomach, with non-effective local endoscopic heamostasis we performed laparoscopic resection the fundal part of stomach Results: Endoscopic local heamostasis were successful (in 85\%) in 588 cases. The relapse of bleeding were in 85 patients. 25 patients died. There was no mortality after laparoscopic operations. There were 7 cases for trocar wounds infection, 3 cases of subphrenic abscess.

Conclusion: Endoscopic band ligation and placement of Danis stents are the most effective methods of local heamostasis. Laparascopic operation is an effective method to prevent the rebleeding from varices veins in the patients with cirrhosis.

\section{P319-HEPATO-BILIAIRY \& PANCREAS-Liver}

\section{LAPAROSCOPIC RESECTION OF CAUDADO LOBE AND ADENOPATHY OF RENAL VEIN IN HEPATOCOLANGIOCARCINOMA}

A. Pinillos, A. Escartin, M. Pablo, M.G. Marta, M. Santamaría, J.T. Jaume, M. Mireia, J. Jorge, Cirugía General

\section{Hospital Universitario Arnau de Vilanova, LLEIDA, Spain}

Goals: The advance of laparoscopic surgery also includes the more complex procedures of abdominal surgery such as those affecting the liver and pancreas. There are multiple indications that laparoscopy has in hepatobiliopancreatic surgery, both in benign and malignant pathologies. Material and methods: We present the video of a 78-year-old male patient with a history of right hemicolectomy due to disease-free intestinal lymphoma who, in the control analysis by his attending physician, detects the elevation of tumor markers. An extension study was started showing a hepatic lesion in the caudate lobe with a pathological anatomy suggestive of hepatocarcinoma and an adenopathy suspicious for malignancy adjacent to the right renal vein. The clinical case is presented in a multidisciplinary tumor committee and it is decided to perform surgery.

A laparoscopic caudate lobe resection was performed, previously performing intraoperative ultrasound and a lymphadenectomy of the portal territory, vena cava and exeresis of adenopathy of the right renal vein.

Results: The surgery lasted $270 \mathrm{~min}$ with a blood loss of $450 \mathrm{cc}$ 's. A total of 3 intermittent clamps were made: $15+15+15$. Total hospital stay of five days with the first $48 \mathrm{~h}$ in the ICU. The final pathological anatomy was compatible for hepatocolangiocarcinoma with margin of resection of hepatic parenchyma free of neoplasia. Adenopathy of the renal vein was positive for hepatocarcinoma metastasis. Currently the patient is on cancer treatment.

Conclusions: Hepatobiliopancreatic surgery is a complex surgery with a non-negligible rate of morbidity and mortality in which the use of laparoscopy for both benign and malignant pathologies is increasingly being implemented. Laparoscopy presents better results in terms of postoperative patient and oncological results not inferior to open surgery. In our case, laparoscopy in hepatobiliopancreatic disease is a safe and effective technique with good results in the hands of qualified centers and expert surgeons.

\section{P320-HEPATO-BILIAIRY \& PANCREAS-Liver}

ARE WE READY FOR THE MAJOR VESSEL INJURY? STRESS LEVEL DURING LAPAROSCOPIC SIMULATION IN A LIVE ANIMAL MODEL

O. Potapov ${ }^{1}$, J. Mitus ${ }^{2}$, M. Marino ${ }^{3}$, M.A. Sanchez Hurtado ${ }^{3}$, F.M. Sánchez Margallo ${ }^{4}$, A.L. Komorowski ${ }^{5}$

${ }^{1}$ Minimally invasive surgery, Center for Innovative Medical Technologies of the National Academy of Sciences o, KYIV, Ukraine; ${ }^{2}$ Cancer Centre, Maria Sklodowska-Curie Memorial Institute of Oncology, KRAKOW, Poland; ${ }^{3}$ Minimally Invasive Surgery Center Jesús Usón, CÁCERES, Spain; ${ }^{4} \mathrm{Head}$ of department, Minimally Invasive Surgery Center Jesús Usón, CÁCERES, Spain; ${ }^{5}$ Minimally invasive surgery, St.Raphael Hospital, KRAKOW, Poland

Introduction: Major vascular complications during laparoscopic surgery occur approximately in one in 1000 cases, but mortality rate can reach $8-17 \%$. Most major vascular injuries lead to conversion to laparotomy but successful laparoscopic repair is also possible.

Simulation training improves laparoscopic performance and possibly reduces surgeons mental strain

Materials \& Methods: During two editions of advanced laparoscopic training course 12 participants had a task to control a major vessel damage (DAMAGE). Before the task an educational video explaining the methods of obtaining haemostasis was shown.

The algorithm of the 'DAMAGE' task was as follows: without previous preparation a $1 \mathrm{~cm}$ injury of a major vessel was done with L-hook electrocautery. After the injury participants were free to control the damage the way they wanted.

Heart rate of the participants was measured with an ear electrode.

Measurements were carried out 3 times—before the injury, immediately after, and afterwards obtaining vessel control.

After participants were interviewed for their feelings after the 'DAMAGE' task

Results: There were 12 vessel injuries in 10 animals. One animal died during the 'DAMAGE' task $20 \mathrm{~min}$ after desuflation due to relapse of bleeding. There was no conversion to open procedure. Temporary vessel control was obtained with different methods. All Participants used Vicryl 2.0 or PDS II 3.0 suture for final hemostatic purposes.

Heart rate of the participants before injury were $52-85 \pm 3.33 \mathrm{bpm}$, immediately after the injury it rose to $75-120 \pm 4.31 \mathrm{bpm}$, and after obtaining vessel control were in the range $50-100 \pm 4.83 \mathrm{bpm}$. A statistically significant difference was found between the ratio of the firs and second HR measurement $(\mathrm{p}=0.01, \mathrm{t}=-9.727)$, and second compared to the third ( $\mathrm{p}=0.02, \mathrm{t}=4.177$ ) measurement.

Participants judged their experience on a 5-point scale (1- was not helpful at all; 5- was extremely educative). The educational value of the task received 5 points in 11 cases and 3 points in one case.

Conclusion: Participants feel stress during major vessel bleeding even in animal model, and this stress can result in a serious intraoperative mental strain and significantly increase heart rate. Participants found the 'DAMAGE' task very useful for their daily practice. 


\section{P321-HEPATO-BILIAIRY \& PANCREAS-Liver}

\section{TREATMENT OF HEPATIC ECHINOCOCCAL CYSTS USING OF ARGON PLASMA COAGULATION}

\author{
V.V. Petrushenko, D.I. Grebeniuk, V.I. Stoika, S. Stukan, \\ O.V. Levadnyi
}

Department of Endoscopic and Cardiovascular Surgery, National Pirogov Memorial Medical University, Vinnytsya, VINNYTSYA, Ukraine

The aim of study was to improve the results of treatment of patients with hepatic echinococcal cysts by using of argon plasma coagulation.

Methods: The analysis of treatment results of 66 patients was put into the basis of this study. It was $12(18.2 \%)$ men and $54(81.8 \%)$ women in total. An average age of them was $47.7 \pm 15.9$ years.

The main difference between groups was a way of liver parenchyma coagulation in order to make reliable hemostasis. In main group the final stage of surgical intervention on liver was argon plasma coagulation. It was performed to $45(68.2 \%)$ patients. Alternatively, monopolar coagulation was performed to 21 (31.8\%) patients (comparison group).

Results: In main group in the $86.6 \%$ cases pericystectomy was conducted. The resecting surgeries was performed to $13.4 \%$ cases. In comparison group was conducted in $28.6 \%$ cases. In early postoperative period in main group the complications were observed in $4.4 \%$ of cases. The same parameter was $4.8 \%$ in comparison group. It led to relaparomies.

The forming of external biliary fistulas was observed in $2(4.4 \%)$ patients in main group and in 3 (14.3\%) patients in comparison group. However, all the fistulas have closed spontaneously on 7th-10th day in both groups.

Hernias of abdominal wall and peritoneal adhesions that manifested by intestinal obstruction of different degree were considered as complications of late postoperative period. These values were $0 \%$ and $4.4 \%$ in main group versus $19 \%$ and $14.3 \%$ in comparison group, respectively.

Conclusion: The resection of hepatic echinococcal cysts with further application of argon plasma coagulation on the cyst bed was accompanied by complications quantity decrease in patients that underwent surgery in early as well as in late postoperative period. In this case more positive dynamics of functional liver values improvements was observed.

\section{P323-HEPATO-BILIAIRY \& PANCREAS-Liver}

ANALYSIS OF THE FACTORS OF SUCCESSFUL INDOCYANINE GREEN FLUORESCENCE IN LAPAROSCOPIC HEPATECTOMY

M. Harada, K. Hashida, Y. Nagahisa, M. Yokota, M. Okabe, H. Kitagawa, K. Kawamoto

Surgery, Kurashiki Central Hospital, KURASHIKI-SHI, OKAYAMA-KEN, Japan

Aims: Indocyanine green (ICG) fluorescence imaging has been reported as a reliable and safe navigation tool in laparoscopic hepatectomy. However, the factors affecting the sensitivity of tumor detection with ICG fluorescence imaging is relatively unclear. The aim of the present study is to analyze the factors of successful ICG fluorescence in laparoscopic hepatectomy.

Methods: This is a retrospective single-center study. This study population consisted of 80 laparoscopic hepatectomies from January 2018 to November 2018 undertaken at Kurashiki Central Hospital. We excluded patients whose tumors were located more than $10 \mathrm{~mm}$ from the liver surface, those who did not receive ICG fluorescence imaging, and those who were not injected with ICG dye $(0.5 \mathrm{mg} / \mathrm{kg})$ intravenously within 7 days of surgery. The PINPOINT Endoscopic Fluorescence Imaging System was used to detect the tumor location. We evaluated the relationship between successful fluorescence and the timing of injecting ICG before operation, tumor size, ICG R15, liver damage and BMI.

Results: Following exclusion, 15 patients were eligible for analysis. Among the 16 tumors resected, ICG fluorescence imaging detected 9 tumors (56.3\%), including 6 hepatocellular carcinomas and 3 liver metastases. ICG fluorescence imaging detected all 9 tumors in the patients injected with ICG 2 to 5 days before hepatectomies. ICG fluorescence imaging detected all 9 tumors which were more than $10 \mathrm{~mm}$ in diameter. There was no relationship between indocyanine green fluorescence with ICG R15, liver damage and BMI.

Conclusions: The injection of ICG 2 to 5 days before operation and a tumor size of more than $10 \mathrm{~mm}$ can be factors in successful fluorescence in laparoscopic hepatectomy.

\section{P322-HEPATO-BILIAIRY \& PANCREAS-Liver}

LAPAROSCOPIC LIVER RESECTION WITH SIMULTANEOUS DIAPHRAGM RESECTION FOR COLORECTAL METASTASES

\author{
$\underline{\text { A.M. Kazaryan }^{1}}$, D.L. Aghayan ${ }^{2}$, Å.A. Fretland ${ }^{2}$, B. Edwin ${ }^{2}$
}

${ }^{1}$ Department of Surgery, Fonna Hospital Trust, STORD, Norway; ${ }^{2}$ Department of HPB Surgery, Oslo University Hospital, OSLO, Norway

Background: Liver resection or ablation remains the only chance for cure for patients with colorectal metastases. Simultaneous resection of tumours with invasion to adjacent organs is challenging. In this study we investigate patients who underwent laparoscopic liver resection with simultaneous diaphragm resections.

Methods: From 1998 to 2018, 787 patients with colorectal metastases underwent laparoscopic liver resection. Patients who underwent simultaneous diaphragm resection were identified and included in this study. Perioperative and oncologic outcomes were analysed. The Accordion classification was used to grade postoperative complications. The Kaplan-Meier method was used for survival analyses. The median follow-up was 26 months (range 6-123).

Results: A total 12 patients underwent laparoscopic simultaneous liver and diaphragm resection due to suspicion of tumour invasion to the diaphragm. Histology confirmed the diaphragm invasion in $9(75 \%)$ of these cases. R0 resections was achieved in $11(92.5 \%)$ cases, including 9 $(88.9 \%)$ cases with confirmed diaphragm invasion. The median operation time was $153 \mathrm{~min}$ (range 105-210) and the median blood loss compiled $200 \mathrm{ml}$ (range 20-1200). Two patients developed postoperative complications. The median postoperative hospital stay was 3 days (range 2-14). There were no 90-days mortality in this study. Five-year overall survival was $66 \%$. Conclusion: Laparoscopic simultaneous liver and diaphragm resection can be performed safely with the good surgical and immediate and long-term oncological outcomes.

\section{P324-HEPATO-BILIAIRY \& PANCREAS-Liver}

\section{GIANT LIVER CYST MANEGEMENT: A CASE REPORT}

S. Cerrato Deldado, M.D. Casado Maestre, J. Varela Recio, S. Ayllon Gamez, A. Fierro Aguilar, C. Peña Barturen, J.M. Pacheco García, Cirugía General

\section{Hospital Universitario Puerta del Mar, CADIZ, Spain}

Introduction: Cysts in the liver have a wide variety of aetiologies. It is important to characterize the cystic lesion before treating it. The simple cyst has a low prevalence and is more frequent in women. Fenestration is a useful option for the treatment of simple cysts in selected patients. Case presentation: A 40-year-old woman was referred to our hospital with a one-year history of intermittent, right upper quadrant pain, with no other associated symptoms. Computed tomography and magnetic resonance imaging showed a large cyst $(14,4 \times 13,2 \mathrm{~cm})$ in the right of the liver. The cyst presented lobulated morphology, smooth edges and well delimited. There were other smaller cysts in the left lobe. Hepatic function in blood analysis was normal. Biomarkers, tumor markers and hepatitis virus markers were negative. Outpatient follow-up and symptomatic treatment of pain was decided. After six months of follow-up, the pain persisted, so surgical treatment was proposed. A laparoscopic fenestration was performed, widely resecting the free wall of the cyst. There was no evidence of a connection to the bile duct. There were no complications. On 3 days she was discharged.

Discussion: Some giant hepatics cysts become symptomatic due to mass effect. Persistence of pain is an indication of surgical treatment. Laparoscopic fenestration is an alternative for the management of simple hepatic cysts. 


\section{P325-HEPATO-BILIAIRY \& PANCREAS-Liver}

\section{NEAREST RESULTS OF THE COMBINED TREATMENT OF PATIENTS WITH UNRESECTABLE LIVER CANCER METASTASES USING SELECTIVE CHEMOEMBOLIZATION} OF LIVER ARTERIES

D.P. Lebedev ${ }^{1}$, D. Astakhov ${ }^{1}$, Y.V. Ivanov², A.G. Kedrova ${ }^{3}$, D.N. Panchenkov ${ }^{1}$

${ }^{1}$ Laboratory of Minimally Invasive Surgery, A.I. Evdokimov Moscow State University of Medicine and Dentistry, MOSCOW, Russia; ${ }^{2}$ Surgical department, A.I. Evdokimov Moscow State University of Medicine and Dentistry, MOSCOW, Russia; ${ }^{3}$ Oncological department, Federal Research Clinical Center for Specialized Health Care and Medical Technol, MOSCOW, Russia

Aim: To assess nearest results of the combined treatment in patients with unresectable liver metastatic cancer of various primary localizations in combination with selective chemoembolization of liver arteries.

Methods: Between January 2015 and November 2018, 25 patients underwent 32 procedures of arterial chemoembolization with drug-saturated microspheres as a part of the treatment of metastatic cancer of various primary localizations. There were 11 men (age from 52 to 81 years, mean 66.5), and 14 women (age from 43 to 78 years, mean 60.5). In 10 cases, femoral artery access was used, and in 15 cases, radial artery access was used. In 22 patients, one vial of HepaSphere (in 14 cases saturated with $50 \mathrm{mg}$, in 13 cases of $75 \mathrm{mg}$ of Doxorubicin and 5 cases of $100 \mathrm{mg}$ of Irinotecan) was co-administered in the hepatic arteries. Three patients required the introduction of two vials of microspheres.

Results: In all cases, chemoembolization supplemented the ongoing systemic treatment, with the result that it was possible to achieve stabilization of the process for a period of 30 days to 371 days (observation continues). The clinical effect at the november 2018: in the form of stabilization of the process in 11 patients $(44.0 \%)$, in 3 patients $(12.0 \%)$ partial clinical regression was observed, 3 patients complete regression $(12.0 \%), 8$ patients $(32.0 \%)$ died from the progression of the disease. Median survival not achieved. In 7 cases, arterial chemoembolization was performed twice at an interval of $62,153,197,296,310,329$ and 371 days.

Conclusion: The method of arterial chemoembolization of the liver with drug-saturated microspheres allows a deep cytotoxic effect on the metastasis of cancer in the liver, improving the immediate results of treatment, manifested in regression and stabilization of the tumor process.

\section{P326-HEPATO-BILIAIRY \& PANCREAS-Liver}

COMPARISON OF PURE LAPAROSCOPIC AND OPEN LIVING DONOR RIGHT HEPATECTOMY AFTER A LEARNING CURVE

\section{B.R. Lee, Y.R. Choi}

General surgery

Department of Surgery, Seoul National University Bundang Hospital, GYEONGGI-DO, Korea

Background: To compare the early outcomes after learning curve of pure laparoscopic living donor right hepatectomy (PLDRH) to those of open living donor right hepatectomy (ODRH). Methods: Our analysis was based on 78 consecutive cases of living liver donor, who underwent right hepatectomy, of which 43 underwent ODRH and 35 PLDRH. The learning curve for each group was analyzed.

Results: Donor characteristics were comparable between the two groups. Two donors in the PLDRH required conversion to an open due to bleeding and large graft size (Open conversion rate $: 6.06 \%$ ). The following outcomes during the study period were comparable between the two groups: operative time $(P=0.64)$; estimated blood loss (EBL; $P=0.86)$; intra-operative transfusion $(P=0.57)$; hospital stay $(P=0.41)$, and postoperative complications $(P=0.51)$. The operative time stabilized for the ODRH group after 17 cases and for the PLDRH group after 15 cases. After the learning curve, the EBL was lower for PLDRH than ODRH $(P=0.04)$. Conclusion: PLDRH can be performed as safely as ODRH and with a lower volume of EBL once the surgeon has attained an appropriate level of learning.

\section{P327-HEPATO-BILIAIRY \& PANCREAS-Liver}

SHORT AND LONG TERM OUTCOMES OF LAPAROSCOPIC LIVER RESECTION FOR COLORECTAL LIVER METASTASES OUR 8 YEARS EXPERIENCE

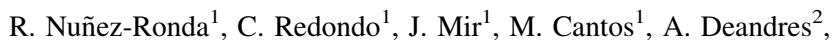
E. Artigues ${ }^{1}$, R. Fabra ${ }^{1}$

${ }^{1}$ General Surgery, Hospital General Universitario de Valencia, VALENCIA, Spain; ${ }^{2}$ General Surgery, Hospital Francesc de Borja, GANDÍA, Spain

Aim: Laparoscopic liver resection for malignant pathology such as colorectal cancer metastases has been a matter of discussion for several groups in the last years. It has been proposed as a safe and feasible treatment but subjects like short and long term outcomes and oncologic results have not been adequately assessed.

Methods: We performed an observacional retrospective study of patients undergoing laparoscopic liver resection for colorectal metastases in our center. From November 2007 to Novembe 2018 a total of 113 patients underwent laparoscopic liver resection. Data for resection margin, hepatic and extrahepatic recurrence and both disease free survival and overal survival were collected. Patients were discussed in a multidisciplinary group with oncologist, radioterapic oncologist and surgeons. The surgical procedures were perfomed by the same team in all the cases to minimize bias.

Results: A total of 9 patients $(7.9 \%)$ were non resectable at the time of surgery.The mean overal survival was 19 months with a maximum of 132 months. We got a mean of disease free survival in our patients of 11.7 months. The hepatic recurrence was $28 \%$, most of them in high risk patients, and from this group $67.74 \%$ underwent a new liver resection. Major complications took place in 9 patients $(7.96 \%)$ two biliar leaks, one bowel perforation, two hepatic failure, one evisceration and three respiratory insufficiency needing urgent surgery in three of the cases. Mean hospital stay was 5.84 days. A mean of 2 days of this stay were in an intensive care unit. Conclusions: Laparoscopic liver resection for colorectal liver metastases could be a feasible technique when perfomed by trained surgeons. It improves the postoperatory recovery with a reduction of hospital stay and less postoperatory pain without increasing the development of major complications or mortality in the first 30 days after surgery. We got good oncological results that have been improving with the experience acquisition of the surgical team.

\section{P328-HEPATO-BILIAIRY \& PANCREAS-Liver}

\section{COMPLICATIONS AFTER LAPAROSCOPIC AND CONVENTIONAL LIVER RESECTIONS FOR METASTATIC COLORECTAL CANCER}

\section{N. Belev}

Surgical Department, Eurohospital, PLOVDIV, Bulgaria

Aim: Comparison of complications and ways to reduce them after laparoscopic and conventional LR for MCC.

Object: Safety of planned liver surgery is continuously improving over the last 30 years. While in 1970 s mortality after liver surgery reached $13 \%$ nowadays results today are more optimistic - $0 \%$ to $4 \%$ mortality and complications in $19.6 \%$ to $35 \%$. These better postoperative results are due to liver surgery refinement in specialized centers, better patient selection regarding liver function and accompaning diseases, the advancement of surgical technique and better understandment of liver segmental anatomy as well as new instruments of parenchymal transection. Furthermore anesthesiology and intensive care in liver surgery have seen drastic improvement.

Materials and methods: For the period of 01.03.2012 until 31.12.2017 we have analyzed retrospectively the immediate results and complications of 156 liver resections for MCC. 88(56\%) of them are simultaneous liver and colorectal resections (34-39\% laparoscopic and 54-61\% conventional and $68(43 \%)$ liver resections for metachronous metastasis (20 laparoscopic and $48(29 \%)$ conventional). We evaluated patients performance status as 0,1,2 according to the European Oncology Study Group, liver function according to MELD criteria, ASA score for anesthesiology risk assessment. Morbidity is classified according to the Clavien-Dindo classification.

Results: Registered mortality is $1.4 \%$. Complications are 23\% (36 patients)-19\% (30 patients) had operation related complications and $4 \%$ (6 patients) had general complications. According to the Clavien-Dindo classification complication were distributed the following way-laparoscopic surgery patients had $19.6 \%$ complications classified as mainly Grade A and B according to the Dindo classification. Mean blood loss in laparoscopic surgery was $80 \mathrm{ml}$ while $150 \mathrm{ml}$ was registered in open surgery. Mean hospital stay is 6.5 days in the laparoscopic group and 9.5 days in the open surgery group. Conclusion: Laparoscopic LR generates better postoperative results (less blood loss, les haemotransfusion requirement, shorter hospital stay. According to our study laparoscopic LR results in lower percent of postoperative morbidity with more complications registered as low grade according to the Dindo classification. 


\section{P329-HEPATO-BILIAIRY \& PANCREAS-Liver}

COMPLEX MINI-INVASIVE SURGICAL CORRECTION OF REFRACTORY ASCITES IN PATIENTS WITH LIVER CIRRHOSIS

G. Anghelici ${ }^{1}$, S. Pisarenco ${ }^{2}$, O. Crudu ${ }^{2}$, T. Zugrav ${ }^{2}$, G. Lupu ${ }^{3}$, V. Roman ${ }^{2}$

${ }^{1}$ Surgical Clinic „C.?îbîrna,, Laboratory of Liver Surgery, State Medical University, N.Testemiţanu, CHISINAU, Moldova;

${ }^{2}$ Laboratory of Liver Surgery, State Medical University,

N.Testemițanu, Moldova, CHISINAU, Moldova

Aim: Occurrence of refractory ascites in liver cirrhosis presents considerable difficulties fo treatment. Evolution of cirrhotic ascites is closely interrelated with violations of peritoneal absorption and blockage of lymph circulation.

Objective: To determine possibilities of surgical correction of refractory ascites in patients with decompensate liver cirrhosis by laparoscopic sanitation with post-surgery fractional rinsing of abdominal cavity, with simultaneous decompression of thoracic lymphatic duct.

Materials and methods: From 2014 to 2018, 98 patients (53 men (54.1\%) and 45 women (45.9\%),) aged 29 to 71 underwent surgery for cirrhosis with massive refractory ascites Child C (9-10), withou obvious signs of hepatic encephalopathy. Major etiological factors were: viral hepatitis $\mathrm{C}(47$ patients $(48.0 \%))$, B (29 patients $(29.6 \%))$ B + D (17 patients $(17.3 \%))$, toxicity (5 patients $(5,1 \%))$. To prevent possible bleeding at the first stage, endoscopic filling of esophageal varices with fibrin glue was performed in 81 patients $(82.7 \%)$. After testing the effectiveness of varices filling, in the following 5-7 days decompression surgery of thoracic lymphatic duct was performed under local anesthesia to improve lymphatic drainage from liver and abdominal organs. Simultaneously, laparoscopic sanitation of abdominal cavity was performed, with complete evacuation of ascites fluid, rinsing and drainage. Fractional post-surgery rinsing was repeated daily for 3-5 days towards removing peritoneum edema and improving its absorptive properties. Results evaluation was performed 3, 6 and 12 months after surgery, based on criteria of liver reserves and ascites volume. Results: Post-surgery mortality from liver failure was $5.1 \%$ (5 patients). 7 other patients died of the same cause the following 3-6 months. Annual survival rate was 87.6\%. Complete ascites regression over 3-12 months after surgery was noted in 53 patients $(55.8 \%)$, significant regression and stabilization in $25(25.6 \%)$, moderate regression with need for periodic decompressive laparocentesis in 8 cases. In all patients, functional liver reserves and life quality significantly improved.

Conclusions: The use of the given technique of refractory ascites correction, in patients with depleted liver cirrhosis, by laparoscopic sanitation with post-surgery fractional rinsing of abdominal cavity, with simultaneous decompression of thoracic lymphatic duct showed very high efficiency and deserves establishment as a clinical practice.

\section{P330-HEPATO-BILIAIRY \& PANCREAS-Liver}

\section{LAPAROSCOPIC ANATOMICAL LIVER RESECTION USING INDOCYANINE GREEN FLUORESCENCE IMAGING}

T. Urade, Hepato-Biliary-Pancreatic Surgery, Kobe University, KOBE, Japan

Aim: Anatomical liver resections guided by a demarcation line after portal staining or inflow clamping of the target territory were established as essential methods for the curative treatment of hepatocellular carcinoma (HCC) and then subsequently applied to other malignancies. However, laparoscopic anatomical liver resection (LALR) is much more difficult to reproduce these procedures and to confirm demarcation of the hepatic segment visually on the monitor. Recently, laparoscopic fluorescence imaging system has been used as a tool for real-time intraoperative navigation in LLR. The aim of this study is to demonstrate how to perform LALR using indocyanine green (ICG) fluorescence imaging.

Methods: Three patients underwent pure LALR using ICG fluorescence imaging. The following operative procedures were performed: 1 partial liver resection for $\mathrm{HCC}, 1$ segmentectomy for liver metastasis and right anterior sectionectomy for HCC. In all patients, preoperative 3D simulation images from dynamic CT were reconstructed using a 3D workstation to decide on cutting points of the Glissonean branches. After mobilization of the liver, intraoperative ultrasonography was performed to identify the location of the tumor and Glissonean pedicles corresponding to the tumor-bearing hepatic region. We dissected or transected the hepatic parenchyma to encircle the Glissonean pedicles. After clamping or closure of them, $2.5 \mathrm{mg}$ of ICG was injected intravenously to identify the boundaries of the hepatic segments under near-infrared light. Parenchymal transection was started according to the demarcation on the liver surface. The lateral aspect of the parenchymal transection was carried out based on the demarcation between non-fluorescing and fluorescing liver parenchyma as far as possible.

Results: In all the 3 cases, demarcation lines on the liver surface could be visualized clearly after injection of ICG. In addition, boundaries of cone units, segments and sections could be recognized to some extent because the tumor-bearing hepatic region became non-fluorescing parenchyma during parenchymal transection. These procedures were completed successfully, and the postoperative courses were almost uneventful.

Conclusion: LALR using ICG fluorescence imaging is a feasible and useful procedure to eliminate the tumor-bearing hepatic region. ICG fluorescence imaging facilitates surgeons' visualization of the demarcation line and identification of the boundaries of the hepatic regions.

\section{P331-HEPATO-BILIAIRY \& PANCREAS-Liver}

\section{LAPAROSCOPIC RESECTION OF INTRAHEPATIC CHOLANGIOCARCINOMA ON CIRRHOSIS AND PORTAL HYPERTENSION}

\section{P. Beltran Miranda, G. Suarez Artacho, C. Cepeda Franco, L.M. Marin Gomez, C. Bernal Bellido, J.M. Alamo Martinez, M.A. Gomez Bravo, F.J. Padillo Ruiz}

HPB and Trasplant Surgery, Virgen del Rocio Hospital, SEVILLA, Spain

Aim: sIntrahepatic cholangiocarcinoma is the second most common primary liver cancer after hepatocellular carcinoma (HCC). Although the laparoscopic approach of these tumours is not frequent due to its complexity, it is performed increasingly by hepatic surgeons.Traditionally, the abdominal surgery in cirrhotic patients has been reserved to selected cases secondary to the high rate of complications. The advance on the treatment of the $\mathrm{HCC}$ on liver cirrhosis and the higher safety when performed by laparoscopic approach has encourage some surgeons to extend surgery to Child B-C or portal hypertension patients.

Methods: We present a male of 56 years old, diagnosed in 2010 of liver cirrhosis accompanied with portal hypertension. On MRI in 2012 was found a solid lesion of $25 \mathrm{~mm}$ located on segment II hepatic. Biopsy confirmed the diagnostic of intrahepatic cholangiocarcinoma. After a liver function evaluation (Child C, Meld 17), an hepatic chemoembolization was performed. Sequentially CT scans indicated a complete radiologic response. After 6 years of follow up, MRI showed a recurrence of $25 \mathrm{~mm}$ between segment II and III of the liver.On multidisciplinary committee liver resection was decided due to suitable liver function and low aggressiveness of the tumour. A laparoscopic left lobe liver resection was performed. Sonastar ${ }^{\circledR}$ and Ligasure ${ }^{\mathrm{TM}}$ were used to perform the liver transection and Endo GIA ${ }^{\mathrm{TM}}$ for portal and hepatic veins sections. The surgery develop was complicated due to trend to bleeding that finally was achieve through cauterization.

Results: Early after the surgery, the patient presented a haematic debt through the drain of $900 \mathrm{cc}$ accompanied of hypotension, therefore an emergent surgery was indicated. An exploratory laparoscopy was performed finding hemoperitoneum and diffuse bleeding of the liver surface that was controlled. The patient had a proper recovery and was discharged on the $11^{\text {th }}$ day post-surgery. The analysis of the specimen showed a $6.5 \mathrm{~cm}$ cholangiocarcinoma with a $0.4 \mathrm{~cm}$ margin of resection. Conclusion: There is an augmented risk of complications on liver resection of cirrhotic patients with portal hypertension. The laparoscopic approach allows to reduce potential complications, despite bleeding continuous to jeopardize this surgery, this option could be proposed on selected patients.

\section{P332-HEPATO-BILIAIRY \& PANCREAS-Pancreas}

\section{ECTOPIC SPLEEN SIMULATING PANCREATIC NEUROENDOCRINE TUMOR. A CASE REPORT}

\author{
P. Martinez, E. Toledo, R. Fernandez, S. Castanedo, J. Garcia, \\ J.C. Rodriguez
}

\section{General Surgery, Humv, SANTANDER, Spain}

Introduction: Accessory spleen itself is found in approximately $7 \%$ to $15 \%$ of the population. Most $(80 \%)$ are located near the splenic hilum but intrapancreatic accessory spleens (IPAS) are the second most frequent location $(16.8 \%)$ of accessory spleens. In adults, IPAS are clinically silent. They may become clinically important because of their radiographic similar appearence of cancer. Intrapancreatic accessory spleen is a rare cause of pancreatic pseudotumors and is located in the pancreatic tail in approximately $1 \%$ to $2 \%$. IPAS can be difficult to differentiate radiologically from hypervascular pancreatic tumors such as pancreatic endocrine neoplasms because theycan share a similar enhancement pattern. As a result, most of the reported cases of IPAS have been diagnosedonly after distal pancreatectomy was completed.

Material and Methods: We present the case of a 55-year-old male patient with a history of large vessel vasculitis followed-up for Rheumatology, which showed a pancreatic nodule in a control CT so he was referred to Digestive for study. An echoendoscopy was performed. It showed, at the level of the tail, in the third distal, a lesion of $16 \times 12 \mathrm{~mm}$, hypoechoic, with rounded morphology and well-defined edges that can not be biopsied given the absence of adequate window for the realization of fine needle aspiration biopsy (FNAB). Based on these radiographic findings, the differential diagnosis included a pancreatic endocrine tumor. Due to the high suspicion of malignancy and the absence of biopsy, he was referred to General Surgery for scheduled surgery. A laparoscopic corporocaudal pancreatectomy was performed without incidents and the definitive histology showed an intrapancreatic accessory spleen in the pancreatic tail that excluded the presence of cancer.

Conclusion: Intrapanceratic accesory spleen is a challenging diagnosis to make and it should be included in the differential diagnosis of pancreatic neoplasm. Its early identification precludes surgical resection. However, the preoperative diagnosis of IPASmay be difficult, and distal pancreatectomy is a safe and relatively simple operation, most of the reported cases of IPAS being diagnosed correctly only after surgery 


\section{P333-HEPATO-BILIAIRY \& PANCREAS-Pancreas}

\section{LAPAROSCOPIC REDO HEPATICOJEJUNOSTOMY FOR STENOTIC HEPATICOJEJUNOSTOMY}

\section{Hussein}

Surgery, American University of Beirut Medical Center, BEIRUT, Lebanon

Aims: Redo Hepaticojejunostomy for Stenotic Hepaticojejunostomy.

Methods: I report a case 56 years old post laparoscopic Whipple procedure for pancreatic mass that revealed to be chronic pancreatitis. Patient presented 1-year post operation with history of repeated episodes of cholangitis. CT scan revealed severe stenosis and PTC failed to clear the intra hepatic and extra heptatic biliary tree from impacted biliary stones.This video shows the various steps used through 5 trocars to release the adhesion and explore the Hepaticojejunostomy, division of the Hepaticojejunostomy, stone extraction from the extra hepatic and intra hepatic biliary system through choledochoscope using Dormia basket and fogarty catheter number 5, and the redo anastomosis performed using intracorpeal suturing of 3-0 PDS

Results: Patient had smooth postoperative course and discharged 4 days post-operative.

Conclusion: Therefore, even with most complicated cases can be handled laparoscopically in Advanced Centers of Laparoscopy

\section{P335-HEPATO-BILIAIRY \& PANCREAS-Pancreas}

\section{LAPAROSCOPIC DRAINAGE OF PANCREATIC PSEUDOCYSTS-A THERAPEUTIC DILEMMA}

S. Xenaki, K. Lasithiotakis, S. Gorgoraptis, E. Athanasakis, $\overline{\text { E. Chrysos }}$

Department of General Surgery, University Hospital of Heraklion Crete, HERAKLION, Greece

Aim: Pancreatic pseudocysts (PPs) are formed after acute, chronic and usually as a result of worsening chronic pancreatitis. Their impact ranges from 0.5 to 1 per 100,000 people per year. There are various options for treating PPs. This paper describes our tailored and methodological approach to laparoscopic drainage of pancreatic pseudocysts based on an anatomical classification.

Methods: We adopted the laparoscopic approach in 28 patients who had PPs requiring surgical drainage. The laparoscopic method had been decided according to preoperative computed tomography (CT) and intraoperative findings. The results shown represent median (range).

Results: Between 2004 and 2018, 30 laparoscopic drainage procedures for PPs were performed in 28 consecutive patients. The surgical approach included complete drainage of the pseudocysts. The operative time was $118(25-300) \mathrm{min}$. There were two conversions to laparotomy (4\%), low morbidity (3\%), and $1 \%$ mortality. The postoperative hospital stay was $2(1-7)$ days. At a followup of $15(1-48)$ months there were no major complications or reccurence.

Conclusion: CT findings and laparoscopic exploration demonstrate the anatomical characteristics of PPs and enable successful planning and execution of their laparoscopic drainage. Laparoscopic drainage of PPs benefit comparing to the open approach concerning minor complications.

\section{P336-HEPATO-BILIAIRY \& PANCREAS-Pancreas}

\section{P334-HEPATO-BILIAIRY \& PANCREAS-Pancreas}

\section{LAPAROSCOPIC DISTAL PANCREATECTOMY WITH SPLENECTOMY FOR A PANCREATIC NEUROENDOCRINE TUMOUR}

J. Tur-Martínez ${ }^{1}$, A. Escartín ${ }^{1}$, P. Muriel ${ }^{1}$, M. González ${ }^{1}$, A. Pinillos ${ }^{2}$, M. Santamaría ${ }^{1}$, J.J. Olsina ${ }^{1}$

${ }^{1}$ Hepatobiliary and Pancreatic Surgery Unit, Hospital Universitari Arnau de Vilanova, LLEIDA, Spain; ${ }^{2}$ General and Digestive Surgery Service, Hospital Universitari Arnau de Vilanova, LLEIDA, Spain

Aim: Laparoscopic surgery for pancreatic diseases is increasingly. Laparoscopic distal pancreatectomy (LDP) with or without spleen preservation, is one of the first laparoscopic approach for distal pancreatic tumours and offers the same oncological results as an open surgery.

The aim of this communication is to present a surgical technique video showing a LDP withou spleen preservation for a neuroendrine tumour located in the body of the pancreas with suspicious of splenic artery infiltration.

Methods: A surgical technique video is presented showing the main steps for the LDP without spleen preservation.

Results: A 73 years old man with a past medical history of arterial hypertension, type 2 Diabetes Mellitus, chronic renal failure and a gastric adenocarcinoma (pTis) resected by endoscopy a few months ago. During the study for the gastric cancer, a $18 \mathrm{~mm}$ hipervascular tumour was detected by the CT scan at the body of the pancreas with suspicious of splenic artery infiltration. A somatostatin- octreotide scan was performed showing a slight activity in the body of the pancreas, without other abnormal activity. Chromogranin A $155 \mathrm{ng} / \mathrm{ml}$ and normal tumoral markers. After a multidisciplinary committee evaluation, the patient was proposed for a surgical treatment. A laparoscopic approach was made with an umbilical Hasson trocar, two $12 \mathrm{~mm}$ trocars and a $5 \mathrm{~mm}$ trocar. A complete mobilization of transverse and left colon was made. Identification and ligation of Splenic artery and vein. Section of the body of pancreas with endo-GIA and medial to lateral dissection to complete de distal pancreatectomy with splenectomy. The patient was discharged the $5^{\text {th }}$ postoperative day without complications.

Definitive anatomopathological exam: neuroendocrine tumour (Chromogranin + , synaptophysin + ), grade $1, \mathrm{Ki}-67<3 \%, 18 \times 10 \mathrm{~mm}$. Free margins. pT1N0 (0/8).

No adjuvant treatment required.

Conclusion: LDP remains the gold standard treatment for distal pancreatic tumours, offering similar oncologic outcomes and all the advantages of the laparoscopic approach. LDP should be performed in centres with high experience in hepatobiliary surgery and in advanced laparoscopy.

\section{TOTAL LAPAROSCOPIC PANCREATECTOMY IN A PATIENT WITH SYNCHRONOUS SOLID PSEUDOPAPILLARY NEOPLASM AND INTRADUCTAL PAPILLARY MUCINOUS NEOPLASM OF PANCREAS}

V. Drakopoulos, N. Roukounakis, K. Botsakis, S. Voulgaris, V. Lygizos, A. Bakalis, V. Vougas, 1st Department of Surgery and Transplantation Unit, District General Hospital of Athens « Evangelismos », ATHENS, Greece

Aim: To report and discuss the first case in the literature of a 39-year-old pregnant woman suffering synchronous solid pseudopapillary neoplasm (SPN) and intraductal papillar mucinous neoplasm (IPMN) of pancreas treated successfully with total laparoscopic pancreatectomy postpartum

Method: A 39-year-old woman was admitted to our hospital due to abdominal pain. The CT-scan demonstrated a mixed type lesion (solid-cystic) in the body to tail of pancreas. The EUS-FNA was not diagnostic. One month later the PET-scan confirmed the CT-scan finding and the presence of a fetus. At this time the patient refused any surgical intervention. After her childbirth the new investigation (CT-scan, PET-scan, EUS-FNA) revealed the presence of synchronous SPN and IPMN of pancreas. She underwent total laparoscopic pancreatectomy using 6 trocars (one $10 \mathrm{~mm}$ in umbilicus as it is introduced in single incision laparoscopic surgery, one hypoxiphoid $12 \mathrm{~mm}, 2$ suprapubic $5 \mathrm{~mm}, 2$ in the middle clavicle line $5-12 \mathrm{~mm}$ proximally to the suprapubics ones.

Results: The microscopic examination confirmed the synchronous presence of two distinct primary neoplasms of the pancreas. A branch-duct type papillary mucinous neoplasm of the head of the pancreas of gastric type and a solid pseudopapillary neoplasm in the body to the tail of the pancreas. The postoperative period was uncomplicated. Nine months after the operation the patient is in good health and according to MRI investigation disease free.

Conclusion: The synchronous presence of SPN and IPMN has reported only in two cases, both in men, until now. The existence of SPN in pregnancy has been discussed in the view of potentia rapid growth and spontaneous rupture of the lesion. No IPMN lesion has been reported in pregnancy. Laparoscopic total pancreatectomy constitutes an effective and safe minimally invasive surgical procedure. Many studies have shown its advantages compared to open surgery regarding blood loss and hospital stay. 


\section{P337-HEPATO-BILIAIRY \& PANCREAS-Pancreas}

\section{MINIINVASIVE OPERATIONS IN PATIENTS WITH MIXED TRAUMA}

\section{Yu.V. Grubnik $^{1}$, Yu.V. Yuzvak ${ }^{1}$, A.V. Plotnikov ${ }^{2}$}

${ }^{1}$ Surgery department 3, Odessa National Medical University, Odessa, Ukraine; ${ }^{2}$ Surgery department, Odessa National Medical University, ODESSA, Ukraine

The aim of this work was to decrease mortality and morbidity in patients with combined trauma. Methods and material: For 5 years 667 patients were brought to our clinic with combined trauma. Everybody was performed CT and ultrasound examination. 286 patients were performed open laparatomic operation due to massive liver rupture, spleen rupture and massive trauma of bowels, pancreas and kidney with massive bleeding. In 106 circumstances we didn't found the trauma of the abdominal organs and the massive abdominal bleeding after $\mathrm{CT}$ observation. Those patients were cured conservatively. In 275 circumstances with combined trauma after CT examination we performed laparoscopic operation. In 97 circumstances from the 275 patients, who we started laparoscopic operation in, we conversed to laparotomy, due to massive liver rupture, and trauma spleen and hollow organs. In those 112 circumstances we performed urgent laparotomies with suture ligation of bleeding points, suturing of liver and hollow organs and drainage of abdomen cavity.

Results: We performed laparoscopic operation in 178 patients. In 107 circumstances with trauma of liver we performed laparoscopic electro coagulation and argon-plasma coagulation. In 66 circumstances with trauma of liver we performed electro coagulation with packing the omenture to its surface. In 5 circumstances with trauma of spleen we performed argon plasma coagulation and used fibrin glue. After laparotomic operations mortality were in 35 circumstances, morbidity were in 87 patients. After laparoscopic operation mortality were in 5 circumstances of severe combined trauma with multiple abdominal trauma and morbidity in 11 patients.

Conclusion: Laparoscopic operations in patients with combined trauma decrease mortality and morbility.

\section{P338-HEPATO-BILIAIRY \& PANCREAS-Pancreas}

\section{THREE-STEP MANEUVER FOR LAPAROSCOPIC DISTAL PANCREATECTOMY}

\author{
K. Hashida, M. Yokota, Y. Nagahisa, M. Okabe, H. Kitagawa, \\ K. Kawamoto
}

\section{Surgery, Kurashiki Central Hospital, KURASHIKI CITY, Japan}

Aims: In laparoscopic distal pancreatectomy, getting away liver and stomach from the surface of the pancreas is sometimes difficult. When we separate the pancreatic body from the retroperitoneum, we must not injure the pancreas to prevent breaking a tumor. When we cut the dorsal side of the spleen from the retroperitoneum, we rarely cut into the spleen accidentally. Based on our experiences, we gradually explored a set of procedural operation steps to resolve these problems. Our three-step maneuver simplifies the procedure and improves the efficiency and safety of laparoscopic distal pancreatectomy

Methods: As the first step, to get away the liver we sutured the round ligament of liver and crus of the diaphragm using 3-0 PDS and the both ends were tugged form the outside of the body through both side of the xiphoid process. And the stomach was hung from the outside using two nylon thread like a bridge, so we could see the surface of the pancreas body with a good view. The second step was a rolling up maneuver of the pancreas. When we separate the pancreatic body and tail from the retroperitoneum, we rolled the pancreas with gauze for use in laparoscopic surgery and lifted the gauze up in only one assistant's forceps. Then we could find the correct line for dissection clearly. The last step was a hanging maneuver of the spleen. When we cut the dorsal side of the spleen from the retroperitoneum, we hanged the hilum of spleen with cotton tape. With this technique we could find easily the correct line to dissect.

Results: The operation time was $4 \mathrm{~h}$ and $7 \mathrm{~min}$ and the estimated blood loss was a little. We did not injure the tumor or spleen in this operation. The patient recovered uneventfully after short hospitalization.

Conclusion: Our three-step maneuver can be effective to perform laparoscopic distal pancreatectomy.

\section{P339-HEPATO-BILIAIRY \& PANCREAS-Pancreas}

ENDOSCOPIC ASSITED LAPAROSCOPIC DRAINAGE OF A WALLED OFF NECROSIS

G. Arredondo ${ }^{1}$, M. Franklin ${ }^{2}$, H. Segura ${ }^{1}$

${ }^{1}$ General Surgery, Escuela de Medicina y Ciencias de la Salud del Tecnológico de Monterrey, MONTERREY, Mexico; ${ }^{2}$ General Surgery, Texas Endosurgery Institute, SAN ANTONIO, United States of America

About $10-20 \%$ of patients with pancreatic collections will develop walled off necrosis, with an associated 8-39\% mortality. There are multiple options for intervention and drainage, usually the outcomes after endoscopic drainage are related with the nature of the collections. Aims: To evaluate and present the rol of endoscopy in pseudocyst and walled off necrosis treatment, and favorable outcomes.

Methods and results: We present a case of a 48 years old male, who presented biliary pancreatitis treated with cholecystectomy and transoperative cholangiogram 6 weeks ago. He continued with persistent abdominal pain; his CT scan showed a big walled off necrosis; he was taken to surgery for an endoscopy-assisted laparoscopic cystogastrostomy with necrosectomy, he was discharged 14 days PO.

Conclusions: The step-up management of walled off necrosis has proven to be a better option than conventional surgical or endoscopical techniques alone; by reducing complications and mortality vs conventional necrosectomy. The use of endoscopic treatments reduce the pro-inflamatory response. Drainage of walled off necrosis can be done by a transpapilar or transmural endoscopic apporach each one with its own advantages. Some authors avoid the use of endoscopy in walled off necrosis because of a higher rate of complications, re-interventions and a greater lenght hospital stay. In our experience, we have achieved excellent results with this combined technique.

\section{P340-HEPATO-BILIAIRY \& PANCREAS-Pancreas}

NEAREST AND LONG-TERM Results: OF THE COMBINED TREATMENT OF PATIENTS WITH LOCALLY ADVANCED PANCREATIC CANCER USING THE NANOKNIFE SYSTEM

D. Astakhov ${ }^{1}$, Y.V. Ivanov ${ }^{2}$, O.R. Shablovsky ${ }^{3}$, N.A. Soloviev ${ }^{2}$, A. Nechunaev ${ }^{2}$, A.I. Zlobin ${ }^{1}$, D.P. Lebedev ${ }^{4}$, D.N. Panchenkov ${ }^{1}$

${ }^{1}$ Laboratory of Minimally Invasive Surgery, A.I. Evdokimov Moscow State University of Medicine and Dentistry, MOSCOW, Russia; ${ }^{2}$ Surgical department, A.I. Evdokimov Moscow State University of Medicine and Dentistry, MOSCOW, Russia; ${ }^{3}$ Surgical department, Federal Research Clinical Center for Specialized Health Care and Medical Technol, MOSCOW, Russia; ${ }^{4}$ Department of interventional diagnosis and treatment, A.I. Evdokimov Moscow State University of Medicine and Dentistry, MOSCOW, Russia

Aim: To assess nearest and long-term results of the combined treatment in patients with locally advanced pancreatic cancer who underwent irreversible electroporation of the tumor in combination with chemotherapy.

Methods: It were analyzed the time to progression and overall survival in 23 patients who underwent irreversible electroporation in combination with chemotherapy in cases of unresectable pancreatic cancer for the period from May 2012 to March 2017. Control group consisted of 35 patients with pancreatic cancer stage III who received standard chemotherapy alone.

Results: Mean age of patients was 61 years (range 45-80). All procedures were successful. Fifteen patients had pancreatic head cancer, Eight patients-cancer of pancreatic body. 20 (86.9\%) patients received preoperative chemotherapy in for 4 months prior to surgery. 17 (73\%) patients underwent chemotherapy after electroporation procedure. 90 day mortality was $4.3 \%$ $(n=1)$ in electroporation group. It was found that Erreversible electroporation improved loca recurrence-free survival ( 12 and 6 months, respectively, $\mathrm{p}=0.01$ ) and distant recurrence free survival ( 15 and 8 months, respectively, $\mathrm{p}=0.03$ ). Overall survival was 18 and 11 months, respectively $(\mathrm{p}=0.03)$

Conclusion: Irreversible electroporation of locally advanced pancreatic cancer is safe. Four month chemotherapy followed by surgical procedure is associated with good local response and better overall survival compared with chemotherapy alone. These data will be validated in further multicenter study. 


\section{P341-HEPATO-BILIAIRY \& PANCREAS-Pancreas}

\section{MINIMALLY INVASIVE DRAINAGE OF A PANCREATIC PSEUDOCYST}

D.E. Dinu ${ }^{1}$, M. Greere ${ }^{2}$, F. Chiru ${ }^{3}$, C. Gindea ${ }^{1}$, A. Constantin ${ }^{1}$, S.M. Constantinoiu ${ }^{1}$

${ }^{1}$ General and Esophageal Surgery Clinic, UMF „Carol Davila,, „Sf Maria,, Clinical Hospital, BUCHAREST, Romania;

${ }^{2}$ Gastroenterology, „Sf Maria,, Clinical Hospital, BUCHAREST, Romania; ${ }^{3}$ General and Esophageal Surgery Clinic, „Sf Maria, Clinical Hospital, BUCHAREST, Romania

Introduction: Pancreatic pseudocysts are the most frequent complication of acute or chronic pancreatitis. Usually asymptomatic, they can be managed conservative or, in case of complications, by several methods, endoscopic, percutaneous or by surgery.

Material and method: We present the case of a 41 years old patient known with an episode of acute pancreatitis five years ago, who was hospitalised now for an upper gastrointestinal bleeding with hematemesis. The upper endoscopy showed a subcardial bulking with an erosion of the posterior gastric wall, with signs of recent bleeding, managed by clipping. Patient work-up showed a $12 \mathrm{~cm}$ pancreatic pseudocyst at endoscopic ultrasound. Taking into consideration the history of the patient, the size and the complication of the cyst, the patient was proposed for a drainage intervention.

Results: A minimally invasive approach was decided. Using ultrasonography guidance, a posterior gastrotomy was performed with the cystotome, establishing the comunication with the pancreatic pseudocyst. Dilatation of the path with $8 \mathrm{~mm}$ CRE baloon, with partial evacuation of turbid liquid. The drainage consisted in 2 pigtail $10 \mathrm{Fr}$ plastic stents. The patient was discharged the following day in a good health condition.The endoscopic ultrasound control at 3 weeks showed complete resolution of the pancreatic cyst and was followed by stent removal Conclusion: The endoscopic drainage of the pancreatic pseudocyst represents the first treatment option as an alternative to the surgical intervention, being minimally invasive, with low risk and fast recovery.

\section{P342-HEPATO-BILIAIRY \& PANCREAS-Pancreas}

\section{VIDEO-ASSISTED RETROPERITONEAL DEBRIDEMENT FOR RIGHT COLLECTION IN STEP-UP APPROACH MANAGEMENT OF ACUTE PANCREATITIS}

S. Cerrato Deldado, A. Valverde Martinez, S. Ayllon Gamez,

C. Peña Barturen, J. Varela Recio, A. Fierro Aguilar,

J.M. Pacheco García, T. Gomez Sanchez, Cirugía General,

Hospital Universitario Puerta del Mar, CADIZ, Spain

Introduction: The benefits of video-assisted retroperitoneal debridement (VARD) in the Step-up Approach management of necrotic pancreatitis have been demonstrated. However, certain authors are not against the use of VARD for right collections for possible complications that may occur. Clinical case report: A 69-year-old man was admitted to the hospital with a diagnosis of severe acute pancreatitis and multi-organ failure. During the first month patient has in UCI and non invasive procedures were attempted: enteral feeding by a nasoduodenal tube was started and antibiotics were administered to control sepsis. On day 30, percutaneous drainage was performed for large retroperitoneal abscess. On 67 days, endoscopic transgastric necrosectomy was performed and the left collection was resolved. Due to the multi-organ failure persistence and the evidence of size increase of the right retroperitoneal collection, a VARD was decided.The right collection was accessed following the previously pigtail catheter. A $12 \mathrm{~mm}$ trocar was placed to create retro-pneumoperitoneum with a pressure between $6-8 \mathrm{mmHg}$. A trocar of $5 \mathrm{mmHg}$ was placed, purulent content was aspirated and a debridement was performed. Irrigation and aspirate was performed with normal saline and povidone-iodine solution. Drainage was used to perform washes with physiological saline and urokinase.On 146 days, the CT confirmed collection resolution. On 190 days he was discharged. After 8 months, the patient is in good clinical condition. Discussion: Drainage of the retroperitoneal abscesses via laparotomy is highly invasive and risky. VARD enables radical necrosectomy and drainage less invasively.

In this patient, the complete resolution of the right collection is obtained with retroperitoneal debridement without complications. We conclude that careful retroperitoneal necrosectomy is a valid alternative for the management of right collections.

\section{P343-HEPATO-BILIAIRY \& PANCREAS-Pancreas}

IMPACT OF INDIVIDUAL SURGEON EXPERIENCE ON THE OUTCOMES OF MINIMALLY INVASIVE DISTAL PANCREATECTOMIES

B.K. Goh, T. Kabir, Y.X. Koh, J.Y. Teo, S.Y. Lee, J.H. Kam, A. Chung, C.Y. Chan, Hepatopancreatobiliary and Transplant Surgery, Singapore General Hospital, SINGAPORE, Singapore

Background: Presently, there are limited studies analyzing the learning experience of minimallyinvasive distal pancreatectomies (MIDP) and these frequently focused on a single surgeon or institution learning curve. This study aims to critically analyze the impact of individual surgeon experience on the outcomes of MIDP based on the collective experiences of multiple surgeons at single institution.

Methods: Retrospective review of 90 consecutive MIDP from 2006 to 2018 was performed. These cases were performed by 13 surgeons over various time periods. The cohort was stratified into 4 groups according to individual surgeon experience. The case experience of these surgeons was as follows: $<5$ cases, $\mathrm{n}=8 ; 6-10$ cases, $\mathrm{n}=2 ; 11-15$ cases, $\mathrm{n}=2$ and 30 cases, $\mathrm{n}=1$. Results: The distribution of the 90 cases were as follows: experience $<5$ cases $(n=44) ; 6-10$ cases $(\mathrm{n}=20)$; $11-15$ cases $(\mathrm{n}=11)$ and 15 cases $(\mathrm{n}=15)$. As individual surgeons gained increasing experience, this was significantly associated with increasingly difficult resections performed, increased frequency of the use of robotic assistance and decreasing open conversion rates $(20.5 \%$ vs $10 \%$ vs $9.1 \%$ vs $0, P=0.038)$. There was no significant difference in other perioperative outcomes. These findings suggest that the outcomes of MIDP in terms of open conversion rate could be optimized after 15 cases. Subset analyses suggested that the learning curve for MIDP of low difficulty was only 5 cases.

Conclusion: MIDP can be safely adopted today and the individual surgeon learning curve in terms of open conversion rate can be overcome after 15 cases.

\section{P344-HEPATO-BILIAIRY \& PANCREAS-Pancreas}

\section{LAPAROSCOPIC SURGERY IN HEPATOCELLULAR CARCINOMA. CONSECUTIVE CASES STUDY}

A. Ríos, C.R. Redondo, J. Mir, R. Gómez, M. Cantos, R. Fabra, C. Zaragoza, General and Digestive Surgery, Hospital General Universitario de Valencia, VALENCIA, Spain

Aims: In this study we analyze laparoscopic approach for hepatocellular carcinoma in order to clarify iwe can take advantage in some outcomes as complications, postoperative recovery or long-term survival outcomes.

Methods: A retrospective case consecutive study has been taken analyzing: age, sex, body max index, comorbidity, surgical extension and tumor size. The outcomes analyzed were: operation time, intraoperative blood loss, blood transfusion, postoperative morbidity and mortality, intensive care stay, hospital stay, tumor size, R0 resection, conversion rate, early reintervention, disease-free survival rate, overall survival rate

Results: In this study 15 patients were analyzed 12 males and 3 females with ages between 54 and 73 years (mean age 62 ) and diverse comorbidities: arterial high pressure $(7 / 15 ; 46 \%)$, diabetes $(2 / 15 ; 13,3 \%)$; dislipemy $(5 / 15 ; 33,3 \%)$, hepatophaty measured as liver cirrhosis $(14 / 15$; $93,3 \%)$. All of them underwent laparoscopic liver surgery, in 9 cases non-anatomical resection was performed while in the other 6 a segmentectomy was performed. In 12 cases the laparoscopic was strict, in 3 and assistance incision was needed. Operative time was 120-525 min (mean: 282 $\mathrm{min}$ ). Blood loss mean was $1,2 \mathrm{~g} / \mathrm{dl}$ and only 2 intraoperative transfusion were needed. Massive blood loss was reported in 1 case. Postoperative medical complications were observed: hepatic failure and renal insufficiency and in 1 case we observed a postoperative hemorrhage that needed an urgent reintervention. The mean of intensive care stay was 1 day and hospital stay was 4.93 days. About oncological outcomes R0 resection was achieve in $9 / 15(60 \%)$, R1 in $6 / 15(40 \%)$. At 3 years $9 / 15$ cases were free disease, 3 dead by progression of disease and 2 dead by other causes.

Conclusions: Laparoscopic approach in hepatocellular carcinoma has showed similar results to open surgery in quality of resection and long-term free disease survival and better results has been reported in blood loss, hospital stay and recovery 


\section{P345-HEPATO-BILIAIRY \& PANCREAS-Pancreas}

\section{LAPAROSCOPIC DOUDENOPANCREATECTOMY-OUR} APPROACH. HOW TO DO IT?

\section{N. Belev}

\section{Surgical Department, Eurohospital, PLOVDIV, Bulgaria}

Aim: The purpose of this study is to analyze our initial experience with laparoscopic duodenopancreatic resection.

Introduction: Laparoscopic procedures have advanced to represent the new gold standard in many surgical fields. Laparoscopic pancreatoduodenectomy and laparoscopic distal pancreatectomy(LDP) are advocated to improved perioperative outcomes, including decreased blood loss, shorter length of stay, reduced postoperative pain and expedited time to functional recovery. However, the indication to minimally invasive approach for pancreatic surgery is often benign or low grade malignances. Material and Method. The steps of LDP procedures are similar to the open procedure. We perform destructive part of procedure totally laparoscopically and we prefer to do reconstructive part of procedure using hand-assisted techniques. For the period 2014-2017, we have been perform 76 PD, 24(32\%) we have done with laparoscopic approach. $6(31 \%)$ of patients were operated totally laparoscopic and $18(69 \%)$ of patients were operated by handassisted techniques.

Results: We found longer operative time in laparoscopic group, $385 \mathrm{~min}$. vs $210 \mathrm{~min}$ in open group. Mean blood loss was $260 \mathrm{ml}$ in laparoscopic operated patients and $430 \mathrm{ml}$ in open group. Mean length of stay was 8 days in laparoscopic group vs 14 days in open group. Overall morbidity in laparoscopic group was 19\%. One patient with superior vein thrombosis(Clavien-DindoII), One patient with postoperative acute necrotizing pancreatitis(Clavien-Dindo-IVa), a 2(two) patient with low debit pancreatic fistula(Clavien-Dindo-II). Mortality rate was 7\%(1 patient was died in early postoperative period from venous mesenterial thrombosis and multiorgan dysfunction.

Conclusion: LPD can be done laparoscopically in selected patients by experienced surgeons (in $\overline{\text { laparoscopic }}$ and open surgery too), but clear advantages remain to be defined.

\section{P346-HEPATO-BILIAIRY \& PANCREAS-Pancreas}

\section{ACUTE PANCREATITIS AFTER ERCP IN THE MANAGEMENT OF PATIENTS UNDERGOING LAPAROSCOPIC CHOLECYSTECTOMY}

D. Micic, B. Oluic, V. Arsenijevic, S. Mijatovic, Clinic for Emergency surgery, Clinical center Serbia, BELGRADE, Serbia

Introduction: Acute cholecystitis is one of the most common gastrointestinal diseases which require hospitalisation and surgical treatment. ERCP is a useful adjunct in the management of patients undergoing laparoscopic cholecystectomy who have common duct stones. It is also necessary for the management of residual stones and complications after laparoscopic cholecystectomy.

Material and methods: This study consisted of 200 patients who underwent laparoscopic cholecystectomy due to cholecystitis and choledocholythiasis.

Results: A significantly higher conversion rate was encountered when LC was done 2-6 weeks after ES, as compared to 1 week after ERCP. It is estimated that pancreatitis after ERCP affects roughly three to 10 percent of patients and many endoscopists quote a post-ERCP pancreatitis rate of $3-5 \%$. However, $10-15 \%$ is probably a more realistic answer for the majority of ERCP endoscopists. Wise endoscopists inform their patients that there is a spectrum of post ERCP pancreatitis severity, from mild ( $>95 \%$ of cases) to severe (1-5\% of cases). In mild forms, pancreatitis after ERCP may resolve itself.

Conclusion: Endoscopic retrograde cholangiopancreatography is a procedure used to diagnose and treat disorders involving the pancreatic and bile ducts. Acute pancreatitis is the most common and feared complication of endoscopic retrograde cholangiopancreatography.

Keywords: laparoscopic cholecystectomy, ERCP, pancreatitis, choledocholyhiasis

\section{P347-HEPATO-BILIAIRY \& PANCREAS-Pancreas \\ COMPARISON OF OPEN AND LAPAROSCOPIC PANCREATODODENECTOMY. INITIAL EXPERIENCE}

\section{Sikorszki, G. Szabadkai, L. Venczel, L. Füstös}

General Surgery, Bács Kiskun County Hospital, KECSKEMÉT, Hungary

Introduction: Even during pancreatic resection, minimal invasive surgery is gaining ground, laparoscopically or even more robotic. Our goal was to analyze our initial experiences. Method We performed 32 pancreatododenectomy between 2017.09.01.-2018.12.01.32. Out of these 14 patients underwent laparoscopic whereas 18 underwent an open resection. We analyzed the intraoperative and postoperative complications, and the early oncological findings.

Results: In the laparoscopic group the mean surgical time was $335 \mathrm{~min}$ and in the open group it was $215 \mathrm{~min}$. The difference is significant. The laparoscopic group had 2 conversions due to vascular injury and tumor infiltration of the portal vein. In the laparoscopic group there was no fatal outcome. However there was one death in the open group on the $8^{\text {th }}$ postoperative day. In that case, hepatic resection has occurred, the patient has had neoadjuvant treatment, after which severe liver involvement of oxaliplatin has been detected and eventually mesenteric circulatory disturbance and MOF caused the death of the patient. In the laparoscopic group, a sentine bleeding caused DSA embolization, and the patient recovered after 3 weeks of drainage.

There was no hernia formation and wound complications in the laparoscopic group, but on the other hand there were 4 wound suppurations and 6 postoperative hernia in the open group. Laparoscopic patients had a median length of hospital stay of 8 days, compared with 12 days for the open patients. In the open and laparoscopic group, hepatic metastases were also detected during the oncological care in 1 case. There was the possibity of local recurrence of the truncus coeliacus in the laparoscopic group in one case, however the biopsy did not confirm it. Among the open surgical procedures there was one case with mesenterial local recurrence. Conclusions: The assumption is that the duration of the laparoscopic method is longer, but on the other hand the patient have better wound healing and fewer possibility of developing postoperative hernia . The postoperative period is much more simple due to the significantly shorter hospitalization and the faster recovery, and according to patients the level of pain is much smaller as well. However the oncology results are the same.

\section{P348-HERNIA-ADHESIONS-Abdominal wall hernia}

\section{LAPAROSCOPIC APPROACH OF BOWEL OBSTRUCTION DUE} TO SPIEGEL'S HERNIA. A CASE REPORT

P. Martinez, E. Toledo, C. Gil, E. Gonzalez, A. Lozano, M. Lainez, J.C. Rodriguez

\section{General Surgery, HUMV, SANTANDER, Spain}

Introduction: Spiegel hernias are a rare, representing only between $0.1 \%$ and $2 \%$ of all abdominal wall hernias. Due to its location, below the Spiegel line, its diagnosis requires a high index of suspicion. The physical examination only detects $50 \%$ of the Spiegel hernias and, in many occasions, imaging tests are necessary for the diagnosis.

Goals: Our objective is to describe the case of an urgent laparoscopic repair of a case of high grade bowel obstruction secondary to a Spiegel hernia.

Material and Methods: We present the case of a 75-year-old male patient with no medical history that comes to the emergency department of our center due to an eight hour evolution of abdominal discomfort associated with nausea without vomiting or other symptoms. The patien was afebrile and hemodynamically stable at all time. On physical examination, the abdomen is soft and depressible, painful on the left flank where a tumor compatible with Spiegel's hernia is palpable. In the blood count there is no leukocytosis nor alteration of inflammatory parameters. An abdominal computed tomography (CT) scan was requested from the emergency departmen which demonstrated a high-grade small bowel obstruction caused by an entrapped loop of distal jejunum conditioned by a left-sided spiegel hernia. Given the situation, an informed consent was obtained, and the patient was taken to the operating room for emergency laparoscopic repair. We performed a laparoscopic hernioplasty with Ventralpatch mesh between oblique major and transverse and primary closure of defect in continuous suture. After this, the evolution of the patient is favorable, with good oral tolerance and re-establishment of intestinal transit, being able to be discharged $48 \mathrm{~h}$ after surgery.

Conclusions: The Spiegel hernia is a rare entity that requires a high index of suspicion for its diagnosis. Despite the limited evidence published in the literature on the laparoscopic repair of incarcerated Spiegel hernias, the studies published so far suggest that the laparoscopic repair is a valid alternative to the classic approach when it is performed by a well-trained laparoscopic surgeon. 


\section{P349-HERNIA-ADHESIONS-Abdominal wall hernia}

MINIMALLY INVASIVE APPROACH TO LATERAL ABDOMINAL WALL HERNIAS-THE EXPERIENCE OF AN ASIAN CENTRE

\section{F. Leong, S. Wijerathne, D. Lomanto}

Department of Surgery, National University Hospital, SINGAPORE

Introduction: Repair of lateral abdominal wall hernias (both primary and incisional) can be challenging due to the complexity of anatomy, issues with fixation and the low incidence of such cases. A good understanding of abdominal wall and retroperitoneal anatomy, coupled with proficient laparoscopic technique is essential for successful repair via the minimally invasive approach.

Methods: A retrospective review of a prospectively maintained database was performed to identify patients with lateral abdominal wall hernias who underwent laparoscopic repair from January 2015 to July 2018

Results: 11 patients with 12 hernias were identified ( 6 primary, 6 incisional). Mean patient age was 60 (range 22-81) and mean BMI was $27.1 \mathrm{~kg} / \mathrm{m}^{2}$ (range 18.2-34.6). According to EHS classification, the incisional hernia defects were located at subcostal $(\mathrm{L} 1, \mathrm{n}=1)$, flank (L2, $\mathrm{n}=3)$, iliac ( $\mathrm{L} 3, \mathrm{n}=1)$ and lumbar $(\mathrm{L} 4, \mathrm{n}=1)$ regions. The incisional hernias were secondary to either open appendicectomy or port sites from various minimally invasive procedures. Primary lateral abdominal wall hernias included 5 Spigelian hernias and 1 lumbar hernia. The mean defect size was $22 \mathrm{~cm}^{2}$ (range 4-68). Mesh repair was used for all patients. Repair type was either intraperitoneal on-lay mesh (IPOM, $\mathrm{n}=3$ ), extended totally extra-peritoneal approach (e-TEP, $\mathrm{n}=5$ ) or trans-abdominal partial extra-peritoneal (TAPE, $n=4)$ approach. 2 patients had seromas and 1 patient had a surgical site infection, all were managed conservatively with antibiotics. No other complication or recurrence was reported with a mean follow up of 10 months (range 1-31). Conclusion: Our study demonstrates the safety and feasibility of minimally invasive repair for lateral abdominal wall hernias. We suggest that repair of these hernias should be attempted only by surgeons who are proficient and comfortable with the various intra-peritoneal and extraperitoneal techniques.

\section{P350-HERNIA-ADHESIONS-Abdominal wall hernia}

\section{TROCAR-SITE INCISIONAL HERNIA-ANALYSIS 256 CASES}

\section{G. Rustamov ${ }^{1}$,E. Rustamov ${ }^{2}$}

${ }^{1}$ GENERAL SURGERY, BAKU CASPIAN HOSPITAL, BAKU, Azerbaijan; ${ }^{2}$ General surgery, BAKU CASPIAN HOSPITAL, BAKU, Azerbaijan

Background: It is commonly admitted that laparoscopic surgery has the advantage of abdominal wall preservation. However, the increased use of laparoscopy has resulted in certain complications specifically associated with the laparoscopic approach, such as trocar-site incisional hernia. Until today, it is not finally clarified 'patient-dependent' factors contributing to the occurrence of postoperative hernia after laparoscopic abdominal surgery.

Methods: Between 1996 and 2017, 256 patients were operated due to trocar-site incisional hernia in one surgical centre. 'The patient-depending' factors which caused postoperative trocar site incisional hernia data was collected and retrospectivily analysed.

Results: Port site incisional hernia occurred in $98 \%$ ( 250 patients) after the use of trocars with $10 \mathrm{~mm}$ or larger diameter. The presence of metabolic syndrome was the decisive factor in the development of postoperative incisional hernia in $62 \%$ (159 patients). In $15 \%$ (38 patients) the postoperative hernia occurred on the background of a long cough symptoms caused by chronic obstructive pulmonary diseases. The cause of postoperative hernia in 13\% (33 patients) of patients was the condition of lifting a one-time severity or heavy physical work. In $10 \%$ (26 patients) of postoperative patients hernia developed due to prolonged constipation of chronic inflammatory colon diseases.

Conclusions: Thus, when the aponeurosis of the trocars is adequately closed, the reason of the occurrence of postoperative hernias was caused by patient-dependent factors which increase intra-abdominal pressure.

\section{P351-HERNIA-ADHESIONS-Abdominal wall hernia}

INTRODUCING SINGLE INCISION LAPAROSCOPIC SURGERY (SILS) INTO RIVES STOPPA REPAIR OF VENTRAL HERNIA

\section{T. Nagahama}

Surgery, Kudanzaka Hospital, TOKYO, Japan

Aims: TEP Rives-Stoppa repair is established method for the repair of ventral hernia. However, there are some technical difficulties like midline crossover or dissection of intestinal adhesion around the hernia orifice. We have introduced SILS into Rives-Stoppa repair (SILS R-S repair) to solve problems mentioned above.

Method: From 2016/11 12 patients of midline incisional hernia, umbilical hernia, epigastric hernia underwent SILS R-S method.

For this method, small midline incision $3 \mathrm{~cm}$ in length $5-6 \mathrm{~cm}$ away from hernia orifice was carried out initially. Dissection of intraperitoneal adhesion was carried out by SILS with SILS device. Subsequently after closure of initial laparotomy unilateral anterior rectus sheath was incised from the same incision and dissection of retro-rectus space up to preperitoneal space was done under laparoscopic vision. Dissecting the other side was carried out by same fashion. Initia dissection of linea alba could be done by open surgery from initial incision. Further dissection of linea alba, retro-rectus space, and hernia orifice was carried out by SILS. Defect closure of anterior and posterior rectus sheath using barbed suture was also done by SILS and self-grip mesh was inserted. Additional trocar to assist retro-rectus dissection, defect closure, and decompression of intraperitoneal cavity was inserted as required.

Result: For 12 patients SILS R-S method completed successfully. Duration of surgery was 98-260 $\mathrm{min}$ (mean $164 \mathrm{~min}$ ) and transverse diameter of hernia orifice was 20-85 $\mathrm{mm}$ (mean $53 \mathrm{~mm}$ ). Number of additional trocar inserted to carry out adhesiolysis was 0-3 (mean 1.5) Three patients received laparoscopic bilateral Trans Abdominal Release (TAR). Persistent wound pain controllable by oral pain killer was most common adverse event. During follow up ranged 3 to 22 months (mean 10.5 months), we have experienced no case of recurrence and wound infection. Discussion: Our SILS R-S method demonstrated some solution for introducing complex RivesStoppa procedures. SILS TEP device enabled no balloon dissector and dissection of linea alba under direct vision contributed to easier preparation of whole retro-rectus spaces.

Conclusion: SILS R-S method can assist introducing of Laparoscopic Rives-Stoppa procedure easier.

\section{P352-HERNIA-ADHESIONS-Abdominal wall hernia}

HOW LAPARO-ENDOSCOPIC SURGERY REINVENTS
RETZIUS AND BOGROS SPACES?

D. Ion, R.V. Stoian, B.M. Serban, D.N. Paduraru, O. Andronic

General Surgery and Emergency Clinic III, The Emergency University Hospital of Bucharest, BUCHAREST, Romania

Aims: The laparo-endocsopic approach of inguinal hernia contiue to bring many clarifications concerning inter-parieto-peritoneal space of this region through in vivo exploration, obtained by magnification by means of specific optic intrumentation. Our study aimed to revalue the in vivo fascias, to establish their embryological correspondences and to reunite the variable nomenclature existing in the classical anatomy of this region. These observations find their applicability in TAPP and TEP hernia procedures, as the old anatomical descriptions are no longer operative. Methods: We have tried to identify the structures that delimit the anatomical regions of Retzius and Bogros in 20 recording of TAPP procedures performed on men, on the right side, for small indirect hernias on patintes with clear view of the structures. Additional, a review of literature on this subject has been performed through a search in the detabases according to the following keywords: Bogros space, Retzius space, preperitoneal approach, urogenital fascia. Results: Retzius and Bogros are the medial and lateral compartments of the inter-parietalperitoneal space, located between the transversal fascia and the parietal peritoneum. These narrow, virtual spaces are best highlighted today with the help of insufflation techniques during laparo-endoscopic procedures. A competent and careful dissection confirms a 'deep and superficial' stratification, highlighting embryonic relics derived from the uro-genital fascia: urinaryprevesical fascia and spermatic fascia. In addition, the real Retzius space is located previously and the real Bogros space is located behind this strcuture. The confluence area of the two spaces is a critical point of laparo-endoscopic dissection, its non-recognition may 'wander' the dissection.

Conclusions: Literature data in this topic reflects a certain terminological confusion using general terms such as 'preperitoneal tissue' or 'arreolar tissue' to denote what we consider to be the urogenital fascia or its prologations. The data obtained were synthesized in several drawings and diagrams very useful in training surgeons to use TAPP / TEP techniques. 


\section{P353-HERNIA-ADHESIONS-Abdominal wall hernia}

\section{EARLY OUTCOMES OF OPEN VS. LAPAROSCOPIC IPOM REPAIR FOR SMALL VENTRAL HERNIAS}

W.Q. Leong, S.A. Lui, S. Wijerathne, L. Loo, W.B. Tan, D. Lomanto, Minimally Invasive Surgical Centre

Department of Surgery, National University Health System, SINGAPORE

According to the 2014 International Endohernia Society (IEHS) guidelines, mesh repair is recommended for abdominal wall hernias with defects larger than $2 \mathrm{~cm}$, and those larger than $3 \mathrm{~cm}$ can be considered for laparoscopic mesh repair. In our study, we sought to compare the outcomes of open versus laparoscopic intraperitoneal onlay mesh (IPOM) repair in small ventral hernias. Data of all patients with a single umbilical defect of less than $4 \mathrm{~cm}$ who underwent surgical mesh repair between January 2015 and September 2017 was collected. Patient demographics, operative findings and regular post-operative follow-up details up to 3 months, including pain scores, recurrence rates, and complications, were recorded.

Forty-one patients underwent laparoscopic IPOM repair (Symbotex mesh, Medtronic, USA) and 47 patients underwent open IPOM repair (Ventralex, BD, USA or Ventral-Patch, Medtronic, USA). The mean age for the study population was 51.5 years (range 26-78). There were 50 $(57 \%)$ males and $38(43 \%)$ females. Para-umbilical hernias were more common $(54 \%)$. The mean defect size was $2.1 \mathrm{~cm}$ in the group that underwent open repair and $2.8 \mathrm{~cm}$ in the group that underwent laparoscopic repair. The median surgery duration for open IPOM repair was significantly shorter ( $53 \mathrm{~min}$ vs. $73 \mathrm{~min} ; \mathrm{p}=0.001)$. There was no significant difference in pain scores and incidence of seroma formation and surgical site infections post-operatively after 2 weeks. There was also no significant difference in both groups in terms of chronic pain and recurrence at 3 months follow-up.

Open IPOM repair for small ventral hernias (less than $4 \mathrm{~cm}$ ) may be superior to laparoscopic IPOM repair in terms of the shorter operative duration, single incision and no additional risk of port site hernias. Moreover, there was no difference in pain scores, wound infection and recurrence rate between the two groups.

\section{P354-HERNIA-ADHESIONS-Abdominal wall hernia}

\section{E-TEP IN VENTRAL AND INCISIONAL HERNIA REPAIR- OUR} EXPERIENCES

\section{B. Radovanovic, M. Mirkovic, N. Davidovic}

Department of Surgery, General hospital Pozarevac, POZAREVAC, Serbia

Aims: Rives-Stoppa repair has become standard in repairing ventral and incisional hernias. Endoscopic retromuscular approach has the same benefits and offers advantages of minimal invasive surgery.

Methods: The technique is based on the retromuscular approach to the linea semilunaris (longitudinal technique) or linea semicircularis (transverse technique). The incision is made on the anterior rectus sheath, and the trocar is placed below the muscle. Retromuscular space is created and the neck of the hernia sac can be reached. Upon the release of the hernia, the sheath of the opposite rectus muscle opens up entirely up to the semilunar line, allowing the creation of enough space for placing the mesh. Mesh can be fixed using transcutaneous sutures, the glue or non-fixed. Closing of the defect is not always necessary.

Results: Between 2003. and 2017. we performed 108 operations. We had 35 umbilical, 17 epigastric, one Spigelian and 55 incisional hernias. There were no intraoperative complications in ventral hernias, and one bowel injury in incisional hernia group. Five conversions. Four recurrences. All of them caused by small mesh, after insufficient dissection. There were no infections. Conclusion: E-TEP (the enhanced or extended view totally extraperitoneal technique) will probably reach the results and benefits of retromuscular open technique.
P355-HERNIA-ADHESIONS-Abdominal wall hernia

PREVENTING POSTOPERATIVE SEROMA FORMATION IN ABDOMINAL WALL HERNIA BY INTRAOPERATIVE HYPERTONIC SALINE IRRIGATION

\author{
M. Dudai, K. Ittah Gilboa
}

Surgery, MERAV Medical Center, Hernia Excellence, TEL AVIV, Israel

Aims: Seroma formation (SF) is a frequent post-operative complication as in Abdominal Wall Hernias (AWH) and reconstructive surgeries, where extensive dissection take place. It increases overall morbidity and can be challenging to manage. Drains, aspirations and sclerotherapy are established for treating postoperative seromas and increase the risk for infections. No study has yet to describe the use of intraoperative preventive measures for SF. The purpose of this report is to describe a novel method of Intraoperative Hypertonic Saline Irrigation (IHSI) to abdominal wall subcutaneous surgical cavity, which prevent SF and enables a shorter drainage usage due to early drain removal.

Methods: This study includes seven patients who undergone the Extended Endoscopic Hernia \& Linea Alba Reconstruction Glue surgery (eEHLARglue), for AWH and Rectus Muscles Separation (RMS). An extensive Endoscopic dissection of the anterior Rectus fascia is performed prio to dissecting and reducing into the abdominal cavity any hernia followed by closing of the RMS A mesh is placed over the repaired RMS and is fused into the muscles by Fibrin Glue. The novel preventive method is based on Intraoperative Irrigation of the subcutaneous cavity through two $7 \mathrm{~mm}$ closed system drains with $20 \mathrm{cc}$ of $\mathrm{NaCl} 10 \%$ which are left in place for $10 \mathrm{~min}$. Results: Our early results with all our 7 patients over three months of follow up, show seroma prevention, lower drain secretion rate of $20 \mathrm{cc}$ in $10 \mathrm{~h}$ and drains removal within 20-24 h. Conclusions: IHSI enhances fusion adhesions and reduce secretion rate and therefore enables early drain removal and prevent SF. As a result, reducing overall morbidity and hospitalization period, decreasing inconveniency and cost saving of multiple outpatient visits or additional surgery. This novel technique could be used in addition to AWH in many others potential postoperative SF surgeries. Further research is advised.

\section{P356-HERNIA-ADHESIONS-Abdominal wall hernia}

\section{SPIGELIAN HERNIA CONTAINING EPIPLOIC APPENDAGE, LAPAROSCOPIC REPAIR}

\author{
M.Y. Uzunoglu ${ }^{1}$, F. Altintoprak ${ }^{2}$, B. Kamburoglu ${ }^{3}$, K. Gundogdu ${ }^{4}$
}

${ }^{1}$ General Surgery, Siirt State Hospital, SIIRT, Turkey; ${ }^{2}$ General Surgery, Istinye University, School of Medicine, ISTANBUL, Turkey; ${ }^{3}$ General Surgery, Sakarya university, School of Medicine, SAKARYA, Turkey; ${ }^{4}$ General Surgery, Sakarya University, Research and Educational Hospital, SAKARYA, Turkey

Aim: Spigelian hernia containing epiploic appendage is really rare entity. In this paper, we present a very rare case of Spigelian hernia involving epiploic appendage performed laparoscopic hernia repair.

Case report: A 60-year-old woman presented to the emergency department with sudden onset abdominal pain in the left lower quadrant. On physical examination, she had a small, palpable tender mass in the left lower abdominal quadrant. Temperature and white blood cell count were normal. An inflamed epiploic appendage with an oval shape, a fatty core, and a central thin hyperdense line in the hernia sac was detected on abdominal computed tomography. Its intraabdominal relationship with the normal wall of the sigmoid colon was well appreciated (Figure 1A, 1B). Diagnostic laparoscopy was performed. (Figure 2) Adhesions between the sac and epiploic appendage are released using sharp dissection. A peritoneal flap is then created (Figure 3). Laparoscopic TAPP repair was used without closing the defect (Figure 4). The patient was discharged on 3th days uneventfully.

Discussion: Spigelian hernia (SH) is a rare type of anterior abdominal wall hernia. The most frequent hernial contents are omentum, small intestine and colon. Epiploic appendage (EA) is very rare content of SH. EAs are pedunculated fatty structures protruding from the serosal surface of the colon, most are localized in the caecum and sigmoid colon along the taenia omentalis and taenia libera. SH have a wide clinical spectrum and difficulties in preoperative diagnosis and a high incarceration and obstruction risk. The treatment of SH is surgical and can be performed either by classic open or laparoscopic technique.

Conclusion: Laparoscopic SH repair is safe and effective although surgical technique depends on patient characteristics, type of hernia and surgeon experience. 


\section{P357-HERNIA-ADHESIONS-Abdominal wall hernia}

THE Method: OF LAPAROSCOPIC PLASTIC OF POSTOPERATIVE VENTRAL HERNIAS

M.V. Zinovsky ${ }^{1}$, Y.V. Ivanov ${ }^{2}$, A.A. Terekhin ${ }^{3}$, G.A. Vityazev ${ }^{3}$, D.N. Panchenkov ${ }^{1}$

${ }^{1}$ Laboratory of Minimally Invasive Surgery, A.I. Evdokimov Moscow State University of Medicine and Dentistry, MOSCOW, Russia; ${ }^{2}$ Surgical department, A.I. Evdokimov Moscow State University of Medicine and Dentistry, MOSCOW, Russia; ${ }^{3}$ Surgical department, Federal Research Clinical Center for Specialized Health Care and Medical Technol, MOSCOW, Russia

Aim: Development of a method for plastics of ventral hernias that increases the effectiveness of treatment, and reduces the likelihood of postoperative complications.

Methods: The method includes the introduction of trocars in the left hypochondrium and on the left side of the abdominal wall, the imposition of pneumoperitoneum, review laparoscopy, dissection of adhesions, determination of the size of the hernia gate, implantation and fixation of the mesh on the abdominal wall with overlap of the hernia gate at $3-5 \mathrm{~cm}$. New is that after dissection of adhesions, the hernia sac is transported into the abdominal cavity until its cavity is eliminated. Then the tissue of the peritoneum of the hernial sac is fixed along a part or along the entire perimeter of the hernial ring, and the mesh is additionally fixed to the peritoneum of the hernial sac. In this method, 30 patients with ventral hernias were operated on.

Results: Patients were discharged after surgery for 2-5 days. The intensity of the pain syndrome corresponded to 2-3 points on a 10 pointed visual analogue scale, was stopped by non-steroidal anti-inflammatory drugs. During planned follow-up examinations after 1 month, 2 seromas were identified in the projection of the implant. Solved conservatively. Authors supposed that complications associated with the material of the used mesh implant with anti-adhesive coating. After 3 months there were no complications associated with the use of this method.

Conclusion: The application of the proposed method allows to increase the efficiency of treatment of patients with ventral hernias by preventing the formation of seromas in the postoperative period, to reduce the likelihood of complications, hernia relapse.

\section{P358-HERNIA-ADHESIONS-Abdominal wall hernia}

\section{LAPAROSCOPIC APPROACH FOR MORGAGNI'S HERNIA REPAIR}

J. Pintor-Tortolero, M. Gómez Infante, F.J. Padillo-Ruiz, General and Digestive Surgery Department, University Hospital Virgen del Rocío, SEVILLE, Spain

Aims: Morgagni's hernia is an in infrequent, congenital, anterior or retrosternal diaphragmatic defect. The right side is the most frequently affected, up to $90 \%$ of cases. It represents between 2 and $5 \%$ of congenital diaphragmatic hernias. In childhood, they usually attend asymptomatically or with respiratory symptoms. Up to 5\% are diagnosed in adulthood, incidentally or after gastrointestinal obstruction debut. The treatment is surgery, which can be by laparoscopic or open approach.We present a case of laparoscopic approach with intra-abdominal mesh placement of giant Morgagni's hernia diagnosed in senile age.

Methods: 84-year-old woman with a history of advanced Alzheimer's dementia, partially dependent in daily life activities and institutionalized who consulted for intermittent episodes of oral diet intolerance associated with vomits of one month of evolution. Abdominal examination was anodine. Chest radiograph revealed a right lower lung field mass with fluid collected. Thoracoabdominal scan showed small bilateral pleural effusion and large, right anterolateral morgagni's hernia, which contains dilated segment of transverse colon and greater omentum . Results: Laparoscopic approach was performed. Hernia was reduced and hernia sac was removed. The defect was repaired with a dual-component (absorbable and non absorbable) mesh anchored with intracorporeal suture. Patient recovered and was discharged 4 days after surgery. Conclusion: Laparoscopic approach for morgagni's hernia reapir is secure and offers the advantages of less post-opertive pain, faster recovery and short postopatory stay.

\section{P359-HERNIA-ADHESIONS-Abdominal wall hernia}

FAILURE ON CONVENTIONAL DEFECT CLOSURE IN LAPAROSCOPIC VENTRAL HERNIA REPAIR.. CAN I PREDICT IT?

J. Gomez Menchero ${ }^{1}$, A. Gila Bohorquez ${ }^{1}$, J.M. Suarez Grau ${ }^{1}$, ${ }_{\text {I. Alarcon del Agua }}^{2}$, E. Licardie Bolaños ${ }^{3}$, A. Fernandez Carazo, J.A. Bellido Luque ${ }^{5}$, M. Sanchez Ramirez ${ }^{5}$, A. Bellido Luque ${ }^{3}$, S. Morales Conde ${ }^{2}$

${ }^{1}$ Surgery, Hospital de Riotinto, MINAS DE RIOTINTO, Spain; ${ }^{2}$ Surgery, Hospital Virgen del Rocio Sevilla, SEVILLA, Spain; ${ }^{3}$ Surgery, Hospital Quiron Sagrado Corazon, SEVILLA, Spain; ${ }^{4}$ Departamento Metodos cuantitativos, Departamento metodos cuantitativos de la Universidad Pablo de Olavide, SEVILLA, Spain; ${ }^{5}$ Surgery, Hospital Virgen Macarena, SEVILLA, Spain

Aims: Closing the Defect (CD) during Laparoscopic Ventral Hernia Repair (LVHR) could be related to a reduction of seroma formation or bulging (hernia mesh) compared to conventional LVHR. Tension originated in the midline for some authors have been identified as a Risk Factor, but there is not consensus about the size of the defects that can be closed with a low rate of complications. We analyzed those factors that might increase a CD failure in order to prevent it. Methods: We conducted a prospective controlled study of LVHR with CCD from January 2014 to December 2016. We evaluated defect closure failure as a recurrence rate and bulging (return to prior distance among rectus muscles with the mesh in the sac in Computerized Tomography(CT) that didn't need surgical treatment.). We analyzed different variables as defect width (DW) and Transverse Diameter of Abdominal Cavity in hernia site (TDAC), Age, sex, Body Mass Index, diabetes, smoking, Chronic Obstructive pulmonary disease (COPD), type of hernia in order to identify those factors more relevant in order to prevent a CCD failure.

Results: We enrolled a total of 60 patients. There were 12 cases of bulging (20\%) and 4 cases of recurrence (6.7\%), detected by CT scan at 1 year follow-up. CCD failure in percent terms was most prevalent in range of defects between 6 and $9 \mathrm{cms}$ both included (33.3\%). Mean BMI in CCD Failure group was $33.6 \mathrm{Kg} / \mathrm{m} 2$. Bulging in smoking was present in (36.4\%), diabetes $(15.4 \%)$, COPD $(50 \%)$, type of hernia primary (21.4\%) incisional (41.9\%)or recurrent hernia (35.7\%). We have developed a SCALE to complete before the procedure to identify individual risk factors of CCD Failure. Conclusion: We believe that is very important to identify those patients with a higher risk of CCD failure before we perform this procedure. A scale prior to the surgery can be useful to decide to perform another techniques without tension as a Component Separation.

\section{P360-HERNIA-ADHESIONS-Abdominal wall hernia}

\section{RETROPROSTHETIC SEROMA IN LAPAROSCOPIC VENTRAL} HERNIA REPAIR... AN UNKNOWN ENTITY

J. Gomez Menchero ${ }^{1}$, G. Ramos Porras ${ }^{2}$, A. Gila Bohorquez ${ }^{1}$, J.M. Suarez Grau ${ }^{1}$, I. Alarcon del Agua ${ }^{2}$, E. Licardie Bolaños ${ }^{3}$ A. Tejada Gomez ${ }^{3}$, S. Morales Conde ${ }^{2}$

${ }^{1}$ Surgery, Hospital de Riotinto, MINAS DE RIOTINTO, Spain; ${ }^{2}$ Surgery, Hospital Virgen del Rocio Sevilla, SEVILLA, Spain; ${ }^{3}$ Surgery, Hospital Quiron Sagrado Corazon, SEVILLA, Spain

Aims: Retroprosthetic seroma (RS) is defined as a fluid retention between the bowel and the prosthesis in the abdominal cavity after a Laparoscopic Ventral Hernia Repair (LVHR).

It is an unknown entity with only a few references in the literature. Our main objective is to analyze the influence of the use of different meshes in LVHR as a risk factor to develop the RS Methods: We conducted a prospective controlled study of LVHR following the Double Crown technique criteria and we compare the use of different intraperitoneal meshes. All the cases were evaluated by physical examination at 1 day, 7 days and 30 days to evaluate clinical seroma, pain (Visual Analogue Scale) and regularization of abdominal perimeter (subjective criteria of the patient of restoring the abdominal wall midline anatomy with no mass in surgical site). A Computerized Tomography (CT) was performed al 30 days to evaluate seroma formation and volume. Results: We enrolled a total of 60 patients. The incidence of RS at 30 days after LVHR was $21 \%$ (13 cases) but only $7.69 \%$ ( 1 case) was symptomatic and needed surgical treatment secondary to a recurrence. A statistical relationship was found between DualMesh ${ }^{\circledR}$ (PTFEe) Group and RS formation $(\mathrm{p}=005)$

Conclusion: DualMesh ${ }^{\circledR}$ (PTFEe) might be considered a risk factor in RS formation compared to others prosthesis. Although 1 case was symptomatic and needed surgical treatment secondary a recurrence in most cases undergoes with no complications 


\section{P361-HERNIA-ADHESIONS-Adhesions}

THE USEFULLNESS OF THE LAPAROSCOPIC OPERATIONS FOR ILEUS

K. Kawasaki, R. Asakura, K. Yamada, Y. Ohwada, M. Hosono, T. Okazaki, T. Ienaga

\section{Surgery, Takatsuki Hospital, TAKATUSKI, Japan}

Introduction: Recently, laparoscopic operations for ileus are increasing. We have undergone laparoscopic operation to adhesive ileus with umbilicar incision at the beginning. The umbilicar incision at the beginning makes it possible to secure the laparoscopic field by peeling the adhesion under direct view, and makes it easy to repair damage to the intestinal tract. Surgical Procedure: At first, the umbilicus 3-4 cm incision was made and peeled the adhesion as much as possible under direct vision. Secondly, EZ access was set and inserted one $12 \mathrm{~mm}$ port, therefore laparoscopic operation was performed with 2 or 3 pieces of $5 \mathrm{~mm}$ ports. When the repair or resection of small intestinal due to damage is necessary, it is pulled out through the EZ access.

Objective: To investigate the possibility of problems of laparoscopic ileus operation to adhesion ileus by umbilicar incision at the beginning.

Methods: We examined the results of 12 cases of the perioperative factors at our hospital from May 2016 to November 2018

Background: Age was 72 years old (same as median, 18-81 years old), surgery history was 1 case of appendectomy, prostate cancer, sigmoid colon cancer, ovarian cancer, ascending colon cancer, uterine cancer, inguinal hernia, esophageal cancer, gastroduodenal ulcer, uterine cancer and stomach cancer.

Surgical results: Medial operative time was $86.5 \mathrm{~min}$ (28-153 $\mathrm{min}$ ), intraoperative bleeding was $0 \mathrm{ml}(0-268 \mathrm{ml})$. Two cases were converted from laparoscopic operation to open operation, because of insufficient decompression of the intestinal tract, 10 cases completed by laparoscopy. Among laparoscopic completion examples, the intestinal tract dissection from the umbilical layer was one case, and the intestinal repair was seven cases.

Perioperative results: Three cases had complications (stenosis of anastomotic site, intussusception of the intestinal tract and paralytic ileus). The postoperative hospital stay (8.5 days, 6-94 days). There was no recurrence of ileus.

Conclusions: Laparoscopic ileus operation were adequate for decompressed adherent ileus. The umbilicar incision at the beginning was seemed to be effective. The intussusception of the intestinal tract due to the ileus tube was the problem to be covered.

\section{P362-HERNIA-ADHESIONS-Adhesions}

\section{BOWEL OBSTRUCTION AND PREGNANCY.}

A LAPAROSCOPIC APPROACH IS A SAFE ALTERNATIVE

P. Martinez ${ }^{1}$, C. Gil ${ }^{1}$, D. Morales ${ }^{1}$, S. Castanedo ${ }^{1}$, J. Jimeno ${ }^{1}$, A. Lozano $^{1}$, J.C. Rodriguez ${ }^{1}$, Y. Jubete ${ }^{2}$

${ }^{1}$ General Surgery, HUMV, SANTANDER, Spain ; ${ }^{2}$ Gynaecology and Obstetrics, HUMV, SANTANDER, Spain

Introduction: Small bowel obstruction (SBO) during pregnancy is a rare condition with an incidence of $0.001-0.003 \%$ and in around $70 \%$ of cases it is most caused by adhesions from previous abdominal surgery. Other diagnosis, such as, hernias, malignancy, volvulus or intussusception are extremely rare. When SBO occurs in pregnancy, it carries a significant risk to mother and fetus. Its diagnosis of can be difficult to make as symptoms are often attributed mistakenly to the pregnancy.

Goals: A case report of congenital bowel obstruction during the second trimester of pregnancy handled by laparoscopy.

Material and Methods: We report the case of a 38 year old woman with a history of chronic lung disease, pregnant because in vitro fertilization $(17+3$ weeks $)$ who attended the emergency department with abdominal pain and bloating accompanied by nausea and vomiting for two days. On physical examination she showed a distended, soft, depressible and painful abdomen without peritonism. Laboratory tests were normal. A nasogastric tube was placed with generous output fecaloid intestinal contents. Abdominal ultrasound by expert radiologists in abdomen showed a moderate amount of free abdominal fluid with normal uterus moderate and SBO to the ileum because of intestinal adhesion. This results were confirmed with an magnetic resonance imaging (MRI)

Results: The patient was operated by laparoscopic approach with three trocars. The main problem was discovered. We founded a congenital adhesion which conditionated the obstructive syndrome. Postoperative recovery was uneventful and the patient was discharged $48 \mathrm{~h}$ after surgery.

Conclusion: The non-obstetrical acute abdomen in pregnant patient is a reality that occurs in one of every 500 pregnancies. Its diagnosis in more difficult than in nonpregnant patients requiring or high index of suspicion. The laparoscopic approach of acute abdomen during pregnancy is a valid and safe option, even in the early hours after diagnosis of bowel obstruction when it is performed by a well-trained laparoscopic surgeon.

\section{P363-HERNIA-ADHESIONS-Adhesions}

\section{ADULT INTESTINAL MALROTATION SYNDROME; LAPAROSCOPIC APPROACH AND OUTCOMES}

M.G. Pramateftakis, S. Simeonidis, E. Kotidis, S. Angelopoulos, L. Loutzidou, K. Tsalis

4th Surgical Department, Aristotle University of Thessaloniki, THESSALONIKI, Greece

Aim: Intestinal malrotation (IM) without midgut volvulus in adults is a rare clinical entity, which is the result of an incomplete rotation of the small bowel during embryogenesis, due to the nonlysis of the Ladd bands. These ligaments spread between the duodenum and caecum and do not allow the gastrointestinal tract to take its normal position into the peritoneal cavity. IM appears in 1 to $300-500$ newborns and is usually asymptomatic. Diagnosis is usually made in the first month, and presents with findings of an acute abdomen, small bowel ileus and volvulus. IM in adults is a rare entity. Most of the times it is asymptomatic, but it can cause chronic abdominal discomfort and constipation. We present the laparoscopic management of an adult patient with intestinal malrotation.

Methods: Our patient, a 22 year old female, presented to the emergency room with a 3-month history of abdominal pain and nausea. All blood tests were normal. An abdominal MRI showed intestinal malrotation without volvulus. Due to persisting symptoms, she underwent a diagnostic laparoscopy with complete lysis of the Ladd bands. The only unusual finding was a slight oedema of the duodenum.

Results: Her symptoms settled postoperatively and she was discharged on the $2^{\text {nd }}$ postoperative day. Since her discharge, she has not developed any similar abdominal pains or complaints. Conclusions: Symptomatic intestinal malrotation in adults is an unusual clinical entity, but it is definitely one of the differential diagnoses we need to consider in case of chronic abdominal symptoms. The management consists of the division of the Ladd bands, and this procedure can be performed safely with laparoscopy.

\section{P364-HERNIA-ADHESIONS-Adhesions}

\section{THE POSSIBILITY OF A LAPAROSCOPIC APPROACH IN INTESTINAL OBSTRUCTION DUE TO FLANGES}

A. Lozano, C. Magadan, E. Garcia, A. Lopez, E. Gonzalez, E. Toledo, J. García, S. Castanedo, M. Lainez, P. Martínez, J.C. Rodriguez

General Surgery, University Hospital Marques de Valdecilla, SANTANDER, Spain

Aims: The objective is to present two cases of intestinal obstruction treated by laparoscopic approach. This topic has been chosen because there are data on the safety of the laparoscopic approach in the intestinal obstruction. It should be noted, the low percentage of patients who are operated by laparoscopic approach. The open approach of adhesiolysis has been the most widely accepted and practiced method. Some reasons is due to the difficulty to move of the dilated small bowel, the greater fragility of the intestinal walls and the smaller space of work due to the distension of small bowel. Despite this, the growing experience in laparoscopic surgery and the improvement of surgical instruments have increased the percentage of cases operated by laparoscopic approach.

Methods: The case of two women of 90 and 81 years is presented. They go to the emergency room due to they are vomiting and they do not have intestinal transit. On examination they have distended and painful abdomen, without peritoneal reaction. In the abdominal CT, dilatation of small bowel is observed with change in caliber in the proximal ileum.

Results: In both cases, surgical intervention is performed using laparoscopic approach. A pneumoperitoneum is performed with a Veress needle. Three 5-mm trocars are placed in the umbilical region, left lumbar region and left iliac region. A flange is the cause of intestinal obstruction. There is no suffering from small bowel so the flange is cut. There are not complications in the postoperative period.

Conclusions: Laparoscopic adhesiolysis can be a safe and effective technique in patients diagnosed with bowel obstruction due to flanges. This approach is beneficial for patients because i allows to evaluate the state of the abdominal cavity, the cause of intestinal obstruction and to determine the type of surgery. In addition, it generates lower postoperative morbidity and reduces the likelihood of new postoperative adhesions. Nevertheless, it should be noted the careful selection of patients and the experience of the surgeon are very important to obtain a high success rate in surgery. 


\section{P365-HERNIA-ADHESIONS-Adhesions}

\section{LAPAROSCOPIC SURGERY FOR A SMALL BOWEL OBSTRUCTION WITHOUT PREVIOUS LAPAROTOMY}

K. Ietsugu, Y. Yoshida, S. Tabata, N. Ota, Z. Nozaki, Y. Asaumi, H. Sugawara, K. Kiyohara

General surgery, Tonami general hospital, TONAMI, Japan

Many small intestinal obstructions are due to adhesions after laparotomy, but small bowel obstructions without history of open surgery is relatively few. In diagnostic imaging such as preoperative CT examination, the cause is diagnosed to some extent, but details are sometimes unknown unless operative observation is actually made. In many institutions, laparoscopic surgery is also actively introduced into the operation to relieve bowel obstruction, and its effectiveness is beginning to be recognized. We examined the usefulness of laparoscopic surgery for patients with small bowel obstruction without history of laparotomy from experience in our hospital.

Aim: From December 2000 to October 2018, we searched cases of laparoscopic surgery for small bowel obstruction without previous laparotomy at our hospital, and clinical findings, surgical results, and postoperative course were examined.

Results: There were ten cases. Eight men and two women. The median age was 57 years (15-90 yrs.). Reasons for intestinal obstruction were adhesions 5 cases, internal hernia 3 cases, persimmon stones 1 case, small intestine tumor 1 case. Four cases of adhesions were emergency surgery. There were 7 cases of emergency surgery and 3 waiting surgery. Five laparoscopic operations were completed and five cases during laparotomy transition. The median surgical

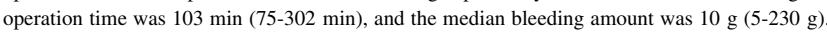
There was no fatal case after operation, only one complications of ileus. The median length of hospital stay was 14 days (10-29 days)

Conclusion: Laparoscopic surgery for intestinal obstruction with no history of laparotomy was thought to be a safe and effective procedure. Although the transition to laparotomy would be higher in case of emergency, but there was no case of large incisional laparotomy.

\section{P366-HERNIA-ADHESIONS-Adhesions}

\section{COMPARISON OF CLINICAL OUTCOMES FOLLOWING LAPAROSCOPIC AND OPEN SURGICAL MANAGEMENT OF SMALL BOWEL OBSTRUCTION}

\section{T. Kaetsu, M. Mizutani, K. Hatta, K. Yokomizo, M. Nagaya, K.} Shimizu, M. Kikuichi, Digestive Center

Department of Surgery, Kikuna Memorial Hospital, YOKOHAMA, Japan

Background: Recently, the efficacy of laparoscopic approach for small bowel obstruction (SBO) have been reported, however, laparoscopic treatment is still controversial in regard to long-term outcome and the indication for SBO. The aim of this study is to compare postoperative outcomes of laparoscopic versus open surgical management of SBO.

Methods: A retrospective analysis of patients underwent surgical treatment for SBO during 2011-2018 was made by a comprehensive search of medical records. Cases were classified as either laparoscopic or open surgical treatment groups and evaluated with each variable using perioperative factors, postoperative complications and recurrences.Postoperative outcomes were overall complication rate, operative time, blood loss, postoperative length of stay (LOS), and rate of recurrences.

Results: Of the 89 SBO included in the analysis, 30 (33.7\%) adhesiolysis procedures were performed laparoscopically. Patients were older $(60.4 \pm 18.6$ vs. $74.8 \pm 12.8 \mathrm{P}<0.0001)$ in laparoscopic group and laparoscopic surgery was less in the number of previous operation $(0.60 \pm 0.77$ vs. $0.93 \pm 0.78 \mathrm{p}<0.05)$. Patients undergoing laparoscopic procedures had lower mean blood loss $(19.6$ vs. $162 \mathrm{ml}, \mathrm{P}<0.0001)$, decreased postoperative length of stay $(11.0 \mathrm{vs}$. 19.0 days, $\mathrm{p}<0.01)$ and fewer rate of recurrences $(6.7 \%$ vs. $28.8 \%)$

Conclusions: Laparoscopic surgery for SBO reduces postoperative complications and contributes to shortening the postoperative hospital stay and to decreasing the rate of recurrences, although it is a retrospective study, which is a safe and a useful approach. Furthermore, first episode of SBO without previous operation seems to be an appropriate indication for laparoscopic surgery.

\section{P367-HERNIA-ADHESIONS-Adhesions}

AN EASY AND EFFECTIVE TECHNIQUE FOR THE PLACEMENT OF ANT-ADHESIVE BARRIER IN LAPAROSCOPIC GASTROINTESTINAL SURGERY

T. Kanazawa, K. Kawabe, Y. Mihara, M. Takahashi, Gastrointestinal Surgery, JR Tokyo General Hospital, TOKYO, Japan

Background: Postoperative adhesion after abdominal surgery may cause intestinal obstruction, chronic pain, or female infertility, which constitutes the major problems after surgery. Adhesion formation are reported to be reduced by laparoscopic surgery and the use of anti-adhesion barriers. Seprafilm composed of sodium hyaluronate carboxymethylcellulose bioresorbable membrane has been widely used to date, especially in open surgery. The characteristics of Seprafilm, which is easily stick when wet, conversely brittle when dry cause it difficult to delive into the abdominal cavity via the small incision in laparoscopic surgery. Therefore, Seprafilm is not much used in laparoscopic surgery. Although various methods of insertion of Seprafilm have been reported, some need special devices, or some acquire skill.

Methods: We adopted the pre-moistening technique for the replacement of Seprafilm in 210 consecutive cases of laparoscopic gastrointestinal surgery. A sheet of Seprafilm was cut into 4 equal pieces. To soften the sheets, one of the pieces was placed on a folded wet gauze until it became naturally curled then it was reversed, and the same procedure was repeated. Softened sheet is easily to deliver into the abdominal cavity via a small incision by pushing with digital finger. Moistened sheet expands naturally in the abdominal cavity. One or two pieces were needed to cover the incision. This process took only a few minutes.

Results: In all cases, the sheets were successfully introduced into the abdomen and spread widely enough to cover the incision. There have been no adverse effects, no postoperative complications, or gastrointestinal obstruction due to adhesion in the observation period of median two years. Conclusions: Short term outcomes were good after applying this technique. However, to record the incidents of intestinal obstruction and chronic pain, over 10 years observation is indispensable.

Long term follow-up studies are required to clarify the usefulness of the anti-adhesive barrier in gastrointestinal surgery.

\section{P368-HERNIA-ADHESIONS-Adhesions}

\section{CURRENT INDICATION FOR IPOM-ARE THERE ANY LEFT?}

B. East, 3rd department of surgery, Motol Faculty Hospital, PRAGUE, Czech Republic

Aim: Since 1994 when the IPOM acronym was used for the first time our views at intraperitoneal mesh positioning has changed several times. Despite growing evidence on its possible long term consequences it is still preferred method at some centres for large number of patients. The aim of this study is to point out the pitfalls of this method but also show that IPOM is a good technique but only for highly selected cohort of patients.

Methods: This is a review of the literature focusing on the indications and complications of IPOM pointing out controversies among the published articles over last two decades. Some mesh material characteristics are being discussed as they are basic for understanding this complex and highly sensitive issue.

Results: A wide range on indications of IPOM from little umbilical to large incisional hernias is advocated by many. However, some opinion leaders promoting this technique as universal and ideal for everyone just few years ago are advising to avoid it if possible lately. A necessary overlap has also been questioned recently. Despite improving anti-adhesion barriers and methods of fixation in May 2017 a surgical mesh has become classified as Risk class III by the EU Parliament and Council on medical devices hoping to prevent Physiomesh like incidents in the future. The need for post market registries and long term follow up is obvious.

Conclusion: Us as surgeons implant a mesh in our patients and therefore we should be aware of its possible long term effects. No mesh on the market has a long term safety evidence especially in the intraperitoneal space. IPOM is a good technique but possess a significant risk of long life complications and therefore should be spared only for those unfit for other methods of repair patients with too high mesh infection risk, obese or older patients. 


\section{P369-HERNIA-ADHESIONS-Emergency surgery}

PERFORATED JEJUNAL DIVERTICULUM: A CASE REPORT ON A RARE 6 X4 CM PERFORATED JEJUNAL DIVERTICULUM DISGUISED AS A CONTAINED GASTRIC PERFORATION

\section{Cartier, J. Gumer, S. Nazir, S. Liu}

General Surgery, NYU, BROOKLYN, United States of America

A 40 year old female with a past surgical history of laparoscopic hiatal hernia repair in 2013 presents with a complaint of 1 day of worsening abdominal pain. Patient admitted to vague abdominal pain for months that would self-resolve. Chest XRAY was performed that showed a small amount of intraperitoneal free air beneath the left hemi diaphragm. On exam patient was comfortable in no apparent distress, and her abdomen was soft, mildly distended, with left abdominal tenderness, non peritoneal. At this time patient was normotensive, afebrile, with tachycardia in the low $100 \mathrm{~s}$. CTAP was performed showing a foci of pneumoperitoneum in the left subphrenic space concerning for bowel perforation with mesenteric stranding. Upper gastrointestinal series was performed showing a small pocket of contrast near the gastric fundus most likely representing an area of localized perforation, with no free flow of contrast into cavity. Patient was hospitalized for 7 days and discharged home once tolerating a liquid diet. Eleven days later the patient represented with a complaint of 2 days of emesis and increasing abdominal pain. On arrival patient was found to be hypotensive, with labs significant for a white blood cell count of 15 and a creatinine of 2.7 (baseline 0.8). On exam patient was in apparent distress with tachycardia in the $110 \mathrm{~s}$. Her abdomen was peritoneal: distended, with mid-epigastric tenderness to palpation and positive rebound tenderness. CTAP was performed showing a collection of air in the proximal small bowel extending into the mesentery in the left abdomen with adjacent mesenteric inflammation, concerning for contained perforation or proximal small bowel ischemic changes. Patient was urgently taken to the operating room for exploratory laparotomy. Upon entering the abdomen a large $6 \times 4 \mathrm{~cm}$ perforated jejunal diverticulum with surrounding pus and adhesive bands was encountered. The jejunum was found to have fulminant diverticular disease, extending almost the entire length and $95 \mathrm{~cm}$ of diseased jejunum was resected. Final surgical pathology resulted as small intestine with focal transmural necrosis suggestion of perforation, subserosal abscesses, and diverticulosis, without tumor.

\section{P370-HERNIA-ADHESIONS-Emergency surgery}

THE ROLE OF ROUTINE COLONOSCOPY IN THE FOLLOW-UP AFTER ACUTE APPENDICITIS IN ELDERLY PATIENTS: A SINGLE CENTRE'S EXPERIENCE

\section{M.S. Salama ${ }^{1}$, A.. Salama ${ }^{2}$, A. Ibrahim ${ }^{1}$, A.R. Nasr $^{1}$ \\ ${ }^{1}$ Surgery, Our lady of lourdes hospital, BETTYSTOWN, Ireland; ${ }^{2}$ Medical Student, NUI Galway, GALWAY, Ireland}

Introduction: Acute appendicitis in elderly patients is relatively uncommon and could represent an underlying neoplasm. Hence patients over the age of 40 are often referred for a follow-up colonoscopy after management of acute appendicitis. The current routine use of computed tomography (CT) scans in the evaluation of suspected acute appendicitis in elderly patients prior to surgery coupled with intra-operative findings at laparoscopy question the role of follow-up colonoscopy for these patients.

Aims: To determine the role and optimal timing of colonoscopy in early detection of colorectal neoplasia after treatment of acute appendicitis in elderly patients.

Methods: All patients aged 40 years and above with confirmed appendicitis admitted to our hospital during the period $1 / 1 / 15$ to $30 / 9 / 17$ were included. Follow-up colonoscopy, diagnosis of colorectal neoplasia and its location in this patient cohort was evaluated.

Results: Number of people aged 40 and above in OLOL who had appendectomies from the dates $1 / 1 / 15$ to $30 / 9 / 17=184$. Out of them $44 / 184(24 \%)$ had full colonoscopy within 2 years of the appendectomy.Of them 25 of the 44 colonoscopies done were maleand 19 were females. $30 / 44(68 \%)$ of these colonoscopies were completely normal.1 colonoscopy identified colorectal carcinoma in ascending colon (2.3\%). Other pathologies identified included: Benign polyp 3 (7\%), polyp with low grade dysplasia $4(9 \%)$ and others 6 (13.6\%) (Lymphocytic colitis, ulcerative colitis, medication related ulceration, diverticulosis, melanosis coli, haemorrhoids). Conclusions: In elderly patients above 40 years of age:

There may be an increased risk of colorectal cancer after acute appendicitis.

Only $24 \%$ of this patient cohort underwent colonoscopy after appendectomy.

The current recommendations suggest the need for follow-up colonoscopy in elderly patients post acute appendicitis.

Further studies are needed to decide whether routine colonoscopy is indicated after acute appendicitis patients over 40 years.

\section{P371-HERNIA-ADHESIONS-Emergency surgery}

\section{ASSOCIATION BETWEEN APPENDICOLITH AND APPENDICITIS: CAUSATION OR COINCIDENCE? A SINGLE CENTRE'S EXPERIENCE}

\author{
M.S. Salama ${ }^{1}$, A.. Salama ${ }^{2}$, P. Anant ${ }^{1}$, S. Babur ${ }^{1}$, H.K. Perthiani ${ }^{1}$ \\ ${ }^{1}$ Surgery, Our lady of lourdes hospital, BETTYSTOWN, Ireland; \\ ${ }^{2}$ Medical Student, NUI Galway, GALWAY, Ireland
}

Introduction: It is generally accepted that the main aetiology of appendicitis is obstruction due to appendicoliths in adults and lymphoid hyperplasia in children. In contrast, incidental appendicoliths have been reported to occur in up to $32 \%$ of the asymptomatic population. Controversy still exists regarding the association of appendicolith and appendicitis. Is the appendicolith a causative factor or merely an incidental finding?

Aims: To determine the association between the presence of appendicolith and acute appendicitis (perforated or non-perforated) vs healthy appendix.

Methods: We collected the data retrospectively from the electronic records of all appendicectomie performed between January 2012 and December 2016 in our institution. Data collected included: age, sex, appendix histology and the presence of appendicolith. Interval or incidental appendicectomies were excluded from this study. We analysed the data using SPSS software version 2010

Results: During the study period 2348 appendectomies were performed (males: 1137, females: 1211, age range: $2-89$ years). 1794 cases were histologically confirmed cases of acute appendicitis and of these, 61 were perforated. A normal appendix was identified in 288 cases. The remaining 266 cases were due to chronic appendicitis, sub-acute appendicitis, lymphoid hyperplasia, parasitic infestation, and neoplasm. Appendicolith was found in 43 cases, of which 33 were found in a normal appendix and 10 were found in an inflamed appendix.

Out of the 33 cases of appendicolith with normal appendix: 23 cases were aged between 1 and 20 years old, 9 cases were aged between 21 and 40 years old and 1 case was aged between 40 and 60 years old. Out of 10 cases of appendicolith with acute appendicitis, 7 cases were aged between 1 and 20 years old, 2 cases were aged between 21 and 40 years old and 1 case was aged over 41 . Conclusions: Appendicolith may merely be an incidental finding and is not the primary cause of appendicitis.

No significant correlation between gangrenous/perforated appendicitis and the presence of appendicolith. Contrary to popular belief appendicoliths are more common in paediatric appendicitis than in adult cases. Further research is recommended.

\section{P372-HERNIA-ADHESIONS—Emergency surgery}

PATIENT SATISFACTION WITH ACUTE SURGICAL ASSESSMENT UNIT (ASAU) DURING ITS FIRST YEAR OF OPERATION AT OUR INSTITUTION

\section{Mohamed S. Salama ${ }^{1}$, Mahmoud S. Salama ${ }^{2}$, Ahmed Salama ${ }^{3}$ \\ ${ }^{1}$ Surgery, Our lady of lourdes hospital, BETTYSTOWN, Ireland; ${ }^{2}$ Medical student, Trinity college, DUBLIN, Ireland; ${ }^{3}$ Medical Student, NUI Galway, GALWAY, Ireland}

Introduction: Patient satisfaction has always been an important issue for hospital management Over the last 20 years, patient satisfaction surveys have gained increased popularity. Nowadays, respect for patients' needs is central to our health care system. Hospitals use patient satisfaction surveys to assess quality of care. Many hospitals routinely survey patient satisfaction but relatively little data has been published. Our Acute surgical assessment unit operates from 8am to 6 pm Monday to Friday and in its first year saw 2079 surgical patients, of whom 1308 were discharged and 771 were admitted to the hospital for further management.

Aims: To assess the levels of satisfaction of patients attending ASAU at Our Lady of Lourdes Hospital Methods: A random sample of patients seen in the ASAU was surveyed to determine their level of satisfaction and the experience they had whilst attending ASAU. A novel self-reported patien satisfaction questionnaire was developed and used to assess patients' opinion regarding the treatment they received, the doctor's explanation of their condition, the waiting time and the service in ASAU. Also the questionnaire encouraged patients to suggest improvements to the service.

Results: 200 patients were included in the random sample. 199 (99.5\%) were satisfied with their treatment while 1 case $(0.5 \%)$ was not. $194(97 \%)$ were satisfied with the doctor's explanation of their condition and 4 cases (2\%) were dissatisfied. 191 (95.5\%) were satisfied with the waiting time and 1 case $(0.5 \%)$ was dissatisfied. 197 (98.5\%) were satisfied with the service while 2 cases $(1 \%)$ were not. The reasons for dissatisfaction were waiting time in A\&E department before the patient was transferred to ASAU and waiting time for scan results in ASAU. 68\% of patients offered suggestions to improve the service all of which are under consideration. Conclusion: Almost $99 \%$ of patients were satisfied with the ASAU service. The patients are the primary consumer of a hospital's services and as such are in a unique position to assess the hospital's performance. Thus assessing patient satisfaction should become routine. The patient satisfaction survey is essential for identifying deficiencies in the existing service and suggestions for improvement. 


\section{P373-HERNIA-ADHESIONS-Emergency surgery}

RIGHT-SIDED BOCHDALEK HERNIA IN AN ADULT: REPORT OF A CASE

\section{J.O. Kim, S.K. Kee, S.Y. Nam, Y.Y. Choi}

Department of Surgery, CHA University, Gumi CHA Hospital, GUMI, Korea

Introduction: Bochdalek hernia is a type of congenital diaphragmatic hernia. In most cases, it is diagnosed during the neonatal period. We present a case of laparoscopically treated congenital Bochdalek hernia that led to jejunal strangulation in an adult

Case: An fifty eight year old obese $(\mathrm{BMI}=38.3)$ female admitted for gradually worsening right flank pain, vomiting and respiratory distress for one day. There was no trauma history to chest or abdomen. The past medical history were old CVA, well controlled hypertension and DM. She was hemodynamically stable. Her right flank was very tender. There was no abdominal distension. Initial CBC tests showed leukocytosis $(15,200 / \mathrm{uL})$, but electrolytes were normal. Chest PA revealed right side diaphragmatic hernia. There was poorly enhanced, herniated small bowel in the right hemithorax on chest CT scan. The patient was taken for emergency operation. On laparoscopy, the normal liver was displaced leftward because of herniated bowel. There was incarcerated jejunum and omentum which could not be reduced. So we widened a $3 \mathrm{~cm}$ sized posterolateral diaphragmatic defect first, and then we could reduce the strangulated jejunum $(45 \mathrm{~cm}$ in length) and omentum. There was no hernial sac. The defect was closed with 2-0 prolene. Finally, the strangulated jejunum was resected and anastomosed extracorporeally. The hospital progress of the patient was not eventful. On post-operative day 4, the patient was allowed soft diet. The patient was discharged on post-operative day 8 without any complication. Conclusion: Congenital diaphragmatic hernia is an uncommon condition in adults, but you should keep in mind the diaphragmatic hernia as a cause of intestinal obstruction and respiratory distress in an adult. Prompt surgical intervention is required to a favorable outcome. Laparoscopic repair of Bochdalek hernia is a good management option.

\section{P375-HERNIA-ADHESIONS-Emergency surgery \\ POSSUM AS A SCALE TO PREDICT THE RISK OF MORTALITY IN PATIENTS OLDER THAN 80 YEARS OLD}

\section{N. Dowgiallo-Wnukiewicz, P. Lech, M. Michalik}

Department of General, Minimally Invasive and Elderly Surgery, University of Warmia and Mazury in Olsztyn, OLSZTYN, Poland

Aim: The aim of the study was to evaluate whether Physiologic and Operative Severity Score for the enumeration of Mortality and Morbidity (POSSUM) is useful to predict the risk of complications in patients older than 80 years.

Methods: We performed a retrospective study of 13 patients older than 80 years old diagnosed with acute abdomen who were admitted to the Department of General, Minimally Invasive and Elderly Surgery in Olsztyn between May and October 2017.

Results: The most common disagnosis was ileus. The mortality rate in Surgery Department was $31 \%$.After relocation to the Intensive Care Unit, the overall mortality rate was $53.9 \%$. The patients who died a short time after surgery had mortality rates greater than $95 \%$ and morbidity rates greater than $60 \%$ according to POSSUM.

Conclusions: This study shows that POSSUM seems to be a valuable scale to predict the risk of death after surgery in older patients. Patients with higher mortality and morbidity scores should be very carefully selected for surgery.

\section{P374-HERNIA-ADHESIONS—Emergency surgery}

\section{LAPAROSCOPIC APPROACH OF ADHESIVE SMALL BOWEL OBSTRUCTION. SAFE TREATMENT IN SELECTED CASES}

M. Retamar Gentil, B. López Durán, B. Marenco de la Cuadra, M. Sánchez Ramirez, A. Cano Matías, E. Perez Margallo, F. Oliva Mompean, J.A. López Ruiz, Emergency Surgery Department, HUV Macarena, SEVILLA, Spain

Aims: Intestinal obstruction is one of the most frequent abdominal conditions in the Emergency Department (ED). Up to $93 \%$ of patients having undergone a laparotomy will have an episode throughout their lives, of which $60 \%$ up to $70 \%$ will respond to conservative management. The laparoscopic approach is widely accepted and supported by the studies published up todate. It is recommended in patients with suspected single band, who have less than one previous laparotomy and less than $24 \mathrm{~h}$ of clinical evolution. Our objective is to validate in our experience that these premises are the appropriate ones in the election of candidates for a minimally invasive approach.

Methods: We present a series of 26 cases admitted with symptoms compatible with adhesive intestinal obstruction in the ED of a third level hospital during 16 months. All patients underwent abdominal CT to rule out the possible causes of obstruction. Emergency surgery was indicated because of failure of a conservative medical treatment or for the findings of the complementary tests.

Results: A initial laparoscopic approach was performed in the 26 patients, with a conversion rate of $38 \%$ of the cases (resection and anastomosis was required in 7 patients, due to loop suffering or intestinal tumor not seen in the CT). Among the patients who required laparotomy, $80 \%$ had more han $24 \mathrm{~h}$ of clinical evolution before de surgery and $20 \%$ had free fluid in the TC. As surgical complications: 5 intestinal perforations were produced secondary to the manipulation. There was 3 recurrences of obstruction in the following 3 months.

Conclusion: The laparoscopic approach is feasible in selected cases and experienced hands. Acording to our results it is recommended to perform it in only in patients with less than $24 \mathrm{~h}$ evolution and with a single band image in the CT without free fluid. The intestine should be explored avoiding the manipulation of the most extensive loops to prevent complications and keep in mind the possible conversion to laparotomy in case of complications.

\section{P376-HERNIA-ADHESIONS-Emergency surgery}

\section{LAPAROSCOPIC MESH REPAIR OF ADULT ONSET, NON- TRAUMATIC DIAPHRAGMATIC HERNIA, IN A PATIENT WITH SPLENOMEGALY}

M. Bonomaully, F. Jackson-Spence, H. Malik, E. Richards, D. Khoo, S. Randhawa

Upper GI surgery, Queen's hospital, Romford, LONDON, United Kingdom

Aims: Diaphragmatic hernia in adulthood is rare. The most common causes are blunt and penetrating trauma. We present an intraoperative video of the laparoscopic repair of an adult onset, non-traumatic, diaphragmatic hernia in a patient with splenomegaly.

Method: A 66 year old woman was referred to upper gastrointestinal surgery with epigastric burning and pain in the left side of her chest, radiating to the left shoulder, for one year. There was no recent or distant history of trauma. She has a past medical history of treated hepatitis C, cirrhotic liver disease, splenomegaly, thrombocytopenia and iron deficiency anaemia. Gastroscopy was interpreted as a fundal diverticulum. CT abdomen/pelvis with intravenous (IV) contrast showed this to be a left diaphragmatic defect with herniated stomach causing a volvulus, which lay immediately above an enlarged spleen. A CT three years prior to this showed no diaphragmatic hernia. The patient had some symptomatic relief with a proton pump inhibitor and oral antacids, however due to her persistent symptoms surgery was undertaken. The patient had laparoscopic repair of the diaphragmatic hernia. Ports were as follows: $10 \mathrm{~mm}$ umbilical, $12 \mathrm{~mm}$ left upper quadrant, $5 \mathrm{~mm}$ right upper quadrant, $5 \mathrm{~mm}$ left iliac fossa. A left posterior diaphragmatic defect was found, just above the enlarged spleen, containing the incarcerated fundus of the stomach. The hernia was reduced by gradual dissection of the sac. A $15 \times 15 \mathrm{~cm}$ nonabsorbable polypropylene mesh (Proceed) was used to cover the defect a $4 \mathrm{~cm}$ margin. This was tacked in place with Protack. A single 16 French Robinson drain was left in situ.

Results: The procedure was uncomplicated. Oral diet was introduced on post-operative day 1 and the drain was removed and patient discharged on day 4. There were no post-operative complications.

Conclusions: The video shows an effective dissection of a left sided diaphragmatic hernia and mesh repair, overcoming multiple technical challenges secondary to splenomegaly and portal hypertension. 


\section{P377-HERNIA-ADHESIONS-Emergency surgery}

\section{LAPAROSCOPIC APPROACH OF A SMALL BOWEL HERNIATION THROUGH A BROAD LIGAMENT DEFECT: A CASE REPORT}

J. Sánchez Segura, P. Fernández Galeano, A. Rodriguez Cañete, A. Martinez Ferriz, P. Gutierrez Delgado, I. Mirón Fernández, M. Reyes Perez, J. Santoyo Santoyo, Cirugía General, Digestiva y Trasplantes, Hospital Regional Universitario de Málaga, MÁLAGA, Spain

Aim: sIntestinal obstruction is a very common cause of presentation to an emergency department. The most common cause in patients with prior abdominal surgery are adhesions, but the list of differential diagnosis is large. Internal hernia is a very rare cause of obstruction, with a reported incidence of between 0.2 and $0.9 \%$. The herniation related with broad ligament defects is even more uncommon.

Methods: We report the case of a 63-years-old woman with antecedents of liver transplant, tubal ligation and appendectomy. The patient was admitted refering abdominal pain in the epigastrium of $24 \mathrm{~h}$ duration, accompanied by nausea and vomiting. On physical examination, abdomen was depressible, tender in the right low quadrant, without evidence of peritoneal irritation. Laboratory studies were normal except for an elevated leukocyte count with a left shift. Computed tomography (CT) revealed dilated small bowel loops with a transition point in right lower quadrant. Radiological diagnosis was intestinal obstruction, with fibrous adhesion as the most probably aetiology. Management was conservative at the beginning, with intravenous hydration, nasogastric tube and administration of gastrografin (diatrizoate) without a good response.

Results: At $24 \mathrm{~h}$, an exploratory laparoscopy was perform, finding dilatation of small bowel loops and a $3 \mathrm{~cm}$ defect in the right broad ligament in which a segment of ileum was herniated. Ileal segment was liberated without evidence of ischemia. The hernial defect was closed by laparoscopy with simple silk stitches. The postoperative course was excellent, tolerating oral feeding next morning. The patient was discharged $72 \mathrm{~h}$ after surgery.

Conclusions: Internal hernias of the broad ligament are an extremely rare cause of intestinal obstruction, but must be added to the differential diagnosis for female patients due to the risk of intestinal strangulation and perforation. Even if clinical and radiological diagnose is difficult, CT is the best tool to delineate the cause and location of the obstruction. Laparoscopy allows reduction of the hernia and closure of the defect with minimal invasiveness. Because of that, the laparoscopic approach of bowel obstruction should be considered as the first choice if there is the suspicion of an internal hernia, without signs of necrosis or perforation.

\section{P379-HERNIA-ADHESIONS-Emergency surgery}

USE OF LAPAROSCOPY IN SURGICAL EMERGENCIES. A COMPARATIVE STUDY BETWEEN AN ACUTE CARE SURGERY UNIT AND GENERAL SURGEONS

A. Sánchez Arteaga, L. Tallón Aguilar, V. Durán Muñoz-Cruzado, J. Tinoco González, J. Pintor Tortolero, E. Perea del Pozo, F. Pareja Ciuró, J. Padillo Ruiz

General Surgery, Hospital Universitario Virgen del Rocío, SEVILLE, El Salvador

Aims: Laparoscopic surgery is considered the gold standard of care for many procedures, including those for emergency surgery as acute cholecystitis or acute appendicitis. Acute care surgery (ACS) units have demonstrated to improve outcomes for abdominal emergency patients. Our aim is to compare the use of laparoscopy in the emergency setting, between an ACS unit and general surgeons from different units.

Methods: Descriptive study from the general surgery data registry from a tertiary hospital, from January 2011 to November 2018. ACS unit was integrated by surgeons trained in surgical emergencies with 8-hour working days and 24-h shifts.

Results: From 7085 patients undergoing surgery during the study period, 4939 were described as amenable for laparoscopic surgery. 3417 patients were operated by an ACS unit and the rest of them were operated by general surgeons not belonging to ACS unit. Acute cholecystitis was the condition where laparoscopic approach was more frequently used in both groups $(83.72 \%$ ACS unit and $78.39 \%$ not-ACS unit). Acute appendicitis also had a high laparoscopy rate (78.27\% ACS unit VS $69.85 \%$ not-ACS unit). The use of laparoscopy in other pathologies, such as gastroduodenal perforation $\left(43^{\prime} 18 \%\right.$ vs $\left.20 \%\right)$, diverticulitis $(23.68 \%$ vs $12.5 \%)$, pancreatitis (6.97\% vs $0 \%)$, small bowel obstruction $(11.20 \%$ vs $9.09 \%)$, and colorectal cancer obstruction (9.09\% vs $8.23 \%$ ) was found higher for ACS unit group, and also progressively higher during the last years.

Conclusion: According to our study, laparoscopic approach in abdominal emergencies shows an upward trend, and surgeons from ACS units seem to have higher rates of laparoscopy than general surgeons in emergency procedures.

\section{P378-HERNIA-ADHESIONS-Emergency surgery}

\section{ROL OF LAPAROSCOPIC SURGERY IN POSTSURGICAL COMPLICATIONS. INSTITUTIONAL EXPERIENCE}

\section{A. Tita, M. Yapur, J.I. Moreno, Digestive and Endocrine Surgery,} Sanatorio NOSTI, RAFAELA, Argentina

Background: Many of the complications after abdominal surgery may require a reoperation to be resolved which leads to a significant associated morbidity and mortality. In recent years, miniinvasive procedures have become popular, with numeours reports of percutaneous a endoscopic procedures applied to the treatment of postoperative complicactions. However, in the current literature, there are few reports of the use fo laparoscopic approach to postsurgical complications from both laparoscopic and open surgery.

Objective: To evaluate the results obtained from laparoscopic reoperations in the management of early surgical complications and define it's current role at our institution.

Material and Methods: Retrospective evaluation of patients undergoing laparoscopic reoperation for early postoperative complications (30 days), in the period from January 2014 to November 2018. Results: In a total of 3615 major surgeries there were 53 laparoscopic reoperations in 48 patients. $54 \%$ for infectious complications, including anastomotic leaks, and $22 \%$ for bowell obstructions. The diagnostic accuracy of laparoscopy was $100 \%$. The average time until reoperation was 2.5 days. We only performed 3 non-therapeutic laparoscopies. Eight patients $(15 \%)$ were converted to an open procedure. Five patients needed complementary relaparoscopy. Morbidity was $21.8 \%$. Average hospital stay was 3.5 days. Only 1 death.

Conclusions: The laparoscopic approach is a safe and effective tool in the management of postoperative complications. It is well tolerated in critically ill patients and avoids respiratory and wound related morbidity associated with laparotomy. It also reduces diagnostic delay and a considerable number of unnecessary laparotomies, with a high resolution rate and minimal morbidity. It thus represents a valid and necessary alternative in surgeon's armamentarium. In the management algorithm of our institution we always choose the laparoscopic technique as the fisrt tool in case a reoperation is necessary.

\section{P380-HERNIA-ADHESIONS—Emergency surgery}

\section{EMERGENT LAPAROSCOPIC REPAIR OF INCARCERATED} HERNIAS - SAFETY AND SHORT TERM Results:

\author{
J. Rachmuth, C. El Nakiv, I. Carmeli, A. Keidar, Y. Manuskin
}

Surgery, Assuta Asdod Medical Center, JERUSALEM, Israel

Background: Incarcerated and strangulated hernias present a major problem in emergency medicine. There is scarce data about the role of laparoscopy in the management of these patients Laparoscopic repair offers the benefits of the ability to survey the incarcerated organ and to evaluate its viability, apart from the obvious advantages of laparoscopic surgery. The use of mesh repair in these emergent operations is also a major concern, due to the un-sterile conditions in which they are performed.

Objective: To evaluate the safety and short-term efficacy of laparoscopic emergent repair of incarcerated hernias.

Methods: Retrospective review of prospectively collected data of all the patients who underwent emergent laparoscopy due to an incarcerated hernia between November 2017 and October 2018. Results: During the study period, 13 patients underwent emergent laparoscopy due to incarcerated hernias ( 5 females, 8 males). 10 had incarcerated inguinal hernias, and 3 had incarcerated umbilical hernias. Mean age was 63.3. All inguinal hernias were repaired in the TAPP approach, and using an absorbable mesh. All umbilical hernias were repaired using the IPOM approach. 7 patients had bowel obstruction, 5 had incarcerated omentum, and one patient had incarcerated urinary bladder. 3 patients underwent resection of an ischemic organ (1 bowel, 1 urinary bladder 1 omentum). Mean hospital LOS was 2.6 days. During the follow up period there were no mortalities, and no recurrences. One patient had a wound infection that resolved with antibiotics. Conclusion: laparoscopic emergent repair of incarcerated hernias is a safe and feasible approach Further studies with longer follow up time need to be conducted, in order to evaluate the added benefit of the laparoscopic approach. 


\section{P381-HERNIA-ADHESIONS-Emergency surgery}

\section{CHALLENGES FACED WHEN DELIVERING A GENERAL SURGICAL SERVICE IN AN ISOLATED CENTER}

\section{C.A.W. Macano, N. Coleman, R. Edmonds, M. Robba, A. Antequera General Surgery, GHA, GIBRALTAR, Gibraltar}

Gibraltar is a small overseas British territory with a residential population of approximately 30,000 inhabitants, that increases up to 50,000 daily due to incoming tourists and cross-frontie workers. As a geographically isolated center we have to provide a varied service including emergency surgery, and elective operating such as colectomies, gastrectomy's etc. One of the challenges faced is the limited stock of red blood cell (RBC) units within Gibraltar and reliance on platelets (PLT) from across the border from Spain. Given the immanent Brexit we need to prepare for the challenges we will face in these times of political and distribution uncertainty. A prospective audit of all blood use within Gibraltar was carried out over 4 months. The number and type of units requested, the number of units given, the speciality, location and indication for requests was recorded. The Blood Transfusion NICE guidelines, 2015 were used as the standard. A total of 168 requests were made. 321 units of RBC were requested and a total of 234 units were transfused, $(72.8 \%)$. We could expect $>500$ requests and $>900$ units requested and processed per year. Only $16.9 \%$ of $\mathrm{Hb}$ values met the NICE criteria for red cell transfusion. $25.6 \%$ of requests had a post transfusion $\mathrm{Hb}$ within the 70-90 range. Over $1 / 4$ of RBC units requested were not transfused. Anaemia was the most common indicator $(66 \%)$, followed by active bleeding $(18 \%)$. Wide variability was seen between the months which could pose an issue with future planning.

This audit has given us an insight into the use of blood products in Gibraltar. It has highlighted issues with current practice but also raised wider issues of resource procurement and distribution. With political unrest and the need for products to be delivered across borders, we need to develop new strategies to ensure continuing supply to Gibraltar especially in cases of unstable emergency laparotomies where we are only able to provide 8 units of FBC before having to resort to bringing in live donors.

\section{P382-HERNIA-ADHESIONS-Emergency surgery}

\section{LAPAROSCOPY TRAINING IN EMERGENCY SURGERY UNIT IN THIRD LEVEL CENTERS}

E. Perea del Pozo ${ }^{1}$, V. Duran Muñoz-Cruzado ${ }^{1}$, L. Tallón Aguilar ${ }^{1}$, J. Tinoco Gonzalez $^{1}$, A. Sanchez Arteaga ${ }^{1}$, F. Pareja Ciuró ${ }^{2}$, J. Padillo Ruiz $^{2}$

${ }^{1}$ Trauma and emergency surgery unit, Hospital Virgen del Rocío, SEVILLE, Spain; ${ }^{2}$ General Surgery, Hospital Virgen del Rocío, SEVILLE, Spain

Introduction: The main problem of the incorporation of laparoscopic to the usual clinical practice is the slow 'learning curve' that characterizes them, having already assumed a progressive adaptation of the staff of the surgery services and making necessary a training program for residents.

Material and methods: We performed a retrospective analysis of laparoscopic surgery interventions performed by residents who were trained in the period of time between January 2009 and December 2018 in the General Surgery Service of the Virgen del Rocio University Hospital. The Department of General Surgery and Gastroenterology has 120 beds, 8 scheduled daily operating rooms (one of them for major surgery Ambulatory and two of urgencies) where more than 5700 patients intervene each year

Results: During the 11 years studied, a total of 3368 patients with abdominal pathology subsidiary of laparoscopy under emergency treatment were operated, of which 2376 were performed by laparoscopy, $70.56 \%$. The residents performed 2028 of the indicated interferences, $60.21 \%$ of the total activity of the Emergency Surgery unit, of the total 1408 were laparoscopies, assuming $59.25 \%$ of the laparoscopies of the unit. The rate of surgeries performed by residents has increased over the years, in pathologies such as appendicitis or cholecystitis with a laparoscopic approach in $91 \%$ of cases and $84 \%$ respectively in our series, residents have performed $77.6 \%$ of laparoscopic appendicitis and 62\% of cholecystitis on average in 2016, 2017 and 2018. The data are different in intestinal neoplasms, obstruction by flanges, intestinal perforations, acute pancreatitis and diverticulitis, reaching $10 \%$ of laparoscopies in the latter by senior residents and in selected cases. All these data have been compared with the training program of the specialty of General Surgery and Digestive Apparatus published in the BOE on May 8, 2007.

Discussion: Although laparoscopic surgery has spread among an increasing number of services and general surgeons, this technique involves certain difficulties that require adequate learning by staff and residents, such as the need to become familiar with the procedure, technical material used, gestures and movements, the use of the camera and the use of traction and contraction.

\section{P383-HERNIA-ADHESIONS-Emergency surgery}

ANALYSIS OF THE USE OF LAPAROSCOPY IN ABDOMINAL TRAUMA IN THE MAIN CENTERS OF ANDALUSIA

E. Perea del Pozo ${ }^{1}$, A. Sanchez Arteaga ${ }^{1}$, L. Tallón Aguilar ${ }^{1}$, J. Tinoco Gonzalez ${ }^{1}$, J.A. López Ruiz ${ }^{2}$, L. Romacho López ${ }^{3}$, F. Jimenez Armientos ${ }^{4}$, F. Turégano Fuentes ${ }^{5}$, M.D. Perez Díaz ${ }^{5}$, F. Pareja Ciuró ${ }^{6}$, J. Padillo Ruiz $^{6}$

${ }^{1}$ Trauma and emergency surgery unit, Hospital Virgen del Rocío, SEVILLE, Spain; ${ }^{2}$ Emergency Surgery, Hospital Virgen Macarena, SEVILLE, Spain; ${ }^{3}$ Emergency Surgery, Hospital Regional Universitario de Málaga, MÁLAGA, Spain; ${ }^{4}$ Emergency surgery, Complejo Hospitalario de Jaén, JAÉN, Spain; ${ }^{5}$ General Surgery, Hospital general Universitario Gregorio Marañón, MADRID, Spain; ${ }^{6}$ General Surgery, Hospital Virgen del Rocío, SEVILLE, Spain

Introduction: The use of laparoscopic surgery in abdominal emergencies, such as in trauma, has had a slow acceptance. The advantages with this approach include less postoperative pain, faster recovery, quicker return to everyday activities, and fewer complications. We have collected the cases and indications of laparoscopy in abdominal trauma in the main hospitals in the Andalusian capitals and compared with the national registry

Material and methods: A total of 25 patients who underwent laparoscopic surgery in the main hospitals of Seville, Cordoba, Malaga, Cadiz, Huelva, Jaen, Granada and Almeria were analyzed. They have been compared with the 567 traumas archived nationally by the Spanish Association of Surgeons taking into account age, sex, score of the American Society of Anesthesiologists, hemodynamic stability and mechanism of injury. The intra and postoperative variables were compared between groups.

Results: At the national level, the main cause of abdominal trauma were traffic accidents, therefore, it was the patients who had a greater number of laparoscopies $(35.3 \%)$, followed by stab wounds $(9,03 \%)$ and run over $(11.2 \%)$. In our series, the average age of the patients is 41 years and $68 \%$ are male. Only ECO-FAST was performed in $45 \%$ of the patients, being positive in $15.9 \%$ of the cases. As they were stable patients, in $95 \%$ of the cases a tac was possible. In our data, $60 \%$ of the laparoscopies were performed for therapeutic purposes as well as being diagnostic, thus avoiding a posterior laparotomy.

Conclusion: sLaparoscopic surgery for abdominal trauma, either blunt or penetrating, is safe and technically feasible in hemodynamically stable patients. We found that laparoscopic surgery was associated with shorter operative time, lower estimated blood loss and faster return to normal diet. Based on our findings we establish the indications of laparoscopy in these patients 


\section{P384-HERNIA-ADHESIONS-Emergency surgery}

\section{INITIAL LAPAROSCOPIC EXPERIENCE IN EMERGENCY INGUINAL REGION HERNIA REPAIR}

A. Suárez Cabrera, M. Sánchez Ramírez, B. Marenco de la Cuadra, M. Retamar Gentil, A. Cano Matías, J.A. López Ruiz, F. Oliva Mompeán

General surgery, Hospital universitario Virgen Macarena, SEVILLE, Spain

Aims: Strangulated hernia is one of the commonest causes of small bowel obstruction. For groin hernia the yearly strangulation risk is around $1-3 \%$. These patients have a wide spectrum of presentation, ranging from painful groin lump to severe sepsis in case of ischemic perforated bowel. Traditional management for emergency groin hernia conditions involve open anterior repair. Most of these patients are elderly, strangulated hernia is associated with higher postoperative morbidity and mortality, particularly for those who required laparotomy, bowel resection, or those who suffered from perforated bowel with peritoneal contamination. With the experience obtained in elective laparoscopic groin hernias repair, there is increasing confidence on both of the surgical technique and understanding of the pre-peritoneal anatomy. This has led expert surgeons to perform laparoscopic treatment for emergency groin hernia conditions. Methods: We present four cases of incarcerated or strangulated groin hernia resolved by laparoscopic aapproach. Three women between 50 and 82 years, and a 55 years old man. Two cases were inguinal hernias and two crural hernias.

Results: In all the cases laparoscopic transabdominal pre-peritoneal technique was performed, with polypropilene mesh made by us. Intestinal resection was not needed. There were no complications of bleeding, wound infection o intraabdominal infections in any case. No recurrences per month or year. Conclusions: The laparoscopic approach is feasible in incarcerated or strangulated groin hernia resolution. This approach is able to detect any concurrent ipsilateral and contralateral hernias intra-operatively. Its advantage is even more significant in cases of femoral and obturatriz hernias. More time is available to evaluate de bowel viability. The mesh placement in laparoscopic repair is superior to open approach since it covers all the potential hernia orifices including inguinal, femoral and obturator canal. The complications, recurrence, and hospital stay are very close to the rates documented in open repair for strangulated/incarcerated hernias. We have to take into consideration the knowledge of anatomy and expertise in laparoscopic surgery. Further randomized controlled trials are needed to confirm the superiority of the laparoscopic approach over the open approach in managing these presentations.

\section{P385-HERNIA-ADHESIONS-Emergency surgery}

\section{A MINIMALLY INVASIVE STRATEGY FOR RUPTURED SUBMUCOSAL ANEURYSM OF SMALL INTESTINE: LAPAROSCOPIC APPROACH FOLLOWING ANGIOGRAPHY AND ENTEROSCOPY}

\section{K. Doden, H. Kitamura, T. Tsuji, D. Yamamoto, H. Bando}

Department of Gastroenterological Surgery, Ishikawa Prefectural Central Hospital, KANAZAWA, Japan

Aims: Submucosal aneurysm of small intestine is extremely rare, but its rapture can be lifethreatening. Due to the unstable hemodynamics and unknown site of bleeding, emergency laparotomy has been widely performed for the rupture. We will present case reports and show the strategy for minimally invasive treatment for ruptured aneurysm.

Methods: We experienced two cases of ruptured submucosal aneurysm resected by laparoscopic surgery. Case 1 is a 16-year-old male who was taken to our ER with massive hematochezia. CT showed arterial bleeding in the small intestine and angiography revealed bleeding from the ilial artery. Selective embolization using gelatin sponge and micro coil was performed and hemostasis was obtained. Video capsule endoscopy found the hemispheric elevated lesion with protrusion at the top in the ileum. Using balloon assisted enteroscopy, the site of aneurysm was marked with injecting India ink, which allows surgeons to accurately and easily identify the part of small intestine with aneurysm. Subsequently, a single incisional laparoscopic assisted partial ileectomy was performed for the purpose of definitive diagnosis and preventing re-bleeding. The ileum with aneurysm was easily identified in laparoscopic exploration owing to the marking, and it was taken out from the incision to perform resection. Case 2 is a 21-year-old female who was transferred to our emergency department with sudden onset of massive melena. CT and angiography were perfomed, and bleeding from the $3^{\text {rd }}$ jejunal artery were confirmed. Subsequently, therapeutic embolization was performed in the same way as case 1. Enteroscopy revealed submucosal elevation similar to case1 in the jejunum. We carried out endoscopic tattooing, followed by single incisional laparoscopic assisted partial jejunectomy. Results: The operative time in case 1 and case 2 were $130 \mathrm{~min}$ and $68 \mathrm{~min}$, respectively, and the amount of blood loss was both $5 \mathrm{ml}$. The postoperative course was uneventful in both cases. Case 1 was discharged on the postoperative day 7, and case 2 was on postoperative day 6 . Conclusions: Our experience indicates that ruptured submucosal aneurysm of the small intestine can be effectively managed by a laparoscopic surgery with combination of therapeutic embolization and enteroscopic evaluation, which is safe and minimally invasive.

\section{P386-HERNIA-ADHESIONS-Inguinal hernia}

BILATERAL GROIN HERNIA REPAIR WITH ONE LARGE SELF-FIXATING MESH: PROSPECTIVE OBSERVATIONAL STUDY WITH PATIENT REPORTED OUTCOME OF UROLOGICAL SYMPTOMS F. Muysoms ${ }^{1}$, M.J. Dewulf ${ }^{1}$, I. Kyle-Leinhase ${ }^{1}$, R. Baumgartner ${ }^{1}$,
F. Ameye

${ }^{1}$ General surgery, Az Maria Middelares, GENT, Belgium; ${ }^{2}$ Urology, Az Maria Middelares Gent-Belgium, GENT, Belgium

Background: Laparoscopic bilateral inguinal hernia repair may be completed with one large selffixating mesh crossing the midline in front of the bladder. No studies have investigated in detai whether preperitoneal mesh placement induces temporary or more lasting urinary symptoms. Methods: Urinary and hernia related symptoms were evaluated preoperatively and postoperatively at 1,3 and 12 months in 100 patients using the ICIQ-MLUTS questionnaire and EuraHSQoL score.

Results: Voiding symptoms and bother scores were unchanged at 1 or 3 months, but there was significant improvement at 12 months compared with preoperative findings (symptoms $\mathrm{P}<0.001$; bother score $\mathrm{P}<0.01)$. Incontinence symptoms improved at 1 month $(\mathrm{P}<0.05)$ but not at 3 or 12 months, with a bother score significantly improved at 1 month $(\mathrm{P}<0.01)$ and 12 months $(\mathrm{P}<0.01)$. Diurnal and nocturnal frequency did not change significantly postoperatively, but 12 months nocturnal bother score was decreased $(\mathrm{P}<0.05)$.

EuraHS-QoL scores showed significant improvement in all 3 domains for all measurements compared to previous measurements. Postoperative symptoms were improved at 12 months, compared with preoperative pain scores $(-6.1)$, restriction of activity $(-10.1)$ and cosmetic scores $(-4.7)$ These findings were statistically significantly $(\mathrm{P}<0.001)$. At 12 months, there were no patients with severe discomfort (score $=5$ ) for any of 3 domains. No recurrences were diagnosed with $95 \%$ clinical follow-up at 12 months.

Conclusion: Placing a large preperitoneal self-fixating mesh for bilateral groin hernia repair did not cause new urinary symptoms and demonstrated significant improvement in voiding symptoms at 12 months. Incontinence and nocturnal bother score were significantly improved.

\section{P387-HERNIA-ADHESIONS—Inguinal hernia}

EARLY AND LATE POST-OPERATIVE COMPLICATIONS AFTER TEP / TAPP HERNIA REPAIR-IS THE PROBLEM IN THE MESH?

M. Sokolov ${ }^{1}$, P. Gribnev ${ }^{1}$, K. Angelov ${ }^{1}$, S. Maslyankov ${ }^{1}$, M.P. Atanasova ${ }^{2}$, D. Tzoneva ${ }^{2}$, G. Todorov ${ }^{1}$

${ }^{1}$ Surgery, Medical University of Sofia, SOFIA, Bulgaria; ${ }^{2}$ Anesthesiology and Intensive Care, Medical University of Sofia, SOFIA, Bulgaria

Introduction: TEP/TAPP hernia repair is an increasingly widely used surgical methods for minimally invasive treatment of inguinal hernia. TEP advantages to TAPP are noincision of the parietal peritonea sheet, therefore no need for its recovery—sewing or sticking at the end of the procedure, and no need for attachment of the prosthetic mesh to the structures of the anterior abdominal wall, which results in a reduction in the financial cost of operation.Various types of meshes with different characteristics are used, depending on the surgeon's preferences.The aim of this study is to highlight mesh-related postoperative complications, which can be serious and life-threatening.

Material and methods: A retrospective cohort study of 68 cases of unilateral or bilateral TEP and TAPP hernia repair performed at the University Hospital for the period 2016-2018 with a study of early and late postoperative complications potentially causally related to the implanted prosthetic mesh and methods of their treatment.

Results: For a 3-year period 22 TAPP ( 12 bilateral) and 46 TEP ( 33 bilateral) have been performed. Three complications (Clavien-Dindo IVa, IVb and V) were found, of which 2 were early postoperative (up to $20 \mathrm{POD}$ ) - one in TAPP-5POD small bowel adhesive ileus due to suture dehyscense of the peritoneal sheet and adhesion of a bowel loop to the surface of polypropylene mesh.One in TEP-2POD—a large preperitoneal hematoma with haemorrhagic shock at 86 years old female in anticoagulant therapy-an open revision of the preperitoneal space and definitive haemostasis; followed in 12 POD established bladder lesion from erosion from the edge of self-locking polypropylene mesh. Suture and drainage performed, but the patient died of decompensation of concomitant diseases. A late complication—11 months after bilateral TEP—erosion of soft polypropylene mesh of sigma (probable undetectable lesion of the peritoneum) with faecal peritonitis-Hartmann procedure with laparostoma followed by restitution but persistent chroniosepsis with established abscess in Retzii. 24 months after-revision with abscess incision and extraction of infected meshes. Discussion: Use of biologic meshes is quite expensive, however synthetic non-resorbable meshes implanted in preperitoneal layout is a prerequisite for specific severe postoperative complications. 


\section{P388-HERNIA-ADHESIONS-Inguinal hernia}

DO WE NEED TO FIX THE MESHES IN LAPAROSCOPIC INGUINAL HERNIOPLASTY IN LARGE DEFECTS? STUDY IN MECHANICAL MODEL WITH MESHES J.L. García Moreno, I. Duran Ferreras, P. Landra Dulato, J. Ferrufino General Surgery, Hospital Riotinto, MINAS DE RIOTINTO, Spain

Introduction: In Inguinal hernioplasty by laparoscopy in hernias of type M1-2 L1-2, we usefixation with glues or not using fixation. M3-L3 hernias usually require traumatic fixation. Generally, although this is a great reason for controversy. We make a mechanical model in which we test in hole type 3 (hernial hole that exceeds two fingers or twice the size of the mouth of the instrument laparoscopic grasp) to check the need for the use of traumatic fixation versus nonfixation.

Material and methods: We used 3 tests to test different meshes used in laparoscopic surgery of the abdominal wall: Dyanmesh-endolap, Bard 3D and 3Dligth, Progrip and Polypropylene(average pore, $82 \mathrm{kD}$ ).1. Traction test with hydraulic press of each mesh to check the resistance of each mesh to thesimple traction.2. Pressure test or fatigue test on a device created with a synthetic skin (double) and a system ofdynamic suspension with springs that supports more than $1 \mathrm{~kg}$ per $\mathrm{cm} 2$ of pressure. Pressure is exertedinconstant and successive of more than 14 Newton $\times \mathrm{cm}^{2}$ for $10 \mathrm{~min}$. 3. Test of constant and maintained pressure of more than 100 Newtonsx $\mathrm{cm} 2$ for $10 \mathrm{~s}$. Results: In the first test it is verified that all the meshes have a resistance immensely superior to what isaccurate in biological wall models(more than $100 \mathrm{~N} \times \mathrm{mm}$ ). By means of the fatigue test with one we can verify that in type 1 and 2 defects there is no mobilization of themesh. But in type 3 defects there is a slight displacement $( \pm 1.5 \mathrm{~cm})$. In the constant pressure test there is noextrusion of the mesh through the hole, but obviously there is mobilization of the mesh Conclusion: sIt is feasible not to fix the meshes in type 1 and 2 defects, being potentially dangerous in type 3 by the displacement especially in sudden changes of pressure. The traumatic fixation in type 3 hernias would be recommended since it is the only defect where displacement existedslight in all the meshes in the two pressure tests.
J.M. Suárez Grau, J. Gómez Menchero, A. Gila Bohorquez,

\section{P390-HERNIA-ADHESIONS-Inguinal hernia}

IS LAPAROSCOPIC INGUINAL HERNIA REPAIR ADOPTED IN A TERTIARY HOSPITAL IN THE MIDDLE EAST?

A.M. AlMuhsin, M.S. Umerani, A.M. al Mubayedh, H.A. Qomawi, R.M. Albaqami, M. Aba Aljaysh, H.. al Zahrani, A. Abouleid

General Surgery, King Fahd Military Medical Complex, DHAHRAN, Saudi Arabia

Introduction: Recent guidelines stated that laparoscopic inguinal hernia repair (IHR) has added benefits of lesser pain, short hospital stay and early resumption of normal daily activities, however it was associated with longer learning curve. Laparoscopic repair is suggested for unilateral and bilateral hernia provided that the surgeon has specific expertise and sufficient resources.

Aim: The aim of this study is to assess our practice for IHR at a tertiary hospital in the Middle East where 4 trained laparoscopic surgeons are operating.

Methods: Retrospective analysis of prospectively maintained data of all patients, who had open or laparoscopic IHR, at a tertiary hospital in the Middle East between January 2013 to December 2017. Data was retrieved via case note review and electronic maintained data.

Results: A total of 181 patients had IHR. $96.6 \%$ were males and $3.4 \%$ were females. The mean age for all patients was 46.9 years. 174/181 were elective admissions (96.1\%) and $7 / 181$ were emergency cases (3.9\%). 145/181 had open IHR (80.1\%) while 36/181 had laparoscopic IHR $(19.9 \%) .127 / 145$ of the open group were unilateral $(87.6 \%), 8$ were bilateral $(5.5 \%)$ and 10 were recurrent $(6.9 \%)$, while $24 / 36$ the laparoscopic group were unilateral $(66.7 \%)$, 10 were bilateral (27.8\%) and 2 were recurrent (5.6\%). 1/36 of laparoscopic group was converted to open surgery (2.8\%). No intraopertive complications in both groups. The mean operative time for the open group was $95.3 \mathrm{~min}$ versus $136.5 \mathrm{~min}$ for the laparoscopic group while the mean hospital stay was 3.2 days for the open group versus 3.9 days for the laparoscopic group. 12/145 of the open group developed early postoperative complications $(8.3 \%)$ versus $3 / 36$ in the laparoscopic group ( $8.3 \%$ ). 30-day readmission was only recorded in open surgery group with $3 / 145(2.1 \%)$ for wound infection and hematoma.

Conclusion: Most of the inguinal IHR is done by open tehnique in a tertiary hospital in the Middle East although 4 trained laparoscopic surgeons are operating. More efforts are required to increase the volume of the laparoscopic repair of the inguinal hernia to follow the guidelines and to achieve better patients outcome.

\section{P391-HERNIA-ADHESIONS-Inguinal hernia}

\section{OPEN INGUINAL HERNIA REPAIR TRAINING IN} A TEACHING HOSPITAL IN THE MIDDLE EAST

\section{IN A TERTIARY HOSPITAL IN THE MIDDLE EAST?}

M.S. Umerani, H. al Zahrani, A. Alkhaldi, B.H. Bugis, A. Alruwaili, A. Alanazi, A.H. Abouleid

General Surgery, King Fahd Military Medical Complex, DHAHRAN, Saudi Arabia

Introduction: Inguinal Hernia repair (IHR) as a day case has become increasingly common over the last decade. Recent guidelines stated that repair of inguinal hernia, as a day surgery, is as safe and effective as that in an inpatient setting and more cost-effective.

Aim: The aim of this study is to evaluate and assess the outcome of all cases of IHR done at a tertiary hospital in the Middle East between January 2013 and December 2018.

Methods: Retrospective analysis of prospectively maintained data of all patients who had IHR at a tertiary hospital in the Middle East between January 2013 and December 2018 . Data was retrieved via case note review and electronic maintained data.

Results: Total number of 181 IHR were done during this period. 175/181 cases were done as inpatient $(97.2 \%)$ while $6 / 181$ cases as day case $(3.3 \%)$. In the inpatient group the Mean age was 47 years (96.5\% Males and 3.4\% females). 68/175 patients had co-morbidities (38\%.8). 143/175 cases were unilateral repair $(81.7 \%), 20 / 175$ bilateral $(11.4 \%)$ and 12/175 Recurrence case (6.9\%). 169/175 cases were Elective (96.6\%) and 6/175 cases were Emergency (3.4\%). 139/175 patients had Open repair $(79.5 \%)$ and $36 / 175$ were Laparoscopic $(20.5 \%)$. The Mean operative time was 102 mins with no intra operative complications $(0 \%) .15 / 175$ had early post operative complications $(8.6 \%)$. The Mean hospital stay was 3.2 days with 3 cases of hospital re-admission $(1.7 \%)$. In the Day case group the Mean age was 32 years (100\% Males) with no co-morbidities $(0 \%)$. All cases were unilateral repair $(100 \%)$ and Elective $(100 \%) .5 / 6$ patients had Open repair $(83.3 \%)$ while $1 / 6$ were Laparoscopic $(16.7 \%)$. The Mean operative time was 111 mins with no intra or postoperative complications $(0 \%)$. The Mean hospital stay was 1 day with no cases of hospital re-admission $(0 \%)$

Conclusion: IHR as day case is not adopted in most of the cases in a tertiary hospital in the Middle East inspite of its reasonable outcomes. More efforts are needed to adopt the day case inguinal hernia repair policy with effective optimisation of the patients.
M.S. Umerani, A. Altaweel, B. Almatar, M.K. Alomair, H. Saeed, I.O. Jabra, A. Abouleid

General Surgery, King Fahd Military Medical Complex, DHAHRAN, Saudi Arabia

Introduction: Open Inguinal Hernia repair (OIHR) is a common procedure that is done as an elective or emergency. It is considered an integral procedure that must performed efficiently and promptly by residents.

Aim: The aim of this study is to evaluate and assess the outcome of Residents supervised by Board Certified Surgeons (Senior Registrars and Consultants) versus Senior registrars and Consultants for OIHR done at a teaching hospital in the Middle East between January 2013 and December 2018

Methods: Retrospective analysis of prospectively maintained data of all patients who had OIHR at a tertiary hospital in the Middle East between January 2013 and December 2018 . Data was retrieved via case note review and electronic maintained data.

Results: Total number of 145 OIHR were done during this period. Two groups were assigned, Residents group (RG) 43/145(29.7\%) and Senior Registrar/Consultant group (SR/C G) 102/145 (70.3\%). The mean age of patients in the RG was 46 years versus 46.5 years SR/C G. $88.3 \%$ of OIHR done by residents were unilateral versus $80.4 \%$ for SR/C G. $43 / 43$ of OIHR by RG are elective (100\%) versus $95 / 102(93.1 \%)$ by SR/C G. The mean operative time for the RG is 108.8 min versus $95.8 \mathrm{~min}$ for the SR/C C. Intra-operative complications were $(0 \%)$ in both groups. The had postoperative complications (7\%) versus $7 / 102(6.97 \%)$ in the SRC G. $1 / 43$ patient was readmitted after OIHR in RG $(2.3 \%)$ while $2 / 102$ was readmitted in the SR/C G $(2 \%)$.

Conclusion: The outcomes of OIHR done by residents supervised by Board certified surgeons are comparable to that done by the Board certified surgeons themselves except for longer operative time.The residents should be encouraged to do more cases of OIHR supervised by senior surgeons for the sake of their training. mean hospital stay of patients for RG was 3.6 days versus 3.4 days for the SR/C G.3/43 in the RG 


\section{P392-HERNIA-ADHESIONS-Inguinal hernia}

FEASIBILITY OF SINGLE-INCISION LPEC FOR TREATMENT OF INCARCERATED INGUINAL HERNIA

\section{IN ADOLESCENTS AND YOUNG ADULTS}

\section{N. Kameyama}

\section{Surgery, KKR Tachikawa hospital, TOKYO, Japan}

Purpose: To examine the feasibility of single-incision laparoscopic percutaneous extraperitoneal closure (SLPEC) for treatment of incarcerated inguinal hernia repair in adolescents and young adults (15-39 years: AYA generation).

Methods: A $0.6 \mathrm{~cm}$ incision was made at the bottom of umbilicus. After small laparotomy, we inserted a $5 \mathrm{~mm}$ port into the abdominal cavity. After insufflation to $6-8 \mathrm{mmHg}$, we inserted a $5 \mathrm{~mm} 30$ degrees laparoscope and detected the inguinal hernia. Our indication for SLPEC in AYA generation is small incarcerated inguinal herniae under $2 \mathrm{~cm}$. If the inguinal hernia did not fit this indication on laparoscopic examination, we performed other methods, such as transabdominal pre-peritoneal repair (TAPP) or totally extra-peritoneal repair (TEP).

For small incarcerated inguinal hernia fitting the criteria, we inserted $2.1 \mathrm{~mm}$ forceps into the abdominal cavity through the same transumbilical incision. A 19-gauge LPEC needle with nonabsorbable suture material was inserted into the inguinal region. Using LPEC needle and forceps, the hernia orifice was closed extraperitoneally with circuit suturing without leaving a peritoneal gap.

Results: We performed surgery for incarcerated inguinal hernia in 11 patients in the AYA generation at our hospital between January, 2017 and December, 2018. The procedures perSLPEC was significantly shorter than the other procedures. There were no complications and no evidence of early recurrence in any patients.

Conclusion: SLPEC is feasible and safe for incarcerated inguinal hernia repair in the AYA generation. formed were; 5 SLPEC, 4 TAPP, 1 TEP, and 1 Lichtenstein procedure. The operation time in

\section{P394-HERNIA-ADHESIONS—Inguinal hernia}

INDIRECT INGUINAL HERNIA SAC COULD BE A SAFE FIRST TROCAR PORT BY USING THE PURSE-STRING SUTURE IN THE LAP TREATMENT OF INCARCERATED INGUINAL HERNIA

J. Su${ }^{1}$, C.M. Mai ${ }^{1}$, W. Phin ${ }^{1}$, M.C. Hung ${ }^{1}$, C.H. Tsai ${ }^{1}$, D.A. Chou ${ }^{1}$,

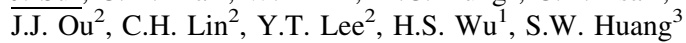

${ }^{1}$ Department of Surgery, Show Chwan Memorial Hospital, Taiwan; ${ }^{2}$ Department of Surgery, Show Chang Bing Show Chwan Memorial Hospital, Taiwan; ${ }^{3}$ IRCAD, Asian Institute of TeleSurgery, IRCAD, Taiwan

Aims: Introduce a suture technique to fix a trocar in the indirect inguinal sac, providing a good route to create pneumoperitoneum and perform laparoscopic operation in the incarcerated inguinal hernia.

Methods: Between Dec. 2015 and Nov. 2018, 10 patients (9 male, 1 female, 11 indirect hernia sacs) with incarcerated inguinal hernia underwent emergency surgery. 8 patients underwent the operation under the general anesthesia. The rest 2 patients received the operation under the spinal anesthesia The initial operative procedures were like traditional repair of indirect inguinal hernia. Once the indirect inguinal hernia sac was identified and opened, the trocar $(5-11 \mathrm{~mm})$ was inserted in the hernia sac and then fixed by purse-string suture of indirect inguinal sac. The pneumoperitoneum is created under laparoscopic vision, which the pressure is from 2 to $15 \mathrm{mmHg}$.

Results: The trocars were fixed well through the hernia sac in the all patients. Three patients didn't experience pneumoperitoneum after laparoscopic inspection of intestine. Three patients who have ischemic small bowel and adhesive omentum underwent pneumoperitoneum and laparoscopic adhesiolysis, resection of ischemic small bowel and anastomosis. One received targeted minimally laparotomy (5 $\mathrm{cm}$ in length) in the lower abdomen. The hernia-repair varied from self-tissue repair (Bassini, Shoudice) to mesh indwelling. One recurrent inguinal hernia (Only repair by high ligation of sac) was noted after two years follow-up.

Conclusions: In patients who have incarcerated inguinal hernia, it is hard to performed laparoscopic operation because distended bowel loop and increased intra-abdominal pressure. The indirect inguinal sac could play a safe role to performed laparoscopic operation by the purse-string suture technique. And it may be a route to perform single-port operation by our limited experience.

\section{P395-HERNIA-ADHESIONS-Inguinal hernia}

\section{P393-HERNIA-ADHESIONS-Inguinal hernia \\ ACHIEVING THE LEARNING CURVE FOR OPEN INGUINAL HERNIA REPAIR}

G. Merola ${ }^{1}$, J. Andreuccetti ${ }^{2}$, G. Pignata ${ }^{3}$, A. Sciuto ${ }^{4}$, G. Cavallaro ${ }^{5}$, C. Stabilini ${ }^{6}$, U. Bracale ${ }^{7}$

${ }^{1}$ Gastroenterology, Endocrinology and Surgical Endoscopy, AUOP Federico II, NAPOLI, Italy; ${ }^{2}$ General and Mininvasive surgery, San Camillo Hospital, TRENTO, Italy; ${ }^{3}$ General Surgery II, Civil Hospital of Brescia, BRESCIA, Italy; ${ }^{4}$ General Surgery, Santa Maria delle Grazie, POZZUOLI, Italy; ${ }^{5}$ General Surgery, University Hospital „La Sapienza,,, ROMA, Italy; ${ }^{6}$ General Surgery, University Hospital of Genoa, GENOVA, Italy; ${ }^{7}$ Gastroenterology, Endocrinology and Surgical Endoscopy, University Federico II, NAPOLI, Italy

Inguinal hernia repair is one of the most performed procedure all over the world, with more than 20 million procedures performed each year, it represents one of the top three most performed procedures. The Lichtenstein procedure is one of the first procedures that a young trainee in general surgery learn, not only for its reproducibility and for the great numbers of procedures that could be done in each Department, but also because during inguinal hernia repair the trainee learn a lot of skills which are the basis of major surgical interventions. The surgeon's performance for any procedure could be evaluated by way of established learning curves that can predict the minimum number of procedures required to reach the same intra and post-operative outcomes as an experienced surgeon performing the same technique. The aim of our multicentre study was to analyse how many cases are required to stabilize operating time (OT) and intra and post-operative complication rates over the course of the learning curve period for a Lichtenstein procedure. From January 2014 to December 2018 all Lichtenstein procedures from four different institutions were recorded in a prospective maintained computer database. The results of the first 100 consecutive procedures performed by three different trainees (Group A; Group B; Group C) were compared with the same numbers of procedures by two senior surgeons of the same institutions (Group E, Group F). CUSUM analysis was performed to evaluate the achieving of learning curve. No differences in terms of biometric and hernia type were recorded between the five groups. CUSUM analysis showed that the trainees achieve the learning curve between the 37-41 procedures. No intra or post-opertive complications were recorded during the training period.In conclusion after our analysis we found that at least 40 procedures are needed for the trainees to achieve the learning curve for Lichtenstein procedures.

\section{AN UNCOMMON CASE OF URETHRAL INJURY ENCOUNTERED DURING LAPAROSCOPIC TOTAL EXTRA- PERITONEAL REPAIR OF AN INGUINAL HERNIA}

\section{J. Yau, A.M. Oo}

General Surgery, Tan Tock Seng Hospital, SINGAPORE, Singapore

Background: Since its first description in the 1990 s, the Total Extraperitoneal (TEP) technique has established itself as a popular endoscopic method for the repair of inguinal hernias. The TEP repair is generally viewed as a technically-demanding procedure requiring adequate experience to minimize and handle complications. In this case report, we describe an uncommon complication of urethral injury, which was successfully repaired laparoscopically.

Case report: $\mathrm{Mr} \mathrm{R}$ is a 25 year old gentleman with no significant past medical history who presents to the Department of General Surgery, Tan Tock Seng Hospital, with a two-month history of a reducible right inguinal hernia, associated with some tenderness. An ultrasonography confirmed the diagnosis of a fat-containing indirect right inguinal hernia. In view of persistent pain, Mr R was counseled for a laparoscopic repair of his right inguinal hernia.

As Mr R was able to empty his bladder just prior to surgery, no urinary indwelling catheter (IDC) was inserted. An infra-umbilical incision was made to access the posterior rectus sheath and a balloon was used to bluntly dissect the pre-peritoneal plane. On inspection of the operating field, persistent pooling of blood was noted in the retropubic space. Careful inspection revealed a defect in a tubular structure just inferior to the bladder neck. An IDC was inserted, which confirmed a $1.5 \mathrm{~cm}$ defect in the pre-prostatic urethra. Decision was made for primary repair using absorbable sutures in two layers. The bladder was subsequently filled via the IDC, which did not reveal any leak. We then completed the right inguinal hernia repair using a mesh. Mr R made an uneventful recovery and was discharged on post-operative day 1 with instructions to keep the IDC in-situ for two weeks. The IDC was removed after two weeks and a micturating cystourethrogram was performed, which showed no filling defects along the urethra and no contrast leaks.

Discussion: Though uncommon, urethral injuries can be a complication of laparoscopic TEP repair. The key to managing these complications is in the early identification of such injuries intra-operatively. With early recognition and careful assessment, such complications can be managed laparoscopically with minimal post-operative morbidity. 


\section{P396-HERNIA-ADHESIONS-Inguinal hernia}

SURGICAL TECHNIQUE AND OUTCOME OF HYBRID TAPP FOR COMPLICATED INGUINAL HERNIA

M. Ouchi ${ }^{1}$, Y. Ishizaki ${ }^{1}$, K. Nagakari ${ }^{1}$, N. Inaki ${ }^{1}$, S. Yoshikawa ${ }^{1}$, M. Fukunaga ${ }^{2}$

${ }^{1}$ Department of Gastrointestinal Surgery, Juntendo University Urayasu Hospita, CHIBA, Japan; ${ }^{2}$ Department of Gastrointestinal Surgery, Juntendo Tokyo Koto Geriatric Medical Center, TOKYO, Japan

Aim: The purpose of this study is to report surgical technique and outcome of Hybrid TAPP procedure (a combination of TAPP and IPOM) for inguinal hernia patients complicated with preperitoneal space adhesion.

Methods: Hybrid TAPP procedure is applied if peritoneal dissection or closure of the peritoneum is difficult due to severe adhesion. The peritoneum should be dissected as much as possible. For the site where adequate dissection was achieved, the collagen mesh is placed outside the peritoneum. In the part where dissection was difficult it is placed inside the peritoneal cavity. In order to prevent mesh migration, the mesh should be directly fixed to the Cooper's ligament with a tacker. For this purpose, the peritoneum around the Cooper's ligament must be well-dissected, even if it is strongly adhered, so that the ligament can be exposed. The crucial points in the hybrid TAPP procedure are fixation of the mesh and prevention of the bowel herniation into the preperitoneal space. At the site where peritoneal dissection is possible, the mesh is directly fixed on the fascia using a tacker. If it is difficult, the mesh is placed in the peritoneal cavity and fixed over the peritoneum. If there is a risk of migration along with peritoneum, transcutaneous full-thickness fixation can be performed using non-absorbable sutures. The preperitoneal space should be closed tightly as soon as possible in order to prevent the bowel herniation into the preperitoneal space. At closure of the preperitoneal space, the peritoneum is fixed on the collagen mesh using non-absorbable sutures. Results: Between May 2014 and November 2018, 15 patients underwent the hybrid TAPP. 11 patients had a history of prostate cancer surgery and 4 patients suffered recurrence of hernia. In all patients postoperative course was uneventful and nobody showed recurrence of hernia or chronic pain related to the surgical procedure.

Conclusion: Although TAPP has not yet been established for patients with preperitoneal fibrillization due to previous prostate cancer surgery or hernia recurrence, Hybrid TAPP procedure could be an alternative option in such conditions.

\section{P397-HERNIA-ADHESIONS-Inguinal hernia}

SELF-FIXANTING MESH AND SUTURELESS BIPOLAR PERITONEAL CLOSURE: AN ALTERNATIVE TRANSABDOMINAL PREPERITONEAL(TAPP) HERNIA REPAIR

A. Paz Yáñez, P. Bretcha Boix, M.Z. Duarte Llanos, J. Farré Alegre, General and Digestive Surgery, HOSPITAL QUIRONSALUD TORREVIEJA, TORREVIEJA, Spain

Objective: Show a TAPP approach using a self-fixating mesh $\left(15 \times 10 \mathrm{~cm}\right.$. Progrip ${ }^{\mathrm{TM}}$ laparoscopic self-fixating mesh, Medtronic) with bipolar peritoneal defect sealing, avoiding the use of tackers and performing an easy and sutureless peritoneal closure.

Material and Methods: 62 years old male, ASA II, medical history of beta-latacm allergy, high blood pressure, dyslipidemia and bilateral knee surgery. Diagnosed of bilateral inguinal hernia at consultation due to inguinal disconfort. Surgical site infection prophylaxis with iv Vancomycin. Balanced general anesthesia. Supine decubitus position with shoulder supporting to allow a forced Trendelemburg. 30 degree optical device with 3 trocars disposition: one $11 \mathrm{~mm}$ umbilical trocar and 2 $5 \mathrm{~mm}$ trocar in both flanks, same distance and height to umbilical trocar. Peritoneal opening and flap creation with monopolar energy, blunt maneuvers and pneumoperitoneum dissection. Anatomical landmarks identification(Cooper's ligament, epigastric and iliac vessels, hernia defect and spermatic cord elements). Reduction of hernia sac content(pseudosac in this case, direct hernia) and complete peritoneal dissection to achive a correct mesh placing. Mesh is folded in 3 parts(one inferior part, two superior parts) in vertical axis outside the abdomen to facilitate the posterior intraabdominal maneuvers. Introduction: into abdominal cavity with grasping forceps and correct unfolding mesh assesment: medially(pubic bone), caudal(Cooper's ligament) cranial(more than $5 \mathrm{~cm}$ of hernia defect/ deep inguinal ring) and lateral(anterior superior iliac spine). Finally, we use a bipolar forceps to close de peritoneal defect. In order to facilitate this step, its necessary to decrease pneumoperitoneum pressure and to use the grasping forceps to bring together both peritoneal flap edges prior to bipolar energy sealing.

Results: 60 min. surgical procedure. $24 \mathrm{~h}$ hospital discharge, no complications. Routine outpatient follow up(week, month, 3 months and 6 month later) with an epididymitis episode 2 months after surgery(treated with oral Ciprofloxacin).

Conclusions:- This procedure is an easy implementation technique once the intraabdominal mesh unfolding procedure control is reached.- The use of a self-fixating mesh avoid the use of tackers and its potential disadvantages(e.g. increasing postoperative pain).-Bipolar peritoneal sealing offers a quick, easy, cheap and safe peritoneal closure, avoiding the contact of the mesh with the viscera in the same manner.

\section{P398-HERNIA-ADHESIONS-Inguinal hernia}

TRANSABDOMINAL PREPERITONEAL (TAPP) REPAIR FOR ESTRANGULATED INGUINAL HERNIA AND INTESTINAL BLOOD FLOW ASSESSMENT USING INDOCYANINE GREEN FLUORESCENCE

P. García-Muñoz, N. García-Fernández, E. Licardie, A. Senent-Boza, T. Yang, L. Tallón, J. Tinoco, I. Alarcón, J. Padillo, S. MoralesConde, General and Digestive Surgery, HUVR, SEVILLE, Spain

Aims: Although the laparoscopic approach for elective inguinal hernia repair has been accepted in the surgical practice, the use of this technique for acute incarcerated or estrangulated inguinal hernia repair is still controversial. This approach has significant advantages such as direct visualization of the incarcerated or strangulated organ. The use of indocyanine green (ICG) fluorescence allows to evaluate the vascular perfusion and viability of the bowell strangulated after the reduction.

Methods: A 52-year-old male with a recurrent right inguinal hernia as admitted with groin pain and sign of bowel occlusion due to strangulation of the groin hernia. Physical examination showed no signs of peritonisms. Convention x-ray showed slight distension of the small bowel with no sign of pneumoperitoneum. A diagnostic laparoscopy with evaluation of small bowe using ICG was proposed together with transabdominal preperitoneal (TAPP) if it could be performed.

Results: Surgery was performed by laparoscopic approach without the need to convert to an open technique. An abdominal examination was performed to identify the hernia and its content. The small bowel strangulated was reduced and dark-red discoloration was observed. The remaining steps were performed as per standard TAPP approach. A polypropylene mesh used was used being fixed using tackers ${ }^{\mathrm{TM}}$. Afterwards, ICG was injected intravenously and fluorescence was seen throughout the small bowel wall, thus indicating the absence of irreversible ischemic changes.

Conclusions: The TAPP procedure can be proposed for emergency treatment of inguino-crura incarcerated hernias, allowing not only hernia correction, but also visual control of the hernia content. ICG fluorescence may be useful for evaluating intestinal blood flow in patients with suspected visceral ischemic changes.

\section{P399-HERNIA-ADHESIONS-Inguinal hernia}

\section{LAPAROSCOPIC TRANSABDOMINAL PREPERITONEAL REPAIR FOR GROIN HERNIAS: IMMEDIATE AND EARLY POSTOPERATIVE OUTCOMES}

Y. Ben Safta ${ }^{1}$, M.D. Maatouk ${ }^{2}$, A. Mabrouk ${ }^{3}$, G. Hamdi El Kebir ${ }^{3}$, A. Anis $^{3}$, S. Daldoul ${ }^{3}$, S. Sayari ${ }^{3}$, K. Haouat ${ }^{3}$, M. Ben Moussa ${ }^{3}$

${ }^{1}$ Surgery A, Charles Nicolle Hospital, Tunis El Manar University, TUNIS, Tunisia; ${ }^{2}$ Surgery A, Charles Nicolle Hospital, TUNIS, Tunisia; ${ }^{3}$ Surgery, Charles Nicolle Hospital, TUNIS, Tunisia

Aim: The postoperative results of Laparoscopic Transabdominal preperitoneal (TAPP) technique in the treatment of groin Hernia are variable and sometimes controversial reflecting variations in experiences and available resources. The objective of this study is to describe, through our 2 years' experience, the postoperative outcomes of this technique and to try to identify possible factors significantly affecting the occurrence of postoperative morbidity and mortality.

Methods: We retrospectively analayzed all the patients operated for groin hernias by the TAPP technique, in the department 'A' of general surgery of Charles Nicolle Hospital of tunis between January 2014 and December 2016.

Results: We performed 53 procedures within 42 patients. The average age was 54 years. Twenty six percent of hernias were bilateral, $11,3 \%$ were inguinoscrotal and $56 \%$ in the right side. The median ASA score was 1 . The conversion rate was 3,7\%. The average duration of the procedure was $88,21 \mathrm{~min} 34 \mathrm{~min}$. Overall morbidity was $19 \%$. There were 5 seromas $(9,4 \%)$. On 2-year follow-up, one recurrence $(1,8 \%)$ was found and chronic postoperative pain in one case . We had no mortality. In the univariate analysis, male sex, inguinoscrotal hernias, hernias classified as Nyhus $3 \mathrm{~A}$ were significantly associated with overall postoperative morbidity. A chronic obstructive pulmonary disease was the only variable significantly associated with the occurrence of medical complications.

Conclusion: Given these results, the TAPP technique is a good alternative in the treatment of groin hernias. However, enhancing this approach is essential to reduce the operating time and the postoperative outcomes. 


\section{P400-ROBOTICS \& NEW TECHNIQUES-Basic} and Technical research

\section{THREE- AND TWO-DIMENSIONAL LAPAROSCOPY IN CHOLECYSTECTOMY (LCC) AND HERNIA REPAIR (TAPP)— TWO RANDOMIZED PROSPECTIVE TRIALS}

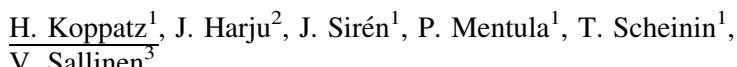

${ }^{1}$ Dpt of Abdominal Surgery, Helsinki University Hospital, HELSINKI, Finland; ${ }^{2}$ Dpt of Abdominal Surgery, Helsinki University Hospital/Jorvi Hospital, ESPOO, Finland; ${ }^{3}$ Dpt of Abdominal Surgery, dpt of Transplantation and Liver Surgery, Helsinki University Hospital, HELSINKI, Finland

Aims: The predominance of 3D equipment is shown in multiple non-clinical research. Few clinical studies have been published and the superiority of 3D is not yet clear. Therefore, we designed two trials to compare $3 \mathrm{D}$ to $2 \mathrm{D}$ laparoscopy in two common abdominal procedure.

Methods: These were randomized prospective studies.We randomized 210 and 278 patients to 3D or 2D laparoscopiy for cholecystectomy (LCC-trial) and inguinal hernia repair (TAPP-trial) between February 2015 and October 2017. Patients were randomized 1:1 to either 2D or 3D operation with block randomization. Patients were operated by 13 residents and 4 attendings, who all were examined with $\operatorname{Randot}{ }^{\circledR}$ stereotest for stereoscopic vision. The primary endpoint was operation time.

Results: Operation times were similar in the LCC 3D and 2D laparoscopy groups (49.0 min vs $48.0 \mathrm{~min}, \mathrm{p}=.703)$, but were shorter in the TAPP 3D laparoscopy group $(56.0 \mathrm{~min}$ vs $68 \mathrm{~min}$, $\mathrm{p}<.001)$. No differences in intra- or postoperative complications were noted between the $3 \mathrm{D}$ and 2D groups in LCC and TAPP trials. In the TAPP trial subgroup analysis, operation time remained shorter in $3 \mathrm{D}$ vs. $2 \mathrm{D}$ laparoscopy among attendings, residents, female surgeons, surgeons with perfect stereovision, surgeons with $>503 \mathrm{D}$ laparoscopic procedures, surgeons with any experience in TAPP, patients with body-mass-indices $<30$, and bilateral inguinal hernia repairs. Operation times were similar between the 3D and 2D laparoscopy groups in all subgroup analyses in the LCC trial.

Conclusion: Three-dimensional laparoscopy improves surgical efficiency in spatially more complex TAPP cases but not in simpler LCC cases. Short term surgical safety profiles were similar between 3D and 2D laparoscopies in both LCC and TAPP trials.

\section{P401-ROBOTICS \& NEW TECHNIQUES-Basic and Technical research}

\section{SURVEYING THEATRE STAFF'S ATTITUDE TOWARDS PATIENT SAFETY CULTURE AND THE USE OF A BLACK BOX AT THE OPERATING ROOM}

\section{A.S.H.M. van Dalen, E.Z. Barsom, M.P. Schijven}

Department of Surgery, Amsterdam Gastroenterology and Metabolism, Amsterdam UMC, University of Amsterdam, AMSTERDAM, The Netherlands

Introduction: Studies have emphasized the impact of a strong safety culture on patient outcomes. Consequently, many interventions focus on improving the safety culture, of which teamwork and safety climate are important ingredients. It is known that differences in culture and safety attitudes may also impact teamwork. Implementations of safety interventions, such as a 'Black Box', are dependent upon these differences. The aim of this study was to assess the safety culture at the operating theatre complex, along with the theatre staff's attitude towards a specific quality improvement intervention, a Black Box in the operating room as a tool for structured team debriefing.

Methods: The validated Dutch version of the Hospital Survey on Patient Safety Culture was administered to all healthcare professionals working in the operating room complex at one academic medical centre. This survey was supplemented with 10 questions regarding the use of a 'Black Box', a medical data recorder in the operating room, to measure the staff's attitude towards this quality improvement tool and its potential contribution to patient safety.

Results: The survey was sent to 26 surgical staff members, 6 surgical fellows, 23 surgical residents, 6 surgical physician assistants, 70 staff anaesthesiologist, 85 anaesthesiology residents, 85 anaesthesiology technicians and 150 operating theatre nurses. Results: are presented. Conclusion: Safety attitudes among theatre staff may impact their performance and need to be carefully taken into consideration when implementing safety and quality improvement initiatives. Future studies are needed to determine the true impact of the use of a Black Box in the operating theatre for structured postoperative team debriefing on patient safety.

\section{P402-ROBOTICS \& NEW TECHNIQUES-Basic} and Technical research

\section{SURGICAL TREATMENT OF DUNBAR SYNDROME}

N. Dowgiallo-Wnukiewicz, P. Lech, M. Michalik ,Department of General, Minimally Invasive and Elderly Surgery, University of Warmia and Mazury in Olsztyn, OLSZTYN, Poland

Aims: The aim of the study was to compare two methods of treatment of Dunbar syndrome thelaparoscopic release of median arcuate ligament alone and the hybrid method consisting ofsurgery and percutaneous stent implantation to celiac trunk.

Methods: We performed 6 laparoscopic release of CT in the Department of General, MinimallyInvasive and Elderly Surgery in Olsztyn in 2016-2018. All of patients suffered from severepain of abdominal cavity before the surgery. Three patients underwent Doppler percutaneousangioplasty of the CT with stent implantation one month after the laparoscopy. Results: All patients reported relief of symptoms in the first days after the operation. In two cases fromboth groups, there were a complete remission of the symptoms. In one case respectively,there was an improvement. There were no postoperative complications.

Conclusions: The results of both methods do not show the differences therefore the surgery alone seems tobe a safe and feasible procedure. It increases the comfort of the patient and brings theopportunity for normal functioning.

\section{P403-ROBOTICS \& NEW TECHNIQUES-Basic and Technical research}

RESULTS OF APPLICATION OF EXPANDED ATTITUDE
PULMONARY RESECTION OF PATIENTS WITH LIMITING
FORMS OF CHEMIORESISTANT TUBERCULOSIS

E.N. Maietnyi ${ }^{1}$, I.A. Kalabukha ${ }^{2}$, O.V. Khmel ${ }^{1}$, V.E. Ivashchenko ${ }^{1}$, R.A. Veremeenko ${ }^{1}$, J.M. Voloshyn ${ }^{1}$

${ }^{1}$ Department of Thoracic Surgery, State organization „National Institute of Phthisiology and Pulmonology FG Yanovs, KYIV, Ukraine; ${ }^{2}$ Surgical, State organization „National Institute of Phthisiology and Pulmonology FG Yanovs, KYIV, Ukraine

The method of wedge resection of lungs in patients with limited forms of chemo-resistan pulmonary tuberculosis is developed. In order to evaluate the efficacy, 80 patients underwent surgery (the main group). For comparison, the data on similar operations in 100 patients, made according to the traditional method (with the help of a cardboard weaving machine YO-60) were selected. Compared the duration of the stage of resection itself, the frequency of need for additional hemostasis of the parenchyma sutures, the degree of deformation of the pulmonary tissue in the seam area, the frequency of postoperative complications and reoperations, the duration of postoperative inpatient treatment. The developed method, in comparison with the traditional one, has the following advantages: simultaneously leak proofness and hemostasis with minimal electrothermal damage to tissues are provided and there is no need for additional hemostasis, there are no negative effects of manual stitching of parenchyma of lung with abandonment of foreign material, a significant reduction in the duration of wedge resection of the lung from 27.5 to $9.2 \mathrm{~min}$, a decrease in the number of postoperative pulmonary-pleural complications is achieved by $96.4 \%$ and caused by them reoperations-by $99.1 \%$, shortening the duration of postoperative inpatient period of treatment from 20.7 to 14.5 days. 


\section{P404-ROBOTICS \& NEW TECHNIQUES-Basic} and Technical research

\section{LAPAROSCOPIC APPROACH IN EMERGENCY SETTINGS: EXPERIENCE OF A SURGICAL EMERGENCY UNIT}

M.J. Cuevas López, V. Durán Muñoz-Cruzado, L. Tallón Aguilar, J. Tinoco González, J. Padillo Ruiz, F. Pareja Ciuró, Cirugía general, Hospital Universitario Virgen del Rocío, SEVILLA, Spain

Introduction/Aims: Laparoscopy is a diagnostic and therapeutic resource that is largely used in elective gastrointestinal surgery due to its well-known advantages over the classic open approach. Nevertheless, there is still some discussion about its application in emergency surgery. Our aim is to analize the use of the laparoscopic approach by the members of the Surgical Emergency Unit from our medical center.

Methods: A descriptive research based on the data of 12920 patients who required emergency surgery, that was performed by the members of the Surgical Emergency Unit of a spanish hospital between November 2000 and May 2018, was conducted. These data were analyzed according the pathology that motivated the surgical procedure and the chosen form of surgical approach (open versus laparoscopic).

Results: Out of the 12920 patients in whom emergency surgery was performed, 9712 suffered from a pathology that actually allowed the laparoscopic treatment. Laparoscopy was used in $38.8 \%$ of these patients. According to pathology, the most common were acute appendicitis and cholecystitis, in which the laparoscopic approach was used, respectively, in $56 \%$ and $62 \%$ of the cases. Regarding other less frequent pathologies, such as gastroduodenal perforation, bowel obstruction, diverticulitis and pancreatitis, laparoscopy had a less significant role. According to the year, a general tendency to increase the use of the laparoscopic approach was found, most notably in the cases of acute appendicitis and cholecystitis (with rates above 90\% in 2018). Conclusions: Despite our positive results in the terms of the implementation of the laparoscopic approach in emergency surgery, there is still room for improvement, especially in regards of the less common pathologies. Furtheremore, additional studies are needed in order to identify the factors that have had an effect, in favour or detriment, in the development of emergency laparoscopy in our center.

\section{P405-ROBOTICS \& NEW TECHNIQUES-Basic} and Technical research

\section{EFFECTIVENESS OF PIVOT RESTRAINT DEVICE FOR LAPAROSCOPIC ACCURATE PROCEDURE}

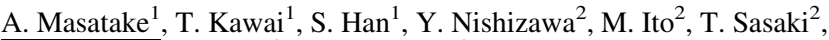
Y. Tsukada ${ }^{2}$ K. Ikeda ${ }^{2}$, H. Hasegawa ${ }^{2}$

${ }^{1}$ Robotics and Design, Osaka Institute of Technology, OSAKA, Japan; ${ }^{2}$ Colorectal Surgery, National Cancer Center Hospital East, KASHIWA, Japan

Aims: Laparoscopic surgery, which produces small scars, has become widespread. When performing surgery through small laparoscopic incisions, a surgeon manipulates tools inserted into the abdomen through ports. For minimally invasive accurate procedure, the port as the pivot point should be stabilized on the abdominal wall. However, these laparoscopic incisions are loaded while manipulation because it is difficult for the port to be fixed on. Thus, it is necessary for the patient friendly manipulation to be fixed the port mechanically. We developed a new pivot restraint device (PRD) attached to a trocar for guiding the tool. The purpose of this study is to evaluate both of reducing the operating time and the load of the port with the PRD experimentally.

Methods: The PRD uses gimbal mechanism for two rotating axes and a linear guide mechanism for the insertion axis though into the forceps. In the experiment, the left hand forceps with or without the PRD and the right hand forceps without the PRD were set on the training box. The box had a measuring system created with a pressure sensitive sensor for the continuous force (resolution $0.1 \mathrm{~N}, 30 \mathrm{fps}$ ) applied to abdominal wall fulcrum. The experiment task was performed as following three steps. (1) The surgeon lifted the $225 \mathrm{~g}$ weight for $5 \mathrm{~s}$ at the initial position using the right hand forceps. (2) The weight was transferred from the right hand forceps to the left hand forceps, and held for $5 \mathrm{~s}$. (3) The weight was moved to the predetermined position, held for $5 \mathrm{~s}$, and returned to the initial position. The surgeons were five endoscopic specialists and five non-specialists. The operating time and the time ratio exceeded $1 \mathrm{~N}$ for the left hand forceps were measured. Two grouped datasets with or without the PRD were compared using two-sided t-test. Results: The PRD was associated with both of reducing the operating time $(33.2 \mathrm{~s}$ vs. $38.2 \mathrm{~s}$; $\mathrm{p}<0.01)$, and the load of the port $(13.3 \%$ vs. $58.0 \% ; \mathrm{p}<0.01)$ at the statistical analysis. Conclusion: The PRD could be used for reducing the operating time and the load of the port in minimally invasive accurate procedure.

\section{P406-ROBOTICS \& NEW TECHNIQUES-Basic} and Technical research

\section{CEREBRAL PATHOPHYSIOLOGIC CHANGES DURING INCREASED INTRA-ABDOMINAL PRESSURE AND LAPAROSCOPIC SURGERY}

A. Bickel ${ }^{1}$, E. Andreas ${ }^{2}$, A. Gavrilov ${ }^{3}$, Z. Segal ${ }^{2}$, T. Abulhiga ${ }^{4}$, M. Weiss ${ }^{5}$, E. Kakiashvilli ${ }^{5}$, N. Intrator

${ }^{1}$ Departments of Surgery, Galilee Medical Center, Bar-Ilan University, NAHARIYA, Israel; ${ }^{2}$ Ophtalmology, Galilee Medical Center, NAHARIYA, Israel; ${ }^{3}$ Department of Anesthesiology, Galilee Medical Center, NAHARIYA, Israel; ${ }^{4}$ Neurosurgery, Galilee Medical Center, NAHARIYA, Israel; ${ }^{5}$ Surgery, Galilee Medical Center, NAHARIYA, Israel; ${ }^{6}$ Faculty of Computer Sciences, Tel-Aviv University, TEL AVIV, Israel

Background: Pathophysiological changes during laparoscopic surgery and positive pressure pneumoperitoneum (PP) may include (beside cardiovascular changes) elevated intra-thoracic as well as intracranial pressures. However, the possibility of physiological and functional cerebral impairment under PP is still debated.

Aim: To study the effects of PP on brain activity during different modes of anesthesia and ventilation during laparoscopic cholecystectomy (LC).

Patients and methods: Thirty patients undergoing elective $\mathrm{LC}$ were divided to those who were ventilated by intermittent positive pressure ventilation (IPPV, 16 pt.) and by high frequency jet ventilation (HFJV, 16 pt.). In those under HFJV we used total intravenous anesthesia (TIVA). In those under IPPV we either used inhalational anesthesia or TIVA. Intra-ocular pressures were detected in both eyes, trans-cranial Doppler was used to measure the changes in flow of the middle cerebral artery, and cerebral oxygenation ( $\mathrm{O} 2$ saturation) was measured too. Each parameter was detected during anesthesia before surgery, several times during surgery under PP and after $\mathrm{CO} 2$ evacuation. A novel computerized signal analysis by a continuous recording through a single electrode was done to explore cerebral cognitive activity during surgery.

Results: All surgeries went uneventful and without complications, PP was set to $14 \mathrm{mmHg}$, and each patient was positioned in a 15 degree anti-Trendelenburg posture. Cerebral perfusion and oxygenation were not changed significantly during PP. Intra-ocular pressures decreased during anesthesia and increased during PP, but to a lesser extent under TIVA. However, pressures during PP did not exceed pre-surgical values. We did not observe changes in cognitive brain activity during PP, although enhanced cerebral activity was seen under HFJV.

Conclusions: Increased intra-abdominal pressure during laparoscopic surgery was not accompanied by decreased cerebral functions, maybe due to cerebral circulatory auto-regulation. Changes in cerebral cognitive functions under HFJV might be explained either by the different cerebral effects of TIVA in comparison to inhalational anesthesia, or due to dissimilar hemodynamic changes during HFJV. 


\section{P407-ROBOTICS \& NEW TECHNIQUES-Basic} and Technical research

\section{LAPAROSCOPIC APPROACH FOR GALLSTONE ILEUS, OUR EXPERIENCE}

B.L. Lopez Duran ${ }^{1}$, A. Suarez Cabrera ${ }^{1}$, B. Marenco de la Cuadra ${ }^{2}$, M. Retamar Gentil ${ }^{2}$, M. Sanchez Ramirez ${ }^{2}$, A. Cano Matias ${ }^{2}$, E. Perez Margallo ${ }^{2}$, F. Oliva Mompean ${ }^{1}$, J.A. López Ruiz ${ }^{2}$

${ }^{1}$ General and Digestive Surgery, Hospital Universitario Virgen Macarena, SEVILLA, Spain; ${ }^{2}$ Emergency Surgery Unit, Hospital Universitario Virgen Macarena, SEVILLA, Spain

Aims: Gallstone ileus (GI) is a rare complication of cholelithiasis and accounts for 0.1-5\% of small bowel obstructions. Intermittent and non-specific presentation often results in late diagnosis. The triad of Rigler is pathognomonic (pneumobilia, small bowel obstruction and ectopic gallstones), so an image test is usually mandatory in order to assure the diagnose.

Our aim is to expose our experience regarding this topic to show that a minimally invasive approach is feasible in selected cases.

Methods: Since January 2016 we treated 12 cases of GI, 5 of whom (42\%) underwent laparoscopic surgery. In all cases a CT was made to reach diagnosis. Enterolithotomy alone is our preferred procedure for the resolution of this pathology.

Here we present a descriptive analysis of our data in those cases where a laparoscopic treatment was attempted. Epidemiological variables, surgical technique, postoperative complications, days until hospital discharge, recurrence, etc. has been collected.

Results: $80 \%$ of patients were female(4) and $20 \%$ male (1). Mean age was 68 . Size of gallstones varied from 20 to $34 \mathrm{~mm}$ and $\mathrm{CT}$ located them all in the ileum.

Two conversions to open surgery were made $(40 \%)$, in one case because the gallstone could not be found and in the other case due to the need of an intestinal resection. In two cases (40\%) la aparoscopic-assisted surgery was performed using a Pfannestiel incision for the gallstone extraction and enterorrhaphy. Only one case was total laparoscopic approach $(20 \%)$

Two cases needed an intestinal resection and anastomosis, one of them was complicated with a leak that needed reintervention.

There were two cases of recurrence during the follow-up time.

Hospital stay varied from 4 to 27 days, mean of 10 days.

Conclusion: The widespread use of CT facilitates early diagnosis with high sensitivity detecting Rigler's triad. A totally laparoscopic procedure might be ideal for patients specially with solitary stones even though a laparoscopic-assisted approach is an easier technique for surgeons with less experience in laparoscopic surgery. Although experience in minimally invasive surgical treatment of GI is still developing, it may be recommended in selected cases and experienced hands.

\section{P408-ROBOTICS \& NEW TECHNIQUES-Basic and Technical research}

\section{THERMO-ADJUSTABLE ENDOSCOPE HEATER SYSTEM} USING A MICROCONTROLLER FOR ANTI-FOGGING

\section{Sanchez-Ramirez ${ }^{1}$, I. Alarcon-del Agua ${ }^{2}$, E. Licardie ${ }^{3}$,} S. Morales-Conde ${ }^{2}$

${ }^{1}$ Emergency Surgery, Hospital Universitario Virgen Macarena, SEVILLA, Spain; ${ }^{2}$ Upper Gi Surgery, Hospital Universitario Virgen Del Rocio, SEVILLA, Spain; ${ }^{3}$ General Surgery, Hospital Quiron, SEVILLA, Spain

Introduction: Most of surgical interventions in hospitals in the world, where laparoscope is used, it is common that the vision inside the human body is constantly interrupted by fogging in laparoscope tip. The laparoscope fogging is caused by the difference of temperatures between the optic tip and the abdominal cavity.

Material and Method: We replaces the traditional laparoscope for the EHS (Endoscope Heate System) with resistance between the internal and external tube that maintains the temperature of laparoscope at $\left(30-50^{\circ}\right.$ Celsius $)$ without modifying the external architecture of traditional laparoscope.

Results: EHS does not generates any waste like other anti-fog systems, like liquids, plastics covers or electric heater. Reduces intervention time, can keep same instruments or accessories for the intervention. All of the above means a saving of resources with have a positive environmental impact. Conclusions: The discomfort transmitted by surgeons about the fogging in laparoscopy tip make success of the product and it will replace the current laparoscope which is fogged.

\section{P409-ROBOTICS \& NEW TECHNIQUES-Colorectal}

SIMULTANEOUS ROBOTIC TOTAL MESORECTAL EXCISION AND RADICAL PROSTATECTOMY FOR SYNCHRONOUS ADENOCARCINOMAS

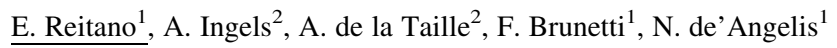

${ }^{1}$ Digestive Surgery, Henri Mondor Hospital, AP-HP, University of Paris Est, UPEC, PARIS, France; ${ }^{2}$ Urology, Henri Mondor Hospital, AP-HP, University of Paris Est, UPEC, PARIS, France

Aim: Synchronous locally-advanced low rectal cancer and prostate adenocarcinoma represent a rare condition and a challenging situation for colorectal surgeons and urologists. The simultaneous resection of both adenocarcinomas after long-course chemoradiation therapy combines two major surgical procedures associated with a potentially increased postoperative morbidity. In the other hand, simultaneous resections minimize the risk of difficult dissections, which are expected if the two procedures are scheduled sequentially.

In the past decade, robotic-assisted minimally-invasive surgical techniques have been increasingly used to treat both rectal and prostatic malignancies. Especially in case of prostatic malignancy, the robotic approach is considered the treatment of choice because it is associated with significantly lower blood loss and transfusion rate, and much greater functional outcomes compared to laparoscopy.

Methods: We present the case of a 66-year-old male patient (BMI: 30.6) diagnosed with a histologically proven locally-advanced rectal adenocarcinoma (cT3aN0) located at $5 \mathrm{~cm}$ from the anal verge and concurrent histologically proven prostatic adenocarcinoma [Gleason score of 8 $(4+4)$ ] located in the postero-basal right lobe. The preoperative total-body computed tomography (CT) scan showed no evidence of metastatic disease. After discussion in a multidisciplinary meeting, the patient received a long-course neoadjuvant chemoradiation therapy (NCRT). At the restaging positron emission tomography / magnetic resonance imaging (PET-MRI), the recta lesion was classified as ymrT0NO. Preoperatively, the surgical difficulty was assessed as high, based on the calculation of the EuMaRCS score (equal to 6/10). Moreover, due to the high-risk status of the prostate cancer (Gleason 8), it was decided not to preserve the neuro-vescular bundles during the radical prostatectomy.

Results: The patient was operated on after 12 weeks from completion of NCRT by using the $d a$ Vinci robot system $\mathrm{Si}$ with a single docking approach, as previously described, in order to address both cancers.

Conclusions: This video shows the main surgical steps of the simultaneous robotic resection of the low rectal adenocarcinoma first, of the prostatic carcinoma then, and the mechanical colo-anal anastomosis followed by drain positioning and ileostomy. This video demonstrates the perioperative safety and feasibility of the minimally invasive robotic approach in case of extended and challenging oncologic resections.

\section{P410—ROBOTICS \& NEW TECHNIQUES-Colorectal ROBOTIC-ASSISTED RIGHT HEMICOLECTOMY FOR COLON CANCER}

\section{E. Kakiashvili ${ }^{1}$, E. Brauner ${ }^{2}$}

${ }^{1}$ General Surgery, Galilee Medical Center, KIRIAT MOZKIN, Israel; ${ }^{2}$ General Surgery, Rambam Medical Center, HAIFA, Israel

75 year old, male patient presented with melena, without abdominal pain, nausea or vomiting. Patient underwent colonoscopy and tumor was found in ascending colon (near the hepatic flexure). Biopsy from the tumor has showed moderately differentiated adenocarcinoma.

His blood laboratory examinations were within normal limits except of Hgb level-11.0. CEA and CEA19-9 were normal.

Abdominal computed tomography was normal.

Patient underwent da Vinci robot-assisted Right hemicolectomy with extracorporeal anastomosis Total operating time was $150 \mathrm{~min}$. Three days after operation patient started regular diet and was discharged home on day four.

Final pathology result confirmed diagnosis of moderately differentiated adenocarcinoma. 


\section{P411-ROBOTICS \& NEW TECHNIQUES-Colorectal}

\section{INFLUENCE OF FLUORESCENCE IN LAPAROSCOPIC COLORECTAL ANASTOMOSIS: A CUASI- EXPERIMENTAL STUDY}

V. Camacho, T. Yang, I. Alarcón, F. Lopez, M. Socas, A. Barranco, J. Padillo, S. Morales- Conde,

Unidad de Innovación en CMI. Servicio de Cirugía General y Digestiva., Hospital Universitario Virgen del Rocío, SEVILLA, Spain

Introduction: One of the goals of colorectal surgery is to decrease the number of leaks once an anastomosis has been performed. This life-threating entity after elective surgery has been related to the clinical history of the patients, the location of the tumor and to technical reasons, specially due to tension in the anastomosis or to lack of vascularization. Tension could be identified during surgery, while vascular supply is evaluated by the surgeons based on a subjective analysis of the color of the colon/ileum. Fluorescence tries to make these subjective parameter more objective in order to avoid an anastomosis with lack of vascularization, decreasing the numbers of leaks related to this factor.

Patients and method: The study presents a quasi-experimental analysis made from January 2009 to October 2017 in two hundred and eighty- five patients who underwent elective colerectal surgery, performing either a colo-rectal, ileo-rectal or intracorporeal ileo-colic anastomosis. Vascular supply was eveluated using Indocianyne Green (ICG) in one hundred and forty-five patients, while one hundred and forty subjects were operated in a previous period without using this technology, being considered the control group. The number of time that the attitude changed and the number of leaks were collected.

Results: Out of the 285 cases performed, 80 were right colectomies (RC), 162 left colectomies (LC) and 43 rectal excision (RE). In $20 \%$ the transection line was changed ( $2,8 \%$ in RC, $11,1 \%$ in LC and 6,3\% in RR). In comparison with the control group, the ICG group had a significantly less indicence of anastomotic leak compared to the control group $(2,8 \%$ vs. $8,6 \%, p=0,04)$, lower rate of terminal stoma after reoperation $(0,7 \%$ vs. $5,7 \%, p=0,018)$, a shorter length of hospital stay ( 4 days vs. 5 days, $\mathrm{p}=0,02$ respectively), and a low morbidity and mortality.

Conclusions: The rate of leaks after colorectal surgery decrease using ICG to detect the proper transsection line before to perform the anastomosis in comparison with control group. These findings might influence in the final results although it is necessary in the future to find a system that provides greater objectivity by quantifying ICG.

\section{P412-ROBOTICS \& NEW TECHNIQUES-Colorectal}

\section{WHERE DOES ICG FLUORESCENCE ANGIOGRAPHY HAVE MORE VALUE IN COLORECTAL SURGERY?}

E. Licardie ${ }^{1}$, T. Yang ${ }^{2}$, V. Camacho ${ }^{2}$, I. Alarcón ${ }^{2}$, M. Sánchez ${ }^{2}$,

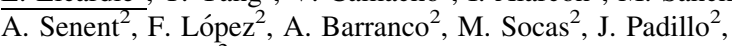
S. Morales-Conde ${ }^{2}$

${ }^{1}$ Sevilla, Hospital Quirónsalud Sagrado Corazón, SEVILLA, Spain; ${ }^{2}$ Unit of innovation in Minimally Invasive Surgery and Unit of General Surgery, University Hospital Virgen del Rocío, SEVILLA, Spain

Aims: Anastomotic leaks continue being one of the most important complications when a colorectal surgery is performed. This complication is usually related to the level and type of resection, the patient clinical history and surgical technique, where tension and vascular supply are the most important. Indocyanine green (ICG) fluorescence angiography seems to be helpful in order to evaluate the vascularization at the resection margins.

Methods: We have collected data on 187 colorectal procedures that were performed by the same surgeon using ICG fluorescence angiography to evaluate vascular supply to the anastomosis. In order to asses in which of the different type of colorectal procedure has more value to be used, we analyzed the type of surgical procedure, the percentage change in the resection margin and the number of anastomotic leaks (AL).

Results: All of the 187 cases were performed by laparoscopic approach: 77 left colonic resection (LC), 66 right colonic resection (RC), 9 splenic flexure partial resection (SF), 15 low anterior resection with partial mesorectal escision (LAR), 19 ultra low anterior resection with total mesorectal escision (ULAR) and 1 total colectomy (TC). There was a change of transection line (CTL) in $21 \mathrm{LC}(27,2 \%), 4 \mathrm{RC}(6 \%), 1 \mathrm{SF}(11,1 \%)$ and $10(28,5 \%)$ in rectal anastomosis (LAR, ULAR and TC). As far as AL we found: LC $1(1,2 \%), \mathrm{RC} 2(3 \%)$ and 2,8\% in rectal procedures. LC, $\mathrm{SF}$ and rectal procedure showed more $\mathrm{CTL}$ and less $\mathrm{AL}$, while RC showed less $\mathrm{CTL}$ and more $\mathrm{AL}$. Conclusion: ICG fluorescence angiography as an additional tool to try to reduce the anastomtic leak rate seems to have more value in the procedures that involve the left colon and the rectum, since that is where we have observed the greatest number of CTL, this could be explained by the Riolan's arcade and the variability of the vascular anatomy. However, it seems that this is a line of research should continue developing with longer and larger studies, so in that way we can have more significant results.

\section{P413-ROBOTICS \& NEW TECHNIQUES-Colorectal}

RESECTION OF RETRORECTAL TUMOR BY TRANSANAL MINIMALLY INVASIVE APPROACH

P. Imma, M. Wenceslao, G. Carlos Javier, S. Gemma, M. Socías, $\overline{\text { R. Josep }}$

Surgery, Fundació Hospital Sant Joan de Deu de Martorell, BARCELONA, Spain

Retrorectal tumors ara rare and often found incidentally. The majority of retrorectal tumours are benign, but they have potential for malignant transformation and therefore should be resected when found. A case of a 44-year-old female patient with a retrorectal tumor is showed. The tumor was found incidentally on CT scan of the abdomen for evaluation of non specific right side abdominal pain. A MRI was also performed and imaging was informed as a probably congenital retrorectal tumor (tailgut cyst) There was no evidence of involvement or invasion of other structures The tumor was palpable at rectal examination. A transanal minimally invasive surgery (TAMIS) approach was proposed. Preoperative preparation was done with a full mechanical and oral antibiotic bowel preparation. Preoperative parenteral antibiotics werw administred. Under general anesthesia, lithotomy position. The contour of the tumor is not visible due to the smal size. Palpation of tumor and placement of clips to lolocate was done. Placement of Gel Point path and rectal insufflation. A longitudinal incision was made to the posterior left side of rectal wall. The insufflation of the perirectal extraperitoneal space allowe for excellent exposure of the tumor The tumor was disected with ligasure. Then the tumor was extracted transanally.The proctotomy was closed in a single layer with reabsorbible monofilament continuous suture (PDS). No complications after the procedure. The patient was discharged at 2 days.

Discusion: Traditionally, the retrorectal tumors have been resected using a posterio parasacrococcygeal approach, an abdominal approach or a combined abdominal and posterior approach. With the advent of minimally invasive surgery, laparoscopyc approach has been described too. However, TAMIS approach is feasible, with low pain, morbidity, fester recovery and excellent cosmetic (no scare) results. It can be accomplished using standard laparoscopic equipment, with transanal access. We think that perhaps it could be the gold standar approach for this tumors.

\section{P414-ROBOTICS \& NEW TECHNIQUES-Colorectal}

\section{SHORT-TERM OUTCOMES OF ROBOTIC-ASSISTED} LAPAROSCOPIC SURGERY FOR RECTAL TUMORS

T. Yamanashi, T. Nakamura, T. Sato, M. Naito, H. Miura, K. Kojo, M. Shimazu, M. Watanabe, Colorectal surgery, Kitasato University School of Medicine, SAGAMIHARA, Japan

Aimes: Robotic-assisted laparoscopic surgery (RALS) is a promising advanced technology that can overcome the inherent limitations of conventional laparoscopic surgery (CLS). Its advantage includes free-moving multijoint forceps, a motion scaling function, high-quality three-dimensional imaging, and stable camera work by an operator. This study aimed to clarify the short-term outcomes of RALS for rectal tumors.

Methods: This study group comprised 25 patients who underwent RALS for rectal tumors (cancer in 24 patients and gastrointestinal stromal tumor in 1 patient), excluding ones with distan metastasis from November 2016 through December 2018. The clinicopathological findings and short-term outcomes in rectal tumors were analyzed.

Results: The median operative time was $372 \mathrm{~min}$ (309-682). The median console time was 207 min with a median blood loss was $5 \mathrm{ml}(5-394)$. Conversion rate was $0.0 \%(0 / 25)$. The median postoperative hospital stay was 11 days (6-17). 2 patients (8.0\%) had postoperative complications. 9 patients $(36.0 \%)$ had lymph nodes metastases. The mean harvested lymph node was 17.6 The R0 resection rate was $96 \%$ (24/25)

Conclusions: These results suggest that RALS for rectal tumors is safe and feasible, and the perioperative outcomes are acceptable. 


\section{P415-ROBOTICS \& NEW TECHNIQUES-Colorectal}

\section{NEAR-INFRARED IMAGING IN ROBOTIC RECTAL CANCER SURGERY}

\section{Langer, J. Kalvach, M. Ryska}

Surgery, Central Military Hospital, PRAGUE, Czech Republic

Introduction: Anastomotic healing defects are a feared complication which might have a fatal impact on the patient. Fundamental conditions for proper anastomotic healing include sufficient blood supply. Fluorescent angiography using indocyanine green in the spectrum of near infrared light facilitates the monitoring of tissue perfusion during a surgery.

Aim: A presentation of the results of our non-randomized study in which we assessed prospectively obtained data from a perioperative assessment of anastomosis perfusion by fluorescent angiography using indocyanine green during robotic rectal cancer surgery.

Method: Thirty patients with rectal cancer, who underwent a robotic resection with primary anastomosis, were consecutively included in the study between April 1, 2017 and June 21, 2018. The study included patients facing a least invasive surgery with a guaranteed payment by a health insurance company. During the surgery, we monitored and assessed the quality of the perfusion of the resection line of the sigmoid colon and subsequent anastomosis by means of fluorescent angiography using indocyanine green in the spectrum of near infrared light. The data were obtained prospectively and subsequently analyzed.

Results: Between April 1, 2017 and June 21, 2018, we consecutively included 30 rectal cancer patients in the project: 16 men and 14 women. Monitoring of the perfusion of the resection line and anastomosis was successful in all cases and perfusion quality was satisfactory across the sample. Perfusion insufficiency requiring a change in the resection line level or anastomosis adjustments was not detected with any patient. In two cases (6.7\%) of TME, we gave up the planned protective ileostomy owing to quality perfusion of the anastomosis. One patient (3.3\%) suffered from defective anastomosis healing without clinical symptomatology (type A). We found no technical complications related to fluorescent angiography or undesirable effects due to the application of indocyanine green.

Conclusion: Even though we did not register insufficient perfusion in our sample and hence we did not have to change the resection line level or adjust the anastomosis, we may state that fluorescent angiography performed by an experienced colorectal surgeon may potentially reduce the frequency of complications linked to defective anastomosis healing.Supported by MO 1012

\section{P416-ROBOTICS \& NEW TECHNIQUES-Colorectal}

\section{INFLUENCE OF ROBOTICS IN SURGICAL COMPLICATION} RATE IN ELDERLY POPULATION WITH RECTAL CANCER

I. Ramallo Solis, R. Jimenez Rodriguez, P. García Muñoz, M.L. Reyes Díaz, J. Tinoco Gonzalez, J.M. Vazquez Monchul, A.M. Garcia Cabrera, J.M. Díaz Pavón, F. de la Portilla, J. Padillo Ruiz

Surgery, Hospital Universitario Virgen del Rocío, SEVILLA, Spain

Aims: The aim of our study is to demonstrate whether robotic surgery has any influence on the reduction of complications in the aged population undergoing rectal cancer.

Methods: We performed a retrospective analysis of a prospective database of 151 patients who underwent robotic surgery for rectal cancer. We divided our population in 3 groups: under 65 year old, between 65 and 80 year old and above 80 year old. We recorded complications in each group intra and post procedure. Qualitative variables were expressed in terms of absolute frequencies and percentages and mean values and standard deviation were used to express quantitative variables. The analysis of data was applying Fisher's exact test or Chi-squared test for qualitative variables and variance analysis or Student'-t test for quantitative variables. Statistically significant values of $\mathrm{p}<0.05$ underwent multivariate logistic regression analysis. Results: The present study included 151 patients (94 males).Seventy seven patients were under 65 year old, 73 patients were between 66 and 80 year old and 11 patients were above 80 year old. The analysis showed conversion rates of $10.38 \%, 13.69 \%, 27.27 \%$, and complication rate of $23.4 \%, 23.8 \%$, and $27.3 \%$ in each group. Univariate analysis showed no differences between the three groups. Nevertheless, there were statistical differences from BMI, ASA and neoadjuvant therapy. In multivariant analysis only neoadjuvant therapy was significant.

Conclusions: Robotic approach do not decrease complications in elderly population.

\section{P417-ROBOTICS \& NEW TECHNIQUES-Colorectal}

TRANSANAL MINIMALLY INVASIVE SURGERY, MORE THAN FOR TATME: REPAIR OF RECTOVAGINAL FISTULA AND REDO ANASTOMOSIS FOR RECTAL STENOSIS

\author{
P. Imma, M. Wenceslao, G. Carlos Javier, S. Gemma, M. Socías, \\ R. Josep
}

Surgery, Fundació Hospital Sant Joan de Deu de Martorell, BARCELONA, Spain

Introduction: It has been described the advantages of total transanal mesorectal excision (TaTME), with better visualization and access to the lower rectum. We use this access whith the Gel Point Path device, to repair a rectovaginal fistula with stenosis of low rectal anastomosis in two patients, that would be difficult by conventional abdominal approach

Method: we show our surgical technique for repair a rectovaginal fistula with stenosis of low rectal anastomosis in two female patients operated due to rectal neoplasia. One of the patients underwent prior chemo-radiotheratpy. Rectoscopy and image test was performed at the patients prior the intervention. No recurrence signs are recorded at MRI.We describe the operation technique: A new anterior rectal resection was performed with a combined transanal (Gel Point Path) and abdominal minimally invasive approach. Redo anastomosis whith EEA 31 stappler was performed, vaginal repair and epiploplasty. The intervention was especially laborious due to the fibrous tissue. Pathology: fistulous path without tumor infiltration in the two patients. At two months, a Opaque enema show permeability and absence of leaks in the two patients. The ileostomy was closed at three months.

Discusion: We believe that transanal access through the Gel point Path can be a good option for rectovaginal fistula and stenosis of low rectal anastomosis, allowing a better visualization and acces, and making more easy a very difficult intervention.

\section{P418-ROBOTICS \& NEW TECHNIQUES-Colorectal}

ROBOTIC APPROACH FOR TRANSANAL POLYP RESECTION: STEP BY STEP TECHNIQUE

R.M. Jimenez-Rodriguez, G. Anguiano, J.M. Diaz-Pavon, I. RamalloSolis, M.L. Reyes-Diaz, A.M. Garcia-Cabrera, J.M. VazquezMonchul, F. de la Portilla, J. Padillo

Surgery, Hospital Universitario Virgen del Rocio, SEVILLA, Spain

Introduction: TAMIS or transanal minimally invasive surgery for polyp resection has increased fame for several situations in which adenomas with or without dysplasia cannot be removed with conventional colonoscopy. In this video we show the step by step technique performed with the Da Vinci Xi system.

Material and Methods: In this video we show the setting and the location of the patient-side cart and the arms to perform the resection of polyps in different patients and how to develope the procedure.

Results: After placing the patient-side cart the arms are connected to 3 ports and the camera, double fenestrated grasper and scissors are connected to the arms through a transanal gel-port device. A line is described around the polyp with monopolar energy to determine the place of the dissection. The scissor is exchanged by a robotic Harmonic wrist instrument and the complete dissection is performed. The wound is closed using a robotic needle holder and a suture. Results: Transanal robotic surgery could be safely performed after a standardized technique is stablished. 


\section{P419-ROBOTICS \& NEW TECHNIQUES-Colorectal}

\section{NOMOGRAM TO PREDICT CONVERSIONS IN ROBOTIC} SURGERY FOR RECTAL CANCER

G. Anguiano, R.M. Jiménez-Rodríguez, J. Tinoco-González, J.M. Diaz-Pavon, I. Ramallo-Solis, M.L. Reyes-Díaz, J.M. VázquezMonchul, A.M. García-Cabrera, F. de-la-Portilla, J. Padillo

General Surgery, HOSPITAL UNIVERSITARIO VIRGEN DEL ROCÍO, SEVILLE, Spain

Aims: Robotic rectal cancer surgery has demonstrated to obtain at least the same results than laparoscopic surgery. However, robotic surgery is associated with high rates of costs, specially when conversion to opened surgery occurs. The goal of this study is to create a predictor nomogram of conversions for robotic rectal cancer surgery.

Methods: We performed a retrospective analysis of a prospective database of patients who underwent robotic surgery for rectal cancer from October 2008 to November 2017. We performed a bivariant analysis and detected the variables which were related with the conversion: Body Mass Index (BMI) and the T. We divided the patients of the population in two groups depends on obesity (BMI of $\mathrm{Kg} / \mathrm{m} 2$ ) and on $\mathrm{T}(\mathrm{T} 1-2 / \mathrm{T} 3-4)$. We registered conversions in each group calculating the pretest risk. We performed likelihood index ( $\mathrm{LR}+/$-) for under and above $30 \mathrm{~kg} / \mathrm{m} 2$ of BMI, adding in a second step the LR of T; obtaining the prediction index for four groups by using a standardize nomogram.

Results: The present study included 194 patients (128 males). 143 were under BMI of $30 \mathrm{~kg} / \mathrm{m} 2$ and 51 above. Regarding T, 54 were with a tumor of T1-2 and 150 with T3-4. The analysis showed a conversion rate of the statistical sample of $14 \%$. Univariant analysis showed significative differences in the BMI $(\mathrm{p}=0.005)$ and $\mathrm{T}(\mathrm{p}=0.022)$. A nomogram was performed; as regards the BMI, the positive likelihood index in the group of BMI $>30$ a prediction index of conversion of $50 \%(\mathrm{LR}+4,95)$ and in $\mathrm{BMI}<30$ the prediction index of conversion is $5 \%$ (LR0,52 ). Adding the $\mathrm{T}$ group data, for $\mathrm{BMI}>30$ and $\mathrm{T} 1-2$ the conversion prediction rate is $3.5 \%$ (LR- 1,2); for BMI $>30$ and T3-4 the conversion prediction is $92 \%(\mathrm{LR}+5,6)$. BMI $<30$ and T1-2 the conversion prediction is $2 \%$ (LR- 1,2); IMC $<30$ and T3-4, the conversion prediction is $30 \%$.

Conclusion: A standardize nomogram with the variable BMI and T facilitates the selection of patients for robotic surgery in rectal cancer avoiding conversion to open surgery.

\section{P420-ROBOTICS \& NEW TECHNIQUES-Colorectal}

\section{D- LAPAROSCOPIC RIGHT HEMICOLECTOMY VS HD LAPAROSCOPIC RIGHT HEMICOLECTOMY}

I.O. Avram, R. Metzger, F. Schütze, C. Lamberty, Klinik für Allgemein- und Viszeralchirurgie, CaritasKlinikum Saarbrücken, SAARBRÜCKEN, Germany

Background: 3D-laparoscopy is proven to improve performance in dry laboratory settings, especially for novice surgeons due to better depth perception. However, the benefits for experienced laparoscopic surgeons are still discussed.

Aim: The aim of this study is to compare the results of right hemicolectomy (RC) using a conventional (2D HD) laparoscopic system with RC performed using a 3D laparoscopic system in terms of duration, complications and results.

Material and methods: From all laparoscopic right hemicolectomies performed in our clinic we selected all procedures performed by the same team of 2 consultant surgeons using the same technique and divided them in 2 groups. The study group comprised of all patients operated using our 3D Einstein Vision 2.0 system; all other patients which were operated using our standard Wolf HD laparoscopy system comprised the control group. All patients were retrospectively analyzed in terms of patients characteristic, OR time, duration of operation, intra- and postoperative complications, length of hospitalization, pain score, necessity of analgesics and number of lymph nodes retrived. Risk factors for complications (BMI, smoker, diabetes, COPD, BPH) were also registered.

Results: There were 54 patients included in the study group, while the control group comprised of 98 patients. Mean operation time in the study group was $123.3 \mathrm{~min}$ in the study group, while mean OR time was $157.4 \mathrm{~min}$. Mean operation time in the control group was $128.1 \mathrm{~min}$, while mean OR time $145.4 \mathrm{~min}$. One reintervention was noted in the control group and two in the the study group; no conversion to open surgery was noted. There were no significant differences regarding patient characteristics, pain score, wound complications, hernia rate, length of hospitalization or number of lymph nodes removed.

Conclusions: There were no significant differences regarding the outcome of RC using 3D laparoscopy; total OR time was significantly higher in the study group due to the time needed to set up the 3D-laparoscopy unit. This is biased by the fact that the 3D system needs to be set up manually while the conventional HD system is integrated in the OR. Also, there was no significant difference in complication rate.

\section{P421-ROBOTICS \& NEW TECHNIQUES-Colorectal}

\section{ROBOTIC SALVAGE SURGERY FOR THE TREATMENT OF RECURRENT RECTAL CANCER}

H.J. Kim, G.S. Choi, J.S. Park, S.Y. Park, Colorectal Cancer Center, Kyungpook National University Chilgok Hospital, DAEGU, Korea

Background/Purpose: Robotic approach can be a treatment option for patients with pelvic recurrence after primary resection for rectal cancer. However, data regarding patient selection, complication rates, and oncologic outcomes are rarely reported. We aimed to present initial experience and to evaluate feasibility, safety, and oncologic outcomes of robotic salvage surgery for recurrent rectal cancer.

Methods: Ten patients who underwent robotic salvage surgery for local recurrence at the anastomotic site, lateral pelvic side-wall, or lateral pelvic lymph nodes (LPNs) were retrospectively evaluated from a prospectively maintained database.

Results: Two patients underwent pelvic mass excision with en bloc resection of anastomosis and redo-anastomosis, and eight patients underwent lateral pelvic lymph node dissection (LPND) for LPN metastasis; one of these eight patient underwent additional en bloc resection of anastomosis. All patients achieved R0 resection. The median operation time was 165 min and the median estimated blood loss was $50 \mathrm{ml}$. There were no conversions. As for intraoperative complications, one patient experienced ureter injury during LPND because the metastatic LPN was closely abutting to the ureter. The median hospital stay was 7 days. In six patients who underwent LPND, the median number of harvested lymph nodes was 7 (range 2-13) and the median number of metastatic lymph nodes was 1 (range 0-2). With median follow-up 26 months, one patient developed lung and pelvic recurrence at 36 months after salvage operation and seven patients remained in disease-free state at the last follow-up.

Conclusion: Initial experience of robotic salvage surgery for pelvic recurrence in rectal cancer indicated that it is safe and feasible. Therefore, the robotic approach can be considered as a treatment option for the treatment of local recurrence in selected patients.

\section{P422-ROBOTICS \& NEW TECHNIQUES-Education}

\section{ASSESSING COGNITIVE LOAD AND EMOTION IN TRAINING SURGEONS EXPOSED TO MORTALITY IN THE ANIMAL LAB}

P. Martinez $^{1}$, J.C. Manuel-Palazuelos ${ }^{1}$, J.M. Maestre ${ }^{2}$, L. Peñate ${ }^{1}$, J.C. González-Cueli ${ }^{1}$, C. Redondo-Figuero ${ }^{1}$, J.I. Martin-Parra ${ }^{1}$

${ }^{1}$ General Surgery, HUMV, SANTANDER, Spain; ${ }^{2}$ Anaesthesia, HUMV, SANTANDER, Spain

Introduction: There is uncertainty regarding the effects of simulated patient death. Several reports showed increased cognitive load and poorer learning outcomes, and others increased performance without causing stress to learners. We have not found any report studying the impact of animal death in the simulation lab.

Methods: This was an observational cohort study to assess the emotional and cognitive load of surgeons who experienced animal death in the simulation lab. Seventy-four faculty and residents from different surgical specialties training minimally invasive surgery participated in the study. One cohort consisted of surgeons whose animal died during surgery, and the other by those whose animal survived. Emotions were assessed using the scale for mood assessment and cognitive load with NASA task load index.

Results: Twenty percent of participants experienced mortality while training anti-reflux surgery (11 cases) and other procedures ( 3 cases). Causes of death included intraoperative pneumothorax $(\mathrm{n}=10)$, hemorrhage $(\mathrm{n}=1)$, and cardiac dysrhythmias $(\mathrm{n}=3)$. Participants exposed to animal death had higher levels of sadness and anxiety, and lower levels of happiness $(p>0.05)$. Cognitive load was slightly higher in the exposed cohort $(\mathrm{p}>0.05)$.

Conclusions: These findings suggest that mortality in the animal lab do not have a significant effect on cognitive workload and emotions of surgeons training complex laparoscopic procedures. 


\section{P423-ROBOTICS \& NEW TECHNIQUES-Education}

CAN VISUOSPATIAL TESTING DISTINGUISH BETWEEN EXPERIENCED LAPAROSCOPIC AND NOVICE' SURGEONS?

$\underline{\text { T. Vajsbaher }}{ }^{1}$, H. Schultheis ${ }^{2}$, V. Uslar ${ }^{3}$, D. Weyhe ${ }^{3}$, N.K. Francis ${ }^{4}$

${ }^{1}$ Spatial Cognition Center \& Department of Human and Health Sciences, University of Bremen, BREMEN, Germany; ${ }^{2}$ Spatial Cognition Center \& The Institute for Artificial Intelligence, University of Bremen, BREMEN, Germany; ${ }^{3}$ University Hospital for Visceral Surgery, Pius-Hospital Oldenburg

Department of Human Medicine, University of Oldenburg, OLDENBURG, Germany; ${ }^{4}$ Department of General Surgery, Yeovil District Hospital, YEOVIL, United Kingdom

Introduction: The visuospatial profiles of expert laparoscopic surgeons remain unaccounted in the current literature for as the influence of visuospatial ability on laparoscopic learning has mainly been investigated in medical students or novice surgeons and using simulators as means of performance measurement. Such knowledge is critical, as without understanding how clinical experience may impact visuospatial processes in surgeons, we hinder our efforts to utilize the available knowledge to support surgical education for the future. This study is aiming to explore the development and influence of visuospatial processes on intraoperative laparoscopic learning. Method: The study reports the interim baseline results from the ongoing longitudinal study throughout a 2-year period of training on laparoscopic surgery. Data from 35 surgeons including 17 residents undergoing training were captured and compared to 18 specialists who are working in departments of General and Visceral surgery at two large Hospitals. The mean experience of the surgical residents was 4 years. The mean laparoscopic experience among the senior surgeons is 17 years, with each surgeon performing an average of 6 laparoscopic procedures per week. Visuospatial ability was tested using Mental Rotation Test (MRT), Guay Visualization of Views Tests (GVVT), Spatial Perspective Taking and Spatial Orientation Test (PTOST) and Pictorial Surface Orientation (PicSOr). Spearman Correlation coefficient was used in this study with a p-value of significance at $<0.05$.

Results: Senior surgeons have an overall good visuospatial profile, in the sense that they performed close to optimum on all measurement scales. The Spearman rho revealed a significant correlation between scores on GVVT and PicSOr $(r=0.607, p=0.012)$ and between PTOST and PicSOr $(r=-.686, p=0.003)$. A significant correlation between years of laparoscopic experience and PTOST score was also observed $(r=0.587, p=0.035)$. When comparing residents and senior surgeons, no significant difference on the MRT was observed $(M=10.2, \mathrm{SD}=4.33)$, nor between baseline scores of senior surgeons and resident surgeons on all tests.

Conclusion: The results of this study carry important clinical and theoretical implications, as the results hint towards the idea that intraoperative laparoscopic experience lends little to no influence over the development of visuospatial ability.

\section{P424-ROBOTICS \& NEW TECHNIQUES-Education}

LEARNING MODELS AND LAPAROSCOPIC TECHNICAL SKILLS, HOW TO ADAPT EACH CASE TO IMPROVE COMPETENCES

P. Martinez, J.I. Martin-Parra, E. Toledo, M. Cañón,

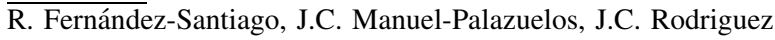

General Surgery, HUMV, SANTANDER, Spain

Objectives: According to DA. Kolb learning is the result of how people perceive and then process what they have perceived. The aim of this study is to identify the personal characteristics of learning in of the participants in a course of laparoscopic technical skills according to the styles described by Kolb.

Methods: Between June 2016 and November 2017, 35 participants performed a 50 h course distributed over five consecutive days performing laparoscopic manual intestinal anastomosis in endotrainer. They all filled in Kolb's learning style test adapted to Spanish. The anastomoses were performed in 'ex-vivo' swine intestines. In each anastomosis we evaluated the quality at the end and execution time. The test and quality variables were analyzed through statistical studies. Results: In our study, $69 \%$ of the participants were women and $31 \%$ wew men. $49 \%$ were staff surgeons and $58 \%$ were resident. The median age among residents was 29 years and among the staff 39 years. The most frequent learning model in the sample studied was converging (31\%). The predominant model among women was assimilating (37\%), which, however, represented only $8 \%$ in men. In men, converging model was predominant (39\%). Among the staff, the most frequent model was diverging (35\%). Adaptation style prevailed among residents (39\%), being rare among the staff $(12 \%)$. The mean time of the anastomosis was $74 \mathrm{~min}$ for both the adapter model and the assimilator, $68 \mathrm{~min}$, for the convergent and divergent models. The quality of the anastomosis performed by each participant was $80 \%$ for the adapter model, $37 \%$ for the assimilator model, $42 \%$ for the convergent model and $45 \%$ for the divergent model.

Conclusions: The predominant style in our study was convergent. Among women, the most frequent model was assimilator wheras in men it was the least frequent. In the residents, the most frequent model was adapter however, it was very rare in adjuncts. Among residents we do not find divergent styles. The highest quality of the anastomosis was achieved by those who worked with an assimilating style. Knowing previously the training style we can individualize the teaching methodology in order to improve competences.

\section{P425withdr-ROBOTICS \& NEW TECHNIQUES-Education}

COMPARING OUTCOMES OF DIFFERENT GRADES OF SURGEONS PERFORMING LAPAROSCOPIC AND OPEN APPENDICECTOMIES AT A SINGLE CENTRE OVER A TENMONTH PERIOD

\section{A.B. Talbot, P. Asaad, N. Iqbal}

Department of Colorectal \& General Surgery, Wrightington, Wigan and Leigh NHS Foundation Trust, WIGAN, United Kingdom

Aims: Assess whether laparoscopic appendicectomies (LA) are a superior option to open appendicectomies (OA). Specifically, comparing the time taken, complication rates and whether it is more appropriate to perform an LA overnight, as opposed to OA. Finally, to find out how a range of outcomes differs between different grades of surgeon.

Methods: An information request was sent to the clinical coding department to derive patien identification numbers for all appendicectomies over a ten-month period (180 total surgeries). These numbers were then inputted into the hospital information system where the electronic operation note is present, and specific outcomes were derived and analysed.

Results: $68 \%$ of operations were OA and $32 \%$ were LA. Mean LA times for consultants, SAS and SpR were 88.4, 78 and 92 min respectively and OA 63, 57 and 59 min respectively. Their respective conversion rates were $27 \%, 16 \%$ and $0 \%$. OA had a complication rate of $16.3 \%$, LA was $10.2 \%$.

Conclusion: OA are performed more than LA. $\mathrm{SpR}$ doctors had the slowest completion times for LA but the lowest conversion rates. SAS doctors had the fastest completion times for LA and OA but higher conversion. LA takes longer than OA but has lower complication rates; key factors when performing at night.

Key statement: Laparoscopic appendicectomies require more surgeon-hours and have the potential to be converted to open, however the rates of complications and serious complications are significantly lower. 


\section{P426-ROBOTICS \& NEW TECHNIQUES-Education}

INTEGRATED EDUCATION FOR COLORECTAL DISEASE-A DIGITAL SOLUTION FOR A DIGITAL AGE

\section{J. Waterman $^{1}$, M. Abdeldayem ${ }^{1}$, D. Brown ${ }^{2}$, P.N. Haray ${ }^{3}$}

${ }^{1}$ Colorectal Surgery, Prince Charles Hospital, MERTHYR TYDFIL, United Kingdom; ${ }^{2}$ Digimed ${ }^{\circledR}$, ESSEX, United Kingdom; ${ }^{3}$ Colorectal Surgery, University of South Wales, WALES, United Kingdom

Background: Paper based resources have been the standard sources for information for centuries. However, more and more people (patients and staff alike) are looking online for information. While the internet often provides excellent resources, there is often conflicting and confusing material of doubtful veracity. Trainee staff and patients/carers should be able to access reliable resources whenever and wherever they are. The aim of this project was to create a high-quality resource fulfilling these needs.

Aim: We present a video demonstrating our Integrated Colorectal Education Website ( http://www.colorectaleducation.com/).

Our Approach: High quality health care provision requires highly trained staff as well as wellinformed patients. Information resources for these two groups are usually accessible from different repositories. Our Integrated Website provides a common platform for all those involved in colorectal surgery, to use, learn and reflect on. Users are directed to separate sections for patients and colorectal professionals. Multiple disclaimers prevent patients accidentally stumbling across clinical/ operative information, whilst providing access to those who wish to do so. Trainees struggle with balancing their educational needs with their service commitments. This website gives them the opportunity to view detailed operative training videos on the go. Many of videos are chapter based allowing them to stop and re-start with ease. Modules are also available for nurses providing them access to relevant educational material. The modular design of the website allows us to build upon it with more topics planned to be added over the next eighteen months. The resource also has detailed chapterised videos for patients due to undergo various colorectal procedures. All have been approved by a multi-professional panel including patients and are designed to provide information, offer support and to allay any anxiety. Videos with the care pathway and previous patients' experiences are accessible on demand.

Conclusion: On demand information has now become the norm with the use of smart phones/ tablets. This website provides patients, surgical trainees and other healthcare professionals access to information and education in a clear and reliable format anywhere in the world. Colorectal Education, on demand and just a click away!

\section{P427-ROBOTICS \& NEW TECHNIQUES-Education}

\section{AN ORGANIZATIONAL MODEL TO IMPROVE THE ROBOTIC SYSTEM IN GENERAL SURGERY}

V. Ferri, E. Vicente, Y. Quijano, H. Duran, B. Ielpo, E. Diaz, I. Fabra, L. Malave, R. Isernia, E. Pinna, R. Caruso

General Surgery, Sanchinarro University Hospital, MADRID, Spain

Objective: In the last decade the growing interest in robotic surgery is evident as shown by several published articles. The aim of the present study is to evaluate the main outcome of a single center experience and to describe the organizational system we have progressively established in our center in order to improve the development of Robotic program in all surgical area.

Materials and methods: We report a case series of patients who underwent robot-assisted surgery at Sanchinarro University Hospital since the beginning of the program (October 2010) until november 2018 Main patient demographic characteristics, type of surgery, peri and postoperative data and follow-up were evaluated.

Results: A total of 326 robotic procedures were performed for a total of 323 patients. The prevalence of malignant disease was $86 \%$.

A total of 72 pancreatic surgery were performes; 22 liver resections (mean operating time: 190 min); 33 gastrectomy (mean operating time $310 \mathrm{~min}$ ); 18 esophagectomy (mean operating time: $490 \mathrm{~min}$ ); 152 colorectal resections (100 rectal resections, 23 sigmoidectomy 19 hemicolectomies right, 10 left colectomy) (mean operating time: $220 \mathrm{~min}$ ); 6 Nissen procedures (mean operating time: $130 \mathrm{~min}$ ), 2 esofagheous myomectomy for achalasia (operating time: $90 \mathrm{~min}$ ); 3 adrenalectomy (mean operating time: $240 \mathrm{~min}$ ); three biliary surgery for benign desease, 2 splenectomy. Eight partial resection of the duodenum, one yeyunal resection, one mesenteric cyst resection and 3 retroperitoneal tumor have been performed. Conversion rate was $6 \%$, total morbidity have been $17 \%$. There has been no peri and postoperative mortality up to 30 days after surgery. The average hospital stay and intensive care were respectively 16 days (range 6-45 days) and 1.9 days (range $0-12$ days).

Conclusions: The organizational model defined in our center is facilitating the constant and progressive development of the robotic program. A broad and flexible availability of the robotic system, a progressive increase of young surgeons joining this technology as well as the institutional and departmental economical effort are the points with which the robotic system may increase its development in a surgical department.

\section{P428-ROBOTICS \& NEW TECHNIQUES-Education}

THE NATIONAL SURVEY FOR THE CURRENT STATUS OF ENDOSCOPIC SURGERY TRAINING IN JAPAN

Y. Kurashima $^{1}$, S. Yamaguchi ${ }^{2}$, S. Hirano ${ }^{1}$

${ }^{1}$ Gastroenterological Surgery II, Hokkaido University, SAPPORO, Japan; ${ }^{2}$ Education Committee, Japan Society for Endoscopic Surgery, TOKYO, Japan

Aims: Endoscopic surgery has been widespread in the field of general surgery. However, in Japan, there is no standard program for endoscopic surgery training, and its competency has not been considered for the acquisition of Board Certified Surgeon. The purpose of this survey was to investigate the current situation of endoscopic surgery training and autonomy of young surgeons for endoscopic surgery in Japan.

Methods: The survey was planned to target general surgery members of the Japan Society for Endoscopic Surgery (JSES) who was post graduate year 10 or less. After approval by the Ethics Committee of JSES, the request for the participating in survey was mailed to 2296 object members. Questionnaire responses were available in print or online media. The contents of the questionnaire consisted of 19 items, about the conditions of endoscopic surgical training, experienced case number, and the self-assessment of autonomy from 1 to 4 point by Zwisch scale in 9 specific procedures of endoscopic surgery.

Results: The total response rate was $28.5 \%$ (645/2296). Sixty five answers were excluded due to inadequate response and 580 answers were analyzed. Of the questionnaire respondents, $87 \%$ were male and $13 \%$ were female. The ratio of Board Certified Surgeon was $67 \%$. Although $87 \%$ of the teaching hospitals had simulators for basic training of endoscopic surgery and $94 \%$ of the respondents practiced basic skill of endoscopic surgery, only $34 \%$ teaching hospitals had specific training programs for endoscopic surgery. The surgeons who operated 20 cases of laparoscopic appendectomy and inguinal hernia repair and 50 cases of laparoscopic cholecystectomy, right hemicolectomy and sigmoidectomy, felt confident to perform each procedure independently. Regarding with laparoscopic rectal resection and gastrectomy, even though the surgeons who had 50 cases of experience, they didn't had confidence to perform those procedures independently. Conclusions: This study is the first national survey to investigate the status of endoscopic surgery training in Japan and the autonomy of young surgeons for endoscopic surgery. In order to develop a training system for not only basic skills but also advanced procedures of endoscopic surgery, cooperation of each teaching hospital, academic surgical society, medical specialty board is necessary.

\section{P429-ROBOTICS \& NEW TECHNIQUES-Education}

\section{COMPARISON OF LAPAROSCOPIC SKILLS BEFORE AND AFTER CALL, THE IMPACT OF SLEEP DEPRIVATION ON THE PERFORMANCE OF GENERAL SURGERY RESIDENTS}

\section{H. Segura-Marin, G. Arredondo-Saldaña, J.A. Diaz-Elizondo,} E. Flores-Villaba

General Surgery, Escuela de medicina del Tecnologico de Monterrey, MONTERREY, Mexico

Currently there is a debate about what is the most optimal work schedule for residents of general surgery, It is important to respect the free time of residents to avoid Burnout, however it is also important have enough exposition to clinical cases that allow a satisfactory development in the clinical practice. This becomes even more important when we talk about the learning of surgical skills. This is where the laparoscopic simulation industry opens a large area of opportunity, For a reasonable price it is possible to practice basic laparoscopic skills without compromising patient safety. This is a pilot study that was carried out during the period from January 2018 to June 2018, In a public hospital in Monterrey, NL, Mexico, The composition between the execution of the standardized exercises of the FLS (Fundamental laparoscopic surgery) in an endoscopic simulator was performed to 20 residents of general surgery (from first to fifth year) 24 hrs before being on call vs these same residents post call. A series of questions was asked to each resident in each measurement, so in this way they answered the same questions twice, then a comparison of the results of both questionnaires was made. The results of the exercises were assessed and rated by the same person using the criteria established in the FLS for the scores of each exercise and for the final grade.

An average age of 27 years was obtained, measurements were taken of 20 residents of which 16 are male and 4 female. On average, the residents before be on call performed the exercises with 7 $\mathrm{h}$ of having slept while the post call performed the exercises with $2.15 \mathrm{~h}$ of having slept, the residents before be on call had on average $10.9 \mathrm{~h}$ without sleep while the Post call had $25 \mathrm{~h}$ without sleep. The average number of hours worked per week is $111 \mathrm{~h}$, measured by the time in and out of the hospital.

In this study, conclusive results were obtained regarding the null relationship of sleep deprivation with the performance of laparoscopic skills in surgical residents. 


\section{P430-ROBOTICS \& NEW TECHNIQUES-Education}

DEVELOPING AN ASSESSMENT TOOL FOR LAPAROSCOPIC PRECISION CUTTING

C.C. $\mathrm{Yeh}^{1}$, C.Y. Lee ${ }^{2}$, C.Y. Hsiao ${ }^{3}$, T.W. Tseng ${ }^{1}$, I.R. Lai ${ }^{4}$

${ }^{1}$ Department of Medical Education, National Taiwan University Hospital, TAIPEI, Taiwan; ${ }^{2}$ Department of Surgery, National Taiwan University Hospital, TAIPEI, Taiwan; ${ }^{3}$ Department of Surgery, National Taiwan University Hospital Yun-Lin Branch, DOULIOU CITY OF YUNLIN COUNTY, Taiwan; ${ }^{4}$ Department of Anatomy and Cell Biology, National Taiwan University Hospital and College of Medicine, National Taiwan Uni, TAIPEI, Taiwan

Aim: 'Precision cutting' is one of skills tasks of the Fundamentals of Laparoscopic Surgery (FLS) Program, which is cutting a circle on a piece of gauze under laparoscope and assessed by completing time (maximum time limit: $300 \mathrm{~s}$ ). There is no definition of quality of the final product. The aim of this study is to develop an assessment tool of laparoscopic precision cutting and test its reliability.

Method: An assessment tool of laparoscopic precision cutting was developed with four items based on completion, degree of deformation, degree of being pulled, and overall appearance of the final product of laparoscopic precision cutting by experts' meetings. The scale of each item was 5 points Likert scale. A descriptive sheet with a legend and a text description for each scale (Fig) was attached for assessors' reference.

For our high school entry medical students, they gained hands-on experiences of laparoscopic skills first time by attending a 1-hour course at Minimally Invasive Surgery Training Center, National Taiwan University Hospital (NTUH). We invited students to participate this study after this training. We collected participants' final products of ' precision cutting' station and assessed them by using this assessment tool. This study was proved by Institutional Review Board, NTUH (IRB No:201512051RINB). Results: 35 students were enrolled between February 2016 to June 2016. Two non-medical assessors and a senior surgeon were invited to assess the products. The mean score and Cronbach' s alpha value of each item were as followed: completion $2.2 \pm 0.9,0.91$; degree of deformation $2.11 \pm 0.9,0.96$; degree of being pulled $3.1 \pm 1.1,0.85$; and overall appearance $2.6 \pm 0.9,0.95$.

Conclusions: In summary, we successfully developed an assessment tool for laparoscopic 'precision cutting' and showed its reliability. The tool could provide qualitative descriptions for objective feedbacks. Validating this tool in a large scale is undergoing.

\section{P431-ROBOTICS \& NEW TECHNIQUES-Education}

DEVELOPMENT AND VALIDATION OF AN OBJECTIVE STRUCTURED ASSESSMENT OF TECHNICAL SKILL SCORE FOR LAPAROSCOPIC LINEAR-STAPLED ANASTOMOSIS

M.W. Schmidt ${ }^{1}$, C.M. Haney ${ }^{1}$, K.F. Kowalewski ${ }^{1}$, V. Bintintan ${ }^{2}$, L. Fischer ${ }^{3}$, B.P. Müller ${ }^{1}$, F. Nickel ${ }^{1}$

${ }^{1}$ General, Visceral and Transplant Surgery, Section Minimally Invasive Surgery, Heidelberg University Hospital, HEIDELBERG, Germany; ${ }^{2}$ Department of Surgery, Iuliu Hatieganu University, CLUJ-NAPOCA, Romania; ${ }^{3}$ General and Visceral Surgery, Klinikum Mittelbaden, BADEN-BADEN, Germany

Aims: Creating laparoscopic anastomosis is a challenging surgical skill with high clinical relevance. To assure efficient training and enhanced learning curves, constructive and objective feedback is essential. Currently there is no appropriate instrument to assess the surgical performance while creating laparoscopic anastomosis. The aim of this study is to develop and validate the Anastomosis-Objective Structured Assessment of Technical Skill (A-OSATS) score.

Methods: To obtain an international expert consensus for a procedure specific checklist (PSC) for laparoscopic anastomosis, a modified Delphi Survey with an integrated Analytic Hierarchy Process is currently being performed. Each A-OSATS sub step is assigned a specific weight to determine its importance to the final outcome of the anastomosis. To validate the A-OSATS score, a laparoscopic side-to-side small bowl anastomosis with a linear stapler and hand-sewn closure of the enterotomy was chosen and is performed by surgeons with varying degrees of laparoscopic experience on a live porcine model. All performances are recorded and rated twice using the A-OSATS by two blinded experts.

Results: The final A-OSATS score includes a weighted PSC developed by the modified Delphi survey and the already validated global rating scale of previously published OSATS scores. Four key steps (Bowel placement, creation of enterotomies, stapling, closure of enterotomy) and sub steps, as well as their definitions, were established during the Delphi survey. To validate the A-OSATS, 16 surgeons (4 Experts, 9 Intermediates, 3 Novices) have participated in the study so far. Preliminary results showed significant differences between all three levels of laparoscopic experience (Novices: $73.6 \pm 8.9$; Intermediates: $89.1 \pm 8.7$, Experts: $110.2 \pm 5.6$; $\mathrm{p}<0.001)$ for the overall A-OSATS score as well as the PSC itself (Novices: $52.0 \pm 7.8$, Intermediates: $63.4 \pm 6.2$, Experts: $76.5 \pm 4.6$; $\mathrm{p}=0.001$ ).

Conclusions: The A-OSATS is a weighted score that objectively assesses surgical skill during the creation of laparoscopic anastomosis. Preliminary results confirm construct validity of the proposed score. Furthermore, by offering the possibility to differentiate single aspects during the procedure, the A-OSATS allows focused feedback to enhance one's performance. Minor changes in weights are expected after the last round of the Delphi survey. Interand intrarater reliability will be assessed after final inclusion of all participants. 


\section{P432-ROBOTICS \& NEW TECHNIQUES-Education}

RECALLING OF AN INTERVENTIONAL SCENARIO FOR TESTING SITUATIONAL AWARENESS OF SURGICAL TRAINEES IN AN INTRA-OPERATIVE DECISION MAKING TRAINING COURSE

\section{C.C. $\mathrm{Yeh}^{1}$, T.W. Tseng ${ }^{1}$, W.C. $\mathrm{Ho}^{2}$, C.N. Chen ${ }^{2}$}

${ }^{1}$ Department of Medical Education, National Taiwan University Hospital, TAIPEI, Taiwan; ${ }^{2}$ Department of Surgery, National Taiwan University Hospital, TAIPEI, Taiwan

Purpose: To evaluate whether the participants who experienced this scenario could recall an interventional scenario for testing trainees' situational awareness and intra-operative decision making when they participated this training again.

Methods: We designed an IODM training course for junior surgical trainees and nurses by using live pigs since Sep 2016. In the first simulation, we created an interventional scenario and then provided an educational session. A researcher disconnected the EKG monitor on purpose for creating a scenario that the pig would lose vital signs when the team nearly finished a diagnostic laparoscopy. If the team did not aware the situation after $1.5 \mathrm{~min}$, a researcher would remind the team (Fig). We used a new developed assessment tool of IODM and an assessment tool for nontechnical skills for surgeons (NOTSS) for self-evaluations and objective assessments. We also discussed with them about their reactions while encountering this interventional scenario. Results: Between Sep 2017 to June 2018, 14 teams participated this training and experienced this interventional scenario. Fourteen $2^{\text {nd }}$ year surgical trainees have experienced it before. Only one participant $(7 \%)$ recalled it and made a quick decision while encountering this interventional scenario again. The results of IODM assessment and NOTSS did not show statistical difference comparing their self-assessments in the first and second year. Based on the analysis of the discussions, most of them remembered this this interventional scenario and reminded themselves to react it properly before the simulation. However, when they were the primary surgeon of diagnostic laparoscopy, they focused on performing this procedure and tutoring their junior trainee. They had no capacity in their brain to notice the change of vital signs. In addition, although they increased their situation awareness in clinical settings after the 1st time IODM training, they did not show this ability in the simulation.

Conclusions: Recalling of an interventional scenario for testing situational awareness of surgical trainees was very poor $(1 / 14,7 \%)$ among the 2 nd year surgical trainees. Qualitative analysis of discussions showed their brain capacities were occupied by performing new procedures and tutoring others. How to enhance trainees' situational awareness should be addressed.

\section{P433-ROBOTICS \& NEW TECHNIQUES-Education}

\section{THE LEARNING CURVES OF MINIMALLY INVASIVE} SURGERY: AN ANALYSIS OF THIS CONFUSED FIELD OF SURGICAL EDUCATION

E. Reitano ${ }^{1}$, E. Schembari ${ }^{2}$, A. Pesce ${ }^{3}$, C. Bortolussi ${ }^{3}$, S. Latteri ${ }^{2}$, D. Russello $^{2}$, G. la Greca ${ }^{2}$

${ }^{1}$ Digestive Surgery, Henri Mondor Hospital, AP-HP, University of Paris Est, UPEC, PARIS, France; ${ }^{2}$ Department of General Surgery, Cannizzaro Hospital, University of Catania, CATANIA, Italy; ${ }^{3}$ Department of General Surgery, Policlinico Hospital, University of Catania, CATANIA, Italy

Aims: A well-designed learning curve is essential to measure the progress of surgical abilities. Learning curves are very important to test the skills of trainees. However, there are still no welldefined criteria for developing good learning curves. As a result, many authors use subjective evaluation criteria. The purpose of this review is to analyse this field of surgical education and to identify the key criteria for good learning curves.

Methods: Learning curves were investigated in the field of laparoscopic and robotic minimally invasive surgery. Surgery of appendectomy, cholecystectomy, cholectomy, inguinal hernia repair and gastrectomy were considered. The type of surgery, the year of publication, the design of the study, the surgeon's experience (resident, young or senior), the surgical technique, the number of patients involved in the study and the suggested learning curve by the different studies were taken into account. In the selection of articles, more importance was given to those based on the activity of young surgeons or residents.

Results: The literature analysis showed conflicting results. The different learning curves for the same surgery may be due to the different evaluation criteria considered. Only a few studies investigate the learning curves of young surgeons and residents.

Conclusions: The data available in the literature on learning curves are contradictory. Several factors need to be evaluated in order to create more accurate learning curves. We suggest the introduction of checklists with a score for each parameter to be examined, in order to develop more objective and standardized learning curves.

\section{P434-ROBOTICS \& NEW TECHNIQUES-Education}

THE DEVELOPMENT AND APPLICATION OF VIDEOASSISTED FEEDBACK DURING A NATIONAL TRAINING PROGRAMME FOR TRANSANAL TOTAL MESORECTAL EXCISION

M. Penna ${ }^{1}$, R. Hompes ${ }^{2}$, N. Mortensen ${ }^{3}$, P.P. Tekkis ${ }^{4}$, N. Francis ${ }^{5}$, G.B. Hanna ${ }^{6}$

${ }^{1}$ Academic Surgery, Imperial College London, LONDON, United Kingdom; ${ }^{2}$ Colorectal surgery, Amsterdam UMC, AMSTERDAM, The Netherlands; ${ }^{3}$ Colorectal surgery, University of Oxford, OXFORD, Uganda; ${ }^{4}$ Colorectal surgery, Imperial College London, LONDON, United Kingdom; ${ }^{5}$ Colorectal surgery, Yeovil Hospital, YEOVIL, United Kingdom; ${ }^{6}$ Cancer and Surgery, Imperial College London, LONDON, United Kingdom

Aim: The UK training programme for Transanal Total Mesorectal Excision (TaTME) has completed its first round of training. The study aim was to design a reporting platform that provided trainees with video-assisted feedback in a clear, concise and useful manner to support their training.

Methods: An established method of video analysis called Observational Clinical Human Reliability Analysis (OCHRA) was used to assess the surgical performance of the trainees during their clinical TaTME cases. A reporting form for the OCHRA results was designed identifying areas of difficulties in each procedure and providing error reduction mechanisms. This was piloted during the national training programme for TaTME in the UK.

Results: The OCHRA reporting form underwent three modifications before the content and format was agreed upon. The final version is divided into three sections: A. Case details, B. OCHRA findings, and C. Suggested Error-Reducing Mechanisms. For part B the TaTME procedure was divided into four phases of the operation: 1. Pursestring, 2. Rectotomy, 3. TME dissection, and 4. Connected phase when the abdominal and transanal teams work together synchronously. For each phase, OCHRA findings described the most frequently occurring technical inaccuracies/errors, number of consequential errors/adverse events and the most frequent and serious consequences encountered. Suggested error-reducing mechanisms in part $C$ were developed and established by an expert workshop and individual interviews with international surgeons experienced in TaTME.Trainee and mentor feedback stated that the reporting form had a clear format, easy to follow and understand. The error-reducing mechanisms were particularly useful and allowed the trainee to focus on improving specific technical aspects in their subsequent cases.

Conclusion: Video analysis using OCHRA can provide a wealth of information on surgical performance, especially for trainees at the start of their learning curve. As an exploratory study, validation of the reporting platform is required; however, its potential to offer detailed, individualised feedback to enhance training is promising. 


\section{P435-ROBOTICS \& NEW TECHNIQUES-Education}

LAPAROSCOPIC PELVIC SURGERY TRAINING PROGRAMUSING A NEW CONCEPT 3D-PRINTED VERSATILE PELVITRAINER

\section{R.C. Elisei ${ }^{1}$, F. Graur ${ }^{2}$, C. Popa $^{2}$, E. Mois ${ }^{2}$, L. Furcea ${ }^{2}$, N. al Hajjar ${ }^{2}$}

${ }^{1}$ General Surgery, Bistrita Emergency County Hospital, BISTRITA, Romania; ${ }^{2}$ General Surgery, Regional Institute of Gastroenterology and Hepathology „Prof. O. Fodor,, CLUJ-NAPOCA, Romania

Pelvic laparoscopic surgery (rectal, urological, or gynecological laparoscopic surgery) is an advanced surgery which require advanced skills, not easy to acquire. There are a lot of training programs for advanced laparoscopic skills but many of them are not affordable for most of surgery residents in Eastern Europe, where the training programs are far behind from those in Western Europe. Because of that those training programs need to be improved and optimized. In the European Union we want equal and high skilled surgeons. This is why we designed a new concept of pelvi-trainer, a versatile one in order to offer the residents the possibility to achieve advanced laparoscopic skills like perfect coordination, precise movements, ability to cut and suture after a well defined route, all of them in the pelvis tight space. We 3D-printed this pelvitrainer which has multiple characteristics: cheap and easy to produce, easy to be used, versatile because offer the possibility to achieve the skills named above, and many others, but also to train on real ex vivo animal rectum (suine model). We also believe that with a proper training a medical student and a young surgery resident are able to achieve the same skills like experienced surgery residents or specialists. In order to demonstrate that we need a study to compare the time to perform 4 or more exercises in this new concept pelvi-trainer by the medical students, young and experienced residents and surgery specialists.

What we want to achieve with this training program project is to have more and more skilled surgeons in advanced laparoscopy and an equal laparoscopic surgery training all over the country, close to the level of training in the Western Europe. Also we want this training program to make a standardization of the pelvic laparoscopic surgery training first in our country and then in other countries if possible.

\section{P437-ROBOTICS \& NEW TECHNIQUES-Education}

\section{A NEW WAY OF LEARNING SWINE LIVER ANATOMY FOR RESIDENTS IN TRAINING}

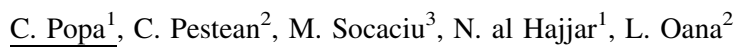

${ }^{1}$ Surgery, Regional Institute of Gastroenterology and Hepatology, CLUJ-NAPOCA, Romania; ${ }^{2}$ Anesthesiology, University of Agricultural Sciences and Veterinary Medicine, CLUJ-NAPOCA, Romania; ${ }^{3}$ Radiology, Regional Institute of Gastroenterology and Hepatology, CLUJ-NAPOCA, Romania

Aims: The aim of the study was to create a new easy learning method of swine liver anatomy for residents in training. Based on human liver surgical anatomy we put 'face to face' the similar structures and also the differences using ex vivo porcine models and $\mathrm{CT}$ reconstructions from live pigs. Methods: Having in mind the human liver anatomy, in the first stage we used data obtained from dissection of twelve porcine liver models to create an anatomical pattern, which summarized the most important surgical information. In the second stage, anatomical data obtained from CT scans of twelve living anesthetized pigs were analyzed. The CT reconstructions and volumetry data were added to the gross anatomy pattern to create a more complex learning module.

Results: The residents established the most frequent description of swine liver anatomy by putting together the information from ex vivo model dissection. The liver parenchyma is divided into fou main anatomic lobes: left lateral, left medial, right medial and right lateral. All those lobes are connected only in the posterior part, which allows a very good separation between them by deep fissures. Just as in humans, we found eight distinct segments with independent vascularization and biliary drainage. Portal vein has a specific ' $S$ ' shape; in most cases hepatic artery was found like a trifurcation and extrahepatic biliary tree has a very thin wall. In the right hemi-liver, the inferior vena cava passes through the liver parenchyma. Most frequent, we found five hepatic veins which are running completely intraparenchymal. The imagistic data offered a very useful 3D reconstruction with anatomical positions of the vascular-biliary tree and liver segmentation and gave us the possibility to create practical scenarios for resections. Perhaps the most important information was to discover and see the section plan and to calculate the volume of the remaining liver after resection. Conclusions: The anatomical-imagistic pattern based on $<\mathrm{i}>\mathrm{ex}$ vivo $</ \mathrm{i}>$ model disections combined with imagistic data offers a unique mindset before intervention. The concept 'human $<\mathrm{i}>\mathrm{vs}<\mathrm{i}>$ swine' to create an easy method of learning for residents in training can be applied to swine liver anatomy.

\section{P438-ROBOTICS \& NEW TECHNIQUES-Education}

\section{SURGICAL TRAINING: FROM A BEHAVIORISTS PERSPECTIVE: IS THERE ROOM FOR PRODUCTIVE ERROR?}

\section{M. di Giuseppe ${ }^{1}$, M. Fitzgerald ${ }^{1}$, F. Mongelli ${ }^{2}$}

${ }^{1}$ Surgery, San Giovanni Hospital-EOC-Bellinzona $(\mathrm{CH})$, BELLINZONA, Switzerland; ${ }^{2}$ Surgery, Ospedale Regionale di Bellinzona e Valli, BELLINZONA, Switzerland

The learning of surgery is traditionally based on the behaviourist model . Goals are set, standards of care fixed, with regular assessments of the level achieved. The teacher exercises control over the student, imposing rules and models, supported by 'reinforcing' actions (reward or punishment). The theory of Skinner's Program of Education, from 1954, is reflected in surgical learning. It foresees a gradual progression by level of difficulty, following a transmission-imitation model These theories seem currently outdated to face the new challenges of medicine and surgery and to keep up with technological developments.

Bruner, one of the theorists of the constructivist model, proposed in 1990 a method of collaborative learning between those who teach and those who learn. The goal of the method was to improve strategic problem solving. The comparison between various perspectives (between teacher and student), allowed the learner to better absorb knowledge and improve critical thinking. In 2008 Kapur published on the theory of 'productive failure'. This model makes the error of a single person useful for all his colleagues, privileges the practice of theoretical knowledge, contextualised learning as opposed to abstract learning, and 'guided' practice compared to a 'guided' theory. Bruner and Kapur's systems favour creativity, critical analysis of a problems origin, and the practical use of knowledge. They represent a hypothesis of learning, based on constructive discussion and a continuos 'give-take' feedback system.

In order to put these new models into practice in the clinical context, one may hypothesise and propose the adoption of a formal discussion of clinical cases that are complicated or difficult. Thereby making the theoretical lessons more collaborative, intuitive and inclusive. In the surgical field, one could adapt such a concept to surgery simulation, virtual reality and anatomical models. 


\section{P439-ROBOTICS \& NEW TECHNIQUES-Flexible surgery}

ROBOTIC-ASSISTED TYPE IV HIATAL HERNIA REPAIR WITH GORETEX MESH

\section{E. Reitano, N. Petrucciani, F. Brunetti, N. de'Angelis}

Digestive Surgery, Henri Mondor Hospital, AP-HP, University of Paris Est, UPEC, PARIS, France

Aim: Large hiatal hernias have a surgical indication when the patients suffering disabling symptoms such as anaemia, dyspnea, chest pain, gastric reflux. Several studies showed that in the case of large hernias the placement of a prosthesis was safe and could protect against recurrence. Mini-invasive surgery is the preferred approach for hiatal hernia repair and anti-reflux procedure and the Toupet fundoplication has been shown to be the best surgical technique for the hiatal hernias repair.The laparoscopic approach is currently the surgical gold standard but is burdened by technical difficulties especially in the case of large hiatal hernias. The robotic system is designed to overcome some technical difficulties of laparoscopy and the studies available in literature report the safety and effectiveness of the robotic approach in complex hiatal hernias repair.

Methods: We present the case of a grade IV hiatal hernia treated with a robotic approach in a 73 years old woman (BMI: $31 \mathrm{~kg} / \mathrm{m}^{2}$ ). The medical history consisted of a road accident with probable mechanism of deceleration, three years before. The patient had been suffering from dyspnea for three years. Due to the recent discovery of an anaemia, the patient was subjected to an endoscopic examination with the identification of a voluminous grade IV hiatal hernia. A subsequent computed tomography (CT) scan showed also the partial herniation of the transverse colon.

Results: The patient underwent to surgery by using the $d a$ Vinci robot system Si ${ }^{\circledR}$ (Intuitive Surgical, Sunnyvale, USA) with a single docking approach. The surgery consisted in the liberation of the hernial sac, the placement of a Goretex prosthesis and the packaging of a Toupet fundoplicatio. The surgery was performed without complication.

Conclusions: The robotic approach in the hiatal hernia surgery seems to be a valid alternative to laparoscopy, especially in complex cases. The surgical ability in robotic surgery is of paramount importance.

\section{P440—ROBOTICS \& NEW TECHNIQUES-Flexible surgery}

\section{VIDEO-ASSISTED THORACOSCOPIC THYMECTOMY USING} CARBON DIOXIDE INSUFFLATION IN OUR EARLY EXPERIENCE

\section{Naruke}

General Thoracic Surgery, Kawasaki municipal hospital, TOKYO, Japan

Aim: Video-assisted thoracoscopic surgery (VATS) with Carbon dioxide $\left(\mathrm{CO}^{2}\right)$ for mediastinal surgery is known to improve the visualization of medaistinal space. We report our experiences with two cases that underwent VATS thymectomy using $\mathrm{CO}^{2}$ insufflation under the one-lung ventilation general anesthesia by double lumen tube.

Methods: The instruments that were used for VATS thymectomy were only the 5-mm 30-degree rigid thoracoscope, Maryland jaw energy device, cotton made-dissectors, and straight endoscopic grasping forceps. They were used through sealed ports designed for laparoscopic surgery. Lowpressure $\mathrm{CO}^{2}$ insufflation set at $8 \mathrm{mmHg}$ were used for compression of surround tissue of mediastinal tumor during the releasing procedure.

Results: The patients were an 81-year-old male and a 54-year-old female. Thoracoscope with the $8 \mathrm{mmHg} \mathrm{CO}{ }^{2}$ insufflation provides excellent visualization of the medaistinal space and operation could be done smoothly without any hemodynamic compromise. Their pathological diagnoses were thymic cancer and thymoma, Type B1. The operative times were $115 \mathrm{~min}$ and $66 \mathrm{~min}$. The postoperative courses were uneventful and the patients were discharged on day 10 th and $3^{\text {rd }}$. Conclusion: We have just begun to routinely use $\mathrm{CO}^{2}$ insufflation for mediastinal tomorectomy and present our early experiences of successful VATS thymectomy by utilizing $\mathrm{CO}<\operatorname{su} 2</$ su insufflation
P441-ROBOTICS \& NEW TECHNIQUES-Flexible surgery

PRELIMINARY RESULTS OF SINGLE-INCISION THORACOSCOPIC SURGERY FOR PRIMARY SPONTANEOUS PNEUMOTHORAX USING A MULTICHANNEL PORT

D. Hokka, N. Hayate, K. Sanae, M. Suguru, M. Gaku, S. Shuto, M. Kazuhiro, K. Kenji, O. Takeshi, S. Nahoko, D. Takeshi, Y. Tanaka, M. Yoshimasa

Thoracic Surgery, Kobe University Hospital, KOBE CITY, Japan

Aims: This retrospective study aims to evaluate the feasibility of single-incision thoracoscopic surgery (SITS) for primary spontaneous pneumothorax (PSP), using a novel multichannel port ( $x$ Gate $($ ) $)$. Methods: Between October 2015 and November 2018, ten patients who underwent SITS using $x$ Gate ${ }^{\circledR}$. Nine patients were male and 1 was female, with mean age of $22.6 \pm 8.7$ years old. A $2.5 \mathrm{~cm}$ incision is placed in the middle axillary line on the 4th or 5th intercostal space, depending on the lesions. Postoperative outcomes of these patients were compared with those of 33 patients with PSP who underwent conventional three-port video-assisted thoracic surgery (VATS).

Results: There were no conversions from SITS to VATS. Mean operative time of SITS group was significantly shorter than that of three-port VATS group (55.4 \pm 14.3 min vs $79.8 \pm 26.0$ min, $p=0.003)$. Mean number of staplers used in surgery was $2.5(1-4)$ in SITS group and $3(1-5)$ in VATS group $(p=0.698)$. Mean duration of postoperative drainage was also shorter in SITS group $(1.0 \pm 0$ days vs $1.3 \pm 0.6$ days, $\mathrm{p}=0.05)$. No recurrence and wound infection were observed in SITS group.

Conclusion: SITS using $x$ Gate ${ }^{\circledR}$ is feasible when performed for selected patients with PSP. $\mathrm{X}$ Gate ${ }^{\circledR}$ provides good visualization of intrapleural space and esthetic outcomes, as well as a superb maneuverability by decreasing mutual interference of surgical instruments. Although conventional three-port VATS for PSP is well established, SITS using $x$ Gate ${ }^{\circledR}$ can be a permissible alternative. Further examinations are required to evaluate efficacy of SITS using $x$ Gate ${ }^{\circledR}$

\section{P442-ROBOTICS \& NEW TECHNIQUES-Flexible surgery}

THE EVOLVING ROLE OF ENDOVASCULAR STENTS IN ABDOMINOPELVIC TRAUMATIC VENOUS INJURIES?

\section{$\underline{\text { W.A. Butterworth }}{ }^{1}$, K. Khan ${ }^{2}$}

${ }^{1}$ General surgery, Guys hospital, LONDON, United Kingdom; ${ }^{2}$ Trauma, St marys, LONDON, United Kingdom

Aims: Haemorrhage remains a leading cause of potentially preventable death in trauma. In particular non-compressible torso haemorrhage is approximated to cause $60-70 \%$ of mortality in civilian trauma patients with otherwise survivable injuries and $80 \%$ in war setting. We performed a literature review to assess the potential for using endovascular stenting in traumatic venous injuries and explore the evidence of their efficacy and safety with different venous injury patterns. Methods: Systematic online search of PubMed performed using key words'endovascular stent', 'venous injury', trauma, penetrating, blunt, abdominal and pelvic. Inclusion criteria included al studies that explored the use of endovascular stents following traumatic abdominopelvic venous injuries. English language studies were used. Results were presented according to PRISMA guidelines.

Results: Of the 112 studies generated by the search,there were only four case reports in the literature documenting the use of endovascular stents in traumatic venous injuries dating back to 1997 and most recently 2009. The four cases included three retrohepatic IVC injuries, two secondary to blunt trauma and one penetrating; whilst the final case a blunt injury at the ilio-caval bifurcation. All four cases reported successful deployment of stents via the femoral or internal jugular veins, with subsequent resolution of haemorrhage. Length of time taken for stent insertion ranged from 9 to $52 \mathrm{~min}$. Three of four patients made full recoveries and discharged from hospital, with one patient subsequently dying of a brain injury independent of the successfu venous stent insertion. No complications were reported at up to 8 months follow up in remaining cases including stent leak, stenosis or migration.

Conclusion: Endovascular venous stents have been used successfully in managing complex abdominopelvic traumatic venous injuries. In particular retrohepatic venous injuries refractory to hepatic packing and vessel embolization, which are not amenable to direct surgical repair due to anatomical location. However before endovascular stenting can be added to the arsenal of interventional radiologists for abdomino-pelvic trauma, further development of stents custom made for venous injuries as well as prospective studies examining their long term safety and outcomes is needed. 


\section{P443-ROBOTICS \& NEW TECHNIQUES-Flexible surgery}

IMMEDIATELY REBUILD THE AIRWAY BY BRONCHOSCOPIC CRYOTHERAPY IN PATIENT WITH TRACHEAL PAPILLOMA

\section{P.H. Chen}

Thoracic Surgery, Hsinchu Mackay Memorial Hospital, HSINCHU, Taiwan

Tracheal papilloma is a rare neoplasm growing from the tracheal or bronchial epithelium and has no specific clinical presentations. This is a 40 -year-old female who complained of progressive dyspnea for about 2 months. Physical examination was unremarkable and the there was no abnormal finding by the chest plain film. Chest computed tomography was arranged and revealed a mass lesion located at the tracheal lumen with more than $80 \%$ luminal obstruction. We used fiberoptic bronchoscopy to evaluate the airway and found a mass lesion with pedicle originated from the posterior tracheal wall. Cryotherapy was considered for the tumor mass removing to establish a patent airway. The pathologic report revealed tracheal papillomatosis without any malignant component. Dyspnea was immediately improved and the patient chose closely observation after the bronchoscopic cryotherapy.

\section{P444-ROBOTICS \& NEW TECHNIQUES-Flexible surgery}

\section{ROBOTIC LIKE SUTURING WITH FLEXDEX FOR DIFFICULT} LAPAROSCOPIC SUTURE (PRESENTATION WITH VIDEO)

L. Castro Diez, M.L. García Jimenez, C. Mosquera Fernández, D.P. Bravo Beltran, J. Aguirrezabalaga González,

F.J. Noguera Aguilar,

Cirugía General y del Aparato Digestivo, Complejo Hospitalario Universitario de A Coruña, A CORUÑA, Spain

Aims: Recent advances in laparoscopic surgery, both in techniques and instrumentation material, have led to the emergence of innovative technological fields, among which Robotic Surgery stands out.One of the handicaps of this surgery is its high cost as well as the long learning curve. In this stage a new tool arises, the FlexDex semi robotic arm, which combines the precision and the range of movements of robotic surgery with the greater availability, simplicity of use and learning of conventional laparoscopic surgery.The objective of this study is to evaluate the efficacy and safety of the FlexDex device in different laparoscopic procedures.

Methods: FlexDex's is a three-axis gimbal technological device integrated in a conventional laparoscopic instrument that translates the surgeon's hand, wrist, and arm movements from outside the patient into corresponding movements of an end-effector inside the patient's body.The greater accessibility provided by the FlexDex allows the surgeon to perform sutures in areas of difficult access where mobility with conventional laparoscopic instruments is not optimal. The comfort of the surgeon remains fundamental in any type of surgery, even more when we are in anatomical locations with complex access, especially for the realization of sutures. Here is where surgical innovation instruments such as FlexDex provides ergonomic comfort for the surgeon and improves the patient's safety, especially in high-risk situations, such as when performing anastomosis.

Results: We present a prospective series of 10 laparoscopic procedures carried out by the same surgical team being the initial experience in our environment in the use of the Flexdex semi robotic arm for the realization of complex anatomical sutures.This is a case series of 10 patients to whom different surgical techniques requiring manual suture have been performed. These being 2 TAPP procedures, 6 Nissen-type fundoplicature and 2 reinforcements of colorectal anastomosis. It is important to note that in none of the cases complications were recorded

Conclusions: FlexDex can provide an excellent alternative to the robotic systems in complex surgical procedures, offering surgeons the precision and control they desire while maintaining the balance of cost, outcome and patient benefit.
P445-ROBOTICS \& NEW TECHNIQUES-Flexible surgery

NEW SINGLE-INCISION DEVICE FOR FLEXIBLE-SINGLEINCISION-SURGERY: VALIDATION IN SIMULATOR AND ANIMAL MODEL

J.F. Noguera $^{1}$, J. Aguirrezabalaga ${ }^{1}$, A. Centeno ${ }^{2}$, G. Fernandez ${ }^{3}$, A. Gómez

${ }^{1}$ Surgery, Complejo Hospitalario Universitario A Coruña, A CORUÑA, Spain; ${ }^{2}$ Centro Tecnológico de Formación, Complejo Hospitalario Universitario A Coruña, A CORUÑA, Spain;

${ }^{3}$ Fundación Profesor Novoa Santos, Complejo Hospitalario Universitario A Coruña, A CORUÑA, Spain

Background: A new single-port device (FSIS-Flexible-Single-Incision-Surgery) is presented This new platform has three working channels, two for rigid instruments and one for the flexible endoscope. The channel for flexible instruments offers a pneumatic sealing to avoid the air's leak of the cavity (abdomen, rectum, vagina). In this study the preclinical data are shown testing the feasibility and safety for laparo-endoscopic instruments.

Methods: Experimental evaluation of feasibility and safety in two stages. In the first stage a working channel with pneumatic sealing was tested in simulators to use a flexible endoscope. In the second stage (animal model) the single incision device that makes possible to use laparoscopic instruments and flexible endoscopes was tested. The measured variables were: time of the procedure, $\mathrm{CO} 2$ employed, adverse intraoperative events, grip's losing, losing of pneumatic sealing, feasibility and safety of the procedure for the surgeon.

Results: The hysterectomy and double adnexectomy was done with a median time of $7.1 \mathrm{~min}$. The median of the $\mathrm{CO} 2$ consumption was 32.5 litres. Only in one case (16.6\%) the surgeon had problems with the abdominal navigation of the endoscope that was easily solved. The grip's lose wasn't a major problem. The median size of the skin incision was $5.4 \mathrm{~cm}$. The median surgeon' score for the feasibility was 10 and for the safety was 9.6 .

Conclusions: The surgeons considered that the use of the device was very feasible and safe. The FSIS-device is a universal platform for Single-Incision-Surgery for surgeons and gastroenterologists and for abdominal, rectal and vaginal access.

\section{P446-ROBOTICS \& NEW TECHNIQUES-Liver}

DIRECT INTRAGALLBLADDER NIRF CHOLANGIOGRAPHY WITH THE ARROW-KARLAN ${ }^{\mathrm{TM}}$ BALLOON CHOLANGIOGRAPHY CATHETER

Picchetto $^{1}$, M. Diana ${ }^{2}$, S. la Rocca ${ }^{1}$, L. Rossi ${ }^{1}$, F. de Laurentis ${ }^{1}$, G. d'Ambrosio ${ }^{1}$

${ }^{1}$ Department of General Surgery and Organ Transplantation, Sapienza University of Rome, ROMA, Italy; ${ }^{2}$ IHU-Strasbourg, Institute of Image-Guided Surgery, IRCAD, Research Institute against Cancer of the Digestive System, STRASBOURG, France

Aim: Despite the Near-infrared Fluorescence (NIRF) via the intravenous administration of indocyanine green (ICG) improves the visualisation of the cystic duct (CD) and the extrahepatic biliary tract (EBT), the back fluorescence of the liver reduces the signal-to-noise ratio.We have modified the technique of NIRF cholecystocholangiography with intragallbladder ICG injection by using the Arrow-Karlan ${ }^{\mathrm{TM}}$ Balloon Cholangiography Catheter instead of the purse string at the gallbladder's fundus. This procedure allows a high rate of visualisation of the EBT, with few cases of ICG leakage.Aim: of this study is to confirm the feasibility of this different technique and to analyse the ICG spillage from the gallbladder and to identify the EBT.

Methods: We enrolled nine patients undergoing laparoscopic cholecystectomy for cholelithiasis The gallbladder was perforated with the cholangiogram catheter, the balloon inflated with $0.5 \mathrm{ml}$ of Saline and tightened. The bile was drained and the ICG bolus injected. A titanium clip was the placed on the catheter strict closely to the gallbladder in order to prevent the catheter dislocation. Results: The CD and the EBT were visible before dissection in 6/9 and 8/9 patients respectively. After dissection the $\mathrm{CD}$ was visible in all the patients and the EBT again in $8 / 9$ patients. There was only one ICG spillage due to a tardive positioning of the clip. In a case of inflamed gallbladder this technique helped in the identification of the dissection plane.

Conclusions: Our preliminary results of this ongoing study confirm the feasibility of this different approach as a possible alternative to the purse string and a good visualisation of EBT. 


\section{P447-ROBOTICS \& NEW TECHNIQUES-Pancreas}

ROBOTIC-ASSISTED UPPER GI AND HBP SURGERY AT A CENTER OF EXCELLENCE FOR MINIMALLY INVASIVE SURGERY: ESTABLISHMENT AND FIRST-YEAR RESULTS

M. Thomaschewski, M. Zimmermann, C. Müller-Debus, L. Frohneberg, K. Honselmann, D. Ellebrecht, R. Hummel, T. Keck, D. Bausch

Surgery, University Medical Center Schleswig-Holstein, Campus Lübeck, LÜBECK, Germany

Introduction: Robotic-assisted surgery is a promising technique for overcoming the limitations of laparoscopic surgery, especially with regards to complex and advanced surgical procedures. Here, we describe the establishment and implementation of our robotic upper gastrointestinal (GI) and hepato-pancreato-biliary (HPB) surgery program within our center of excellence for minimally invasive surgery as well as the first-year results.

Method: Robotic-assisted surgery was performed using the daVinci Xi Surgical System ${ }^{\mathrm{TM}}$ and performed by two surgeons specialized in minimally invasive surgery (DB and TK). Our robotic surgery program of upper GI and HPB surgery was established in three steps: (1) First, surgical procedures with easier degree of difficulty were performed robotically, including cholecystectomy, minor gastric resections and fundoplications. (2) Then, pancreatic distal resections, enucleations, adrenalectomies and atypical liver resections were robotically performed, as procedures with moderate degree of difficulty. (3) Finally, advanced and highly complex procedures were performed, including right hemihepatectomy, complex pancreatic head resections (including portal vein resections), total gastrectomy and esophagectomy. Data collected from July 2017 till July 2018 were retrospectively analyzed with regard to conversion rate, morbidity (Clavien Dindo grade $£ 3$ ) and mortality.

Results: Within the first year, a total of 66 robotic assisted upper GI and HPB resections were performed. The first step of establishing our robotic surgical program included eight procedures. Here, conversion rate, morbidity and mortality were $0 \%$. Within the second step of establishment 31 procedures were performed. Conversion rate, morbidity and mortality were $27 \%, 10 \%$ and $0 \%$. The last step included 27 of advanced and highly complex procedures. These procedures resulted in a conversion rate of $48 \%, 30 \%$ morbidity and $0 \%$ mortality.

Conclusion: Our stepwise approach enables a safe implementation of a robotic surgical program for upper GI and HPB surgery with low morbidity and no mortality even for highly complex procedures. However, highly complex procedures required a high conversion rate, which might be caused by the early stage of experience.

\section{P448-ROBOTICS \& NEW TECHNIQUES-Pancreas}

ROBOTIC RESECTION OF CHOLEDOCHAL CYST WITH ROUX-EN-Y HEPATICOJEJUNOSTOMY IN ADULTS: CONSECUTIVE 22 CASES WITH COMPARISON OF LAPAROSCOPIC Method:

\section{J.H. Lee}

Surgery (HBP), Asan medical center, SEOUL, Korea

The standard surgical procedure of choledochal cyst is a complete excision of the cyst with Rouxen-Y hepaticojejunostomy and laparoscopic surgery had been increasingly used. This is still a challenging way to perform anastomosis due to the small diameter of bile duct and the possibility of bile leak or stricture. Robotic system can overcome the shortcomings of laparoscopy with providing three-dimensional view, magnification, and articulated instruments. From Jan 2014 to Dec 2017, 22 patients underwent robotic cyst excision and hepaticojejunostomy by single surgeon. We reviewed the clinical data and compared with laparoscopic outcomes of early (from 2009 to 2011) and late (from 2014 to 2017) group, retrospectively. Patients of robotic series were all female with mean age 35.3 years and BMI 23.7. The mean size of cyst was $3.2 \times 5.0 \mathrm{~cm}$, and Todani type Ia 10, Ic 6 and IVa 6 , respectively. Total 5 trocars were used with 3 robotic working arm and 1 assist and 1 camera. The mean operative time $248.5 \pm 52.9 \mathrm{~min}$, and it was similar with late laparoscopic group $(236 \pm 62.9 \mathrm{~min})$ and significantly shorter than early group $(395 \pm 85.9 \mathrm{~min})$.There were no open conversion in robotic and late laparoscopic group, however, the early laparoscopic group involved $35 \%$ of conversion rates. The hospital length was $7 \pm 3.8$ days in robotic group, and it was similar with late group $(7 \pm 3.5)$ and more shorter than early group $(9.3 \pm 6.8)$. In robotic series, postoperative complications occurred 3 patients. One case included cholangitis which was resolved after conservative treatment. Bile leakage was developed in 1 patient, and treated with drain that inserted intraoperatively. Last cases showed incisional hernia at postoperative 4 months, and was corrected by laparoscopic herniorrahphy. Complications $(n=7)$ in late laparoscopic group included hepaticojejunostomy stricture and stone, bleeding of jejunal branch, portal vein thromobosis, acute pancreatitis, and adhesive ileus. There were no mortaility case in any groups.Robotic surgery of choledochal cyst is a safe and feasible option with short-term results that are comparable to laparoscopic approach.

\section{P449-ROBOTICS \& NEW TECHNIQUES-Pancreas}

\section{ROBOTIC PANCREATIC ENUCLATION: EXPERIENCE} OF A SINGLE CENTRE

V. Ferri, E. Vicente, Y. Quijano, D. Hipolito, B. Ielpo, E. Diaz, I. Fabra, L. Malave, R. Isernia, E. Pinna, R. Caruso

General Surgery, Sanchinarro University Hospital, MADRID, Spain

Background: The incidental detection of benign to low-grade malignant small pancreatic neoplasms increased in the last decades. The surgical management of these patients is still under debate. The aim of this paper is to evaluate the safety and feasibility of robotic enucleations. Methods: We retrospectively reviewed our prospectively databases from November 2018 Demographics, pathological characteristics, perioperative outcome, and medium- term follow-up of patients who underwent robotic pancreatic enucleations were collected.

Results: 18 patients were included. The mean age of the patients was 61 years (48-74). The median body mass index was 26 (24-29). Ten lesions were located in the pancreatic head, 4 in the pancreatic body, 4 in the pancreatic tail. Operative time was 250 min (range 114-356), no intraoperative transfusion were needed and in one patient conversion to open approach was needed. In three patients grade B pancreatic fistula occurred. The mean postoperative stay was 8,4 days.

Conclusions: Robotic enucleation is a feasible and safe approach, with low incidence of morbidity.

\section{P450—ROBOTICS \& NEW TECHNIQUES-Solid organs}

\section{LONG-TERM Results: OF SURGICAL INTERVENTIONS FOR} PULMONARY TUBERCULOSIS

E.N. Maietnyi ${ }^{1}$, I.A. Kalabukha ${ }^{2}$, O.V. Khmel $^{1}$, V.E. Ivashchenko ${ }^{1}$, R.A. Veremeenko ${ }^{1}$, J.M. Voloshyn ${ }^{1}$

${ }^{1}$ Department of Thoracic Surgery, State organization „National Institute of Phthisiology and Pulmonology FG Yanovs, KYIV, Ukraine; ${ }^{2}$ Surgical, State organization „National Institute of Phthisiology and Pulmonology FG Yanovs, KYIV, Ukraine

The results of surgical treatment of patients with pulmonary tuberculosis were evaluated depending on the prevalence of the tuberculosis process and the type of surgical intervention used. According to the results of the questionnaire, 556 people operated on pulmonary tuberculosis in the period from 1 to 9 years ago, the frequency of cases of tuberculosis reactivation, the complicated course of the remote postoperative period, as well as the mortality and causes of lethal outcomes were assessed. It was found that after sublobular resection and lobectomy, treatment failure was noted at $3.2 \%$, relapse of tuberculosis- $2.2 \%$, pleural empyema- $-1.1 \%$, bronchial fistula $-0.8 \%$, cardiovascular insufficiency-in $1.3 \%$ operated. The mortality rate was $3.2 \%$ with a total clinical efficacy of $96.0 \%$. After combined resection and bylobectomy, treatment failure was noted at $8.9 \%$, relapse of tuberculosis- $15.0 \%$, pleural empyema- $12.0 \%$, bronchial fistulae $-6.3 \%$, cardiovascular failure $-5.0 \%$ operated. The mortality rate was $12.6 \%$ with a total clinical efficacy of $87.4 \%$. After pneumonectomy, treatment failure was noted at $5.6 \%$, relapse of tuberculosis- $5.6 \%$, pleural empyema $-3.8 \%$, bronchial fistulae-3.8\%, cardiovascular failure $-3.8 \%$ operated. The mortality rate was $10.0 \%$ with a total clinical efficacy of $90.0 \%$ 


\section{P451-ROBOTICS \& NEW TECHNIQUES-Solid organs}

ROBOTIC REDUCED-PORT SPLENECTOMY USING SINGLESITE PLATFORM

\section{J.H. Lee}

Surgery (HBP), Asan medical center, SEOUL, Korea

Background: In the era of minimal invasive surgery, single incision laparoscopic splenectomy can offer some advantages compared to conventional laparoscopic splenectomy. But it requires expertise in minimally invasive techniques due to technical difficulties. The Da Vinci Robotic reduced-port splenectomy using Single-site platform permits greater freedom of movement and higher levels of accuracy than previous laparoscopic surgery through two small incisions.

Methods: We performed a retrospective review of all patients who underwent Robotic reduced-port splenectomy using Single-site platform at our institution between January,2015 and November,2018. One $3 \mathrm{~cm}$ periumbilical incision was made for Glove port insertion and the other incision was made at left side of abdomen for additional $8 \mathrm{~mm}$ port insertion. The surgical technique is much same as open procedure. Short gastric artery was ligated, firstly. Splenic artery and vein were ligated individually. During the surgery, any stapling device was not used. Vessel sealer was used for hemostasis and mobilization of spleen. A specimen was removed through umbilical port site within Lap-bag.

Result: Eight patients ( 6 female and 2 male) with median age of 33.5 years underwent Robotic reducedport splenectomy using single-site platform (One case with combined robotic cholecystectomy for gall bladder stones without additional trocar). The indications were; hematological disease $(\mathrm{N}=3)$, splenic mass (benign $N=4$, malignant $N=1$ ). Preoperatively measured spleen size was ranged $5.5 \mathrm{~cm}$ to $16 \mathrm{~cm}$ $($ mean $11 \mathrm{~cm})$. There were no intraoperative complications and open conversion. Mean operative time was $132 \mathrm{~min}$. (range 74-206 min) including docking (mean $19 \mathrm{~min}$ ) and console time (mean $62 \mathrm{~min}$ ) Mean blood loss was under $10 \mathrm{ml}$. Mean hospital stay was 5.2 days after surgery. One patient underwent oral anticoagulation therapy only for portal vein thromobisis without any symptoms, and thromobisis was resolved at 1 month follow-up CT scan. There were no Clavien-dindo class III or above postoperative complication

Conclusions: Robotic reduced-port splenectomy using single-site platform seems to be feasible and effective. It seems to overcome certain limits of previous robotic or conventional single-site laparoscopic splenectomy and single-site only robotic splenectomy. We think $8 \mathrm{~mm}$ additional port allows to use Endo-wrist Da Vinci instruments such as Vessel sealer which enhances dissection efficiency andsafety of procedures.

\section{P452-ROBOTICS \& NEW TECHNIQUES-Technology}

\section{ROLE OF SMOKE EVACUATOR IN LAPAROSCOPIC} SURGERY

M. Hirota ${ }^{1}$, H. Takahashi ${ }^{2}$, Y. Miyazaki ${ }^{2}$, T. Takahashi ${ }^{2}$ Y. Kurokawa ${ }^{2}$, M. Yamasaki ${ }^{2}$, Y. Doki ${ }^{2}$, K. Nakajima ${ }^{3}$

${ }^{1}$ Surgery, Toyonaka Municipal Hospital, TOYONAKA CITY, OSAKA, Japan; ${ }^{2}$ Gastroenterological Surgery, Osaka University, OSAKA, Japan; ${ }^{3}$ Next Generation Endoscopic Intervention, Osaka University, OSAKA, Japan

Aims: Surgical plume has problem in poor visibility of the operative field, inclusion of harmful chemical substances, and biological risk. It is desirable that plume should be removed appropriately to minimize these risks. We assessed whether these problems can be solved by using commercialized evacuator.

Methods: Laparoscopic surgery was set up with porcine models and electrocautery (EC:VIO300D; ERBE) or ultrasonic scalpel (US. Sonicision, Covidien) was activated on the liver surface for a minute to generate surgical plume. In this model, we evaluated the following value with or without a smoke evacuator equipped with ULPA filter (RapidVac, Covidien): (1) Laparoscopic visualization: was evaluated by five surgeons (scored by 4 grades). (2) Semi-quantification of residual chemicals in the abdominal cavity: was performed using industrial smoke tester by aspirating the intra-abdominal plume onto filter papers and digitizing the stains. (3) Detection of DNA in the exhausted gas from the evacuator: the HEPA filter, which was interposed at the inlet or outlet of the evacuator, was analyzed using PCR method to detect any DNA derived from porcine tissues.

Results: (1) Laparoscopic visualization: judgement score were 2.2 vs. 1.0 for EC and 3.8 vs. 1.8 for US (evacuator: on vs. off, both $p<0.0001$ ), indicating the visualization was significantly better in the use of the evacuator on both devices. (2) Semi-quantification of residual chemicals: measured values are $17.3 \mathrm{vs} .67 .2$ for $\mathrm{EC}$ and 8.9 vs. 32.0 for US (evacuator: on vs. off, $\mathrm{p}=0.0002$ and $\mathrm{p}=0.0126$, respectively), indicating the chemicals were significantly less remained in the use of evacuator on both devices. (3) Detection of DNA in the exhaust: regardless the devices, porcine derived DNA was detected from all filters placed at the inlet of the evacuator (EC 3/3, US $3 / 3$ samples), and was not detected from any of the filter placed at the outlet (EC $0 / 3$, US $0 / 3$ samples), indicating that the exhaust from the evacuator is clean enough to be released into OR atmosphere. Conclusions: The evacuator improved visibility, reduced the amount of floating chemical substances, and successfully removed DNA so that the exhaust becomes safe and clean enough to be released in the air.

\section{P453-ROBOTICS \& NEW TECHNIQUES-Technology}

AUGMENTED REALITY TECHNOLOGY APPLIED TO SURGICAL PLANNING IN COLORECTAL SURGERY

J. Fernando Trebolle ${ }^{1}$, N. Pérez Serrano ${ }^{2}$, F.M. Sánchez Margallo ${ }^{3}$, A. Navarro Barles ${ }^{4}$, R. Ferrer Sotelo ${ }^{1}$, J. Escartín Valderrama ${ }^{1}$, J. García Egea ${ }^{1}$, P. Palacios Gasós ${ }^{1}$, B. Cros Montalbán ${ }^{1}$, C. Yánez Benítez ${ }^{1}$, M. Valero Sabater ${ }^{1}$, S. Borlán Ansón ${ }^{1}$, P. Menal Muñoz ${ }^{5}$, M.A. Sánchez Hurtado ${ }^{3}$, A. Blasco Satué ${ }^{5}$ A. Rodríguez Borobia ${ }^{5}$, J.L. García Calleja ${ }^{1}$, A. García García ${ }^{1}$, E. Gonzalvo González ${ }^{1}$, J. Usón Gargallo ${ }^{3}$, J.L. Blas Laína ${ }^{1}$

${ }^{1}$ General Surgery, Royo Villanova Hospital, ZARAGOZA, Spain; ${ }^{2}$ General Surgery, San Pedro Hospital, LOGROÑO, Spain;

${ }^{3}$ Minimally Invasive Surgery Centre, Jesús Usón Minimally Invasive Surgery Centre, CÁCERES, Spain; ${ }^{4}$ General Surgery, Ernest Lluch Hospital, CALATAYUD, Spain; ${ }^{5}$ Radiology, Royo Villanova Hospital, ZARAGOZA, Spain

Aims: To apply augmented reality technology from three-dimensional colon models as preoperative planning method in colorectal surgery.

Method: From three-dimensional anatomical models of the colon we have developed holograms of augmented reality. The models were obtained from CT images (Siemens Somatom Perspective 64®) with abdominal image cuts with $1 \mathrm{~mm}$ thick. The recovery of the images was in DICOM format and the processing to achieve the three-dimensional reconstruction was performed with the programs OsiriX® and Horos ${ }^{\circledR}$, which made a complete segmentation of the colon surface, and a modification of the image density. In this way models 3D were obtained of the isolated colon, and in relationship with the bone structure. The application Colon 3D AR was designed (increased hyper experience- visualizer with SLAM technology) creating a hologram of augmented reality to scale 1:1 from each three dimensional model to make projection of it on the abdomen of the patient by modifying the position in height of the reconstruction, using the bone pelvis as anatomic reference point to calibrate the placement of the hologram.

Results: In the preliminary phase (from October to December 2018) holograms of augmented reality were developed in 6 patients with colorectal cancer (right colon, left colon, transverse colon and rectum) to complement the radiological reconstruction with the virtual model. In the application phase (from January 2019) the holograms developed are going to be applied as a method to improve preoperative study. Conclusions: Three dimensional reconstruction of the tumor in the preoperative plan of colorrectal surgery combined with hyperreality technology allows to develop models of augmented reality in order to improve colon anatomy knowledge and to plan the surgical technique.

\section{P454-ROBOTICS \& NEW TECHNIQUES-Technology}

\section{SHORT TERM OUTOCOMES OF ROBOTIC GASTECTOMY FOR GASTRIC CANCER OF OUR INSTITUTE}

S. Yagi, K. Sato, M. Ohbatake, A. Sakamoto, H. Kawasaki

Digestive Surgery, Ehime Prefectural Central Hospital, MATSUYAMA, Japan

In several reports, robotic gastrectomy for gastric cancer with da Vinchi Suregical system have advantages due to $3 \mathrm{D}$ vision system, wristed instruments and removing tremor system. So this system can reduce complication like as post operative pancreatic fistura. In our institute,we started robotic gastrectomy for gastric cancer on 22 August 2017. We performed this operation using Harmonic Scarpel for lymphadenectomy at infrapyloric lesion,and without this at suprapancreatic lesion. We have performed 15 cases of robotic gastrectomy for gastric cancer. We have no intraoperative complication,but one case of deep vein thrombosis as postoperative complication. In our cases,the mean bleeding amount was $1 \mathrm{ml}$, and mean operative time was $307 \mathrm{~min}$. Mean after operative hospital stay was 10 days.In our institute, robotic gastrectomy for gastric cancer was safely and feasibly performed. 


\section{P455-ROBOTICS \& NEW TECHNIQUES-Technology}

MINIMALLY INVASIVE INGUINAL LYMPH NODE DISSECTION VERSUS OPEN APPROACH-COMPARISON OF SURGICAL OUTCOMES

\section{R. Canotilho, M. Peyroteo, C. Ribeiro, J. Abreu de Sousa}

Surgical Oncology, Instituto Português de Oncologia do Porto, PORTO, Portugal

Aims: Inguinal lymph node dissection carries an important risk of post-operative complications, mainly related with wound complications and long term lymphedema. The minimally invasive approach aims to reduce the morbidity of this procedure, avoiding the traditional groin incision but still allowing a full access to the lymph node basin. The authors aimed to describe their videoassisted inguinal lymph node dissection (VILND) cases, comparing the surgical outcomes with a sample of open inguinal lymph node dissection (OILND) cases.

Methods: We performed a retrospective descriptive study that compared the data from patient submitted to VILND since 2017 (the year in which this technique was first performed in our institution) with the patients submitted to OILND in 2015 and 2016. Gynaecologic and urologic malignancies were excluded. The statistical analysis was performed using SPSSv25 $\odot$, with a $\mathrm{p}$ value $<0.05$ indicating statistical significance.

Results: A total of 62 cases of inguinal lymph node dissection were analysed, $33.9 \%$ of which VILND (none of them requiring conversion to the open approach). Melanoma was the primary tumour in $87 \%$ of patients. The VILND and OILND groups had no statistically significant difference between them regarding age, body mass index, smoking status or the reason for lymph node dissection—clinically detected lymph node vs. positive sentinel node biopsy. The mean of isolated lymph nodes in the VILND (7.71) and OILND (9.63) groups was also not statistically different $(p=0.109)$. There was no difference in the rate of post-operative seroma, wound dehiscence or lymphedema. The rate of surgical site infections was higher in the OILND group$34 \%$ vs. $9.5 \%$ during post-operative hospital admission $(\mathrm{p}=0.045) ; 29.3 \%$ vs. $4.8 \%$ after discharge $(\mathrm{p}=0.036)$

Conclusions: In our population of patients we conclude that the main advantage of the videoassisted approach regarding surgical morbidity lies in the reduction of the infection rate, as the published literature also confirms. The equivalent number of lymph nodes retrieved in both groups points toward the oncological safety of the minimally invasive procedure, that we hope to study further in the future after a longer follow up period.

\section{P456-ROBOTICS \& NEW TECHNIQUES-Technology}

FEASIBILITY STUDY FOR TUMOR LOCALIZATION TECHNIQUE USING RADIO-FREQUENCY IDENTIFICATION CLIP MARKER: RESULT OF IN-VIVO TEST IN PORCINE MODEL

C.I. Choi ${ }^{1}$, C.R. Park ${ }^{2}$, H.Y. Joo ${ }^{2}$, D.H. Kim ${ }^{1}$, T.Y. Jeon ${ }^{1}$, S.Y. Ahn ${ }^{2}$, D.H. Kim

${ }^{1}$ Surgery, Pusan National University Hospital, PUSAN, Korea;

${ }^{2}$ Mechanical engineering, Pusan National University, PUSAN, Korea

Objectives: To evaluate the clinical feasibility of tumor localization technique with radio-frequency identification (RFID) clip marker

Methods: We developed the proto-type RFID integrated endoscopic clip (RFID-clip) and probe to detect it on serosa surface during the laparoscopic surgery. A pig weighing $40 \mathrm{~kg}$ was used as the specimen for the in-vivo test. Endoscopist performed the application of the RFID-clip on porcine gastric mucosa. After then, the surgeon tried to find the location of RFID-clip using the detection probe and marked with the electrocautery. After the gastrectomy with $3 \mathrm{~cm}$ margin (each to proximal and distal), we confirmed the prediction of RFID-clip location and accuracy of resection.

Results: RFID-clip location was detected and recorded on the exact site of clip application. Detection range was very short and we confirmed there are almost no differences between actual clip location and our prediction. This result might arise from using the low-frequency RFID tag to increase the accuracy through reduction of the range. However, some RFID-clip were not detected because of the issue of clipping trouble, not RFID tag.

Conclusions: This is a basic study to evaluate the clinical usefulness and feasibility of the new localizing technique. We confirmed the possibilities of this system and it could be the helpful option to provide the information of exact location for the minimally invasive surgery or early gastrointestinal tumors.

\section{P457-SOLID ORGANS-Adrenal}

BENEFITS OF ROBOTIC POSTERIOR RETROPERITONEAL ADRENALECTOMY OVER LAPAROSCOPIC POSTERIOR RETROPERITONEAL ADRENALECTOMY: A SINGLE CENTER EXPERIENCE

T.Y. Sung, W.W. Kim, Endocrine Surgery, Asan Medical Center, University of Ulsan College of Medicine,Seoul, SEOUL, Korea

Background: The advantages of laparoscopic posterior retroperitoneal adrenalectomy (LPRA) have been described in the literature. The aim of this study was to compare the clinical outcomes of LPRA and robotic posterior retroperitoneal adrenalectomy (RPRA) and determine the differences that could affect the outcomes.

Methods: We retrospectively analyzed 253 adrenalectomy cases at Asan Medical Center from 2014 to 2017. There were 190 LPRA and 63 RPRA cases, and their clinicopathological features and surgical outcomes were compared.

Results: In LPRA, there was a positive relationship between operation time and male gender early period of experience, adrenal tumor size, and pheochromocytoma. In RPRA, adrenal tumor size and pheochromocytoma were the only factors affecting the operation time. When the adrenal tumor size was $=5.5 \mathrm{~cm}$, the operation time of LPRA was shorter than that of RPRA $(p=0.001)$ When the tumor size was $>5.5 \mathrm{~cm}$, there was no significant difference in the operation time of LPRA and RPRA $(p=0.102)$

Conclusions: RPRA is a feasible and technically safe approach for benign adrenal diseases. The use of RPRA could benefit patients with large tumors and provide comfort by overcoming the factors contributing to a longer operation time in the laparoscopic technique.

\section{P458-SOLID ORGANS-Adrenal}

\section{LAPAROSCOPIC REDO LEFT ADRENALECTOMY FOR A 15CM PHEOCHROMOCYTOMA USING LATERAL POSITION AND THROUGH THREE TROCAR TECHNIQUE}

\section{Hussein}

Surgery, American University of Beirut Medical Center, BEIRUT, Lebanon

Aims: Laparoscopic Redo Left Adrenalectomy for a $15 \mathrm{~cm}$ pheochromocytoma using lateral position and through three trocar technique.

Methods: Twenty years experience at the American University of Beirut Medical Center for Laparoscopic Adrenalectomy. A total of 65 cases were done laparoscopically with no conversion and minimal complication. The average operative time is 40 mins. The video will show the various steps used for Lap redo (Lt) adrenalectomy for a $15 \mathrm{~cm}$ pheochromocytoma using the lateral position and through 3 trocars. Attempt to remove the pheochromocytoma in Iraque was complicated by cardiac arrest treated successfully and patient referred to the American University of Beirut Medical Center.

Results: Patient had smooth postoperative course following laparoscopic adrenalectomy and patient discharged 3 days later with no complications.

Conclusions: Even large adrenal masses can be completed laparoscopically in advanced experienced centers in laparoscopy. 


\section{P459-SOLID ORGANS-Adrenal}

LAPAROSOCPIC RIGHT ADRENALECTOMY USING LATERAL POSITON AND THROUGH TROCAR TECHNIQUE

\section{Hussein}

Surgery, American University of Beirut Medical Center, BEIRUT, Lebanon

Aims: Laparoscopic Right Adrenalectomy using Lateral position and through Trocar Technique. Methods: I report my experience at the American University of Beirut Medical Center for Laparoscopic Adrenalectomy 65 cases, 35 left adrenalectomy and 30 cases for right adrenalectomy. Three out of the series are large adrenal of $15 \mathrm{~cm}$, and all of these were completed laparoscopically.The video will show the steps of this procedure.A large Rt. adrenal mass measuring $15 \mathrm{~cm}$, Wt.750gm was removed laparoscopically using 4 trocar techniques. The lateral position facilitated the exposure and ease of dissection. The mass was removed by extending one of the trocar site with muscle splitting using Endocatch $15 \mathrm{~mm}$.

Results: Patient was discharged home 3 days after surgery. The operative time was 1 hour. Pathology revealed carcinoma with no involvement of the capsule or vascular invasion.

Conclusion: Therefore, large adrenal mass (CA) can be treated laparoscopically without violation of oncological principle.

\section{P460-SOLID ORGANS-Adrenal}

COMPARISON OF LAPAROSCOPIC TRANSPERITONEAL AND RETROPERITONEOSCOPIC ADRENALECTOMIES- A SINGLE INSTITUTION STUDY OF 20 YEARS

\section{A. Ottlakan, A. Paszt, Zs. Simonka, Sz. Abraham, B. Borda, M. Vas,} Gy. Lazar

Department of Surgery, University of Szeged, SZEGED, Hungary

Aims: Among the most popular methods of minimally invasive approaches, it is still unclea whether laparoscopic transperitoneal (TP), or retroperitoneoscopic (RP) adrenalectomy maintains the most advantage

Methods: Between January 1998 and April 2018, 163 patients (male: $\mathrm{n}=39$; female: $\mathrm{n}=124$ ) underwent minimally invasive adrenalectomy (TP: $n=135 ;$ RP: $n=28$ ) at our Institute. Mean patient age was 53.8 years (21-82 years). Besides comparing operative (intraoperative blood loss, previous abdominal surgeries, conversion rate, operative time, tumor size) and perioperative factors (time of hospitalization, time to oral intake, histology, postoperative complications) in each group, perioperative outcomes of a learning curve (lc) - the first 28 procedures in both groups- was also analyzed.

Results: Mean operative time (TP: $78.51+12.38 \mathrm{~min}$ vs RP: $134.5 \pm 12.4 \mathrm{~min} ; \mathrm{p}=0.019$ ) an rate of previous abdominal surgeries [TP: $61(45.18 \%)$ vs RP: $4(14 \%) ; p=0.038$ ] showed significant difference, favoring the TP approach. In terms of tumor size, significantly larger lesions were removed with TP (TP: $48.18 \pm 22.8 \mathrm{~mm}$ vs RP: $34.8 \pm 11.2 \mathrm{~mm}$; $=0.028$ ). The number of ASA (American Society of Anesthesiologists) II patients were significantly higher in the TP group [ASA II- TP: $51(37.77 \%)$ vs RP: $6(23 \%)$; $=0.048$ ], while there were significantly more ASA III patients in the RP group [TP: $57(42.22 \%)$ vs RP: $17(58 \%) ; \mathrm{p}=0.029)$ ] Conversions (TP: $6(4.44 \%)$ vs RP: $5(18 \%) ; \mathrm{p}=0.257)$, intraoperative blood loss (TP. $65.7 \pm 8.45 \mathrm{ml}$ vs. RP: $50.2 \pm 10.78 \mathrm{ml} ; \mathrm{p}=0.147$ ), mean time of hospitalization (TP $4.25 \pm 1.58$ days vs RP $4.61 \pm 2.24$ days; $p=0.237$ ) showed no significant difference. The analysis of lc showed a significant difference in previous abdominal surgeries [TP: $10(35.7 \%)$ vs RP: $4(14 \%) ; \mathrm{p}=0.015]$, conversion rate [TP: 0 vs RP: $5(18 \%) ; \mathrm{p}=0.011]$ and mean operative time [TP: $110 \pm 8.1 \mathrm{~min}$ vs RP: $134.5 \pm 12.4 \mathrm{~min} ; \mathrm{p}=0.023$ ] all favoring the TP approach.

Conclusion: Both methods proved to be feasible and safe in terms of minimally invasive adrenalectomy. Based on our own experience the TP approach resulted in improved operative time and conversion rates, with the successful removal of large, even malignant lesions.

\section{P461-SOLID ORGANS-Adrenal}

LAPAROSCOPIC ADRENAL GLAND SURGERY: CARCINOMA OF UNCERTAIN MALIGNANCY, AN ENTITY TO DISCOVER

M. Fornell Ariza ${ }^{1}$, A. Bengoechea Trujillo ${ }^{1}$, C. Bazan Hinojo ${ }^{2}$, A. Mayo Ossorio , S. Roldan Ortiz ${ }^{2}$, J.M. Pacheco Garcia ${ }^{1}$

${ }^{1}$ Unidad de Cirugia Esofagogastrica y Bariatrica, Hospital Universitario Puerta del Mar, CADIZ, Spain; ${ }^{2}$ Unidad de Cirugia Colorectal, Hospital Universitario Puerta del Mar, CADIZ, Spain

Aims: The adrenocortical of uncertain malignancy neoplasm is a spectrum of classification for adrenal tumors whose histopathological diagnosis is uncertain.

Clinical case: we present a 65 year old patient with constitutional syndrome and severe hypercortisolism and hypokalemia reason why she was admitted to ICU for episodes of ventricular fibrillation. No other medical history of interest except refractory hypertension to treatment. The TC showed a left adrenal mass of $6.5 \times 4.5 \times 5 \mathrm{~cm}$ with microcalcifications, areas of necrosis and hemorrhage, no infiltrating, without disease to distance. The surgery was a laparoscopic left adrenalectomy with no evidence of infiltration and no lymph nodes. The histopathology lesion presented a dense proliferation cellular of cortical type, with incomplete fibrous, without vascular or capsular invasion, with a $30 \%$ ki67; positivity vimentin and CD56. All epithelial markers, were negative. All this leads to the diagnosis of a neoplasm of uncertain malignancy potential adrenocortical. During the postoperative period, the patient presents a crisis of adrenal insufficiency that was treated with intravenous replenishment corticoidea and later orally with good clinical response. Discussion: The adrenal carcinoma has a low incidence $(0.1 \%)$, incidence peak around the 50 years, the most frequent is the mixed secretory. They are $2-5 \%$ of the adrenal incidentalomas. It is usually presented to the diagnosis as a locally advanced tumor with metastases (to liver, lung, retroperitoneal ganglia and bone). May present clinically due to hormonal hyperproduction; or be non-functioning tumors. The adrenal carcinoma poses a great difficulty at the time of the diagnosis pathological, and includes as differential diagnosis to other abdominal tumors. The distinction between corticoadrenal adenoma and adrenal carcinoma is sometimes difficult, so it has been defined a spectrum of intermediate category called Adrenocortical neoplasm of intermediate or uncertain malignancy. It is obtained with the Weiss criteria, being necessary at least 3 of them for confirm the diagnosis of adrenal carcinoma. This category has a low risk of local recurrence or metastasis, but it needs a narrow follow-up.

Conclusion: Adrenal carcinoma of uncertain malignancy implies a new category in those tumors of difficult classification.

\section{P462-SOLID ORGANS-Adrenal}

\section{BILATERAL LAPAROSCOPIC SYNCHRONOUS ADRENALECTOMY IN MEN TYPE2}

M.A. Mayo-Ossorio ${ }^{1}$, M. Fornell-Ariza ${ }^{2}$, D. Perez-Gomar ${ }^{2}$, A. Bengoechea-Trujillo ${ }^{2}$, S. Cerrato-Delgado ${ }^{2}$, A. Fierro-Aguilar ${ }^{2}$, J. Varela-Recio ${ }^{2}$, C. Peña- Barturen ${ }^{2}$, J.M. Pacheco-Garcia ${ }^{2}$

${ }^{1}$ Cirugía General Y Del Aparato Digestivo, Hospital Universitario Puerta Del Mar Cadiz, CADIZ, Spain; ${ }^{2}$ General Surgery, Hospital Universitario Puerta Del Mar Cadiz, CADIZ, Spain

Aims: Multiple endocrine neoplasia type 2 (MEN2) is an autosomal dominant disorder with an estimated prevalence of 1 per 30,000 in the general population. Among patients suspected to have a pheochromocytoma, the diagnosis is rarely confirmed and only $10 \%$ is presented bilaterally. We present bilateral laparoscopic adrenalectomy in patients with MEN2.

Method: A 77-year-old woman with a family history of medullary thyroid cancer and breast cancer. Personal history: hypertension, medullary thyroid cancer, breast cancer, laparoscopic cholecystectomy. appendectomy. After a study by endocrinology and suspicion of bilateral pheochromocytoma, discussing the case in a multidisciplinary committee, bilateral adrenalectomy was decided by laparoscopic approach. Selective alpha-1-adrenergic blocking agent (doxazosin) were utilized before surgery. Under general anesthesia left adrenalectomy was performed first in right lateral decubitus position. $15 \mathrm{mmHg}$ Pneumoperitoneum was started with the Verres needle and 3 trocars $(11 \mathrm{~mm}$ umbilical, $5 \mathrm{~mm}$ subxifoid and $12 \mathrm{~mm}$ left subcostal). Once dissection was completed the gland was placed in a plastic bag and extracted through one of the trocars incisions, then the position of the patient was changed to left lateral decubitus for the right adrenal approach. Another right subcostal $5 \mathrm{~mm}$ trocar was used. Adhesiolysis of previous cholecystectomy was performed to right adrenal approach. Adrenal veins were divided between metallic clips.No drainage was employed.

Results: The procedures were successfully performed without conversion. Surgical time was 150 min and hospital stay was 2 days. Had a clinical reversion with control of blood pressure monitored by endocrinology

Conclusions: Currently, the laparoscopic approach is the technique of choice for the management of adrenal pathology.Lateral decubitus transperitoneal approach is the procedure of choice in most cases. Bilateral laparoscopic synchronous adrenalectomy is feasible and safe with good results as in our patient. 


\section{P463-SOLID ORGANS-Adrenal}

\section{LAPAROSCOPIC TRANSPERITONEAL ADRENALECTOMY FOR LEFT ADRENAL ADENOMA}

V. Drakopoulos, V. Lygizos, S. Voulgaris, I. Iliadis, P. Trakosari, A. Bakalis, V. Vougas

1st Department of Surgery and Transplantation Unit, District General Hospital of Athens « Evangelismos », ATHENS, Greece

Aims: Laparoscopic adrenalectomy has become the standard of care for most adrenal masses. We report a case of laparoscopic adrenalectomy for left adrenal adenoma.

Methods: We present the case of a 72-year-old Caucasian female patient with an asymptomatic, left-sided adenoma, that was incidentally detected during abdominal ultrasound. No headaches, palpitations, tachycardia, tremor, dizziness or vomiting were reported. Pre-operative blood tests confirmed that the tumor was a non-secreting one and a CT-scan revealed a $2.9 \times 2.2 \mathrm{~cm}$ left adrenal mass. Laparoscopic surgical excision was proposed. The patient was placed in semilateral right-sided decubitus position. Four trocars ( 1 epigastric $-10 \mathrm{~mm} \& 3$ subcostals $-10 \mathrm{~mm}$ \& $25 \mathrm{~mm}$ ) were used, without the use of a liver retractor. The adrenal vessels were clipped not only with the standard laparoscopic clips, but also with the Hem-o-Lok ligation system.

Results: The operation lasted for $2^{1 / 2} \mathrm{~h}$ with minimal blood loss. The patient's post-operative course was uneventful and she was finally discharged four days post-operatively. Histology report ensured that it was adenoma of the adrenal cortex.

\section{P464-SOLID ORGANS-Adrenal}

\section{STANDARDIZED LEFT LAPAROSCOPIC ADRENALECTOMY, LESS IS MORE!}

J. Hernandez Gutierrez, A.F. Aranzana Gomez, J.S. Malo Corral, B. Muñoz Jimenez, G. Krasniqi, R. Lopez Pardo, P. Toral Guinea

\section{General Surgery, C.H.Toledo, TOLEDO, Spain}

Aims: Since the first laparoscopic adrenalectomy in 1992 (Gagner), the laparoscopic lateral transabdominal approach has proved to be the one of choice. It provides an easy anatomical orientation, overall the technique is similar to other traditional laparoscopic procedures. On the other hand, the posterior retroperitoneoscopic adrenalectomy (PRA), described in 1995 (Waltz), has proven to be a safe technique and effective for the surgical management of several adrenal pathologies. The advantages include direct access to the adrenal gland, without the need for visceral mobilization or lysis of adhesions from previous abdominal operations and the ability to perform a bilateral adrenalectomy without repositioning the patient. Currently there is controversy about which is the approach of choice, having to take into account the learning curve necessary for the retroperitoneal approach and the reduced number of patients with adrenal pathology subsidiary of surgical management.

The objetive is to demonstrate the safety and efficacy of the standardized laparoscopic approach of the left adrenal gland with 3 trocars for selected cases.

Methods: Clinical case: 43-year-old man, resistant hypertension despite concurrent use of three antihypertensive agents, with biochemical and radiological diagnosis of left adrenal adenoma with primary hyperaldosteronism. Demonstrative video of the technical steps in a standardized way that we propose for laparoscopic left adrenalectomy only using 3 trocars.

Results: Full laparoscopic surgical approach in right lateral decubitus position: 3 trocars-lateral transabdominal approach. Steps: 1. Laparoscopic liberation of the splenic flexure of the colon for the colo-spleen-pancreato-gastric en block mobilization until identification of the left pillar, 2. dissection of the medial border of the gland, identification of left renal and diaphragmatic vein, as well as the adrenal vein which is dissected and clipped, 3. dissection of the lateral edge of the adrenal gland, 4. lower pole dissection of the gland completing the resection with Ligasure ${ }^{\circ}$. The patient presented a successful postoperative recovery, being discharged $24 \mathrm{~h}$ after the intervention. Asymptomatic, the patient does not need antihypertensive drugs at 1 year follow-up. Conclusion(s): The standardization of the procedure allows reducing the number of trocars, maintaining the safety and effectiveness of the minimally invasive approach.

\section{P465-SOLID ORGANS-Adrenal}

POSTERIOR RETROPERITONEAL ENDOSCOPIC APPROACH FACILITATES FUNCTIONAL CONSERVATION OF THE ADRENAL CORTEX

E. Delgado-Oliver ${ }^{1}$, R. Diaz del Gobbo ${ }^{2}$, M. Manyalic-Blasi ${ }^{3}$,

F. Hanzu ${ }^{4}$, M. Squarcia ${ }^{5}$, D. Martínez ${ }^{6}$, D. Fuster ${ }^{7}$,

C. Fondevila ${ }^{3}$, O. Vidal ${ }^{3}$

${ }^{1}$ General Surgery, Hospital Universitario Miguel Servet,

ZARAGOZA, Spain; ${ }^{2}$ General Surgery, Hospital Mutua De Terrassa, BARCELONA, Spain; ${ }^{3}$ General Surgery, Hospital Clinic De Barcelona, BARCELONA, Spain; ${ }^{4}$ Endocrinology and Nutrition, Hospital Clinic De Barcelona, BARCELONA, Spain;

${ }^{5}$ Radiodiagnosis, Hospital Clinic De Barcelona, BARCELONA, Spain; ${ }^{6}$ Pathological anatomy, Hospital Clinic De Barcelona, BARCELONA, Spain; ${ }^{7}$ Nuclear medicine, Hospital Clinic De Barcelona, BARCELONA, Spain

Aims: Cortical-sparing adrenalectomy is a suitable treatment for hereditary and sporadic bilatera pheochromocytoma, in cases of low risk of malignancy, to reduce the possibility of adrenal insufficiency assuming the chance of local recurrence. The aim of the study is to analyze the functional results of partial adrenalectomy by retroperitoneal endoscopic approach in singleadrenal patients or patients requiring bilateral adrenalectomy.

Methods: Prospective study between January 2015 and October 2017 including pheochromocytoma patients diagnosed with low risk of malignant mutations. All patients agreed to be included in the study. Experienced endocrine surgeons who have been trained in minimally invasive endocrine surgery performed the procedure using the same surgical technique. Demographic variables and clinical characteristics were collected, subsequently carrying out the descriptive analysis of the data. Results: A total of eight patients were registered, five associated with MEN type 2 syndrome and three in the context of VHL syndrome. Retroperitoneoscopic resection was performed without laparoscopic or open conversion and no postoperative complications; the average hospital stay was 2.2 days. Preservation of the functional cortex without corticosteroids was achieved in 7 $(87.5 \%)$ of out 8 cases with a follow-up of $37.5 \pm 4$ months. Today, these seven patients have a preserved adrenal function without hormone replacement.

Conclusions: cortical-sparing adrenalectomy by the retroperitoneal endoscopic approach, in exper hands, is safe and feasible for the treatment of hereditary and sporadic pheochromocytoma in a contex of low malignancy, making it possible to avoid the need for corticoid replacement in most cases.

\section{P466-SOLID ORGANS-Adrenal}

\section{MINIMALLY INVASIVE PARTIAL ADRENALECTOMY \\ IN THE TREATMENT OF HEREDITARY PHEOCHROMOCYTOMA: SYSTEMATIC REVIEW}

\section{E. Yiannakopoulou}

\section{Biomedical Sciences, University of West Attica, ATHENS, Greece}

Partial adrenalectomy has been suggested for patients benign adrenal tumors especially in the case of hereditary syndromes, like multiple endocrine neoplasia type 2, Von Hippel-Lindau disease and neurofibromatosis type I.

Aims: This systematic review aimed to investigate the role of partial adrenalectomy in the treatment of hereditary pheochromocytoma.

Methods: Electronic databases were searched with the search terms 'MEN II', 'Von Hippel Lindau', 'neurofibromatosis', 'laparoscopic partial adrenalectomy', 'robotic assisted partial adrenalectomy' for the time period up to and including December 2018. Full publications, including clinical trials randomized or not, retrospective studies, case series, case reports that provided relevant data met inclusion criteria

Results: Thirty five possibly relevant studies were identified. Abstracts were reviewed and fourteen articles were excluded as they were review articles or articles presenting data on open partia adrenalectomy. Twenty one studies, that met inclusion criteria were retrieved in full text and included in the systematic review. Eight studies presented data on partial adrenalectomy in patients with von Hippel Lindau including two case series with median follow up ranging from 5 to 7.2 years and six case reports. Thirteen studies presented data on partial adrenalectomy in patients with MEN II including two case series and eleven case reports. Recurrence rate was estimated at about $10 \%$ for pheochromocytoma. Overall steroid dependence rate was estimated at $90 \%$.

Conclusion: Minimally invasive partial adrenalectomy is a therapeutic option especially in patients with heritable pheochromocytoma, given that tumors are often bilateral, tumors are commonly benign and severe morbidity and mortality may be associated with life-long steroid replacement therapy such as the possibly lethal Addisonian crisis . However, data are limited, follow up is not standardized and not appropriately reported and RCTs are difficult to be done due to the rarity of the disease. A multinational registry on the short term and long term outcomes of partial adrenalectomy in hereditary pheochromocytoma would be a significant source of knowledge. 


\section{P467-SOLID ORGANS-Adrenal}

FOCUSED MINIMALLY INVASIVE PARATHYROIDECTOMY IN THE TREATMENT OF PATIENTS WITH FAMILIAL AND HEREDITARY HYPERPARATHYROIDISM: SYSTEMATIC REVIEW

\section{E. Yiannakopoulou}

\section{Biomedical Sciences, University of West Attica, ATHENS, Greece}

Traditionally the treatment of hyperparathyroidism for patients with familial hyperparathyroidism was subtotal parathyroidectomy or total parathyroidectomy and auto transplantation. In the era of minimally invasive parathyroidectomy, the removal of only abnormal glands guided by preoperative localizing studies has been suggested.

Aims: This systematic review aimed to investigate the role of focused minimally invasive parathyroidectomy in the treatment of patients with familial hyperparathyroidism.

Methods: Electronic databases were searched with the search terms 'MEN I', 'familial hyperparathyroidism', 'MEN2A','hyperparathyroidism-jaw tumor syndrome', 'parathyroidectomy', 'minimally invasive ', for the time period up to and including December 2018. Full publications, including clinical trials randomized or not, retrospective studies, case series, case reports that provided relevant data met inclusion criteria.

Results: Thirty five possibly relevant studies were identified. Abstracts were reviewed and fifteen articles were excluded. Twenty studies, that met inclusion criteria were retrieved in full text and included in the systematic review, including three retrospective cohort studies i.e. two presenting data on MENI associated hyperparathyroidism and the third study on familial hyperparathyroidism and seventeen small case series or case reports. The two retrospective studies on MENI hyperparathyroidism included 125 patients treated either with focused minimally invasive parathyroidectomy or with the conventional approach. These studies presented conflicting data with one supporting and the other negating the focused minimally invasive parathyroidectomy due to the failure of localization studies to identify enlarged parathyroid glands in a great number of patients.

Conclusion: Undoubtedly, the idea of minimally invasive parathyroidectomy in patients with hereditary and familial hyperparathyroidism is interesting. This idea is especially challenging in the case of MENI. Existing data suggest that focused mimimally invasive parathyroidectomy is feasible under the condition of exact preoperative localization studies. The main advantage of this approach is the minimization of the risk of postoperative hypoparathyroidism. However, data are limited and further research is needed before valid conclusions can be drawn on the suitability of this approach.

\section{P468-SOLID ORGANS-Adrenal}

\section{SURGERY FOR PHEOCHROMOCYTOMA: A SINGLE INSTITUTION 20- YEAR EXPERIENCE}

\section{Parianos, C. Aggeli, G. Ntokos, K. Pateas, G. Zografos}

3rd Department of Surgery, General hospital of Athens, ATHENS, Greece

Objective: Resection of pheochromocytomas is a challenging procedure due to hemodynamic lability, tumor vascularity and malignant potential.Given the technical challenges for resection of large pheochromocytomas, there were hesitations about using the laparoscopic approach for these tumors during the first decade of laparoscopic surgery. However, improvement in imaging modalities,better pharmacological preparation,advances in anaesthesia and laparoscopic surgery rendered laparoscopic surgery for pheochromocytomas safe and efficient. Our aim was to evaluate surgical outcomes in 86 patients with pheochromocytoma and to validate the role of laparoscopic surgery in the treatment of these tumors.

Design: A total of 85 procedures for pheochromocytoma were performed between January 1998-September 2018. The preoperative diagnosis, operative details, complications, length of hospital stay, morbidity and follow up were retrieved from the hospital records of 668 patients who underwent 686 adrenalectomies for benign and malignant adrenal tumors in the same period. Preoperative localization was established in all patients with computerized tomography (CT) or magnetic resonance imaging (MRI), while iodine -123-metaiodobenzyguanidine(MIBG) scan was reserved for ambiguous cases where paraganglioma or metastatic disease was suspected. Endocrinological evaluation and complete adrenal dynamic testing were performed to determine whether the tumor was functional or not.

Results: Eighty-seven tumors were removed from 85 patients. One patient with MENIIA underwent bilateral resection of pheochromocytomas in two stages. Tumor size in laparoscopic procedures ranged from $1.2 \mathrm{~cm}$ to $11.0 \mathrm{~cm}$ (mean $5.87 \mathrm{~cm}$ ). Forty-three patients had benign disease, 41 potentially malignant (based on PASS), 1 malignant with metastasis. Eight were in the context of a familial syndrome. Sixty -eight patients underwent laparoscopic adrenalectomy, 8 patients had open approach from the start for recurrent pheochromocytoma or large benign tumor, 1 patient had open approach due to inoperable malignant pheochromocytoma and 10 patients had conversions from laparoscopic to open procedure. Nine patients received sodium nitroprusside intraoperatively to treat hypertension. One patient developed pulmonary embolism, and succumbed 1 month later. There were no recurrences for the benign tumors during the follow-up period.

Conclusions: Laparoscopic resection of pheochromocytomas despite its increased level of difficulty compared to that of other adrenal tumors, is a safe and effective procedure.

\section{P469-SOLID ORGANS-Adrenal}

\section{LAPAROSCOPIC ADRENALECTOMY. HOW MUCH DOES THE SIZE MATTER?}

V. Pino Diaz ${ }^{1}$, M. Rubio-Manzanares Dorado ${ }^{1}$, V. Camacho Marente $^{1}$, A. Senent Boza ${ }^{1}$, F.J. Padillo Ruiz ${ }^{1}$, .J.M. Martos Martinez ${ }^{2}$

${ }^{1}$ Cirugia General, Hospital Universitario Virgen Del Rocio, SEVILLA, Spain; ${ }^{2}$ Cirugia Endocrina, Hospital Universitario Virgen Del Rocio, SEVILLA, Spain

Aim: The concept 'large' in transperitoneal lateral laparosopic adrenalectomy (TLLA) has been evolving along time, ranging from 5 to $8-10 \mathrm{~cm}$ depending on different authors. On the other hand, some authors discourage laparoscopic surgery in larger tumors due to the increased risk of malignancy in those larger than $5-6 \mathrm{~cm}$, referring to malignancy in 1 out of 3 or 4 cases. The aim of this study is to evaluate the results of TLLA in larger tumors.

Methods: In a prospectively collected database of TLLA operated at our center from 2007 to 2017 , records of patients with tumors $=51 \mathrm{~mm}$ were included for analysis. Only patients with evident primary adrenal malignant tumors at CT and/or MRI (invasion or lymph node or distan metastasis) had been excluded for TLLA.

Results: Fourty-four patients among $189(23.28 \%)$ presented at pathology study a tumor greater than $51 \mathrm{~mm} .21$ were men and 23 women. Mean age was $48.91 \pm 17.84$ y.o. Mean BMI was $27.61 \pm 5.71$ ASA risk scale was I 6.8\%, II 17\%, III 52.3\% and IV 2.3\%. 50\% were non-functioning tumors. Mean size was $68.39 \pm 22.25 \mathrm{~mm}(51-150 \mathrm{~mm})$, being 29 of them $(65.9 \%)$ larger than $61 \mathrm{~mm}$ and 9 $(20.45 \%)$ larger than $80 \mathrm{~mm}$. Four patients $(9.1 \%)$ required conversion to open surgery. Conversions were not related to size ( 83 vs $67 \mathrm{~mm}$, not significant), laterality ( $\mathrm{p}=0.667$ ) or other non-tumor depending factors (sex, age, BMI or ASA risk). Mean operative time was $104.55 \pm 29.62 \mathrm{~min}$ and did not correlate with tumor size or differ significantly in converted cases. Mean postoperative stay was 3.81 days. Excluding a patient who presented in the postoperative period a jejunal perforation due to ischemia not related to surgery, mean stay was 2.80 days. Regarding to malignancy, 2 patient presented previously known adrenal metastases and only one of the 42 remaining patients ( $2.38 \%$ ) presented a primary adrenal carcinoma, currently asymptomatic after a follow-up of 26 months. Conclusions: In our experience, when an adequate preoperative assessment is performed, malignancy risk is very low. Patients with adrenal tumors should not be excluded for laparoscopic approach based only in its larger size.

\section{P470-SOLID ORGANS-Adrenal}

\section{LAPAROSCOPIC RESECTION OF FUNCTIONAL INTER- AORTOCAVA PARAGANGLIOMA}

\section{Jiménez Díaz, A. Acosta Mérida, M. Callejón Cara, J. Silvestre Rodríguez, J. Marchena Gómez}

General And Digestive Surgery, Hospital Universitario De Gran Canaria Doctor Negrín, LAS PALMAS DE GRAN CANARIA, Spain

Paragangliomas are rare tumors originated in extra-adrenal chromaffin cells, with an incidence of 2-8 cases per million inhabitants. They can appear in any location between neck and pelvis. Sympathetic paragangliomas are usually functional and catecholamines producers.

We present a movie of surgical intervention of a 22 -year-old patient who, in study for refractory hypertension, presented paraganglioma producing norepinephrine, whose approach was performed laparoscopically. 22-year-old woman studied by Nephrology for refractory hypertension. On physical examination, only obesity standed out. In blood exams, levels of normetanephrine were observed in plasma of $1950 \mathrm{pg} / \mathrm{ml}$ and aldosterone $832 \mathrm{pg} / \mathrm{ml}$. Abdominal scintigraphy was performed in which there was no evidence of increased activity at adrenal level. Abdominal CT shows retroperitoneal extra-adrenal tumor of inter-aortocava location immediately below renal vessels with dimensions of $3.6 \times 2.1 \times 6 \mathrm{~cm}$. After preparation, she was operated. Laparoscopic access was performed under exhaustive monitoring. An heterogeneous, polylobulated tumor of $6 \mathrm{~cm}$, located interaortocava, intimately adhered to left renal vascular pedicle, was observed. A Cattell-Braash and Kocher maneuver was performed, with exposure of inferior cava and aorta to iliac bifurcation. Complete tumor excision was performed after clipping arterial and venous tributary branches. After the operation, the pacient presented favorable evolution being discharge on the second postoperative day with good control of blood pressure levels. Laparoscopic approach of retroperitoneal paragangliomas is a safe technique, which allows minimally invasive access, with consequent improvement in postoperative results. The exact location of lesions and their relationships with surrounding structures, as well as their functional behavior, are very important when considering the best therapeutic strategy for these patients. 


\section{P471-SOLID ORGANS-Adrenal}

\section{A CLOSE ENCOUNTER: LEFT BASAL PNEUMONIA AND PANCREATIC FISTULA AFTER LAPAROSCOPIC LEFT ADRENALECTOMY}

\author{
O. Enciu, E.A. Toma, A. Miron
}

Surgery, Elias University Emergency Hospital, BUCHAREST, Romania

We present the case of a 50-year-old obese male patient referred for adrenalectomy after being diagnosed with left adrenal incidentaloma. Abdominal MRI showed a 4.7/4.1/3.9 left adrenal mass with normal hormonal levels.

After preoperative workup, the patient underwent standard laparoscopic adrenalectomy. The lateral to medial dissection and mobilization of the spleen and pancreatic tail was difficult due to the abundance of peritoneal and pararenal fat. The anatomy was peculiar: the bulky pancreatic tail was located well inferior to the splenic hilum and was visible throughout the intervention and the spleen was quite elongated-long axis $=15 \mathrm{~cm}$. The exposure of the adrenal gland was therefore cumbersome. The operating time was $128 \mathrm{~min}$ and blood loss $170 \mathrm{ml}$. The abdominal drainage was maintained for $48 \mathrm{~h}$. Before discharge the patient underwent a control abdominal US examination that only showed a thin line of left pleural fluid.

The patient was readmitted 6 days after discharge for chest pain, fever $\left(38.9^{\circ} \mathrm{C}\right)$ and malaise with no abdominal signs. The emergency CT scan diagnosed left basal pneumonia with minimal pleural effusion and a 7/1 cm fluid collection between the spleen and diaphragm while the blood test showed leukocytosis. The patient was treated for pneumonia with an apparent clinical benefit for three days and lowered white cell count but his condition worsened during the forth day. Repeat abdominal US demonstrated that the abdominal collection increased in size therefore the patient underwent emergency surgery. During laparoscopic exploration, the collection was unveiled as being pancreatic juice (more than 7 times the normal serum levels of lipase and amylase). After thorough lavage, two drainage tubes were positioned in the left subphrenic space. The postoperative course was uneventful under antibiotic treatment for pneumonia and pancreatic antisecretory medication. The patient was discharged after 7 days with minimal pancreatic drainage and the drainage tube was extracted after 5 more days.

\section{P472-SOLID ORGANS-Gynaecology}

\section{TREATMENT OF UTERUS LEUOMYOMA AND ADENOMYOSIS USING ENDOSCOPIC Method: S DEPENDING ON REPRODUCTIVE PLANS}

\section{V.P. Mishchenko}

Department of Obstetrics and Gynecology ?1., Odessa medical University, ODESSA, Ukraine

The aim of the study was to develop the algorithm and the choice of the method of endoscopic treatment of a combined pathology of uterine leiomyoma and adenomyosis depending on the reproductive plans.

Methods: The study involved 60 patients with a combined pathology of uterine leiomyoma and adenomyosis. Indications for conservative myomectomy were: the size of the uterus is more than 13 weeks. pregnancy; multiple leuomatous nodes and adenomyotic foci up to $5 \mathrm{~cm}$ in size; hemorrhagic and pain syndromes, anemia, compression of the adjacent organs; suspected node malfunction; submucous leiomyoma deforming the uterine cavity with foci of adenomyosis; subserous, cervical isthus nodes and foci of adenomyosis; the presence of endometrial hyperplasia, tumors of uterine appendages; growth rate of uterine leiomyoma more than 4 weeks pregnancy for the year; the growth of uterine leiomyoma on the background of drug treatment; infertility associated with leiomyoma and uterine adenomyosis. The laparoscopic myomectomy of the subserous node on the 'leg' with a size of more than $6 \mathrm{~cm}$ and nodes of more than $8 \mathrm{~cm}$ of intramural location is shown with an interest in preserving the organ.The hysterectomy is indicated for women after 43 years of age who insist on hysterectomy, with a combination of uterine leiomyoma with atypical endometrial hyperplasia.

Results: The conservative myomectomy and removal of adenomyotic foci were performed in 24 $(40.5 \%)$ patients: from hysteroscopic access - 4, vaginal access-3, laparoscopic access-13, abdominal access -5 in the presence of reproductive plans. The hysteroscopic myomectomy was performed in $8(21.1 \%)$ patients, hysterectomy in $30(78.9 \%)$ patients: from laparoscopic access -23 , from vaginal access -3 , from abdominal access -4 in the absence of reproductive plans.

Conclusions: The choice of surgical treatment of uterine leiomyoma and adenomyosis depends on the reproductive plans of the woman and the severity of the lesion.The laparoscopic method of treating a combined pathology of uterine leiomyoma and adenomyosis in the presence and absence of reproductive plans is a priority for women.

\section{P473-SOLID ORGANS-Gynaecology \\ PERSPECTIVE OF SURGICAL TREATMENT IN PATIENTS WITH MUTATION IN BRCA1 AND BRCA2 GENES}

\section{T. Fontana}

Surgery, Policlinico „Paolo Giaccone,,, PALERMO, Italy

Background: Breast cancer in females represents the most frequent neoplasm in all age groups The risk of getting breast cancer (MC) increases with age. The BRCA1 and the BRCA2 genes (tumor-suppressor genes, autosomal dominant transmission at high penetrance) alone justify from $30 \%$ to $70 \%$ of cases of hereditary breast cancer.

Methods: From 1 January 2011 to 1 June 2017 we have analyzed 18 patients with BRCA mutation. All 18 patients had in common a genetic mutation of BRCA1 or BRCA2 tumor suppressor genes.

Results: The frequency of germline mutation on BRCA1 (9 patients: 50\%) was identical to BRCA2 gene ( 9 patients: $50 \%$ ). 13 of the analyzed patients were women $(72.2 \%$ of patients) 9 BRCA1 and 4 BRCA2, and 5 men (27.8\%) all with BRCA2 mutation.

Conclusions: Prophylactic surgery must be seen as a way to put the patient in the condition to implement the most appropriate treatment. Further studies will be necessary to support the validity of prophylactic surgery in patients with mutations in BRCA1 and BRCA2 genes.

\section{P474-SOLID ORGANS-Gynaecology}

TOTAL LAPAROSCOPIC HYSTERECTOMY WITH BILATERAL SALPINGO-OOPHORECTOMY

V. Drakopoulos, V. Lygizos, S. Voulgaris, I. Iliadis, K. Botsakis, K. Sarafi, N. Morfis, E. Daskalaki, V. Vougas

1st Department of Surgery and Transplantation Unit, District General Hospital of Athens « Evangelismos », ATHENS, Greece

Introduction: Laparoscopic hysterectomy is a safe surgical technique for removing the uterus with or without including the ovaries and fallopian tubes. Laparoscopic surgery of endometrial cancer is a safe method, with the mean time of recovery being two days only.

Material-Method: The case of a $55 \mathrm{yr}$ old woman with metrorrhagia and anaemia ( $\mathrm{Ht} 24,5 \%)$ due to adenocarcinoma of the endometrius is presented. The patient underwent a laparoscopic hysterectomy and oophorectomy. 4 trocar ports were used during the procedure (a $10 \mathrm{~mm}$ transumbilical port, similar to the port used in single incision laparoscopic operations, two $5 \mathrm{~mm}$ ports at the level of the anterior superior iliac spines, and a $10 \mathrm{~mm}$ port in the middle of the imaginary line between the pubic symphisis and the umbilicus). The uterine vessels and the uterine ligaments were ligated and dissected by using a thermal energy source. The patient's postoperavite course was uneventful. The patient continues to be in good condition, 6 months post-surgery.

Conclusion: Laparoscopic hysterectomy seems to be a safe method for addressing endometrial cancer, as it offers the surgeon a better surgical field, is tissue friendly and causes fewer postoperative complications. It is considered to be a less traumatic operative method, as due to zooming in the picture there is greater accuracy in handling the tissue, and blood loss is minimal. 


\section{P475-SOLID ORGANS-Gynaecology}

\section{LAPAROSCOPIC VERSUS OPEN HYSTERECTOMIES IN OBESE PATIENTS}

\section{Shahin}

General surgery, Menoufia university hospital, SHIBIN ELKOM, Egypt

Background: Hysterectomy is one of the most frequently performed surgical procedure. Though there are three approaches in hysterectomy (open, vaginal and laparoscopic), still there are controversies regarding the optimal route for performing it.

Methods: This prospective comparative study included 42 obese patients subjected for panhysterectomy as a treatment. The forty-two patients were allocated into two groups: group (A) subjected to laparoscopic pan-hysterectomy, group (B) subjected to open pan-hysterectomy. Results: There was significant difference between the two groups regarding mean operative time, blood loss, analgesic requirements and hospital stay, while no significant difference regarding intra-operative complications.

Conclusions: Laparoscopic hysterectomy in obese patients has emerged as a viable, safe and better alternative to open hysterectomy amongst appropriately trained surgeons.

\section{P477-SOLID ORGANS-Gynaecology}

INDOCYANINE GREEN MEDIATED SENTINEL LYMPH NODE BIOPSY FOR ENDOMETRAL CANCER-PRELIMINARY EXPERIENCE

\author{
G. Filip, I. Balescu, S. Filip, C. Copaescu
}

Surgery, Ponderas Academic Hospital, BUCHAREST, Romania

Introduction: Sentinel node biopsy is the newest accepted method for surgical staging of early stage endometrial and cervical cancer.

Aim: To evaluate the role of the technique of indocyanine green (ICG) identification of the sentinel lymph nodes in cases of early endometrial cancer.

Material and method: Five patients with early endometrial and cervical cancer were introduced in a prospective study. ICG was locally injected during the laparoscopic exploration. Novadac Pinpoint near to red technology was used. Guided biopsies were performed into the marked sentinel nodes and histological results were evaluated.

Results: Sentinel lymph nodes were easily identified by using ICG and near-infrared technology. Technical details are described. No associated complication was encountered.

Conclusion: SLN mapping using ICG in uterine cancers is demonstrated as an effective and safe procedure.

\section{P476-SOLID ORGANS-Gynaecology}

\section{ENDOMETRIOSIS OF THE UTERUS ROUND LIGAMENT MIMICKING INGUINAL HERNIA}

G. Ayiomamitis, E. Koutroumanos, C. Kontopoulou, E. Bourbouteli, M. Apostolidi, N. Paschlidis ,

1st Surgical Department, Tzaneio General Hopsital, PIRAEUS, Greece

General: Endometriosis in the inguinal region is rare. The usual presentation is that of a woman in the reproductive age group. It accounts for $0.3-0.6 \%$ of patients affected by endometriosis. The groin swelling is usually slow growing, painful with exacerbations during menses. The incidence of inguinal endometriosis on the right side is $90-94 \%$ as compared to the left.

Aim: To present our laparoscopic approach for the treatment of the diagnostic dilemma.

Case presentation: A 40-year-old woman presented with a palpable mass in the right groin. The swelling was associated with a dull aching pain. The patient was suffering from increasing pain over the swelling during menstruation. She had undergone cesarean section some years ago and the scar had healed by primary intention. MRI scan revealed a nodular hypoechoic lesion at the level of the internal inguinal ring with the absence of vascular flow around the lesion.

Results: Since inguinal endometriosis was in the differential diagnosis and it may be associated with pelvic or intraperitoneal endometriosis, a laparoscopic approach was decided. The procedure was successfully completed laparoscopically following the transabdominal preperitoneal approach. The endometriosis was found, after dissecting the internal inguinal ring, firmly adhered to the round ligament. It was excised en bloc with the round ligament. A preperitoneal polypropylene mesh was inserted to protect for future inguinal hernias due to extensive dissection at the level of the internal inguinal ring. No intraperitoneal endometriosis was appreciated. Histopathology revealed endometriosis of the round ligament. The patient was uneventfully discharged the next day. On follow up the patient was asymptomatic.

Conclusions: Round ligament endometriosis is a rare entity. It is a disease of specific interest to the physician. It can be confused with an inguinal hernia and thereby pose a diagnostic dilemma. We recommend considering endometriosis in the differential diagnosis of groin swellings in women. The transabdominal preperitoneal approach is feasible and safe in the hands of an advanced laparoscopic surgeon.

\section{P478-SOLID ORGANS-Kidney \\ LAPARASCOPIC EXTRACTION OF AN INTRAPERITONEAL GOSSYPIBOMA FOLLOWING C/S AND A RETROPERITONEAL GOSSYPIBOMA FOLLOWING PYELOPLASTY}

\section{N. Ozlem}

General surgery department, Ahievran University, KIRSEHIR, Turkey

Gossypibomas are forgatten foreign bodies,iatrogenic.their symptoms are different where they are. they extracted with laparotomy in the past but now we can some article mentioned their extraction was made with laparoscopy

Case 1: 33 Y O female has abdominal pain after c/s for 2.5 years. A gossypiboma was extracted with laparoscopy above umblicus.A superficial surgical site infection existed,drained,subsided. Case 2: $46 \mathrm{YO}$ M had a pyleoplasty operation 8 years ago.a gossypiboma was extracted with retroperitonescopy,no postoperative event. Basibuyuk et al reported retroperionescopic extraction of a gossypiboma from single port in first time.Althoug every effort taken the incidence of foreign body detected in the body is about $0.03-0.1 \%$. They are most frequently localized in the intraabdominal cavity followed by tracheobronchial area,pleural cavity,pararenal area, vagina,spinal chord, neck, femur,breast,bladder,pancreas,and they may cause local irritation,and infection.Tactile sense is absent in laparoscopy. All radiologic examinations(usg ct pet mri etc) be used to detect.we used usg ct.in the end laparoscopy make the diagnosis and remove gossypibomas in our cases with less postoperative pain and cosmosis. Justo et al the computerized tomography (CT) scan is the most useful method for diagnosis; however, sometimes the preoperative diagnosis remains uncertain even after the imaging exam. In that case, laparoscopy arises as a valuable diagnostic tool, as well as a prompt treatment option. Concerning gossypiboma, prevention is preferred rather than treatment. Notwithstanding, there is no highly reliable prevention system. Counting sponges is a method based on staff communication during the surgery with only $77 \%$ sensibility. Routine surgical postoperative X-ray (SPOX) constitutes an early detection system, but the need to incorporate a radiopaque marker and to expose the whole surgical field to maximize its efficacy limits its use. More recently, electronic dispositives based on barcode detection and other technological adjuncts for counting sponges are being developed. None of these prevention systems are reliable when used alone. Our education and research clinic was a state hospital before. No surgeon followed above instruction.but now we use all. Multiple procedures and surgical teams, long operations and non-elective operations are the evidenced risk factors.c/s operation was learned full opened of ostium of cervix of the patient. 


\section{P479-SOLID ORGANS-Kidney}

\section{THE RELATIONSHIP BETWEEN ROBOTIC PARTIAL NEPHRECTOMY OUTCOMES AND DAP AND RENAL NEPHRECTOMY SCORE}

Y. Yamauchi, A. Asano, S. Ishiguro, K. Hattori, R. Ishida, Y. Yamada, N. Nishikimi, K. Kobayashi

\section{Urology, Japan, NAGOYA, Japan}

Aims: Some scoring systems have been suggested to standardize the renal tumor characteristics. Among them, RENAL score is widely used in partial nephrectomy. Whereas diameter-axis-polar (DAP) score was developed to be more significantly related with postoperative renal function. Our study compared DAP score with RENAL score in robotic partial nephrectomy (RPN) outcomes.

Methods: Records of patients who underwent RPN at Nagoya Daini Red Cross Hospital between April 2016 to October 2018 were analyzed retrospectively. Those include three oncocytomas. Accordingly, we calculated the estimated glomerular filtration rate (eGFR) just before RPN and 1 month postoperatively in 51 patients. We compared two nephrometry scores with warm ischemic time and change in eGFR.

Results: In our institution, four surgeons performed RPN. According to DAP score, 14 patients were high, 19 were middle and 18 were low. According to RENAL score, 1 were high, 26 were middle and 24 were low. The median warm ischemic time was $20 \mathrm{~min}$ (11-35). The median eGFR decreased from $67.9(23.2-127.3)$ to $57.8(10.7-116.9) \mathrm{ml} / \mathrm{min} / 1.73 \mathrm{~m}^{3}$. There were no significant differences in warm ischemic time and percentage change in eGFR between RENAL score groups $(P=0.38$ and 0.87$)$ but significant differences between DAP score groups $(P<0.05$ and $\mathrm{P}<0.05$ ). Univariate and multivariate analyses were used to identify factors influencing postoperative renal function. That confirmed that DAP score was independent poor predictors of change in eGFR after RPN.

Conclusions: DAP score is simpler estimate system than RENAL score. Our study suggested that DAP score is a useful scoring system for preoperative evaluation of renal tumor for RPN. Further investigation is needed to better understand preoperative DAP score.

\section{P481—SOLID ORGANS-Parathyroid}

\section{NEW APPROACH IN SINGLE INCISION ENDOSCOPIC PARATHYROIDECTOMY USING LIFTING METHOD BY ORIGINAL RETRACTOR VIA CHEST}

\section{K. Kayano, K. Takao, J. Kohmoto, Y. Yaginuma, I. Suzuka,} M. Kojo

Surgery, Ako central hospital, AKO, Japan

We started endoscopic thyroidectomy using the lifting method in 2001 and have developed single incision endoscopic thyroidectomy (SIET) via chest (C-) or axillary incision (A-) by our original retractor since 2007. We created a new approach in 2010. Recently, we have applied this method to parathyroid surgery. In this study, we present our method and results in parathyroid surgery with regard to surgical outcome and patients' complaints.

Method: Endoscopic parathyroidectomy of C-SIET was performed in 6 patients with hyperparathyroidism (primary 4, secondary 2 ) in new approach (mean age 69, Male 1 Female 5). Single parathyroid adenoma was diagnosed using ultrasonic device, preoperatively. The patient is placed in a supine position with the neck extended. $30 \mathrm{~mm}$ vertical incision is made in anterior chest. Flexible endoscope (Olympus Co. Japan) is used through $5 \mathrm{~mm}$ trocar detached the retractor. In new approach, the parathyroid and thyroid are exposed through the avascular space between sternal head and clavicular head of sternocleidomastoid muscle. Both of the skin and sternal head are lifted up by our original retractor (Takasago Medical Co. Japan). Parathyroid adenoma behind the thyroid is resected using an ultrasonic scalpel. I would like to present our C-SIET procedure. Results: No scars in the neck were left in all cases. Benign and hemi lateral parathyroid adenoma sized from $8 \mathrm{~mm}$ to $25 \mathrm{~mm}$ (mean: $16.5 \mathrm{~mm}$ ) were operated. Mean operation time is $123 \mathrm{~min}$. in new approach. There was no complication. Parathyroid hormone levels decreased in all patients immediately after operation.

Conclusion: It is a little possible to make recurrent nerve palsy in this approach. New approach is useful to operate and make the working space wider without stress to find out of parathyroid adenoma. Our original retractor can be introduced easily in most hospital, because it is not so expensive. Most of women satisfied cosmetic results because of hidden scars.

\section{P480-SOLID ORGANS-Kidney}

\section{MINIMALLY INVASIVE APPROACH FOR THE SURGICAL TREATMENT OF A RETROPERITONEAL TUMOR}

J. Hernandez Gutierrez, A.F. Aranzana Gomez, B. Muñoz Jimenez, J.S. Malo Corral, P. Toral Guinea, G. Krasniqi, R. Lopez Pardo

General Surgery, C.H.Toledo, TOLEDO, Spain

Aims: Retroperitoneal primary tumors comprise a great variety of neoplasm with different histological typologies, with insidious clinical symptoms and little specificity in most cases. It diagnosis is established through imaging tests and anatomopathological study is needed so complete surgical resection is the treatment of choice.

The aim of the video is to demonstrate the safety and efficacy of the minimally invasive approach in patients with retroperitoneal lesions.

Methods: A 66-year-old female patient who, in the course of an abdominal pain at the right iliac fossa suspected of possible acute appendicitis, is diagnosed with a right retroperitoneal tumor, compatible with primary neurogenic tumor on a CT. Radiographic imaging is a key component of the evaluation of a patient with a retroperitoneal mass, a CT scan is necessary to evaluate the primary site as well as to rule out metastatic disease. After complete biochemical study, nonfunctioning tumor is determined. The study is completed with MRI where the lesion is located below the right kidney, in front of the right psoas muscle and lateral to the inferior vena cava, and without contact with these structures. ??It is in intimate contact with the ovarian vein. The complementary tests and iconography of interest of the case are exposed. Surgical intervention is proposed with a laparoscopic approach.

Results: Full minimally invasive approach in left lateral decubitus position: 4 trocars-lateral laparoscopic transabdominal approach. Laparoscopic liberation of the right colon, Kocher maneuver until the inferior vena cava is visualized, identification of a tumor of approximately $5 \mathrm{~cm}$ in the right infrarenal region, lateral to the right ureter, which includes the gonadal vessels. Resection of the tumor in block with margins previous dissection and clipping of the proximal and distal gonadal vessels with Ligasure ${ }^{\circledR}$. The patient presented a successful postoperative recovery, being discharged $24 \mathrm{~h}$ after the intervention. Definitive result of the specimen: Leiomyosarcoma, grade 2 of the FNCLCC with negative margin.

Conclusion(s): The laparoscopic approach is a safe and effective technique in the approximation of retroperitoneal tumors, a radical oncological criterion is always needed with correct margins of resection especially in those of uncertain etiology.

\section{P482-SOLID ORGANS-Parathyroid}

RADIOFREQUENCY ABLATION OF PARATHYROID
ADENOMA IN THE CONTEXT OF SECONDARY
HYPERPARATHYROIDISM TO CHRONIC RENAL DISEASE

\section{HYPERPARATHYROIDISM TO CHRONIC RENAL DISEASE}

I.M. Cornejo Jurado, B. López Durán, M. Díaz Rodríguez, C. Sacristán Pérez, F. Oliva Mompeán, C. Marín Velarde

General Surgery, Hospital Universitario Virgen Macarena, SEVILLE, Spain

Objectives: Radiofrequency ablation (RFA) is a novel and developing technique for the treat ment of parathyroid hyperplasia/adenoma in the context of secondary hyperparathyroidism (HPT) to chronic kidney disease (CKD) and there is little literature on the subject. The purpose of this study is to determine its usefulness by contributing a case carried out in our hospital. Methods: We selected a case of secondary HTP in a patient of 62 years old with CKD who presented a parathyroid adenoma detected clearly by ultrasound scanning. The patient was dismissed for surgery due to high surgical risk due to his comorbidities. RFA of a right inferior parathiroid adenoma was performed. Intact parathyroid hormone (iPTH) was measured before $\mathrm{ARF}$ and $10 \mathrm{~min}$ after de procedure, calcium and phosphorus were measured the day after. The treatment was considered effective if iPTH levels decreased at least 50\% 10 min after RFA and calcium levels decreased the day after.

Results: iPTH level before RFA was $1985 \mathrm{pg} / \mathrm{ml}$. iPTH level after $10 \mathrm{~min}$ of RFA was $835 \mathrm{pg} /$ $\mathrm{ml}$, this meant a $58 \%$ reduction (normal values $15-65 \mathrm{pg} / \mathrm{ml}$ ). Calcium levels were from 10.2 at the baseline to 8.6 the day after (normal values $8.5-10.5 \mathrm{mg} / \mathrm{dl}$ ) and phosphorus from 4.5 to $5.4 \mathrm{mg} / \mathrm{dl}$ (normal values $2.5-4.5 \mathrm{mg} / \mathrm{dl}$ ). The patient presented dysphonia as a complication that improved with corticosteroid therapy. We are currently waiting for the next analytical controls at 3, 6 and 12 months after the proceidure.

Conclusions: RFA of parathiroid adenomas for treating secondary HPT in patients with CKD is feasible in selected patients. This treatment may reduce the morbidity that surgery supposes, it is developed in an outpatient regime avoiding hospital admission and this contributes to a reduction of health costs. However, a longer follow-up is necessary to verify the good results in our case 


\section{P483-SOLID ORGANS-Spleen}

\section{LAPAROSCOPIC SPLENECTOMY IN CIRRHOTIC PATIENTS AMELIORATES LIVER FIBROSIS AND STIMULATE PROLIFERATION OF PROGENITOR-LIKE CELLS}

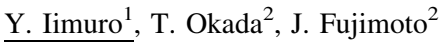 \\ ${ }^{1}$ Surgery, Yamanashi Central Hospital, KOFU, Japan; ${ }^{2}$ Surgery, \\ Hyogo College of Medicine, NISHINOMIYA, Japan
}

Splenectomy is one of the treatment strategy for advanced portal hypertension due to liver cirrhosis. After splenectomy, thrombocytopenia is dramatically ameliorated, and liver function parameters have also been improved in several clinical settings. However, the mechanism underlying such a phenomenon remains unclear.

The aims of the present study was to analyze histological changes of the liver after splenectomy in human, and to speculate the underlying mechanism.

Subjects and methods: Cirrhotic patients with hepatocellular carcinoma (HCC) who had undergone laparoscopic splenectomy prior (4 weeks-52 months) to hepatic resection were analyzed $(\mathrm{n}=15)$. Non-tumorous liver specimens obtained at hepatectomy were histologically investigated. Liver tissues from cirrhotic HCC patients who underwent only hepatectomy were used as controls $(\mathrm{n}=15)$.

Results: After splenectomy, significant leukocytosis, especially increase in monocytes, was observed in addition to thrombocytosis. In the non-cancerous liver tissues, many round-shaped CD68-positive macrophages accumulated after splenectomy, while this phenomenon was merely observed in patients without splenectomy. The macrophages were $\mathrm{CD}_{163}{ }^{+}\left(\mathrm{M} 2\right.$ marker) and $\mathrm{CD} 14^{-} \mathrm{CD} 16^{+}$, suggesting their anti-fibrotic population. The accumulated macrophages existed around fibrous scar as well as CK $19^{+} \mathrm{EpCAM}^{+}$cells spreading out from the ductular reactions (DR). As a result, the number of Ki67-positive hepatocytes significantly increased after splenectomy. The amount of platelets detected in the liver did not change even after splenectomy. Finally, remarked attenuation of the established liver fibrosis was detected after relatively long duration. The accumulated macrophages expressed metalloproteinase (MMP)-1 and fibroblast growth factor (FGF)-7, suggesting these molecules may possibly participate in resolution of established fibrosis and hepatocyte proliferation. Conclusion: Splenectomy in cirrhotic patients with portal hypertension ameliorate liver fibrosis, and stimulate liver regeneration. The mechanism possibly include hepatic accumulation of anti-fibrotic CD163-positive macrophages and stimulation of DR-derived $\mathrm{CK} 19^{+} \mathrm{EpCAM}^{+}$progenitor-like cells. In patients with advanced splenic fibrosis, splenectomy could be a feasible therapeutic modality.

\section{P484-SOLID ORGANS-Spleen}

\section{PURPOSE AND ROLE OF LAPAROSCOPY IN ABDOMINAL CONTUSIONS}

D. Cartu ${ }^{1}$, A.M. Goganau ${ }^{1}$, S.I. Bordu ${ }^{1}$, D. Marinescu ${ }^{1}$, S. Mucenic ${ }^{2}$, A. Nicolaescu ${ }^{2}$, D. Belivaca ${ }^{2}$, E. Georgescu ${ }^{1}$, M.V. Surlin ${ }^{1}$

${ }^{1}$ General Surgery, UMF Craiova / Clinical Emergency Hospital of Craiova / General Surgery 1, CRAIOVA, Romania; ${ }^{2}$ General Surgery, Clinical Emergency Hospital of Craiova / General Surgery 1, CRAIOVA, Romania

The paper tries to establish the role and the opportunity of using laparoscopy in regard with abdominal contusions, as well as its indications or contraindications, combined in a therapeutic algorithm. We analyzed two groups of patients with abdominal contusions divided over two 5-year periods, 2008-2012 (51 patients) and 2013-2017 (60 patients) respectively. We have separated the two periods because starting from 2013 we have established a strategy for dealing with cases of abdominal contusions where we included diagnostic and / or therapeutic laparoscopy and nonoperative management. The investigation was done by FAST echography, CT scan, simple abdominal radiography, peritoneal lavage puncture, and sometimes arteriography. In the second period we determined the diagnostic and therapeutic laparoscopy indications: suspicion of hollow or parenchymal organ injury, or mesentery injury, the presence of hemoperitoneum or fluid in the peritoneal cavity in a stable patient without major hemorrhage, apparent with unique injuries, without immediate vital risk and without other associated severe trauma. We have associated in this last period the nonoperative management for patients with grade 1 and 2 lesions of parenchymal organs that do not have fluid in the peritoneum, or only a very discreet quantity. In the first period, all 51 patients were treated by classic surgery, resulting in 4 unnecessary laparotomies where no visceral lesions were revealed. In the second period, we applied non-operative management to 8 patients out of 60,2 patients with grade 1 and 2 splenic injuries, and 6 patients with grade 1 and 2 hepatic lesions. Diagnostic laparoscopy was performed in 5 cases, in 2 of them without evidence of lesions, and in 3 other cases of grade 1 lesions no therapeutic action was required. Therapeutic laparoscopy was required for one case of splenectomy and one of hepatorrhaphy. Diagnostic laparoscopy is useful in abdominal contusions, if certain indications are followed and in selected patients. In our study, with the introduction of modern therapeutic strategies, unnecessary laparotomies were completely avoided, some lesions being even treated by laparoscopy. The new algorithm introduced allowed $23 \%$ of patients to avoid laparotomy.

\section{P485-SOLID ORGANS-Spleen \\ SPLENIC HAMARTOMA, A RARE CAUSE OF SPLENOMEGALY}

J. Hernandez Gutierrez, A.F. Aranzana Gomez, J.S. Malo Corral, B. Muñoz Jimenez, G. Krasniqi, R. Lopez Pardo, P. Toral Guinea

\section{General Surgery, C.H.Toledo, TOLEDO, Spain}

Aims: About 150 cases of splenic hamartoma have been described in the literature since it was first described by Rokitansky in 1861, it is a rare benign tumor. It is usually a casual finding in laparotomies or autopsies. They are usually asymptomatic, but there are few symptomatic splenic hamartomas and they can be associated with haematological alterations, being in some cases associated with spontaneous splenic rupture and acute abdomen, two thirds of them have multiple tumors.

There are no specific data that allow the preoperative diagnosis of this entity, which is performed after the anatomopathological study of the surgical specimen, which must be extracted entirely, this together with the size of the spleen makes the laparoscopic approach difficult.

The aim of this video is to demonstrate the surgical technique of a complete laparoscopic approach for this type of lesions, without the need for assistance laparotomies (handport).

Methods: Clinical case: A 44-year-old man admitted to Internal Medicine due to fever and left lumbar pain. Additional explorations of interest are discussed, including: Thrombopenia of probable peripheral origin secondary hypersplenism (FNA of bone marrow), CT: Splenomegaly with 4 splenic masses, which deform the splenic contour, compatible with atypical hemangiomas, without being able to discard other vascular splenic tumors.

Results: Complete semi-laparoscopic approach, 4 trocars, Multilobulated splenomegaly $(19 \times 16 \mathrm{~cm}$.), mechanical vascular section, complete bag extraction after minilaparotomy on the left flank. The patient presented a successful postoperative recovery, being discharged on the 4th PO day. Abdominal ultrasound at 1st week with portal vein thrombosis, which resolves after treatment with heparin. Definitive result of the specimen: multiple splenic hamartoma. Asymptomatic one year after surgery.

Conclusion(s): The laparoscopic approach is a valid and effective alternative to splenic benign tumor lesions. The size does not contraindicate this type of approach, although the complete extraction of the spleen is recommended for its pathologic study. We recommend eco-Dopple control per week, given the risk of portal thrombosis with an existing laparoscopic post-splenectomy.

\section{P486-SOLID ORGANS-Spleen \\ GIANT CONGENITAL SPLENIC CYST AND TREATED BY DEROOFING TECHNIQUE}

A. Vílchez Rabelo ${ }^{1}$, L. Vázquez Medina ${ }^{1}$, N. Pineda Navarro ${ }^{1}$, $\vec{A}$. Vílchez Casares ${ }^{2}$

${ }^{1}$ Cirugía General y del Aparato Digestivo, Servicio Andaluz de Salud, LINARES JAÉN, Spain; ${ }^{2}$ Traductor, Servicio Andaluz de Salud, LINARES JAÉN, Spain

Objectives: Splenic cysts are a rare entity, currently described between $800-1000$ cases in literature. A female patient's case is hereby presented, giant splenic cyst treated by conservative laparoscopic surgery obtaining good results.

Method: 30 years old female, without any relevant medical history, examined after abdominal pain on the left hypochondriac region, nausea, postprandial swelling and mass sensation. After exploration the presence of such mass was ratified, the rest of exploration found no relevant findings, no record of previous traumatism nor any other relevant incidence. Diagnosis was made through ultrasound and computerized tomography, the existence of a big splenic cyst is confirmed, $19 \mathrm{~cm}$ by $14 \mathrm{~cm}$, on the superior section of the spleen, negative results after parasitism test, normal haemogram, coagulation and biochemistry levels. Patient was intervened using laparoscopic surgery, performing the deroofing technique on the cyst (two liters of orangey amber serous liquid that was sent for analysis) as well as extirpation of superior wall of the cyst, which was sent to Pathological Anatomy, a saline solution was used to cleanse the cavity, omentum and drainage were then set in place.

Results: Patient evolved satisfactorily, hospital discharge and drainage withdrawal after $48 \mathrm{~h}$. Regular check-ups, after 12 and 24 months, patient presents no symptoms nor recurrence. Pathological Anatomy confirmed primary splenic cyst and the extracted liquid as cystic.

Conclusion: Splenic cysts are primary $(25 \%)$ or secondary $(75 \%)$. Diagnosis is performed through imagery tests, CAT scan the being standard test used. Regarding her treatment there is no clear consensus, due to the fact that up to a few years ago, complete splenectomy was the recommended treatment, techniques with preservation of the spleen are currently being widely recommended through laparoscopy in literature. Among the conservative techniques percutaneous aspiration, with or without the injection of a sclerosing agent, partial splenectomy, marsupialisation, cystectomy, decapsulation, unroofing or fenestration can be found. The main issue is recurrence rates. Few cases of primary giant splenic cysts treated by laparoscopic decapsulation can be found in literature, this treatment being simple and quick to perform, resenting a recurrence rate lower than other techniques such as aspiration and marsupialization. 


\section{P487-SOLID ORGANS-Spleen}

\section{WHAT IS THE ESPLENIC SIZE LIMIT FOR LAPAROSCOPIC} APPROACH?

A.F. Aranzana Gómez, J. Malo Corral, J. Hernandez Gutierrez, B. Muñoz Jimenez, I. Fraile Alonso, A. Trinidad Borras, A. Muñoz Tébar, B. Muñoz Jimenez, M.A. Morlan Lopez General Surgery, Complejo Hospitalario Toledo, TOLEDO, Spain

Objective: To demonstrate the safety and efficacy of the laparoscopic approach in the treatment of large splenomegaly. Currently, this approach is recognized as the one of choice in benign splenic pathology, being controversial in the face of a massive splenomegaly or neoplastic pathology.

Material and method: Clinical case: A 38-year-old man followed in the Dept. of Internal Medicine for a hepatosplenomegaly of probable lymphoproliferative origin. Additional explorations of interest are provided.

Result: Intervention: Complete laparoscopic approach, right lateral partial decubitus, massive splenomegaly, $+23 \mathrm{~cm}$, splenuncle of $3-4 \mathrm{~cm}$ that is resected, section of short vessels, dissection of the splenic hilum, vascular section with Endogias, splenectomy with full extraction in a pocket through reduced laparotomy in the left flank for anatomopathological study, drainage placement. Correct surgery, discharge the 3rd day.

Conclusions: In teams with experience in laparoscopy and selected patients with massive splenomegaly, the laparoscopic approach is a valid and safe alternative.

\section{P489-SOLID ORGANS-Thyroid}

TRANSAXILLARY THYROIDECTOMY: WHAT ADVANTAGES DOES IT OFFER?

M. Manyalich Blasi, V. Gonzabay, R. Termes, J. Farguell, A. Torroella, S. Espinoza, Y.C. Guzman, G. Cardenas, O. Vidal

General Surgery, Hospital Clinic de Barcelona, BARCELONA, Spain

Introduction: Technology's progress and its application in the minimally invasive surgery of the thyroid gland offers us new surgical approache's like the transaxillary approach. This new technic still unusual in our environment and has recently begun to be incorporated into our surgical practice. The objective of this case is to explain step by step how to carry out a right transaxillary thyroidectomy and emphasize in the most relevant tips to take into account. Also we going to review the main limitations we observed so far.

Statement of the case: We present the case of a 49-year-old woman referred for evaluation of a left thyroid nodule without associated symptomatology. The blood test shows normal thyroid profile. Cervical ultrasound is performed identifying a $3.5 \mathrm{~cm}$ single right nodule with welldefined edges and presence of peripheral vascularization . No other nodules are identified. FNA of the nodule describes a Bethesda III. After evaluation we decide to perform a left transaxillary thyroidectomy.

Discussion: Surgical treatment of the thyroid gland by transaxillary approach may be indicated in previously selected patients, offering the advantages from minimally invasive techniques (shorter recovery time, shorter incision length, etc.). Surely, more evidence and experiencie is required to make a better assessment of minimally invasive approaches in thyroid surgery.

\section{P488-SOLID ORGANS-Spleen}

\section{MASSIVE SPLENOMEGALY DUE TO} LYMPHOPROLIFERATIVE DISORDER T ASSOCIATED WITH EBV

J. Hernandez Gutierrez, A.F. Aranzana Gomez, J.S. Malo Corral, B. Muñoz Jimenez, R. Lopez Pardo, P. Toral Guinea, G. Krasniqi

\section{General Surgery, C.H.Toledo, TOLEDO, Spain}

Aims: The aim of this video is to demonstrate the safety and efficacy of the laparoscopic approach in the treatment of large splenomegaly. Currently, this approach is recognized as the one of choice in benign splenic pathology, being controversial in the case of a massive splenomegaly or neoplastic pathology.

Methods: Clinical case: A 38-year-old man studied by Internal Medicine for a hepatosplenomegaly of probable lymphoproliferative origin. Additional explorations of interest are provided.

Results: Full laparoscopic surgical approach in right lateral partial decubitus position: massive splenomegaly $(>23 \mathrm{~cm})$, splenuncle of $3-4 \mathrm{~cm} .4$ trocars-lateral transabdominal approach Laparoscopic section of short vessels, dissection of the splenic hilum, vascular section with EndoGIA, splenectomy with full extraction in a bag through reduced laparotomy in the left flank for pathological study. Correct postoperative recovery, discharge on the 3rd day.

Conclusion(s): In experienced laparoscopic surgical teams and selected patients with massive splenomegaly, the laparoscopic approach is a valid and safe alternative.

\section{P490-SOLID ORGANS-Thyroid}

\section{COMPARISON OF THE TRANSORAL AND BILATERAL AXILLO-BREAST APPROACHES IN ENDOSCOPIC THYROIDECTOMY}

\section{$\underline{\text { S.H.H. Huang }}{ }^{1}$, H.Y. Huang ${ }^{2}$, S.L. Wang ${ }^{2}$}

${ }^{1}$ Surgery, Taipi City Hospital, Yan-Ming Branch., TAIPEI, Taiwan; ${ }^{2}$ Surgery, Taipei City Hospital, TAIPEI, Taiwan

The first endoscopic thyroidectomy was performed in 1997 using a cervical approach. Since then, various remote-access method, have been developed for thyroid surgery to avoid scarring of the neck. Trans axillary approach(TAA),bilateral axillo-breast approach(BABA),and retroauricular approach(RAA) are common in use. The main benefit of these procedure is that there are no visible scar that is one of the drawbacks of conventional kocher's incision. However,these methods require more dissection and longer operation time than conventional thyroidectomy Transoral thyroidectomy(TOVET) is a new approach and has become popular in recent years, However,most surgeons peformed a single procedure because of the limited patients and the learning periods

Sine 2017,more than 100 cases were performed,patients received Endoscopic Thyroidectomy(ET) procedure at our hospital. We compare the surgical procedure of Bilateral AxilloBreast Approach(BABA) with Transoral Vestiblar Approach(TOVET) in our hospital both performed by one single surgeon. The surgen has expended eaqual amounts of time with these two procedures. The patient seletion process,operation time, operation procedure and approach,learnig experience, consmetic effect,onaologic consideration and surgical outcome were discussed yhroughly. 


\section{P491-SOLID ORGANS-Thyroid}

THYROID METASTASIS FROM OVARIAN NEOPLASM, A SINGULAR CASE

B.L. Lopez Duran ${ }^{1}$, I. Cornejo Jurado ${ }^{1}$, E. Perea del Pozo ${ }^{2}$, M. Diaz Rodriguez ${ }^{2}$, C. Sacristan Perez ${ }^{2}$, F. Oliva Mompean ${ }^{1}$, C. Marin Velarde ${ }^{2}$

${ }^{1}$ General and Digestive Surgery, Hospital Universitario Virgen Macarena, SEVILLA, Spain; ${ }^{2}$ Endocrine Surgery Unit, Hospital Universitario Virgen Macarena, SEVILLA, Spain

Presenting a case of a thyroid metastasis from an ovarian carcinoma, we conducted a review of the literature without finding similar reported cases.

Case: A 43-year-old woman consults for progressive asthenia, weight loss and ascites. Abdominal CT finds a conglomerate in the pelvis involving the ovaries and peritoneal implants, the largest up to $10 \mathrm{~cm}$. An omental epigastric lesion biopsy and paracentesis is performed resulting in adenocarcinoma and omental metastasis from ovarian neoplasm, associated with Ca125 of 4553 . Patient starts neoadjuvant therapy with Carboplatin-Paclitaxel. In image controls there is a favorable response. Three months later, intervention was carried out; Laparotomy Hysterectomy + double anexectomy + omentectomy + appendectomy + pelvic and paraaortic lymphadenectomy. The anatomopathological study shows a low-differentiated endometrioid carcinoma, omentum infiltration and absence of metastatic lymphatic involvement. While getting the maintenance treatment with Bevacizumab the patient presented symptoms of arthritis and hypercalcemia was detected (11.4) with PTH 268. A Gammagraphy was performed and an increased uptake area was detected in the lower pole of RTL, suggestive of a parathyroid adenoma. We initially proposed the possibility of performing radiofrequency ablation but in a previous thyroid ultrasound we visualize 3 nodular lesions in RTL compatible with adenoma and a mass in the superior mediastinum that seems to correspond the area of greatest uptake in the Gammagraphy so finally the procedure is dismissed and surgery is proposed. During the intervention we found a hard consistency nodule in the inferior pole RTL and lymphadenopathies of hard consistency in right VI area that are sent for intraoperative anatomopathological study with the result of adenocarcinoma metastasis without identifying origin.

A total thyroidectomy, parathyroidectomy and central ganglion drainage is performed with the result of a Parathyroid Adenoma, Lymphatic invasion of ovarian-grade latent carcinoma and EXTENSIVE VASCULAR PERMEATION BY CARCINOMA OF THE THYROID. The patient maintains oncological treatment with Carboplatin-Caelix. In the last follow-up, the PTH and calcemia remains normal. Conclussion: Although some cases of neoplasic thyroid involvement associated with Struma ovarii have been published, no cases similar to the one described are found, neither in our experience, which is why it is an exceptional case.

\section{P492-UPPER GI-Benign Esophageal disorders}

\section{A COMPARATIVE STUDY OF SHORT AND LONG-TERM OUTCOMES FOR TRANSCERVICAL VERSUS TRANSORAL SURGERY FOR ZENKER DIVERTICULUM}

L. Andrasi ${ }^{1}$, A. Paszt ${ }^{1}$, Zs. Simonka ${ }^{1}$, Sz. Abraham ${ }^{1}$, L. Rovo ${ }^{2}$, Gy. Lazar

${ }^{1}$ Department of Surgery, University of Szeged, SZEGED, Hungary; ${ }^{2}$ Department of Oto-Rhino- Laryngology and Head- Neck Surgery, University of Szeged, SZEGED, Hungary

Aims: We present our experience with open (transcervical diverticulectomy, cricomyotomy) and transoral surgery (transoral stapler diverticulostomy) for Zenker diverticulums.

Methods: Between 1 January 2006 and 31 December 2016, 29 patients were examined with a symptom-causing Zenker diverticulum. In 13 cases, transcervical surgery, in 16 cases, transoral surgery were performed. Perioperative and long-term results were evaluated and compared. Results: Patients were operated on after an average of 31 months with complaints. In both groups, the leading symptoms were severe dysphagia and severe regurgitation. No intraoperative complication was detected. In the transoral group, one patient had to be reoperated on for bleeding, another patient developed pneumonia in the transcervical group. The average duration of the surgeries $(42.5$ vs. $98 \mathrm{~min}, \mathrm{p}<0,001)$, the time to oral feeding $(2.9$ vs. 4.6 days, $\mathrm{p}<0,001)$ and the mean hospital stay $(7.3$ vs. 9.7 days, $\mathrm{p}<0,001)$ were significantly shorter in the transoral group than the transcervical group. 15 patients were completely symptomless postoperatively. After transcervical treatment, complaints were developed in 2 cases (moderate dysphagia and hoarseness). After transoral surgery, recurrent symptoms were observed in 6 patients, 4 had to be reoperated transcervically due to severe regurgitation.

Conclusion: Transoral stapler diverticulostomy is a fast procedure and offers short hospital stay especially in comorbid, aged patients and intermedium diverticulum size. In the long term, some of the patients may require reintervention due to persistent regurgitation. The transcervical approach has higher perioperative morbidity, which can be performed in patients with less than $3 \mathrm{~cm}$ or large diverticulum size.

\section{P493-UPPER GI-Benign Esophageal disorders}

USE OF ABSORBABLE MESH WITH ON-LAY APPLIANCE FOR THE RECONSTRUCTION OF COMPLEX HIATAL HERNIAS. LAP \& ROBOTIC APPROACH

H. Konstantinidis, C. Charisis

Robotic General and Oncologic Surgical Department, Interbalkan Medical Centre, THESSALONIKI, Greece

Aims: Complex hiatal hernias, either implicating large hiatal defects or concerning cases of recurrence, often need apart from the primary closure of the hiatal gap, the re-enforcement of the crura with the use of meshes. Our aim is to demonstrate the surgical technique for the on-lay placement of the absorbable mesh (Phasix ${ }^{\mathrm{TM}}$ ST Mesh /Bard) in challenging cases, presenting both the laparoscopic and the robotic approach.

Methods: We present video fragments from procedures of laparoscopic and robotic reconstruction of complex hiatal hernias, performed by our team, in which an absorbable mesh was utilized in an on-lay fashion.

Results: Patients having undergone a minimally invasive surgical approach (laparoscopic or robotic) for the treatment of complex hiatal hernias with the use of an absorbable mesh, had an uneventful post-operative course and very short hospital stay and recovery time. The 6-month follow up revealed no recurrences or late complications.

Conclusions: Treating complex cases of hiatal hernias with a minimally invasive approach can be proven quite challenging, with high recurrences and possible complications rate. A proper surgical technique, either laparoscopic or better (based in our primary experience) robotic, by experienced surgical teams and the use of meshes with the right strategy, minimizes the complications, offers all the benefits of minimally invasive surgery and reduces the recurrence rates.

\section{P494-UPPER GI-Benign Esophageal disorders}

\section{ENDOSCOPIC THULIUM LASER TREATMENT FOR ZENKER'S} DIVERTICULUM: RESULTS OF A PILOT STUDY

S. Zanghì, S. Siboni, C.G. Riva, P. Milito, M. Sozzi, D.T. Bernardi, L. Bonavina

Surgery, IRCCS Policlinico San Donato, SAN DONATO MILANESE, Italy

Aims: Several flexible endoscopic techniques for symptomatic Zenker's diverticulum have been developed during the last decade. Thulium laser has limited tissue penetration and may decrease the risk of perforation. This study reports the first use of thulium laser through flexible endoscopy for cricopharyngeal (CP) myotomy. Aims were safety and efficacy of flexible endoscopic Thulium laser myotomy and quality of life (QoL) changes after treatment.

Methods: A retrospective review of a prospectively collected database of 19 patients who underwent Thulium laser septum division for symptomatic Zenker's diverticulum was done. Demographic data, presenting symptoms, diverticulum characteristics, and intraoperative data were analyzed. Functional Outcome Swallowing Scale (FOSS) and M.D. Anderson Dysphagia Inventory (MDADI) questionnaires were administered to determine severity of dysphagia and its effect on QoL, both preoperatively and during follow-up visits. All the operations were carried out under general anesthesia. A continuous laser configuration and an emission power of $9 \mathrm{~W}$ was used in non-contact mode. Once the mucosa was opened, the fibers of the cricopharyngeal muscle were divided until the buccopharyngeal fascia was visibile. Results: Between March 2017 and September 2018, 19 patients (12 males) underwent flexible endoscopic CP myotomy with thulium laser. Mean age was $72 \pm 10.6$, mostly males $(68.4 \%)$. Seven patients $(36.8 \%)$ presented with recurrent diverticulum after previous transoral or open treatment. Mean diverticulum size was $2.5 \pm 0.8 \mathrm{~cm}$. Preoperative main symptoms were dysphagia $(94.7 \%)$, regurgitation $(68.4 \%)$, and cough $(47.3 \%)$. FOSS score was $=2$ in 12 patients (66.7\%). Mean MDADI global and composite score were $47.8 \pm 25.8$ and $59.7 \pm 9.4$. Complete division of the septum was achieved in all patients. Mean hospital stay was $2.83 \pm 1.62$ days. There was only one perforation treated conservatively. No 90-days mortality was observed. At median follow-up of 7 months, FOSS was $=2$ in $1(5.6 \%)$ patient and MDADI global and composite score were $90.0 \pm 12.4$ and $89.5 \pm 7.7$. All main symptoms were significantly reduced and QoL significantly increased.

Conclusions: Flexible endoscopic approach with Thulium laser is a safe and effective treatment option for Zenker's diverticulum either as a primary treatment or as a rescue therapy. 


\section{P495-UPPER GI-Benign Esophageal disorders}

\section{PREVENTION OF RECURRENT BLEEDING IN PATIENTS WITH PORTAL HYPERTENSION}

V.V. Petrushenko, D.I. Grebeniuk, I.V. Radoga, M.O. Melnychuk, S.A. Cheshenchuk

Department of Endoscopic and Cardiovascular Surgery, National Pirogov Memorial Medical University, Vinnytsya, VINNYTSYA, Ukraine

The aim of the study was to evaluate the effectiveness of the use of embolization of the splenic artery in order to prevent portal bleeding.

Methods: The study included 96 patients, who had esophageal varices bleeding, which developed as a result of decompensated cirrhosis of the liver of various etiologies of classes B and C according to Child-Pugh.

Patients were divided into 2 groups. The main group included $71(73.95 \%)$ patients who underwent endoscopic ligating of bleeding varix and in order to prevent recurrence of bleedingembolization of the splenic artery with Gianturco coils. The comparison group consisted of 25 $(26.05 \%)$ patients who received only drug therapy.

To assess the effectiveness of the treatment, the patient's condition was monitored for 6 months. Results: The average age of patients in the comparison group was $56.8 \pm 4.4$ years. Using only drug therapy, we stopped bleeding in $54(76.1 \%)$ patients. In all cases, at the end of treatment, we received an improvement in clinical and laboratory parameters. $17(23.9 \%)$ patients died. The duration of treatment was $10.1 \pm 2.4$ days.

The average age of patients in main group was $55.2 \pm 5.6$ years. Performing endoscopic ligation of bleeding varices, we stopped bleeding in $23(92.0 \%)$ patients. In all cases, at the end of treatment, we received an improvement in clinical and laboratory parameters. $2(8.0 \%)$ patients died. The duration of treatment was $6.5 \pm 2.7$ days.

A statistical analysis of mortality and duration of treatment revealed a significant difference $(\mathrm{p}<0.01)$ between the groups in both indicators.

After splenic artery embolization in all cases managed to achieve a reduction in blood flow of $60-80 \%$.

After 6 months among 54 patients in the comparison group, bleeding relapse occurred in 12 $(22.2 \%)$ cases. In the main group, this indicator was $8.7 \%$ ( 2 patients). The indicator in the main group was significantly $(\mathrm{p}<0.01)$ different from the same indicator in the comparison group. Conclusion: Performing embolization of the splenic artery in patients after endoscopic hemostasis of variceal bleeding allows to reduce the pressure in the portal system, which in turn leads to a decrease in the frequency of bleeding recurrences.

\section{P496-UPPER GI-Benign Esophageal disorders \\ THORACOSCOPIC ESOPHAGECTOMY FOR AORTOESOPHAGEAL FISTULA}

Y. Ebihara, T. Shichinohe, Y. Kurashima, S. Murakami, Surgery II, Hokkaido University, SAPPORO, Japan

Background: Aortoesophageal fistula (AEF) is an uncommon but one of highly fatal conditions. There are surgical, endoscopic and interventional radiological treatment options, however, definitive treatment is the surgical intervention. Video-assisted thoracoscopic surgery (VATS) has been gradually accepted as a substitution for thoracotomy to reduce the invasiveness of the surgery as radical surgery for esophageal cancer. We aimed to evaluate a feasibility of VATSesophagectomy (VATS-E) for AEF in this study.

Material and methods: Between 2009 and 2017, we retrospectively reviewed clinical charts of six patients who underwent VATS-E for AEF.

Results: The median thoracoscopic time was $146 \mathrm{~min}$ (range, 114-178 min). None of the patients were converted to open surgery. Thoracic endovascular aortic repair (TEVAR) was performed five patients $(83.3 \%$ ). Four patients underwent aortic replacement with artificial graft. Esophageal reconstruction was possible in three patients.

Conclusion: TEVAR and VATS-E are feasible for the treatment of AFE. Further accumulation of cases is necessary to establish safe and secure surgical strategy for AEF.

\section{P498-UPPER GI-Benign Esophageal disorders \\ BASIC SURGICAL STEPS FOR LAPAROSCOPIC RE-DO SURGERY FOR RECURRENT ACHALASIA}

$<\mathrm{u}>$ M.P. Fernández $</ \mathrm{u}>^{1}$, V. Sosa ${ }^{2}$, I. Alarcon ${ }^{2}$, M. Socas ${ }^{1}$, A. Barranco 3 , S. Morales-Conde ${ }^{4}$

${ }^{1}$ Unidad de Innovación en cirugía mínimamente invasiva. Servicio de cirugía general, Hospital Universitario Virgen del Rocío, SEVILLA, Spain; ${ }^{2}$ Cirugía general y digestiva, Hospital universitario de Cabueñes, GIJÓN, Spain; ${ }^{3}$ Unidad de innovación técnica en CMI. Servicio de cirugía general y digestiva, Hospital Universitario Virgen del Rocío, SEVILLA, Spain; ${ }^{4}$ Unidad de innovación en CMI. Servicio de Cirugía general y digestiva, Hospital Universitario Virgen del Rocío, SEVILLA, Spain

Introduction: Achalasia is the most common motility disorder of the esophagus. Heller's cardiomyotomy associated with a antireflux technique is the treatment of choice in patients with this disease; however, a small group of patients could present a recurrence of the symptoms being necesary a new surgery, what is an important challenge for most of the surgeons. We report the case of recurrence after a laparoscopic miotomy and Dor fundoplication as a paradigm for the appropiate management in this kind of patients.

Methods: A 63 years old female, who underwent a previous miotomy and a Dor fundoplication in 2011 due to an Achalasia.Six years after surgery, the patient showed epigastric pain and dysphagia. The study of the patient was performed with: barium swalow, pHmetry, manometry, CT-scan and MRI showing a recurrence of her disease.The patient was transfered to our center where she underwent a new surgery. The key points of the new surgery includes the next steps: Dissection of the previous adhesions, Dissection of the Dor's partial fundoplication, avoid dissection of the anterior esophageal wall at the leve lof the hiatus (the area of previous myotomy) in order to avoid perforation of the esophagus, Lateral and posterior dissection of the distal esophagus, lateral myotomy at the rigth wall of the esophagus and a Toupet's Funduplicatury. All of thisis procedures are done under intraoperative endoscopy in order to confirm a Good passage to the estmach and to identify a perforationic supervision.

Results: Following theseis steps several patients have been operated in our center with excellent results. In all of these cases, including the patiente presented previously, the symptoms have dissapeared. Conclusions: Achalasia is a rare motility disorder of the esophagus, being recurrences an important challenge for surgeons. A great proper therapeutic strategy using the different diagnostic exams and the supervison by a group of experts in this kind of entity are the basis in order to obtain good results in these situations.

\section{P499-UPPER GI-Benign Esophageal disorders}

\section{LAPAROSCOPIC RE-DO FUNDOPLICATION FOR PROGRESSIVE DYSPHAGIA—CONVERSION FROM NISSEN TO TOUPET}

\section{A.M. Harris}

General Surgery, Hinchingbrooke Hospital, HUNTINGDON, United Kingdom

Aims: Re-do fundoplication is usually performed for recurrent reflux symptoms due to wrap failure or recurrent hiatus hernia. Conversely, persistent dysphagia may occur early due to tight wrap/crural repair which should be avoided by good surgical technique. A small group of patients however may suffer progressive dysphagia due to weakening motility (especially in older patients), fibrosis of the wrap or a combination of the two. This video demonstrates the successful treatment of this problem with a laparoscopic conversion from Nissen to posterior Toupet fundoplication.

Methods: A 72 year old man underwent an uncomplicated laparoscopic Nissen fundoplication in 2015 with complete resolution of reflux symptoms. He re-presented 2 years later, still free of reflux but suffering progressive dysphagia and troublesome regurgitation. Investigations demonstrated intact wrap and no mechanical obstruction, but confirmed low-amplitude peristalsis. A trial endoscopic dilatation improved symptoms for 11 days before recurrence, suggesting likely wrap fibrosis (which would reduce elasticity and impede passage of food bolus), justifying consideration for a conversion from Nissen to Toupet.

Results: This video demonstrates the expected adhesions between fundoplication and inferior surface of left lobe liver, mobilisation and division of the Nissen fundoplication, and reconstitution of a posterior Toupet fundoplication. The patient made a good recovery and was discharged the following day. Three- and six-month follow-up confirmed complete resolution of symptoms with no recurrence of reflux.

Conclusion: Laparoscopic re-do surgery for late-onset progressive dysphagia is a safe and viable option. Patients must be thoroughly investigated and carefully selected for an appropriately tailored procedure. They should also be advised of the increased risks associated with re-do surgery. The anatomy can be unpredictably distorted by variable adhesions and this operation should therefore only be performed by laparoscopic surgeons experienced in both primary and re-do fundoplication. 


\section{P500-UPPER GI-Esophageal cancer}

LAPAROSCOPIC TRANS HIATAL ESOPHAGECTOMY WITH IATROGEN ESOPHAGEAL INJURY POST THYROIDECTOMY

\section{Hussein}

Surgery, American University of Beirut Medical Center, BEIRUT, Lebanon

Aims: Laparoscopic Trans Hiatal Esophagectomy with gastric pull up to neck for the treatment of

Methods: I report unusual iatrogenic injury of cervical esophagus that resulted with complete resection post total thyroidectomy for papillary CA of thyroid patient presented 4 days post surgery to our center. The video will show the steps used to treat this unusual complication by neck exploration, Laparoscopic Trans hiatal Esophagectomy with creation of gastric tube with preservation of the right gastroepiploic artery and the neck anastomosis between the cervical esophagus and stomach.

Results: Patient did well and discharged from hospital 1 week post surgery with no complication.On follow-up, Gastrograffin Swallow and CT Scan was negative for leak GASTRIC PULL UP TO NECK FOR THE TREATMENT OF Iatrogenic Esophageal injury post Thyroidectomy.

\section{P502-UPPER GI-Esophageal cancer}

THORACOSCOPIC APPROACH IN ESOPHAGECTOMY FOR ESOPHAGEAL CANCER: SINGLE CENTER EXPERIENCE

M. Peyroteo, R. Canotilho, M. Correia, A. Ramalho, A. Sousa

Surgical Oncology, IPO Porto, PORTO, Portugal

Aims: There has been an increasing tendency towards minimally invasive surgery for esophageal cancer. Our aim was to evaluate the results of the thoracoscopic approach (TA) and compare them with the ones of open approach (OA) at our institution

Methods: Retrospective review of all patients who underwent esophagectomy due to esophageal cancer (adenocarcinoma or squamous cell) between 2013 and 2017 were included. Patients with Siewert III tumors and those who didn't need a thoracic approach were excluded.

Results: During the study period were performed 83 esophagectomies, 23 through TA. In $43.5 \%$ of these, the abdominal stage was done by laparoscopy. When comparing TA versus OA, there were no statistically significant differences in the baseline characteristics of the two groups (mean age, median body mass index, ECOG performance status, ASA score, smoking status, diabetes mellitus, pulmonary disease, histologic type, clinical staging and neoadjuvant chemo and radiotherapy). Regarding outcomes, there were no significant differences in need of intraoperative transfusion, median intraoperative blood loss, operative time and length of stay. Although no significant, in TA group there was a tendency for higher overall morbidity $(69.6 \%$ versus $58.3 \%$, $\mathrm{p}=0.347)$; major morbidity-CTCAE $3-5(56.5 \%$ versus $38.3 \%, \mathrm{p}=0.135)$; anastomotic leak ( $34.8 \%$ versus $16.7 \%, \mathrm{p}=0.084)$ and re-intervention rate $(17.4 \%$ versus $15 \%, \mathrm{p}=0.748)$. On the other hand, in TA group there was a tendency (although not significant) towards lower rate of respiratory complications $(17.4 \%$ versus $33.3 \%, \mathrm{p}=0.152)$, lower rate of $\mathrm{R} 1$ margins $(4.3 \%$ versus $13.3 \%, \mathrm{p}=0.433$ ) and higher median of lymph nodes removed ( 22 versus $18, \mathrm{p}=0.083$ ) Conclusions: In our series, outcomes of TA were similar to OA, with a tendency towards lower respiratory complications, lower rate of R1 margins and higher number of lymph nodes removed in TA group. The impact of these findings in survival remains to be seen. The tendency towards higher morbidity may be related to the learning curve, since this were the first cases performed a our center.

\section{P503-UPPER GI-Esophageal cancer}

\section{P501-UPPER GI-Esophageal cancer}

\section{COMPARING MINIMALLY INVASIVE AND OPEN ESOPHAGECTOMIES}

Z.J. Lee, T.H. Tan, K.W. Lim, W.H. Chan, C.H. Lim, N.G. Tan, K.H. Eng, W.K. Wong, H.S. Ong

Department of Upper GI Surgery, Singhealth, SINGAPORE, Singapore

Introduction: With the advent of minimally invasive techniques, open surgical procedures are slowly replaced as the surgical modality of choice. It has been proven to bring about improvements such as better pain control, early mobility and improved physiological recovery. In particular, open esophagectomies require open thoracotomies which impacts significantly on the patient's post operative pain. Minimally invasive techniques have been adopted to address these issues with faster time to recovery, better pain control and its associated advantages although there are still concerns regarding the perioperative mortality and morbidity. This study aims to compare both the surgical and anesthetic outcomes between minimally invasive and open esophagectomies.

Methodology: A retrospective review of all minimally invasive and open esophagectomies performed in the Singapore General Hospital between 2016 to 2018. Patient demographics, histopathological, surgical and anesthesia data were collected. Subsequent analysis were done on factors that influence the outcomes of the patients.

Results: A total of 27 esophagectomies were performed between 2016 and 2018, of which 14 were open and 13 minimally invasive esophagectomies. Of the 27 patients, 10 were for squamous cell carcinoma, 13 were adenocarcinoma and 3 were of other histological diagnosis such as gastrointestinal stromal tumor and schwannoma. The median length of stay for patients who underwent minimally invasive esophagectomies was 9 days (9 to 120 days) while the median length of stay for patients who underwent open esophagectomies was 15 days ( 9 to 45 days). The minimally invasive group had a shorter icu stay of 1 day. For 30 day morbidity, the minimally invasive esophagectomy group had 2 patients who encountered anastomotic leaks, 1 with post operative pneumonia while the open esophagectomy group had 1 patient with anastomotic leak, 1 patient with post operative stricture and 1 patient with delayed gastric emptying. There were 2 mortalities in the minimally invasive group while there were no mortalities in the open group. Conclusion: Our data show that patients who underwent minimally invasive esophagectomies had a shorter duration of hospitalization with similar perioperative morbidity rates. Minimally invasive esophagectomy is a viable surgical option for a select group of patients.

\section{THE IMPACT OF ELDERLY ON SURGICAL OUTCOMES AFTER IVOR-LEWIS ESOPHAGECTOMY: REVIEW OF A SINGLE INSTITUTION EXPERIENCE}

A. Cossu, P. Parise, F. Puccetti, U. Elmore, R. Rosati, Gastrointestinal Surgery, San Raffaele Hospital, MILAN, Italy

Background: Esophagectomy is a surgical procedureburdened by a high morbidity rate. The effect of minimally invasive (MI) approach on elderly patients is still not clear. Aim: of this study was to analyze the impact of MI approach on post-operative course according to the patient age. Methods: A consecutive series of 692 patients underwent to elective oncological esophagectomy between 1997 and 2017. All data were entered into a prospective database. Patients submitted to 3-flield or trans-hiatal esophagectomywere excluded andonly Ivor-Lewisopen, hybrid or totally minimally invasive esophagectomywere. Patients were stratified according to age in 3 groups:Group A( $=50$ years $) 53$ patients, Group B $(>51$ and $<70$ years $) 269$ and Group $C$ (were $=71$ years) 126.Clinical and pathological factors influencing surgical outcome were evaluated. Complications were classified according to Clavien-Dindo (CD).

Results: As expected outcomes worsened with patients age $(\mathrm{CD}=3 \mathrm{~b}: 7.5 \%$ group $\mathrm{A}, 13 \%$ group $\mathrm{B}$ and $21 \%$ group C. $\mathrm{p}=0.001)$, mortality $(0 \%$ group $\mathrm{A}, 3 \%$ group $\mathrm{B}$ and $5.5 \%$ group $\mathrm{C}$ $\mathrm{p}=0.035)$ and length of stay (10 days group $\mathrm{A}, 11$ days group $\mathrm{B}$ and 13 days group $\mathrm{C}$ $\mathrm{p}=0.001)$.A statistically significant higher incidence of anastomosticleaks was observed among patients submitted to totally MI esophagectomy in group C vs A and B that were respectively $12,5 \%, 0 \%$ and $7 \%$. Major respiratory complications were not statistically different among these 3 three sub-group.

Conclusions: Old age has a significant impact on outcomes afteresophagectomy. In this subset of patients a MI approachcould also increasepostoperative morbidity. Elderly patients should be carefully selected before to be submitted to MI esophagectomy. 


\section{P504-UPPER GI-Esophageal cancer}

\section{ADVANTAGES OF TOTALLY MINIMALLY INVASIVE ESOPHAGECTOMY IN THE TREATMENT OF ESOPHAGEAL CANCER}

S. Constantinoiu, F. Achim, A. Constantin, R. Birla, P. Hoara, M. Gheorghe, R. Tomsa

\section{Center of Excellence in Esophageal Surgery, Carol Davila UMF,} BUCHAREST, Romania

Introduction: Esophagectomy is a major surgical procedure with morbidity and mortality related to the patient's condition, stage of the disease, complementary treatments, and surgical experience. Minimally invasive esophagectomy (MIE) may lead to a reduction in perioperative morbidity and mortality with very good quality of life.

Material and Method: We present the experience of the Center of Excellence in Esophageal Surgery regarding totally MIE through thoracolaparoscopic modified McKeown three-stage approach followed by esophageal reconstruction by gastric intrathoracic pull-up and cervical esophagogastric anastomosis used for the treatment of thoracic esophageal cancer.

Results: In the last 4 years, MIE was performed initial, in our clinic with extracorporeal preparation of the gastric conduit with reduced lung complications and hospital stay. We introduced the totally minimally invasive esophagectomy with laparoscopic-assisted feeding jejunostomy using a 3D high definition camera. Operative times were: thoracic $-120 \mathrm{~min}$, abdominal $-130 \mathrm{~min}$ and cervical $-50 \mathrm{~min}$ with a total of $300 \mathrm{~min}$. The augmented 3D high definition image provided an excellent visual field, that allowed an accurate identification of dissection plans and extensive periesophageal and perigastric lymphadenectomy. The short-term outcomes of the totally minimally invasive esophagectomy procedure were very encouraging with early feeding on jejunostomy and the control of cervical anastomosis was usually performed in the 5th day postoperative and the patients were discharged in the 9th day postoperative without any symptomatology. At the first and third-month follow-up was not reported any major complications. The long-term oncological results are being evaluated.

Conclusions: The totally minimally invasive approach using advanced technology of endoscopic surgery allowed for these patients a simple postoperative evolution, no major complications, and a good recovery after an extensive surgery. The solid experience in open esophageal surgery of the upper gastrointestinal surgeons provides a fast learning curve of complex minimally invasive surgical procedures with reduced perioperative morbidity. Long-term follow-up should confirm the results from the literature regarding the survival, which is expected to be for these patients at least equivalent with outcomes after open esophagectomy.

\section{P505-UPPER GI-Esophageal cancer}

\section{ENDOSCOPIC MANAGEMENT OF ESOPHAGEAL LEAKS}

P.A. Hoara ${ }^{1}$, C. Rosianu ${ }^{2}$, R.D. Birla ${ }^{1}$, D.E. Dinu ${ }^{1}$, M. Gheorghe ${ }^{1}$, R. Tomsa $^{1}$, A. Caragui ${ }^{1}$, A. Alkadour ${ }^{1}$, S.M. Constantinoiu ${ }^{1}$

${ }^{1}$ General and Esophageal Surgery Clinic, UMF „Carol Davila,,, , St Mary, Clinical, BUCHAREST, Romania; ${ }^{2}$ Gastroenterology, „St Mary,, Clinical Hospital, BUCHAREST, Romania

Introduction: Esophageal fistulas, benign or malignant, represent a real challenge for the surgeons and gastroenterologists, regarding the treatment and the outcome. In these cases, endoscopic treatment is the first line approach, being less invasive and sometimes avoiding the need for surgery. This includes clips, stents, glue and even suture.

Material and method: we have analyzed 9 esophageal fistulas in patients with benign or malignant pathology, diagnosed and treated in the first 6 months of 2018. The management of this complication included a self-expandable esophageal metallic stent. We have evaluated the diagnosis, the surgical intervention, the timing until the development of the leak, the localization and management of the fistula.

Results: 5 were postoperative leaks and 4 spontaneous esophageal fistulas. The localization was cervical in one case, thoracic in 5 cases and abdominal in 3 cases. For the postoperative fistulas, in 4 patients the treatment included at least one surgical reintervention with lavage and drainage, beside the insertion of an esophageal metallic stent. In the other cases, endoscopic treatment and antibiotic therapy was enough. In 2 cases, the stent migrated needing repositioning. 30 days mortality was $22 \%$, both patients from postoperative group.

Conclusions: Esophageal fistulas represent a severe complication, usually in patients already immunocompromised. Endoscopic management, including self expandable esophageal metallic stent, can be the main approach, by stopping the contamination and by permitting the early per oral feeding. Disadvantages include the possibility of migration and the need of removal after 6-8 weeks.

\section{P506-UPPER GI-Esophageal cancer}

RISK FACTORS OF POSTOPERATIVE COMPLICATIONS AND DIFFICULTY OF MINIMALLY INVASIVE ESOPHAGECTOMY FOR ESOPHAGEAL CANNER

\author{
A. Saito, K. Otsuka, S. Goto, T. Aoki, M. Murakami;
}

Gastroenterological and General Surgery, Showa University, SHINAGAWA, TOKYO, Japan

Aims: To consider the risk factors of postoperative complications and the factors of difficulty of Minimally Invasive Esophagectomy (MIE) for esophageal canner

Methods: Five hundreds and one patients with esophageal cancer who underwent MIE from 2010 to 2016 at our department were eligible. We considered the risk factors of complications of pneumonia, anastomotic leakage, and hoarseness after surgery, and the risk factors of difficulty of surgery.

Results: The risk factors of postoperative complications in univariate analysis were more than 75 years old (odds ratio: $2.1, \mathrm{P}=0.01$ ), more than II in ASA-PS (odds: $3.1, \mathrm{P}<0.01$ ), more than $300 \mathrm{~g}$ of bleeding (odds: $2.1, \mathrm{P}=0.01$ ), more than $450 \mathrm{~min}$. of operation time (odds: 2.2 , $\mathrm{P}<0.01$ ), and colon reconstruction (odds: $3.2, \mathrm{P}=0.02$ ). The one in multivatiate analysis was more than II in ASA-PS (Odds: 3.2, P $=0.01$ ). The risk factors of much bleeding were colon reconstruction (odds: $6.5, \mathrm{P}<0.01$ ), and more than 50 of lymph node dissection (odds: 1.5 , $\mathrm{P}=0.05$ ). The risk factors of long operation time without cervical lymph node dissection were neo-adjuvant therapy (odds: $8.1, \mathrm{p}<0.01$ ), more than 60 of lymph node dissection (odds: 3.0 , $\mathrm{P}=0.01$ ), and colon reconstruction (odds: $8.1, \mathrm{P}<0.01$ ). The ones with cervical lymph node dissection were more than pStage III (odds: $2.4, \mathrm{P}<0.01$ ) and more than 60 of lymph node dissection (odds: $2.8, \mathrm{P}=0.03$ )

Conclusions: Considering those risk factors, we should perform perioperative management more carefully.

\section{P507-UPPER GI_Esophageal cancer}

\section{ONCOLOGICAL RESECTION OF EPIDERMOID ESOPHAGUS CARCINOMA WITH IVOR-LEWIS TORACOSCOPIC AND LAPAROSCOPIC SURGERY}

E. Vidaña Márquez, P.A. Sánchez Fuentes, J. Jorge Cerrudo, F. Rubio Gil, I. Blesa Sierra, M. Ferrer Márquez, R. Belda Lozano, P.J. Moya Forcén, A. Reina Duarte, R. Rosado Cobián

Cirugía General y del Aparato Digestivo, Hospital Universitario Torrecárdenas, ALMERÍA, Spain

Aims: To present the surgical procedure of Ivor-Lewis by endoscopic thoracic and abdomina surgery, fulfilling oncological criteria, carried out in the General Surgery Service of the Hospital of Torrecárdenas.

Method: SA 74-year-old man with a tobacco and alcoholic habit was suspended for years, unde treatment for arterial hypertension, who consults for a logical dysphagia of 4 months of evolution. He is diagnosed of stenosing esophageal distal third epidermoid carcinoma TxN1M0. It is decided to place a prosthesis that is effective and subsequent neoadjuvant QT-RT, after 6 weeks of its completion the surgery is performed.

Results: The surgery is performed in 2 times, initially by laparoscopy. The esophageal hiatus and the greater curvature are dissected preserving the right gastroepiploic, and lymphadenectomy of the celiac trunk with pedicle section of the left gastric. Gastric plasty is performed with a section of lesser curvature towards fundus. It is continued by thoracoscopy. A section of the azygos vein is performed, dissection of the esophageal middle and lower third and lymphadenectomy. Gastric plasty is promoted, proximal esophagus section and latero-lateral intrathoracic gastro-oesophageal anastomosis. The anatomopathological study reports ypT3 and pN2 with 4/33 adenopathies, and disease-free surgical margins. He was discharged without complications on the 12th day and did not require re-entry.

Conclusions: Ivor-Lewis endoscopic surgery is safe and meets oncological criteria in selected patients with distal esophageal neoplasia and performed by an experienced esophagogastric unit. 


\section{P508-UPPER GI-Esophageal cancer}

\section{PROGRESSION OF BARRETT'S ESOPHAGUS AFTER LAPAROSCOPIC ANTIREFLUX SURGERY}

Zs. Simonka ${ }^{1}$, A. Paszt ${ }^{2}$, E. Man ${ }^{1}$, I. Toth ${ }^{1}$, Z. Horvath ${ }^{1}$, J. Tajti ${ }^{1}$, I. Petho ${ }^{1}$, L. Tiszlavicz ${ }^{2}$, I. Nemeth ${ }^{3}$, F. Izbeki ${ }^{4}$, A. Rosztoczy ${ }^{4}$, T. Wittmann ${ }^{4}$, Gy. Lazar ${ }^{1}$

${ }^{1}$ Department of Surgery, University of Szeged, SZEGED, Hungary; ${ }^{2}$ Department of Pathology, University of Szeged, SZEGED, Hungary; ${ }^{3}$ Department of Dermatology, University of Szeged, SZEGED, Hungary; ${ }^{4} 1$ st Department of Internal Medicine, University of Szeged, SZEGED, Hungary

Introduction: Gastroesophageal re?ux disease (GERD) is one of the most common upper gastrointestinal diseases in western countries. Barrett's esophagus (BE) is a proved precancerosis. It can transform into adenocarcinoma.

Patients and methods: Between 2001 and 2008 we performed laparoscopic Nissen fundoplication (LARS) in 254 cases of GERD. In 78 cases of GERD patients BE was proved by endoscopy and histological examination. The DeMeester score was higher $(18.9$ versus $41.9, \mathrm{p}<0.001)$, and bile re?ux was measured more frequently among the $\mathrm{BE}$ patients. The pressure of the lower oesophageal sphincter were lower than normal (GERD 12.1 vs BE $12.6 \mathrm{Hgmm}, \mathrm{p}=0.892$ ).

Results: The postoperative functional follow up con?rmed an increased sphincter pressure in the lower esophagus (GERD 17.6 Hgmm versus BE $18.7 \mathrm{Hgmm}, \mathrm{p}<0.001$ ), and a decreased acid reflux (DeMeester score GERD 7.7 vs BE 12.7, p $<0.001$ ). Regression of BE was observed in one third among the BE patients in the short follow up period. On the other hand during the 8.5 years long endoscopic follow up early Barrett carcinoma developed in 2 patients, 38.5 months after the LARS. Both patients underwent a limited surgical resection of the distal esophagus and esophagogastric junction, regional lymphadenectomy, and reconstruction by interposition of an isoperistaltic jejunal segment. There were no complication. Histological examination was shown pT1N0 stage disease in both cases. Oncological follow up was 82 months long (6.8y) and both patients are still disease free.

Conclusions: Although LARS can affect regression in a part of BE patients, progression to adenenocarcinoma can also occur. Endoscopic surveillance is important in the case of BE to recognize early cancer, to perform limited surgical resection with low morbidity and long overall- and disease free survival.

\section{P509-UPPER GI-Esophageal cancer}

\section{THORACOSCOPIC ESOPHAGECTOMY WITH RADICAL LYMPH NODE DISSECTION FOR THORACIC ESOPHAGEAL} CARCINOMA IN THE LEFT LATERAL DECUBITUS POSITION

H. Sato, Y. Miyawaki, N. Fujiwara, H. Sugita, M. Aikawa, S. Sakuramoto, K. Okamoto, S. Yamaguchi, I. Koyama ,

Gastroenterological Surgery, Saitama Medical University International Medical Center, HIDAKA-SHI, SAITAMA, Japan

Background: The rates of thoracoscopic esophagectomy performed in the prone and left lateral decubitus positions are similar in Japan. We retrospectively reviewed short term outcomes of thoracoscopic esophagectomy for esophageal cancer performed in the left lateral decubitus position under artificial pneumothorax by $\mathrm{CO} 2$ insufflation in a single institution. This study aimed to evaluate the feasibility of applying this procedure.

Methods: Between July 2013 and December 2017, 124 patients with esophageal cancer underwent thoracoscopic esophagectomy in the left lateral decubitus position under artificial pneumothorax by $\mathrm{CO} 2$ insufflation. The thoracic procedure is performed as follows:The lymph nodes around the right recurrent laryngeal nerve are dissected. On the cranial side, the lymph node dissection is advanced to the level of the inferior thyroid artery. Then, the assistant rotates the trachea toward the ventral side, and the lymph nodes around the left recurrent laryngeal nerve are dissected. The middle and inferior mediastinal lymph nodes are dissected including supradiaphragmatic lymph nodes and the dorsal lymph nodes around the thoracic descending aorta. Then, the esophagus is transected using an automatic suture device. Finally, the tracheal bifurcation area lymph nodes are dissected. We retrospectively analyzed these patients.

Results: The completion rate of thoracoscopic esophagectomy was $92.0 \%$, and the procedure was converted to thoracotomy in five patients, due to hemorrhage,severe adhesion. The mean intrathoracic operative time, intrathoracic blood loss, and number of dissected mediastinal lymph nodes were $210.5 \mathrm{~min}, 120.5 \mathrm{~mL}$, and 23.0, respectively. Postoperative complications included pneumonia $(13.7 \%)$, anastomotic leakage $(16.9 \%)$, and recurrent nerve paralysis $(16.1 \%)$. Postoperative (30d) mortality was $2 / 124(1.6 \%)$ due to ARDS and NOMI, respectively. Conclusions: Standardization of the procedure for thoracoscopic esophagectomy in the left lateral decubitus position under artificial pneumothorax by $\mathrm{CO} 2$ insufflation, with a standardized clinical pathway for perioperative care led to favorable surgical outcomes.

\section{P510-UPPER GI-Esophageal cancer}

\section{A CASE OF THORACOSCOPIC ESOPHAGECTOMY FOR THORACIC ESOPHAGEAL CANCER WITH CHEST DESCENDING AORTIC SACCULAR ANEURYSM}

Y. Miyawaki, H. Sato, N. Fujiwara, S. Sakuramoto, S. Hirohumi, K. Okamoto, S. Yamaguchi, I. Kayama

Gastroenterological Surgery, Saitama medical University International Medical Center, SAITAMA, Japan

Introduction: Recently thoracoscopic surgery has become widespread even in chest procedure in thoracic esophageal cancer surgery. As an advantage of minimally invasive esophagectomy, it is possible to perform sophisticated procedures due to its magnified visual effects. On the other hand, short-term perioperative safety and oncological safety are still unclear. In cases where abnormal anatomy or comorbidity in the thoracic cavity is observed, it is thought that it is necessary to carry out thoracic surgery which ensures safety while keeping in mind the transition to transthoracic surgery. Here, we report on esophageal resection of the thoracic esophageal cancer accompanied by a $20 \mathrm{~mm}$ saccular aneurysm inside the aortic arch.

Patient: A 67-year-old man visited a nearby doctor with a chief complaint of discomfort during swallowing. Upper gastrointestinal endoscopy examined middle cervical esophageal cancer and received referral to our hospital. CT revealed a $20 \mathrm{~mm}$ saccular aneurysm inside the descending aorta in contact with the thoracic esophagus. Preoperative diagnosis was Middle thoracic esophageal cancer; 0-IIc cT1bN0M0 StageIA (UICC 8th). We performed thoracoscopic esophagectomy and lymph node dissection as curative surgery. The anterior surface of the aorta was exposed from the lower mediastinum and descended ascendingly, reaching the lower end of the saccular anus at the head level of the lower pulmonary vein. Peeling off the esophagus dorsal side along the margin of the saccular sac and performing esophageal resection.

Conclusion: We reported thoracoscopic esophageal resection for thoracic esophageal cancer with chest descending aortic saccular aneurysm. Thoracoscopic surgery, which can fully exploit close magnification effect, seemed to be useful for anatomically disqualified cases.

\section{P511-UPPER GI-Gastric cancer}

\section{OESOPHAGO-JEJUNAL ANASTOMOSIS LEAK-AN INNOVATIVE ENDOLUMINAL AND RADIOLOGICALLY TECHNIQUE TO INSERT DOUBLE PIG-TAILED CATHETER APPROACH}

\section{B.C. Toh, B.P.M. Yeung, J.T.H. Tan}

General Surgery, Sengkang General Hospital, SINGAPORE, Singapore

Introduction: Anastomotic leakage from oesophagojejunal $(\mathrm{OJ})$ anastomosis after total gastrectomy is associated with a high morbidity and mortality rate. Leakage rates reported vary between $3 ? \%$ and $11 ? \%$ but lack of consensus in management. In the past, it often required surgical intervention or radiologically abscess drainage that will keep patients fasted with external drain for a long duration. Recently, variable endoscopic options-oesophageal stents, clips, fibrin glue and endoluminal vacuum therapy had been introduced with variable outcomes. Here, we presented a case of OJ anastomotic leak management with combination innovative endoluminal and radiologically technique to insert double Pig-tailed catheter. Aim: To introduce the feasibility of double Pig-tailed catheter for drainage and management of OJ anastomosis leak.

Methods: A 70 year old man presented with two months history of dysphagia. Upper endoscopy (OGD) showed suspicious cardio-oesophageal lesion. Histology biopsy confirmed with Adenocarcinoma. CT-scan of thorax, abdomen and pelvic showed irregular thickening at cardiooesophageal junction with regional lymphadenopathy. No distant metastases.

He underwent uneventful D2 total gastrectomy. On 5th post-operative day, patient had spike fever and newly developed atrial fibrillation. Urgent CT-thorax, abdomen and pelvis with oral Omnipaque. It showed lower mediastinal gas-containing fluid adjacent to OJ anastomosis within the left retrocrural space suspicious for leak.

OGD evaluation showed pin-hole OJ leak. Guidewire inserted via endoscopy into left retrocural space under radiologically guidance. Double pig-tail $7 \mathrm{Fr} 5 \mathrm{~cm}$ subsequently inserted via seldinger approach over guide wire. The proximal end of pig-tail pushed into left retrocural space and distal end positioned into efferent jejunal limb with crocodile jaw through endoscope. Diluted contrast injected and passed down to efferent limb with minimal leak.

Outcome: After double pig-tail insertion, patient started on clear feed on 1st day post-insertion. One week later, he was started on full feed. Repeat upper endoscopy and stent removal done two weeks later. Contrast injection showed small blind ended sinus tract from anastomosis toward left pleural space without obvious leak.

Conclusion: Radio-endoscopic is a novel minimally invasive technique that allows insertion of double pig-tailed internal drainage to control OJ anastomosis leak. It allows early enteral nutritional feeding and avoid external drainage. 


\section{P512-UPPER GI-Gastric cancer}

\section{POSTOPERATIVE DYNAMIC SURVIVAL OF GASTRIC CANCER PATIENTS: A MULTI-INSTITUTIONAL, INTERNATIONAL ANALYSIS OF 22265 PATIENTS}

C. Huang ${ }^{1}$, Q. Chen ${ }^{1}$, Q. Zhong ${ }^{1}$, W. Wei ${ }^{2}$, D. Jacopo ${ }^{3}$, P. Li ${ }^{1}$, J. Xie ${ }^{1}$, J. Wang ${ }^{1}$, J. Lin ${ }^{1}$, J. Lu ${ }^{1}$, C. Zheng ${ }^{1}$, J. Peng ${ }^{4}$, Z. Zhou ${ }^{2}$, P. Amilcare ${ }^{3}$

${ }^{1}$ Department of Gastric Surgery, Fujian Medical University Union Hospital, FUZHOU, China; ${ }^{2}$ Department of Gastric Surgery, Sun Yatsen University Cancer Center, GUANGZHOU, China; ${ }^{3}$ Department of Digestive Surgery, St. Mary's Hospital, University of Perugia, TERNI, Italy; ${ }^{4}$ Department of Esophageal and Gastrointestinal Surgery, Fujian Medical University Union Hospital, FUZHOU, China

Background: The number of gastric cancer (GC) survivors, especially long-term survivors, is increasing. How best to evaluate the diseasespecific survival (DSS) of GC survivors over time is unclear. We aimed to assess changes in the conditional survival of patients with GC after curative intend gastrectomy and the evolution of the impact of well-known risk factors.

Methods: Clinicopathological data from 22,265 patients who underwent curative intend resection for GC at four specialized centres (three in China and one in Italy) and from the Surveillance, Epidemiology, and End results (SEER) database were retrospectively analysed. Changes in the patients' 3 -year conditional disease-specific survival (CS3) were analysed. We used time-dependent Cox regression to analyse which variables had long-term effects on DSS and devised an accurate, dynamic DSS predictive model based on the length of survival.

Results: The median follow-up time was 74 months, and disease-specific death occurred in 9,927 cases (44.6\%). The DSS of the patients after surgery was dynamic, and most of the disease-specific deaths occurred within the first 3 years after surgery. Based on 1-, 2-, 3-, 4- and 5-year survivorships, the CS3 of the population increased gradually from $62 \%$ to $68.1 \%, 77.3 \%, 83.7 \%, 87.6 \%$, and $90.6 \%$, respectively. Subgroup analysis showed that the CS3 of patients who had poor prognostic factors initially demonstrated the greatest increase in postoperative survival time (e.g., N3b: $26.6 \%-84.1 \%, ? 57.5 \%$ vs. N0: $84.1 \%-93.3 \%, ? 9.2 \%$ ). Time-dependent Cox regression analysis showed the following predictor variables constantly affecting DSS: age, the number of examined lymph nodes, $\mathrm{T}$ stage, $\mathrm{N}$ stage and site ( $\mathrm{P}$ all $<0.05,5$ years after gastrectomy).

Conclusions: The influence of prognostic factors on DSS and CS3 changed dramatically over time. Based on data from several large global centres, we developed an effective model for predicting the DSS of GC patients based on the length of survival time. This model can provide personalized long-term follow-up strategies for patients.

\section{P513-UPPER GI-Gastric cancer}

DEVELOPMENT AND EXTERNAL VALIDATION OF WEBBASED MODELS TO PREDICT THE PROGNOSIS OF REMNANT GASTRIC CANCER AFTER SURGERY: A MULTICENTER STUDY

C. Huang ${ }^{1}$, Q. Chen ${ }^{1}$, Q. Zhong ${ }^{1}$, Q. $\mathrm{He}^{2}$, W. $\mathrm{Lin}^{3}$, G. Su${ }^{4}$, D. $\mathrm{Xu}^{5}$,

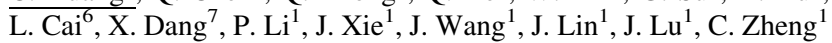

${ }^{1}$ Department of Gastric Surgery, Fujian Medical University Union Hospital, FUZHOU, China; ${ }^{2}$ Department of gastrointestinal surgery, Fujian Medical University Union Hospital, FUZHOU, China;

${ }^{3}$ Department of Gastrointestinal Surgery and Gastrointestinal Surgery Research Ins, The Affiliated Hospital of Putian University, PUTIAN, China; ${ }^{4}$ Department of Gastrointestinal Surgery, The First Affiliated Hospital of Xiamen University, XIAMEN, China; ${ }^{5}$ Department of Gastrointestinal Surgery, Longyan First Hospital Affiliated to Fujian Medical University, LONGYAN, China; ${ }^{6}$ Department of General Surgery Unit 4, ZhangZhou Affiliated Hospital of Fujian Medical University, ZHANGZHOU, China; ${ }^{7}$ Department of General Surgery, Shanxi Provincial Cancer Hospital, TAIYUAN, China

Background: Remnant gastric cancer (RGC) is a rare malignant tumor with poor prognosis. There is no universally accepted prognostic model for RGC.

Methods: We retrospectively analyzed clinicopathological data for 253 RGC patients who underwent radical gastrectomy from 6 centers. The prognosis prediction performances of the AJCC7th and AJCC8th TNM staging systems and the TRM staging system for RGC patients were evaluated. Web-based prediction models based on independent prognostic factors were developed to predict the survival of the RGC patients. External validation was performed using a cohort of 49 Chinese patients. Result: The mean number of retrieved lymph nodes was 16.1 , and in $54.2 \%$ of patients, the number was $=15$. The predictive abilities of the AJCC8th and TRM staging systems were no better than those of the AJCC7th staging system (c-index: AJCC7th vs. AJCC8th vs. TRM, 0.743 vs. 0.732 vs. $0.744 ; \mathrm{P}>0.05)$. Within each staging system, the survival of the two adjacent stages was not well discriminated $(\mathrm{P}>0.05)$. Multivariate analysis showed that age, tumor size, $\mathrm{T}$ stage and $\mathrm{N}$ stage were independent prognostic factors for overall survival (OS), disease-specific survival (DSS) and disease-specific survival (DFS). Based on the above variables, we developed 3 web-based prediction models, the Huang OS model, the Huang DSS model and the Huang DFS model, which were superior to the AJCC7th staging system in their discriminatory ability (Cindex), predictive homogeneity (likelihood ratio chi-square), predictive accuracy (AIC, BIC), and model stability (time-dependent ROC curves). The stratified analysis showed that regardless of whether more or fewer than 15 lymph nodes were retrieved, the predictive performances of the web-based prediction models were still better than those of the other three staging systems. A decision curve analysis showed that the Huang model provided better net benefits than the other three staging systems. External validation showed predictable accuracies of $0.780,0.822$ and 0.700 , respectively, in predicting OS, DSS and DFS.

Conclusion: The AJCC TNM staging system and the TRM staging system did not enable good distinction among the RGC patients. We have developed and validated visual web-based prediction models that are superior to these staging systems. 


\section{P514-UPPER GI-Gastric cancer}

DEVELOPMENT A NOMOGRAM FOR PREDICTING THE CONDITIONAL PROBABILITY OF SURVIVAL AFTER D2 LYMPHADENECTOMY FOR GASTRIC CANCER

C. Huang ${ }^{1}$, Q. Zhong ${ }^{1}$, Q. Chen ${ }^{1}$, C. Zheng ${ }^{1}$, W. Wang ${ }^{2}$, D. Jacopo ${ }^{3}$, J. Lin ${ }^{1}$, J. Lu ${ }^{1}$, Z. Zhou ${ }^{2}$, P. Amilcare ${ }^{3}, \mathrm{P}^{2} \mathrm{Li}^{1}$

${ }^{1}$ Department of Gastric Surgery, Fujian Medical University Union Hospital, FUZHOU, China; ${ }^{2}$ Department of Gastric Surgery, Sun Yatsen University Cancer Center, China; ${ }^{3}$ Department of Digestive Surgery, St. Mary's Hospital, University of Perugia, TERNI, Italy

Background: Previous studies have elucidated that on average, long-term cancer survivors have better prognoses than newly diagnosed individuals. This study aimed to devise a nomogram to predict the conditional probability of cancer-specific survival (CPCS) in gastric cancer (GC) patients after gastrectomy with D2 lymphadenectomy.

Methods: Clinicopathological data for 2,596 GC patients who underwent D2 lymphadenectomy in a large-volume Eastern institution (the training cohort) were analysed. Cancer-specific survival (CSS) was predicted using Cox regression models. A conditional survival nomogram was constructed to predict CPCS at 3 and 5 years post-gastrectomy. Two external validations were performed using a cohort of 2,198 Chinese patients and a cohort of 504 Italian patients.

Results: In the training cohort, the 5-year CPCS was $59.2 \%$ immediately post-gastrectomy and increased to $68.8 \%, 79.7 \%, 88.8 \%$ and $95.1 \%$ at $1,2,3$ and 4 years post-gastrectomy, respectively. Multivariate Cox regression analyses showed that age; tumour site, size and invasion depth; numbers of examined and metastatic lymph nodes; and surgical margins were independent prognostic factors of cancer-specific survival (all $P<0.05$ ) and formed the nomogram predictor variables. Internal validation showed that the conditional nomogram exhibited good discrimination ability at 3 and 5 years post-gastrectomy (concordance index, 0.794 and 0.789 , respectively). External validation showed a 3- and 5-year concordance index of 0.788 and 0.785 , respectively, in the Chinese cohort and 0.792 and 0.787 , respectively, in the Italian cohort. Calibration of the nomogram predicted that survival corresponded closely with actual survival. Conclusions: We developed and externally validated a robust nomogram to predict CPCS after D2 lymphadenectomy for GC based on survival duration. This nomogram will facilitate survival assessments and surveillance planning.

\section{P515-UPPER GI-Gastric cancer}

\section{DOES NON-COMPLIANCE IN LYMPH NODE DISSECTION AFFECT ONCOLOGICAL EFFICACY IN GASTRIC CANCER PATIENTS UNDERGOING RADICAL GASTRECTOMY?}

C. Huang, Q. Chen, Q. Zhong, Z. Liu, C. Zheng, P. Li, J. Xie, J. Wang, J. Lin, J. Lu, L. Cao

Department of Gastric Surgery, Fujian Medical University Union Hospital, FUZHOU, China

Background: Few reports have examined the prognosis of or possible remedial treatments for patients with non-complaint D2 lymphadenectomy. Here, we investigate the effect of noncompliance in lymph node (LN) dissection on long-term survival in gastric cancer (GC) patients after radical gastrectomy and explore intervention measures.

Methods: The clinicopathological data were retrospectively analysed in 2401 patients who underwent radical gastrectomy for GC. Non-compliance was defined as patients with more than 1 empty LN station, as described in the protocol of the Japanese GC Association.

Results: The overall non-compliance rate was $49.1 \%$. The 3-year overall survival (OS) rate was significantly better in compliant patients than in non-compliant patients $(74.0 \%$ vs. $60.1 \%$, $\mathrm{P}<0.001)$. Univariate and multivariate analyses revealed that non-compliance was an independent risk factor for OS. Logistic regression analysis demonstrated that the extent of gastrectomy, primary tumour site, history of intraperitoneal surgery, BMI and open gastrectomy were independent preoperative predictive factors for non-compliance. Cox analysis demonstrated that age, $\mathrm{pT}, \mathrm{pN}$, and the extent of gastrectomy independently affected OS in patients with noncomplaint lymphadenectomy. However, OS was significantly better in the compliant group than in the non-compliant group regardless of the recommendation for chemotherapy. Stratified analysis demonstrated that OS was significantly better in chemotherapy patients than in patients without chemotherapy and stage II patients (pT1N2/N3M0 and pT3N0M0) in whom chemotherapy was not recommended.

Conclusion: Non-compliance is an independent risk factor after radical gastrectomy for GC. Adjuvant chemotherapy improved the prognosis of patients with pT1N2/N3M0 and pT3NOM0 disease who underwent non-compliant D2 lymphadenectomy.

\section{P516-UPPER GI-Gastric cancer}

\section{A PREDICTION MODEL FOR THE POTENTIAL INTRAOPERATIVE LAPAROSCOPIC HAEMOSTASIS IN SPLEEN-PRESERVING NO.10 LYMPHADENECTOMY FOR PROXIMAL GASTRIC CANCER}

C. Huang, Q. Chen, Q. Zhong, Z. Liu, C. Zheng, P. Li, J. Xie, J. Wang, J. Lin, J. Lu, L. Cao

Department of Gastric Surgery, Fujian Medical University Union Hospital, FUZHOU, China

Purpose: To identify the risk factors for intraoperative laparoscopic haemostasis during laparoscopic spleen-preserving splenic hilar lymph node dissection (LSPSD) for proximal gastric cancer (GC) .In addition, the study was designed to develop and validate a model to estimate the risk of intraoperative laparoscopic haemostasis.

Methods: Laparoscopic haemostasis is defined as a vascular injury that requireds compression with laparoscopic instruments or gauze, clipping by an absorbed or titanium clip, or laparoscopic ligation during LSPSD, without conversion to open laparotomy or splenectomy.Between January 2011 and December 2014, we prospectively collected and retrospectively analysed the medical records of 398 patients with proximal GC who underwent LSPSD. The data were split 75/25, with one group used for model development and the other for validation testing.

Results: Of the 398 patients enrolled in this study, $174(43.7 \%)$ required laparoscopic haemostasis treatment. A multivariate analysis determined the following preoperative adverse risk factors for the model group: gender, preoperative $\mathrm{N}$ stage, and terminal branches of the splenic artery (SpA), and we developed a scoring system based on these findings. Each of these factors contributed 1 point to the risk score. The intraoperative laparoscopy hemostasis rates were $11.5,33.6,58.5$, and $73.5 \%$ for the low-, intermediate-, high-, and extremely high-risk categories, respectively. There were statistically significant differences among groups $(\mathrm{p}<0.001)$. With the increase in risk, both blood loss volume (BLV) and operative time ( $\mathrm{min}$ ) of LSPSD increased significantly $(\mathrm{p}<0.001)$.The area under the receiver operating characteristic curve for the score of intraoperative laparoscopic haemostasis was 0.700 . The observed and predicted incidence rates for intraoperative laparoscopic haemostasis were parallel in the validation set.

Conclusions: This simple, efficient scoring system using the factors of gender, preoperative $\mathrm{N}$ stage, and terminal branches of the SpA could accurately predict the risk of intraoperative laparoscopic haemostasis during LSPSD to improve surgical safety.

\section{P517-UPPER GI-Gastric cancer}

IMPACT OF INCREASING AGE ON CANCER- AND NONCANCER-SPECIFIC MORTALITY IN PATIENTS WITH GASTRIC CANCER : A COMPETING RISK ANALYSIS

C. Huang ${ }^{1}$, L. Cao ${ }^{1}$, J. Lu ${ }^{1}$, C. Zheng ${ }^{1}$, P. Li ${ }^{1}$, J. Xie $^{1}$, J. Wang ${ }^{1}$, J. Lin ${ }^{1}$, Q. Chen ${ }^{1}$, M. Lin' ${ }^{1}$, R. Tu ${ }^{2}$

${ }^{1}$ Department of Gastric Surgery, Fujian Medical University Union Hospital, FUZHOU, China; ${ }^{2}$ Fujian Medical University Union Hospital, FUZHOU, China

Objective: To perform competing risk analysis and evaluate cancer- and noncancer-specific mortality in patients with gastric cancer after radical surgery.

Methods: A total of 5051 patients from our department (as training set) and a total of 7123 patients from the Surveillance, Epidemiology, and End results (SEER) database (as validation set) were enrolled in the study. The cumulative incidence of cancer and noncancer-specific mortality was determined by univariate and multivariate competing risk analysis.

Results: The five-year cancer- and noncancer-specific cumulative incidence of death (CID) in the training set were $36.9 \%$ and $2.5 \%$, respectively, which were significantly lower than that in validation set ( $48.2 \%$ and $8.6 \%$, respectively). Multivariable analysis showed that age, tumor site, tumor size and pTNM stage were independent predictors of gastric cancer-specific mortality and overall survival, whereas age was an independent predictor of gastric noncancer-specific mortality. Noncancer-specific CID surpassed cancer-specific CID for pTNM stage I patients after approximately 8 years of surgery, but never for stage II and III patients. Moreover, for stage I patients, the time point when noncancer-specific CID surpassed cancer-specific CID become earlier as age increasing, with only 3.5 years after surgery for patients more than 74 years of age. Conclusions: Age is an independent predictor of gastric cancer- and noncancer specific mortality and overall survival for patients after radical surgery. For patients with stage I gastric cancer, noncancer-specific mortality is a significant competing event, with an increasing impact as age increases. 


\section{P518-UPPER GI-Gastric cancer}

\section{A NOMOGRAM FOR PREDICTING THE BENEFIT OF ADJUVANT CHEMOTHERAPY AFTER RESECTION IN PATIENTS WITH BORRMANN TYPE IV GASTRIC CANCER}

\author{
C. Huang ${ }^{1}$, J. Lu ${ }^{1}$, Y. Dai ${ }^{1}$, C. Zheng ${ }^{1}$, P. $\mathrm{Li}^{1}$, J. Xie ${ }^{1}$, J. Lin ${ }^{1}$,

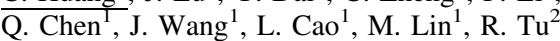

${ }^{1}$ Department of Gastric Surgery, Fujian Medical University Union Hospital, FUZHOU, China; ${ }^{2}$ Fujian Medical University Union Hospital, FUZHOU, China

Objective: This study sought to explore prognostic factors for patients with Borrmann type IV gastric cancer and to establish a predictive model for survival benefit of postoperative adjuvant chemotherapy in such patients.

Method: This study reviewed the clinical data of patients who underwent curative surgery at Fujian Medical University Union Hospital from 2006 to 2014 for Borrmann type IV gastric cancer using a prospective database. Cox regression analyses were performed to identify prognostic factors that formed the basis for a nomogram and risk groups. Establishment of risk groups to identify patients with Borrmann type IV gastric cancer who would benefit from adjuvant chemotherapy.

Results: 265 patients who underwent R0 resection were included in this study.Multivariate analysis showed that BMI, tumour differentiation, $\mathrm{pT}$ stage, $\mathrm{pN}$ stage, and ASA score were independent prognostic factors. Patients in the ACT-group had longer OS than patients in the SAgroup, although the p-value for this difference was marginally above the threshold for statistical significance $(23.8 \%$ vs. $10.9 \%, p=0.057)$. Stratified analysis showed that there was no significant difference in OS between the ACT-group and the SA-group for each AJCC stage (stage II: $40.6 \%$ vs. $29.8 \%, p=0.44$; stage III: $21.4 \%$ vs. $9.7 \%, p=0.056$ ). A nomogram was established based on these independent risk factors, and nomogram scores were used to divide all patients into a high-risk group (score $>16$ ), an intermediate-risk group $(8<$ score $=16$ ) and a low-risk group (score $=8$ ). Further stratified analysis based on AJCC stage showed that the 3-year survival rate was higher in the adjuvant chemotherapy group than in the surgery alone group for low- and intermediate-risk patients in each AJCC stage, while high-risk patients in stage III did not significantly differ.

Conclusion: The nomogram that we established may effectively be used to identify patients with Borrmann type IV gastric cancer who would benefit from postoperative adjuvant chemotherapy. Postoperative adjuvant chemotherapy can improve survival in low- and intermediate-risk patients.

\section{P519-UPPER GI-Gastric cancer}

\section{DOES SMOKING MODIFY THE ASSOCIATION BETWEEN CHEMOTHERAPY AND SURVIVAL IN GASTRIC CANCER AFTER R0 RESECTION? A DECISION TREE ANALYSIS}

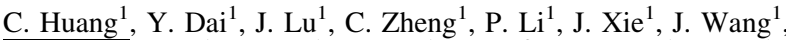

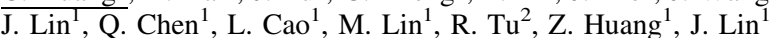

${ }^{1}$ Department of Gastric Surgery, Fujian Medical University Union Hospital, FUZHOU, China; ${ }^{2}$ Fujian Medical University Union Hospital, FUZHOU, China

Objective: This study sought to explore the prognostic factors for smoking patients with gastric cancer and to establish a predictive model for the survival benefit of postoperative adjuvant chemotherapy in such patients.

Methods: We studied 2081 patients who were diagnosed from September 2009 to Septembe 2014 at Union Hospital of Fujian Medical University. Cox regression analyses were performed to identify prognostic factors. The Kaplan-Meier method was used to assess the effect of smoking history on the benefit of adjuvant chemotherapy after gastric cancer surgery. A decision tree algorithm was used to identify smoking patients who benefited from postoperative adjuvant chemotherapy

Results: The median follow-up time for the whole group was 42.5 months, and the average age of all the included patients was 61.5 years.Multivariate analysis showed that age $(\mathrm{p}<0.001)$, BMI $(\mathrm{p}<0.001)$, degree of tumor cell differentiation $(\mathrm{p}<0.01)$, and AJCC stage $(\mathrm{p}<0.001)$ were independent risk factors for the prognosis of smoking patients. Based on these independen risk factors, a decision tree model for the benefit of adjuvant chemotherapy for smokers with gastric cancer was established, and the smoking patients were divided into the low-risk patients (3-year OS, 78.7\%), medium-risk patients (3-year OS, 51.3\%) and high-risk patients (3-year OS, $28.4 \%)(\mathrm{p}<0.001)$

Conclusion: Cigarette smoking may reduce the efficacy of adjuvant chemotherapy after gastric cancer surgery. Our decision tree model is simple and effective for identifying smokers who would benefit from adjuvant chemotherapy.

\section{P520-UPPER GI-Gastric cancer}

LYMPH NODE NONCOMPLIANCE AFFECTS THE LONGTERM PROGNOSIS OF PATIENTS WITH GASTRIC CANCER AFTER LAPAROSCOPIC TOTAL GASTRECTOMY

C. Huang ${ }^{1}$, G. Lin ${ }^{1}$, Q. Chen ${ }^{1}$, Q. Zhong ${ }^{1}$, C. Zheng ${ }^{1}$, P. Li ${ }^{1}$, J. Xie ${ }^{1}$,

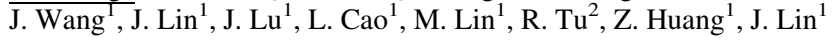

${ }^{1}$ Department of Gastric Surgery, Fujian Medical University Union Hospital, FUZHOU, China; ${ }^{2}$ Fujian Medical University Union Hospital, FUZHOU, China

Objective: Our study investigated the effect of lymph node (LN) noncompliance on the longterm prognosis of patients after laparoscopic total gastrectomy (LTG) and explored the risk factors of LN noncompliance.

Methods: The clinicopathological data of gastric cancer (GC) patients who underwent LTG with D2 lymphadenectomy from June 2007 to December 2014 were prospectively collected and retrospectively analyzed. The effects of $\mathrm{LN}$ noncompliance on the long-term prognosis of patients with GC after LTG were explored.

Results: The overall LN noncompliance rate was $51.9 \%$. LN noncompliance was significantly correlated with age, BMI, ASA score, tumor size, macroscopic tumor type and TNM staging ( values $<0.05$ ). The survival rate of patients after LTG with LN compliance was significantly superior to that of patients with LN noncompliance $(\mathrm{p}=0.013)$. The stratified analysis of TNM stage indicated that there was no difference between the OS of stage I patients with LN compliance and those with LN noncompliance; OS of stage II/III patients with LN compliance was significantly better than that of those with LN noncompliance. Cox regression analyses showed that LN noncompliance was an independent risk factor for OS. Logistic regression analysis showed that high BMI $\left(>25 \mathrm{~kg} / \mathrm{m}^{2}\right)$ was an independent risk factor for preoperative prediction of LN noncompliance in cStage II/III patients. Compared with patients with a low BMI (BMI < $25 \mathrm{~kg} / \mathrm{m}^{2}$ ), those with a high BMI were more likely to show LN noncompliance during surgery, especially during the dissections of \#6, \#8a and \#12a LN stations.

Conclusion: LN noncompliance was an independent risk factor for poor prognosis in patients with advanced gastric cancer (AGC) after LTG. Patients with high BMI were more likely to have LN noncompliance, especially during the dissections of \#6, \#8a and \#12a LN stations. LN tracing was recommended for these patients to reduce the rate of $\mathrm{LN}$ noncompliance.

\section{P521-UPPER GI-Gastric cancer}

DIFFERENCES IN PATHOLOGY, SURVIVAL AND
RECURRENCE BETWEEN SPECIAL AND NONSPECIAL
REMNANT GASTRIC CANCER: A MULTICENTER
RETROSPECTIVE STUDY IN CHINA

C. Huang ${ }^{1}$, Z. Huang ${ }^{1}$, Q. Chen ${ }^{1}$, C. Zheng ${ }^{1}$, P. $\mathrm{Li}^{1}$, J. Xie ${ }^{1}$, J. Wang ${ }^{1}$,

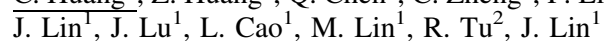

${ }^{1}$ Department of Gastric Surgery, Fujian Medical University Union Hospital, FUZHOU, China; ${ }^{2}$ Fujian Medical University Union Hospital, FUZHOU, China

Aim: To study the differences in pathology, survival, and recurrence between special remnan gastric cancer (sRGC) and nonspecial RGC (nRGC).

Method: A total of 366 RGC patients were analyzed in 7 hospitals in China from January 2003 to July 2015.We compared the 3-year overall survival (OS) disease-free survival (DFS) rates and used two-step regression explore the influence of the RGC categories on patient outcomes.

Results: All of the patients divided into sRGC Group (Group S) $(n=200)$ and nRGC Group (Group N) $(\mathrm{n}=166)$. The R0 resection rate and lymph node (LN) dissection number of Group $S$ were significantly higher than Group $\mathrm{N}(\mathrm{P}<0.05)$. The difference in 3-year OS was not significant $(P=0.282)$, but the 3-year DFS of Group S was worse than Group N $(P=0.042)$. Twostep multivariate analyses showed $\mathrm{nRGC}$ was an independent risk factor for poor DFS. Of the 225 patients who had undergone R0 resection, 74 patients $(32.89 \%)$,suffered recurrence, and the recurrence rate of Group $\mathrm{S}$ was significantly higher than Group N $(\mathrm{P}=0.039)$, moreover, the LN recurrence rate of Group $\mathrm{S}$ was significantly higher than Group N $(\mathrm{P}=0.027)$. Cox regression analysis showed that age, CA199 level, N stage and category of RGC were independent risk factors for $\mathrm{RGC}$ recurrence.

Conclusion: sRGC has a higher R0 resection rate and LN dissection number than nRGC, bu among patients who had undergone radical gastrectomy, sRGC patients had worse DFS and a higher tendency for LN recurrence; thus, they should be treated differently in the clinic. 


\section{P522-UPPER GI-Gastric cancer}

BMI-ADJUSTED PROGNOSIS OF SIGNET RING CELL CARCINOMA IN PATIENTS UNDERGOING RADICAL GASTRECTOMY FOR GASTRIC ADENOCARCINOMA

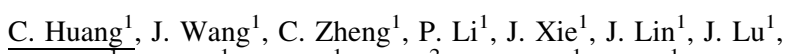

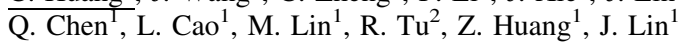

${ }^{1}$ Department of Gastric Surgery, Fujian Medical University Union Hospital, FUZHOU, China; ${ }^{2}$ Fujian Medical University Union Hospital, FUZHOU, China

Background: Compared with other histologic types, signet ring cell gastric carcinoma (SRC) has unique oncological characteristics, and its implication on the prognosis of gastric cancer patients remains unclear. The purpose of this study was to evaluate the prognostic impact of body mass index (BMI) on SRC patients.

Methods: A retrospective analysis was performed using the clinical records of 3342 patients with SRC or tubular adenocarcinoma who underwent radical gastrectomy between 2000 and 2014 Patients were divided into three groups according to histologic subtype: SRC, well-to-moderately differentiated adenocarcinoma (WMD), and poorly differentiated adenocarcinoma (PD). We compared the survival of SRC patients with that of tubular adenocarcinoma patients according to BMI

Results: The 5-year survival of SRC was significantly worse than that of WMD $(\mathrm{P}<0.001)$ but superior to that of $\mathrm{PD}(\mathrm{P}<0.001)$. BMI-stratified analysis showed that in the high-BMI group, the prognosis of SRC was similar to that of WMD $(\mathrm{P}>0.05)$ and better than that of PD $(\mathrm{P}<0.001)$. In normal-BMI patients, SRC had a worse prognosis than WMD $(\mathrm{P}<0.001)$ but a more favorable prognosis than $\mathrm{PD}(\mathrm{P}<0.001)$. SRC among low-BMI patients displayed much poorer survival than did both WMD $(\mathrm{P}<0.001)$ and $\mathrm{PD}(\mathrm{P}=0.005)$. Multivariate analysis indicated that the risk of death was lowest for SRC patients with a high BMI and highest for SRC patients with a low BMI (low-BMI hazard ratio: SRC 1 vs. WMD 0.51 and PD 0.53). Conclusion: SRC has worse prognostic impact as BMI decreases. BMI leads to differing prognosis of SRC compared with tubular adenocarcinoma.

\section{P523-UPPER GI-Gastric cancer}

SHORT-TERM EFFICACY OF SPLEEN-PRESERVING SPLENIC HILAR LYMPHADENECTOMY FOR ADVANCED UPPER GASTRIC CANCER: ROBOTIC VERSUS LAPAROSCOPYASSISTED

C. Huang, Z. Liu, Q. Chen, C. Zheng, P. Li, J. Xie, J. Wang, J. Lin, J. Lu, L. Cao, M. Lin, Z. Huang, J. Lin

Department of Gastric Surgery, Fujian Medical University Union Hospital, FUZHOU, China

Background: To investigate the short-term efficacies of robotic spleen-preserving splenic hilar lymphadenectomy (RSPSHL) and laparoscopic spleen-preserving splenic hilar lymphadenectomy (LSPSHL) for advanced gastric cancer using Huang's three-step maneuver.

Methods: Between April 2012 and July 2017, 643 patients who underwent spleen-preserving splenic hilar lymphadenectomy (SPSHL) were recruited. Baseline characteristics were compared in a 35-patient RSPSHL cohort and a 608-patient LSPSHL cohort. One-to-four propensity score matching was performed to determine between-group differences.

Result: In total, 175 patients were matched, including 35 patients who underwent RSPSHL and 140 who underwent LSPSHL. No significant differences in baseline characteristics were observed between these groups after matching. Significant differences in total operative time, estimated blood loss (EBL), splenic hilar blood loss (SHBL), splenic hilar dissection time (SHDT), and splenic trunk dissection time were detected between these groups (all $\mathrm{P}<0.05$ ). Furthermore, no significant differences were evident between RSPSHL and LSPSHL in the overall noncompliance rate of lymph node $(\mathrm{LN})$ dissection $(62.9 \%$ vs $60 \%, \mathrm{P}=0.757)$, No. $10 \mathrm{LN}$ retrieval $(3.1 \pm 1.4$ vs $3.3 \pm 2.5, \mathrm{P}=0.650)$, total retrieved $\mathrm{LNs}(37.8 \pm 13.1$ vs $40.6 \pm 13.6, \mathrm{P}=0.274)$, and postoperative complications $(14.3 \%$ vs $17.9 \%, \mathrm{P}=0.616)$ were found. A stratified analysis dividing RSPSHL into an early group (EG) and late group (LG) showed that with increasing surgical cases, EBL and SHBL were significantly lower in the EG and LG than in the matched LSPSHL group. Additionally, the LG showed significant improvements in EBL and SHBL compared with those in the EG (all P $<0.05$ ). Logistic analysis revealed that RSPSHL was a protective factor for decreased SHDT and SHBL (all $\mathrm{P}<0.05$ ).

Conclusion: RSPSHL is safe and feasible, resulting in surgical outcomes with a shorter SHDT and less SHBL than LSPSHL and a radical curative effect equivalent to that of LSPSHL.

\section{P524-UPPER GI-Gastric cancer}

EFFECT OF HIGH POSTOPERATIVE BODY TEMPERATURE ON LONG-TERM PROGNOSIS IN PATIENTS WITH GASTRIC CANCER AFTER RADICAL RESECTION

C. Huang ${ }^{1}$, H. Zheng ${ }^{1}$, J. Lu ${ }^{1}$, C. Zheng ${ }^{1},{\mathrm{P} . \mathrm{Li}^{1}, \mathrm{~J} . \mathrm{Xie}^{1} \text {, J. Wang }}^{1}$, J. Lin ${ }^{1}$, Q. Chen ${ }^{1}$, L. Cao ${ }^{1}$, M. Lin ${ }^{1}$, R. Tu ${ }^{2}$, Z. Huang ${ }^{1}$, J. Lin ${ }^{1}$

${ }^{1}$ Department of Gastric Surgery, Fujian Medical University Union Hospital, FUZHOU, China; ${ }^{2}$ Fujian Medical University Union Hospital, FUZHOU, China

Background: There is a lack of data on the effect of high postoperative body temperature on DFS in patients following a potentially curative resection of gastric adenocarcinoma.

Methods: The clinical and pathological data were selected based on patients who underwent radical gastrectomy from January 2006 to December 2011. The highest body temperature within 1 week after operation was used to establish diagnostic thresholds for high body temperature and low body temperature, which was obtained by X-tile software. The study used COX regression to analyze the influence of high body temperature on 5-year DFS.

Results: A total of 1396 patients were included in the analysis. The diagnostic threshold for high body temperature was defined as $38^{\circ} \mathrm{C} ; 370$ patients with a high postoperative body temperature were allocated to the high temperature group (HTG), while another 1026 patients were allocated to the low temperature group (LTG). Clinicopathologic data between the two groups showed no significant differences. The incidence of overall complications $(22.7 \%$ VS $8.5 \%, \mathrm{P}<0.001)$ and infectious complications $(18.9 \%$ VS $5.1 \%, \mathrm{P}<0.001$ ) in the HTG were significantly higher than those in the LTG. For all patients, survival analysis showed that 5-year DFS in the HTG was significantly lowe than that for the LTG $(55.6 \%$ VS $63.9 \%, \mathrm{P}=0.007)$. Multivariate analysis revealed that high postoperative body temperature was an independent prognostic risk factor for 5-year DFS (HR = 1.244 (1.032-1.499), $\mathrm{P}=0.022$ ). For patients with no complications, a survival analysis showed that the 5-year DFS rate in the HTG was lower than that for the LTG $(57.5 \%$ VS $64.4 \%, P=0.051)$, especially in patients with stage III gastric cancer $(31.3 \%$ VS $41.7 \%, \mathrm{P}=0.037)$. In addition, a multivariate COX regression analysis showed that high postoperative body temperature was an independent prognostic risk factor for 5-year DFS ( $\mathrm{HR}=1.302(1.031-1.646), \mathrm{P}=0.027$ ).

Conclusion: As an easily accessed index, high postoperative body temperature is an independent prognostic risk factor for gastric adenocarcinoma patients. For patients with stage III gastric cancer and no complications, high postoperative body temperature can significantly reduce the 5 -year DFS rate. Improved follow-up in these patients after operation should be encouraged.

\section{P525-UPPER GI-Gastric cancer}

'FIVE STEP' LAPAROSCOPIC-ASSISTED TOTAL GASTRECTOMY WITH LYMPH NODE DISSECTION FOR REMNANT GASTRIC CANCER FOLLOWING BILLROTH-II GASTRECTOMY

C. Huang, Z. Zheng, J. Lu, C. Zheng, P. Li, J. Xie, J. Wang, J. Lin, Q. Chen, L. Cao

Department of Gastric Surgery, Fujian Medical University Union Hospital, FUZHOU, China

Background: Laparoscopic surgery for remnant gastric cancer (RGC) is gaining interest ${ }^{1-4}$ However, due to adhesions to adjacent organs, displacement of anatomical structures, and changes in lymphatic flow triangulation, laparoscopic-assisted total gastrectomy (LATG) for RGC was considered challenging. In this study, we report our experience performing LATG with lymph node dissection for RGC following Billroth-II gastrectomy.

Methods: First step: exploration and separation of adhesions and the greater omentum. Second step: dissection of the lymph nodes (LNs) in the suprapancreatic area. Third step: baring of the right side of the esophagus. Fourth step: exposure of left gastroepiploic vessels and LNs dissection in the splenic hilar area. Fifth step: baring of the left side of the esophagus. The above procedure was performed for 45 RGC patients with stage cT1-4aNO/+ disease.

Results: There was no conversion to open surgery. Mean operation time was $195.0 \pm 52.5 \mathrm{~min}$, mean blood loss was $104.3 \pm 90.4 \mathrm{ml}$, and mean times to first flatus, fluid diet, and soft diet were $3.6 \pm 1.1$ days, $4.5 \pm 1.4$ days, and $9.0 \pm 5.1$ days, respectively. A mean of $19.8 \pm 12.7$ LNs were retrieved. The overall postoperative morbidity rate, major postoperative morbidity (ClavienDindo classification $\left.=\mathrm{III}^{5}\right)$ rate and mortality rate were $22.2 \%(10$ of 45$), 11.1 \%(5$ of 45$)$, and $0 \%$, respectively. At a median follow-up of 47 months, cumulative 3 -year overall survival was $56.8 \%$.

Conclusions: The novel 'Five step' LATG was technically safe and feasible, with acceptable surgical outcomes, in RGC patients following Billroth-II gastrectomy. 


\section{P526-UPPER GI-Gastric cancer}

\section{A NOVEL PROGNOSIS PREDICTION MODEL AFTER GASTRECTOMY FOR REMNANT GASTRIC CANCER: DEVELOPMENT AND VALIDATION USING INTERNATIONAL MULTICENTER DATABASES}

C. Huang ${ }^{1}$, Z. Zheng ${ }^{1}$, J. Lu ${ }^{1}$, C. Zheng ${ }^{1}$, Q. $\mathrm{He}^{2}$, W. $\mathrm{Lin}^{3}$, P. $\mathrm{Li}^{1}$, J. Xie ${ }^{1}$, J. Lin ${ }^{1}$, Q. Chen ${ }^{1}$, L. Cao ${ }^{1}$

${ }^{1}$ Department of Gastric Surgery, Fujian Medical University Union Hospital, FUZHOU, China; ${ }^{2}$ Department of gastrointestinal surgery, Fujian Medical University Union Hospital, FUZHOU, China; ${ }^{3}$ Department of Gastrointestinal Surgery and Gastrointestinal Surgery Research Ins, The Affiliated Hospital of Putian University, PUTIAN, China

Objective: To create a simple and universally applicable prediction model for remnant gastric cancer (RGC) patients after gastrectomy.

Background: Examined lymph node (ELN) counts of RGC patients are often insufficient. Therefore, the prognostic ability of TNM staging is limited.

Methods: A 5-year overall survival (OS) prediction model for RGC patients was developed using a test dataset of 148 consecutive patients. Model coefficients were obtained based on Cox analysis of clinicopathological factors. Prognostic performance was assessed with the concordance index (C-statistic) and decision curve analysis (DCA). For internal validation, the bootstrap method and calibration assessment were used. The model was validated using 2 external cohorts from China (First Affiliated Hospital of Fujian Medical University, $\mathrm{n}=46$ ) and USA (Mayo Clinic, $\mathrm{n}=20$ ). Result: pT stage, No. metastatic lymph nodes, pM stage and surgical duration were independent prognostic factors. Our model's C-statistic $(0.761)$ showed better discrimination power than the $8<\sup >$ th $<$ sup $>$-TNM staging $(0.714, \mathrm{P}=0.001)$. The model calibration was accurate in predicting 5-year survival. DCA showed that the model has a greater benefit. The results were also confirmed by bootstrap internal validation. In external validation, C-statistics and DCA showed good prognostic performance in patient datasets from 2 participating institutions. Moreover, we verified reliability of the model in an analysis of patients with different ELN counts $(>15$ or $=15)$. Conclusion: Utilizing clinically practical information, we developed a universally applicable prediction model for accurately determining the 5-year OS of RGC patients after gastrectomy. Our predictive model outperformed TNM staging in diverse international data sets regardless of ELN counts.

\section{P527-UPPER GI-Gastric cancer}

\section{A NOVEL ABDOMINAL NEGATIVE PRESSURE LAVAGE- DRAINAGE SYSTEM FOR ANASTOMOTIC LEAKAGE AFTER R0 RESECTION FOR GASTRIC CANCER}

\section{Huang, J. Lu, Z. Zheng, C. Zheng, P. Li, J. Xie, J. Wang, J. Lin, Q. Chen}

Department of Gastric Surgery, Fujian Medical University Union Hospital, FUZHOU, China

Objective: The aim of this study was to report our institution's experience with a novel abdominal negative pressure lavage-drainage system (ANPLDS) for anastomotic leakage (AL) after radical gastrectomy (RG) for gastric cancer (GC).

Background: AL is a severe complication associated with high morbidity and mortality after RG for GC. The optimal creation of drainage in AL patients after RG remains controversial.

Methods: The study enrolled 4173 patients who underwent R0 resection for GC at our institution between 2009 and 2016. ANPLDS was routinely used for patients with AL after January 2014 $\mathrm{AL}$ rates and postoperative outcome were compared before and after the ANPLDS therapy. We used multivariate analyses to evaluate clinicopathological and perioperative factors for associations with $\mathrm{AL}$ and failure-to-rescue (FTR) after AL.

Results: AL occurred in 83 patients $(83 / 4173,2 \%$ ), leading to 7 deaths. The AL rate was similar before (2009-2013, period 1) and after (2014-2016, period 2) the implementation of ANPLDS $(1.7 \%$ vs $2.3 \%, \mathrm{p}=0.121)$. Age and malnourished were independently associated with AL. The FTR rate and abdominal bleeding rate after AL occurred were respectively $8.4 \%$ and $9.6 \%$ for the entire period, but compared with period 1 , it significantly decreased at period $2(16.2 \%$ vs $2.2 \%$, $\mathrm{p}=0.041 ; 18.9 \%$ vs $2.2 \%, \mathrm{p}=0.020$, respectively). What's more, only ANPLDS therapy was an independent protective factor for FTR after AL.

Conclusion: Our experience demonstrates that ANPLDS is feasible and cost-effective for the management of AL after RG for GC

\section{P528-UPPER GI-Gastric cancer}

\section{A NOVEL TNM STAGING SYSTEM FOR GASTRIC CANCER BASED ON THE 'METRO-TICKET' PARADIGM:} A COMPARATIVE STUDY WITH THE AJCC-TNM STAGING SYSTEM

C. Huang, J. Lu, Z. Zheng, C. Zheng, P. Li, J. Xie, J. Wang, J. Lin, Q. Chen, L. Cao

Department of Gastric Surgery, Fujian Medical University Union Hospital, FUZHOU, China

Objective: To apply the principles of the 'Metro-ticket' paradigm to develop a novel TNM staging system (nTNM) for gastric cancer (GC)

Background: The 'Metro-ticket' prognostic tool for hepatocellular carcinoma has been proven to predict outcome, but a similar concept has not been investigated for GC

Methods: The nTNM considered the distance from the origin on a Cartesian plane incorporating the $\mathrm{pN}$ (x-axis) and $\mathrm{pT}$ (y-axis) stages. GC patients undergoing radical resection at Fujian Medical University Union Hospital (FMUUH) $(\mathrm{n}=4267)$ were included. The nTNM was validated using 2 external cohorts from the Sun Yat-sen University Cancer Center (SYSUCC) $(\mathrm{n}=1800)$ and Surveillance, Epidemiology, and End Results (SEER) $(\mathrm{n}=3227)$ databases.

Results: nTNM classes with the same distance from the origin have same stage; the stage increases with this distance. Among all patients, $48.0 \%(\mathrm{n}=2049)$ were restaged in the $\mathrm{nTNM}$ compared with the 7th edition of the AJCC-TNM classification; $26.2 \%(\mathrm{n}=1116)$ were downstaged in the nTNM compared with the 8 th edition. The nTNM provides significant survival differences between stages (all $\mathrm{P}<0.001$ ). The survival difference between stages IB and IIA was especially large for the nTNM $(P<0.001)$ compared to the 7 th and 8 th editions $(P=0.073)$ The concordance index and hazard ratio increased successively with the nTNM stage. Similar findings were observed in both external cohorts.

Conclusion: Compared with the AJCC-TNM classification, the 'Metro-ticket' nTNM for GC is easier to remember and provides some improvements; therefore, the nTNM may be considered for adoption in future editions of the AJCC-TNM classification.

\section{P529-UPPER GI-Gastric cancer}

\section{LONG-TERM USE OF PROTON PUMP INHIBITORS MAY INCREASE THE INCIDENCE OF NON-CARDIAC GASTRIC CANCER}

C. Huang, J. Lin, J. Lin, C. Zheng, P. Li, J. Xie, J. Wang, J. Lu, Q. Chen, L. Cao, M. Lin

Department of Gastric Surgery, Fujian Medical University Union Hospital, FUZHOU, China

Background: Studies have shown that long-term use of proton pump inhibitors may increase the risk of gastric cancer, but these studies remains controversial. We performed a systematic review with a meta-analysis of observational studies assess the risk for gastric cancer in PPI users compared with non- PPI users.

Methods: We searched the PUBMED, Cochrane Library, EMBASE and clinicaltrials.gov for relevant studies published between through January 1987 and May 2018. We calculated pooled risk ratio for gastric cancer in PPI users compared with non- PPI users by random-effects models. Two independently researchers search literatures, extracted data and evaluated quality according to inclusion and exclusion criteria. All analyses were performed with STATA version 13.0 (Stata, College Station, TX). Results: We analyzed data from 8 studies, comprising more than 927,684 patients, including 3 casecontrol studies and 5 cohort studies. The risk of gastric cancer in PPI users compared was significantly higher than non- PPI users $[\mathrm{RR}=2.10,95 \% \mathrm{CI}(1.17,3.97), \mathrm{P}=0.013]$ ? We also performed durationresponse relationship in this meta-analysis. Risk of gastric cancer was similar between the 2 groups when duration $=1$ year $[\mathrm{RR}=2.18,95 \% \mathrm{CI}(0.66-7.11), \mathrm{P}=0.199] ;$ While risk of gastric cancer for $\mathrm{PPI}$ users was higher than non- PPI users when duration between $1-3$ year, $=1$ year, $=3$ year and $=5$ year. The risk of gastric cancer when duration $=5$ year $(R R=2.03)$ and duration $=3$ year $(R R=1.95)$ are higher than risk of gastric cancer when duration between 1-3 year $(\mathrm{RR}=1.74)$. According to location subgroups meta-analysis,risk of non-cardiac gastric cancer for PPI users higher than non- PPI users[RR $=2.66,95 \% \mathrm{CI}(1.66-4.27)]$,risk of non-cardiac gastric cancer for PPI users higher than non- PPI users when duration $=1$ year[RR $=1.99,95 \% \mathrm{CI}(1.03-3.83)]$, while risk for cardiac gastric cancer was similar between the 2 groups $[\mathrm{RR}=1.86,95 \% \mathrm{CI}(0.71-4.89)]$. Risk of gastric cancer for PPI users is statistically significant higher than non-PPI users $[\mathrm{RR}=4.8$, $95 \% \mathrm{CI}(1.82-12.67)]$ prior helicobacter pylori infection, while risk of gastric cancer was similar between the 2 groups when helicobacter pylori infection $[\mathrm{RR}=0.91,95 \% \mathrm{CI}(0.17-4.90)]$.

Conclusion: Based on a systematic review with meta-analysis, we found the correlation between long-term use of PPI and the risk of gastric cancer and long-term use of PPI may increase the risk of non-cardiac gastric cancer when duration $=1$ year 


\section{P530-UPPER GI-Gastric cancer}

COMPLETE BLOOD COUNT-BASED INFLAMMATORY SCORE (CBCS) IS A NOVEL PROGNOSTIC MARKER FOR GASTRIC CANCER PATIENTS AFTER CURATIVE RESECTION

C. Huang ${ }^{1}$, J. Lin ${ }^{1}$, J. Lin ${ }^{1}$, C. Zheng ${ }^{1}$, P. Li ${ }^{1}$, J. Xie ${ }^{1}$, J. Wang ${ }^{1}$, J. $\mathrm{Lu}^{1}$, Q. Chen ${ }^{1}$, L. Cao ${ }^{1}, \mathrm{M} \cdot \mathrm{Lin}^{1}, \mathrm{R} . \mathrm{Tu}^{2}$

${ }^{1}$ Department of Gastric Surgery, Fujian Medical University Union Hospital, FUZHOU, China; ${ }^{2}$ Fujian Medical University Union Hospital, FUZHOU, China

Objective: To investigate the prognostic value of complete blood count (CBC)-based biomarkers for patients with resectable gastric cancer (GC).

Methods: Patients with GC who underwent curative resection between December 2008 to December 2013 were included. Estimated area under the curve (AUC) and multivariate Cox regression models were used to identify the best $\mathrm{CBC}$-based biomarker. Time-dependent receiver operating characteristics (t-ROC) analysis was used to compare the prognostic impact.

Results: Based on multivariate analysis, the lymphocyte-monocyte ratio (LMR) and hemoglobin $(\mathrm{Hb})$ level were the independent prognostic factors (both $\mathrm{P}<0.05$ ). Based on the LMR and $\mathrm{Hb}$ level, we established the CBC-based inflammatory score (CBCS). Higher CBCS was associated with older age, female sex, higher American Society of Anesthesiologists (ASA) score, proximal tumor location, larger tumor size, later stage and vascular involvement (all $\mathrm{P}<0.05$ ). Univariate analyses showed that higher CBCS was also associated with poorer overall survival (OS), which was consistent in each stage (all $\mathrm{P}<0.05$ ). Multivariate analysis revealed that the $\mathrm{CBCS}$ was a significant independent biomarker $(\mathrm{P}<0.05)$. Furthermore, $\mathrm{t}$-ROC curve of the CBCS was superior to that of the prognostic nutritional index (PNI), systemic immune-inflammation index (SII), modified Glasgow prognostic score (mGPS) and C-reactive protein/albumin ratio (CRP/ Alb) throughout the observation period.

Conclusion: Preoperative LMR and $\mathrm{Hb}$ were optimal CBC-based biomarkers for predicting OS in GC patients after curative resection. Based on the LMR and $\mathrm{Hb}$, we developed a novel and easily obtainable prognostic score called the CBCS, which may improve the prediction of clinical outcomes.

\section{P531-UPPER GI-Gastric cancer}

IS THE AJCC TNM STAGING SYSTEM STILL APPROPRIATE FOR GASTRIC CANCER PATIENTS SURVIVAL AFTER 5 YEARS?

C. Huang, J. Lin, J. Lin, C. Zheng, P. Li, J. Xie, J. Wang, J. Lu, Q. Chen, L. Cao, M. Lin

Department of Gastric Surgery, Fujian Medical University Union Hospital, FUZHOU, China

Purpose: The aim of this study was to evaluate the prognostic value of the eighth AJCC TNM staging classification for patients with gastric cancer who had already survived for 5 years. Patients and Methods: Patients who underwent radical gastrectomy at a large eastern center were considered. The prognostic value of staging systems were assessed and compared. Additional external validation was performed using a dataset from the Surveillance, Epidemiology, and End Result (SEER) database.

Results: The 5-year overall survival (OS) rate for patients in the training set was $59.4 \%$. With the prolongation of the survival time after surgery, the 5 -year OS improved significantly $(\mathrm{P}<0.05)$. However, there were no significant differences in survival curves among patients who have survived 5 years after surgery. The AUC and $\mathrm{c} 2$ of the eighth AJCC classification for predicting of 5-year OS decreased gradually after surgery and appeared stable after 5 years. For patients who survived 5 years after surgery, we constructed a new TNM staging system (nTNM) according to the survival curves of $\mathrm{T}$ stage and $\mathrm{N}$ stage. A 2-step multivariate analysis showed that nTNM, age and sex were independent prognostic factors. The nTNM demonstrated superior prognostic stratification, with higher c-statistic and likelihood ratio chi-square scores and lower AIC values than those of the AJCC classification. Similar results were observed in the external validation set.

Conclusion: The nTNM predicted an additional survival more accurately than did the AJCC classification for patients who have survived 5 years after surgery; this may guide decisions regarding surveillance.

\section{P532-UPPER GI-Gastric cancer}

PROGNOSTIC VALUE AND ASSOCIATION OF SARCOPENIA AND SYSTEMIC INFLAMMATION FOR GASTRIC CANCER PATIENTS FOLLOWING RADICAL GASTRECTOMY

C. Huang ${ }^{1}$, J. Lin ${ }^{1}$, J. Lin ${ }^{1}$, C. Zheng ${ }^{1}$, P. $\mathrm{Li}^{1}$, J. Xie ${ }^{1}$, J. Wang ${ }^{1}$, J. Lu ${ }^{1}$, Q. Chen ${ }^{1}$, L. Cao ${ }^{1}$, M. $\operatorname{Lin}^{1}$, R. Tu ${ }^{2}$

${ }^{1}$ Department of Gastric Surgery, Fujian Medical University Union Hospital, FUZHOU, China; ${ }^{2}$ Fujian Medical University Union Hospital, FUZHOU, China

Objective: To investigate the relationship between preoperative sarcopenia and systemic inflammation and evaluate the prognostic impact of these factors on patients with resectable gastric cancer (GC).

Methods: Patients with GC who underwent radical gastrectomy between December 2009 and December 2013 were included. A multivariate Cox regression analysis was performed to identify the prognostic factors. A novel prognostic score (SLMR) was developed based on preoperative sarcopenia and the lymphocyte-monocyte ratio (LMR), and its prognostic value was evaluated. Results: In total, 1167 patients with resectable GC were included in the study. On multivariate analysis, preoperative sarcopenia and the LMR were shown to be independent prognostic factors (both $\mathrm{P}<0.001)$. A low LMR was an independent predictor from sarcopenia $(\mathrm{P}<0.001)$. Based on preoperative sarcopenia and the LMR, we established the SLMR. An elevated SLMR was associated with older age, higher ASA scores, larger tumor size, advanced stages and vascular invasion (all $\mathrm{P}<0.05$ ). Multivariate analysis revealed that the SLMR was a significant independent predictor $(\mathrm{P}<0.001)$. We incorporated the SLMR into a prognostic model that included tumor size and TNM stage and generated a nomogram, which accurately predicted 3- and 5-year survival for GC patients.

Conclusion: Preoperative systemic inflammation is significantly associated with sarcopenia. The LMR combined with sarcopenia could enhance prognostication for GC patients who underwent radical gastrectomy and may open new therapeutic avenues to improve cancer outcomes.

\section{P533-UPPER GI-Gastric cancer}

ARE PATIENTS AGED LESS THAN 50 YEARS WITH PT1N0-3 AND PT2/3N0 GASTRIC CANCER ROUTINELY RECEIVING ADJUVANT CHEMOTHERAPY?

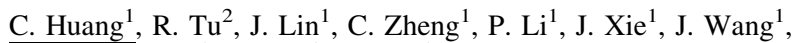
J. Lu ${ }^{1}$, Q. Chen ${ }^{1}$, L. Cao ${ }^{1}$, M. Lin ${ }^{1}$

${ }^{1}$ Department of Gastric Surgery, Fujian Medical University Union Hospital, FUZHOU, China; ${ }^{2}$ Fujian Medical University Union Hospital, Fujian Medical University Union Hospital, FUZHOU, China

Objective: To explore whether adjuvant chemotherapy is still needed in patients aged less than 50 years with pT1N0-3 and pT2/3N0 gastric cancer.

Methods: Multi-center cohort data of patients with gastric cancer who underwent radical gastrectomy were analyzed. Kaplan-Meier curves and Cox regression were used to analyze the relationships between chemotherapy and prognosis. Additionally, nomograms to predict the benefit of chemotherapy were established.

Results: In total, 1,432 patients with pT1N0-3 and pT2/3N0 gastric cancer were included. 217 patients $(15.2 \%)$ were aged $<50$ years. The 5 -year overall survival (OS) was not significantly different between the $<50$ years of age group and $=50$ years of age group $(92.6 \%$ vs. $90.4 \%$, respectively; $\mathrm{p}=0.249$ ). Lymph node $(\mathrm{LN})$ metastases (HR 5.054; $\mathrm{p}=0.002$ ) and $\mathrm{LN}$ dissection number $<15$ (HR 6.944; $\mathrm{p}<0.001$ ) were independent risk factors for the OS of patients aged $<50$ years. Adjuvant chemotherapy did not improve the 5 -year OS for patients aged $<$ 50 years with pT1N0-3 and pT2/3N0 gastric cancer $(\mathrm{p}=0.218)$. However, Chemotherapy showed a significant benefit $(\mathrm{p}=0.042)$ when there were $\mathrm{LN}$ metastases and/or $\mathrm{LN}$ dissection number was $<15$. Two nomograms were constructed, and the calculated difference was the potential benefit of adjuvant chemotherapy for the patients aged $<50$ years.

Conclusions: $\mathrm{LN}$ metastases and LN dissection number $<15$ were independent prognostic risk factors of patients aged $<50$ years with pT1N0-3 and pT2/3N0 gastric cancer. Patients with these risk factors may benefit from the addition of adjuvant chemotherapy. 


\section{P534-UPPER GI-Gastric cancer}

AGE-ADJUSTED CHARLSON COMORBIDITY INDEX (ACCI) IS PATIENTS WITH GASTRIC CANCER J. $\mathrm{Lu}^{1}, \mathrm{Q} . \mathrm{Chen}^{1}, \mathrm{~L} . \mathrm{Cao}^{1}, \mathrm{R} . \mathrm{Tu}^{2}$

${ }^{1}$ Department of Gastric Surgery, Fujian Medical University Union Hospital, FUZHOU, China; ${ }^{2}$ Fujian Medical University Union Hospital, FUZHOU, China

Objective: To assess the ability of the Age-Adjusted Charlson Comorbidity Index (ACCI) to predict survival after radical gastrectomy in patients with gastric cancer.

Method: Data from patients with gastric cancer (GC) who underwent radical gastrectomy from January 2008 to December 2012 in Fujian Medical University Union Hospital were retrospectively analyzed. Patients were categorized into either the high-ACCI group or low-ACCI group based on the effect of ACCI on long-term GC prognosis. The predictive value of the ACC relative to survival was further evaluated by the area under the curve (AUC) of receiver operating characteristic (ROC) curves and Harrell's concordance index (C-index).

Results: There were 1476 patients included in the analysis. The high-ACCI and low-ACCI groups had significant differences in preoperative abdominal surgery history, ASA grade, tumor size, tumor stage, histologic type, age and comorbidity (all $\mathrm{P}<0.05$ ). The incidence of postoperative complications was $17.9 \%$ in the high-ACCI group and was significantly higher than that in the low-ACCI group $(\mathrm{P}=0.001)$. The overall survival rate $(\mathrm{OS})$ and cancer-specific survival (CSS) rate in the low-ACCI group were both higher than those in the high-ACCI group $(\mathrm{P}<0.05)$. Univariate and multivariate analyses showed that the ACCI was an independent risk factor for OS and CSS $(\mathrm{P}<0.05)$. Furthermore, a combination of the TNM staging system and ACCI showed a trend toward higher prognostic value and higher AUC for OS and CSS than the TNM staging system alone $(\mathrm{P}<0.05)$

Conclusions: The ACCI was an independent risk factor for the long-term prognosis of GC patients after radical gastrectomy that could effectively improve the predictive efficacy of the TNM staging system for gastric cancer. A SIGNIFICANT FACTOR FOR PREDICTING SURVIVAL IN

C. Huang ${ }^{1}$, Y. Huang ${ }^{1}$, J. Lin ${ }^{1}$, C. Zheng ${ }^{1}$, P. Li ${ }^{1}$, J. Xie ${ }^{1}$, J. Wang ${ }^{1}$,

\section{P536-UPPER GI-Gastric cancer}

DEVELOPMENT AND VALIDATION OF A NEW STAGING SYSTEM FOR NODE-NEGATIVE GASTRIC CANCER BASED ON RECURSIVE PARTITIONING ANALYSIS

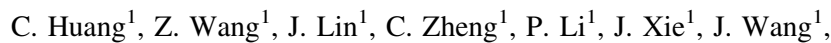
J. Lu ${ }^{1}$, M. Lin ${ }^{1}$, L. Cao ${ }^{1}$, R. Tu ${ }^{2}$, Z. Huang ${ }^{1}$, J. Lin ${ }^{1}$

${ }^{1}$ Department of Gastric Surgery, Fujian Medical University Union Hospital, FUZHOU, China; ${ }^{2}$ Fujian Medical University Union Hospital, FUZHOU, China

Aims: To establish a reasonable staging system for patients with node-negative gastric cancer (GC) based on recursive partitioning analysis (RPA).

Background: Whether the tumor-node-metastasis (TNM) staging system is suitable for patients with node-negative GC is still controversial. The modified staging system established by RPA showed good prognostic performance in a variety of cancers. The application of RPA has not been reported in the prognostic prediction of GC.

Methods: Node-negative GC patients who underwent radical resection at Fujian Medical University Union Hospital $(\mathrm{n}=862)$ and Sun Yat-sen University Cancer Center $(\mathrm{n}=311)$ with an at least 5-year follow-up information were selected as the training set. RPA was used to develop a modified staging system. Patients from the Surveillance, Epidemiology, and End Results databases $(n=1415)$ were selected as the external validation set.

Results: The 5-year overall survival (OS) rates of patients with 8th AJCC-TNM stage IA-IIIA in the training set were IA 95\%, IB 87\%, IIA 78\%, IIB 76\% and IIIA 73\%. Multivariate analysis (MVA) showed that larger tumor size, older age, and deeper depth of invasion were independent risk factors for OS in patients with node-negative GC (all P $<0.05$ ). Patients were reclassified into RPA I, RPA II, RPA III, and RPA IV stage based on RPA, the 5-year OS rates were $96 \%, 87 \%, 81 \%$, and $64 \%$, respectively, with significantly difference $(\mathrm{P}<0.05)$. Two-step MVA showed that the RPA staging system was an independent predictor for OS $(\mathrm{P}<0.05)$. Compared with the 8th AJCC-TNM staging system, the RPA staging system had a smaller AIC value ( 2544.863 vs. 2576.259 , respectively), higher c2 score (104.245 vs. 69.592 , respectively) and Harrell's C-index (0.697 vs. 0.669 , respectively). The same result was obtained in the validation set.

Conclusions: A new staging system for node-negative GC was successfully developed and validated, which has better prognostic performance than the 8th AJCC-TNM staging system.

\section{P537-UPPER GI-Gastric cancer}

\section{CLINICOPATHOLOGICAL FEATURES AND IMPACT} OF ADJUVANT CHEMOTHERAPY ON THE LONG-TERM SURVIVAL OF PATIENTS WITH MULTIPLE GASTRIC CANCERS

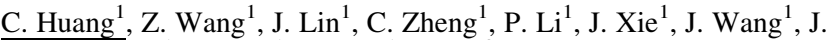

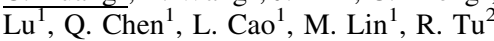

${ }^{1}$ Department of Gastric Surgery, Fujian Medical University Union Hospital, FUZHOU, China; ${ }^{2}$ Fujian Medical University Union Hospital, FUZHOU, China

Background: Little is known about the clinicopathological features and prognostic factors of multiple gastric cancers (MGC). We aimed to investigate the clinicopathological features and prognosis of patients with MGC and the impact of postoperative adjuvant chemotherapy on long-term survival.

Methods: The clinical and pathological data of patients diagnosed with gastric adenocarcinoma and undergoing radical gastrectomy from January 2007 to December 2016 were retrospectively analyzed. The clinicopathological features were analyzed, and prognostic differences between MGC and solitary gastric cancers (SGC) were compared after propensity score matching. Results: Among the 4107 patients investigated in this study, the incidence of MGC was 3.2\% (133/ 4107). Before matching, patients with MGC and SGC had differences in the type of gastrectomy, pathological tumor stage ( $\mathrm{pT}$ stage), pathological node stage ( $\mathrm{pN}$ stage), and pathological tumornode-metastasis stage (pTNM stage). After 1:4 matching the clinical data of 133 cases of MGC and 532 cases of SGC were comparable. The 5-year overall survival (OS) rate was $56.6 \%$ in the whole group, $48.1 \%$ in the MGC group and $58.7 \%$ in the SGC group, respectively, with a statistically significant difference. $(P=0.013)$. Multivariate analysis revealed that MGC, age, $\mathrm{pT}$ stage, $\mathrm{pN}$ stage, and adjuvant chemotherapy were independent predictors of OS (all $P<0.05$ ). Stratified analysis showed that, in advanced gastric cancer (AGC), the 5-year OS rates of MGC without adjuvant chemotherapy and SGC without adjuvant chemotherapy were $34.0 \%$ and $46.1 \%$, respectively, with a statistically significant difference $(P=0.025)$. The 5 -year OS rates of advanced MGC after adjuvant chemotherapy and of advanced SGC after adjuvant chemotherapy were $48.0 \%$ and $53.3 \%$, respectively, and the difference was not statistically significant $(P=0.292)$. The 5 -year OS rate of advanced MGC after adjuvant chemotherapy was significantly higher than that of patients without adjuvant chemotherapy $(48.0 \%$ vs. $34.0 \%, P=0.026)$

Conclusions: MGC is a poor prognostic factor after radical gastrectomy for gastric cancer. Postoperative adjuvant chemotherapy can improve the long-term prognosis of patients with advanced MGC

\section{HIGH BODY MASS INDEX DOES NOT AFFECT THE SURGICAL OUTCOME OF LAPAROSCOPIC TOTAL GASTRECTOMY: A MULTICENTER RETROSPECTIVE STUDY}

M. Miyasaka, Y. Ebihara, K. Tanaka, Y. Nakanishi, T. Asano, T. Noji, Y. Kurashima, S. Murakami, T. Nakamura, T. Tsuchikawa, K. Okamura, T. Shichinohe, S. Hirano

Department of Gastroenterological Surgery II, Hokkaido University Faculty of Medicine, SAPPORO, HOKKAIDO, Japan

Background: Obesity is considered one of the risk factors for poor outcome after surgery Although, laparoscopic total gastrectomy (LTG) for gastric cancer has been become popular in recent years, few studies have been conducted concerning outcomes of patients with obesity. The purpose of the study was to evaluate the relationship between high body mass index (BMI) and outcomes of LTG

Methods: The data of the patients who underwent LTG for gastric cancer at six institutions between January 2004 and October 2018 were retrospectively collected. Patients were classified into two groups according to BMI of $<25 \mathrm{~kg} / \mathrm{m}^{2}$ (332 patients; High BMI group) and $=25 \mathrm{~kg}$ / $\mathrm{m}^{2}$ (108 patients; Low BMI group). For these 440 patients, clinicopathological variables were analyzed using propensity score matching to mitigate the selection bias: sex, age, ASA physical states, clinical stage, laparoscopy-assisted total gastrectomy (LATG) or totally laparoscopic total gastrectomy (TLTG), D2 lymph node dissection, combined resection of other organs, method of anastomosis, jejunal pouch reconstruction. The surgical results and postoperative outcomes were compared and examined between the two groups.

Results: A total of 152 patients were matched for the analysis. Contrary to our expectations, there were no differences in the surgical results about operative time and estimated blood loss (low BMI $336.3 \pm 72.0 \mathrm{~min}$, high BMI $354.5 \pm 85.3 \mathrm{~min} ; \mathrm{p}=0.479$, low BMI $144.2 \pm 300.8 \mathrm{~g}$, high BMI $112.6 \pm 155.8 \mathrm{~g} ; \mathrm{p}=0.695$, respectively). Furthermore, there was no significant difference in postoperative outcome of complication (Clavian-Dindo $>$ IIIa) and the length of postoperative hospital stays (low BMI 13 cases, high BMI 10 cases; $p=0.522$, low BMI $18.7 \pm 14.3$ days, high BMI $19.0 \pm 19.7$ days; $\mathrm{p}=0.246$, respectively).

Conclusions: LTG for gastric cancer could be performed safely even in obesity patients in comparison with non-obesity patients. 


\section{P538-UPPER GI-Gastric cancer}

THE OUTCOME FOR BILLROTH II RECONSTRUCTION AFTER LAPAROSCOPIC DISTAL GASTRECTOMY IN OUR DEPARTMENT

\section{T. Tsuji}

Gastroenterological Surgery, Ishikawa Prefectural Central Hospital, KANAZAWA, Japan

Objective: The choice of reconstruction after distal gastrectomy remains controversial. We have performed Roux-en-Y (R-Y) method after laparoscopic distal gastrectomy(LDG) as a standard since 2008, but we have performed Billroth II (B-II) method in an increasing number of cases, depending on the patient. We retrospectively investigated the outcomes of patients with B-I method after laparoscopic distal gastrectomy in our hospital.

Methods: Patients who underwent B-II and R-Y reconstruction after LDG from January 2008 to December 2015 were included. The patient characteristics, surgical outcomes, and postoperative outcomes between the 2 procedures were retrospectively analyzed. We also compared extend of gastritis on endoscopy and loss of body weight after surgery at 1 year.

Results: B-II / R-Y :110/307. B-II was selected in the elderly patients with poor ASA-PS $(\mathrm{p}<0.001)$. In surgical outcomes, operative time was shorter for B-II than $\mathrm{R}-\mathrm{Y}(\mathrm{p}<0.001)$, and blood loss was also smaller $(p=0.023)$. In postoperative outcomes, there were significant differences in complications (?grade3) (B-II vs. R-Y: 0.9 vs. $6.8 \%, \mathrm{p}=0.013$ ) and length of stay (BII vs. R-Y: median 10.5 vs. 14-day, $\mathrm{p}<0.001)$. There was significant difference in presence of gastritis between B-II (35.4\%) and R-Y $(11.0 \%)(\mathrm{p}<0.001)$, but no significant difference in loss of body weight $(\mathrm{p}=0.105)$.

Conclusion: B-II reconstruction may be an adequate procedure for high-risk cases because of its shorter operative time and the absence of severe complications.

\section{P540-UPPER GI-Gastric cancer}

DELTA-SHAPED ANASTOMOSIS VERSUS CIRCULAR STAPLER ANASTOMOSIS AFTER LAPAROSCOPIC DISTAL GASTRECTOMY WITH BILLROTH I RECONSTRUCTION

$<\mathrm{u}>$ K. Hosoda $</ \mathrm{u}>^{1}$, H. Mieno ${ }^{2}$, A. Ema ${ }^{2}$, H. Ushiku ${ }^{2}$, M. Washio ${ }^{2}$, K. Yamashita ${ }^{2}$, M. Watanabe ${ }^{3}$

${ }^{1}$ Upper gastrointestinal surgery, Kitasato University School of Medicine, SAGAMIHARA, Japan; ${ }^{2}$ Upper gastrointestinal surgery, Kitasato University, SAGAMIHARA, Japan; ${ }^{3}$ Surgery, Kitasato University, SAGAMIHARA, Japan

Purpose: The aim of this study is to evaluate the efficacy of delta-shaped anastomosis compared to circular stapler anastomosis in laparoscopic distal gastrectomy with Billroth I reconstruction (LADG-BI)

Method: This is a single-center randomized controlled study. Eligibility criteria included histologically proven gastric adenocarcinoma in the lower third of the stomach, clinical stage tumor. Patients were preoperatively randomized to circular stapler anastomosis or delta-shaped anastomosis. The primary endpoint is the number of analgesics use during 3 days after surgery. We compared the surgical outcomes of the two groups. Postoperative QOL was evaluated using the Postgastrectomy Syndrome Assessment Scale-45. This trial was registered at the UMIN Clinical Trials Registry as UMIN000016496.

Results: Between December 2016 and September 2018, 39 patients (delta-shaped anastomosis 18, circular stapler anastomosis 21) were enrolled. There was no difference in the number of analgesics use during 3 day after surgery (median 9: delta-shaped anastomosis vs. 7: circular stapler anastomosis, $\mathrm{P}=0.91$ ). There was no difference in the overall proportion with in-hospital grade II-IIIB surgical complications (11\%: delta-shaped anastomosis, $14 \%$ : circular staple anastomosis). There was no operation-related death in either arm. Regarding postoperative QOL evaluated 1 month after surgery, diarrhea subscale was significantly worse in delta-shaped anastomosis than in circular stapler anastomosis.

Conclusion: We did not demonstrate the advantage of delta-shaped anastomosis in terms of postoperative pain. Since delta-shaped anastomosis tended to cause postoperative abdominal symptoms related to diarrhea, we should carefully apply the delta-shaped anastomosis to LADG.

\section{P539-UPPER GI-Gastric cancer}

COMPARISON OF RECURRENCE BETWEEN 3D AND 2D LAPAROSCOPIC RADICAL GASTRECTOMY: USING RANDOMIZED PHASE III TRIAL DATA

\author{
C. Huang, B. Xu, J. Lu, P. Li, C. Zheng, J. Xie
}

Department of Gastric Surgery, Fujian Medical University Union Hospital, FUZHOU, China

Background: Numerous studies have shown that the short-term efficacy of three-dimensional (3D) laparoscopic radical gastrectomy (LG) is comparable to that of two-dimensional (2D)-LG. Whether 3D-LG affects the recurrence pattern after surgery has not been investigated. Using data from a prospective clinical trial, the present study compares the recurrence patterns between 2DLG and 3D-LG.

Methods: From January 2015 to April 2016, a total of 419 patients were recruited for the clinical trial (NCT02327481). The recurrence types, the first recurrence time and recurrence-free survival (RFS) were compared between the two groups. Multivariate analyses of factors associated with RFS were performed to identify whether 3D-LG affects the recurrence patterns.

Results: Ultimately, 401 patients were analyzed (197 in the 2D-LG group and 204 in the 3D-LG group), and there were no differences in the clinicopathological data between the two groups. Distant metastasis was the most common type of recurrence. There were no significant differences between the two groups in the recurrence types, the first recurrence time or RFS (all $\mathrm{p}>0.05$ ). According to the 7th American Joint Committee on Cancer tumor-node-metastasi (TNM) staging system, both groups were stratified into pathological (p) I, II, and III stages. The stratified analysis showed that there were no statistically significant differences in RFS between the $2 \mathrm{D}$ group and the $3 \mathrm{D}$ group among patients in each subgroup (all $\mathrm{p}>0.05$ ). The multivariate analysis of RFS showed that pathological TNM (pTNM) stage and lymphovascular invasion were independent risk factors (all $\mathrm{p}<0.05$ ). The multivariate analysis of post-recurrence survival (PRS) showed that adjuvant chemotherapy was an independent protective factor $(\mathrm{p}=0.043)$. Conclusions: Distant metastasis was the most common type of recurrence after LG. The postoperative recurrence patterns, RFS and PRS after 3D-LG were similar to those after 2D-LG.

\section{P541-UPPER GI-Gastric cancer}

\section{THREE-DIMENSIONAL (3D) VISUALIZATION PROVIDES BETTER OUTCOME IN SINGLE-PORT LAPAROSCOPIC DISTAL GASTRECTOMY}

S.H. Kang, Y.S. Cho, S.H. Min, Y.S. Park, S.H. Ahn, D.J. Park, H.H. Kim

Department of Surgery, Seoul National University Bundang Hospital, 13620, Korea

Introduction: The use of a three-dimensional(3D) camera for laparoscopic surgery has been reported in literature. However, there are only few comparative studies demonstrating its benefits, and no reports on the application of 3D vision to single-incision laparoscopic surgery. This study aims to compare 3D vision to the previous two-dimensional(2D) system in solo single-incision laparoscopic distal gastrectomy(SIDG).

Methods: Medical charts of 179 gastric cancer patients who underwent solo SIDG from February 2014 to December 2017 were retrospectively reviewed. Patients were grouped into either 2D group or 3D group depending on the type of camera used. All the operations were performed by a single surgeon using a flexible camera(Olympus, Japan), fixed onto a passive scope holder without the use of a scopist or an assistant. Operative data, postoperative outcome, and early complication were analyzed.

Results: Ninety had their operations under 2D vision and 89 used the 3D scope. In both groups, there was no difference in age, body mass index, staging, and other demographic or histopathologic criteria. Operative time was significantly faster in the 3D group $(115.6 \pm 34.0 \mathrm{vs}$. $129.4 \pm 38.5$ mins., $\mathrm{p}=0.012)$ and EBL was also less $(20.7 \pm 30.0$ vs. $35.1 \pm 56.0 \mathrm{ml}$, $\mathrm{p}=0.034)$. Patients in the $3 \mathrm{D}$ group started small fluid diet faster $(2.5 \pm 0.9$ vs. $3.0 \pm 1.1$ postoperative days, $\mathrm{p}=0.006)$, and were discharged faster $(4.4 \pm 1.7$ vs. $5.2 \pm 3.1$ postoperative days, $\mathrm{p}=0.024)$. Early complication was also less in the $3 \mathrm{D}$ group $(2.2 \%$ vs. $6.7 \%)$ but there was no statistical significance $(\mathrm{p}=0.140)$.

Conclusion: The use of the 3D camera improves operative outcome and hospital stay in patients undergoing solo SIDG. 


\section{P542-UPPER GI-Gastric cancer}

\section{SENTINEL LYMPH NODE AND FUNCTION-PRESERVING GASTRECTOMY IN THE PATIENTS WITH EARLY GASTRIC CANCER}

\author{
V.V. Grubnik ${ }^{1}$, Y.V. Grubnik ${ }^{2}$, V.V. Grubnyk ${ }^{3}$, V.V. Ilyashenko ${ }^{3}$, \\ R.P. Nikitenko ${ }^{3}$
}

${ }^{1}$ Department of surgery, Odessa medical university, ODESSA, Ukraine; ${ }^{2}$ Departmen of surgery \#2, Odessa medical university, ODESSA, Ukraine; ${ }^{3}$ Department of surgery \#1, Odessa medical university, ODESSA, Ukraine sentinel lymph node (SLN) concept.

Methods: During last 5 years in two clinics Odessa National Medical University we used mapping procedures in the 25 patients with early gastric cancer. There were 11 men and 14 women, age 52 to 85 years, mean age $56.8 \pm 8.2$ years. Blue dye was injected into 4 quadrants of the submucosal layer surrounding the primary lesion using an endoscopic puncture needle in 16 patients. Blue lymphatic vessels and blue-stained lymph nodes can be identified by laparoscopy within $15 \mathrm{~min}$. of the blue dye injection. We used $0.5 \%$ indocyanine green in 9 patients, which we injected by intraoperative endoscopy. New technology indocyanine green (ICG) fluorescent imaging was used for SLN mapping in this 9 patients.

Results: Amany 16 patients, in which we used blue dye for mapping SLN, positive SLN was in 5 patients, negative - in 11 patients. In all 16 patients distal gastrectomy (DG) was performed with D2 lymphdissection. From 11 patients with negative SLN in 3 patients metastasis in other lymph nodes were detected.Among 9 patients in whom we used ICG fluorescent mapping positive SLN were detected in 2 patients. Laparoscopic-assisted distal gastrectomy with D2 lymph node dissection was performed in these patients. In 7 patients with negative SLN partial wedge resection was performed in 2 patients, segmental pylorus preserving gastrectomy was performed in 5 patients. During follow-up period from 3 to 24 months no recurrences or metastasis were detected in these group of patients. QoL in this group of patients was much better, than in patients with conventional distal gastrectomy.

Conclusions: ICG fluorescent method is highly effective for detection of SLN. In the patients with early gastric cancer function preserving gastrectomy based on SLN navigation may be promising strategy to achieve better results.

\section{P543-UPPER GI-Gastric cancer}

\section{LAPAROSCOPIC PROCEDURE TAKING ADVANTAGE OF ROBOTIC GASTRECTOMY FOR GASTRIC CANCER TO PREVENT PANCREATIC FISTULA}

\section{Y. Watanabe}

Gastrointestinal surgery and surgical oncology, Ehime University, TOON-CITY, Japan

Backgrounds and Aims: Analysis of Japanese national clinical database (NCD) showed that laparoscopic gastrectomy(LG) had rather increased pancreatic fistula (PF) compared with open gastrectomy. On the other hand, last year, multicenter collaborative research result of robotic gastric cancer surgery(RG)was shown that the complications including PF were significantly decreased as compared with LG. In this study, we have employed a new easy to use device in LG to minimize $\mathrm{PF}$ during suprapancreatic lymph nodes dissection requiring pancreatic retraction and compared with conventional LG and RG.

Materials and methods: Internal organ retractor (Aesculap $\left.{ }^{\circledR}\right)$ to grasp the gastropancreatic fold and the suprapancreatic peritoneum to imitate daVinci's forceps was guided with a thread outside the body. 104 patients(Jan.2016 Nov.2018) were divided into three groups as follows, Group LG-1(N = 40), LG using the standard devices, Group LG-2(N $=40)$, LG using organ retractor, Group RG $(\mathrm{N}=24)$. Amylase value in drain(d-amylase) and the volume in drainage, intraoperative bleeding, postoperative hospital stay, incidence of $\mathrm{CD}$ ( $\geqq$ grade III) were compared among three groups.

Results: Data are indicated as LG-1/LG-2/RG(mean $\pm \mathrm{SD}$ ), respectively. On the day and third day after surgery, d-amylase were $1203 \pm 260 / 645 \pm 148 / 608 \pm 285$ and $383 \pm 228$ / $323 \pm 136,176 \pm 98(\mathrm{IU} / \mathrm{L})$. d-Amylase was significantly lower in LG-2 and RG group than in LG-1 the day after surgery. The operation time was significantly longer in RG, $318 \pm 38$ / $290 \pm 64 / 396 \pm 47$ ( $\mathrm{min}$ ). Bleeding volume and hospital stay did not differ among 3 groups. Pancreatic fistula (CD $\geq$ grade III)was observed only in LG-1 group at 5(\%).

Discussion: $\mathrm{PF}$ (grade $\geqq \mathrm{CDIII})$, which may lead to mortality, occurred in LG-1 group. A significant elevation of d-amylase on the 1st postoperative day was prevented in LG-2 just like RG, which seemed to lead to prevent PF afterwards. The multijoint forceps is known to be an advantage of RG but it cannot be reproduced by LG using a linear forceps. However, another advantage such as vertical grasping and lifting of the gastropancreatic fold at rest could be mimicked by LG using this device, which seemed to enable a safe lymph node dissection and lead to reduce the pancreatic damage. Conclusion: This inexpensive and easy to use method taking the advantage of RG seems to reduce surgeon's fatigue and tissue damage(PF).

\section{P544-UPPER GI-Gastric cancer}

'MANY WAYS TO SKIN GASTRIC CANCER'-ROBOTIC VERSUS LAPAROSCOPIC VERSUS OPEN GASTRECTOMY

E. Kakiashvili ${ }^{1}$, E. Brauner ${ }^{2}$, O.B. Ben Ishai ${ }^{2}$

${ }^{1}$ General Surgery, Galilee Medical Center, KIRIAT MOZKIN, Israel; ${ }^{2}$ General Surgery, Rambam Medical Center, HAIFA, Israel

Aim: robotic techniques relevance in gastric cancer surgery is being examined.

The study presents comparison of perioperative outcome between different surgical approaches for gastric adenocarcinoma (AC).

Methods: retrospective cohort of 85 patients that underwent gastrectomy for (AC) at Rambam Hospital during 2012-2016. Patients data was collected based on demographic characteristics, BMI, operating room time (ORT), number of lymph nodes (LN), length of hospitalization (LOH), and perioperative complications.

Results: study population included 55 patients after total gastrectomies, 10 of them robotic and 30 partial gastrectomies, 12 of them robotic. Age, gender and BMI were similar between patients who underwent any type of procedures.

Median length of hospitalization ( $\mathrm{LOH}$ ) for robotic total gastrectomy was 4.5 days and it was significantly shorter than both laparoscopic total gastrectomy (LTG) 7.0 days $(p=0.003)$ and open total gastrectomy (OTG) 9.0 days $(\mathrm{p}<0.001)$. Similar significant differences in $(\mathrm{LOH})$ between the groups were observed among patients who underwent partial gastrectomy, but the comparison between robotic and laparoscopic procedures was limited due to small numbers of (LPG)

Median(ORT) was significantly longer among robotic gastrectomies compared to open, the difference was $64 \mathrm{~min}$ in total gastrectomy group and $145 \mathrm{~min}$ in partial gastrectomy group ( $p<0.001$ for both differences), but the difference in(ORT) between laparoscopic and robotic procedures were smaller and non-significant.

The number of dissected (LN) was similar between the 3 procedures in total gasrectomies. In partial gastrectomies, the number of dissected (LN) was even higher among both laparoscopic and robotic gastrectomies compared to open $(\mathrm{p}<0.001)$.)

Conclusions: robotic total and partial gastrectomies for gastric (AC) are associated with oncologically adequate lymphadenectomy and faster patient recovery, but longer ORT.

\section{P545-UPPER GI-Gastric cancer}

\section{TECHNIQUE OF ESOPHAGOJEJUNOSTOMY USING ORVIL AFTER LAPAROSCOPY ASSISTED TOTAL GASTRECTOMY (LATG) FOR GASTRIC CANCER}

S. Sakuramoto, H. Sugita, K. Watanabe, S.I. Ishii, S.I. Itou, N. Fujiwara, Y.M. Miyawaki, H. Sato, K. Okamoto, S. Yamaguchi, I. Koyama

Gastroenterological Surgery, Saitama Medical University International Medical Center, HIDAKA, Japan

Objectives: During esophagojejunostomy using a circular stapler after LATG, placement of the anvil head via the transabdominal approach proved difficult. The authors report on a method modified for laparoscopy-assisted, esophagojejunostomy performed by placing the pretilted anvil head(OrVil) via the transoral approach.

Methods: Between January 2013 and November 2018, esophagojejunostomy was performed using OrVil in 99 patients after LATG. The anesthesiologist introduced the anvil while observing its passage through the pharynx. During the anastomosis, we kept the jejunum fixed in position with a silicone band Lig-A-Loops, thereby preventing the intestine from slipping off the shaft of the stapler.

Results: Esophagojejunostomy using the OrVil was achieved successfully in all patients. No other complications, such as hypopharyngeal perforation and/or esophageal mucosal injury, occurred during passage. The postoperative complications of anastomosis were leakage in two patients and stenosis in 5 patients, in whom mild relief was achieved using a bougie. Conclusions: Esophagojejunostomy using the OrVil is a simple and safe technique. 


\section{P546-UPPER GI-Gastric cancer}

\section{FLUORESCENT LYMPHOGRAPHY-GUIDED LYMPHADENECTOMY DURING GASTRECTOMY FOR GASTRIC CANCER}

B. Molteni, P. Porsio, S. Molfino, S. Benedicenti, M.S. Alfano, N. Portolani, G. Baiocchi

General Surgery, University of Brescia-Spedali Civili, BRESCIA, Italy

Background and aim: Recently indocyanine green (ICG) was introduced in clinical practice as a fluorescent tracer. The use of ICG for sentinel lymph node (LN) mapping was investigated in lots of fields such as breast, melanoma, gynecological, urological, gastric, colo-rectal cancer. Recently ICG lymphography was introduced to investigate the feasibility of fluorescence-guided lymphadenectomy. Our study aims to investigate the feasibility of ICG fluorescence to detect lymphatic drainage in all $\mathrm{T}$ stage gastric cancer.

Methods: We conduced a single center prospective trial. We included patients with gastric cancer candidate to surgery. ICG was injected intraoperative or the day before surgery, via submucosal or subserosal. Total or subtotal gastrectomy was performed open, laparoscopic or video-assisted access. During gastric cancer standard lymphadenectomy we studied lymphatic flow and LN bright in vivo and ex vivo. After specimen removal we re-checked the operatory field to detect residual fluorescence.

Results: We included 13 patients. A total of 100 lymph node stations have been removed, for a total of 501 lymph nodes. 70 stations resulted being fluorescent (70\%) including 398 lymph nodes. 76 lymph node were metastatic, none resulted belonging to a lymph node station outside bright lymphatic basin. The mean number of $\mathrm{LN}$ dissected per patient was 38,5. In 2 cases fluorescence was positive in station $11 \mathrm{p}$ and in 1 case in $8 \mathrm{p}$ the results was $1 \mathrm{LN} 11 \mathrm{p}$ positive and the second $11 \mathrm{p} \mathrm{LN}$ station and $8 \mathrm{p}$ were negatives.

Conclusion: Fluorescent lymphography-guidedlymphadenectomy is a new promising technique. Until now no metastatic LN were found outside bright station. Maybe this technique can help the surgeon to perform a complete lymphadenectomy and decision making in extend extra D2 lymphadenectomy during gastric cancer surgery.

\section{P547-UPPER GI-Gastric cancer}

\section{LAPAROSCOPIC GASTRECTOMY FOR CANCER IN ELDERLY PATIENTS}

R. Mikami, E. Tanaka, S. Ishida, Y. Matsui, K. Horita, M. Yamada, T. Murakami, T. Kan, R. Nitta, T. Harada

\section{Surgery, Kobe City Medical Center West Hospital, KOBE, Japan}

Introduction: In Japan, the number of elderly patients with gastric cancer has been increasing in correlation with the increase in average age of the population. The aim of this study is to assess the safety and efficacy of laparoscopic gastrectomy for cancer in elderly patients compared with the short-term outcome in the nonelderly.

Method: We reviewed 231 patients who underwent laparoscopic gastrectomy (dital gastrectomy,proximal gastrectomy,total gastrectomy)between January 2010 and December 2016.Of these, 41 patients(aged 80 years or more) were compared with 190 younger patients. Patient characteristics,operative factor and short-term outcomes were analyzed.

Results: ASA-PS $\geq$ GradeII was more common in elderly patients than in the nonelderly $(87.8 \%$ versus $48.9 \% ; \mathrm{P}<0.001)$.Pulmonary and cardiovascular disease were more common in elderly patients than in the nonelderly $(19.5 \%$ versus $7.9 \% ; \mathrm{P}=0.012, \quad 60.9 \%$ versus $41.6 \% ; \mathrm{P}=0.021)$. The incidence of advanced cancer(StageIIor more)was higher in elderly patients $(56.1 \%$ versus $32.6 \% \cdot \mathrm{P}=0.002) \mathrm{D} 2$ lymph node dissection was performed less in the elderly patients than in nonelderly $(2.4 \%$ versus $28.4 \% ; \mathrm{P}<0.001)$. There were no significant differences in the operating time,blood loss and postoperative hospital stay. There were no significant differences in the incidence of postoperative morbidity.

Conclusion: In elderly patients, there was a tendency of reduction surgery being selected according to individual condition, but there was no significant difference in the short-term outcome.Hence,we conclude that laparoscopic gastrectomy is indicated even in elderly patients.

\section{P548-UPPER GI-Gastric cancer}

\section{IMPROVED TECHNIQUE OF VACUUM THERAPY IN ANASTOMOTIC LEAKAGE AFTER GASTRECTOMY}

A. Voynovskiy, E. Kryukov, A. Chuprina, E. Artemkin, A. Sokolov, S. Kozyrev, K. Tychinskaya

Abdominal, Named after N.N. Burdenko Main Military Clinical Hospital,, MOSCOW, Russia

The frequency of anastomotic leakage after gastrectomyreaches $7-8 \%$. At the same time, mortality in this group of patients reaches $30 \%$, and the use of aggressive methods of surgical treatment for the treatment of anastomotic leakage increases the mortality rate from 20 to $64 \%$. Since 2006, vacuum-assisted closure has been used to treat anastomotic leakage of various localizations. The essence of this method is based on the creating a local negative pressure, which is transmitted to the drip cavity through a special porous spongy system. The negative pressure created in the closed cavity, allows you to remove exudate, helps to reduce tissue swelling, improvesmicrocirculation, which in turn contributes to the development of granulations and wound healing with separation of the fistulous course. Failures in using the method of vacuum therapy in anastomotic leakage are associated with the great difficulty of delivering a polyurethane sponge with a drainage tube to the leakage zone. In this regard, we have developed an improved method of endoscopic local vacuum therapy, in which the delivery of a polyurethane sponge was carried out with the help of a thread through a pharyngeal ring, a leakage zone and brought out through a drainage tube.

This technique has been successfully used in the treatment of four patients with anastomotic leakage after operations on the upper part of the digestive tract. For complete healing of the cavity of the leakage and defect of the organ wall, it took $6,9,10$ and 5 sessions of replacing the VAC system, respectively (average $7.5 \pm 2.4$ ). There were no complications during the endoscopic local vacuum therapy. When the control endoscopic studies after 3 months after the completion of the treatment at the site of defects of the seams of the anastomoses formed tender scar tissue without signs of narrowing of the organ.

\section{P549-UPPER GI-Gastric cancer}

\section{EARLY RED FLAGS ASSOCIATED WITH DELAYED DISCHARGE IN PATIENTS SUBMITTED TO GASTRECTOMY: ANALYSIS OF PERIOPERATIVE VARIABLES AND ERAS PROTOCOL ITEMS}

P. Parise, A. Cossu, F. Puccetti, M. Palucci, R. Cerchione, L. Cinelli, R. Rosati, Gastrointestinal Surgery Unit, San Raffaele Hospital, MILAN, Italy

Aims: Enhanced Recovery After Surgery pathways are safe and effective for patients undergoing gastrectomy. This study aimed to identify perioperative factors influencing the adherence to the protocol, the postoperative course, and the consequent length of stay.

Methods: Between 2014 and 2017, 201 patients were referred to our institution for gastric cancer. Among these, 21 patients underwent atypical gastric resection and were excluded from this analysis. 187 were assigned to either total or distal gastrectomy and represent the study population. All patients were managed with a standardised perioperative pathway according to ERAS principles. According to data from the literature and based on our clinical experience, patients with optimal adherence to ERAS protocol may fit the criteria for discharge within ninth postoperative day, that was considered our ideal threshold for hospital discharge.

Data were retrospectively collected and analysed from a prospectively maintained database. Statistical analyses were performed using SPSS version 24 for Macintosh. The $\chi^{2}$ test, with a significance level of 0.05 , was used to investigate the association between the outcome and perioperative categorical variables. When parametric assumptions were met, Student's two-tailed t-test was used to compare the means of continuous variables; otherwise, the Mann-Whitney test was performed. A significance level of 0.05 was chosen. Logistic binary regression with a backward selection procedure and selection criteria of $\mathrm{p}$-value $<0.05$ were exploited to determine significant predictors

Results: 44 preoperative, intraoperative and early postoperative variables were considered. Among all, multivariate regression analysis revealed that incomplete preoperative immunonutrition, failure to extubate the patient at the end of surgery, intraoperative crystalloids infusions $>2150 \mathrm{ml}$ and blood transfusion $>268 \mathrm{ml}$, surgery duration $>195 \mathrm{~min}$, and failure to mobilise patients within $24 \mathrm{~h}$ from surgery were associated with delayed discharge. The logistic regression model was statistically significant $(\mathrm{p}<0.001)$ and correctly classified $73.6 \%$ of cases. Sensitivity and specificity were $74.1 \%$ and $73.2 \%$, respectively.

Conclusions: Results seem to be clinically rational and focus the attention on the importance of some perioperative clinical issues for the management of postoperative course. These variables could be considered as clinical goals to be reached in order to get an early discharge. 


\section{P550-UPPER GI-Gastric cancer}

\section{SHORT-TERM OUTCOMES OF LAPAROSCOPIC TOTAL GASTRECTOMY IN OUR HOSPITAL}

\section{K. Watanabe, S. Shinichi, S. Yamaguchi, I. Koyama}

Digestive surgery, Saitama Medical University International Medical Center, SAITAMA HIDAKA CITY, Japan

Introduction: We have worked on stereotyping of a hand skill by making it a coincidence way using Circular stapler (CS) like laparotomy up to now at this hospital. A coincidence way using Linear stapler (LS) is being also taken in in recent years. It was compared and examined according to the coincidence way about short-term results in LTG this time.

Method: 115 patients diagnosed as the cancer of the stomach (less than cT 2 and N 1) by this hospital from January, 2013 to April, 2018 and carried out LTG . A patient factor (the gender, the age and BMI), an operation factor (operation time, the bleeding amount, lymph node dissection and conjurer), a coincidence related complication (Clavien Dindo classification, sutural insufficiency of Grade more than 2, anastomotic stricture, anastomotic region bleeding and reflux esophagitis) and the post-operatively length of stay were considered

Result: CS crowd met 98 cases (85.2\%) and LS cluster (14.8\%) 17 cases. 70 years old of age medians (38-84), men and women were 93 examples (83.4\%), 22 examples (16.6\%) and BMI median 22.5 (16.2-31.1) by a patient factor, and a significant difference didn't admit by two groups. 74:13, D2 cleanup was 24:4, and a operater (Expert:Trainee) was 62:36, LS group by CS group, and D1 + dissection was 15:2 for 55:42 and a lymph node dissection for 325:286 and the bleeding amount (ml) in the average operation time (the part) by an operation factor in CS group :LS group. leackage in CS group :LS group generally admitted a coincidence related complication in $8.7 \%(10 / 115)$, and (5/98):0\% of 5.1\% (0/17) and a reflux esophagitis were (6/98):0\% of $6.1 \%(0 / 17)$, and anastomotic region bleeding was two groups, and didn't admit (4/98):0\% of $4.1 \%(0 / 17)$ and an anastomotic stricture. For 13 days, the post-operatively average length of stay was 11 days by LS group by CS group.

Conclusion: operation time was short for a coincidence by Linear stapler more than a coincidence by Circular stapler in comparison of an esophagoenterostomy way in LTG, and a coincidence related complication was little.

\section{P551-UPPER GI-Gastric cancer}

\section{STOMACH RESECTION WITH INTRAOPERATIVE FLUOROSCOPY IN LAPAROSCOPIC DISTAL GASTRECTOMY FOR EARLY GASTRIC CANCER}

J. Kawachi, H. Kashiwagi, R. Shimoyama, N. Isogai, K. Miyake, N. Kume, T. Murata, T. Nishida, R. Fukai, Y. Nagata, N. Shinozaki

Surgery, Shonan Kamakura General Hospital, KAMAKURA, Japan

laparoscopic distal gastrectomy (LDG) is common in Japan for early gastric cancer. Formerly, we used to verify the location of the marking clip to decide the proximal incision line with our hand, through a small epigastric incision. In 2015 we introduced intracorporeal reconstruction and started to decide the incisional line using intraoperative fluoroscopy.

We aimed to evaluate the efficacy and safety of intraoperative fluoroscopy in LDG. A total of 47 patients were included in this retrospective study. On the day before the operation, we endoscopically clipped several points located $2 \mathrm{~cm}$ proximal to the tumor edge to cover about half of the tumor. After lymph node dissection, we incised the stomach with an endoscopic linear stapling device,including the previously placed clips. Reconstruction was performed in all patients who underwent Billroth I or Roux-en-Y procedures.

Result: No complications were observed during pre-operative endoscopic clipping or intraoperatively. All resected specimens had negative margins and the mean distance from the tumor edge was $30.3 \pm 17.2(10-75) \mathrm{mm}$. stomach resection with intraoperative fluoroscopic guidance was safe and effective.

\section{P552-UPPER GI-Gastric cancer}

LAPAROSCOPIC GASTRECTOMY WITH SIMULTANEOUS INTRAPERITONEAL CHEMOTHERAPY MAY BE CONSIDERED AS A TREATMENT FOR FAR ADVANCED GASTRIC CANCER

Y.J. Won ${ }^{1}$, Y.S. Cho ${ }^{1}$, S.H. Min ${ }^{1}$, Y.S. Park ${ }^{1}$, S.H. $\mathrm{Ahn}^{2}$, D.J. Park ${ }^{2}$ H.H. Kim ${ }^{2}$

${ }^{1}$ Surgery, Seoul National University Bundang Hospital, SEONGNAM, Korea; ${ }^{2}$ Surgery, Seoul National University College of Medicine, SEOUL, Korea

Objectives: The purpose of this study is to confirm the safety of laparoscopic gastrectomy with intraperitoneal cisplatin administration as a treatment for advanced gastric cancer with potential for peritoneal seeding.

Methods: From July 2014 to August 2018, 56 patients with advanced gastric cancer who underwent IP chemotherapy after diagnostic laparoscopy were retrospectively studied. Al patients underwent laparoscopic gastrectomy with IP chemotherapy or IP chemotherapy alone after a diagnostic laparoscopy. Gastrectomy was performed for palliative purposes even with seeding.

Results: The average age of the patients was 56 years. Eight patients (14.3\%) had preop chemotherapy. Curative resection (R0) was performed in 31 patients $(55.4 \%)$. In diagnostic laparoscopy, cytology was performed in 38 patients $(67.9 \%)$ and CY1 was $10(26.3 \%)$. Peritoneal metastasis was detected in 35 patients (62.5\%). Of the total cohort, the 2 year OS rate was $54.5 \%$ and the median survival time was 19 months. In the case of Stage IIIb and below, the 2-year OS rate was $83 \%$, but it was $42 \%$ in Stage IIIc-IV group. When the R0 resection group and the R1-2 resection group were compared, the 2 -year OS rates were $70.7 \%$ and $26.7 \%$, respectively. Hematological toxicity such as neutropenia was not seen in all patients. The mean hospital stay was 8.2 days and adjuvant chemotherapy was performed in 35 patients $(62.5 \%)$.

Conlusions: Laparoscopic gastrectomy with IP cisplatin is safe and feasible. Considering the rapid recovery of laparoscopic surgery compared to open surgery, it may be effective for the early implementation of adjuvant chemotherapy.

\section{P553-UPPER GI-Gastric cancer}

\section{LAPAROSCOPIC PROXIMAL GASTRECTOMY FOR GASTRIC CANCER: TECHNIQUE AND PERIOPERATIVE OUTCOME}

\section{I.R. Lai, C.C. Yeh}

\section{Surgery, National Taiwan University Hospital, TAIPEI, Taiwan}

Background: Radical proximal gastrectomy (PG) and lymph nodes dissection are indicated for selected gastric cancers at the upper third of the stomach. With the advent of laparoscopic surgeries, more and more PG were performed by laparoscopic apporaches. In the past 5 years, our team has accomplished and reported the oncological outcome of laparoscopic distal gastrectomies in 100 cases of clinical stage I gastric cancer in Taiwan. Through the evolution of surgical trechniques and team work, we have cruised the learning curve of laparoscopic gastrectomy and reconstruction.

Materials and methods: In this report,we would like to present our surgical experience of laparoscopic proximal gastrectomy for gastric cancer patients. From 2005 to 2018,192 pateints with gastric cancer underwent laparoscopic gastrectomies by the same surgical team at the National Taiwan University. Among them,six consecutive pateints (male:female $=3: 3$ ) with gastric adenocarcinoma of the upper stomach underwent laparoscopic PG in 2018. The demographics, dissection, reconstruction methods and peri-operative outcome are presented. All six patients tolerated the procedure well, onepatient had mild anastomotic stenosis and improved with one session of endoscopic dilatation. One patient needed temporary proton pump inhibitor for controlloing acid reflux. Four of the 6 patients were pathological stage I, and the rest two pateint were stage IIA and IIIA disease. There was no tumor recurrence until now.

Summary: Laparoscopic proximal gastrectomy is technically safe for treating upper third gastric cancers. The long term oncological outcome deserve further observation. 


\section{P554-UPPER GI-Gastric cancer}

\section{A CASE OF JUVENILE GASTRIC POLYPOSIS WHICH PERFORMED LAPAROSCOPIC TOTAL GASTRECTOMY}

\author{
H. Onozawa ${ }^{1}$, M. Ito $^{2}$, M. Saito ${ }^{3}$, H. Ami $^{3}$, Y. Koyama ${ }^{3}$, K. Kono ${ }^{2}$
}

${ }^{1}$ Surgery, Aizu chuo hospital, AIZUWAKAMATSU-SHI, Japan;

${ }^{2}$ Gastrointestinal tract surgery, Fukushima medical university, FUKUSHIMA-SHI, Japan; ${ }^{3}$ Surgery, Ohara general Hospital, FUKUSHIMA-SHI, Japan

Background: Juvenile polyposis of the stomach is a very rare disease, and its malignant potentia has been reported previously and total gastrectomy has been recommended as a standard treatment. Recently, the usefulness of laparoscopic surgery for this case has been reported, however this type of surgery is thought that maintaining the surgical space is difficult because of distended and thickening stomach.

Case presentation: Eight years ago, a 64-year-old woman who had no family history of gastrointestinal polyposis had been diagnosed with gastric polyposis and polyp-related anemia and received twice endoscopic submucosal dissection to early gastric cancer in another hospital. She had received an annual upper gastrointestinal endoscopy and she had taken iron supplements for anemia caused from the occasional bleeding from the polyps. However, the number of the polyps had increased over time. Because she had a loss of appetite, she admitted to our hospital. Enhanced computed tomography showed gastric wall thickening and multiple gastric polyps without lymphadenopathy or distant metastasis. Colonoscopy showed no specific findings. She was diagnosed as the juvenile polyposis of the stomach, and she received laparoscopic total gastrectomy with Roux-en Y esophagojejunostomy. In operative findings, although there were the excessive distention and congestion of the stomach, standard laparoscopic surgery could be performed. The resected specimen revealed multiple variously sized polyps throughout the stomach except for lesser curvature and fundus and the histopathological examination revealed that all polyps were hyperplastic polyps without containing cancer. She was discharged on postoperative day 10

Conclusion: We successfully performed laparoscopic surgery to treat a rare case of juvenile gastric polyposis.

\section{P556-UPPER GI-Gastric cancer}

\section{LAPAROSCOPIC AND OPEN GASTRECTOMIES IN LITHUANIA: LONG TERM FOLLOW UP}

\author{
$\underline{\text { M. Ambrazevicius }}{ }^{1}$, N. Kaselis ${ }^{1}$, A. Maleckas ${ }^{2}$
}

${ }^{1}$ Abdominal and endoscopic surgery, Klaipeda Republican Hospital, KLAIPEDA, Lithuania, ${ }^{2}$ Abdominal surgery, Lithuanian University of Health Sciences, KAUNAS, Lithuania

Introduction: Despite being the pioneer in laparoscopic surgery, Europe did not have similar surgical experience compared to East Asia due to decreased exposure to gastric cancer. Several studies on minimally invasive gastrectomy for gastric cancer have been conducted in Europe. However, some of them did not analyse total gastrectomy as a distinct entity combining both distal and total gastrectomies; moreover, most of them do not provide data on full five-year follow up for each patient.

Baltic countries stand in between East and West in terms of gastric cancer incidence: incidence rate per 100,000 is 10.6 in United Kingdom, 26.3 in Lithuania and 85.3 in Japan. This exposure to gastric cancer provides unique opportunity to investigate the role of laparoscopic gastrectomy. Therefore, a case-control study was designed to evaluate laparoscopic (LTG) versus open total gastrectomy (OTG), comparing short-term surgical and long-term oncologic outcomes.

Methods: 34 patients with stage T1-2 gastric cancer underwent total gastrectomy from October 2004 to July 2009 in Klaipeda Republican Hospital and the Hospital of Lithuanian University of Health Sciences. 17 patients having a laparoscopic approach were retrospectively compared to homogenous group of patients, who undergone open total gastrectomy, paired for age, stage of disease and comorbidities.

Results: Median operating time was $270 \mathrm{~min}$ in LTG group and $210 \mathrm{~min}$ in OTG group $(\mathrm{P}<0.05)$. There were 20 (5-39) lymph nodes harvested laparoscopically and $22(12-51)$ in using open approach $(\mathrm{p}=0.35)$. Median length of stay was $14(4-53)$ days in LTG group and 14 (9-67) in OTG group $(\mathrm{p}=0.64)$. Time to resume liquids was $3(1-7)$ days in laparoscopic surgery group and $6(2-8)$ in open surgery group $(\mathrm{p}=0.00)$. Complication rate did not differ significantly between the groups. 5-year overall survival was 76\% in LTG group and 53\% in OTG group. Conclusions: Laparoscopic total gastrectomy is safe and has similar long term outcomes to open gastrectomy. Further European studies, and particularly randomized controlled trials, are needed comparing laparoscopic gastrectomies with open gastrectomies.

\section{P555-UPPER GI-Gastric cancer}

\section{MINIMALLY INVASIVE ABDOMINAL AND LEFT THORACIC APPROACH (MALTA) FOR LOCALLY ADVANCED ADENOCARCINOMA OF ESOPHAGOGASTRIC JUNCTION: A CASE REPORT}

Y. Takeuchi, Y. Ebihara, K. Tanaka, Y. Nakanishi, T. Asano, T. Noji, Y. Kurashima, S. Murakami, T. Nakamura, T. Tsuchikawa, K. Okamura, T. Shichinohe, S. Hirano

Department of Gastroenterological Surgery II, Hokkaido University Faculty of Medicine, SAPPORO, HOKKAIDO, Japan

Introduction: We report a novel technique for combined use of laparo and thoracoscopy for faradvanced adenocarcinoma of esophagogastric junction (AEG).

Case presentation: A 50's years old man presented with far-advanced AEG. An esophagogastroduodenoscopy revealed a type 2 lesion with the entire circumference around esophagogastric junction (EGJ). Contrast radiography revealed a severe stenosis in the EGJ and wall irregularity from EGJ to cardia. Computed tomography revealed a stenosis of EGJ, suspected invasion into the left side diaphragm and some lymph nodes metastases at the abdomen. We diagnosed Siewert type II AEG (cT4aN1M0, cStage IIIA : Japanese Classification of Gastric Carcinoma ver.14). Surgical technique :The patient was placed in the reverse-Trendelenburg position with the left upper body lifted and legs spread, under general anesthesia. The tumor was huge, exposed from the serous membrane and invaded the left crus. First we performed from laparoscopic proximal gastrectomy using five ports. Then, three ports were added in the 8th, 9th, and 11th intercostal spaces with the patient in the same body position, and performed thoracoscopic lower esophagectomy under artificial pneumothorax with intrathoracic pressure of $8-10 \mathrm{mmHg}$, which allows the ventilation of both lungs. The lower esophagus was resected under the thoracoscopic view to ensure an adequate margin. Following this resection, Intrathoracic esophagojejunostomy was performed by using the laparo- and thoracoscopic techniques. The operative time was $439 \mathrm{~min}$, and the blood loss was $15 \mathrm{~g}$.

He was discharged on the 15 th day after the operation without any postoperative morbidity. The histopathological diagnosis was pT4bN3aM1, P1, pStage IV. After adjuvant chemotherapy with capecitabine and oxaliplatin, ramcilumab monotherapy is undertaken now. CT revealed solitary lung metastasis in 24 months after the operation.

Conclusion: MALTA for locally advanced AEG invading the surroundings could be performed safely.

\section{P557-UPPER GI-Gastric cancer}

\section{PROGNOSTIC VALUE OF MEAN CORPUSULAR VOLUME IN GASTRIC CANCER}

\section{I.H. Jeong ${ }^{1}$, D.H. Lee ${ }^{1}$, S.S. Kim ${ }^{2}$}

${ }^{1}$ Surgery, Jeju National University, School of medicine, JEJU, Korea; ${ }^{2}$ Surgery, Chosun University, school of medicine, GWANGJU, Korea

Objective: Although mcv (mean corpuscular volume) levels are known to be associated with the prognosis of various diseases, few study investigated $\mathrm{MCV}$ as prognostic factor after gastric cancer surgery. The aim of this study is to address the prognostic value of mcv in gastric cancer who underwent curative gastric cancer surgery.

Methods: 286 patients (June 2009—December 2015) with stage I, II, and III cancer were consecutively included in this study. All patients underwent curative gastric cancer surgery including subtotal gastrectomy or total gastrectomy. Overall survival (OS), disease-free survival (DFS) and postoperative complications rate were compared between mcv $>94$ group and $=93$ group

Results: Of all patients, the mean MCV was $89 \mathrm{fL}$ (normal range, 80 to $100 \mathrm{fL}$ ). The DFS was significantly higher in the high-mev $(>94)$ than low-mev group $(=93)(\mathrm{p}<0.05)$ group. There was no significant difference in postoperative complications when compared with Clavien-Dindo scale. The survival rate of the high mcv group was higher but there was no significant difference. Conclusions: MCV may be a predictive factor after gastric cancer surgery. Unlike previous studies, patients with low MCV group showed lower DFS. More research is needed on the significance of mcv in variety of disease. 


\section{P558-UPPER GI-Gastric cancer}

\section{SMALL INTESTINAL TUMORS AFTER LAPAROSCOPIC SURGERY IN OUR HOSPITAL}

M. Kawai ${ }^{1}$, K. Sakamoto ${ }^{1}$, T. Hukunaga ${ }^{2}$, Y. Tomiki ${ }^{1}$, Y. Kojima ${ }^{1}$, H. Kamiyama $^{1}$, K. Sugimoto ${ }^{1}$, Y. Okazawa ${ }^{1}$, K. Amemiya ${ }^{1}$

${ }^{1}$ Coloproctological Surgery, Juntendo univercity, TOKYO, Japan; ${ }^{2}$ Gastroenterological Surgery, Juntendo univercity, TOKYO, Japan

Introduction: Small intestinal tumors are rarely observed, accounting for about 3-6\% (malignant cases: $1-2 \%$ ) of all gastrointestinal tumors. Therefore, occasionally, their diagnoses can be difficult. However, recently, capsule and balloon endoscopes have been widely employed, facilitating their diagnoses.

Subjects \& Methods: Twenty patients with small intestinal tumors, treated in our department between 2009 and 2018, were examined regarding patient backgrounds, diagnostic methods, pathological findings, postoperative courses, and prognoses.

Results: The subjects consisted of 15 males and 5 females, with a mean age of 63 years. Their chief complaints were black stools (blood in stools) (7 cases), anemia (6 cases) and abdominal pain ( 5 cases). Fourteen $(70.0 \%)$ were diagnosed using a small intestine endoscope. The affected lesions were the jejunum ( 9 cases; $45.0 \%$ ), ileum ( 9 cases; $45.0 \%$ ), and multiple sites ( 2 cases; $10.0 \%)$. The median distance from the Treitz ligament or Bauhin valve was $50 \mathrm{~cm}(2-200)$. Surgical procedures were partial resection of the small intestine (14 cases; $70.0 \%)$, resection of the ileocecum (6 cases; $30.0 \%$ ). Single Incision Laparoscopic Surgery (SILS) eight were soon (40.0\%). Postoperative complications were abdominal abscess ( 2 cases; $10.0 \%$ ) and surgical site infection (SSI), hemorrhage, and paralytic ileus (1 case each; 5.0\%). Pathological diagnoses were lymphoma ( 5 cases; $25.0 \%$ ), GIST ( 4 cases; $20.0 \%$ ), primary small intestinal cancer ( 3 cases; $15.0 \%$ ), metastatic small intestinal tumor ( 2 cases; $10.0 \%$ ), and granuloma, lipoma, Peutz-Jeghers polyp, clear cell sarcoma, malignant mesothelioma, and ectopic pancreas (1 case each; $5.0 \%$ ). Postoperative hospital stays and survival periods were 9.5 (5-238) and 632.5 (35-2,006) days, respectively.

Discussion: The incidence of small intestinal tumors is very low (20 patients in our hospital over the past 10 years). Most patients were diagnosed in bleeding, complicated by anemia and black stools. However, as most tumors were relatively close to the Treitz ligament and Bauhin valve, almost a half could be diagnosed with a small intestine endoscope before surgery. Also, for examination of the upper and lower gastrointestinal tracts, the small intestine should be aggressively investigated in cases of prolonged anemia of unknown cause.

\section{P559-UPPER GI-Gastric cancer}

\section{INFLUENCE OF ELECTROSURGICAL DEVICES ON} POSTOPERATIVE PANCREATIC FISTULA FOLLOWING LAPAROSCOPY-ASSISTED GASTRECTOMY FOR EARLY GASTRIC CANCER

\section{K. Ito, M. Nagai, Y. Nomura}

Department of Surgery, Asahi General Hospital, CHIBA, Japan

Background: Postoperative pancreatic fistula (POPF) is a severe complication following laparoscopy-assisted gastrectomy (LAG).However, the influence of electrosurgical devices on POPF following LAG remains unclear. This study aimed to investigate the influences of electrosurgical devices on the incidence of POPF following LAG.

Methods: We retrospectively reviewed 92 patients who underwent LAG between January 2013 and December 2014. Patients were classified as POPF and No-POPF according to their grade B or $\mathrm{C}$ POPF status. POPF was diagnosed according to International Study Group of Pancreatic Fistula (ISGPF) criteria or clinical findings. Patient characteristics, intraoperative parameters, electrosurgical device type, pathological findings, and early postoperative outcomes were compared. Electrosurgical devices were classified asTHUNDERBEAT (TB) or laparosonic coagulating shears (LCS) based on energy sources.

Results: Eighteen patients developed grade B or C POPF. Among them, $12(66.7 \%)$ and 6 $(33.3 \%)$ were diagnosed with POPF according to ISGPF criteria and clinical findings, respectively.Twelve $(66.7 \%)$ and $6(33.3 \%)$ received grades of $\mathrm{B}$ and $\mathrm{C}$, respectively. Univariate analyses revealed that visceral fat area $(\mathrm{VFA} ; \mathrm{P}=0.011)$, operation time $(\mathrm{P}=0.03)$ and electrosurgical device type $(\mathrm{P}=0.005)$ were significant risk factors for POPF following LAG. Multivariate analysis revealed that operation time (odds ratio $[\mathrm{OR}], 1.007 ; 95 \%$ confidence interval $[\mathrm{CI}], 1.001-1.015 ; \mathrm{P}=0.017)$ and TB device $(\mathrm{OR}, 6.80 ; 95 \% \mathrm{CI}, 1.95-28.61 ; \mathrm{P}=0.002)$ were independent risk factors for POPF following LAG.

Conclusions: Operation time and TB use significantly affect the risk of POPF and should be considered in future clinical studies.

\section{P560-UPPER GI-Gastric cancer}

FEASIBILITY AND NUTRITIONAL BENEFITS OF DOUBLE FLAP WITH NO-KNIFE STAPLER RECONSTRUCTION AFTER LAPAROSCOPIC PROXIMAL GASTRECTOMY FOR GASTRIC CANCER

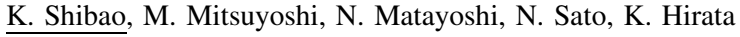

Department of Surgery I, University of Occupational and Environmental Health, KITAKYUSHU, Japan

Background: Laparoscopic proximal gastrectomy (LPG) with double flap method is known to reduce reflux symptoms, which is a major concern after proximal gastrectomy. However, in case of combined resection of distal ehophagectomy, extra efforts is need to complete the double flap method by transhiatal approach because of technical difficulty. Therefore, we modified this reconstruction method by adding no-knife stapler fixation between esophagus and remnant stomach prior to esophagogastrostomy (Double Flap with No-knife Stapler Reconstruction: DFNSR) to make procedures easier. This study aimed to clarify the feasibility and nutritional benefits of LPG with DFNSR over laparoscopic total gastrectomy (LTG).

Methods: A retrospective review of the prospectively established database identified patients with esophagogastric junctional cancer/ gastric cancer in the upper stomach who underwent LPG with DFNSR (LPD group, $\mathrm{n}=17$ ) or LTG with Roux-en-Y reconstruction (LTR group, $\mathrm{n}=46$ ) between January 2006 and July 2017. Baseline characteristics and surgical outcome, changes in body weight, and nutritional status after surgery were compared at 6 and 12 months after the surgery were compared between two groups.

Results: Operating time was significantly longer in the LPD group ( $488 \pm 53.7$ vs $400 \pm 42.8 \mathrm{~min}, \mathrm{p}=0.03$ ). The incidence of $\mathrm{CD}$ grade III or more complications and the hospital stay were comparable between two groups. Postoperative levels of hemoglobin and serum albumin were similar in LPD and LTR groups. However, postoperative changes in body weight a 6 and 12 months after surgery were consistently less in LPD group than in LTR group (8.5\% vs. $12.7 \%, 7.8 \%$ vs. $15.4 \%$, respectively, $\mathrm{p}=0.01)$. Especially, at 12 months after surgery, LTR group showed postoperative weight loss in all cases, but 6 cases (35.3\%) in LPD group did not show any postoperative weight loss.

Conclusion: LPG with DFNSR is feasible and may be the preferred procedure for cancer of the esophagogastric junction from the viewpoint of postoperative nutritional status, with less weight loss than LTG.

\section{P561-UPPER GI-Gastric cancer}

\section{PREOPERATIVE AND POSTOPERATIVE C-REACTIVE PROTEIN LEVELS PREDICT RECURRENCE AND CHEMOTHERAPY BENEFIT IN GASTRIC CANCER}

\section{Huang, B. Xu, J. Lu, C. Zheng, P. Li}

Department of Gastric Surgery, Fujian Medical University Union Hospital, FUZHOU, China

Background: Preoperative and postoperative C-reactive protein (CRP) are related to the prognosis of patients with malignant tumors. This study aimed to explore the predictive value of combining the two in gastric cancer(GC) patients.

Methods: A total of 419 gastric cancer patients in a clinical trial(NCT02327481) from January 2015 to April 2016 were analyzed. Receiver operating characteristic curves were generated, and by calculating the areas under the curve(AUC) and the C-index, the discriminative ability of CRPs during different periods were compared, including pre-CRP, postoperative days 1, 3,5 and postoperative maximum CRP (post-CRP $\mathrm{P}_{\max }$ ). A decision curve analysis was performed to evaluate the clinical utility.

Result: Ultimately, 401 patients were included this study and the median follow-up time was 29 (3-41) months. For postoperative recurrence, the AUC and C-index of pre-CRP were 0.692 and 0.678 , respectively, significantly higher than the other CRPs, all $\mathrm{p}<0.05$. among $=$ " “ “"the $=$ " " post-crps $=$ " “ post-crp $=$ " " sub $=$ " " " $>\max$ had the highest AUC $(0.591)$ and C-index (0.585). The optimal cut-off point values of the two for predicting postoperative recurrence were $3.1 \mathrm{mg} / \mathrm{L}$ and $77.1 \mathrm{mg} / \mathrm{L}$, respectively. Multivariate analysis of recurrence-free survival (RFS) showed that both pre-CRP $\geq 3.1 \mathrm{mg} / \mathrm{L}$ (high-pre-CRP) and post-CRP ${ }_{\max } \geq 77.1 \mathrm{mg} / \mathrm{L}$ (highpost-CRP $\mathrm{max}_{\mathrm{max}}$ ) were independent prognostic factors for recurrence. The risk model consisting of the pre-CRP, post-CRP ${ }_{\max }$ and TNM had a higher predictive ability and clinical utility. Further adjuvant chemotherapy(ACT) benefit analysis for stage II/III GC showed that patients with pre$\mathrm{CRP}<3.1 \mathrm{mg} / \mathrm{L}$ couldn't benefit from chemotherapy (RFS:90.0\%(non-chemotherapy group) vs $80.7 \%$ (chemotherapy group), $\mathrm{p}=0.557$ ). In the high-pre-CRP group, only high-post-CRP $\mathrm{m}_{\max }$ but not post-CRP ${ }_{\max }<77.1 \mathrm{mg} / \mathrm{L}$ could benefit from chemotherapy (RFS:33.2\%(non-chemotherapy group) vs $49.9 \%$ (chemotherapy group), $\mathrm{p}=0.037$, respectively). Similar findings were observed for overall survival.

Conclusion: Both pre-CRP and post-CRP ${ }_{\max }$, cheap and easily obtained, are independent predictors of recurrence for GC. ACT significantly prolonged the RFS for stage II/III GC patients with high-prep-CRP and high-post-CRP ${ }_{\max }$ after R0 resection. 


\section{P562-UPPER GI-Gastric cancer}

\section{COMPARISON OF LAPAROSCOPIC VERSUS OPEN} GASTRECTOMY FOR TREATMENT OF GASTRIC CANCER. ANALYSIS OF A TEXTBOOK OUTCOME

P. Priego, A. Puerta, A. González Barranquero, S. Sánchez Picot, L. Blazquez, M. Cuadrado, J. Galindo

Division of Esophagogastric and Bariatric Surgery, Ramón y Cajal University Hospital, MADRID, Spain

Introduction: Open gastrectomy (OG) has long been the preferred surgical approach worldwide for treatment of gastric cancer (GC). Nowadays, several randomized, prospective trials have confirmed improvements in postoperative outcomes for laparoscopic gastrectomy (LG) compared to open procedures, with similar oncologic outcomes. However, most part of these studies comes from the Eastern countries.

Material and methods: A prospective non randomized study was conducted with all patients operated of GC at Ramón y Cajal University Hospital from January 2015 to December 2017. Over 96 patients enrolled, 47 patients underwent LG and 49 OG. Textbook outcome was defined as the percentage of patients who underwent a complete tumour resection with at least $15 \mathrm{lymph}$ nodes in the resected specimen and an uneventful postoperative course, without hospital readmission.

Results: A textbook outcome was achieved in $51.04 \%$ of patients operated of GC. The outcome parameter 'no severe postoperative complication' had the greatest negative impact on the textbook outcome. A statistically higher number of patients with early cancer $(40 \%$ vs. $16.3 \%)$ and subtotal gastrectomy $(57.5 \%$ vs. $34.7 \%)$ were found in the laparoscopic group. No statistically differences were found between open and laparoscopic approach regarding operating time, rate of microscopic margin positivity, hospital stay, number of retrieved lymph nodes, complications, reinterventions, mortality and readmissions. No statistical differences in textbook outcome were found between both groups $(57.14 \%$ vs. $45 \% ; \mathrm{p}=0.25)$.

Conclusions: Laparoscopic gastrectomy for treatment of gastric cancer seems to be safe and feasible with similar textbook outcomes compared to open gastrectomy.

\section{P563-UPPER GI-Gastric cancer}

\section{OUTCOMES OF THE LEARNING CURVE IN OUR FIRST 100 CONSECUTIVE LAPAROSCOPIC GASTRECTOMIES}

P. Priego, A. Puerta, A. González Barranquero, S. Sánchez Picot, L. Blazquez, M. Cuadrado, J. Galindo

Division of Esophagogastric and Bariatric Surgery, Ramón y Cajal University Hospital, MADRID, Spain

Introduction: Laparoscopic surgery has been increasing for treatment of gastric cancer. However, standardization of this minimally invasive approach has not been reached yet because of its technical difficulties and the concern about oncological safety. The aim of the study was to analyze the outcomes of our learning curve in this complex surgical technique.

Material and methods: The first consecutive 100 cases of Laparoscopic Gastrectomy (LG) performed at our Hospital from November 2008 to February 2018 were enrolled. Patients were divided into two groups based on the period they were operated. Training phase (TP) was considered between 2008 and 2014 (46 cases) and more-developed phase (MDP) between 2015 and 2018 (54 cases). Conversion, lymphadenectomy and retrieved lymph nodes (LN), hospital length of stay, mean operative time, complications, reintervention and mortality rates were compared between the two phases of learning curve.

Results: The number of retrieved LN was higher in the MDP ( $17 \pm 8,6$ vs. $23,3 \pm 10,4$ $p=0,004)$. Furthermore, we have also found less complications $(47,8 \%$ vs. $27,8 \% ; p=0,038)$, a decreased reintervention rate $(15,2 \%$ vs. $1,85 \% ; \mathrm{p}=0,023)$ and overall mortality $(8,7 \%$ vs. $0 \%$; $\mathrm{p}=0,003)$ in the MDP. There were no significant differences in conversion rate, mean operative time, and hospital length of stay between phases.

Conclusion: Although we consider that our learning curve is not completed yet because the average of monitored parameters have not reached a steady state, the improvement on surgical parameters and postoperative course in the last two years have showed our results are near to the best results published in the literature.

\section{P564-UPPER GI-Gastric cancer}

\section{LAPAROSCOPIC DISTAL GASTRECTOMY USING INDOCYANINE GREEN LYMPHOGRAPHY FOR LYMPH NODE DISSECTION}

M. Bailon-Cuadrado ${ }^{1}$, M. Toledano-Trincado ${ }^{1}$, J.M. Garcia-Abril ${ }^{1}$, J. Sanchez-Gonzalez ${ }^{1}$, M.L. Martin-Esteban ${ }^{2}$, P. Concejo-Cutoli ${ }^{2}$, J.R. Gomez-Lopez ${ }^{2}$, M.A. Montenegro-Martin ${ }^{2}$, J.C. Martin-del Olmo ${ }^{2}$, D. Pacheco-Sanchez ${ }^{1}$

${ }^{1}$ General and Digestive Surgery, Rio Hortega University Hospital, VALLADOLID, Spain; ${ }^{2}$ General and Digestive Surgery, Hospital of Medina del Campo, MEDINA DEL CAMPO, Spain

Aims: Lymph node $(\mathrm{LN})$ dissection proves to be essential for oncological gastrectomy, given that the presence of LN metastases is very high, even for early gastric cancer (4.9\% for T1a and $21.4 \%$ for T1b). This way, D2 dissection for advanced gastric cancer and D1 + for early gastric cancer are the gold standard procedures. Some teams are using indocyanine green (ICG) lymphography to improve their LN dissections, claiming that this technique facilitates the harvesting of small fluorescent LN that, otherwise, would be difficult to identify by conventional laparoscopic methods.

Methods: We herein present the case of a 60-year-old man with a T1b distal gastric cancer. Endoscopic ultrasound discarded the presence of metastatic LN and CT scan showed no distant metastases. ICG was administrated endoscopically the day before the surgery, an amount of $6 \mathrm{mg}$ was injected along the submucosal layer around the tumour. In the video we can see how we perform a laparoscopic distal gastrectomy with D1 + LN dissection and Roux-en-Y reconstruction. ICG lymphography helped us to complete our expected LN harvesting, especially for groups 6 (infrapyloric) and 7 (left gastric artery). Thanks to this technique, we could resect $\mathrm{LN}$ that we might have obviate during a usual laparoscopic procedure.

Results: Patient was discharged home on the sixth postoperative day without complications and with adequate oral tolerance.

Conclusions: We present a case in which we have performed a laparoscopic distal gastrectomy with D1 + dissection and Roux-en-Y reconstruction. We used ICG lymphography to help us to improve our LN harvesting. Although it is soon to assess if this technique may increase the number of retrieved LN and in which stations might be more useful, we consider this is a harmless method that may help gastric teams to complete their expected LN dissections.

\section{P565-UPPER GI-Gastric cancer}

\section{GIST GASTROINTESTINAL STROMAL TUMORS}

G. Contreras Gonzalez, O.s. Chau, M. Kushinakajo, A. Martin, W. Jimenez

\section{Surgery, Clinica Guemes, LUJAN BUENOS AIRES, Argentina}

Introduction: Gastrointestinal stromal tumor (GIST) represents around $0.1 \%$ to $3 \%$ of gastrointestinal neoplasms, with the mesenchymal tumor being more frequent than the digestive one.The GIST can be produced from the esophagus to the anus, at any point, being the stomach of ( 39 to $60 \%$ ) and the small intestine ( 30 to $42 \%$ ) more frequent sites.It is characterized by the expression of the tyrosine kinase growth factor receptor,CD117,differentiating it from other mesenchymal tumors,which do not express it.It is accepted that its origin corresponds to the interstitial cells of Ramón y Cajal,which act as a pacemaker for intestinal motility.They are very heterogeneous tumors, which vary in size,morphology and biological behavior,being neoplasms with uncertain malignant potential.The incidence is between the fourth and sixth decades,being the distribution by gender similar. Clinical case: Female patient of 70 years, who goes to the general surgery service,as interconsultation,after a Veda,by dyspepsia.It is reported Stomach:ceiling mucosa without alterations,at the level of the greater curvature is seen a tumor of $5 \mathrm{~cm}$,hard to the touch with the biopsy forceps,slightly irregular covered with mucosa of normal appearance.

Computed Tomography: Stomach body:rounded image of nodular aspect which does not present heterogeneous enhancement after administration intravenous iodine contrast extending to peritoneal region, measures $44 \times 33 \times 32 \mathrm{~mm}$

Liver:hypodense image without heterogeneous enhancement adjacent to this, a $10 \mathrm{~mm}$ rounded image that is suggested to be studied with NMR.

Gadolinium NMR Liver hypodense image with well-defined limits without heterogeneous enhancement of cystic aspect.

Gastric roof,heterogeneous formation,which enhances with gadolinium $37 \mathrm{~mm} \times 38 \mathrm{~m}$ $\mathrm{mx} 40 \mathrm{~mm}$,having to discard a GIST.

Surgical Technique

Laparoscopic partial gastrectomy.

Pathological Anatomy and Immunohistochemistry

$1.5 \mathrm{~cm}$ injury with net edges.Uncertain Malignant Fusocellular Nodule, CD117 +++ actinDOG1 +++ S100-No mitosis or invasion of the mucosa is observed.

Conclusion: A case of stomach GIST is presented,which,the main symptom was dyspepsia,being the clinical presentation very variable,in relation to the place in which it is located.

There is Fletcher criteria for the risk of malignancy,this being less than $1.5 \mathrm{~cm}$,very low risk,less than $2 \mathrm{~cm}$, the patient evolved favorably,without surgical complications. 


\section{P566-UPPER GI-Gastric cancer}

ROBOT-ASSISTED GASTRODUODENAL SURGERY: A SINGLE CENTER EXPERIENCE

R. Caruso, E. Vicente, Y. Quijano, H. Duran, B. Ielpo, I. Fabra, E. Diaz, L. Malave, R. Isernia, E. Pinna, V. Ferri

General Surgery, Sanchinarro University Hospital, MADRID, Spain

Objective: Robot-assisted gastroduodenal surgery (RAS) was introduced to overcome the technical limitations of conventional laparoscopy. It provides a 3D-amplified view to the surgeons and an increased ability to control the operative field by manipulating optics, as well as enhanced mobility and precision of instruments. The aim of the present study is to evaluate the main outcome of a single center experience in gastroduodenal robotic surgery.

Materials and methods: We report a case series of patients who underwent robot-assisted gastroduodenal surgery at Sanchinarro University Hospital between January 2010 and September 2018. Main patient demographic characteristics, type of surgery, peri and post-operative data and follow-up were evaluated.

Results: A total of 35 consecutive robotic gastroduodenal resections were analyzed, 16 women and 19 men, with a mean age of 61 years (22-80). According to the anesthetic classification, 7 patients were ASA I, 23 patients ASA II and only 5 patients ASA III. In $90 \%$ of patients the surgical indication was for malignant disease: 22 gastric adenocarcinomas, 8 GIST, 2 neuroendocrine tumor, 1 submucosal lipoma and 2 pancreatic heterotopia was. Were performed 22 total gastrectomy, 6 subtotal gastrectomy, 4 enucleations of duodenal tumors and 3 atypical partial resections of gastric wall. Conversion to open procedure was required in 6 patients. The mean operative time was $340 \mathrm{~min}$ (range 210-600 $\mathrm{min}$ ). The mean stay in intensive care was 1,3 (1-9) days and the mean hospital length of stay was 11 days (3-23). Complications occurred in three patients, of which 1 case was due to an intra-abdominal collection. Another complication was stenosis of jejuno-jejunal anastomosis caused by torsion with ischaemic damage which required surgical reinterventation. The last complication was due to acute pulmonary edema secondary to heart failure. Anastomotic leakages occurred in 4 patients. No case of postoperative mortality was recorded.

Conclusions: Robot-assisted gastroduodenal surgery is a safe and feasible technique in experienced centers with advanced robotic skills. In the literature, there are only few reports of robotic assisted gastroduodenal resection. Further studies are necessary to better confirme our results.

\section{P567-UPPER GI-Gastric cancer}

\section{ATYPICAL PARTIAL GASTRECTOMY OF GIST IN LESSER GASTRIC CURVATURE WITH LAPAROSCOPIC SURGERY}

E. Vidaña Márquez, P.A. Sánchez Fuentes, J. Jorge Cerrudo, F. Rubio Gil, I. Blesa Sierra, M. Ferrer Márquez, R. Belda Lozano, P.J. Moya Forcén, A. Reina Duarte, R. Rosado Cobián

Cirugía General y del Aparato Digestivo, Hospital Universitario Torrecárdenas, ALMERÍA, Spain

Aims: To present the surgical procedure of resection of the lesser gastric curvature and its pedicle with laparoscopic surgery, fulfilling oncological criteria, carried out in the General Surgery Service of the Hospital of Torrecárdenas.

Methods: An 85-year-old man with prostate cancer treated with complete hormonal block and EPOC, who consults for rectal bleeding of 1 week of evolution. It is diagnosed of GIST in gastric lesser curvature, $9 \times 8 \times 11 \mathrm{~cm}$, very vascularized and infiltrates the wall producing marked imprint on the fundus. It is tributary of left gastric artery. Precise blood transfusion and presents hemodynamic stability, is decided surgical resection scheduled.

Results: The surgery is performed by laparoscopy, with a tumor of approximately $10 \mathrm{~cm}$, which is dependent on the lesser curvature. The esophageal hiatus and the lesser curvature are dissected with section of the left gastric pedicle. Atypical gastrectomy of the lesser curvature including GIST, making a gastric sleeve dependent on the greater curvature. The anatomopathological study reports pT4 pN0 with 22 lymph nodes without adenopathies, and disease-free surgical margins. He was discharged without complications on the 6th day and did not require re-entry. Conclusions: The laparoscopy surgery for atypical gastrectomy of lesser curvature is safe and meets oncological criteria in selected patients and performed by an experienced esophagogastric unit.

\section{P568-UPPER GI-Gastric cancer}

TOTAL GASTRECTOMY WITH COMPLETE D2 LINFADENECTOMY IN GASTRIC ADENOCARCINOMA WITH LAPAROSCOPIC SURGERY

E. Vidaña Márquez, P.A. Sánchez Fuentes, J. Jorge Cerrudo, F. Rubio Gil, I. Blesa Sierra, M. Ferrer Márquez, R. Belda Lozano, P.J. Moya Forcén, A. Reina Duarte, R. Rosado Cobián

Cirugía General y del Aparato Digestivo, Hospital Universitario Torrecárdenas, ALMERIA, Spain

Aims: To present the surgical procedure of total gastrectomy with D2 lymphadenectomy with laparoscopic surgery, fulfilling oncological criteria, carried out in the General Surgery Service of the Hospital of Torrecárdenas.

Methods: A 35-year-old male with a tobacco habit who consults due to epigastric pain and constitutional syndrome of 6 months of evolution. It is diagnosed of gastric adenocarcinoma T3N2M0. Neoadjuvant QT is decided, after 4 weeks of its completion, scheduled surgery is performed.

Results: The surgery is performed by laparoscopy, showing a stenosing tumor in at gastric antrum of approximately $8 \mathrm{~cm}$. Dissection of the greater curvature with section of the right gastroepiploic at its birth and duodenal section is performed. Dissection of the lesser curvature with D2 lymphadenectomy, section of the pedicle of the left gastric and the distal esophagus. The transit is restored with latero-lateral esophageal-jejunal anastomosis and jejunojejunostomy. The anatomopathological study reports ypT4a and $\mathrm{pN} 2$ with $3 / 36$ adenopathies, and disease-free surgical margins. He was discharged without complications on the 7th day and did not require reentry.

Conclusions: The laparoscopy surgery for total gastrectomy with complete D2 lymphadenectomy is safe and meets oncological criteria in selected patients and performed by an experienced esophagogastric unit.

\section{P569-UPPER GI-Gastric cancer}

AN EVALUATION OF PHOTO-DOCUMENTATION QUALITY DURING OESOPHAGOGASTRODUODENOSCOPY (OGD). A SINGLE CENTRE EXPERIENCE

\section{E. Burke, F. Kazi, M. Salama, I. Ahmed}

Surgery, HSE- Our Lady of Lourdes Hospital Drogheda, DROGHEDA, Ireland

Introduction: Although OGD remains the gold standard for diagnosing oesophago-gastro-duodenal pathology, lesions can easily be missed particularly at the upper oesophagus and incisura. Recently, endoscopic imaging documentation has gained an important role in OGD reporting and is an integral aspect of quality control. Currently there is no globally accepted standardised protocol for photo-documentation. In Japan 20-40 photos are taken at OGD. In contrast the recen guidelines from the British Society of Gastroenterology (BSG) recommend photo-documentation of 8 anatomic landmarks including upper oesophagus (UO), lower oesophagus (LO), fundus (F), gastric body (GB), incisura (IN), antrum (A) duodenal bulb (DB) and distal duodenum (DD). Aims: To assess the practice of OGD imaging documentation in our endoscopy unit in light of the current guidelines.

Methods: Retrospective review of OGD reports before and after the introduction of the new guidelines. Inclusion criteria: all elective OGDs. Exclusion criteria: emergency OGDs and elective therapeutic OGDs. Data recorded: patient demographics, endoscopist, indication, number of photos, anatomical site photographed, pathology identified and whether pathology photographed or not.

Results: 1099 OGDs reviewed, 790 before the guidelines (Group 1) and 309 afterwards (Group 2). The most common indication was reflux $166(21 \%)$ in group 1 and anaemia $67(22 \%)$ in group 2. Average number of photographs taken in group 1 was 4 (Surgery 4.8 , Gastroenterology 2.8 ) and 4.7 in group 2 (Surgery 5.5 Gastroenterology 3.5). Number of OGDs with photo-documentation of the recommended anatomic landmarks in group 1: UO $79(10 \%)$, LO $500(63 \%), \mathrm{F}$ $519(66 \%)$, GB $117(15 \%)$, IN $10(1 \%)$, AN $445(56 \%)$, DB $88(11 \%)$ and DD $578(73 \%)$ and in group 2 : UO 33 (11\%), LO 222 (72\%), F 245 (79\%), GB 59 (19\%), IN 0 (0\%), A $192(62 \%)$, DB $17(6 \%)$, DD $249(81 \%)$. Pathology reported in $603(76 \%)$ of group 1 and $245(79 \%)$ of group 2 however no photo was taken of this in $89(15 \%)$ in group 1 and $26(8 \%)$ in group 2.

Conclusion: Currently our centre is not meeting the guideline recommendations. We will begin a program of education to improve performance and will re-audit following same. 


\section{P570-UPPER GI-Gastric cancer}

\section{ADVANCED GASTRIC ADENOCARCINOMA IN MOROCCO: CLINICAL UTILITY OF SYSTEMATIC PRE TREATMENT STAGING LAPAROSCOPIC EXPLORATION}

\author{
A. Souadka, F. Alazaoui, M.A. Majbar, B. Benkabbou, R. Mohsine
}

Surgical department, National Institut of oncology, RABAT, Morocco

Background: The preoperative staging has limitations on finding peritoneal dissemination in gastric cancer patients. Systematic laparoscopic exploration can discover occult carcinomatosis not described on the imaging. This retrospective study aimed to determine the clinical value of staging laparoscopy for advanced gastric cancer.

Methods: All locally advanced gastric adenocarcinoma managed in surgical oncological unit between 1st January and 30th November were prospectively enrolled in the study. In the absence of emergency surgery or preoperative contraindications, all patients with curative intent underwent either preoperative chemotherapy followed by surgical exploration in the intent of curative gastrectomy (G)or systematic pretreatment laparoscopic exploration (L).Disease stages were compared with those obtained by conventional methods.

Results: From sixty seven locally advanced cancer, 11 were operated with an emergent indication (haemorrhage) and 17 in a palliative intent. Twenty fore patients were in the $\mathrm{L}$ group vs 15 patients in the G group. Peritoneal carcinomatosis was discovered in 5/24 (20\%) patients in the L group and 5/15 (33\%)patients in G group. All the 10 patients received palliative chemotherapy, however in the L group these patients begun treatment earlier than in the G group.

Conclusion: We showed the utility of systematic laparoscopic pretreatment exploration not only to avoid negative laparotomy but also to avoid the delay of the beginning of chemotherapy in case of palliative intent. In the setting of developing country these results may help to improve the optimisation of all ressources in the management of locally advanced gastric cancer.

\section{P572-UPPER GI-Gastric cancer}

\section{SHORT TERM OUTCOME OF ROBOTIC ASSISTED GASTRECTOMY FOR GASTRIC CANCER}

N. Inaki, R. Mastui, T. Otsuka, J. Nomoto, S. Kohama, D. Azuma, K. Honjou, Y. Gyoda, K. Takehara, M. Ouchi, K. Nagakari, Y. Ishizaki, Digestive and General Surgery, Juntendo University Urayasu Hospital, URAYASU, Japan

Aims: Robotic surgery has been introduced for gastric cancer treatment. However, its validity against conventional laparoscopic surgery is still controversial. We examined the short-term outcome of robotic assisted gastrectomy (RAG) for gastric cancer from our experiences.

Methods: We examined series of 30 RAG and 88 laparoscopic gastrectomy (LG) for gastric cancer cases without neoadjuvant chemotherapy from October 2016 to June 2018. The patient background (age, gender, BMI) and c-stage of the preoperative factor were matched using Propensity score matching method, and the surgical results were compared and examined.

Results: Thirty RG groups matched RAG 30 cases. The operation time (RAG / LG) was significantly longer in the RAG group as $309.2 \pm 47.9 \mathrm{~min} / 220.6 \pm 55.2 \mathrm{~min}(\mathrm{p}<0.05)$. Amoun of blood loss was not significantly different each other; $10 \mathrm{ml} / 9 \mathrm{ml}(\mathrm{p}=0.693)$. Pathologically T4a case was involved in 4 cases in RAG and 5 cases in LG. The extent of lymph node dissection (D1 + / D2) was $23 / 7$ cases in both groups. The number of retrieved lymph node was also $42.7 \pm 17.9 / 43.6 \pm 18.7(\mathrm{P}=0.855)$. There is no significant difference between the two groups, regarding the frequency of postoperative complications $(\mathrm{p}=0.1967)$, the first flatus date $(p=0.49)$, the frequency of analgesic use $(p=0.895)$ after the fifth postoperative day, and the number of hospital stays $(\mathrm{p}=0.153)$.

Conclusions: RAG in our clinical experiences can be safely introduced and short-term results are comparable to those of LG. Verification of superiority of robotic surgery including long-term results seems to influence the future of robotic surgery.

\section{P573-UPPER GI-Gastric cancer}

TOTALLY LAPAROSCOPIC GASTRECTOMY WITH VARIOUS TYPES OF INTRACOPOREAL ANASTOMOSIS USING BY LAPAROSCOPIC LINEAR STAPLERS

\section{C.H. Kim}

Upper GI surgery, The catholic University of Korea, Incheon St. Mary's hospital, INCHEON CITY, Korea

Purpose: The aim of this study is to compare the surgical outcomes of totally laparoscopic gastrectomy in 10 years of single center experience.566 111 .

Materials and methods: We retrospectively collected data from 677 patients who underwen totally laparoscopic gastrectomy for gastric cancer patients including total and distal gastrectomy between January 2005 and June 2015. The evaluated parameters included patients and tumor characteristics, operative details, postoperative complications classified according to ClavienDindo classification

Results: Totally laparoscopic total gastrectomy and distal gastrectomy were performed in Billroth-I, Billroth-II and Roux-en-Y were performed in $111(16.4 \%)$ and $566(83.6 \%)$, respectively. Billroth-II reconstruction were more frequent in advanced stage $(31.4 \%, p<0.001)$ and more than D2 lymph node dissection $(65.6 \%, p=0.008)$. Billroth-I $(91.2 \%)$ and uncut Roux-en-Y $(81.6 \%)$ were more frequent in early stage $(p<0.001)$. Uncut Roux-en-Y were more frequent in young age $(54.9 \pm 10.2, p<0.001)$. Anastomosis time was significantly shorter in Billroth-I $(3.5 \pm 5.1, p=0.021)$. There were no significant differenece in another operative details. In tota gastrectomy, the number of lymph node retrieval $(47.0 \pm 20.9, p=0.048)$ and more than D2 lymph node dissection $(90.0 \%, p=0.003)$ were more frequent in advanced gastric cancer than early gastric cancer. Postoperative complications, more than Clavien-Dindo grade III, occurred in $60(8.9 \%)$ patients without statistically significant differences among groups $(p=0.797)$

Conclusions: Totally laparoscopic gastrectomy is feasible method in terms of surgical outcomes. Furthermore, totally laparoscopic total gastrectomy is not technically difficult in advanced gastric cancer such as early gastric cancer and safety method.

Key words: Gastrectomy, Reconstruction, Laparoscopic surgery, Stomach neoplasm 


\section{P574-UPPER GI-Gastric cancer}

\section{THE SAFETY AND FEASIBILITY OF LAPAROSCOPIC GASTRECTOMY BY ICG FLUORESCENCE NAVIGATION Method: FOR GASTRIC CANCER}

Y. Aoyama, T. Omori, K. Yamamoto, Y. Yanagimoto, N. Shinno, H. Miyata, K. Sugimura, O. Masayuki, M. Yano

Department of Surgery, Osaka International Cancer Institute, OSAKA, Japan

Background: In gastric cancer surgery, to secure surgical margin, it is necessary to accurately judge the position of the tumor. However, with conventional marking clips, it is difficult to identify the exact location of the tumor during laparoscopic surgery.

Purpose: We investigate whether ICG (indocyanine green) fluorescence navigation method is effective and safe for determination of cutting line in laparoscopic gastrectomy.

Patients and Methods: 428 subjects underwent laparoscopic gastrectomy (including robot-assisted surgery) based on the ICG method for gastric cancer in the period from April 2017 to December 2018. The day before surgery, ICG diluted 50 times $(0.2 \mathrm{ml}$ of reagent $+9.8 \mathrm{ml}$ of distilled water) was injected at $1 \mathrm{~cm}$ from the tumor edge and $0.5 \mathrm{ml}$ at the four submucosal layers around. Then clip to the same part. Gastrectomy based on standard surgery is performed, and the position of the tumor and spread of ICG are confirmed by ICG fluorescence navigation during operation, and a cutting line is determined. The extent of ICG from the tumor is again measured with the excised specimen and compared with the pathological margin.

Results: Among the patients who underwent intraoperative pathological examination, they were negative in all cases except one. The spread of ICG was $2.5 \mathrm{~cm}$ on average, and considering the marking position $(1 \mathrm{~cm})$ from the tumor edge, securing of $3.5 \mathrm{~cm}$ or more was possible. The operation time was $230.0 \pm 92.7 \mathrm{~min}$ and the estimated bleeding loss was $24.6 \pm 120.9 \mathrm{ml}$. Conclusion: Laparoscopic gastrectomy with ICG method can evaluate tumor position and spread laparoscopic gastrectomy. easily and in real time during operation and it was effective for determining the cutting line in

\section{P576-UPPER GI-Gastroduodenal diseases}

CYSTIC LYMPHANGIOMA IN JEJUNAL MESENTERY, AS A CAUSE OF PAIN AND PALPABLE ABDOMINAL MASS, IN RELATION TO A CASE

L.A. Vega Rojas, S. Sánchez Cordero, R. Claveria, P. Besora, K. Oh-Uiginn, C. Galmes, D. Carmona, R. Rodriguez, H. Janafse, D.L. Luckute, J. Camps

General Surgery, Consorcio Sanitario de la Anoia, IGUALADA, Spain

Aims: To present a representative clinical case of cystic lymphangioma

Methods: Clinical case diagnosed and treated in our service and bibliographic review.

Results: A 35-year-old female with no medical history of interest or allergies to medications, who consulted for palpable mass at mesogastric level to the left of the midline associated with abdominal pain of 3-6 months of evolution, without concomitants or relationship with the intake, Valsalva or physical efforts, without change in the depositional habit or toxic syndrome. The abdominal CT (Computed Tomography) revealed a cystic mass in jejunum mesentery, defined edges, about $6 \mathrm{~cm}$ in diameter and that does not capture contrast; likewise, there is no ascites, retroperitoneal adenopathies or other intra-abdominal or pelvic masses, radiology recommends completing the study with abdominal MRI (Magnetic Resonance Imaging) that informs of possible lymphangioma at the level of the jejune mesentery. Surgical exeresis was decided, which was carried out by laparoscopic approach, with emptying of the lesion and enucleation of the lesion without incidents, the postoperative evolution was favorable being discharged at $48 \mathrm{~h}$. The pathological anatomy reported fibro-adipose tissue with presence of lymphatic dilatations associated with a cystic lesion without epithelial lining, with serous fluid and abundant macrophagic reaction compatible with mesenteric lymphangioma.

Conclusions: The mesenteric cyst is a rare pathology with an incidence ranging from 1/27,000 to $1 /$ 250,000 , predominating in the fourth decade of life. It is defined as any cystic lesion in the mesentery, and is subdivided according to its origin into lymphatic, mesothelial, urogenital, dermoid, and enteric and pseudocysts. Most of the time they are asymptomatic although they can (as in our case) present with abdominal pain and even produce complications such as intestinal obstruction, volvulus, intracystic hemorrhage, infection, rupture, and even malignant transformation. For the diagnosis, the palpation can be of great help, showing mass of well-defined limits and partially mobile. The imaging test of choice is abdominal ultrasound / abdominal CT, supplemented by magnetic resonance imaging. The recommended treatment is surgical exeresis, considering laparoscopy as the first option; if it is complete, it can be considered as a curative treatment.

\section{P577-UPPER GI-Gastroduodenal diseases}

\section{P575-UPPER GI-Gastric cancer}

\section{ROBOT-ASSISTED TOTAL GASTRECTOMY FOR EPSTEIN- BARR VIRUS-ASSOCIATED MULTIPLE GASTRIC CARCINOMA}

K. Kimura, Y. Ebihara, K. Tanaka, Y. Nakanishi, T. Asano, T. Noji, Y. Kurashima, S. Murakami, T. Nakamura, T. Tsuchikawa, K. Okamura, T. Shichinohe, S. Hirano

Department of Gastroenterological Surgery II, Hokkaido University Faculty School of Medicine, HOKKAIDO, Japan

Epstein-Barr virus (EBV) has been known as one of causal virus of gastric cancer. EBV-related gastric cancer considered to be about $10 \%$ of the entire gastric cancer, and it is rare that EBVrelated gastric cancer has multiple lesions. The patient was 60 years old female. She was diagnosed with upper gastrointestinal endoscopy with lesion in the lower major stomach body, lower anterior wall of the stomach body, rear wall in the middle part of the stomach, rear wall in the middle part of the stomach, and lesser curvature of the stomach angle, as a result of biopsy, adenocarcinoma was observed from the former four. The patient underwent a robot-assisted total gastrectomy. Adding a newly found lesion, the histopathological diagnosis was pT1b in the lower major stomach body, pT1b in the lower anterior wall of the stomach body, pT1b on rear wall in the middle part of the stomach, pT1b on rear wall in the middle part of the stomach, and pT1a in lesser stomach body, pNO, pStageIA. Pathological examination results showed that the four lesions were positive for tumor cells in EBER in situ hybridization and were considered to be EBV—related gastric cancers. She was discharged on the 12th day after the operation without any postoperative morbidities. There has been no sign of recurrence without postoperative therapy for 12 months.

\section{CLINICAL OUTCOMES IN MINIMAL INVASIVE SURGERY OF MECKEL'S DIVERTICULUM : A RETROSPECTIVE MULTICENTER STUDY}

\section{J.W. Kim ${ }^{1}$, H.S. Jung ${ }^{2}$, J.Y. Kim ${ }^{3}$, S.G. Park ${ }^{3}$}

${ }^{1}$ Department of Surgery, Hallym University College of Medicine, HWASEONG-SI, Korea; ${ }^{2}$ Department of Surgery, Dongtan Sacred Heart Hospital, HWASEONG-SI, Korea; ${ }^{3}$ Department of Surgery, Dongtan Sacred Heart Hospital, Hallym University College of Medicine, HWASEONG-SI, Korea

Aims: Meckel's diverticulum (MD) is one of the most common congenital anomalies of the small intestine caused by an obliteration defect of omphalomesenteric duct. The objective of this study was to review surgical treatment and clinical outcomes of MD, and evaluate the safety and feasibility of minimal invasive surgery (MIS) in MD.

Methods: We performed a retrospective analysis of medical record for patients who underwent Meckel's diverticulectomy at six Hallym-University-affiliated hospitals between January 2008 and December 2017. The patients were underwent either open or MIS. Then patients in MIS group were divided into laparoscopic-only diverticulectomy (LOD) group or laparoscopic-assisted diverticulectomy (LAD) group in more detail.

Results: Of 65 patients underwent Meckel's diverticulectomy, 22 underwent open surgery and 43 underwent MIS. There were no differences in the operation time, complication, and the length of hospital stay between the two groups. Time to flatus, and time to soft food intake were shorter in the MIS group than in the open group ( 3.1 vs. 4.1 days, $\mathrm{P}=0.028 ; 4.9$ vs 6.8 days, $\mathrm{P}=0.003$, respectively). In three-group analysis, $\mathrm{LOD}$ group showed the fastest recovery, including time to gasout and time to soft food intake ( 3.0 vs $4.1,3.3$ days, $P=0.008,4.4$ vs $6.8,5.3$ days. $P=0.001$ ) and the shortest length of postoperative hospital stay compared with the open group and LAD group (6.2 vs $10.4,8.6$ days, $\mathrm{P}=0.041$ ). Multivariate analysis showed that old age and ileus as preoperative diagnosis were independent predictors of complications $(\mathrm{P}=0.034$ and 0.006 , respectively). Operation type, including open, LOD, and LAD was not associated with complications. Conclusion: The present study showed that MIS is associated with faster recovery without increasing complications. Therefore, MIS can be a safe alternative to open surgery in MD. 


\section{P578-UPPER GI-Gastroduodenal diseases}

\section{NEW LAPAROSCOPIC GASTROSTOMY PROCEDURE DESIGNED FOR BASIC LAPAROSCOPIC SUTURE TRAINING}

\section{Y.Y. Liu}

General surgery, Chang gung memorial hospital kaohsiung division, KAOHSIUNG, Taiwan

Purpose: Gastrostomy(OG) is an alternative method for nutrition support, especial for the patients with oral-esophagus route obstruction or dysfunction. The Most operation were conducted by young surgeon or residents. Laparoscopic gastrostomy(LG) was a new coming procedure and the skillful suture techniques were needed. The most the residents can't be qualified for this operation. We designed the method for laparoscopic gastrostomy to provide the traning opportunity of suture skill training and guarantee the patient's safety.

Material and method: laparoscopic gastrostomy procedure was done with two $5 \mathrm{~mm}$ trocar. The cutaneous fixation. The straight needle with 2-0 Prolene was inserted into peritoneal cavity from upper point, then punctured the sero-muscular layer of stomach. The needle was retrieved out from the same point by guidance of 18 gauge needle. The same way was used for other three points. One purse string around gastrostomy was created by one hand suture method and fastened by Köckerling knot tier after insertion of 20 Fr Foley tube. Finally, the peritonization was finished by hand tie externally and knot were keep in subcutaneous layer

Results: Since $2017-2018$ (divided into 4 phase), total 124 patients (84 LG, 40 OG) received gastrostomy as treatment. $54 \mathrm{LG}$ were done by senior endoscopic surgeon. Median operative time was $27 \mathrm{~min}$. Other 70 gastrostomy done by residents including $40 \mathrm{OG}$ and $30 \mathrm{LG}$. Percentage of LG done by residents in the phase I to IV was $0 \%, 10 \%, 40 \%$ and $97 \%$; Median operative time $127,112,97$ and $65 \mathrm{~min}$. No mortality and re-operatiion case. Wound erosion rate is about $12 \%$ without significance between LG and OG.

Conclusions: New designed laparoscopic gastrostomy by 2 trocar ports procedure provided the training opportunity of suture skill training and guarantee the patient's safety lower body of stomach was chose. Four Point around gastrostomy wound were chose for sub-

\section{P580_-UPPER GI-Gastroduodenal diseases}

SUPERIOR MESENTERIC ARTERY SYNDROME: A RETROSPECTIVE DESCRIPTIVE STUDY

\section{$\underline{\text { M.R. Elkeleny }}{ }^{1}$, R. Awaludin ${ }^{2}$}

${ }^{1}$ GIT and bariatric surgery, Faculty of medicine, Alexandria University, ALEXANDRIA, Egypt; ${ }^{2}$ GIT surgery, Faculty of medicine, ALEXANDRIA, Egypt

Background: Superior mesenteric artery syndrome is best described as compression of the thir part of duodenum by the superior mesenteric artery, resulting in obstruction. The study of this rare medical condition was carried out since decades yet remain obscure. This study aimed to analyze different clinical presentations, diagnostic modalities, treatment approaches and outcomes, as well as to emphasize the importance of long term follow up.

Methods: Thirty-five superior mesenteric artery syndrome cases were collected retrospectively from a Facebook group called 'Superior Mesenteric Artery Syndrome Awareness \& Support'. A questionnaire was designed using Google form to obtain the demographics, presenting symptoms, risk factors and co-morbidities, investigations, means of treatment and the outcomes. Data was entered into Microsoft Office Excel for statistical analysis.

Results: The median age at diagnosis was 22 years. The median body mass index was $20.8 \mathrm{~kg} /$ $\mathrm{m}^{2}$;. The median time interval from symptom onset to initial diagnosis was 22 months. The major presenting symptoms were abdominal pain (82.86\%), nausea (77.14\%), and vomiting $(65.71 \%$ ) Abdominal computed tomography scan with contrast $(82.86 \%)$ was commonly used for confirmation of diagnosis. Thirteen cases $(37.14 \%)$ were congenital. Thirty patients $(85.71 \%)$ had received treatment. The overall management success was only $13.33 \%$. Surgical management (34.29\%) was the most used regimen.

Conclusion: Diagnosis of superior mesenteric artery syndrome is established after a thorough assessment of the clinical presentations and confirmation with suitable imaging modalities. The choice of treatment should be dependent on the causes and severity as different patients respond differently to therapy. Recurrence is possible in all patients thus a long-term follow up is required.

\section{P581-UPPER GI-Gastroduodenal diseases}

\section{LAPAROSCOPIC TOTAL GASTRECTOMY WITH OMEGA RECONSTRUCTION}

V. Drakopoulos, I. Iliadis, K. Botsakis, S. Petsa-Poutouri, K. Sarafi, N. Morfis, K. Rekouna, V. Vougas

1st Department of Surgery and Transplantation Unit, District General Hospital of Athens « Evangelismos », ATHENS, Greece

Introduction: Laparoscopic surgery is gaining acceptance in the treatment of stomach cancer. Laparoscopic treatment of cancer of the fundus and gastroesophangeal junction as well as restoration of the continuity of the gastrointestinal tube after the resection often presents technical difficulties.

Material-Method: We present the case of a 85 year old woman who presented with melena, hematemesis, anemia (Ht 12.5\%) and being haemodynamically unstable. After the stabilization of the patient, a gastroscopic examination followed, where it revealed a tumor of the fundus (adenocarcinoma). The patient was submitted to laparoscopic total gastrectomy and oesophagojejunal anastomosis, Omega type $(\mathrm{O})$, and intestinal anastomosis Braun, with the usage of 3 trocars (umbilical $10 \mathrm{~mm}$ as inserted in laparoscopic surgery of a single incision, and two $12 \mathrm{~mm}$ in the midclavicular line bilaterally). The oesophagojejunal anastomosis was conducted with the use of a linear stapler for the posterior wall and the convergence of the anterior wall with laparoscopic sutures in two layers. Patient remains in well condition, 6 months after the operation.

Conclusion: Laparoscopic approach seems to be safe for treatment of gastric cancer of the fundus and of the gastroesophangeal junction, as it offers better surgical field view and less postoperative complications. The restoration of the continuity of the gastrointestinal tube with anastomosis of $\mathrm{W}$ type is considered safe alternative to the classic Roux-en-Y anastomosis.

\section{LAPAROSCOPIC TREATMENT OF PERFORATED BLEEDING GASTRIC ULCER}

F.J. Buils Vilalta, J.J. Sánchez Cano, E. Homs, E. Bartra, P. Martínez, C. Morales, D. del Castillo

General and Digestive Surgery, University Hospital of Sant Joan. Faculty of Medicine Rovira i Virgili, REUS, Spain

Aims: In the last hundred years much has been written on peptic ulcer disease and the treatmen options for one of its most common complications: perforation. Laparoscopic repair of perforate peptic ulcer has been gaining popularity in recent years. Treatment for perforated ulcer can be performed laparoscopically in $85 \%$ of cases, making it possible to avoid a median laparotomy which can lead to wound infection and late eventration.

Methods: A 77-year-old male presented to emergency room with a three-hour history of progressively worsening epigastric pain and nausea. Physical examination revealed rebound tenderness compatible with an acute abdomen. A CT scan showed: important pneumoperitoneum unable to define the drilling point; distended stomach with plenty of fluid inside and dense content fundus / body suggestive of active arterial bleeding

Results: The patient was emergently taken to the operating room for diagnostic laparoscopy Perforation shown in greater gastric curvature associated blood remnants. Gastrotomy for clot removal is done without observing active bleeding. The gastrotomy was repaired using standard stitches. All exudate was aspirated and the peritoneal cavity was irrigated with warm saline solution The patient had an uncomplicated post-operative course. JP drain was removed and he was discharged one week after surgery.

Conclusion: The role of laparoscopic surgery in emergencies is well documented. Laparoscopic approach is indicated in any case of suspected gastroduodenal perforation and seems to offer the same advantages as for the vast majority of laparoscopic procedures. Laparoscopic surgery may therefore have a real place in the treatment of perforated peptic ulcer. 


\section{P582-UPPER GI-Gastroduodenal diseases}

\section{LAPAROSCOPIC AND ENDOSCOPIC OPERATION IN THE PATIENTS WITH GIST}

\section{Yu.V. Grubnik, Yu.V. Yuzvak, V.A. Fomenko}

Surgery department 3, Odessa National Medical University, ODESSA, Ukraine

Methods: and materials. For 6 years we observed 11 cases with GIST of stomach and duodenum. Seven patients were brought to clinic with the bleeding and two patients were brought to clinic with vomiting and compensate stenosis. In all circumstances we done the CT, MRT and endoscopic examinations of stomach and duodenum with biopsy. In two circumstances we performed endoscopic operation. In one circumstance we successfully take off the GIST from the duodenum endoscopically. During the operation we use the endoscopic instruments. In another circumstances, after endoscopic excision the tumor appear the bleeding which was stopped by endoscopic local heamostasis, by putting clipps on the vessels. In 9 circumstances the tumors were in stomach. In 4 circumstances we performed laparoscopic wedge resection the tumors by staplers. In 3 circumstances when the tumor was very big and situated in the fundus of stomach, we performed laparoscopic resection of the fundal part of stomach by using laparoscopic staplers and 'Liga Sure' sealing. In 2 circumstance we took off the tumor by putting laparoscopic trocars inside the stomach for instruments and for visualization tumor. After excision the tumor and took it of the stomach we sutured the holes in the stomach.

Results: We have no mortality after laparoscopic operation. There were no malignisation in all 9 circumstances. We have 3 cases morbidity. In 2 circumstance the bleeding from the stomach that was stopped endoscopically. In 1 circumstance there was wound infection.

Conclusion: Laparoscopic and endoscopic operations are successful methods for the curing the GIST.

\section{P583-UPPER GI-Gastroduodenal diseases}

\section{LAPOROSCOPIC OPERATION IN THE PATIENTS WITH PERFORATED ULCER OF THE STOMACH AND DUODENUM}

Yu.V. Grubnik, Yu.V. Yuzvak², V.A. Fomenko ${ }^{2}$, Surgery department 3, Odessa National Medical University, ODESSA, Ukraine

The aim of the study to decrease the morbidity in the patients with perforated ulcers of the stomach and duodenum. We observed 107 patients with perforated ulcers of stomach and duodenum. Women were 45 , men were 62. Average age about 45 years. 91patients had perforation ulcer of stomach and duodenum. 26 patients had perforations with bleeding. All patients were divided in two groups. The first groups 59 patient operated laporocopically, in the second group 48 patients operated traditionally. Results: There were no mortality in the group that operated laparoscopically.

In the group that were operated traditionally one patient died after rebleeding. The average stay in hospital in the group that were operated laporoscopically about 2 days. In the groups with traditional operations, were about 8 days. The morbidity in the first group were in 5 cases. Pneumonia in 2 cases, suppuration of the troacar points were in 3 cases. In the second group pneumonia were in 3 cases, suppuration of the operation wound were in 5 cases, subdiaphragmatic abscess was in 1 cases.

Conclusion: Laporoscopic operation in during treatment decrease the mortality, morbidity and hospital staying in the patients the perforated ulcer of stomach and duodenum .

\section{P584-UPPER GI-Gastroduodenal diseases}

\section{COMPARISON OF DIFFERENT TREATMENT TACTICS IN PATIENTS WITH HIGH RISK OF REBLEEDING OF PEPTIC ULCER HEMORRHAGE}

V.V. Petrushenko, D.I. Grebeniuk, I.V. Radoga, V. Sobko, V.M. Koval, N.A. Liakhovchenko

Department of Endoscopic and Cardiovascular Surgery, National Pirogov Memorial Medical University, Vinnytsya, VINNYTSYA, Ukraine

Aim: of the study was to compare of different tactics of treatment in patients with high risk of rebleeding after peptic ulcer hemorrhage.

Methods: Analysis of treatment of 302 patients was conducted during the study. 154 cases of patients admitted in a state of hemorrhagic shock were selected. All these patients had a high risk of rebleeding, which was estimated using predictive scales Rockall (minimum score-4). Group 1 includes 45 patients who underwent endoscopic hemostasis. Group 2 includes 101 patients with hemorrhagic shock and treated with drug therapy. Group 3 includes 32 patients operated on profuse bleeding or rebleeding. The compare criteria's were rebleeding incidence, outcome and hospital treatment duration.

Results: Of the 45 patients of the group 1 endoscopic hemostasis in 31 $(68.9 \%)$ cases were made during the initial endoscopy and in $14(31.1 \%)$ patients - in second endoscopy. The average time of bleeding recurrence was $1.5 \pm 0.4$ days. $2(4.44 \%)$ patients were operated on for recurrent bleeding. $3(2.5 \%)$ patients died. The average time spent in the hospital for deceased patients is $1.2 \pm 0.6$ days. The average treatment time for patients in group 2 was $6.4 \pm 2.1$ days.

Among patients in group 2, bleeding recurrence was recorded in 34 cases $(33.7 \%)$. The average time of bleeding recurrence was $1.4 \pm 0.6$ days. 22 $(21.8 \%)$ patients were operated on for recurrent bleeding. $16(15.8 \%)$ patients died. The average time spent in the hospital for deceased patients is $1.3 \pm 0.4$ days. The average treatment time for patients in group 2 was $6.7 \pm 1.9$ days.

Of the 32 patients of the third group $22(68.75 \%)$ were operated about ulcer rebleeding in the hospital, and $10(31.25 \%)$-about the profuse bleeding ulcer. Noonr patient had recurrent bleeding. The average treatment time for patients in group 2 was $12.5 \pm 3.2$ days.

Conclusions: The development of hemorrhagic shock in patients with peptic ulcer bleeding significantly increases the risk of rebleeding and mortality. The application of endoscopic hemostasis allows to reduce the risk of rebleeding and mortality compared with conservative antiulcer therapy.

Surgical treatment can achieve reliable hemostasis, but accompanied by higher mortality and longer duration of hospital treatment. 


\section{P585-UPPER GI-Gastroduodenal diseases}

EFFECTIVENESS OF LOCAL INJECTION OF PLATELET-RICH PLASMA FOR TREATMENT OF PEPTIC ULCER BLEEDING WITH HEMORRHAGIC SHOCK (EXPERIMENTAL STUDY)

\section{V.V. Petrushenko ${ }^{1}$, D.I. Grebeniuk ${ }^{1}$, I.V. Taran ${ }^{2}$, V. Sobko ${ }^{1}$,} I.V. Radoga ${ }^{1}$

${ }^{1}$ Department of Endoscopic and Cardiovascular Surgery, National Pirogov Memorial Medical University, Vinnytsya, VINNYTSYA, Ukraine; ${ }^{2}$ Department of Farmacology, National Pirogov Memorial Medical University, Vinnytsya, VINNYTSYA, Ukraine

The aim: Of our study was to evaluate of effectiveness of local injection of platelet-rich plasma for treatment of peptic ulcer bleeding with hemorrhagic shock in experiment.

Methods: The study was performed on $60 \mathrm{Wistar}$ rats according to local and international rules for working with experimental animals. The average weight of animals was $183 \pm 16$ grams. In all animals our modification of type 2 acetic acid ulcer (Susumu Okabe, 2005) was modeled. We randomly divide all animals in 3 groups. 20 rats with only modeled ulcer were included in group 1. 20 rats with modeled ulcer and hemorrhagic shock after 3-3.5 ml blood sampling were included in group 2. In group 3 we included 20 rats with modeled ulcer and hemorrhagic shock and performed local injection of platelet-rich plasma (local periulcelar injection of $0.1 \mathrm{ml}$ of autologous platelet-rich plasma). On 1st, 7 th and 14th day measurement of the ulcers square and morphological study were performed.

Results: The data we have received demonstrate a tendency of decrease of ulcers' square in all groups with time flow. We also compared sizes of ulcerative defects in all groups at every point of the study. On the $1^{\text {st }}$ day of investigation there were no differences $(\mathrm{p}>0.05)$ between ulcers' square in all groups. On the $7^{\text {th }}$ day we found out more rapid decrease of size in group 3 $(p>0.05)$. However, this tendency had no statistical significance. On the 14 th day difference was larger and it was statistically significant this time $(\mathrm{p}<0.01)$

Also the better ability to stimulate the activity of fibroblasts and revascularization in the young connective tissue with improving oxygenation in the ulcers and enhancing of cell proliferation, differentiation and accelerating of maturation of connective tissue and healing of ulcers was demonstrated in group 3 .

Conclusion: Platelet rich plasma reduces inflammatory response and stimulates proliferation of gastric epithelial cells on $7^{\text {th }}$ day with the restoration of secretory activity and epithelialization of ulcers in $71.4 \%$ of experimental animals on $14^{\text {th }}$ day, the activation of the fibroblastic reaction during the all experiment and decreasing of ulcers' square.

\section{P586-UPPER GI-Gastroduodenal diseases}

\section{REDUCED PORT LAPAROSCOPY AND ENDOSCOPY COOPERATIVE SURGERY (RP-LECS) FOR GASTRIC GIST AIDED BY FREE JAW CLIP AND FREE LOOP PLUS}

H. Fujii, Depat. of Surgery, Japanese Red Cross Fukui Hospital, FUKUI, Japan

Introduction: In conjunction with Charmant, a local eyeglass frame manufacturer, we developed novel devices called the FJ (Free Jaw) Clip to grasp organs in the abdominal cavity and the F (Free) Loop Plus to pull thread extracorporeally from within the abdominal cavity.

Product Summary: The FJ Clip is used to grasp organs in the abdominal cavity, a stainless steel, removable forceps for use in laparoscopic surgery. It provides a strong grip but rarely crushes organ tissue. The clip comes in two sizes, one for use in a 5-mm port and the other for use in a 12-mm port, and in two lengths, $29.4 \mathrm{~mm}$ and $35.6 \mathrm{~mm}$, respectively. To pull out thread tied to the FJ Clip, we developed the F Loop Plus, which is a $21 \mathrm{G}$ by 90 -mm-long special stainless needle with f0.1-mm NiTi alloy thread which is used pull suture threads from inside the abdominal cavity to outside the body. Case: We performed 9 cases of reduced port laparoscopic and endoscopic cooperative surgery (LECS). We performed reduced port surgery (RPS) by making a $1.5-\mathrm{cm}$ incision at the umbilicus, inserting 2 trocars $(12 \mathrm{~mm}$ and $5 \mathrm{~mm}$ ), and inserting another trocar $(5 \mathrm{~mm})$ at the left side of the abdomen. We expanded the left hepatic lobe with a 12-mm FJ Clip for Penrose drain placement, grasped the front wall of the gastric body with a $12-\mathrm{mm}$ FJ Clip, applying traction toward the legs to pull up the tissues around the tumor, and resected all layers of the tumor via oral endoscopic submucosal dissection technique. The resected area was closed with a suturing device or interrupted sutures in the abdominal cavity. Results: The average operation time was $221 \mathrm{~min}$ and postoperative hospital stay was 10.4 days. There were no complications after surgery, all patients have been satisfied for their cosmetics. Conclusion: We believe the FJ Clip and F Loop Plus are effective for use in LECS and RPS.

\section{P587-UPPER GI-Gastroduodenal diseases}

FISH BONE PENETRATION THROUGH STOMACH INTO SUPERIOR MESENTERIC VEIN CAUSING SMV THROMBOSIS FORMATION: CASE REPORT

\section{J.H. Kang, I.S. Chen}

General Surgery, Kaohsiung Veterans General Hospital, KAOHSIUNG, Taiwan

A 75 year-old female was admitted to the emergency department with complaints of abdominal cramping pain, back pain and diarrhea for one day. She also had fever, ever up to $39^{\circ}$. In these two weeks, she felt occasionally epigastric pain. Her past medical history included hypertension. On physical examination, she was conscious and alert. Abdominal examination revealed diffuse tenderness and knocking pain over right flank. Laboratory tests indicated an degraded white cell count of $2890 /$ cumm with $22 \%$ band forms, C-reactive protein of $25 \mathrm{mg} / \mathrm{dL}$ and abdominal liver function tests (Alanine Aminotransferase: $149 \mathrm{U} / \mathrm{L}$, alkaline phosphatase: $249 \mathrm{U} / \mathrm{L}$, gammaglutamyl transferase: $175 \mathrm{U} / \mathrm{L}$ ) without hyperbilirubinemia. Abdominal X-ray showed paralytic ileus. Our presumptive diagnosis was acute peritonitis, based on the patient's symptoms. Empirical antibiotics were administered immediately, and a computed tomography (CT) imaging study was performed. The CT scan showed a stick like foreign body noted between ventral side of pylorus and SMV lumen, about $1.5 \mathrm{~cm}$ in length and associated with perifocal infiltration and segmental SMV thrombus formation.(Fig. 1) However, there is no obvious pneumoperitoneum and no evident ascites is associated. An emergency exploratory laparotomy was performed, revealing stomach perforation at posterior wall with a $3 \mathrm{~cm}$ fish bone thourgh pancreas into SMV Localized inflammation and fibrosis were identified without obvious fluid accumulation(Fig. 24). Removal of fish bone and simple closure of stomach perforation were performed. Blood cultures revealed Bacteroides thetaiotaomicron. Three weeks later, she received a follow-up CT scan which showed SMV obliteration with chronic pylephlebitis.

\section{P588-UPPER GI-Gastroduodenal diseases}

\section{ENDOSCOPIC APPROACH FOR IATROGENIC GASTRIC PERFORATION SECONDARY TO CHEST DRAIN INSERTION}

A. Sánchez Arteaga, V. Durán Muñoz-Cruzado, J. Tinoco González, V. Camacho Marente, J. Pintor Tortolero, G. Anguiano Díaz, L. Tallón Aguilar, F. Pareja Ciuró, J. Padillo Ruiz

General Surgery, Hospital Universitario Virgen del Rocío, SEVILLE, El Salvador

Aim: Here we present a case report about the endoscopic treatment for iatrogenic gastric perforation secondary to a chest tube insertion.

Methods: A case report of a 24-year-old male with history of a road traffic accident. Described injuries were severe brain injury with GCS $<8$ at pre-hospital care arrival, thoracic injury with several rib fractures on the left hemithorax and hypoventilation on the left side. Prior to hospital transfer a chest drain was inserted on the left side, and the patient was intubated.

Results: At hospital admission, the patient was hemodynamically stable and connected to a mechanical ventilator. Thoracic exam showed persistent hypoventilation on the left chest. No other abdominal or pelvic injuries were found in the physical exam. A frontal chest x-ray revealed pneumothorax and the chest tube was not viewable. A further CT scan showed the chest drain placed in the abdominal cavity, into the stomach, besides a subdural hematoma, comminuted pelvic fracture of the pubic rami and a left sacroiliac fracture.

During the first $24 \mathrm{~h}$ in the ICU, neurological worsening was observed, and a new cranial CT revealed enlargement of the subdural hematoma, for what the patient underwent decompressive craniectomy, with improvement thereafter. Following a five-day period of stabilization after surgery, the patient was evolving satisfactorily, and the removal of the intragastric chest drain was considered. Endoscopy was performed to confirm the placement of the drain, and it was removed under direct vision. Approximately twenty five centimeters of the catheter were visualized in the gastric lumen, and then successfully removed. The patient recovered well and was discharged from ICU to medical hospital ward after fourteen days, and a week later he was discharged home.

Conclusion: Endoscopic management for gastric perforation after a chest drain insertion may result effective and can prevent open surgery morbidity. 


\section{P589-UPPER GI-Gastroduodenal diseases}

STEP BY STEP INSTRUCTIONAL VIDEO OF LAPAROSCOPIC REPAIR OF PERFORATED DUODENAL ULCER FOR SURGEONS IN TRAINING IN A SINGAPORE TERTIARY INSTITUTION

\section{A.M. Oo}

Department of General Surgery, Tan Tock Seng Hospital, SINGAPORE

Tan Tock Seng Hospital is second largest hospital in Singapore. It is affiliated to two medical schools in Singapore and it is a training hospital for both undergraduates and postgraduates. Minimally Invasive surgery for both benign and malignant diseases of upper gastrointestinal tract becomes more and more popular nowadays. In our department, all the residents have to view the step by step instructional videos of mininally invasive surgeries before they can assist in the cases or perform on their own under the supervision of consultant surgeons. The viewing of the instructional videos help them with better understanding of the procedures. The viewing of videos help them with the importance of steps, standardization of steps. With the help of instructional video, they can not only assist better in the surgery but also reduce the learning curve when they start doing the procedure themselves after the graduation from the residency programme. This is the step by step instructional video of laparoscopic repair of perforated duodena ulcer for surgeons-in-training rotated to our department.

\section{P591-UPPER GI-Gastroduodenal diseases}

FEASIBILITY AND SAFETY OF NON-EXPOSED DUODENUM LAPAROSCOPIC AND ENDOSCOPIC COOPERATIVE SURGERY: A NOVEL TECHNIQUE FOR SUPERFICIAL DUODENAL TUMORS

Y. Yanagimoto, T. Omori, N. Shinno, K. Yamamoto, K. Sugimura, H. Miyata, M. Ohue, M. Yano

Gastroenterological Surgery, Osaka International Cancer Institute, OSAKA, Japan

Background: Pancreatoduodenectomy is considered to be very invasive for early superficia duodenal tumors (SDTs), which have a lower risk of lymph node metastasis. Partial duodenal resection with endoscopic submucosal dissection for SDTs is an attractive technique but it is associated with a high risk of complications. The full-thickness resection of the duodenum wal including laparoscopic and endoscopic cooperative surgery has risk of spreading tumor cells and digestive juices into the abdominal cavity. We have developed novel technique for SDTs to decrease the risk of exposure to abdominal cavity of tumor cells and digestive juices, called nonexposed duodenum laparoscopic and endoscopic cooperative surgery (Neo-DLECS). Aim: The aim of this study is to evaluate the feasibility and safety of Neo-DLECS for SDTs. Surgical Procedure: The attachment of the transverse mesocolon was freed from the head of the pancreas and retroperitoneal tissues under laparoscopy. The duodenum and the head of the pancreas were mobilized from the retroperitoneum using the Kocher maneuver. A standard ESD was performed for the SDT using endoscope. The serosa of the ESD ulcer bed was reinforced using the laparoscopic hand-sewn suturing technique in the seromuscular layer around the resected area. After completing the procedure, the endoscope was inserted and passed over the resected area to confirm that there was no stenosis or leakage.

Methods: Ten consecutive patients with SDT underwent Neo-DLECS in our institute between March 2015 and March 2017. The clinicopathological features of the patients and surgical outcomes were prospectively collected and retrospectively analyzed.

Results: Pathological diagnosis was adenocarcinoma for six patients, adenoma for three patients, and neuroendocrine tumor grade 1 for one patient. The median tumor size was $36(20-54) \mathrm{mm}$. The median operative time was 227.5 (180-390) min. The median blood loss was $0(0-175) \mathrm{g}$. There were no conversions to open surgery in this series. Intraoperative perforation was found in two cases during the ESD procedure. However, all perforations were closed and reinforced using hand-sewn sutures. No postoperative complications were above grade 2 in the Clavien-Dindo classification system.

Conclusions: Neo-DLECS is safe and feasible and can be an option for surgical SDT resection.

\section{P592-UPPER GI-Gastroduodenal diseases}

\section{P590-UPPER GI-Gastroduodenal diseases \\ LAPAROSCOPIC TRANSGASTRIC ENUCLEATION OF HETEROTOPIC PANCREATIC CYSTIC LESION CAUSING GASTRIC OUTLET OBSTRUCTION}

G. Ayiomamitis ${ }^{1}$, P. Grivas ${ }^{1}$, S. Paravas ${ }^{1}$, A. Liarakos ${ }^{1}$, K. Tsavari ${ }^{2}$, K. Manoloudaki ${ }^{2}$, N. Paschalidis

${ }^{1}$ 1st Surgical Department, Tzaneio General Hopsital, PIRAEUS, Greece; ${ }^{2}$ Pathology Department, Tzaneio General Hopsital, PIRAEUS, Greece

In general duplication cysts are rare developmental congenital disorders of the GI tract. Three morphological criteria should be met in order to confirm the pathological diagnosis: 1 . they should be attached to the stomach's wall and should be the continuation of it, 2. at least one of the muscle layers of the stomach's wall should be included and 3.it should have normal gastric mucosa. The treatment is either enucleation or partial gastrectomy.

Aim: Present our minimally invasive approach to a rare prepyloric submucosal cystic lesion causing gastric outlet obstruction.

Case Report: A 27-year-old female with vomiting, weight loss and in bad general condition was diagnosed after a full work-up (blood tests, endoscopies, EUS, CT and MRI) with a submucosal cystic tumor. This cyst first was thought to be a duplication cyst. Since the patient was young, our intention was to offer the least invasive surgical technique in order to spare gastrectomy and Billroth anastomosis.

Results: The procedure was completed laparoscopically with enucleation of the cyst through a gastrotomy on the anterior wall of the stomach. After the enucleation of the cyst the gastric mucosa was sutured back and then the gastrotomy was closed with continuous sutures. The result of the pathological report confirmed a rare case of a heterotopic pancreatic cystic lesion. The postoperative course of the patient was uneventful and was discharged with instruction for her diet the $4^{\text {th }}$ postoperative day. The patient 3 months post-operative has no symptoms.

Conclusion: In such benign conditions and especially in young patients, gastrectomies could be avoided if possible and give their place to less invasive approaches in order to reduce lifelong risks and morbidity. Trangastric enucleation of the cyst although a demanding approach is safe and could be considered as a 'gentler' technique with reduced morbidity.

\section{IS THE LAPAROSCOPIC DUODENOJEJUNOSTOMY SAFE AND EFFECTIVE FOR THE CHRONIC SYMPTOMS OF WILKIE'S SYNDROME?}

S. Dios-Barbeito, F. López, F. Moreno, N. García, P. García, M. Socas, A. Barranco, I. Alarcón, J. Padillo, S. Morales-Conde

Digestive Surgery, Hospital Universitario Virgen del Rocío, SEVILLE, Spain

Aims: Wilkie's syndrome is caused by the entrapment of the $3^{\text {rd }}$ part of the duodenum between the aorta and the Superior Mesenteric Artery (SMA). Surgery is indicated for chronic cases and failure of conservative management, being reported a laparoscopic duodenojejunostomy as a minimally invasive option.

Methods: All cases treated by laparoscopic duodenojejunostomy in our centre because of chronic Wilkie's syndrome were recorded.

Results: 3 females and 1 male underwent a laparoscopic duodenojejunostomy, with a mean age of 32 years (range 19-47). All patients presented abdominal pain, and weight loss was identified in most of them. A reduced aortomesenteric angle measured by CT scan was the key for the diagnosis (mean angle 22.5 degrees, range 21-24).

Conventional laparoscopic approach was performed in two patients, the other two patients underwent a SILS port approach. Mean time of surgery was 62.5 min (range 35-100) and length of stay was 5 days (range 2-13). After a mean follow-up of 47.5 months (range 11-69), 3 patients improved their symptoms.

Conclusions: Surgery is the mainstay in complicated or refractory cases of SMA. Laparoscopic duodenojejunostomy has the advantages of the laparoscopic approach (including rapid recovery time, reduced post-operative pain and shorter hospital stay) and it is feasible, safe and effective. 


\section{P593-UPPER GI-Gastroduodenal diseases}

RADICAL OPEN REPAIR VERSUS CONSERVATIVE LAPAROSCOPIC REPAIR OF PERFORATED PEPTIC ULCER DISEASE

Y. Ben Safta ${ }^{1}$, M.D. Maatouk ${ }^{2}$, N.S. Tounsi ${ }^{3}$, A. Mabrouk ${ }^{4}$, A. Anis ${ }^{4}$, S. Daldoul ${ }^{4}$, S. Sayari ${ }^{4}$, M. Ben Moussa ${ }^{4}$

${ }^{1}$ Surgery A, Charles Nicolle Hospital, Tunis El Manar University, TUNIS, Tunisia; ${ }^{2}$ Surgery A, Charles Nicolle Hospital, TUNIS, Tunisia; ${ }^{3}$ Surgery, Salah Azaiz Institute Surgery, TUNIS, Tunisia; ${ }^{4}$ Surgery, Charles Nicolle Hospital, TUNIS, Tunisia

Aims: During the last two decades, the incidence of perforated peptic ulcer PPU disease remains relatively unchanged, despite the advancement of medical treatment. Despite the significant progress of laparoscopic surgery for peptic ulcer disease, no consensus conclusion favoring its application has been reached. The aim is to compare the open radical technique to laparoscopic treatment with only a suture in terms of morbidity and mortality.

Methods: In this retrospective and consecutive study, all patients who underwenteither open or laparoscopic were identified and extracted from database from January 2005 to December 2014 in our surgical unit.

Results: A total of 159 patients were diagnosed with PPU during the study period.The mean patient age was 41 years. Laparoscopic repair was performed for 65 patients $(41 \%)$, and the remaining patients underwent open repair. There were no conversions in the laparoscopic groups. The mean operative time for laparoscopic repair was $(151 \mathrm{~min})$, significantly shorter than open repair time $(216 \mathrm{~min})$. The average of nasogastric tube duration was shorter in laparoscopic group (4.04 d Vs $3.18 \mathrm{~d}$ ), as well as the average of drainage stay. Patients who underwent laparoscopic repair required significantly less parenteral analgesics than the open group.The mean postoperative stay was significantly shorter for laparoscopic group (mean, $5.48 \mathrm{~d}$ ) than the open one.Morbidity of medical and surgical complication was higher in open groups ( 23 Vs 2 ). The most common complication in both groups was medical complication.More case of pneumonia was occurred in open groups compared to laparoscopic groups. Specific complication was higher in open .There were two $(2.1 \%)$ patients in open groupe that had intra-abdominal abscess. Two patients $(2.2 \%)$ had a surgical site infection in the open group. Revision surgery for suture-site leakage was occurred in three patients in open groups. There was no death in the laparoscopic group while 6 deaths $(5.7 \%)$ in the open group.

Conclusion: Our series shows to ensure that the laparoscopic repair became a gold standard in PPU.

\section{P594-UPPER GI-Gastroduodenal diseases}

\section{MECKEL'S DIVERTICULUM: OUR EXPERIENCE}

S. Dios-Barbeito, P. García-Muñoz, J. Tinoco-González,

A. Sánchez-Arteaga, L. Navarro-Morales, S. Martínez-Núñez,

N. García-Fernández, M.J. Cuevas, A. García-León, E. Perea,

J. Padillo-Ruiz,

L. Tallón-Aguilar

Digestive Surgery, Hospital Universitario Virgen del Rocío, SEVILLE, Spain

Aims: The aim of this study is to describe all the cases of Meckel's diverticulum (MD) resected in our centre.

Methods: A retrospective study using our prospective database was designed to analyse all the resected MD in our centre. Epidemiological data, clinical setting, diagnostic test and histological results were reported.

Results: MD was resected in 112 patients, 80 males and 32 females, with a mean age of 13.00 years (3.25-59.50). In 35 cases, a laparoscopic approach was chosen.

Eighty-seven percent of the patients had a presurgical imaging test (ultrasounds, CT-scan or Meckel's scan), but this only was diagnostic in the $18.1 \%$.

MD was resected as an incidental found in 80 patients, while in symptomatic patients the most common surgical found was a perforated diverticulum.

After pathologic examination, $78.6 \%$ of the diverticulum showed a normal aspect, and $68.8 \%$ had no heterotopic mucosa, while heterotopic gastric mucosa was found in the $25.9 \%$ of the cases. The average hospital stay after resection was 6 days (4-12) and complications were more commonly associated with the pathology that stablished the surgical indication.

Conclusions: MD is more common in males and incidentally found when operating another abdominal pathology. The pathological examination showed more commonly a normal appearance with no heterotopic mucosa. About complications, they were usually related to other surgical gestures, more than with the MD resection.

\section{P595-UPPER GI-Gastroduodenal diseases}

PERFORATED PEPTIC ULCER WITH DIFFUSE PERITONITIS: CAN PROGNOSTIC SCALES PREDICT POSTOPERATIVE MORBIDITY?

\author{
A. Sazhin, E. Stradymov, G. Ivakhov
}

Faculty surgery, Russian State National Medical University, MOSCOW, Russia

Background: perforated peptic ulcer (PPU) is a substantial health problem with significant postoperative morbidity up to $63 \%$ and mortality up to $40 \%$ worldwide.

Aims: this study aimed to estimate the sensitivity scoring systems for prognosis morbidity of patients operated for PPU with diffuse peritonitis.

Methods: a total of 153 patients were underwent emergency repair for PPU with diffuse peritonitis in Pirogov Russian National Research Medical University's surgical clinics during 2014-2016 years. Different scoring systems used to predict outcome in PPU patients were identified: Boey score, peptic ulcer perforation (PULP) score, ASA, Mannheim Peritonitis Index (MPI), World society of Emergency Surgery sepsis severity score (WSES score). To quantify the strength of the concatenation of prognostic score and morbidity we use odds ratio (OR) with $95 \%$ CI.

Results: 114 patients with PPU and diffuse peritonitis underwent laparoscopic repair (74,5\%), 19 patients-open approach (12,4\%), 20 patients underwent PPU repair after conversion $(13,1 \%)$. Overall morbidity rate was $27 \%$. All postoperative complications were classified according to Clavien-Dindo classification (CDC). 42 patients had 79 complications by CDC: I class-1,3\%, II-40,5\%, IIIA-16,5\%, IIIB-8,9\%, IV-6,3\%, V-26,6\% respectively. Boey 2 is significant risk factor of morbidity, the odds ratio (OR) is 67,7 with $95 \% \mathrm{CI}$ of $[8.6,533.8](\mathrm{p}<0,05)$ and Boey 1 has OR 5,1 with 95\% CI of $[2.2,11.9](\mathrm{p}<0,05)$. Also, MPI $>21$ and WSES SSS $>5$ are risks factors of morbidity, the OR are 41,6 with $95 \%$ CI of $[14.8,116.4]$ and $36,395 \%$ CI of $[13.6,97](\mathrm{p}<0,05)$, respectively. PULP score and ASA score have good prognostic value in relation to morbidity, but less than Boey, MPI and WSES SSS. Patients with PULP $>7$ had OR 27 with $95 \%$ CI of $[7.4,99.1](\mathrm{p}<0,05)$ and ASA $>3$ had OR 16,1 with $95 \%$ CI of $[4.3,60.5]$ $(\mathrm{p}<0,05)$.

Conclusion: prognostic scales for PPU patients with diffuse peritonitis could help us to identify high-risk patients. We suggested that the Boey score 2, MPI $>21$ and WSES SSS $>5$ have the best prognostic value to predict morbidity.

\section{P596-UPPER GI-Gastroduodenal diseases}

\section{GASTROSTOMY TUBE PLACED BY LAPAROSCOPY AS A NEW THERAPEUTIC OPTION FOR CONTINUOUS INTESTINAL INFUSION TREATMENT WITH LEVODOPA/ CARBIDOPA}

P. Rodríguez González ${ }^{1}$ M. Delgado Morales², A. De la Rosa Baez ${ }^{2}$, J. Guadalajara Jurado ${ }^{1}$, R. Balongo García ${ }^{1}$

${ }^{1}$ General and Digestive Surgery, Juan Ramón Jiménez, HUELVA, Spain; ${ }^{2}$ General and Digestive Surgery, Infanta Elena, HUELVA, Spain

Aims: Intestinal infusion treatment with levodopa/carbidopa (Duodopa) is a therapeutic option concerning the Advanced Parkinson disease cases with no response to the conventional treatment. The drug requires carrying out a gastrostomy either by percutaneous endoscopy way, or by laparoscopy -if the first one is not possible-. Later, a duodenum-yeyunum tube is placed in order to infuse the Duodopa gel continuously by a portable bomb. In this report, we explain the laparoscopic gastrostomy technique.

Method: SIn this report, we include two patients with advanced Parkinson disease: the first one is a 61 year-old female patient suffering from an important gait disorder; and the second one is a 71 year-old male patient with uncontrolled motor fluctuations. In both cases, a percutaneous endoscopic gastrostomy was proposed, but neither was feasible because of the non-traslumination between the gastric and the abdominal wall. Under general anesthesia, neumoperitoneum by Veress needle was performed. Three main trocars and one accessory were placed. At the level of the gastric antrum, a $1 \mathrm{~cm}$ incision was conducted to insert a gastrostomy tube, to be the guide for the drug infusion catheter. Next, the gastrostomy is fixed to the abdominal wall by the Stamm technique, externalizing the catheter through the accessory trocar in the medial line.

Results: On the first post-operative day, a duodenum-yeyunum tube is placed by endoscopic control through the gastric device. Both patients got well satisfactorily, and no complications were described; and they develop a total normal life within the limitations of their underlying disease.

Conclusions: The Duodopa intestinal infusion shows a significative improvement for the advanced Parkinson disease symptoms, compared with oral medication; appreciating positive results referring to life quality. When the catheter placement by endoscopy way does not seem posible, gastrostomy by laparoscopy constitutes a valuable surgical option for the treatment of this kind of patients. 


\section{P597-UPPER GI-Gastroduodenal diseases}

LAPAROSCOPY-ASSISTED DISTAL GASTRECTOMY WITH D2 LYMPH NODE DISSECTION FOR EARLY GASTRIC CANCER

G. Arredondo ${ }^{1}$, H. Segura ${ }^{1}$, J. Rojas ${ }^{2}$

${ }^{1}$ General Surgery, Escuela de Medicina y Ciencias de la Salud del Tecnológico de Monterrey, MONTERREY, Mexico; ${ }^{2}$ General Surgery, Hospital San José TecSalud, MONTERREY, Mexico

In Mexico in 2013, gastric cancer represented the 3rd cause of death; it may manifest in a variety of histologic, anatomic, and genetic patterns, which influences the surgical approach. Until now gastrectomy with curative intent is the only treatment that offers potential cure in gastric cancer. In recent years, laparoscopy has emerged as an important modality in the surgical management. In multiple trials no significant difference in recurrence, long-term survival and disease-free survival was observed when compared to the standard open gastrectomy.

We present the case of a 62 year old man. With a smoking history of 30 pack years, suspended 12 years earlier. He presented unspecific upper gastrointestinal symptoms; an upper endoscopy was made observing a suspicious depressed lesion of $3 \mathrm{~cm}$ located in the greater curvature between the body and the antrum, the biopsy resulted in a poorly diferentiated signet-ring cell carcinoma of the stomach. An endoscopic ultrasound and a thoracoabdominal CT scan showed no evidence of enlarged adenopaties or metastatic disease. Initially a diagnostic laparoscopy was made, there was no evidence of carcinomatosis, nor free intraperitoneal fluid; so the greater omentum was dissected towards the splenic and hepatic flexure; a D2 lymph node dissection was performed, and a subtotal gastrectomy with reconstruction of Roux en $\mathrm{Y}$ was done; intraoperative endoscopy was done to identify the lesion, so adequate margins could be obtained. The patient had a good post operative evolution and was discharged home at 4th day tolerating oral intake. Minimally invasive techniques have proved equivalency of oncologic results when compared to the conventional approach; these techniques are becoming the preferred approach in the treatment of well-selected patients with gastric cancer and have a role in definitive staging, curative resection, and lymphadenectomy. Appropriate selection of patients and optimal technical approach are paramount for good outcomes. Most data of laparoscopic gastrectomy come from Eastern countries, where the prevalence is higher; however Western experience is growing along with evolution and development in surgical instruments and new technology.

\section{P598-UPPER GI-Gastroduodenal diseases}

\section{WILKIE SYNDROME A RARE CAUSE OF HIGH INTESTINAL OBSTRUCTION SOLVED BY A LAPAROSCOPIC STRONG PROCEDURE}

F. Grasa Gonzalez ${ }^{1}$, D. Palomo Torrero ${ }^{2}$, M. Lopez Zurera $^{2}$, $\overline{\text { L. Bollici Martinez }}^{2}$, S. Antunez Martos ${ }^{2}$, F. Serratosa Gutierrez ${ }^{2}$, M. Pradas Caravaca ${ }^{2}$

${ }^{1}$ Cirugia General Y Aparato Digestivo, Sistema Andaluz de Salud / Hospital Serranía Ronda, RONDA, Spain; ${ }^{2}$ Cirugia General Y Aparato Digestivo, Hospital Serrania Ronda, RONDA, Spain

Wilkie Syndrome is a rare cause of high intestinal obstruction, resulting from the compression of the duodenum between the abdominal aorta and the superior mesenteric artery. The main symptoms are nausea and vomiting, weight loss, early satiety, abdominal distension and epigastric pain. Historically, the barium study and arteriography were the diagnostic tests used; more recently the angioTAC has shown greater sensitivity. The diagnostic criteria are: dilated duodenum, duodenal compression by the superior mesenteric artery and aortomesenteric angle less than 20 degrees. Patients with an acute condition usually respond to conservative treatment (decompression, correction of hydroelectrolyte alterations, nutritional support...). However, those with chronic symptoms usually require surgery preferably with laparoscopic approaches of duodenojejunostomy or the Strong's procedure.

The Strong procedure mobilizes the duodenum by dividing the ligament of Treitz. Once the duodenal-jejunal junction is mobilized, the duodenum is positioned to the right of the superior mesenteric artery and it is preferred because it provides less morbidity due of the maintaining of the integrity of the gastrointestinal tract, but it has a failure rate of $25 \%$. Gastrojejunostomy allows gastric decompression, but does not relieve duodenal compression, so digestive symptoms may persist, leading to the appearance of a blind loop syndrome or recurrent peptic ulcers. On the other hand, the duodenojejunostomy, which according to some series may be the procedure of choice, may obtain a success rate higher than $90 \%$. We advocate to initiate the surgical approach with the Strong procedure and if it fails to perform to a duodenojejunostomy.

\section{P599-UPPER GI-Gastroduodenal diseases}

ROBOT-ASSISTED RESECTION OF GASTROINTESTINAL STROMAL TUMORS (GIST): A SINGLE CENTER EXPERIENCE

Y. Quijano, E. Vicente, H. Duran, E. Diaz, I. Fabra, R. Caruso, L. Malave, V. Ferri

General Surgery, Sanchinarro University Hospital, MADRID, Spain

Background: Robotic techniques are claimed to be an alternative to laparoscopic and open approaches for gastrointestinal stromal tumors (GIST) treatment. This video shows the surgical technique used in patients with GIST in the duodenum and stomach. Robotic surgical resection was performed.

Materials and methods: Robotic assisted resection was performed in patients with gastric and duodenal GIST. The procedure was carried out using the da Vinci Surgical System.

Results: The procedures were successfully performed without postoperative complications. None of the interventions needed conversion to open surgery. All lesions had a microscopically negative margin of resection. Mean follow-up was 24 months (8-33) with a disease-free survival rate of $100 \%$. Conclusions: A robotic approach for GIST tumors is a safe and feasible procedure with a wellaccepted oncological surgical result.

\section{P600-UPPER GI-Gastroduodenal diseases}

\section{LAPAROSCOPIC SURGICAL TREATMENT IN PEPTIC GASTRODUODENAL PERFORATIONS: AN EARLY EXPERIENCE IN OUR HOSPITAL}

B.L. Lopez Duran ${ }^{1}$, C. Dominguez Sanchez ${ }^{1}$, A. Cano Matias ${ }^{2}$, B. Marenco de la Cuadra ${ }^{2}$, M. Retamar Gentil ${ }^{2}$, M. Sanchez Ramirez ${ }^{2}$, E. Perez Margallo², F. Oliva Mompean ${ }^{1}$, J.A. López Ruiz ${ }^{2}$

${ }^{1}$ General and Digestive Surgery, Hospital Universitario Virgen Macarena, SEVILLA, Spain; ${ }^{2}$ Emergency Surgery Unit, Hospital Universitario Virgen Macarena, SEVILLA, Spain

Peptic perforated ulcus (PPU) is a common surgical emergency and laparoscopic repair has been introduced as an alternative to open repair. It has shown good results and allows closure and peritoneal lavage, just like the open repair does but with the advantages of a minimally invasive surgery. The objective is to report the outcome of laparoscopic PPU in our Hospital.

Methods: From January 2015 to October 2018, 16 patients with a clinical diagnosis of PPU were assigned to undergo laparoscopic repair. This retrospective study included all HUSM patients who underwent laparoscopic PPU repair by emergency surgeons. Minimum follow-up of 3 months is carried out

Results: Of the 16 patients in this series, $70 \%$ were men and 30\% were women, between 15 and 80 years of age at the time of surgery, average of 48 years. The time between the manifestation of symptoms and surgery was $>$ to $24 \mathrm{~h}$ in $70 \%$ of patients. In 6 patients there was a history of previous ulcer or non-steroidal anti-inflammatories intake and up to $50 \%$ were smokers.

A CT scan was performed in all cases to reach the diagnosis

Primary closure with simple suture plus omental patch was the elected technic $(90 \%)$. The approach was performed with 3 trocars in $44 \%, 4$ trocars in $50 \%$ and 5 in 1 case.

13 cases $(81 \%)$ were gastric ulcer, 2 duodenal cases $(13 \%)$ and in one case no perforation was found. The conversion rate was $19 \%$, in two cases due to technical difficulty and in the other case because the level of the perforation was not found

The median postoperative stay was 7 days although there were 2 cases with intrabdominal complications. There was an exitus due to a metastasic pulmonary neoplasia diagnosed in the immediate postoperative period. There were no cases of recurrence in the follow-up time. Conclusion: In most centers, including ours, the rate of laparoscopic management has gradually increased along with the improvement of technical skills. Improvements in the outcome of laparoscopic PPU repair are to be expected with more experience surgeon and a good selection of the cases. 


\section{P601-UPPER GI-Gastroduodenal diseases}

CASE REPORT: LAPAROSCOPIC RESECTION OF THE GASTRIC DIVERTICULUM

\section{Todorovic, S. Lovric, S. Petrovic}

Department of General Surgery, JZU Hospital „Sveti Vracevi,, BIJELJINA, Bosnia-Herzegovina

Introduction: Diverticulum is an outpouching of a hollow organ. Gastric diverticulum is rare form od this disease. Incidence of detection varies depending on investigation method. It has been reported in $0.02 \%$ cases of autopsies, $0.04 \%$ cases of gastroduodenal roentgenographies with contrast, and $0.01-0.11 \%$ cases of upper endoscopies.Small diverticula are usually asimptomatic, but bigger diverticula can cause variable symptoms such as abdominal pain, feeling of epigastric fullness right after meal, feeling of discomfort in upper parts of abdomen, and severe 'foetor ex ore' .Diagnosis is usually established in procedures such as gastroduodenal roentgenographies with contrast, upper endoscopies and abdominal CT scan.

Case report: A 57-year-old woman came to our hospital because of feeling of discomfort and mild pain in upper abdomen that lasted for last year. Diagnosis is established after CT scan of abdomen and upper endoscopy procedure. Initially she has been prescribed conservative therapy (proton pump inhibitors). Since the symptoms persisted, laparoscopic resection of the gastric divertuculum was performed using EndoGIA stapler. Considering the feeling of discomfort and abdominal pain dissapeared, the patient was discharged from hospital on the fourth postoperative day.

Conclusion: Asymptomatic gastric diverticula doesn't require treatment. Since gastric diverticulum can have complications such as bleeding, perforation and neoplasia, patient without symptoms should be monitored. Initial therapy for symptomatic diverticula is conservative therapy (proton pump inhibitors). If conserative therapy doesn't procude expected results, laparoscopic resection of the diverticulum should be considered.

\section{P602-UPPER GI-Gastroduodenal diseases}

\section{PERFORATED GASTRIC PEPTIC ULCER WITH SEVERE PERITONITIS: STANDARDIZED LAPAROSCOPIC TREATMENT}

A.F. Aranzana Gómez, A.F. Aranzana Gómez, J. Malo Corral, J. Hernandez Gutierrez, B. Muñoz Jimenez, A. Muñoz Tébar, A. Trinidad Borras, M.A. Morlan Lopez, A.F. Aranzana Gómez

\section{GENERAL SURGERY, COMPLEJO HOSPITALARIO TOLEDO,} TOLEDO, Spain

Introduction: The acute perforation of a gastric ulcer is a serious entity that requires urgent surgical treatment in most of the occasions, it is increasingly accepted that the approach of choice is laparoscopic, depending, above all, on the time of evolution of the process.

Objectives: To demonstrate the safety and efficacy of the laparoscopic approach in the perforation of a pyloric peptic ulcer, even in cases of severe peritonitis, by means of a standardized procedure, insisting on the sequential thorough washing of the cavity.Material and methods: We present a video of the surgical intervention of a patient with acute abdomen, with a history of NSAID ingestion, exploration and CT-analysis compatible with perforation of hollow viscus, probably of gastric origin.

Results: Intervention: complete laparoscopic approach, 4 trocars. Severe biliopurunitic peritonitis, by pyloric perforation 'acute', liquid culture, suture of the perforation, epipoplasty, sequential thorough washing of the cavity with physiological saline and placement of drainages.Correct postoperative period, discharge from the hospital on the 7th day after completing antibiotic treatment. Endoscopy and helycobacter test are performed on an outpatient basis with normal results.

Conclusion: The laparoscopic approach is safe and effective in acute and complicated gastric ulcer disease, even in cases of severe peritonitis.

\section{P603-UPPER GI-Gastroduodenal diseases}

COMBINATION OF LAPAROSCOPIC AND ENDOSCOPIC APPROACHES TO NEOPLASIA WITH NON-EXPOSURE TECHNIQUE (CLEAN-NET) FOR GASTRIC SUBMUCOSAL TUMOR

K. Nabeshima ${ }^{1}$, M. Ogimi ${ }^{1}$, K. Nakamura ${ }^{1}$, H. Hara ${ }^{1}$, M. Tomioku ${ }^{1}$, E. Nomura ${ }^{2}$, T. Nakagori ${ }^{1}$, S. Ozawa ${ }^{1}$

${ }^{1}$ Department of surgery, Tokai University, ISEHARA, Japan; ${ }^{2}$ Department of surgery, Tokai university Hachioji hosipital, HACHIOJI, Japan

Background: Laparoscopic and endoscopic cooperative surgery (LECS) was reported in 2008 (Hiki. $\mathrm{N}$ et al.) and LECS related procedures are developed. In our institute, we underwent LECS for gastric submucosal tumor (SMT), especially combination of laparoscopic and endoscopic approaches to neoplasia with non-exposure technique (CLEAN- NET, Inoue $\mathrm{H}$ et al.) as an indication for gastric SMT with ulceration.

Aim: We report the surgical procedure and asses the outcome of CLEAN-NET for gastric SMT Patients and methods: From the laparoscopic surgery for the gastric SMT at Tokai university hospital from January 2014 to June 2018, 6 patients underwent CLEAN-NET.

Surgical procedure: The CLEAN-NET procedure involves the selective dissection of both the serosa and muscle layer using a laparoscopic monopolar endoscopic scissor. The preserved mucosal layer provides a mechanical barrier between the gastric lumen and peritoneal cavity that aids in the prevention of peritoneal cavity contamination with gastric contents. Tumors are observed with an upper gastrointestinal endoscope with the injection of indocyanine green (ICG) into peri-tumoral submucosal layers at 4 points. Selective seromuscular dissection is performed using a laparoscopic electrocautery monopolar scissor. The mucosa surrounding the GIST is then resected using a endoscopic mechanical stapler to prevent exposure of the gastric lumen to the peritoneal cavity and peritoneal tumor cell seeding.

Results: There were 5 males and 1 female, and the average age was 65 years. The operation time was $186 \mathrm{~min}$, the average bleeding volume was $14.6 \mathrm{ml}$, the postoperative hospital stay was 7.8 days. The mean tumor diameter was $32.3 \mathrm{~mm}$, the final histopathological diagnosis was 5 GIST, 1 schwannoma. There were no postoperative complications of Clavien-Dindo classification 2 or more.

Conclusion: CLEAN-NET was found to be safe and useful for the treatment of gastric SMT with ulceration.

\section{P605-UPPER GI-Reflux-Achalasia}

\section{YEAR OUTCOMES: LAPAROSCOPIC HELLER MYOTOMY STANDS THE TEST OF TIME}

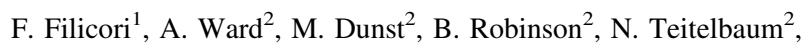
F. Abdelmoaty ${ }^{2}$, R. Demeester ${ }^{2}$, M. Zihni ${ }^{2}$, M. Reavis ${ }^{2}$, L. Swanstrom ${ }^{2}$

${ }^{1}$ Surgery, Lenox Hill Hospital, Hofstra/Northwell School of Medicine, NEW YORK, United States of America; ${ }^{2}$ Surgery, The Oregon Clinic, PORTLAND, United States of America

Aims: Laparoscopic Heller myotomy (LHM) has become the gold standard for the treatment of achalasia. We present 20 year outcomes of our initial consecutive patient cohort. Methods: Patients were identified in a prospectively maintained IRB-approved database (19931998). Post-operative Eckardt scores and a 5-point validated system questionnaires were obtained via telephone interviews. Success was defined by the lack of re-intervention as well as 20 year Eckardt scores $<3$. Patients were invited to obtain an up-to-date esophagogastroduodenoscopy (EGD).

Results: Thirty-three patients had a LHM (14 lost to follow-up, 5 deceased). Fourteen patient were included in the study with a median follow-up of 20.1 years. Indications for LHM included achalasia (12) and diffuse esophageal spasm (2). One patient required reoperation for failed myotomy. The mean Eckardt score at 20 years was $0.81( \pm 0.21)$, with all fourteen patients having an Eckardt score $<3$. All patients reported significant improvement in their quality of life. Classic GERD symptoms (heartburn and regurgitation) were present in $3(21.4 \%)$ patients Proton-pump inhibitors are being used by $35 \%$ of with patients with excellent symptom control. Seven patients returned for a repeat EGD (median 18.3yrs) with 5 patients having normal anatomy and 2 having LA grade A esophagitis (1 patient on PPI). Barrett's esophagus was not detected.

Conclusion: Long-term results from our early experience with LHM are excellent and durable with only one patient requiring re-intervention in 20 years. All patients in this study are satisfied with their operation and confirm it has led to a significant improvement in their quality of life. 


\section{P606-UPPER GI-Reflux-Achalasia}

MINIINVASIVE TREATMENT OF END-STAGE ACHALASIA: EXPERIENCE SINGLE CENTER

\section{Koshkin ${ }^{1}$, R. Izrailov ${ }^{1}$, O. Vasnev ${ }^{1}$, K. Shishin ${ }^{2}$, S. Kazakova ${ }^{2}$}

${ }^{1}$ High-tech surgery, Moscow Clinical Scientific Centre, MOSCOW, Russia; ${ }^{2}$ Endoscopic surgery, Moscow Clinical Scientific Centre, MOSCOW, Russia

Until recently the esophagectomy was the only choice in treatment of patients with end-stage achalasia. Developing of minimally invasive techniques such as a laparoscopic Heller miotomy and peroral endoscopic myotomy (POEM) allowed to use them as a treatment options.

Aim: to present an experience of treatment of patents with end-stage cardiac achalasia.

Materials and methods: since 07.2013 till the 12.2018 eleven patients with end-stage cardiac achalasia were operated on. Among them $2(15 \%)$ were males and $11(85 \%)$ were females. Mean age was 54 years $(26-81)$.

Results: POEM was performed in 7 patients, laparoscopic Heller myotomy was performed in 3 , and esophagectomies were performed in 3 patients with failed previously myotomy made in other clinics. Gastric tube was used to replace the esophagus in patients underwent esophagectomy. Mean operative time was $90 \mathrm{~min}$ for POEM, $172 \mathrm{~min}$ for laparoscopic Heller procedure and 422 min for laparoscopic esophagectomy. Blood loss was insignificant in all patients. There were no clinically significant complications during the postoperative course of all patients. Mean postoperative hospital stay was 2 days after POEM, 4 days after laparoscopic Heller procedure and 11 days after esophagectomy. To asses the dysphagia after POEM and Heller procedure Eckardt scale was used. In all cases it was rated as grade 0 .

Conclusion: minimally invasive techniques such as POEM and laparoscopic Heller procedure should be the first options in surgical treatment of patients with end-stage cardiac achalasia. In case of ineffectiveness of these options esophagectomy should be performed.

\section{P607-UPPER GI-Reflux-Achalasia}

GASTRO-ESOPHAGEAL REFLUX TEST DECIDING SURGICAL INDICATION AND Results: OF LAPAROSCOPIC NISSEN FUNDOPLICATION FOR GERD PATIENTS

T. Suwa, A. Obana, N. Mizuguchi, K. Kitamura, T. Matsumura, M. Nakayama, K. Karikomi, M. Koyama, Y. Sato, R. Yoshida,

H. Suzuki, S. Masamura

\section{Surgery, Kashiwa Kousei General Hospital, KASHIWA, Japan}

Introduction: The indication of laparoscopic anti-reflux surgery for GERD patients is difficult. We have established 'Reflux Test' as the tool for the decision in the surgical indication for GERD patients.

\section{Surgical Indication: Reflux Test}

At the standing position a patient swallows $300 \mathrm{ml}$ barium solution. After total solution goes into stomach, a patient lies down at the flat position.

Then a patient changes the position to left lateral decubitus position, flat position, right lateral decubitus position and flat position again every $10 \mathrm{~s}$ in the order.

During this procedure, gastro-esophageal reflux was evaluated and assigned to severe, moderate and slight category. If the reflux was observed slightly up to cervical esophagus, the case was assigned to moderate category. If the reflux was observed intensely up to cervical esophagus, the position was returned to head high position for the safety and the case was assigned to severe category. The anti-reflux surgery was considered in the moderate and severe categories.

Results: We have performed laparoscopic Nissen procedure in 95 cases. The outcome was assessed by Reflux Test performed on 4-5 postoperative day, and the results showed the reflux was disappeared in every cases. Median follow-up period of this study was 56 months (3110 months). In 11 cases (11.6\%) PPI was restarted before 6 months after the anti-reflux surgery. In 25 cases $(26.3 \%)$ PPI was restarted after the anti-reflux surgery during the whole follow-up period of this study.

The BMI of the patients had no relationship to the needed restart of PPI

To evaluate the degree of esophagitis objectively before and after the anti-reflux surgery we designed 'the esophagitis score'. In this scoring method, a number from 0-5 was assigned according to the degree of esophagitis along with the LA classification. The results of the study have shown that the reflux esophagitis was improved obviously after the anti-reflux surgery even in the PPI restarted group $(\mathrm{p}<0.001)$.

Discussion: To extract the GERD patients who really need anti-reflux surgery is important. Reflux Test is feasible because of its convenience and visual effects for the patients. The results of the laparoscopic Nissen fundoplication were good.

\section{P608-UPPER GI-Reflux-Achalasia}

\section{LAPAROSCOPIC CARDIOMYOTOMY FOR ACHALASIA}

\section{Hussein}

Surgery, American University of Beirut Medical Center, BEIRUT, Lebanon

Aims: Laparoscopic Cardiomyotomy leads to excellent relief of dysphagia in 95\% of patients and avoids thoracotomy or laparotomy.

Methods: We present a video illustration of the procedure that was modified at the American University of Beirut Medical Center. So far, 129 patients underwent Laparoscopic cardiomyotomy, age range of 14 to 76 years, with 56 males and 73 females. Most of them have had previous balloon dilatation.

Results: All cases were successfully completed laparoscopically without complications. Followup of 2 months to 15 years revealed excellent results with complete resolution of symptoms and no need for further medications. This will result in minimal post-operative pain and very short recovery period and is associated with low complication rate.

Conclusion: Cardiomyotomy for achalasia is ideal for Laparoscopic approach. Magnification allows for precise division of muscle fibers. The new technique of hydro dissection and Enseal for division of esophageal muscle allows for completion of the procedure without injury of the mucosa. Therefore, adequate release of the obstructing segment followed by anti reflux procedure toupet will lead to excellent results with minimal morbidity and no mortality.

P609-UPPER GI-Reflux-Achalasia

\section{LAPAROSCOPIC REPAIR OF HUGE HIATUS HERNIA}

\section{Hussein}

Surgery, American University of Beirut Medical Center, BEIRUT, Lebanon

Aims: Laparoscopic Repair of Huge Hiatus Hernia

Methods: Twenty two cases of Huge Hiatus Hernia presented to the American University of Beirut Medical Center. Patients underwent through 5 trocars in the upper abdomen reduction of the hernial sac from the chest. Special care was taken in the dissection of the mediastinum to keep the thoracic fascia and pleura intact. The defect was sutured primarily by 0-Ethibond sutures reinforced by onlay prolene mesh $\mathrm{u}$-shaped was fixed at the Rt. and Lt. crus and a floppy Nissen Fundoplication performed

Results: The video presentation includes the technical aspects and the method of reducing and repair of huge hiatus hernia. 


\section{P610-UPPER GI-Reflux-Achalasia}

THE NEW TECHNIQUE OF LAPAROSCOPIC HIATAL HERNIA REPAIR AND NISSEN FUNDOPLICATION

\section{A.V. Malynovskyi, S.Yu. Badion, M.M. Galimon}

Department of Robotic and Endoscopic Surgery, Odessa national medical university, ODESSA, Ukraine

Aims: Mesh repair is recommended for large hiatal hernias, but the search for the safest and most effective technique is ongoing. The aim of study was to access the first results of a new technique of laparoscopic hiatal hernia repair using retrocrural placement of small patches of Parietene Progrip self-fixating mesh and V-Loc self-grippind continious suture.

Methods: Four pationts with type 1 hiatal hernias with mean hiatal surface area of 12 sq.cm (820) underwent laparoscopic hiatal repair using the new technique called 'posterior buttress of crural repair'. Mean age was 45 years old (range, 39-55 years). There were 2 women and 2 men. After skeletization of crura posteriorly to esophagus, two separated rectangular patches of Parietene Progrip mesh (Covidien) measuring 1 $2.5-3 \mathrm{~cm}$ were attached to the posterior surfaces of the crura. The patches were fixated themselves due to special hooks. Than continious twodirections suture was placed through both crura along with the patches using self-gripping V-Loc 2-0 suture (Covidien). The same suture was used for construction of Nissen fundoplication wrap $3.5 \mathrm{~cm}$ long. Aditional anchoring stich through the wrap and esophageal wall was placed using Ti-Cron 2-0 suture (Covidien). 3D laparoscopy was used while suturing using Richard Wolf Epic system.

Results: All the procedures were performed successfully. There were no cases of bleeding from the suturing points either from the crura and the fundus wall. There were no crural dehiscence while suturing, even if the distance between crura was more than $4 \mathrm{~cm}$. The mean duration of suturing facilitated by 3D laparoscopy was $15 \mathrm{~min}$ (range, 12-20 $\mathrm{min}$ ) for crural repair, and $10 \mathrm{~min}$ (range, $8-25 \mathrm{~min}$ ) for fundoplication. There were no excessive postoperative pain in all the patients. There were no disphagia 1 month postop in every patient.

Conclusions: 1 . The new technique of posterior buttress of crural repair using small patches of Parietene Progrip mesh and V-loc suture showed feasibility and safety. 2. The use of 3D laparoscopy facilitates suturing while performing hiatal repair and fundoplication. 3. More cases collection and long-term follow-up is needed to support the new technique and comparing it with the existing methods.

\section{P611-UPPER GI-Reflux-Achalasia}

\section{TREATMENT OF LARYNGOPHARYNGEAL REFLUX IN PATIENTS WITHOUT LARGE SIZE OF HIATAL HERNIA}

\section{Paranyak, V. Grubnyk}

Surgery, Odessa national medical university, ODESSA, Ukraine

Background: Laryngopharyngeal reflux (LPR) is described as the retrograde flow of stomach content to the larynx and pharynx. In such case most commonly manifested symptoms are cough, sore throat, hoarseness, dysphonia, globus and only $40 \%$ patients with LPR have typical GERD symptoms. Also PPI therapy are less effective in patients with LPR in comparison with patients which have typical features of GERD.

Purpose: To compare the outcomes between surgical treatment and conservative therapy in patients with laryngopharyngeal reflux.

Materials and methods: For the period March 2014 to April 2017 in Odessa Regional Hospital we perform study, where selected patients reported chronic cough and globus pharyngeus, with LPR which was diagnosed according to the 24-hour impedance-pH monitoring. Excluded criteria for our study ware diagnosed hiatal hernia $(\mathrm{HH})$ type II or III. We offered for patients two ways of treating the disease: surgical method and PPIs therapy (where emphasize that conservative therapy could be for life). In this way 38 patients (30- $\mathrm{HH}$ type I, 8- without features of $\mathrm{HH}$ ) underwent laparoscopic Nissen fundoplication (LNF) without cruroraphy. PPIs therapy group include 18 patients (6- without $\mathrm{HH}, 12-\mathrm{HH}$ type I). We assessed 24-h impedance-pH monitoring, quality of life (GERD-HRQL) questionnaires.

Results: Baseline characteristics were similar between groups. After a median follow-up of 27 months the prevalence of cough $(13.2 \%$ vs $27.8 \%$; p < 0.05$)$, globus pharyngeus $(10.5 \%$ vs $38.9 \% ; \mathrm{p}<0.05$ ), was significantly lower in patients who underwent LNF. RSI improved from $22.5 \pm 5.3$ to $11.92 \pm 4.7(\mathrm{p}<0.05)$ in the first group and from $17,4 \pm 4,3$ to $10,8 \pm 3,1$ $(\mathrm{P}<0,05)$ in the conservative therapy group. Based on the questionnaire GERD-HRQL results, in the I group quality of life improved from $16.7 \pm 5.4$ to $6.2 \pm 1.1(\mathrm{P}<0.05)$, in the II group from $15.2 \pm 6.7$ to $11.1 \pm 1.2(\mathrm{P}<0.05)$

Conclusion: LNF had higher efficacy in long-term eliminating of LFR symptoms in comparison with PPIs in patients without large size of HH. Also LNF show better results in quality of life improvement in patients with LFR than PPIs.

\section{P612-UPPER GI-Reflux-Achalasia}

\section{ROBOT-ASSISTED SURGERY IN PATIENTS WITH GIANT HIATAL HERNIA AND REFLUX-ESOPHAGITIS}

F.P. Vetshev, A.F. Chernousov, S.V. Osminin, T.V. Khorobryh, A.A. Chesarev

Faculty Surgery \#1, Federal State Autonomous Educational Institution of Higher Education I.M. Sechen, MOSCOW, Russia

Aim: Nowadays, there is little experience in the world of applying robotic surgical system (RSS) in treatment of patients with hiatal hernia (HH) and reflux-esophagitis (RE). The aim of study was to determine the possibility and feasibility of using RSS in treatment of patients with $\mathrm{HH}$. Materials and methods: A total of 41 patients underwent robot-assisted HH repair without mesh, followed by fundoplication with our original method $\left(360^{\circ}\right.$ full symmetric wrap). The clinical and technical analysis did not reveal any advantages over similar laparoscopic procedures, so we abandoned the use of RSS for HH type I, and these 4 patients were excluded. There were $32(86 \%)$ patients with HH type III and 5(14\%) type IV. The surgeries were performed by experienced robotic upper gastrointestinal surgeon and conducted with the daVinci Si Surgical System (Intuitive Surgical, Sunnyvale, CA).

Results: Average operation time was $118 \pm 37$ (62-173) min. The respondents' mean age was $56.2 \pm 10.9$ years (range 29-68) and BMI was $30.8 \pm 7.1$ (range 17.1-44.3) $\mathrm{cm} / \mathrm{kg}^{2}$. Average blood loss was $20 \pm 9(5-70) \mathrm{mL}$. Average hospital stay was $5 \pm 1.3(1-15)$ days. The average follow-up time was $14 \pm 3.6$ (6-24) months. Postoperative X-Ray imaging and upper GI endoscopy was conducted in all $37(100 \%)$ patients. There was no HH recurrence diagnosed. We did not observe a relapse of $\mathrm{HH}$ or clinical manifestations of RE in the early (less than 30 days) and long-term (more than 6 months) postoperative periods.

Conclusion: We can conclude that robot-assisted surgery is safe, appropriate and justified in patients with HH type III and IV. All procedures performed to the patients with giant HH revealed clear technical advantages of RSS over similar laparoscopic operations: an enlarged 3D HD image, bendable instruments with EndoWrist technology allowed for precise dissection of tissues (hernial sac, cicatricial adhesions) in a narrow anatomical space—posterior mediastinum without damage to pleura, pericardium and vagus nerves. We believe that use of RSS in treatmen of patients with reflux esophagitis and/or HH type I is unjustified, due to the lack of proven advantages over laparoscopy.

\section{P613-UPPER GI-Reflux-Achalasia}

\section{BENEFITS OF LAPAROENDOSCOPIC REPAIR OF HIATAL HERNIA IN THE PRESENCE OF MAJOR ANATOMICAL OBSTACLES}

\section{M.Y. Fanous}

Surgery, Aspirus Iron River Hospital, IRON RIVER, United States of America

Introduction: The presence of major anatomical obstacles -such as massive caudate lobe- in the confined operative field of laparoscopic hiatal hernia repair (LHHR) poses significant challenge to the foregut surgeon.

Aim: To provide a safe alternative for LHHR using a laparoendoscopic approach.

Method: This patient is a 60 year old female, with BMI of 32.2. Her past Medical history includes diabetes, hypertension and hyperlipidemia. She had GERD for 20 years. Her EGD showed $5 \times 4 \mathrm{~cm}$ hiatal hernia and class B esophagitis. Manometry showed ineffective esophageal motility.

We used the classic five ports approach for LHHR. We found a massive caudate lobe which was comparable to the size of an already enlarged left lobe of the liver.

The operative strategies: Terminating the procedure

Proceeding with the standard approach and taking the risk of bleeding from the caudate lobe itself or the inferior vena cava (IVC) with possible catastrophic outcome.

Using the laparoendoscopic approach.

The following three steps facilitated the performance of safe and effective surgery. Additional liver retractor This improved exposure and minimized manipulation of the caudate lobe. Extracorporeal sliding arthroscopic knots (ESAK) ESAK are similar to the knots used in endoloop. They are tied extracorporeally and require a single insertion of the knot pusher as they do not unravel.

Transoral incisionless fundoplication (TIF) We performed TIF to avoid a limited operative field and to prevent excessive tissue manipulations associated with laparoscopic fundoplication. TIF also preserves the angle of His and produces partial fundoplication which has less side effects of dysphagia and gas bloat syndrome.

Results: The operative time was $98 \mathrm{~min}$ (LHHR $78 \mathrm{~min}$ and TIF $20 \mathrm{~min}$ ). There were no complications. Patient discontinued omeprazole which she used daily for 20 years. At 6 months follow up, her GERD related quality of life (HRQL) and GERD symptom (GERSS) scores were (30 vs 5 ) and (40 vs 8$)$.

Conclusion: The laparoendoscopic repair of hiatal hernia in the presence of anatomical obstacles is safe and effective. Longer follow up is needed to assess the durability of this repair. 


\section{P614-UPPER GI-Reflux-Achalasia}

\section{A CASE OF A PRIMARY PARAHIATAL HERNIA ASSOCIATED WITH A TYPE I HIATAL HERNIA}

V.M. Surlin ${ }^{1}$, S.D. Preda ${ }^{1}$, D.M. Albulescu ${ }^{2}$, S. Patrascu ${ }^{1}$,

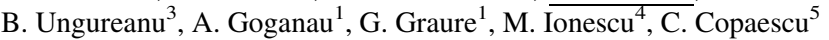

${ }^{1}$ Surgery, Emergency County Hospital Craiova, CRAIOVA, Romania; ${ }^{2}$ Radiology, Emergency County Hospital Craiova, CRAIOVA, Romania; ${ }^{3}$ Gastroenterology, Emergency County Hospital Craiova, CRAIOVA, Romania; ${ }^{4}$ Anesthesiology, Emergency County Hospital Craiova, CRAIOVA, Romania; ${ }^{5}$ Surgery, Ponderas Academic Hospital, BUCHAREST, Romania

Parahiatal hernia is a rare disease that occurs when an abdominal organ protrudes through an opening adjacent to an anatomically intact esophageal hiatus. The herniated organ is usually the stomach, although cases of omental and colonic herniation exist. The reported incidence is 0.2 $0.35 \%$ in patients undergoing surgery for hiatal hernia. They can be classified as primary/congenital or secondary/acquired. We report the case of a 60-year-old woman which accused epigastric pain, starting 2 years prior, pseudo-angina, heartburn and bloating. Based on imagistic findings the patient was diagnosed with a parahiatal hernia and an associated type I hiatal hernia. Patient underwent surgery and a $7 \mathrm{~cm}$ diameter defect in the diaphragm lateral to the left crus was discovered, through which $40-50 \%$ of the stomach had herniated. The hiatal orifice was slightly enlarged but anatomically intact, with an associated small sliding hiatal hernia. We performed closure of the defect, hiatoplasty and a Floppy-Nissen Fundoplication. Postoperative outcome was uneventful, with the patient discharged on the 5th postoperative day.

\section{P615-UPPER GI-Reflux-Achalasia}

\section{THE LAPAROSCOPIC APPROACH TO THE TREATMENT OF REFLUX ESOPHAGITIS RESULTING FROM RECURRENT HIATAL HERNIA AFTER TRANSTHORACIC NISSEN FUNDOPLICATION}

\section{Agapov, V.V. Kakotkin, E.A. Gallyamov}

General surgery, Medical Research Educational Center. M.V. Lomonosov Moscow State University., MOSCOW, Russia

Gastroesophageal reflux disease (GERD) is a condition that reduces the quality of life and can causedisorders associated with acid reflux, such as bronchial asthma, Barrett's esophagus and esophagealadenocarcinoma. GERD is often caused by existing of hiatal hernia. Nowadays, some surgeons haddifficulties with the laparoscopic approach to treatment of recurrent hiatal hernias.Patient was a 30-year-old man. He requested medical assistance with dysphagia, nausea after eatingand heartburn getting worse in a horizontal position. Conservative treatment was not effective.Transthoracic Nissen fundoplication was performed in 2017. The main complaints of the patientpersisted during the postoperative period. The upper half of stomach and S-like curved esophagus werelocated in the mediastinum according to multislice computed tomography of the thorax in 2018 August.In our clinical center was performed laparoscopic cruroraphy, cardiomyotomy and Nissen fundoplicationin 2018 November. During the surgery the normal anatomical position of stomach has been restored, S-like curve of esophagus has been removed; a gastric cuff (Collis-Nissen) has been created and anteriorand posterior cruroraphy has been performed. The patient was in intensive care during $8 \mathrm{~h}$. Anasogastric tube feeding was continued during the first $48 \mathrm{~h}$. Passage of the contrast through theesophagogastric junction was free within $72 \mathrm{~h}$ after surgery. Patients had been discharged within 5 days after surgery.This case report shows that at the current stage of surgery laparoscopic approach can be useful not onlyfor treatment of primary hiatal hernias - but also for treatment of recurrent ones.

\section{P616-UPPER GI-Reflux-Achalasia \\ LAPAROSCOPIC HELLER-DOR TECHNIQUE FOR ESOPHAGEAL ACHALASIA}

V. Drakopoulos, V. Lygizos, S. Voulgaris, I. Iliadis, K. Rekouna, P. Trakosari, A. Bakalis, V. Vougas

1st Department of Surgery and Transplantation Unit, District General Hospital of Athens « Evangelismos », ATHENS, Greece

Aims: Laparoscopic Heller myotomy procedure, completed with an anti-reflux procedure is technically demanding. We report a case of laparoscopic Heller myotomy followed by a Dor anterior fundoplication.

Methods: This is the case of a 57-year-old Caucasian woman with gradual dysphagia for solids and liquids, accompanied by severe regurgitation and chest pain. An initial diagnosis of achalasia was made in 2010, with the use of manometry and barium swallow. Endoscopic dilatations were attempted pre-operatively with no clinical improvement. Decision was made to perform a laparoscopic Heller myotomy, combined with a standard Dor anterior fundoplication. A 4-ports operation took place (one intra-umbilical $10 \mathrm{~mm}$ trocar-Single Incision Laparoscopic Surgery (SILS) technique, two 5-mm subcostal trocars, and one another $10 \mathrm{~mm}$ subcostal trocar for the use of liver retractor). The operation lasted $2 \mathrm{~h}$ and $15 \mathrm{~min}$.

Results: No post-operative complications were noted. The post-operative swallow test showed improvement of the esophageal patency. The patient started a liquid diet three days later and was discharged six days post-operatively. Two months later the patient presented no complications. Conclusions: Heller's myotomy has demonstrated good long-term results in the treatment of esophageal achalasia and the laparoscopic approach has been well established in the last two decades. It is a very demanding operation to perform and the disease is relatively rare, making the learning curve difficult to achieve.

\section{P617-UPPER GI-Reflux-Achalasia}

\section{GREAT HIATAL HERNIA ASSOCIATED TO ACALASIA, VERY DANGEROUS}

J. Hernandez Gutierrez, A.F. Aranzana Gomez, B. Muñoz Jimenez, J.S. Malo Corral, P. Toral Guinea, G. Krasniqi, R. Lopez Pardo

\section{General Surgery, C.H.Toledo, TOLEDO, Spain}

Aims: Achalasia is a type of motor disorder of the esophagus due to degeneration of ganglion cells in the myenteric plexus, leading to failure of relaxation of the lower esophageal sphincter, accompanied by a loss of peristalsis in the distal esophagus.

The association of a long-term achalasia and a large size hiatus hernia is an infrequent entity Among the therapeutic options is medical treatment, endoscopic treatment and surgical treatment associated with an antireflux procedure. The laparoscopic approach being the more indicated due to its better results in terms of morbidity, mortality and recurrences.

The aim of the VIDEO is to show the effectiveness and safety of the laparoscopic approach in this infrequent pathology, pointing out the importance of performing a standardized procedure. Methods: 73-year-old male patient, with personal history of chronic ischemic heart disease and obesity, diagnosed with long-term achalasia with moderate dilatation of the esophagus associated with giant hiatus hernia. The complementary explorations and iconography of interest are exposed.

Results: Intervention: complete endoscopic approach, 5 trocars. Reduction of hernial content into the abdominal cavity, dissection of the hernial sac and esophageal lipoma. Extended mediastinal esophageal dissection. Complete resection of both the sac and lipoma, respecting the posterior vagus. Heller's myotomy of $10 \mathrm{~cm}$, including $3 \mathrm{~cm}$ distal to the UEG, perforation of $3 \mathrm{~mm}$ of the mucosa at the UEG level, suture and blue methylene verification of the sealing. Hiatorraphy and Dor-type anterior fundoplication as antireflux technique. Correct postoperative, with EGD control on the 3rd PO day and discharge on the 6th. Asymptomatic at 24 months after surgery. Conclusion(s): For achalasia laparoscopic Heller myotomy with a partial fundoplication should be the treatment of choice in patients who are at low surgical risk. The length of the myotomy, especially distal to UEG is one of the most important aspects of the surgery, to achieve an effective disruption of the LES. The presence of a giant hiatus hernia makes the procedure difficult, increasing the risk of complications, such as perforation. Standardization is essential to increase safety and efficacy in these complex cases. 


\section{P618-UPPER GI-Reflux-Achalasia}

\section{ASSESSMENT OF SHORT-TERM OUTCOMES WITH TIO2 MESH IN LAPAROSCOPIC REPAIR OF LARGE PARAESOPHAGEAL HIATAL HERNIAS}

P. Priego, A. Puerta, A. González Barranquero, S. Sánchez Picot, L. Blazquez, M. Cuadrado, J. Galindo

Division of Esophagogastric and Bariatric Surgery, Ramón y Cajal University Hospital, MADRID, Spain

Purpose: There is evidence that the application of mesh-reinforced hiatal repair has resulted in a significant reduction in recurrence rates in comparison with primary suture repair, at least in short-term follow-up. However, and instead of this, the standard of care for repairing large paraesophageal hiatal hernias (LPHH) remains controversial because no clear guidelines are given regarding indications, mesh type, shape and position. The aim of this study is to evaluate our short-term outcomes in management of LPHH with a biosynthetic monofilament polypropylene mesh surrounded by a high-purity and adherent titanium dioxide surface coating to enhance the biocompatibility $\left(\mathrm{TiO}^{2} \mathrm{Mesh}^{\mathrm{TM}}\right.$ ).

Methods: A retrospective study was conducted on our institution between December 2014 and October 2018. Data were collected on 27 patients with LPHH greater than $5 \mathrm{~cm}$ in which a laparoscopic repair was carried on by primary suture and additional reinforcement with a $\mathrm{TiO}^{2} \mathrm{Mesh}^{\mathrm{TM}}$. Clinical and radiological recurrences, dysphagia and mesh-related complications were investigated.

Results: There were 17 females and 10 males with a mean age of 73 years (Range, 63-79 years). All operations were completed laparoscopically. Median postoperative stay was 3 days. After a mean follow-up of 16 months, 3 patients developed clinical recurrence of reflux symptoms $(11.1 \%)$ and 2 radiological recurrences $(7.4 \%)$. There were no mesh-related complications. Conclusions: The use of $\mathrm{TiO}^{2} \mathrm{Mesh}^{\mathrm{TM}}$ for laparoscopic repair of LPHH is suited and with a reasonably low recurrence rate in this short-term study. Additional long-term studies with enormous numbers carried out for years will be necessary to affirm whether this mesh is convenient in the prevention of recurrences and mesh related complications.

\section{P619-UPPER GI-Reflux-Achalasia}

\section{OUTCOMES OF LAPAROSCOPIC REDO FUNDOPLICATION}

P. Priego, A. Puerta, A. González Barranquero, S. Sánchez Picot, L. Blazquez, M. Cuadrado, J. Galindo

Division of Esophagogastric and Bariatric Surgery, Ramón y Cajal University Hospital, MADRID, Spain

Background: Surgery for refractory gastroesophageal reflux disease (GERD) has a satisfactory outcome, however sometimes fundoplication fails and redo surgery is required. Several publications have investigated the feasibility of performing reoperative fundoplications using laparoscopic techniques. The aim of this study was to describe our experience in laparoscopic redo fundoplications in the last 4 years.

Material and methods: We retrospectively reviewed 26 consecutive patients who required laparoscopic redo fundoplication from January 2014 to August 2018.The indications were recurrent symptoms of gastroesophageal reflux disease (GERD) (15.4\%), recurrent symptomatic paraesophageal hernia $(42.3 \%)$, dysphagia $(30.8 \%)$ and acute volvulus $(11.5 \%)$.

Results: All redo fundoplications (basically Toupet $69.2 \%$ and Nissen $26.9 \%$ ) were completed laparoscopically. The mean operative time was $120 \mathrm{~min}$ (Range, 100-136.25 min). A mesh was placed in $31 \%$ of cases. Intraoperative and postoperative complication rates were $23.1 \%$ and $3.8 \%$ respectively. The mean hospital stay was 4 days (Range, 3-5 days). One patient (3.8\%) from the laparoscopic group required a third operation-one for acute recurrent paraesophageal herniation of the redo wrap one month after surgery, which was repair laparoscopically again. Symptomatic outcome was successful in $84.6 \%$ without any kind of proton bomb inhibitors therapy.

Conclusion: Laparoscopic redo fundoplication is technically feasible and clinically effective with a reasonable low rate of postoperative complications

\section{P620-UPPER GI-Reflux-Achalasia}

THERAPEUTIC DIFFICULTIES IN APPROACHING A PATIENT WITH IATROGENIC PERFORATION DURING ESOPHAGEAL PNEUMATIC DILATATION FOR ACHALASIA

\section{R.D. Birla ${ }^{1}$, C. Rosianu ${ }^{2}$, A. Constantin ${ }^{1}$, P.A. Hoara ${ }^{1}$, C. Gindea ${ }^{1}$,} E. Vasiliu ${ }^{1}$, S.M. Constantinoiu ${ }^{1}$

${ }^{1}$ General and Esophageal Surgery Clinic, UMF „Carol Davila,, „St Mary, Clinical Hospital, BUCHAREST, Romania;

${ }^{2}$ Gastroenterology, ,St Mary,, Clinical Hospital, BUCHAREST, Romania

Introduction: Achalasia is a primary esophageal motility disorder characterized by the absence of esophageal peristalsis and impaired relaxation of the lower esophageal sphincter (LES) in response to swallowing. The treatment, paliative, is done by endoscopy or surgery. Iatrogenic perforation during esophageal pneumatic dilatation is a rare but challenging complication.

Material and Method: We present a case of a 68 years old, female patient, diagnosed with achalasia, who was admitted on the Gastroenterology unit for endoscopic treatment on 4 July 2018. Pneumatic dilation with $30 \mathrm{~mm}$ balloon was performed under general anesthesia. Radiological contrast contro and endoscopy reevaluation revealed a perforation just above the squamo-columnar junction. A minimally invasive approach was decided. An fully covered esophageal stent was inserted. Radiological control after 2 days reveals left pleurisy and migration of the stent. The same day was performed an endoscopic repositioning of the stent with clip fixation. Left pleural puncture was performed and clear fluid was extracted. The condition of the patient got worse and she was transferred on ICU (08.07). We performed left pleurostomy and initial exploratory laparoscopy—no intraperitoneal lesions. Due to difficult transhiatal access to the inferior mediastinum the surgery was converted to open-perisophageal mediastinal abscess was found, evacuated and drainage and jejunostomy were performed. After a week, the patient presented progressive altered condition, febrile syndrome. Thoraco-abdominal CT-scan showed left pleural effusions. Left pleurostomy was performed, with extraction of fetid fluid. Continuous lavage was instituted. On $1^{\text {st }}$ of August, the pleurostomy tube drained gastric content, and the clinical examination revealed signs of generalized peritonitis. Laparotomy was performed with lavage, drainage and posterior decompression gastrostomy.

Results: Postoperative evolution was favorable, with the suppression of pleural drainage in 13.08 and discharge in 29.08 with alimentation exclusive on jejunostomy. One month later, she had normal clinical and radiological examination. Endoscopic stent and gastrostomy tube extraction was performed, with resumption of normal alimentation. The esophageal passage was normal on barium swallow. Conclusion: Endoscopic balloon treatment in achalasia is frequent the first approach, but, if perforation occurs, management can be very difficult, due to the esophageal dilatation.

\section{P621-UPPER GI-Reflux-Achalasia}

EVALUATION OF EFFECTIVENESS OF DIFFERENT ANTIREFLUX SURGERY FOR THE TREATMENT OF EXTRAESOPHAGEAL SYMPTOMS OF GERD

\section{Barbieri, A. Aiolfi, G.I. Saino, D. Bona}

General and Minimally Invasive Surgery, Istituto Clinico Sant'Ambrogio, MILAN, Italy

Aim:Extra-esophageal symptoms are caused by laringo-pharingeal reflux (LPR) which is caused by the backflow into the aero-digestive tract of weakly-acidic or non-acidic gastro-esophageal refluxes. For this reason, it is not fully treated by PPI, but the effectiveness of surgery for these symptoms is still controversial. Aim of our study is to assess the efficacy of surgery on LPR symptoms and identify the best technique among the ones in use.

Methods: From June 2007 to December 2017, 66 patients with LPR underwent surgery. The techniques involved were a total Nissen fundoplication (18), a partial Toupet fundoplication (29) and esophageal magnetic sphincter augmentation (MSA) implant (19). Evaluation of efficacy was performed with Reflux Symptom Index (RSI) specific for extraesophageal symptoms, subjective satisfaction and occurrence of dysphagia and gas-bloat syndrome. A RSI score $>12$ was considered as pathological

Results: RSI significantly decreased after surgery $(14.53 \pm 3.746$ Vs $3.394 \pm 4.914)$, difference of RSI score before and after surgery are shown in table 1 and found out better results for Toupet and MSA as compared with total fundoplication; $83,3 \%$ of patients were satisfied with surgery: a comparison between techniques showed superiority of MSA for symptoms control compared with the other two $(94.7 \%$ Vs $79.2 \% ; \mathrm{p}=0.0372$ ), while the two fundoplications were equivalent $(82.8$ and $78.8 \%$; $\mathrm{p}$; $0.8189)$. A higher incidence of dysphagia occur after Nissen and MSA compared with Toupet (22 Vs $6.9 \% ; \mathrm{p}=0.02)$. Gas bloat symptoms rate is lower with MSA $(5.2 \mathrm{Vs} 30 \% ; \mathrm{p}=0.0041)$

Conclusion: Anti-reflux surgery is effective not only for typical symptoms of GERD, but also for extra-esophageal symptoms. Among the different techniques offered, a partial fundoplication and MSA implant seems to offer better results in terms of symptom resolution, with fewer complication. A tailored approach based on preoperative clinical and manometric data, should be offered to enhance patient's satisfaction. 


\section{P622-UPPER GI-Reflux-Achalasia}

IMPROVING RATES OF LAPAROSCOPIC FUNDOPLICATION PERFORMED AS A DAY-CASE PROCEDURE IN A NATIONAL SPECIALIST CENTRE

M.A. Tsachiridi ${ }^{1}$, C.J. Kelty ${ }^{2}$, E. Flatt ${ }^{3}$, T. Petropoulou ${ }^{4}$

${ }^{1}$ Upper GI Surgery, Mediterranean Hospital of Cyprus, LIMASSOL, Cyprus; ${ }^{2}$ Upper GI Surgery, Northern General Hospital, SHEFFIELD, United Kingdom; ${ }^{3}$ Orthopaedics, Northern General Hospital, SHEFFIELD, United Kingdom; ${ }^{4}$ Colorectal Surgery, Sheffield Teaching Hospitals, SHEFFIELD, UNITED KINGDOM, United Kingdom

Aims: The British Association of Day Surgery (BADS) defines day surgery as procedures performed for patients admitted and discharged on the same day. Research has shown that day-case laparoscopic fundoplication is feasible and safe if patients are assessed and selected carefully based on surgical, medical and social factors. In this study we aim to demonstrate that we have successfully increased the rate of laparoscopic fundoplication performed as a day-case procedure over a 4-year period, without adversely affecting patient outcomes.

Methods: Retrospective data analysis was performed. All patients undergoing laparoscopic fundoplication under one surgeon from 2013 to 2017 were identified. Demographic details, length of stay, details of complications and re-admissions were obtained from trust electronic records systems. Data was analysed using Windows Excel.

Results: From 2013-2017 a total of 159 patients underwent laparoscopic fundoplication by the same surgeon using the Nissen technique. 88 patients were female $(55.3 \%)$ and 71 male $(44.7 \%)$. The average age of patients undergoing the procedure was 49.4. 67 patients (42.1\%) were ASA grade $1,81(50.9 \%)$ ASA 2, 11 (6.9\%) ASA 3. Across the 4 year period 61 patients were discharged the same day $(38.3 \%)$, with the average length of stay 1.8 days. The rate of surgeries performed successfully as day cases increased steadily across the 4-year period. In $201315.4 \%$ of patients $(n=2)$ required no overnight stay post-op; in 2014 this was $25 \%$ $(\mathrm{n}=10)$, in $201532.5 \%(\mathrm{n}=13)$, in $201645 \%(\mathrm{n}=31)$, and in $201762.8 \%(\mathrm{n}=22)$. Overall the readmission rate was 8 patients (5\%) within 30 days and 5 patients (3.1\%) within 3 months. Reasons for readmission included post-operative pain (30.8\% of readmissions), dysphagia (38.5\%) \& vomiting (15.4\%) and indirectly related conditions including chest infection and myocardial infarction (15.4\%). The rate of readmissions for day-case patients across the 4-year period was $4.9 \%(\mathrm{n}=3)$ and for none day-case patients was $10 \%(\mathrm{n}=10)$.

Conclusion: Across a 4-year period we have been able to increase the number of laparoscopic fundoplications performed as day-case procedures, thus saving the trust cost and bed-space. There was no increase in readmission rates for patients undergoing laparoscopic fundoplication as a day-case.

\section{P623-UPPER GI-Reflux-Achalasia}

STEP BY STEP INSTRUCTIONAL VIDEO OF LAPAROSCOPIC PARAESOPHAGEAL HERNIA REPAIR WITH FUNDOPLICATION FOR SURGEONS-IN-TRAINING IN A SINGAPORE INSTITUTION

\section{A.M. Oo}

Department of General Surgery, Tan Tock Seng Hospital, SINGAPORE

Background: Laparoscopic paraesophageal hernia repair with fundoplication has become more and more popular nowadays due to less morbdity and mortality with shorter length of hospital stay. Discussion: Tan Tock Seng Hospital is the second largest hospital in Singapore. It is affiliated to two medical schools in Singapore and it is a training hospital for both undergraduates and postgraduates. In our department, all the residents have to view the step by step instructional videos of mininally invasive surgeries before they can assist in the cases or perform on their own under the supervision of consultant surgeons. The viewing of the instructional videos help them understand the procedures better. The videos can also help them recognize the important steps and standardized safe approach. With the help of instructional video, they can not only assist better in the surgery but also reduce the learning curve when they start performing the procedure themselves during their training period. This is the step by step instructional video of laparoscopic paraesophageal hernia repair with fundoplication for surgeons-in-training who are posted to our department.

Conclusion: The step by step instructional video on laparoscopic paraesophageal hernia repair with fundoplication can help the surgeons in training reduce their learning curve and improve their surgical skills so that they can perform the procedure safely.

\section{P624-UPPER GI-Reflux-Achalasia}

\section{LAPAROSCOPIC HELLER MYOTOMY WITH TOUPET FUNDOPLICATION IN A PATIENT WITH ESOPHAGEAL DISMOTILITY ASSOCIATED TO HIV}

\section{G. Arredondo ${ }^{1}$, H. Segura ${ }^{1}$, D. Aguirre $\mathrm{Mar}^{2}$, A. Franco ${ }^{2}$}

${ }^{1}$ General Surgery, Escuela de Medicina y Ciencias de la Salud del Tecnológico de Monterrey, MONTERREY, Mexico; ${ }^{2}$ General Surgery, Hospital San José TecSalud, MONTERREY, Mexico

The Human Immunodeficiency Virus (HIV) is a neurotropic virus. There have been reports of patients with HIV who have esophageal motility problems, sometimes associated with opportunistic infections. The absence of contractility is defined as a major motility disorder according to the Chicago v 3.0 classification, which is characterized by normal esophagogastric union relaxation and $100 \%$ peristalsis failure.

We present the case of a 56-year-old male patient with a history of acquired immunodeficiency on treatment with efavirenz, emtricitabine and tenofovir. He presented progressive dysphagia, gastroesophageal reflux and pyrosis of 4 months of evolution. Physical examination showed no alterations. Upper endoscopy is done reporting a normal esophagus and diffuse chronic gastritis. The esophagogram reported inadequate esophageal motility with contrast stasis and a delayed emptying. The esophageal manometry reported an upper esophageal sphincter with high resting pressure. The middle and distal esophagus showed absence of peristalsis with a pan-esophageal pressurization pattern. The lower esophageal sphincter presented normal resting pressure and borderline relaxation $(41 \%)$. The integrated relaxation pressure was less than $15 \mathrm{mmHg}$. The diagnostic impression was absence of contractility (Chicago Classification v 3.0).Medical management was initiated with inhibitors of the proton pump, isosorbide dinitrate and injections of botulinum toxin without success. It was decided to program the patient for a Heller myotomy with Toupet fundoplication. A trans-surgical endoscopy revealed a complete myotomy with no leakage or obstruction. The patient went home on the second postoperative day tolerating a solid diet.Heller myotomy by laparoscopy with partial fundoplication is safe in the treatment of patients with HIV and esophageal motility disorders, reporting a mortality of $0.1 \%$. The effect of endoscopic treatments prior to surgery is controversy

\section{P625-UPPER GI-Reflux-Achalasia}

\section{LAPAROSCOPIC DIVERTICULECTOMY, HELLER MYOMECTOMY AND DOR FUNDOPLICATION FOR ACHALASIA AND SECONDARY EPIPHRENIC DIVERTICULUM}

M. Toledano-Trincado ${ }^{1}$, M. Bailon-Cuadrado ${ }^{1}$, J. Sanchez-Gonzalez ${ }^{1}$, M.L. Martin-Esteban ${ }^{2}$, P. Concejo-Cutoli ${ }^{2}$, J.R. Gomez-Lopez ${ }^{2}$, M.A. Montenegro-Martin ${ }^{2}$, M.A. Cuesta-Valentin ${ }^{3}$, J.C. Martin-del Olmo ${ }^{2}$, D. Pacheco-Sanchez ${ }^{1}$

${ }^{1}$ General and Digestive Surgery, Rio Hortega University Hospital, VALLADOLID, Spain; ${ }^{2}$ General and Digestive Surgery, Hospital of Medina del Campo, MEDINA DEL CAMPO, Spain; ${ }^{3}$ General and Digestive Surgery, Amsterdam University Medical Center Amsterdam, AMSTERDAM, The Netherlands

Aims: Epiphrenic diverticulum represents an infrequent entity and it is usually associated with esophageal motility disorders, such as achalasia, distal esophageal spasm, nutcracker esophagus or hypertensive lower esophageal sphincter. Nowadays, epiphrenic diverticulectomy, esophageal myotomy and partial fundoplication is the gold standard technique; although it supposes a challenging procedure and it may provoke lots of complications. Approach for diverticulectomy usually depends on the distance from the upper border of the diverticulum's neck to gastroesophageal junction, considering that thoracoscopy should be carried out when this distance is more than $5 \mathrm{~cm}$.

Methods: We present the case of a 57-year-old male patient, with a body mass index of 30 and a medical history of diabetes, smoking and alcoholism. His symptoms were mainly regurgitation and dysphagia. Upper endoscopy showed esophageal dilatation and the presence of a diverticulum with its neck $2 \mathrm{~cm}$ over the gastroesophageal junction. CT scan confirmed these findings and manometry showed achalasia. In the video we can see how we perform a laparoscopic diverticulectomy with esophageal myotomy and Dor fundoplication. Results: Patient was discharged home on the second postoperative day with no complication. After more than two years of follow-up, he has not suffered regurgitation, heartburn, dysphagia or chest pain.

Conclusions: We present a case with an epiphrenic diverticulum secondary to achalasia in which we performed a laparoscopic diverticulectomy, esophageal myotomy and Dor fundoplication. Some authors suggest that the correction of the underlying motility disorder is the key in the management of these patients and they do not recommend concomitant diverticulectomy for all cases. However, we consider that the complete procedure, adding diverticulectomy, supposes the gold standard and it is feasible to perform for teams which are skilled in esophageal and gastric laparoscopic surgery, despite its high morbidity rates. 


\section{P626-UPPER GI-Reflux-Achalasia}

\section{BALLOON DILATATION OF SPHINCTER ZONES OF THE ESOPHAGOGASTRODUODENAL REGION}

\section{O. Babii ${ }^{1}$, B. Shevchenko ${ }^{1}$, N. Prolom ${ }^{1}$, S. Tarabarov ${ }^{2}$}

${ }^{1}$ Digestive tract surgery department, SI Institute of Gastroenterology of NAMS of Ukraine, DNIPRO, Ukraine

${ }^{2}$ Department of minimal invasive endoscopic surgery and instrumental diagnostic, SI Institute of Gastroenterology of NAMS of Ukraine, DNIPRO, Ukraine

Objectives: In recent years, balloon dilatation (BD) for diseases requiring correction of the impaired patency of the sphincter zones of the esophagogastroduodenal region has become widespread.

Purpose: to assess the effectiveness of the use of the balloon dilatation in patients with impaired sphincter zones of the esophagogastroduodenal region.

Materials and methods: In the Institute department of surgery for the period from 2006 to 2018 , BD was performed in 245 patients. 210 of them diagnosed with achalasia of cardia (AC): 17-1 stage, $86-2$ stage, $62-3$ stage, $45-4$ stage. 7 patients diagnosed with pylorospasm, 7 patients had compensated stenosis and 21 patients had subcompensated ulcerative pyloroduodenal stenosis. There were 87 males, 158 females, average age ( $45.3 \pm 5.2)$.

BD was performed under endoscopic and / or x-ray control by 'Boston scientific' balloons with a diameter of 18-20 mm, $35 \mathrm{~mm}$ and $40 \mathrm{~mm}$, a course of 3-6 sessions with an interval of 1-3 days and a cylinder exposure of 3-6 min. Evaluation of BD was performed using esophagogastroscopy, balloon manometry and X-ray passage of barium.

Results: In the course of the study, the existing indications were refined and new indications were developed for performing an endoscopic BD in pyloroduodenal stenosis and in AC. In patients with stage 1-2 AC, a positive result was noted in $94.3 \%$ of cases already after the first session of BD. Recurrences of AC after BD for up to 5 years were established in 49 (23.3\%) patients: at stage $1-$ in $12.2 \%$, at stage 2 -in $16.3 \%$, at stage 3 -in $24.5 \%$ and at stage $4-$ in $47.0 \%$. Repeated BD courses in case of AC recurrence in $29(13.8 \%)$ cases turned out to be ineffective. Recurrence of pyloroduodenal subcompensated stenosis was diagnosed in $2.8 \%$ of cases in the period of 24 months after performing BD

Conclusions: BD is an effective method for correcting the permeability of the sphincter zones caused by the pathology of the esophagogastroduodenal region.

Keywords: balloon dilatation, achalasia of cardia, pylorospasm, ulcerative pyloroduodenal stenosis, recurrences.

\section{P627-UPPER GI-Reflux-Achalasia}

\section{LAPAROSCOPIC SURGERY REINTERVENTION OF ANTI- REFLUX SURGERY: A SYSTEMATIC REVIEW}

\section{J.A. Campos-Badillo ${ }^{1}$, K.D. Lopez-Olivera ${ }^{2}$}

${ }^{1}$ Surgery, Universidad Nacional Autónoma de México, EDO OF MEXICO, Mexico; ${ }^{2}$ Emergency Medicine, Universidad Nacional Autónoma de México, EDO OF MEXICO, Mexico

Introduction: The reoperation in antireflux surgery significantly increases morbidity and mortality up to $75-85 \%$, reaching rates of $42 \%$ in patients undergoing 3 or more surgeries. The advantages of laparoscopic surgery used in this surgical technique have amplified its acceptance and use, resembling its results in terms of feasibility, safety and efficacy of laparoscopic surgery to open surgery.Objective: :Evaluate the currently literature about antireflux surgery reintervention, focusing on the main indications of re-intervention, type of approach and morbidity and mortality of laparoscopic antireflux surgery.

Material and Methods: A literature search was conducted in two electronic databases, MEDLINE and EMBASE. The search was limited to the period 2009 to 2016. Terms were used in relation to the procedure or intervention and the underlying disease. We chose observational studies (cohort, cases and controls and series of cases), where the main indication for antireflux surgery would have been gastroesophageal reflux disease.

Results: A total of 19 studies were selected, most of them were case series (57.9\%), cohort studies (31\%) and case-control studies (10.5\%). A total of 1940 patients. The main indications were anatomical faults, of these failures, recurrent hiatus hernia and sliding occupy the highest percentage, while physiological failures, failure in esophageal and gastric motility occur more frequently. The main type of approach was laparoscopic in $85 \%$, the conversion rate was $5.3 \%$ and the open approach was reserved for complex cases with more than one re-intervention $12.9 \%$, for abdomen $8.6 \%$ and chest $3.5 \%$, this last for cases with high esophageal lesions that can not be repaired via trans-abdominal.The main complications were injuries to hollow viscera, such as: esophagus and stomach among others. These complications are related to the complexity of the procedure. Mortality has remained low up to $0.05 \%$, however, the cause of death was due to medical complications and not related to the procedure. Conclusions: This systematic review on reoperation in reflux surgery has confirmed that morbidity after reoperation surgery is higher than after primary surgery and reoperation indications increase with the use of new technologies (manometry) and the laparoscopic approach continues on the rise, with great adaptation to its use and improvement in results.

\section{P628-UPPER GI-Reflux-Achalasia}

\section{GIANT PARAESOPHAGEAL HERNIA: A HAZARDOUS SITUATION}

A.F. Aranzana Gómez, J. Malo Corral, J. Hernandez Gutierrez, P. Toral Guinea, R. Lopez Pardo, A. Trinidad Borras, G. Krasniki, A. Muñoz Tébar, B. Muñoz Jimenez, M.A. Morlan Lopez

\section{General Surgery, Complejo Hospitalario Toledo, TOLEDO, Spain}

Introduction: Currently, the laparoscopic approach is controversial for large hiatal hernia because thethe hiatal defect is greater, the complete resolution being complicated by the greater difficulty in the dissection andidentification of the structures and the tension-free closing of the pillars. In these cases, although the current trend isthe use of mesh-hiatoplasty or perform a Collis-Nissen, its use it is still very controversial since important complications due to migrationerosion have been published.

Objective: To demonstrate the efficacy of hiatorraphy without the use of meshes in thegiant paraesophageal hiatus hernia, as well as the STANDARDIZATION of our technique, with thetechnical steps that we make successively.

Material and method: Clinical cases: 68-year-old man,with symptomatic hiatal hernia with progressive intolerance and dysnea. EGD: the stomach rotated in a giant hiatal hernia.Gastroscopy not completed due to endoscope loop formation within giant hiatal hernia with gastric volvulation. CT: large hiatal hernia,combined volvulation (axial axial mesenteric organ), the stomach in a right subpulmonary situation.

Results: Intervention: Laparoscopic approach.HH of large paraesophageal size,double organoaxial-and-mesenteric volvular component,Gastric walls very thickened and adhered to the mediastinum Reduction of all content and the sac, is adhered to the pleura, EXTENDED MEDIASTINAL ESOPHAGEAL DISECTION, up to vein pulmonary and get enough abdominal esophagus and rule out the presence of an short esophagus,posterior-anterior and left tutorized MODIFIED HIATORRAPHY with stitches in "U" with non-absorbable suture on Teflon reinforcement patches.Nissen fixed to both pillars, intramediastinal drainage.EGD at the 1st day with esophageal stenosis due to inflamation of the Nissen, resolved with medical treatment. Dischage at $5^{\text {th }}$ day.Asymptomatic and without radiological recurrence after 10 months of follow-up. Conclusions: In giant and paraesophageal hiatus hernias, modified primary hiatorraphy together with mediastinal esophageal dissection extended can be an effective and safe alternative, and can be advised as a technical gesture prior to a Collis Nissen and-or placement of a hiatal-hiatoplasty mesh. The use of the meshes in the hiatusfor the treatment of large hiatal hernias should be individualized for each patient.STANDARDIZATION of the procedure facilitates the realization of the same increased safety and effectiveness,especially in cases of greater complexity.

\section{P629-UPPER GI-Reflux-Achalasia}

\section{OUTCOMES AFTER IMPLEMENTATION OF REDUCED OPIOID PRESCRIPTIONS FOR PAIN CONTROL POST LAPAROSCOPIC FOREGUT PROCEDURES}

A. Addo, A.S. Weltz, Z. Sanford, A. Broda, H.R. Zahiri, R. Lu, A. Park

General Surgery, Anne Arundel Medical Center, ANNAPOLIS, United States of America

Aim/Background: Prescribed opioids for pain control have been implicated as major contributors to addiction through their illicit use. Efforts to reduce opioid prescriptions and measure their impact on outcomes are novel. We analyzed how patient outcomes are affected with reduced opioid prescriptions following laparoscopic foregut surgery.

Methods: A prospective study was conducted of 139 patients who underwent laparoscopic Nissen fundoplication and hiatal hernia repair from January 2017 to June 2018. Eighty-five patients received standard postoperative pain prescriptions (NARCs); 54 received a 50\% reduction in opioid prescriptions (NARCr). Primary outcomes were 30-day postoperative number of calls requesting additional opioid prescriptions and patient satisfaction scores during this period. Secondary outcomes included perioperative complication rates, and quality of life (QOL) outcomes assessed utilizing four validated instruments: Reflux Symptom Index (RSI), Laryngopharyngeal Reflux QOL (LPR-QOL), Swallowing QOL (SWAL), and GERD-Health Related QOL (GERD-HRQOL) surveys.

Results: Groups were statistically similar in mean age, gender composition, BMI, ASA scores and comorbidities. Mean prescribed outpatient opioid medications for NARCs vs NARCr were 442 MME vs 263 MME, respectively $(\mathrm{p}<.05)$. There were no requests for additional opioid prescriptions 30 days following discharge for the NARCr group. The two groups were equivalent $(\mathrm{p}>.05)$ in perioperative outcomes including 30-day satisfaction rates (NARCs: $73 \%$ vs. NARCr: $89 \%$ ), length of hospital stay (NARCs: 1.60 days vs. NARCr: 1.62 days), 30-day readmission rates (NARCs: $1 \%$ vs. NARCr: $4 \%$ ) and perioperative complication rates. Additionally, no significant QOL outcome differences between the groups were reported at one month postoperatively.

Conclusion: Our study supports reducing opioid prescriptions as a strategy to counter illicit drug use and addiction, without significant impact on operative outcomes. 


\section{P630-UPPER GI-Reflux-Achalasia}

PARAESOPHAGEAL HERNIA REPAIR IN A KOREAN SINGLE

\section{S. Kim ${ }^{1}$, I.H. Jeong ${ }^{2}$} Jeju national university, JEJU, Korea

Purpose: Although paraesophageal hernias (PEH) occur frequently in the elderly, in Korea, a fundoplication for PEH was performed infrequently. The aim of this study is to analyze our experience with fundoplication for $\mathrm{PEH}$. hernia repair at a tertiary referral center were analyzed retrospectively. Demographic data, ASA classification, characteristics of PEH, onset of symptom, dysphagia severity score, characteristics of fundoplication (partial vs. total; laparotomy vs. laparoscopy; emergency vs. elective) and surgical outcome (length of stay, complication and 30-day mortality) were recorded and reviewed.

Results: 50 patients were included; $88 \%$ were female (Mean age of 76.8 years old and mean body mass index of 21.5). Mean onset of symptom was 2.0 weeks. Type 3 PEH was the most

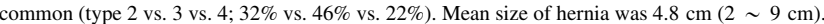
ASA classification was 2.1 (55\% of patients had an ASA 2). $80 \%$ patients underwent laparoscopic fundoplication (partial vs. total; 28 vs. 22). The mean length of stay and Follow-up were 10.0 $(4 \sim 28)$ days and $7(0 \sim 36)$ months respectively. Postoperative complications were $20 \%(10 /$ 50). One mortality happened after fundoplication (due to myocardiac infarction). After PEH repair, dysphagia severity scores were changed 1.8 from 3.7 .

Conclusion: In our series, the dysphagia severity scores reduced after surgery. Although PEH occurred in the elderly, a fundoplication was feasible and acceptable.Keywords : Hiatal hernia; Paraesophageal hernia; Fundoplication CENTER

${ }^{1}$ Surgery, Chosun university hospital, GWANGJU, Korea; ${ }^{2}$ Surgery,

Methods: Beetween April 2008 and October 2018, 50 patients who underwent paraesophageal

\section{P632-UPPER GI-Reflux-Achalasia}

THE NEW SIMPLE DEVICE OF A PUNCTURED STENT SLIDING METHOD IN THE HYBRID LAPAROSCOPIC PANCREATICO-DUODENECTOMY

Y. Sato, T. Matsumura, A. Obana, K. Kitamura, N. Koide, M. Koyama, K. Karikomi, T. Suwa

Surgery, Kashiwa Kousei General Hospital, KASHIWA, Japan

Background: The most critical obstacle is a pancreatic leakage(PL). The most cause of PL might be an activation of pancreatic juice by the mixing of pancreatic juice and intestinal fluid because of the anastomosis technique, the difference of anastomosis between pancreatic duct and caliber of jejunum, and the topple of jejunal mucosa.

Aim: In this study, we devised the new anastomotic method of pancreato-jejunostomy, so called Pancreatic Stent Sliding Guide ' (PSSG) method using a pancreatic duct stent. We would like to demonstrate its method and results. (Operative procedure) The 10cases of hybrid laparoscopic pancreatico-duodenectomies (PD) were done by shuriken-shaped umbilicoplasty with PSSG. The pancreatic duct stent, which is fit for a diameter of pancreatic duct, is used for the direct puncture without any incineration. The aims of direct puncture are both the avoidance of the enlargemen of anastomotic opening and disturbance of blood flow. The contralateral of anastomotic opening is also punctured and the stent is pulled out of the jejunum. The 6-0 PDS with the needles at both ends is used for anastomotic thread. Firstly, the eversion anastomosis of posterior wall is done by sliding the needle on the stent. And then the anastomosis of anterior wall is done by the same way. The stent of contralateral side is cut and the hole is closed.

Materials and methods: The 10 cased of pancreato-jejunostomy by PSSG method were done by February 2019. The average of patient's age was 72 y.o. The disease of patients were pancreatic cancer $(n=4)$, bile duct cancer $(n=5)$, and Papilla vater cancer $(n=1)$. The pancreatic leakage by the ISGPF were Grade 0:10,A:0,B:0,C:0 respectively. In the same periods, we underwent the more ten cases of open PD by PSSG method.

The PL were only one case of grade A and there were none of clinical PL. Conclusion: Our new device of pancreato-jejunostomy by PSSG might be very effective for the decrease of PL from the view point of machanisms of PL even for laparoscopic PD.

\section{P633-UPPER GI-Benign Esophageal disorders} ROBOTIC-ASSISTED PARTIAL GASTRECTOMY WITH
INTRACORPOREAL BILLROTH II GASTOENTEROSTOMY

\section{PROPHYLACTIC ANTI-REFLUX SURGERY AFTER} LAPAROSCOPIC GASTRIC WEDGE RESECTIONS FOR GASTRIC SUBMUCOSAL TUMORS OF GASTROESOPHAGEAL JUNCTION

\section{C.H. Kim}

Upper GI surgery, The catholic University of Korea, Incheon St. Mary's hospital, INCHEON CITY, Korea

Purpose: A laparoscopic wedge resection for a gastric submucosal tumor closed to gastroesophageal junction or involved to gastroesophageal junction is technically challenging and more aggressive compared with tumors in other sites of the stomach. A gastroesophageal reflux disease would be more prevalent after laparoscopic wedge resection of a gastric submucosal tumor in gastroesophageal junction because of the destruction to low esophageal sphincter. We hypothesized that a prophylactic anti-reflux surgery after this surgery would be less prevalent the gastroesophageal reflux disease (GERD) and more improve the quality of life of the patients. The aim of this study is to analyze our experience with prophylactic anti-reflux surgery after laparoscopic wedge resection for a gastric submucosal tumor of gastroesophageal junction Materials and methods: We retrospectively collected data from 51 patients who diagnosed with submucosal tumor of near the gastroesophageal junction underwent laparoscopic wedge resection between January 2000 and December 2017. The patients were divided into 2 groups according to operation with prophylactic anti-reflux surgery (group A) and without one (group B).

Results: There were no difference in the frequency of the preoperative GERD symptoms between the 2 groups, whereas postoperative GERD symptoms and postoperative use of acid suppressive medications were more frequent in the group B $(p=0.032, p=0.036)$. However, there were no differences in the follow-up endoscopic findings in terms of reflux esophagitis and Hill's grade between the 2 groups. In group A, postoperative mean low esophageal sphincter (LES) pressure was $22.0 \pm 13.0$. The LES pressure was dropped until $15 \mathrm{mmHg}$ in the only one patient. However, there was no reflux symptom in this patient.

Conclusions: The prophylactic anti-reflux surgery after laparoscopic gastric wedge resection of gastroesophageal junction is an effective method of prevent gastroesophageal reflux symptoms. Key words: Gastric wedge resection, Esophagogastric Junction, Gastroesophageal reflux

\section{E. Kakiashvili ${ }^{1}$, E. Brauner ${ }^{2}$}

${ }^{1}$ General Surgery, Galilee Medical Center, KIRIAT MOZKIN, Israel; ${ }^{2}$ General Surgery, Rambam Medical Center, HAIFA, Israel

65 year old, male patient presented with upper abdominal discomfort and pain, without nausea, vomiting or weight loss.

An sub mucosal lesion was found on endoscopy examination in first part of the duodenum. Endoscopic ultrasound has showed $2.5 \mathrm{~cm}$ sub mucosal lesion in first part of duodenum (anterio wall and close to pylorus). Cytology examination from the lesion has showed neuroendocrine tumor.

Computed tomography of abdomen and chest were normal.

His blood laboratory examinations were within normal limits.

Patient underwent da Vinci robotic partial gastrectomy with intra corporeal Billroth II gastrojejunostomy.

Total operating time (ORT) was $255 \mathrm{~min}$. Three day after operation patient started regular diet and was discharged home on day fife.

Final pathology report confirmed diagnosis of Carcinoid tumor with Ki67 less than 1\%. 


\section{P634-UPPER GI-Gastric cancer}

ROBOTIC-ASSISTED PARTIAL GASTRECTOMY WITH MODIFIED D2 LYMPHADENECTOMY AND BILROTH II GASTROENTEROSTOMY

\section{E. Kakiashvili ${ }^{1}$, E. Brauner ${ }^{2}$}

${ }^{1}$ General Surgery, Galilee Medical Center, KIRIAT MOZKIN, Israel; ${ }^{2}$ General Surgery, Rambam Medical Center, HAIFA, Israel

58 year old, male patient presented with upper abdominal pain, nausea, vomiting (on an of) and anemia, started two month before admission.

An ulcerative, obstructive lesion was found on endoscopy examination (close to the pylorus). Biopsy from the mass has showed poorly differentiated signet ring cell adenocarcinoma.

Chest computed tomography revealed $38 \mathrm{~mm}$ thoracic aortic aneurism. Abdominal computed tomography showed $43 \mathrm{~mm}$ infra renal aortic aneurism and no evidence of metastatic disease. His blood laboratory examinations showed $\mathrm{Hgb}-9.3$. Serum CEA level was normal.

Patient underwent da Vinci robotic-assisted Partial Gastrectomy with modified D2 lymphadenectomy and Billroth II gastrojejunostomy.

Total operating time (ORT) was $222 \mathrm{~min}$. Three day after operation patient started regular diet and was discharged home on day four.

Final pathology result confirmed poorly differentiated adenocarcinoma with signet ring cells.

\section{P635-UPPER GI-Gastric cancer}

LAPAROSCOPIC PARTIAL GASTRECTOMY WITH MODIFIED

\section{D2 LYMPHADENECTOMY AND BILLROTH II}

\section{GASTROJEJUNOSTOMY}

\section{E. Kakiashvili ${ }^{1}$, E. Brauner ${ }^{2}$}

${ }^{1}$ General Surgery, Galilee Medical Center, KIRIAT MOZKIN, Israel; ${ }^{2}$ General Surgery, Rambam Medical Center, HAIFA, Israel

70 year old, female patient presented with upper abdominal pain, weight loss (10 kg during last three month), without nausea or vomiting.

An ulcerative lesion was found on gastroscopy examination in antrum (on the grate curvature) Biopsy was done and pathology result showed Intestinal type, HER2-negative adenocarcinoma of the stomach.

Chest and abdominal computed tomography (CT) were normal. Endoscopic ultrasound (EUS) revealed $3 \mathrm{~cm}$ lesion with invasion to the muscularis propria (MP), without enlargement of regional lymph nodes (T3NOM0).

Her blood laboratory examinations were within normal limits including serum CEA.

She was treated by neo adjuvant chemotherapy ( 3 cycles Carboplatine $+5 \mathrm{FU})$.

Patient underwent laparoscopic Partial Gastrectomy with modified D2 lymphadenectomy and Billroth II gastrojejunostomy.

Total operating time (ORT) was $320 \mathrm{~min}$. Three day after operation patient started regular diet and was discharged home on day fife.

Final pathology result confirmed intestinal type, modified differentiated adenocarcinoma of the stomach.

\section{P636-HEPATO-BILIAIRY \& PANCREAS-Gallbladder}

\section{MODIFIED ERAS PROTOCOL IN PATIENTS WITH ACUTE CHOLECYSTITIS}

\author{
M. Terzopoulou, K. Alexiou, D. Bethani, N. Economou
}

1st Surgical Department, Sismanoglion G.H.A., ATHENS, Greece

Aims: ERAS protocol is not commonly used in acute emergency procedures. Elective lc is commonly performed as one day surgery, while in an emergency setting of acute cholecystitis, the in hospital stay averages 4,5 days. The aim of this trial is the application of ERAS protocol in patients with acute cholecystitis, undergoing laparoscopic cholecystectomy.

Methods: A randomized prospective trial was conducted in first surgical department of Sismanogleion G.H.A. The study included 96 patients, who were admitted with acute cholecystitis and underwent lc into $24 \mathrm{~h}$ from their admission. Preoperatively, they all received crystalloid isotonic solutions and antibiotics. $5.3 \%$ were submitted to ERCP, preoperatively, due to choledocholithiasis. The postoperative care included early mobilization into $2 \mathrm{~h}$ after surgery, early fluid intake (into $4 \mathrm{~h}$ ) and early liquid food intake (into $6 \mathrm{~h}$ ). They all received systematically antibiotics, analgesics and antiemetic on demand. ASA score was not an exclusion criterion. Results: Conversion to open procedure was necessary in $6.5 \%$ of patients, whom were excluded from the study. All the rest were discharged into $24 \mathrm{~h}$ from the surgery with the guidance to receive oral antibiotics for 3 more days. Readmission was necessary for 2 patients, one week after the operation. The first one presented with bile leak and submitted to ERCP with stent placemen and percutaneous drainage of the intrabdominal collection. The second one presented with choledocholithiasis and underwent ERCP with balloon catheterization.

Conclusion: It is commonly accepted that ERAS protocol in elective procedure enhances the postoperative recovery while reduces the in hospital stay and cost. In emergency condition ERAS cannot be applicated preoperatively. However, a modified post surgery application seems to have advantages equal to those observed in elective procedures.

\section{P638-HEPATO-BILIAIRY \& PANCREAS-Pancreas}

\section{ELECTIVE LAPAROSCOPIC CHOLECYSTECTOMY WITH AND WITHOUT CHEMOPROPHYLAXIS: A PROSPECTIVE RANDOMIZED TRIAL}

D. Bethani, K. Alexiou, M. Terzopoulou, N. Economou

1st Surgical Department, Sismanoglion G.H.A., ATHENS, Greece

Aim: Laparoscopic cholecystectomy is the gold standard for the treatment of symptomatic cholelithiasis. Administration of one single dose of chemoprophylaxis before an elective laparoscopic cholecystectomy is a broadly accepted practice. However, its value is currently questioned, especially in low risk patients.

Method: This study was conducted in a high volume surgical department. One hundred and twelve patients submitted to elective laparoscopic cholecystectomy were included in this research. A written consent was acquired after thorough patient briefing. Half the patients that underwent surgical operation received one dose of antibiotics $30 \mathrm{~min}$ prior to the incision and the other half did not receive any chemoprophylaxis.

Results: The age ranges from 16 to 81 years old. Commonest concomitant diseases were arterial hypertension, type 11 diabetes, hypothyroidism and respiratory deficiency. Approximately $30 \%$ of patients were smokers and $11 \%$ were obese (BMI $>30$ ). The duration of the operations was between 20 and $85 \mathrm{~min}$. Intra-operative gallbladder rupture was observed in 36 patients (rate $32 \%$ ). All the patients were discharged the first post-operative day and their monitoring continued for 30 more days. In the chemoprophylaxis group, no surgical site infection or other major complication was observed. From the group that did not receive any antibiotics, one patien developed surgical site infection and specifically infection of the surgical port in the epigastrium, which was treated with drainage of the abscess and oral antibiotics administration. No other complications were recorded.

Conclusion: Our study concluded no statistically significant difference between the two patient groups, which depicts that chemoprophylaxis may not be necessary in elective cholecystectomy operations. On the contrary, antibiotics increase the cost of hospital stay and are often accompanied by multiple mild or severe side effects.

Publisher's Note Springer Nature remains neutral with regard to jurisdictional claims in published maps and institutional affiliations. 